\title{
Evolutionary dynamics of spliceosomal intron revealed by in silico analyses of the P-Type ATPase superfamily genes
}

Toshiyuki Oda ${ }^{1}$, Ryosuke L. Ohniwa ${ }^{2}$, Yuki Suzuki ${ }^{1}$, Masatsugu Denawa ${ }^{1}$, Masahiro Kumeta ${ }^{1}$, Hideyuki Okamura ${ }^{3}$ and Kunio Takeyasu ${ }^{1}$

\author{
${ }^{1}$ Laboratory of Plasma Membrane and Nuclear Signaling, Kyoto University Graduate School of Biostudies, Yoshida-Konoe- \\ cho, Sakyo-ku, Kyoto 606-8501, Japan \\ ${ }^{2}$ Institute of Basic Medical Sciences, Graduate School of Comprehensive Human Sciences, University of Tsukuba, Tennoh- \\ dai, Tsukuba, Ibaragi 305-8575, Japan \\ ${ }^{3}$ Department of Biology, Osaka Dental University, Kuzuhahanazono-cho 8-1, Hirakata, Osaka 573-1121, Japan
}

\section{Corresponding author}

Toshiyuki Oda

Laboratory of Plasma Membrane and Nuclear Signaling, Graduate School of Biostudies, Kyoto University, Yoshida-Konoecho, Sakyo-ku, Kyoto 606-8501, Japan

Phone \& Fax: +81-75-753-7905

E-mail: toda.m08@lif.kyoto-u.ac.jp

\begin{abstract}
It has been long debated whether spliceosomal introns originated in the common ancestor of eukaryotes and prokaryotes. In this study, we tested the possibility that extant introns were inherited from the common ancestor of eukaryotes and prokaryotes using in silico simulation. We first identified 21 intron positions that are shared among different families of the P-Type ATPase superfamily, some of which are known to have diverged before the separation of prokaryotes and eukaryotes. Theoretical estimates of the expected number of intron positions shared by different genes suggest that the introns at those 21 positions were inserted independently. There seems to be no intron that arose from before the diversification of the PType ATPase superfamily. Namely, the present introns were inserted after the separation of eukaryotes and prokaryotes.
\end{abstract}




\section{Keywords}

Spliceosomal intron $\cdot$ Introns -late theory $\cdot$ Introns -early theory $\cdot$ Simulation $\cdot$ Proto -splice site $\cdot \mathrm{P}$-Type ATPase 


\section{Introduction}

Eukaryotic genes often contain non-coding regions called introns that interrupt the protein-coding regions of these genes. Introns are transcribed along with the coding regions into nuclear pre-mRNA and spliced out by the spliceosome, a eukaryota-specific component, during mRNA maturation. These introns are called spliceosomal introns.

Spliceosomal introns are often found at the same positions in homologous genes and even in ancient genes that diverged before the separation of prokaryotes and eukaryotes [1,2]. Therefore, some researchers believe that these introns existed in the common ancestor to prokaryotes and eukaryotes before the divergence. On the other hand, it is known that insertion and deletion of spliceosomal introns occurs frequently [3,4], and, therefore, it is possible that these introns were inserted independently at identical positions in ancient homologous genes after the separation of prokaryotes and eukaryotes [5]. The origin of these introns has long been controversial and is referred to as the "introns-late" versus "introns-early" debate [6].

Several researchers have independently attempted, by statistical approaches, to identify the origin of introns in genes that diverged before and after the separation of prokaryotes and eukaryotes, but their conclusions are inconsistent $[1,5,7,8]$. These contradictory results may be due to the researchers' distinct assumptions of the positions that allow intron insertion in genes. Iwabe et al. and Kersanach et al. assumed all the nucleotide-nucleotide junctions allow intron insertion [1,8], and their results supported "introns-early" theory. In contrast, "introns-late” theory was supported by Sverdlov et al. , who estimated only "proto-splice sites" are available for intron insertion [7]. Thus, the researchers who set relatively many positions for intron insertion in genes reached "introns-early" theory, and the researchers who set a few positions proposed “introns-late" theory.

In this study, we focused on the P-Type ATPase superfamily, one of the largest gene superfamilies found in both prokaryotes and eukaryotes. This superfamily consists of 5 families. Family 1 comprise the $\mathrm{K}^{+}$and heavy metal ion transporters, Family 2 the $\mathrm{Ca}^{2+}$ ion transporters, Family 3 the $\mathrm{H}^{+}$transporters, Family 4 the lipid translocators, and Family 5 comprise unknown substrates. All the families are found in eukaryotes, and Families 1-3 are also found in prokaryotes [9,10]. Therefore, Families 1-3 are believed to have diverged before the separation of prokaryotes and eukaryotes. Furthermore, although the amino acid sequences share only 20\% identity, the protein structures of all P-Type ATPases are basically similar. Nevertheless, eukaryotic P-Type ATPase genes contain many spliceosomal introns, and some of which exist at the same positions in homologous genes diverged before the separation of prokaryotes and eukaryotes. Now, we can obtain over 100 genes in P-Type ATPase from the gene database. Taking advantage of this large superfamily, we addressed the question about "introns-early" vs "introns-late" theory by our newly established method which enables to eliminate the 
unknown variables such as number of positions in the estimation of introns insertion. 


\section{Materials and Methods}

\section{P-type ATPase superfamily}

Gene sequences of the P-Type ATPase superfamily were obtained by database search in GeneBank [11]. The search was conducted in the genomes of 7 animals, 2 plants, 4 fungi, and 5 protists in Dec. 2007. It covered all the eukaryotic organisms whose complete or draft genomes were available at that time. All the P-Type ATPase genes of those organisms referred as RefSeq [12] were collected, except the genes which had stop codons in its coding regions or lacked extremely large regions because these were probably the pseudo genes.

\section{Intron Mapping}

The gene sequences were aligned based on their translated amino acid sequences by MAFFT [13], and the alignment was manually modified. Sequences were reverse-translated into nucleotide sequences, and introns were mapped on the alignment.

Note that we applied two different rules, strict and generous rules, for selecting intron positions. Under the strict rule, intron positions in well-aligned regions without any gaps were selected (see Supplemental Data 1). Under the generous rule, all the positions, except the gap-rich regions where intron positions were hardly comparable, were selected.

\section{Genetic Distance}

Genetic distance is the phylogenetic distance within a pair of genes calculated by the PROTDIST program in the PHYLIP package [14]. Jones-Taylor-Thornton matrix was used as the scoring matrix.

\section{Index to evaluate the degree of intron inheritance}

If introns are independently inserted into two distinct genes, the probability for their insertion at identical positions is calculated as

$$
\operatorname{prob}_{A B(i)}=\left({ }_{I_{a}} C_{i} \times_{\left(I_{A B_{-} a l l}-I_{a}\right)} C_{\left(I_{b}-i\right)}\right) /_{I_{A B_{-} a l l}} C_{I_{b}} \quad \text { Eq (1) }
$$

where $\operatorname{prob}_{A B(i)}$ is the probability that intron position $i$ is common to genes $\mathrm{A}$ and $\mathrm{B}, I_{a}$ and $I_{b}$ are the numbers of introns in 
genes $\mathrm{A}$ and $\mathrm{B}$, respectively, and $I_{A B_{a} a l}$ is the number of the nucleotide-nucleotide junctions allowing intron insertion in genes A and B. In this formula, all nucleotide-nucleotide junctions are assumed to allow the intron insertion with the same probability.

The expected value for the number of intron positions shared by genes $\mathrm{A}$ and $\mathrm{B}\left(E_{A B}\right)$ is calculated with the following formula.

$E_{A} \quad \bar{B} \sum_{i=1}^{i \leq I_{b}}\left(i \times p \quad r_{A} \varphi_{B}\right)$

Here, we introduce an index, Ratio $(R)$, to evaluate the degree of intron inheritance from their ancestor in a pair of genes. $R$ for gene pair $\mathrm{A}$ and $\mathrm{B}$ is calculated as

$R_{A B}=O_{A B} / E_{A \text { I }}$ Eq (3)

where $O_{A B}$ is the actual number of intron positions shared by gene pair A and B.

\section{Tree-based weight}

Due to the inclusion of highly homologous sequences such as isoforms and paralogs in the sequence population, it is erroneous to treat all of the sequences equally [15]. In this study, we introduced tree-based weight (Fig. 1).

Weight was assigned based on branch length of phylogenetic trees of each family, using kdpB, P-Type ATPase of Escherichia coli (NP_415225) as an outgroup. The trees are made by the NEIGHBOR program in the PHYLIP package [14]. Negative branch lengths were set to zero. This method is the same as the method used in CLUSTAL W [16] with the single modification of excluding a normalization step in order to reduce round-off errors.

The tree-based weight of the gene pair A and B is given by

$W_{A B}=\left(V_{A} \times V_{B}\right)^{1 / 2}$

where $V_{A}$ and $V_{B}$ are the tree-based weights of genes $\mathrm{A}$ and $\mathrm{B}$, respectively. 


\section{Simulation test of the $R$ values}

One 3522 bp (average length of P-type ATPase genes analyzed in this study) gene with 16 introns (average number of introns present in the P-type ATPase genes) was artificially constructed as an ancestral gene for in silico simulation. The genes were repeatedly duplicated in silico with the mutation rate per nucleotide at 0.001 , and the $R$ values were calculated under different conditions considering the rate of gain and loss of introns and the position preference of intron insertion. The mutation rate was arbitrarily selected because this rate itself never affected the convergence of the $R$ values (data not shown). The detailed procedures are as following.

i) Effect of the rates of gain and loss of introns

After the duplication of the gene, introns were removed on the rates of intron loss (High $=0.6$, Middle $=0.4$, Low $=$ 0.3 per intron), and new introns were inserted on the rates of intron gain $($ High $=0.006$, Middle $=0.004$, Low $=0.003$ per nucleotide-nucleotide junction). Position preference was not considered (all the nucleotide-nucleotide junctions allow the intron insertion with the same probability). These steps were repeated 8 times and 256 genes with different number of introns were obtained. Then, $R$ values were calculated in each case.

ii) Effect of position preferences for intron insertion

After the duplication of the gene, introns were removed on the rates of intron loss ( 0.4 per intron). Then, new introns were inserted into duplicated genes on the rates of intron gain (0.004 per nucleotide-nucleotide junction). In this step, the positions of intron insertion were restricted to $25 \%, 50 \%$ and $100 \%$ of the nucleotide-nucleotide junctions. These steps were repeated 8 times and 256 genes with different number of introns were obtained. Then, $R$ values were calculated in each case. 


\section{Results and Discussions}

We collected sequences of 354 eukaryotic genes encoding P-Type ATPases by database search, and identified the positions of all introns in those 354 genes (Table 1). We also investigated introns in prokaryotic P-Type ATPase gene and found no introns. Under the strict rule (using well-aligned region among P-Type ATPases. See Intron Mapping section in Materials and Methods), there were 949 introns mapped into 111 positions and under the generous rule (using whole regions except for gap-rich regions in the alignment), there were 4,120 introns mapped into 631 positions (Supplemental Table 1). The 949 introns under the strict rule are a subset of the 4,120 introns under the generous rule. Among the 111 and 631 intron positions identified, 21 and 67 positions were shared by at least two different families, respectively (Fig. 2, Table 2). The numbers 21 and 67 are larger than those reported in similar analyses [1,5,17].

In the case that introns are independently inserted into two different genes, the expected value for "the number of intron positions shared by two genes” can be estimated by Eq (2). If the number of actual intron positions shared by two genes is larger than this expected value, it is reasonable to judge that one or more of the introns have been inherited from a common ancestor (in this paper, we call such introns as "inherited introns”). Therefore, we introduced an index, Ratio (R) (Eq (3)), to evaluate whether the introns are likely to have been inherited from a common ancestor or to have been inserted independently. Homologous genes that have diverged recently, such as isoforms, are expected to have high $R$ values because almost all the introns will be conserved. In contrast, the $R$ values for homologous genes that diverged early in the evolution likely to be small, because many insertion/deletion events are thought to have occurred since their divergence. Most of the introns in distant homologues likely to have been inserted after divergence, and, therefore, the $O_{A B}$ values are expected to be close to the $E_{A B}$ values.

The $R$ values of all gene pairs (354x353/2=62,481 pairs) were calculated except for 30,096 pairs under the strict rule and 6,688 pairs under the generous rule. To analyze the ancestry of inherited introns, the $R$ values were plotted against the genetic distance of each pair, where genetic distance was used to represent the relative time since divergence (Fig. 3a, 3b). The majority of $R$ values were almost zero, while some pairs had relatively high $R$ values. Since $O$ is the actual value in Eq (3) and there will be variation in the $O$ value, resulting in scattering in the distribution of $R$ values. For example, if genes A and B independently gain 6 and 4 introns, respectively, the possible number of the intron positions shared by the two genes varies from 0 to 4 . In this case, there is a high probability of an $R$ value of 0 and a very low probability that the $R$ value will be extremely high (Table 3). Thus, the relatively high $R$ values in Fig. 3a and 3b may not due to the existence of inherited introns, but caused by the existence of introns independently inserted to the identical positions which will occur 
with small probability.

Therefore, we averaged the $R$ values at every genetic distance to assess the overall trends in $R$ dynamics (Fig. 3c, 3d, 3e, 3f). To avoid over-estimation of $R$ values associated with the presence of many closely related homologues like isoforms, we applied tree-based weights in the average calculation. The $R$ values of intra-family pairs showed a drastic decline with longer genetic distances. This result suggests that recently diverged pairs inherit most of the introns from a common ancestor and that the inherited introns were removed soon after the divergence. In the case of inter-family pairs, the $R$ value stays low and constant. This trend did not change when pair selection was restricted to the genes that had diverged before the separation of prokaryotes and eukaryotes (Fig. 3e, 3f). Since the $R$ value becomes small when one or both members of pair lose inherited introns, these results indicate that the deletion of inherited introns have occurred quickly under the assumption that new intron insertions/deletions occur continuously, and consequently the $R$ values become constant.

According to the definition of $R$, the $R$ value should stay as 1 if all the introns are inserted independently. Nevertheless, for most genetic distances, the $R$ values of inter-family pairs were greater than 1 (Fig. 3c, 3d, 3e, 3f). There are two possible explanations for such a result. One possibility is, of course, that inherited introns preferentially remain in the genes. In this case, particular pairs of inherited introns should remain regardless of the genetic distance. However, there is no such intron in the P-type ATPases identified (Supplemental Table 1), and, therefore, it is unlikely that introns inherited from an ancestral gene of each family are conserved.

There are several important factors for in silico intron analysis such as the rate of gain and loss of introns and the position preference of intron insertions $[17,18]$. Here, we simulated how the rate of gain and loss affects to the $R$ values with the set of hypothetical genes and introns (Figure 4a). While the rate affects the declining speed of the $R$ values, it never affects the convergence values $(R=1)$. Therefore, our observation that the $R$ values of P-Type ATPase stayed as over 1 were not be caused by the different rates of gain and loss of the introns.

In contrast, the position preference affected the convergence values of $R$ (Fig. 4b). The simulation showed that the $R$ values became larger than 1 if the positions of intron insertion were restricted. This is well consistent with our result that $R$ value of P-type ATPase stayed at over 1. Therefore, it is likely that there are particular positions that allow intron insertion more frequently than others in P-Type ATPase genes (insertion hot spots).

It has been reported that introns often found at the particular nucleotide sequence: A[A/C]AG![A/G]T [19-21] or !CTC [20] (! is the positions where introns are found). These sequences are believed to act as the "proto-splice site" and allow for easy intron insertion. In the case of the P-Type ATPase, the nucleotide preference at -4 to +4 positions before/after the introns are A[A/C]AG!GTT (Table 4), and are compatible to the reported sequences in other genes [19-21]. In addition, introns are often found in the phase 1 codon of conserved glycines [22]. The P-Type ATPase superfamily has 50 amino acids 
conserved in over $80 \%$ members (Table 5), and 11 out of 13 conserved glycine contain introns at Phase 1 . Since the glycine codon is GGN, it is reasonable that Phase 1 of glycine naturally constitutes proto-splice sites and potential intron insertion hot spots. Such a relationship between amino acid conservation and intron insertion is also observed with other amino acids, including lysine, which occupies 3 out of the 50 conserved positions in the P-Type ATPase superfamily. The lysine codons are AA[A/G], and phase 3 of all 3 lysine codons have been the target of intron insertion (Table 5). Thus, such conserved amino acids may provide the positions for intron insertion hot spots. Other factors, such as "domains / modules" of proteins $[2,23,24]$ and "phase" in the codons [25] have been reported to affect to the presence of introns. Those factors may also provide the positions for intron insertions hot spots and be responsible for high $R$ values.

\section{Concluding Remarks}

In conclusion, the introns found at the same positions among the inter-families pairs were not inherited from a common ancestor but were inserted independently after the divergence. Thus, the introns found in the genes belonging to the P-Type ATPase superfamily were independently acquired since the separation of prokaryotes and eukaryotes, supporting the "introns-late" theory. As shown in this study, particular sites on genes accumulate more introns than others. This bias may result in independently inserted introns appearing to be conserved since the separation of prokaryotes and eukaryotes. 


\section{References}

[1] Kersanach R, Brinkmann H, Liaud M, Zhang D, Martin W, Cerff R (1994) Five identical intron positions in ancient duplicated genes of eubacterial origin. Nature 367:387-389

[2] de Roos A (2007) Conserved intron positions in ancient protein modules. Biol Direct 2:7

[3] Rogozin I, Wolf Y, Sorokin A, Mirkin B, Koonin E (2003) Remarkable interkingdom conservation of intron positions and massive, lineage-specific intron loss and gain in eukaryotic evolution. Curr Biol 13:1512-1517

[4] Nguyen H, Yoshihama M, Kenmochi N (2005) New maximum likelihood estimators for eukaryotic intron evolution. PLoS Comput Biol 1:e79

[5] Rzhetsky A, Ayala F, Hsu L, Chang C, Yoshida A (1997) Exon/intron structure of aldehyde dehydrogenase genes supports the "introns-late" theory. Proc Natl Acad Sci U S A 94:6820-6825

[6] de Souza S (2003) The emergence of a synthetic theory of intron evolution. Genetica 118:117-121

[7] Sverdlov A, Csuros M, Rogozin I, Koonin E (2007) A glimpse of a putative pre-intron phase of eukaryotic evolution. Trends Genet 23:105-108

[8] Iwabe N, Kuma K, Kishino H, Hasegawa M, Miyata T (1990) Compartmentalized isozyme genes and the origin of introns. J Mol Evol 31:205-210

[9] Axelsen K, Palmgren M (1998) Evolution of substrate specificities in the P-type ATPase superfamily. J Mol Evol 46:84-101

[10] Okamura H, Denawa M, Ohniwa R, Takeyasu K (2003) P-type ATPase superfamily: evidence for critical roles for kingdom evolution. Ann N Y Acad Sci 986:219-223

[11] Benson D, Karsch-Mizrachi I, Lipman D, Ostell J, Wheeler D (2007) GenBank. Nucleic Acids Res 35:D21-25

[12] Pruitt K, Tatusova T, and Maglott D (2007) NCBI reference sequences (RefSeq): a curated non-redundant sequence database of genomes, transcripts and proteins. Nucleic Acids Res 35: D61-5.

[13] Katoh K, Misawa K, Kuma K, Miyata T (2002) MAFFT: a novel method for rapid multiple sequence alignment based on fast Fourier transform. Nucleic Acids Res 30:3059-3066

[14] Felsenstein J (1989) PHYLIP - Phylogeny Inference Package (Version 3.2). Cladistics 5, 164-166.

[15] Vingron M, Sibbald P (1993) Weighting in sequence space: a comparison of methods in terms of generalized sequences. Proc Natl Acad Sci U S A 90:8777-8781

[16] Higgins D, Thompson J, Gibson T (1996) Using CLUSTAL for multiple sequence alignments. Methods Enzymol 266:383-402

[17] Cho G, Doolittle R (1997) Intron distribution in ancient paralogs supports random insertion and not random loss. J Mol Evol 44:573-584

[18] Roy S, Gilbert W (2005) Rates of intron loss and gain: implications for early eukaryotic evolution. Proc Natl Acad Sci U S A 102: 5773-8

[19] Dibb N, Newman A (1989) Evidence that introns arose at proto-splice sites. EMBO J 8:2015-2021

[20] Tomita M, Shimizu N, Brutlag D (1996) Introns and reading frames: correlation between splicing sites and their codon positions. Mol Biol Evol 13:1219-1223

[21] Long M, de Souza S, Rosenberg C, Gilbert W (1998) Relationship between "proto-splice sites" and intron phases: evidence from dicodon analysis. Proc Natl Acad Sci U S A 95:219-223

[22] Endo T, Fedorov A, de Souza S, Gilbert W (2002) Do introns favor or avoid regions of amino acid conservation? Mol Biol Evol 19:521-252

[23] Roy S, Nosaka M, de Souza S, Gilbert W (1999) Centripetal modules and ancient introns. Gene 238:85-91

[24] Kaessmann H, Zollner S, Nekrutenko A, Li W (2002) Signatures of domain shuffling in the human genome. Genome Res 12:1642-1650

[25] Long M, Deutsch M (1999) Association of intron phases with conservation at splice site sequences and evolution of spliceosomal introns. Mol Biol Evol 16:1528-1534 
Fig. 1. Scheme of tree-based weights

g1, g2 and g3 represent genes, and b1, b2, b3 and b4 represent provisional branch names. Values in parentheses indicate the branch lengths. The tree-based weight is the summation of the weight of each branch, which is calculated as (length of the branch)/(number of genes which belong to the branch).

For the representative tree shown here, the tree-based weight of g3 is estimated as (length of b2)/2 + (length of b4) $=1.5 / 2+$ $0.5=1.25$; likewise, that of $\mathrm{g} 1$ is 2.0 .

Fig. 2. An example of the intron positions in the alignment of P-Type ATPase.

The first three letters of the gene name represent the species name (osa; Oryza sativa, rno; Rattus norvegicus, ani; Aspergillus nidulans, hsa; Homo sapiens, ath; Arabidopsis thaliana, dre; Danio rerio, gga; Gallus gallus, cne; Cryptococcus neoformans). Roman numbers of the gene name indicate the family of P-type ATPase to which the genes belong to. (I; Family 1, II; Family 2, III; Family 3, VI; Family 4, V; Family 5). The genes shown here are osa_I (NP_001058417.1), rno_I (NP_036643.1), ani_II (EAA64748.1), hsa_II (NP_001001396.1), ath_III (NP_180028.1), osa_III (NP_001067382.1), ani_IV (EAA58087.1), dre_IV (XP_693773.2), gga_V (NP_001026485.1), and cne_V (XP_570514.1). The BLOSUM 62 score table was used for the shading with GeneDoc package (http://www.nrbsc.org/gfx/genedoc/). The arrows indicate intron positions identified by the strict rule. Colors of the arrows represent the phases of introns; white for phase 0 , grey for phase 1 and black for phase 2. Asterisks on the arrows indicate that the introns were conserved across different families.

Fig. 3. Correlation between genetic distance and the index, $R$.

a,b; $R$ of intra-family pairs (circles) and inter-family pairs (triangles) are plotted against their genetic distances. a represents the plots under the strict rule (intra-family pairs; $n=8,895$, inter-family pairs; $n=23,490$ ). b represents the plots under the generous rule(intra-family pairs; $\mathrm{n}=16,440$, inter-family pairs; $\mathrm{n}=39,353)$. c, d; Weighted means of $R$ of the intra-family pairs (circles) and the inter-family pairs (triangles), respectively, calculated at genetic distance intervals of 0.2. Error bars indicate \pm SE which represents the relative scattering of $R$. Magnifications of the line charts are shown in insets to clarify the trend of the $R$ of inter-family pairs. Weighted means of $R$ under the strict rule and under the generous rule are shown in c and $\mathrm{d}$, respectively. e, f; $R$ of the inter-family pairs were classified into two groups: pairs of genes which had diverged before the separation of prokaryotes and eukaryotes (pairs which members belong to Family 1-2, 2-3 or 1-3, point-down triangles) and the other inter-family pairs (diamonds). e represents $R$ under the strict rule (pairs diverged before the separation; $n=4,572$, other inter-family pairs; $\mathrm{n}=18,918$ ). f represents $R$ under the generous rule (pairs diverged before the separation; $\mathrm{n}=7,378$, other inter-family pairs; $n=31,975$ ).

Fig. 4. The effects of "rates of gain and loss of introns" and "position specific preference of intron insertion" on the $R$ values. a; Effects of rates of gain and loss of introns on $R$ values. The $R$ values of hypothetical genes and introns were simulated under the high (circle and times superimposed), middle (circle and plus superimposed) and low (square and plus superimposed) rates of loss and gain of introns. b; Effects of position specific preference of intron insertions on $R$ values. Introns were supposed to be inserted into all the nucleotide-nucleotide junctions (circle and plus superimposed), into $50 \%$ of nucleotide-nucleotide junctions (square and times superimposed) and into only $25 \%$ of nucleotide-nucleotide junctions (diamonds and plus superimposed).

Supplemental Data 1. Alignment of amino acids used in this study.

Amino acids given in upper-case letters were used under the strict rule, while under the generous rule, amino acids given in upper- and lower-case letters, except for " $x$ ", were used. The letter " $x$ " represents amino acids which were not used in any calculations.

Supplemental Table 1. Positions of introns found in the P-Type ATPase genes and the names of genes which have introns at these positions.

The first column indicates positions of nucleotides before the introns, and the second column indicates positions of nucleotides after the introns. The third and subsequent columns indicate the names of genes. 


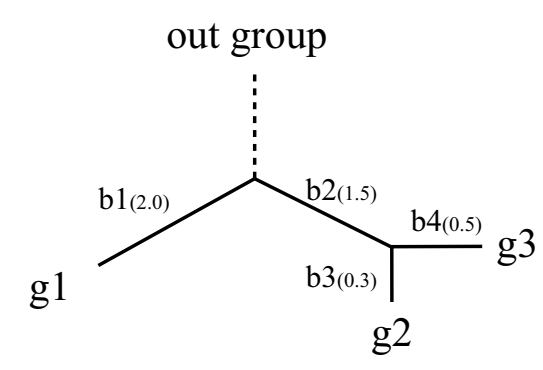



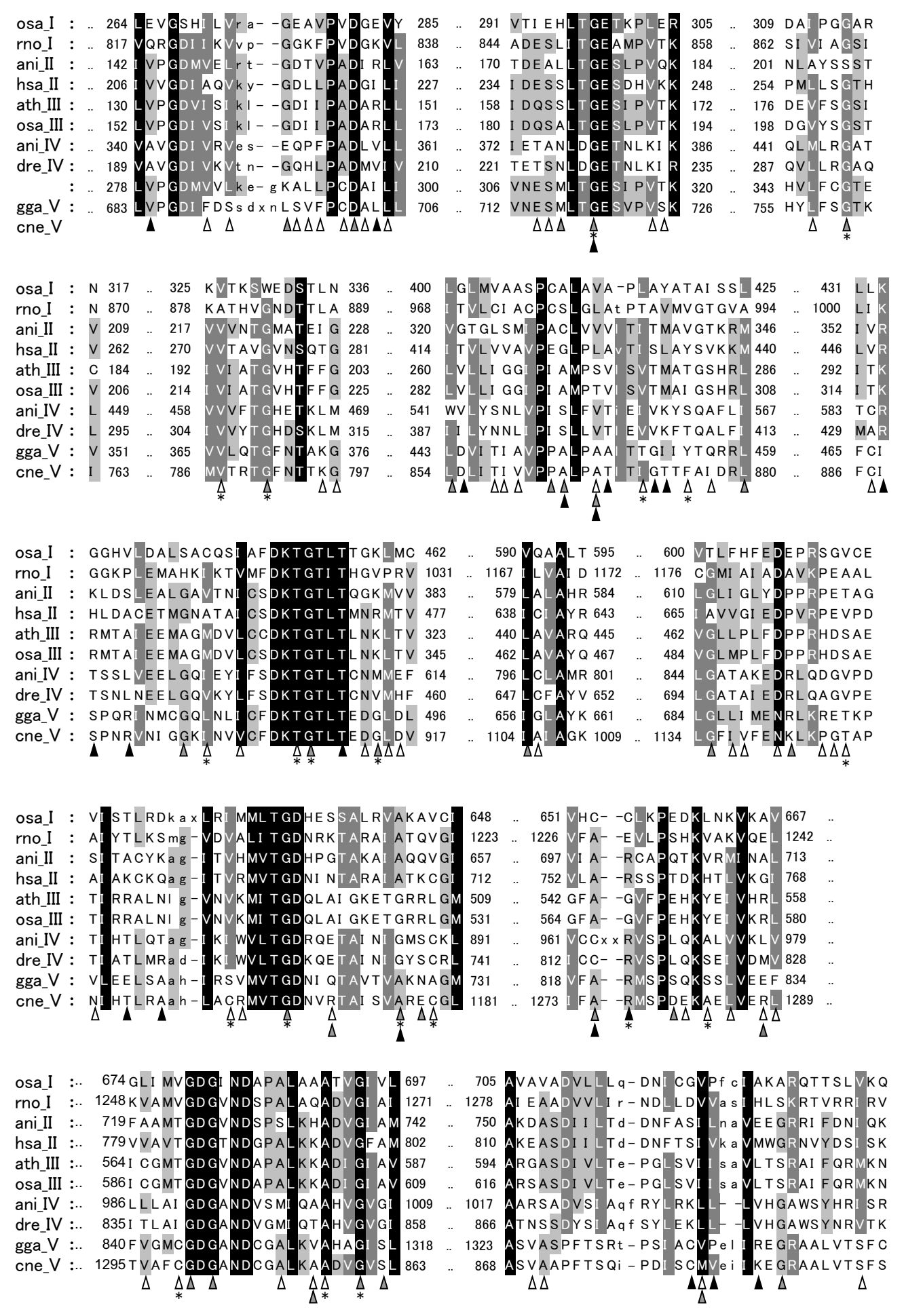

osa_I : SVA 740

rno_I : NLV1313.

ani II : FVL 785

hsa II : FLQ 845

ath_III : YTI 629

osa_III : YTI 651

ani_IV : VI L 1051

dre_IV : CI L 900

gga $\vee$ : MFK 1358.

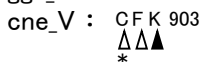




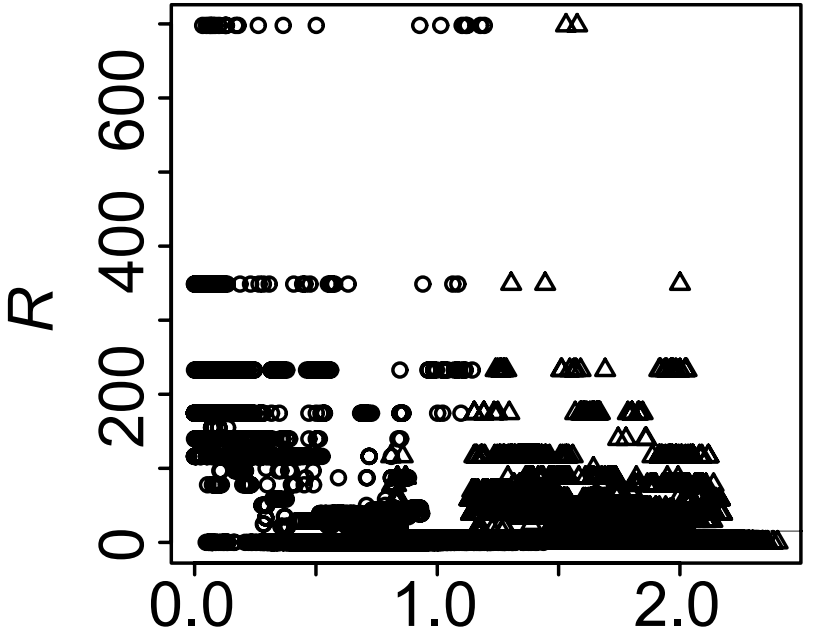

Genetic Distance

C

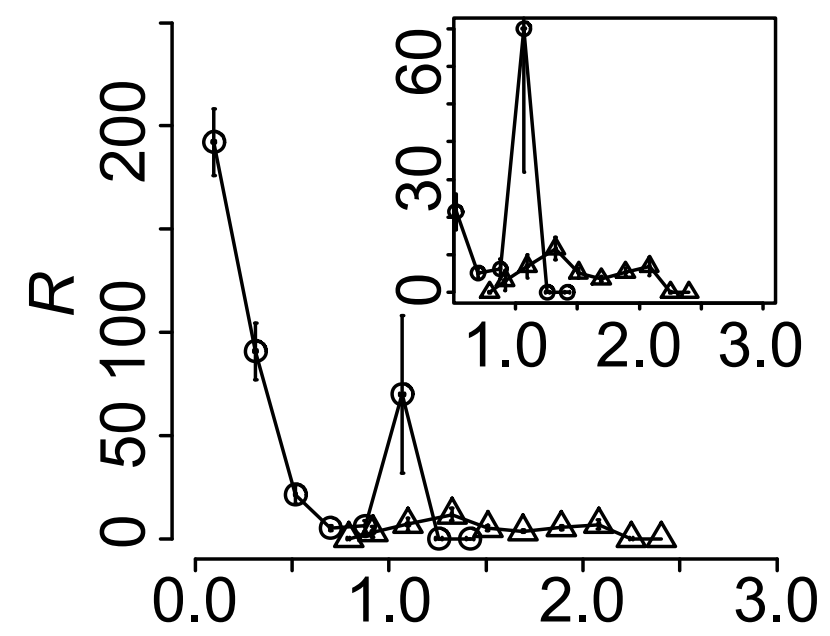

Genetic Distance

e

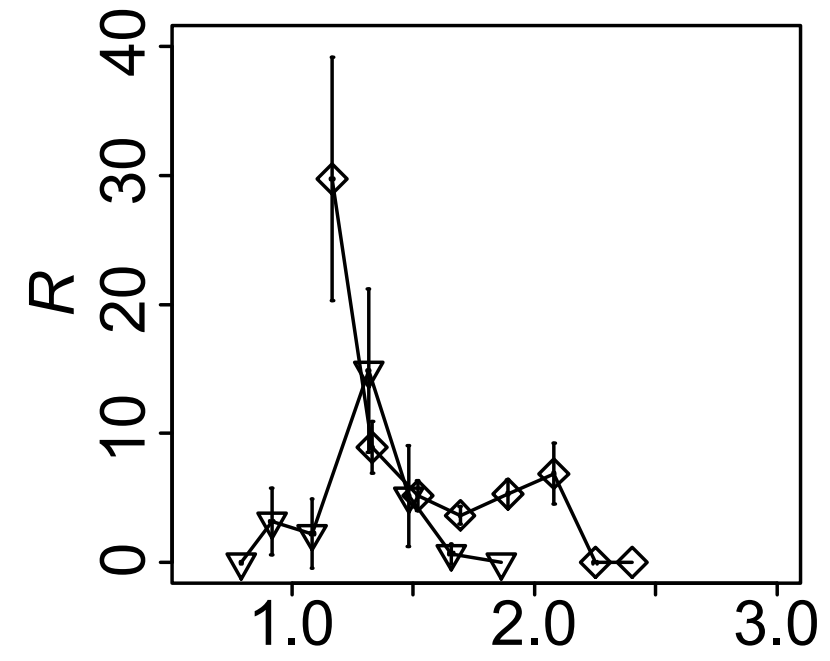

Genetic Distance

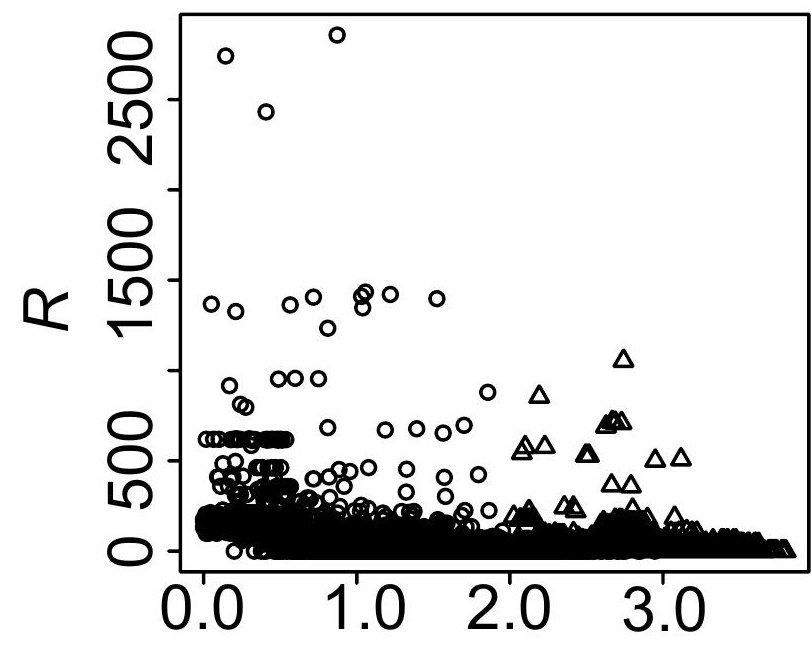

Genetic Distance

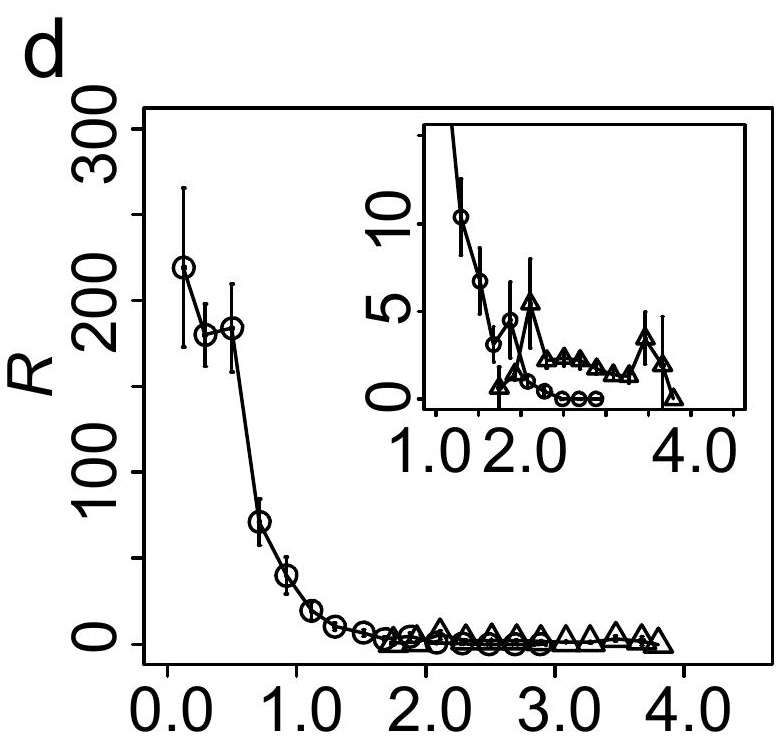

Genetic Distance

f

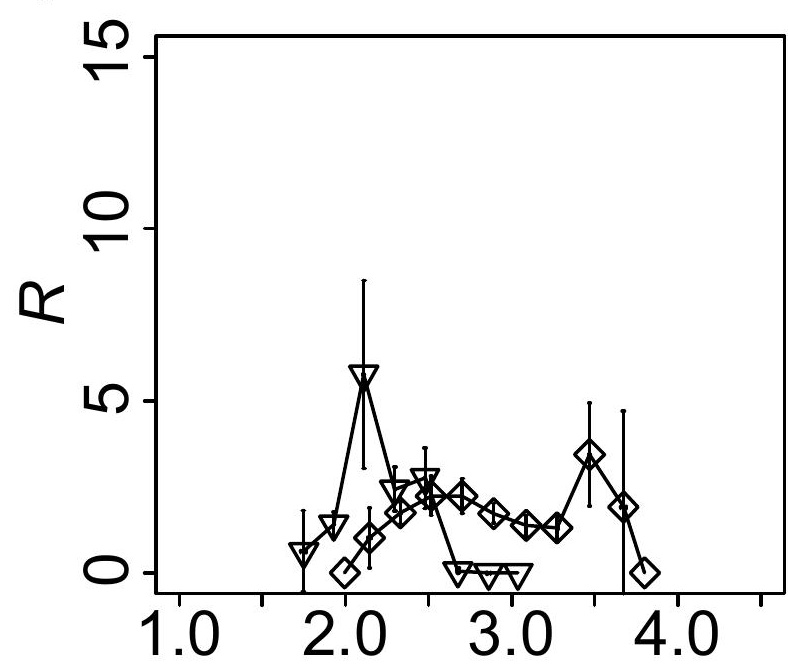

Genetic Distance 
Table 1. Numbers of genes belonging to the P-Type ATPase families in different organisms.

\begin{tabular}{|c|c|c|c|c|c|c|}
\hline Kingdom & Species & Family1 & 2 & 3 & 4 & 5 \\
\hline \multirow[t]{7}{*}{ Animals } & Homo sapiens & 3 & 15 & - & 13 & 5 \\
\hline & Mus musculus & 2 & 13 & - & 12 & 5 \\
\hline & Rattus norvegicus & 1 & 13 & - & 11 & 5 \\
\hline & Gallus gallus & 2 & 8 & - & 10 & 4 \\
\hline & Danio rerio & 2 & 18 & - & 12 & 1 \\
\hline & Drosophila melanogaster & 1 & 4 & - & 6 & 2 \\
\hline & Caenorhabditis elegans & 1 & 9 & - & 5 & 4 \\
\hline \multirow[t]{2}{*}{ Plants } & Arabidopsis thaliana & 7 & 13 & 11 & 12 & 1 \\
\hline & Oryza sativa & 6 & 10 & 9 & 2 & 1 \\
\hline \multirow[t]{4}{*}{ Fungi } & Schizosaccharomyces pombe & - & - & - & 3 & 1 \\
\hline & Magnaporthe grisea & 2 & 9 & 1 & 4 & 1 \\
\hline & Cryptococcus neoformans & 1 & 4 & 2 & 4 & 2 \\
\hline & Aspergillus nidulans & 2 & 10 & 2 & 4 & 1 \\
\hline \multirow[t]{2}{*}{ Amoebozoa } & Entamoeba histolytica & - & - & - & 4 & - \\
\hline & Dictyostelium discoideum & 3 & 5 & 1 & 9 & 4 \\
\hline \multirow[t]{3}{*}{ Chromalveolata } & Plasmodium falciparum & 1 & 1 & - & 2 & 2 \\
\hline & Theileria parva & - & 1 & - & 2 & 2 \\
\hline & Theileria annulata & - & 1 & - & 2 & 2 \\
\hline
\end{tabular}


Table 2. Profiles of intron positions among the P-type ATPase gene families

Intron positions Intron positions

Intron positions shared in different shared in different

found in one gene

genes belong to the

genes belong to the

All intron positions same family different families

generous rules

236

328

67

631

strict rules

29

61

21

111


Table 3. Probability distribution of the number of shared intron positions in a gene pair.

Number of intron positions shared in two genes ${ }^{\mathrm{a}}$

\begin{tabular}{ccc} 
shared in two genes $^{\mathrm{a}}$ & Probability & $\mathrm{R}$ \\
\hline 0 & 0.96598 & 0.00000 \\
\hline 1 & 0.03365 & 29.08333 \\
\hline 2 & $3.66 \mathrm{E}-04$ & 58.16667 \\
\hline 3 & $1.41 \mathrm{E}-06$ & 87.25000 \\
\hline 4 & $1.53 \mathrm{E}-09$ & 116.33333
\end{tabular}

a: An example of one gene with 6 introns and another with 4 introns under the strict rule: the heavy metal transporting ATPase gene of Arabidopsis thaliana (NP_001031920.1) and calcium-transporting ATPase gene of Danio rerio (NP_001007030.1). $I_{A B \_a l l}=689, E_{A B}=0.03438$. 
Table 4. Nucleotide species around the exon-intron borders.

\begin{tabular}{|c|c|c|c|c|c|c|c|c|c|}
\hline \multicolumn{10}{|c|}{ Distance } \\
\hline Nucleotide & -4 & -3 & -2 & -1 & $!$ & +1 & +2 & +3 & +4 \\
\hline A & $31.2 \%$ & $35.0 \%$ & $59.8 \%$ & $10.3 \%$ & & $24.1 \%$ & $21.3 \%$ & $26.6 \%$ & $27.2 \%$ \\
\hline $\mathrm{T}$ & $25.6 \%$ & $13.9 \%$ & $18.1 \%$ & $9.1 \%$ & & $10.9 \%$ & $42.6 \%$ & $30.0 \%$ & $25.9 \%$ \\
\hline $\mathrm{G}$ & $20.9 \%$ & $16.6 \%$ & $11.4 \%$ & $77.6 \%$ & & $54.0 \%$ & $17.6 \%$ & $22.6 \%$ & $25.2 \%$ \\
\hline $\mathrm{C}$ & $22.4 \%$ & $34.5 \%$ & $10.7 \%$ & $2.9 \%$ & & $10.9 \%$ & $18.5 \%$ & $20.9 \%$ & $21.8 \%$ \\
\hline
\end{tabular}


Table 5. Existence of introns in the 50 amino acids conserved in over 80\% P-Type ATPase members.

\begin{tabular}{|c|c|c|c|c|c|}
\hline \multirow[b]{2}{*}{ Amino acid } & \multirow{2}{*}{$\begin{array}{c}\text { Position in the } \\
\text { alignment }\end{array}$} & \multicolumn{4}{|c|}{ Introns are found at } \\
\hline & & Phase 0 & Phase 1 & Phase 2 & Phase 3 \\
\hline $\mathrm{A}$ & 3944 & $-^{\mathrm{a}}$ & - & - & - \\
\hline A & 4439 & - & - & - & 0 \\
\hline A & 4751 & - & - & - & - \\
\hline A & 5752 & ! & - & - & - \\
\hline A & 5898 & 0 & - & - & - \\
\hline $\mathrm{D}$ & 2172 & - & - & - & - \\
\hline $\mathrm{D}$ & 2232 & - & $\square$ & - & 0 \\
\hline $\mathrm{D}$ & 3167 & - & - & - & - \\
\hline $\mathrm{D}$ & 4719 & $\circ$ & - & - & - \\
\hline $\mathrm{D}$ & 4746 & - & - & - & - \\
\hline $\mathrm{D}$ & 5741 & - & - & - & - \\
\hline $\mathrm{D}$ & 5745 & - & - & - & - \\
\hline $\mathrm{E}$ & 2391 & - & - & - & - \\
\hline $\mathrm{E}$ & 3156 & - & - & - & - \\
\hline $\mathrm{F}$ & 4101 & - & - & - & - \\
\hline $\mathrm{G}$ & 2171 & - & - & - & - \\
\hline $\mathrm{G}$ & 2390 & - & $!$ & $\circ$ & - \\
\hline $\mathrm{G}$ & 2799 & - & $!$ & - & - \\
\hline G & 3159 & - & 0 & - & - \\
\hline $\mathrm{G}$ & 3170 & - & $!$ & - & - \\
\hline $\mathrm{G}$ & 4438 & $!$ & $\square$ & - & - \\
\hline G & 4713 & - & 0 & - & - \\
\hline G & 4745 & - & $!$ & - & - \\
\hline G & 5091 & - & - & - & - \\
\hline G & 5740 & - & 0 & - & - \\
\hline G & 5742 & - & 0 & - & - \\
\hline G & 5755 & - & $!$ & - & - \\
\hline G & 5922 & - & 0 & - & - \\
\hline $\mathrm{K}$ & 3168 & - & - & - & $!$ \\
\hline $\mathrm{K}$ & 4342 & - & - & - & $!$ \\
\hline $\mathrm{K}$ & 5660 & - & - & - & $!$ \\
\hline L & 2388 & - & - & - & - \\
\hline $\mathrm{L}$ & 3005 & - & - & - & - \\
\hline $\mathrm{L}$ & 3192 & - & - & - & - \\
\hline M & 1 & - & - & - & - \\
\hline $\mathrm{N}$ & 1530 & - & - & - & $\square$ \\
\hline $\mathrm{N}$ & 5744 & - & - & - & - \\
\hline $\mathrm{P}$ & 2230 & - & - & - & $\circ$ \\
\hline $\mathrm{P}$ & 3002 & - & - & - & - \\
\hline $\mathrm{P}$ & 5657 & - & - & - & - \\
\hline $\mathrm{P}$ & 6044 & - & $\square$ & - & - \\
\hline $\mathrm{R}$ & 4652 & - & - & - & - \\
\hline $\mathrm{R}$ & 5654 & - & - & $!$ & - \\
\hline $\mathrm{T}$ & 3169 & $!$ & - & - & - \\
\hline $\mathrm{T}$ & 3191 & - & - & - & - \\
\hline $\mathrm{T}$ & 3193 & - & - & $\square$ & - \\
\hline $\mathrm{T}$ & 4744 & - & - & - & - \\
\hline $\mathrm{T}$ & 4750 & - & - & - & - \\
\hline $\mathrm{V}$ & 2795 & $!$ & - & - & - \\
\hline V & 5664 & - & - & - & - \\
\hline
\end{tabular}

a: Positions in which no genes have introns are shown by “-”. Introns present in more than two distinct families (!), introns present in the more than two distinct genes in the same family $(\circ)$, and introns present in only one gene ( $\square$ ) are also listed. 
\#Supplemental Data 1

\#Molecular Biology Reports

\#Evolutionary dynamics of spliceosomal intron revealed by in silico analyses of the P-Type ATPase superfamily genes

\#Toshiyuki Oda, Ryosuke L. Ohniwa, Masatsugu Denawa, Hideyuki Okamura and Kunio Takeyasu

>athI_IB101 NP_195444.1

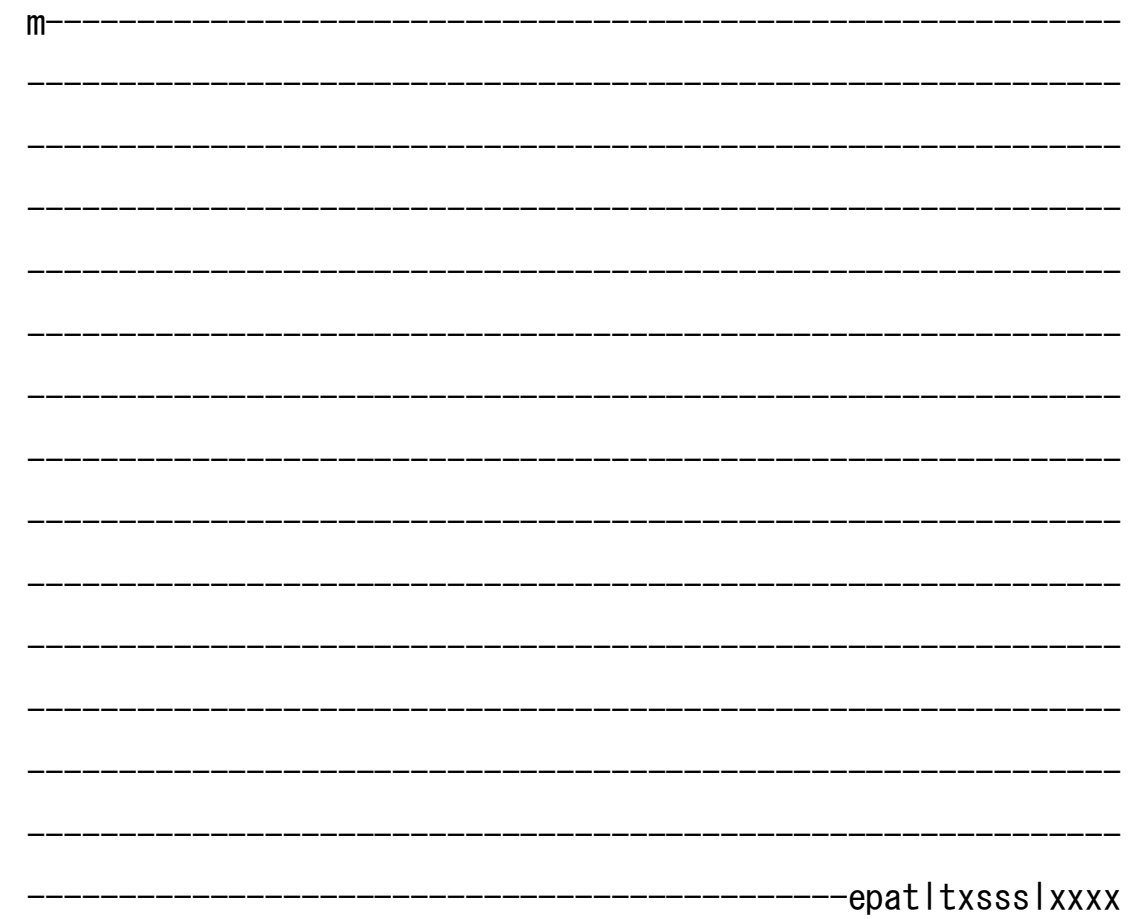

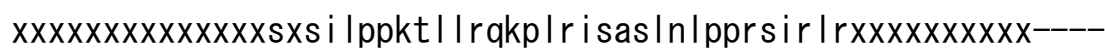
-hddeqdhhnhhhhhhq-

-hgccsve I kaeskpqkm I fgfaka i gwvr lany I xxxxxxxxsaaamf laaavcpy lax

-epy iks I qnaf

-mivgfp Ivgvsas I

-da Imd i aggkv 
-nihvImalaa--fasvf

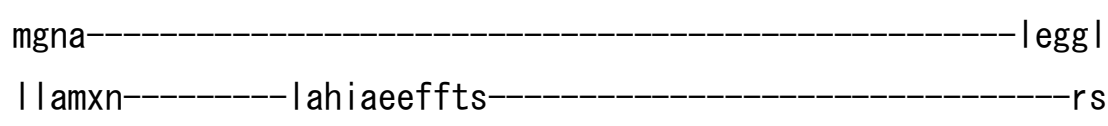

mvdvkelkes-

-xat I TIEHLTGEVKPLEAkx-

-DRVPG--GARN-

Id----grmi--_-_-_-_-_-_-_-_-_-_KATKAWNDSTLNki vq I tee-

-ahsnkpk I qrwIdefgenyskvvvvIs laia

-f I gpf I fkwpf I sta------------_-------_--------acrgsvyraLGLMVAA

SPCALAVA-PLAYATAIS-SCarkg-

-iLLK-GAQVLDALASCHT I AFDKTG-

-TLTTGGLTCka iep iyg

$-x x x x x--x x-x$

$x x x x x x x x$

$-x x$

xxa lava-----aame 


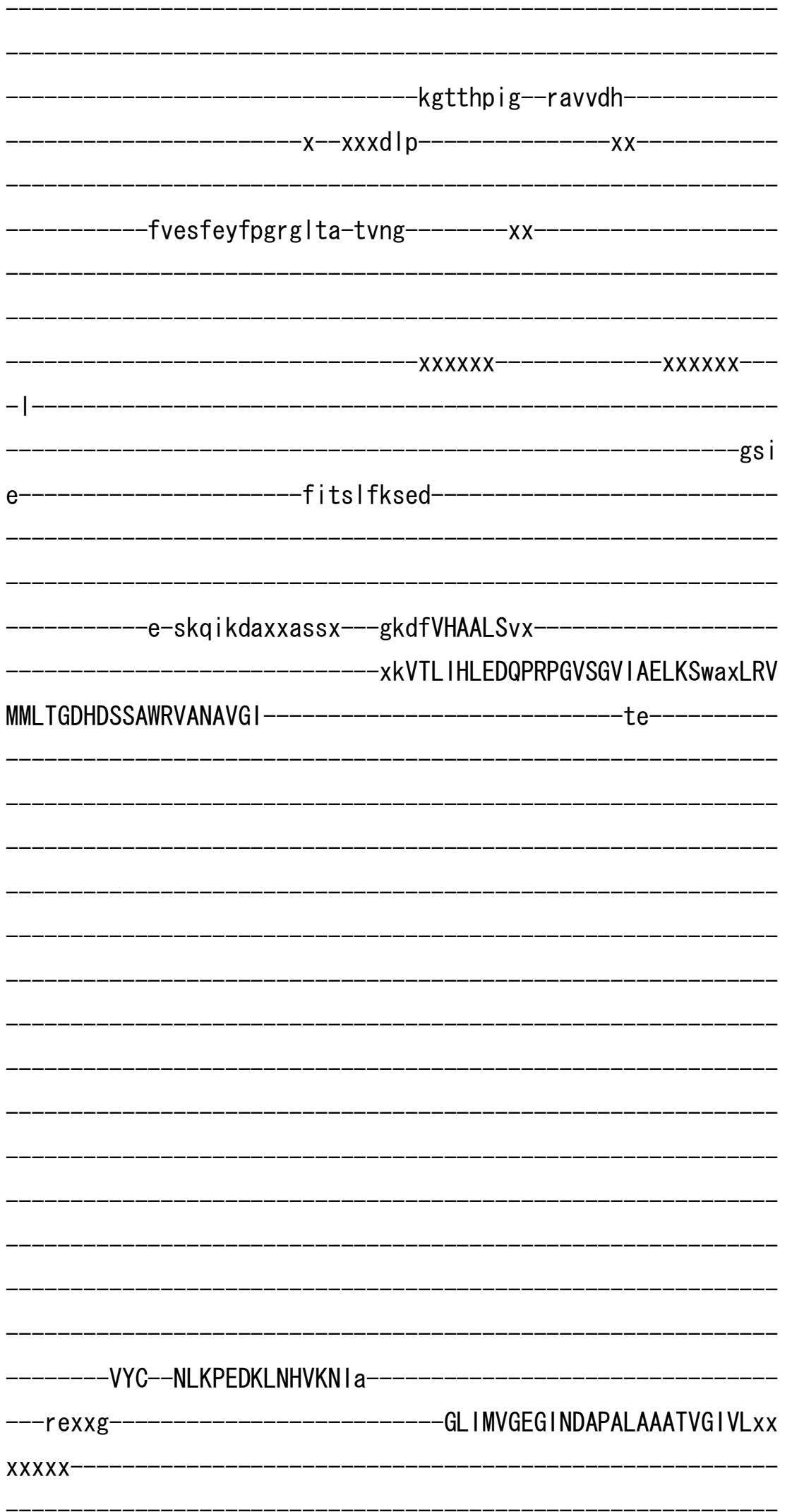

-AIAVADILLLr-DN I TGVPfcVAKSRQTTSLVKQNV-------A 


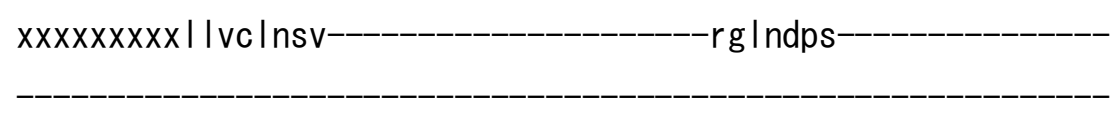

-wswkqdi vhl inx I rxxxxxxxxxxxxxxxx

h

>athI_IB102 NP_179501.1

m- 
en i Iks I dgvkeysvi vpsrtvivvhds I I i sp

fqiakalnear

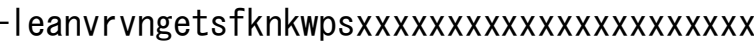

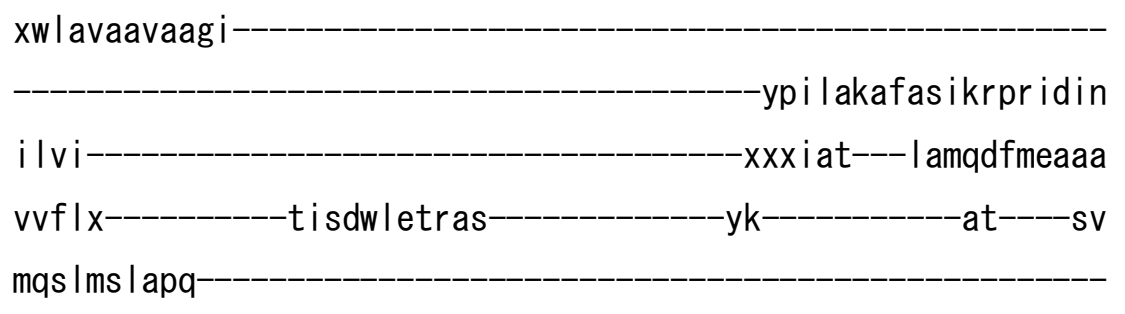

-kaixaet-

$-x \times x-------v$

e-

-xceVDEKTLTGEAFPVPKqx-

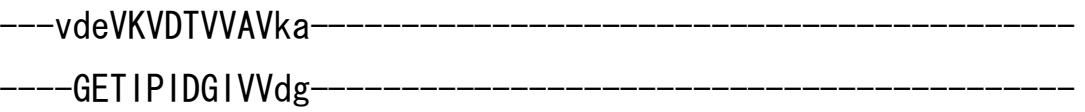

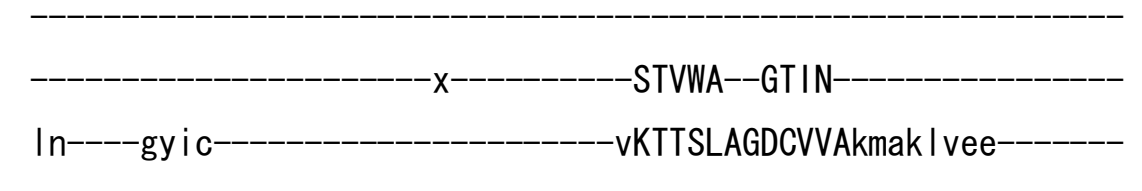

-aqssktksqr I idkcsqyytpa i i vsacva

-ivpvimkvh-nlkhwfhlaLVVLVSG CPCGLILStPVATFCALT-KAatsg- 
ILIK-SADYLDTLSKIKIVAFDKTG-

-TITRGEF IVi

dfks I srdi

xx | | ywv-----ssve-

sksshpma--at i vdy-----akxxx---

xxxrpe

evedyqnfpgeg i yg-kidgxxxxx

-gnk

k

i asragcst

-vpe i evd--tkg----gktvGYVYVGe

$x$ I AGFFNLSDACRSGVSQAMAELKS I g-IKT

AMLTGDNQAAAMHAQEQLGN-

$-v \mid x$ 


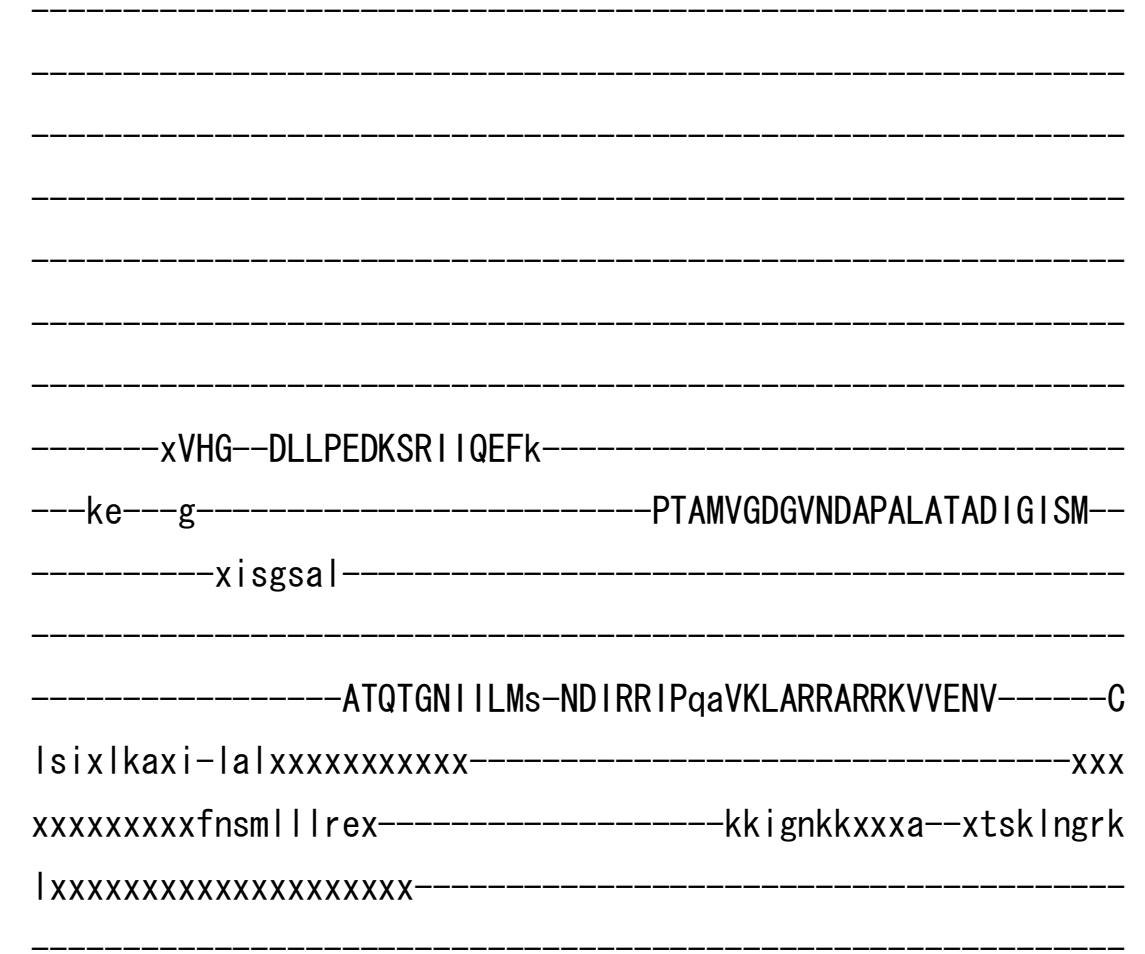

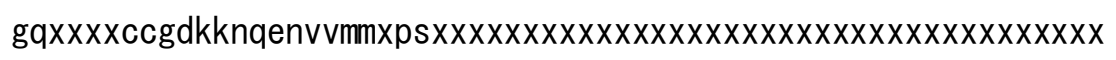

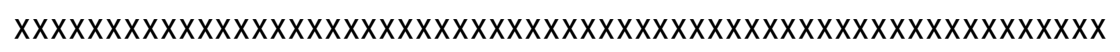

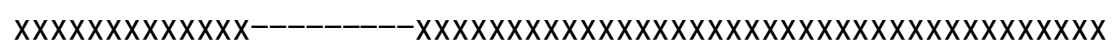
xxxxxxxxxxxxxxxx----------------------------------------

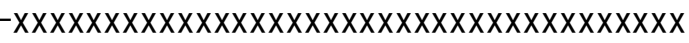

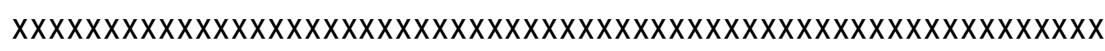

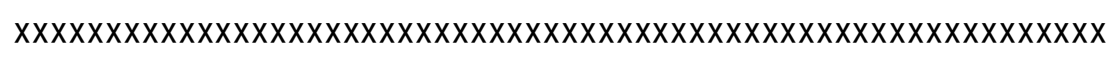

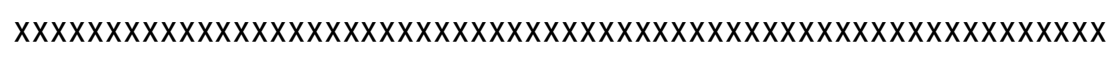

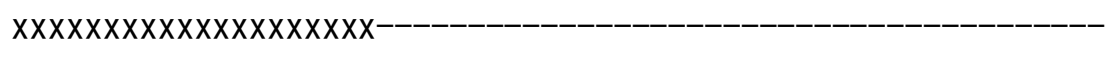

a >athI_IB103 NP_194740. 1 m- 
-mtksyfdv lgicctsevpli--

en i Insmdgvkefsvi vpsrtvivvhdt I i I sq

fqivkalnqaq-

leanvrvtgetnfknkwpsxxxxxxxxxxxxxxxxxxxxxxx

xw lavaavvag $\mathrm{i}$

-yp i lakavas larfridin

ilvv -xxxgat---i gmqdyteaav

vvflxyk -as----av mqs Ims I apq

-kavxaet

$-x X X$ $-\mathrm{v}$

e-

----vdeLKTNTVIAVka

GETIPIDGVVVdg 


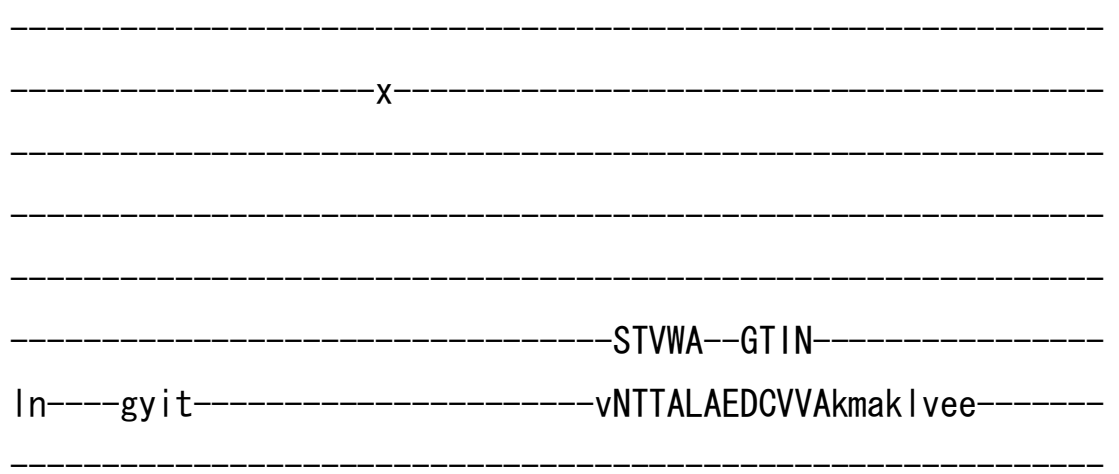

-aqnsktetqrfidkcskyytpa i i i s icfv -aipfalkvh--_-_-_-_-_-_-_-_-_-_-_-_-_-_-_-_n lkhwvh I aLVVLVSA CPCGLILSTPVATFCALT-KAatsg-
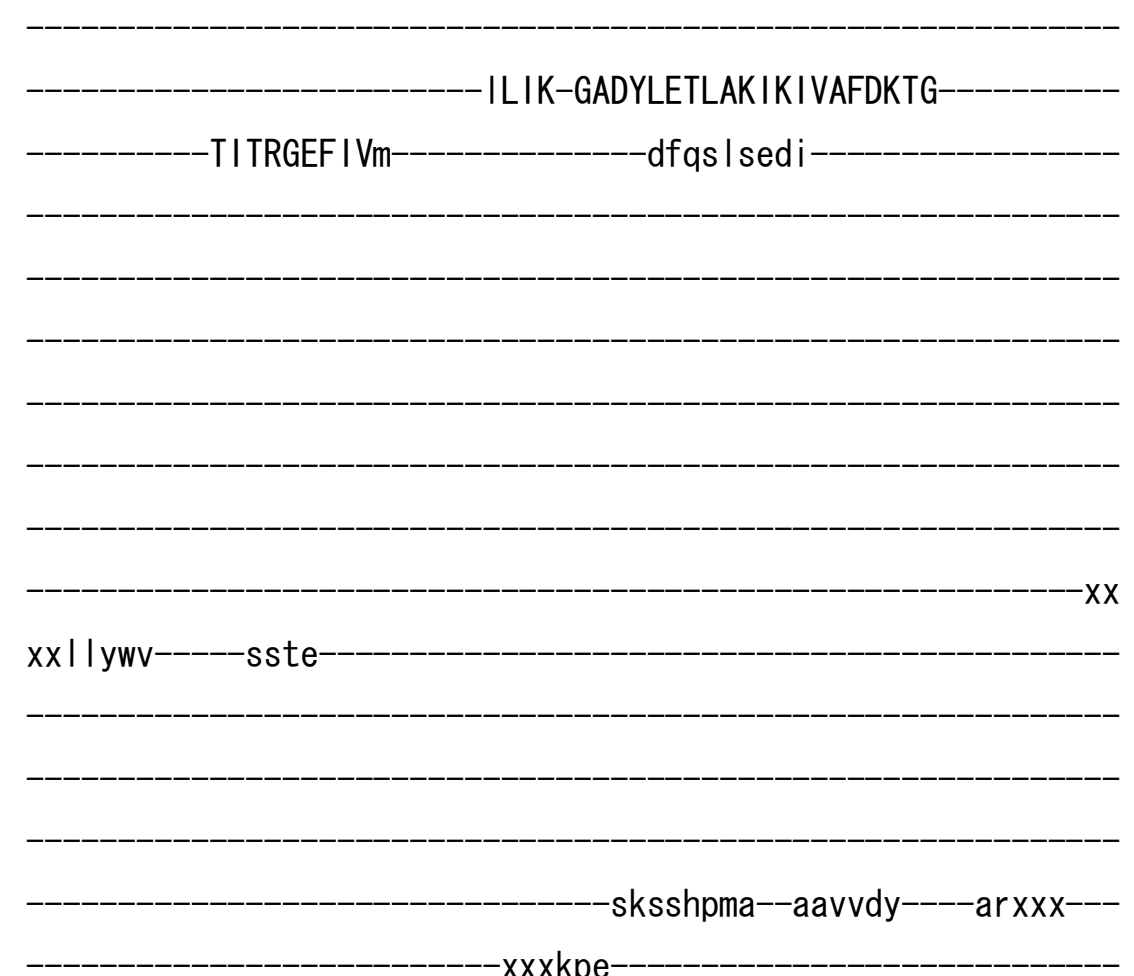

$-x x x k p e$

-avedyqnfpgeg i yg-kidgxxxxx- 
-vpdidvd--tkg----gktiGYVYVGex

- AGVFNLSDACRSGVAQAMKELKS I g-IK I

AMLTGDNHAAAMHAQEQLGN-

amx

-XVRA--ELLPEDKSE I IKQLK

$---r e--e g-$

-PTAMVGDGLNDAPALATAD I G I SM--xvsgsal

ATETGN I ILMs-ND IRR IPqa IKLAKRAKRKVVENV $-\mathrm{V}$

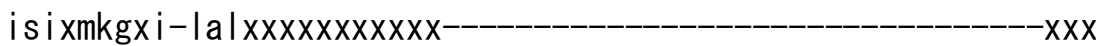
xxxxxxxxx|nsm| | |sd-_-_-_-__-__-_-_-_-_-_khktgnkxxxexxxssv | iaek $1--x x x x x x x x x x x x x x x x x x-$

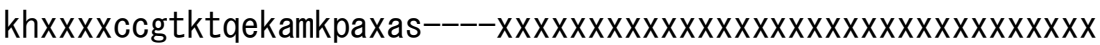
$x x x x x x x x x x x x x x x x x x$ $x x x x x x x x x x x x$

$-X X X X X X X$ 
e

>osal_IB101 NP_001058417. 1

m

ql Itaasaxassaxxxx

$x x x x x x x x x x x x x x x p x \mid r r$ caph|pskp|n|aarsp | | |arrs|pxxxxxxxxxxx

-hghhhhhhghghshhhgpev--

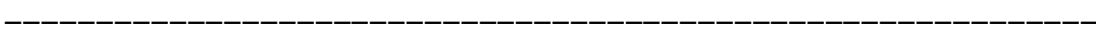

-hgsgggaavmrvaka i gwadvada |xxxxxxxxis |g | |

I i aaacphipx

- nsvrr lqda |

-iavafp I vgvsaa I

-da l vni adgki 
-nihvImalaa--fasif

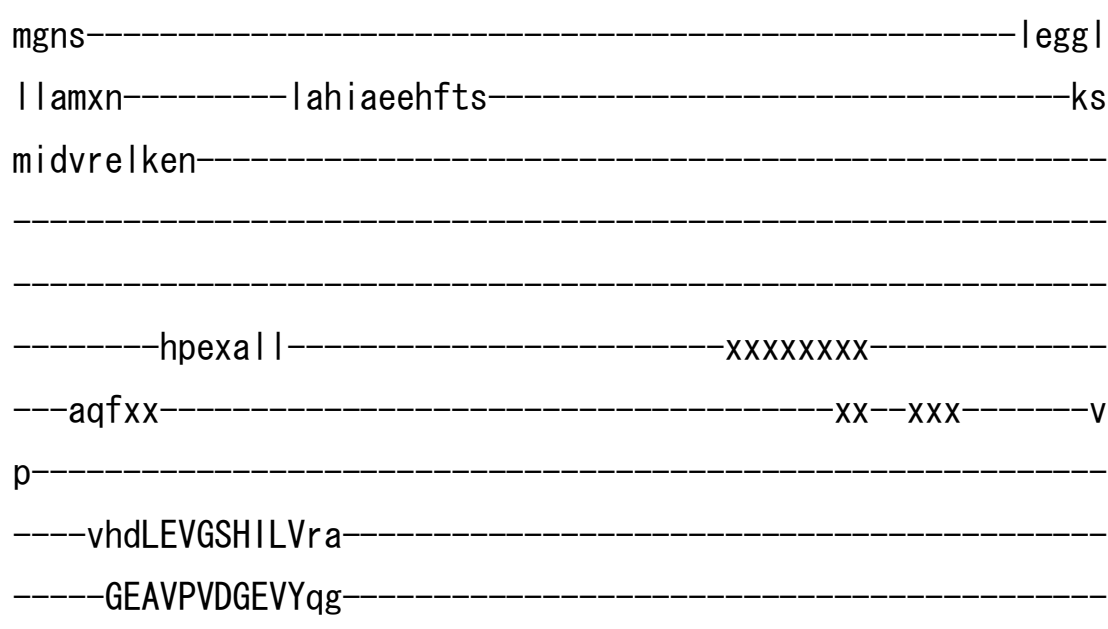

-xstVTIEHLTGETKPLERtx-

$$
-\mathrm{x}
$$$$
\text { DAIPG--GARN- }
$$

le----gmmi -VKVTKSWEDSTLNr i vql tee-

-gqlnkpk larw I defgehysrvvvvIs Ivva - I I gp I I fkwpffgns--------------------_--------vcrgs i yrgLGLMVAA SPCALAVA-PLAYATAIS-SLaskg-

\section{iLLK-GGHVLDALSACQS I AFDKTG}

-TLTTGKLMCkai eping

$-x x x x x--x x x x$

$x x x x x x x x$

xxalava aame 


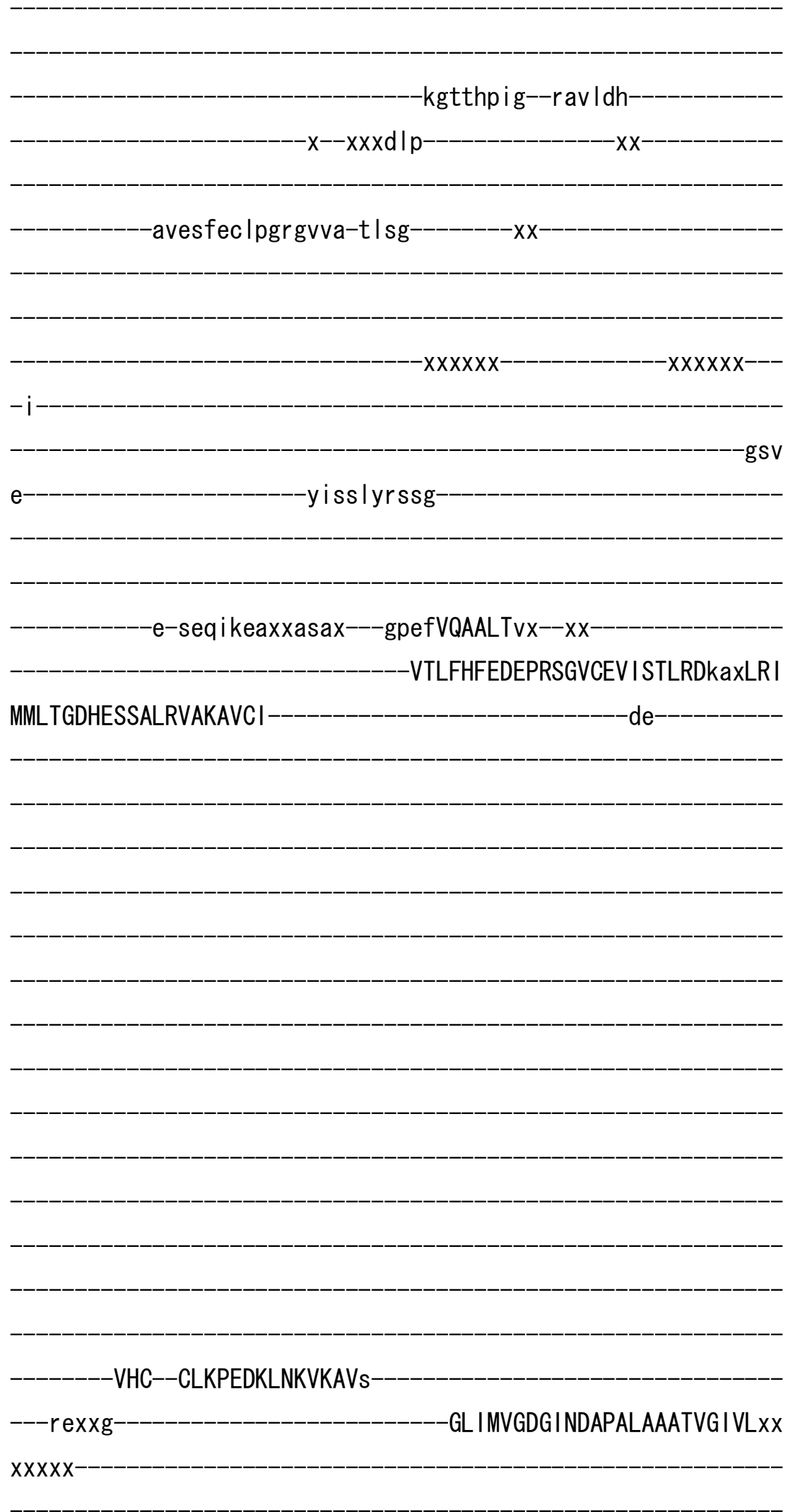

-AVAVADVLLLq-DN I CGVPfc I AKARQTTSLVKQSV------A 


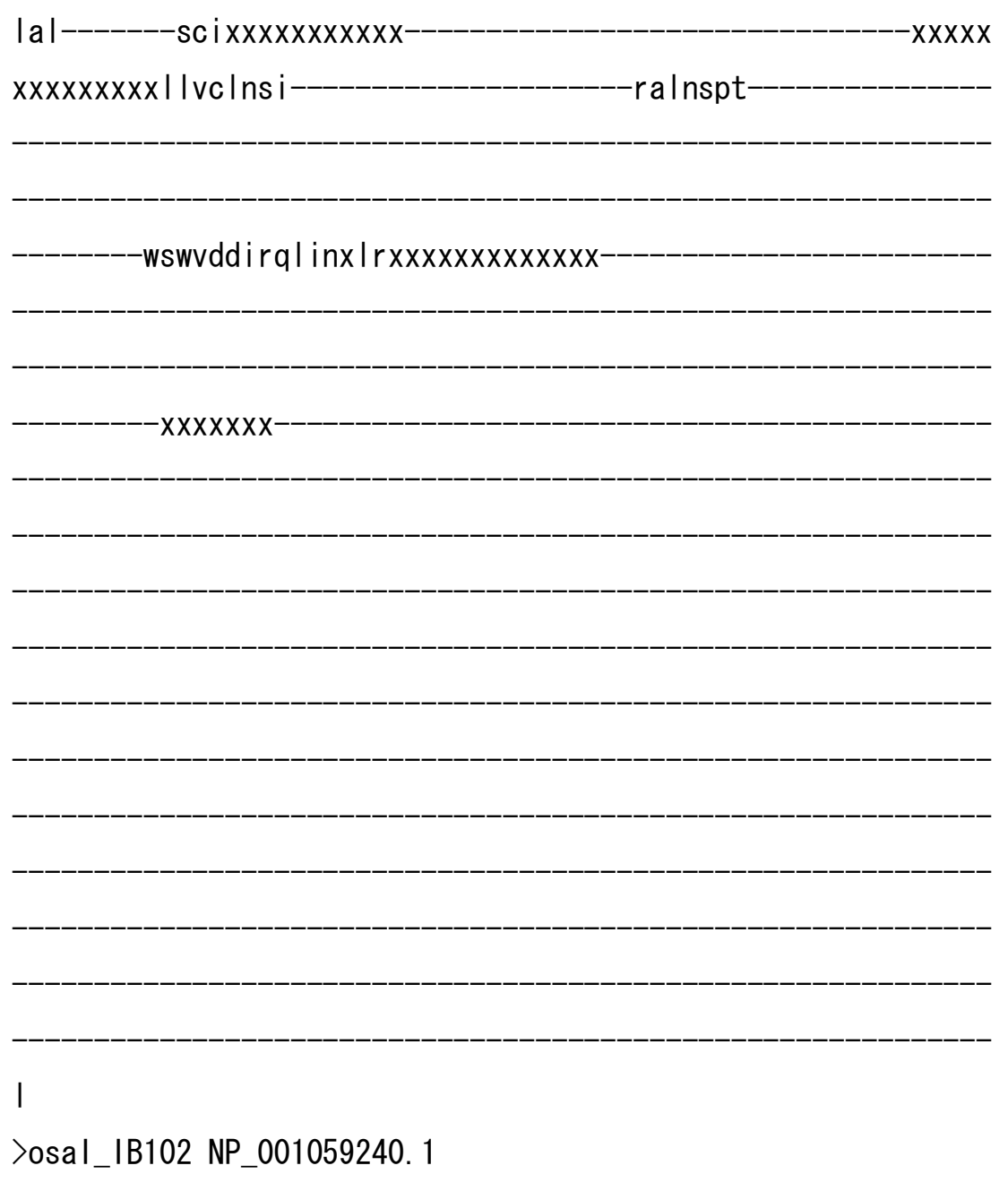

m- 


\section{Imgm I pvkav}

-xseVDERSLTGESFPVPKq--

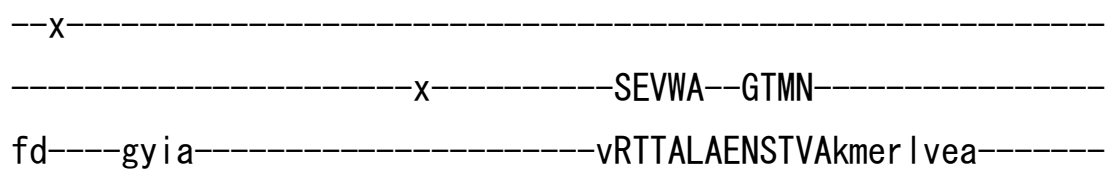

-aqnsr sktqr I idscakyytpavvvvaagva

- I ipal I gadg l eqwwk I aLVMLVSA CPCALVLStPVASFCAML-RAarmg- 
iFIK-GGDVLESLGE IRAVAFDKTG-

-TITRGEFSId-

sfh I vgdhk

$-x x x$

xx | Iywi-----as i e-

-sksshpma--aal vey-----aqxxx---

xxxnpe

-nvgdfr i ypgeg i yg-e i hgxxxxx

-gnr

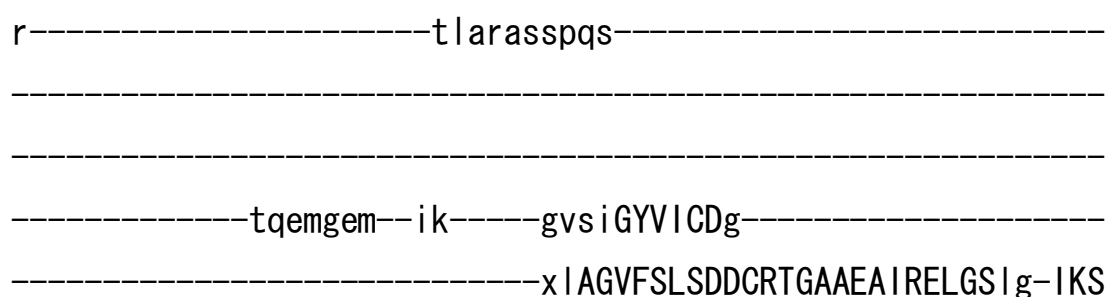

VMLTGDSSAAATHAQGQLGG-

$-\mathrm{vmx}$ 


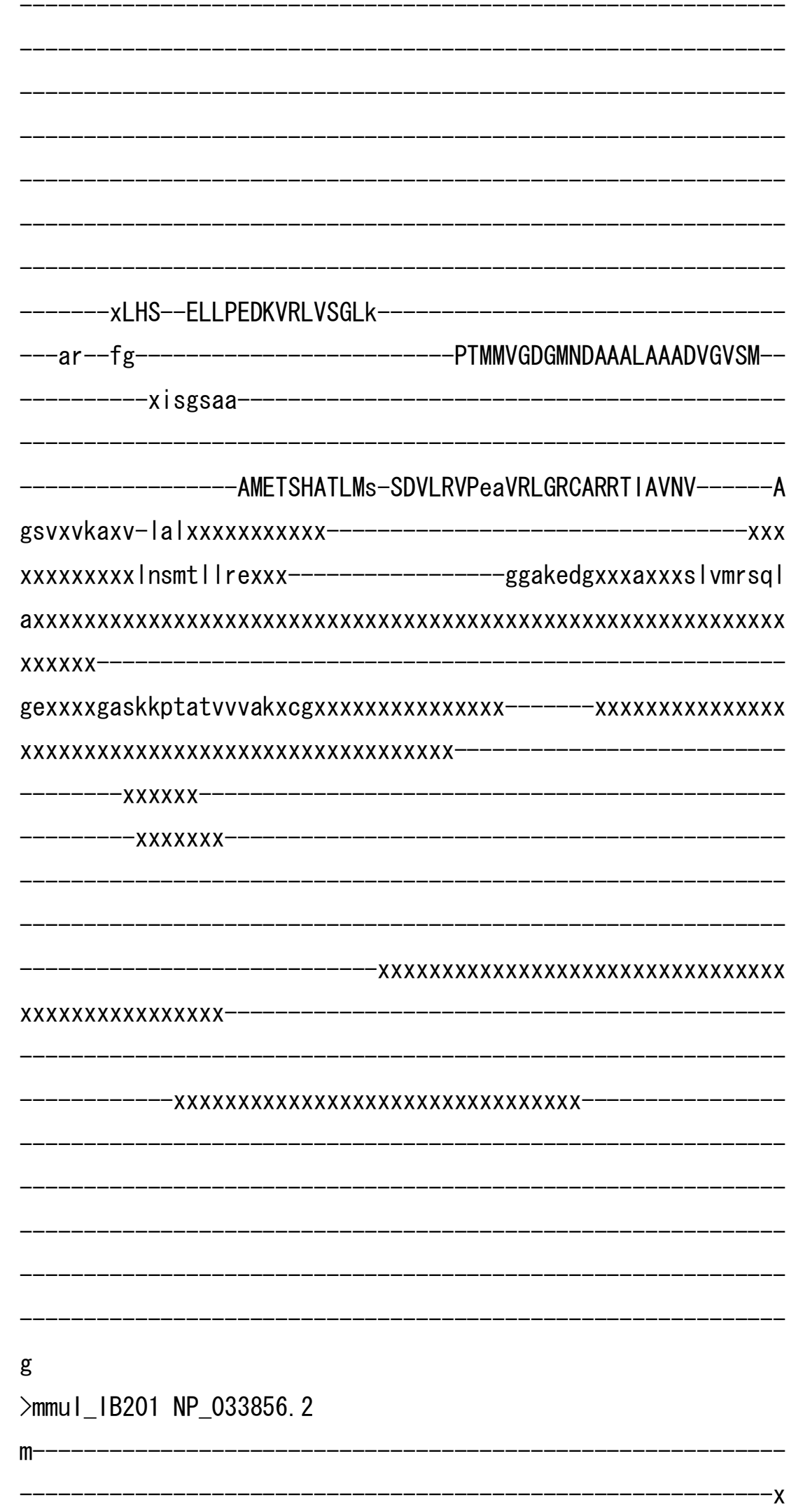

$-\mathrm{X}$

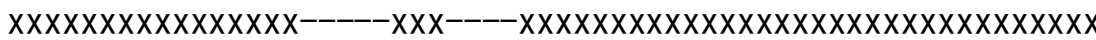




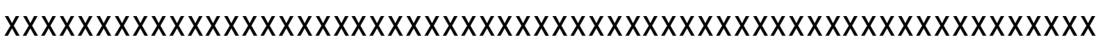

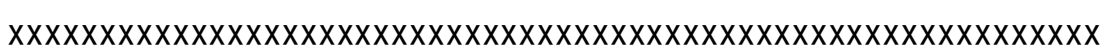

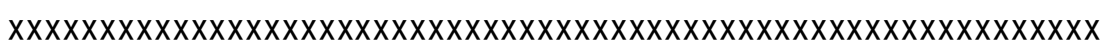

$x x x-$

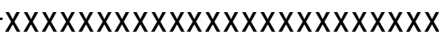

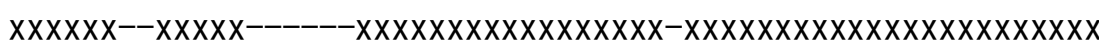

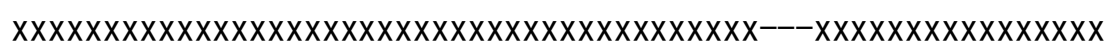

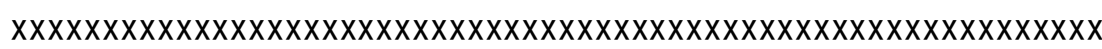

-xxxreai edmgxdaal

-xxxxxp-|vviaqps letp | |pssnel-envmtsvqn

-kcy iqvsgmtcascvani--

ernI r reegiysv I va Imagkaevrynpaviqpr

-viaefi

relgfgamvmx

$-x x x x x x x x x x x x x x x t c a s c v h k i$ est I

$-X X X X X X X X X X X X X X X X X X X X X X X X X X X X X X X$

$-x x x x x x x x$

xxx----------------as I vkkdrsanh I dhkre $\mathrm{i}$

-kqwrgsf|vs|xxxxxx-- $x x x x x x x x x x x x x x x x x x x x x x x x x x x x x x x x x x x x x x x x x$ $x x x x x x x x x x x x x x x x x x x x x x x x x x x x x x x x x x k h-k$ txnmdv l iv latt iafays $\mid v$

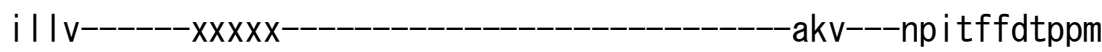

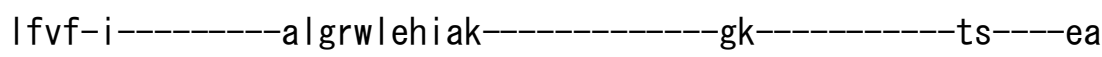
laklis lqat-

eatxvt I

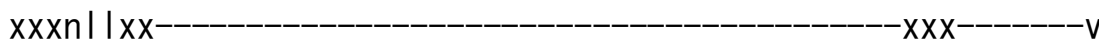

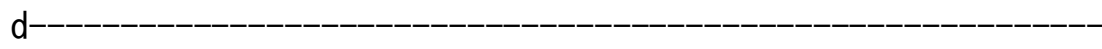

----ve IVQRGD I IKVvp

GGKFPVDGRV I eg 


$$
-x
$$

$-S T V I A--G S I N$

qn----gs I

iRATHVGADTTLSqi vk I vee

-aqtskap iqqfadk I sgyfvpfiv Ivsivt I

-Ivw i i i gfanfe i vexxxxxxxxx--------------xxxxxxi irfafqas I TVLCIA CPCSLGLAtPTAVMVGTG-VGaqng-

iLIK-GGEPLEMAHKVKVVVFDKTG

TI THGTPVVn qvkvIvesn

$-x \times x \times x--x---$

--i laiv-----gtae-

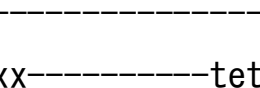

snsehp I g--aavtky

$-\operatorname{ckxx}$

$-x x-$

-tctdfqvvpgcg i sc-kvtnxxxxxxxxxx

$-\mathrm{X}$

$x X X X X X X X X X X X X$

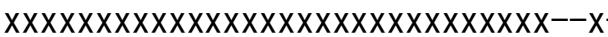
$-x---$

$-\mathrm{i}$

gnr

$\mathrm{e}$

-wmirnglvis 
-xxV-desmi eh--err----grtaVLVTIDdx

I CGL I A I ADTVKPEAELAVHILKSmg-LEV

VLMTGDNSKTARS IASQVGI tk

-VFA--EVLPSHKVAKVKQLq

---ee--gk-

-RVAMVGDGI NDSPALAMANVG I A --

gtgtdv

-AIEAADVVL I r-NDLLDVVas I DLSRKTVKRIRINF$-\mathrm{V}$

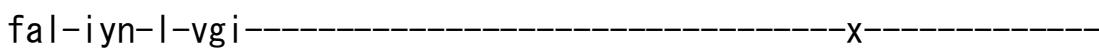

-i aagvf I pi--x--_-_-_-_-_-_-_-_-- | v l qpwm---g---saamaass

$\mathrm{V}$

sv----v|ss|f|k|yrkpt-yd- $x x x x x x x x x x x x x x x x x------x x x x x x x x x x x x x x x$

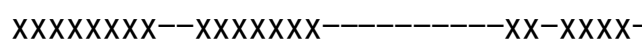

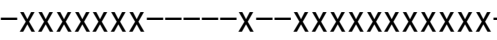

$x x x x x x$ 


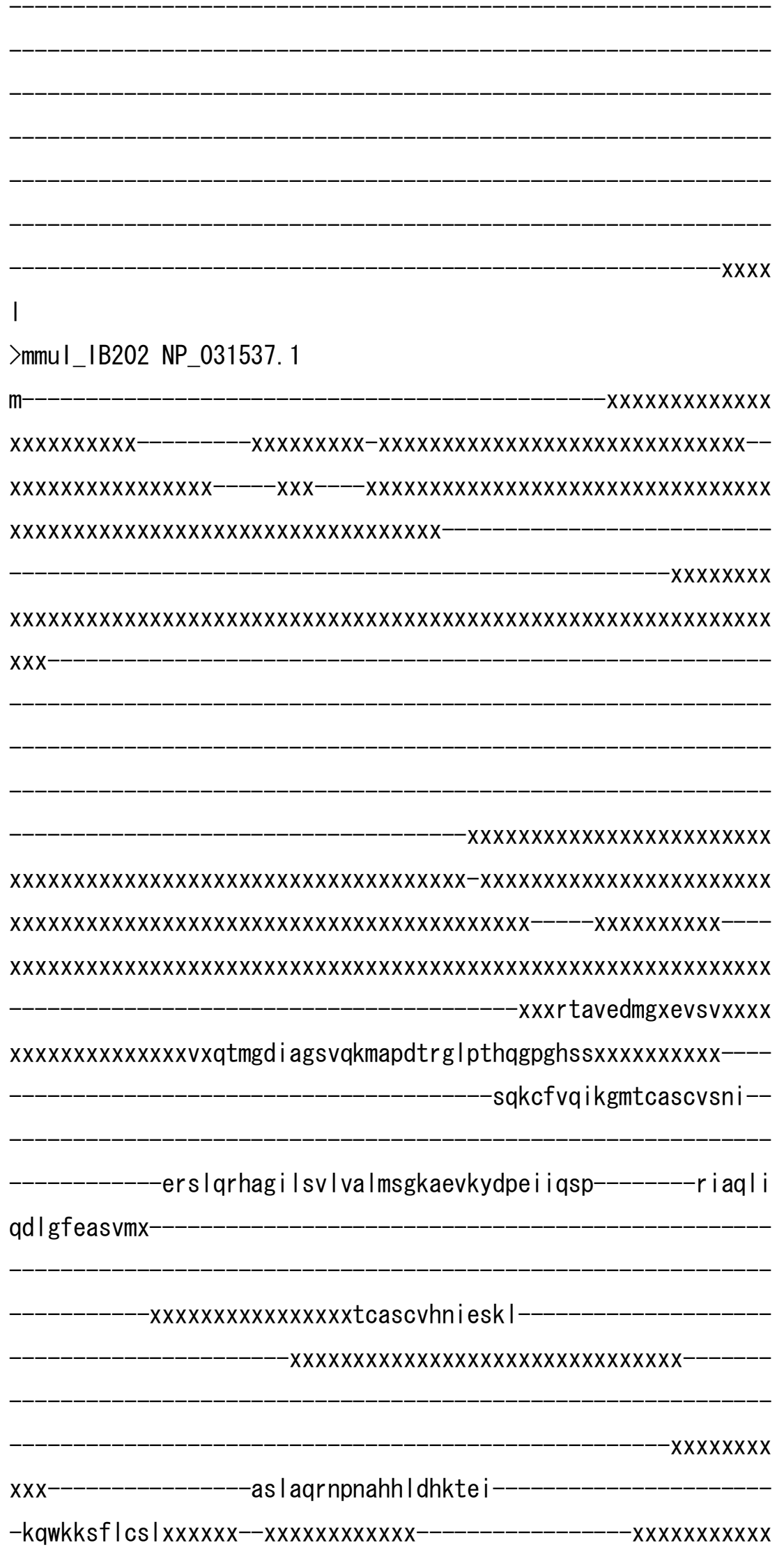


$x x x x x x x x x x x x x x x x x x x x x x x x x x x x x x x x x x x x$ rh- $r$ s $x n m d v \mid$ iv latt $i$ ayays $\mid v$

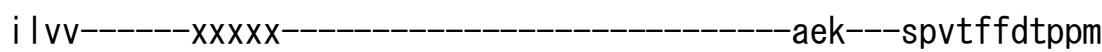

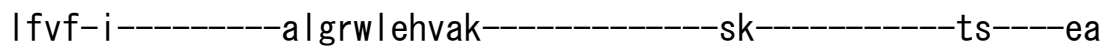
lak Ims lqat -eatxvt I

$x x x n l$ i $x x$ $-\mathrm{XXX}-------V$ $\mathrm{p}$ ----me IVQRGDVIKVvp GGKFPVDGKVLeg

xtmADESL I TGEAMPVTKkx-

SIVIA--GSIN-

ah----gsv I---_-_-_-_-_-_-_-_--_IKATHVGNDTTLAqi vk I vee

-aqmskap iqq ladrfsgyfvpf i i i i st I t I -vvwi vigfvdfgvvqxxxxxxxxxx------------xxxxxxi i rfafqts I TVLCIA CPCSLGLAtPTAVMVGTG-VAaqng-

-vLIK-GGKPLEMAHK IKTVMFDKTG

TI THGVPRVm -rfl I ladva

--vlavv-----gtae- 
--assehp I g--vavtky-----ckxx----

$-x x-$

-tet

$-x x-$

-ystdfqavpgcg i sc-kvsnxxxxxxxxx-

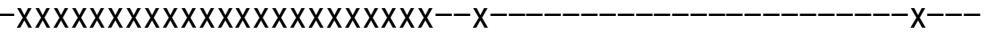

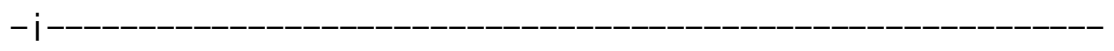

-gnr

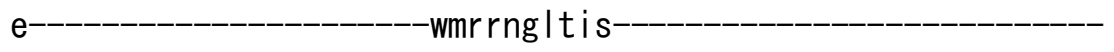

-xxi-sdamtdh--emk----gqta ILVAIDgx

I CGM I A I ADAVKPEAALA I YTLKSmg-VDV

AL I TGDNRKTARA I ATQVGI-

$-n k$

-VFA--EVLPSHKVAKVQELq-

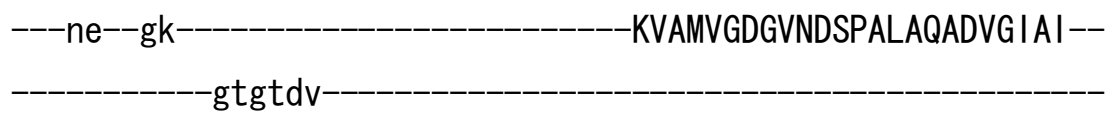

-AIEAADVVL I r-NDLLDVVas I HLSKRTVRR I RVNL------V 
$|a|-i y n-m-v g i$

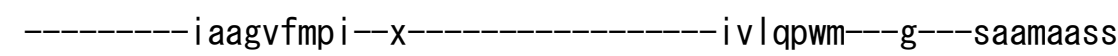

$-\mathrm{x}$

$\mathrm{v}$

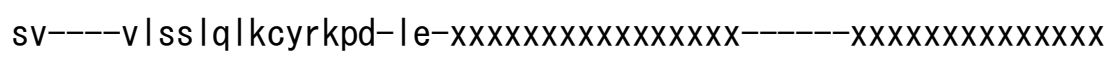

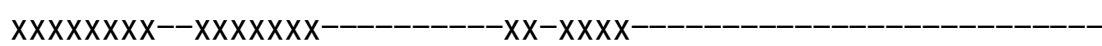

$-x x x x x x x----x x--x x x x x x x x x x x$

$-x x x x$

$-x x x-$

i

>ggal_IB201 XP_420307.2

$\mathrm{m}$

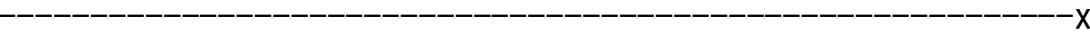

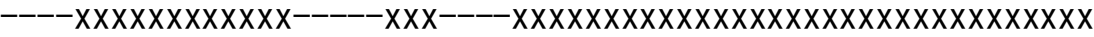

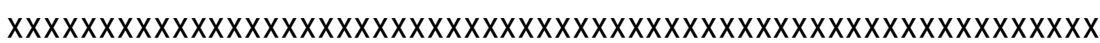

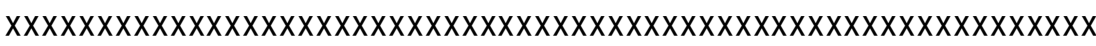

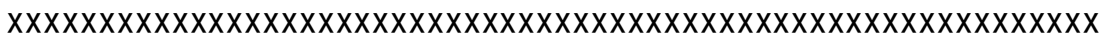
$x x x$

$x x x x x x x x x x x x x x x x x x x x x x x x$

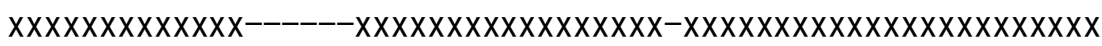

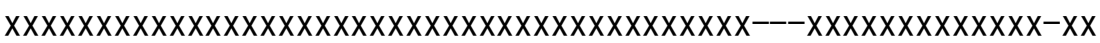

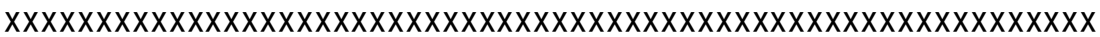
$-x x x r$ ssi enmgxdas $\mid$ 
-xxxxx I-pvgi tqptskeq I--esaeptskm I rsfvaxxxxx-----x---I skcy iqvtgmtcascvan i--

ern I r redgihsv I va Imagkaevrynpavihps aiaeli

relgfgatvmx

$-x x x x x x x x x x x x x x x x t$ saacvhkiest I

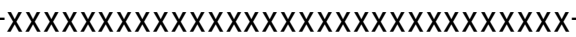

$x x x x x x x x$

xxx-----------------tal vkkdrsash I dhrqe i

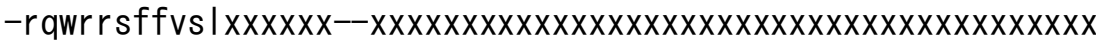

$x x x x x x x x x x x x x x x x x x x x x x x x x x x x x x x x x x x x k h-r$ txnmdv livlatsvaf $v y s f v$

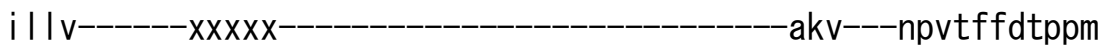

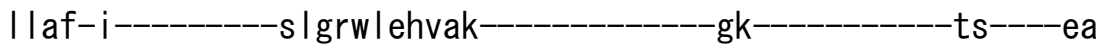
larlis lqat-

-eatxvt I

$x x x n i \mid x x$

$-x \times x-------V$

d

----ve IVQRGDIVKVvp

GGKFPVDGRV I eg

-xsmVDESL I TGEAMPVTKkx-

$$
-\mathrm{X}
$$

NTVIA--GSIN-

qn----g | | |

- iSATHVGADTTLSq i vk I vee

-aqtskap i qqfadk i sgyfvpf i vvvsvvt I

-fawi i i gfvdfe i vexxxxxxxxx-xxxxxx i irfafqas ITVLCIA CPCSLGLAtPTAVMVGTG-VGaqng- 
iLIK-GGEPLEMAHKVNVVVFDKTG

-TI THGTPEVm-

-rvkylvenn

$-x \times x x x--x---$

--mla i v-----otae-

-snsehp I g--aai tky----ckxx----

$x x$

se i

$-x x-$

tctdfavvpgcg i sc-kvtnxxxxxxxxxx

$-x$

$X X X X X X X X X X X X X$

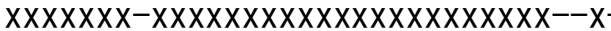

$-\mathrm{x}---$

$-i$

gnr

$\mathrm{e}$

-wmnrng | l vk

-xxV-dkami eh--err----grtaVLVAVDgx

I CGL I A I ADTVKPEAELAVYTLKNmg-LEV

VLMTGDNSKTARSIASQVGI

sk 
-VFA--EVLPSHKVAKVKQLq-

---de--gk------------------------RVAMVGDG I NDSPALAMANVG I Al--

- tgtdv-

-AIEAADVVL Ik-DDLMDVVas IDLSRKTVKRIRINF$-\mathrm{V}$

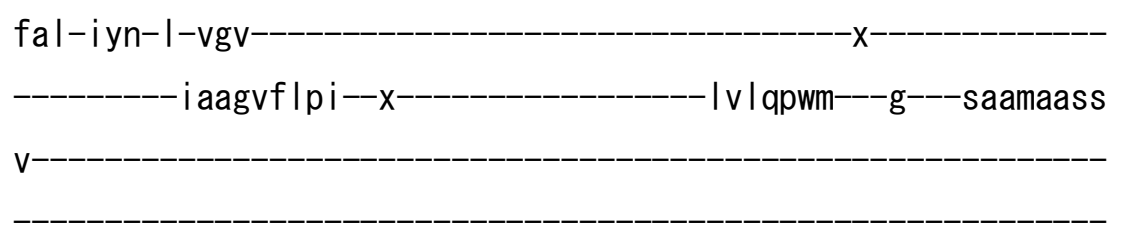

sv-----v|ss | I | kmyqkps-se- $x x x x x x x x x x x x x x x x x------x x x x x x x x x x x x x x x$ $x x x x x x x x--x x x x x x x----------x x-x x x x--$

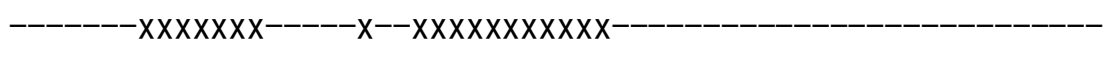
$-x x x x x x$ $\mathrm{xXX}$ $-X X X X$

I

>ggal_IB202 XP_417073. 2

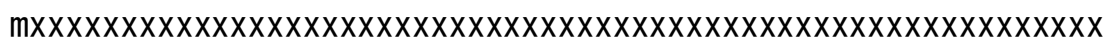

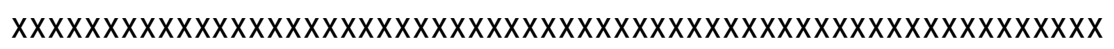

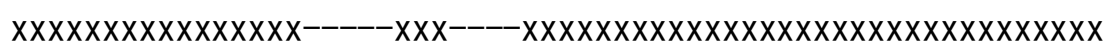




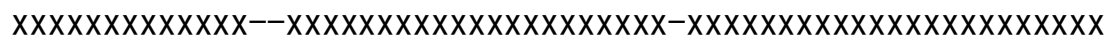

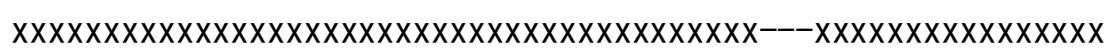

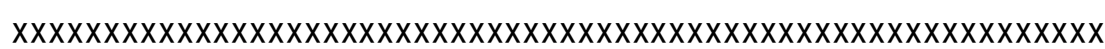
$-x x x$ raai eemgxdas I $x x x x$

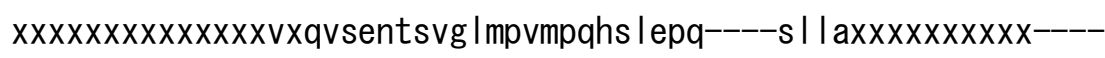
-akkcf Iqitgmtcascvsti-ern I qkedg i i sv I va Imagkae ikykpefi qp I e i aql i qn lgfeatvix $-x x x x x x x x x x x x x x x x t c a s c v h n i$ esk I $-X X X X X X X X X X X X X X X X X X X X X X X X X X X X X X X$

$-x x x x x x x x$ xxx-----------------asvsrrvpnthn I dhkke $\mathrm{i}$

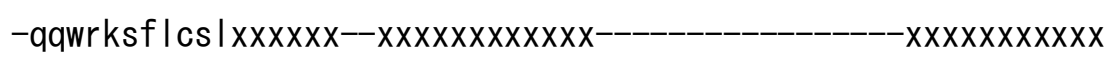
$x x x x x x x x x x x x x x x x x x x x x x x x x x x x x x x x x x x x x x k h-k a x n m d v$ l i v latt $i$ ay $y y s c v$

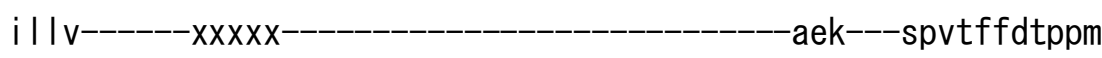

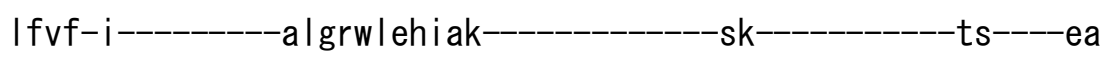
lak I is lqat-eatxvt I

xxxhs ixx $-\mathrm{xxx}--------\mathrm{V}$ $\mathrm{p}$ ----ve IVQRGD IVKVvp GGKFPVDGKV I eg 
-aqmskap i qq ladkfsgyfvpf i i i istvt I - iawitigfinfdi iqxxxxxxxxx-xxxxxxi Irfafats ITVLSIA CPCSLGLAtPTAVMVGTG-VAaqng-

iL IK-GGKPLEMAHK IKTVMFDKTG

-TITCGVPKVm rvl I Igdta

--v lavv-----ogtae-

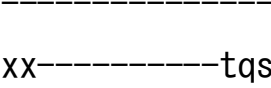

-assehp I g--vavtky-----ckxxtqs $-x x-$

-yctnfqavpgcg i sc-kvggxxxxxxxxxx-

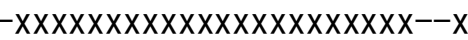

$-\mathrm{i}$ 
-xxv-ndamtdh--etk----gqta ILVAIDgx

ICGMIA I ADTVKQEAALAVHTLKNmg-IDV

VLITGDNRKTAKAIATQVGI

$-k k-$

-VFA--EVLPSHKVAKVQELq

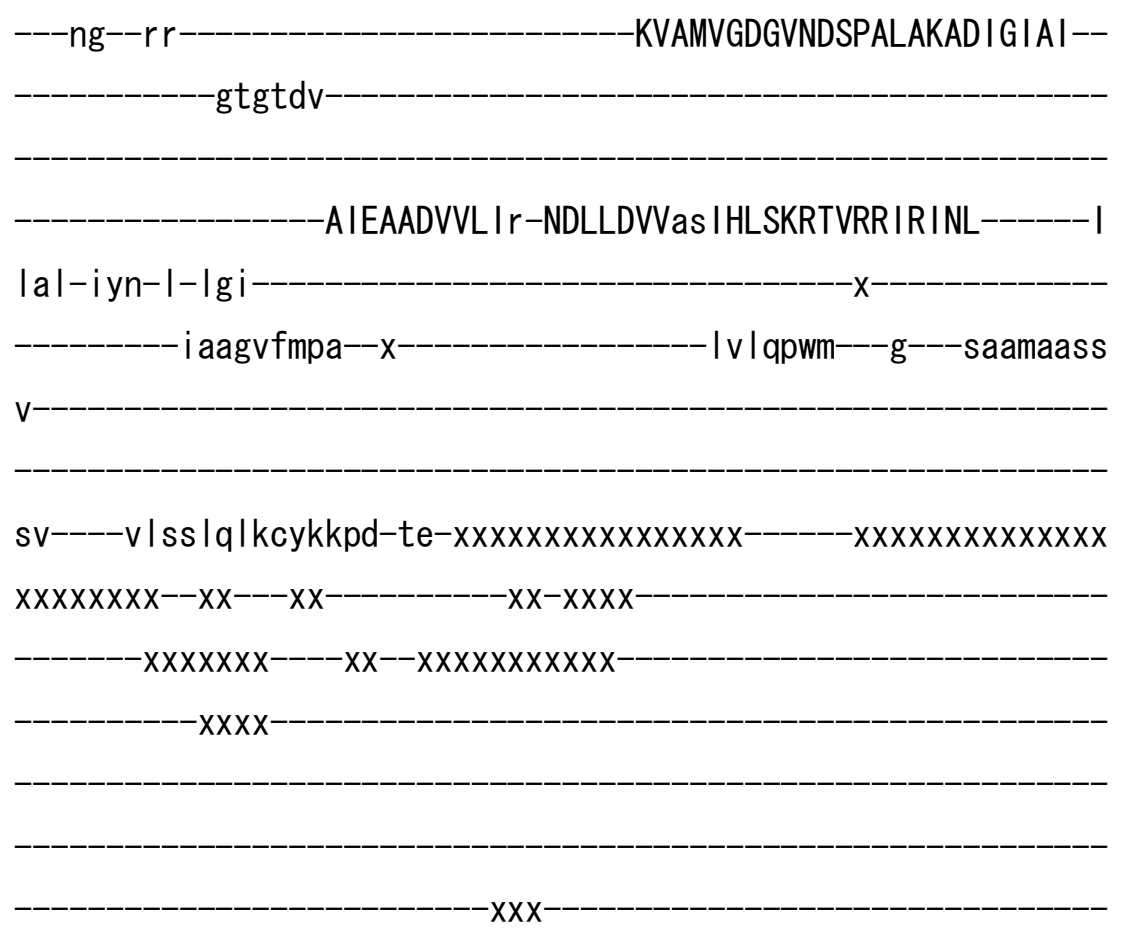




$$
\text { i }
$$

>anil_IB201 EAA63688. 1

$$
\mathrm{m}
$$

$-x x x x x x x x x x$

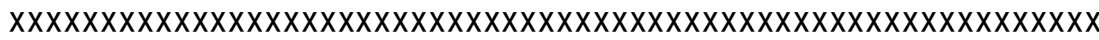
$\mathrm{X}$ $x x x x x x x x x x x x x x x x x$ tatrfphdxrfsi $x x x x$ xxxxxxxxxxxxxxdxcrkegqptdeksqh I snvqgaeaeddsvsxxxxxxxxxxx----kfhar i s i ggmscascsns i $x x$

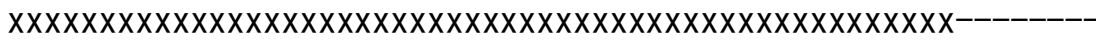
-vdtepsqtan I gy i ae i s i ggmtcgscsgavtqgxxxxxxxxnavvn I mshsaqveldx

$--x x x x x x x x x x x x x x x x x x x x x x x x x k$ kaae $|g s g| e k r t$ $x x x x x x x x x x x x x x x x x x x x x x x x x x x x x$

$-x x x x x x x x$

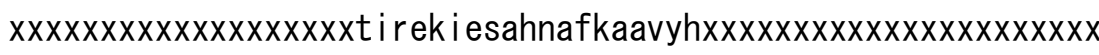

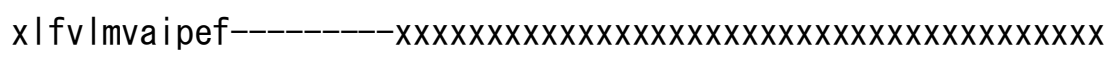


$x x x x x x x x x x x x x x x x x x x x x x x x x x x x x x x x x x x x \mid y-r f x$ smnfl i sagttvayvs la

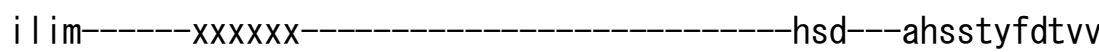

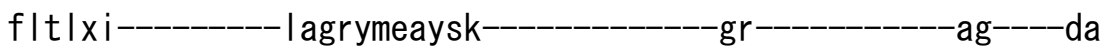
vaalgk $\mid r$

-xxfqFDESSLTGESKPVSKtx-

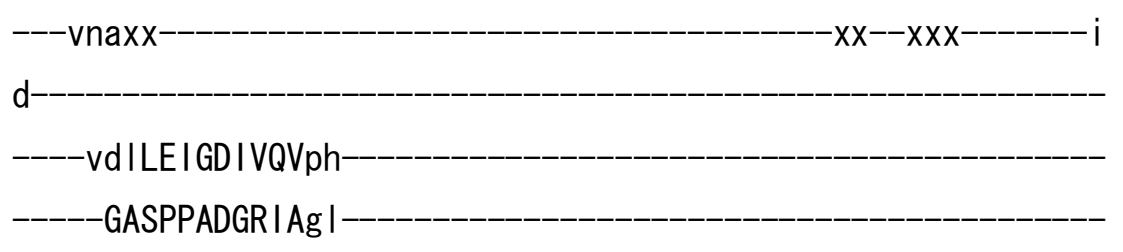

$$
-\mathrm{x}
$$$$
\text { -DQVYT--GSVN- }
$$

vg-----qpve----------_-------------iQITAI GRSSMLDqi i avvre-

-gqgkrap lqrvadt Ivghfvpaitl iav Itf -viwvs I gvsgal psdxxxxxxxx---------------------wpfws I efa I AVFVVA CPCGLALAaPTALFVGGG-LAaryg-

iLVKxGGEAFQEASRLNA IVFDKTG

TLTEGGSLQV xxhes I itdss

xxawava-----rk le 
essnhpia--raiaaf

$-x--x x x p s a$

$x x-$

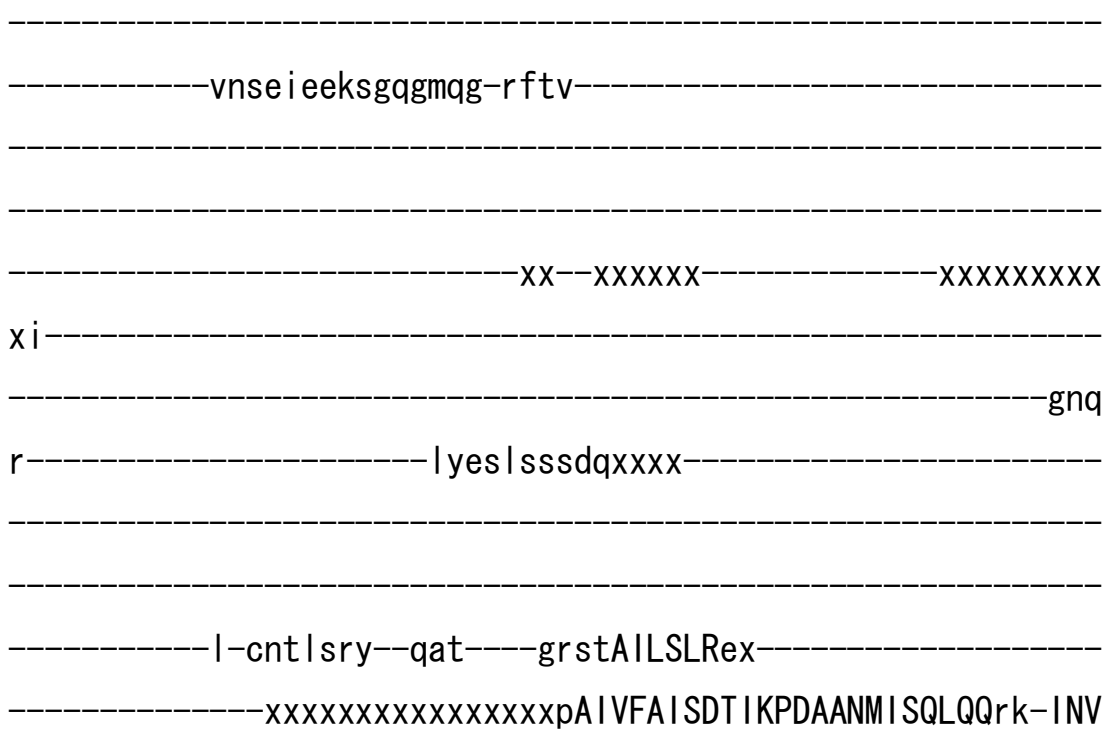

FMCTGDNETTAHAVADMI G I

$-p r x$

-xVLA--NTLPAGKADFVRQIq

---iq--paxxxxxxxxxx-

-IVAFVGDGVNDSPALAAADVSIAM--

asgsdv

-A INSASF I LLn-SELTT ILn I VLLSRRV INR I K INF- 

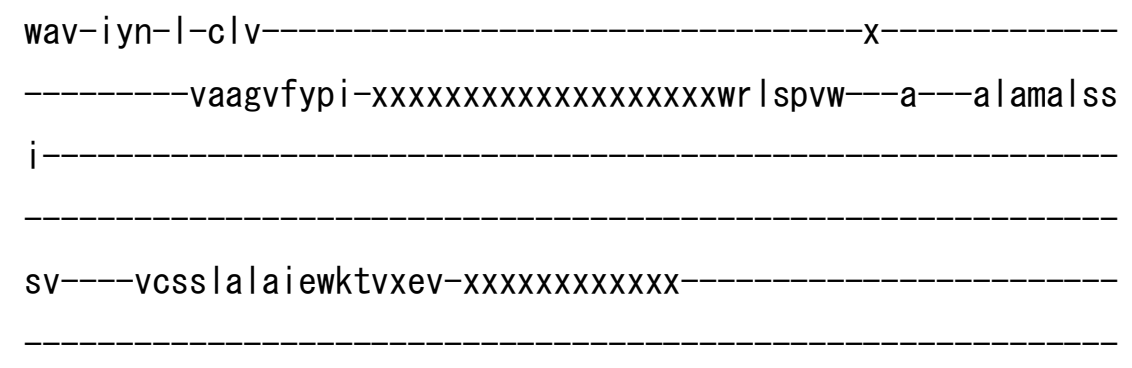

e

>ani I_IB202 EAA59832. 1

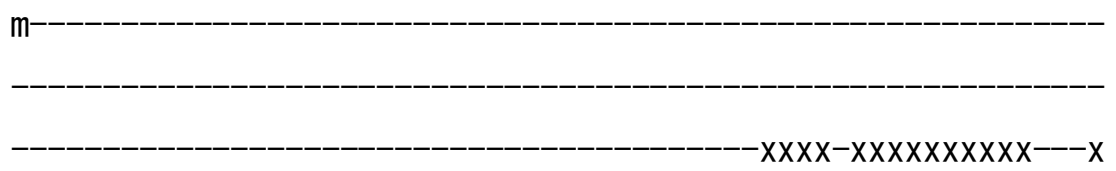

$\mathrm{XXX}$

$-x x-x x x x x$

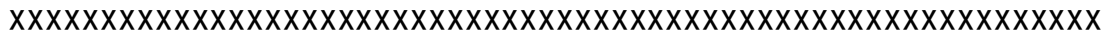
$\mathrm{xXX}$ 
qaafdgvegviqfnis I laera i i thn-pqi lps xkivei i

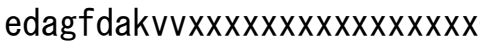

Xxxxxxrdansaaal eds I

$x x x x x x x x x x x x x x x x x x x x x x x x x x x x x x x x$

$x x x x x x x x$

$\mathrm{XXXXXXX}$ -dsddkntqles-laktkev

- lewrrafIfs| $x x x x x x x x x x x x x x x x x x x x x$ $x x x x x$

$x x x x x x x x x x x x x x x x x x x x x x x x x x x x x x x x x x x$ rh-raxtmdv lvm I gtsaaffysvf

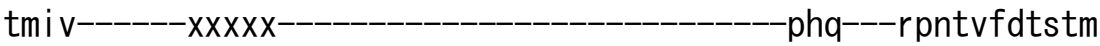

l itf-i----------t l grwlenrak-------------'gq------------ts----aa Isr|ms--|a-

-x-x---tyVDESMI TGEALPIHKax-

$\mathrm{x}--\mathrm{qki}$

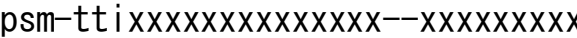

$\mathrm{xxxxxxxxx}$

$-\mathrm{i}$

$\mathrm{p}$

----te I IEVGD IVVLhp

GDKVPADGVVI $r$

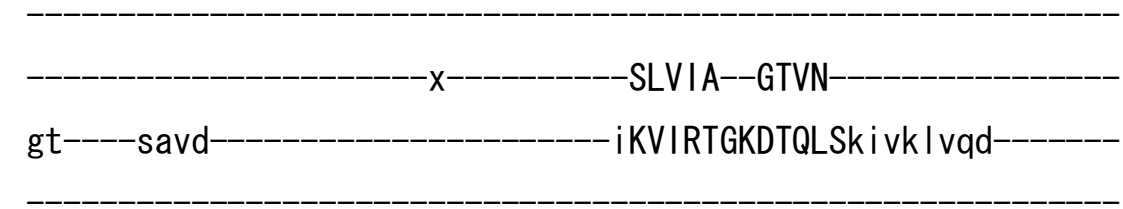

-aqtsras i qrmadi vagyfvps i i I I Ivtf

-fgwmfvsh I lphppkxxxxxxxxx-kvmvc|kIcISVIVFA CPCALGLStPTAVMVGTG-VGatgg- 
iLVK-GGAVLEAATKI THVVYDKTG

-TLTTGQMSVt

ear i ephws

$-x x x x x x$

xxww l i v-----g lae

-mnsehpig--kaihla

$-x x x x x x x--x x x g g \mid$

$-x x-$

-s Igdfear igkgi aa-Ivepxxxxxxxxxx

$-\mathrm{x}$

$-x--$

$-\mathrm{i}$

gna

$a$

$-f$ I qshsvavpx

-xxxt-tpdasgyxxsrv----gitqIHVAID

-xxfAGT I ALQDTVKVTAVAAVAALHRmg-IST

SLITGDSRAAAISIASAVGI

$-a p x$ 
-xVHA--SVSPSDKQSI I ASLq-

--- et--gd-

-RVAMVGDG I NDSPALATASVG I AL--

asgtdv

AMEAAD I VLMr xDDLLSVPasLALSRSVFNR IKLNL $-\mathrm{V}$

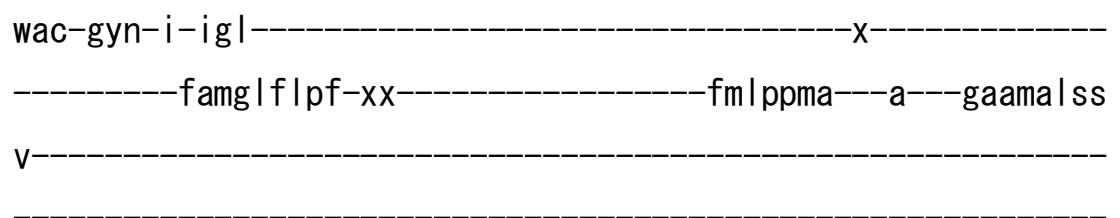

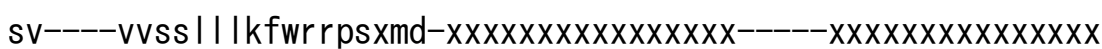
$x x x x x x x x--x x x x x x x-$ $-x x x x x x x$

$-x x x x x x x----x x--x x x x x x x$

V

>mgr I_IB202 XP_361181.1

m- 


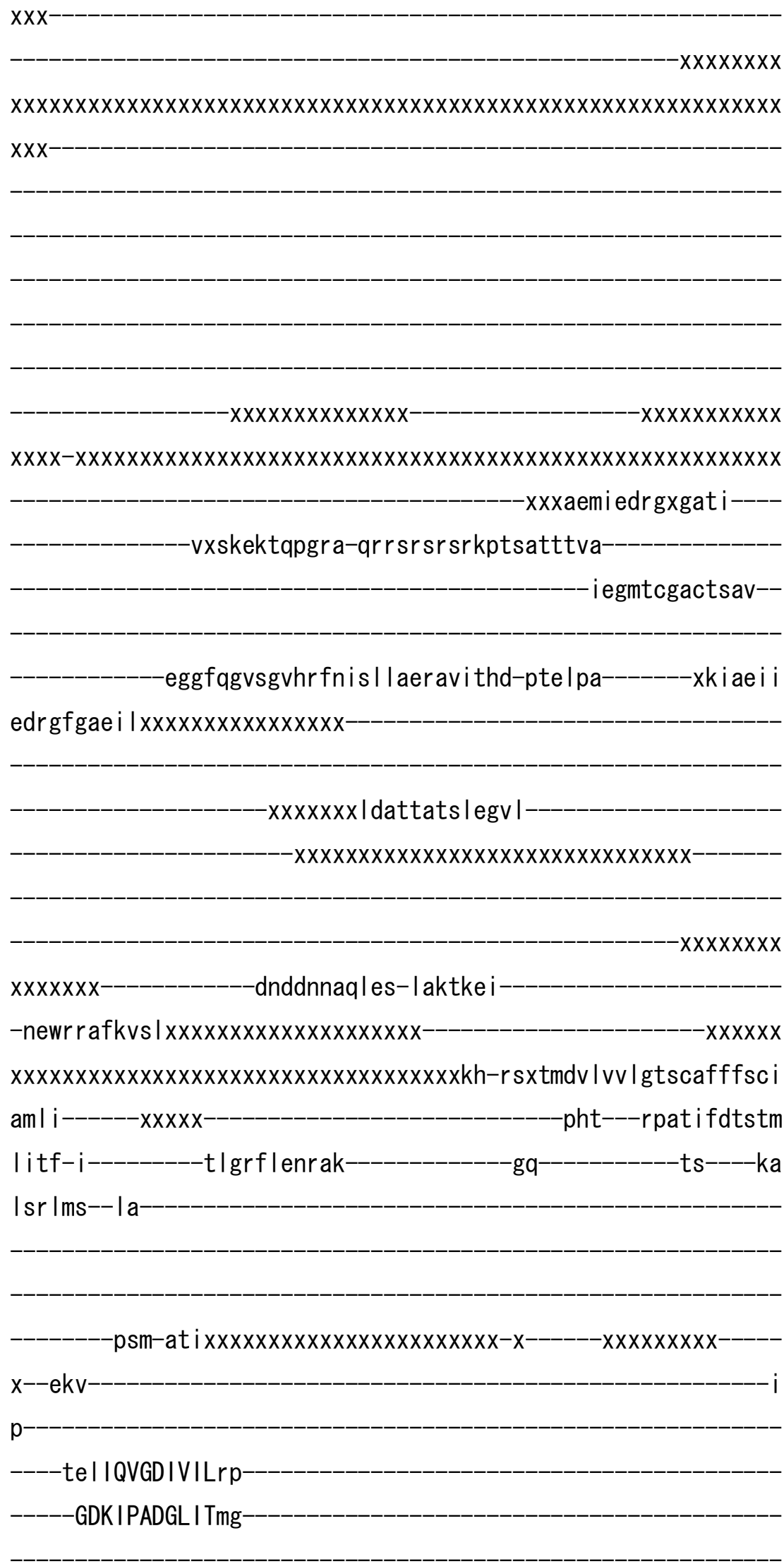




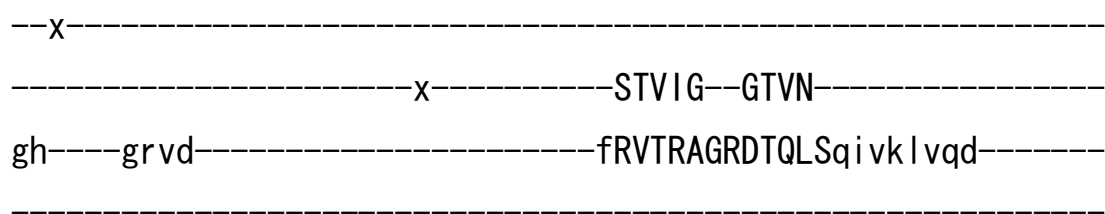

-aqttrapiar ladt lag i fvpti I i lgf Itf -atwmv I shv lanppkxxxxxxxxxx-kvfvclkIcISVIVFA CPCALGLAtPTAVMVGTG-IGaeng-

iLVK-GGAALETATTITKVVLDKTG

TI TYGKMRVa nahi aehwq

$-x X X X X X$

xxwwt i v-----g lae

$-\mathrm{X}$

-

$-\mathrm{V}$

-mgsehpvg--kav lga-

$-x x x x x x x--x x x g t i$

$-x x-$

-svgdfeaavgrg i sa-vvepxxxxxxxxxx

gni

k

-fI rqnnvnvpxxxxx 
xxxxxxxxxxxk-rkagssdxxssa----gttnIFIAIDgx

-yAGHLCLSDT IKEGAAAA I AVLHRmg-IKT

AIVTGDQRSTALAVAASVGI spx

-xVYA--GVSPDQKQA IVKQMq

---eq--ge-----_-_-_-_-_-_-_-_-_-_CVAMVGDG I NDSPALATADVG I AM--

-AMEAADVVLMr xDDLMNIPaaLHLARSIFTR I KMNL------A

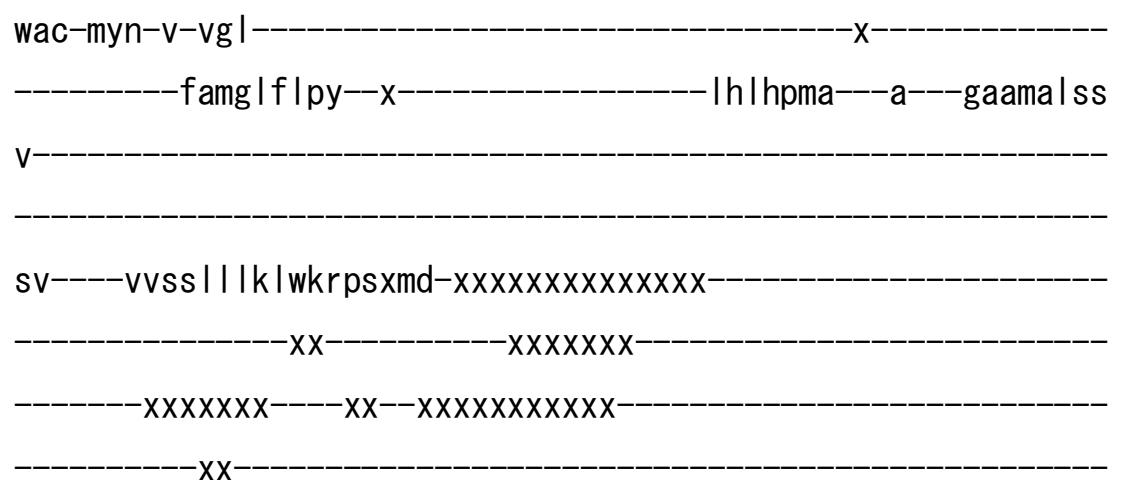

$x x$ 


$$
\text { i }
$$

>mgr I_IB203 XP_361258. 1

$$
\mathrm{m}
$$




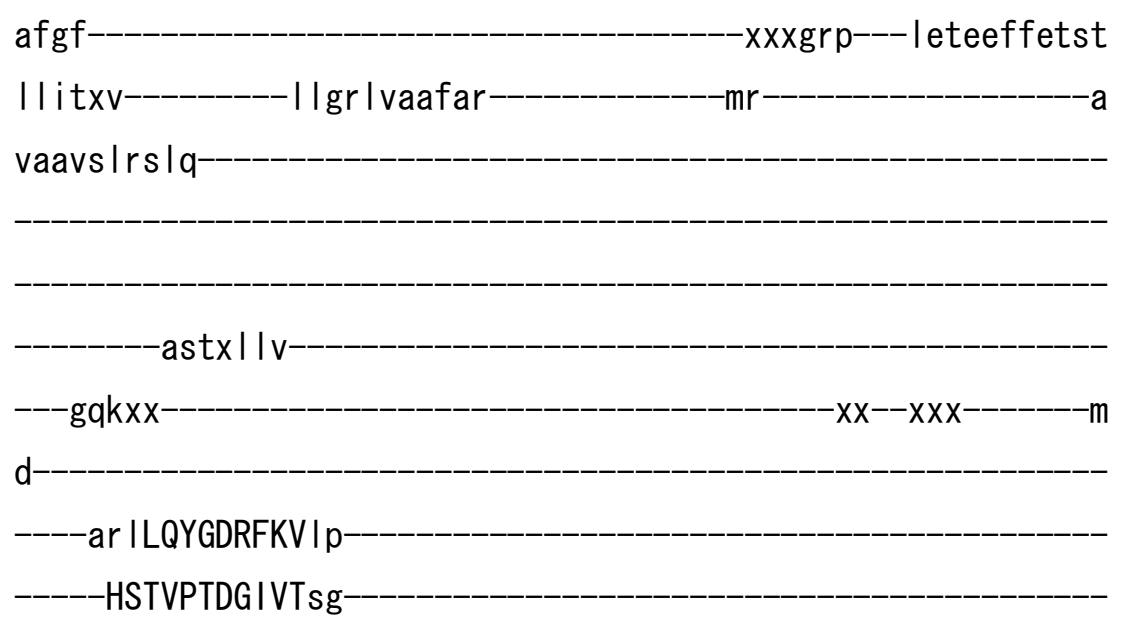

-xseVDESMLTGEAVPVPKex-

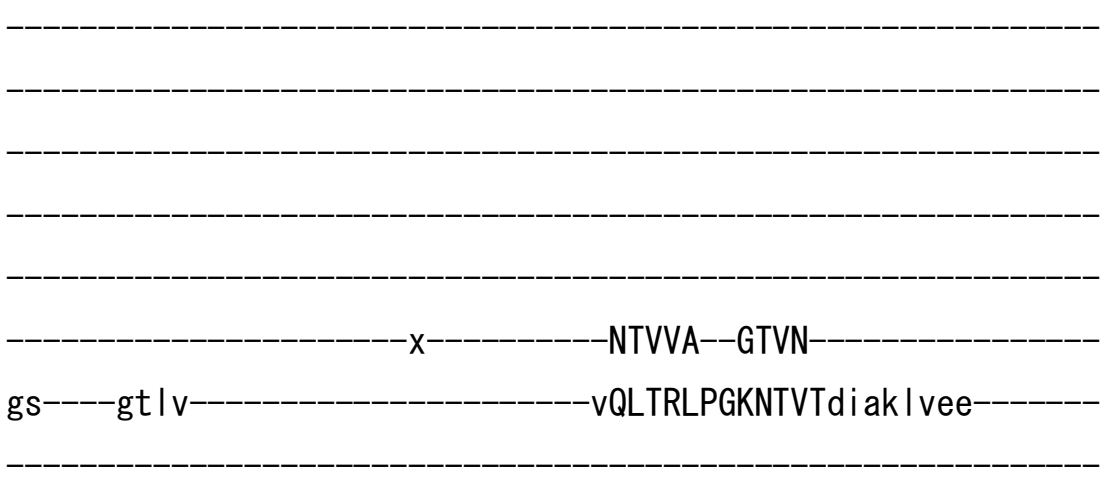

-aanskpkiqdvadrvagwfvpvvtvtai lat

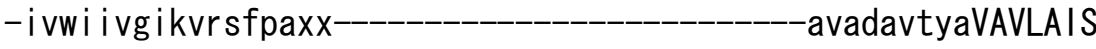

CPCALGLAvPMVLVVAGG-IAargg-

-VVIK-SAESTERARKVTDVVFDKTG

-TITESELDIt------_--_----xdeyp Inh I 
-sgnkhpvs--vavakh-

$-x--x x x t a a$

dI tdvhvvpgfg i ea-kykgxxxxx

-gnp

h

-wtates

-hstvar I--qdq----gmt i FAVSLAdx--x-

IAFYSLRTRLRVDAAAVVKQLQRrg-ISV

HLVSGDQKRAVEAVAAQVGI

XxxxVAS--QYTPAQKREYVAQLm

$-x x x x x x x x$

$x x x q k x x p k$

-VVMFVGDGTNDAVAVAQADVGVQL $x x$

xxxxxxxxxxxaaasev

-TRGAADVVLL--SGLGGVLf ILDISRSASLRMKFNF- 


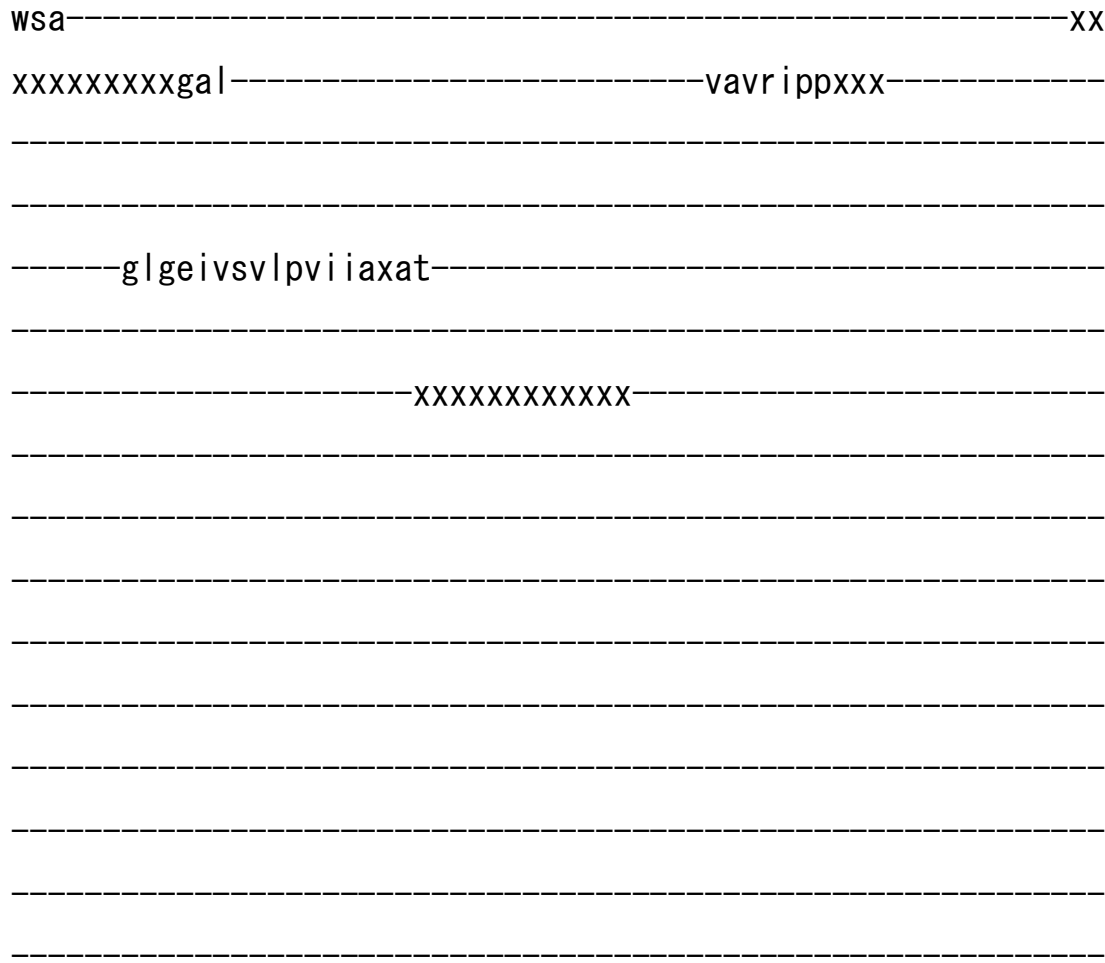

glgeivsvlpvi iaxat

>hsal_IB201 NP_000043. 2

$\mathrm{m}$

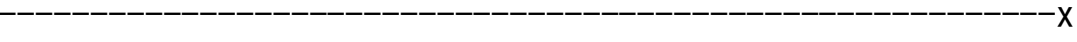

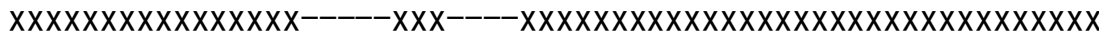

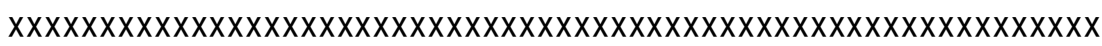

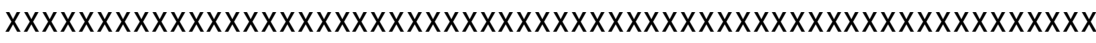

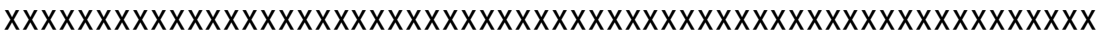
$\mathrm{XXX}$

$x X X X X X X X X X X X X X X X X X X X x X X x$

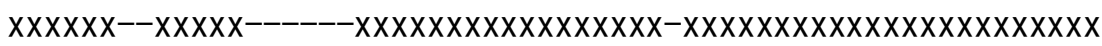

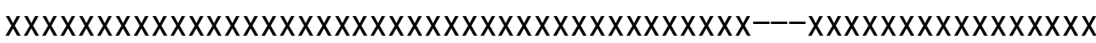

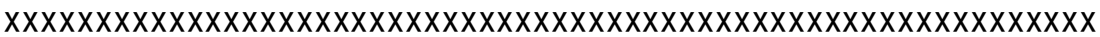
-xxxrgai edmgxdat $1----$ 
-xxxxxp-Ivviaqpssemp I Itstnefytkgmtpvqdxxxxx-----x----sskcy iqvtgmtcascvani-ern I rreegi iss i Ivalmagkaevrynpavi qpp -miaefi

relgfgatvix

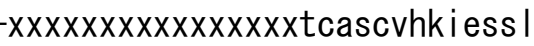

$x x x x x x x x x x x x x x x x x x x x x x x x x x x x x x x x$

$x x x x x x x$

$\mathrm{xxX}$ -as I vkkdrsash I dhkre i

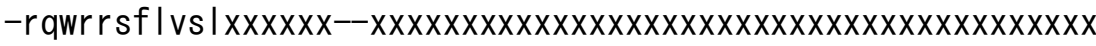
$x x x x x x x x x x x x x x x x x x x x x x x x x x x x x x x x x x x$ kh-kt $x$ nmdv livlatt $i$ afays $\mid \mathrm{i}$

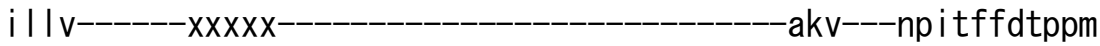
I fvf-i----------a l grw lehi ak-------------gk------------ts----ea lakl is lqat -eatxvt I

xxxni I xx-

$-\mathrm{xxx}--------\mathrm{v}$

$d$

----ve IVQRGD I IKVvp

GGKFPVDGRV I eg

-xsmVDESL I TGEAMPVAKkx-

$$
-x
$$

STVIA--GSIN-

qn----gs |

iCATHVGADTTLSqi vk I vee

-aqtskap iqqfadk I sgyfvpf i vfvs iat I

- IvwivigfInfe ivexxxxxxxxx-xxxxxx i irfafqas ITVLCIA CPCSLGLAtPTAVMVGTG-VGaqng- 
iLIK-GGEPLEMAHKVKVVVFDKTG-

TITHGTPVVn-

qvkvItesn

$-x \times x x x--x--$

--i la iv-----gtae-

-snsehp I g--taitky----ckxx----

$-x x$

tet

$-x X$

-tc idfavvpgcg i sc-kvtnxxxxxxxxxx

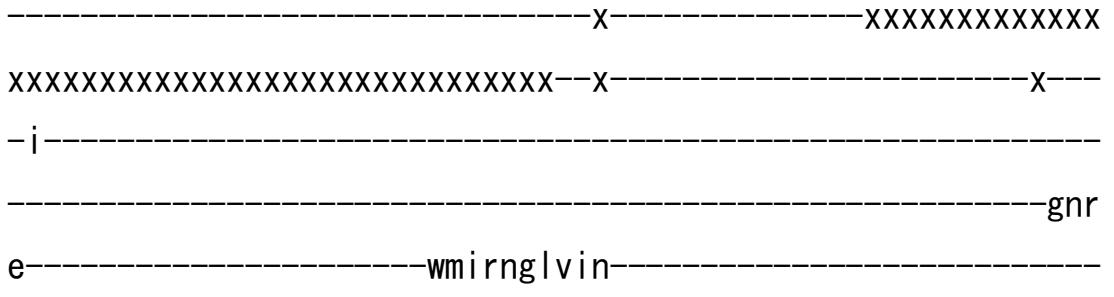

-xxv-ndfmteh--erk----grtaVLVAVDdx

I CGL IA I ADTVKPEAELAIHILKSmg-LEV

VLMTGDNSKTARS I ASQVG I

-tk 


\section{-VFA--EVLPSHKVAKVKQLq}

---ee--gk--_-_-_-_-_-_-_-_-_-_-_--_RVAMVGDG I NDSPALAMANVG I AI --

AIEAADVVL I r-NDLLDVVas IDLSRETVKRIR INF $-\mathrm{V}$

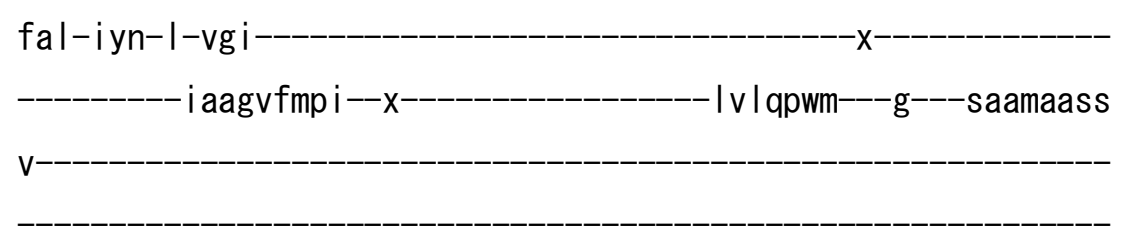

sv-----v|ss|f|k|yrkpt-ye- $x x x x x x x x x x x x x x x x x------x x x x x x x x x x x x x x x$

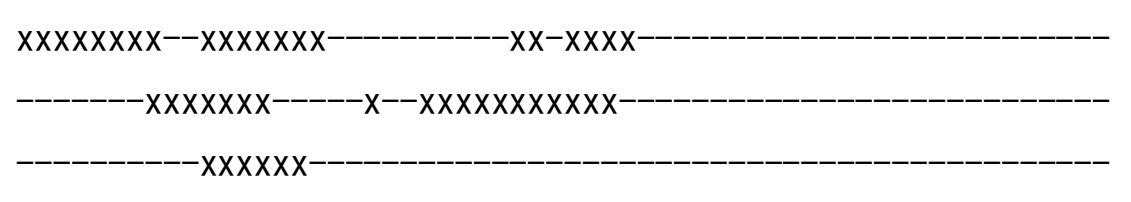
$\mathrm{XXX}$

।

>hsal_IB202 NP_000044. 2

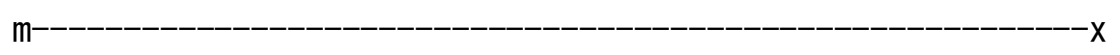

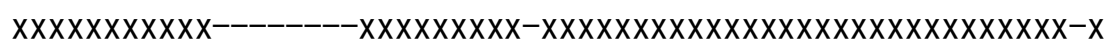

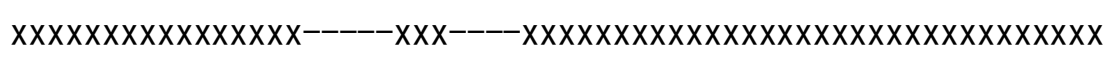




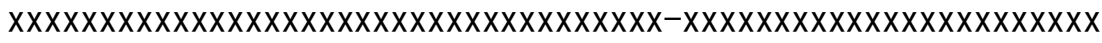

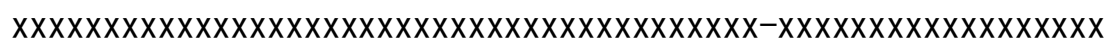

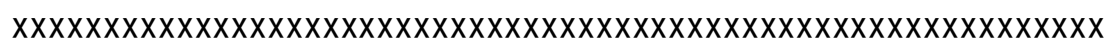

-xxxraai edmgxeasvxxxx xxxxxxxxxxxxxxmxqttdgtptsvqevaphtgr I panhapd i laxxxxxxxxxxx----pqkcf Iqikgmtcascvsni-ern I qkeagv I sv I va I magkae i kydpevi qp I e i aqfi qd I gfeaavmx $-x x x x x x x x x x x x x x x x t$ cascrhniesk

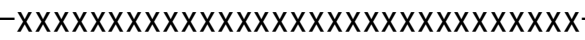

$-x x x x x x x$ $\mathrm{XXX}$ as I aqrnpnahh I dhkme i

-kqwkksflcs|xxxxxx--xxxxxxxxxxxx $x x x x x x x x x x x$

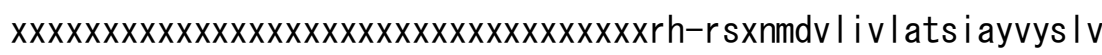

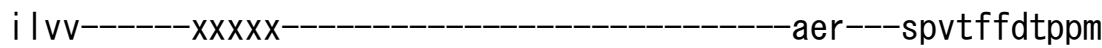

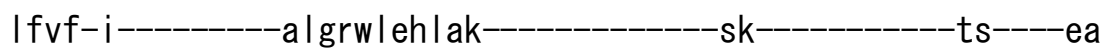
lak Ims lqat-eatxvt I

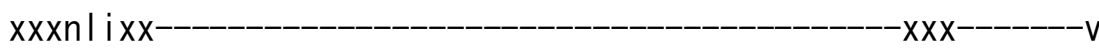

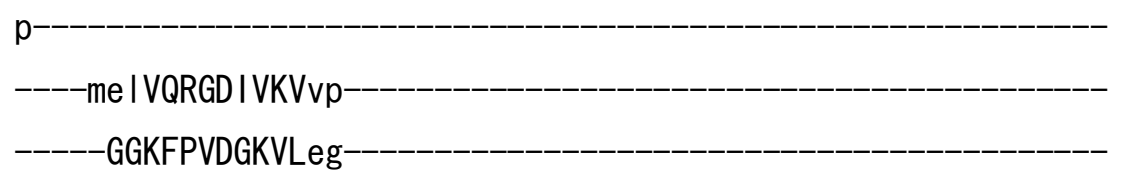


ah----gsvl-

-aqmskap iqq ladrfsgyfvpf i i imst It I -vvwivigfidfgvvqxxxxxxxxx-xxxxxxi irfafats ITVLCIA CPCSLGLAtPTAVMVGTG-VAaqng-

iLIK-GGKPLEMAHK IKTVMFDKTG-TI THGVPRVm-rvIIIgdva -tet$-x x-$

-yctdfqavpgcg i gc-kvsnxxxxxxxxxx

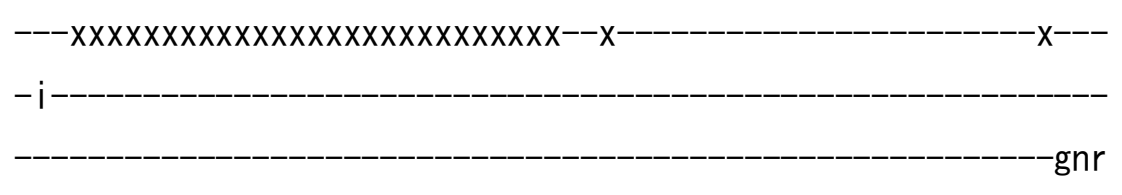
e-wlrrngltis 
-xxv-sdamtdh--emk----gqta ILVAIDgx

I CGMI A I ADAVKQEAALAVHTLQSmg-VDV

VLITGDNRKTARA IATQVGI

$-n k$

-VFA--EVLPSHKVAKVQELq

$---n k--g k$

KVAMVGDGVNDSPALAQADMGVAI--

gtgtdv

-AIEAADVVL I r-NDLLDVVas I HLSKRTVRR IRINL

$-\mathrm{V}$

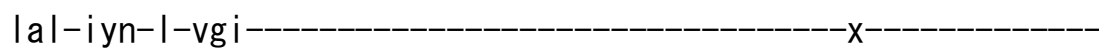

-i aagvfmp i--x--------------------i v l qpwm---g----saamaass

$\mathrm{V}$

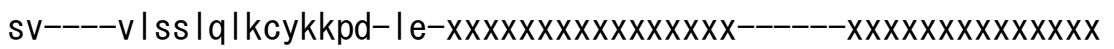
$x x x x x x x x--x x x x x x x---------x x-x x x x$

$-x x x x x x x----x x--x x x x x x x x x x x$

$x \times x x$ 
i

>hsal_IB203 XP_932923. 2

$$
\text { m }
$$

$-\mathrm{XXXXX}$

$-x x x x x x x x x x x x x x x$

-xxxxxp- I vvi aqpssemp I I tstnefytkgmtpvqdxxxxx-----x---_ -sskcy iqvtgmtcascvani--

-ernI rreegi ys i I va Imagkaevrynpavi ipp

$-m i a e f i$

relgfgatvix-

$-x x x x x x x x x x x x x x x x t c a s c v h k i$ ess I

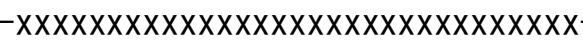

$-x x x x x x x x$

xxx---_-_-_-_----as I vkkdrsash I dhkre $\mathrm{i}$

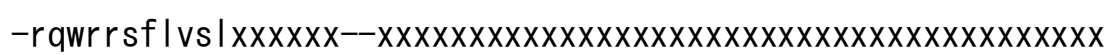


$x x x x x x x x x x x x x x x x x x x x x x x x x x x x x x x x x x x x k h-k$ txnmdv $\mid$ ivlatt $i$ afays $I i$

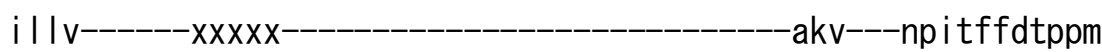
Ifvf-i----------a l grwl ehi ak--------------gk-----------ts----ea lakl is lqat-eatxvt I

xxxni Ixx$-\mathrm{XXX}-------V$ $d$

-xsmVDESL I TGEAMPVAKkx$-\mathrm{x}$ STVIA--GSINqn----gs I I--_-_-_-_-_-_-_-_-_-_iCATHVGADTTLSqi vk I vee-aqtskap iqqfadk I sgyfvpf i vfvsiat I -I vwivigfInfe i vexxxxxxxxx--------------xxxxxx i irfafqas ITVLCIA CPCSLGLAtPTAVMVGTG-VGaqng-

-iLIK-GGEPLEMAHKVKVVVFDKTG

-TI THGTPVVn -qvkvItesn-

--i la iv-----gtae- 


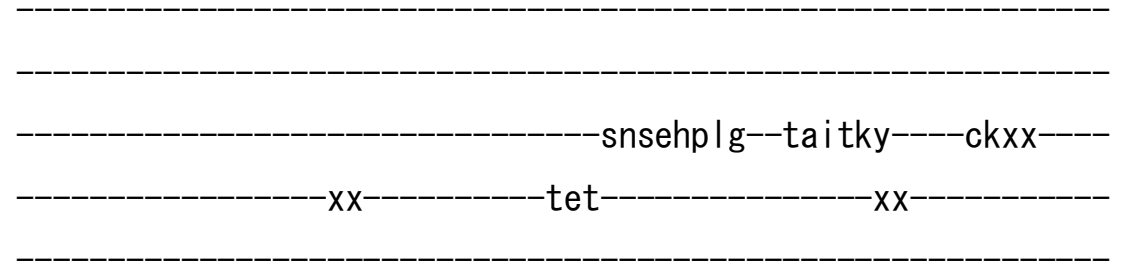

-tc i dfqvvpgcg i sc-kvtnxxxxxxxxxxx

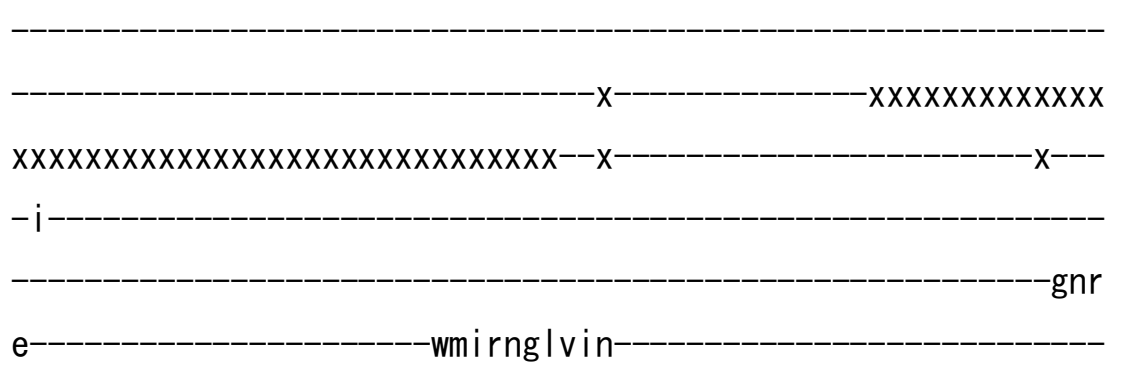

-xxv-ndfmteh--erk----grtaVLAPILdx

I CGL IA I ADTVKPEAELAIHILKSmg-LEV

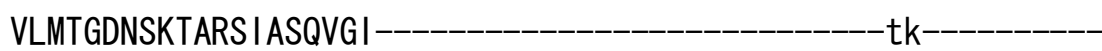

-VFA--EVLPSHKVAKVKQLq

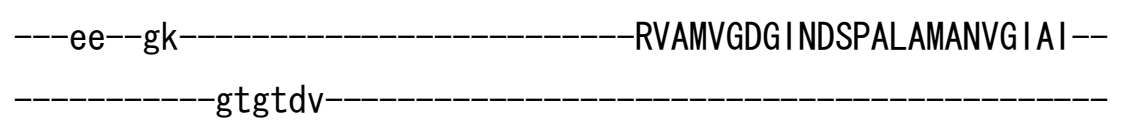

-A IEAADVVL I r-NDLLDVVas I DLSRKTVKRIRINF-------V 
fal-iyn-I-vgi

$-\mathrm{x}$

-i aagvfmp i--x--_-_-_-_-_-_-_-_--|v l qpwm----g---saamaass

$\mathrm{V}$

sv----v|ss|f|k|yrkpt-ye- $x x x x x x x x x x x x x x x x x{ }^{------} x x x x x x x x x x x x x x x$

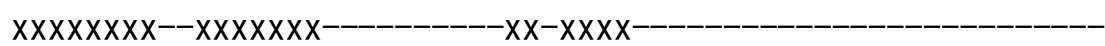
$-x x x x x x x-----x--x x x x x x x x x x x$ $x x x x x x$

$x x x$

।

>dmeI_IB201 NP_572756. 2

$\mathrm{m}$

$-x x x x x x x x x x x x x$

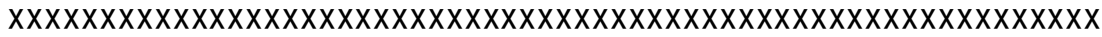
$x x x$

$-x x x x x x x$

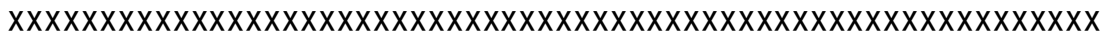
$-x x x a e$ l iddmgxeasvxxxx 


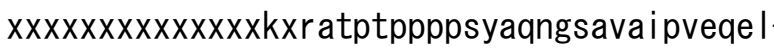

Itkcflhirgmtcascvaai--

ekhckkiyg I ds i I val l aakaevkfnanvtae

-niaksi

telgfptelix

$-x x x x x x x x x x x x x x x x t c a s c v n k i$ eshv-

$x x x x x x x x x x x x x x x x x x x x x x x x x x x x x x x x$

$-x x x x x x x x$

xxxxx---_-_-_-_----Imtgrdkmahny l ehkee

-rkwrnaf|vs| $x x x x x x x x x x x x x x x x x x x x x$

$-x x x x x x x$

$x x x x x x x x x x x x x x x x x x x x x x x x x x x x x x x x x x x x$ h

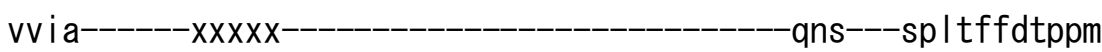

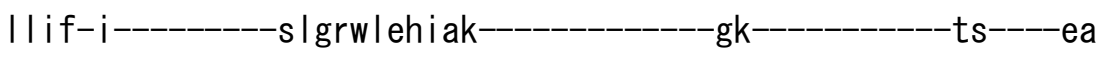
|sk | s lkaa-

dalxve i

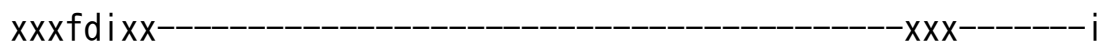

S---_-_-_-_-_-_-_-_-_-_-_-_-_-_-_-_-_-_-_-_-_-_-_-_-_-_-_-_-_-_-

----vdyVQRGDILKV ip

GAKVPVDGKVLyg

-xssCDESL I TGESMPVAKr--

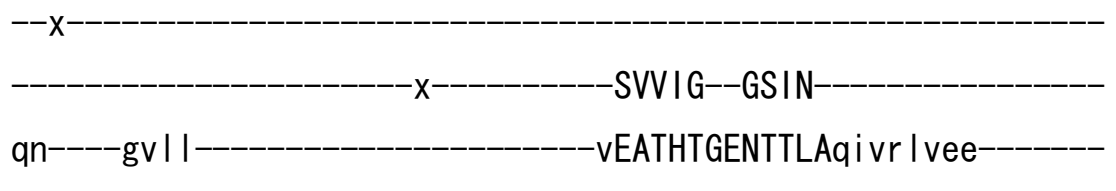

-aqtskap iqq ladr iagyfvpfvvvvssit I

- iaw i i i gfsnpnlvpxxxxxxxxxxxxxxi vsyafkcaLSVLAIA

CPCALGLAtPTAVMVATG-TGaing- 
-vLVK-GATALENAHKVKTVVFDKTG-
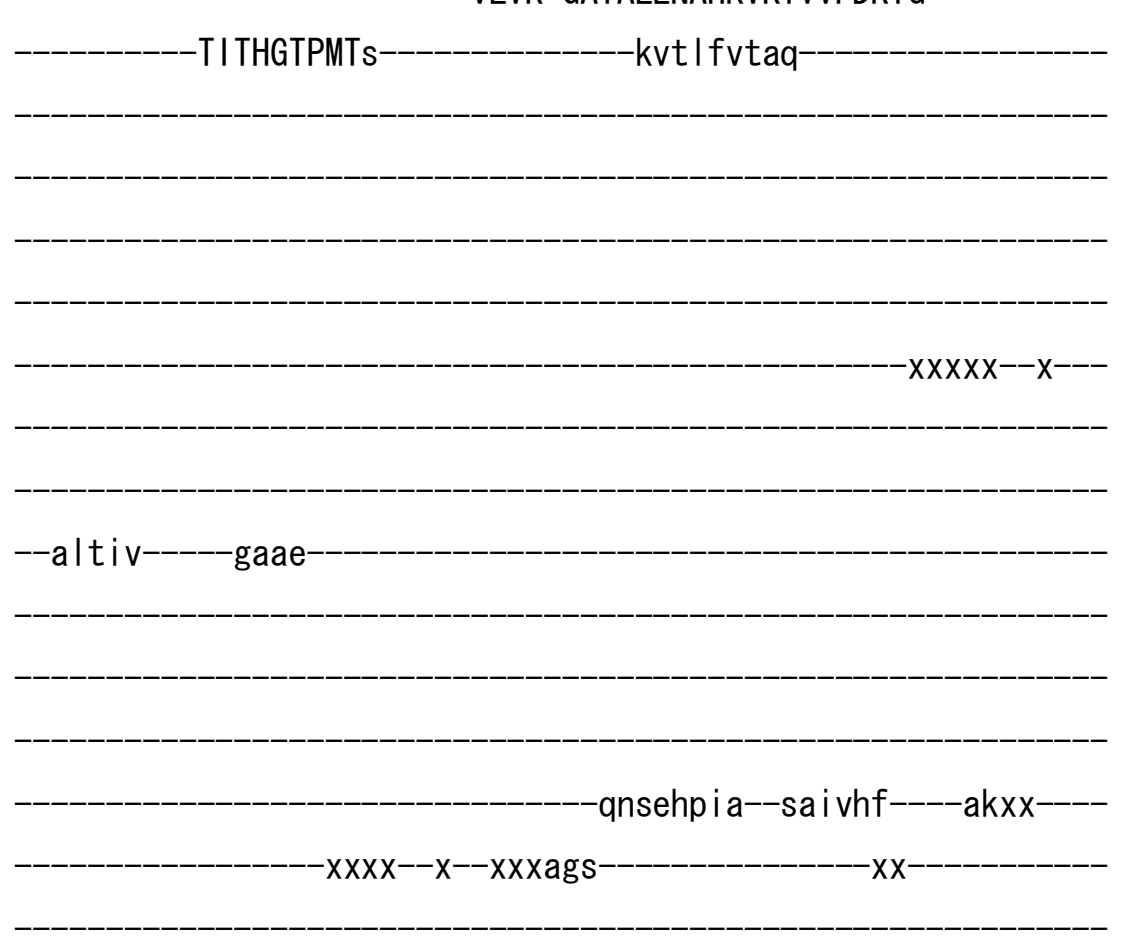

-ksshfqavpgcgirv-tvsnxxxxxxxx -

$x X X X X X X X$
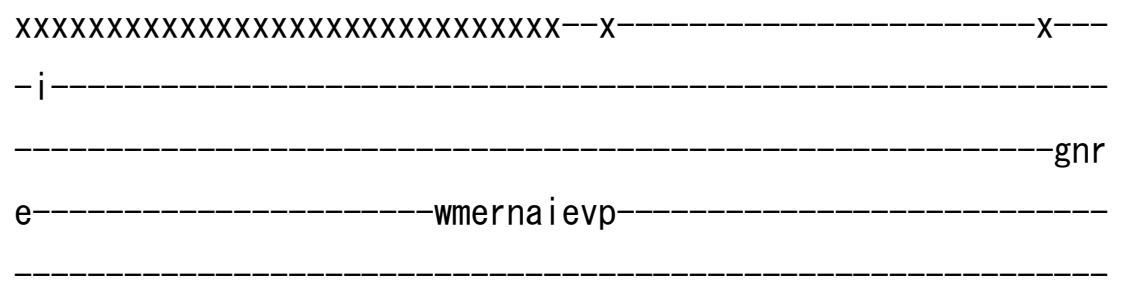

-xxi-sdcmthe--erk----ghtaVLCALNg-

- I IVCMFAVSDMVKPEAHLAVYTLKRmg-IDV

VLLTGDNKNTAAS I AREVG I

$r t-$ 
$-V Y A--E V L P S H K V A K I Q R I q$

---an--gi--------------------------RVAMVGDGVNDSPALAQADVGITI--

-aagtdv-

AAEASD IVLMr-NDLLDVVacLDLSRCTVRR IRYNF$\mathrm{F}$

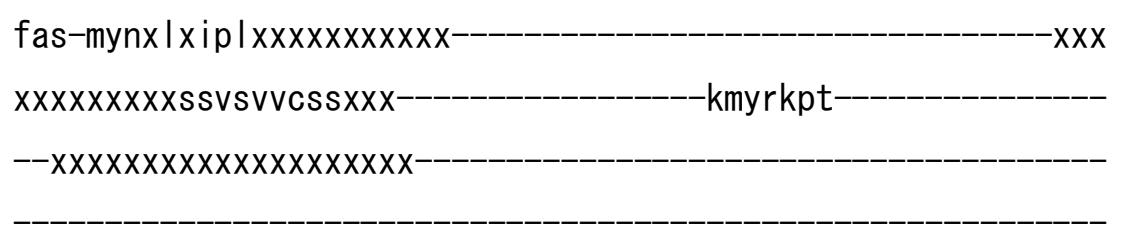

sexxxxk|s|hrg|dd|pekxrm------------------ $x x x x x x x x x x x x x x x$ $x x x x x x x x x x x x x x x x x-$

।

>pfaI_IB201 XP_001351923. 1

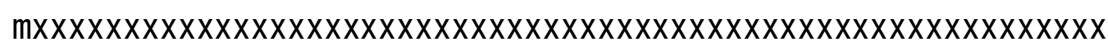

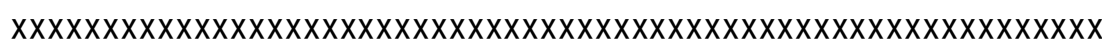
XXXXXXXXXXXXXXXXXXXXXXXXXXXXXXXXXXXXXXXXXXXXXXXXXXXXXXXXXXXX 


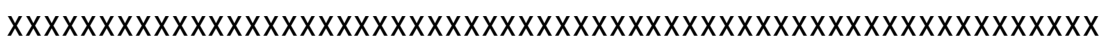

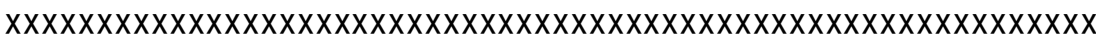
$X X X X X X X X X X X X X X X X X X X X X X X X X X X X X X X X X X X X X X X X X X X X X X X X X X X X X X X X X X X X$

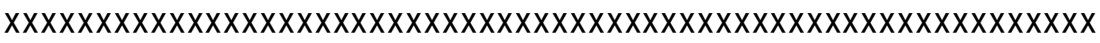

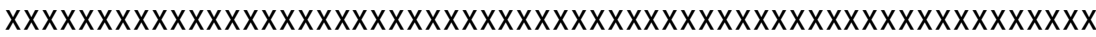

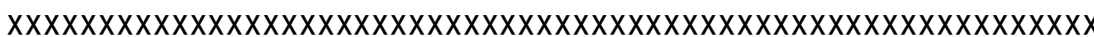

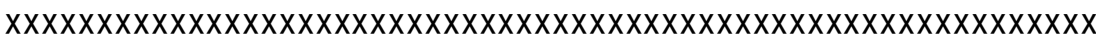

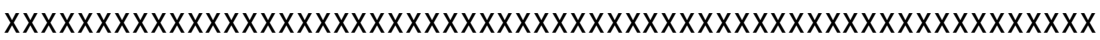

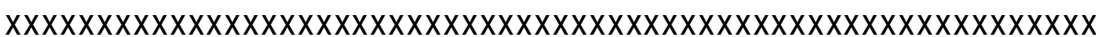

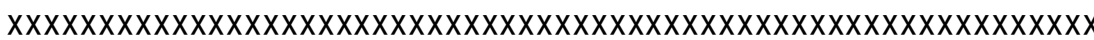

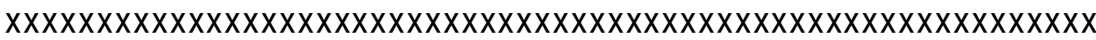

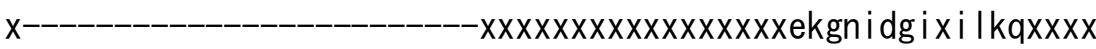
xxxxxxxxxxxxxxsxkeeksdnensnee i dknyny lkrkekdhnsxxxxxxxxxx---$-v \mid$ kenvkeee ihesssneqs $x x$

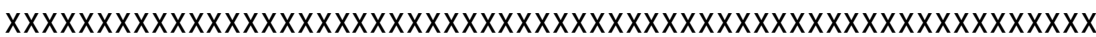

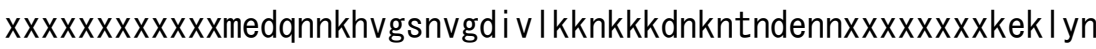

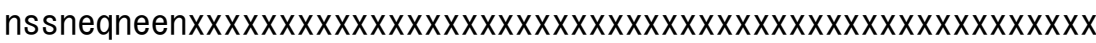

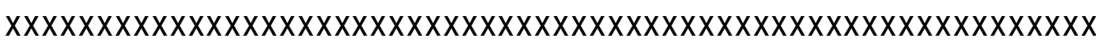

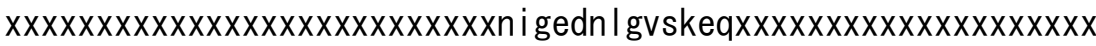

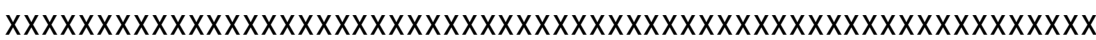

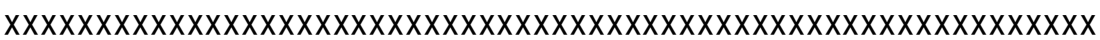

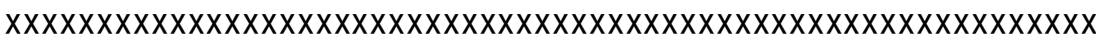

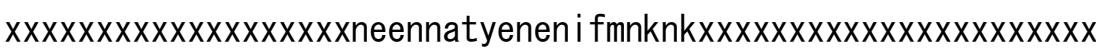

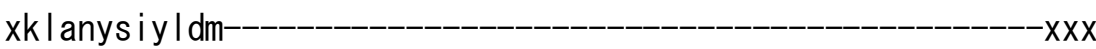
$x x x x x x x x x x x x x x x x x x x x x x x x x x x x x x x x x x x x x x x k n-n m x n m n v \mid$ is isssfsyfys |

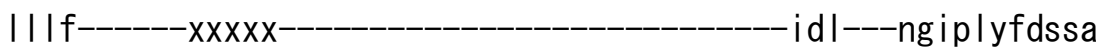

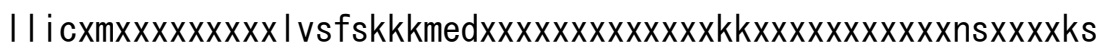
msniskafsk

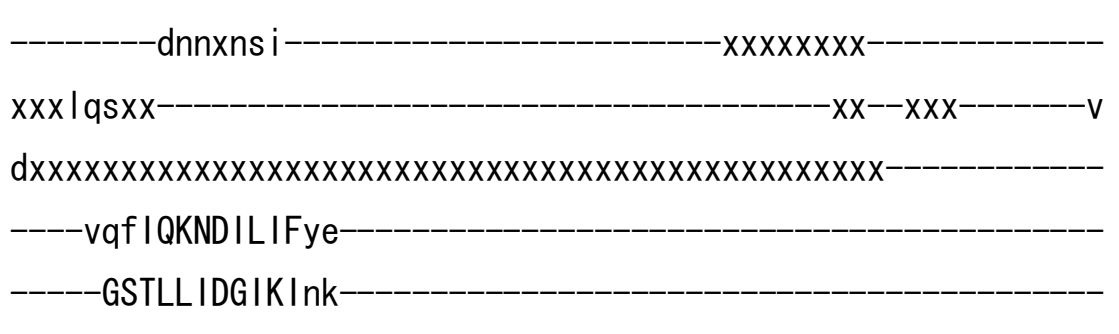




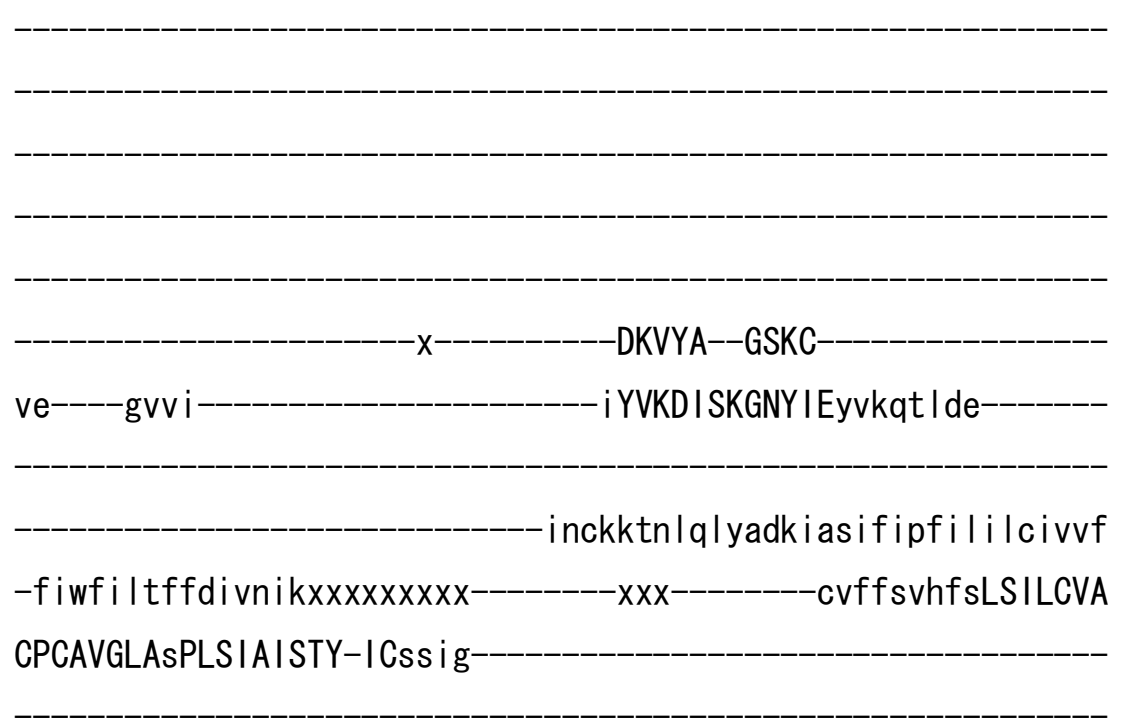

i I IK-NINIFE IFLECKHF IFDKTG-

-TLTVGKPVVn-

$-k i$ isnnid-

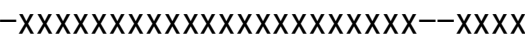

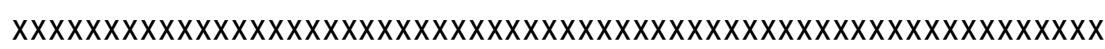

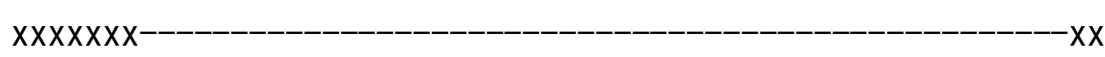

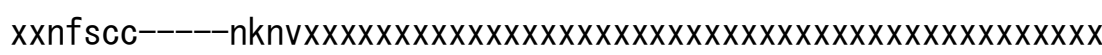

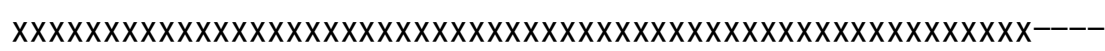

$x x x x x x x x x x x x x x x x x x x x x$

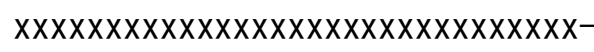

-qnmssp l exxnsymek----ssxxxxxx $-x x x x x x x--x x x s n h-$

$-x x$

$-x x x x x x x x x x x n f$ s inevfdvnn lknxknqgxxxxxxxxxxx

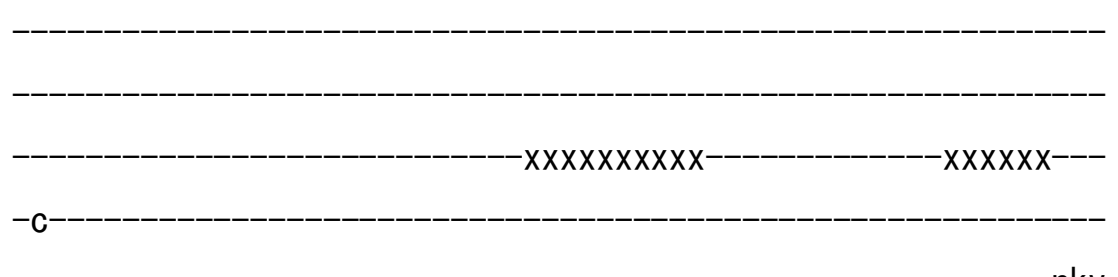
$-n k v$ p i i eek Itvksxxxxx 
$x x x--x x x x x x t$-yqy lysy--snsxxxxsnni IFMCIEgx

-vVGFFTLVDN I KPEVFEL I NFLKRek-KKV

YVCTGDNYMNALYISKILGI

$-k k x$

-xVSS--NTLPLEKVQFVKKVq

---sm-xdg-

-KVCM I GDG I NDCFALKTADLGLSL--

ctrtnv

-VMDSADAC I Vd-NN I NV I I I IFE I SRKTLLV I KFNF

fsf-finxf-

$--x x x x x x x x x x x x-$

xxvfspfIftf Imfcsxi i

$-x x x x x x x x x x$ 


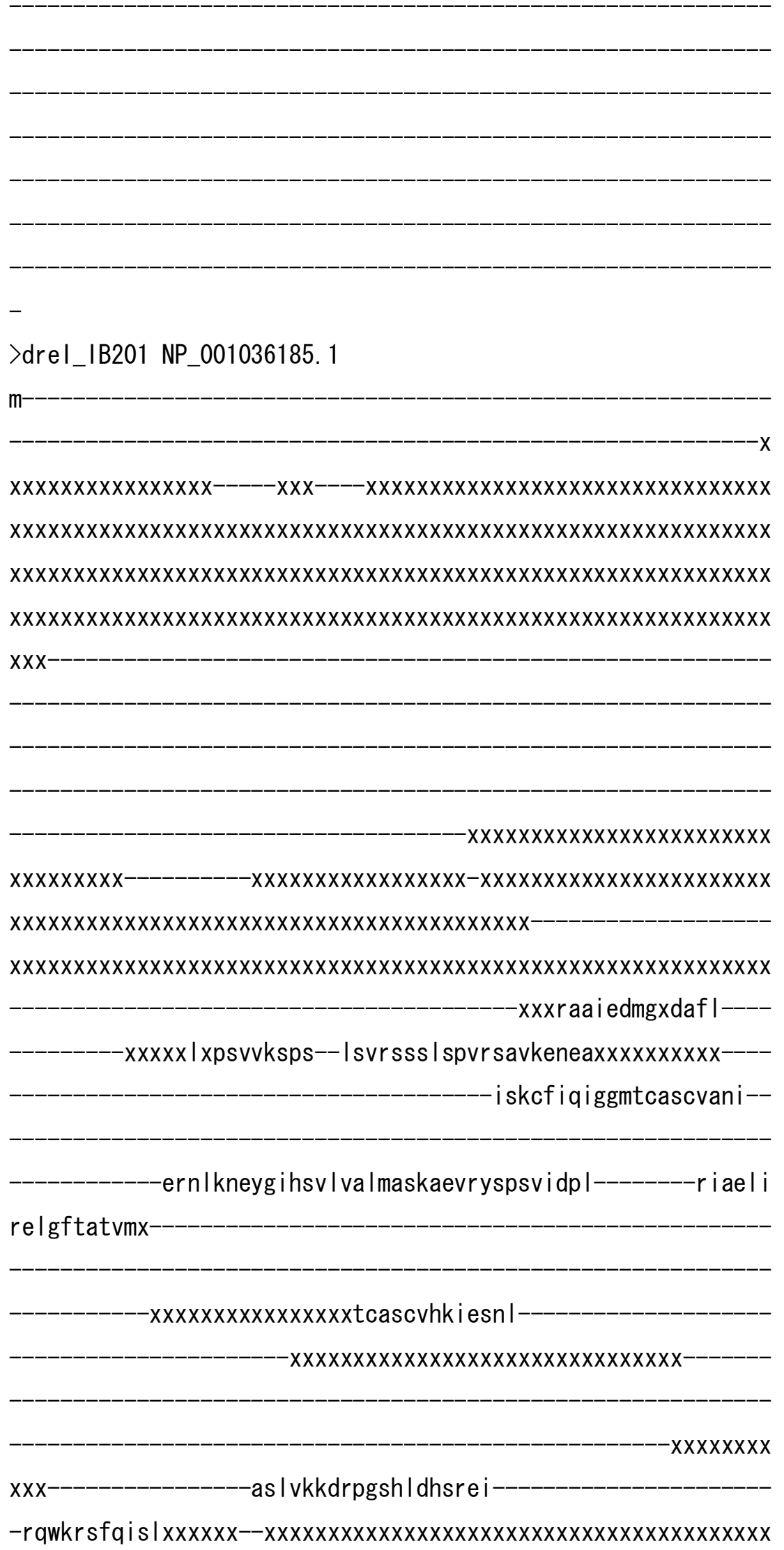


$x x x x x x x x x x x x x x x x x x x x x x x x x x x x x x x x x x x x$ kh- $r$ txnmdv l ivlatt iaftys $v$

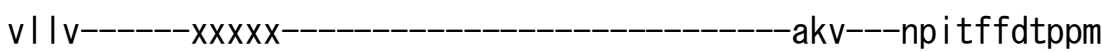

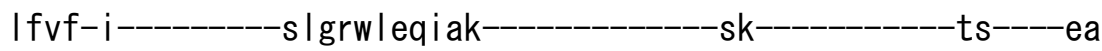

Isk Ims lqat-

eatxvt I

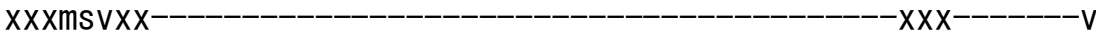

$d$

-xsmADESL I TGEAMPVTKkx-

STVIA--GSIN-

qn----gs | I--_-_-_-_-_-_-_-_-_-_iKATHVGTDTTLSqi vk I vee-

-aqtskap iqqfadk issyfvpfivvisvitl

- law i i i gfvnfs I vqxxxxxxxxx-------------xxxxxxvirfafqas I TVLCIA CPCSLGLAtPTAVMVGTG-VGaqng-

-iLIK-GGEPLEMAHKIQSVVFDKTG

TI TYGAPKVV

qvkmlaegn-

$-\mathrm{XXXXX--X---}$

--l la iv-----gtae- 


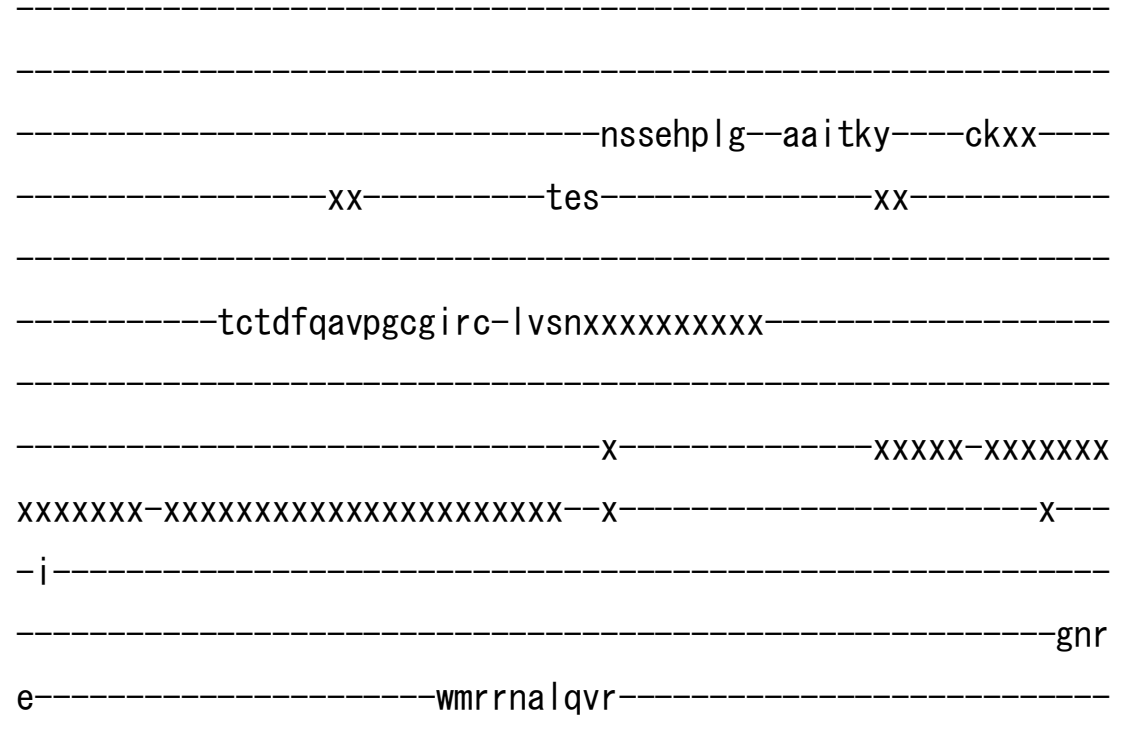

-xxv-deamteh--err----gctaVLVAVDnx-

I CAMVA I ADTVKPEAELAVHVLSAmg-LEV

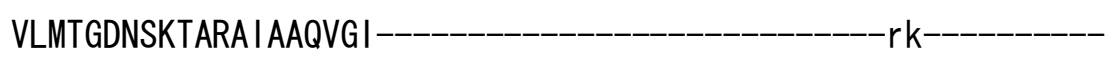

-VFA--EVLPSHKVAKVEQLq-

---qe--gk--_-_-_-_-_-_-_-_-_-_-_-_-_-_RVAMVGDGVNDSPALAMADVGIAI--

-AIEAADVVL I r-NDLLDVVgs IDLSKKTVKR IRINF------V 
fal-myn-I-vg

$-\mathrm{x}$

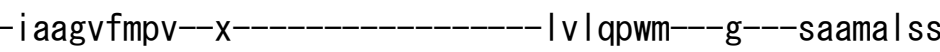

$\mathrm{v}$

sv----v|ss | I lkcytkpt-ve- $x x x x x x x x x x x x x x x x x------x x x x x x x x x x x x x x x$

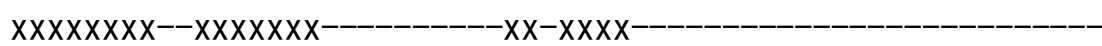

$-x x x x x x x-----x--x x x x x x x x x x x$

$-x x x x x$

$x x x-$

$-x x x^{-}$

V

$>$ dre I_IB203 XP_684415. 2

$\mathrm{m}$

$x x x x x x x x x x x x x x$

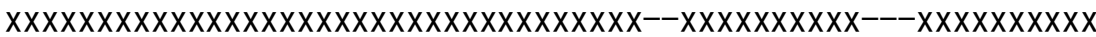

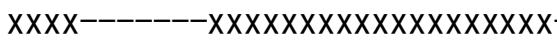

$-x x x x x x x$

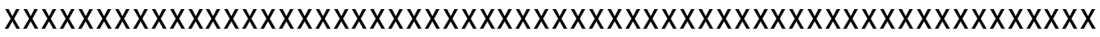
$x x x$

$-X X X X X X X X X X X X X X X X X X X$

$-x x x x x x x x x-x x x x x x x x x x x x x x x x x x x x x x x x$

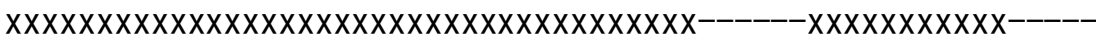

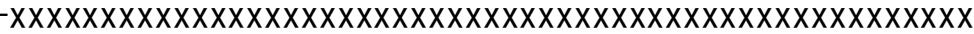
-xxxrae i edmgxeaw $\mid x x x-$ 
-xxxxxv-ssvsqmpsg|kh|-psqrhpskpspspitkxxxxxxxxxx----

Irkcfvhvtgmtcascvsni--

ern I vkheg i ksv I va Imagkaevkydpg I I dpa

qivqli

sh Igfgasvmx

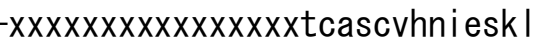

$x x x x x x x x x x x x x x x x x x x x x x x x x x x x x x x x$

$x x x x x x x x$

$\mathrm{xxx}$

-vs I ikneg Innt I dhqee i

-rqwkhsfIfs I $x x x x x x--x x x x x x x x x x x x$

$x x x x x x x x x x x x x x$

$x x x x x x x x x x x x x x x x x x x x x x x x x x x x x x x x x x x x x h$-gvxnmdv livlatt $i$ ay $y y s f t$

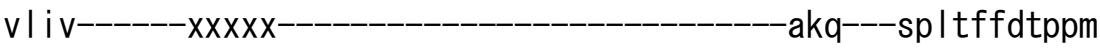

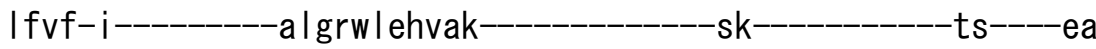

lak Ims lqat

-datxvs I

xxxnt ixx-1

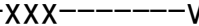

S

----vd IVQRGDVVKVap

GGKFPVDGKV I eg

-xsmADESL I TGEPMPVIKkx-

$$
-\mathrm{x}
$$

SCVIA--GSIN

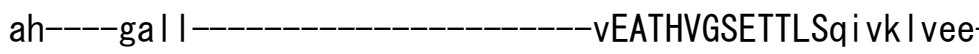

-aqtskap iqq ladk I sgyfvpfivvisi Itv

-tawl i igf Idfdvvsxxxxxxxxx-xxxxxx i vrfafqas ITVLSIA

CPCSLGLAtPTAVMVGTG-VGaqng- 
iL IK-GGEPLEMAHKVGAVMFDKTG-

-TI TNGVPQVt

-rviviwdra

$-x x x x x--x---$

--v lavv-----gtae-

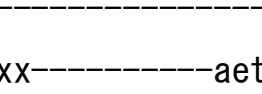

-assehp I g--mavakh

$\operatorname{ckxx}$

$-x x-$

fchdfqavpgcg i sc-kvss xxxxxxxx

$-X X X X X X X X X X X X X X X X X X X X X X X--x$

$-x---$

$-i$

-gnr

q

-wml rnglevt

-xxv-dnamssh--etk----gqta ILVAIDgx

I CAM I AVADTVKAESALAVHTLSSmg-IEV

YMI TGDNRRTARAIATQVGI-

-rk 


\section{-VFA--EVLPSHKVAKVQELq-}

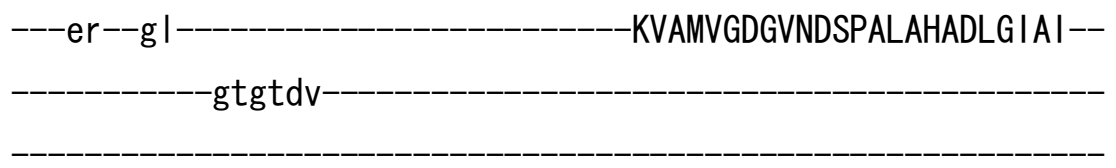

-AIEAAD IVL I r-NDLLDVVas IELSKKTVQR IRINF-------V
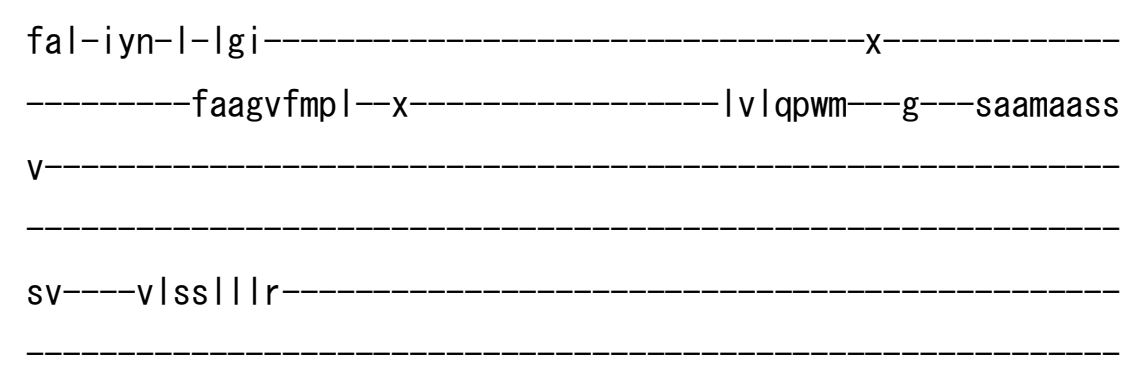

।

>celI_IB201 NP_499778. 1

m- 


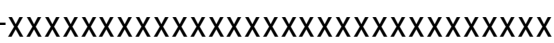

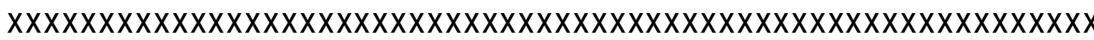

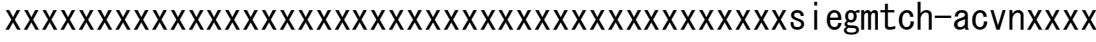
$x x x x x x x x x x x x x x \mid x q k q g t v d y n s e k w n g e$ svaes iddmgfdck $x x x x x x x x x x x x x x$ $x x x x x x x x x x x x x x x x x x x x x x x x x x x x x x x x x x x x x x$ lekctfavegmtcascvqyi-erni sk i egvhs i vval i aakaev i ydgrvtssdxxxxxxxxe I gyka t I Idsmganp $-x x x x x x x x x x x x x x x x x x x x x x$ shv $\mid$ sksgidscn

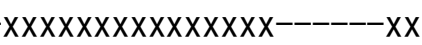

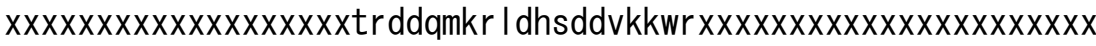

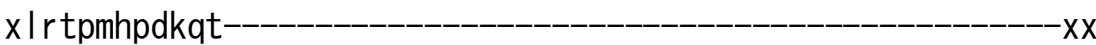

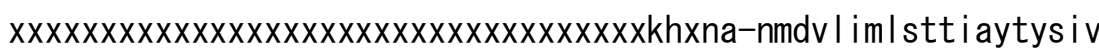

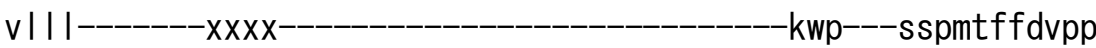

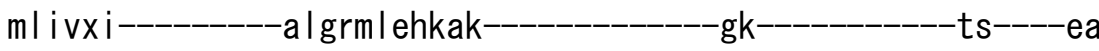
Isk Ims lqakeatxvtm-

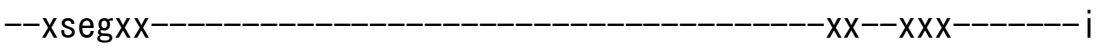
$\mathrm{n}$ ---- i e I VQRNDL I KVvp GAKVPVDGVVVdg 


\section{$-x$}

STVIG--GSVN-

qk----gvl i---_-_-_-_-_-_--_-_----vKATHVGNDSTLSqi vr I vee-

-aqtnrap iqq ladkiagyfvpfvivIs Ift I

-gvwi y i eynsarnanxxxxx

xxxxalkiafeaalTVLAIA

CPCSLGLAtPTAVMVGTG-VGaang-

iLIK-GGEPLESVHKVTT IVFDKTG

TI TEGRPRVv

-qiasfvnps

$-x \times x x x--x---$

--itfls-----gate-

-al sehp i g--navaaf-----akxx-----

$-x x-$

ept-

$-x x-$

ntsrfhvsaghgvtc- $r$ ids $x x x x x--x x x$

$-x$

$X X X X X X X X X X X X$

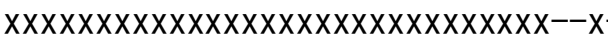
$-x---$

$-i$

\section{$r$}

-mmerhg ipvs 
-xxv-kmt I see--qrk----ghi sVICAINaxVVAVISI ADQVKKEASLAIYTLREmg-LRV

VLLTGDNSKTAESTAKQVGI -de-

-VFA--EVLPNQKQQKIKQLK

$---\mathrm{gy}--\mathrm{kn}-$

KVAMVGDGVNDSPALAEANVGIAI-aagsdv

AIESAGIVLVr-NDLVDVVga IKLSKMTTRRIRLNF $-\mathrm{L}$

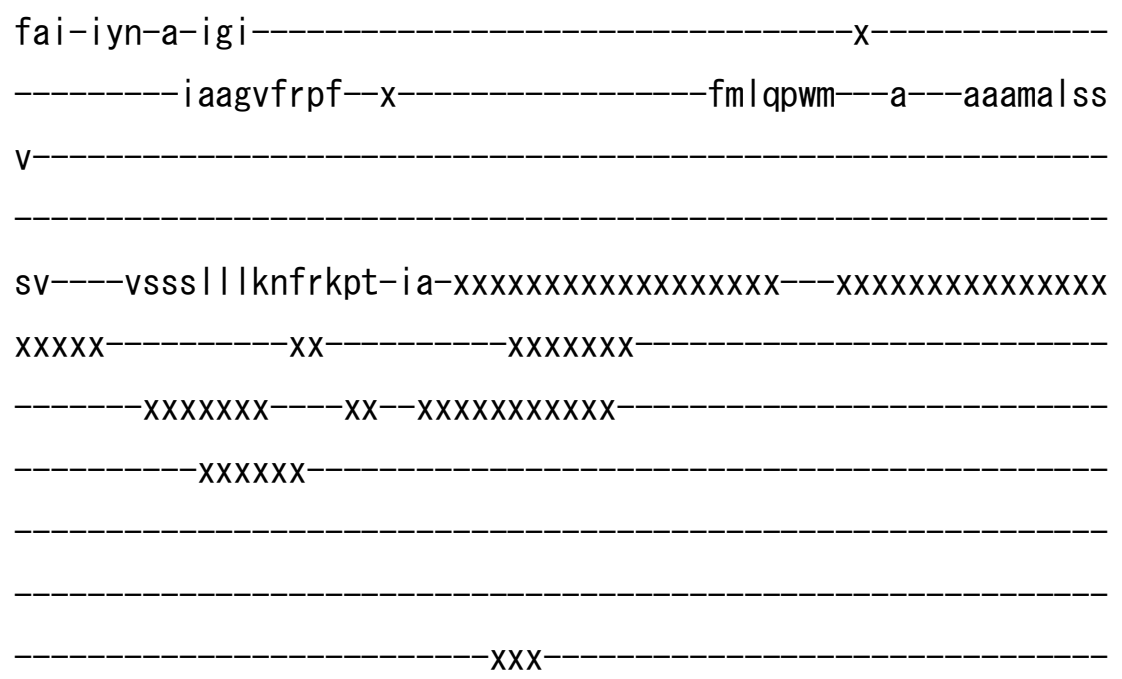


V

$>$ cne I_IB201 XP_568603.1

xxxgspphrqrxasnsxxxx

xxxxxxxxxxxxxxtxprveqrv| leedevp l eegsqs I skeapvg

-Irrvelrvggmtcgacvasi--

-esqlkqpgiksiqis I laergvveydenfvkad-xxxxxxxki aee i edigfeatvvx-

$-x x x x x x x x x x x x x x e n q e$ ivss I Istt-

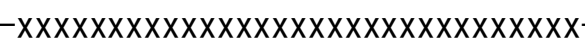

$x x x x x x x x x x x x x x x p v s n n d d s q i a s$ lqkhkea

-alwkrt If I saxxxxxxxxxxxxxxxxxxxx-

$-x x x x x x x$ 
$x x x x x x x x x x x x x x x x x x x x x x x x x x x x x x x x x x x x k h$-gs $x$ tmdv Ivv|gtssafcysv $\mid$

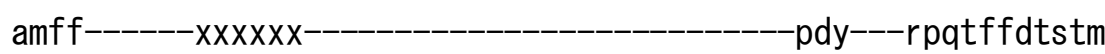

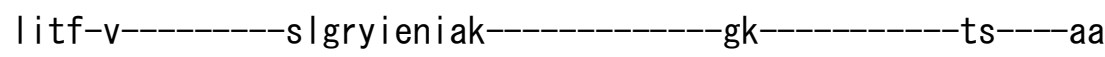

$|t d||s| t p s$

- sat-iyv

$-X X X X X X X X$

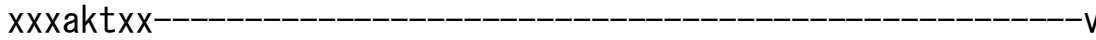

$\mathrm{p}$

----te IVQVGDVVLLvp

GEKIPADGTVLtg

$-x x$ sVDESMVTGEALPMPKtx-

SQV IG--GTVN-

xxgtit-

fRVTRAGADTALSqivk I ved

-aqtskap iqefadrvagifvpivit Is Istf

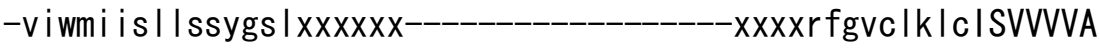

CPCALGLStPTAVMVGTG-VGakng-

iLIK-GGKALEACKGVKRVVLDKTG-

TVTEGRMAV svvwassss

$-x x x x x x x x x x x$

$\operatorname{xx} x \operatorname{xx} x \operatorname{xx} x x x x x$

$-x x$

xxi isli-----slae 
-ar sehp I g--mavaah----grxx----

$-x x x x x x x--x x x g$

-evvefeshtgeglea-vvk|xxxxxxxxxx

$-\mathrm{i}$

-gka

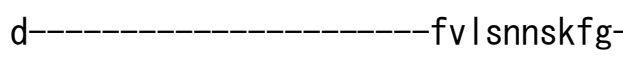

--xxxxxxxxxm-pse i lef--ehdxxxxartvIFVSI I rx

xxxxpVAALSLSDSPKPTSAQAIRALKNmg-IKV

TMLTGDAATAQAVARQVGI

dex

-xVYS--GVSPKGKAKIVQDLd

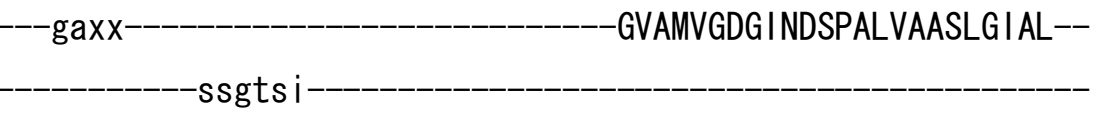

AIEAADVVLVr-SDLLDVVaaLDLGQVIFRK IKANL------। 


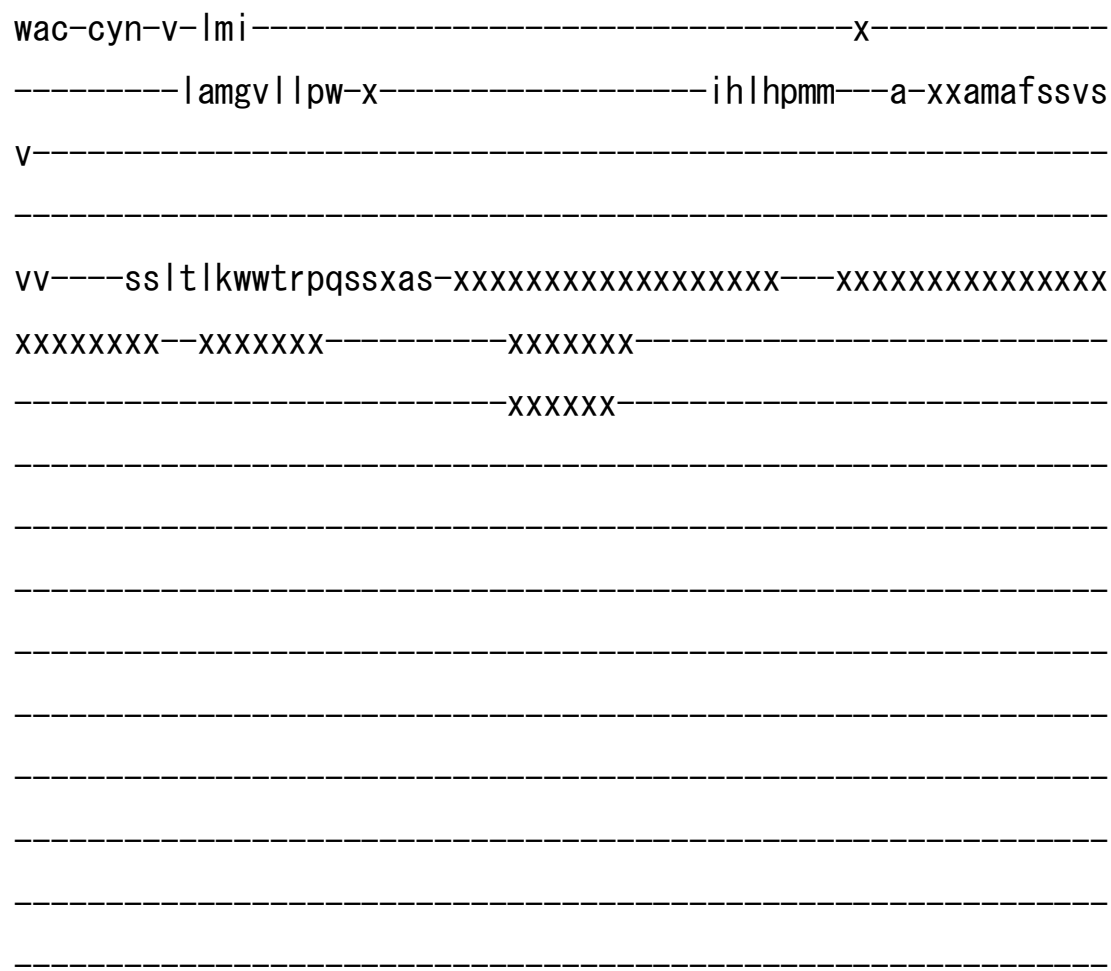

V

>rnol_IB202 NP_036643. 1

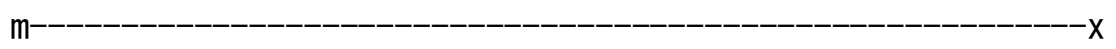

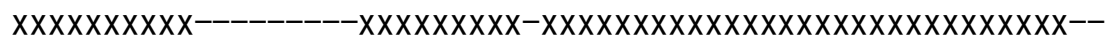

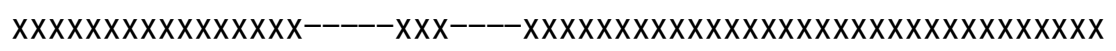

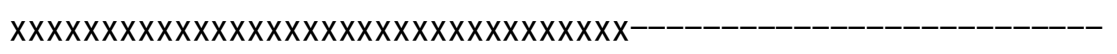

$-x x x x x x x x$

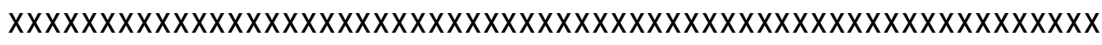
$\mathrm{x} \times \mathrm{X}$

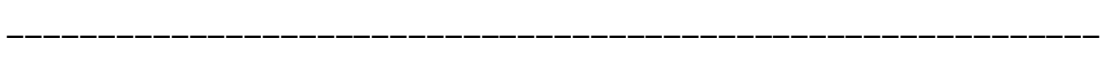

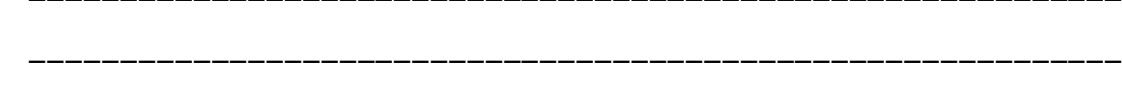

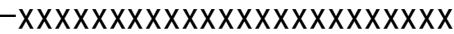

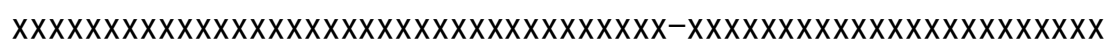

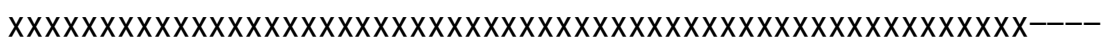

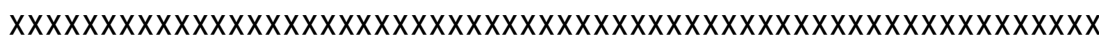




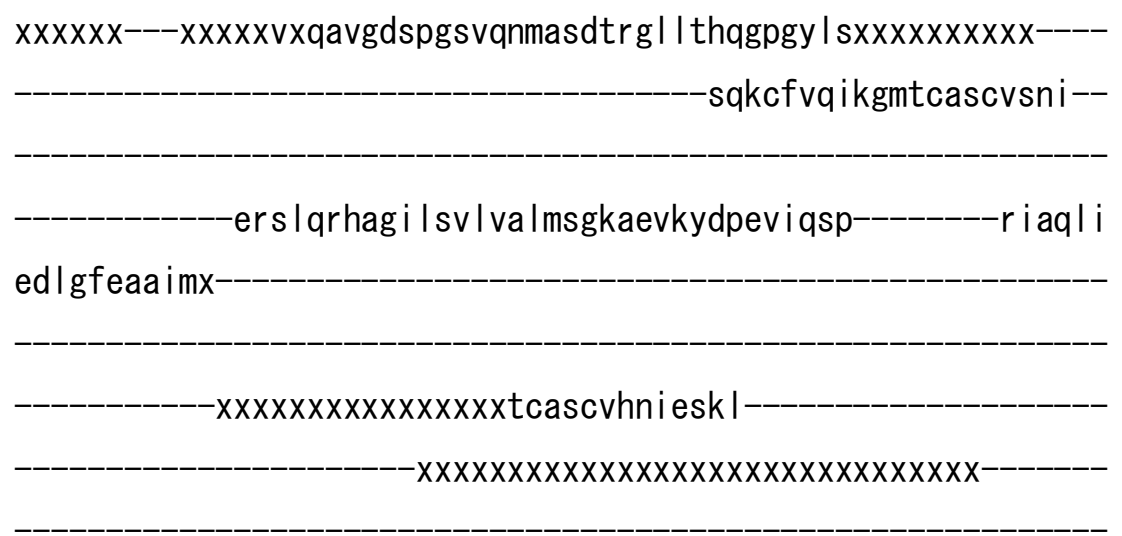

$-x x x x x x x x$ xxx---_-_-_-_-_-----as I ahrnpnahh I dhkte $\mathrm{i}$

-kqwkksflcsl $x x x x x x--x x x x x x x x x x x x$ $x x x x x x x x x x x$

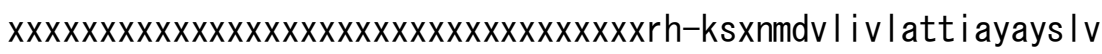

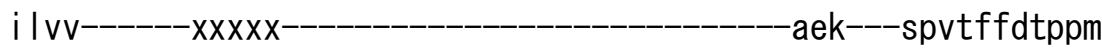

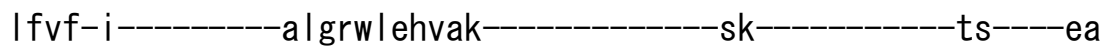
lak Ims lqat-eatxvt I

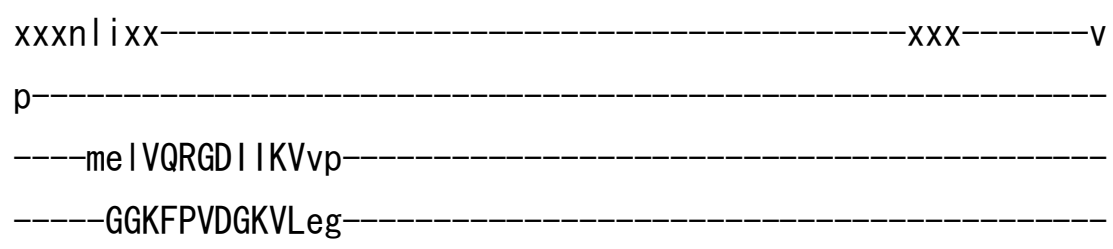

-xtmADESL I TGEAMPVTKkx-

$$
-\mathrm{X}
$$$$
\text { SIVIA--GSIN- }
$$

ah----gsvl----------------------_iKATHVGNDTTLAqi vk I vee-

-aqmskap iqq ladrfsgyfvpf i i i istlt l

-vVwi i i gfvdfg i vqxxxxxxxxxx-------------xxxxxxi i irfafats ITVLCIA CPCSLGLAtPTAVMVGTG-VAaqng- 
-vLIK-GGKPLEMAHK IKTVMFDKTG

-TI THGVPRVm

-rfl IIvdva

$-x \times x x x--x--$

--v lavv-----gtae-

-assehp I g--vavtky-----ckxx----

$x x$

tet

$-x x-$

-ystdfqavpgcg i sc-kvsnxxxxxxxxx

-xxi-sdamtdh--emk----gqta ILVAIDgx

I CGM I A I ADAVKPEAALA IYTLKSmg-VDV

AL I TGDNRKTARA I ATQVGI

$-n k$ 
-VFA--EVLPSHKVAKVQELq

---nk--gk--------------------KVAMVGDGVNDSPALAQADVGIAI--

$-g \operatorname{tgtdv}-$

-A IEAADVVL I r-NDLLDVVas I HLSKRTVRR IRVNL$-\mathrm{V}$

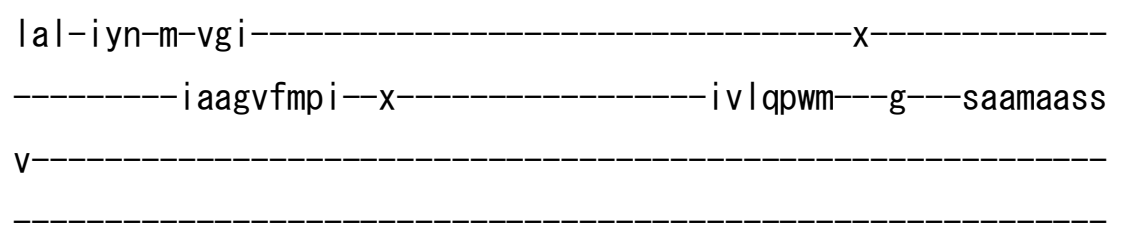

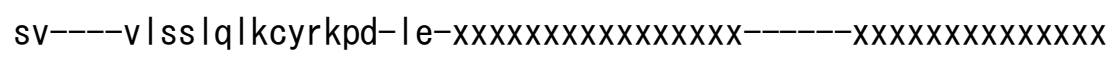

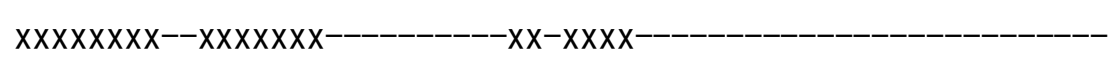
$-------x x x x x x x----x x--x x x x x x x x x x x---------------------$ $-x x x x-$ $-x x x-$

i

>ddi I_IB202 XP_646096. 2

m- 


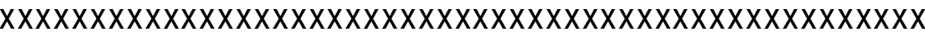

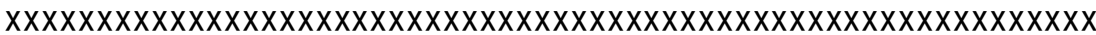

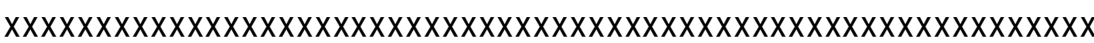

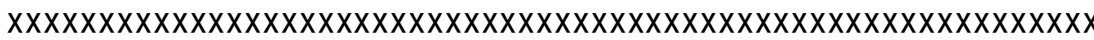

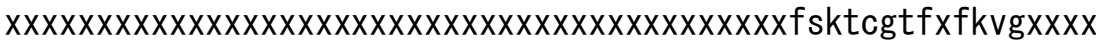

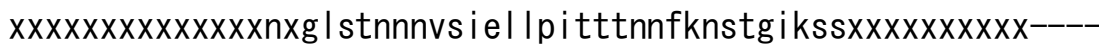
$-k$ velhImisgmtcadcvttI-eqkmks I sgvne iqts I ftgkckinyspmi i qpqxxxxxxxxigfvgk qvhgqssnsnx

$-X X X X X X$ $x x x x x x x x x x x x x x x x x x x x x x x x x x x x \mid$ da $i$ eesgsvndk

$-x x x x x x x$

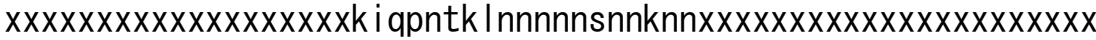
xtilisyifpni $x x x x x x x x x x$

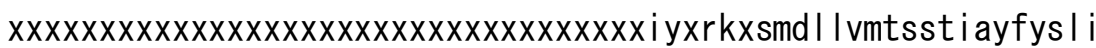

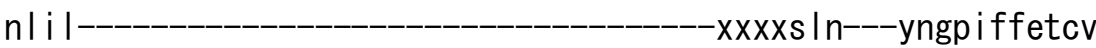

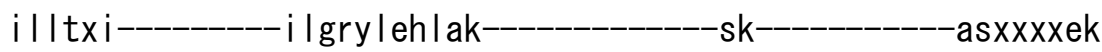
mkqldssnai-

$x x x x x x x x g$ sexe im-

$\mathrm{e}$

----ke I IEKGDILKVIp

FTKIPTDGI I I rg 
---xgy IDESI I TGEANHVYKr--

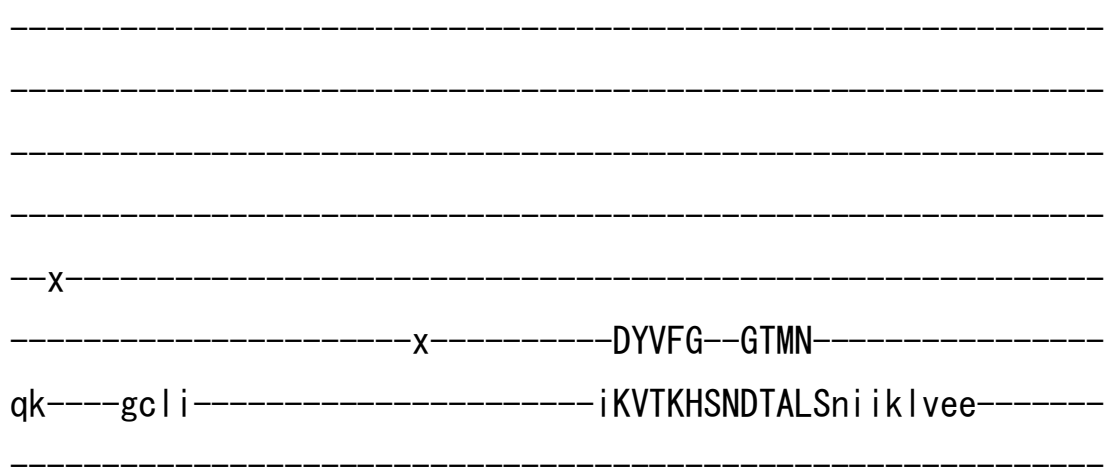

-sqsikpnfqnyadl vasyfvpti l ics i itf

-ivwfcl ihynvviat-

-xxxxaftfaiki sMSLMVIS

CPCAISLAtPTSIMVGSA-IGakkfx-

iLFKxGGQVIELASKVNKVIFDKTG

TLTTGEIDIn

-hfs i ydqsi

$-x x x x x x x x x x x x x x x--x x x x$

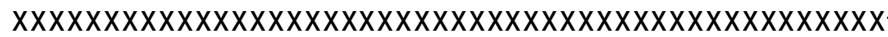

$-x x$

xxqlsnn-----nnk i-

-dscqsp lq--ssql in-----skxxxxxx

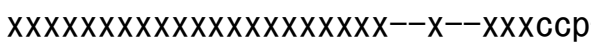

$-x x-$

-aapp i stgngsdckkxccps xxxxxxxxxx

$-x x x x x x x x$

$-\operatorname{cxx} x x---x \times x x x x x x x x x x x-$

- gcc

$\mathrm{s}$

ssssql sksq 
$x x x--x x x x x x i-q n d i d k I--e n q----g k t v$ ILVSMNe

-xi I GLVSLSDKLKPEAKKV I SKLKNng-IDV

WLVSGDNKRATQS IGEQLNI

$-n s x$

$x$ I IG--SALPIDKFNIVRKL $q x x x x x x x x x x x x x x x x x x x x x x x x x x x x x x x x x x$

$x x x k n--k s$

IVVMVGDGVNDSLALVQSDIGISI--

-sngtdi

-AIESSSVILLk-NDLNDILvcFDLSKSIFKTI KFNL------F

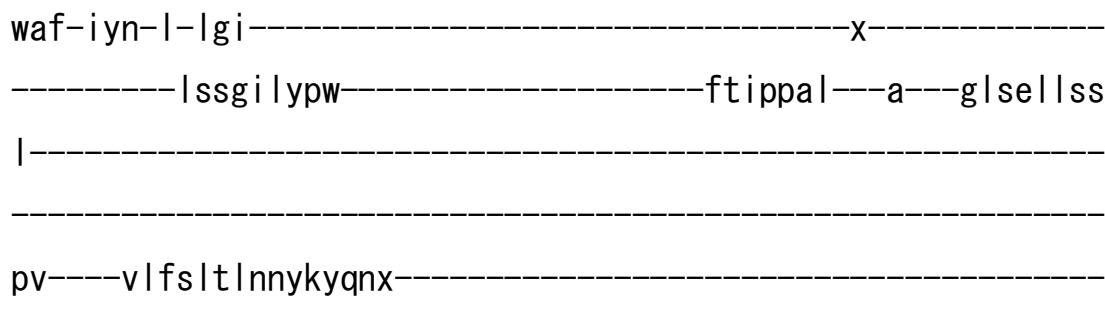


>ddi I_IB203 XP_644796.1

$$
\text { m- }
$$

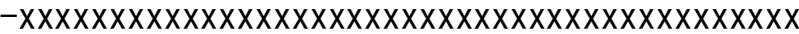

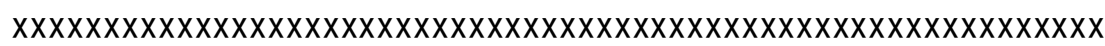

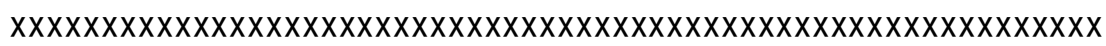

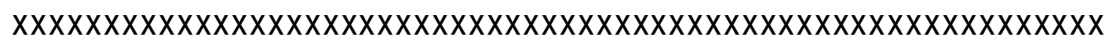

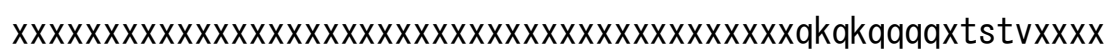
$x x x x x x x x x x x x x x d x s$ s itkspfep isssdeevve innnnkynns $x x x x x x x x x x x x x x$

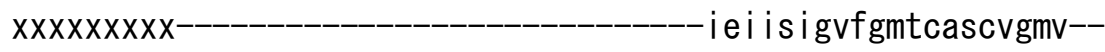

-ehsiksvdgvi ecnvnl laeraeikynssickdvxxxxxxxxi lgfet k I iqqskagtx-

$-x x x x x x x x x x x x x x x x x$

$x x x x x x x x x x x x x x x x x x x x x x x x x x x x$ ssssqkknkknkn-

$-x x \operatorname{xx} x x x x x x x x x x x y---x x x x x$

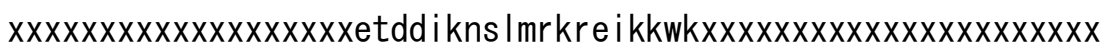
xksikfIhyqla 
-- $x x x x x x x x x x x x x x x x x x x x x x x x x x x x x x x x x k n x h g-n m d$ l I vavgstcay $y s v i$

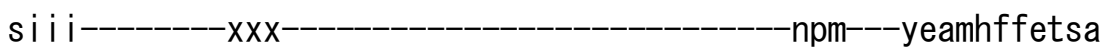

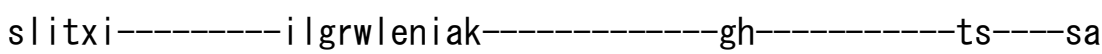

ivk Imnlqsk-

ettxvt I-

$\mathrm{XXXXXXX}$

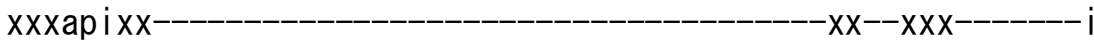

$q^{-}$

----ss I ISYGDLLKV ip

GQSIPTDGIVIng

-xssCDESMI TGESIPVLKk--

$--x$

$x-$

-DSVTG--GTLN-

Id----gv|i

iKANK I GSENTLSq i i I vqq-

-aqtskap iqq ladt i skyfvpi i I I gl itf

-giwi avtetnsvsaexx-----------------------xxxxpf I ysf I ta ISVIVIA

CPCALGLAtPTAVMVGTG-VGas i g-

- LLIK-GGKPLETAHKATAILFDKTG

TITTGKMNVt

dyrvnhqsn-

$-X X X X$

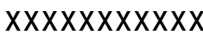

XX

xxffkiv-----gaae- 
-sgsehp i g--ka ivqf----ckxx-

$-x \times x x x x x--x x x s p m$

xxxxxxxpvsdfkgiagrg $\mid$ sc-ivds

$-X X X X$

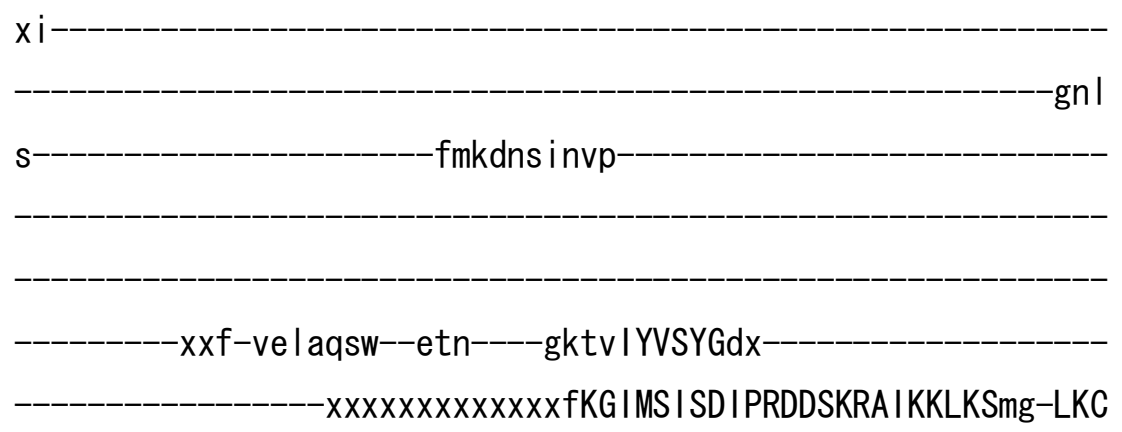

YMVTGDNKRAAKF I ANQVG I

dex

$-x$ IYS--EVIPKEKAEKVSDL

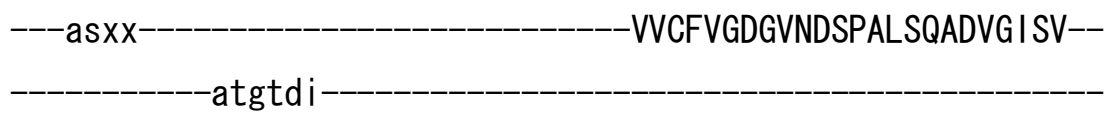

-A IESSS IVLLk-NSLTDVYrs IHLSRVVFRRIK INF------T 


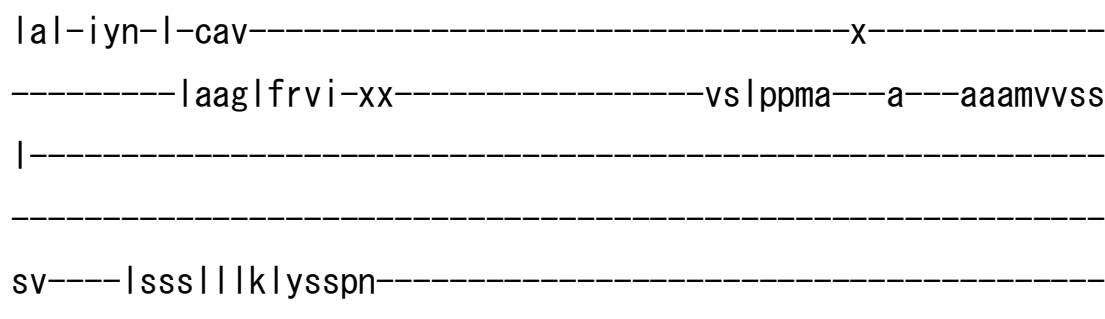

>ddi I_IB204 XP_638720.1

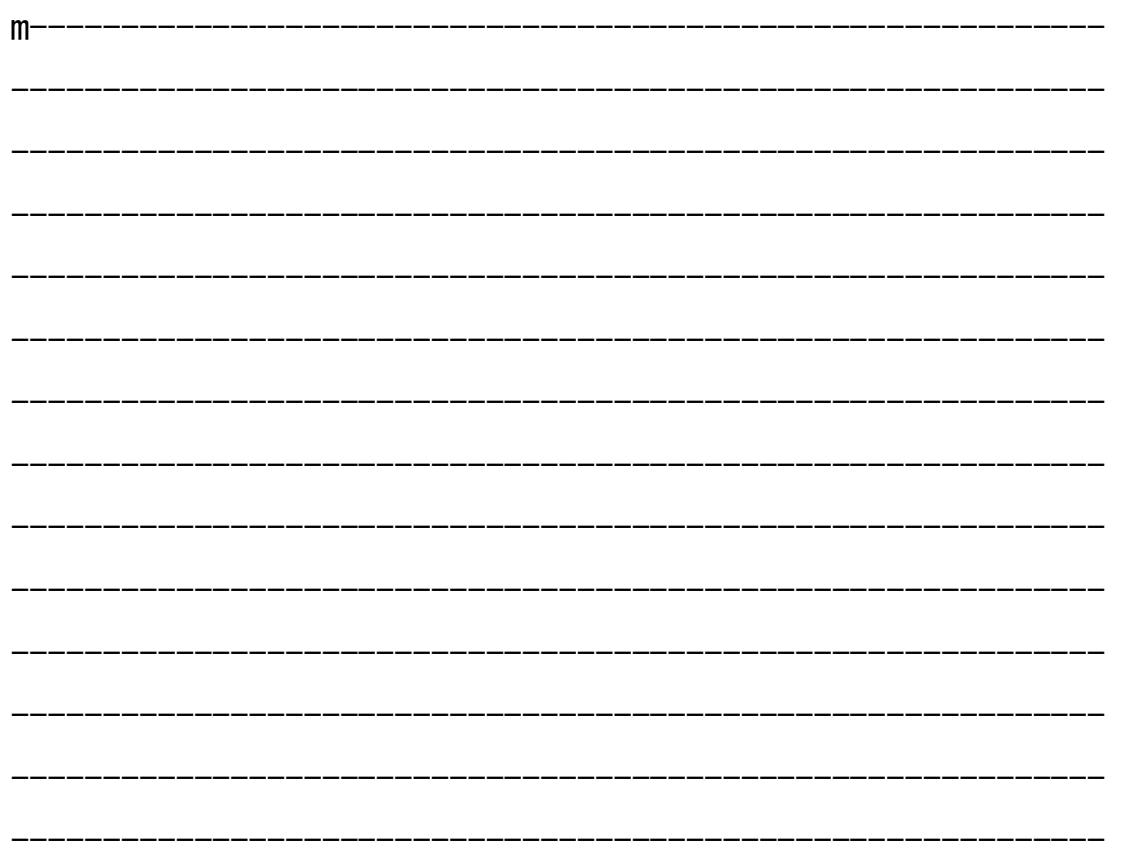


xxxxxxxxxxxxxx|xqetaevkfnp| i I sedd i veq i smvgfeakxxxxxx-

-nnt iv Iniggmtcsscvg i i--

enyvsnvdgv i ecrvnl ametarvvydpd I tgvrxxxxx-xxdvgfta

qipsqnfddt

-kniqkeeaek Ikxxxxxxxxxxxxxxxxxxxxxx

xsffnflytnni

$-x x x x x x x x x x x x x x x x x x x x x x x x x x x x x x x x x x x k h x g a-n m d v \mid$ valgtscayfys $\mid m$ v I Imxxxxxxx---_--_-_-_-_-_-_-_-_-_-_-_-_-_-_--gst----vgmktffdtsa

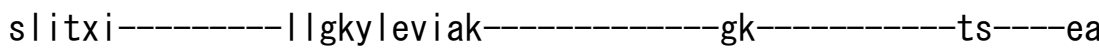

ikk Imglqat-

$-k a|x| t i$

$-x x x x x x x x x x x x$

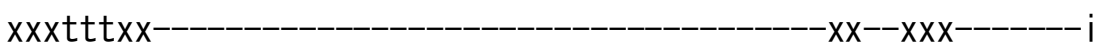

$d$

----idIVQRGDYLKVIp-

-GSKVPTDGIVVsg

-xsh IDESI I TGESLPVSKkx-

$$
-\mathrm{x}
$$

$-D K L I G--G T L N-$

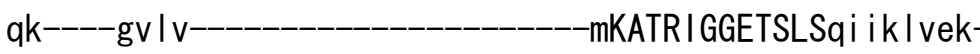

-aqterap iqs ladkvsgyfvpsvitIgIItf

-i vwl i agssgvasdyxxx-xxxxvfafalrnalSVIVIA

CPCALGLAtPTAVMVGTG-IGaqng- 
iLIK-GGSHLETAHK ISAI IFDKTG

TLTTGKPIVs -nvnifgnIn

$x X X X$

$\mathrm{XXXXXXXX}$

xxffqmv-----asae

aasehpla--ga i vny----afxx

$-x--x x x s t$

-pplsfesitgsgira-tlapxxxxx

$-x^{---}$

$-\mathrm{i}$

$-g n \mid$

k

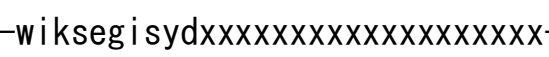

xxxxxxxxxxxi-edqurr|--esd----gntvVYVVIDrx

- IMGY IAISDQLKPEAHAT I TELNKmg-ICT

WMVTGDNPRTANAIAQQVGI-

dq 
-VFA--EVLPSNKSKKVMELk

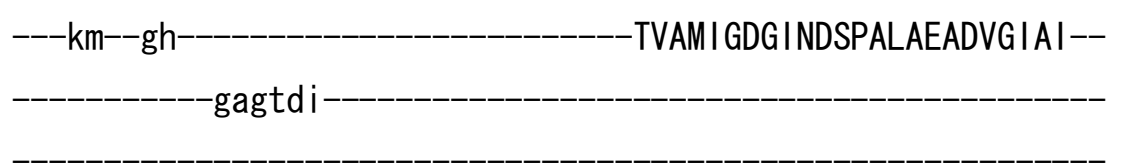

-AIEAAD I VLVk-SDLRDVI ta I SLSKTTFNR IRFNY------L

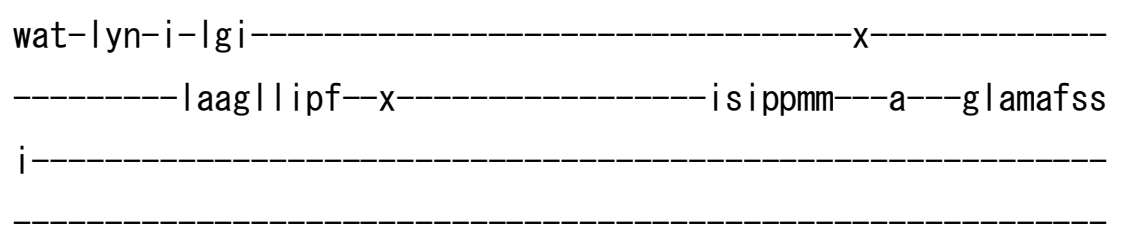

sv----v|sslhlktyqkpdxpi-xxxxxxxxxxxxxxxxx-

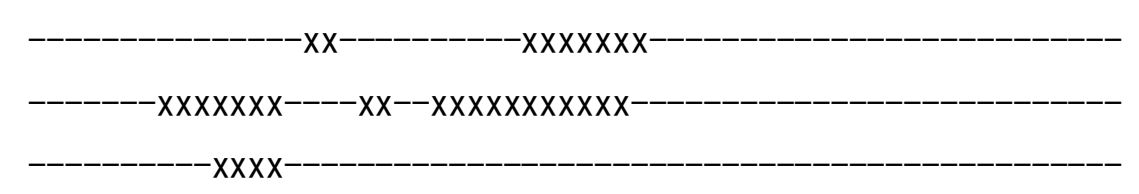

v

>athI_IB201 NP_199292. 1

m- 


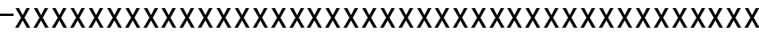

$x x x x x x x x x x x x x$

-xxxxxgvtgmtca-acsnxxxx

xxxxxxxxxxxxxx|xqnradvvfdpn| vkeed i kea i edagfeaexxxxxxxxx-----

-t I vgqft iggmtcaacvnsv--

eg i I rd Ipgvkravva I sts I geveydpnvinkdxxxxx-xxdagfeg

slvqsnqqdk

$-x x x x x x x x x x x x x x x x x x g$ i Itr Ingvrqfr

$-x x x x x x x x x x x x x x x----x x x x$

$X X X X X X X X X X X X X X X X X X X X X m s p y e r \mid s s k d t g e a s n m f X X X X X X X X X X X X X X X X X X X X X X X$

$x a|f d a| l v w r c$

$--x x x x x x x x x x x x x x x x x x x x x x x x x x x x x x x x x x x x x r n x s t-n m d v \mid$ valgtsasyfysvg

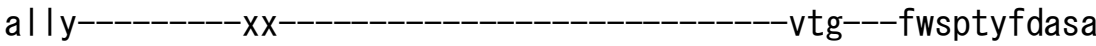

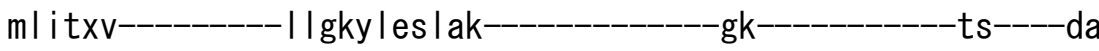
mkk I vql tpa-

$$
\text { tai- } \mid 1 \mathrm{t}
$$

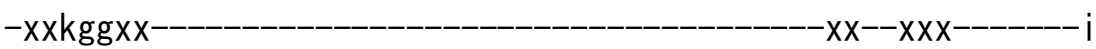

$d-$

----a I I IQPGDTLKVhp

GAKIPADGVVVwg 


\section{-SPVIG--GTINxx}

galh$-m K A T K V G S D A V L S q$ i i s I vet

-aqmskap iqkfadyvas i fvpvvit la lft I

-vgws i ggavgaypdexxx-xxxxhfvfsImfsISVVVIA CPCALGLAtPTAVMVATG-VGatng-

-vLIK-GGDALEKAHKVKYVIFDKTG-

-TLTQGKATVtttkvfsemd

-dtsdfsa Ipgkg i qc-I vne

$$
-x+-x-
$$

$-\mathrm{v}$ 
--xxv-ekfvedl--ees----gktgVIVAYN-xx I VGVMG I ADPLKREAALVVEGLLRmg-VRP

IMVTGDNWRTARAVAKEVGI - ed-

-VRA--EVMPAGKADVIRSLq-

---kd--gs--_-_-_-_-_-_-_-_-_-_-_-_TVAMVGDG I NDSPALAAADVGMAI--gagtdv-

-AIEAADYVLMr-NNLEDVI ta I DLSRKTLTRIRLNY $-V$

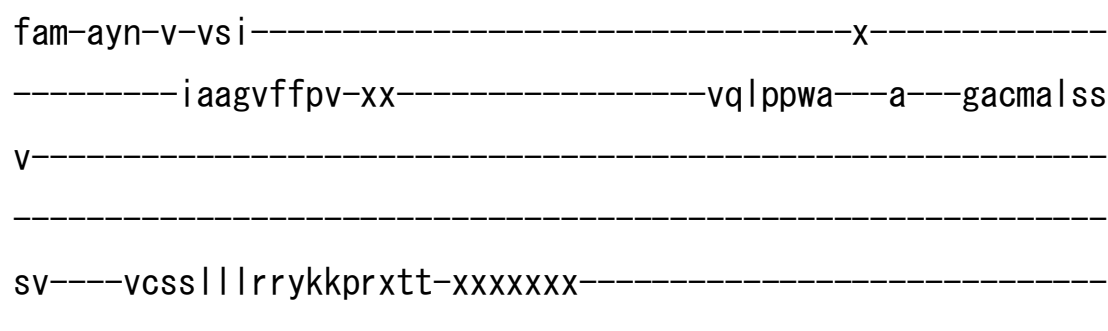


>athI_IB202 NP_974675. 1

$\mathrm{m}$

$x X X X X X X X X X X X X X X X X X X X X X X X X X X X X$

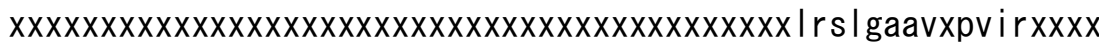

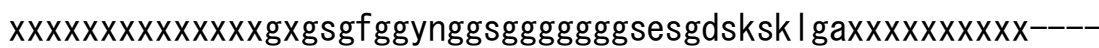
-sdi i i l dvggmtcggcsasv--

-kk i lesqpqvasasvn Itteta i vwpvpeaksvpxxxxxxxxt Ianh I tncgfqstprx

Ivtenffkvfetk-

-tkdkqar lkesgre lavswxxxxxxxxxxxxxxxxxxxxxx

xaihstgfhvsI 
$-x x x x x x x x x x x x x x x x x x x x \mid k$-gsxnmnt Ivg |galssfsvss |

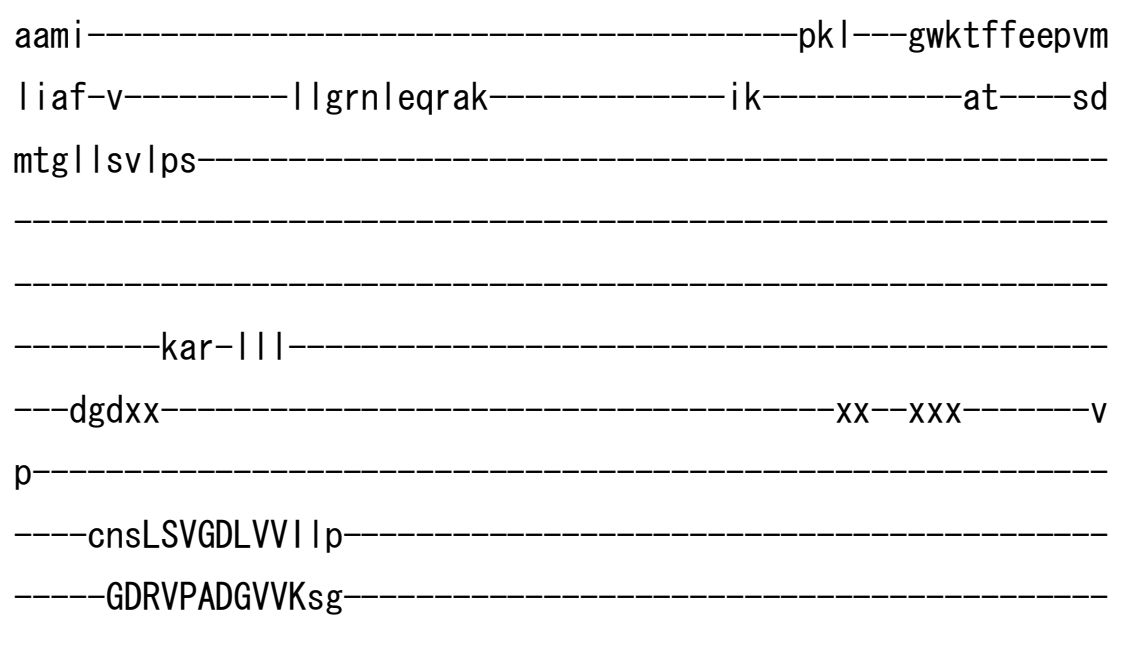

$-x s t$ IDESSFTGEPLPVTKe--

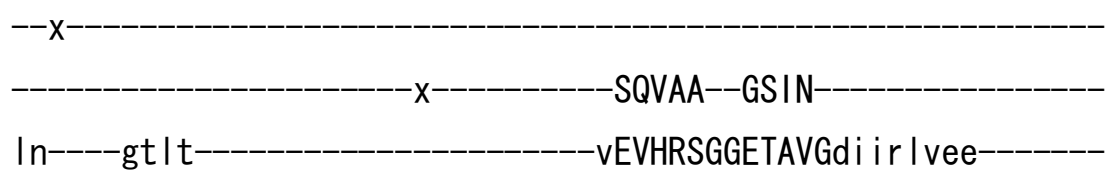

-aqsr eapvqq I vdkvagrftygvma I saatf

-tfwn I fgahv I psal--_-_-_-_-_-_-_-_-_-_-_---Xxxxpms I a l q I sCSVLVVA

CPCALGLATPTAMLVGTS-LGarrg-

ILLR-GGD ILEKFSLVDTVVFDKTG

TLTKGHPVVtevi i penpr

$\mathrm{xxx}$ $-X X$

xxv $|m| a-----a a v e-$ 
-sntthpvg--ka i vka----arxx-

-ncqtmkaedgtfteexgsgaxxxxxxxxxx

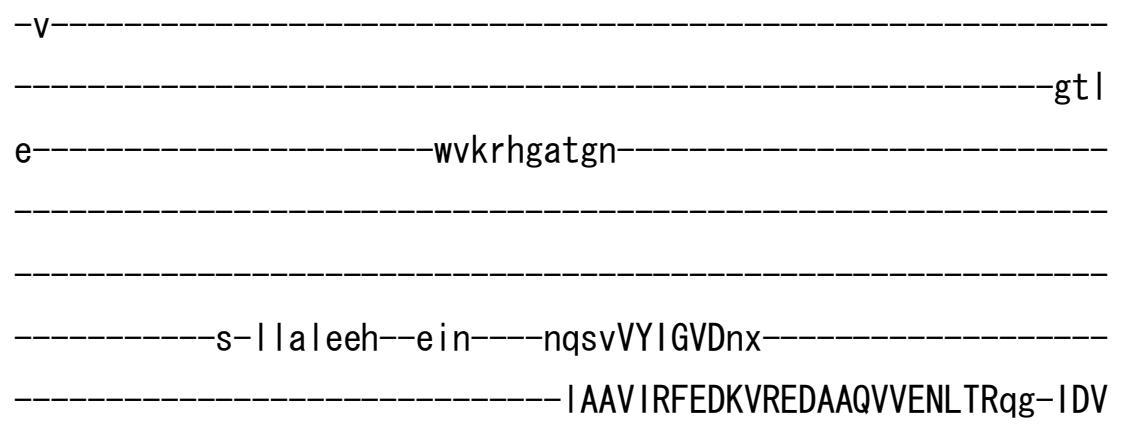

YMLSGDKRNAANYVASVVGI-

$-n h x$

-XVIA--GVKPAEKKNF INEL $q$

----kn--kk---_-_-_-_-_-_-_-_-_-_-_---IVAMVGDG I NDAAALASSNVGVAM--

-ASEVSPVVLMg-NRLTQLLdaMELSRQTMKTVKQNL------W 


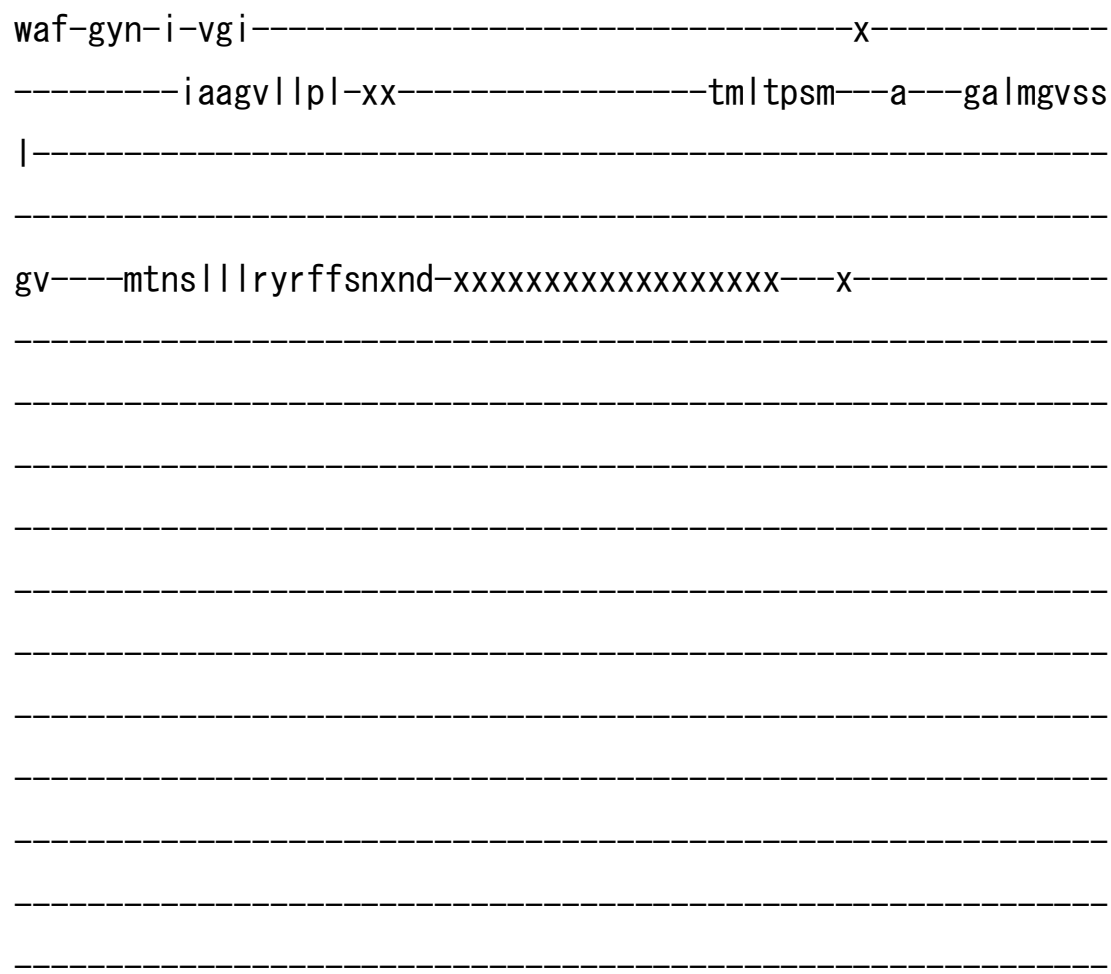

S

>athI_IB203 NP_176533. 1

m

$x x x x x x x x x x x$

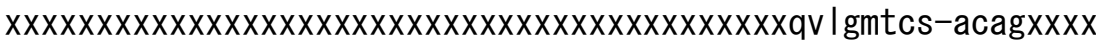


xxxxxxxxxxxxxxaxnnraqi I fypnsvdvet i ret i edagfeasxxxxxxxxxxx----

-rqveriringmtctscssti--

erv l qsvngvqrahva I a i eeaeihydpr Issydxxxxx-xxnagfea

vlistgedvs-

$-x x x x x x x x x x x x x x x x x x x r$ s lealpgvqsve

$x x x x x x x x x x x x x x x x x x x x x x x$

$x x x x x x x x x x x x x x x x x x x x$ seggvgresqkqge $i$ kqyy $x x x x x x x x x x x x x x x x x x x x x x x$

xpgikd I Imfkv-

$-x x x x x x x x x x x x x x x x x x x x x x x x x x x x x x x x x x x r r x s a-n m d v \mid$ ialgtnaayfys $\mid y$

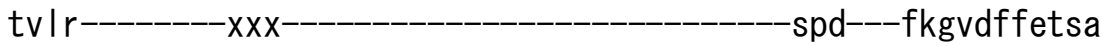

ml isxi--_-_-_--_i l gky l evmak-_-_-_-_-_-_-_gk-_-_-_-_-_--ts----qa

iak Imnlapd-

taix $|s|$

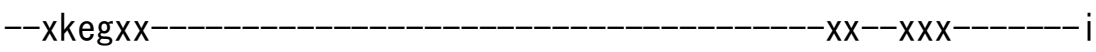

$d$

----gr I IQKNDVIKIvp

GAKVASDGYVI wg

-xshVNESMI TGEARPVAKr--

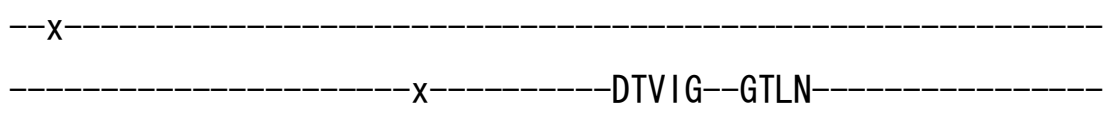

en----gv lh---_--_-_-_-_--_----vKVTRVGSESALAq i vr I ves

-aq lakapvqk Iadr iskffvp IvifIsfstw

- lawf l agk I hwypesxxx xxxxsfelalafgISVMVIA

CPCALGLAtPTAVMVGTG-VGasqg- 
-vLIK-GGQALERAHKVNCIVFDKTG-

TLTMGKPVVV

$-k t k$ I I knmv

xxfyelv-----aate-

-vnsehpla--ka i vey

$-x x--x x x n p a$

$$
-x x-
$$

-eacdfvs i tgkgvka-tvkg

$x x--x$

x---

$-\mathrm{v}$

-gnk

$\mathrm{n}$

Imndhkv i ip

-xxa-ee I lads--edm----aqtg ILVSINs

- I IGVLSVSDPLKPSAREA I ILKSmn-IKS

IMVTGDNWGTANS I AREVGI

ds 
-VIA--EAKPEQKAEKVKELq

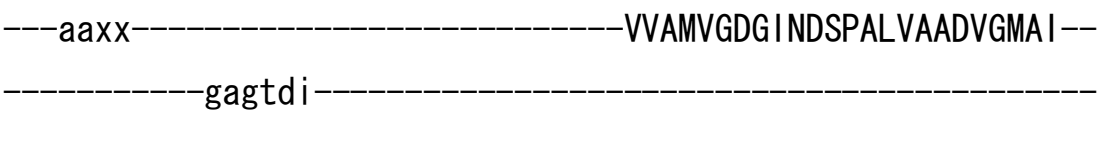

-A IEAAD I VLMk-SNLEDVI ta I DLSRKTFSR IRLNY------V

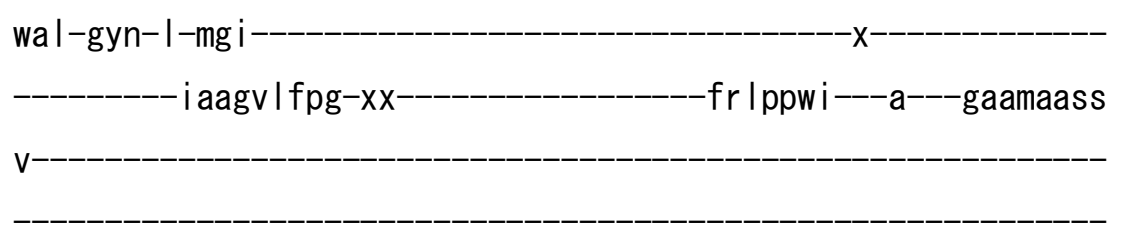

sv----vccs I I IknykrpkxId-Xxxxxxxxxxx-

V

>athI_IB204 NP_001031920.1

$\mathrm{m}-$ 
asnl I rfpxpppsxxxx

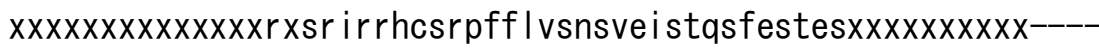
dtp i I lvsgmmcggcvarv--ksv Imsddrvasavvnm I tetaavkfkpevevtaxxxxxxxxr I tesg feakrrvsgmx

$-x x x x x x x x$ xxxxxxxxxxxxxxxxxxxavgal Igpgrel I fdgika-

fgxrsxnmns Ivg Igsmaafs is I i slvn -xele---wdasffdepvm

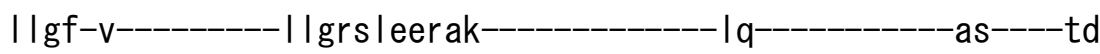
mnellslist-qsrxvit $-x x x x x$

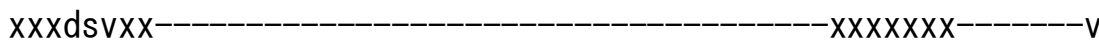
S ----vddIRVGDSLLVIp GETFPVDGSVLag 


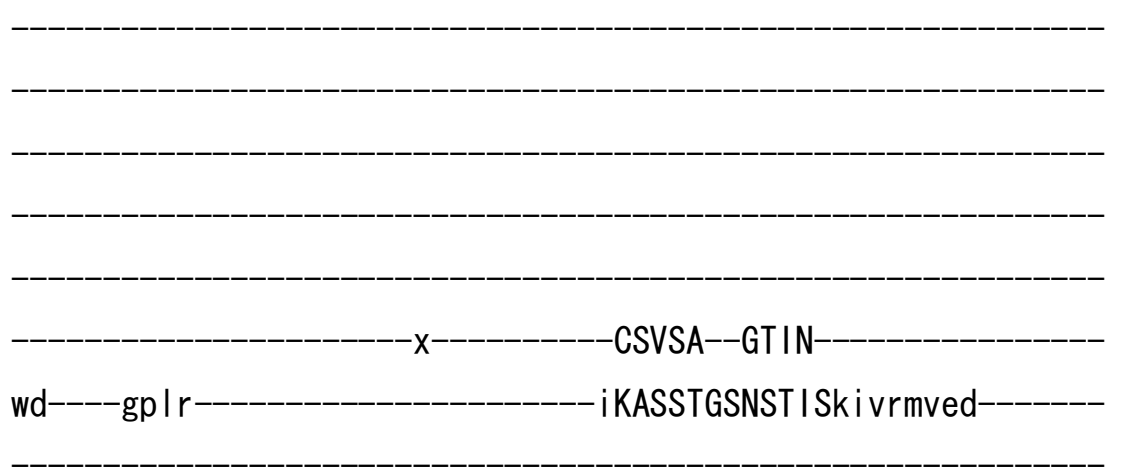

-aqgnaapvar lada i agpf fyt ims I samtf -afwyyvgshifpdv|xxxxxx-xxxxalals lklaVDVLVVS CPCALGLAtPTAIL I GTS-LGakrg-

-yLIR-GGDVLERLASIDCVALDKTG

TLTEGRPVVs gvas I gy

xxv I kma-----aave-

$$
\text { -x--xxxktp----------------x--------- }
$$

-trgql tepgfgt l aexdgrf $-x x$

$-\mathrm{V}$ $\mathrm{e}$ -wvsdrf I kknxxx 


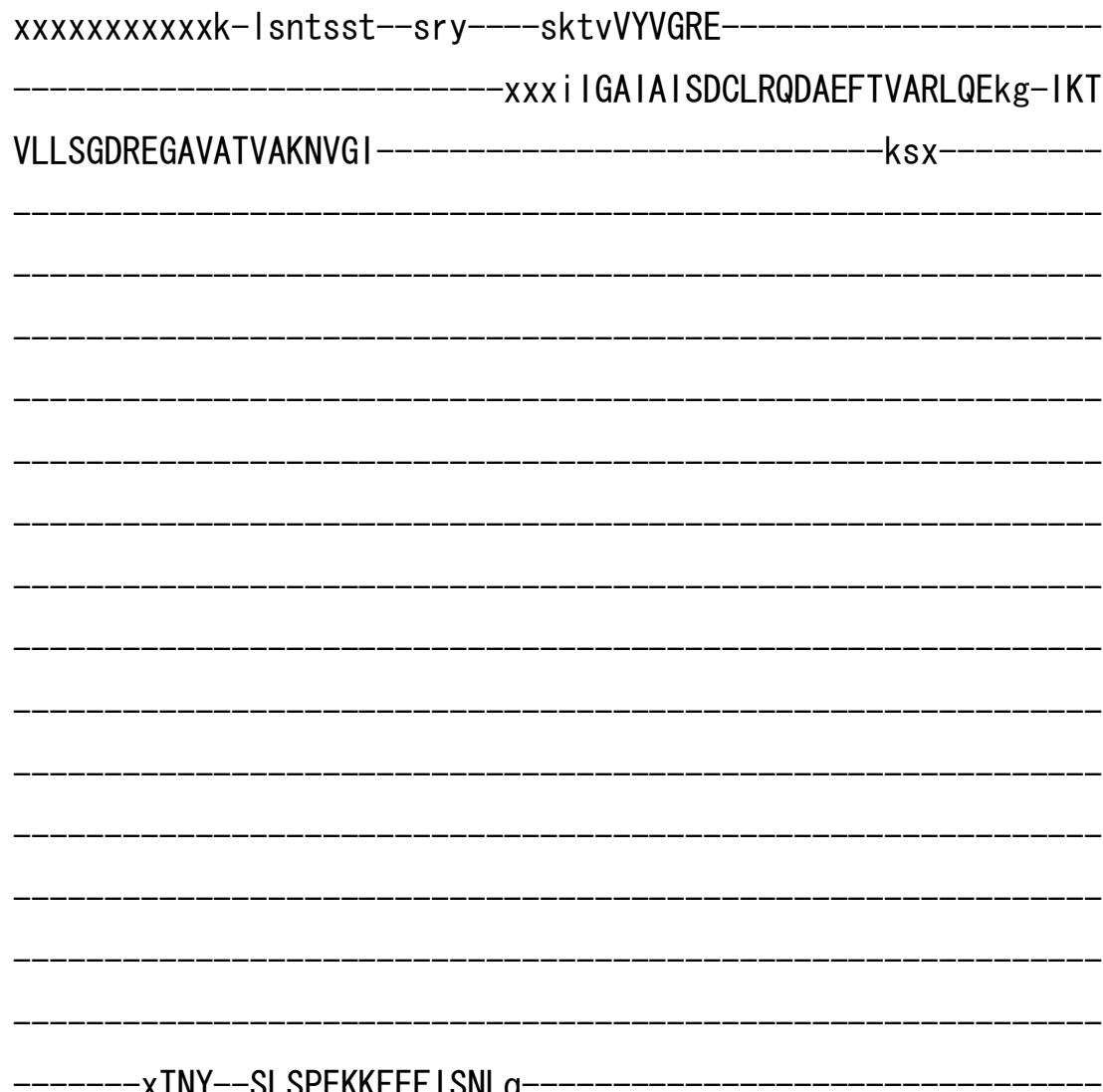

$-x$ TNY--SLSPEKKFEF I SNLq

---ssxx--------_---_----_---_-----RVAMVGDG I NDAPSLAQADVG I AL--

-ASNAASVILVr-NKLSHVVdaLSLAQATMSKVYQNL------A

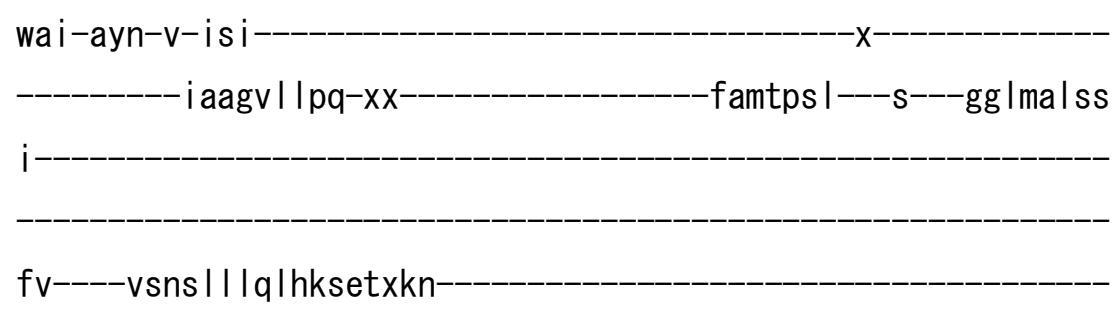


।

>osal_IB201 NP_001046033.1

$\mathrm{XXXXXX}$

$-\mathrm{XXXXXXXXX}$

$x X x$

$-X X X X X X X----X X X X X X X X X$

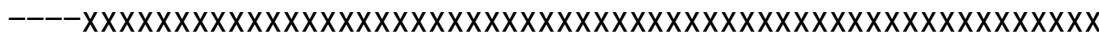
$\mathrm{x}$ $x x x x x x x x x x x x x x x x x$ i eai edagxeae ${ }^{-----~}$

- xdstvsqpk I qnt I sgq

friggmtcaacvnsv--

-egi Ikk Ipgvkravvalats I geveyd-psvisk-

-xe ivqa i

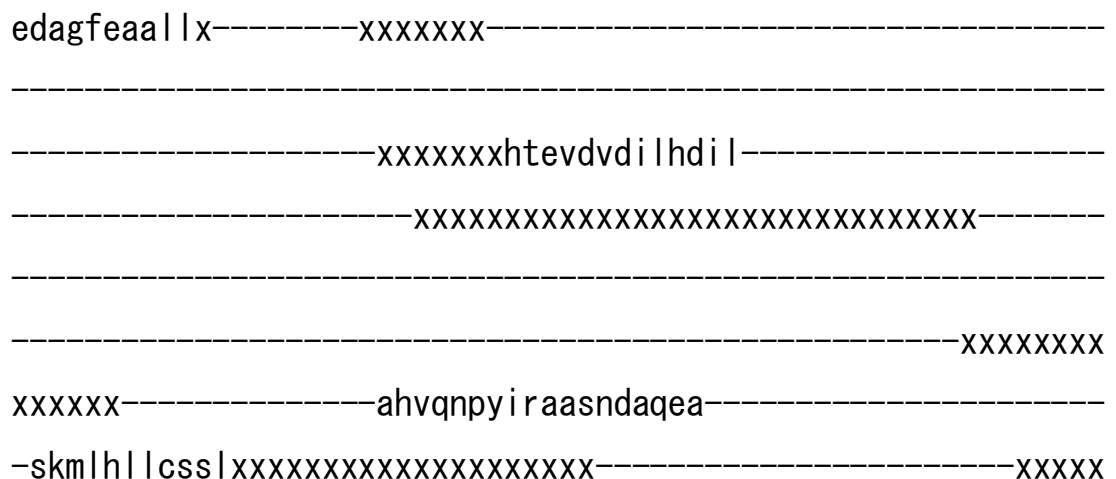


$x x x x x x x x x x x x x x x x x x x x x x x x x x x x x x x x x x x$ rh-gs $x$ mmdv lvvlgttasyvysvc al ly------- $x x x x x----$

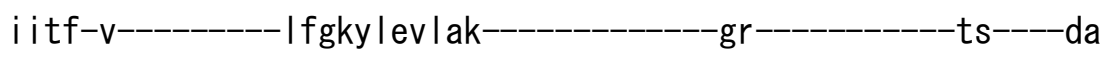
ikklve--Iv-

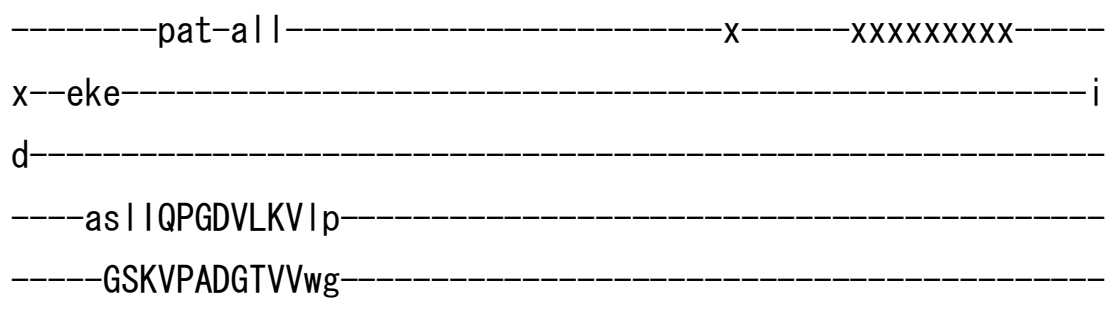

$-x$ shVDESMVTGESAPISKex-

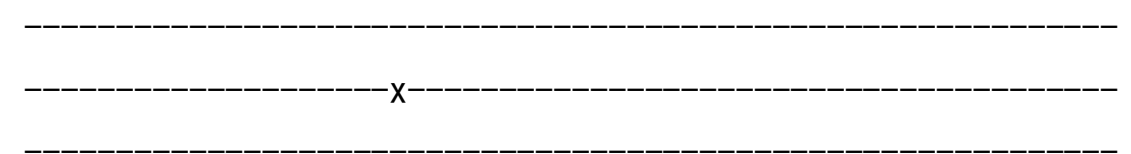

-SIVIG--GTMNxx

gilhiQATKVGSGTVLSqi is I vet

-aqmskap iqkfadyvag i fvpivvt I s Ivtf -i awf I ggs I gaypnsxxx--_-_-_-_-_-_-_-_-_---Xxxxcfvfs Imfs ISVVVIA CPCALGLAtPTAVMVATG-VGanhg-

-vLVK-GGDALERAQNVKYVIFDKTG

-TLTQGKATVtstkvfsgid- 


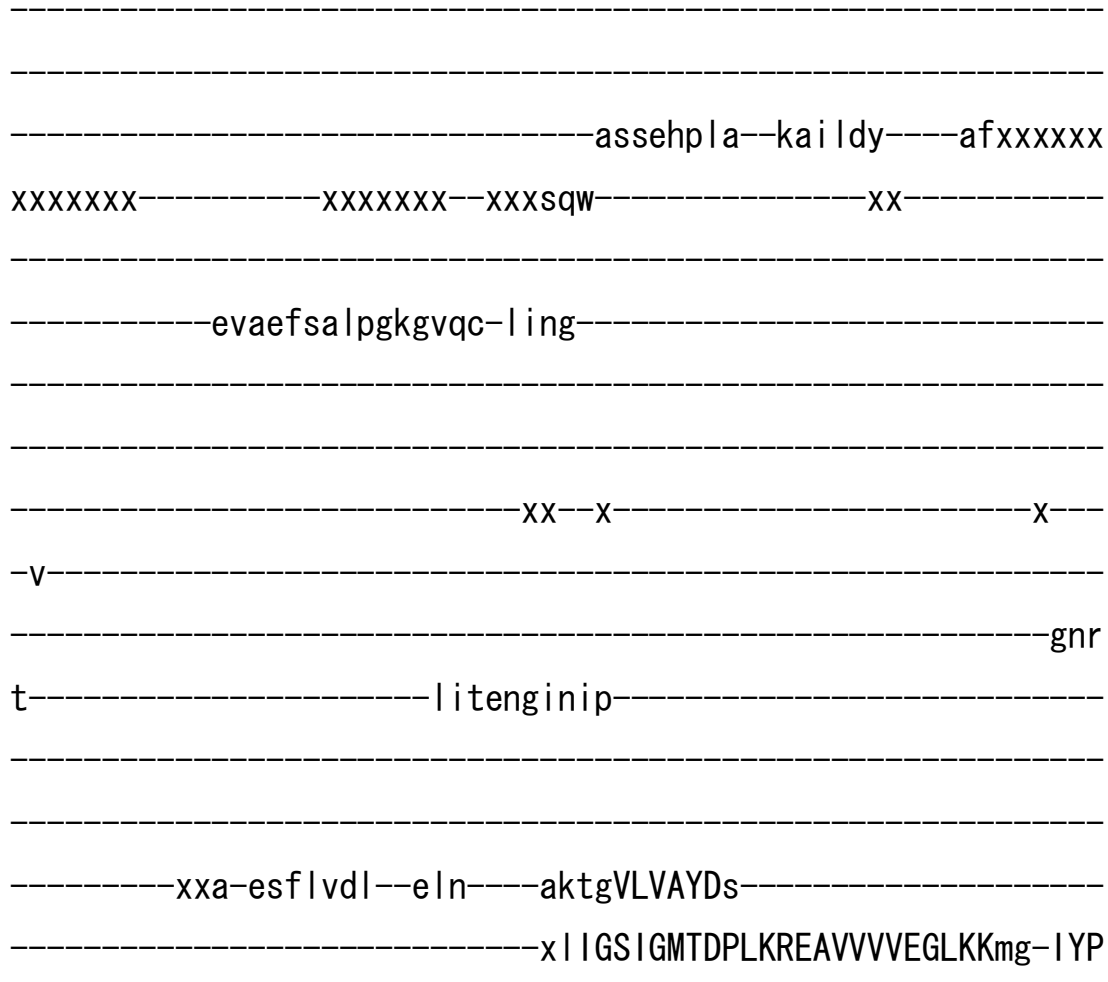

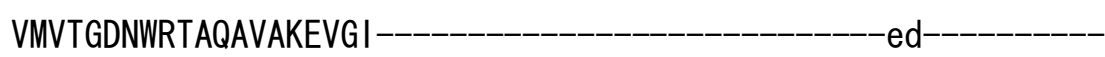

--VRA--EVMPAGKADVVRSLq

---kd--gs--_-_-_-_-_-_-_-_-_-_-_-_-_-_VVAMVGDG I NDSPALAAADVGMAI--

-AIEAADYVLVr-NNLEDVI ta IDLSRKTFSR I RWNY------F 
fam-ayn-i-iai

$-x$

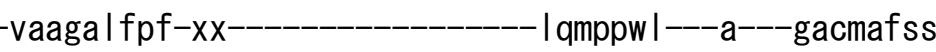

$\mathrm{v}$

sv----vcss Iw $\mid$ rryrkprxtt-xxxxxxxxxxxxxxxxx-

a

>osal_IB202 NP_001046193.1

m

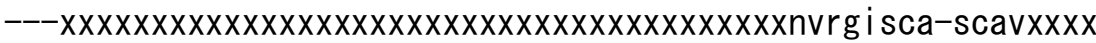




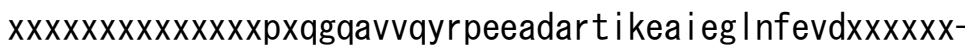

iavcr lqikgmactscsesv--

era l qmvpgvkkaavg I a l eeakvhfdpnitsrdxxxxx-xxdagfga

dl i ssgddvnx

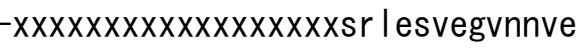

$x x x x x x x x x x x x x x x x x x x x x x x$

$x x x x x x x x x x x x x x x x x x--y$ sppkqreaerhhe $i$ rny $r x x x x x x x x x x x x x x x x x x x x x x$

$x s p f g d w \mid f y k v$

$-x x x x x x x x x x x x x x x x x x x x x x x x x x x x x x x x x x x k r x y s-n m d v$ Ivalgtnaayfysvy i vlk--_-_-_-_-_-_-_-_-_-_-_-_-_-_-_-_-_-_---xxxses----fegqdffetsa

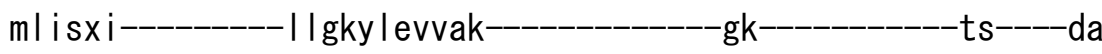

Isk|telape-

$-x-x---$ shVNESMI TGEARPI AKk $x-$

$$
-t a c x|t|
$$

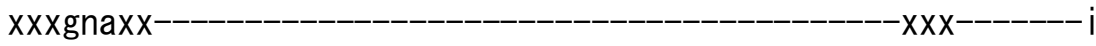

$\mathrm{s}$

----tq ILQRNDV IKI vp---_---_-_-_-_-_-_-_-_-_-_-_-_-_-_-_-_-_-

GEKVPVDGVI $k$

$-\mathrm{x}$

-DKVIG--GTVN-

dn-----gci i

-aq larapvqk ladr isrffuptvvvaaf I tw

- I gwfvagqfdi yprexxx xxxxsfelalafgISVLVVA

CPCALGLAtPTAVMVATG-KGasqg- 
-vLIK-GGNALEKAHKVKA I IFDKTG-

-TLTVGKPSV

-qtkvfskip

$-\mathrm{x}$

xx|cdla-----agae

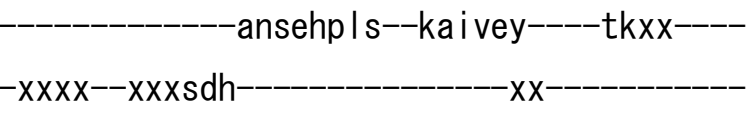

eskdfevhpgagvsa-nveg

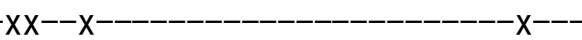

$-\mathrm{v}$

-gnk

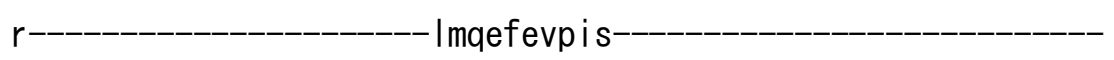

-xxv-eghmset--eel----artcVLVAIDrx

iCGALSVSDPLKPEAGRAISYLSSmg-ISS

IMVTGDNWATAKS I AKEVG I-

$-\mathrm{gt}$ 


\section{-VFA--EIDPVGKAEKIKDLq}

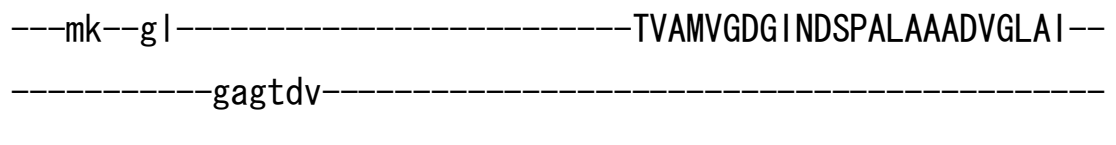

-A IEAAD IVLMr-SSLEDVI ta IDLSRKTLSRIRLNY------V

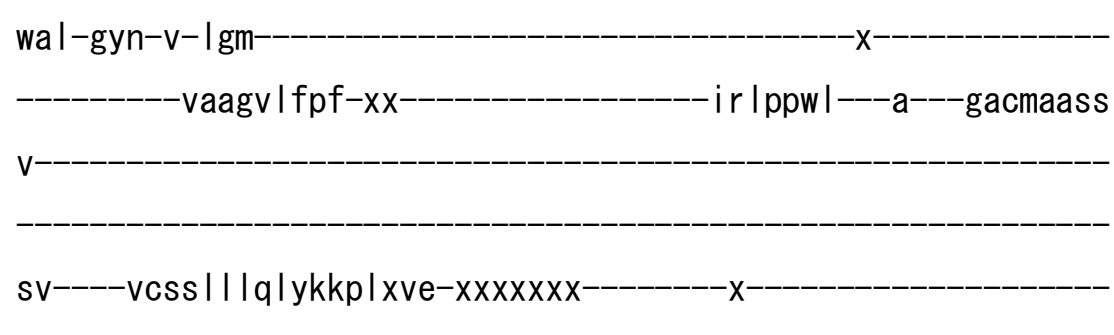

V

>osal_IB203 NP_001049149.1

$\mathrm{m}-$ 


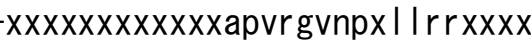

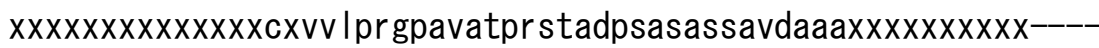
-aatv I I dvsgmmcggcaarv--

rt i l aadervetaavn I laesaavr I rspepaagxxxxxxxxxecgfps varrggaasgx

$-X X X X X X X$

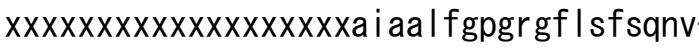

xnvtpdi $|f d g|$

-xxfkxgsxnmns Ivgfgsaaafaissv sIIn xele---wnstffdepvm

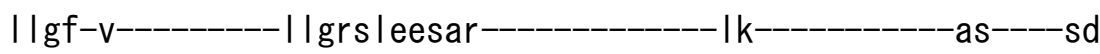
mnelvs| Isp

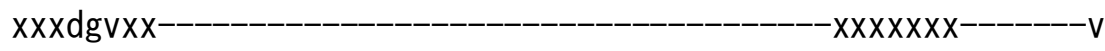

$$
p
$$$$
\text { ----vddVRVGDF ILV I } p
$$
GETIPVDGNVLgg 
X-

-FPVFA--GTVN-

wd----gp lk-

iKATTTGPSSTIAk i vrmved

-aqar eapvqr I ads i agpfvytvmt I saatf

-sfwyy i gth ifpev|xxxxxx -xxxxs|||s|k|aVDVLVVS

CPCALGLAtPTAILI GTS-LGakrg-

ILIR-GGDVLERLAGIDA I VLDKTG

TLTKGRPVVt

sias lay

xxilrla-----aave-

-ktalhpia--na imee----ae-

$-x--x x x d i p$

$-x$

-tsgq | tepgfgc | aexdgc |

$-x x$

$-\mathrm{V}$

$-g$ l

d

-wvhnrfetkaxxx 


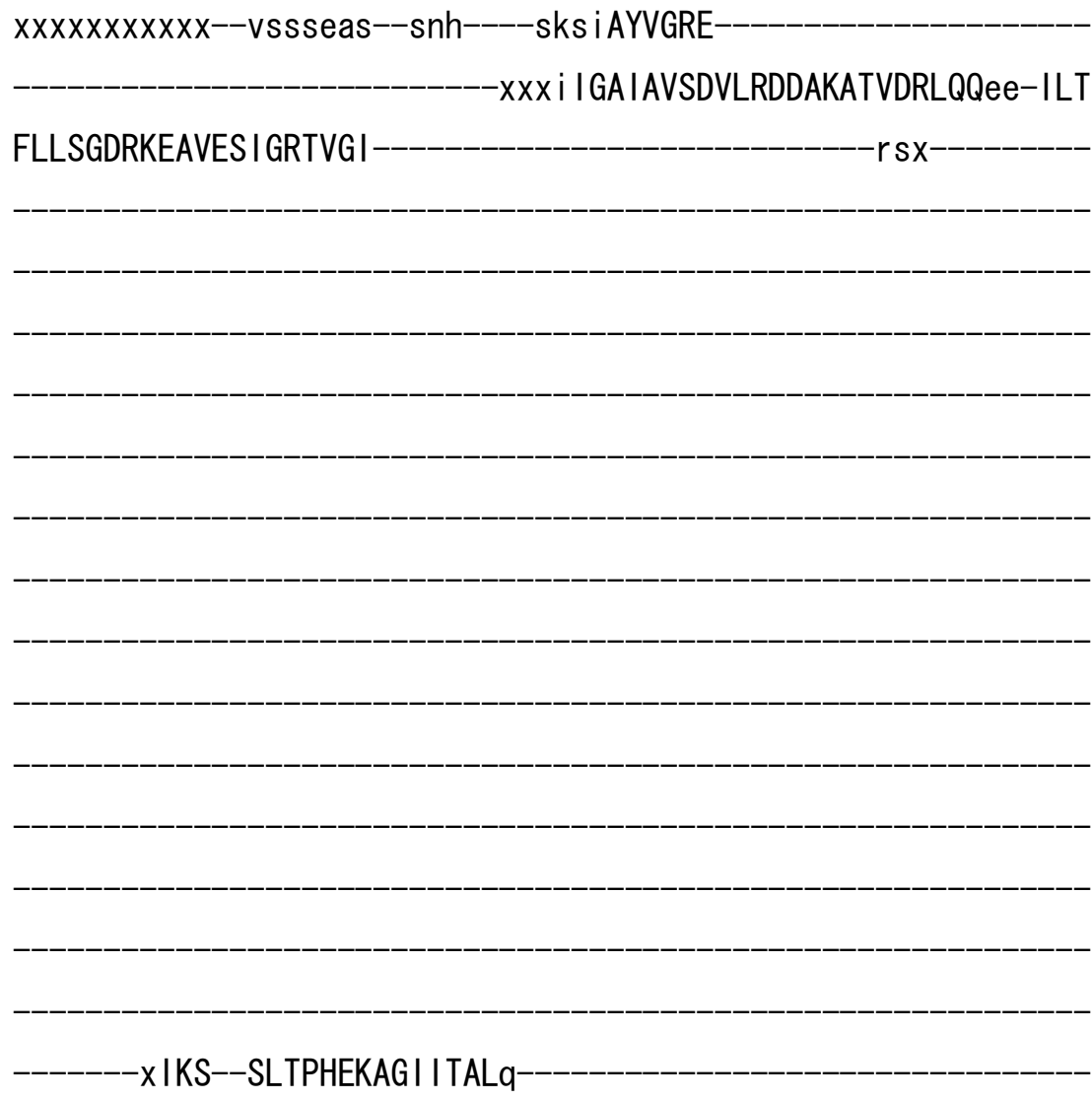

-RVAMVGDGI NDAPSLAAADVGVAM--xxnskesa

ASDAASVVLLg-NRLSQVMdaLSLSKATMAKVHQNL A

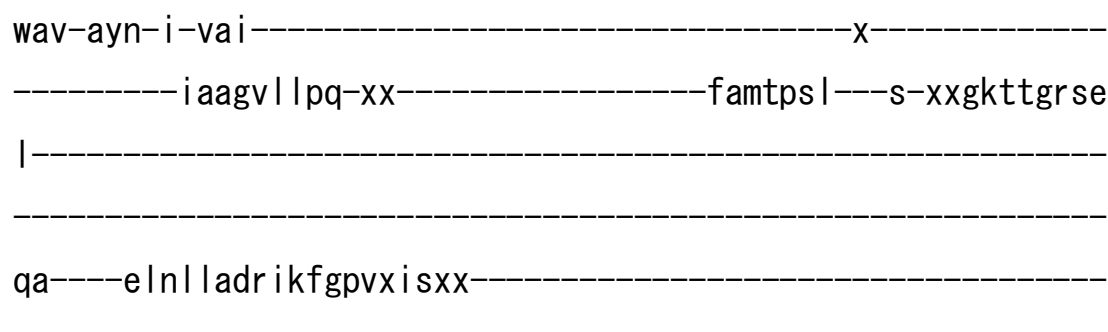


$\mathrm{p}$

>osal_IB204 NP_001058305.1

$\mathrm{m}$

$\mathrm{XX}$

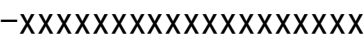

---- $X X----X X X X X X X X X X X X X X X X X X X X X X X X X X X X X X X X X X X X X X X X X X X X X X X X X X$

$x x x$ i ea $i$ edagxdae $i$

-ixdtai sqpkaqkt I saq

-fr iggmtcancvnsv--

-eg i lkr I sgvkgavva l ats I geveyd-psvink

-xe iveai

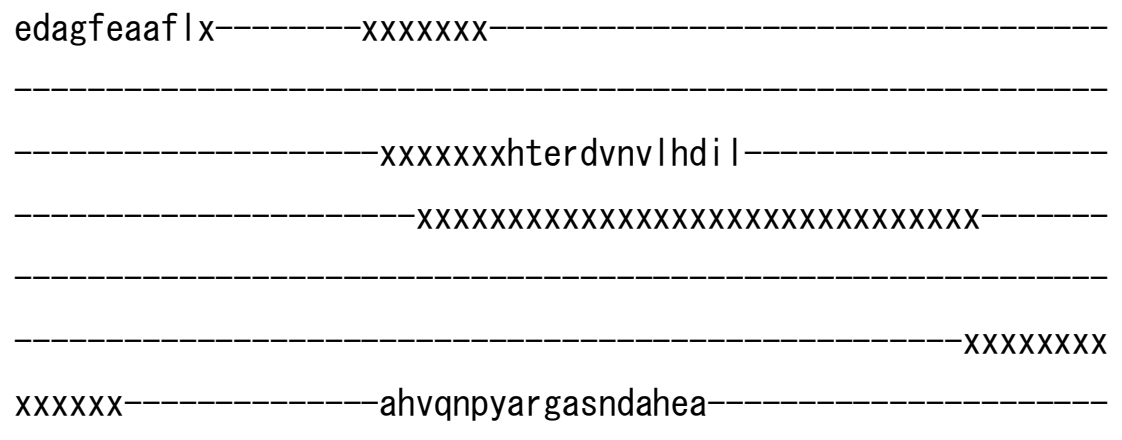

$-a k m|h||r s s| x x x-$ 
-ylevlak

-gk-------------ts-----da

ikkIve--Iv-

-pat-al I

$-x^{-------x x x x x x x x x}$

$\mathrm{x}--\mathrm{ere}----------------------------------------------------\mathrm{i}$

d-

al IVQPGDILKVIp

GSKVPADGVVVwg

$-x$

shVNESMI TGESAPIPKex-

$-S A V I G--G T M N x x$

gvlh-

iQANKVGSETVLSqi is I vet

-aqmskapiqkfadyvasifvpivit Ismitf

- I vwf I cgwvgaypnsxxx---------------_------xxxxcfvfs I mfal AVVVIA

CPCALGLAtPTAVMVATG-VGanhg-

-VLVK-GGDALERAQNVNYVIFDKTG

TLTQGKAVVttakvfsgmd-

\section{xxflt|v-----asae}




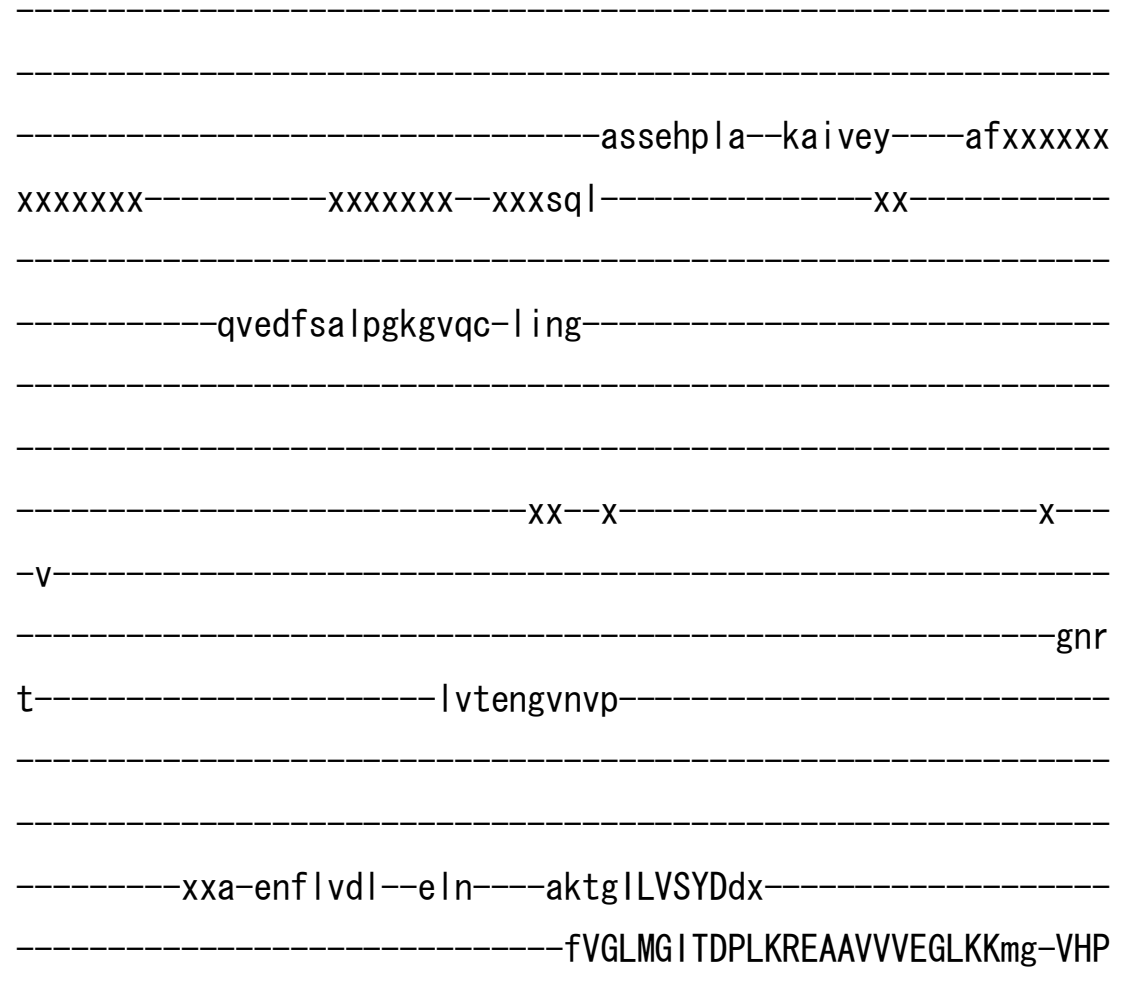

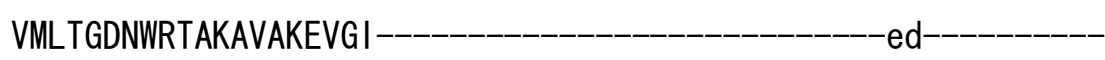

--VRA--EVMPAGKADVVRSLq

---kd--gs--_-_-_-_-_-_-_-_-_-_-_-_-_-_IVAMVGDG I NDSPALAAADVGMAI--

-AIEAADYVLVr-NNLEDVI ta IDLSRKTFSR I RWNY------F 


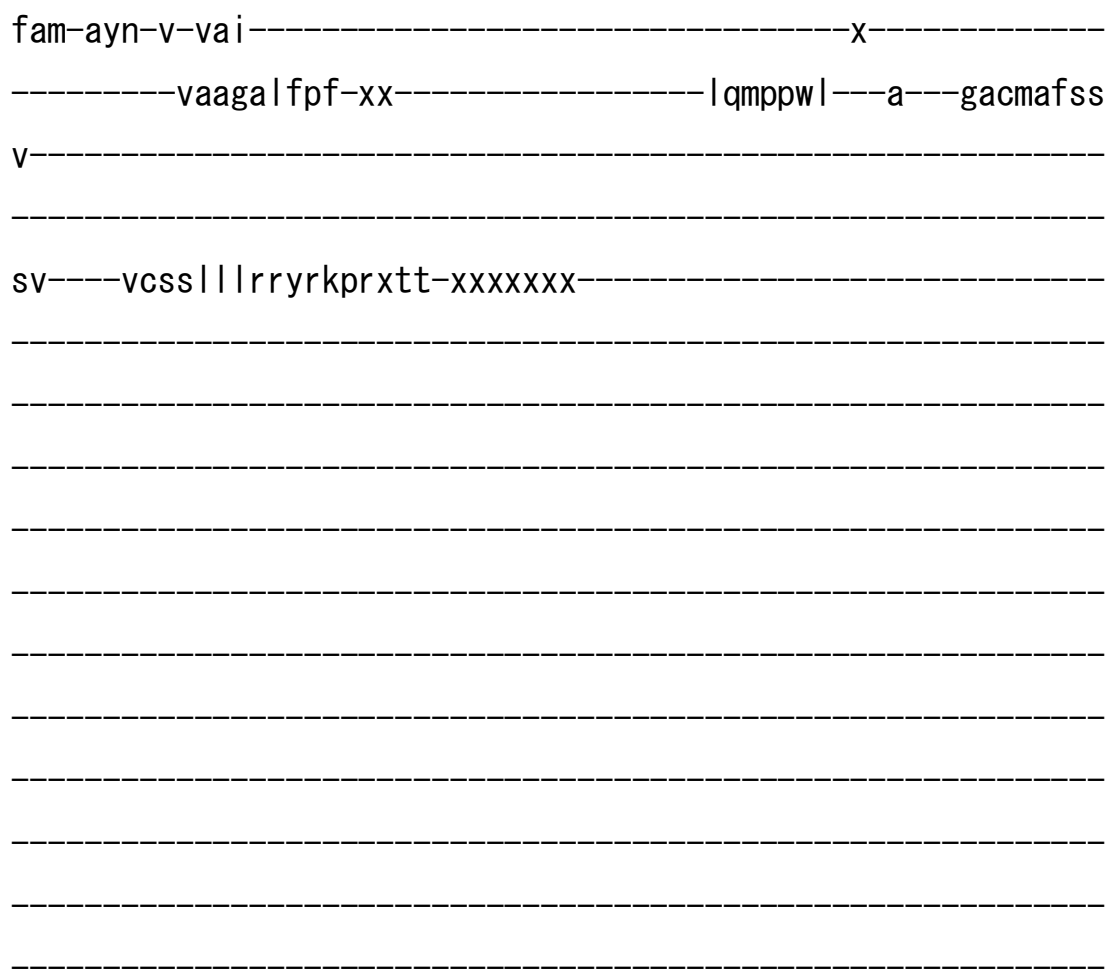

>tanII_IIA101 XP_952193. 1

m-

$-x X$ 


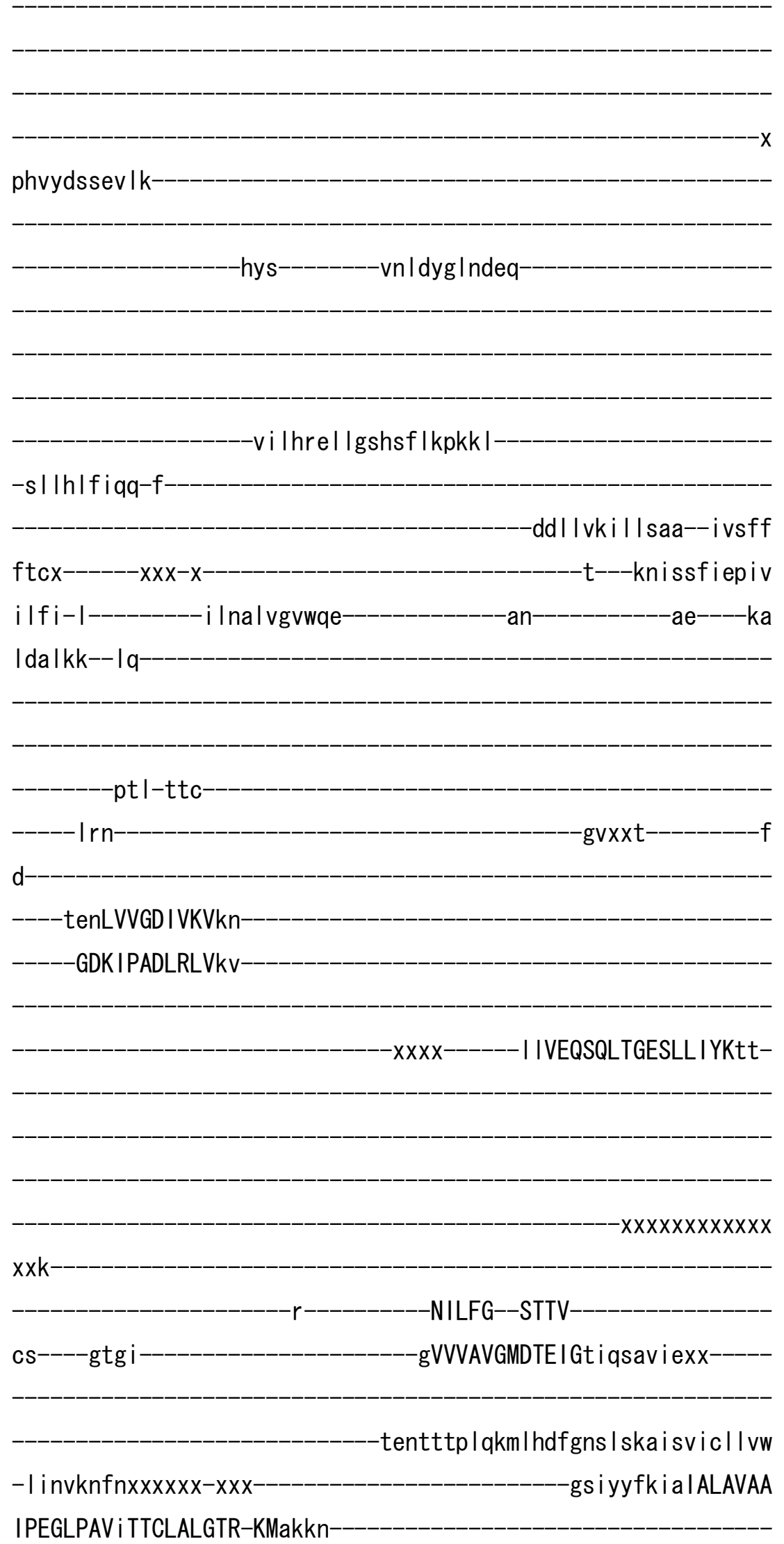


a IVR-KLPSIETLGCTTVICSDKTG-

TLTTNKMTTV -vvn I fnqqn

$-x x x x x x x p x x x$

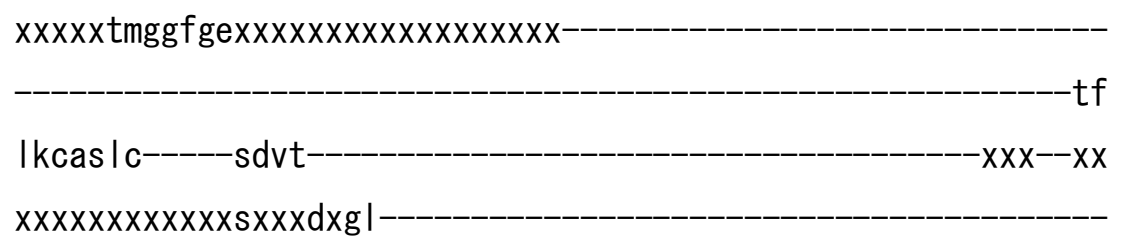

$-v$ legepte--va i i emxxxxxxxxxxxx

$x x x x x x x x x x x x x x x x x$ ennssni--kkmkxx$x x x x x x x x x x x x x$

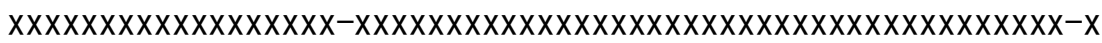

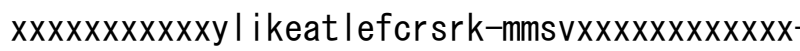

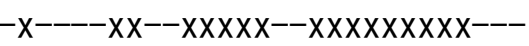

$-\mathrm{s}$ $-\mathrm{k}$

-gap

$\mathrm{e}$

-s i levctsym I pdgx

vnk I sksekte $\mathrm{i}-\mid$ dhvkq |--ane-----al rvLAFSYRsa-- $x x x x x x x x x x x x x x x x x x$ x------xxxxxxtmknnsvfsk-i ekdmvfLGLVGIMDPPRPEVKDSISKCMRag-IRV IMI TGDNKLTAEA I ARKVGI-_-_-_-_-_-_-_-_-_-_-_-_-_-_-_-_iki ixxs--_-_-_

$x x x x x x x x x x x x x x x x$

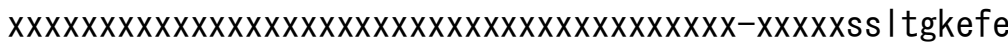
sltqdaqrk 
IItts-xIVFS--RTEPKHKQSIVSILk-

$---d \mid--g e-$

- IVAMTGDGVNDAPALKMADIG I SM--

-xingtev

-AKEASDM I LAd-DNFKT IVsa I EEGRCIYSNMKAF I R

yli-ssn-i-gevvs i fmtam I g i-_--_-_-_-_-_-_-_-_-_---_p--egm I pvq I I wv

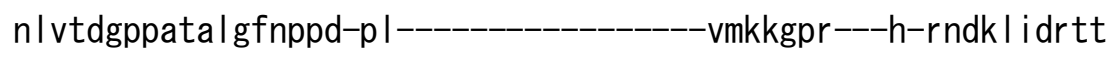

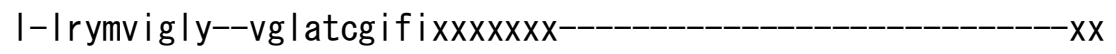

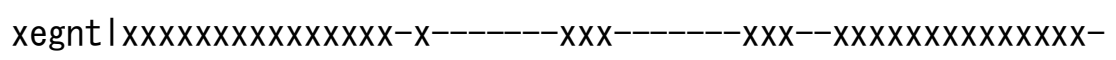
kv----kast Is Itt Ivi lemfn-alnal se--dss i I--xx---ppxsnpy I icaiffs il ihcfi I--yixxfss--_-_-_-_--_ fnvvpl-_-_-_-_-_-_-_-_-_-_-_-_-_

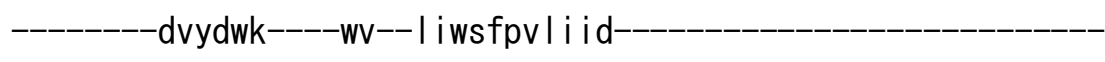
---------ecfkl c----------------------------------$-k r s y k x$ $-x x x x x x x-$ $-\mathrm{XXX}$

\section{$\mathrm{XXXXXXXXXXXXXX-1}$}

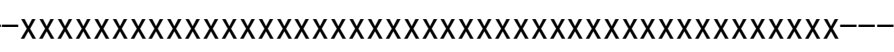

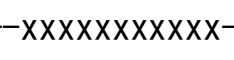

$\mathrm{y}$

>ggalI_IIA101 XP_415130. 2

$\mathrm{m}-$ 
ahtktveevla

-yfg--------vnestg I s leq-

-vkk I kekwgsne I paeegk

-tllelvieq-f

-edllvrilllaa--cisfv

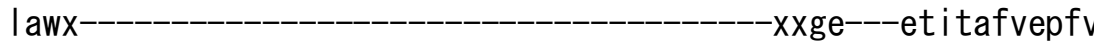

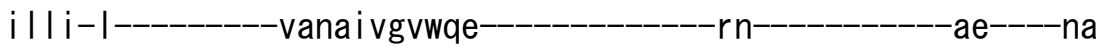
iealke--ye-

-pem-gkv-

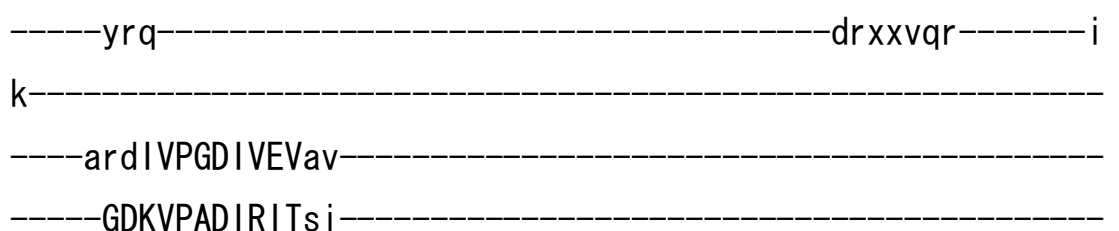

GDKVPADIRITsi 
$-\mathrm{x}---\mathrm{x}-\mathrm{t}--\mathrm{t}$ I rVDQSILTGESVSVIKht-

$-X X X X X X X X X X$

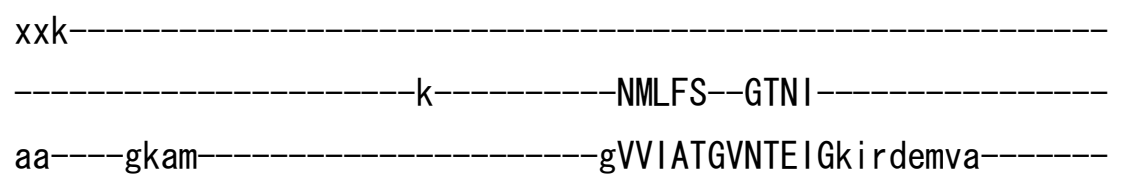

teqertp lqqk I defgeq I skvis I iciavw

-i in i ghfnxxxxxxxxxx----------------_---_-----ga i yyfk i aVALAVAA

IPEGLPAV i TTCLALGTR-RMakkn-

-a IVR-SLPSVETLGCTSVI ICSDKTG

-TLTTNQMSV $--------------r m f$ i l dkve

$-x x x x x x x \operatorname{exxx}$

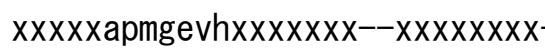

-el

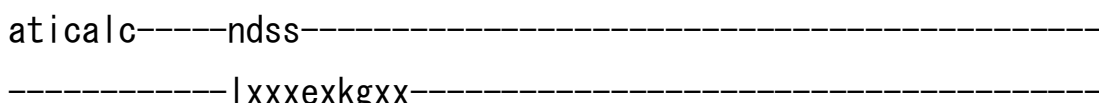

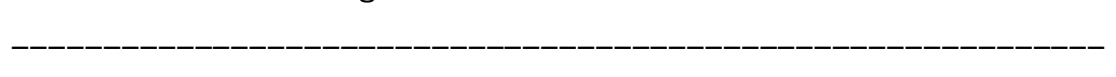

ekvgeate--taltcl-----xxxx----

$-x x x x x x x x x x x x$ ieranac--nsvixx

- Imkkeft lefsrdrk-smsvxxxxx

$-x x \times x x x x x-x \times x---$

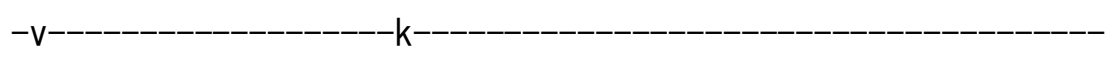

-gap

e---------_---_---_----gvi dr cthvrvgna 
k ip I ssg i kqki-msvi r ewxxgrd----t I rcLALATHdn---x-----------xxx---xxxn ledssnf in-yetn ItfVGCVGMLDPPRIEVASSIKLCKQag-IRV IMI TGDNKGTAVA I CRR I G I fvexxd-

$-X X X$

kaftgrefd

els laaqrd

achha--rCFA--RVEPSHKSKIVEFLq

---sf--de-

- ITAMTGDGVNDAPALKKAE I G I AM--

gsgtav

AKTASEMVLAd-DNFST I VaaVEEGRA I YNNMKQF I

R

y l i-ssn-v-gevvc if I taa l gf--_-_-_--_-_-_-_-_-_-_--p--eal i pvq I l wv n I vtdg I pata I gfnppd-I d------------------i mnkppr---n-pkep I i sgw I f-fryla i gcy--vgaatvgaaaxxxxxxx-----------------------------

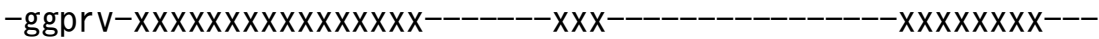

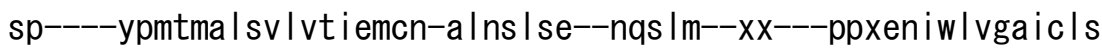
ms Ihf I i l--yvxx Ipi-----------ifqi tp I-

--------nvtqw|----mv--Iki s Ipvi IIdet Ikyv 


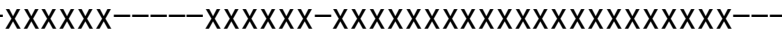

$x x x x x x x x x x x$

$-x X X X X$

S

>ggalI_IIA102 NP_990222. 1

$\mathrm{m}$

ahsvpvqdvIs

-rfg--_-_-_-vaescg I speq

-vrrnrekygpne 


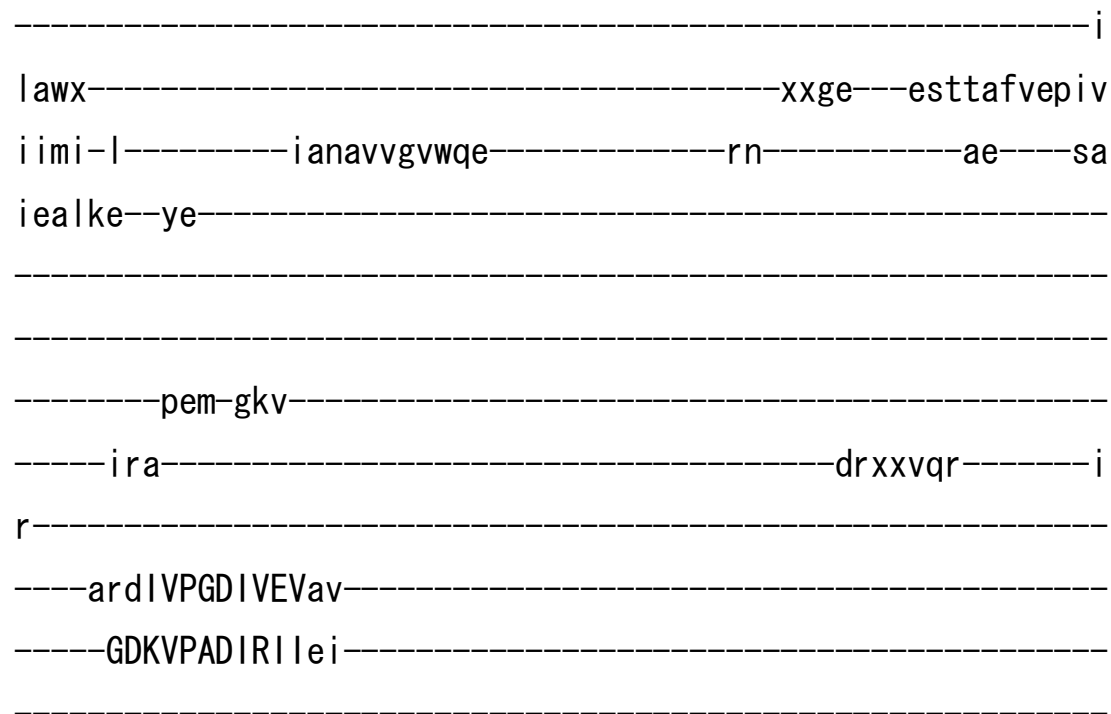

$-\mathrm{x}---\mathrm{x}-\mathrm{t}--\mathrm{t}$ | rVDQSILTGESMSVIKha-

$-x \times X X X X X X X$

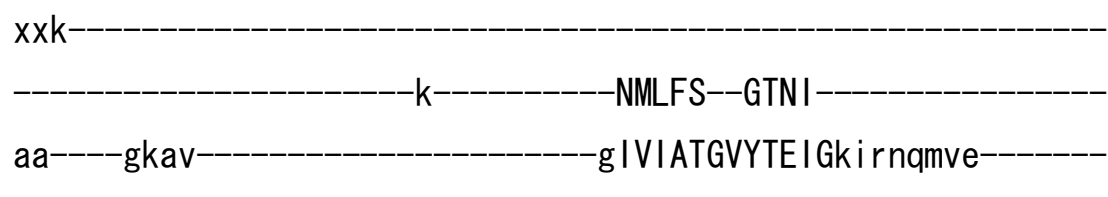

-tepektp Iqqk I defsqq I skvif I vciavw

-vini shfsxxxxxxxxxxx---------------_----------ga i yyfktsVALAVAA

IPEGLPAV i TTCLALGTR-RMakkn-

a IVR-SLPSVETLGCTSVICSDKTG

TLTTNQMSVc

rmf imekve

$-x x x x x x x \operatorname{exx} x$

xxxxxapegq i Ixxxxxxx--xxxxxxxx-

el

at icalc-----ndss

I xxxexkkxx 
-ekvgeate--taltc|-----xxxx----

$-x x x x x x x x x x x x v e r a n a c--n s v i x x$

- Imrkect lefsrdrk-smsvxxxxx

$-x x x x x x x x x x x x--$

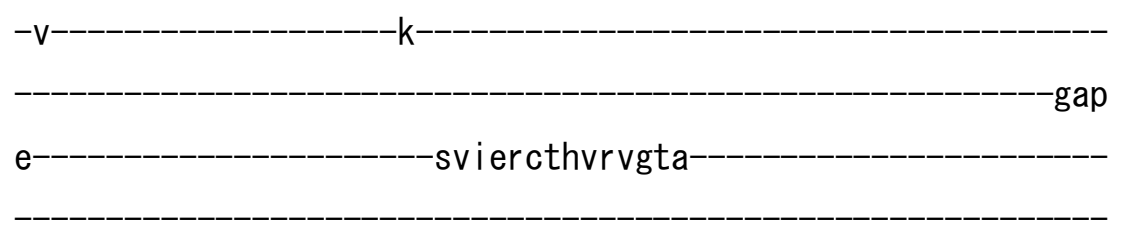

kvp I tppvreki-I sqi rdwxxgtd-----t I rcLALATHda--x-----------xxx---xxxq Ihdsttfth-yetn I tfVGCVGMLDPPRKEVTSSIEMCRKag-IRV

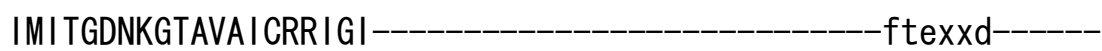

$x x x$

kaytgrefd

el speaqrq

acrea--rCFA--RVEPAHKSRIVEYLq

---sf--se---_-_-_-_-_-_-_-_-_-_-_ITAMTGDGVNDAPALKKAE I G I AM--

gsgtav

-AKSAAEMVLSd-DNFST IVsaVEEGRA I YNNMKQF I 
y l i-ssn-v-gevvc i f I ta i Ig |---_-_-_-_-_-_-_-_---eal i pvq I I wv

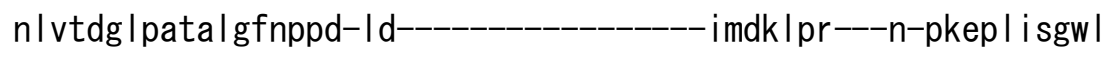

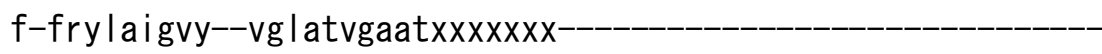

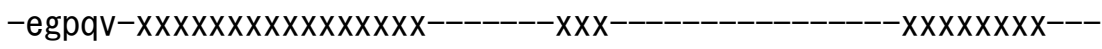
sr----ypttma | sv I vt i emcn-a Insvse--nqs I I--xx---ppx I n iw I I gai vms

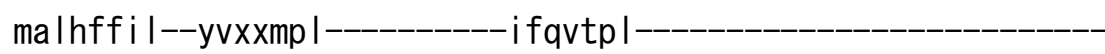

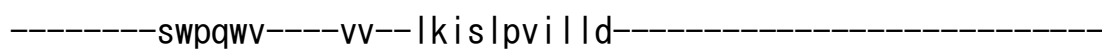
$-e g|k y|-$

srnhl

$-x x x x x x x x x x x x x x x x x x x x x x x x x x x x x x x x x x x x x x x x---$ $-\mathrm{XXXXXXXX}$

d

>mgr II_IIA101 XP_362105. 2

$\mathrm{m}$ 
ayalstsevlk

$-n \mid g$

-vdqnng I seeq

-vtk I rakhgkna i aeeppt

-plwel i leq-f

$-k d q|v|$ i I gsa--avsfv

$|a| x$

-xee---egwsafvdpav

ilti-I-

i Inavvgvsqe

ss

$-a e^{----k a}$

iaalqe--ys

$x x x x$

-faVDQAILTGESESVGKda-

$-x x x x x x x x x x x$

$x \times q$

$-i$

-NMLFS--GTTV-

vt-----grak-

aVVVLTGSNTAI Gdihes i ta

-q i septp Ikqk Indfgdq I akv i tv i cv I vw

-I iniphfsxxxxxx-xxx-gai yylkiaVSLGVAA

IPEGLAVV i TTCLALGTR-KMaakn- 
-aVVR-SLPSVETLGSCSVICSDKTG-

-TLTTNQMSVs

-kivyikeng

$-x x x \operatorname{exxx}$

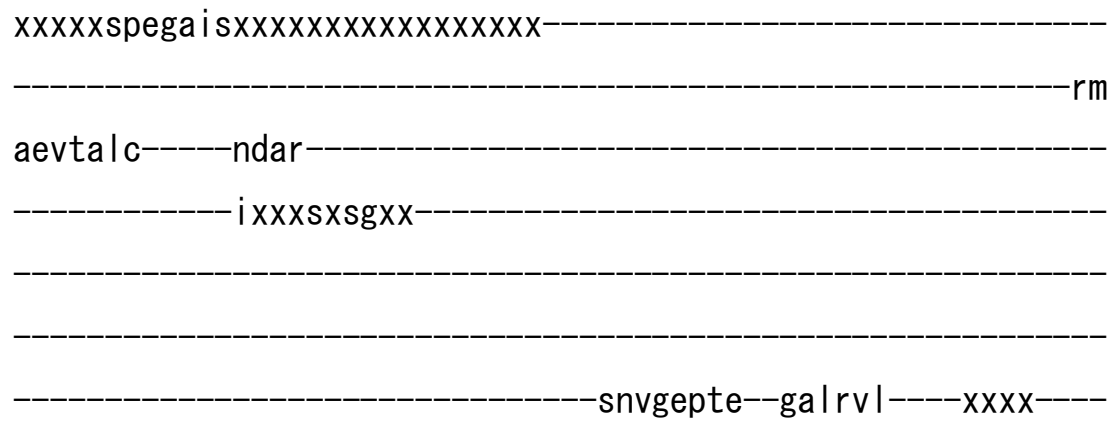

$-x x x x x x x x x x e d r$ thya--sswyxx

qqer iatfefsrdrk-smsvxxxxx

$-x \times x \times x x--$

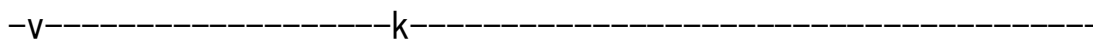

-gap

e-

-s i Idrcshal vgadxx-

kvamnak I sal I-mkevvdy--gnr----g I rv IALAAI dd

xxxxxxxkkakttaeyax I eqnmt ILGLVGMLDPPRPEVPESIRKCKDag-IR I

IVI TGDNRNTAES I CRK I GV-_-_-_-_-_-_-_-_-_-_-_-_-_-_-_-_fgexxd--_-_-_

$x x x$

$-k s f t g r e f d-----$

ql spaqqle- 
aakka--sLFS--RVEPSHKSRLVDLLq-

$---s \mid--$ ge-

-VVAMTGDGVNDAPALKKADIGVAM--

gsgtdv

SKLAADMVLAd-DNFAT IEsa I EEGRSI YNNTQQF I

$-R$

y l i-ssn-i-gevvs if I taamgm--_-_-_-_-_-_-_-_-_----p--eal i pvq I I wv

nl vtdg I patal sfnppd-hd-_-_-_-_-_-_-_-_--imr rqpr---k-rdep I iggw I

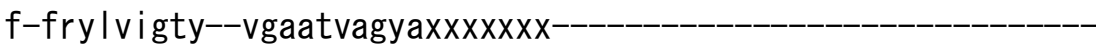

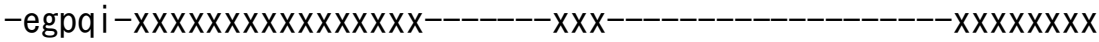

ak----sastvs | si Ivviemfn-avnalss--ses ||--xx---p| xenmm | vyaials malhfal|--ytxx|qs-----------|fsi|p|-

-nt l ewk----av--vy i sapvivid-

eflk|i

errff

$-x x x x x x x x x$

$-x x x x x$

q

>tpalI_IIA101 XP_765090.1

$\mathrm{m}-$ 
phvyeshevln

hys

-vnldyg Indeq

-vI Ihrev|gshsf Ikpkk I

- sl lhlfiqq-f

-ddI Ivki I lsaa--ivsff

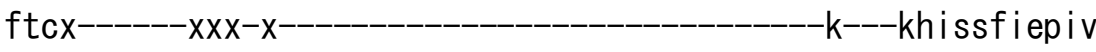

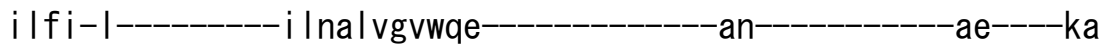

ldalkk--lq

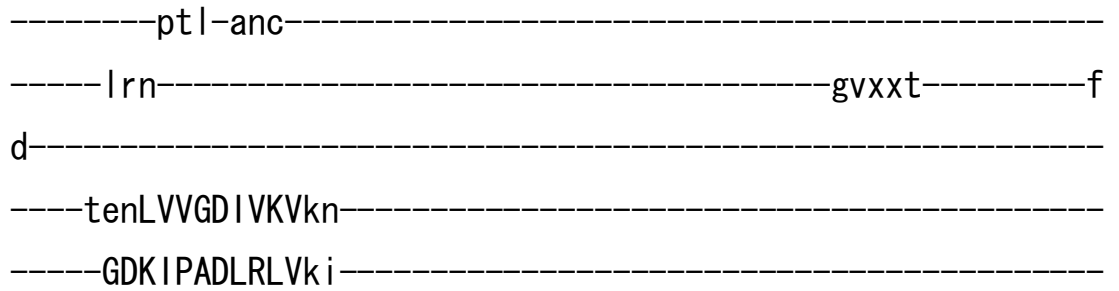


$-x x x x-----$ I IVEQSQLTGESLL IYKtt-

$-X X X X X X X X X X X X$

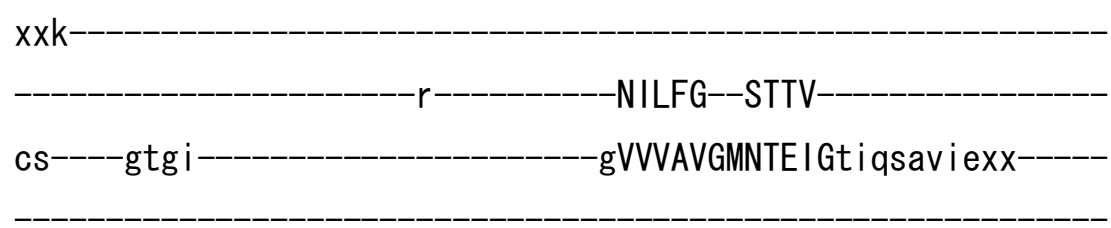

tentatp I qkm I hdfgns I skvi s i ic I l vw - I invknfnxxxxxx-xxx--_-_-_-_-_-_-_-_-_-_-_gc i yyfk i a I ALAVAA IPEGLPAV i TTCLALGTR-KMakkn-

-aIVR-KLPSIETLGCTTVICSDKTG-

-TLTTNKMTTV $-v v n$ I fnqqn

$x x x x x x x p x-x$

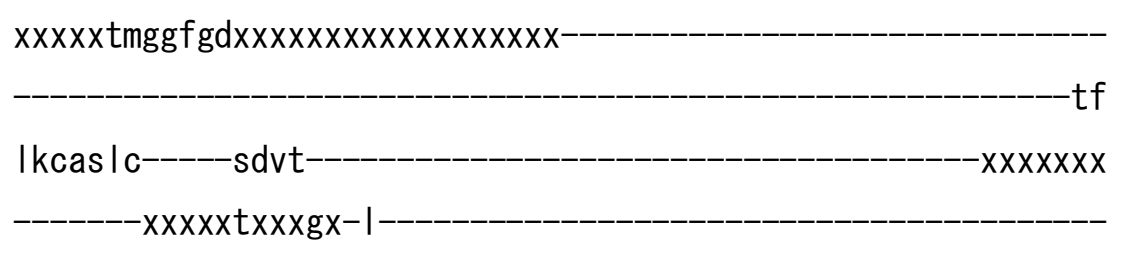

-vlegepte--vai i emxxxxxxxxxxxxx

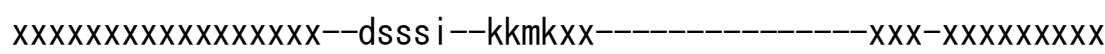

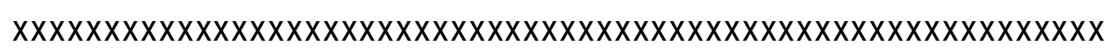
$x x x x x x x x x x x y$ likeat lefortrk-mmsvxxxxxxxxxxxx

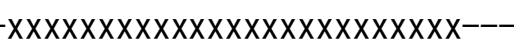

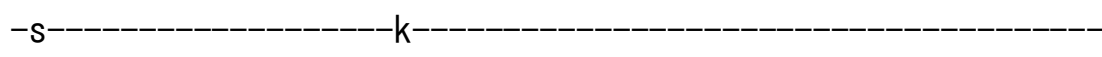

-gap

e------_--_-_-_-_-_-_--s i mevctsym I pdgx 
vnk laksekne i-I dhvkql--ane----al rvLAFSYRqa--xxxxxxxxxxxxxxxxxxx $x x----x x x x x x x s v k t s n v f s r-i$ ekdmtfLGLVG IMDPPRPEVKDS I SKCMRag-IRV IM I TGDNKLTAEA I ARKVGIikpxxr-

$-x x x$

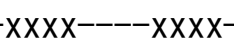

$-x x x x x x x x x x x x x x$ ss Itgkefe

s Issdaqrq

I Itks-x IVFS--RTEPKHKQSIVSILk

$---\mathrm{el--ge-}$

- IVAMTGDGVNDAPALKMADIGVSM--

-xvngtev-

-AKEASDM I LAd-DNFKT IVaa I EEGRC I YSNMKAF I $-R$

y l i-ssn-i-gevvs i fmtam I g i----_-_-_-_-_---_------p--egm I pvq I I wv nlvtdgppatal gfnppd-p I---_-_-_-_-_-_----vmkrepr---h-rndk I idr it I-Irylvigly--vglatcg if i $x x x x x x x-----$

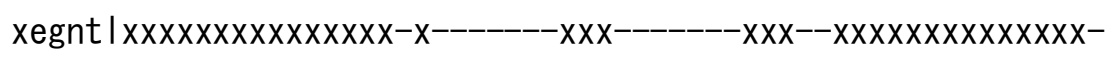

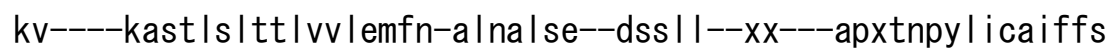

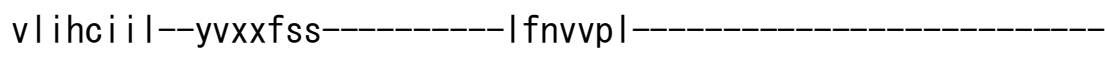

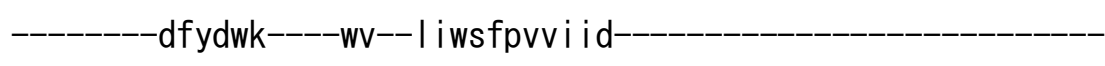
- ecfklc- 
y

>mmulI_IIA101 NP_031530. 2

$$
\mathrm{m}
$$

ahsksteecls

-yfg---------vsettg|tpdq-

-vkrh l ekygpne I paeegk

-s I we Ivveq-f 


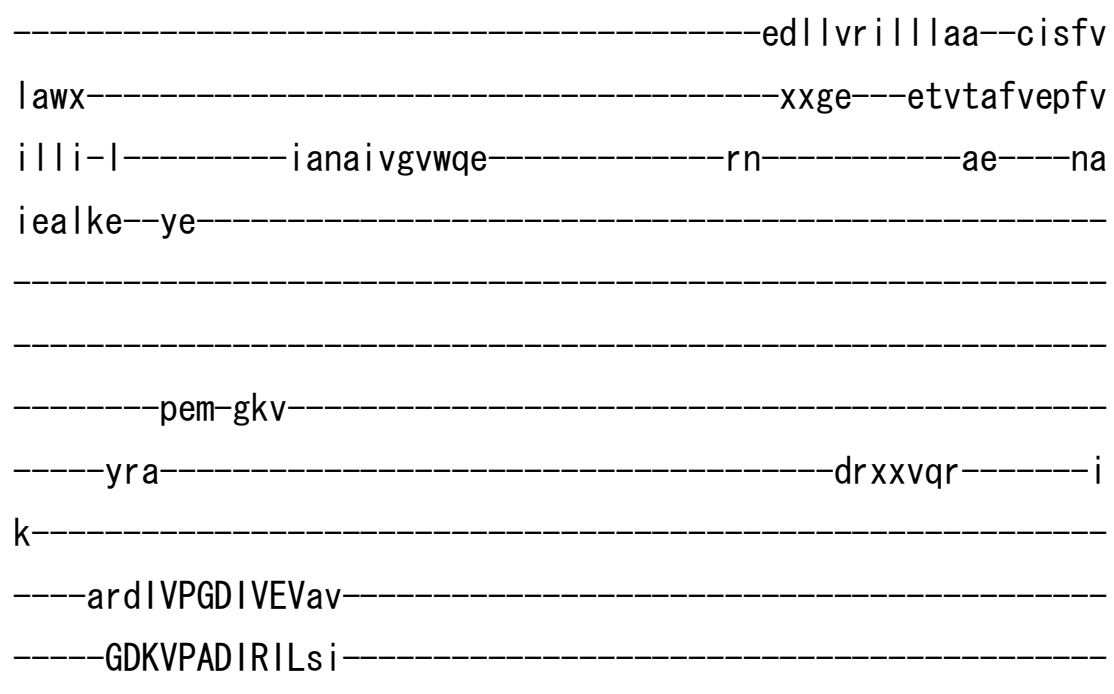

$-\mathrm{x}---\mathrm{x}-\mathrm{t}--\mathrm{t}$ IrVDQSILTGESVSVIKht-

$-x \times X X X X X X X$

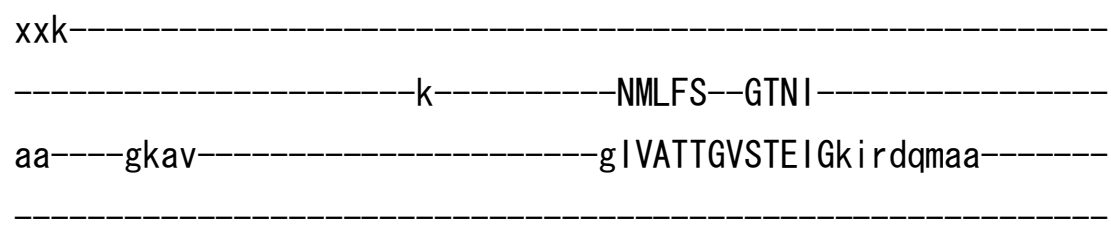

-teqdktp Iqqk I defgeq I skvi s I icvavw

- I inighfnxxxxxxxxxx----_-_-_-_-_-_-_-_---gai yyfk iaVALAVAA

IPEGLPAV i TTCLALGTR-RMakkn-

a IVR-SLPSVETLGCTSVICSDKTG

TLTTNQMSV -

$-k m f$ i idkvd-

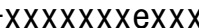

xxxxxapegev Ixxxxxxx--xxxxxxxx-

el

at icalc-----ndss

- xxxexkgxx 
-ekvgeate--taltt|-----xxxx----

$-x x x x x x x x x x x x v e r a n a c--n s v i x x$

- Imkkeft lefsrdrk-smsvxxxxx

$-x x x x x x x x x x x x--$

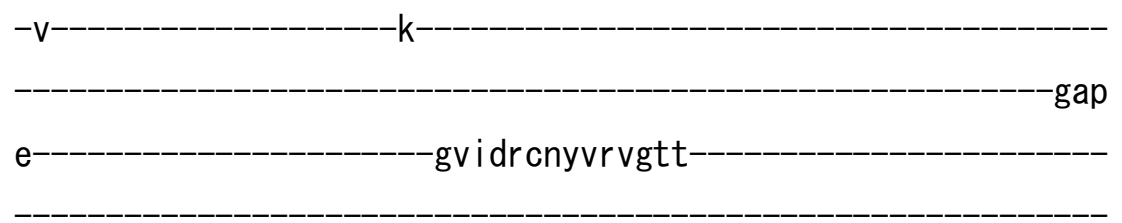

rvp I tgpvkek i-msvi kewxxgrd-----t I rcLALATRdt---x-----------xxx---xxxv I ddsakfme-yemd I tfVGVVGMLDPPRKEVTGSIQLCRDag-IRV

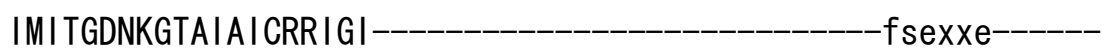

$-x x x$

raytgrefd

dlplaeqre

acr ra--cCFA--RVEPSHKSKIVEYLq-

---sy--de---_--_--_-_-_-_--_-_-----ITAMTGDGVNDAPALKKAE I G I AM--

-gsgtav

-AKTASEMVLAd-DNFST IVaaVEEGRA I YNNMKQF I 


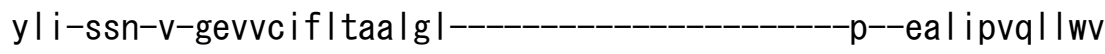
n l vtdg | pata I gfnppd-Id-_-_-_-_-_-_-_-_-_-_imdrppr---s-pkep I i sgw | f-fryma i ggy--vgaatvgaaaxxxxxxx--------------------------------

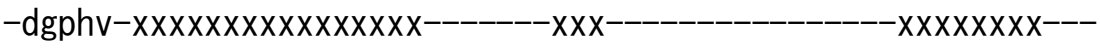
ap----epmtma | sv I vt i emcn-a|ns | se--nqs ||--xx---ppxvniw | gs ic ls ms Ihfli I--yvxx|pm-----------ifk|ral-

- dftqw|----mv--|kis $|p v i g| d$

ellkfi

arnyl

$-x$

g

>mmul I_IIA102 NP_033852. 1

m 
ahtktveevlg

-hfg--------vnestgl s leq

-vkk I kerwgsne I paeegk

-tllelvieq-f

edl Ivrill laa--cisfv

lawx

-xxge---eti tafvepfv

illi-I-

$r n$

-ae----na

iealke--ye-

$-\mathrm{x}---\mathrm{x}-\mathrm{t}--\mathrm{t}$ | rVDQSILTGESVSVIKht-

$-x x x x x x x x x x$

$x x k$

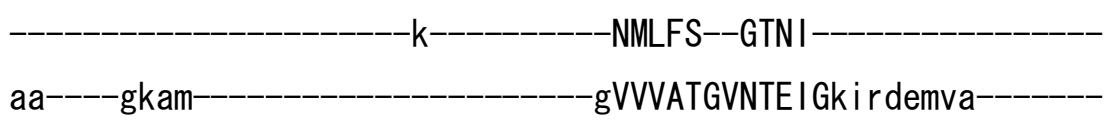

teqertp Iqqk I defgeq I skvis I i c iavw

- i inighfnxxxxxxxxxx-

gai yyfkiaVALAVAA

IPEGLPAV i TTCLALGTR-RMakkn- 
a IVR-SLPSVETLGCTSVICSDKTG-

TLTTNQMSVc

-rmf i ldkve

$x x x x x x x \operatorname{exxx}$

$x x x x x a p$ i gevqxxxxxxx--xxxxxxxx-

el

at ical c-----ndsa-

- Ixxxexkgxx

ekvgeate--taltcl-----xxxx----

$-x x x x x x x x x x x x$ ieranac--nsvixx

- Imkkeft lefsrdrk-smsvxxxxx

$-x x x x x x x x-x x x---$

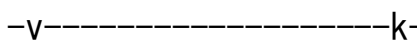

$-k-$

gap

e-gvidrcthirvgst

kvpmtpgvkqki-msvi r rewxxgsd-----t I rcLALATHdn---x-----------xxx---xxxhledsanf ik-yetn ItfVGCVGMLDPPRIEVASSVKLCRQag-IRV IMI TGDNKGTAVA I CRR I GI-_-_-_-_-_-_-_-_-_-_-_-_-_-_-_-_gqxxd--_-_-_

$x x x$

- kaftgrefd-----

el spsaqrd- 
ac Ina--rCFA--RVEPSHKSK IVEFLq-

$---s f--d e-$

- ITAMTGDGVNDAPALKKSE I G I AM--

-gsgtav

-AKTASEMVLAd-DNFST IVaaVEEGRA I YNNMKQF I $-R$

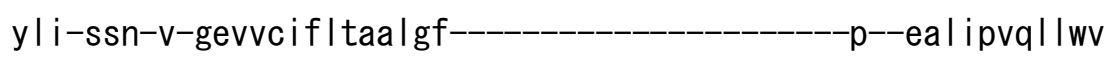
nlvtdg I pata I gfnppd-Id-_-_-_-_-_-_-_-_-_imnkppr---n-pkep I i sgw I f-fryla i gcy--vgaatvgaaaxxxxxxx---_-_-_-_-_-_-_-_-_-_-_-_---

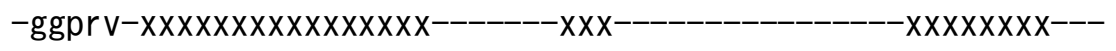
sp----ypmtmal sv I vt i emcn-alns I se--nqs | I--xx---ppxen iw $\mid$ vgs ic Is ms|hflil--yvxx|p|---_--_---ifqitp|-

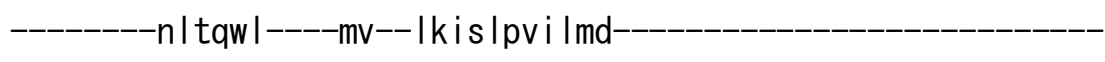
etlkfv-

$-a r n y l$

$-\mathrm{x}------------x x x x x-$

e

>mmul I_IIA103 NP_058025. 2

$\mathrm{m}-$ 
ah I saadv|r

-rfs---------vtaegg|s leq-

-vtdar erygpne lpteegk

-s I welvveq-f

-edIIvrill laa--Ivsfv

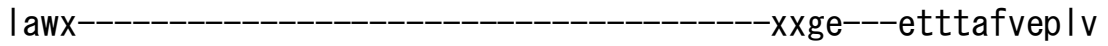

iml i-I--_-_-_---vana i vgvwqe--_-_-_-_-_-_-_rn---_-_-_----ae-----sa

iealke--ye-

-pem-gkv-

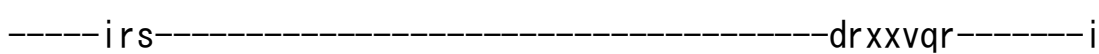

$r$

----ardIVPGD IVEVav

GDKVPADLRL I e i 
$-\mathrm{x}---\mathrm{x}-\mathrm{t}--\mathrm{t}$ I rVDQSILTGESVSVTKht-

$-X X X X X X X X X X$

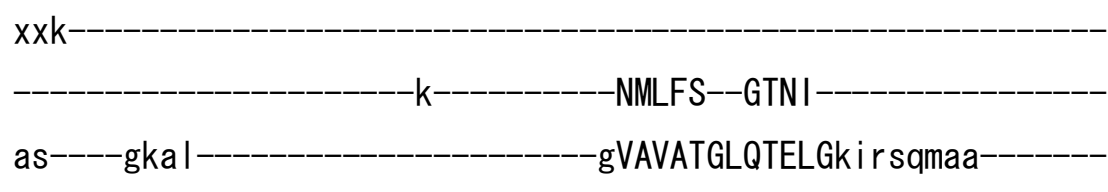

-vepertplark Idefgrql shai svicvavw -vinighfaxxxxxxxxxx--_-_-_-_-_-_-_-_-_-_-_-_--gavyyfk iaVALAVAA IPEGLPAV i TTCLALGTR-RMarkn-

a IVR-SLPSVETLGCTSVICSDKTG

-TLTTNQMSVcrmfvvaeae-

$-x x x x x x x \operatorname{exxx}$

xxxxxtpegevrxxxxxxx--xxxxxxxx-

-el

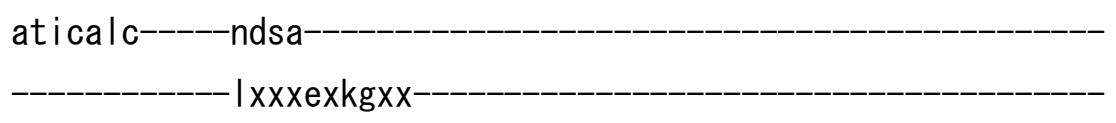

ekvgeate--taltcl-----xxxx----

$-x x x x x x x x x x x x$ veragac--nsvixx-

- Imrkeft lefsrdrk-smsvxxxxx

$-x x x x x x x x x x x x^{---}$

-v-----------------------k-

gap e svi ercssvrvgsr 
tap I sttsrehi-laki rdwxxgsd-----t | rcLALATRdt---x-----------xxx---xxxh I ddcsrfvq-yetd I tfVGCVGMLDPPRPEVAACI TRCSRag-IRV VMI TGDNKGTAVAICRRLGI $f g d x x d$

$-X X X$

kaytgrefd

dl speqqra

acrta--rCFA--RVEPAHKSRIVENLq

---sf--ne-

ITAMTGDGVNDAPALKKAE I G I AM--

gsgtav

AKSAAEMVLSd-DNFAS I VaaVEEGRA I YNNMKQF I

R

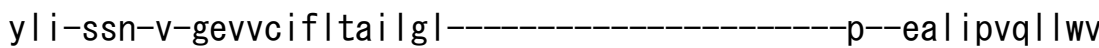
n lvtdg | pata I gfnppd-|d--_-_-_-_-_-_-_-_-_imekppr---n-preal i sgw | f-frylaigvy--vglatvaaatxxxxxxx-

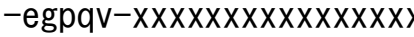
$\mathrm{XXX}$ $-x x x x x x x x---$

sr----fpttma | sv I vt i emcn-a I nsvse--nqs | |--xx---ppx I npw I I gavvms malhf|il--|vxx|p| ifqutp I-

--------sgrqwg-----vv--I qms I pvi I Idealkyl 
d

>rnol I_IIA101 NP_478120.1

$$
\text { m- }
$$

ahsksteecls

-yfg--------vsettg|tpdq-

-vkrh l ekygpne I paeegk

-s I we lvveq-f 


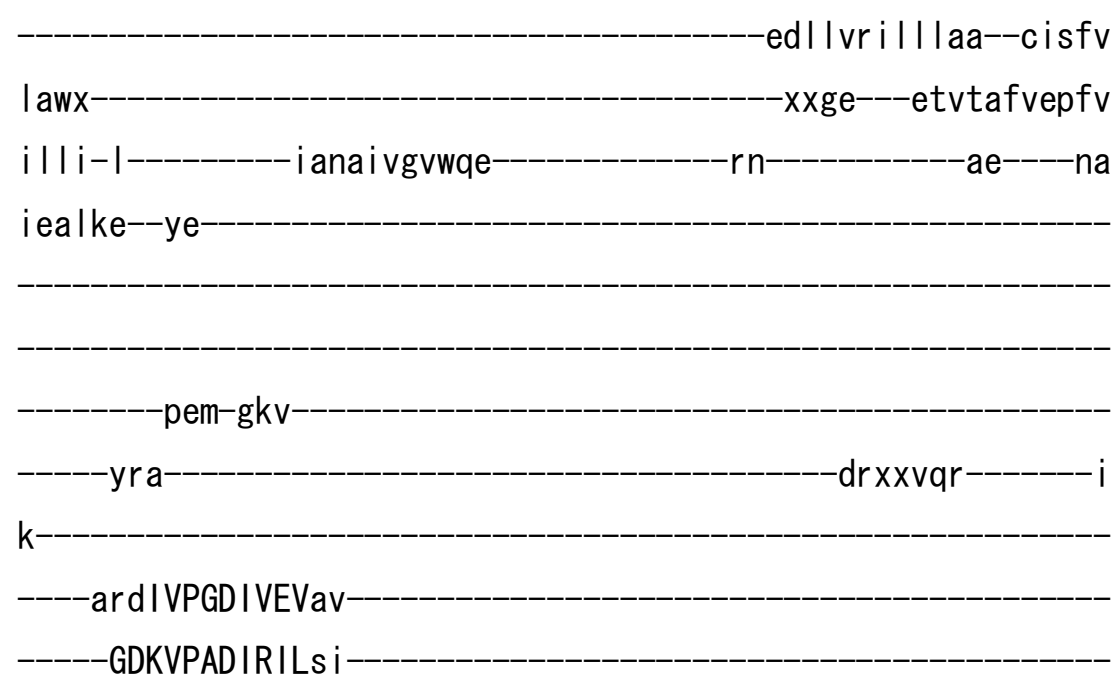

$-\mathrm{x}---\mathrm{x}-\mathrm{t}--\mathrm{t}$ I rVDQSILTGESVSVIKht-

$-x \times X X X X X X X$

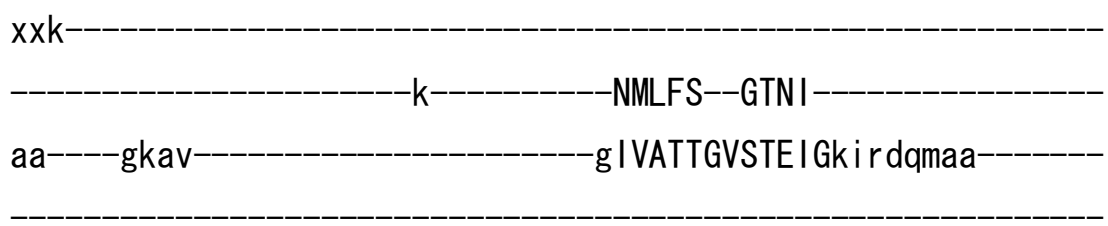

-teqdktp Iqqk I defgeq I skvi s I icvavw

- linighfnxxxxxxxxxx----_-_-_-_-_-_-_-_--'gai yyfk iaVALAVAA

IPEGLPAV i TTCLALGTR-RMakkn-

a IVR-SLPSVETLGCTSVICSDKTG

TLTTNQMSV -

$-k m f$ i idkvd-

$x x x x x x x \operatorname{exxx}$

xxxxxapegev |xxxxxxx--xxxxxxxx-

el

at i cal c-----ndss

- xxxexkgxx 
-ekvgeate--taltt|-----xxxx----

$-x x x x x x x x x x x x v e r a n a c--n s v i x x$

- Imkkeft lefsrdrk-smsvxxxxx

$-x x x x x x x x x x x x--$

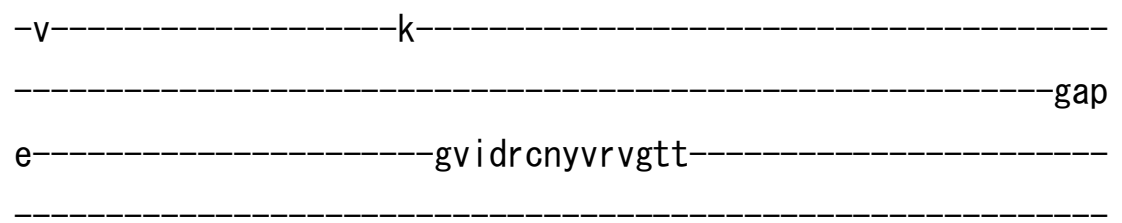

rvp I tgpvkek i-msvi kewxxgrd-----t I rcLALATRdt---x-----------xxx---xxxv I ddsakfme-yemd I tfVGVVGMLDPPRKEVTGSIQLCRDag-IRV

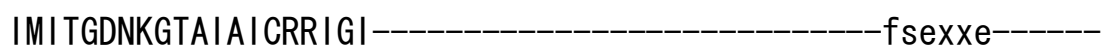

$-x x x$

raytgrefd

dlplaeqre

acr ra--cCFA--RVEPSHKSKIVEYLq-

---sy--de---_--_--_-_-_-_--_-_-----ITAMTGDGVNDAPALKKAE I G I AM--

-gsgtav

-AKTASEMVLAd-DNFST IVaaVEEGRA I YNNMKQF I 


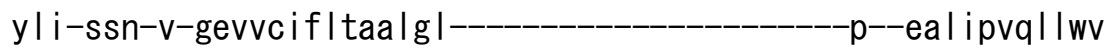
n l vtdg | pata I gfnppd-Id-_-_-_-_-_-_-_-_-_-_imdrppr---s-pkep I i sgw | f-fryma i ggy--vgaatvgaaaxxxxxxx--------------------------------

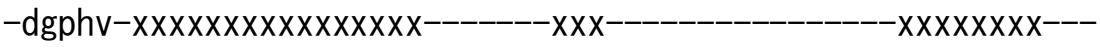
ap----epmtma | sv I vt i emcn-a|ns | se--nqs ||--xx---ppxvniw | gs ic ls ms Ihfli I--yvxx|pm-----------ifk|ral-

- dftqw|----mv--|kis $|p v i g| d$

ellkfi

arnyl

$-x$

g

>rnol I_IIA102 NP_058986. 1

$\mathrm{m}$ 
ahtktveev Ig

-hfg---_-_--vnestg l s leq-

-vkk Ikerwgsn-

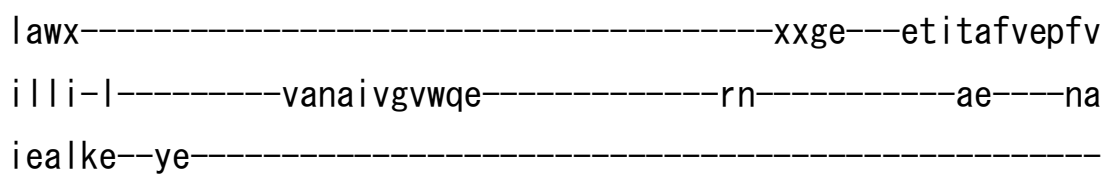

$-\mathrm{x}---\mathrm{x}-\mathrm{t}--\mathrm{t}$ I rVDQSILTGESVSVIKht-

$-x x x x x x x x x x$

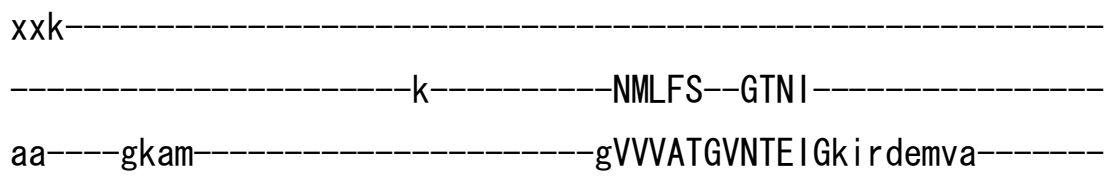

-teqer tp I qqk I defgeq I skvi s I ic iavw

- i in ighfnxxxxxxxxxxxgai yyfki aVALAVAA

IPEGLPAV i TTCLALGTR-RMakkn- 
a IVR-SLPSVETLGCTSVICSDKTG-

TLTTNQMSVc

-rmf i ldkve

$x x x x x x x \operatorname{exxx}$

$x x x x x a p$ i gevqxxxxxxx--xxxxxxxx-

el

at ical c-----ndsa-

- Ixxxexkgxx

ekvgeate--taltcl-----xxxx----

$-x x x x x x x x x x x x$ ieranac--nsvixx

- Imkkeft lefsrdrk-smsvxxxxx

$-x x x x x x x x-x x x---$

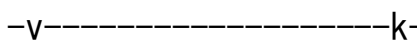

$-k-$

gap

e-gvidrcthirvgst

kvpmtpgvkqki-msvi r rewxxgsd-----t I rcLALATHdn---x-----------xxx---xxxhledsanf ik-yetn ItfVGCVGMLDPPRIEVASSVKLCRQag-IRV IMI TGDNKGTAVA I CRR I GI-_-_-_-_-_-_-_-_-_-_-_-_-_-_-_-_gqxxd--_-_-_

$x x x$

- kaftgrefd-----

el spsaqrd- 
ac Ina--rCFA--RVEPSHKSK IVEFLq-

---sf--de-

- ITAMTGDGVNDAPALKKSE I G I AM--

gsgtav

-AKTASEMVLAd-DNFST IVaaVEEGRA I YNNMKQF I

$-\mathrm{R}$

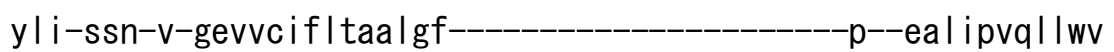

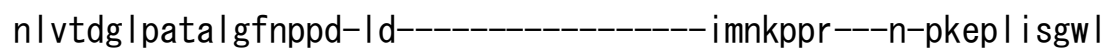

f-fryla i gcy--vgaatvgaaa $x x x x x x$-------------------------------

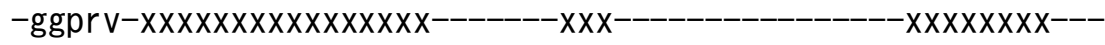

sp----ypmtma | sv | vt i emcn-a |ns | se--nqs | |--xx---ppxen iw I vgsic ls ms $|h f| i|--y v x x| p|----------i f q i t p|-$

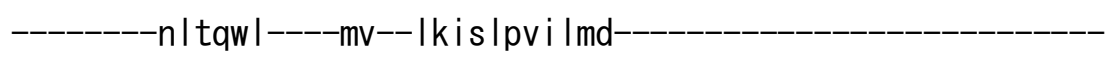

et Ikfv

arnyl

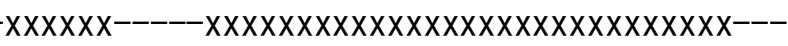

$\mathrm{xxxxxxxxxxx-10}$

$-\mathrm{XXXXX}$

S

>rnolI_IIA103 NP_037046.1

m- 
ahI Isaadv|r

-rfs---------vtaegg|tleq-

-vtdar erygpne lpteegk

-s I welvveq-f

-edlIvrilllaa--Ivsfv

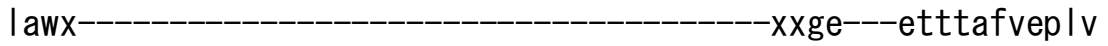

iml i-I--_-_-_---vana i vgvwqe--_-_-_-_-_-_-rn---_-_-_----ae----sa

iealke--ye-

-pem-gkv

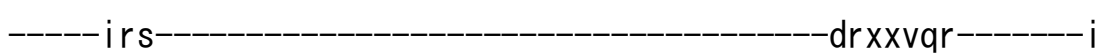

$r$

----ardIVPGDIVEVav

GDKVPADLRL I e i 
$-\mathrm{x}---\mathrm{x}-\mathrm{t}--\mathrm{t}$ I rVDQSILTGESVSVTKht-

$-X X X X X X X X X X$

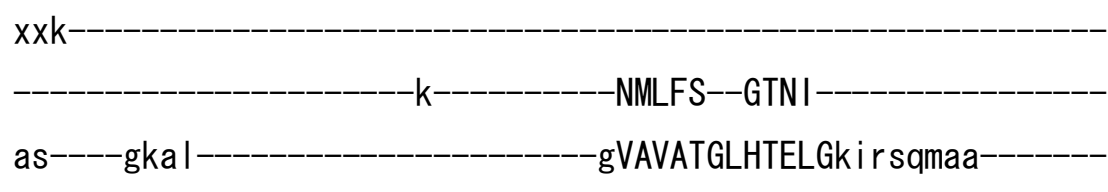

-vepertplark Idefgrql shai svicvavw -vinighfaxxxxxxxxxx--_-_-_-_-_-_-_-_-_-_-_-_--gavyyfk iaVALAVAA IPEGLPAV i TTCLALGTR-RMarkn-

a IVR-SLPSVETLGCTSVICSDKTG

-TLTTNQMSVcrmfvvaeae-

$-x x x x x x x \operatorname{exxx}$

xxxxxtpegevrxxxxxxx--xxxxxxxx-

-el

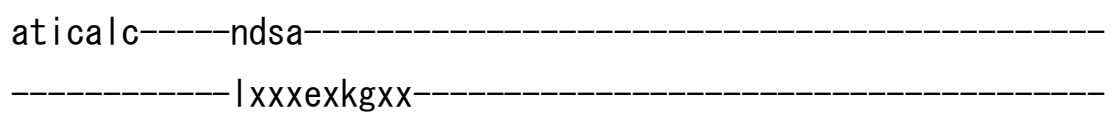

ekvgeate--taltcl-----xxxx----

$-x x x x x x x x x x x x$ veragac--nsvixx-

- Imrkeft lefsrdrk-smsvxxxxx

$-x x x x x x x x x x x x^{---}$

-v-----------------------k-

gap e svi ercssvrvgsr 
tvp I satsrehi-lak i rdwxxgsd-----t I rcLALATRdt---x-----------xxx--xxxq I ddcsqfvq-yetg I tfVGCVGMLDPPRPEVAACI TRCSRag-IRV

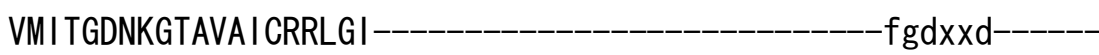

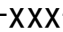

kaytgrefd-

dl speqqra

acrta--rCFA--RVEPAHKSRIVENLq

---sf--ne-

- ITAMTGDGVNDAPALKKAE I G I AM--

-gsgtav

AKSAAEMVLSd-DNFAS IVaaVEEGRA I YNNMKQF I $-R$

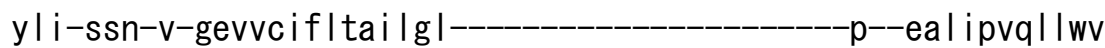
n I vtdg | pata I gfnppd-Id---_--_-_-_-_-_---imek | pr---n-preal i sgw I f-fryla i gvy--vg latvaaatxxxxxxx------------_---_-------------

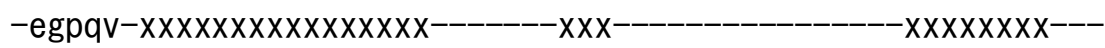
sr----fpttma I sv I vt i emcn-a Insvse--nqs | I--xx---ppx I npw I I gavvms malhfIil--|vxx|p|---_-_-_---ifqvtp |

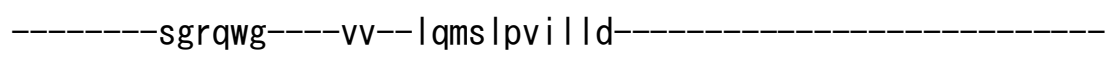
-ealky| 
k

>dme I I_I IA101 NP_476832. 1

m-

ghsktveqs In

ffg--------tdperglt ldq-

ikanqkkygpne I pteegk

-s iwq I leq-f 


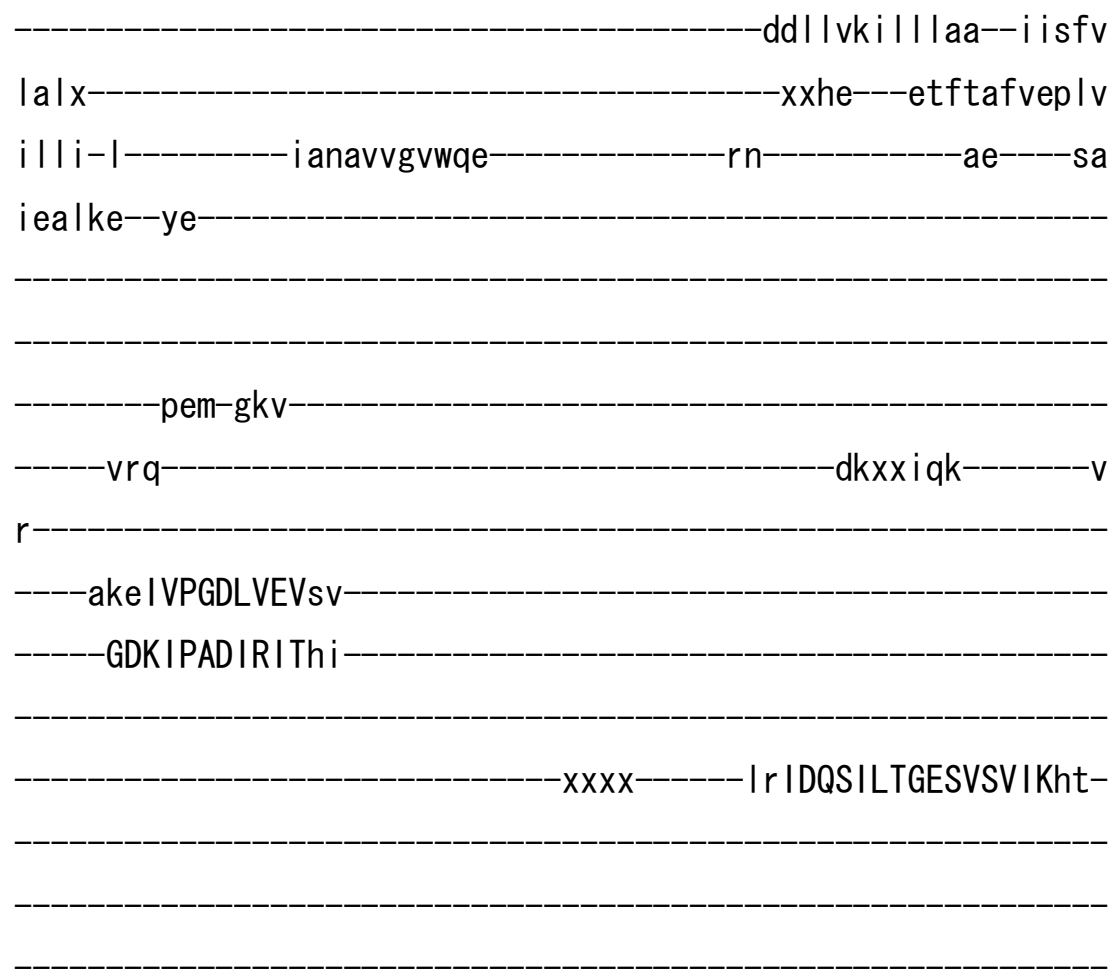

$-x x x x x x x x x x$

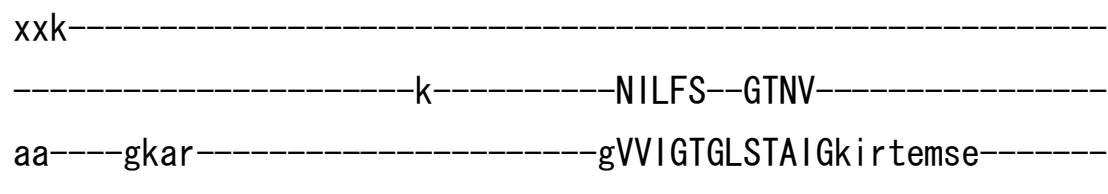

-tee iktp I qqk I defgeq I skv i sv i cvavw

-ainighfnxxxxxxxxxx---------------------------gai yyfk i aVALAVAA

IPEGLPAV i TTCLALGTR-RMakkn-

a IVR-SLPSVETLGCTSVICSDKTG

TLTTNQMSVs

-rmf ifdkve

$-x x x x x x x \operatorname{exx} x$

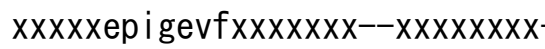

-el

st i c imc-----ndsa-

ixxxexkqxx 
-ekvgeate--tal iv l-----xxxx----

$-x x x x x x x x x x x x r$ saaiac--rge ixx

$-k w k k e f t l e f s r d r k-s m s s x x x x x$

$-x x x x x x x x x x x x--$

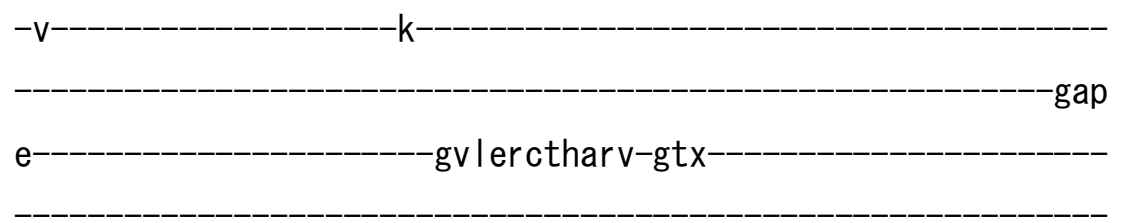

kvp Itsalkaki-lal tgqyxxgrd-----t | rcLALAVAds--x-----------xxx---xxxd I gdstkfyq-yevn I tfVGVVGMLDPPRKEVFDSIVRCRAag-IRV IVI TGDNKATAEA I CRRI GV-_-_-_-_-_-_-_-_-_-_-_-_-_-_-_-_faexxd--_-_-_

$x x x$

ksysgrefd

dl spteqka-

avars--rLFS--RVEPQHKSKIVEFLq

---sm--ne

- ISAMTGDGVNDAPALKKAE I G I AM--

gsgtav

AKSAAEMVLAd-DNFSS IVsaVEEGRA I YNNMKQF I 


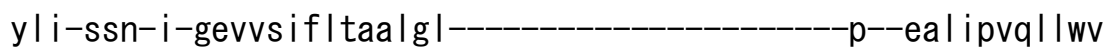

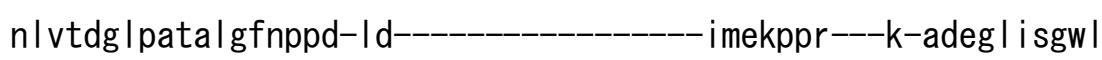
f-fryma i gfy--vgaatvgaaaxxxxxxx--------------------------------

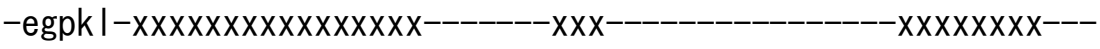
dp----hamtma I sv I vt i em In-amns I se--nqs | i--xx---ppxcn I w I igsma I s ft|hfvil--yvxx|st-----------vfqutp I

-saeewi----tv--mkfs ipvvl Idetlkfv

$-\operatorname{ar}$

$-x x x x x x x$

$-x x x x x$

m

>cellI_IIA101 NP_499385.3

m- 
ahakdanevck

$-f f g$ -tgpe-gltpqq

-vet I rnkygenempaeegk

-s lwe l i leq-f

dd I I vki I I laa--i isfv

$|a| x$ -xxhexxxeavtafvepfv

i I i-I i anatvgvwqe

$-r n$

-ae----sa

i ealke--ye-

$-x x x x$

- ir IDQSILTGESVSV IKht-

$-x x x x x x x x x x$

$x x k$

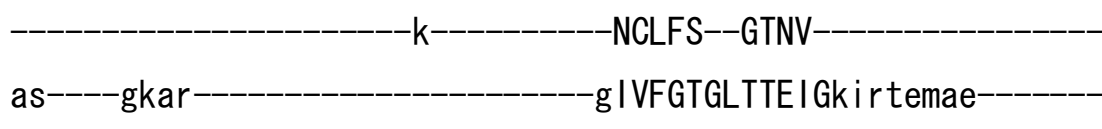

-tenektp I qqk I defgeq I skv i sv i cvavw

-ainighfnxxxxxxxxx gai yyfkiaVALAVAA

IPEGLPAV i TTCLALGTR-RMakkn- 
a IVR-SLPSVETLGCTSVICSDKTG-

-TLTTNQMSVs

-kmf i agqas

$-x x x x x x x \operatorname{exxx}$

$x x x x x e p v g k v s x x x x x x x x x x x x x x x x x$

$-\mathrm{el}$

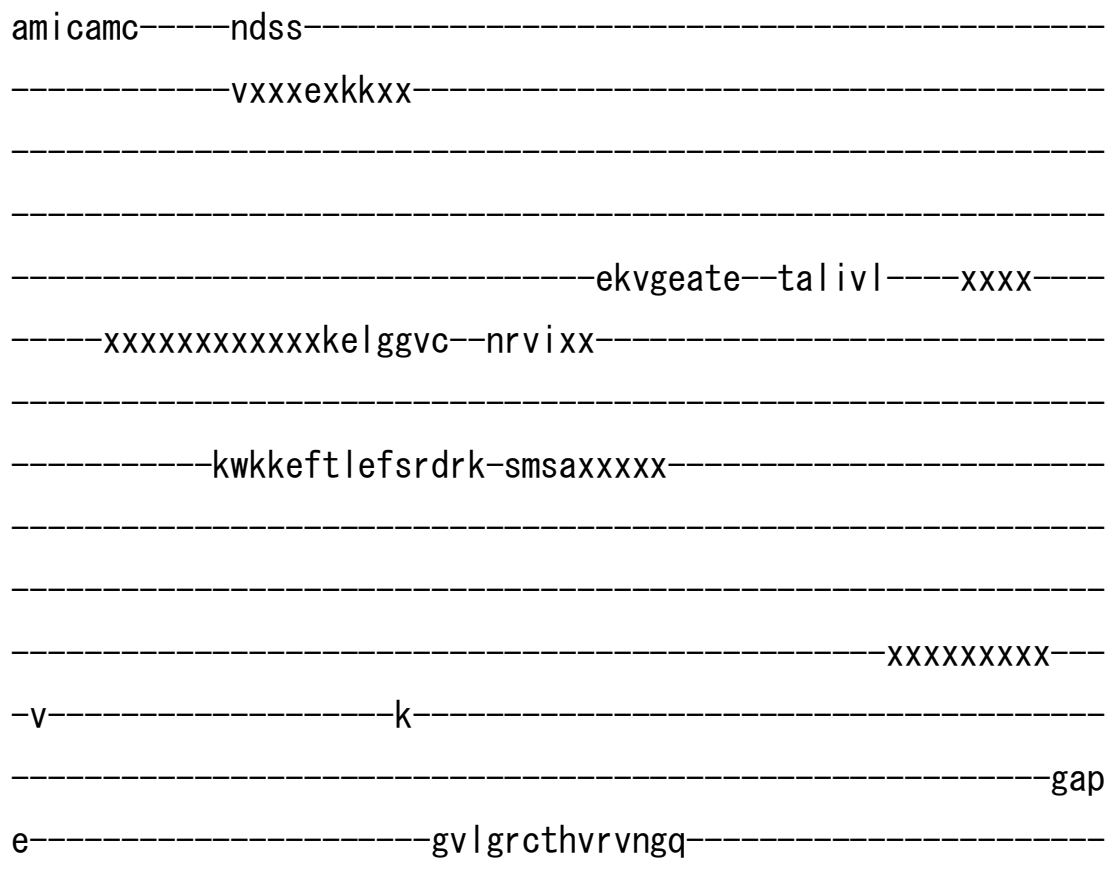

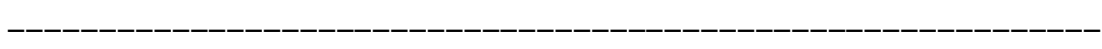

kvp I tsamtqki-vdqcvqyxxgrd-----t I rcLALGT I dt--x-----------xxx---xxxnl edstafvk-yeqd itfVGVVGMLDPPRTEVSDSIKACNHag-IRV I I I TGDNKNTAEA IGRR IGL fgexxd-

$x \times x$

kaytgrefd----- 
acr ra--kLFA--RVEPSHKSK IVDILq-

---sq--ge-

- ITAMTGDGVNDAPALKKAE I G I SM--

-gsgtav

-AKSASEMVLAd-DNFAS IV saVEEGRA IYNNMKQF I $-R$

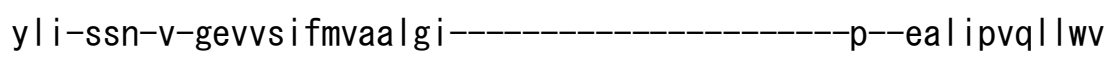
nlvtdg I pata I gfnppd-Id-_-_-_-_-_-_-_-_-_imdrhpr---s-andg I i sgw |

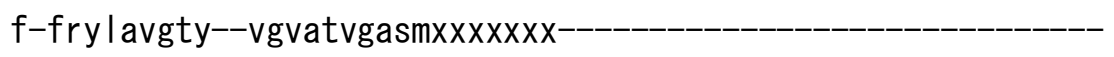

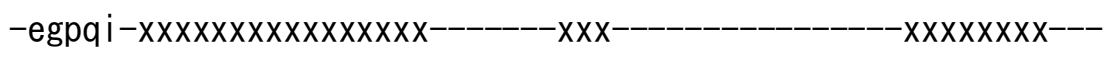
dn----hpnamal sv I vt i em In-ains I se--nqs I I--xx---ppxkn iw Imaa i I s ms Ihfvi I--yvxxmat-_-_-_-_-_-_ifqi tp I--_-_-_-_-_-_-_-_-_-_-_-_-_-_-

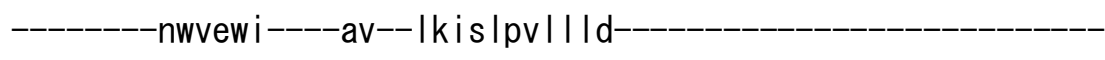
-eilkfi-arny i

d

>hsalI_IIA101 NP_775293.1

$\mathrm{m}-$ 
ahaktteecla

$-y f g--------v s e t t g \mid t p d q-$

-vkrn l ekyg I ne I paeegk

-tIwelvieq-f

-edllvrilllaa--cisfv

lawx-_-_-_-_-_-_-_-_-_-_-_-_-_-_-_-_-_-_-_xxge---et i tafvepfv

i I l i-I--_-_-_--_ i ana i vgvwqe--_-_-_-_-_-_rn--_-_-_-----ae----na iealke--ye-

- pem-gkv-

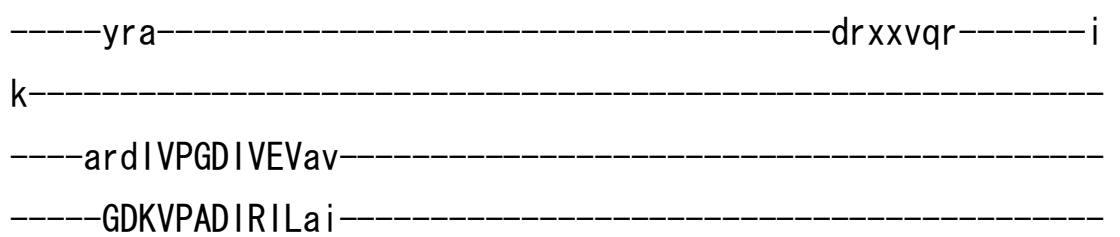

GDKVPADIRILai 
$-\mathrm{x}---\mathrm{x}-\mathrm{t}--\mathrm{t}$ I rVDQSILTGESVSVIKht-

$-X X X X X X X X X X$

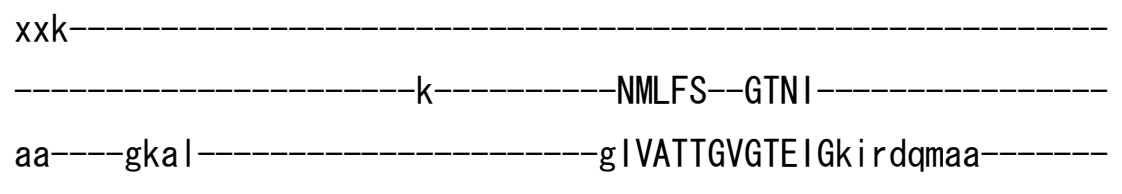

teqdktp Iqqk I defgeq I skvi I I icvavw -I in i ghfnxxxxxxxxxxx--------------------------gai yyfk i aVALAVAA IPEGLPAV i TTCLALGTR-RMakkn-

-a IVR-SLPSVETLGCTSVICSDKTG

-TLTTNQMSV c---------------kmf i i dkvd-

$-x x x x x x x \operatorname{exxx}$

xxxxxapegev|xxxxxxx--xxxxxxxx-

-el

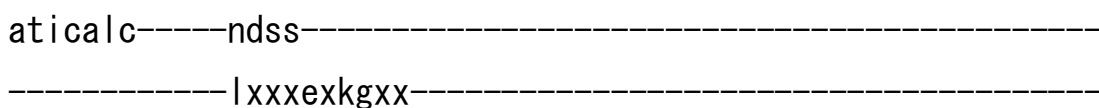

ekvgeate--taltt|----xxxx----

-xxxxxxxxxxxxveranac--nsvixx-

- Imkkeft lefsrdrk-smsvxxxxx

$-x x x x x x x x x x x x^{---}$

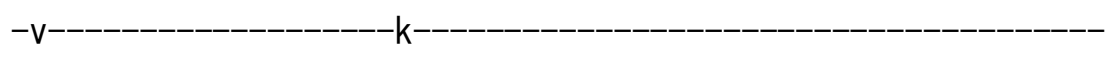

-gap $\mathrm{e}$

-gvidr cnyvrvgtt- 
rvp I tgpvkeki-mavi kewxxgrd-----t I rcLALATRdt---x-----------xxx---xxxv I ddsarf I e-yetd I tfVGVVGMLDPPRKEVTGSIQLCRDag-IRV IMI TGDNKGTAIA I CRR I GI--_-_-_-_-_-_-_-_-_-_-_-_-_-_fgexxe--_-_-

$-X X X$

raytgrefd

dlplaeqre-

acr ra--cCFA--RVEPSHKSKIVEYLq

--- sy-- de-

- ITAMTGDGVNDAPALKKAE I G I AM--

gsgtav-

AKTASEMVLAd-DNFST IVaaVEEGRA I YNNMKQF I

$-R$

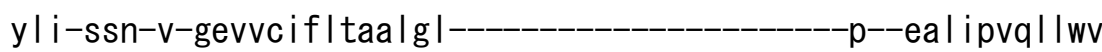
n I vtdg | pata I gfnppd-Id--_-_-_-_-_-_-_-_-_imdrppr---s-pkep I i sgw I

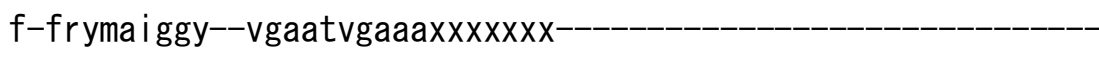

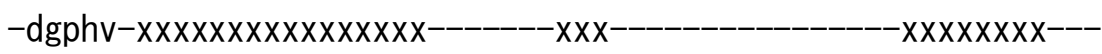

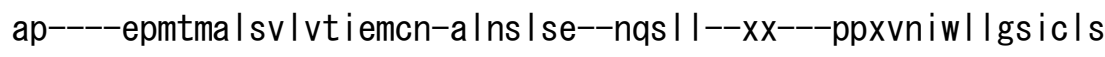

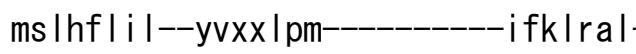

$-d \mid$ tqw I----mv--Ikis $|p v i g| d-$ eilkfv- 
k

>hsal I_IIA102 NP_733765. 1

m-

ahtktveev Ig

-hfg----_---vnestg l s leq-

vkk I kerwgsne I paeegk

-tIlelvieq-f 


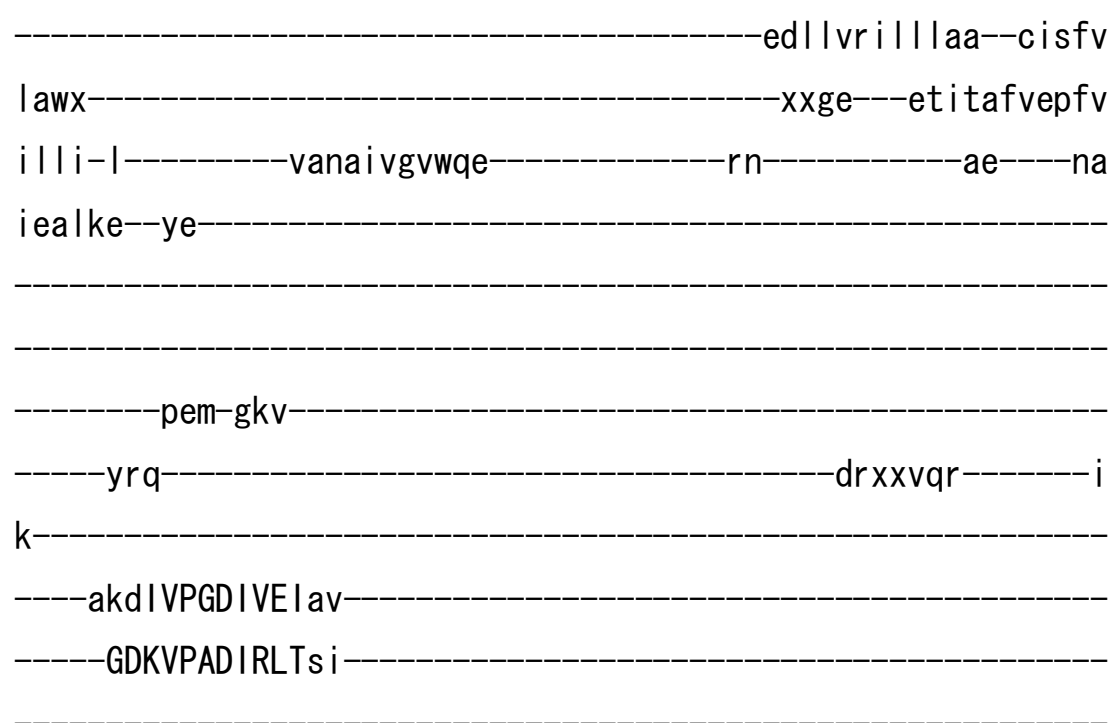

$-\mathrm{x}---\mathrm{x}-\mathrm{t}--\mathrm{t}$ I rVDQSILTGESVSVIKht-

$-x \times x X x X x x x x$

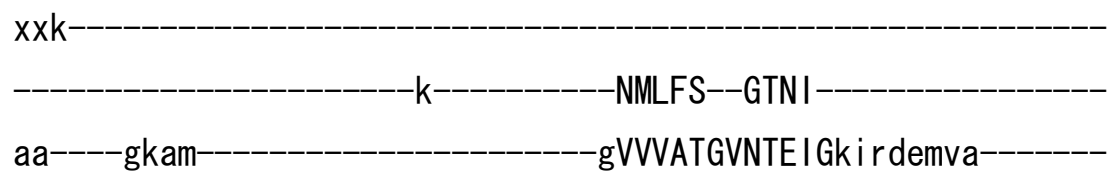

-teqertp Iqqk I defgeq I skvis I ic iavw

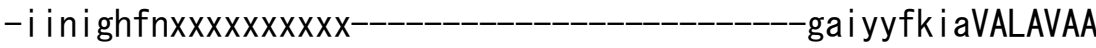

IPEGLPAV i TTCLALGTR-RMakkn-

a IVR-SLPSVETLGCTSVICSDKTG

$-T L T T N Q M S V c$ -rmfildrve-

$-x x x x x x x \operatorname{exx}$

xxxxxap igevhxxxxxxx--xxxxxxxx-

el

at icalc-----ndsa

I xxxexkgxx 
-ekvgeate--taltc|-----xxxx----

-xxxxxxxxxxxxieranac--nsvixx-

Imkkeft lefsrdrk-smsvxxxxx

$-x x x x x x x x-x x x--$

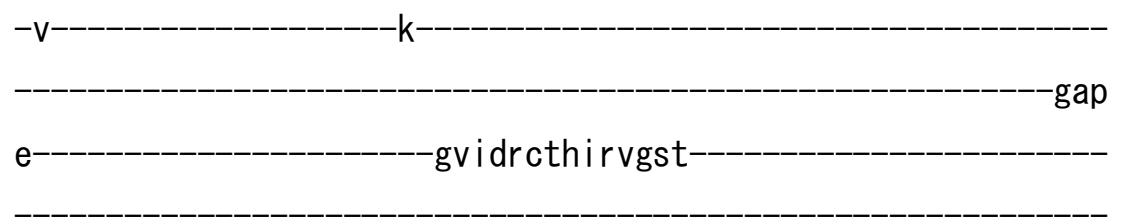

kvpmtsgvkqk i-msvi rewxxgsd-----t I rcLALATHdn---x-----------xxx---xxxhl edsanf ik-yetnItfVGCVGMLDPPRIEVASSVKLCRQag-IRV

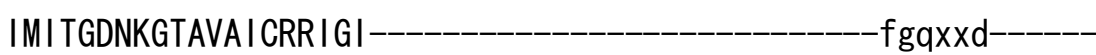

$-x x x-$

kaftgrefd

elnpsaqrd-

ac Ina--rCFA--RVEPSHKSK IVEFLq-

---sf--de---_--_--_-_-_-_--_-_----_ITAMTGDGVNDAPALKKAE I G I AM--

-gsgtav

-AKTASEMVLAd-DNFST IVaaVEEGRA I YNNMKQF I- 
yl i-ssn-v-gevvc if I taa I gf---_-_-_-_-_-_-_-_----p--eal i pvq I I wv

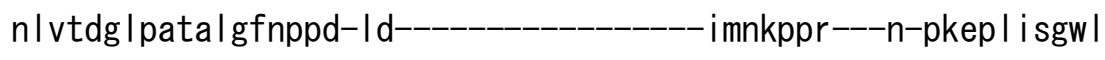
f-fryla i gcy--vgaatvgaaaxxxxxxx--_-_-_-_-_-_-_-_-_-_-_-_-_-_-_-

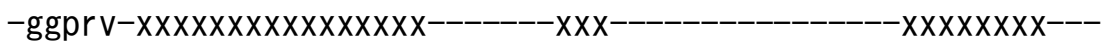

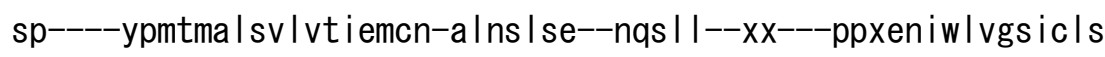

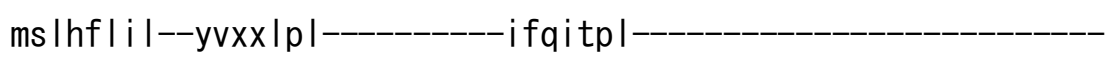
---_----nvtqw I-----mv--Ik i I lpvi Imd-_-_-_-_-_-_-_-_-_-_-_-_-_et lkfv-

arnyl

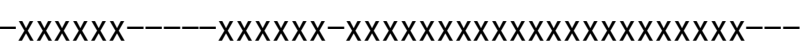
$-x \times X X X X X X X$

$-\mathrm{XXXXX}$

S

>hsalI_IIA103 NP_005164. 2

m- 
ahI Ipaadv|r

hfs -vtaegg I spaq

-vtgar erygpne I pseegk

- slwelvleq-f

edI Ivri II laa--Ivsfv

lawx

-xxge---etttafvep Iv

imli-l

$r n$

ae----sa

iealke--ye-

$-\mathrm{x}---\mathrm{x}-\mathrm{t}--\mathrm{t}$ | rVDQSILTGESVSVTKht-

$-x x x x x x x x x x$

$\mathrm{xxk}$

ts----gkav-----------------------'gVAVATGLHTELGki r sqmaa-------

-veper tp I qrk I defgrq I sha i svi cvavw

-vinighfaxxxxxxxxxxgavyyfkiaVALAVAA

IPEGLPAV i TTCLALGTR-RMarkn- 
a IVR-SLPSVETLGCTSVICSDKTG-

- TLTTNQMSV

rmfvvaead

$-x x x x x x x \operatorname{exx}$

xxxxxtpegevrxxxxxxx--xxxxxxxx-

el

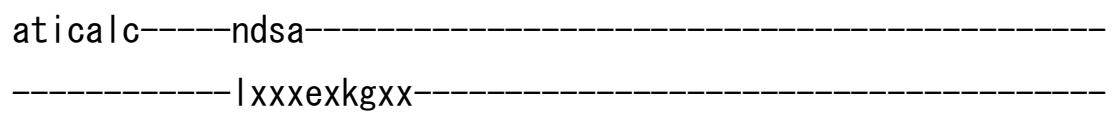

-ekvgeate--taltcl-----xxxx----

xxxxxxxxxxxxveragac--ntvixx-

-Imrkeftlefsrdrk-smsvxxxxx

$-x x x x x x x x x x x x---$

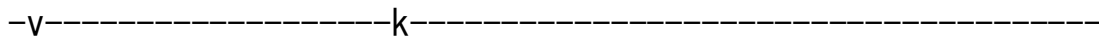

-gap

$\mathrm{e}$

-sviercssvrvgsr

tap I tptsreqi-laki rdwxxgsd-----t I rcLALATRda---x-----------xxx---xxxe Iddcskfvq-yetd I tfVGCVGMLDPPRPEVAACI TRCYQag-IRV VMI TGDNKGTAVA I CRRLGI-_-_-_-_-_-_-_-_-_-_-_-_-_-_-_fgdxxd--_-_-_ 
acrta--rCFA--RVEPAHKSR IVENLq

---sf--ne-

- ITAMTGDGVNDAPALKKAE I G I AM--

gsgtav-

-AKSAAEMVLSd-DNFAS IVaaVEEGRA I YSNMKQF I $-R$

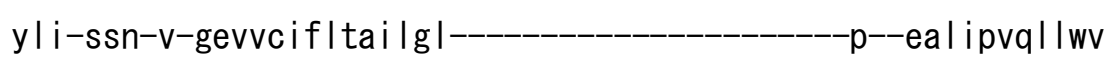
nlvtdg I patal gfnppd-Id-_-_-_-_-_-_-_-_-_imek | pr---s-preal i sgw I

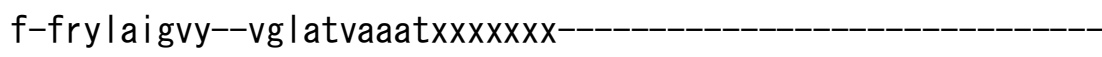

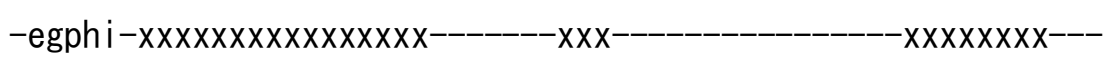
sr-----fpttmal sv I vt i emcn-al nsvse--nqs I I--xx----ppxmnpw I I vavams malhflil--|vxx|p|----------ifqutp|-

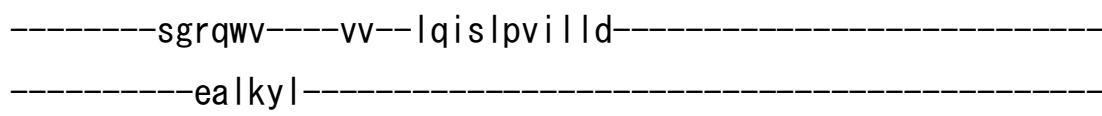

srnhm-

$-\mathrm{x}----------x x x x x-$

k

>osal I_IIA101 NP_001049744.1

$\mathrm{m}-$ 
$x x x x x x x x x x x x x$

$-x x x x x-x x$

$-x$

awartpsecla

-elg--------vaadrg|ssee-

aaar I r rygpne lerhaap

- svwk Ivleq-f

-ddt I vr i l laaa--vvsfv

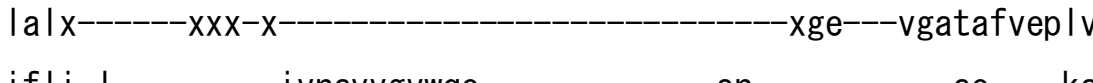

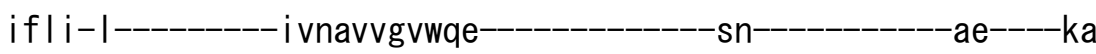

lealke--iq-

seh-atv-

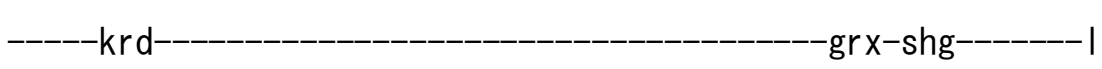

$\mathrm{p}$

$----\operatorname{ardLVPGD~IVELrV}$

GDKVPADMRVLqI 


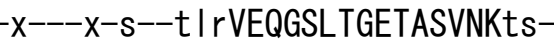

$-x x x x x x x x x x$

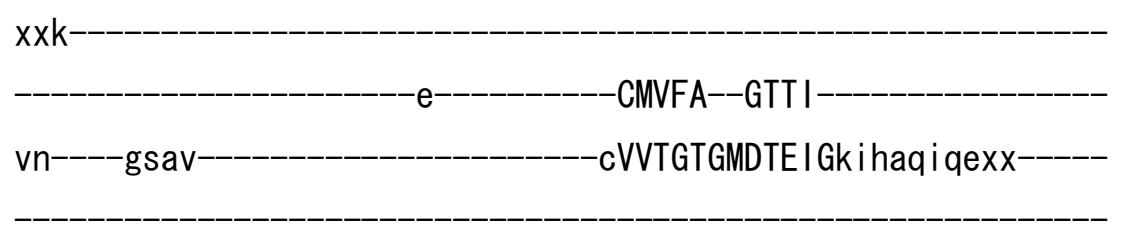

qeeddtp Ikkk Inefgea I ta i igvical vw

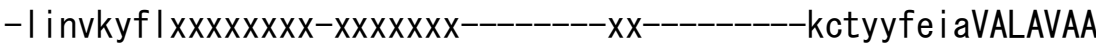
IPEGLPAV i TTCLALGTR-KMaqkn-

-aLVR-KLPSVETLGCTTVICSDKTG

TLTTNQMSAv -kIvai grwp-

$-x x x x s x x x$

$x x x x x d p s d g k i x x x x x x x x x x x x x$

$-\mathrm{mi}$

ak i aavc-----ndas-----------------------------------------------

-vatgmpte--aalkvl-----xxxxxxxx xxxxxxx----------ssd I I r c--cqwwxx----------------------------aakrvat lefdrtrk-smgvxxxxx

$-x \times x x x x x x---$

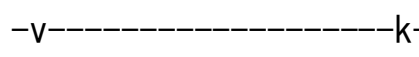
$\mathrm{e}$ - nl lersgyiql Idgx 
vv I I degakal i-I st | rem--sas----al rcLGFAYKedxxxxxxxxx-

-------xxxxxxky I I dpsyysx i esn I i fCGFVGLRDPPREEVHKAIEDCRAag-IRV

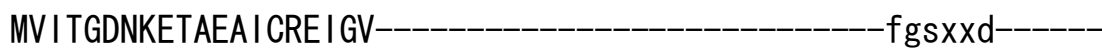

$-x x x$

$-k s f t g k e f m$

$-s \mid--s d k k k$

I rqtxx ILFS--RAEPKHKQE IVRLLk

--- ed--ge-

-VVAMTGDGVNDAPALKLADI GVAM--

$-x$ itgtev

AKEASDMVLAd-DNFST IVaaVGEGRS I YDNMKAF I

$-R$

ymi-ssn-i-gevas i f I tsa I g i--_-_-_-_-_-_-_-_-_-_---p--eg I i pvq I I wv

nlvtdgppatalgfnpp 
>pfalI_IIA101 XP_001350994.1

m-

$-X X X X X X$

ahtydvedv Ik

-fld---------vnkdng | knee-

Iddr r Ikyg I ne levekkk

-sifel i Inq-f 
ddI Ivki I l laa--fisfv

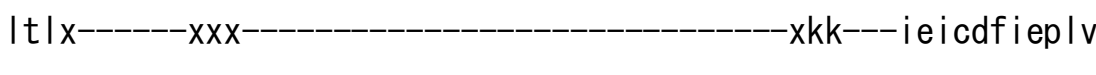

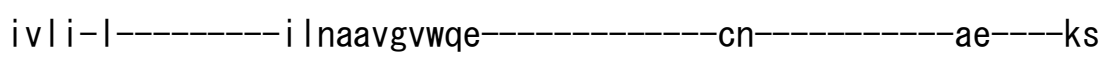

lealke--lq-

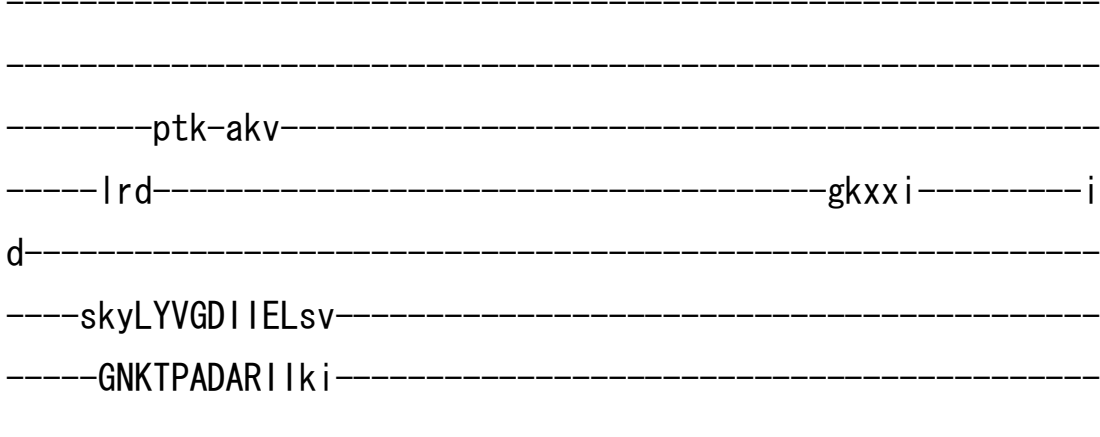

-xxxx------IkVEQSMLTGESCSVDKya-

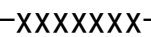

$x x x x x$

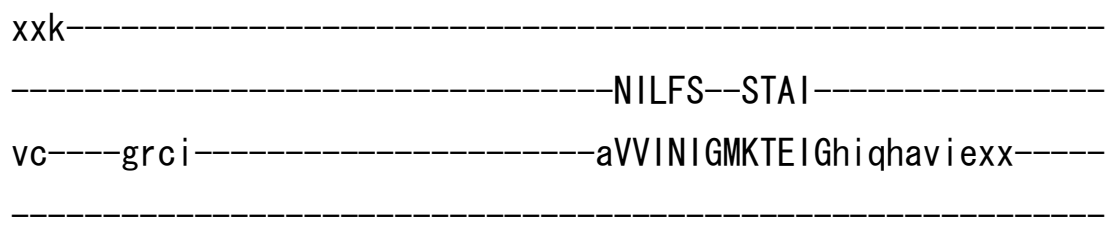

-sedtqtp lqikidl fgqql sk i ifvicvtvw

-i infkhfsxxxxxx-xxx-----_--_-_-_-_-_-_-_-_--_gcl yyfk i sVALAVAA

IPEGLPAV i TTCLALGTR-RMvkkn-

-a IVR-KLQSVETLGCTTVICSDKTG

-TLTTNQMTTt-

vfhIfresd-

$-x x x x x x x c x x x$

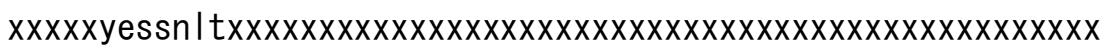

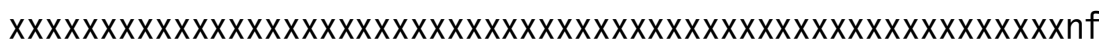
ymc I vnc------nean-

ixxxdxsqxx 
-kkfgdste--lal lhf-----xxxxxxxx

$x x x x x x x x x x x x x x x x x x x p v q s s n k--k d k s x x x x x x x x x x x x x x x x x x x x x x x x x x x x x x x$

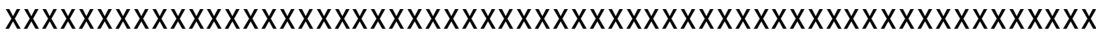

$x x x x x x x x x x x x$ eckqiki ieftrerk-Imsvxxxxxxxxxx

$-\mathrm{X}$

$-X^{--}-$

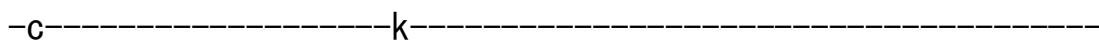

-gap

e----------------------n $\mathrm{n}$ i knckyy I tknd

irp Inet I kne i-hnk i qnm--gkr----al rtLSFAYKk I

-xxxxxnikntddyyk- I eqd I i yLGGLGI IDPPRKYVGRA IRLCHMag-IRV

FMI TGDN INTARA I AKE INI

Inkxxgxx----

$x x x x x x$

xxxxxxccyngrefe

dfs lekqkh-

i I kntxx iVFC--RTEPKHKKQIVKVLk

$---\mathrm{dl}--\mathrm{ge}-$

-TVAMTGDGVNDAPALKSAD I G I AM--

xingtev

-AKEASD I VLAd-DNFNT I Vea I KEGRC I YNNMKAF I 


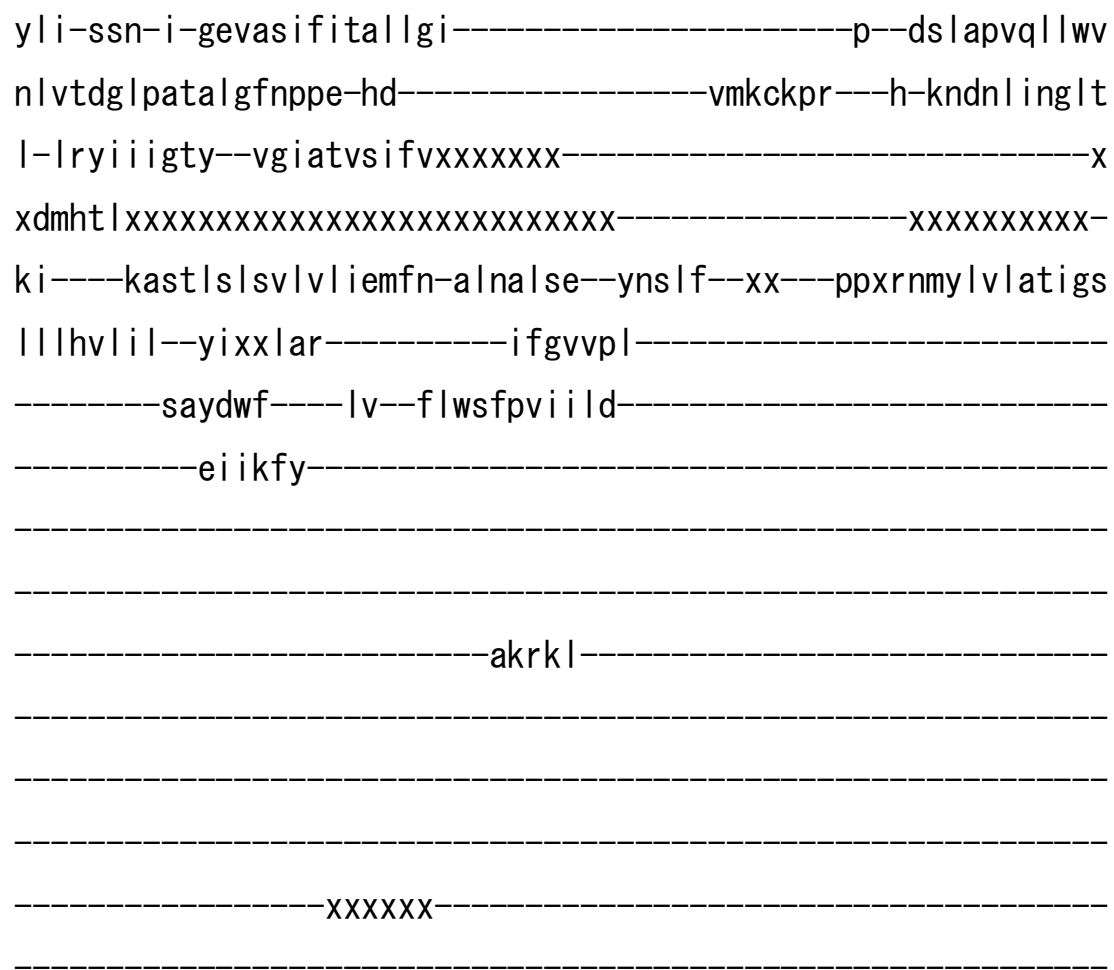
$x x-$ 


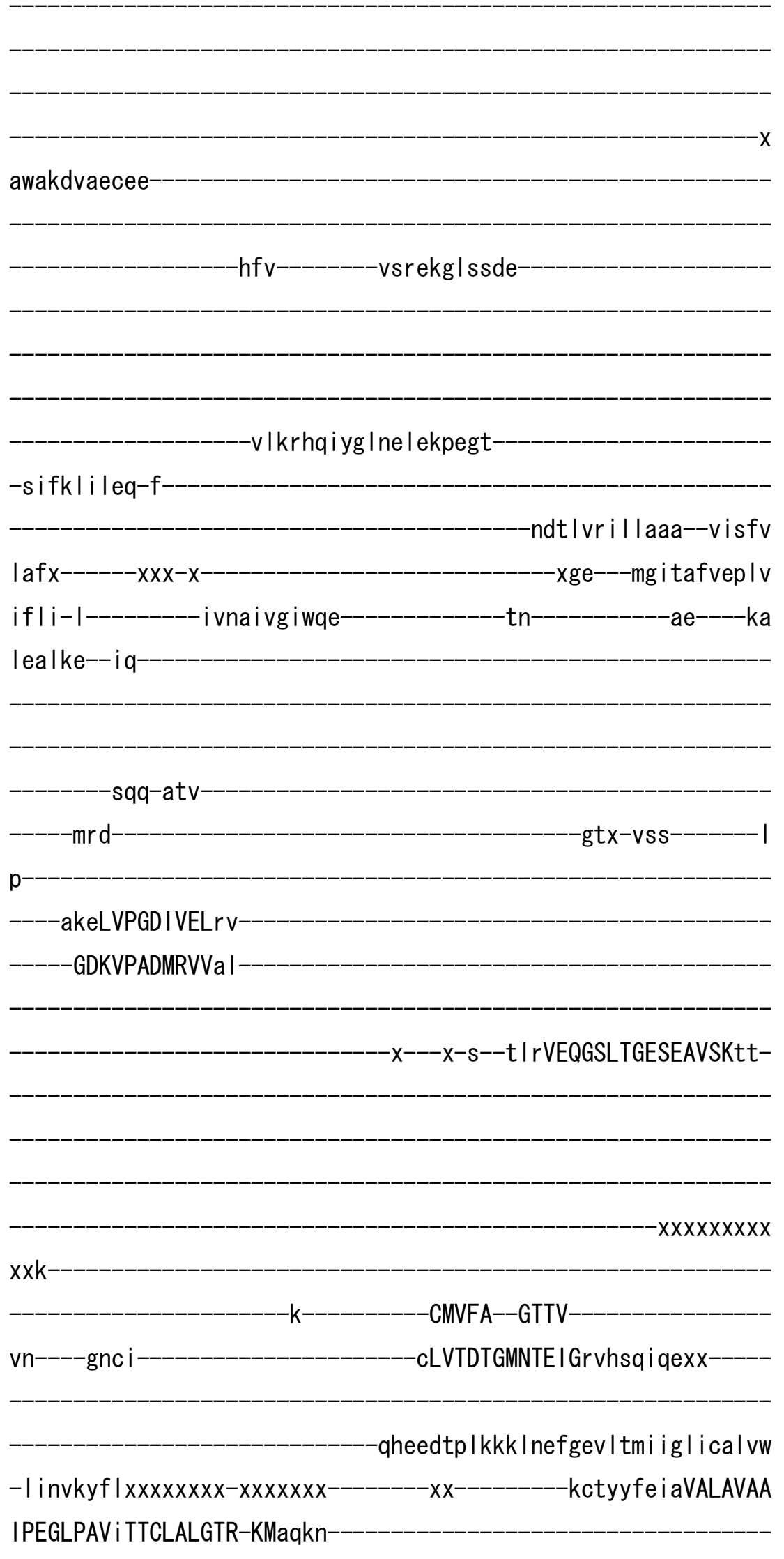


-aLVR-KLPSVETLGCTTVICSDKTG-

-TLTTNQMAVs

-k vamgsr i

$-x x x x \operatorname{xxx}$

$x x x x x d p r d g k$ i $x x x x x x x x x x x x x$

$-\mathrm{mi}$

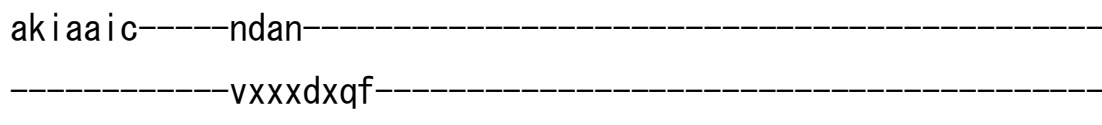

vsrgmpte--aalkvl-----xxxxxxxx

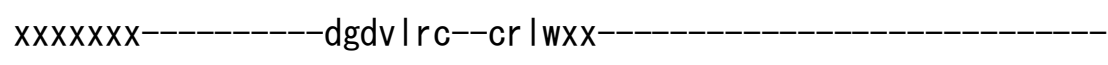

- eqr iat lefdrdrk-smgvxxxxx

$-x x x x x x x---$

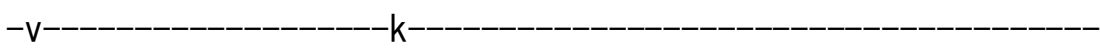

-gav

e-nvlersthiql Idgx

kreldqysrdl i-|qs |rdm--s|s----al rcLGFAYSdvxxxxxxxxx-xxxxxxxqq I Inpsnysx i esn I i fVGFVGLRDPPRKEVRQA I ADCRTag-IRV MV I TGDNKSTAEA I CRE I GV-_-_-_-_-_-_-_-_-_-_-_-_-_-_feaxxd-

$x x x$

-rsltgiefm

$-d v--q d q k n-$ 
h I rqtxx ILFS--RAEPKHKQE IVRLLk-

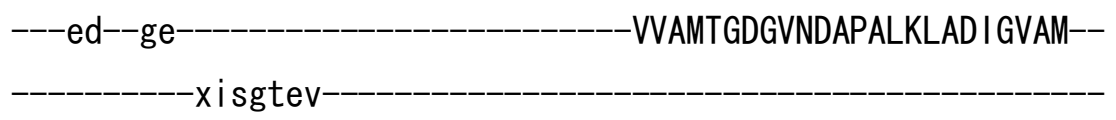

AKEASDMVLAd-DNFST I VaaVGEGRS I YNNMKAF I------R

ymi-ssn-i-gevas if I taa I g i--_-_-_-_-_-_-_-_-_-_----p--egmi pvq I I wv nlvtdgppata I gfnppd-kd--_--_-_-_-_-_-_-_-imkkppr---r-sdds I i taw i

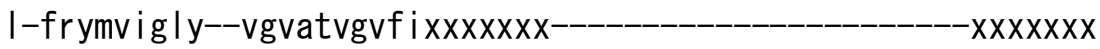
$x$ dghs I $x x x x x x x x x x x x x x x-x-------x x x x x x x x x x x x x--x x x x x x x x x x x x x x-$

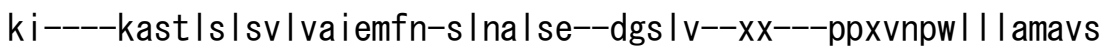
fglhfvil--yvxxlaq----------vfgivpl - s new|----|v--|avs Ipvilidevlkfv $-x$ $x x x x x x x x x x x$

e

>athII_IIA102 NP_191999.1

$\mathrm{m}-$ 
awswsveqc Ik

eyk

tr Idkg|tsed-

-vqir rqkygfne lakekgk

$-p|w h| v \mid$ eq-f

-ddt Ivki I gaa--fisfv

lafx

-xxehxxxsgfeafvepfv

i vl i-I----------i I navvgvwqe--------------sn-------------ae-----ka

lealke--mq-

-ces-akv

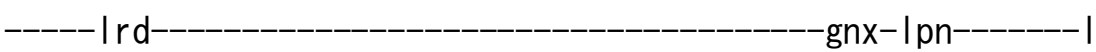

$\mathrm{p}$

----ar eLVPGD IVELnv

GDKVPADMRVSg I 
$-x^{---x-s--t ~ I ~ r V E Q S S L T G E A M P V L K g a-~}$

$\operatorname{xxxxxxxxxxxxx}$

e----------NMVFA--GTTV-

cIVTSI GMDTE I Gk i qrqi ihexx-----

leesetp Ikkk I defgsr Ittai c ivcv Ivw

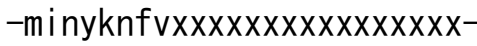

-xx---------kctyyfk i aVALAVAA

IPEGLPAV i TTCLALGTR-KMaqkn-

-a IVR-KLPSVETLGCTTVICSDKTG

-TLTTNQMSAT

efft Iggkt

$-x x x x V x x x$

xxxxxdpkdggi $x x x x x x x x x x x x x$

av

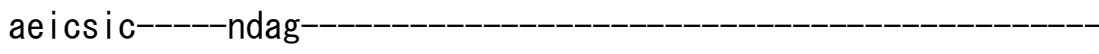

$-v x x x g x \mid f$

ratg $|p t e--a a| k v \mid----x x x x x x x x$

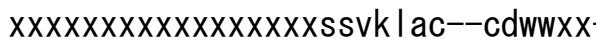

-rskkvat lefdrvrk-smsvxxxxx

$-x \times x \times x x x---$

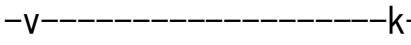

$\mathrm{e}$

-silerssfaqladgx 
I val dessrevi-|kkhsem--tsk----g| rcLGLAYKde--x-xxxxxxxxxxxx---xxxk I I dpssysn-i etn I i fVGVVGLRDPPREEVGRA IEDCRDag-IRV MV I TGDNKSTAEA I CCE I RL--_-_-_-_-_-_-_-_-_-_-_-_---_fsexxd--_-_-

$-\mathrm{XXX}$

ssftgkefm

slpasrre-

i I sksxxkVFS--RAEPRHKQE IVRMLk

$---\mathrm{em}--\mathrm{ge}$

IVAMTGDGVNDAPALKLADI G I AM--

xitgtev

AKEASDMVLAd-DNFST IVsaVAEGRSI YNNMKAF I

$-R$

ym i-ssn-v-gev i s i I t taa I g i-_-_-_-_-_-_-_-_-_-_---p--ecm i pvq I I wv

n I vtdgppata I gfnpad-i d-------------------i mkkppr---k-sddc I idswv

I-irylvigsy--vgvatvg i fvxxxxxxx--------------------- $x x x x x x x$

$x d g h t \mid x x x x x x x x x x x x x x x x x x-------2 x x x x x x x x x x x x x x x x x x x x x x x x x x x x x x-$

kv----kpmt |s | tv |vaiemfn-s | nal se--dns | |--xx---ppxrnpw | |vamtvs falhovil--yvxxlan----------vfgivpl-

-sfrewf-----vv--ilvsfpvilid

ealkfi 


\section{m}

>athII_I IA103 NP_563860.1

$$
\mathrm{m}
$$

ayarsvsevld

-ffg---_-----vdptkg | sdsq-

-vvhhsr I ygrnv I peekrt

$-p f w k|v| k q-f$ 
-ddl Ivki livaa--ivsfv

$|a| x$

-xge---tgltaflepfv

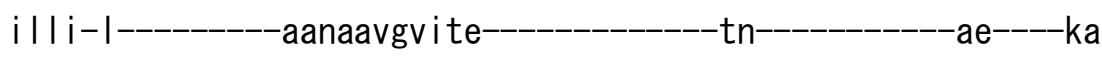

leelra--yq

- an i-atv-

Irn

$-\operatorname{gcxxi}$

$-1$

$\mathrm{p}$

----ateLVPGDIVEVtv

-GCKIPADLRM I em

$-\mathrm{x}---\mathrm{x}-\mathrm{n}--\mathrm{tfrVDQAILTGESCSVEKdv-}$

$-x x x x x x x x x x x x x x$

-NILFS--GTDV-

va-----grgr-----------------------_aVVI GVGSNTAMGs i hdsm I q

-tddeatp Ikkk I defgsf I akviag i cv I vw

-vvnighfsxxxxxx-xxx---_-_-_-_-_-_-_-_-_-_---gai hyfk i aVALAVAA

IPEGLPAVvTTCLALGTK-KMar In-

- IVR-SLPSVETLGCTTVICSDKTG

TLTTNMMSVs

-kicvvqsae-

$x x x x x x \operatorname{exx}$

$x x x x x a p e g t v f x x x x x x x x x x x x x x x x x$

$-\mathrm{hl}$

amcss I c-----nds i

- xxxpxkdxx 
-ekigeste--val rvl-----xxxx----

xxxxxxxxxxxxxxxxxherasyc--nhywxx-

qfkkvyv leftrdrk-mmsvxxxxx

$-x X X X X X--$

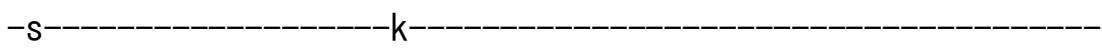

-gap

e------------------------s i i ar cnk i l cngdxx-----------------------

vvp I taagrael-esrfysf--gde----tI rcLALAFKtv

-phgqqt i syxnend I tf I GLVGMLDPPREEVRDAMLACMTag-IRV

IVVTGDNKSTAESLCRKIGA-

$-\mathrm{fdnxxd-}$

$-X X X$

msytasefe

r lpavqqt I

al rrm--tLFS--RVEPSHKRMLVEALq

$---k q--n e-$

-VVAMTGDGVNDAPALKKADIG I AM--

gsgtav

-AKSASDMVLAd-DNFAS IVaaVAEGRA I YNNTKQF I 


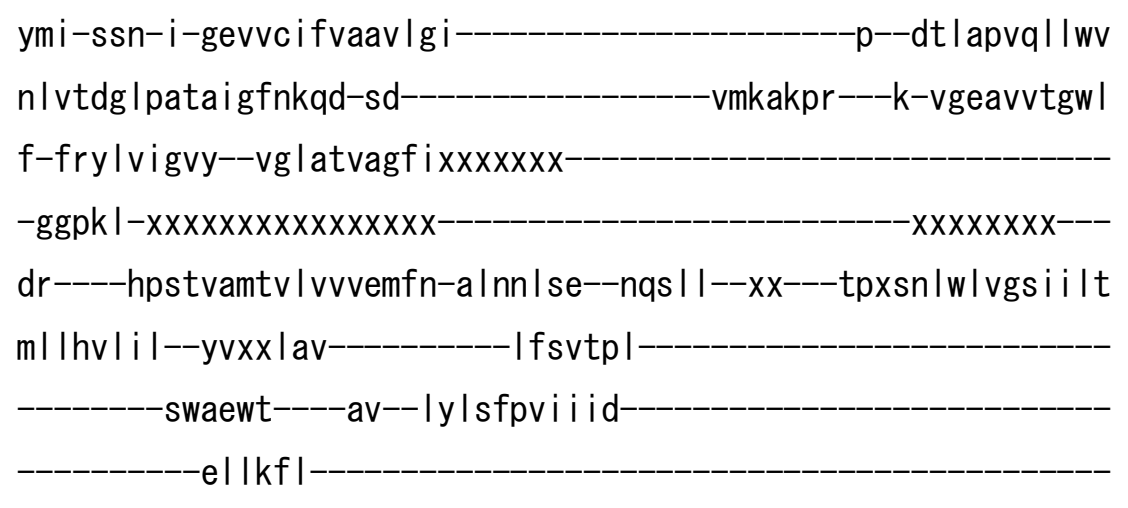

srntg

$-x \times x---$ $-x x x x x x x x x x x$

$-x x x x x$

k

>drelI_IIA101 NP_001007030.1

m- 
ahtketpecla

$-y f g$

-vsestg| tpeq

-vkknqakygfne I paeegk

-siwelvveq-f-

edl Ivrill laa--cisfv

lawx

xxge---etvtafvepfv

illi-I-

i anavvgvwqe

$-r n$

$-a e^{----s a}$

iealke--ye-

$-\mathrm{x}---\mathrm{x}-\mathrm{t}--\mathrm{t}$ I rVDQSILTGESVSVIKht-

$-x x x x x x x x x x$

$x x k$

aa----gka i-----------------------gVVVATGVSTE I Gk i r dqmaa-------

-teqektp I qqk I defgeq I skvi s I icvavw

- I inighfnxxxxxxxxxxgavyyfkiaVALAVAA

IPEGLPAV i TTCLALGTR-RMakkn- 
a IVR-SLPSVETLGCTSVICSDKTG-

-TLTTNQMCVt-

-kmfviekve

$-x x x x x x x q x x x$

xxxxxtpegevtxxxxxxx--xxxxxxxx-

$-\mathrm{el}$

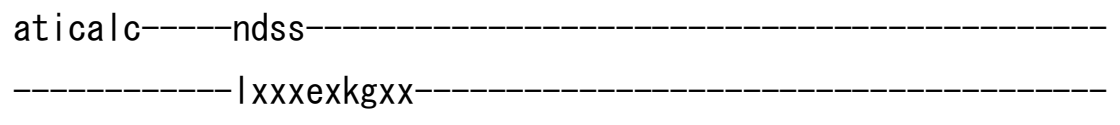

ekvgeate--talccl-----xxxx----

$-x x x x x x x x x x x x$ verantc--cavixx

- Imkkdftlefsrdrk-smsvxxxxx

rvp I tgpvkdk i-mavikewxxgrd-----t I rcLALATRdn---x-----------xxx---xxxn ledstkfae-yetdItfVGCVGMLDPPRKEVVGSIELCRAag-IRV IM I TGDNKGTAVA I CRR I G I--_-_-_-_-_-_-_-_-_-_-_-_-_-_-_fsd

$x x x$

-raftgrefd

-dlplpqqre 
avrka--cCYA--RVEPSHKSK IVEFLq-

$---\mathrm{gf}--\mathrm{de}-$

- ITAMTGDGVNDAPALKKAE I G I AM--

-gsgtav

-AKSASEMVLAd-DNFSS IVaaVEEGRA IYNNMKQF I $-R$

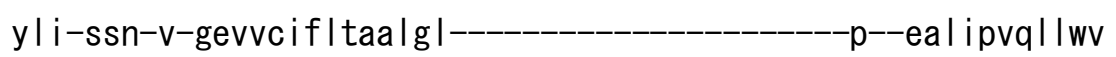
nlvtdg I pata I gfnppd-Id-_-_-_-_-_-_-_-_-_imgkapr---s-pkep I i sgw I

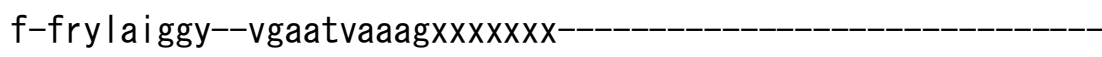

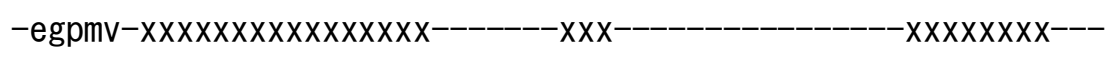
aa-----ppmtma | sv I v t i emcn-a | ns | se--nqs | |--xx---ppxsn I w I agamt I s ms Ihfmi i--yvxx I pm---_-_-_-_-_ifk I th I--_-_-_-_-_-_-_-_-_-_-_-_-_--------nfdqwi----vv--Ik I sfpvi l id--_-_-_-_-_-_-_-_-_-_-_-_-_-_ -ealkfv-arny l $-x$

a >drelI_IIA102 NP_001071001.1 m- 
ahtkgpaec la

$-y f s--------v s e t t g \mid s p e q$

fkkn l akygyne I paeegk

- s iwel i i eq-f

-ed I lvrill laa--cisfv

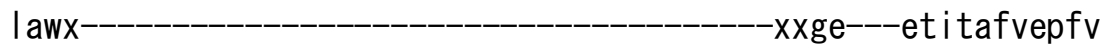

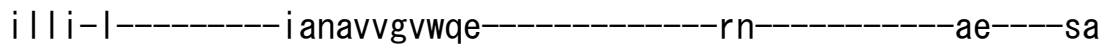

i ealke--ye-

- pem-gkv-

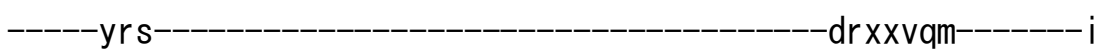

k-

----are IVPGDIVEVsV

GDKVPADIRIThi 
$-x x x x-----\mid$ rVDQSILTGESVSVIKht-

$-X X X X X X X X X X$

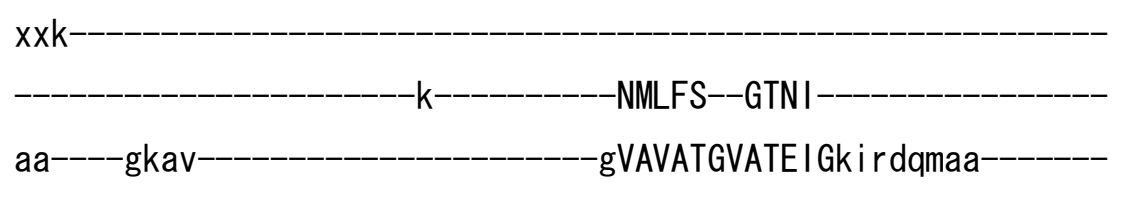

-teqektp Iqqk I defgeq I skvis I icvavw -mini ghfnxxxxxxxxxxx-------------------------gavyyfk i aVALAVAA

IPEGLPAV i TTCLALGTR-RMakkn-

-a IVR-SLPSVETLGCTSVICSDKTG

-TLTTNQMCVt---------------kmfvidrid-

$-x x x x x x x x x x$

$x x x x x$ tpegevtxxxxxxx-- $x x x x x x x x$

-el

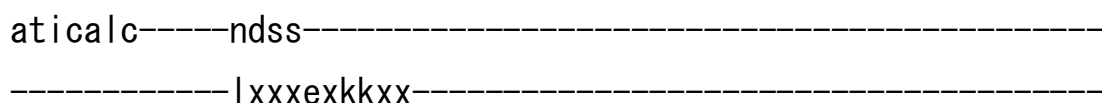

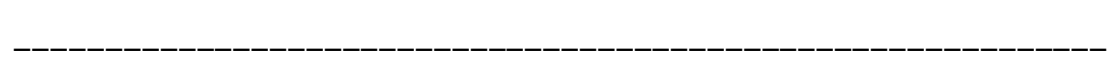

ekvgeate--talccl-----xxxx----

$-x x x x x x x x x x x x v e r a n a c--c s v v x x-$

- Imkknft lefsrdrk-smsvxxxxx

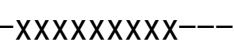

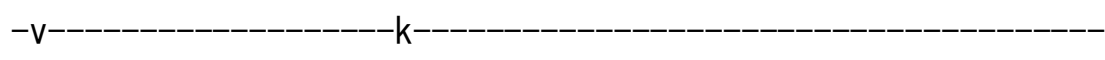

-gap e

-gvi drctyvrvgst 
rvp I tgavkdk i-mnvikewxxgrd-----t I rcLALATRds---x-----------xxx--xxxnl edstkfad-yetdI tfVGCVGMLDPPRKEVTGSIELCRAag-IRV IMI TGDNKGTAVA I CRR I GI--_-_-_-_-_-_-_-_-_-_-_-_-_-_fsexxd--_-_-

$x X X$

kaytgrefd

dl phseqse-

avrra--cCFA--RVEPSHKSKIVEFLq

--- gy-- de-

- ITAMTGDGVNDAPALKKAE I G I AM--

gsgtav

AKSASEMVLAd-DNFSS IVaaVEEGRA I YNNMKQF I

R

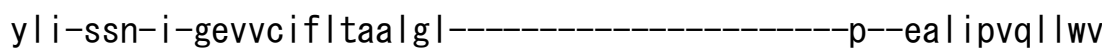

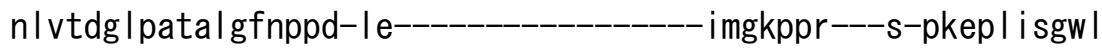

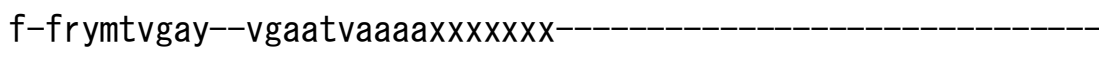

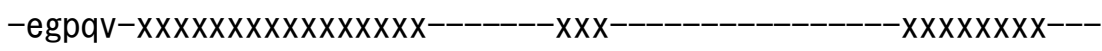
ac----ppmtma | sv I vt i emcn-a | ns | se--nqs I v-- xx----ppxsngw I I samt I s ms Ihfmi i--yvxxmpm----------ifr Ith I-

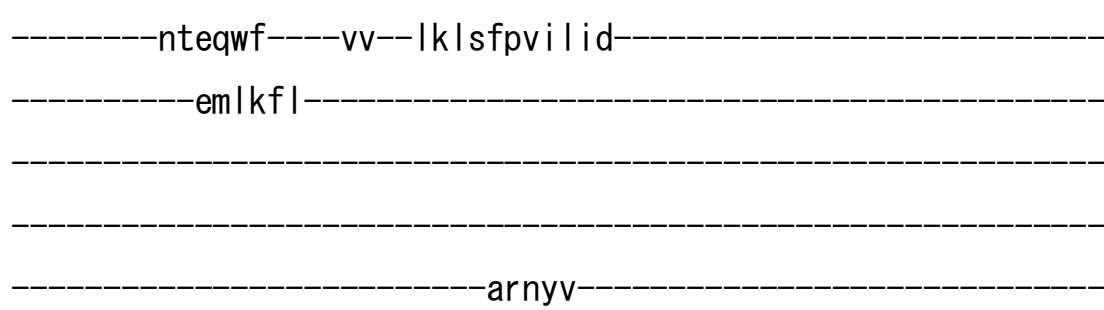


>drel I_IIA103 NP_957259.1

m-

ahtksveevys

nfs---------vnestglt ldq-

-vkrnrdkwgpne I paeegk

-siwelvi eq-f 


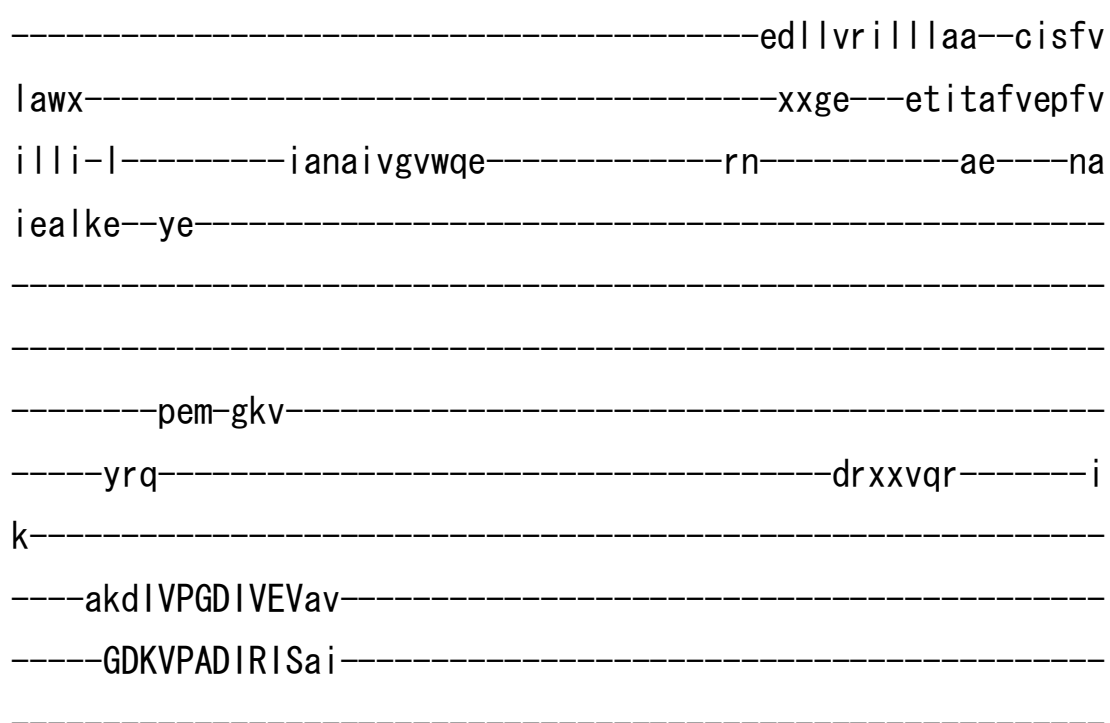

$-\mathrm{x}---\mathrm{x}-\mathrm{t}--\mathrm{t}$ I rVDQSILTGESVSVIKht-

$-x \times x X x X x x x x$

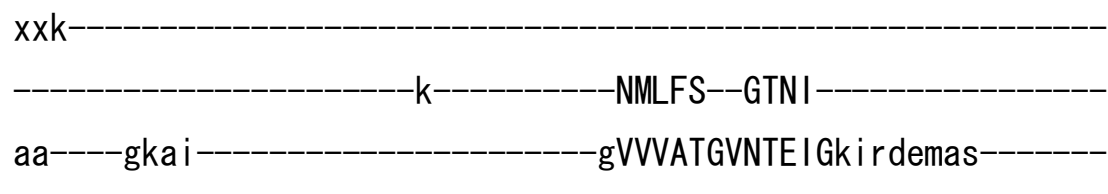

-teqertp Iqqk I defgeq I skvis I ic iavw

-i ini ghfnxxxxxxxxxx---------------------------gavyyfk iaVALAVAA

IPEGLPAV i TTCLALGTR-RMakkn-

a IVR-SLPSVETLGCTSVICSDKTG

TLTTNQMSVc rmf i idkae-

$\operatorname{xxxxxxxexxx}$

$x x x x x a p e g d v c x x x x x x x--x x x x x x x x$

el

at icalc-----ndss

I xxxexkgxx 
-ekvgeate--taltc|-----xxxx----

$-x x x x x x x x x x x x$ ieranac--navixx

Imkkeft lefsrdrk-smsvxxxxx

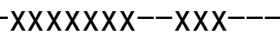

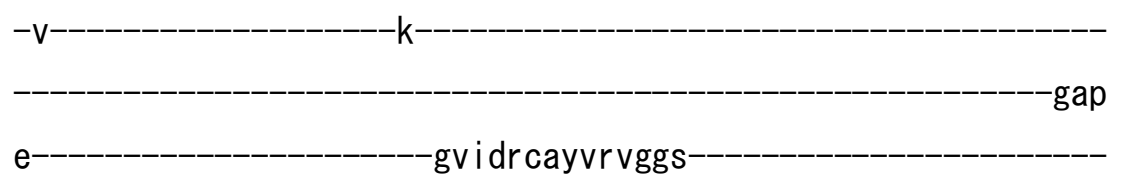

kvp I tqg i kdk i-msvi r eyxxgrd-----t I rcLALATRdn---x-----------xxx---xxxv I sdtarfad-yesd I tfVGCVGMLDPPRTEVAASIKLCRHag-IRV

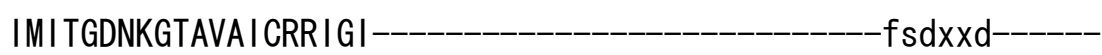

$-x x x$

-maftgrefd

dl sphaqre-

avtva--rCFA--RVEPSHKSKIVEFLq-

---gf--de--_-_--_--_-_-_-_--_-_----ITAMTGDGVNDAPALKKAE I G I AM--

-gsgtav-

-AKTASEMVLAd-DNFST IVaaVEEGRA I YNNMKQF I 
yl i-ssn-v-gevvc if I taa I gf---_-_-_-_-_-_-_-_----p--eal i pvq I I wv

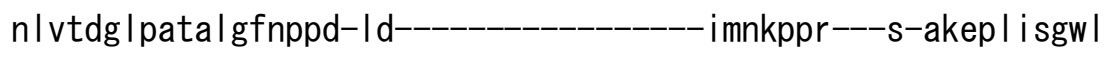
f-fryla i gcy--vgaatvgaaaxxxxxxx---_-_-_-_-_-_-_-_-_-_-_-_-_-_-

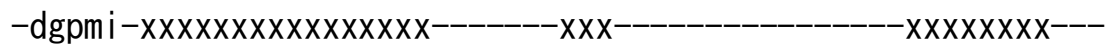
sp----ypmtma | sv I vt i iemcn-a Ins I se--nqs I v--xx---ppxen i w I ga ic Is

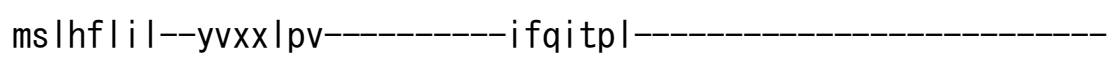
--_-----nvtqwm----mv--Ikis Ipvi I ld-_-_-_-_-_-_-_-_-_-_-_-_-_ ellkfv-

$-a r t y 1$

$-x x x x x$

d

>drelI_IIA104 NP_001025448. 1

m- 
ahtktveevys-

$-f f a-$

-vnestg $|\mathrm{g}|$ eq

-vkrqrekwgpn

gk-

-s I we l vveq-f

edl Ivrill laa--cisfv

lawx

-xxge---eti tafvepfv

illi-I-

- i ana i vgvwqe

$r n$

-ae----na

iealke--ye-

$-\mathrm{x}---\mathrm{x}-\mathrm{t}--\mathrm{t}$ | rVDQSILTGESVSVIKht-

$-x x x x x x x x x x$

$x x k$

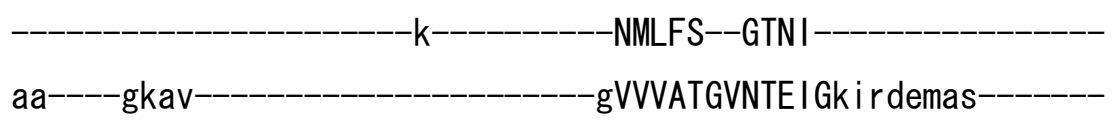

-teqer tp I qqk I defgqq I skvi s I i c iavw

- i inighfnxxxxxxxxxxgavyyfkiaVALAVAA

IPEGLPAV i TTCLALGTR-RMakkn- 
a IVR-SLPSVETLGCTSVICSDKTG-

- TLTTNQMSV

-rmf i vdqan

$-x x x x x x x \operatorname{exx}$

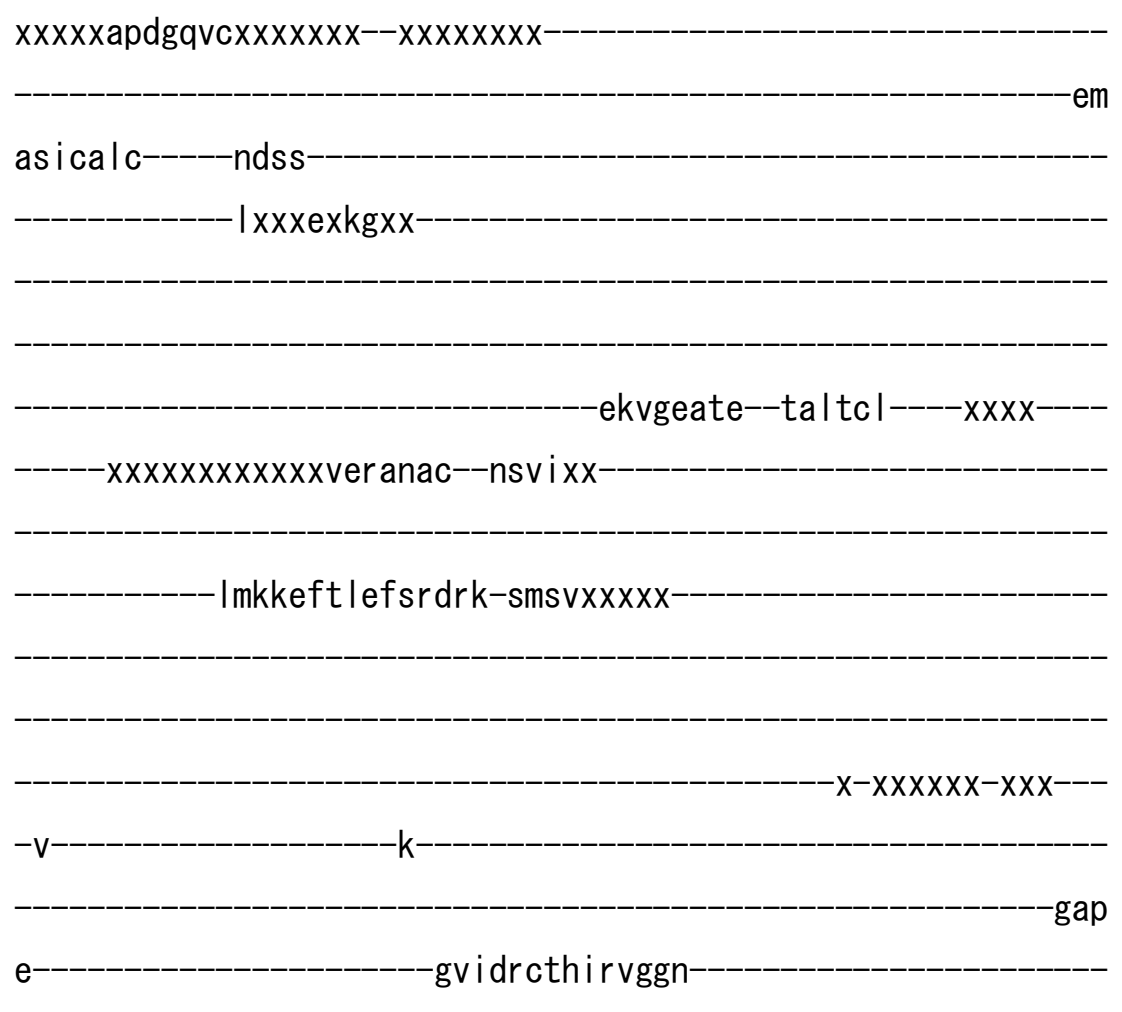

kvpmtpg i kek i-msvi reyxxgrd-----t I rcLALATRdn--x-----------xxx---xxxv ledstrfve-yetd I tfVGCVGMLDPPRAEVAASIKLCRQag-IRV IMI TGDNKGTAVA I CRR IGI--_-_-_-_-_-_-_-_-_-_-_-_-_-_-_fgexxd-_-_-_-

$x x x$

maytgrefd

dl saaaqre- 
av I ta--rCFA--RVEPSHKSK IVEFLq

--- sy-- de-

- ITAMTGDGVNDAPALKKAE I G I AM--

gsgtav

AKTASEMVLAd-DNFST IVaaVEEGRAIYNNMKQF I

$-R$

yl i-ssn-v-gevvc if I taal gf--_-_-_-_-_-_-_-_-_---p--eal ipvq I lwv

n lvtdg | pata I gfnppd-|d--_-_-_-_-_-_-_-_-imskppr---n-arep I isgw |

f-fryl a i gcy--vgaatvgaaaxxxxxxx-------------------------------

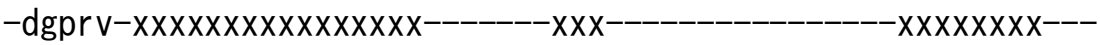

sp----ypmtma | sv I vt i emcn-a | nsvse--nqs | I--xx---ppxenvw I I gaic | s ms Ihfl i I--yvxx|pm-----------ifqitp I-

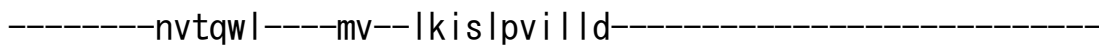

evlkfa-

arnyl

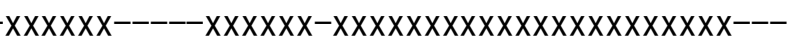

$x x x x x x x x x x x$

$-x x x x x$

S

>drelI_IIA105 XP_684227.1

m- 
ahtktveevlg

-yfs----_----vnettg I sseq

Irksrerwgpne I paeegk

-s Iwelvleq-f

-edllvrilllaa--cisft

lawx-_-_-_-_-_-_-_-_-_-_-_-_-_-_-_-_-_-_-_xxge--- gt i tafvepfv

i I l i-I--_-_-_--_ i ana i vgvwqe--_-_-_-_-_-_rn--_-_-_-----ae----na

iealkq--ye-

- pem-gkv-

-----yrq--_-_-_-_-_-_-_-_-_-_-_-_-_-_-_-_

r

$----a r d I V P G D I V E V a v$

GDKVPADIRLTs i 
$-\mathrm{x}---\mathrm{x}-\mathrm{t}--\mathrm{t}$ I rVDQSILTGESVSVLKht-

$-X X X X X X X X X X$

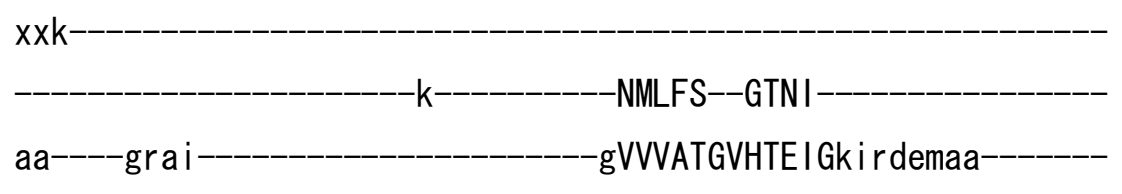

-tdper tp I qqk I dqfgeq I smvi tvicvavw -gini ghfnxxxxxxxxxxx--------------------------gavyyfk i aVALAVAA IPEGLPAV i TTCLALGTR-RMarkn-

a IVR-SLPSVETLGCTSVICSDKTG

-TLTTNQMSVs-

rIfivdmva

$-x x x x x x x \operatorname{exxx}$

$x x x x x$ apegevs $x x x x x x x--x x x x x x x x$

em

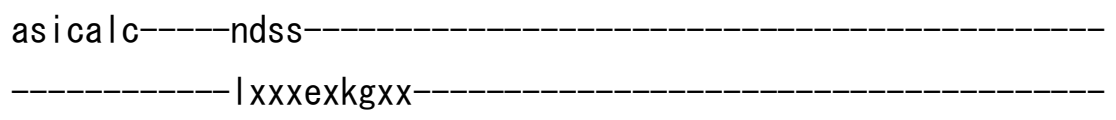

ekvgeate--talccl-----xxxx----

-xxxxxxxxxxxxaeratac--csvixx-

- Imrkelt lefsrdrk-smsvxxxxx

$-x x x x x x x x x x x x---$

-v-----------------------k-

gap

$\mathrm{e}$

-sv lercrwi rvsggx 
rvp Issd | req |-| stvr ewxxgrd-----t I rcLAMATRds---x-----------xxx---xxxn l ensaafse-yesdI tfVGCVGMLDPPRKEVLNAVRMCRQag-IRV

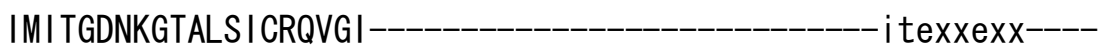

sgltgrefd-

elpphlqrq-

acrta--rCFA--RVEPTHKSRIVEYLq-

$---s \mid--s d-$

- ITAMTGDGVNDAPALKKAE I G I AM--gsgtav

-AKSASEM I LAd-DNFST IVaaVEEGRA I YNNMKQF I

$-R$

y I i-ssn-i-gevvc if I taa I gm--_-_-_-_-_-_-_-_-_-_---eal i pvq I l wv nI vtdgfpatal gfnppd-Id---_--_-_-_-_-_---imsrppr---s-pkep I i ssw I

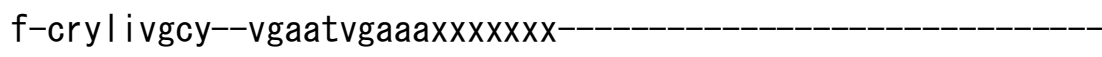

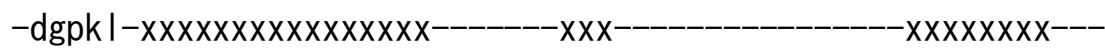
sp----ypmtma | sv I vt i emcn-a | ns | se--nqs I I--xx----ppxsnpw I vgaic Is malhflil--yvxx|pv-----------ifqirpl-

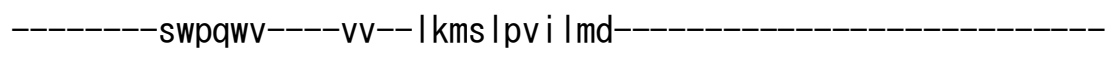
ealkfl 
d

>drelI_IIA106 XP_697108. 2

m

ahtksasevla

-nfg--------vnentg|t leq-

-vknnfdkygpne I pssers

-svwslaitw-f 


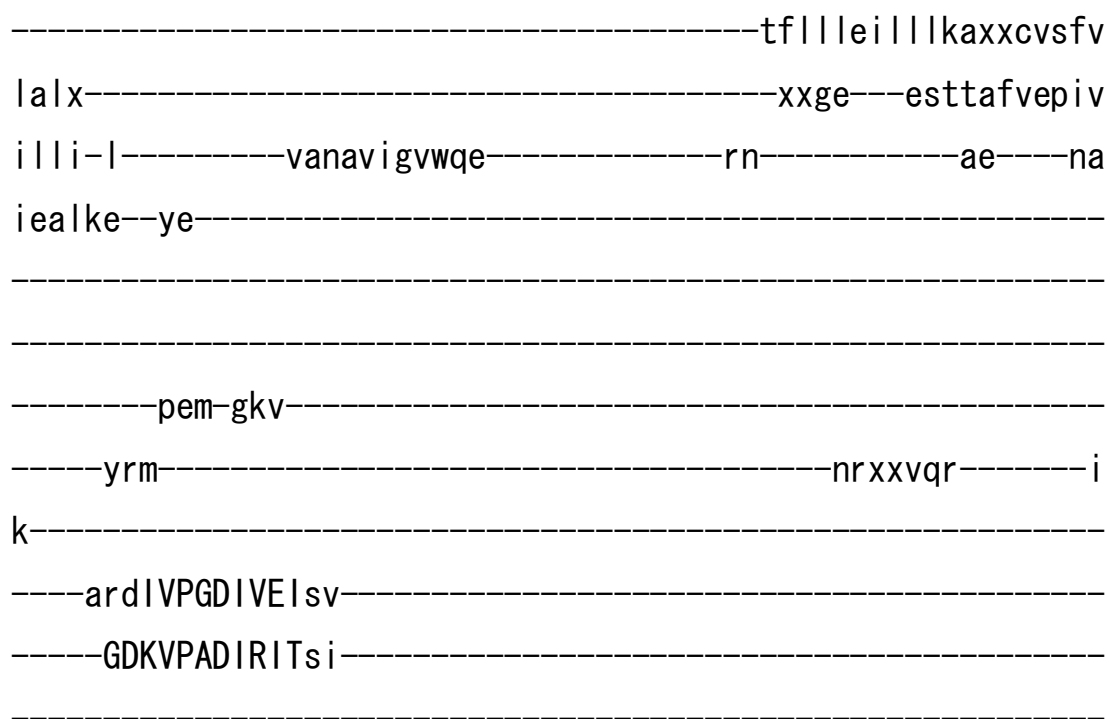

$-\mathrm{x}---\mathrm{x}-\mathrm{t}--\mathrm{t}$ I rVDQSILTGESVSVIKht-

$-x \times X X X X X X X$

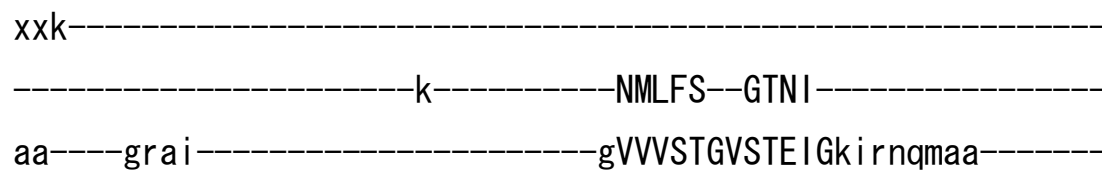

-teqektp Iqqk I defgqq I skvi I I i ciavw

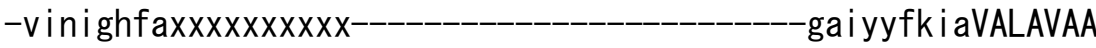

IPEGLPAV i TTCLALGTR-RMakkn-

a IVR-SLPSVETLGCTSVICSDKTG

$-T L T T N Q M S V c$ rmfvvnkad

$-x x x x x x e x x x$

xxxxxapegev Ixxxxxxx--xxxxxxxx-

el

at ics I c-----ndss

- xxxexkgxx 
-ekvgeate--taltt|-----xxxx----

$-x x x x x x x x x x x x v d r a a a c--n l$ i i $x x-$

Imqkkftl lefsrdrk-smsvxxxxx

$-x \times x \times x \times x \times x---$

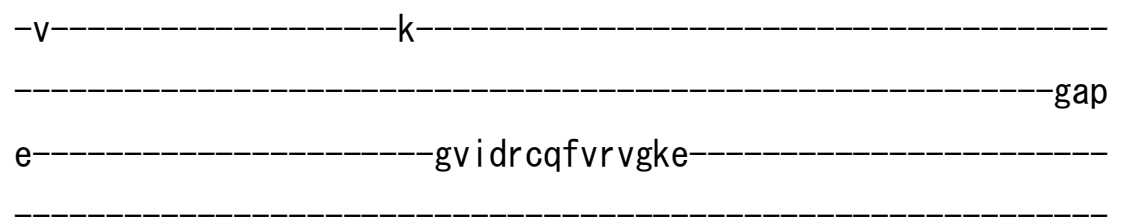

rfp I tmavkee I-mst i rdwxxgrd-----t I rcLALATRds---x-----------xxx---xxxd l enagkfae-yess I tfVGCVGMLDPPRKEVIGSIKLCNKag-IRV

IMI TGDNKGTAVA I CRR I GI--_-_-_-_-_-_-_-_-_-_-_-_-_-_fsexxd---_-_-

$x x x$

raytgrefd

dl apeaqre-

avkra--rCFA--RVEPAHKSKIVAYLq-

---sf--de----_-_-_-_-_-_-_-_-_----ITAMTGDGVNDAPALKKAE I G I AM--

gsgtav

-AKSASEMVLSd-DNFST IVaaVEEGRA I YNNMKQF I 


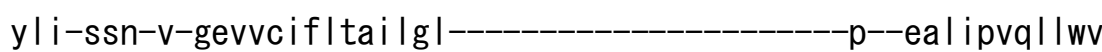
n I vtdg I pata I gfnppd-Id--_-_-_-_-_-_-_-_imdkppr---n-pkep I i sgw I f-fryla i ggy--vg I gtvsaatxxxxxxx-------------------------------

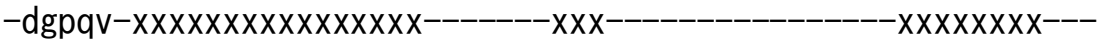
sr----ypttma I sv I vtiemfn-alns I se--nqs ||--xx---ppxvniw I gai i Is |s|hf|i|--hvxx|p|---_------ifqutp| -hfsqwi----iv--fki sipvilldealky i

srhhl

$-x x x x x x x x x x x x x x x$

$f$

$>c n e$ II_IIA101 XP_572412. 1

m 
awtftpqda I g

-yfg---------tnpdtg| teeq-

-vkrnreaygens I pesapn

- slfklilaq-f-

qdqlvli I Igsa--vvsfi

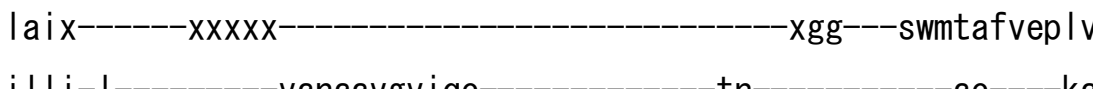

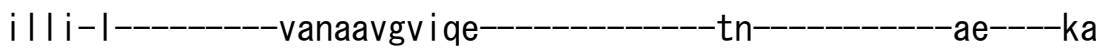
idalke--ys-

$-\mathrm{x}---\mathrm{x}-\mathrm{s}--$ sfrVDQAMLTGESMSVGKtd-

$-x x x x x x x x x$

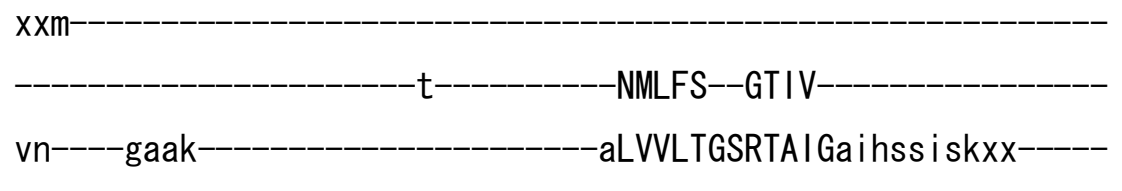

-eeektp Ikrk Iddfgdq lakvisvici I vw

-Ivnirhfnxxxxxx-xxxgai yy lkiaVALAVAA

IPEGLAAV i TACLALGTK-KMakrg- 
a IVR-NLPSVETLGCTNV ICSDKTG-

-TLTTNQMSVs

-rfvtcddag

$-x x \operatorname{exxx}$

xxxxxap igavtxxxxxxxxxxxxxxxxxx

se ica ic-----ndak-

snvgepte--aalkvl-----xxxx----

$-x x x x x x x x x x x x x x x x \mid$ aratav--ndyyxx

-nvkr I Itfefsrdrk-smsvxxxxx

$-\mathrm{XXXXXX---}$

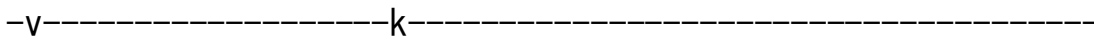

-gap

e----------------------sv lercsnv I I png

vkaftpe |rkk I-eekq ley--ghk----g I r tLALAYVde $-x x x x x x x y k t d r$ sedyvxferdmt fVGL I GMLDPPRPEVRDAI AKCKTag-IRT IV I TGDNKNTAET I CRE I GV-_-_-_-_-_-_-_-_-_-_-_-_-_-_-_fghxxd$-x x x$ -ksytgreld al sheekia- 
avqra--sLFS--RTEPTHKSQLVDLLq-

$---\mathrm{g}|--\mathrm{g}|$

-VVAMTGDGVNDAPALKKADI I I AM--

gtgtdv

-AKLAADMVLAn-DNFAT IEkaVEEGRA I YNNTKQF I $-R$

y I i-ssn-i-gevvs i f I tv I I gm---_-_-_-_-_-_-_-_-_----p--eal i pvq I wv nl itdg I pata I gfnppd-hq-_-_-_-_-_-_-_-_-_imktppr---s-gkep I vggw I

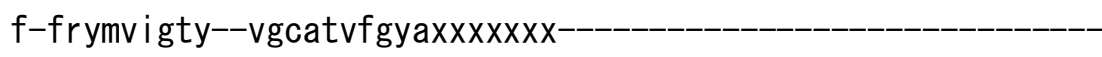

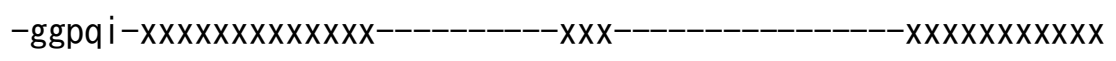
aq----rattvs Is i Ivviemfn-acns | se--nes If---xx---p |xsnpy I vasi i Is malhfmi l--yvxxfre----------mfrital---------nkeewi-----av--i vi sfpvi i id-----------------------------$-e v l k f i$

e

>anilI_IIA101 EAA62836. 1

m- 
syi lapaevlk

hfd

vtersgl ssaq

-vshsrqkygpna laeeppt

$-p \mid w e l v l e q-f$

$-k d q|v|$ i I Igsa--avsfv

lalx

-xes---ddwtvfvdpav

i l ti-l----------i I nai vgvtqe--------------ss------------ae----ka

iaalqe--ys

ane-akv-

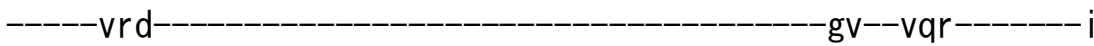

$\mathrm{k}-$

----aedLVPGD IVHVav

GDRVPADCRLLa i 
$-x---x-n--s f r V D Q A I L T G E S E S V A K d t-$

$-X X X X X X X X X X$

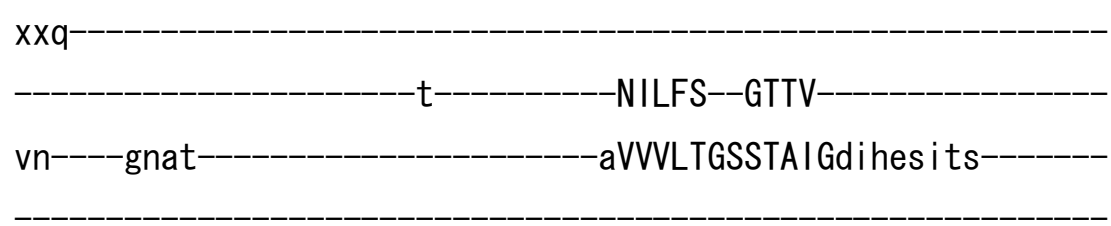

-qi septp Ikqk Indfgdm I akv i tv i c I l vw -viniehfnxxxxxx-xxx-gai yy lkiaVSLGVAA

IPEGLAVV i TTCLALGTR-KMaqkn-

-aVVR-SLPSVETLGSCSVICSDKTG-

TLTTNQMSVg-kivylsqlg

$-x x x x V x x x$

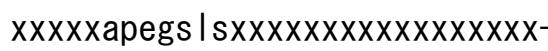

qm

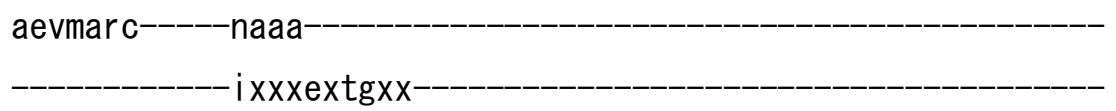

sc igepte--gal rvl-----xxxx----

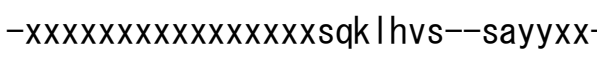

Ip lqatyefsrdrk-smsvxxxxx

$x \times x \times x=--$

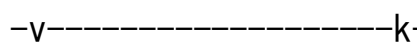

$\mathrm{e}$

s i lercsyal I gpnxx- 
rvs|tkah|d|I-ssevvey--asr----g|rvIALASVdd

------xxxxxxxhkastseeyax I eqnmt I IGLVAMLDPPRVEVADS IKKCAEag-IRV

IVITGDNQNTAES I CRE I GV-_-_-_-_-_-_-_-_-_-_-_-_-_-_--fgkxxd---_-_-

$x x x$

ksftgrefd

s I shneqle-

avksa--sLFS--RTEPSHKSKLVDLLq-

$---s \mid--g h-$

-VVAMTGDGVNDAPALKKSDI GVAM--

gtgtdv

AKLAADMVLVd-DNFAT I TtaVEEGRSIYSNTQQF I $-R$

y l i-ssn-i-gevvs if I taa I gm----_--_-_-_-_-_-_-----p--ea I vpvq I I wv n I vtdg I pata I sfnpad-hd-----_-_-_-_-_----vmr rppr----k-rdep I vggw I I-frylvigty--vgaatvfgyvxxxxxxx-_-_-_-_-_-_-_-_-_-_-_-_-_-_-_

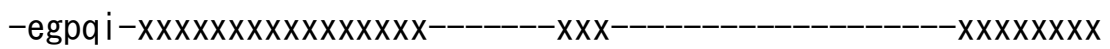
sr----sastvs I s i vvi em In-amnalss--ses | |--xx---g| xnnmm I vyai i Is mt Ihfail--yixxlgg $-\mid$ fai $|p|$ - dwtewk----av-- la i sapvv- 
>drelI_IIA201 XP_695172. 2

$$
\text { m- }
$$

$-x x x x x x x x x x x$

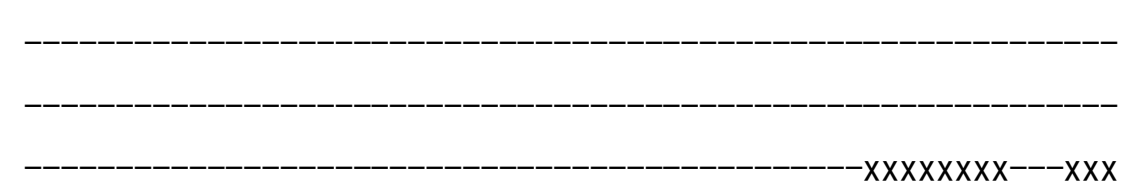

ase Ipvnevac

$-a \mid q$

adlafgInree-

-verr r tyhgwnefdi sede

$-p|w k k y| s q-f$ 
$-k d p$ I i l IIssa--visv I

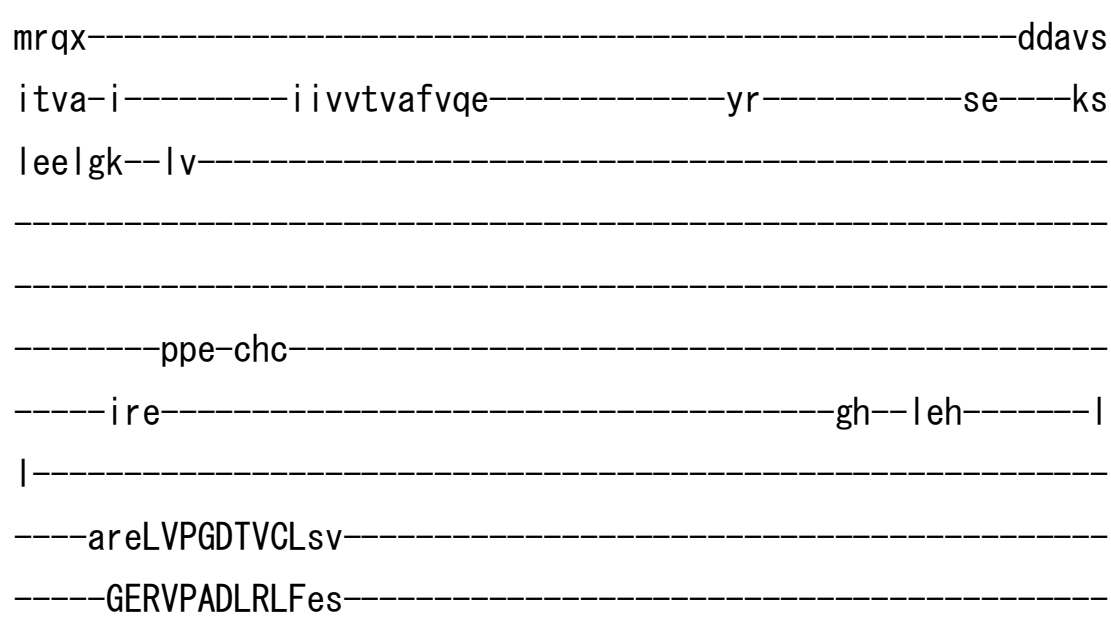

t--d laVDESSLTGETTPCTKts-

$-x x x x x x x x x x x x x x x$

$-N I A F M--G T L V-$

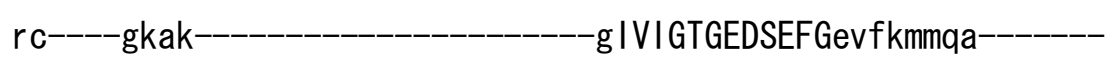

-eeapktp lqksmd। I gkq I s lys I g i igvim

-fvgw l qgk--_-_-_-_-_-_-_-_-_-_-_-_-_-_-_-_-_--y i l dmft i gVSLAVAA

IPEGLPIVvTVTLALGVM-RMvkkr

-a IVK-KLPIVETLSCCNVICSDKTG

-TLTKNEMTVt-

hIftsdgqh-

$-x x x$

xxxxxnnsge $i \mid x--x x x x x x x x x x x x x x$

vevgcvc-----ndam-

ixxx- 
-tImgrpte--galial-----xxxxx---

$\operatorname{eg} \mid x x$

efvr lee ipftseqk-wmavxxxxx

$\mathrm{XXXXXXXXX---}$

$-k$

gay

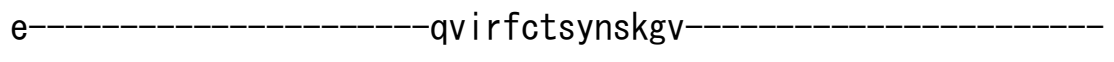

tmp Innqqrdfy-qqqksym--gsg----g I rvLAFASGse

-mgs I tfLGLVGI IDPPRAGVKEAVATL I Ssg-VAV

KMVTGDSEETAVAIASRLGL-

-ytk

xxqc I sgdevd

qmdiqh I sq

i vhrv--vVFY--RASPRHKLKIVKSLq

$---n i--$ ga-

-VVAMTGDGVNDAVALKAAD IGVAM--

xqtgtdv

CKEAADM ILVd-DDFQT ILsa IEEGKGIYNN I KNFV- 

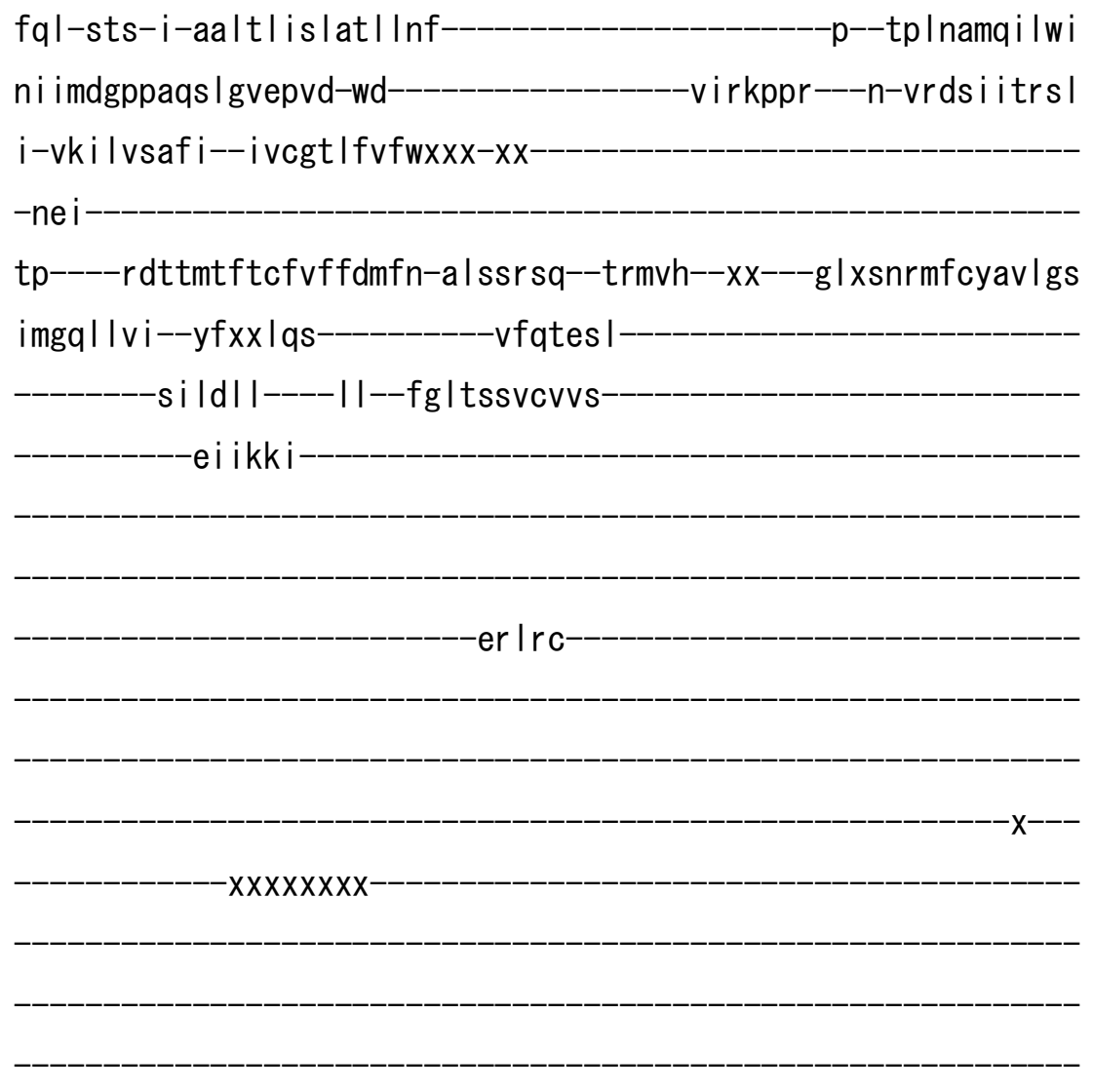

V

>cnelI_IIA201 XP_568029. 1

$\mathrm{m}-$

$-X X X X X X X X X X X X X X X X$

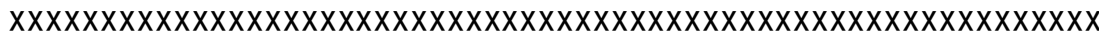

$\mathrm{xxxxx}$ 


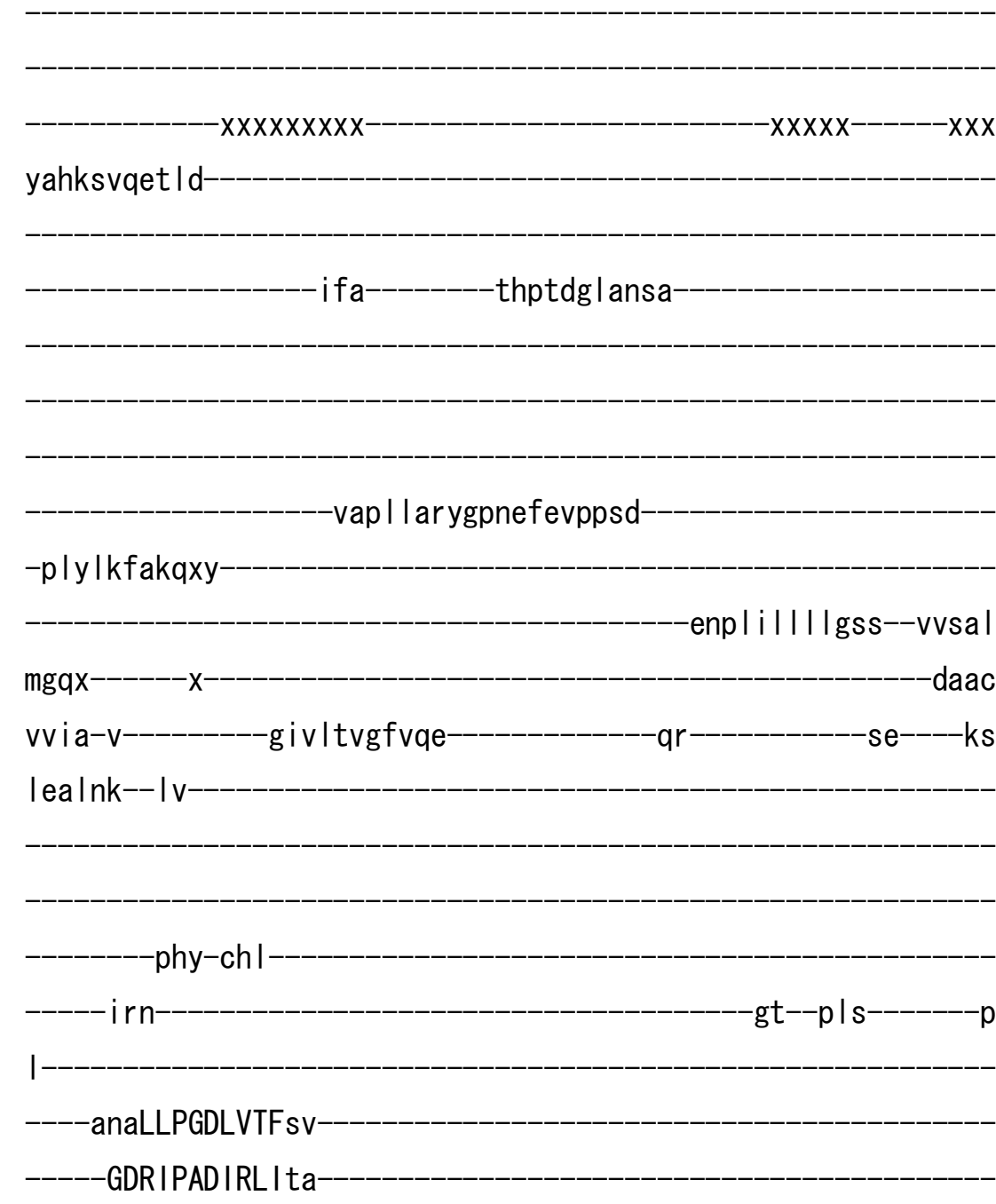

-n--h l e IDESALTGETRPARKnt-

XXXX
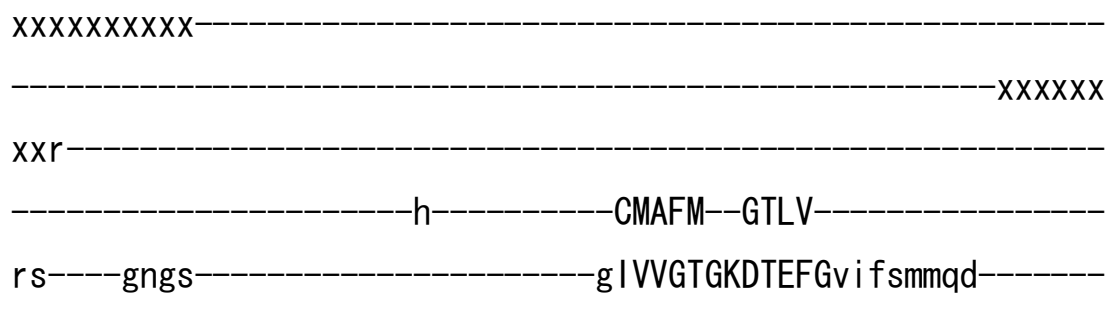

-veekrtp IqIdmdn lakq Isivsfivigfiv

-l igviqkrdw lemft i gVSLAVAA

IPEGLPIVtTVTLALGVL-RMskrk- 
a IVK-KLPSVEALGSVSVICSDKTG-

- TLTKNEMTVt

-hmysvde

$-x x x x x--p x x x$

xxxxxgprrpdsxxxxxxxxxx

$-k v$

alvgnlcxxxxxneqg

invgqate--val Inv

- lpvlkae--dark

-nfirkse ipfssetk-tms i

$-x \times x x x x$

$-x \times x x x x---$

$-1$

$-k$

gav

e-

-qvi arcryyyvtdsx

tps Idtatqki i-Idramev--skr----g I rvIAMAYGfp

-gkgdxepnn I vfVGFEAMMDPPRNGVVHAVSALHSag-VQ I

VMI TGDAEPTAVAIAKQLGL

$-k v s x x t x x$

$-x x x x x x x$

$-x x x x x x s c i \mid$ gsqid

qmtere Ive- 


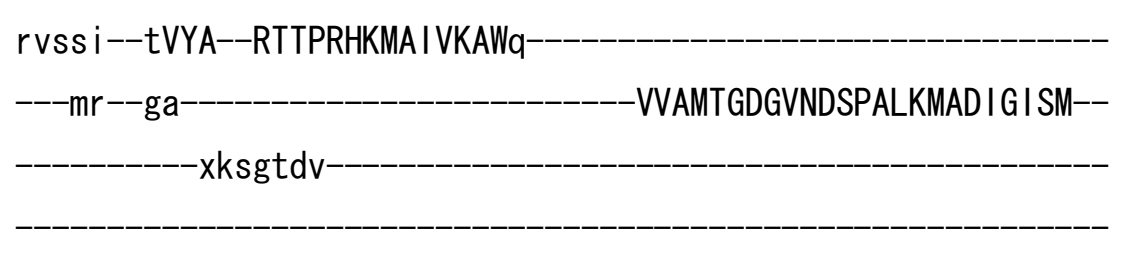

-AKEAADVILVd-DDFASILpaVEEGKSIFYNI QNFL-------S

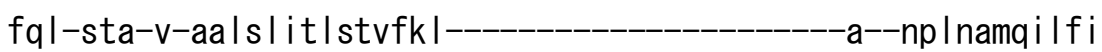
n i Imdgppaqa I gvdpvd-ee--_-_-_-_-_-_-_-_-_ imr qppr----k-kgshv I str I
i-yrv Ifsaam--i v I gt I w i ytxxx-xx-_-_-_-_-_-_-_-_-_-_-_-_-_-_-_-_-

V

>anilI_IIA201 EAA62044. 1

m- 
ysql spqetad

$r \mid q$

-ts|thg I tpae

ae i ry irdgpne I pheepe

$-p|w| r f l k q-f$

ketlillllasa--avsff

mgnx ddavs

it l a-v----------t i vvtvgfvqe---------------yr-------------se-----ks

lealnr--|v-

-phh-ahl

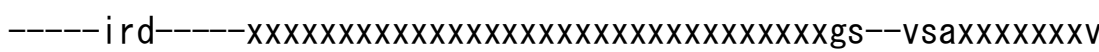

$\mathrm{p}$

----aaeLVPGDLVLFtv

GDRIPADIRITaa 
-t--d I tLDESNLTGENEPVVKyp-

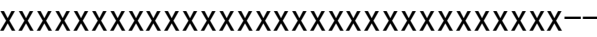

$-X X X X X X$

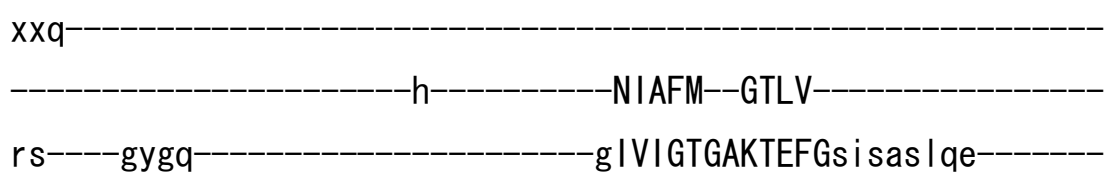

-iesprtp lqlsmdr I gqe I sy isfgvial iv

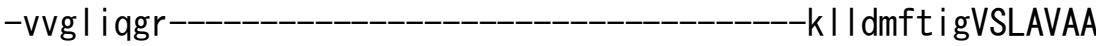

IPEGLPI I vTVTLALGVL-RMasrg-

a IMR-RLPSVETLGSVNVVCSDKTG

TLTLNHMTVt

-kmwhfdcae

$-x x x x x h x x x$

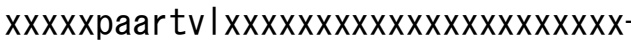

SS

aav I sstxxxxxgsmk

xrwvgqptd--vai Idl-

Idafged--dvrdx

i sarvaetpfsserk-wmgvxxxxx

$-x x x x x x x x x x x x x x x x---$

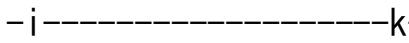

gal

$\mathrm{e}$

qv larcdty Itkdgx 
evi I der r rqvv-rqaaeqm--ase-----g I rvLSFASGav--_-_-_-_-_-_-_----xx

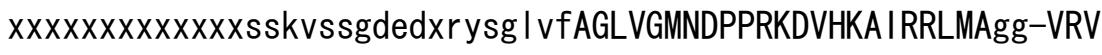
IM I TGDAETTAVA I AKKLGM-piv-

-xxxxxxnv logqe id

rmgtse lsq-

al ssv--s IFA--RTSPDHKMK IVRALq

$---\mathrm{sr}-\mathrm{gd}-$

-VVAMTGDGVNDAPALKKAD I G I AM--

$-x k \mid g t d v$

-AKEAADM ILTd-DDFST IL r I IEQGKG IFYN I QNF I $-\mathrm{T}$

fql-sts-v-aal s I v I I stmfgf--_-_-_-_-_-_-_-_-_---k--np I namq i I w i

ni Imdgppaqs I gvepvd-ps--_-_-_-_-_-_-_-_-_imgrppr---p-ksarv Itkp I

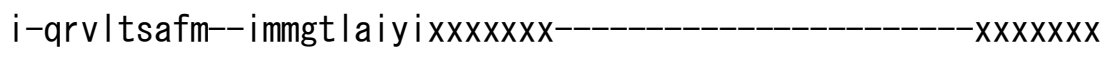
xrvv---_-_-_-_-_-_-_-_-_-_-_-_-_-_-_-_-_-_-_-_-_-_-_-_-_-_

ta----hdttmtftcfv|fdmfn-altcrse--gksv I xxxx---s I xgnkmfnyav I gs lagqacv i--y |xx | qr----------vfqtep I-

--------naah|f-----k|--vciastvfwvd--_-_-_-_-_-_-_-_-_-_-_-_-_-_-egrkyy

qs lar 
V

>ggal I_IIA201 XP_426010. 2

$$
\mathrm{m}
$$

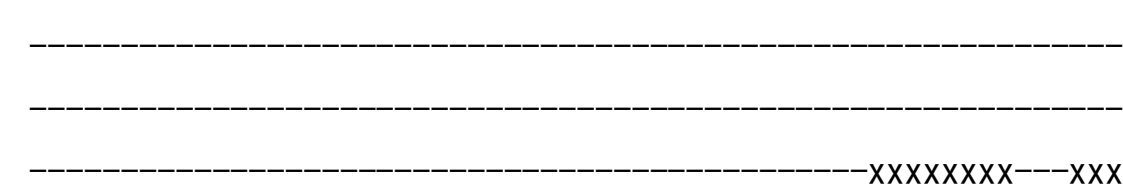

ase Ipvdevas

i l q

-an I qng I knce-

-vchrrafhgwnefdi sede

-p I wkky i sq-f 


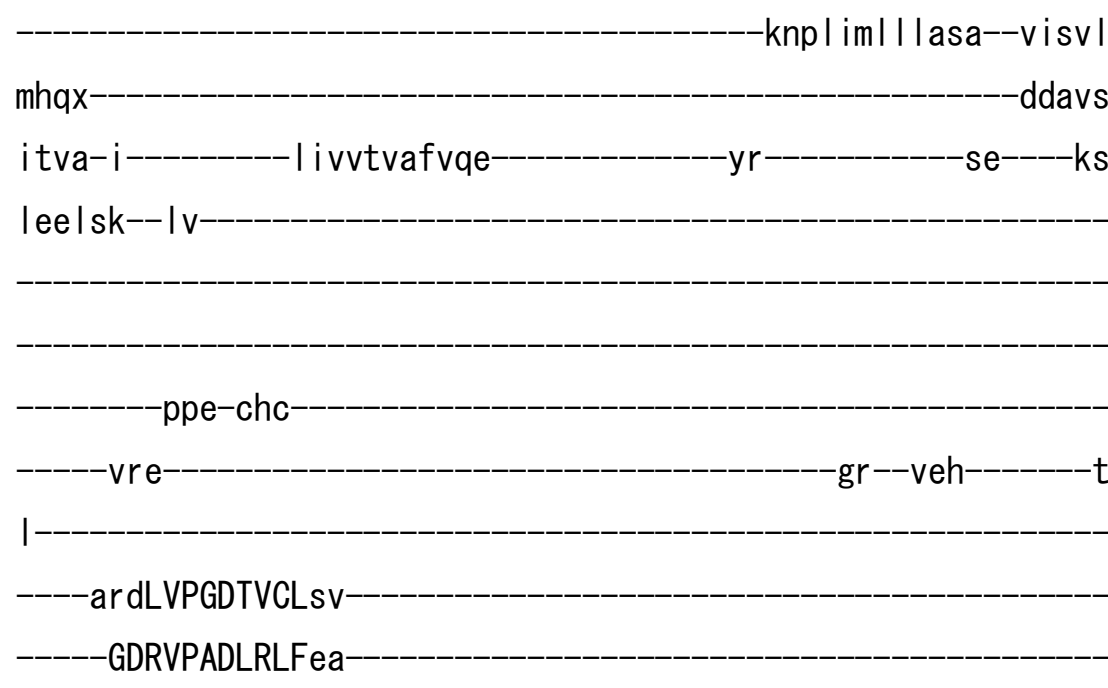

-v--dI sIDESSLTGETAPCAKst-

$-x X X X X X X X X X X X X$

-NIAFM--GTLV-

rc----gkak--------------------gIVIGTGENSEFGevfkmmqa-

-eeapktp lqksmd I gkq Is lysfgi igvim

- I vgw l ggk---_-_-_-_-_-_-_-_ I dmft i gVSLAVAA

IPEGLPIVvTVTLALGVM-RMvkkr

a IVK-KLPIVETLGCCNVICSDKTG

-TLTKNEMTVt

hiftsdggh-

$-\mathrm{XXX}$

xxxxxnrfgdvmx--xxxxxxxxxxxxxx-

veagcvc-----ndal

ixxx 
-t Imgkpte--gal ial-----xxxxx---

$|d g| x x$

dy irkaeypfsseqk-wmavxxxxx

$\mathrm{XXXXXXXXX---}$

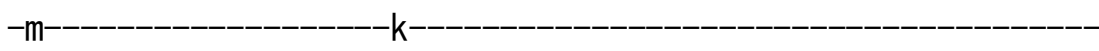

-gay

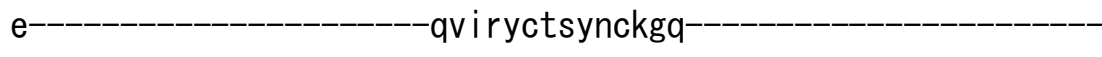

t I p I vqqqr eqy-qqektsm--gsa----g I rvLALASGpe

I gqmtfLGLVG I IDPPRTGVKEAVTTL IMsg-VA I

KMI TGDSQETAVA I ASRLGL-

$-y s k$

xxqa i sgee id

dldiqqlsq

i tpkv--aVFY--RASPRHKLKI IKSLq

$---\mathrm{nn}--\mathrm{ga}$

-VVAMTGDGVNDAVALKAADIGVAM--

xqtgtdv

-CKEAADM I LVd-DDFQT I Msa I EEGKG I YNN I KNFV 


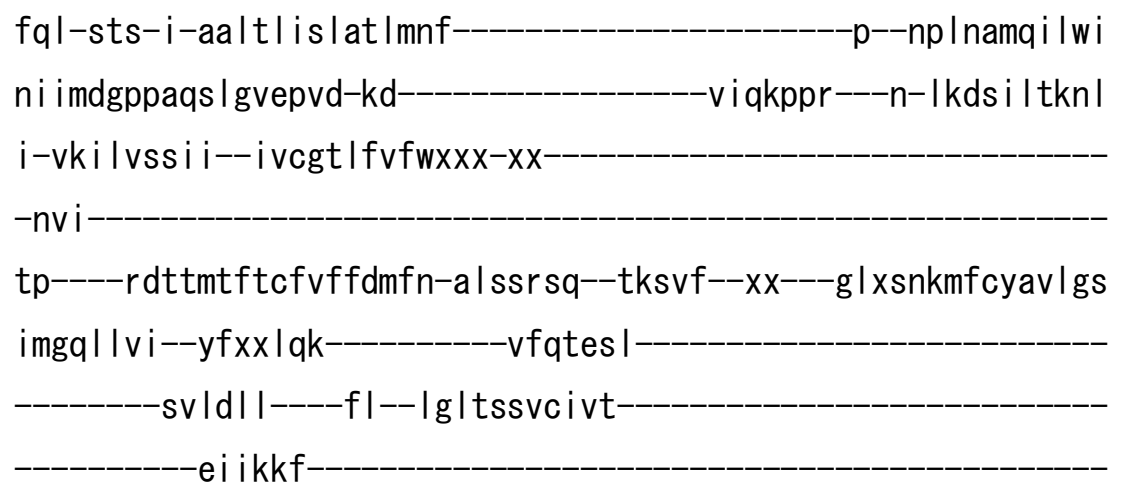

erske

----

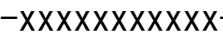

$-\mathrm{XXXX}$

V

>mgr II_IIA201 XP_365047. 2

m- 
$x X X X X X X X X X X X X X$

fsyltasetat

$-r \mid q$

-ts I thg I tate

-al r rqqdhg I ne i pheppe

-plwlrfigq-f

-kep I IIII lasa--aas if

$\operatorname{lgn} x$ ddavs

itva-vtivvtvgfvqe

$-\mathrm{yr}$

se----ks

iealsh--Iv-

$-p d h-a h \mid$

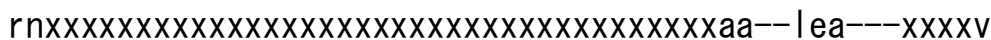

$\mathrm{m}-$

----aeqLVPGDLVIFtt

GDRIPADIRVTka

$-x x$

I t IDASNLTGENEPERLta-

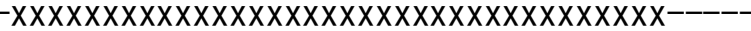

$-x x x x x x$

$\mathrm{xxp}$

-NVAYM--GTLV-

rs----ohgq-

g IVFATGGNTHFGt i s I svsg

-tesprsp Iq I smde Iggq I skfsf i vigf is

- I igf lqgk -kIle ift is ISLAVAA

IPEGLP I IvTVTLALGVH-RMakrn- 
a IVR-KMPKVETLGSVNIVCTDKTG

TLTMNHMTTt-

-kmwyfgqqs

$x x x x x x x d x x x$

xxxxxaat Ir i Ixxxxxxxxxxxxxxxxxxxxxxxx-

vIsst I gxxxxxsayt

rwvgqptd--vam ld I

ldrfkeh--dvrgx

-vgprttetpfsserk-wmgvxxxxxxxxxx

$x \times x x x x---$

$-\mathrm{i}$

$-k-$

gal

d

-rvlascdty itgegx

efv Idsnr rqea-i qaaeqm--aak----g I rvLAFASGav

$-x x x x x x x x x x$

xxxxxxxxxxxxxsedghpatnexpytg I tfAGLVGMSDPPRPGVARS IRKLMRgg-VKV IMI TGDAETTAVA I GRQLGM-

-hiaxxi

$-x x x x$

$-x x x x x x p v \mid r g e e v d$

amteee laq 
amqtt--tVFA--RTNPDHKMK I IRALq

$---\mathrm{mr}--\mathrm{gd}-$

-IVAMTGDGVNDAPALKKAD I G I AM--

$-x r q g t d v$

-AKEAADM I LTd-DDFST I Lha I EEGKG IFNN I QNFL $-\mathrm{T}$
fql-sts-a-ag|s|v|lctalgf-
$-k--$ sp I namqi I wi
ni imdgppaqs I gveavd-kd-----_-_-_-_-_----vmnrppr---k-rndpv I trav
i-qrvaqsat i--vmvgtml vytxxxxxx-
- rqv
tr----rdttmtftcfv Ifdmfn-al acrse--sks i I xxxx---g | xant I fnwavs Is iagql lvi--yfxx|qe-----------vfqteal
- gfmdlv----rl--vmicstvfwad-
elrkw|

$$
-\mathrm{k}-\mathrm{yg}
$$

V

>mmul I_IIA201 NP_778190. 2

$\mathrm{m}-$ 
ase lavsevag

$11 q$

-ad l qng I nkse

-vshr rafhgwnefd i sede

-p lwkky i sq-f

-knp I iml I lasa--visi I

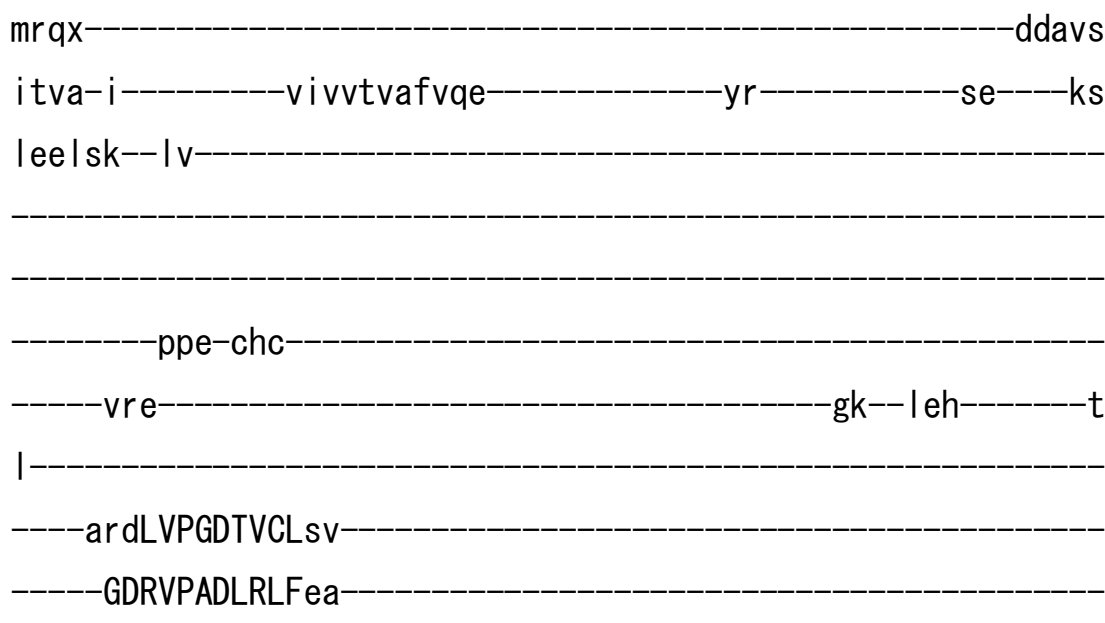


-v--d I sVDESSLTGETAPCSKvt-

$-x x x x x x-x x x x x x x x$

-NIAFM--GTLV-

rc----gkakg IVIGTGENSEFGevfkmmqa

-eeapktp lqksmd। I gkq l sfysfg i i gi im $-|v g w|$ lgk -di l emft i sVSLAVAA

IPEGLPIVvTVTLALGVM-RMvkkr -

-a IVK-KLPIVETLGCCNV I CSDKTG

TLTKNEMTVt

-hi Itsdglh-

$-X X X$

xxxxxnqfgevix--xxxxxxxxxxxxxx-

tImgkpte--gal ial-----xxxxx---

$|d g| x x$

dy i rkaeypfsseqk-wmavxxxxx

$-\mathrm{xxxxxxxxxx---}$

-m----------------------k

gay

$\mathrm{e}$

-qvikycttynskgq 
t la l tqqard ly-qqekarm--gsa----g | rvLALASGpe - gq I tfLGLVG I IDPPRTGVKEAVTTLIAsg-VS I

KMI TGDSQETAIAIASRLGL $-\mathrm{ysk}-$

-xxqsvsgeevd tmevqh Isq

i vpkv--aVFY--RASPRHKMK I IKSLq

$---\mathrm{kn}--\mathrm{ga}$

-VVAMTGDGVNDAVALKAADI GVAM-$-x q \operatorname{tgtd} v$

CKEAADMILVd-DDFQT IMsa IEEGKGIYNN I KNFV R

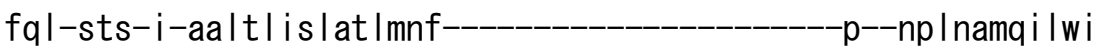
ni imdgppaqs I gvepvd-kd--_---_-_-_-_-_-_--vi r kppr---n-wkds i I tkn I i-Iki lvssi i--ivcgt I fvfwxxx-xx- nvi

tp----rdttmtftcfvffdmfn-al ssrsq--tksvf--xx---g|xsnkmfcyav|gs imgql Ivi--yfxx lqk-----------vfqtes I-

- si $|d||----f|--|g|$ tssvcivs e i ikkv 


$$
\mathrm{V}
$$

>mmulI_IIA202 XP_486167.4$$
\mathrm{m}
$$

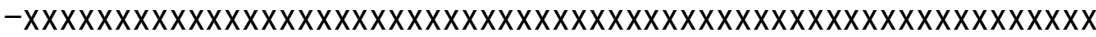

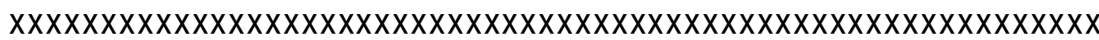

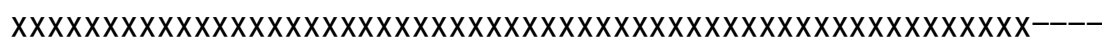
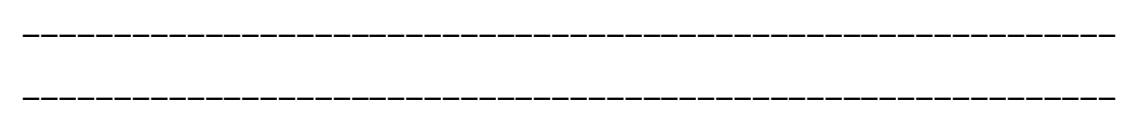

$-x x x x x x x x---x x x$

ackcsreelar

afh -vdldsgl sefa-vaqr I vhgwnefvtdnae $-p v w k k y l d q-f$ 


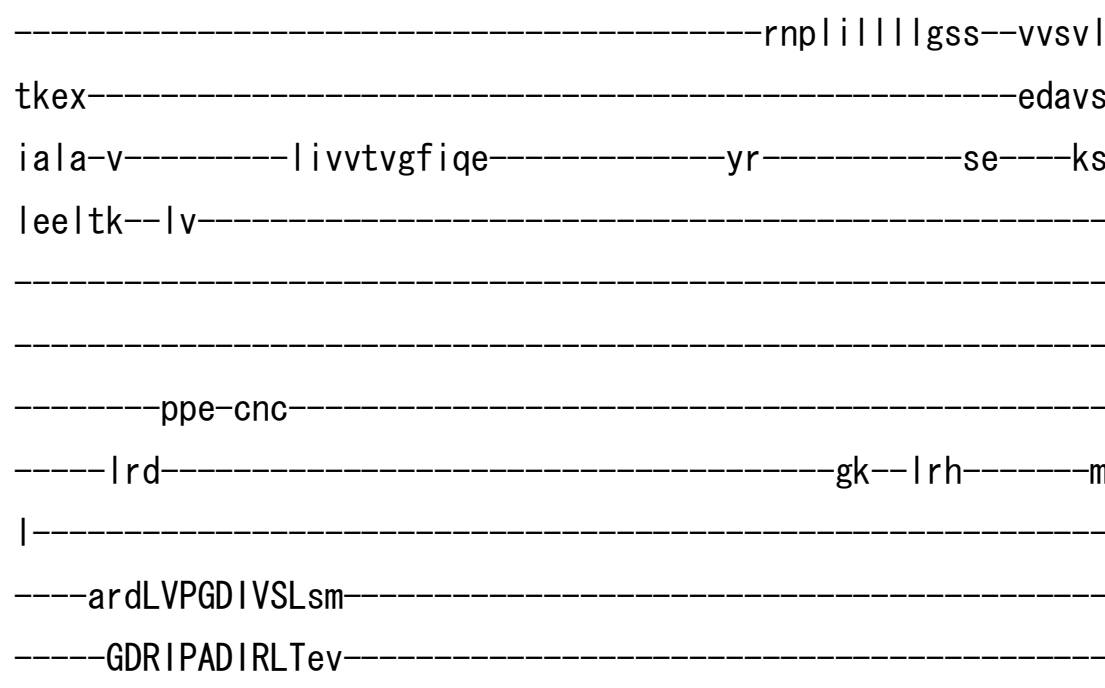

$-x$

d I IVDESSFTGEVEPCGKtd-

$x \times x x x x x x$

$x \mathrm{X} \mid$

-NVVFM--GTLV

qc----gkgq-_--_-_-_-_-_-_-_-_-_---gVVIGTGEQSQFGevfkmmra

-eetpktp lqksmdk I gkq It i fsfg i ig I Im

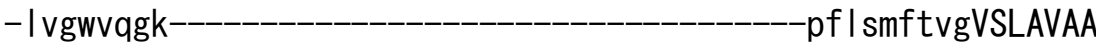

IPEGLPIVvMVTLVLGVL-RMakkr

-v IVK-KLPIVETLGCCNV ICSDKTG

TLTANEMTAt-

qlvtsdgfh

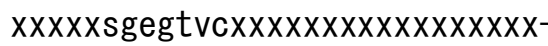

veagcva-----nnav

ixxx 
-avmgqpte--ga| vv I-----xxxxx---

Igsixx

syvrkke ipfsseqk-wmavxxxxx

$-x X X X X X X X X--$

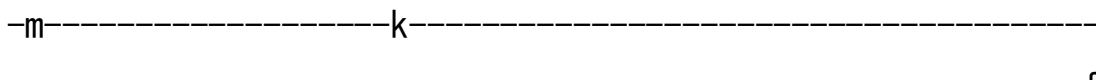

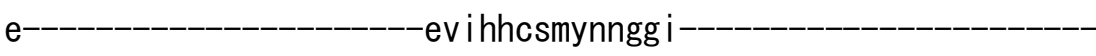

-gaf

plp I tpqqksyc-qqeekkm--gs I----g|rvLALASGpe

- gr I tfLGLVGI IDPPRAGVKEAVQVLSEsg-VSV

KMVTGDALETALAIGRTIGL-

cne

xxkamsgeeve

gteqga laa

rvrqv--sVFF--RTSPKHKVKI IKALq

---es--ga-

-IVAMTGDGVNDSVALKSAD I G I AM--

xqtgtdv 


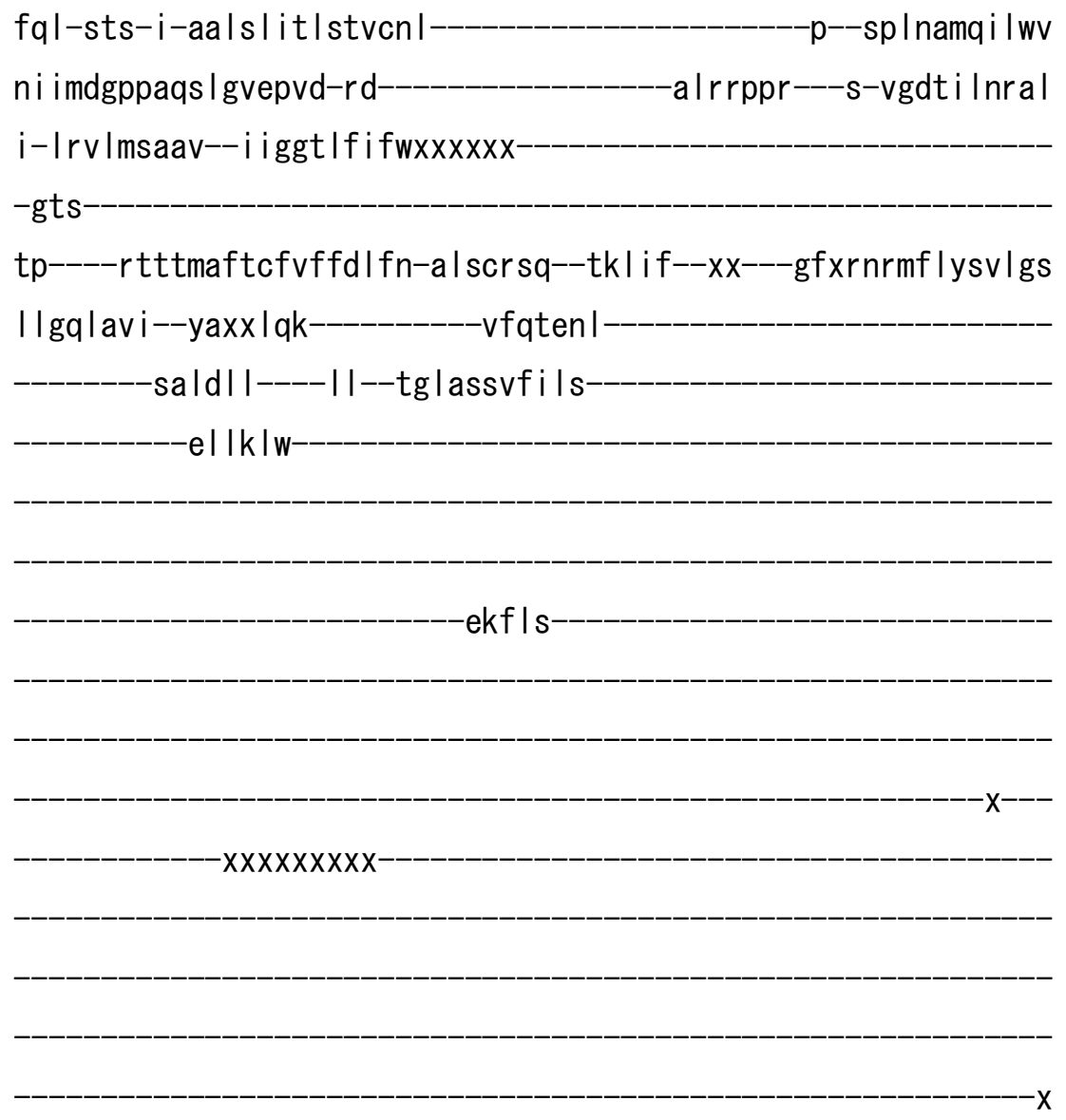

V

>rnolI_IIA201 NP_571982. 2

m- 
$-k d$ l wesklag-

-hlq--------ad l qng Inkse-

-vshrrafhgwnefdi sede

$-p$ lwkky i sq-f

$-k n p \mid$ iml I lasa--visv I

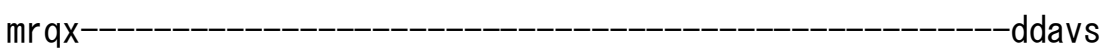

itva-i----------। i vvtvafvqe----------_---yr-----_------se-----ks

leelsk--|v-

$-v--d$ I s IDESSLTGETTPCSKvt-

$-x x x x x x x x x x x x x x x-$

-NIAFM--GTLV-

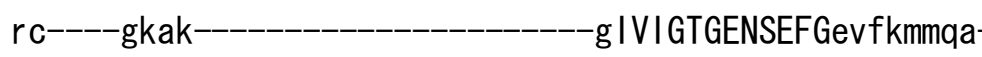

-eeapktp lqksmdl I gkq l sfysfg i i gi im

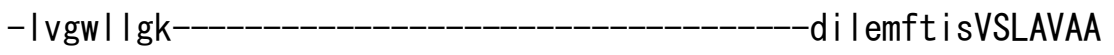

IPEGLPIVvTVTLALGVM-RMvkkr 
a IVK-KLPIVETLGCCNV ICSDKTG-

TLTKNEMTVt

hi Itsdg Ih

$x X X$

xxxxxnqfgevix--xxxxxxxxxxxxxx-

$-r \mathrm{i}$

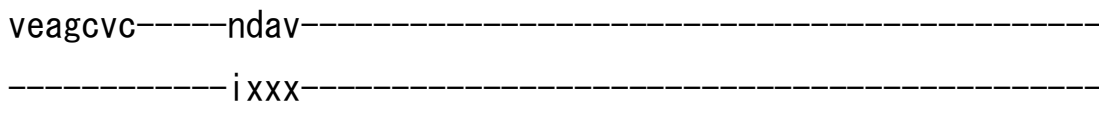

tImgkpte--gal ial-----xxxxx---

$\operatorname{ldg} \mid x x$

dy i rkaeypfsseqk-wmavxxxxx

$-x x x x x x x x x x---$

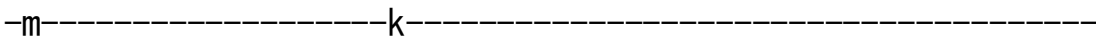

-gay

e---------_-_--_--_----qvi kycttynskgq

t l a l tqqqrd ly-qqekaqm--gsa----g I rvLALASGpd

- gq I tfLGLVG I IDPPRTGVKEAVTTLI Asg-VS I

KMI TGDSQETAIAIASRLGL-

-ysk-

$-x x q s v s g e e v d$

tmevqh Isq 


$$
\begin{aligned}
& \text { i vpkv--aVFY--RASPRHKMK I IKSLq- } \\
& \text {---kn--gs-----_--_-_-_--_--_-_-_-_-_VVAMTGDGVNDAVALKAAD I GVAM-- } \\
& \text { xqtgtdv- }
\end{aligned}
$$

CKEAADM I LVd-DDFQT I Msa I EEGKG I YNN I KNFV-------R

$$
\begin{aligned}
& \text { fql-sts-i-aalt l is lat Imnf-_-_-_-_-_-_-_-_-_---p--np I namq i I wi } \\
& \text { ni imdgppaqs I gvepvd-kd----_-_-_-_-_-_---vi i kppr---n-wkds i Itkn I } \\
& \text { i-Iki Ivssi i--ivcgt I fvfwxxx-xx-_-_-_-_-_-_-_-_-_-_-_-_-_-_-_ } \\
& -n v i- \\
& \text { tp----rdttmtftcfvffdmfn-alssrsq--tksvf--xx---g| xsnkmfcyav I gs }
\end{aligned}
$$

V

>rnolI_IIA202 NP_604457. 1

m- 
ackcskeelar

-tfh--------vdldsgl sefa-

-vaqr r I vhgwnefvtdnte

$-p v w k k y \mid d q-f$

-rnpli III |gss--vvsv|

tkexedais

ia la-v--_-_-_--- l i vvtvgf i iqe--_-_-_-_-_---yr--_-_-_-_---se-----ks

leeltk--|v-

-ppe-cnc-

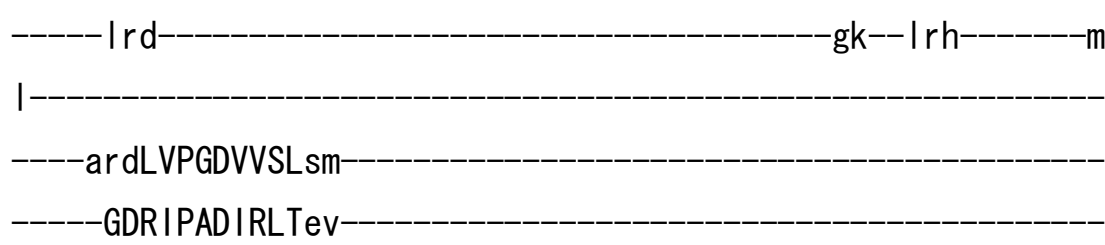


$-X X X X X X X X X$

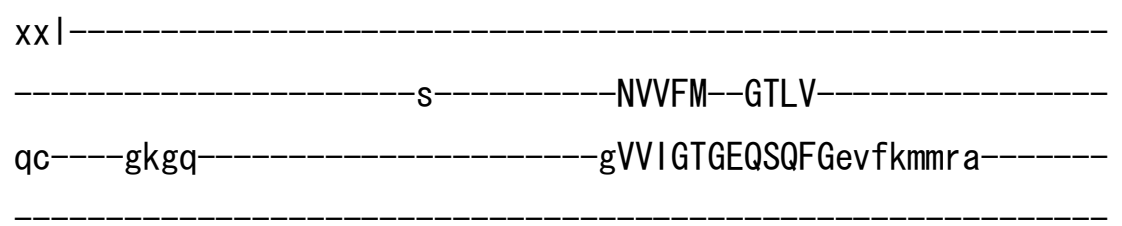

-eetpktp Iqksmdk I gkq I tvfsfg i ig I Im - I vgwvqgk-_-_-_-_-_-_-_-_-_-_-_-_-_-_-_-_-_-_ I I smft i gVSLAVAA IPEGLPIVvMVTLVLGVL-RMakkr-

- VIVK-KLPIVETLGCCNV ICSDKTG-

-TLTANEMTAtqlvtsdgfh-

$-x x x$

$x x x x x$ sgegtvcxxxxxxxxxxxxxxxxxx

veagcva-----nnav----------------------------------------------

-avmgqpte--gal vv I----xxxxx--Igs ixx

sy i rkke ipfsseqk-wmavxxxxx

$-x \times x \times x x \times x x^{---}$

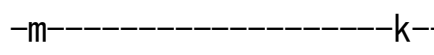

-gaf e -evihhcstynngg i 
p |p| tpqqksyc-qqeekkm--gs |----g | rvLALASGpe

- gr I tfLGLVGI IDPPRAGVKEAVQALSEsd-VSV

KMVTGDALETALA IGRT IGL

cde-

-xxkamsgeeve

gmeqda laa-

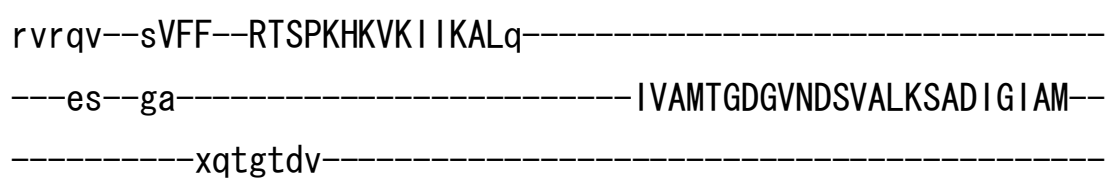

SKEAADM ILVd-DDFSA IMsaVEEGKG IFYN IKNFV------R

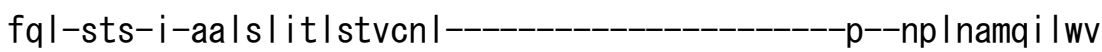
ni imdgppaqs I gvepvd-rd-----_-_-_-_-_-_---alkrppr---s-vkdt i Inra I

i-Ik i Imsaav--i I ggt I f i fwxxxxxx-

$-r$ ts

tp----rtttmaftcfvffdIfn-al scrsq--tkl if--xx---gfxrnrmf Iysi Igs

I I gqlavi--yaxx lqk-----------vfqten I-

--------saldI I-----||--tglassvfi Is-

ellk|c-

ekfcs 


\section{V}

>dme II_I IA201 NP_730745.1

m-

ssthsasevag

$-r \mid q$

-vdvrtglkwte

-akyrak i ighne I I vaed

-ptwkky i eq-f 


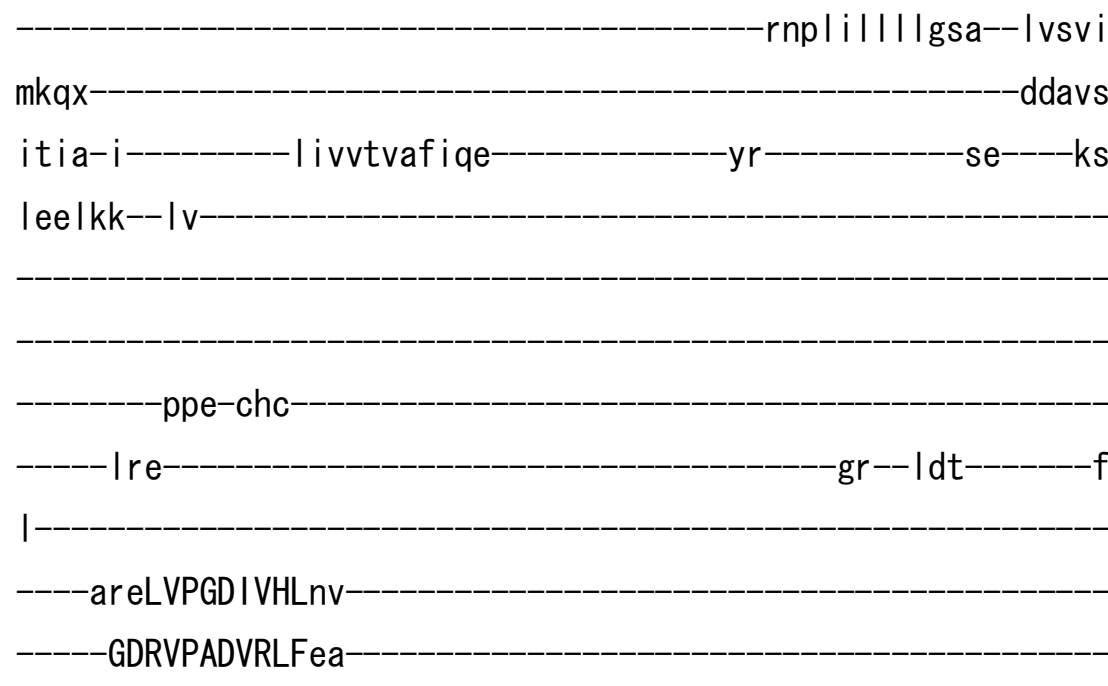

$-v--d$ I s IDESSFTGETEPARK it-

$-X X X X X X X X X X X X$

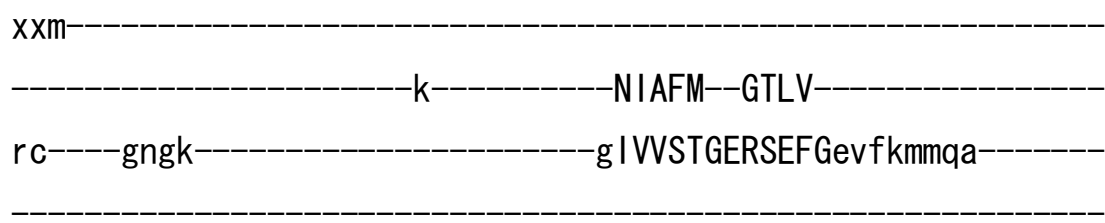

-eeapktp Iqksmd i gaq I sfysf I i igv im

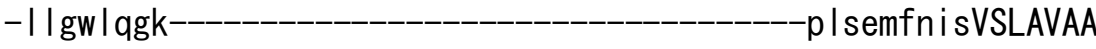

IPEGLPIVvTVTLALGVM-RMakrn-

s IVK-KLPTVETLGCVNV ICSDKTG

TLTKNEMTAt-

- i i tsdgym-

$-X X X$

xxxxxndqge $i h x x x x x x x x x x x x x x$

$-n \mid$

le i gavc-----nnay

i xxx 
-t l gqpte--gal vav-----xxxxx---

myatxx

-nyvr iqeypfsseqk-mmavxxxxx

$\mathrm{XXXXXXXXX---}$

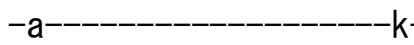

-gal

e-----------------------t I l pqctkyqfgtq

tvp Itkqneaef-laeaye i--grk----g I rvLALAKGrs

-mqd I i yCGLVG I TDPPRPLVRES I EMLMQsg-VRV

KMVTGDAQETALA I ANL I G I

$-d t i$

xxqt I sgqemd

-qmnehq I dk

vannv--sVFY--RVSPRHKLEIVKSLq

$---r s--g n-$

-IVGMTGDGVNDGVALKKAD I G I AM--

xkngtdv

CKEAADM I LVn-DDFHT I I aa I EEGKG IFYN IRNFV 

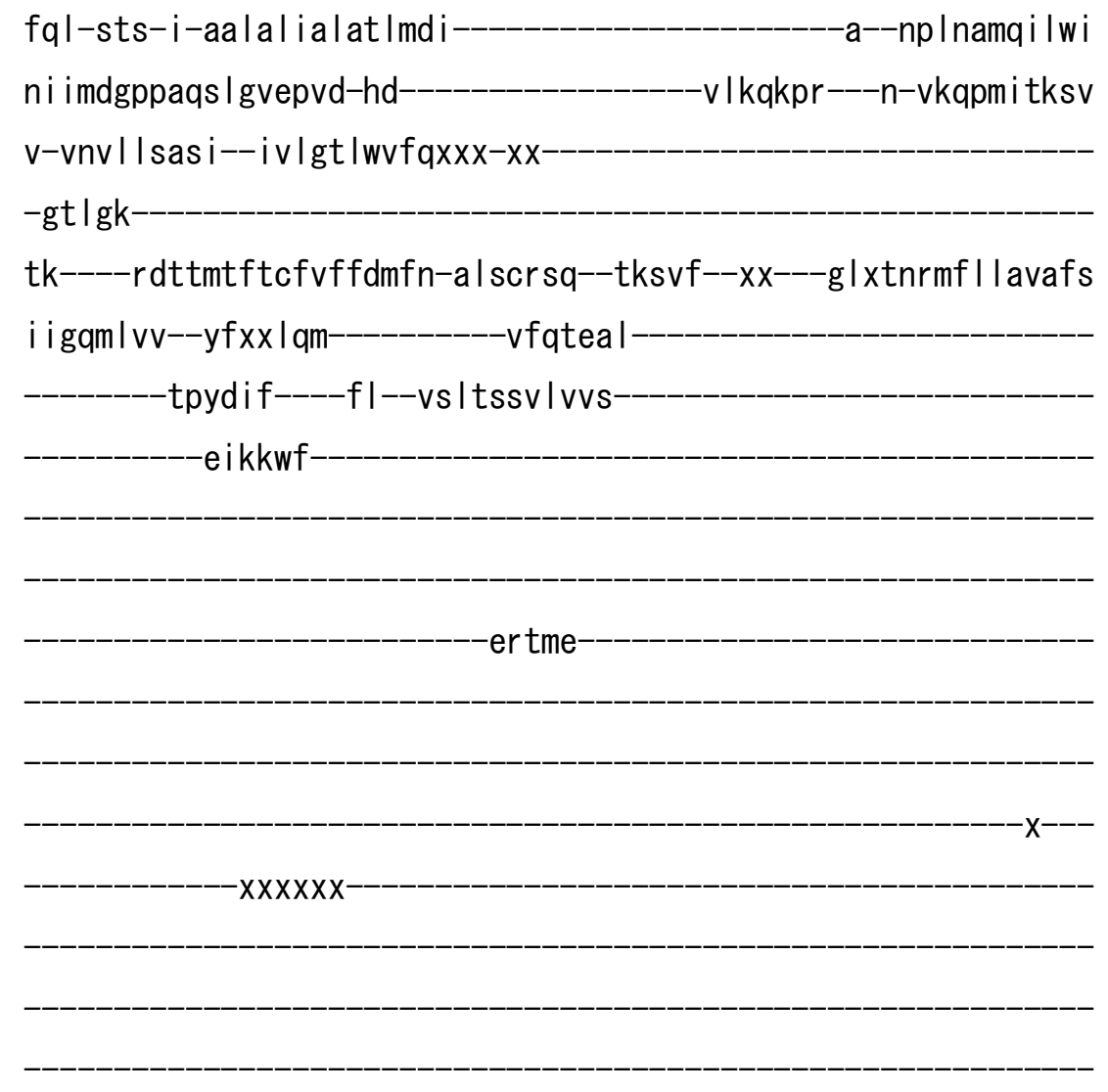

V

>cellI_IIA201 NP_001021862.1

m- 


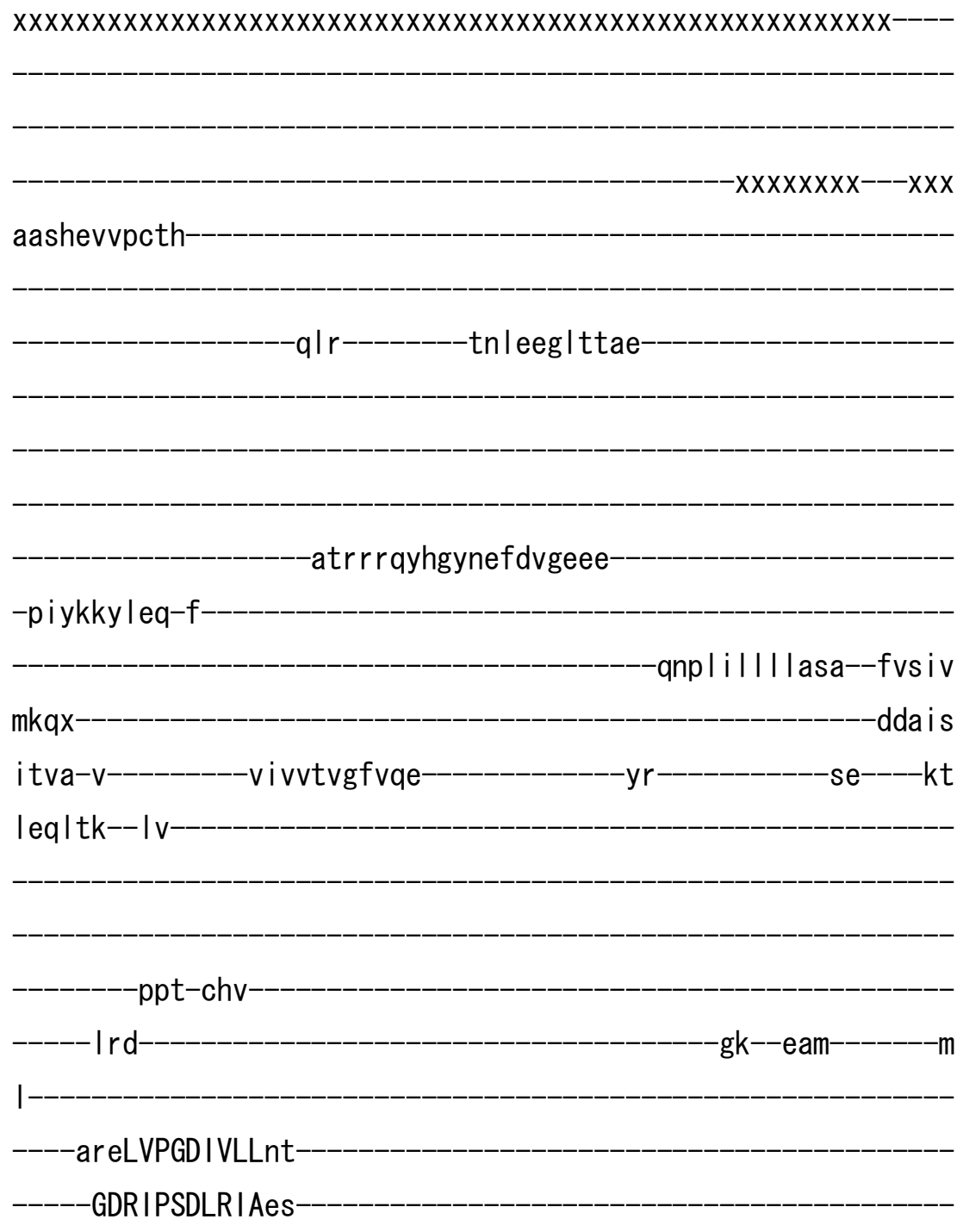

f--s I qIDESSLTGETEPKHKet-

$-x x x x x x x x x x x x x$

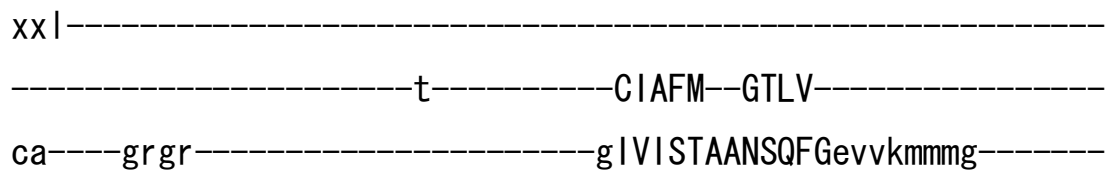

-eespktp I qksmdd I gkq I s i ysfgv i av if

- I i gmfqgr--_-_-_-_-_-_-_-_-_-_-_-_-_-_-_-_-_-_nvvdmft i gVSLAVAA

IPEGLPIVvAVTLAIGVM-RMakr r 
-aVVK-KMPAVETLGCVTVICSDKTG-

-TLTKNEMTAq-

ai atpegk I

$x X X$

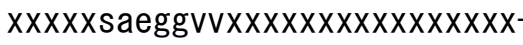

$-r i$

i eag I vc-----nnat

$\mathrm{ixxx}$

-k I i gqpte--ga i vv |----xxxxx---

legvxx

eykr I rempfssdtk-wmgvxxxxx

$-x x x x x x x---$

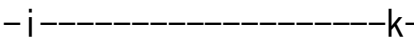

gal

d

-rvldqcgtyyssdnx

rkqcdqysrqhi-le i gke |--gqk----g | rvLGLARGes

-mqs I mfLGM I GMMDPPRPGAADA ISI VKAsg-VDV

KL I TGDAMETAQS I GQSLGI-

Iss

$-x x s c$ I sgaqvd

-amsdhd le I 
virqv--tVFY--RASPRHKLKIVKALq-

---al--ge----------------------VVAMTGDGVNDAVALKKAD I GVAM-$-x v \operatorname{cgtd}-$

-CKEAADM I LCd-DDFSTMT Taa IEEGKA IYHN I TNFV------R

fql-sts-v-aal s I i aastmfkf----_-_-_-_-_-_-_-_-_----d--np I namq i I wi

ni imdgppaqs I gvepvd-dd--_--_-_-_-_-_-_--_i i rarpr---n-tkqpm I tgk I

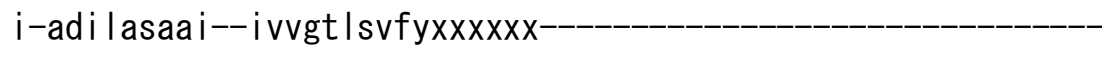

$-n k v$

tp----rdttmtftcfv Ifdmwn-al scrss--rkmi w--xx---g| xrnrmfs lavsas I icql Ivi--ywxx Iqh---------ifateal

-slfdli----fl--ttitssvfifn-

-etrkyf

$-x X X X X X X X X$

$-x X X X X$

i

>hsalI_IIA201 NP_055197. 2

m- 
ase Ipvsevas

ilq

-adlang Inkce

-vshr rafhgwnefdi sede-

$-p \mid w k k y$ i sq-f

$-k n p \mid$ iml I lasa--visv I

mhqx ddavs

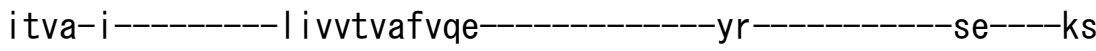
leelsk--Iv-

ppe-chc

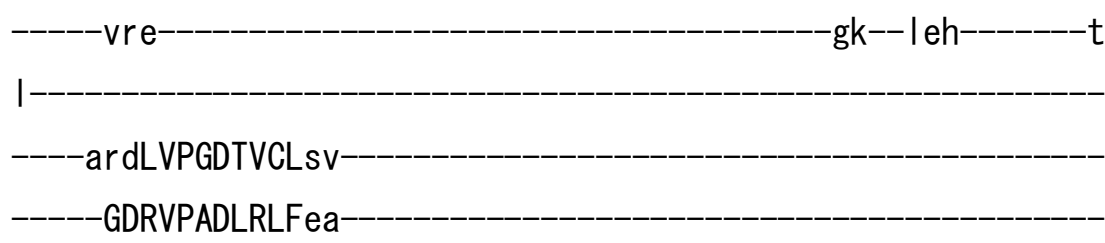


-v--d I I IDESSLTGETTPCSKvt-

$-x x x x x x x x x x x x x x x$

-NIAFM--GTLV-

rc----gkak gVVIGTGENSEFGevfkmmqa

-eeapktp lqksmd। I gkq l sfysfg i i gi im $-|v g w|$ lgk -di l emft i sVSLAVAA

IPEGLPIVvTVTLALGVM-RMvkkr -

-a IVK-KLPIVETLGCCNV I CSDKTG

TLTKNEMTVt

hiftsdglh-

$-X X X$

xxxxxnqfgevix--xxxxxxxxxxxxxx-

tImgkpte--gal ial-----xxxxx---

$|d g| x x$

dy i rkaeypfsseqk-wmavxxxxx

$-\mathrm{xxxxxxxxxx---}$

-m----------------------k

gay

$\mathrm{e}$

-qvikycttyqskgq 
tI t I tqqqrdvy-qqekarm--gsa----g I rvLALASGpe - gq I tfLGLVG I IDPPRTGVKEAVTTLIAsg-VS I

KMI TGDSQETAVAIASRLGL-ysk-

-xxqsvsgee id amdvqq $\mid s q$

i vpkv--aVFY--RASPRHKMKI IKSLq-

$---k n--g s-$

-VVAMTGDGVNDAVALKAADI GVAM--

$-x q \operatorname{tgtd} v$

CKEAADMILVd-DDFQT IMsa IEEGKGIYNN I KNFV$-\mathrm{R}$

fql-sts-i-aalt l i s lat Imnf---_--_-_-_-_-_-_------p--np I namq i I w i ni imdgppaqs I gvepvd-kd------------_------vi rkppr---n-wkds i Itkn I i-Iki lvssi i--ivcgt I fvfwxxx-xx---_-_-_-_-_-_-_-_-_-_-_-_-_-_-_ $-n v i$

tp----rdttmtftcfvffdmfn-alssrsq--tksvf--xx---g|xsnrmfcyavlgs imgq I lvi--yfxx lqk-----------vfqtes I-

-sildI|----fl---|g|tssvcivae i ikkv 
V

>hsal I_I IA202 NP_055676. 1

$$
\mathrm{m}
$$

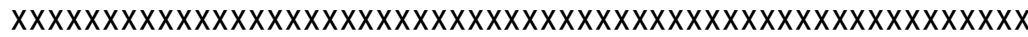

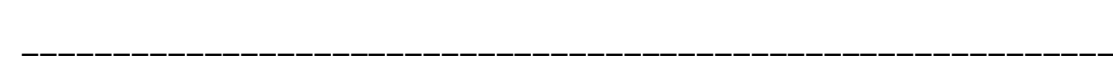

$-x x x x x x x x x x x x x x$

flaapmselvp

$-x x s f a$ -vdlhtgl sefs-

-vtar r lahgwnefvadnse-

$-p v w k k y \mid d q-f$ 


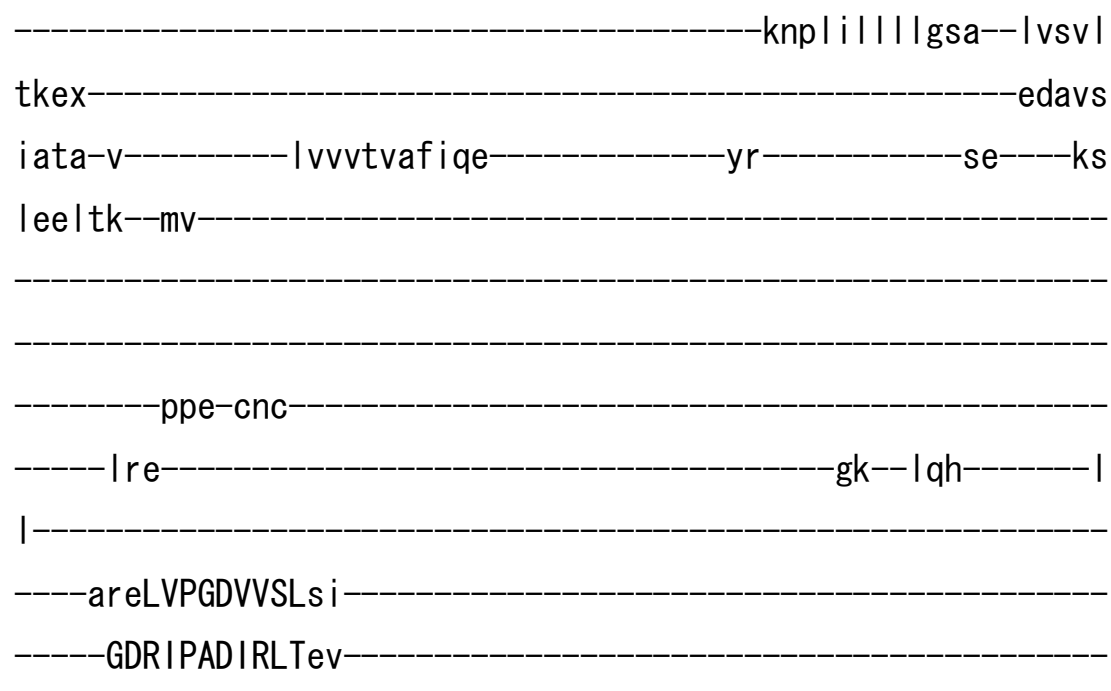

$-x$

d I IVDESSFTGEAEPCSKtd-

$x \times x x x x x x$

$\mathrm{XX} \mid$

$-\mathrm{s}$

qy----grgq-
-N IVFM--GTLV-

gVVI GTGESSQFGevfkmmqa

eetpktp I qksmdr I gkq I t I fsfg i ig I im ql I smft igVSLAVAA

IPEGLPIVvMVTLVLGVL-RMakkr

- IIVK-KLPIVETLGCCSVLCSDKTG

-TLTANEMTVtqlvtsdg $\mid r$

$x x x x x$ dgqgtvc $x x x x x x x x x x x x x x x x x x$

veagcva

nnav

ixxx- 
-avmgqpte--galmal-----xxxxx---

Isdixx

sy irkke ipfsseqk-wmavxxxxx

$-x x x x x x x x x--$

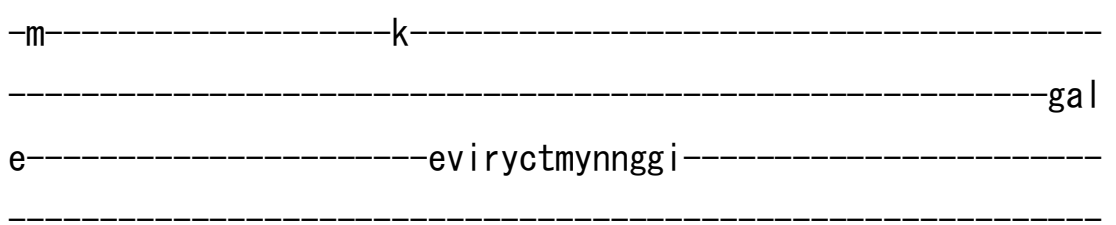

plp|tpqqrsfc-|qeekrm--gs|----g| rvLALASGpe

I gr ItfLGLVGI IDPPRVGVKEAVQVLSEsg-VSV

KMI TGDALETALA I GRN IGL

cng

xxqamsgeevd

svekge lad-

rvgkv--sVFF--RTSPKHKLKI IKALq

---es--ga---_---_--_-_-_-_--_--_---_IVAMTGDGVNDAVALKSAD I G I AM--xqtgtdv 


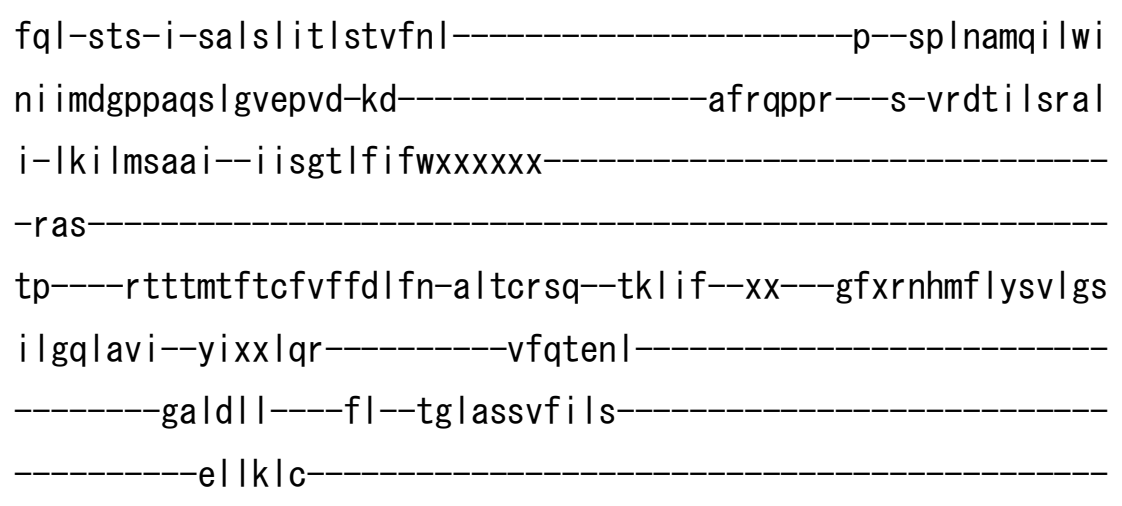

-ekycc

$-\mathrm{x}--$

$-x \times X X X X X X$

$-x$

V

>drelI_IIB03 XP_686848. 2

m- 


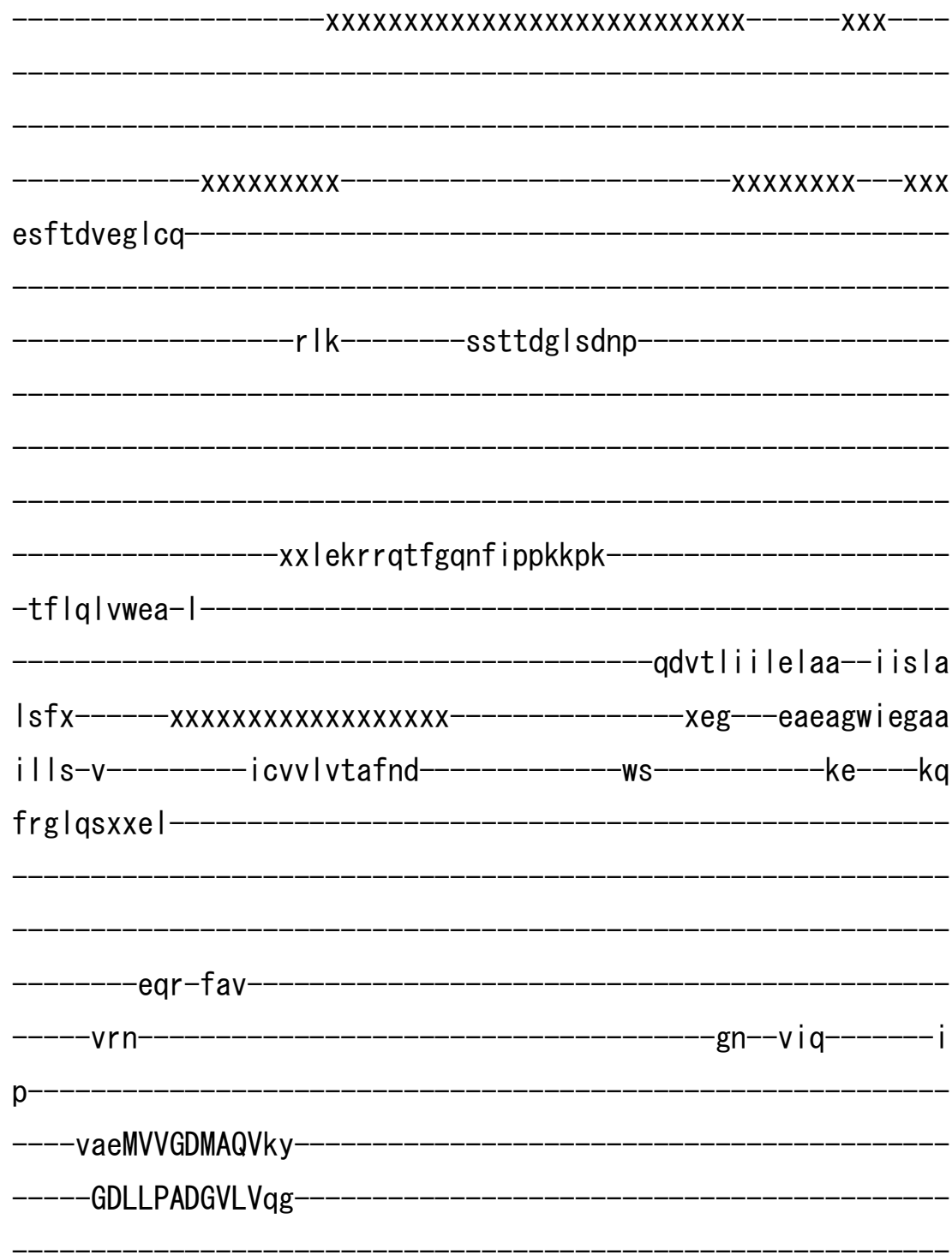

-n--d IkIDESSLTGESDHVRKsv-

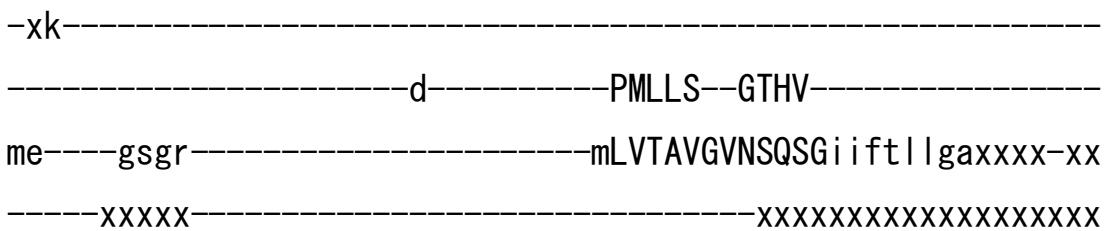
$x x x x x x x x x x x x x x x x x x x x x x x x x x x x x$-pkkeksv lqgk I tk lavqigkag I vmsaitv

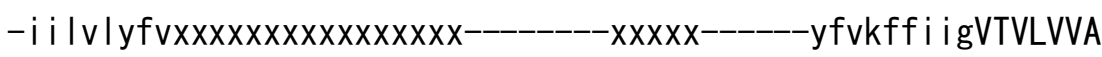
VPEGLPLAvTISLAYSVK-KMmkdn- 
-nLVR-HLDACETMGNATA I CSDKTG-

-TLTTNRMTV

qsy indqhf

$-x-x x x x x s^{--x}$

i spnt le

vnais inxxxxxkimp-

$-p x x \times g x \mid p-$

-kqvgnkte---cg| Igf-----xx-x---Ikrdyap--vreqxx

-eek lykvytfnsvrk-smst

$-v-x x x x--x x----x x x x x x k-$

-gas

e

-ivlkkcssi l gtngx-

arnfrprdrdemxkkvi epm--ace----g|rtICIGYRdI

-xxdpepeweneax i vtd I tc I AVVG IEDPVRPEVPDA IRKCQRag-I IV

RMVTGDN I NTARAIASKCGI -

i qp--g-

$-x x x---|c|$ egkdfnx

$\mathrm{xxxx}$

xxx--------------e i eqer i dk- 
i wpk I--rVLA--RSSPTDKHTLVKGI i

---dsxx l e--------xxx----------------VVAVTGDGTNDGPALKKADVGFAM--

$-x i a g t d v$

-AKEASDI ILTd-DNFSS IVkaVMWGRNVYDSISKFL------Q

fql-tvn-v-vavi vaftgac i tq---_-_-_-_-_-_-_-_-_--_--sp I kavqm I wv

nl imdtfas la lateppt-ea--_-_-_-_-_-_-_-_ I I rkpy---g-rnnp I i s I tm

m-kni I ghgvy--qlvi ift I If

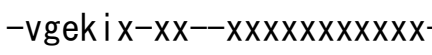

ps----ehyt i ifntfv Imq Ifn-e inarki $x x e r n v f---x---g$ i xanp ifcs i v lgt fgvqvi iv--qf--ggk-----------pfscap I-

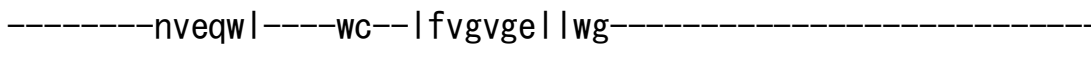

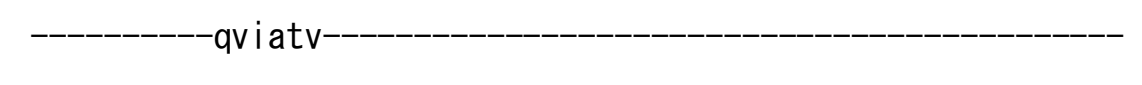

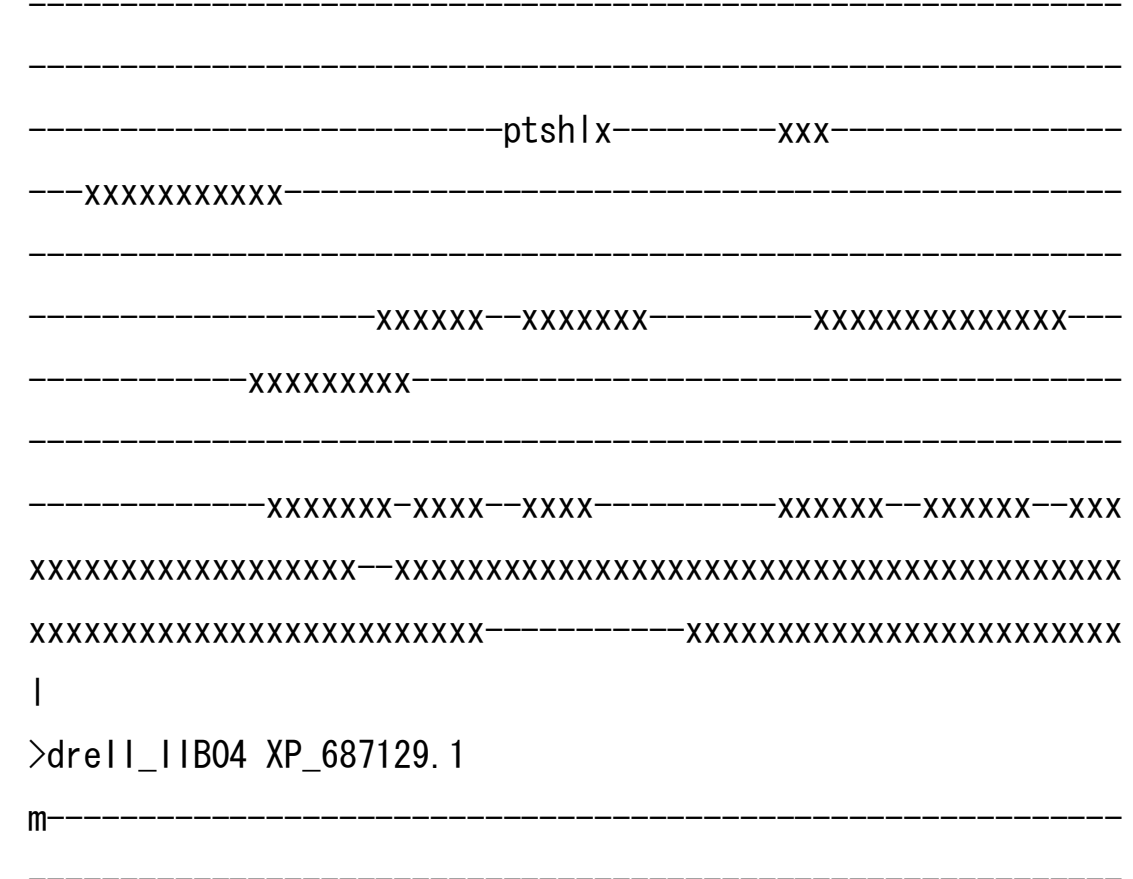


ecygdvag Ics

$-k \mid k$ ssp i eg I sghp

xxiarrkeefgknf i ppkkpk

-tflqlvwea-I

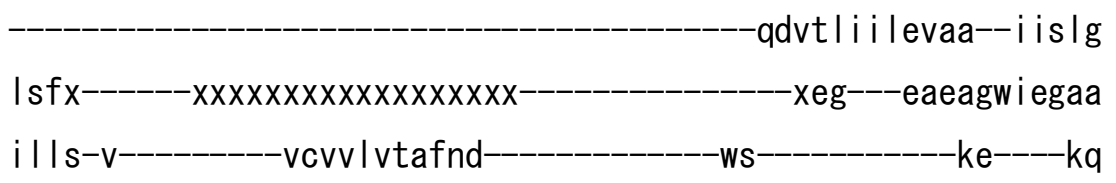

frglqsxxeq-

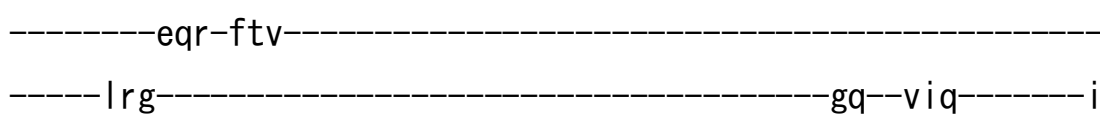

$\mathrm{p}$

----vse IVVGD I AQVky

GDLLPADGVLI qg 
-n--d lkIDESSLTGESDHVKKt I-

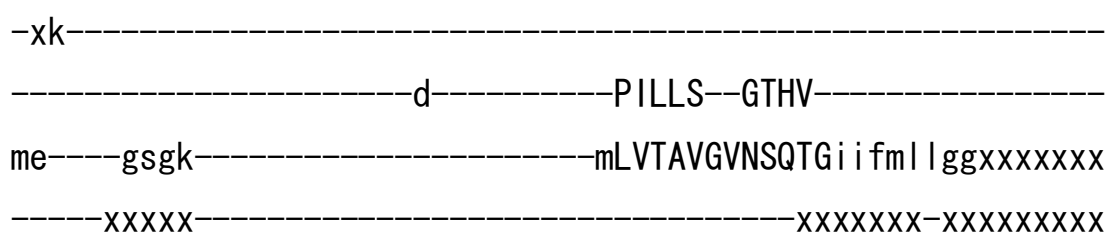

$x x-x x x x x x x x x x x x x x x---x x x x x x-p k k e k s v$ lqgk|tk lavqigkag I fmsaitv

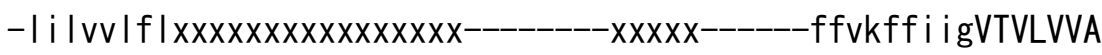
VPEGLPLAvTISLAYSVK-KMmkdn-

-nLVR-HLDACETMGNATA ICSDKTG

TLTMNRMTVV

qvfiagrhf

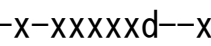

-ipgnimn

vtgigvnxxxxxkims

$-\operatorname{axx} \operatorname{xx} \mid \mathrm{p}$

rqvgnkte--cal lgf-----xx-x----I rkdyqa--ircexx

-eek I ykvytfnsvrk-smst

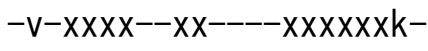

e

-i I lkkccki Itsngx 
akhfrptdrddmxtqvi epm--ase----g I r t ICLAYRdf

-xxxdgepdwdnegx i I tg I tc I CVVG IEDPVRPEVPDA IKKCQRag-I TV

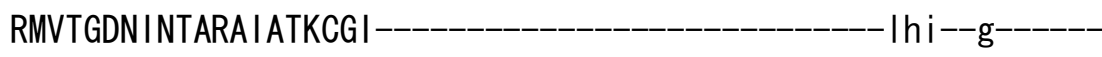

$-x x x---\mid c l e g k e f n x$

$-x x x x$

xxx--------------evskng I dk-

vwpk I--rVLA--RSSPTDKHTLVKGI i

---dsxx le e-------xxx--------------VVAVTGDGTNDGPALKKADVGFAM-$-x i a g t d v$

-AKEASD I ILTd-DNFSS IVkaVMWGRNVYDS I SKFL------Q

fql-tvn-v-vavi vaftgac i tq---_---_--_-_-_-_-_----d---sp I kavqm I wv nl imdtfas la lateppt-es------_--_-------- I I rkpy---g-rnkp I i srtm m-kni I ghavy--qlt i ift I If -ageqi $x-x x--x x x x x x x x x x x$

ps----ehytvvfntfvlmqlfn-e inarki $x x$ ernvf----x---gixnn i ifcsi Ifgt fi iqfvi v---qf--ggk--_-_-_-_---pfscvg I--_-_-_-_-_-_-_-_-_-_-_-_-_-

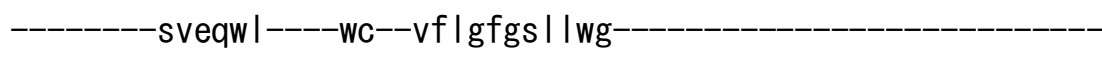
qvistv

ptrrlx $-x x x-$ 


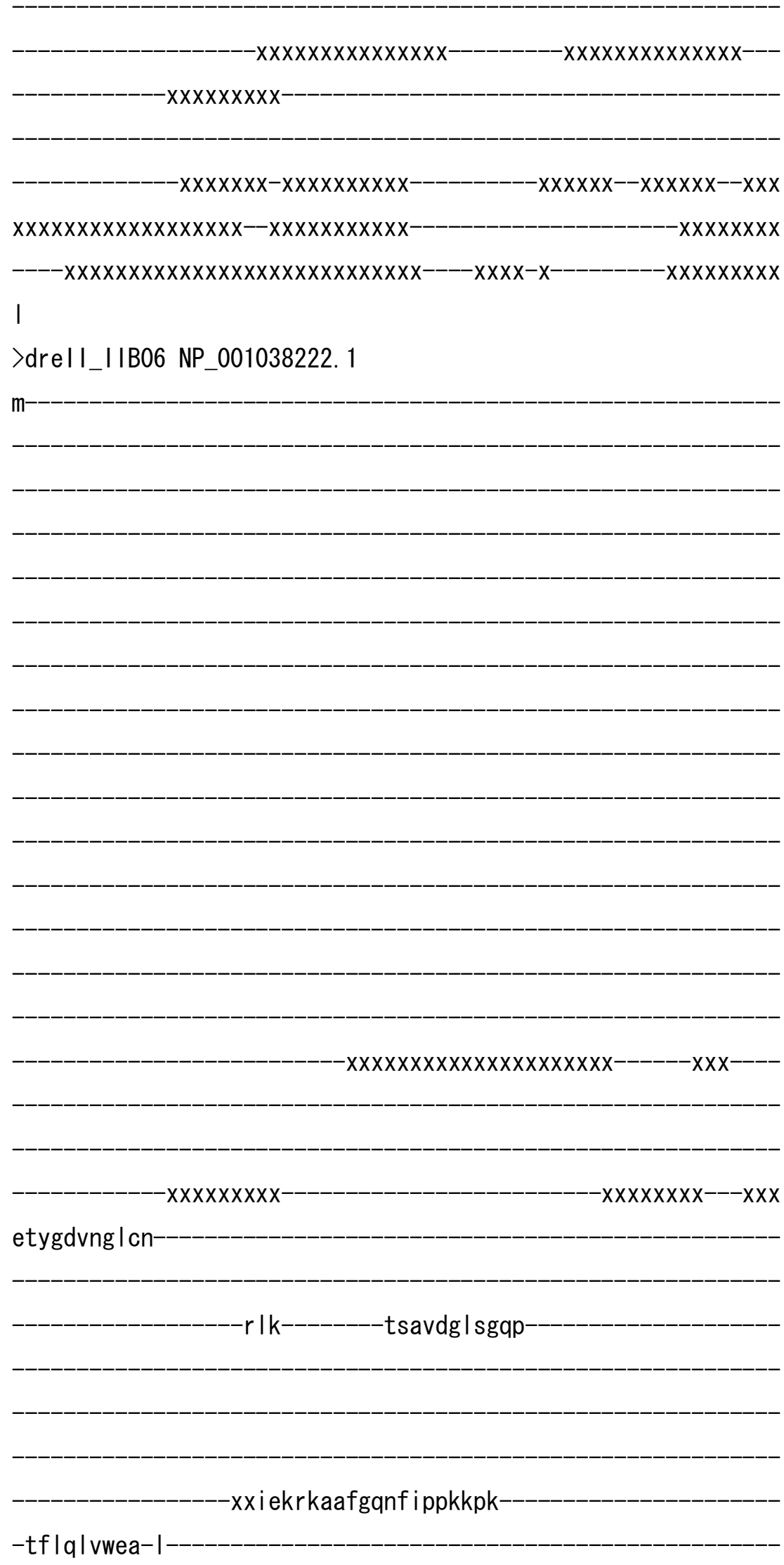




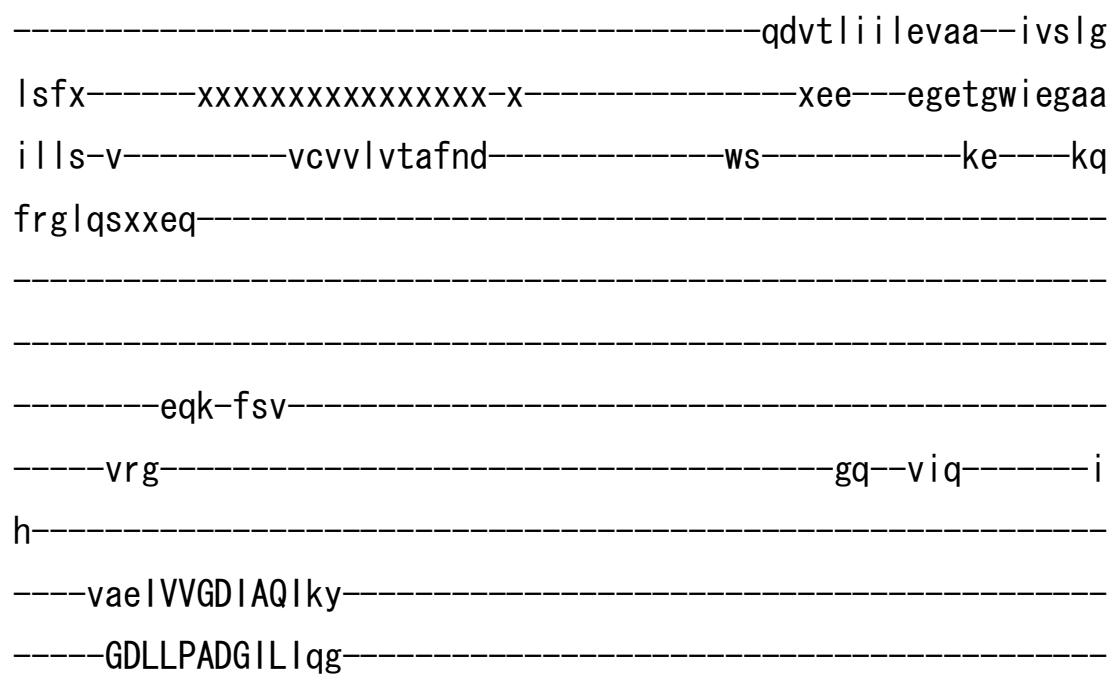

-n--dlkIDESSLTGESDHVKKs I-

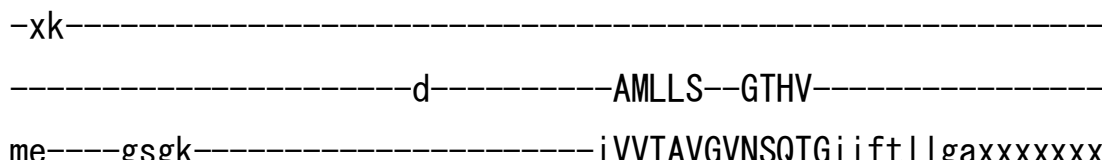

$x x x x x x x x x x x x x x x=--$

$x x-x x x x x x x x x x x x x x x x--x x x x x x x$-pkkeksv lqgk Itk I avq i gkag I Imsaitv

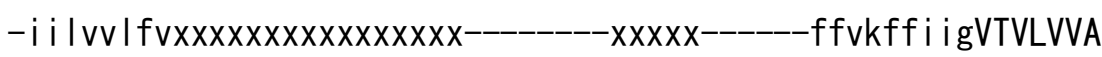
VPEGLPLAvTISLAYSVK-KMmkdn-

-nLVR-HLDACETMGNATAICSDKTG

$-T L T M N R M T V$ qvf i iadkhy

$-x-x x x x x d--x$

vpastmd

ivgi svnxxxxxkims

$-p x x \times g x \ln -$ 
-rqvgnkte--cal Igf-----xx-x-

-lkkdyqa--vrnexx-

-eek Iykvytfnsvrk-smst

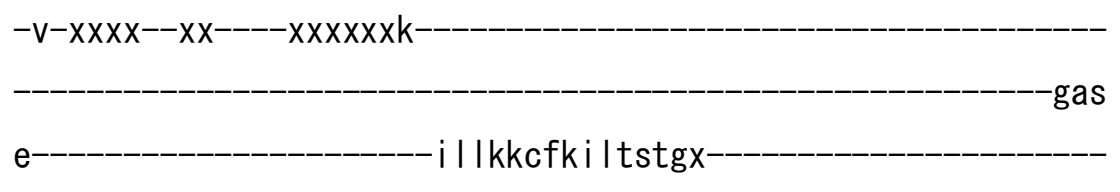

akvfrprdrddmxkrvi epm--ase----g I rt I CLAYRdf

-xxxegepdwdneax i Itr I tcVCVVG IEDPVRPEVPDAIRKCQRag-ITV

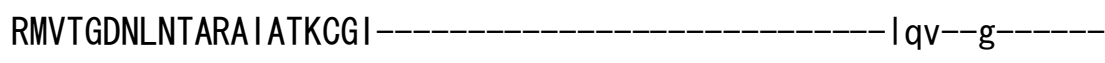

$-x x x---|c|$ egkefnx

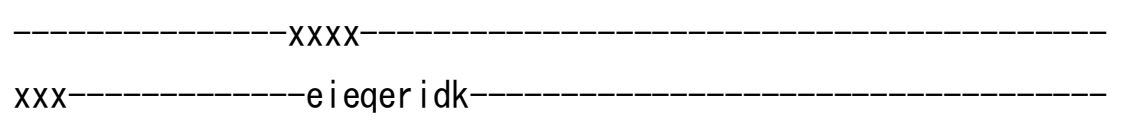

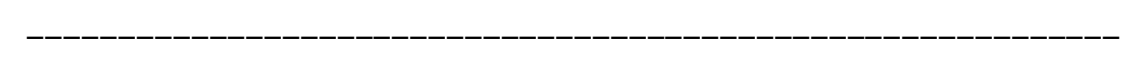

i wpk I--rVLA--RSSPTDKHTLVKGI i

---dsxxve--------xxx----------------VVAVTGDGTNDGPALKKADVGFAM--

-AKEASD I ILTd-DNFSS IVkaVMWGRNVYDSI SKFL------Q 
fql-tvn-v-vavi vaftgac i tq---_-_-_-_-_-_-_-_----d--sp I kavqm I wv nl imdt I as l a lateppt-es--_-_-_-_-_-_-_-_-- I I rkpy---g-rnkp I i sr tm m-kn i I ghavy--q I i i ft I I f--_-_-_-_-_-_-_-_-_-_-_-_-_-_-_-_-_-_-_ -agekmx-xx--xxxxxxxxxxx-----------------------------------ps----ehyt i vfntfvmmq I fn-e inarki $x x e r n v f---x---g i x n n m i$ fct i $f g t$

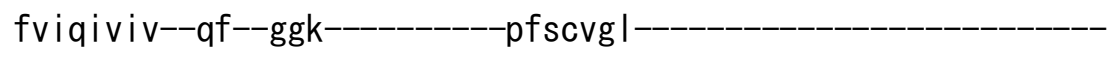

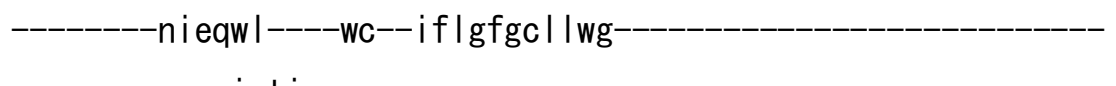

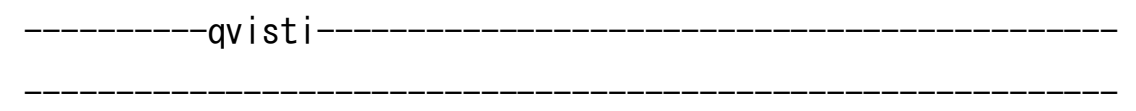

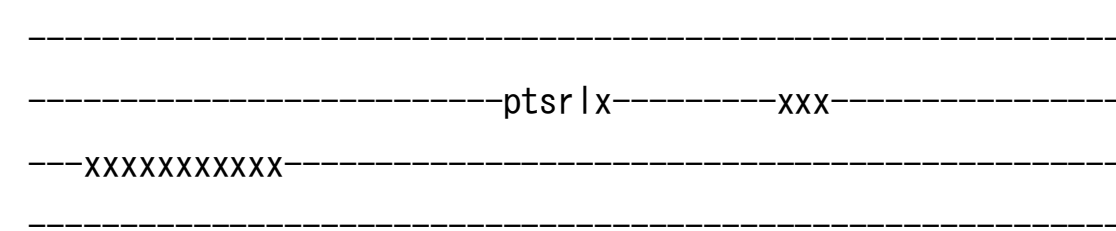

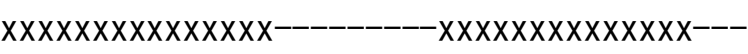
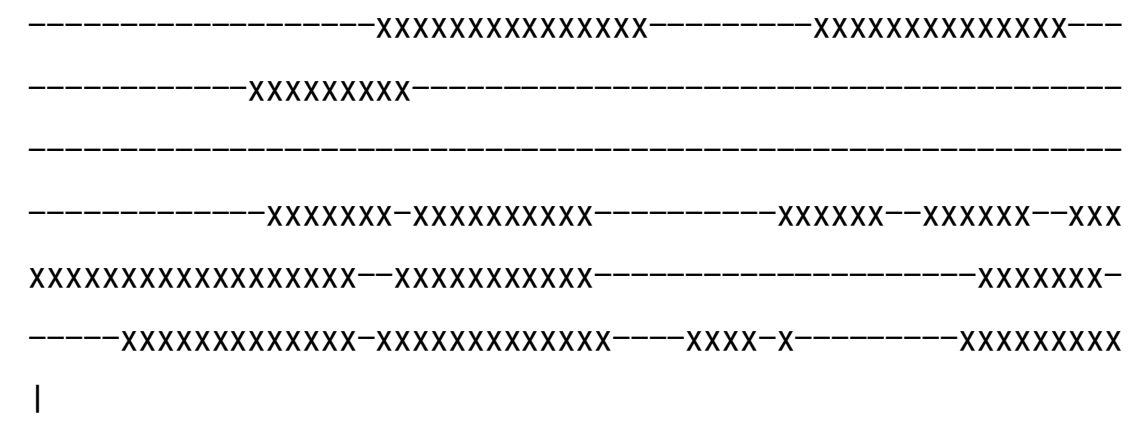

>athII_IIB01 NP_849716.1

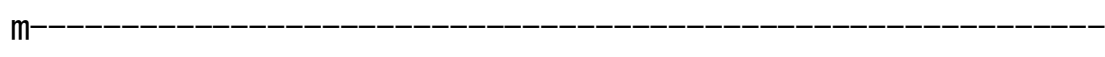

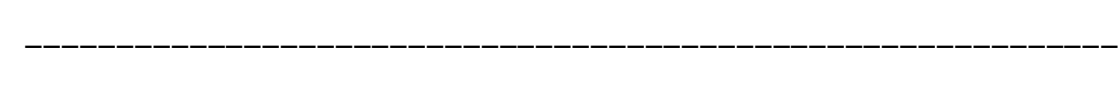




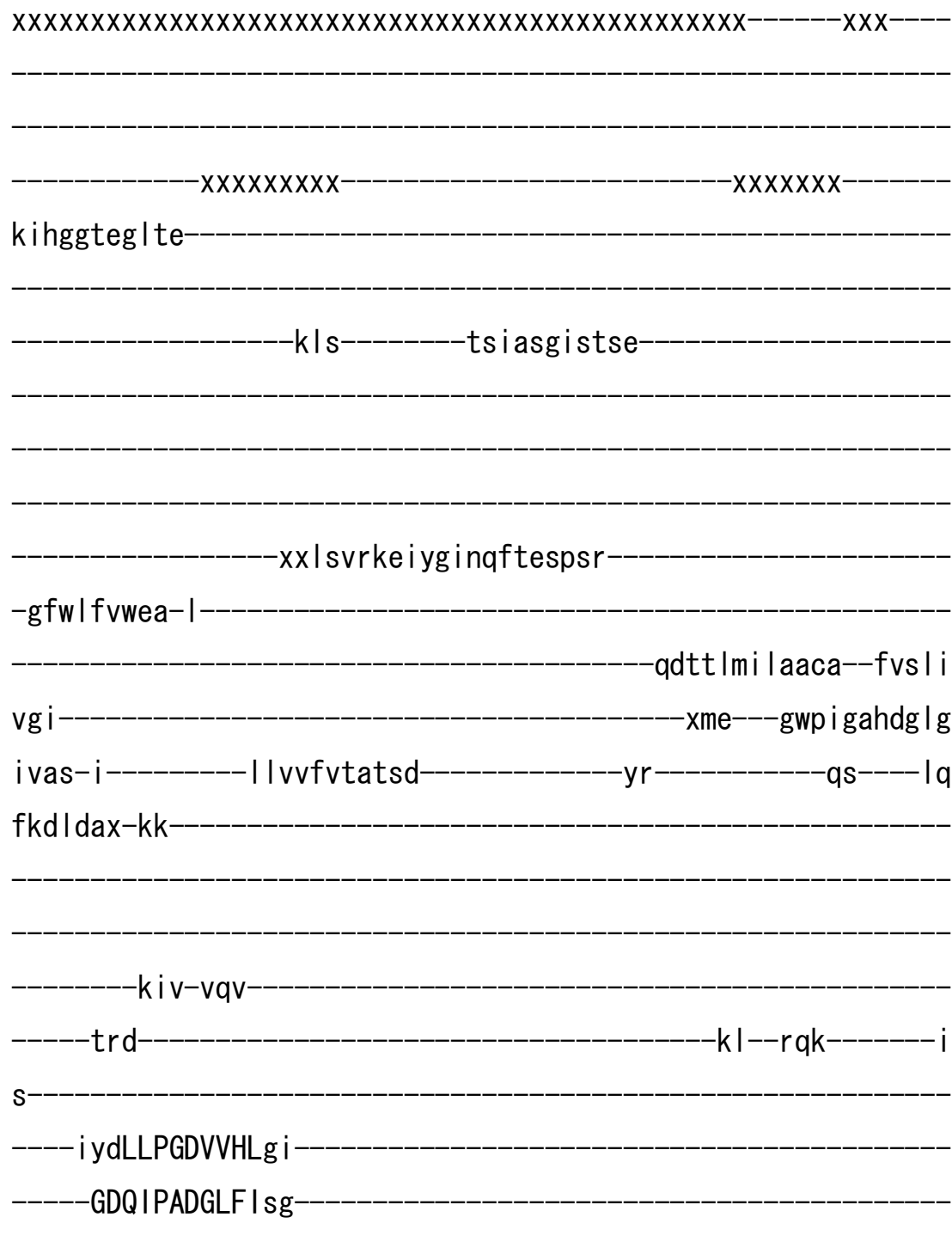

f--sv I INESSLTGESEPVSVsv-

$$
-x x-
$$

PFLLS--GTKV

qd-----gsck--_-_-_-_-_-_-_-_-_-_-_-_mLVTTVGMRTQWGk I mat I se-

-ggddetp I qvk Ingvat i igkig I ffavitf -av I vqg I axxx-xxxxxxxxxxxxx----_----xxx---_----aml eyfavaVTIVVVA VPEGLPLAvTLSLAFAMK-KMmndk 
aLVR-NLAACETMGSATT ICSDKTG-

TLTTNHMTV

-kac i ceqa

$x-x x x x x d x x x$

xxxxx ipesavk

Iqsiftn---xxgeiv

$-v x x \times n-k t-$

e i gtpte--tal lef----xx-x----

Iggdfqe--vrq

asnvvkvepfnstkk-rmgv

$-\mathrm{v}-\mathrm{x} x \mathrm{xx}--\mathrm{x} x----\mathrm{x} x \mathrm{x} x \mathrm{x} x \mathrm{k}$

-gas

$e^{-}$

i v ldscdky inkdgx

vvp I dekstshl-kn i i eef--ase----al rtLCLAYFe i

-gdefs l eap i-psggytc IG IVG IKDPVRPGVKESVA ICKSag-ITV

RMVTGDNLTTAKA I ARECG I

$-\mid t d--d-$

-x-----iai egpefr

-eksdeel lk- 
I ipk |--qVMA--RSSPMDKHTLVRLLr

$---\mathrm{tm}-\mathrm{xqe}-$

-VVAVTGDGTNDAPALHEADIGLAM--

- isgtev

AKESADVI I Ld-DNFST IVtvAKWGRSVYINI QKFV

$-0$

fql-tvn-v-val ivnflsacltg

-n--ap I tavq I Iwv

nmimdt I ga l a lateppq-dd

Imkrspv---g-rkgnf i snvm

w-rni l gqs ly--qlvi iwclat

$-k g k \operatorname{tm} x-x x-----x x x---x x$

dl-----tIntl ifnifvfcqvfn-e i ssremx-kidvf----x---gixknyvfvavltct vvfqvi i i--el--lgtfadttp I

-----_--n I gqw I-----vs-- i i I gf I gmpva

aal kmi

-pvgsh

>athII_IIB02 NP_195479. 1

$\mathrm{m}$ 


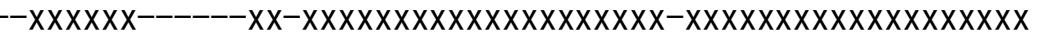

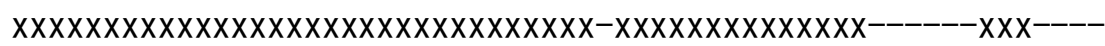

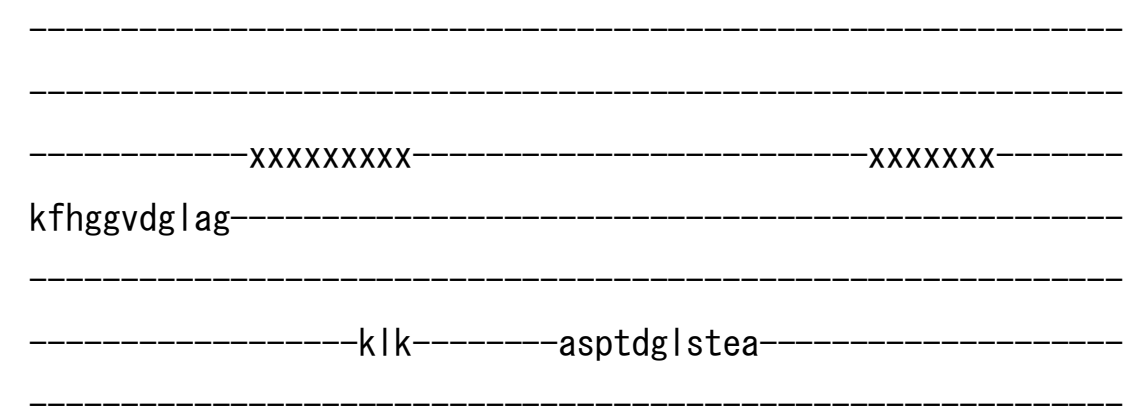

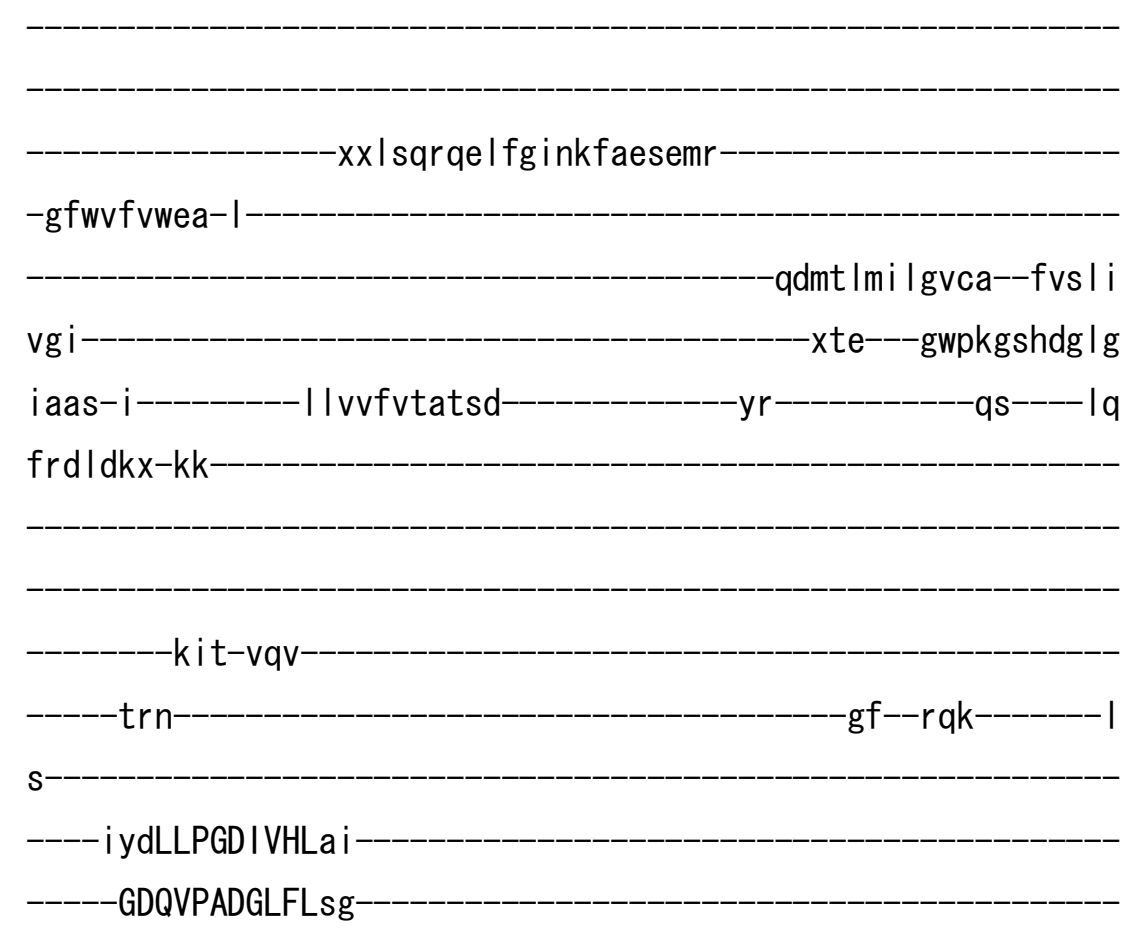


f--svvIDESSLTGESEPVMVna-

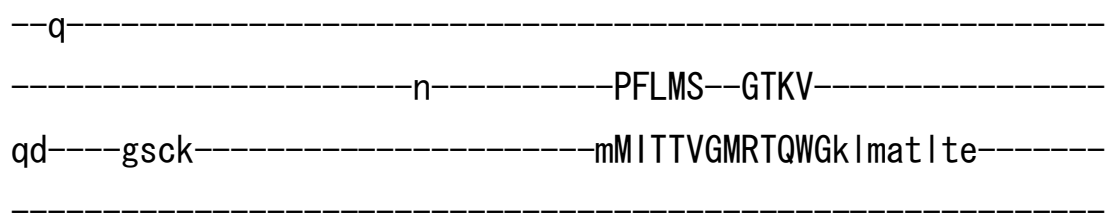

-ggddetp lqvk Ingvat i igkig I ffavvtf

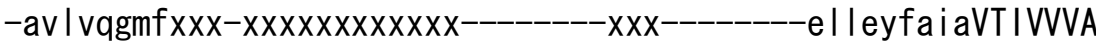
VPEGLPLAvTLSLAFAMK-KMmndk-

aLVR-HLAACETMGSATT I CSDKTG

TLTTNHMTV

$-k s c$ i cmnv

$-x-x x--x n x x x$

xxxxxipesavk

iqsifnn---xxgevv-

$-v x x x g-k t$

el Igtpte--tailel-----xx-x----

- lggkfqe--erk

-sykvikvepfnstkk-rmgv

$-v-x x x x x-x x----x x x x x \times k$

$\mathrm{e}$

iv laacdkvvnssgx 

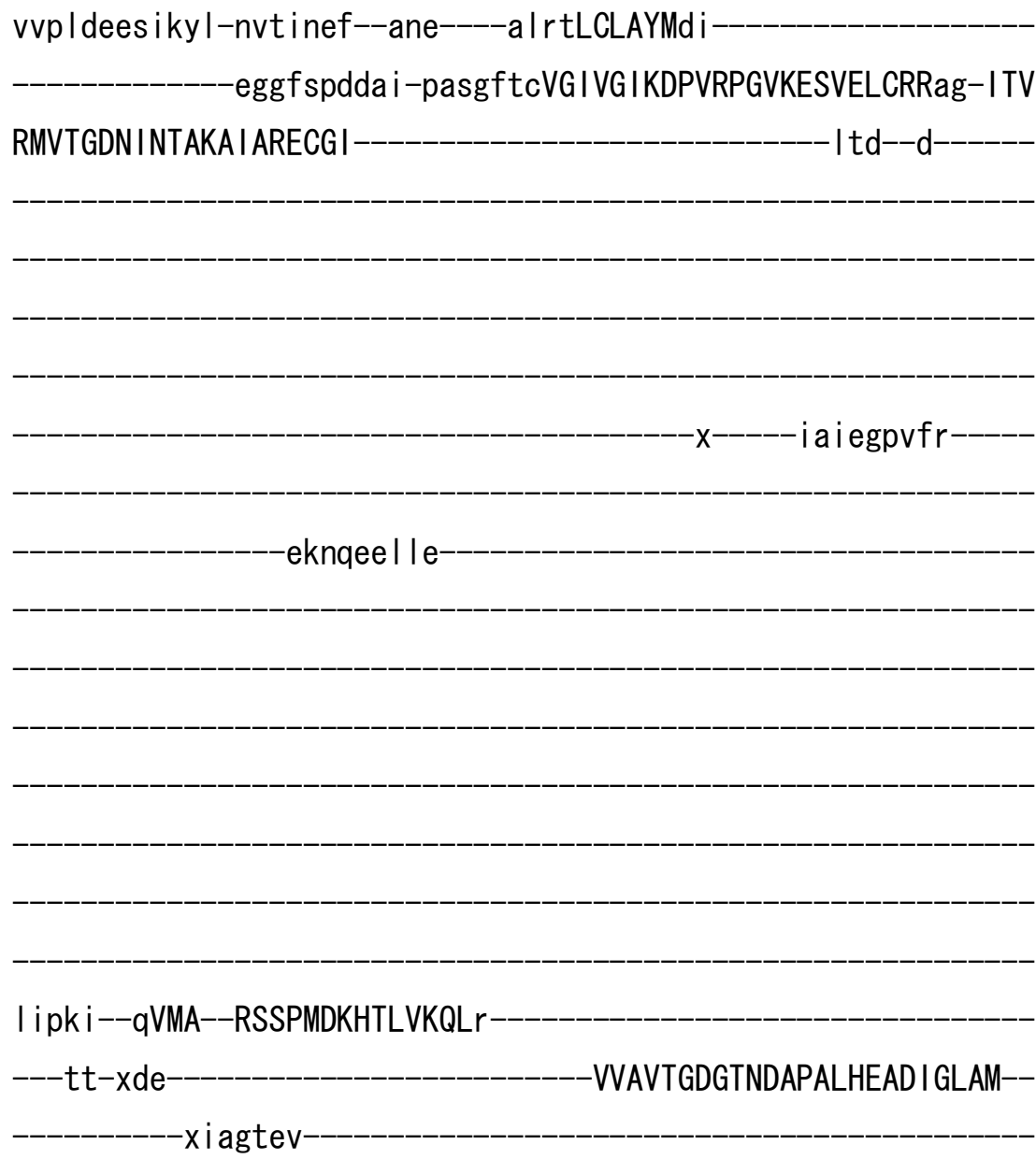

-AKESADV I ILd-DNFST IVtvAKWGRSVYINI QKFV------Q

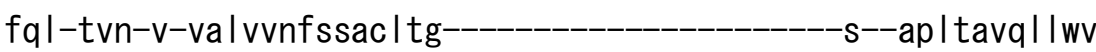
nmimdt I ga la lateppn-de---_-_-_-_-_-_-_-- Imkr I pv---g-rrgnf i tnam w-rni l gqavy--qfi viwi lqa- kgkamx-xx------xxx----xx

tI----mIntl i fncfvfcqvfn-e i ssremx-e idvf----x---gi ixdnyvfvvvigat vffq i i i i--ef--lgt-----------fasttp I-

-ti tqwi----fs--ifigf I gmp i a aglkti 
>athII_IIB03 NP_181687.1

m-

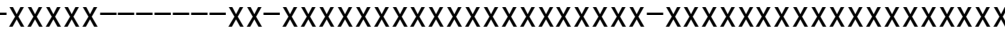

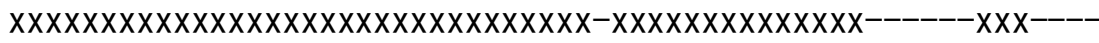

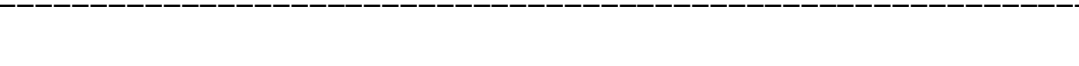

$-x X X X X X X X X$

$\mathrm{XXXXXXX}$

aqkggveelak

kvs

-vs Isegirsse

vpirekifgenrytekpar

-sf Imfvwea-I 


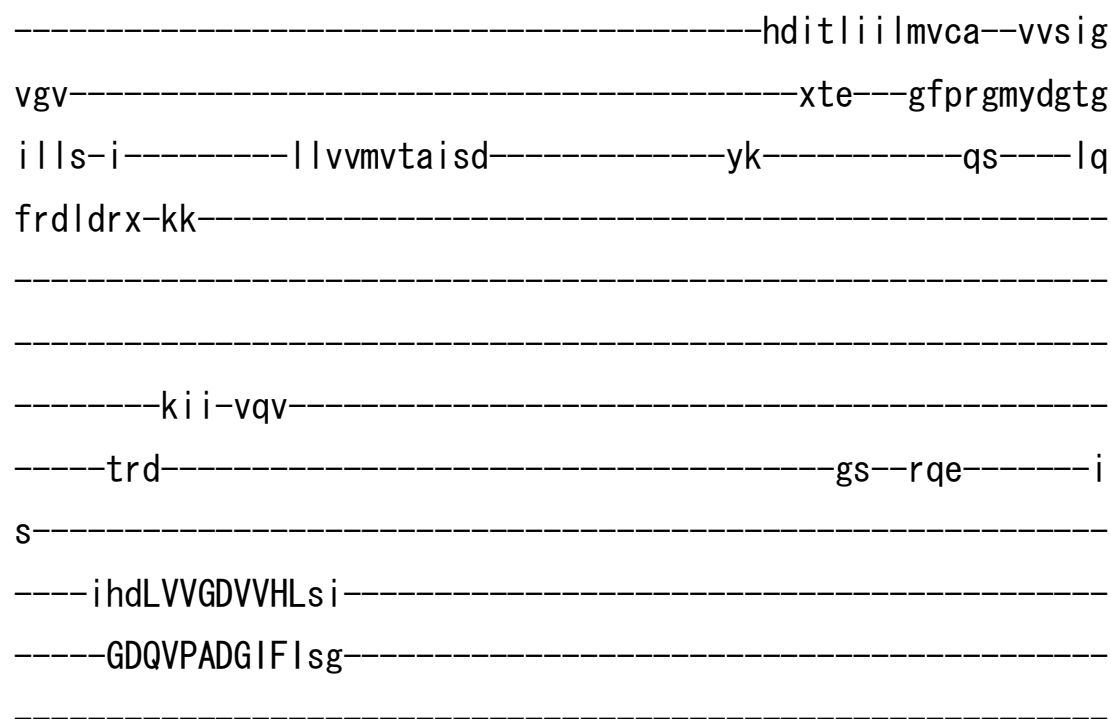

-y--nl e IDESSLSGESEPSHVnk-

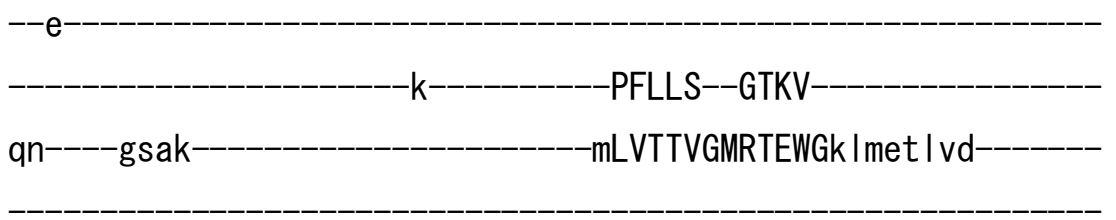

-ggedetp lavk Ingvati igkig Isfavltf -vvlcirfvxxx-xxxxxxxxxxxx-------xxx-------t/IdyfaisVTIIVVA VPEGLPLAvTLSLAFAMK-KLmsdr

aLVR-HLAACETMGSSTCICTDKTG

TLTTNHMVVn$-k v w i c d k v$

$x x x x x g x x x$

$x x x x \mid$ seevqs

lag i fqn---xxsevv

$-k x x x g-n t$ 
qi lgspte--railef-----xx-x

lggdfnt--ark

ehki I k i epfnsdkk-kmsv

$-1-x x x x--x x----x x x x x x k$

-gas

e-------------------------i i l kmcenvvdsngx------------------------

svp Iteer itsi-sdi i egf--ase----al rtLCLVYKdI

deapsgexpdggytmVAVVGIKDPVRPGVREAVQTCQAag-ITV

RMVTGDNISTAKAIAKECG I-

-yte--g------

------lai egsefr-

dl sphemra-

i i pk i--qVMA--RSLPLDKHTLVSNLr

$---k i--g e-$

-VVAVTGDGTNDAPALHEAD IGLAM--

-xiagtev

-AKENADV I I Md-DNFKT I VnvARWGRAVY I I I QKFV- 


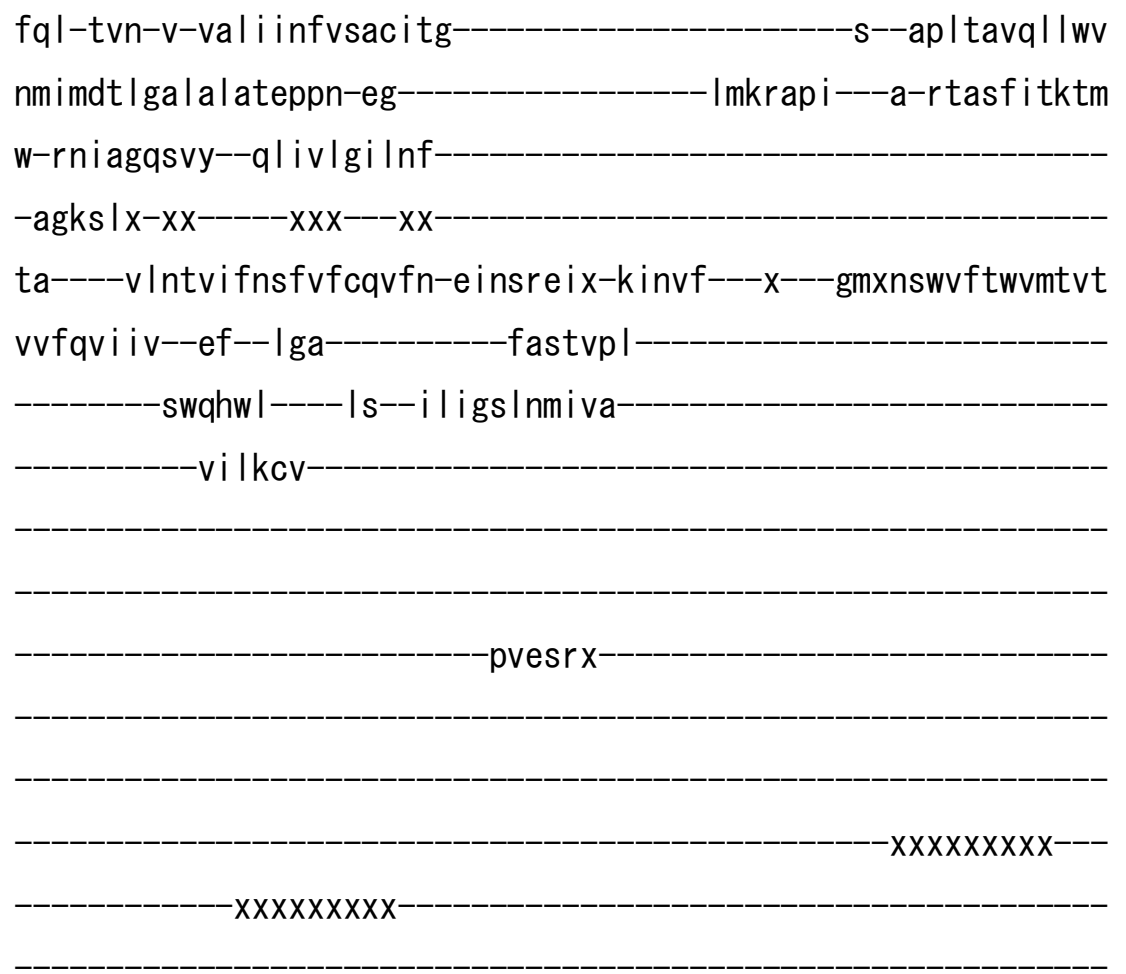




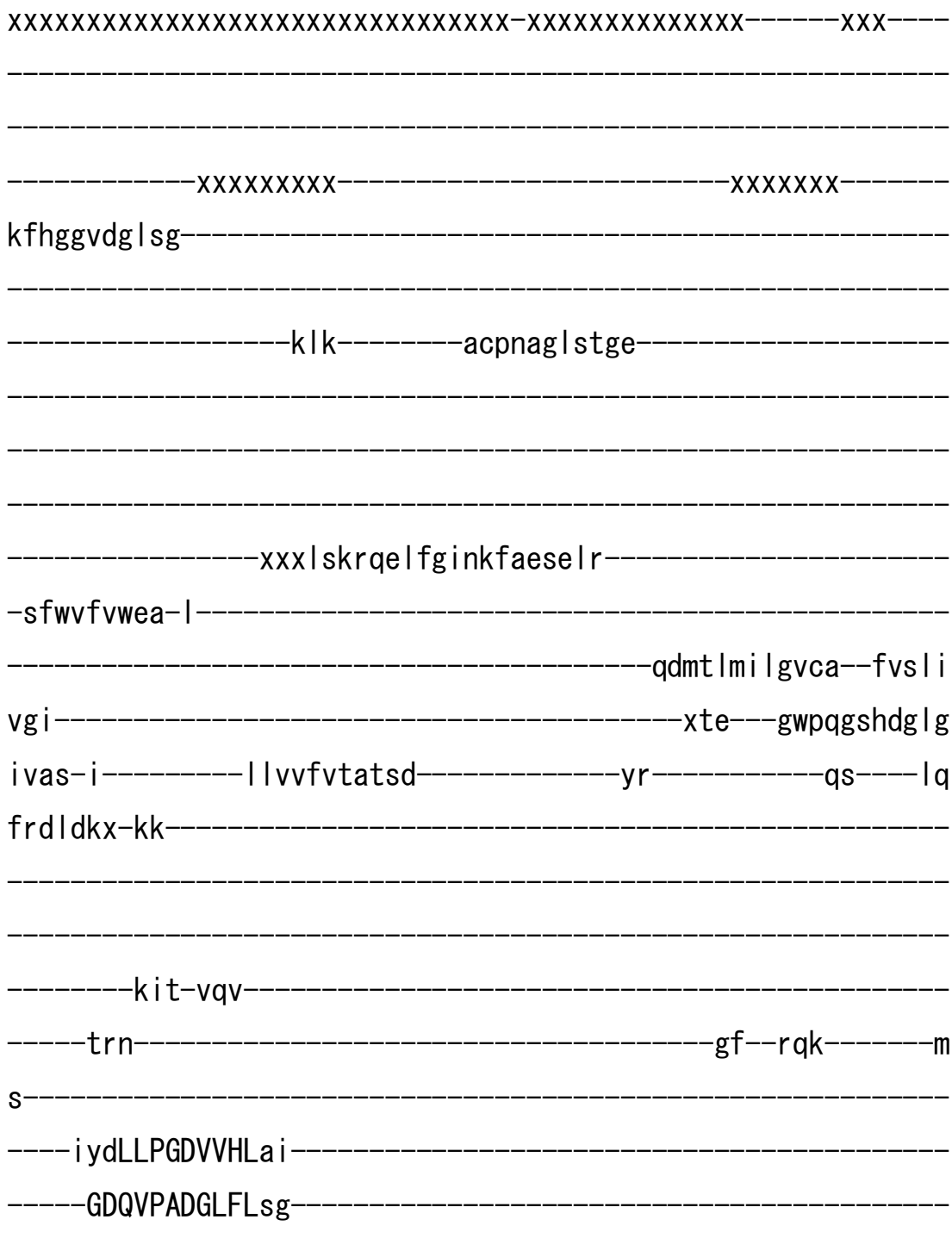

f--svv IDESSLTGESEPVMVta-

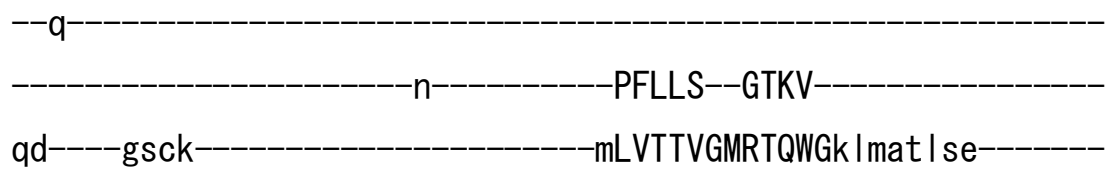

-ggddetp I qvk Ingvat i igkig I sfaivtf -av I vqgmf $x x x-x x x x x x x x x x x x---------x x x--------e l$ l eyfa iaVTIVVVA VPEGLPLAvTLSLAFAMK-KMmndk- 
aLVR-HLAACETMGSATT I CSDKTG

TLTTNHMTVV

$-k s c$ i cmnv

$-x-x x--x s x x x$

xxxxxipeaalk

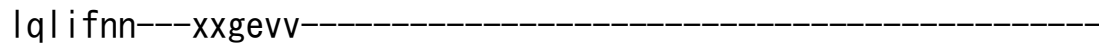

$-v x x x g-k t-$

e i gtpte--tai le l-----xx-x----

Iggkfqe--erq

-snkvikvepfnstkk-rmgv

$-\mathrm{v}-\mathrm{x} x \mathrm{xxx}-\mathrm{xx}----\mathrm{x} x \mathrm{xx} x \mathrm{xk}-$

-gas

e-

- i v l aacdkv inssgx-

vvp Iddes ikfl-nvtidef--ane----al rtLCLAYMdi

-esgfsadeg i-pekgftc IGIVGIKDPVRPGVRESVELCRRag-I MV

RMVTGDN I NTAKA I ARECG I---_-_-_-_-_-_-_-_-_-_-_-_-_-- Itd-- d-

-x-----iai egpvfr

eknqeemle- 
I ipk i--qVMA--RSSPMDKHTLVKQLr

$---\mathrm{tt}-\mathrm{xde}-$

-VVAVTGDGTNDAPALHEADIGLAM--

xiagtev

-AKE I ADV I I Ld-DNFST IVtvAKWGRSVY I I QKFV

$-Q$

fql-tvn-v-val i vnfssacltg -s--ap I tavq I Iwv

nmimdt I ga la lateppn-neImkrmpv---g-rrgnf i tnam w-rni I gqavy--qf i i iwi lqa

- kgksmx-xx----- xxx--- xx

tl----v|ntl i fncfvfcqvfn-evssremx-eidvf----x---gixdnyvfvvvigat vffqi i i i--ef--lgt fasttp I

-t i vqwf----fs--i fvgf I gmp i a

aglkki

pv

>athII_IIB05 NP_200521. 3

$\mathrm{m}$ 
$x X X X X X X X X X X X X X X X X X X X X X X$

$-x X X X X X$

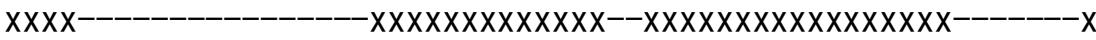

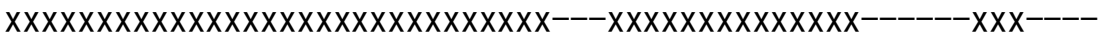

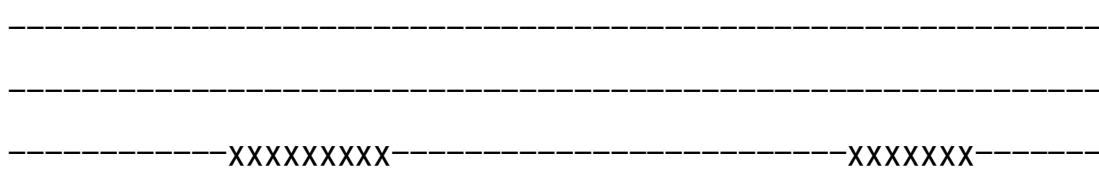

eqyggtag I an

| l k--_-_-_--tnpekg i sgdd-

xxI lkrktiygsntyprkkgk

$-g f|r f| w d a-c$

-hdlt l i Imvaa--vas la

I g i------------------------------------------xte---g i kegwydggs

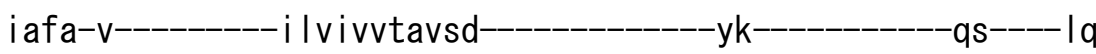

fqn Indx-kr

$-n i h-l e v$

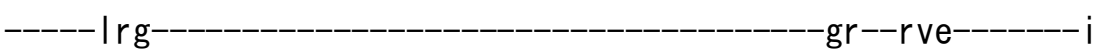

s

----i ydIVVGDVIPLni

GNQVPADGVLI sg 
-h--s I aLDESSMTGESK IVNKda-

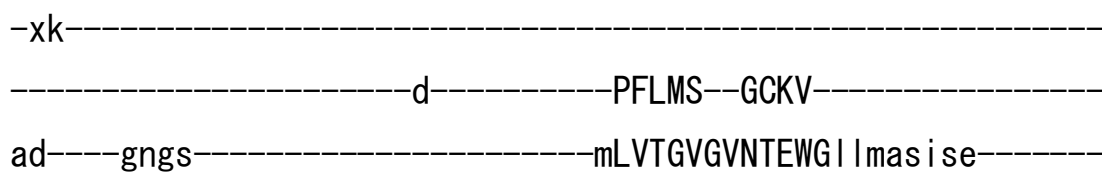

-dngeetp I qvr Ingvatf igs ig I avaaav I

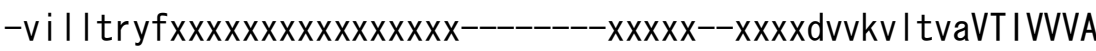
VPEGLPLAvTLTLAYSMR-KMmadk-

-aLVR-RLSACETMGSATT ICSDKTG

TLTLNQMTVV

-esyaggkk

xxxxxlpatits

eysgspte--ka i lgw-----xx-x----

Igmnfet--ars

qss i lhafpfnsekk-rggv

$-a-x x x--x x x----x x x x x x k$

$\mathrm{e}$

-ivlascrsyidedgx 
vapmtddkasff-kng indm--agr----t I rcVALAFRty -xxxxxptgee I skwvxpedd I i ILA IVG IKDPCRPGVKDSVVLCQNag-VKV RMVTGDNVQTARA I ALECG I Iss-- d-

xxxxxxt l i egksfr emtdaerdk-

i sdk i--sVMG--RSSPNDKLLLVQSLr

$---r q--g h-$ -VVAVTGDGTNDAPALHEADIGLAM--xiagtev

AKESSD I I ILd-DNFASVVkvVRWGRSVYAN I QKF I Q

fql-tvn-v-aalvinvvaaissgd--vp I tavq I Iwv

nl imdt I ga la lateppt-dhImgrppv---g-rkeplitnim w-rnll iqaiy--qvsvllt Inf

$-r g$ i si $x-x x-----x x x x x x x$

tr----vknt i i fnafv lcqafn-efnarkpx-ekn if---x---gvxknr I fmg i i vit Ivlqvi iv--ef-- Igk-----------fasttk I$-n w k q w \mid----i c--v g$ i gvi swp la Ivgkfi 


$$
\text { I }
$$

>athII_IIB06 NP_188755. 2

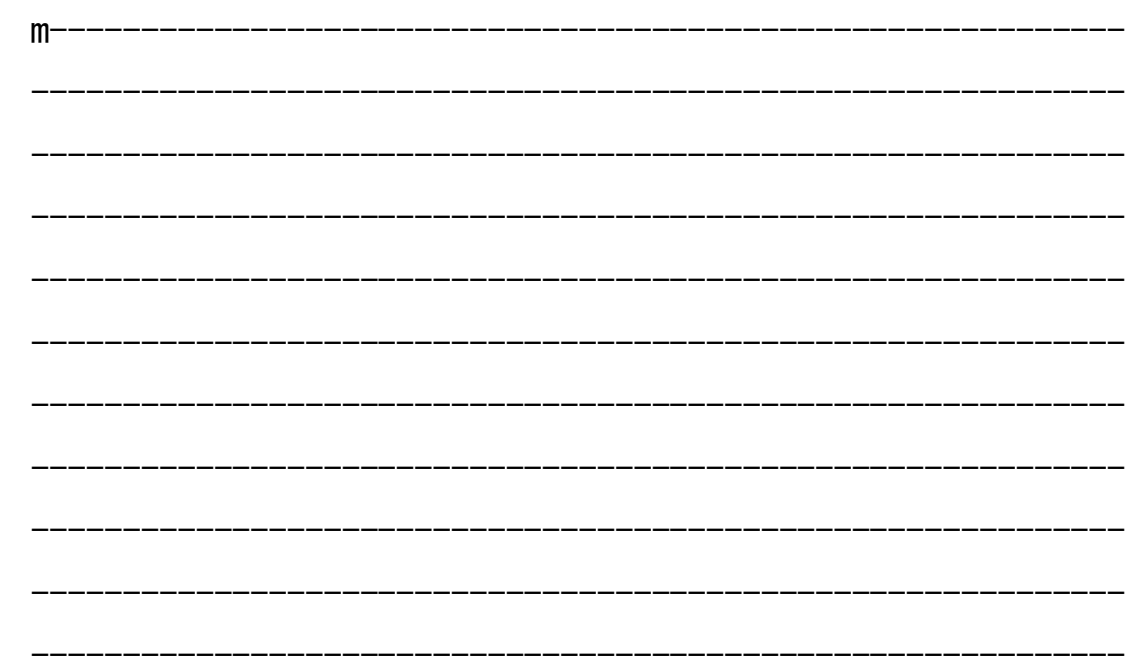

$-\mathrm{x} x \mathrm{xx} x \mathrm{XXX}$

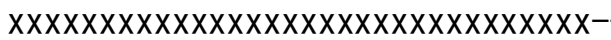

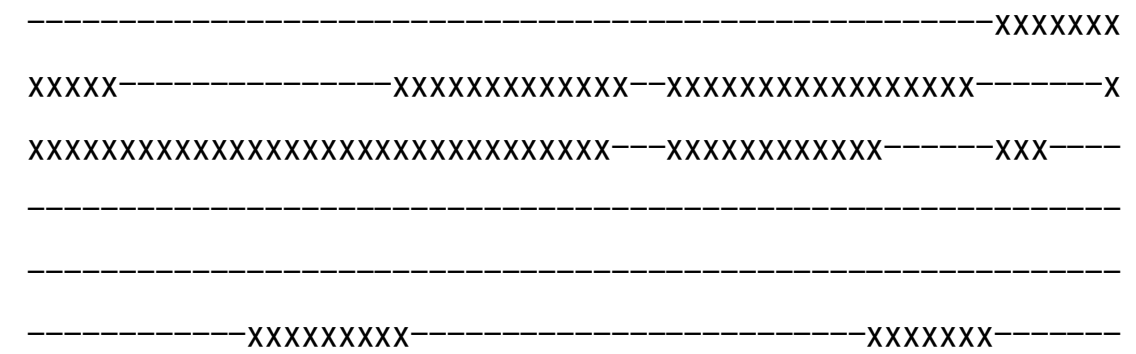

qqyggvkgvae

$k \mid k$

-snmeqg inede

-xxvidrknafgsntypkkkgk

-nffmf I wea-w 


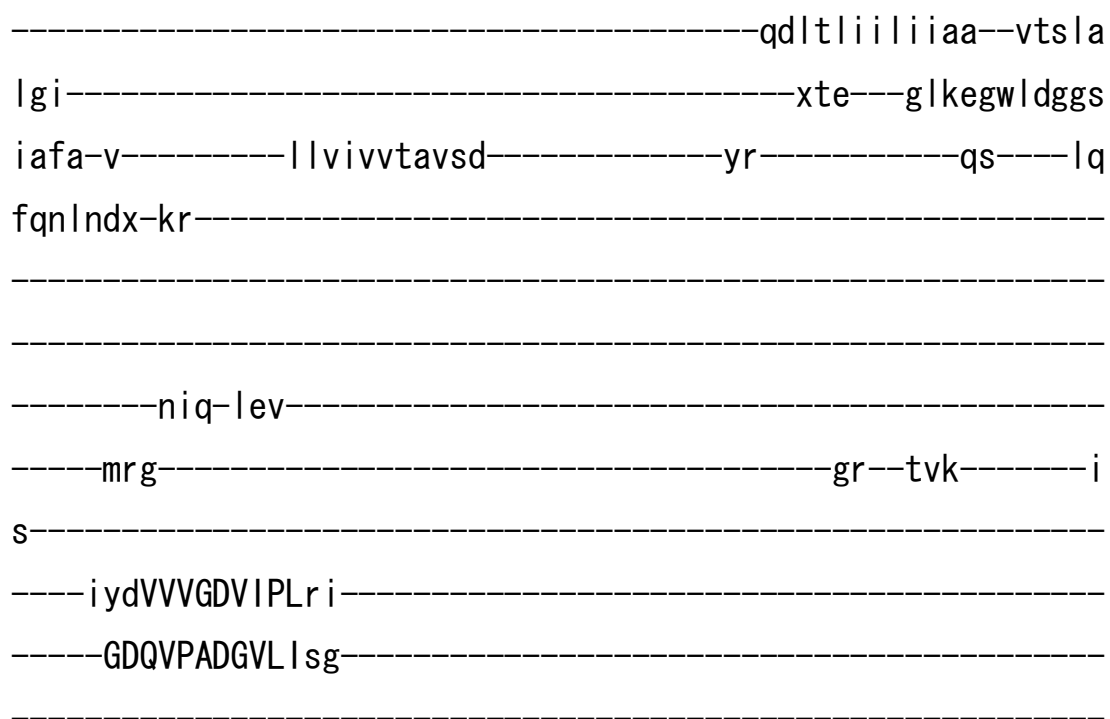

-h--s I a IDESSMTGESK IVHKdq-

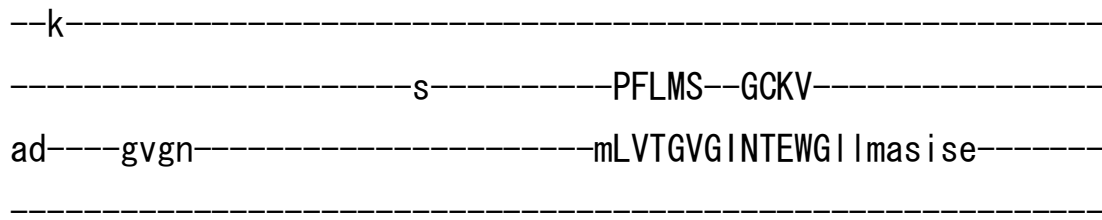

-dtgeetp |qvr Ing |atfigivg I svalvv I

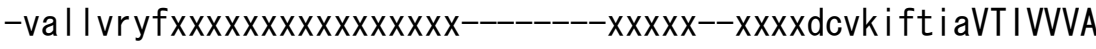
VPEGLPLAvTLTLAYSMR-KMmadk-

aLVR-RLSACETMGSATTICSDKTG

$-T L T L N Q M T V$ etyaggskm-

$-d-x x$

xxxxxIhpk Iva

segvaqn---xxgnif 
l gmkfdt--irs

esai ihafpfnsekk-rggv

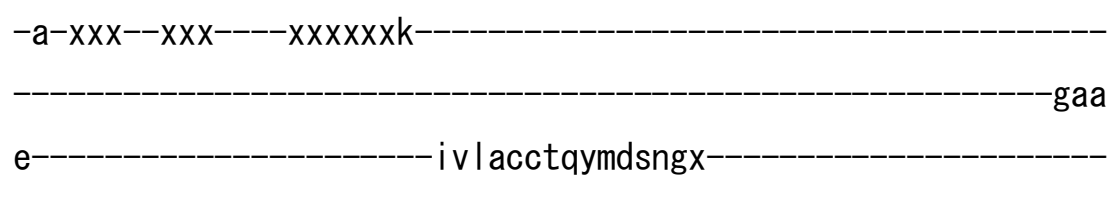

I qs i esqk-eff-rvai dsm--akn----s I rcVAI ACRtq -xxxxxxkeqed I dkwaxpede I i ILA IVG IKDPCRPGVREAVR I CTSag-VKV RMVTGDNLQTAKAIALECGIIss-- d-

-xxxxxxt i i egkvfr

el sekereq

vakk i--tVMG--RSSPNDKLLLVQAL

$---\mathrm{kn}--\mathrm{gd}-$

-VVAVTGDGTNDAPALHEADIGLSM--

-xisgtev 


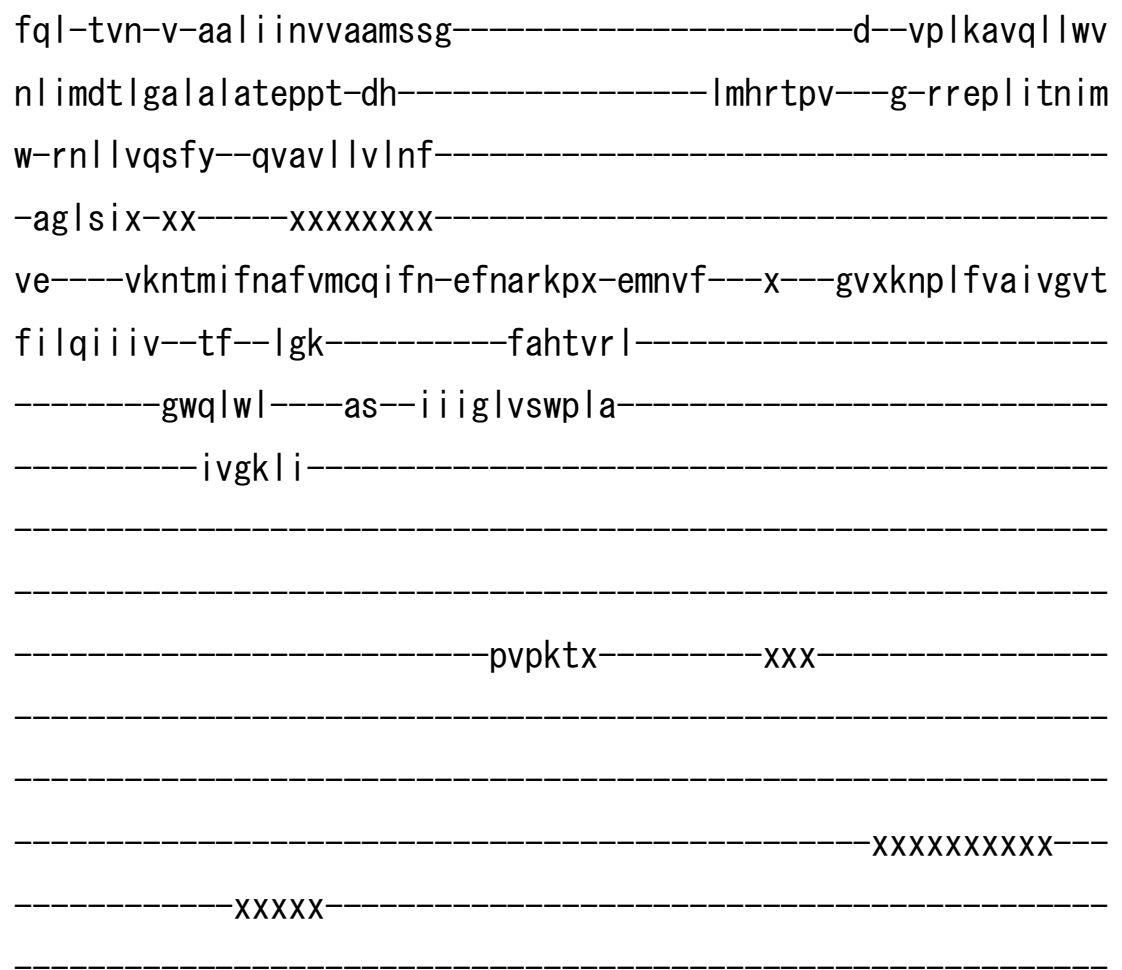

nl imdt I ga la lateppt-dh------------------ Imhrtpv---g-rrep I i tn im w-rnl Ivqsfy--qvavIIvInf-

-aglsix-xx-----xxxxxxxx-

ve----vkntmi fnafvmcqi fn-efnarkpx-emnvf---x---gvxknp I fva i vgvt 


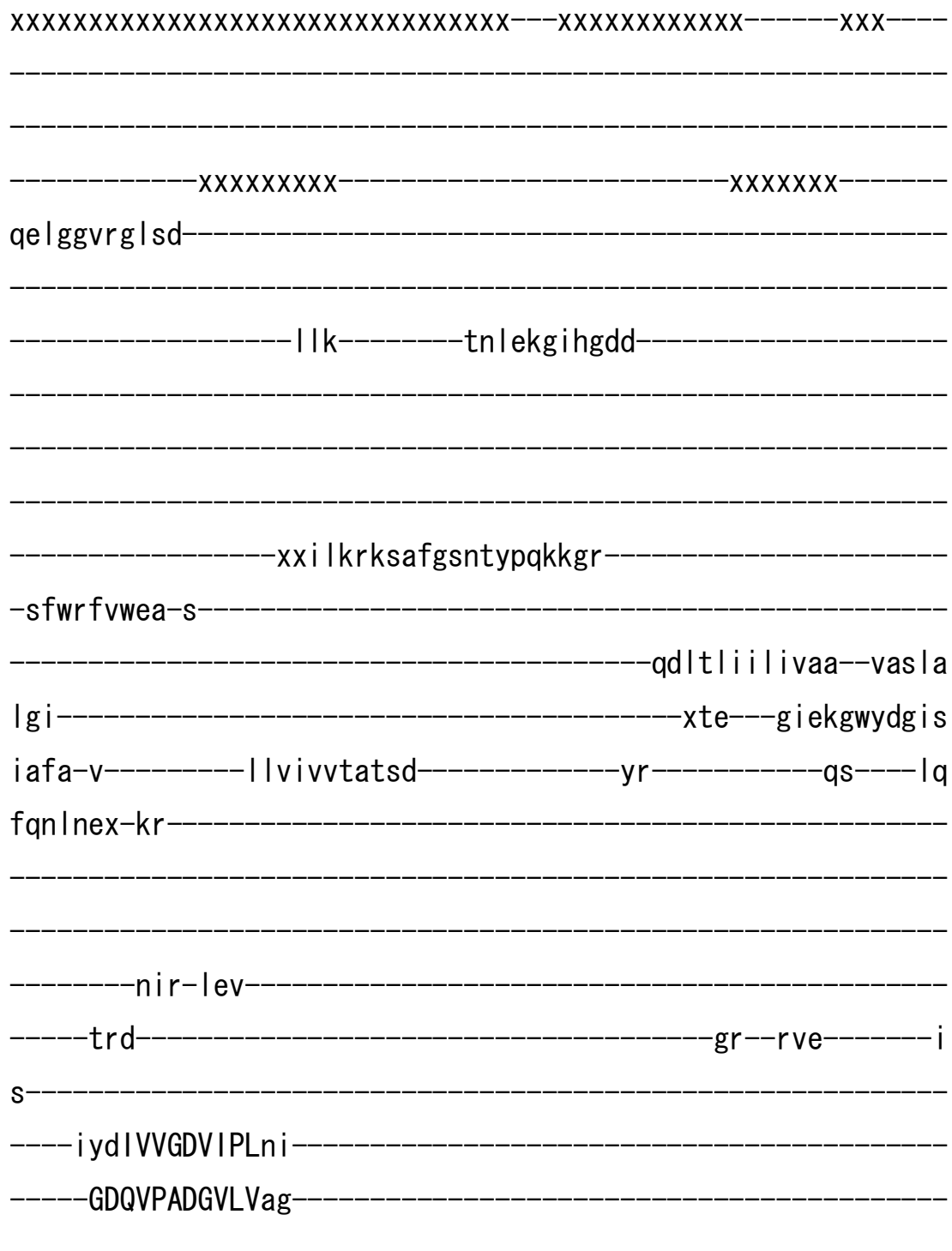

-h--s I aVDESSMTGESK IVQKns-

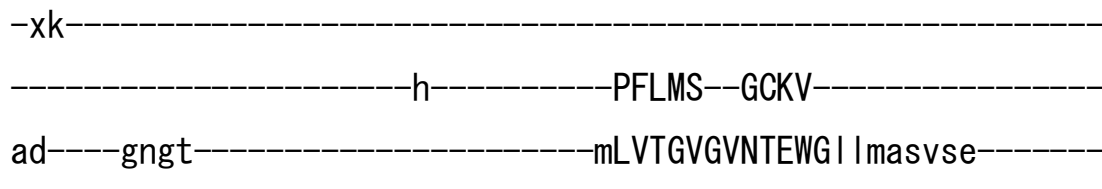

-dnggetp I qvr Ingvatf i givg | tvagvv |

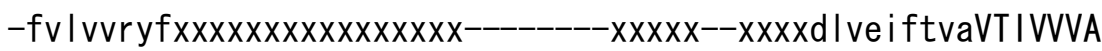
VPEGLPLAvTLTLAYSMR-KMmadk- 
-aLVR-RLSACETMGSATTI CSDKTG-

TLTLNEMTV

ecyag | qkm

$d-x x$

xxxxx|psafts

$-\mathrm{i}$

veg i ahn---xxgsvf

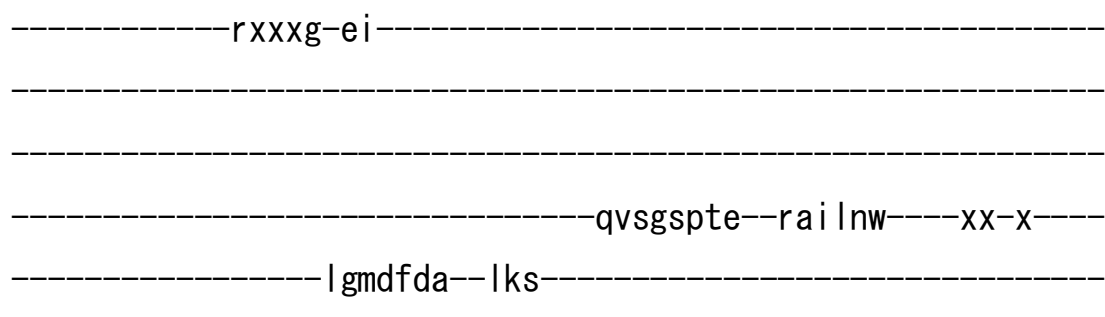

essavqffpfnsekk-rggv

$-a-x x x--x x x----x x x x x x k$

gaa

$e^{-}$

i v I gscthymdesex

fvdmsedkmgg |-kda i ddm---aar-----s | rcVAIAFRtf

xxxxxxtdeeq I srwexpedd I i ILAIVG IKDPCRPGVKNSVLLCQQag-VKV

RMVTGDNIQTAKA IALECGI

las--d -

$-x x x x x x n l$ iegkvfr 


$$
\text { i cee i--sVMG--RSSPNDKLLLVQSLk- }
$$$$
---r r--g h-
$$

-VVAVTGDGTNDAPALHEADIGLAM--

-xiqgtev

-AKEKSD I I ILd-DNFESVVkvVRWGRSVYAN I QKF I

$$
-0
$$

fql-tvn-v-aal v invvaa i sag---_--_--_-_----------e--vp I tavq I I wv

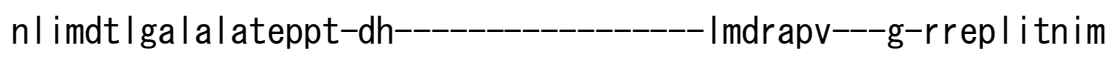
w-rnIfiqamy--qvtvI I I Inf

-rgisix-------xxxxxxxx

er----vkntvifnafvicqvfn-efnarkpx-e inif----x---gvxrnhlfvgi is it ivlqviviv--ef--lgt-----------fasttk |-

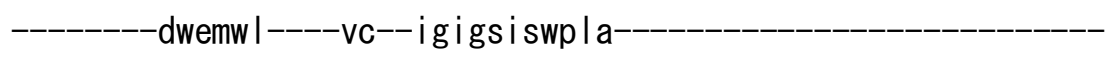
vigkli

>athII_IIB08 NP_191292.1

$\mathrm{m}-$ 


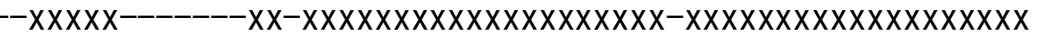

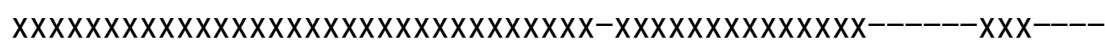

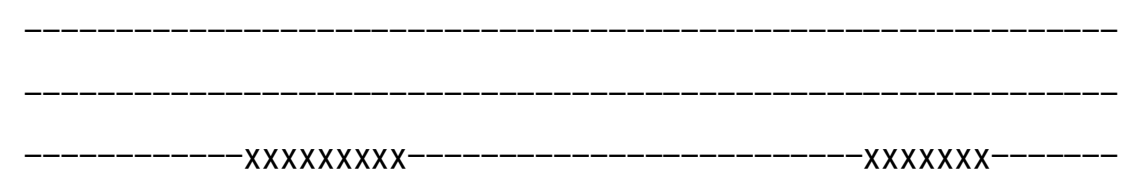

tkiggpeg iaq

kvs-vs l aegvrsse-

Ihireki ygenrytekpar

-sfltfvwea-I-

-qditl i i Imvca--vvsig

vgv -xte---gfpkgmydgtg

i I Is-i-_-_-_-_-_ i l vvmvta i sd-_-_-_-_-_-_--yk-_-_-_-_-_--qs--_- lq frdldrx-kk-

$$
\text { --------k i i-i qv------------------------------------------------- }
$$

s

----ihdLVVGDVVHLs i

GDQVPADG IF I sg 
-y--nl eIDESSLSGESEPSHVnk-

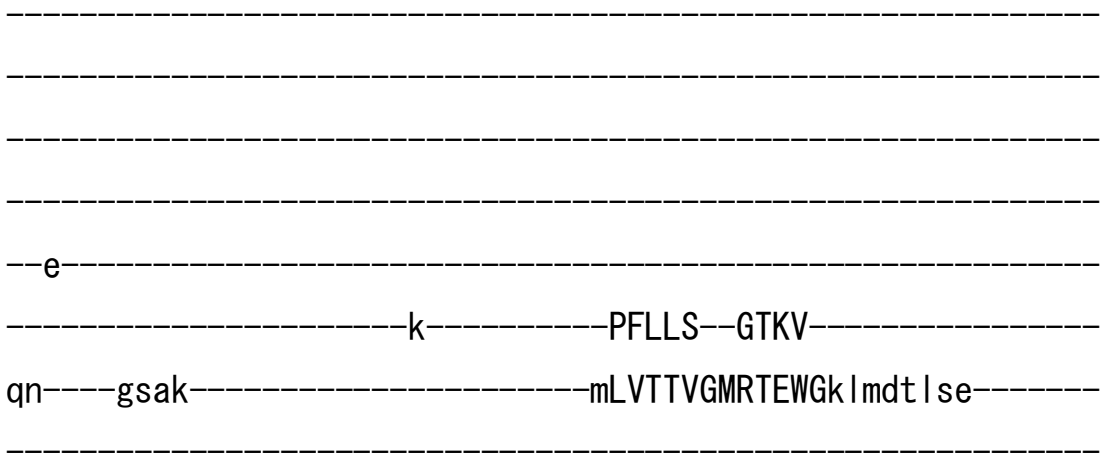

-ggedetp Iqvk Ingvati igkig I gfavItf -vvlcirfvxxx-xxxxxxxxxxxxx--_-_----xxx--------tIIdyfa iaVTIIVVA VPEGLPLAvTLSLAFAMK-QLmsdr-

aLVR-HLAACETMGSSTCICTDKTG

TLTTNHMVVn -kvwiceni

$-x x x x---x$

$x x x x \mid$ seqvkn

iqai fqn---xxsevv-

$-k x x x g-k t$

qi lgspte--rai lef-----xx-x---- ggdvdt--arr

-ehk i lk i epfnsdkk-kmsv

$-1-x x x x--x x----x x x x x x k$ $\mathrm{e}$

iv lkmcekvvdsngx 
svp I seek i as i-sdv i egf--ase----al rtLCLVYTdI deaprgdxpnggyt IVAVVGIKDPVRPGVREAVQTCQAag-I TV RMVTGDN I STAKAIAKECG I Ita--g------

------vai egsdfr nlpphemra-

i l pk i--qVMA--RSLPLDKHTLVNNLr

$---\mathrm{km}--\mathrm{ge}-$ -VVAVTGDGTNDAPALHEADIGLAM-xiagtev

-AKENADV I IMd-DNFAT IVnvAKWGRAVYIN I QKFV $-Q$

fql-tvn-v-val i infvsac i tg--_-_-_-_-_-_-_-_-_-_--s--ap I tavq I I wv nmimdt I ga la lateppn-eg--_-_-_-_-_-_-_---Imkrqp i---g-rtasf i tram w-rni igqsiy--ql iv lgi Inf -agkqi $x-x x-----x x x---x x$ ti----vInti i fnsfvfcqvfn-evnsre ix-kinvf---x---gmxkswvfvavmtat

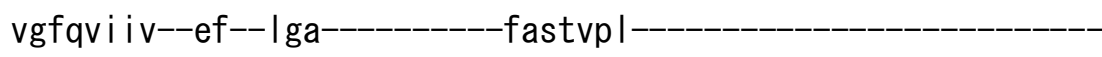
--------swghw |----|lc--i I igsvsmi la$-v g \mid k c i$ 
>athII_IIB09 NP_191897.1

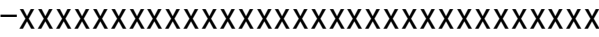

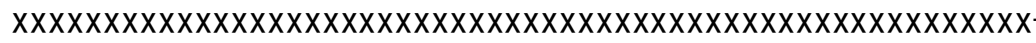
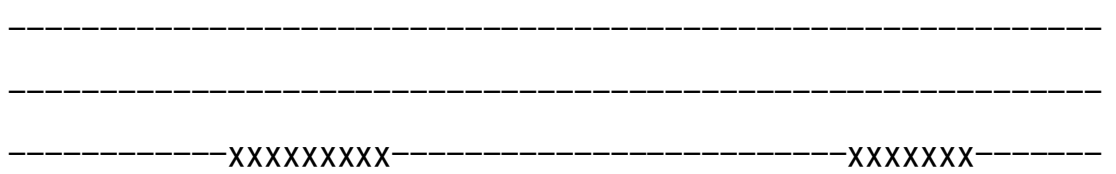

qa l ggvegvaa

$-s \mid r$ -tnptkgihgne-

-xxvsrrrdlfgsntyhkpppk

$-g$ I ffvyea-f 


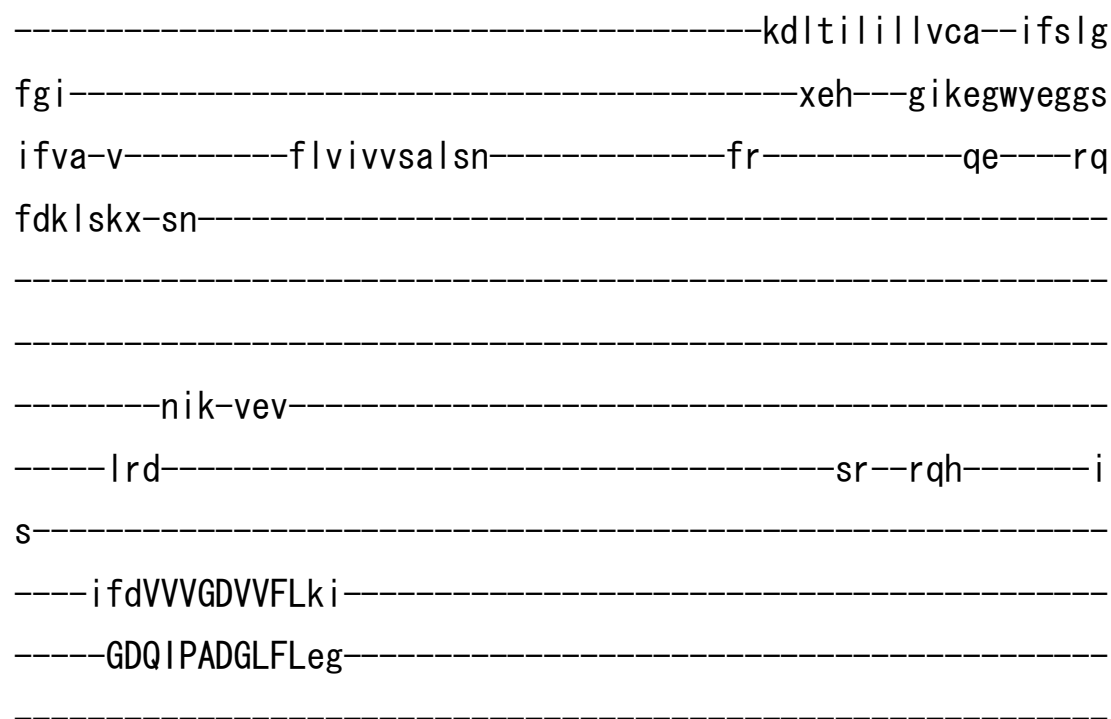

-h--s I qVDESSMTGESDHLEVdh-

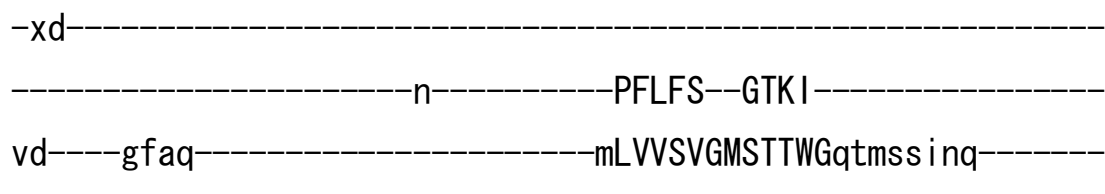

-dssertp|qvr Idt Itstigkig|tvaalv|

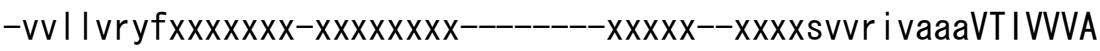
IPEGLPLAvTLTLAYSMK-RMmsdqaMVR-KLSACETMGSATVICTDKTG -TLTLNEMKVt kfwlgqesi

xxxxxispdvld-

yqgtg In---xxgsvc $-v x x x g x t p$ 
I gmdmes--vkq

-khev Irvetfssakk-rsgv

$-1------x x x x x x x x x x x x x k$

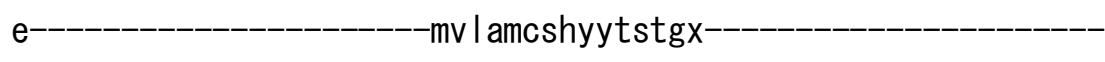

vdImdstaksr i-qa i iqgm--aas-----s I rc IAFAHK i a--xxxxxx-

-eedg I t I MG I VGLKDPCRPGVSKAVETCKLag-VT I

KMI TGDNVFTAKAIAFECGI-

$-1 \mathrm{dh}-\mathrm{-n}-$

-xxxxxavvegvafr

nytdeermq-

kvdki--rVMA--RSSPSDKLLMVKCLr

$---\mid k--g h-$

-VVAVTGDGTNDAPALKEADIGLSM-

-xiqgtev 


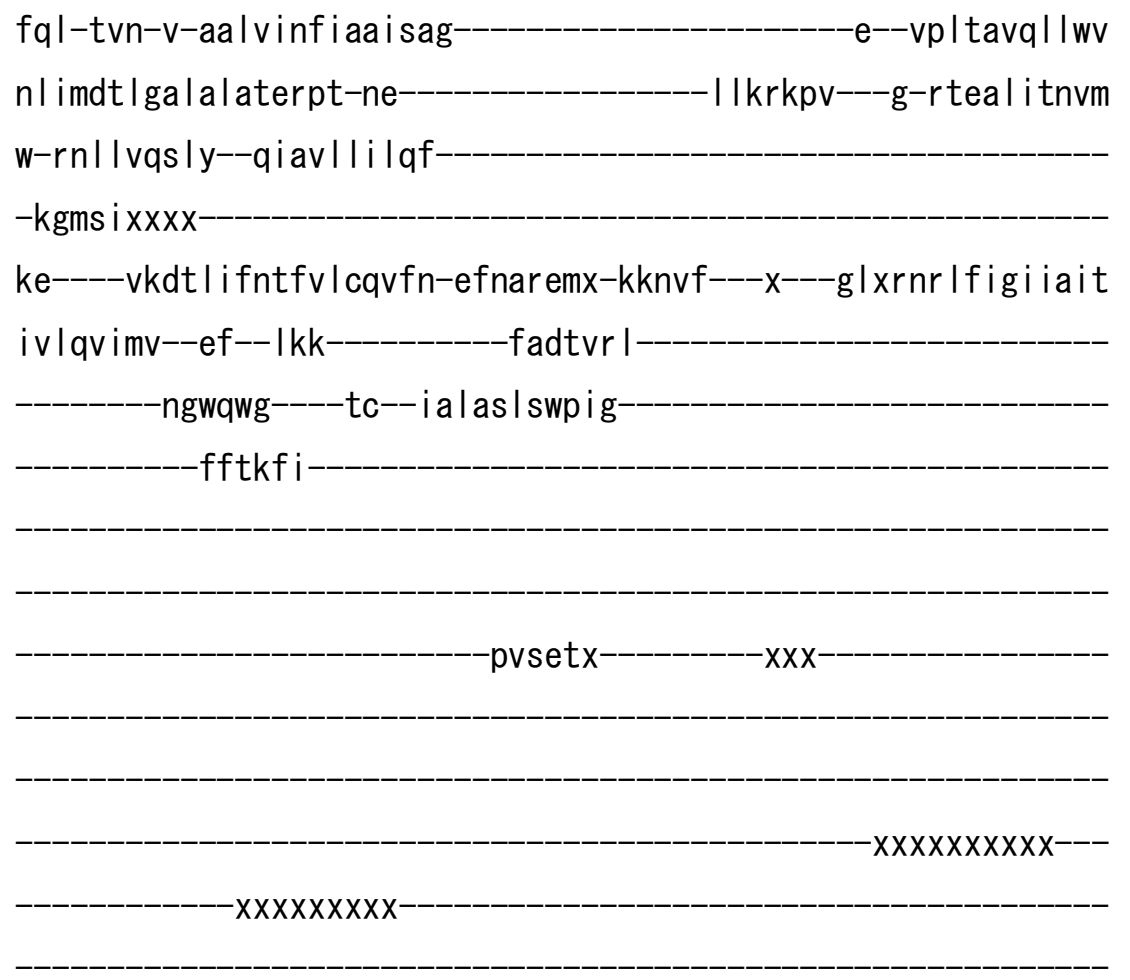

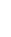




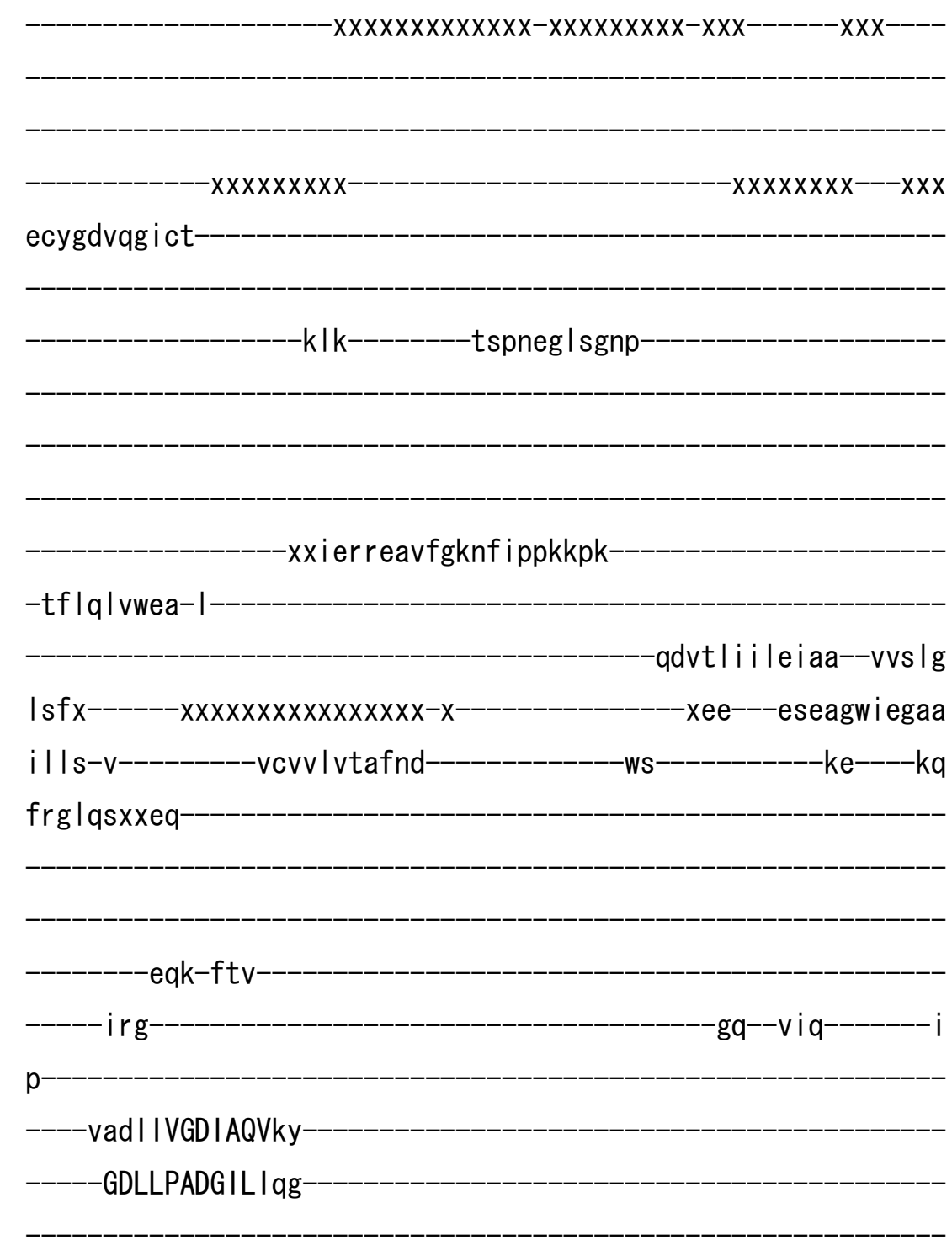

-n--d IkIDESSLTGESDHVKKs I-

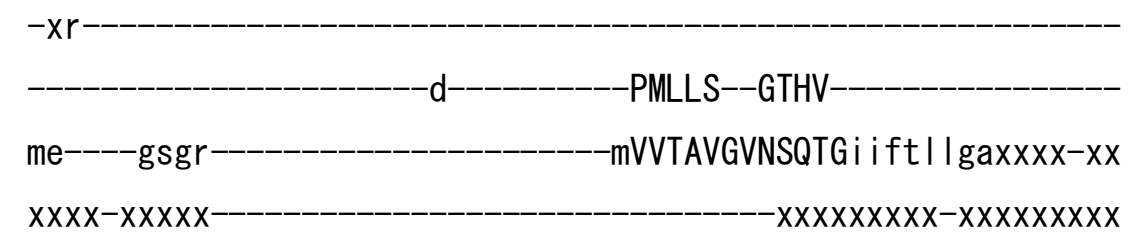

$x x-x x x x x x x x x x x x x x x x x x x x x x x x x$-pkkeksv lqgk|tk lavqigkag I Imsaitv

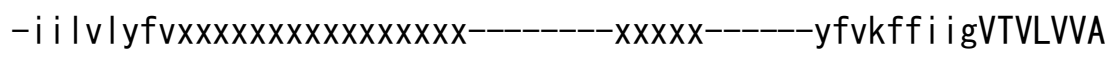
VPEGLPLAvTISLAYSVK-KMmrdn- 
-nLVR-HLDACETMGNATA I CSDKTG-

TLTMNRMTVV

qay i sekhy

$-x-x x x x x e--x$

ipenima

vtg i svnxxxxxki lp

$-p x x x g x \mid p$

-rhvgnkte--cal I gf-----xx-x----

Ikrdyqd--vrnexx

-eekIhkvytfnsvrk-smst

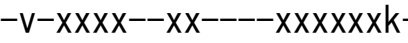

gas

$\mathrm{e}$

ivlkkcfki l sadgx

pkvfrprdrddi ixktvi epm--ase----g I rt ICLAFRdf

-xxxepepewdnenx i vtg I tc I AVVG IEDPVRPEVPDA IKKCQRag-I TV

RMVTGDN INTARA I ALKCGI-

Inp--g-

$-x x x---|c|$ egkdfnx

$\mathrm{XXXX}$

xxx--------_-----e i eqer i dk- 
i wpk I--rVLA--RSSPTDKHTLVKGI i

---dsxxfd--------xxx----------------VVAVTGDGTNDGPALKKADVGFAM--

$-x i a g t d v$

-AKEASDI ILTd-DNFTSIVkaVMWGRNVYDSISKFL-------Q

fql-tvn-v-vavi vaftgac i tq--_-_---_-_-_--_-_-_---d--sp I kavqm I wv

nl imdt l as l a lateppt-ea--_-_-_-_-_-_-_--_ I I rkpy---g-rnkp I i srtm

m-kni I ghafy--qlvvvft I If

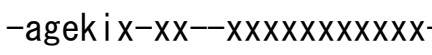

ps-----ehyt i vfntfvmmq I fn-e inarki ixxernvf----x---gixnna ifct iv lgt

fvvqi i i v--qf--ggk-_-_-_-_-_--pfscsk I-_-_-_-_-_-_-_-_-_-_-_-_-_-_-

--------s i eqw I-----ws--vf I gmgt I I wg-

qlisti

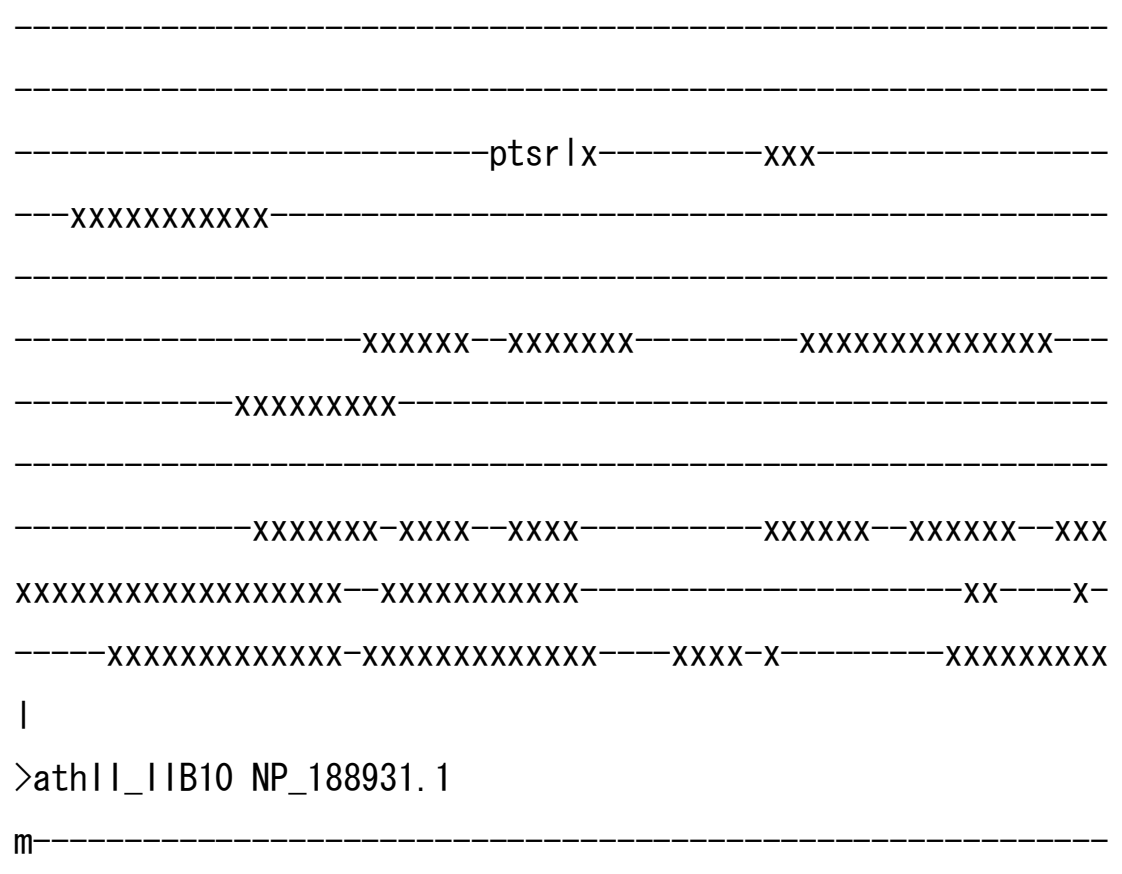


$-X X X X X X X X X X X X X X X X X X X X X X x X X X$

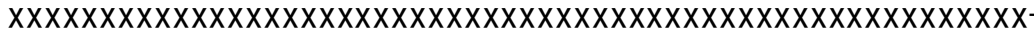

$x X X X X X X X$

$-x \times x \times x x x$

es I ggpng Ivs

$-a l k$ sntrlgineeg

xxiqrrrstfgsntytrqpsk

-g I fhfvvea-f

$-k d|t i l i| l g c a--t|s| g$

$\mathrm{fgi}$ -xeh---glkegwydggs

ifva-v

fIvvavsavsn

$f r$

qn----rq

fdk Iskx-ss-

$-n i k-i d v-$

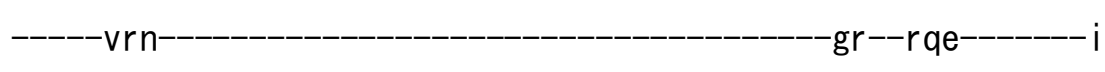

s

----i fdIVVGDIVCLni

GDQVPADGVFVeg 
-h-- I lhVDESSMTGESDHVEVs--

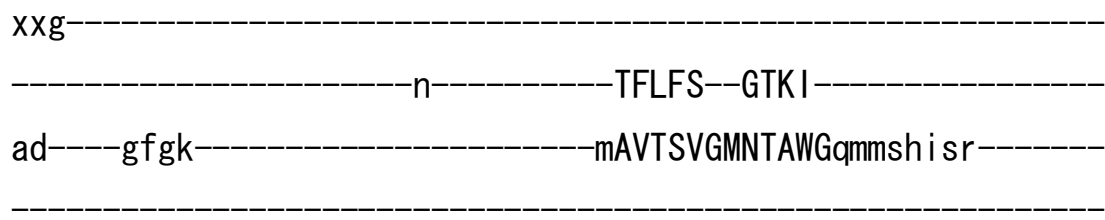

-dtneqtp |qsr Idk | tssigkvg | vaf Iv | -|v|liryfxxxxxxxxxxxxxxxxx--------xxxxx--xxxxavvkmvaaaVTIIVVA IPEGLPLAvTLTLAYSMK-RMmkdn-

-aMVR-KLSACETMGSATVICTDKTG

$-T L T L N Q M K V t-$ -dfwfglesg

-xxxxvsqrvve

hqgvamn---xxgsvf

$-k x x x g x e y$

efsgspte--kai Isw-----xxxx----

lemgmek--vie-

-ehdvvhvegfnsekk-rsgv

$-1----x \times x x x x x x \times x \times x x x \times k$

$\mathrm{e}$

$-k$ i l amcstfcdgsgx 


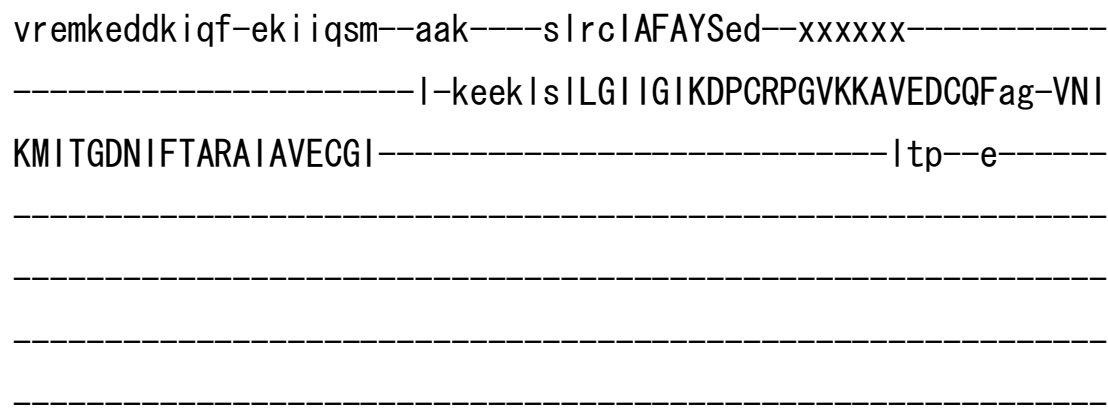

xxxxxxav legekfr

nytqeer le-

kver i--kVMA--RSSPFDKLLMVKCLk

---el--gh-

-VVAVTGDGTNDAPALKEADIGLSM--xiqgtev-

-AKESSDIV I Ld-DNFASVAtvLKWGRCVYNN I QKF I $-0$

fql-tvn-v-aalvinfvaavsag-_-_-_-_-_-_-_-_-_-_-_-_--vp I tavq I I wv nl imdt I ga l a latekpt-nd--_-_-_-_-_-_-_--_ Imkkkp i---g-rvap I i tn im w-rnl laqafy--qisv I Ivlqf -rgrsixxxx-

ek----vknt I ifntfv|cqvfn-efnars Ix-kknvf---x---gl xknr If i gi ivvt vv lqvvmv--ef--Ikr-_-_-_-_-_-_fadter I-_-_-_-_-_-_-_-_-_-_-_-_-_-_-

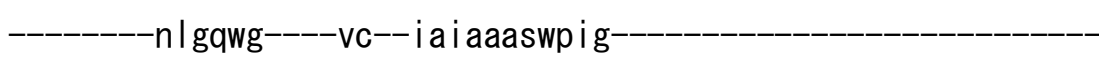
- w l vksv 
s

>ggalI_IIB02 XP_001231642.1

m

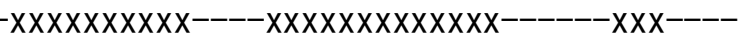

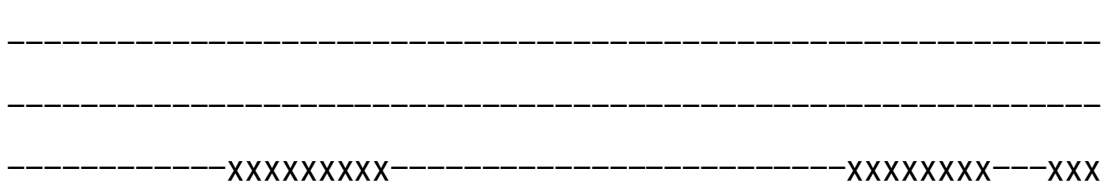

etygeteglor

-hlk--------tspteglagta-

$-x x$ l ekrkl ifgknf ippkkpk

-tfiqlvwea-I 


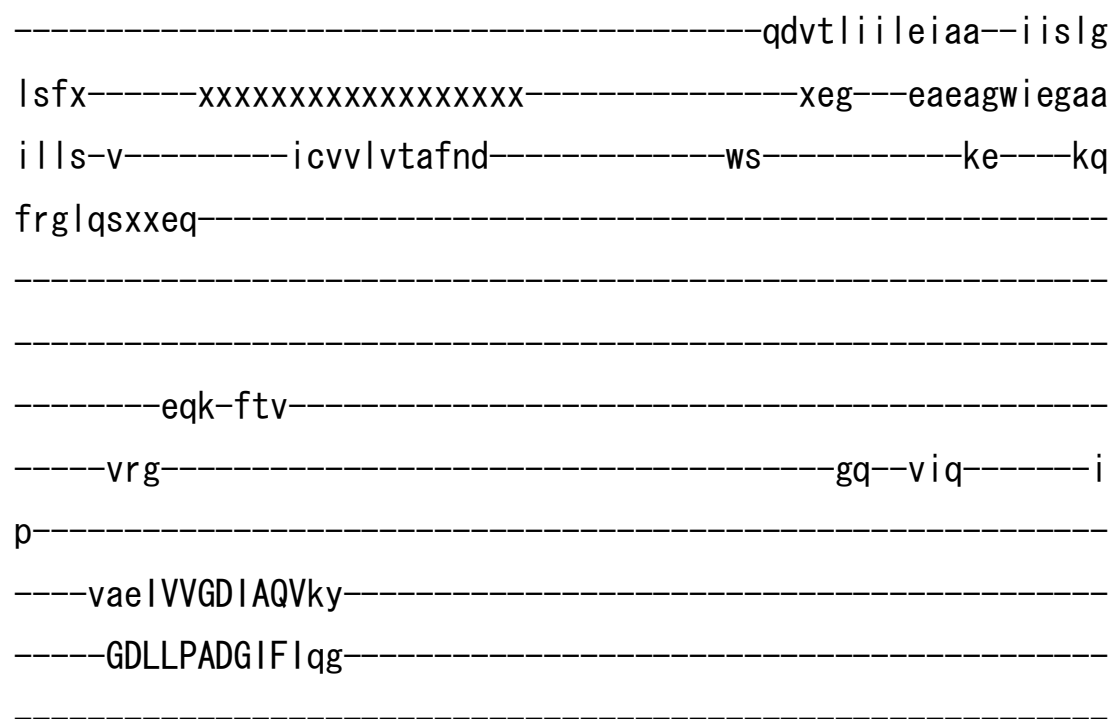

n--d IkIDESSLTGESDQVRKsv-

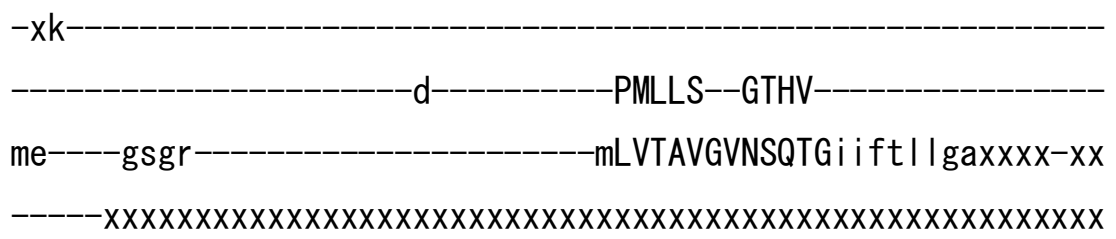
$x x x x x x x x x x x x x x x x x x x x x x x x x x x x$-hkkeksv lqgk Itk l avq i gkag I vmsai tv

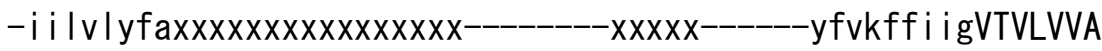
VPEGLPLAvTISLAYSVK-KMmkdn-

nLVR-HLDACETMGNATAI CSDKTG

TLTTNRMTV qay igdvhy-

$-x-x x x x x d--x$

vpakt le

vnaiainxxxxxki Ip $-p x x x g x \mid p$ 
-rqvgnkte--cg| |gf-----xx-x

- kqdyep--vrnlxx-

eeklykvytfnsvrk-smst

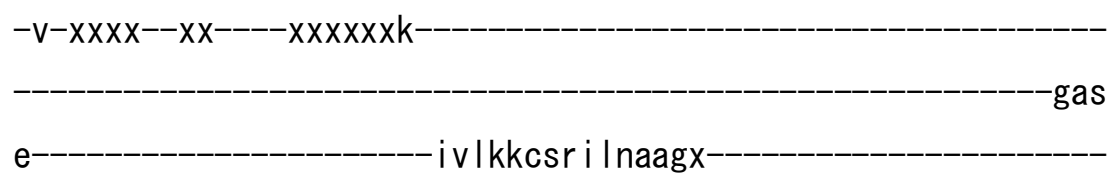

pr ifrprdrdemxkkv i epm--acd----g | rt ICVAFRdf

-xxspepdwdnenx i I sd I tc I CVVG IEDPVRPEVPEA IRKCQRag-ITV

RMVTGDN I NTARA I A IKCGI---_-_-_-_-_-_-_-_-_-_-_-_-_-_-_ihp---g--_-_-

$-x x x---|c|$ egkefnx

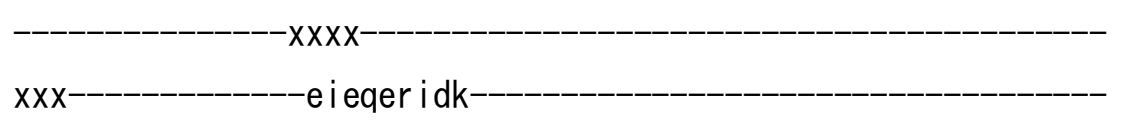

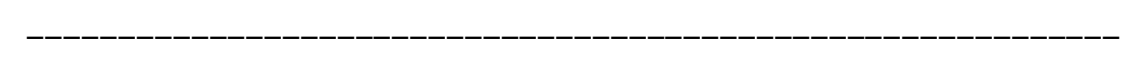

i wpk I--rVLA--RSSPTDKHTLVKGI i

---dsxxve--------xxx----------------VVAVTGDGTNDGPALKKADVGFAM--

-AKEASD I ILTd-DNFSS IVkaVMWGRNVYDSI SKFL------Q 
fql-tvn-i-vavi vaftgac i tq-_-_-_-_-_-_-_-_-_-_-_----sp I kavqm I wv nl imdtfas la lateppt-ea--_-_-_-_-_-_-_-_-- I I rkpy---g-rnkp I i sr tm

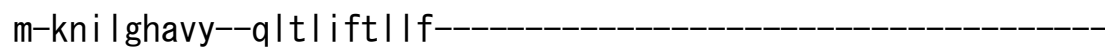

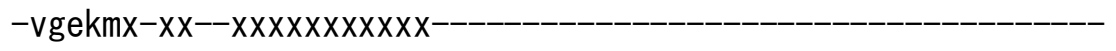
ps----ehyt i i fntfvmmq I fn-e inarkixxernvf----x---gi $x$ rnp i fct iv lgt

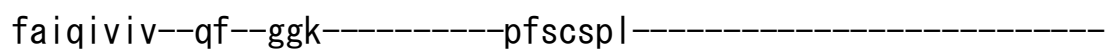

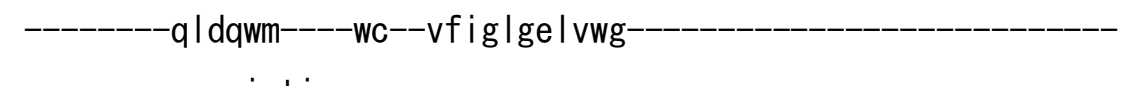
-qviati-
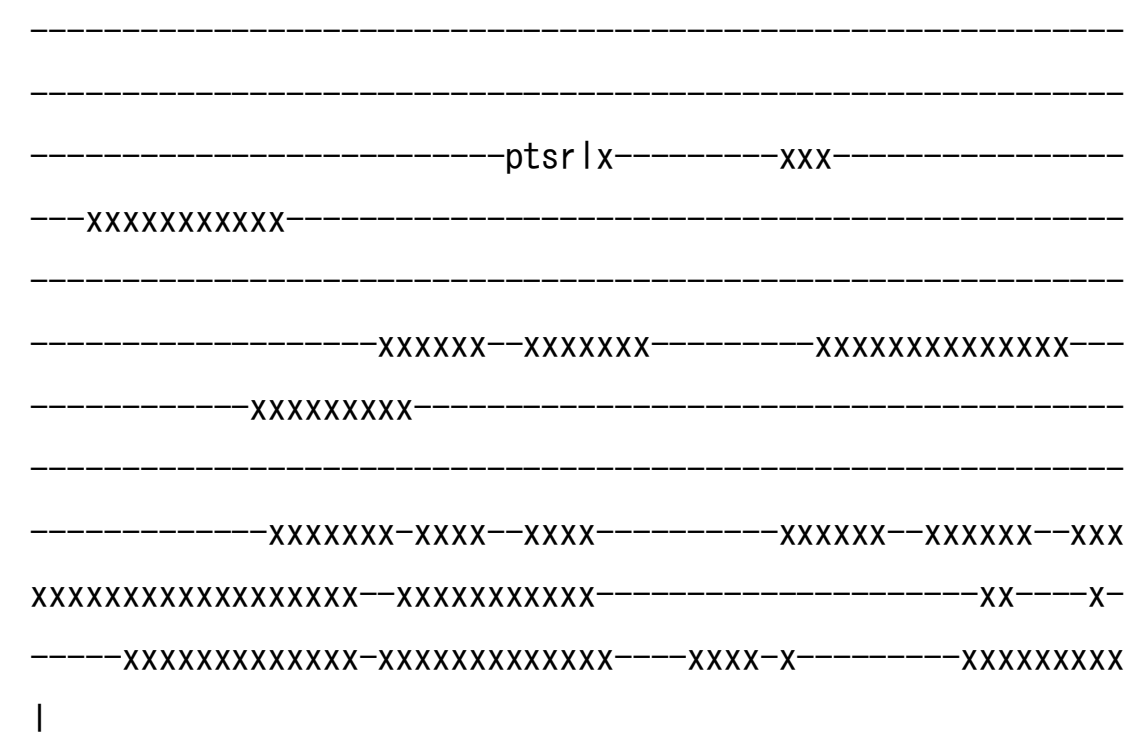

>ggal I_IIB03 XP_418055. 2

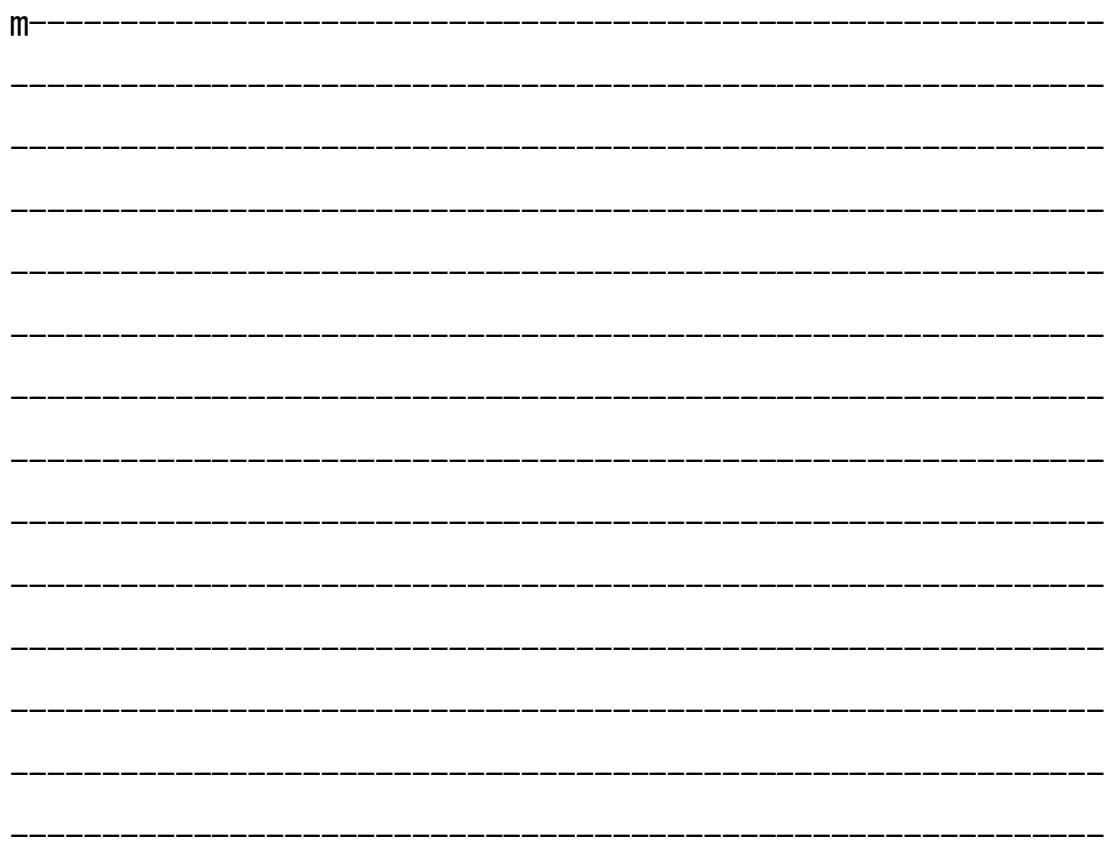




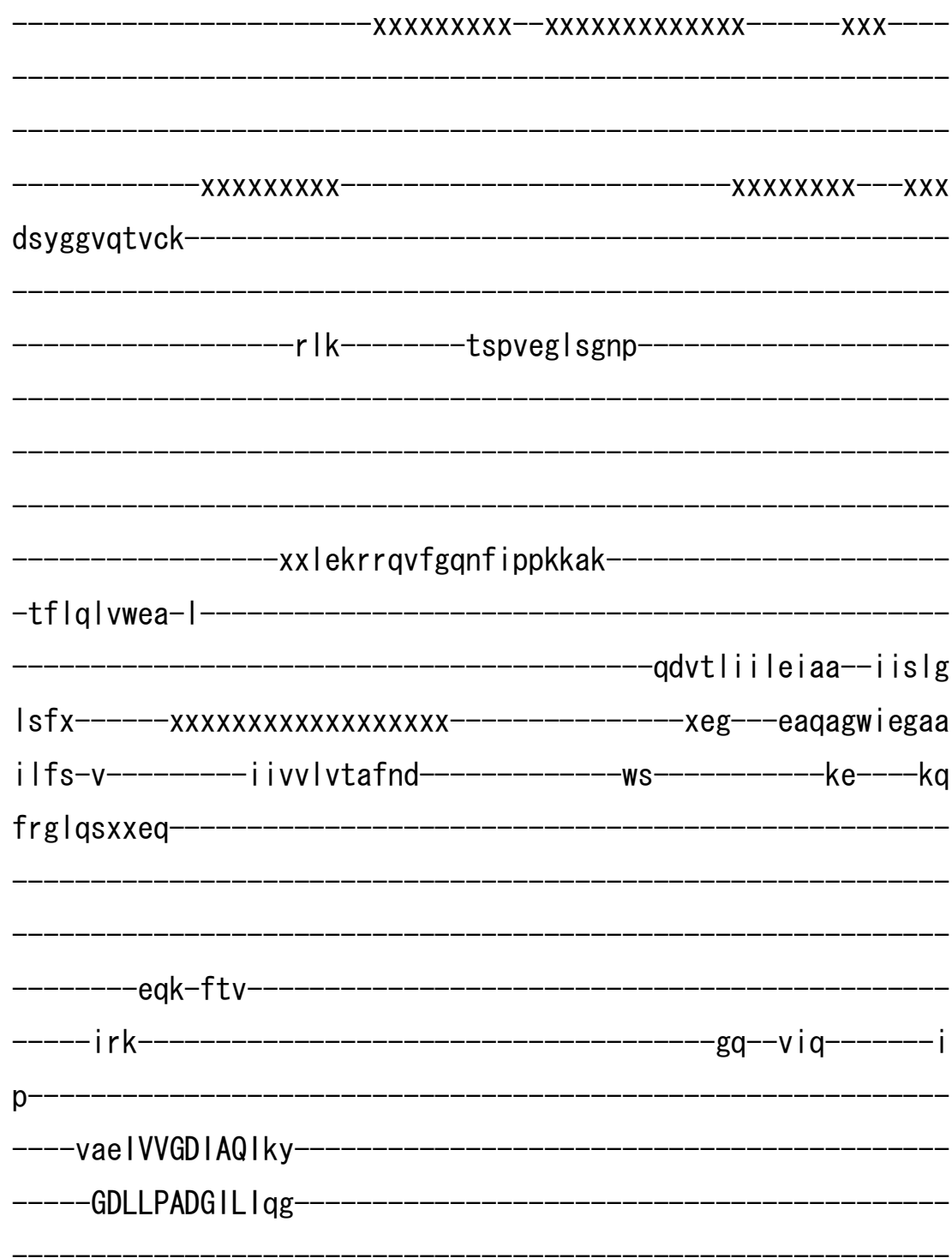

-n--d IkIDESSLTGESDQVKKs I-

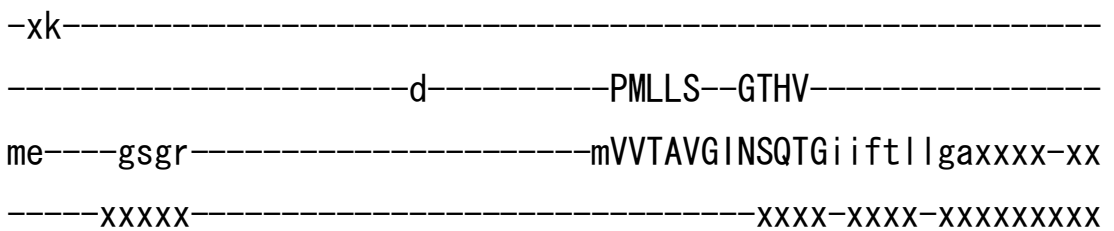

$x x-x x x x x x x x x x x x x x x x x x x x x x x x x x$-pkkeksv lqgk|tr lavqigkag | imsaitv

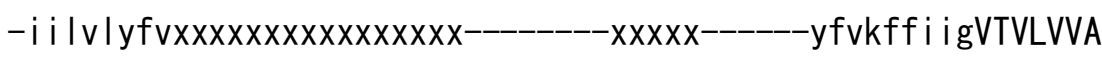
VPEGLPLAvTISLAYSVK-KMmkdn- 
-nLVR-HLDACETMGNATA I CSDKTG-

$-T L T M N R M T V$

qayvgdthy

$-x-x x x x x e--x$

ilpkvld

vngvainxxxxxki Ip

$-p x x x g x \mid p$

-rqvgnkte--cal I gf-----xx-x----

Ikqdyqa--vrnexx

-eek I ykvytfnsvrk-smst

$-v-x x x x--x x----x x x x x \times k-$

gas

e-

- i i l rkctk i l dkngx

prmfkvkdrdemxkkvi epm--ach----g I rt ICLAFRdf

-xxdaepdwdsenx i I sd I tc I AVVG IEDPVRPEVPDA I LKCQRag-I TV

RMVTGDN INTARA I ATKCG I

I p--g-

$-x x x---|c|$ legkefnx

$x x x x$

$\mathrm{xxx}-$--_--_-_-_---eveqeq $\mathrm{dk}$ 
i wpk I--rVLA--RSSPTDKHTLVKGI i

---dsxxgd--------xxx----_----_------VVAVTGDGTNDGPALKKADVGFAM--

$-x i a g t d v$

-AKEASDI ILTd-DNFTSIVkaVMWGRNVYDSISKFL-------Q

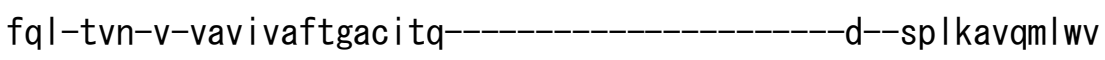

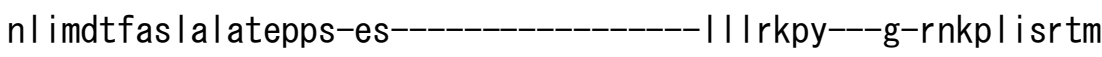

m-kni I ghavy--qlt i ift I I

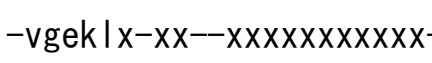

pt----ehyt i $f$ fntfvmmq I fn-e i narki $x x e r n v f---x---a i x r n p$ ifctvv lgt faaq i i iv--ef--ggk----------pfscsg I

---------t I sqwf-----wc--i i i gvge I I wg

-qlictv

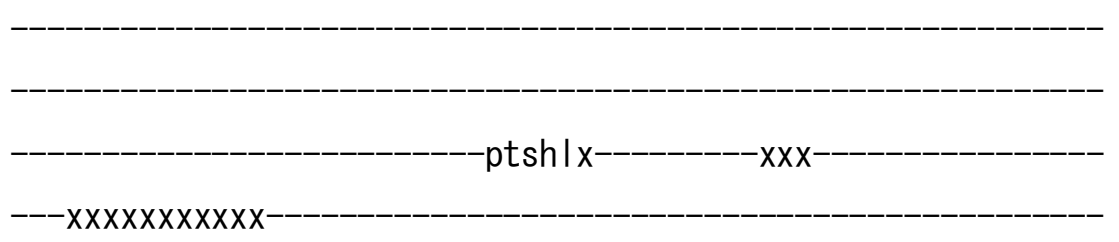

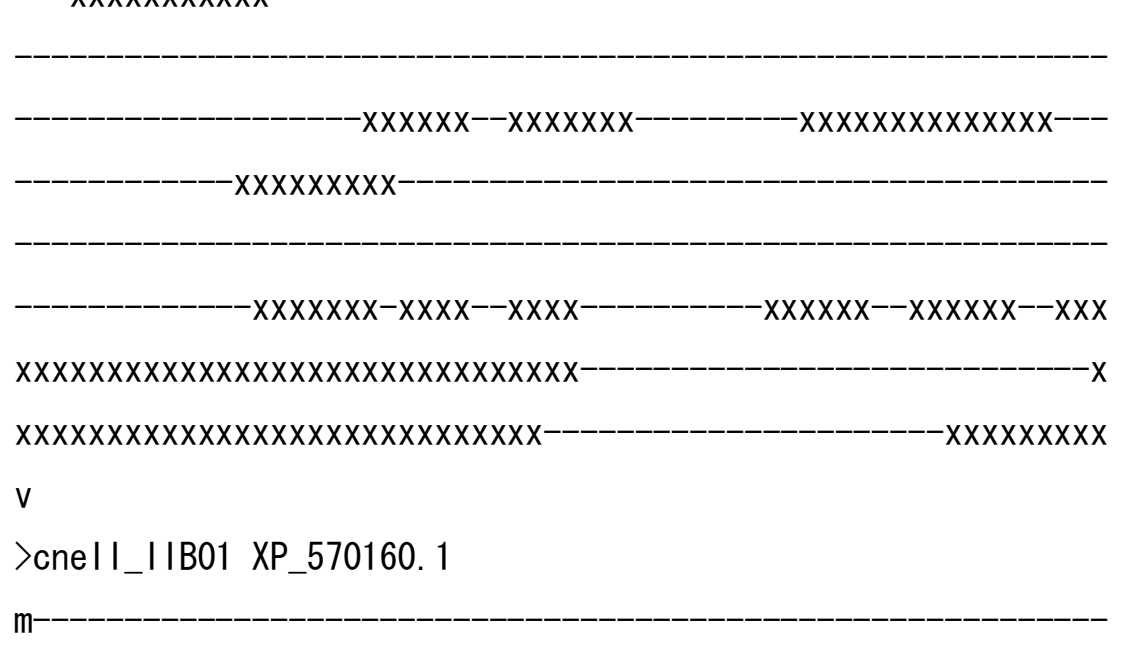




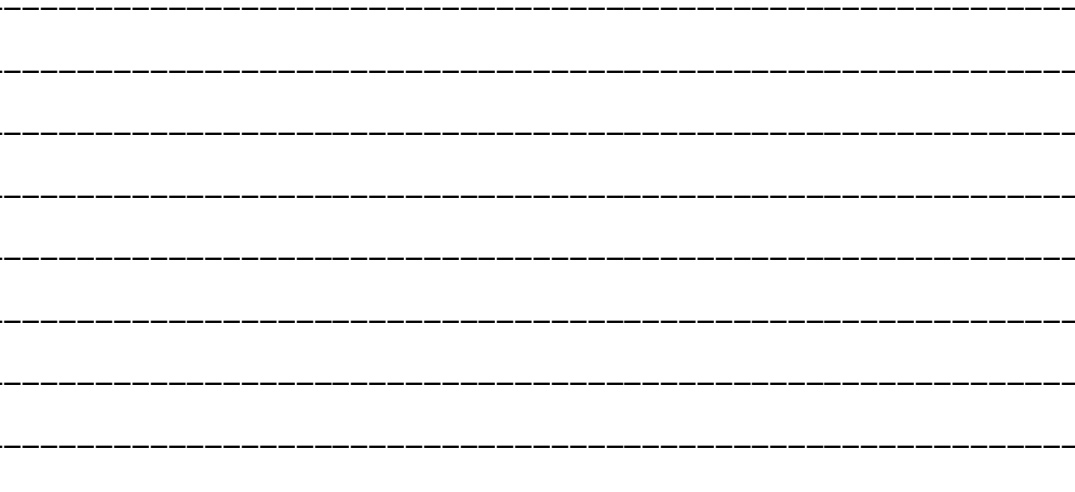

$-x x x x x x x x x x x x x x$

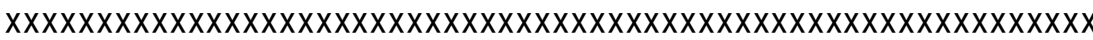

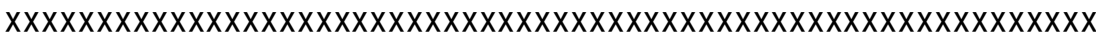

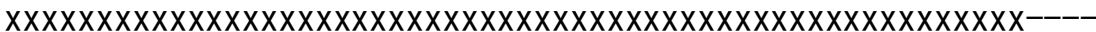

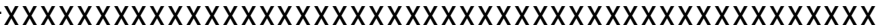

ekiggvsgl le

$\mathrm{g} \mid \mathrm{g}$ -vdgakglavgt

$-X X X X X X X$ $x x x x x x x x x x x x x x x x x x x m d h r$ reiygrndlprrksk

$-s|||| m w \mid a-f$

$-k d k v l i \mid l$ siaa--vvsla

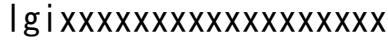
-xce---eaqvdwvegva

ivva-i-----_---i i vvlvgs ind $-w q$ ke----rq

fkkIne--kr

$$
\text { -edrxvkv }
$$

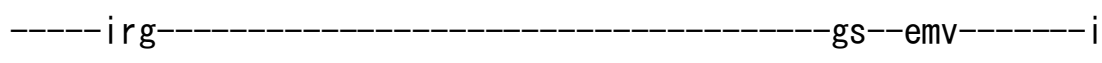

$n$

----vkdVVVGDVCLLep

GEI IPVDG IFLrg 
-h--nvrCDESGATGESDAIKKfs-

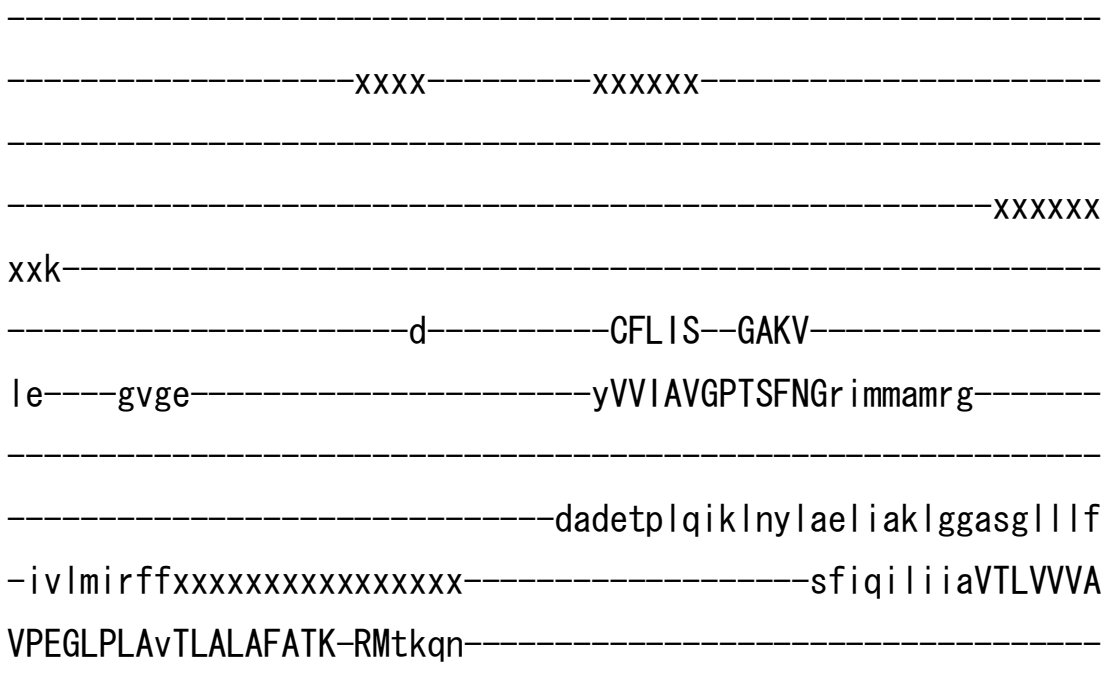

ILVR-VLGSCETMANATVVCTDKTG

TLTQNEMTV

ag

$-\mathrm{X}$

$x x x x x$ f f $v k d l$ s $x x x x x x x x x x x x x x x x x x x x x x x x x x x x x x x x x x x x x x x$

- If

neaicin-----staf

exxxexgkx

nfvgskte--tal Irf-----xxxx----

ewpdyrq--vre-

sae ivqmipfsselk-amgv

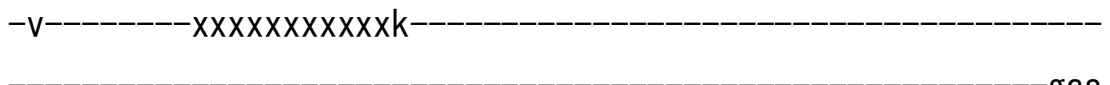

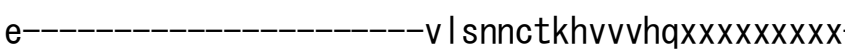


ttefdddamsni-skt i i fy--anq----s I rt IALCYRdf

x--xxxxxx--xxkdeadevpyex i akdmt I I I TG IEDPLRPGVKEAVEKCQLag-VAV

KMCTGDNVLTARS IASQCGI

fta--g------

------vvmegpvfr

$k \mid s d s d r l e-$

i apr I--qILA--RSSPEDKRLLVKTLk-

--- sm--ge-

-VVGVTGDGTNDGPALKLANVGFAM--

-xiagtev

-AKEASD I I LMd-DSFKN IV I a I MWGRCVNDSVKKFL

$-Q$

fqi-svn-i-tavfitf i savass-_-_-_-_-_-_-_-_-_-_-_--sxxsv I tavq I I wv

nl imdtfaa la latdpat-es---_-_-_-_-_-_-_-_--s l drkpd----r-knap I i tvem

f-kmimvqai y--qi ivclvlhf

-aglkixx---------xxxxxxx

nt-----e I gal vfncfvfcq-_-_-_-_-_-_-_-_-_-_-_-_-_-_-_-_-_-_-_-_i |

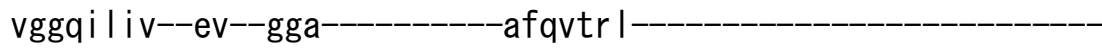

-ggrdwg----it--Ivigals Ipig-

-alvr|t

ptaqf $x$

$x x x x x x x x x x x x x x x x x x x$ 


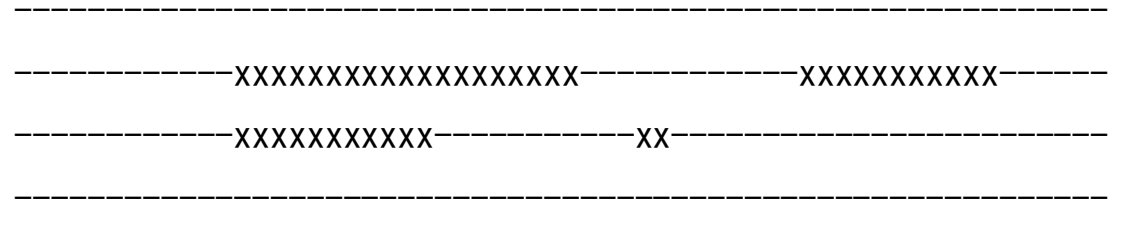

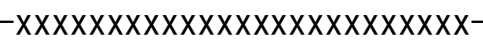

$-x x x x x x x x x x x x x x x x x x$

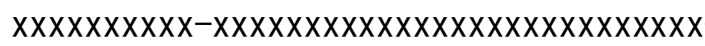

$-x x x x x$

V

>mmul I_I IB01 NP_080758. 1

$$
\mathrm{m}
$$

$-x x x x x x x x x x x x x-x x x x x x x x x x x x x$ $-x \times x----$

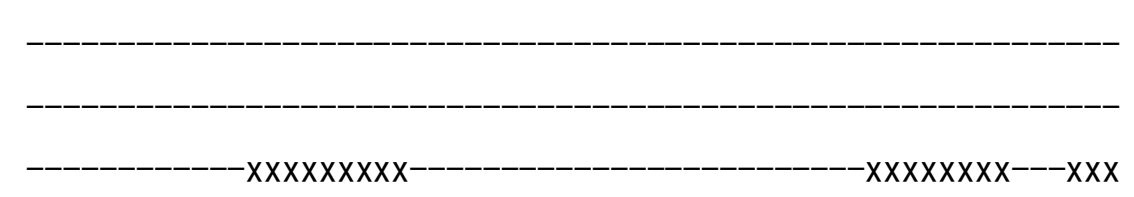

esygdvyg ict

-k lk---_--_-tspneg | sgnp-

-xx lerreavfgknf i ppkkpk

-tf $|q|$ vwea- 


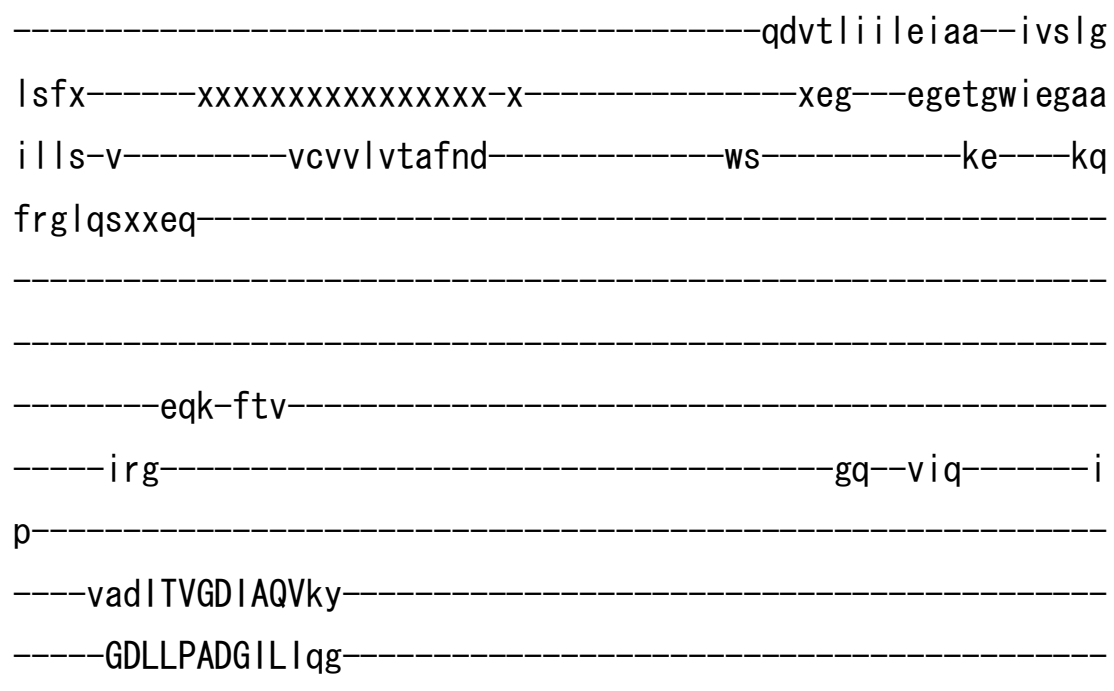

-n--d IkIDESSLTGESDHVKKs I-

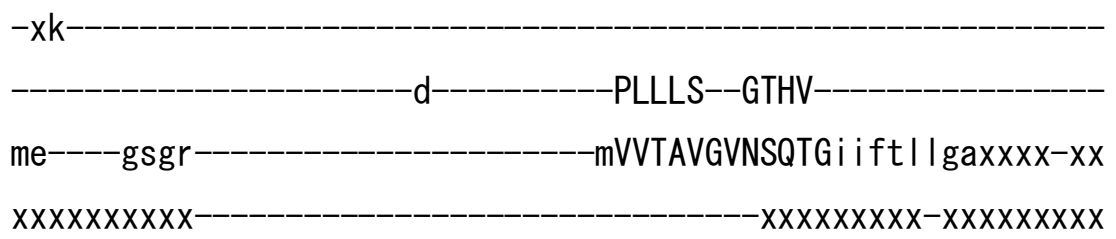

$x x-x x x x x x x x x x x x x x x x x x x x x x x x x x-p k$ keksv lqgk|tk lavqigkag | Imsaitv

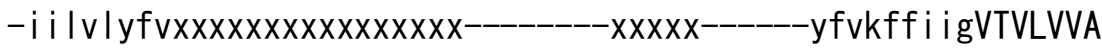
VPEGLPLAvTISLAYSVK-KMmkdn-

nLVR-HLDACETMGNATAI CSDKTG

$-T L T M N R M T V$ qay inekhy

$-x-x x x x x e^{--x}$ ippnils

$-\mathrm{yl}$

vtg i svnxxxxxk i lp $-p x x x g x \mid p$ 
-rhvgnkte--cal Igf-----xx-x

- lkrdyqd--vrnexx-

-eealykvytfnsvrk-smst

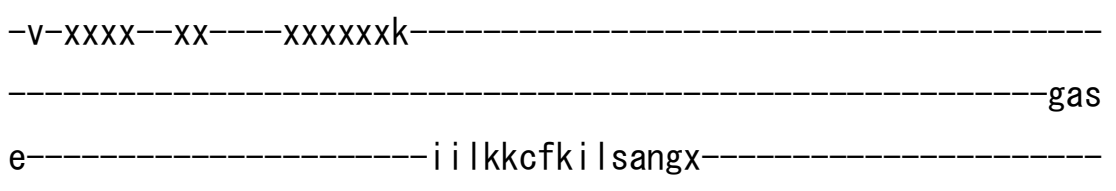

akvfrprdrddi xktv i epm--ase----g I rtICLAFRdf

-xxxepepewdnenxvvtg I tc I AVVG I EDPVRPEVPEA IKKCQRag-I TV

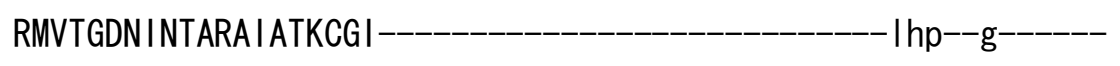

$-\mathrm{xxx}---\mid \mathrm{c}$ legkdfnx

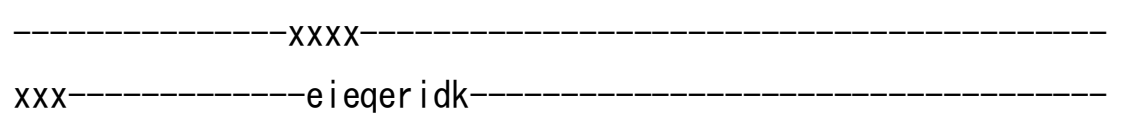

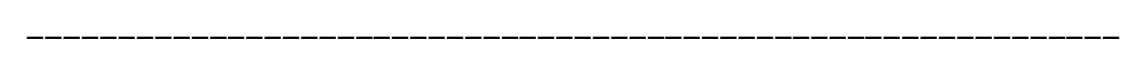

i wpk I--rVLA--RSSPTDKHTLVKGI i

---dsxxse---_---xxx--_-_-_-_-_-_-_VVAVTGDGTNDGPALKKADVGFAM--

-AKEASD I ILTd-DNFTSIVkaVMWGRNVYDSI SKFL------Q 


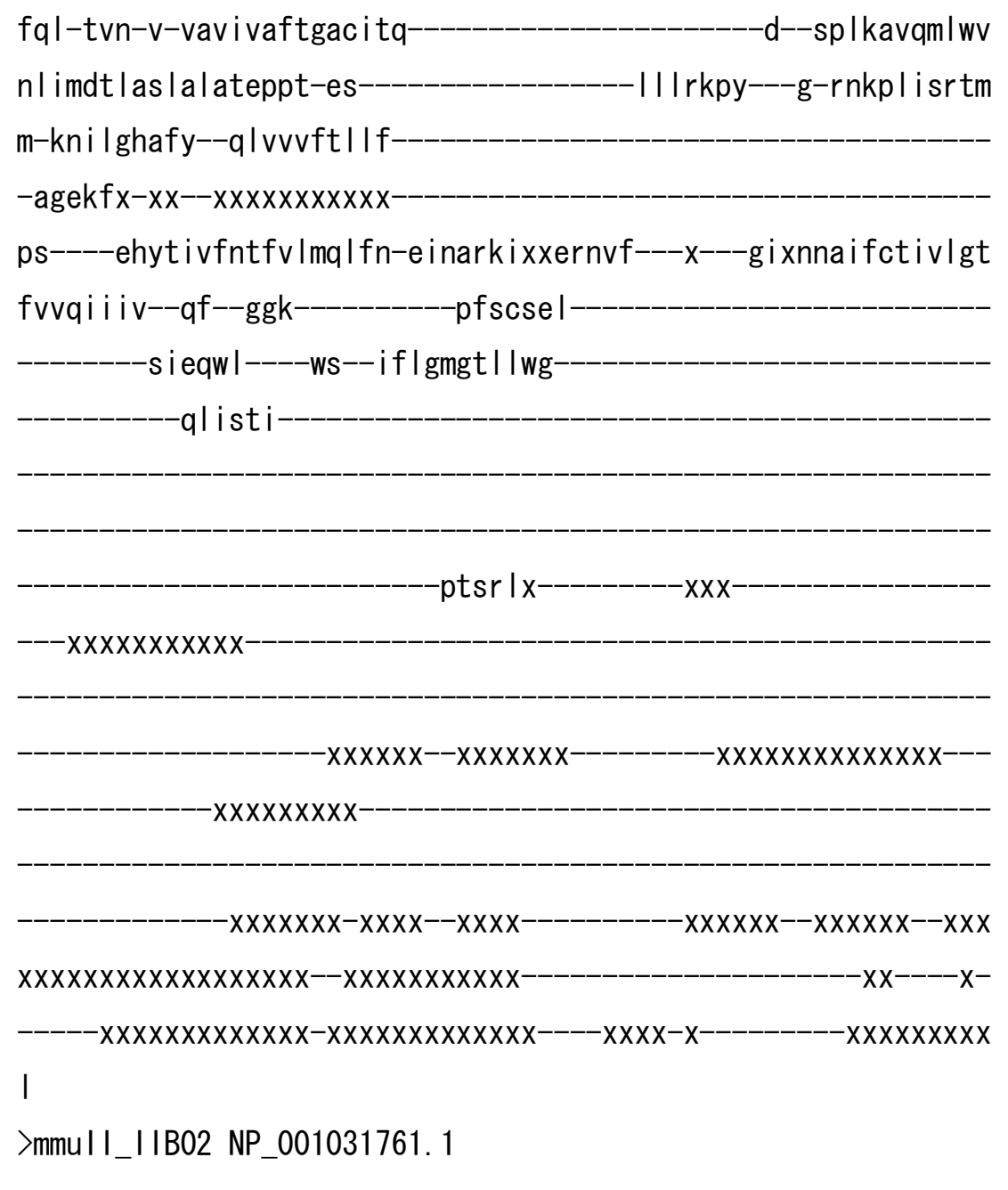
m- 


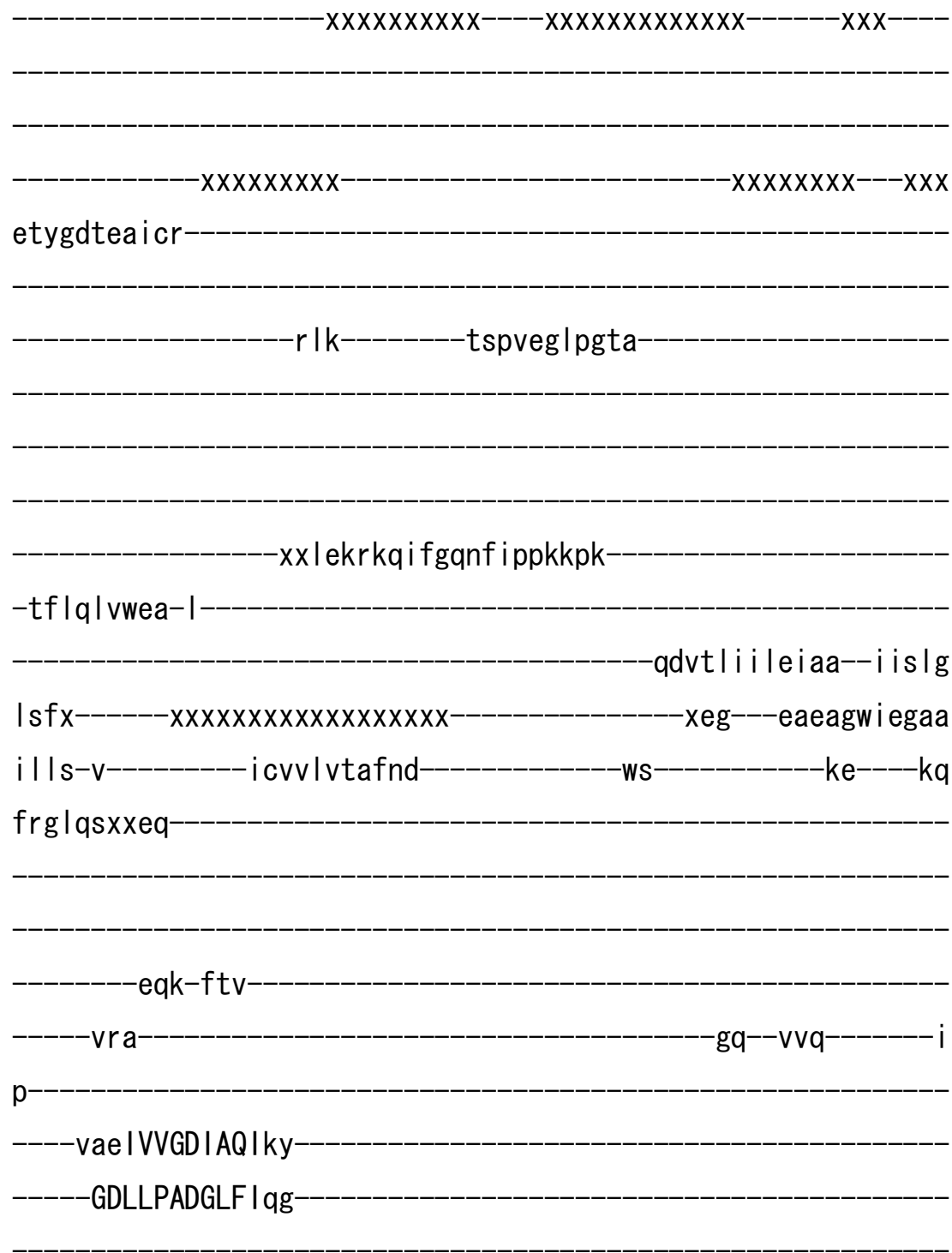

$-n--d$ lkIDESSLTGESDQVRKsv-

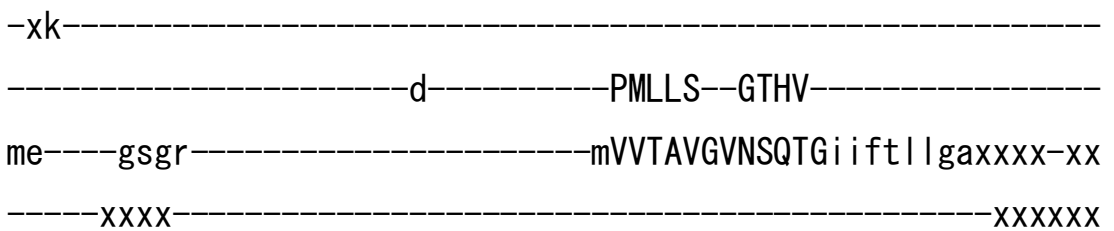
$x x x x x x x x x x x x x x x x x x x--x x x x x x x$-hkkeksv lqgk I tk lavqi gkag I vmsaitv

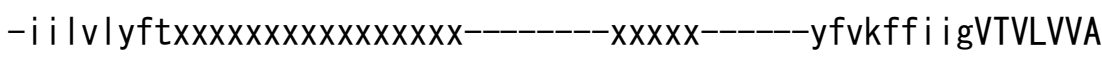
VPEGLPLAvTISLAYSVK-KMmkdn- 
-nLVR-HLDACETMGNATA I CSDKTG-

-TLTTNRMTV

qayvgdvhy

$-x-x x x x x s^{--x}$

inaktle-

vnaiainxxxxxki Ip

$-p x x x g x \mid p$

rqvgnkte--cg | Igf-----xx-x----

Irqdyep--vrsqxx

-eek lykvytfnsvrk-smst

$-v-x x x x--x x----x x x x x \times k-$

-gas

e-

i v I kkcck i I sgagx

arvfrprdrdemxkkvi epm--acd----g|rtICVAYRdf

-xxspepdwdnenx $\mathrm{i}$ I ne I tc I CVVG IEDPVRPEVPEA I RKCQRag-I TV

RMVTGDN INTARA I A IKCG I

ihp--g-

$-x x x---|c|$ egkefnx

$\mathrm{xxxx}$

xxx--------------e e i eqer idk- 
i wpk I--rVLA--RSSPTDKHTLVKGI i

---dsxxte--------xxx----------------VVAVTGDGTNDGPALKKADVGFAM--

$-x i a g t d v$

-AKEASDI ILTd-DNFSSIVkaVMWGRNVYDSISKFL-------Q

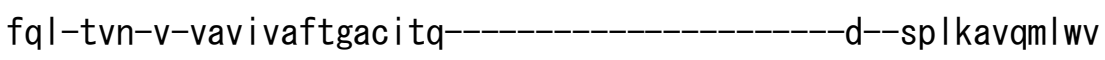

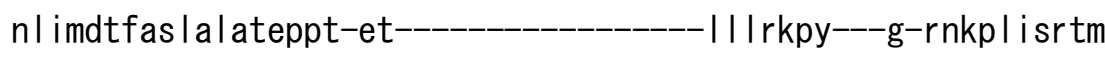

m-kni Ighavy--qlt l ift I If

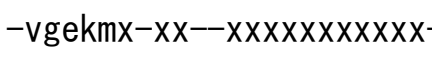

ps----ehyt i ifntfvmmq I fn-e i narkixxernvf----x---gi $x$ rnp i fct iv lgt faiqi viv---qf--ggk--_-_-_-_---pfscsp I--_-_-_-_-_-_-_-_-_-_-_-_-_-_-

---------q l dqwm----wc--if i g I ge l vwg----------------------------

qviati

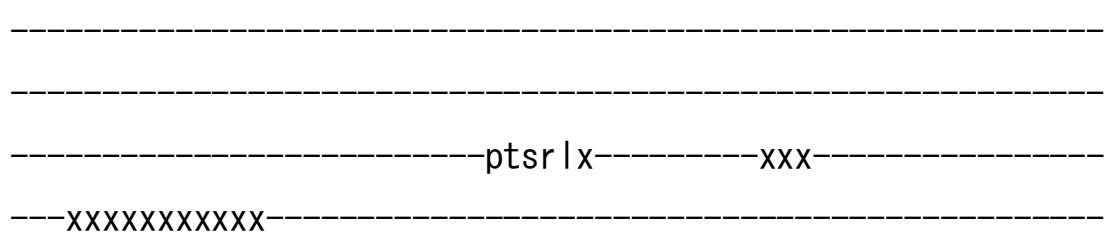

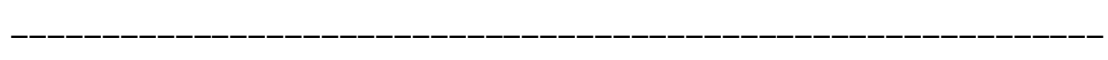

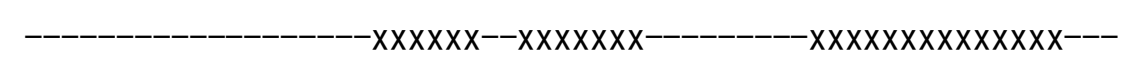

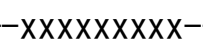

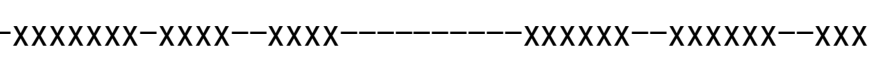

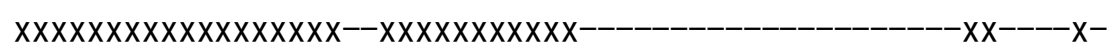

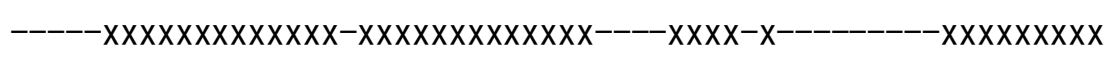

।

>mmul I_IIB03 NP_796210.2

m- 
eaygdvsglor

$-r \mid k$ -tsptegladnt

xxlekrrqiygqnf ippkqpk

-tflqlvwea-I

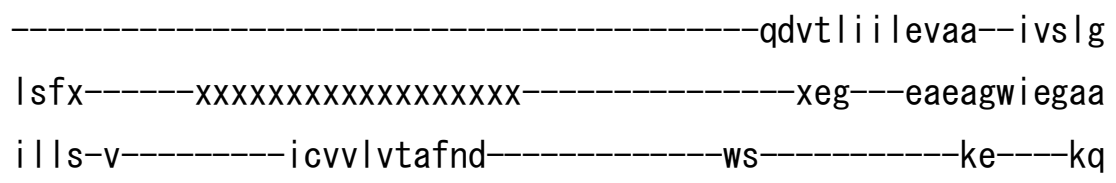

frglqsxxeq-

$-e q k-f t v-$

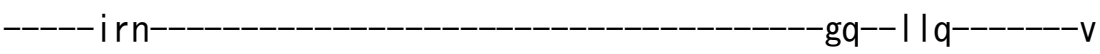

$\mathrm{p}$

----vaaLVVGDIAQVky

GDLLPADGVLI qg 
-n--d IkIDESSLTGESDHVRKsa-

$-x-$

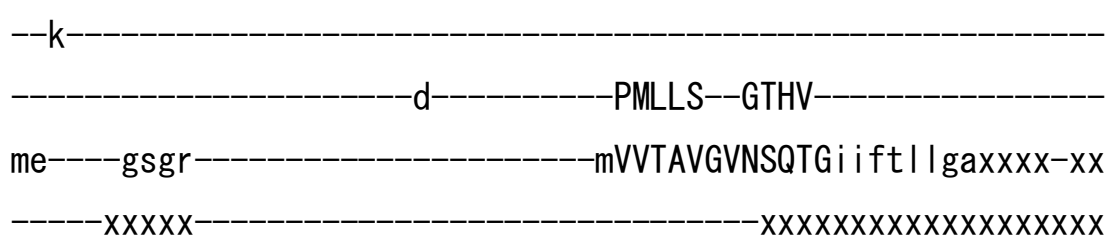

$x x x x x x x x x x x x x x x x x x x x x x x x x x x$-pkkeksv lqgk|tk lavqigkag I vmsaitv

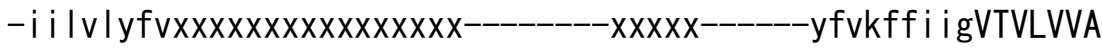
VPEGLPLAvTISLAYSVK-KMmkdn-

-nLVR-HLDACETMGNATA I CSDKTG-

TLTTNRMTV -qsy I gdthy

$-x-x x x x x s^{--x}$

Itpkild

vhaisinxxxxxkilp-

$-p x x x g x \mid p-$

rqvgnkte--cal lgf-----xx-x----Ikrdfap--vreqxx

edqlykvytfnsvrk-smst

$-\mathrm{v}-\mathrm{x} x \mathrm{xx}--\mathrm{xx}-----\mathrm{x} x \mathrm{x} x \mathrm{x} x \mathrm{k}-$ $\mathrm{e}$

-i I lkkctni Insngx 
Irgfrprdrddmxkk i i epm--acd----g I rtICIAYRdf

-xx i qepdwdnenxvvgd I tc I AVVG I EDPVRPEVPEA IRKCQRag-I TV RMVTGDN INTARA I AAKCG I

iqp--g-

$-x x x---|c|$ egkefnx

$-x x x$

xxx--------------e i eqer $\mid \mathrm{dk}$

vwpk I--rVLA--RSSPTDKHTLVKGI i

---dsxxge--------xxx---------------VVAVTGDGTNDGPALKKADVGFAM-$-x$ iagtdv

-AKEASD I ILTd-DNFTS IVkaVMWGRNVYDS I SKFL------Q

fql-tvn-v-vavi vaftgac i tq--_-_-_-_-_-_-_-_-_----d--sp I kavqm I wv

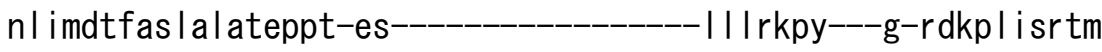
m-kni Ighavy--ql t i ift I If

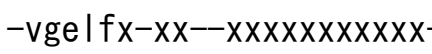

ps-----ehyt i i fntfvmmq I fn-e i nark i xxernvf----x---gi ixsnp ifct iv lgt fgiqiviv--qf--ggk-----------pfscspl-

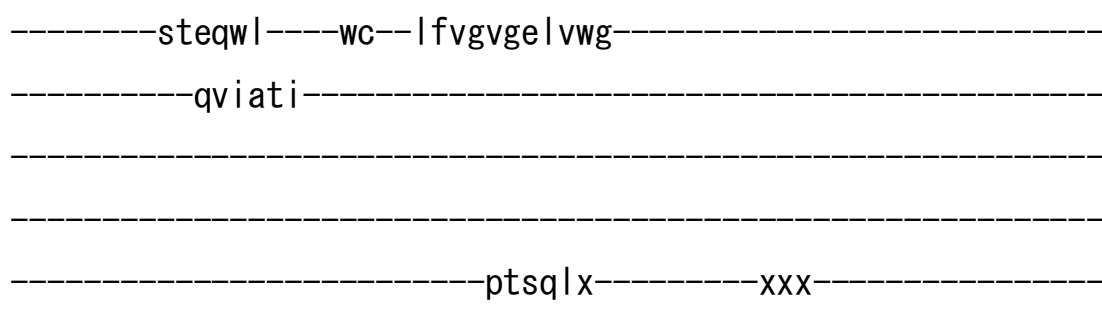




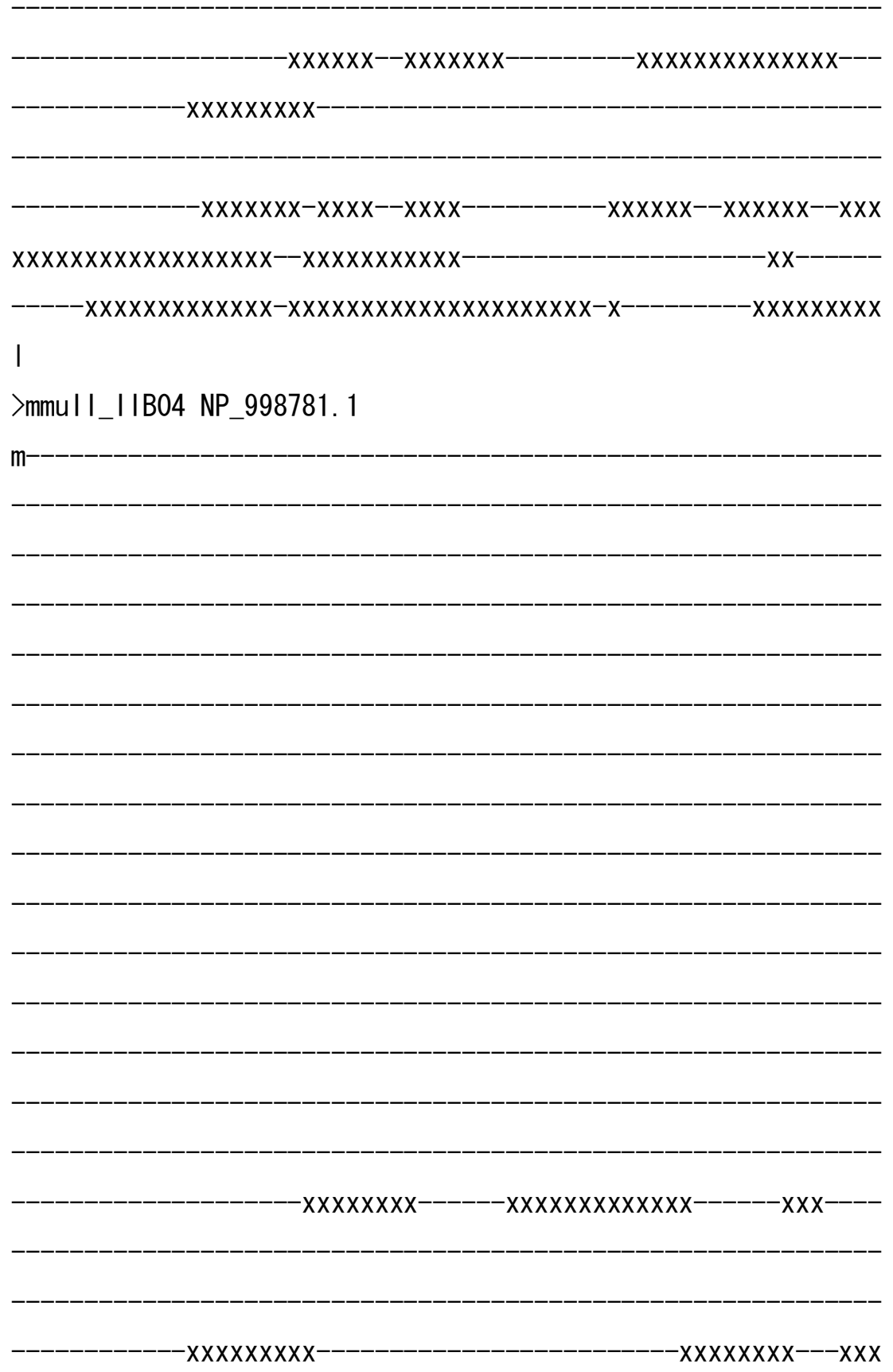

ahyggvae ict

rlk--------tsp i eg I sgnp-

-xx l ekrr I vfgknv i ppkrpk

-tflelvwea-I 


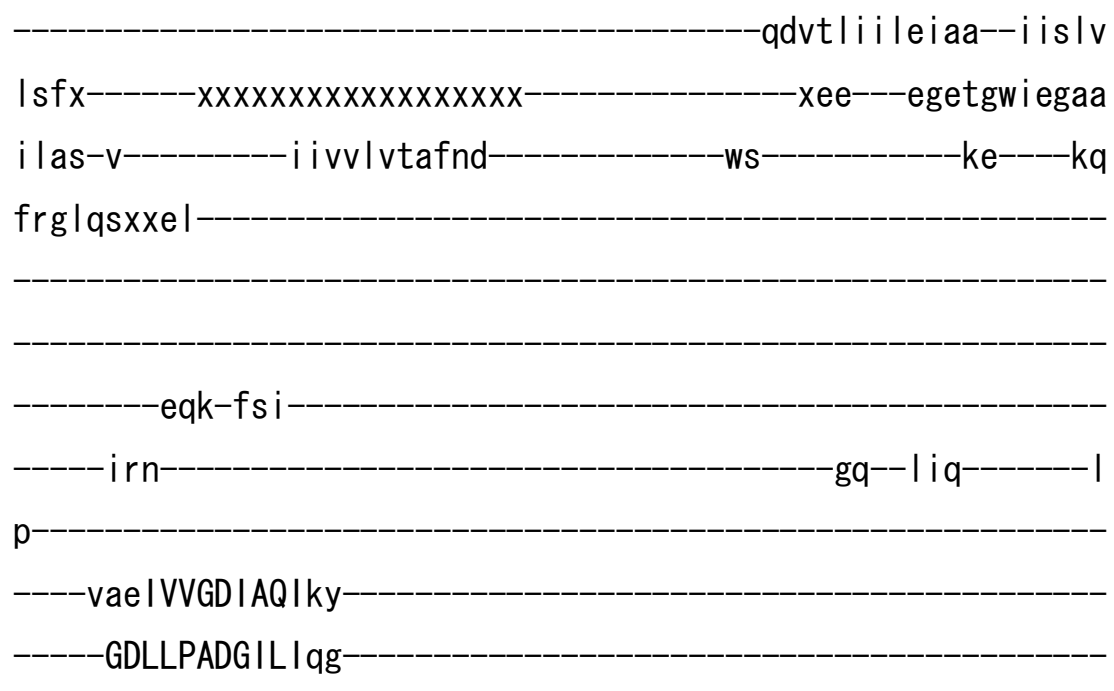

-n--dlkIDESSLTGESDHVKKt I-

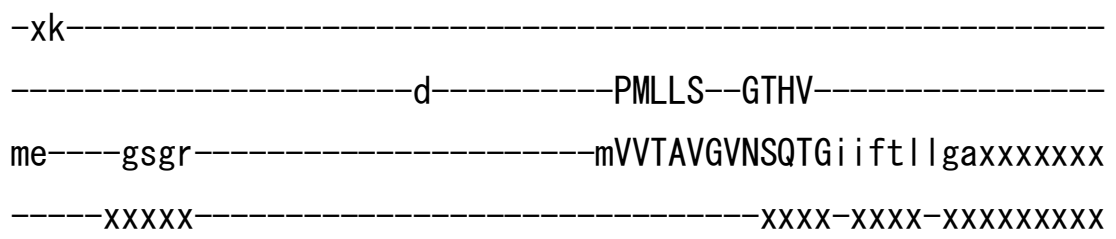

$x x-x x x x x x x x x x x x x x x x x x x x x x x x x x$-pkkeksv lqgk|tr lavqigkag I imsv Itv

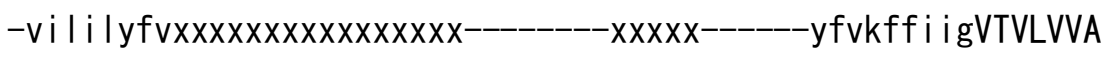
VPEGLPLAvTISLAYSVK-KMmkdn-

nLVR-HLDACETMGNATAI CSDKTG

$-T L T M N R M T V$ -qay i ggthy

$-x-x x x x x d--x$

fppkvle

vng i $\sin x x x x x k$ iqp

$-p x x x g x \mid p$ 
fql-tvn-v-vavi vaftgac i tq---_-_-_-_-_-_-_-_----d--sp I kavqm I wv

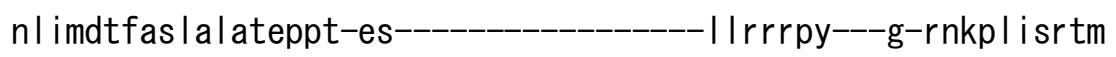
m-kn i I ghavy--q I I i vf I I vf-_-_-_-_-_-_-_-_-_-_-_-_-_-_-_-_-_-_-_-agdt Ix-xx--xxxxxxxxxxx-----------_---------------------

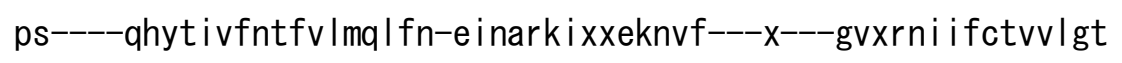

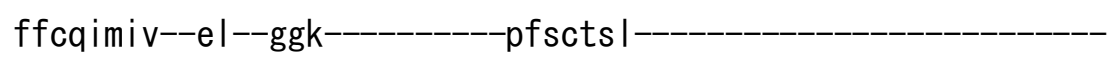
-tmeqwm----wc--I fig ige I I wg-qvisai

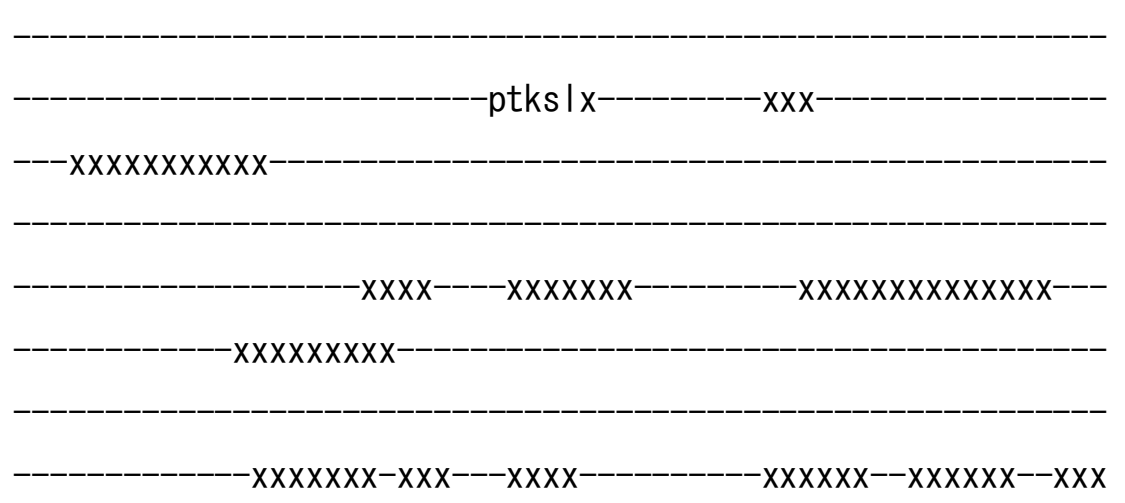
$x x x x x x x x x x x x x x------1 x x x x x x x x x x x--------------------x x x x x x x x$

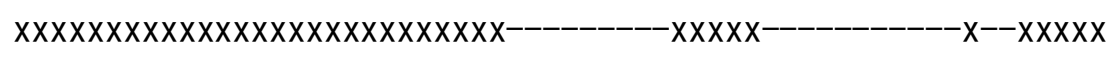
$v$ >anilI_IIB01 EAA66307.1

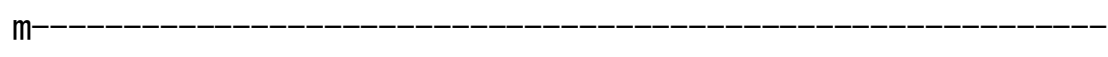

$x x x x x x x$ XXXXXXXXXXXXXXXXXXXXXXXXXXXXXXXXXXXXXXXXXXXXXXXXXXXXXXXXXXX

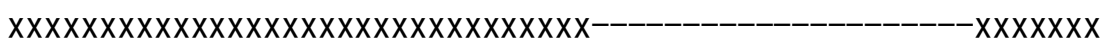

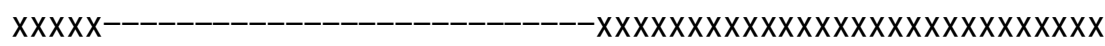



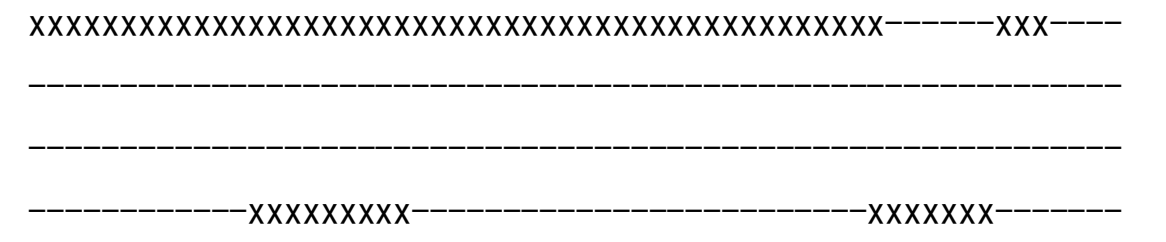

yalgglaglak

$$
\text { -g|r--_-_-_-_tprsg }|\mathrm{s}| \mathrm{de}-
$$

$-x x x x x x x x x x x x x x x x x x x x-$

$-x \times x X X X X X$

$x x x x x x x x x x x x x x x x x x x y$ sdrkrvfgank lpekktk

- silelawla-y-

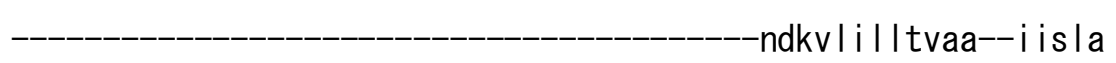

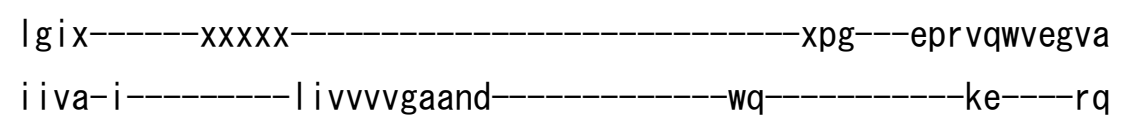

fvkInkx-ke-

$-\mathrm{h}--\mathrm{nvkCDESSATGESDVLRKtp-}$

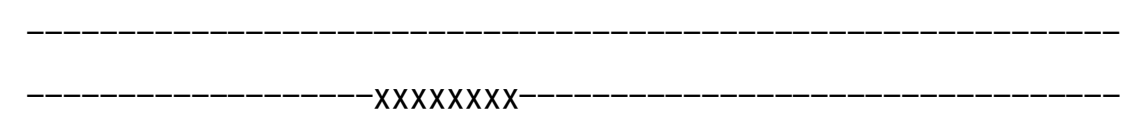

$x x x x x$

$\times x m$

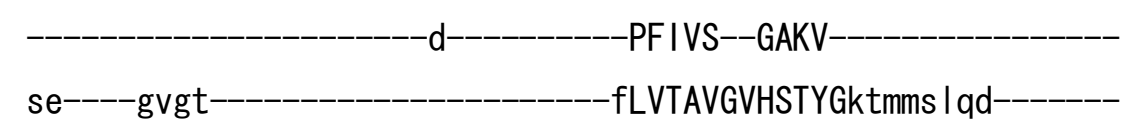

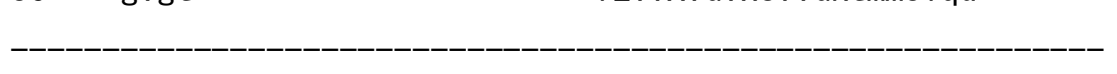

egqttp Iqtk Inv laey iak Ig laag I I If

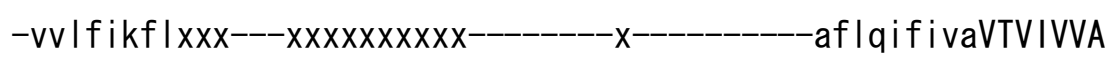

VPEGLPLAvTLALAFATT-RM I $k d n$ 
nLVR-LLRACETMGNATT ICSDKTG

TLTENKMTAv

aat Igtgtr

$-f g g r x x x x x x x x x x x x x x x x x x x x x x x x x x x x x x x x x x x x x$

Idsivin-----staf

exxxextm-

tfigskte--tal Igf-----xxxx----

$\lg |\mathrm{gs}| \mathrm{s}--$ eard

-nas i vqmvpfdsgrk-cmav

$-\mathrm{v}-------x \times x \times x \times x \times x \times x \times x k-$

gas

e-

- v I l akstr i vrnptxxxx

egp Iddkdrsk I-det inky--atq----s I rt IGLVYRdf

x--xxxxxxxxxxeedrs laafdx ifkdmvmFGVFGI QDPLRAGVTESVQQCQRag-VFV

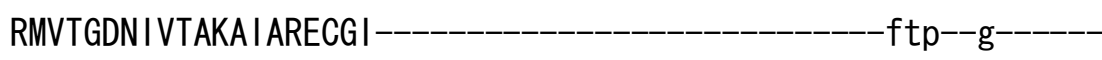

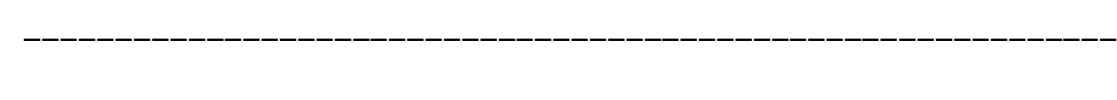

-x-----vai egpkfr

$-k \mid$ ssrqmtq 
i i pr I--qVLA--RSSPDDKKILVSQLk

$---\mathrm{kl}-\mathrm{-ge}$

-TVAVTGDGTNDAQALKTADVGFSM--

xitgtev

-AKEASDI I LMd-DNFAS IVkaMAWGRTVNDAVKKFL $-0$

fqi-tvn-i-tavIItf i savasg--_-_-_-_-_-_-_-_-_-_-_dxxsv I tavq I I wv

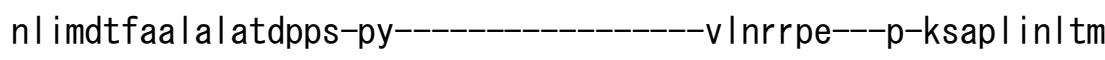
w-kmmigqs i y--qlvvt Iv Inf

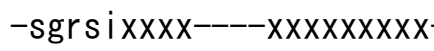

nn----v|ttvvfntfvwmq i fn-qwnsrr Ixxg In if---x---g|xrnrwfigiaf i i vggqi l i i--fv--ggh----------afsvtr I

--------tgaqwa----vc--l i I gvisipvg-

-viirli

- pdef ix $-x x x----x x x x x x x x x x x x$

$x x x x x x x x x x x x x x x x-$

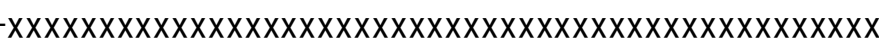

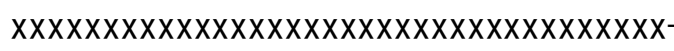

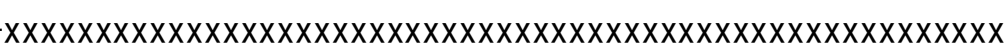
$x x x x x x x x x x x x x x x x x x x x x x x x x x x x x x x x x x x x x x x x$

$-x x x x x$

s

>anilI_IIB02 EAA63398.1

m- 


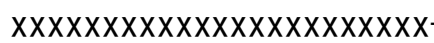

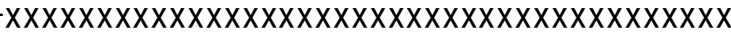

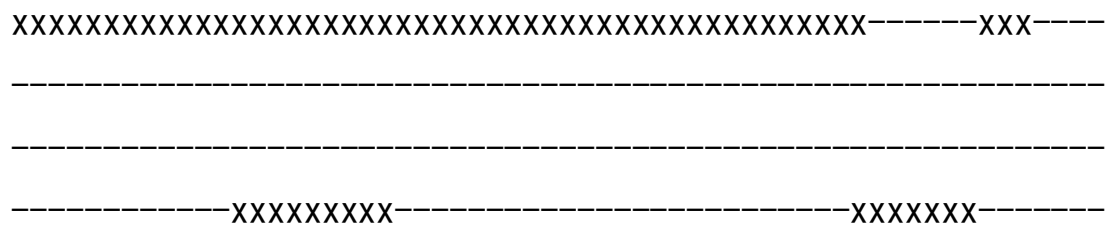

valgg|pg|an-

gl r--------td $\mid$ sag $\mid$ stea-

xxxxxxxxxxxxxfadrirvfkenv lpekkat

$-p|w k| m w \mid a-y$

$-n d k v \mid$ illtaaa--ais la

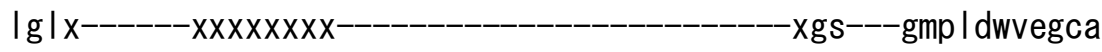

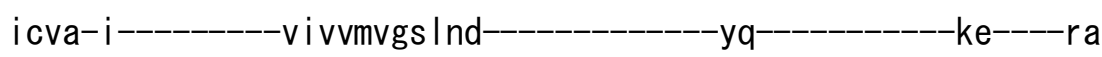

fvk Inkx-ke-

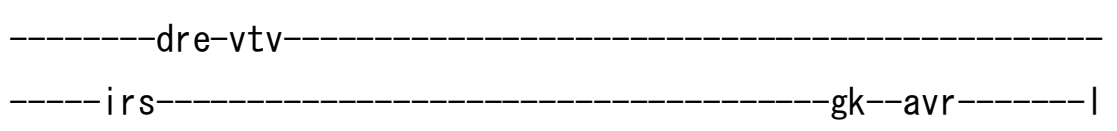

S

----vheVLVGDILHLep

GDLVPVDGIFIdg 
-h--nvkCDESSATGESDQLKKtg-

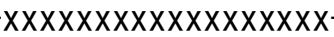

$d-$

le-----gvgt-
-PFI IS--GSKV-

cLVTSVGVNSSYGki I mamrq

-dmeptp I qkk Idr I asa i ak I gassa if If

-lil|frflxxxxxxxxxxxxxxxx-qvtdi l ivalTVIVVA

VPEGLPLAvTLALAFATT-RMvk In-

-nLVR-VLKSCETMGNATTVCSDKTG

TLTTNKMTVV

-tgtfgdedf

i esvain-----staf-

exxxnxvp

gfvgskte--tal Igf-----xxxx----

I gmgp l a--evra-

-natvvq Impfdsgrk-cmga

-v-------- $x x \times x x \times x \times x \times x \times k-$

e

-i I ryssfvwrpsg 
pvdlvsserer I-eqvi I dy--akq----s I rt I ALVSRef

$x--x x x x x x x-x x p d d p s q a d|g x|$ I qdms I I GVVG I QDP IRPGVPEAVAKCHHag-VAV RMVTGDNMVTAKAIATDCGI

$-\mathrm{yt}---\mathrm{g}------$

------i vmegphfr

t Isdaefde-

v lpq I--qVLA--RSSPEDKRILVTKLr

$---\mathrm{al}-\mathrm{ge}-$

IVAVTGDGTNDGPALKAAN IGFSM--

xiagtev

AKEASA I VLMd-DNFSSIL taLMWGRAVNDAVRKFL $-Q$

fqi-tvn-i-tavl Itf i ssvads--_-_-_-_-_-_-_-_-_---exxsv I tavq I I wi

nl imds l aa l a l atdppt-ee--_-_-_-_-_-_-_-_- i lnrkp i---k-ggap I i s i tm w-kmi igqs if--ql ivtlt Ihf

-gprqnxxxx------------- $\mathrm{x}$

dd----vrrsivfntfvwmq i fn-efnnrr|xxrfnif----x---g|xrnwffigincim vgcqi via--fy--gga---_-_-_----afs i vqi--_-_-_-_-_-_-_-_-_-_-_-_-_-_ --_------hdeqwa----_ic--i I vaais I pwa--_-_-_-_-_-_-_-_-_-_-_-_-_-_ $-v v v r \mid f-$ 


\section{$\operatorname{xx} x \operatorname{xx} x x x x x x x x x$}

v

>anilI_IIB03 EAA60998.1

m

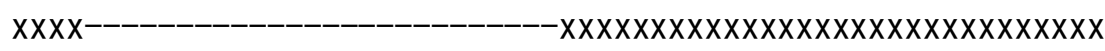

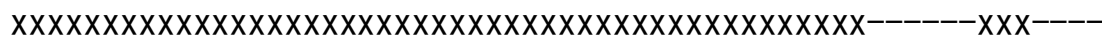
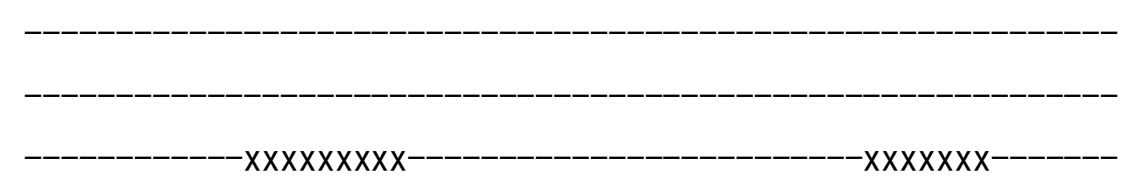

qa $\operatorname{gg}|\mathrm{sg}| \mathrm{aq}$

$-a \mid r$ -tdlksglstde$-x x x x x x x x x x x x x x x$

$---x x x x x x x x x x x x x x x x f s d r$ irvfsqn l parktt $-g f f m \mid$ I wma-y 
-ndki i illtiaa--vvsIs

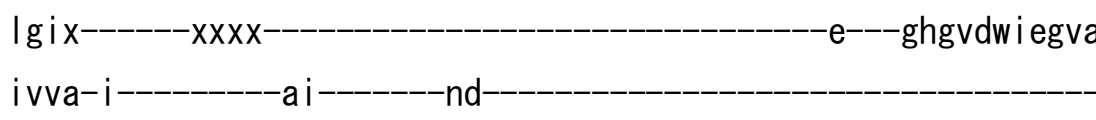

-h--gikCDESSATGESDQMKKtd-

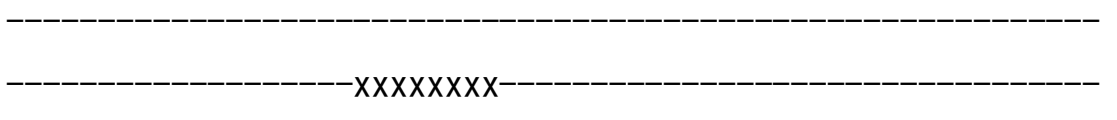

$x x x x x$

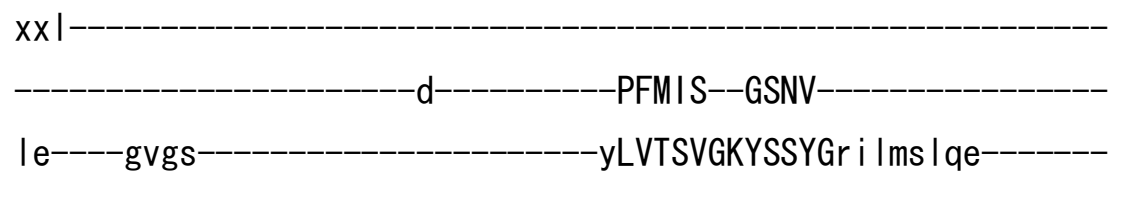

-sndptp I qvk I gr lanwi gw I gssaaiv If

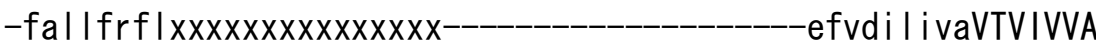
IPEGLPLAvTLALAFATT-RMvken-

nLVR-VLRACETMGNATVICSDKTG

TLTQNKMTV agt lgtkgf-

$-x x x x x x x x x x x x x x x x x x x x x x x x$

vksialn-----staf-

exxxextk- 
I gmdvat--era

-sat i iql ipfdsark-cmgv

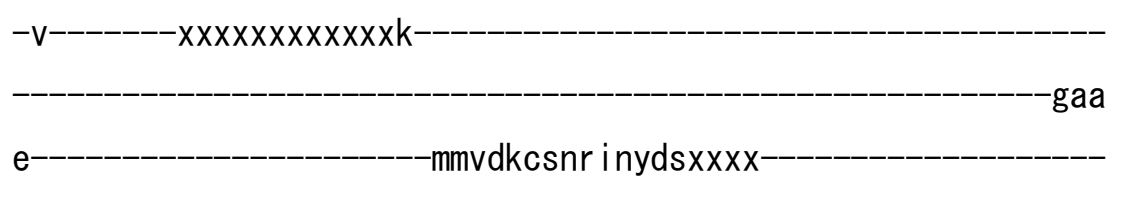

i epaaakdkqe i-le i i esy--akk----s I rtIGLVYKdf

$x x x x x x x x x x x x x q d d p d s a e f d x$ i fhdmtwLGVMG I QDPLRPEVPAAIERCHVag-VQV KMVTGDN INTATAIAESCGI $-k t e^{--} d-$

------i amegptfr

r I seeemdk

vipr I--qVLA--RSSPEDKRILVARLk

$---\mathrm{k} \mid--\mathrm{ge}-$ -TVAVTGDGTNDGPALKTADVGFSM-xiagtev 


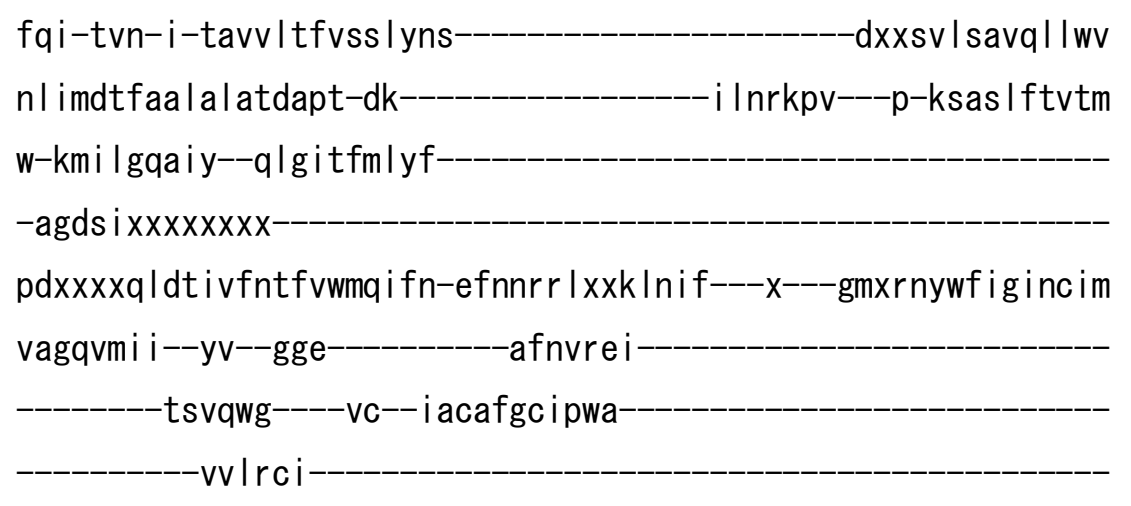

$-p d k p v x$

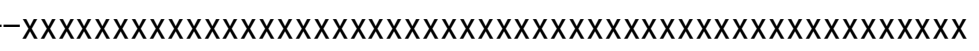

\section{$x x x x x x x x x x x x x x x x x x x x x x x x x$}

r

>anilI_IIB04 EAA60183.1

m- 


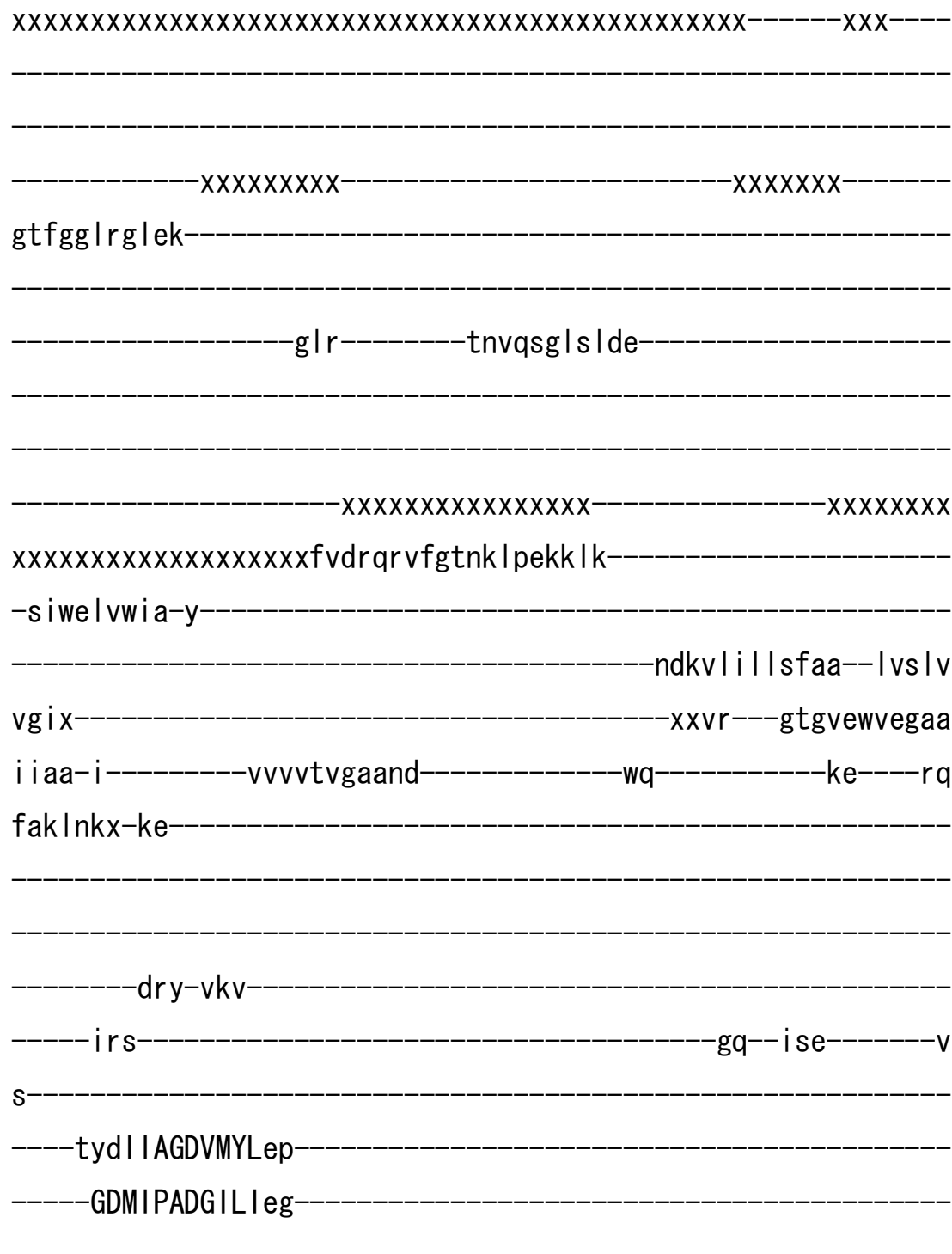

-h--gvkCDESSVTGESDLLRKtp-

\section{$-P F I M S--G S S V$}

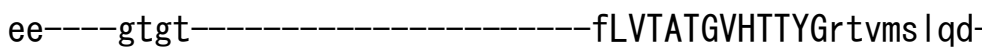

ege i tp Iqvk Inalady iakvg Itsg I i f

-vvlfikfIxxx--xxxxxxxxxxx--------x---------afIrilivaVTIVVVA VPEGLPLAvTLALAFATT-RM ikdn- 
-nLVR-LLRACETMGNATTI CSDKTG-

TLTQNKMIVv-

aat I dtasq

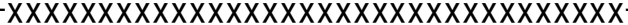

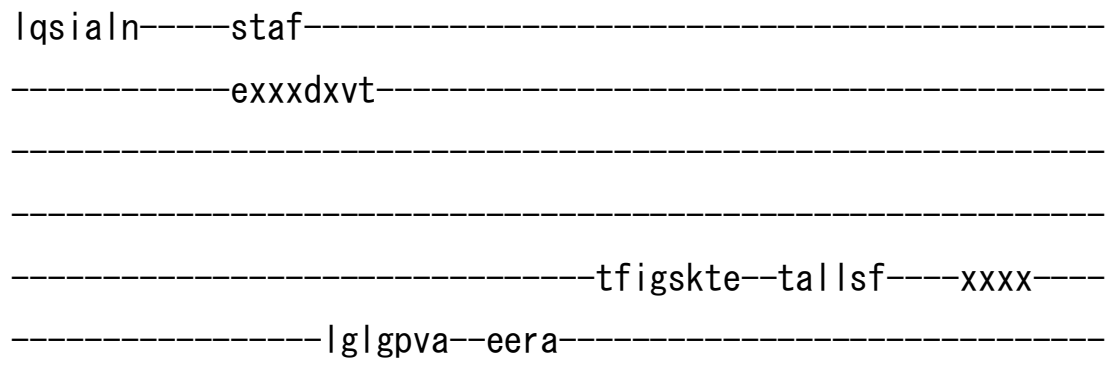

-nae i vqmfpfdssrk-cmav

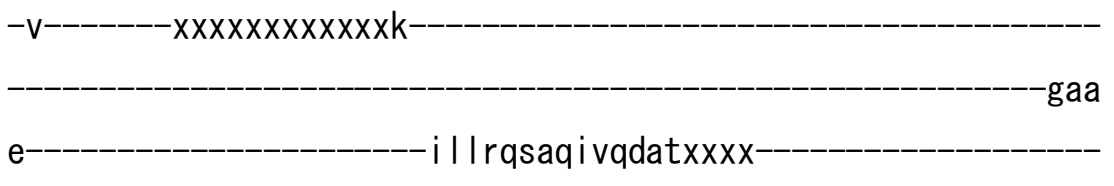

avp I seeak it I-dt i itdy--asr----s I rc IALVHRdf

x--xxxxxxxxxxene--mavfex i fkdmtmLGIFG I QDPVREGVPDAVRQCQHag-VFV RMVTGDN I ITAKA IAQQCG I $-\mathrm{ytp}-\mathrm{g}$

-x-----va i egpefr

el shdqmnk 
I ipr I--qVIA--RSSPDDKKILVSQLK

$---\mathrm{el}-\mathrm{ge}-$

TVAVTGDGTNDAQALKTADVGFAM--

xvagtev

-AKEASD I I IMd-DNFTS IVka I AWGRTVNDAVKKFL

$-Q$

fql-tvn-i-tavi Itfvsavasn--_-_-_-_-_-_-_-_-_---dxxpv I savq I I wv

nl imdtfaa la latdpps-ph---_-_-_-_-_-_---vlerkpe---p-ksap I it l tm

w-kmi i sqa iy--qlavt IvInf

-agqhix-

pkxxxxciqtvvfntfvfmq ifn-qyncrrvxxr Invi---x---gi xnnrwfia iqvi i

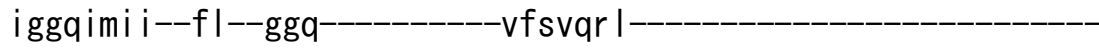

-------xqpsqwa----atxx i y lap l egpra-

rylrgk-

$-p p \mid r v x$

$-x x x x x x x x x x x x x x x x x x x x x x x x x x$

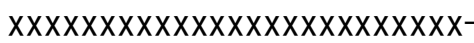

S

>anilI_IIB05 EAA67021.1

$\mathrm{m}$ 
$-x x x x x x x x x x x x x x x x x x x x$

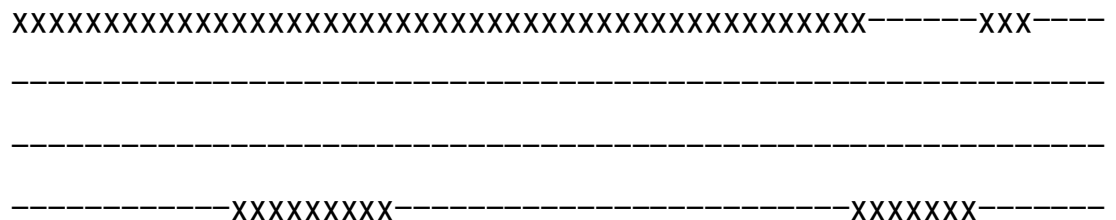

yalgg $\mid$ gg $\mid$ ey

glq--------tdlengl stte-

$-x x x x x x x x x x x x x x x x x x$

$x X X X X X X$

$x x x x x x x x x x x x x x x x x x x f a d r$ trvfgtnalpsapkk

$-k f$ ir I I wda-y

-ndk i i i l t i aa--vvs la

$\lg i x$ -xxxsg---qsqvdwi egva

vcva-i a ivvavtagnd

$-w q$

$-k q----r q$

fgk $|n k x-k|$

--_--_---drs-vra--_-_-_-_

h-

----i teLTVGDIVHLdp

GDAAPADGVI I vn- 
-h--dikCDESTATGESDQVEKvs-

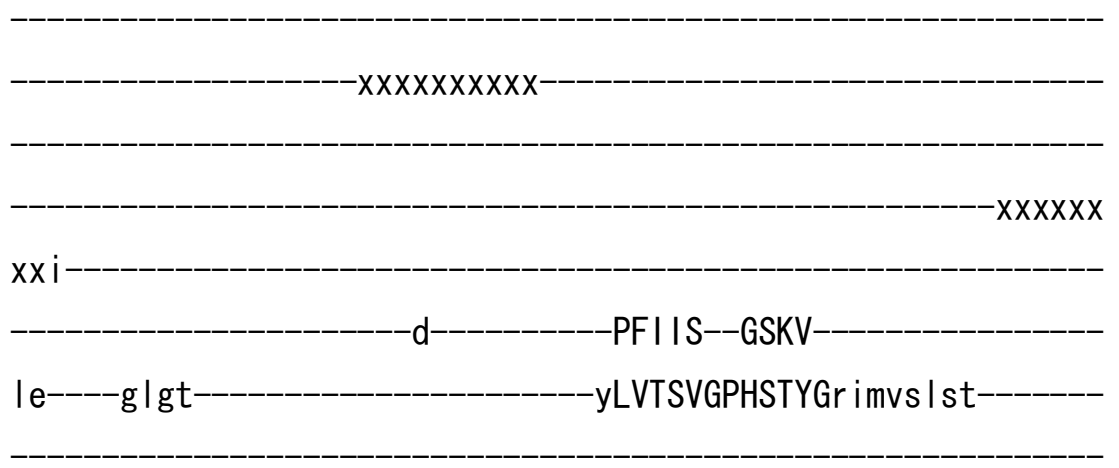

etdptp I qvk Iar I agwi gwfg I gsa I I f -fvIffrfixxxxxxxxxxxxxxxx--------x----_-----hfmdi I i vaVTVIVVA IPEGLPLAvTLALAFATA-RMI ken-

-nLVR-LLRACETMGNATV ICSDKTG

TLTQNKMSVV agfcsages
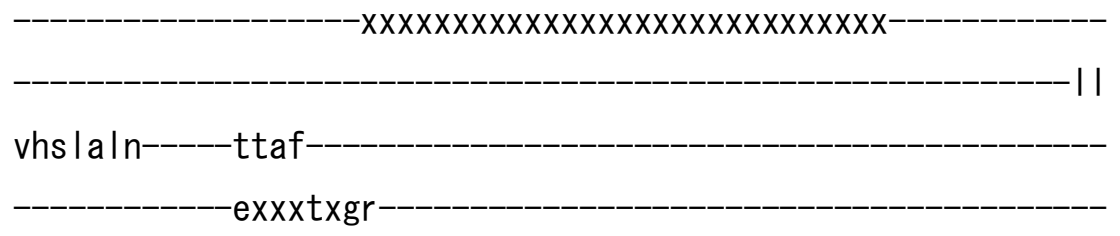

efvgnkte--ial|ql-----xxxx----

| gmd l sr--iqa

dnr i shvypfdssrk-amav

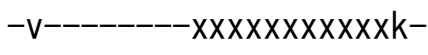
$\mathrm{e}$

i I l daavq i vqpgpxxxxxx 
paq i sdsdrhl i-sgr insy--ara----s I rt IGIAYRdf

-xxxxpnmkr tpnfsx i l ke i tw I GAFG I HDPLRPEVVEA I GNCHSag-VQV KMVTGDN INTALS IAESCGI $-k t e--d-$

------i amegpe $\mid r$

$k|d k d q| d e-$

vvpk I--qVLA--RSSPNDKELLVKHLk

$---r \mid--g e-$

IVAVTGDGTNDGPALKAADVGFSM--

$-x \mid \operatorname{sgtd} v$

AREASS I ILLd-DNFRS IVta I SWGRAVNDAVAKFL

Q

fqi-tvn-i-tavlltvvtaiyns-

rxxsvfravq||w|

nl imdtfaa la latdppt-ad------_--_---_----i l krppt---p-rhap I ftvtm

w-kmi lgqsi iy--klalcfvlyf-

-cghsixxxxxx

syxxxxeldt i ifntfvwmq ifn-efncrrlxxrfnif----x---gixrnvwffvinl im vggq i l i i--fv--gga-----------afgvtr I-

tgrqwg----ic---I gfavvc i pwa

$-a|l k| v$ 
$\mathrm{h}$

>ddi II_IIB01 XP_642164. 2

$\mathrm{m}$

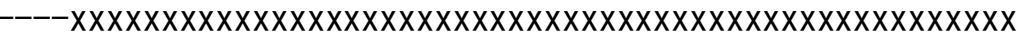

-sniktglplek -

xxxeenrvl kyskni Ipdpphq

-p|wsiv|dax 


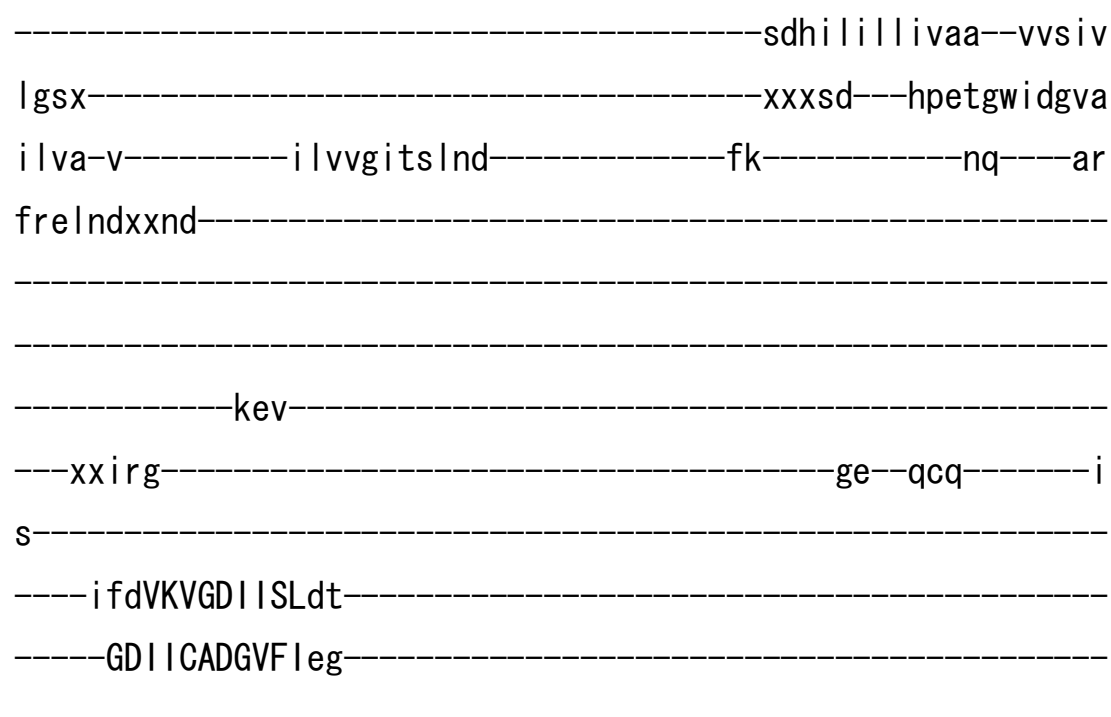

-h--al kCDESSI TGESDP IKKgq-

$-x x x x$

$$
-d-
$$

ie----gfgt-PFLIS--GSMV -mLVTAVGVNSFNGktmmg I rv

-asedtp I qmk I sv lasr igyfgmgaa I Im I

- I ia ipkyfxxxxxxxxxxxxxxxxx-pivqlvisaltIVVVA

VPEGLPLAvTMALAYGMM-KMfken-

-nLVR-NLASCETMGSATT ICSDKTG

$-T L T Q N V M S V V$ tgt icgvfp-

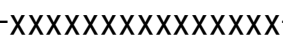

tdgma in------snay

exxxsxgkx 
-efigskte--cal Inf-----xx-x

-fgcdyne--vrk

r levvelypfssark-rmsv

$-\mid--------x x x x x x x x x x x k$

-gas

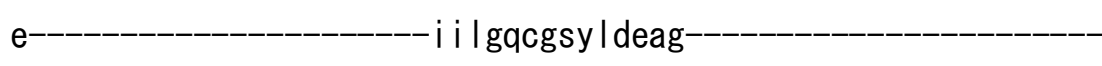

ni rp i seakayf-eeq innf--asd----al rt IGLAYRdf

-qygecdfkexpenn I v I I I VG I KDPLRPEVPEAVE I CKRag-IVV

RMVTGDNLVTAQN I ARNCG I

Ite--g------

------I cmegpkfr

el sqsemda

i l pk |--qVLA--RSSPTDKQLLVGRLk

$---\mathrm{dl}--\mathrm{ge}$

-VVAVTGDGTNDGPALKLANVGFSM--

-xisgtev

-A I AASDVVLLd-DNFAS IVraVLWGRN I YDA I CKFL 
fql-tvn-v-vavtvaf i $g$ I Itsdxxxxxxxxxxxxxxxxxxxxxxxrxxsp I tavq I I wv nl imdt l aa la lateppt-pe---_--_-_-_-_--- I lerppn---g-knap I itrsm w-kni igqaal--qlailfti ly -qghni $x x x x--x x x x x x x x x x x$ gl----hhyt I vfncfvflq Ifn-e inarvl xxrtnpf----x---nfxnnp if i avmift I gvq i i fv--tf--ggs--_-_-_----atstds |--_-_-_-_-_-_-_-_-_-_-_-_-_ -y i vewi----cc--vvvgai s lpvgIllrki

pi repx $-\mathrm{XXX}--------X X X X X X X$

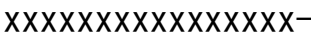

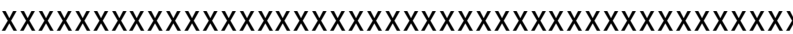
$x x x x x x x x x x x x x x x x x x x x x x x x$

$-\mathrm{X} X X X X X X X X X X X X X X X X X X X X X X X X X X X X X X X X X X X X X X X X$

$\mathrm{n}$

>ddilI_IIB02 XP_636219.1

$\mathrm{m}$ 


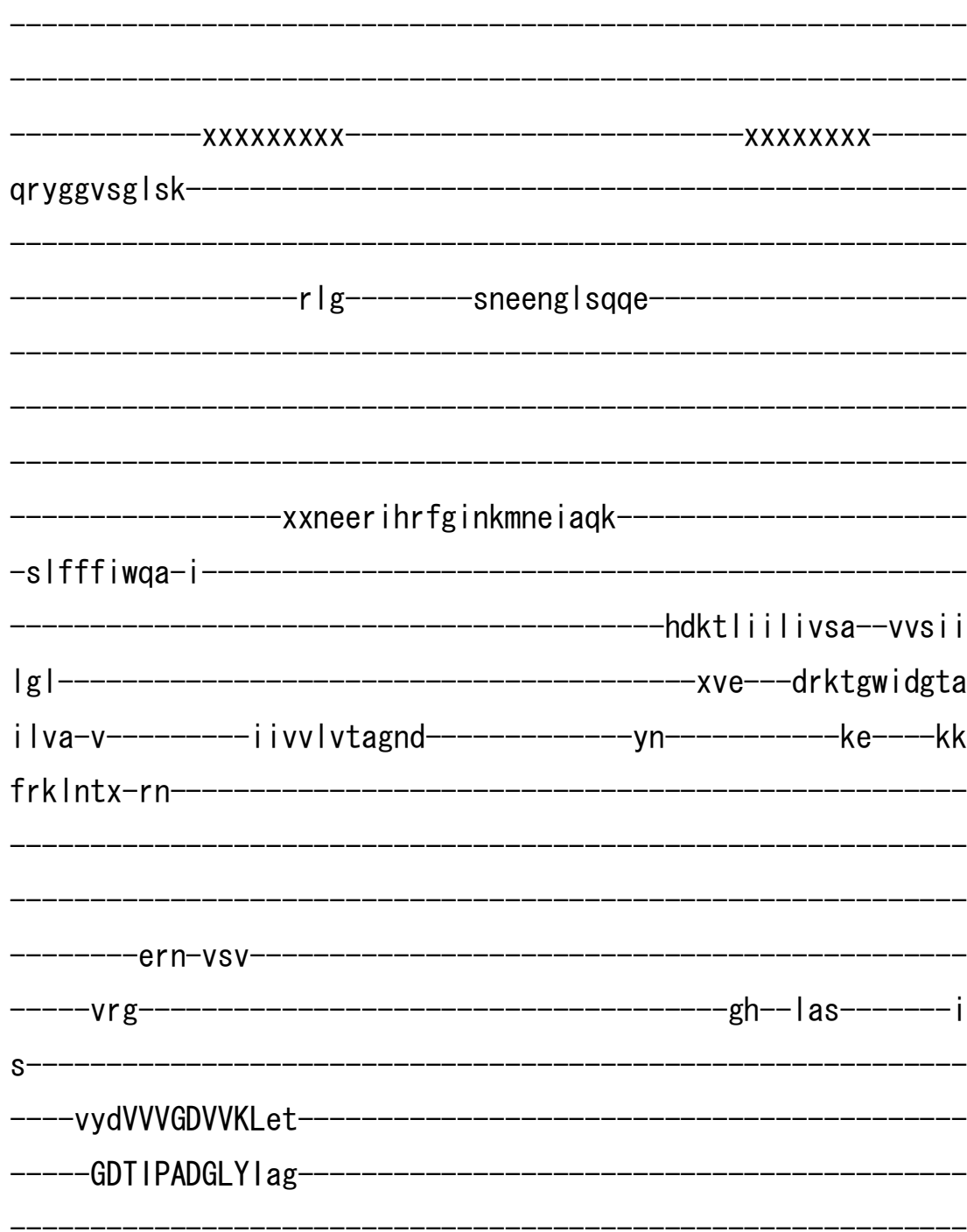

q--s i aVDESSMTGESDQKRKs--

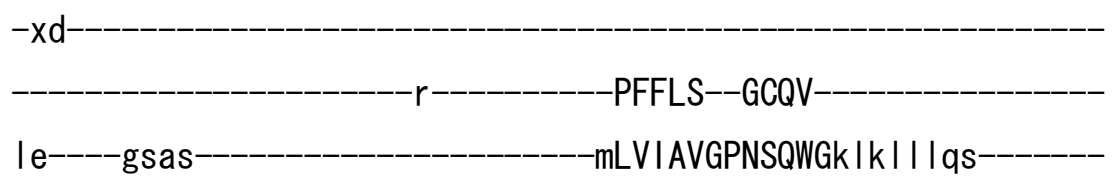

-pdsdtp I tqk l ek laet igkfg I i aai Itf

-gv I I lkyvxxxxxxxxxxxxxxxxtivgfvvtalTIIVVA VPEGLPLAvTISLAYSMM-KMmkdq- 
-nLVR-HLEACETMGGATN I CSDKTG-

TLTQNRMTVV

kk i igks in

$-x x x x x x x x x S x x x$

XXXXX i ysspseXXXXXXXXXXXXXXXXXXXXXXXXXXXXXXXXXXX

aeg i $\ln$------stay

ixxxtxr|x

dh igskte---cal lew-----xxxx----

pnqsyet--vrkex

-kdrvvkvypfssekk-msav

$-\mid \operatorname{xx} x x x x x x x----x x x x x x k$

gaa

e-

iv Innctni vdkngx

stamsrdekm I --qkd i i if--ase-----g I rtLVLAYKe i

-xnepssedeakx i ytg I tfLGLVG IKDPVRKEVPRAVKRCQGag-IFV

RMLTGDN ILTAKN I ARECG I-

lkd--g-

-x-----vai egpqfr

- ltddq Idt 
i i ph I--qVIA--RCSPTDKFRLVHRLr

$---\mathrm{el-}$-ge-

-VVAVTGDGVNDAPQLKEADVGFSM--

xiagtev

-AKEASD I VLLd-DNFNS I AkaV I WGRNVYDS I RKF I

$$
-Q
$$

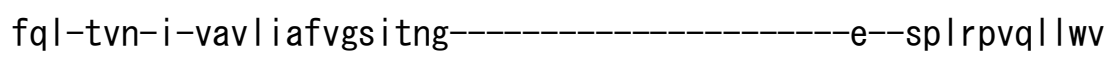
nl imdt I galal stepps-ee--_-_-_-_-_-_-_-_-- I fnrrpy---g-rfds I i tyrm w-rni igqs i y--qiafIfs imy-

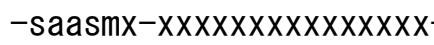

kt----vyht i ifntfvfcqffn-e incrvlxxqlnvf----x---ni $x k s y v f v g v v s f c$ ifiqvilv--ef--gge-----------ffgtrhl-

-dykqwf----fc--i i igfgsl iwg-

$-f c|r| \mid$

$-p l k d r x$

$\operatorname{xxxxxxxxxxxxxxxxxxx}$

$\operatorname{xx} x \operatorname{xx} x \operatorname{xx} x \operatorname{xx} x \operatorname{xx} x \mathrm{x}$

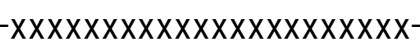

$-x x x x x x x x x x x x x x---$

$-x x x x x x x x x-$

$-\mathrm{XXXXX}$

S

>ddi II_IIB03 XP_638496.1

m- 
-sffsk-Imexf

-qdp I ihi lcval--vitvi

Isfx gyaewfegvg

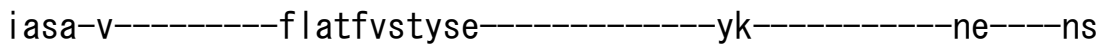

fqelqexxsr

$-v k c$

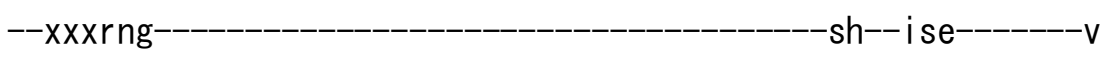

$\mathrm{y}$

----gfdVVVGDLVLLqa

GDKIPADGRLVag 
e IhVNQSTLNGEPHLEKK i v-

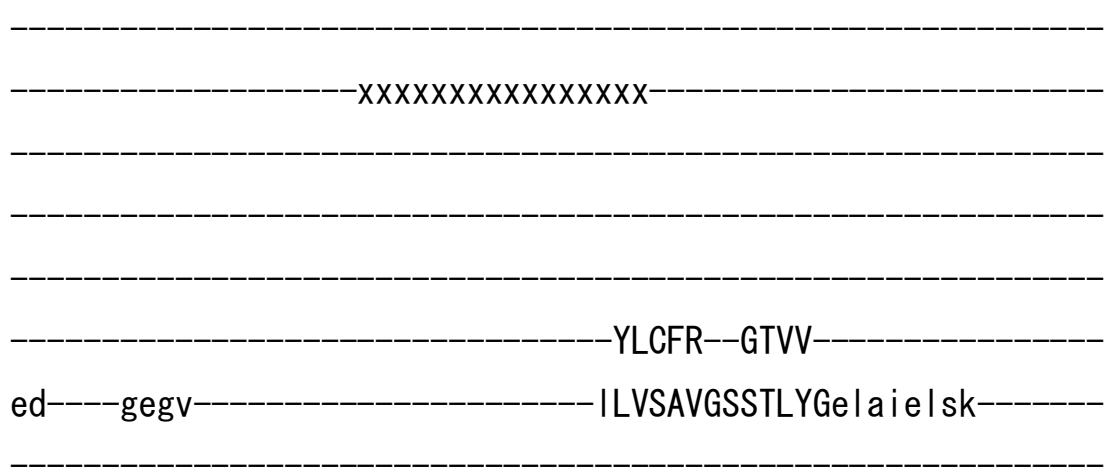

-aderesplqikIsnladg i st igy igagf iv -isflfkqf $x x x x x x x x x x x x x x x x--------x x x x x x x----$ divnsitlall IIVVA VPEGLPMM i A IVLSLNMR-KL I kak-

-vLVR-KLLGIETAGSLDILFVDKTG

TLTKGKFIP -rtfisgsah-

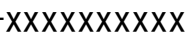

revlsftxxxxxssvi

$-x \operatorname{exgq}$

i vggnasd--ka l lqf

|dkes | |--ady

qvkveke i I fhserkxsaal xxxxx

$x x x x x x x x x x x x x x x x x x x x x x x x x x x x x x$

$\mathrm{xc}-$ $-x k$ $\mathrm{e}$

-ivInrctshfnedgxxxx 
-ipdsvg|-tnsins|--seq----girvIAVAVSre

- I vetex I pns I i IVGIVGVYDEI REESRSSIQTARSag-IQV

VMI TGDKKETAIAVAHQIGL

isp--g-

$-x x x x x x v \mid t s f e l a$

nI sdnk I an-

mlphl--rVVS--RALPSDKVRFVNVAq-

$---s \mid--h k$

-VVGMTGDGVNDSAALKHADVGFAM--

gsgsev

SKEAADIVILd-DNFASI TqaVLYGRTIYKSIQKF I $-\mathrm{V}$

fqs-t in-v-ast I i vf I gpfmgf--_-_-_-_-_-_-_-_-----d--fp I t I iq I w w nI vmdt l aa l a ggepa l-tr---_-_-_-_-_-_----ymqekp i---k-rdqs i i tsrm w-gs i Igggpfxxams i IfItndxxxxxxxxx-

gexxxxafl taffaffiflsvin-afnvrts--r InIfx------hl xdnkgfivvvffi ffvqivft--yi--ggk----------i|rtvg|-

-tssewa----Iv--vamsfsvipfd-

lirkfa- 
>mgr II_IIB01 XP_365785. 2

$\mathrm{m}$

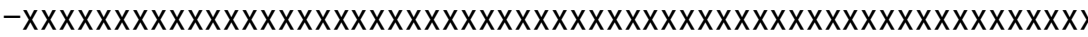

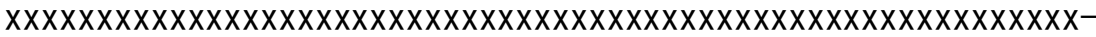

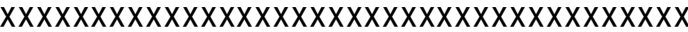

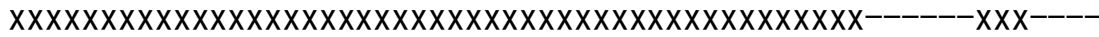

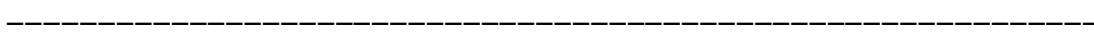

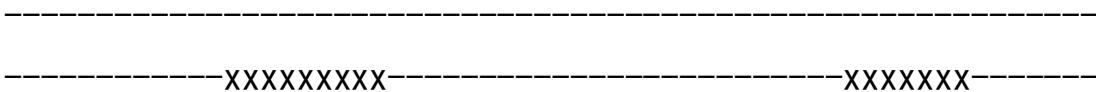
$y k|g g| s g l e k$ gl r--------tdrkag | svde$x x x x x x x x x x x$ $\mathrm{XXXXXXXX}$

$x x x x x x x x x x x x x x x x x x x f v d r k r v y k d n r \mid p e r t g k$ $-s|l q| m w i t-f$ 
-ndk i I I I sgaa--ais la

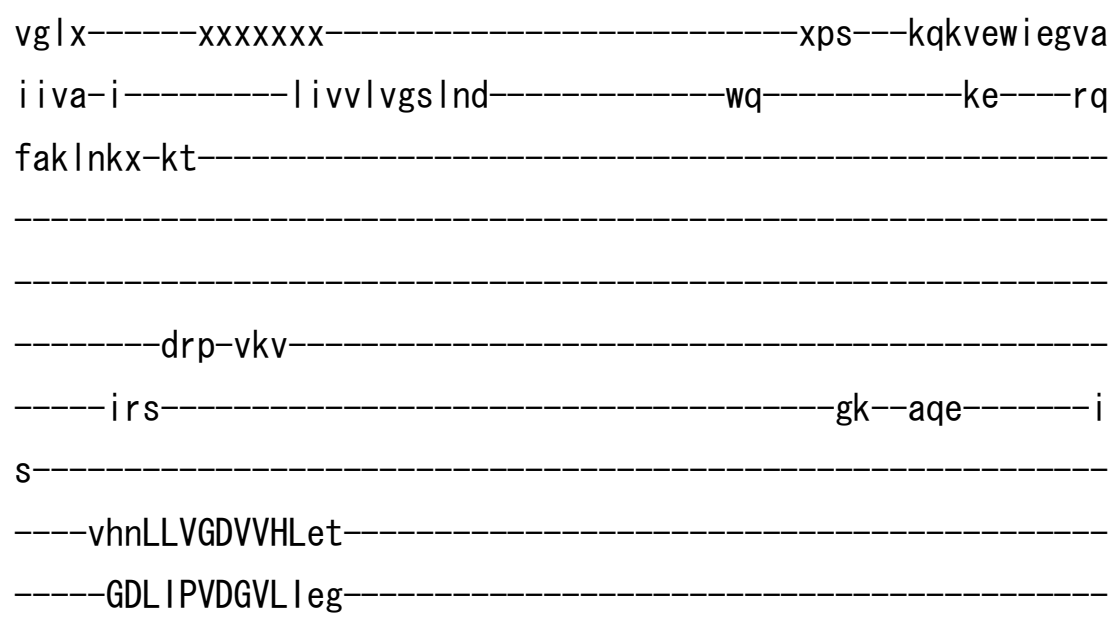

-h--nikCDESQATGESDLIKKr--

$-x x x x x x x x x x x x x x x x x$

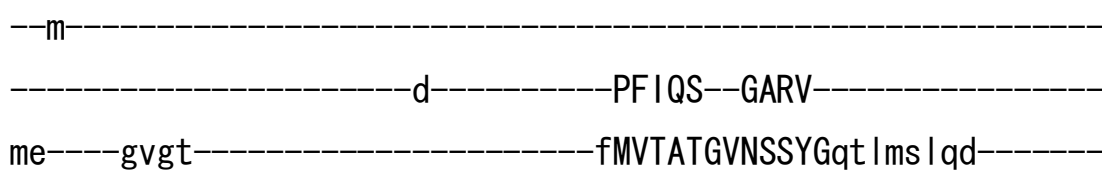

-dpe itp Iqqk Inviadgiakiggaaal IIf

-Ivlfikflxxxxxxxxxxxxxxxx--------x---------qfirifivvVTIIVVA VPEGLPLAvTLALSYATK-KMvkqn-

-nLVR-QLKACEVMGNATTICSDKTG

TLTQNKMKVV-egtvgtthr

$-f x x x x x x x x x x x x x x x x x x x x x x x x x x x x x x x x x x x x$

$-\mathrm{vl}$

Irsiv|n-----staf-

$-e x x x d x e q$ 
-nskt lql ipfdsgrk-cmg i

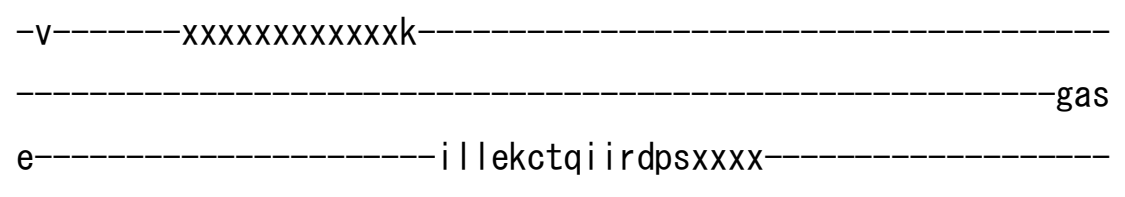

tat Itdenrtg|-ns I i eny--akk----s|rt IGICYRdf

$x--x x x x x x x x x x d g e n d e v k f e x$ i fkqmt ILGVVG IKDPLRDGVREAVKDCQRag-VVV RMVTGDN I MTAEA I ARDCG I Iqp--d-

------i imegpkfr-

nI skreqed-

vvpr I--hVLA--RSSPEDKRVMVKRLk

$---\mathrm{dk}--\mathrm{gh}-$ - IVAVTGDGTNDAPALKMADVGFSM-xvsgtev 
fql-tvn-v-tavl|tfvsavsndrxxsvItatql Iwv

$\mathrm{nl}$ imdt l aa la latdpph-ptvld--pw---n-----y ipav $\mid$ C -nrr|xxrfni I----x---gi ixknp ifia i s lam

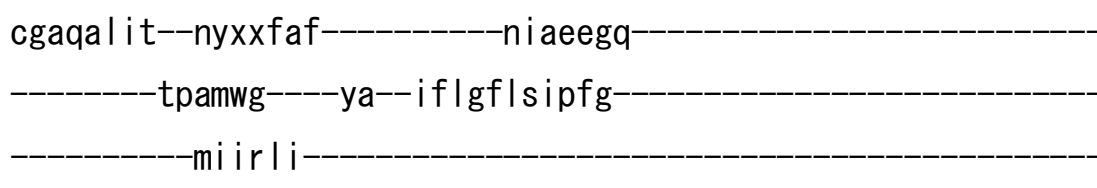
$-p d a f v x$ $-x X X X X X X X X X X X X X X X X X$ $x x x x x x x x x x x x x x x x-$

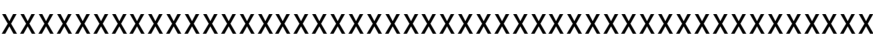

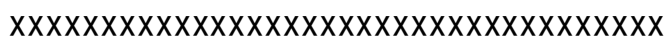
XXXXXXXXXXXXXXXXXXXXXXXXXXXXXXXXXXXXXXXXXXXXXXXXXXXXXXXX

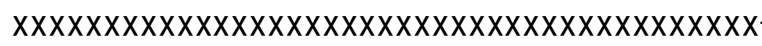
$-x x x x$ e >mgr II_IIB02 XP_359887. 2 $\mathrm{m}-$ 


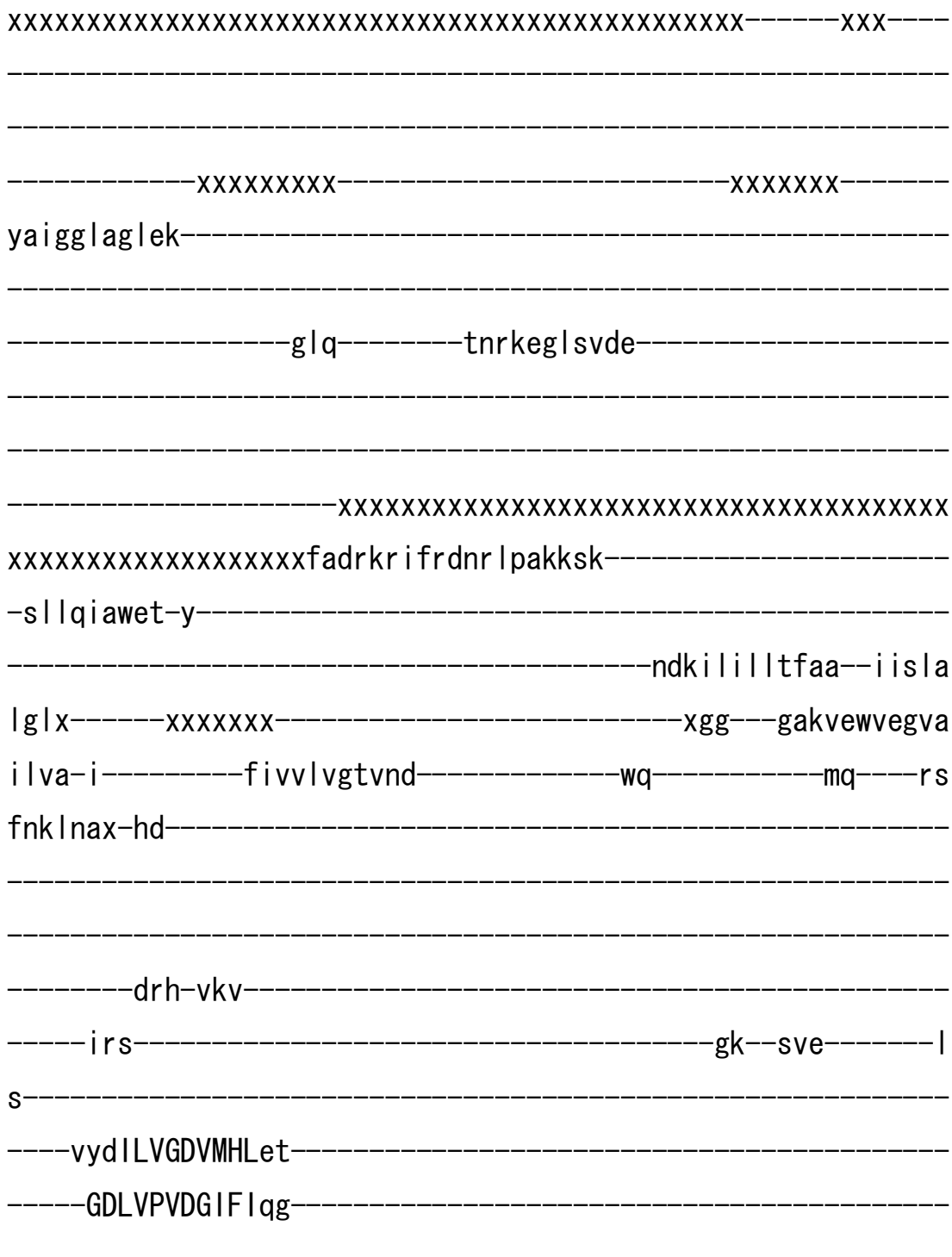

h--gvkCDESSATGESDLLKKtp-

$-X X X X X X X X X X X X X X$

$-X X X X X$

$\mathrm{XXm}$

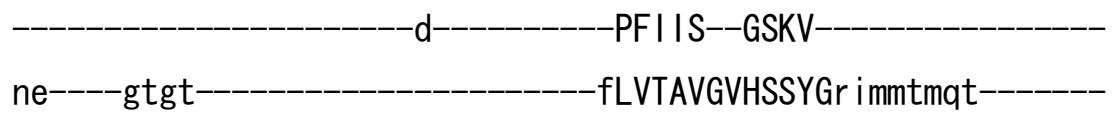

-gqestp Iqqm Ink Iadmi ayagtgsa IIIf

-vvIfikfIxxx--xxxxxxxxxxx--------x----------tfIr IfitaVTVVVVA

VPEGLPLAvTLALAFATT-RMtkdn- 
-nLVR-VLRACETMGNATTI CSDKTG-

$-T L T Q N K M T V$ att Igts Is

---xxgtdem lexxxxxxxxxxxxxxxxxxxxxxxxxxxxxxxxxxxxx-

tyigskte--valltf-----xxxx---- I gappva--evrs-

-nsdvvqvvpfdsalk-ymat

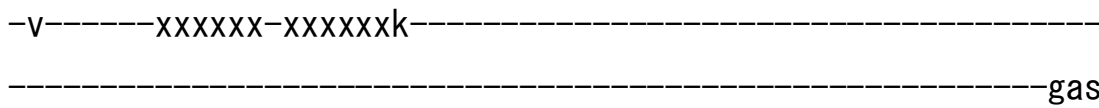

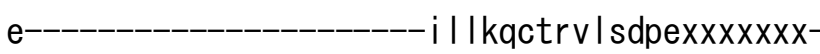

elt--delretf-nstitsy--agq----tIrtISSSYRdf

$x--x x x x x x x-x x k e d p r$ sadfnxvhsdmt I VS IFG I IKDPLRPGVIDA IKDCKRag-VVV

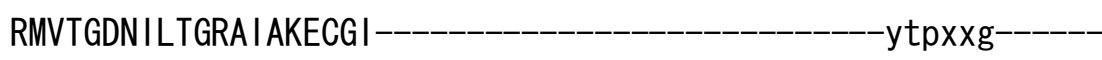

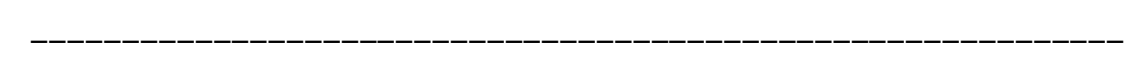

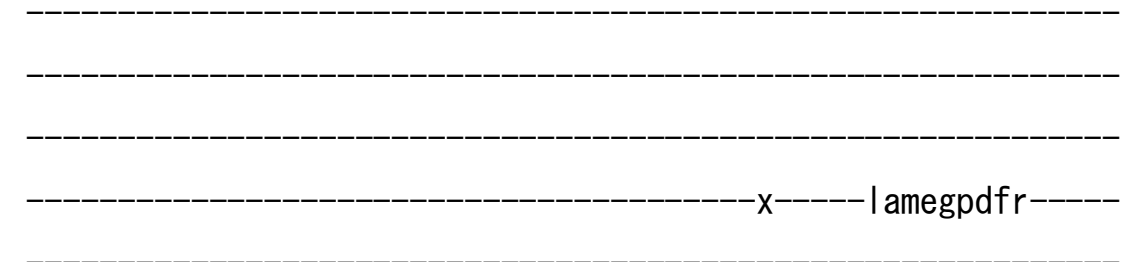

-rkseeelke- 
i apk I--qVLA--RSSPEDKRILVKILk

$---\mathrm{el-}-\mathrm{ge}$

-TVAATGDGTNDAPALKMADIGFAM--

-xiagtev

-AKEAAA I I LMd-DNFAT IVkaMAWGRTVRDAVKKFL $-0$

fql-tvn-v-tavv I vfvsavsss---_-_-_-_-_--_--_-_---txxsv I navq I I wv nl imdtmaa la latdpph-ps---_--_-_-_-_-_-_--i I hrkpd----r-ksas I i tpam a-kmi igqaic--qlait IvInf

-ggys $\mid x x--------x x x x x x x$

ei----rlkt Ivfntfvwlqifn-evnnr Ixxk Ini I---x---gvxknywf I gvnl im i ggqv l i i--fv--gre--_-_-_-_--_afki vp I-_-_-_-_-_-_-_-_-_-_-_-_-_-_-

--------dgkewg----i is--i g I gai s i pwg--_-_-_-_-_-_-_-_-_-_-_-_-_-_

$-\mathrm{mi}$ irli

- pdhw ix $-x x x----x x x x x x x x x x x x$

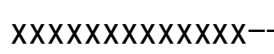

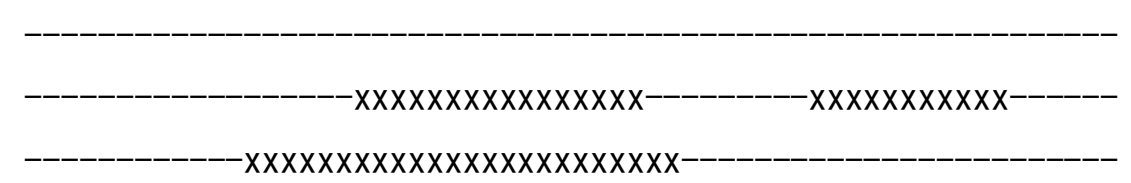

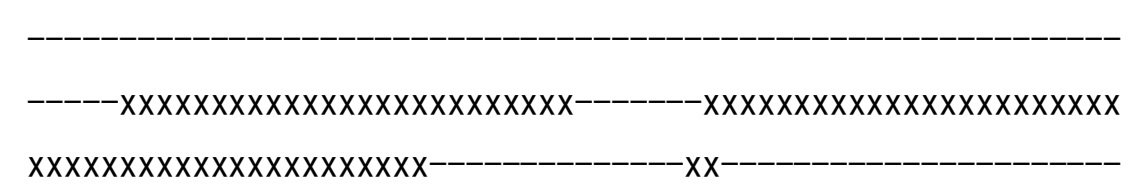

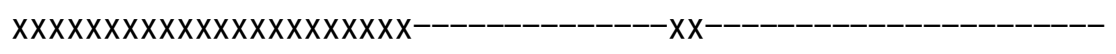
$-x x x x x$

d

>mgr II_IIB03 XP_368067.2

$\mathrm{m}-$ 


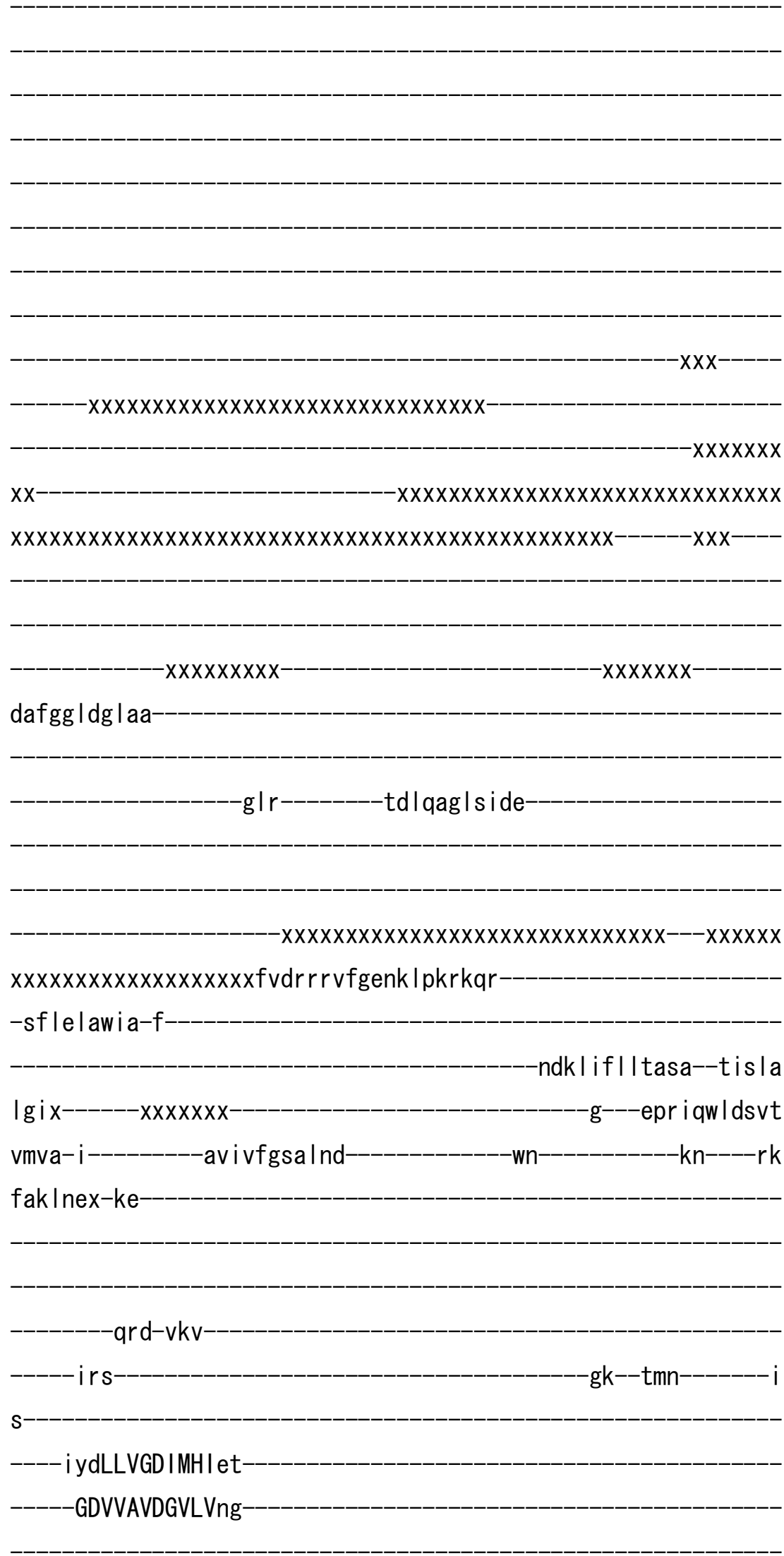


---g iqVDESSLSGESELVHKta-

$-x x x x x x x x x x x x x x x x x x x$

$-X X X X X$

xxs

d---------PFILS--GTTV-

ng----gvgn-----------------------yLVISVGTNSTFGrt I mn I rt

dveetp Iqqk I gk Iarql it Iga Iag Ivff

-I vmf irfcxxxxxxxxxxxxxxx---------------------effkt I i laVTVVVVT

VPEGLALAvTLGLAFATS-RMIkdk-

nLVR-MIRSCEVMGNATCICSDKTG

TLTQNVMTVV

-agn i gaaea

$-x x x x x x x \operatorname{exx} x$

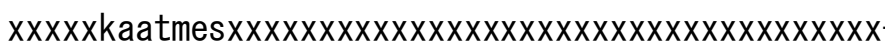

ksgivhn-----ttaf-

$\operatorname{exxx}$

gfvgmste--tal lkf-----xxxx----

- amgp In--eert

nad i vdmfpfdasnk-wmav

$-\mathrm{m}-------x \times x \times x \times x \times x \times x \times x k-$

e

-v I Idqctral sdpkxxxxxxxx 
tedftpemhen I-rqvvqsy--avk----mI rpVAMAYRdf

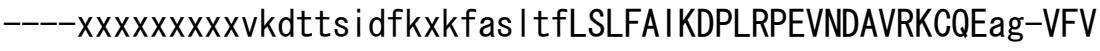
RMVTGDNFLTAKAIATECGI

------i amdgptfr

$-k \mid$ seaq $|d|$

vipr I--qVLA--RSSPEDKLLLVSHLk

---gm--me-

-TVAVTGDGTNDALALKAADVGFAM--

-xiggtev

AKEAASI ILLd-DNFTSIVkaLSWGRTVNDAVRKFL

Q

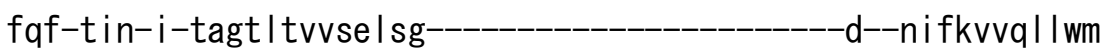

nl imd i fas I g latdyps-pd--_-_-_-_-_-_-_-_-_f | krkpq---p-rkts I vs I tm

w-kmi vgqal $y--q$ l ivift lhy

- gkdal $x x x x x x x x-$

ed----qlqt I vfni yvwmqffn-qhncrrvxxk In iw--xx---gvxrnpwfigvqc/t

fagqmi i i--yk--ggq-----------afqtvp I

dgpqwg----ws--m I fg i l t i p lg

$-v \mid$ irlt 
$-X X X X X X X X X X X X X X X X X X X X X X X X X X X X X X X X X X X X X X X X X X X X X X X X X X X X X X X$

$\mathrm{XXXX}$

$-X X X X X$

e

>cellI_IIB01 NP_501709. 2

$\mathrm{m}$

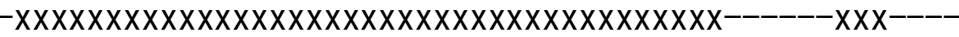

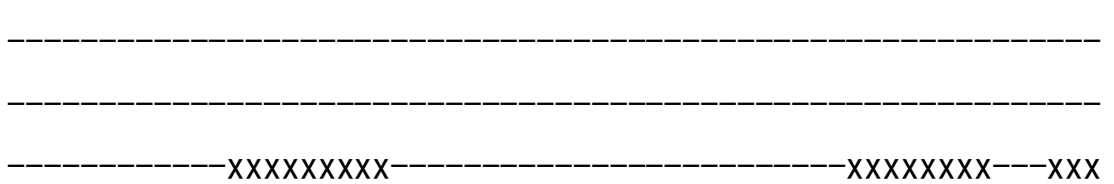

tehegveglck

-k lk----_----tds I vg I ngeq-

xxIdrr rhvygant ippaksk

-gfvrlvida-c 
$-k d p t \mid v i l v l s g--f i n l a$

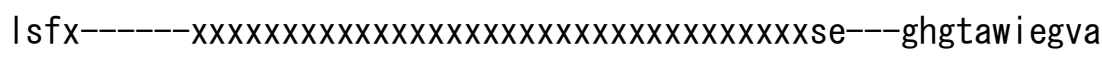

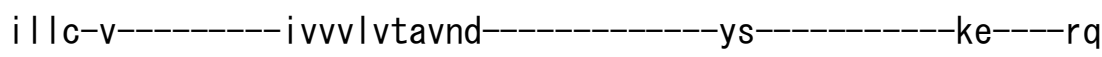
frslqexxet-

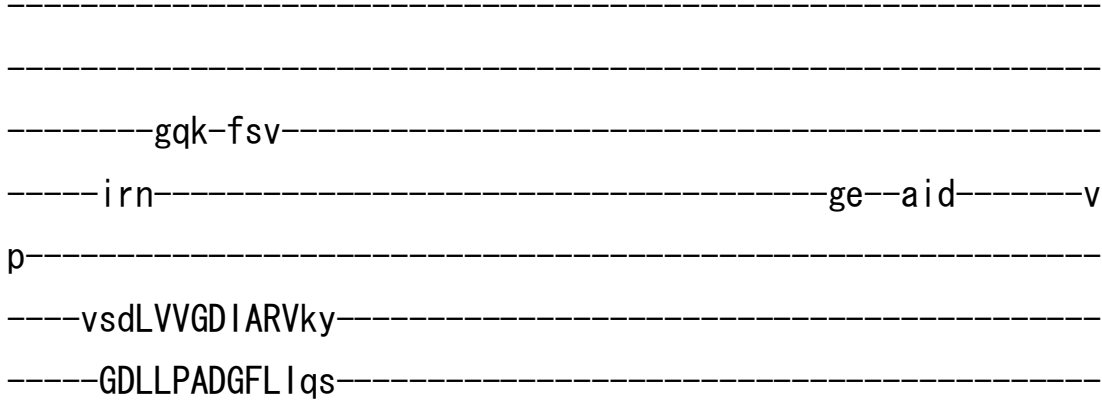

-n--dIkIDESSLTGESDHIKKs i-

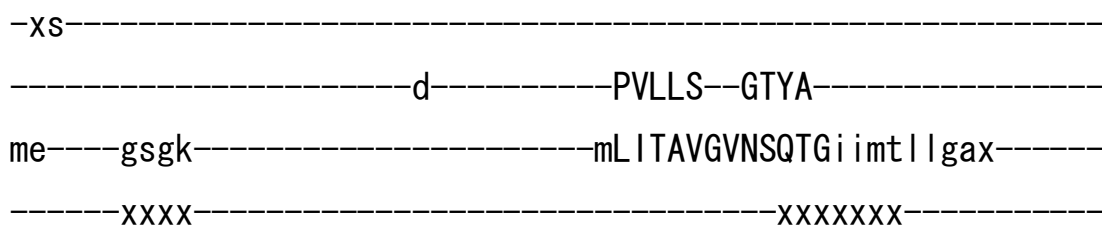

$-x x x x x x x x x x x x x x x x x x x x x x x x x x x x x d$ ltaksv lqaklsklalqi i ycgtt $i$ a i ial -ivlvtrfcxxxxxxxxxxx-----x--------xxxxx------mfvkffi iaVTILVIS IPEGLPLA i ALALTYSVR-KMmhdn-

-nLVR-HLDACETMGNATSICSDKTG

TLTTNRMTV -qsy ingnhy-

$-x x x x x x h x x x$ lpgstgp

meaisvnxxxxxmi ve pxxxgxqi 


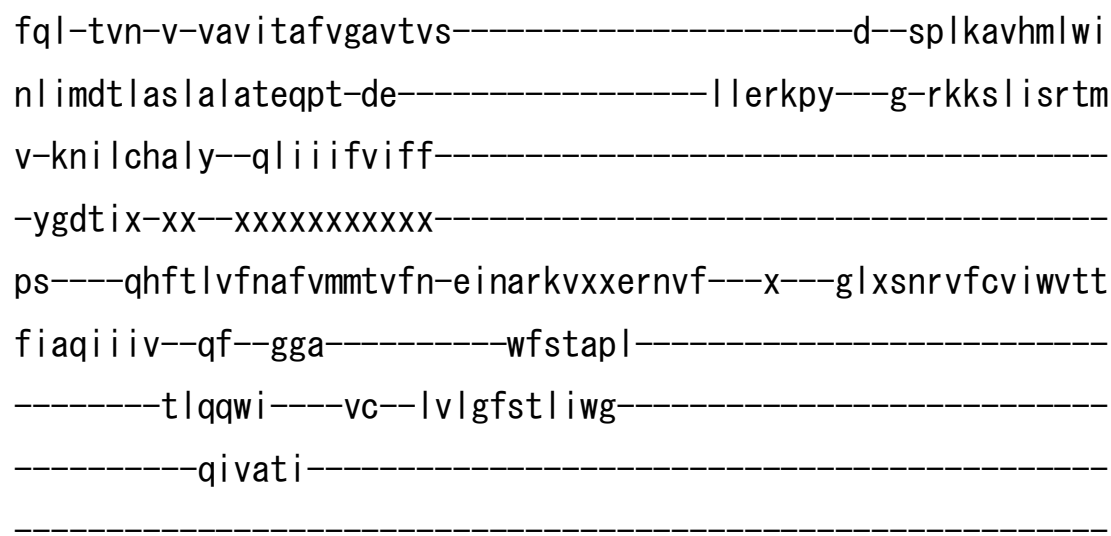

$-p s k k \mid x$

$-x x x-$

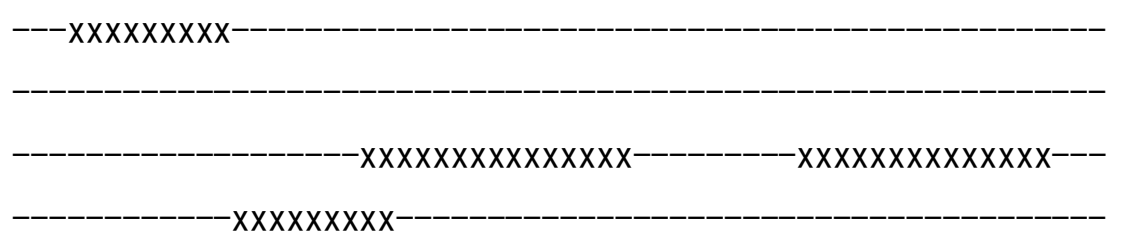

$-x x x x x x x---x x x x x x x x x x x x x x x x x x x x x x x x x x x x x x x x x x x x x x$

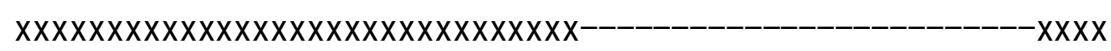

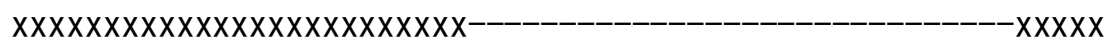

$n$

>cellI_IIB02 NP_500161.1

$\mathrm{m}-$ 


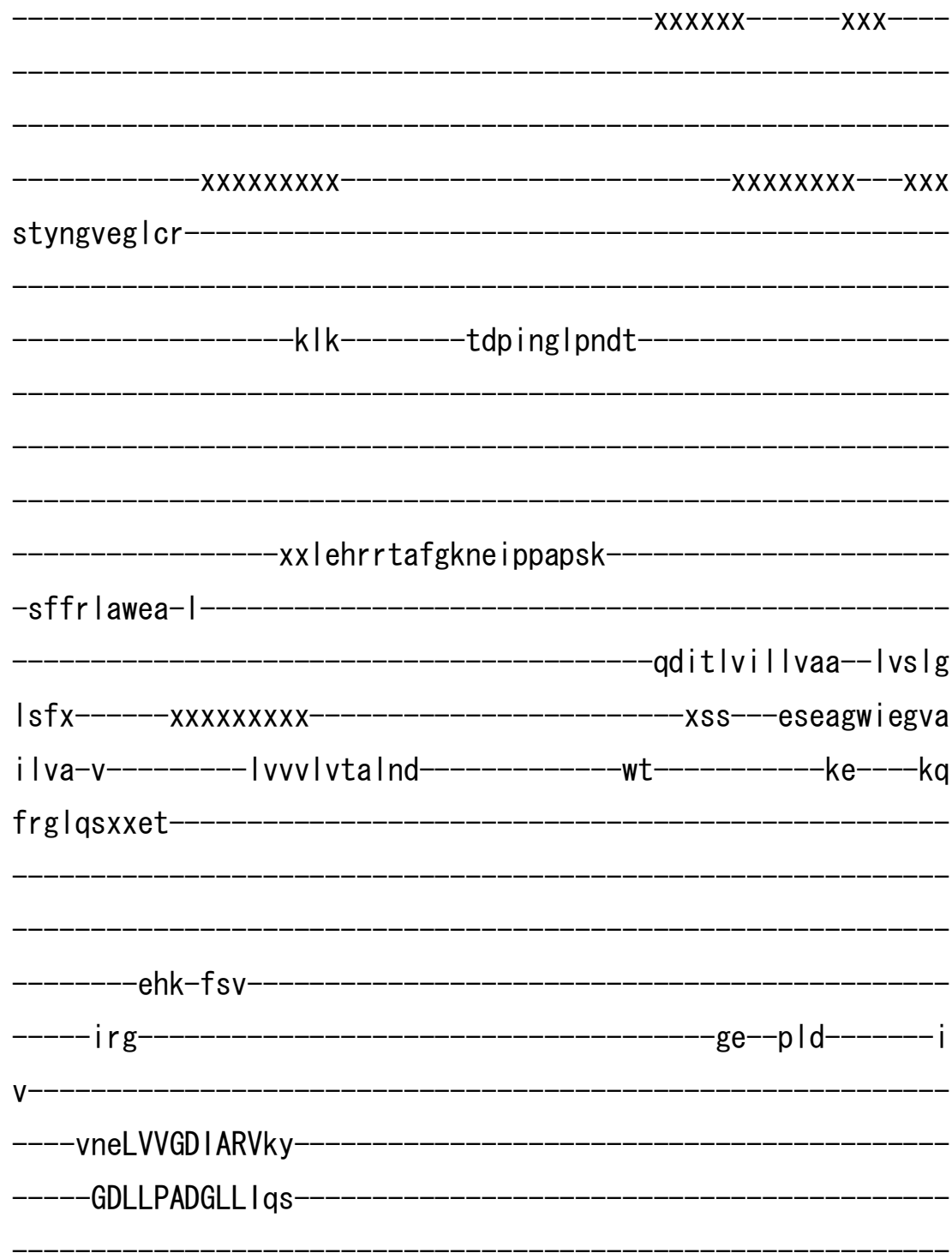

-n--dIkIDESSLTGESDLIRKse-

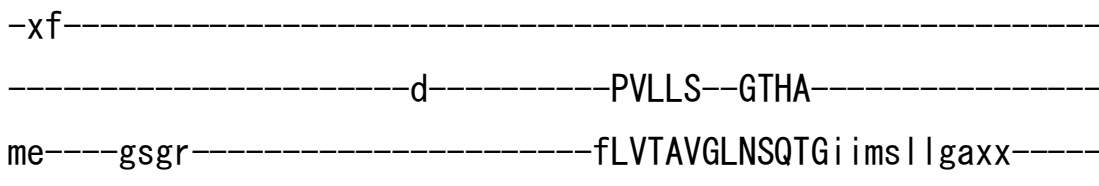

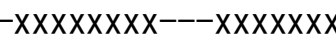

$x x-x x x x x x x x x x x x x x--x x x x x x x x x-$ grmsksv lqtk Isn la lq i gy i gs i vaaatv

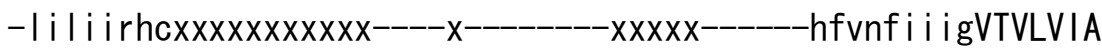
VPEGLPLA i TLALTYSVK-KMmkdn- 
-nLVR-HLDACETMGNATS ICSDKTG-

TLTTNRMTC

qqy insefy

$-x x x x x x x e^{--x}$

- mdpstrd

fngivinxxxxxtvvt

pxxxgxar

gqignkte--cs I Igf-----xx-x----

-sgrsyed--Irrqxx

eek I ykvytfnssrk-smmt

$-\mathrm{V}-\mathrm{xx} x \mathrm{x}--\mathrm{xx}-----\mathrm{xx} x \mathrm{xx} \mathrm{xk}$

gas

$\mathrm{e}$

- i i t trony i fgksgx-

i eqf gpkeaavmxknv i epm--asd----g | r t IGLAFKd I $-\mathrm{X}$

$x x x x x x x x x x x x x d g$ e idwedee $x$ i regqtv I AVMG I QDPVRPEVPAA I AKCQKag-I TV RMVTGDN INTARS I ATQCGI-

$-m q p--g$

$-x x x---\mid$ a legkefnx

$x \times x x-$

xxx--------------kvnqqkfda- 
i wpk I--rVLA--RAQPSDKYVLVKGI i

---esxxtk-------xxx--------------VVAVTGDGTNDAPALKKADVGFAM--

$-x i a g t d v$

-AKEASDI ILTd-DNFSS IVkaVMWGRNVYDSI AKFL------Q

fql-tvn-v-vavt i af i gaca i s---_------_---_--_-----d--sp I kavqm I wv

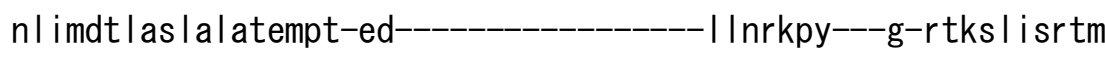
v-kni vghavy--q lai I faimf

-wgdk | xxxx--xxxxxxxxxxx-

ps----ahft i i fnafv Imt I vn-e i nark i xxernvf----x---g i x tnp i fcv i witt I i sh i I iv--qf--ggq-----------wfstap I-

-d l taw i----ic--iccgvge I fwg

qi inc $\mathrm{i}$

-pasilx

$-x x x-$

$---X X X X X X X X X X X X X-$

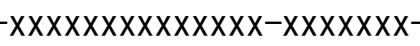

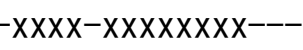

$-x x x x x x x x x-$

$-x x x x x x x-x x x x x x x x x y-$

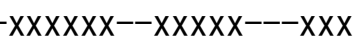

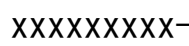

$-x x x x x x x x x x x$

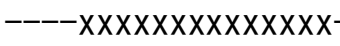

$-x x x x x$

a

>hsalI_IIB01 NP_001673.2

$\mathrm{m}-$ 
esygdvygict

-klk---------tspneg | sgnp-

xx ler reavfgknf i ppkkpk

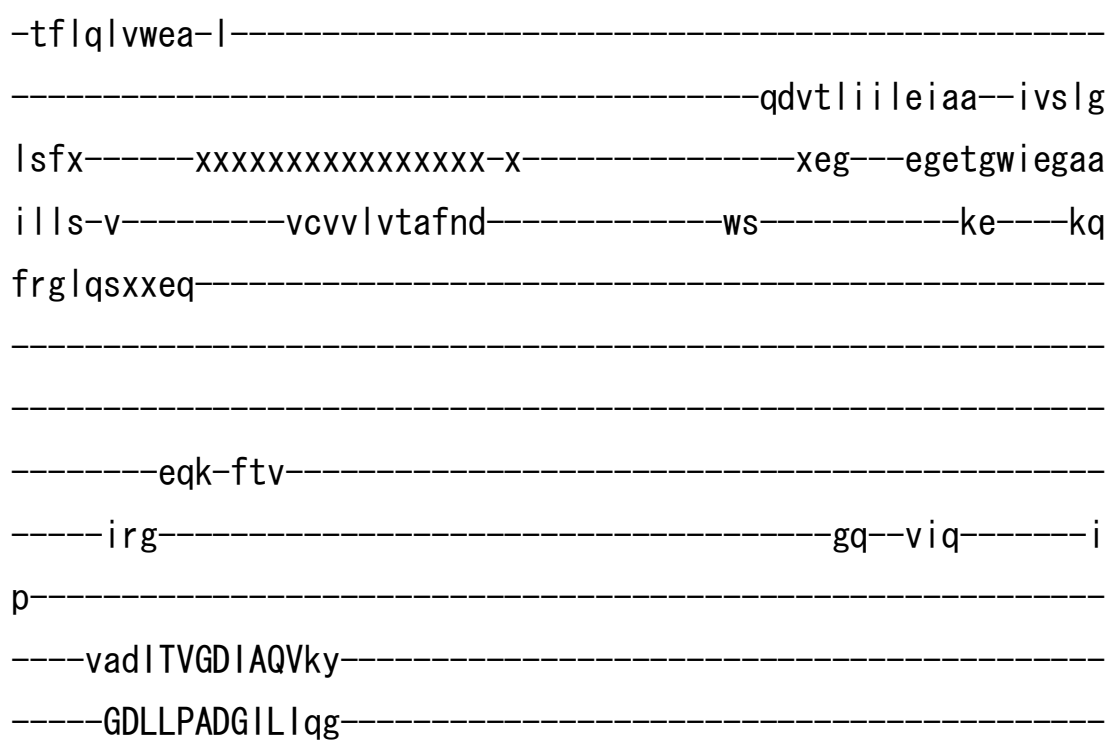


n--dlkIDESSLTGESDHVKKs I-

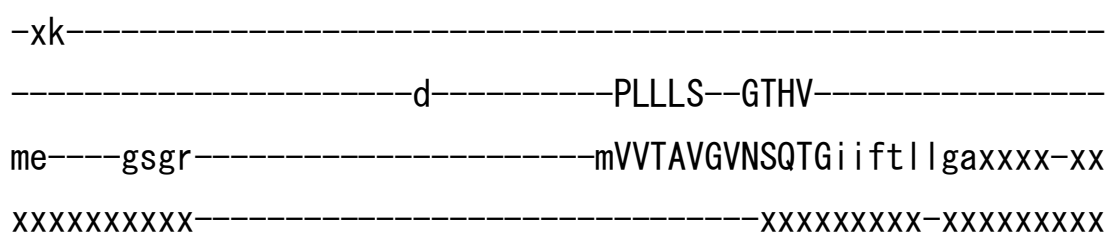

$x x-x x x x x x x x x x x x x x x x x x x x x x x x x$-pkkeksv lqgk Itk I avqigkag I Imsaitv

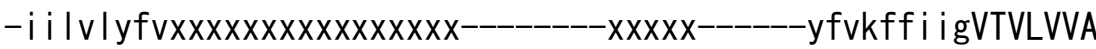
VPEGLPLAvTISLAYSVK-KMmkdn-

nLVR-HLDACETMGNATA ICSDKTG

TLTMNRMTVV

qay inekhy

$-x-x x x x x e^{--}-x$

ippnils

vtgisvnxxxxxki lp

$-p x x x g x \mid p-$

rhvgnkte--cal |g |-----xx-x----

Ikrdyqd--vrnexx

-eealykvytfnsvrk-smst

$-v-x x x x--x x----x x x x x x k-$

$\mathrm{e}$

- i i lkkcfk i I sangx 
akvfrprdrddi xktv i epm--ase----g I r I ICLAFRdf -xxxepepewdnenx i vtg I tc I AVVG I EDPVRPEVPDA IKKCQRag-I TV RMVTGDNINTARA IATKCG I Ihp--g-

xxx--------------e i eqer idk-

i wpk I--rVLA--RSSPTDKHTLVKGI i

---dsxxsd-------xxx---------------VVAVTGDGTNDGPALKKADVGFAM-$-x i a g t d v$

-AKEASD I ILTd-DNFTS IVkaVMWGRNVYDS I SKFL------Q

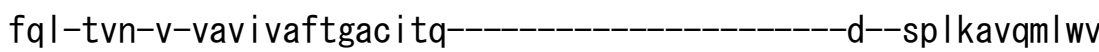
nl imdt l as la lateppt-es-------_--------- I I rkpy---g-rnkp I i srtm m-kni I ghafy--qlvvvft I If

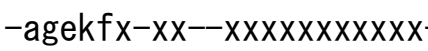

ps-----ehyt i vfntfv Imq I fn-e inarki $x x e r n v f---x---g$ i $x n n a$ ifct iv lgt fvvqi i iv--qf--ggk-----------pfscsel-s i eqw I----ws--i f I gmgt I lwg ql ist i 


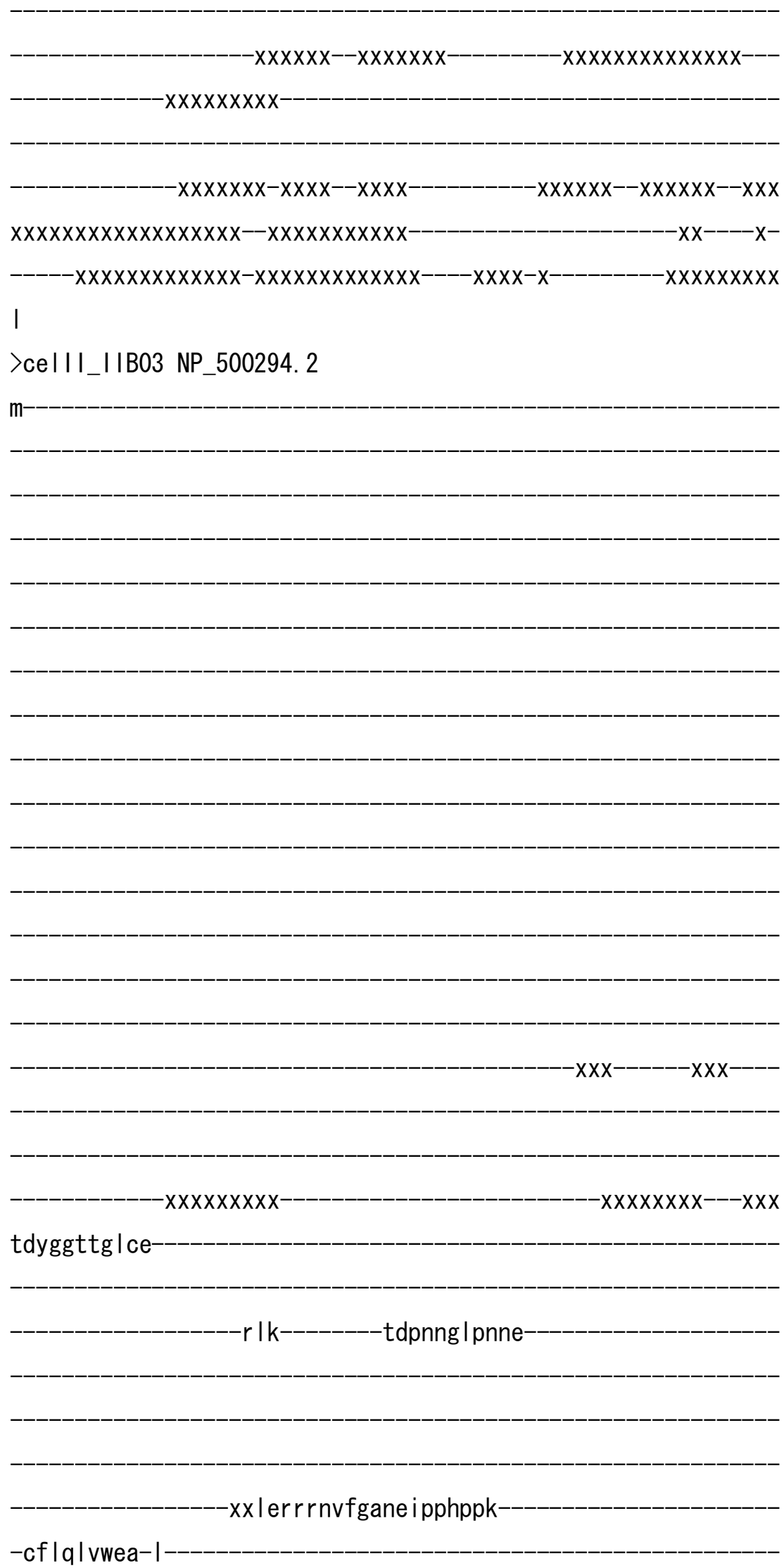




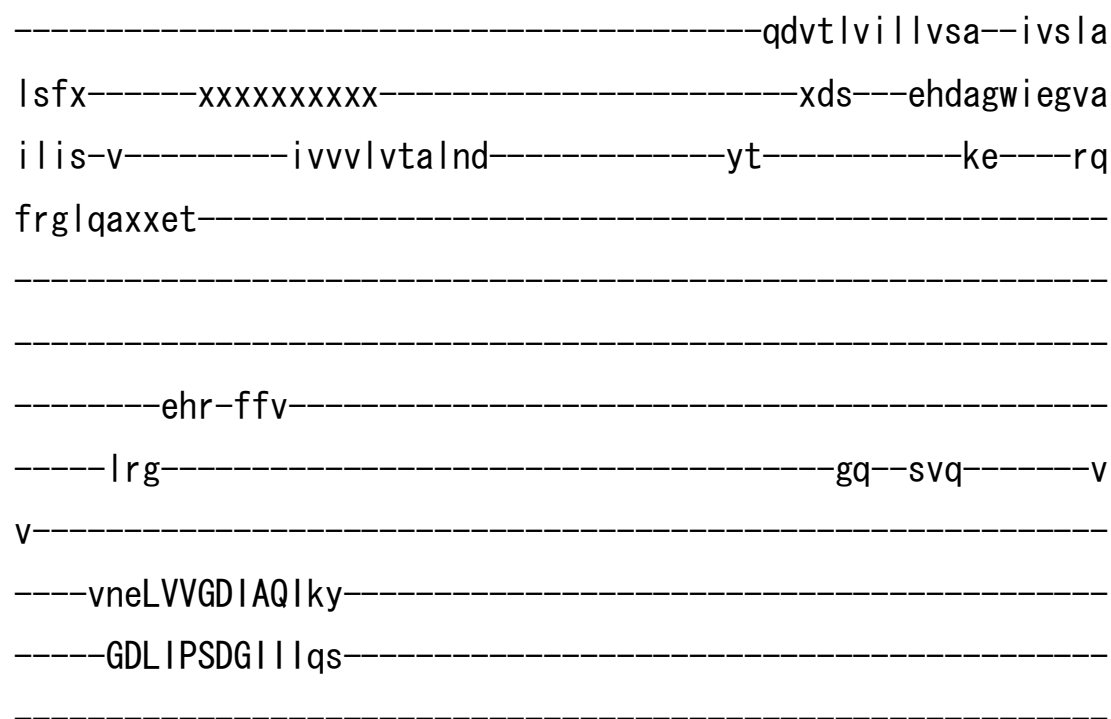

-n--dlkMDESSLTGESDQIRKsp-

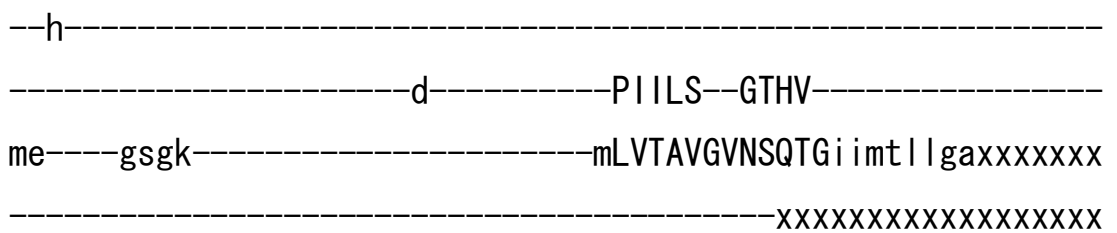

$x x-x x x x x x x x x x x x x x x x x x x x x x x x x x$-gkkersv lqak|tr la iq igyagsfvagctv - I il i irfcxxxxxxxxxxx-----x--------xxxxx------hfinfl i igVTVLVVA VPEGLPLAvTLSLAYSVK-KMmIdn-

nLVR-HLDACETMGNATAI CSDKTG

TLTTNRMTV qsfundvhy

$-x-x x x x x e^{--x}$

Idqntak

$-1 \mathrm{~m}$

mdc i $\sin x x x x x q v i p$

pxxxgxqa 
-tqlgnkte--cgm|gf-----xx-x

- gksyqe--irdrxx-

-eet ipkvytfnsvrk-smst

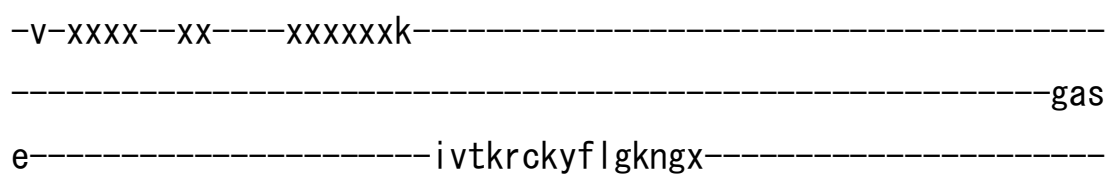

Itkfsskdaen I xrdv i epm---asd-----g | rt ICVAYKdy----_--_------------x xxxxxxxxxxxxxssepdweneex ivgdmta I AVLGI QDPVRPEVPAA I TRCQEag-ITV

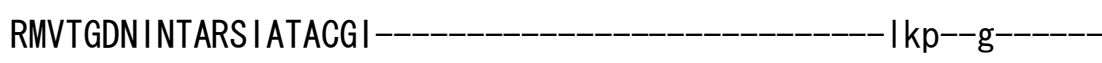

xxx--------------evsqek IdI

i wpk I--rVLA--RAQPSDKYVLVKGI i

---dsxxtd--------xxx---_--_-_-_-----VVAVTGDGTNDGPALKKADVGFAM--

-AKEASDI ILTd-DNFTS IVkaVMWGRNVYDS I AKFL------Q 


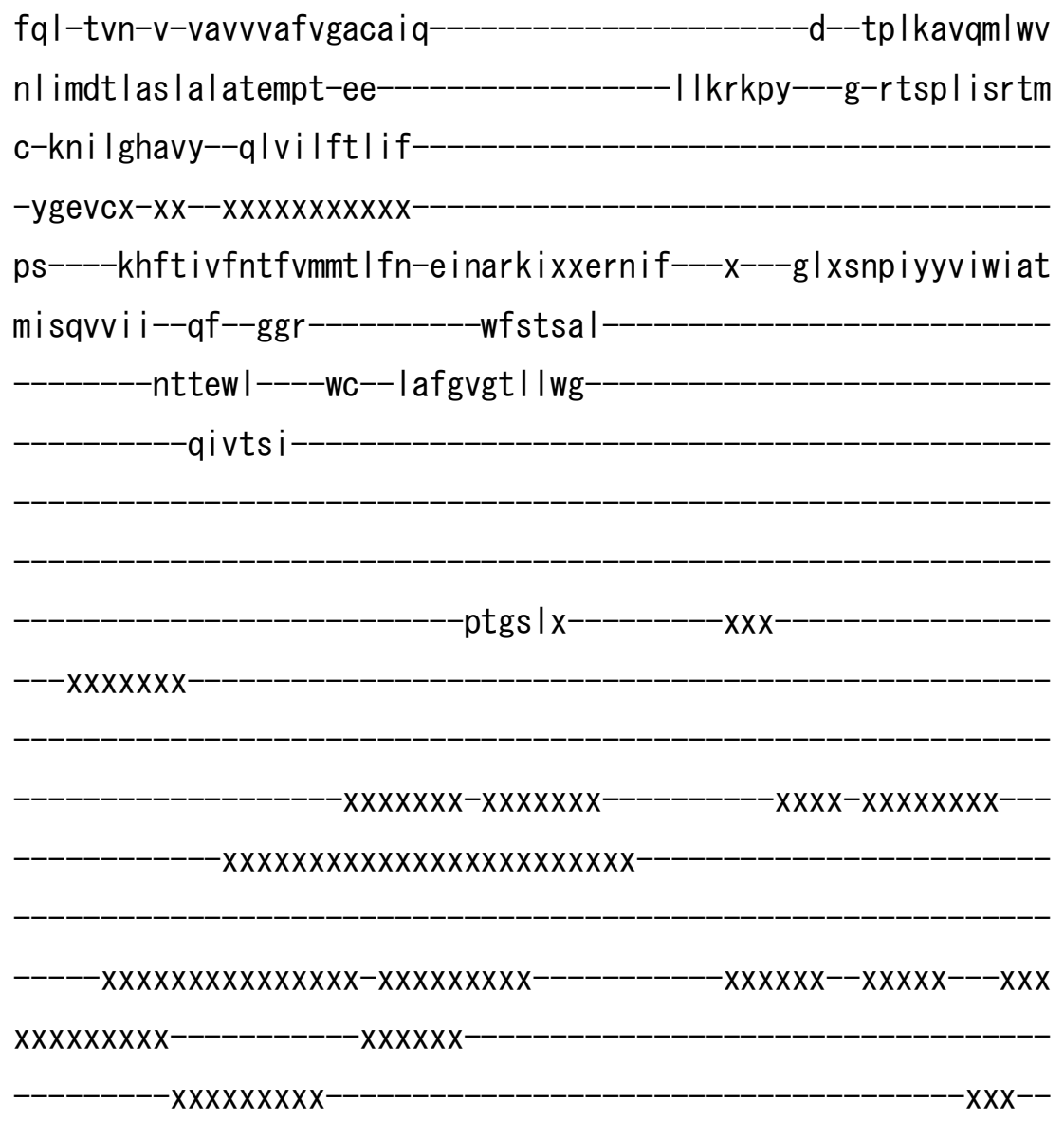

>hsalI_IIB02 NP_001001331.1

m- 


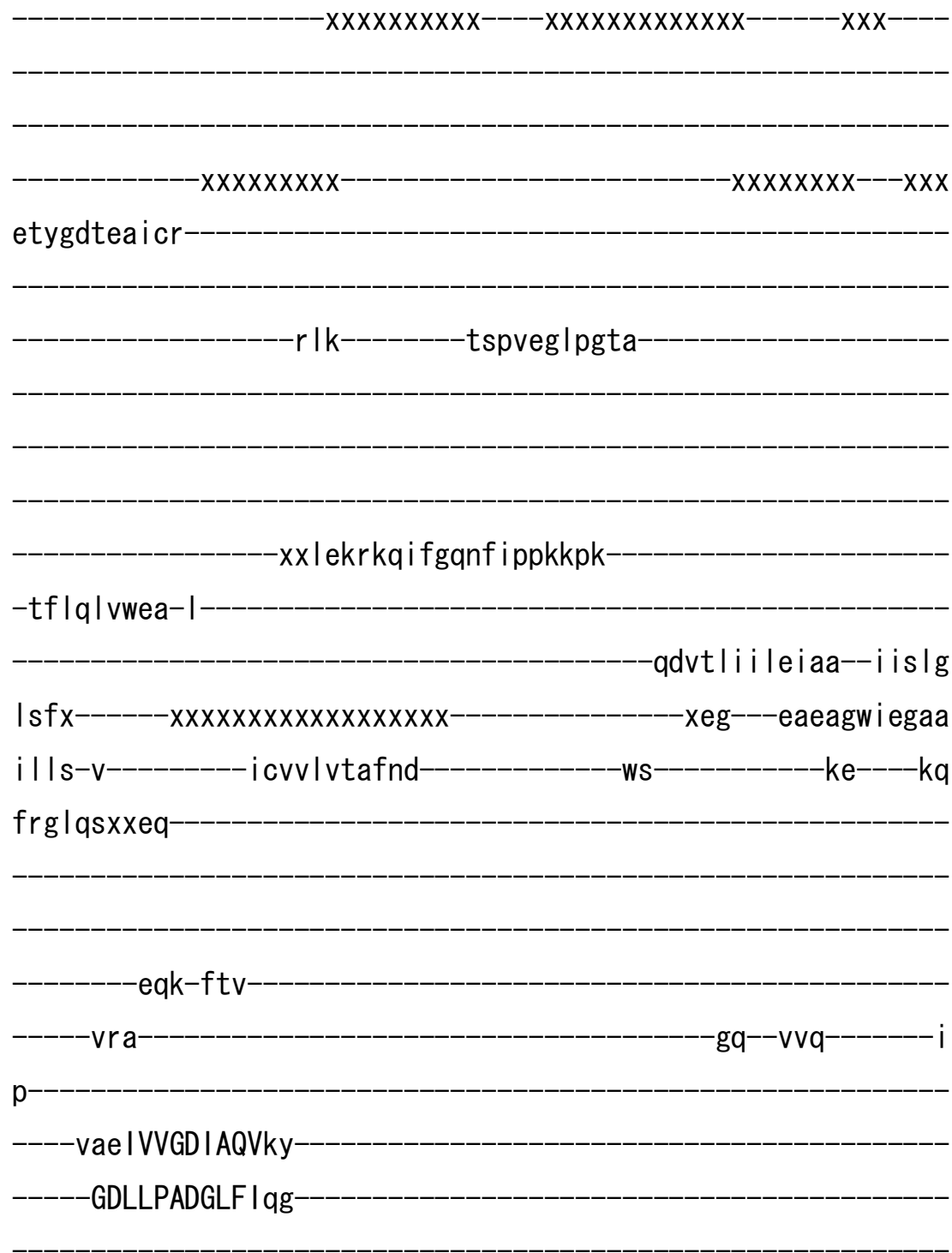

-n--dlkIDESSLTGESDQVRKsv-

$-\mathrm{xk}$

d----------PMLLS--GTHV

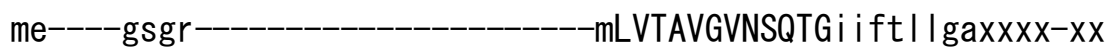

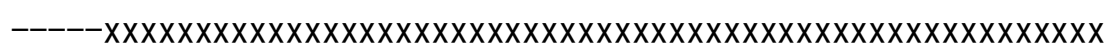
$x x x x x x x x x x x x x x x x x x x x--x x x x x x x-h k k e k s v$ lqgk | tk lavqi gkag I vmsaitv

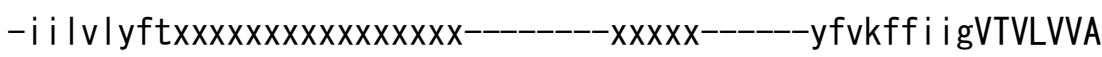
VPEGLPLAvTISLAYSVK-KMmkdn- 
-nLVR-HLDACETMGNATA I CSDKTG-

-TLTTNRMTV

qayvgdvhy

$-x-x x x x x s^{-}-x$

intktme

inaiainxxxxxki Ip

$-p x x \times g x \mid p$

rqvgnkte--cg | Igf-----xx-x----

Ikqdyep--vrsqxx

eek I ykvytfnsvrk-smst

$-\mathrm{v}-\mathrm{xx} x \mathrm{x}--\mathrm{xx}-----\mathrm{xx} x \mathrm{xx} \mathrm{xk}$

gas

e-

i v Ikkcck i Ingagx

prvfrprdrdemxkkvi epm---acd----g I rt I CVAYRdf

xxspepdwdnenx i I ne I tc I CVVG IEDPVRPEVPEA IRKCQRag-I TV

RMVTGDN I NTARA I A I KCG I

ihp--g-

$-x x x---|c|$ legkefnx

$x X X X$

xxx--------------e i eqer i dk- 
i wpk I--rVLA--RSSPTDKHTLVKGI i

---dsxxte--------xxx----------------VVAVTGDGTNDGPALKKADVGFAM--

$-x i a g t d v$

-AKEASDI ILTd-DNFSSIVkaVMWGRNVYDSISKFL-------Q

fql-tvn-v-vavi vaftgac i tq--_-_---_-_-_--_-_-_---d--sp I kavqm I wv

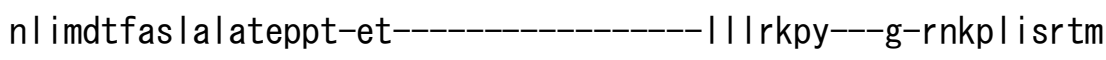

m-kni I ghavy--qlal i ft I I f

-vgekmx-xx--xxxxxxxxxxx-

ps----ehyt i ifntfvmmq I fn-e i narkixxernvf----x---gi $x$ rnp i fct iv lgt faiqi viv---qf--ggk-_-_-_-_----pfscsp I-_-_-_-_-_-_-_-_-_-_-_-_-_-_-_

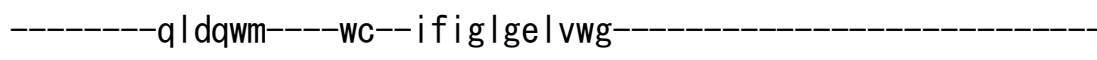

qviati

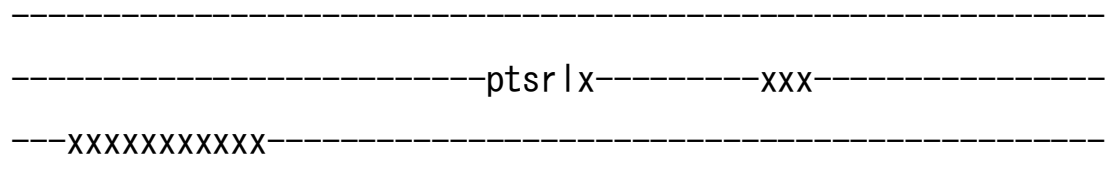

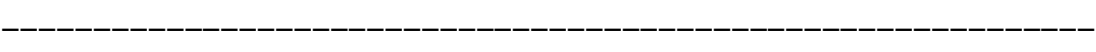

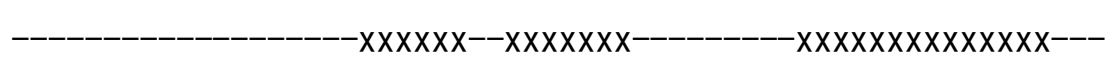
$\mathrm{XXXXXXXX-}$

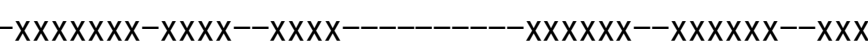

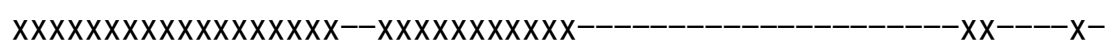

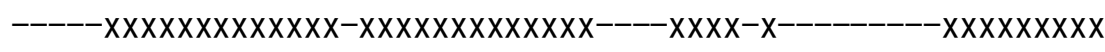

।

>hsalI_IIB03 NP_068768.2

$\mathrm{m}-$ 
eaygdvsglor

$-r \mid k$ -tsptegladnt

xxlekrrqiygqnf ippkqpk

-tflqlvwea-I

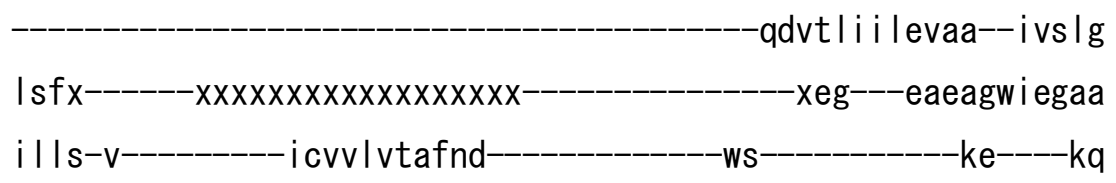

frglqsxxeq-

$-e q k-f t v-$

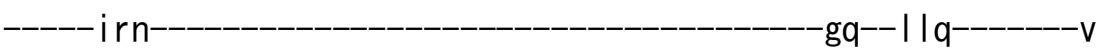

$\mathrm{p}$

----vaaLVVGDIAQVky

GDLLPADGVLI qa 
-n--d IkIDESSLTGESDHVRKsa-

$-x-$

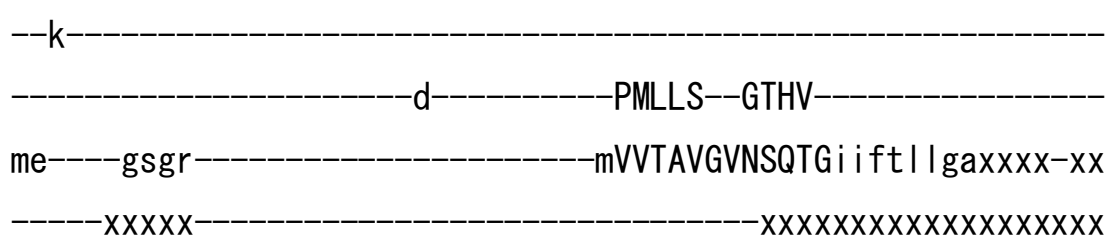

$x x x x x x x x x x x x x x x x x x x x x x x x x x x$-pkkeksv lqgk|tk lavqigkag I vmsaitv

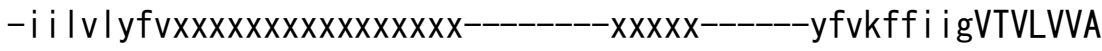
VPEGLPLAvTISLAYSVK-KMmkdn-

-nLVR-HLDACETMGNATA I CSDKTG-

TLTTNRMTV -qsy I gdthy

$-x-x x x x x s^{--x}$

Itpkild

vhaisinxxxxxkilp-

$-p x x x g x \mid p$

rqvgnkte--cal Igf-----xx-x----Ikrdfap--vreqxx

-edk I ykvytfnsvrk-smst

$-\mathrm{v}-\mathrm{x} x \mathrm{xx}--\mathrm{xx}-----\mathrm{x} x \mathrm{x} x \mathrm{x} x \mathrm{k}-$

e-

-i l lkkctni Insngx 
Irgfrprdrddmxrk i i epm--acd----g|rtICIAYRdf

-xxgqepdwdnenxvvgd I tc I AVVG IEDPVRPEVPEA IRKCQRag-I TV RMVTGDN INTARA I AAKCG I

iqp--g-

$-x x x---|c|$ egkefnx

$-x x x$

xxx--------------e i eqer I dk-

vwpk I--rVLA--RSSPTDKHTLVKGI i

---dsxxge-------xxx--------------VVAVTGDGTNDGPALKKADVGFAM-$-x i a g t d v$

-AKEASD I ILTd-DNFTS IVkaVMWGRNVYDS I SKFL------Q

fql-tvn-v-vavi vaftgac i tq--_-_-_-_-_-_-_-_-_---d--sp I kavqm I wv nl imdtfas la lateppt-es--------_---------I I rkpy---g-rdkp I i srtm m-kni I ghavy--q la i ift I If

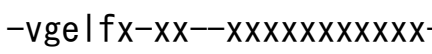

ps-----ehyt i i fntfvmmq I fn-e i nark i xxernvf----x---gi ixsnp ifct iv lgt fgiqiviv--qf--ggk-----------pfscspl-

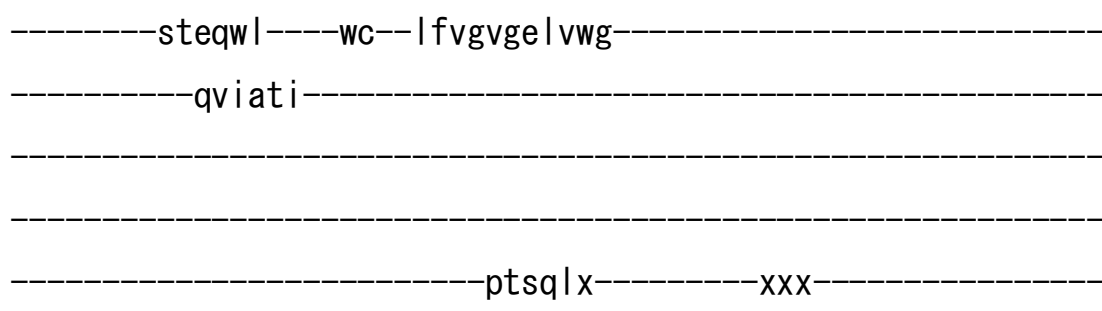




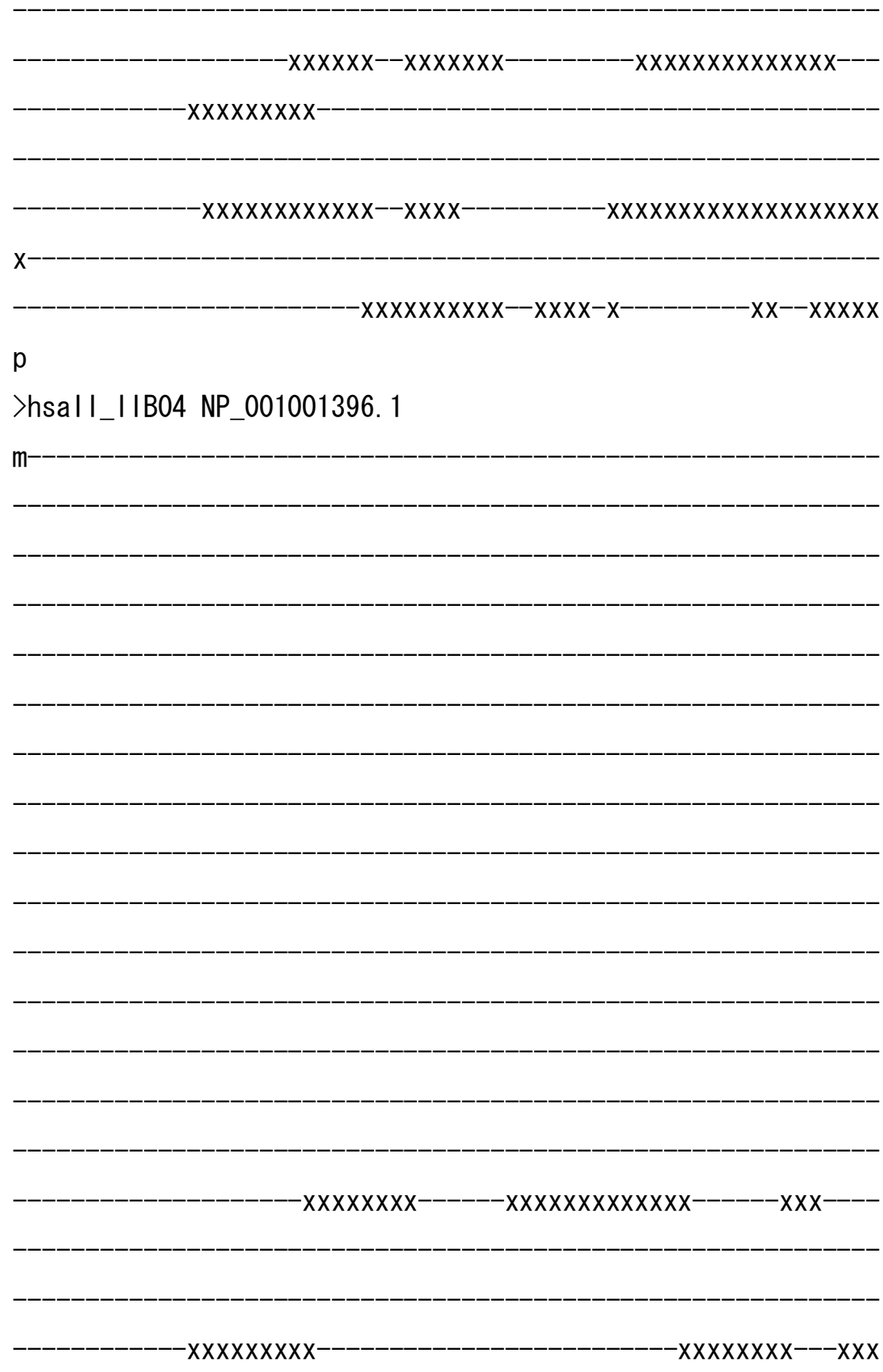

vhyggvanlos

rlk--------tspveg I sgnp-

xx l ekr rqvfghnv i ppkkpk

-tflelvwea-I 


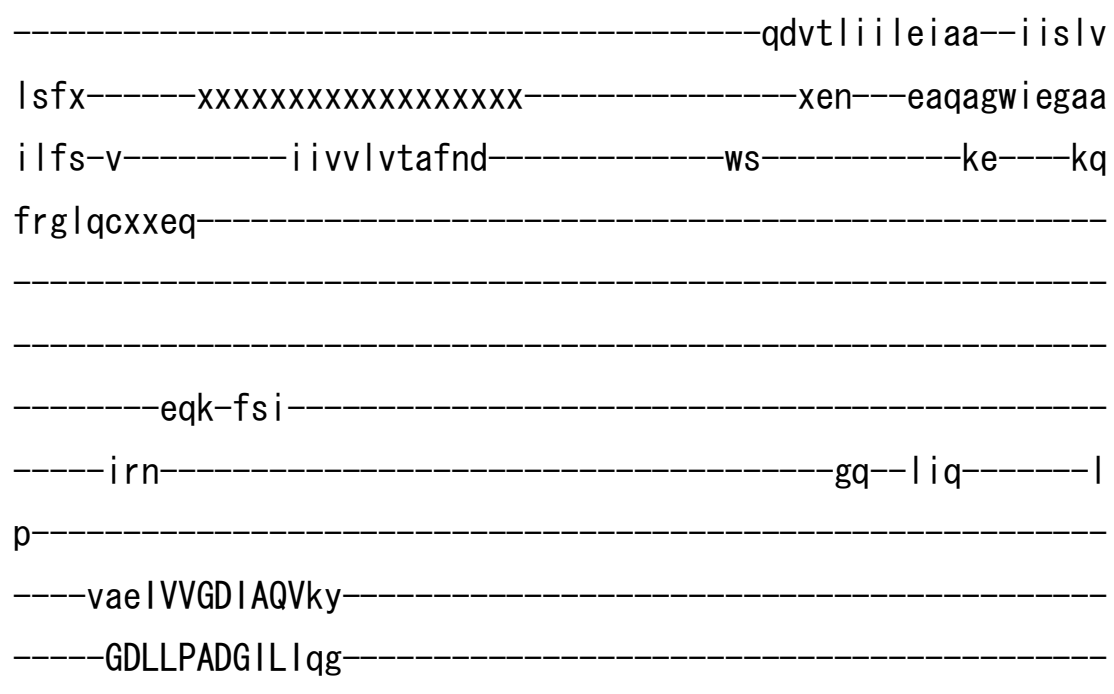

-n--d lkIDESSLTGESDHVKKs I-

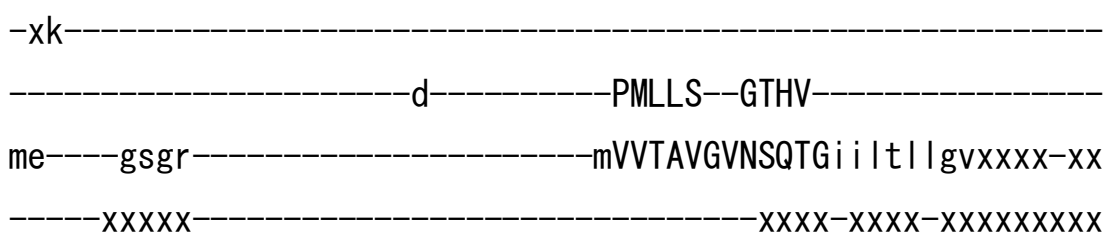

$x x-x x x x x x x x x x x x x x x x x x x x x x x x x$-pkkeksv lqgk Itr lavqigkag I Imsal tv

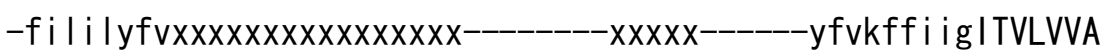
VPEGLPLAvTISLAYSVK-KMmkdn-

nLVR-HLDACETMGNATAI CSDKTG

TLTMNRMTV -qay i gg i hy

$-x-x x x x x d--x$

flpkvld

vng i s inxxxxxk i lp $-p x x x g x \mid p$ 
rqvgnkte--cal Igf-----xx-x-

Ikqdyqa--vrnexx

-eek lykvytfnsvrk-smst

$-v-x x x x--x x----x x x x x x k$

-gas

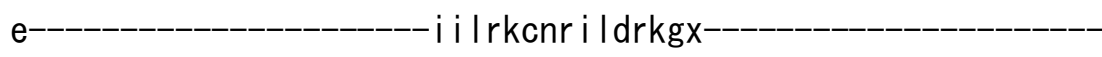

avpfknkdrddmxrtvi epm--acd----g I rtICIAYRdf

xdtepswdnenx i I te I tc I AVVG IEDPVRPEVPDA I AKCKQag-I TV

RMVTGDN I NTARA I ATKCG I-

Itp--g------

$-x x x---|c|$ egkefnx

$-\mathrm{XXXX}$

xxx--------------eveqek / dk-

i wpk I--rVLA--RSSPTDKHTLVKGI i

---dsxxge--------xxx---_----_--_----VVAVTGDGTNDGPALKKADVGFAM--

-AKEASD I ILTd-DNFTS IVkaVMWGRNVYDS I SKFL------Q 


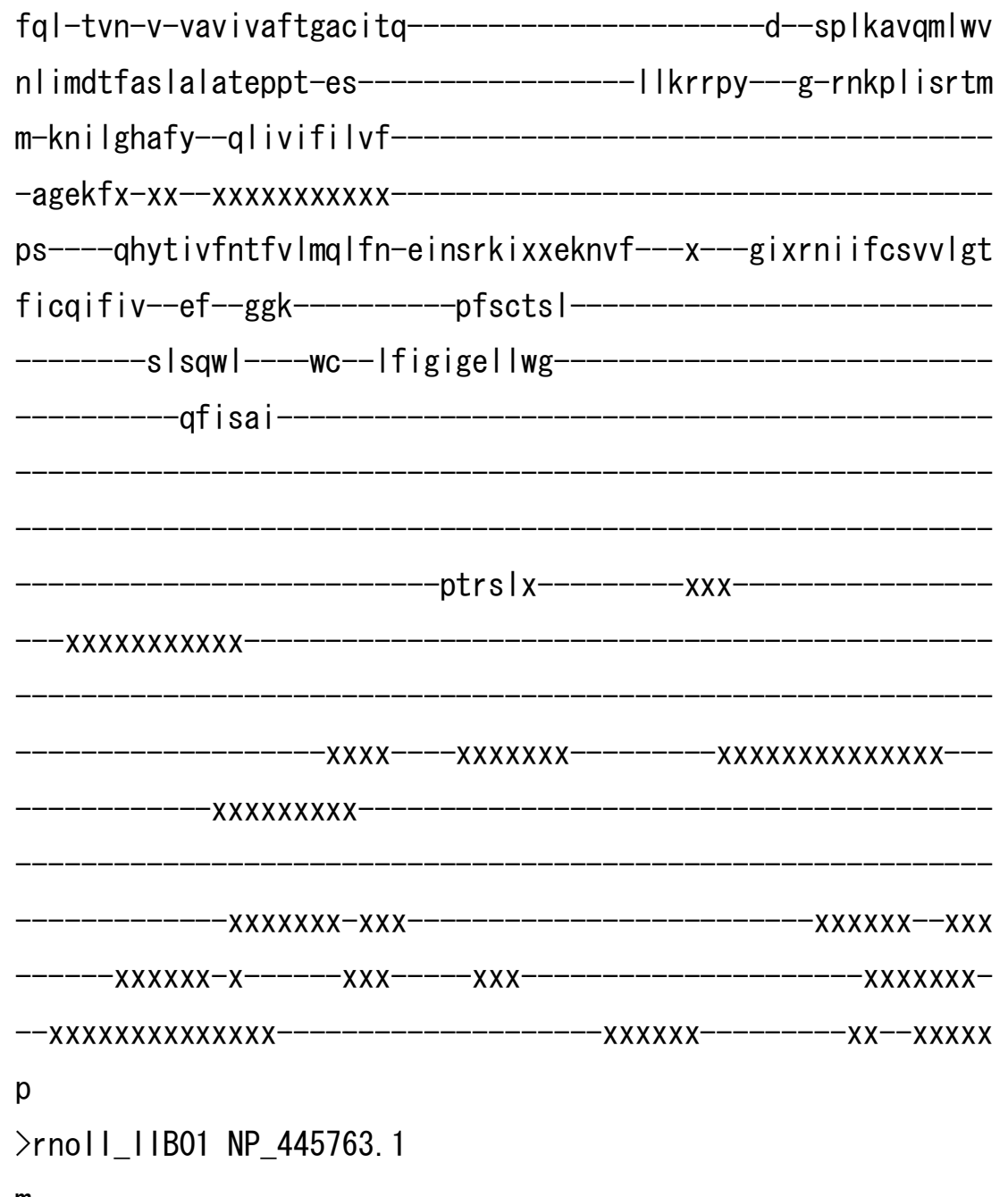

m- 


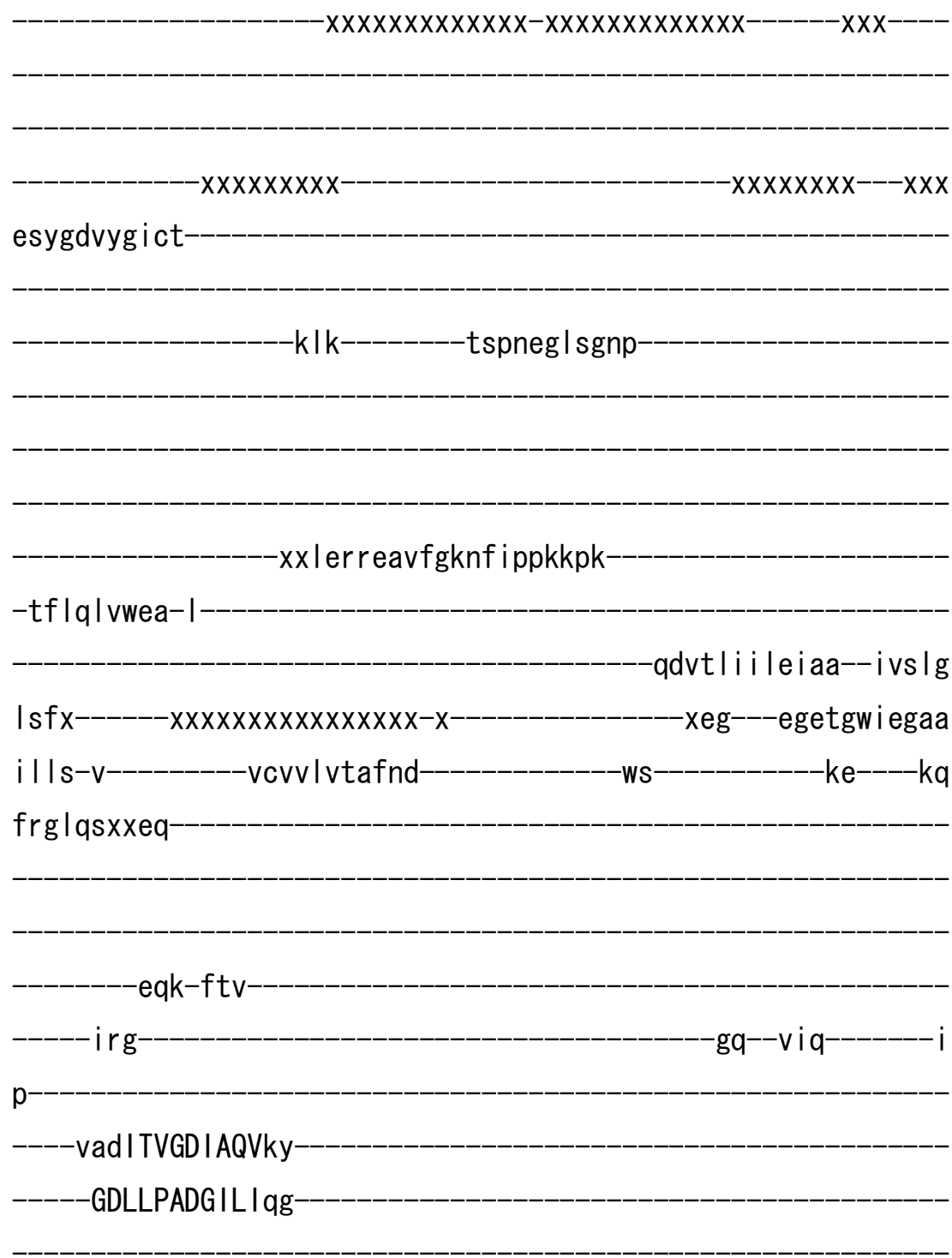

-n--d IkIDESSLTGESDHVKKs I-

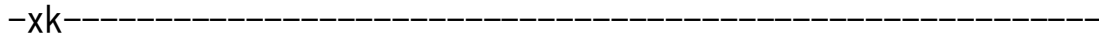

me-----gsgr--_-_-_-_-_-_-_-_-_-_-_mVVTAVGVNSQTG i ift I I gaxxxx- xx

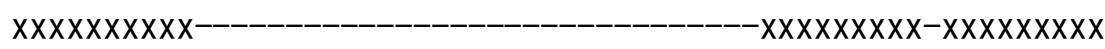
$x x-x x x x x x x x x x x x x x x x x x x x x x x x x x-p k$ keksv lqgk|tk lavqigkag I Imsaitv

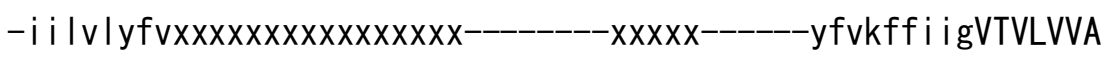
VPEGLPLAvTISLAYSVK-KMmkdn- 
nLVR-HLDACETMGNATA ICSDKTG-

$-T L T M N R M T V$

qay inekhy

$-x-x x x x x e^{--}-x$

ippnils

vtg i svnxxxxxki I

$-p x x x g x \mid p-$

-rhvgnkte--cal Igf-----xx-x----

- lkrdyqd--vrnexx-

-eealykvytfnsvrk-smst

$-v-x x x x--x x----x x x x x x k$

-gas

e-

- i i lkkcfki l sangx-

akvfrprdrddi xktv i epm--ase----g I r tICLAFRdf

-xxxepepewdnenxvvtg I tc I AVVG IEDPVRPEVPEA IKKCQRag-ITV

RMVTGDN I NTARA I ATKCGI -

-lhp--g-

$-x x x---|c|$ egkdfnx

$x x x x$

xxx--------------e i eqer i dk- 
i wpk I--rVLA--RSSPTDKHTLVKGI i

---dsxxse-------xxx--------------VVAVTGDGTNDGPALKKADVGFAM--

$-x i a g t d v$

-AKEASD I ILTd-DNFTS IVkaVMWGRNVYDS I SKFL------Q

fql-tvn-v-vavi vaftgac i tq------_--_-_----------d--sp I kavqm I wv

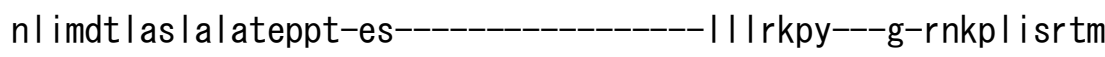

m-kni I ghafy--qlvvvft I If

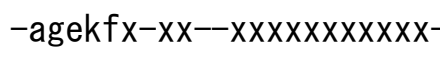

ps----ehyt i $f$ fntfv Imq I fn-e inarkixxernvf----x---gixnna ifctivlgt

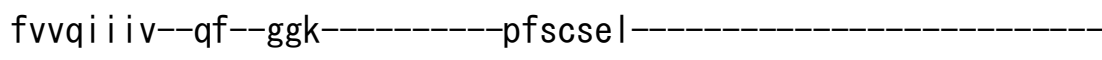

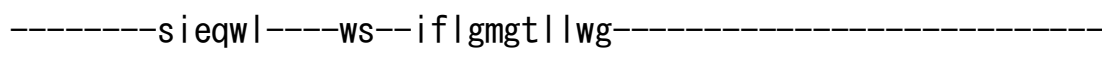

-qlist i

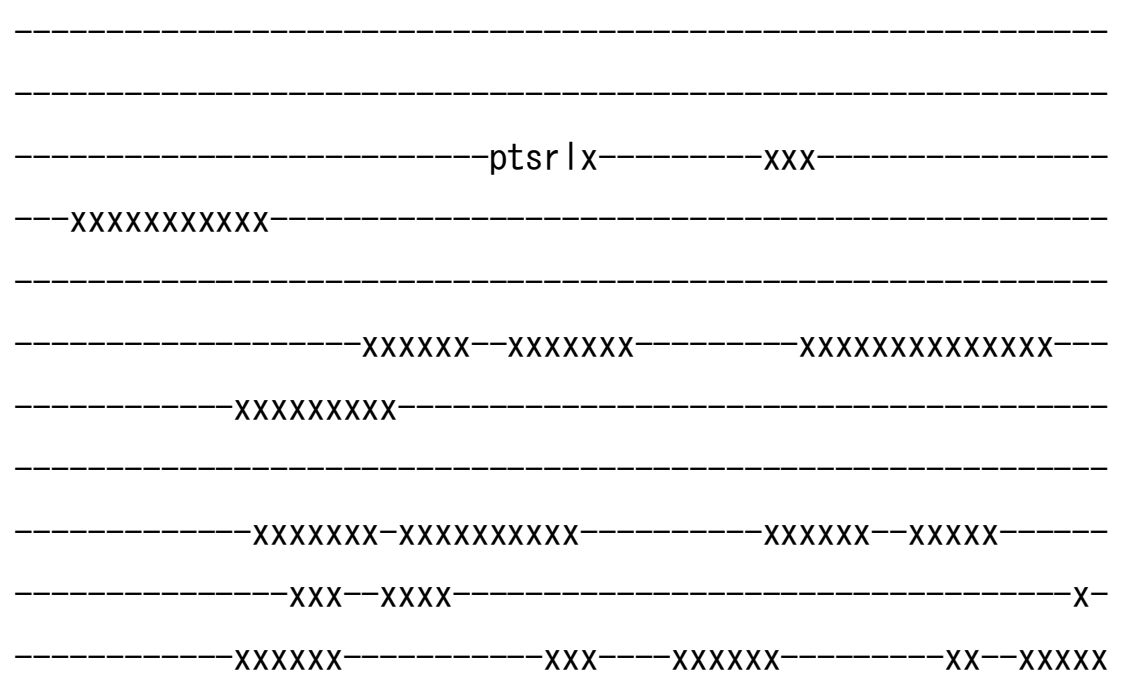

$\mathrm{s}$

>rnolI_IIB03 XP_343840.2

m- 
eaygdvsglor

$-r \mid k$ -tsptegladnt

xxlekrrqiygqnf ippkqpk

-tflqlvwea-I

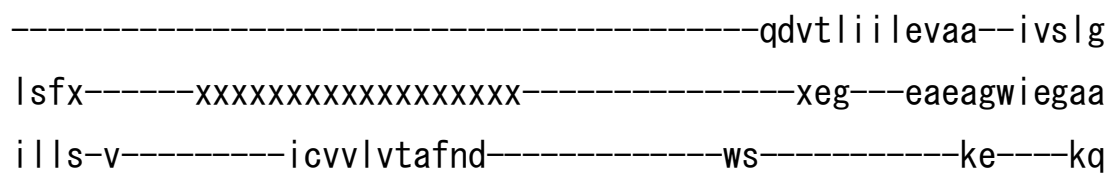

frglqsxxeq-

$-e q k-f t v-$

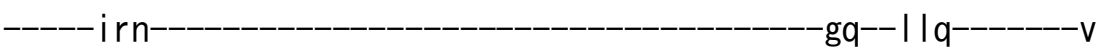

$\mathrm{p}$

----vaaLVVGDIAQVky

GDLLPADGVLI qg 
-n--d IkIDESSLTGESDHVRKsa-

$-x-$

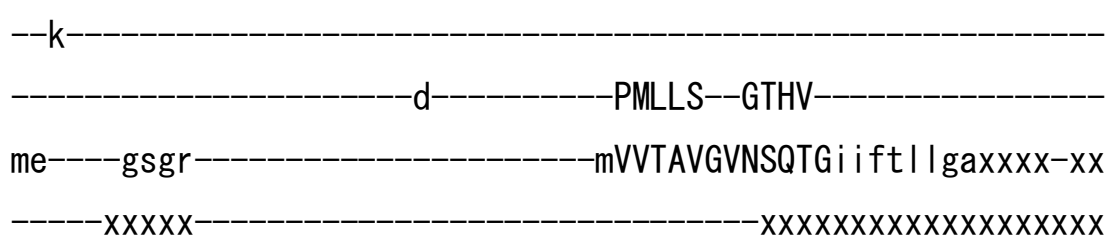

$x x x x x x x x x x x x x x x x x x x x x x x x x x x$-pkkeksv lqgk|tk lavqigkag I vmsaitv

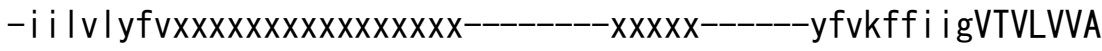
VPEGLPLAvTISLAYSVK-KMmkdn-

-nLVR-HLDACETMGNATA I CSDKTG-

TLTTNRMTV

-qsy I gdthy

$-x-x x x x x s^{--x}$

Itpkild

vhaisinxxxxxkilp-

$-p x x x g x \mid p-$

rqvgnkte--cal lgf-----xx-x----

-Ikrdfap--vreqxx

edq lykvytfnsvrk-smst

$-\mathrm{v}-\mathrm{x} x \mathrm{xx}--\mathrm{xx}-----\mathrm{x} x \mathrm{x} x \mathrm{x} x \mathrm{k}-$

$\mathrm{e}$

-i I lkkctni Insngx 
Irgfrprdrddmxkk i i epm--acd----g I rtICIAYRdf

-xx i qepdwdnenxvvgd I tc I AVVG I EDPVRPEVPEA IRKCQRag-I TV RMVTGDN INTARA I AAKCG I iqp--g-

xxx--------------e i eqer $\mid \mathrm{dk}$

vwpk I--rVLA--RSSPTDKHTLVKGI i

---dsxxge--------xxx--------------VVAVTGDGTNDGPALKKADVGFAM-$-x i a g t d v$

-AKEASD I ILTd-DNFTS IVkaVMWGRNVYDS I SKFL------Q

fql-tvn-v-vav i vaftgac i tq---_---_--_-_-_-_-_----d--sp I kavqm I wv nl imdtfas la lateppt-es---_-_-_-_-_-_-_-_ I I rkpy---g-rdkp I i srtm m-kni Ighavy--ql ti ift I If -vgel $f x-x x--x x x x x x x x x x x$

ps-----ehyt i i fnt fvmmq I fn-e i nark i xxernvf----x---gi ixsnp ifct iv lgt fgiqiviv--qf--ggk----------pfscspl-

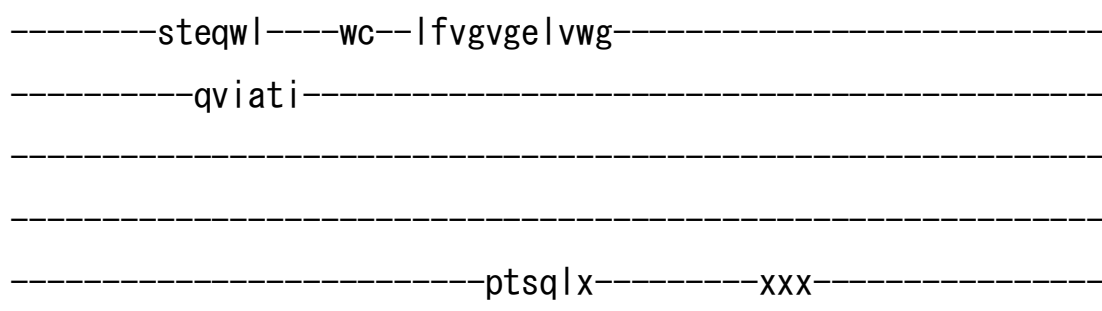




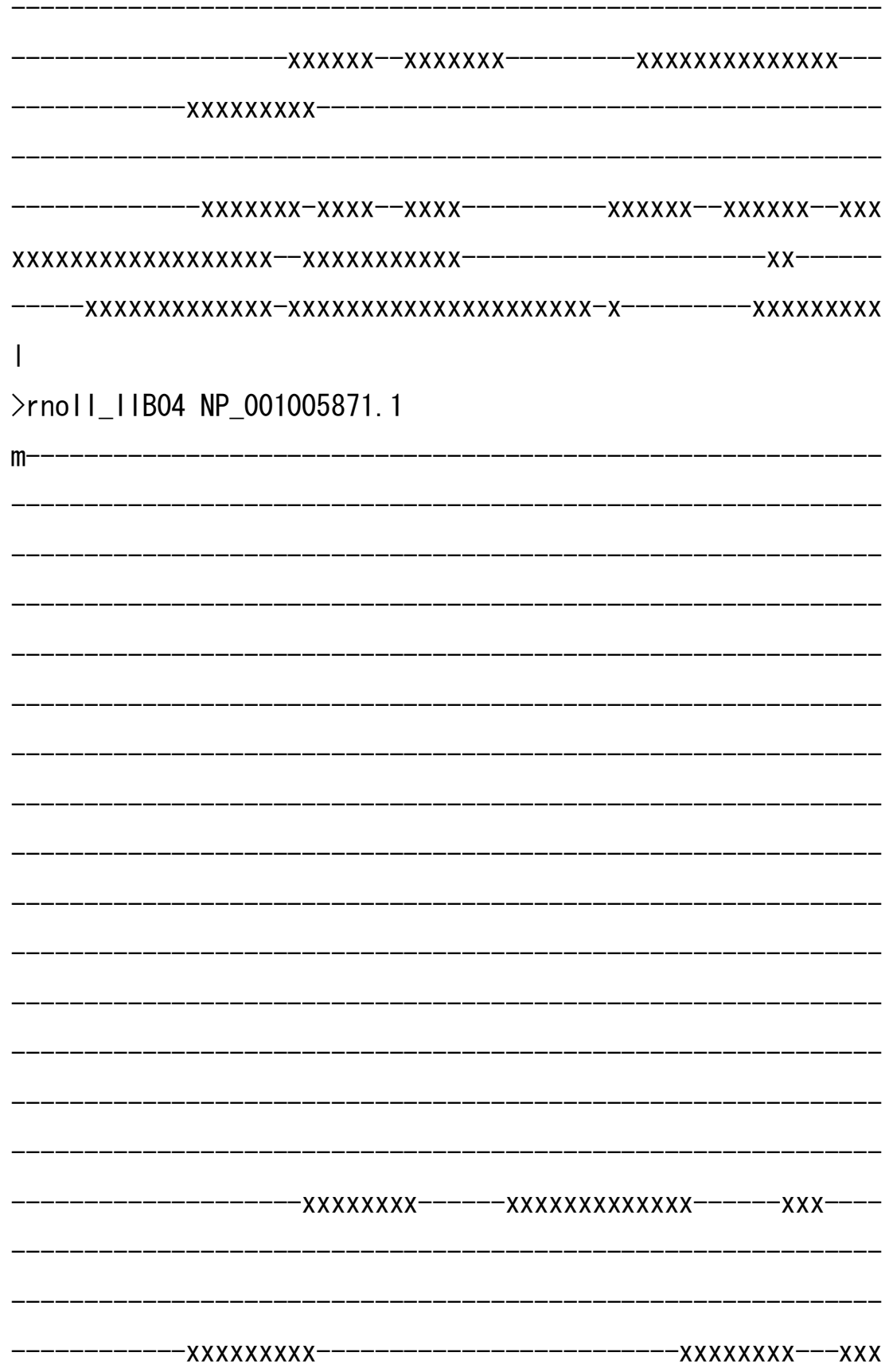

ahygsvqe ica

rlk--------tspveg I sgnp-

-xx l ekr r I vfgknm i ppkkpk

-tflel vwea-I 


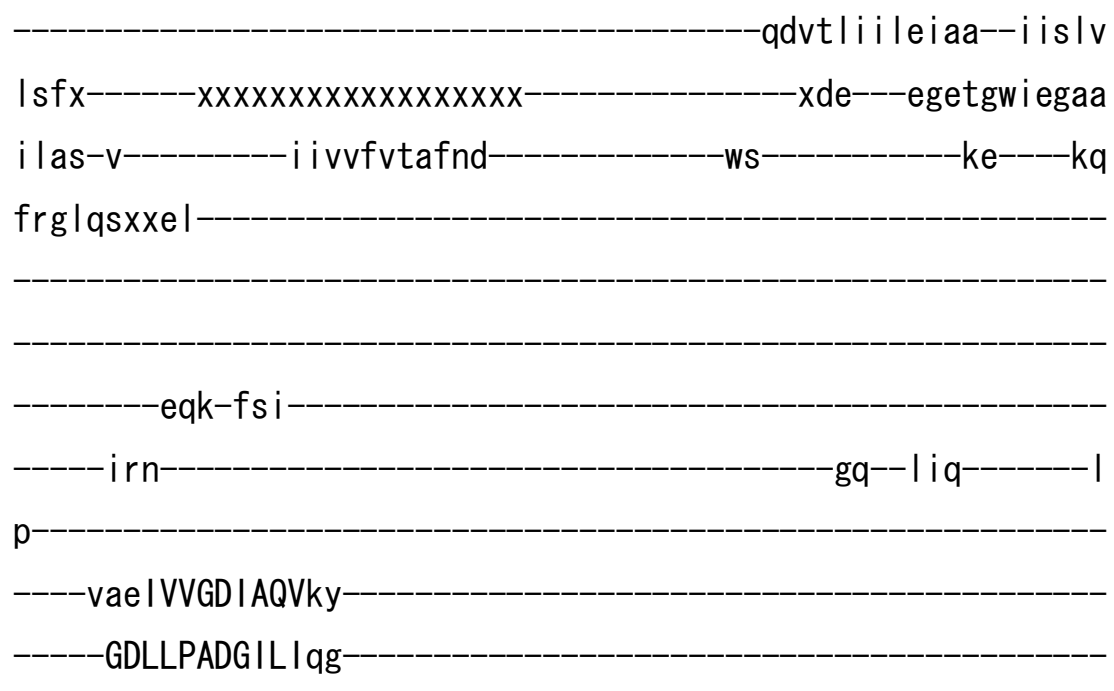

-n--dlkIDESSLTGESDHVKKt I-

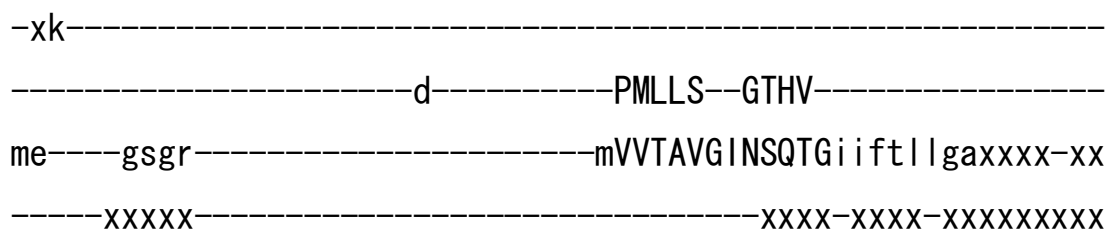

$x x-x x x x x x x x x x x x x x x x x x x x x x x x x x$-pkkeksv lqgk Itr lavqigkag I ims i Itv

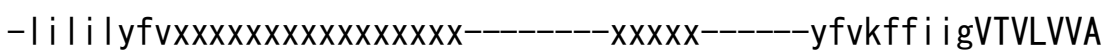
VPEGLPLAvTISLAYSVK-KMmkdn-

nLVR-HLDACETMGNATAI CSDKTG

TLTMNRMTV -qay i ggthy

$-x-x x x x x d--x$

lppnvld

vnsicinxxxxxkilp $-p x x x g x \mid p$ 
-rqvgnkte--cg| |gf-----xx-x-

-Ikqdyqa--vrsexx

-eek I fkvytfnsvrk-smst

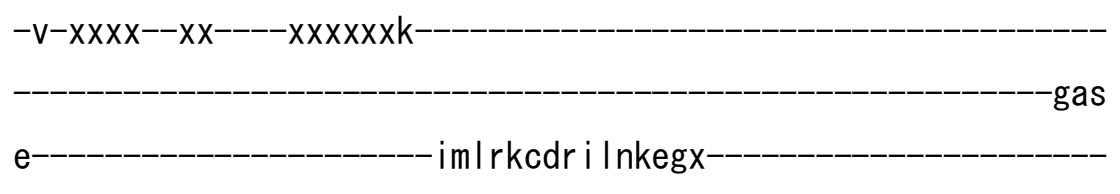

ivpfktkdrdnmxrnvi epm--ase----g | rt IGIAYRdf

xgeepswenenx iftg I vc IAVVG IEDPVRPEVPDA INKCKRag-ITV

RMVTGDNVNTARAIATKCGI

- tp--g------

$-x x x---\mid c l e g k e f n x$

xxx--_-_-_-_-_-_--_eveqek I dk-_-_-_-_-___-_-_-_-_-_-_-_-_-_-_-_-_-_-_

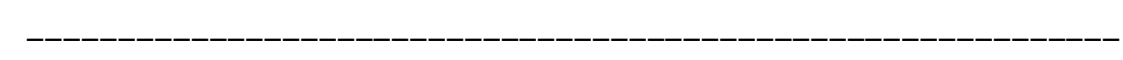

vwpr I--rVLA--RSSPTDKHTLVKGI i

---dsxxge---_---xxx--_-_-_-_-_-_-_VVAVTGDGTNDGPALKKADVGFAM--

-AKEASDI ILTd-DNFTS IVkaVMWGRNVYDS I SKFL------Q 
fql-tvn-v-vavivafsgacitq---_--_-_-_-_-_-_-_---d--sp I kavqm I wv

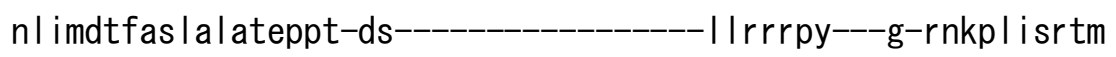

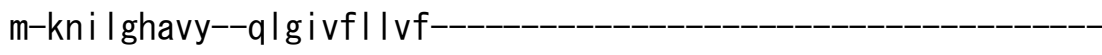
-agdk|x-xx--xxxxxxxxxxx-------------------------------ps----qhyt ivfntfvlmqlfn-einsrkixxeknvf----x---gvxrni ifcsvvlgt ffcqv-

$-p t k s \mid x-$

$-\mathrm{xxx}-$
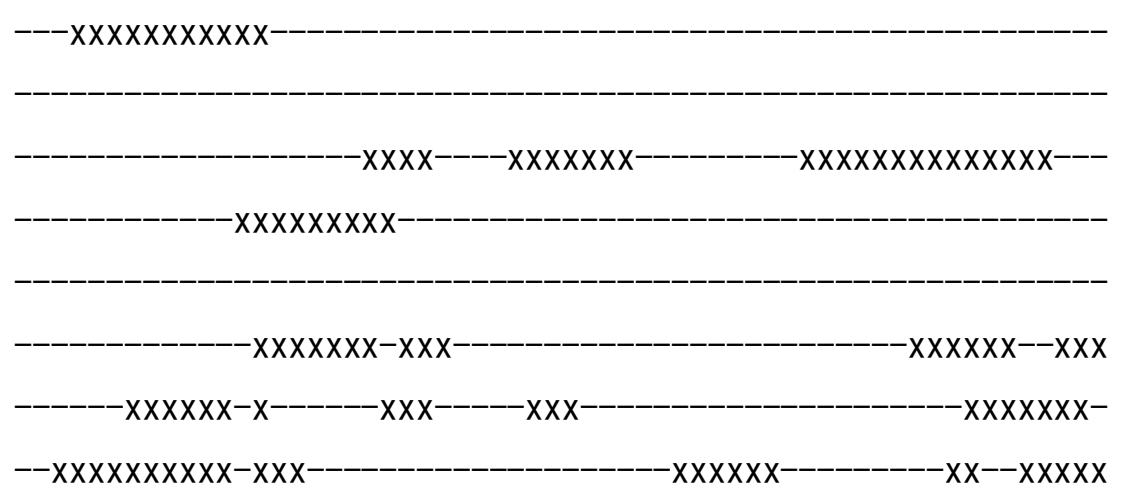

$S$

>osalI_IIB01 NP_001045345.1

m- 


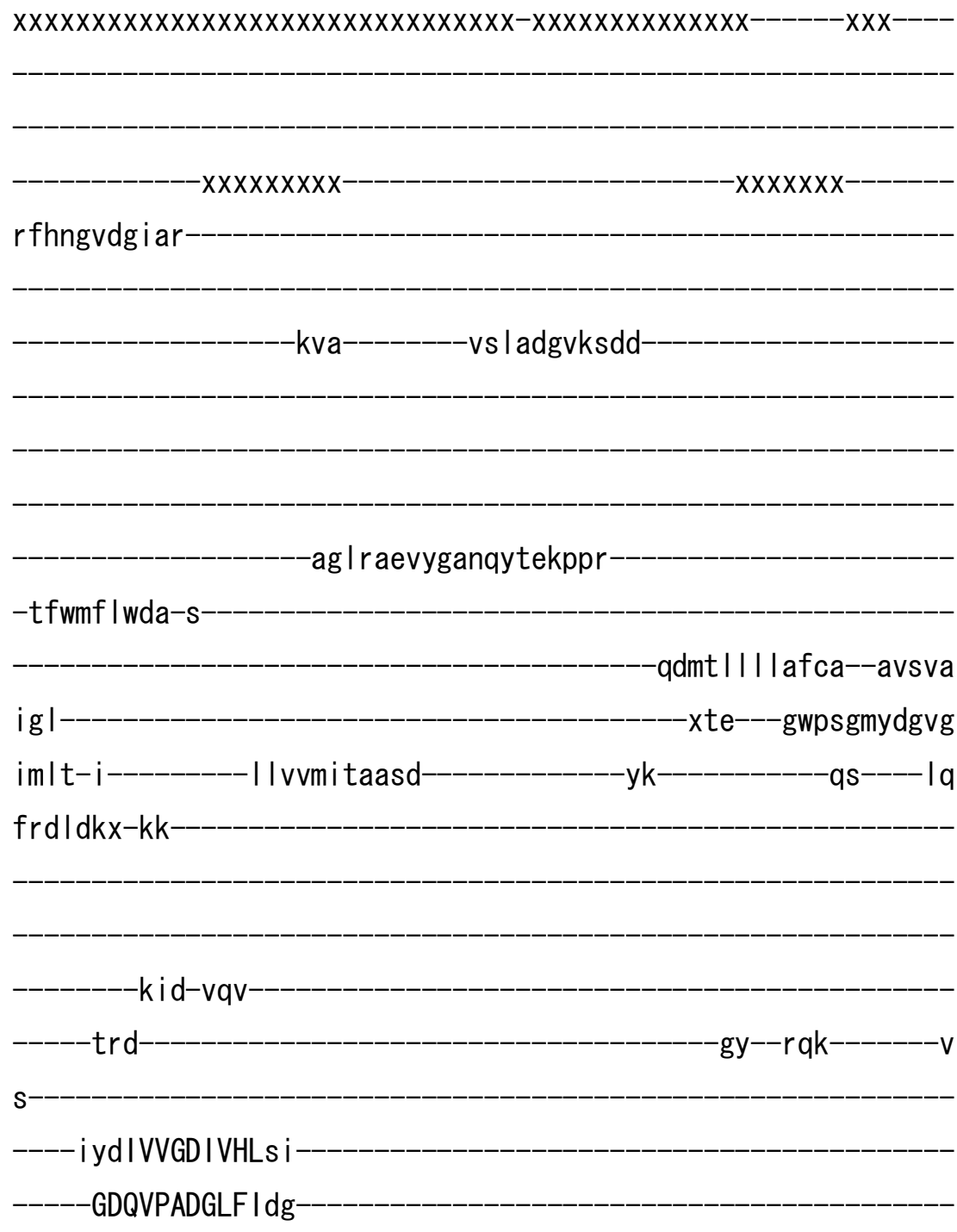

$-y--s f v V D E S N L S G E S E P V H V s t-$
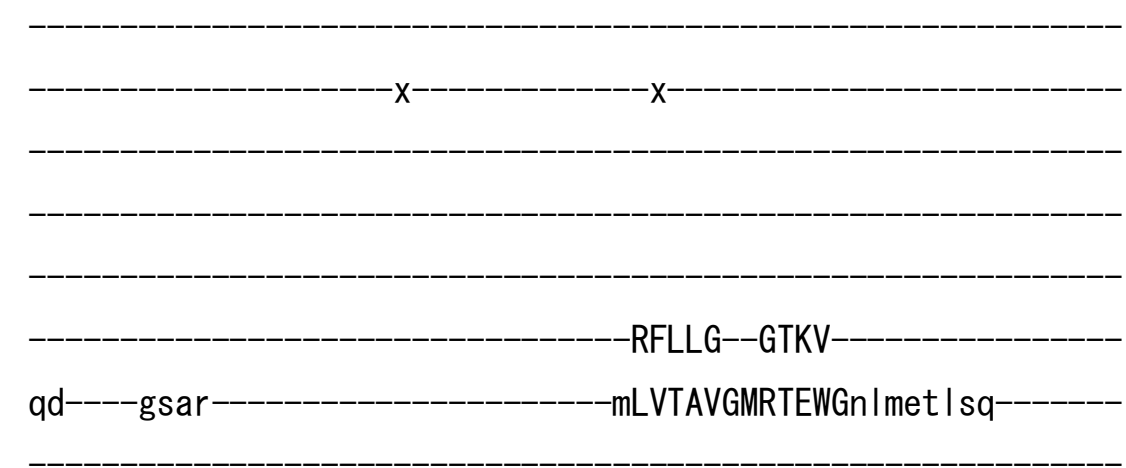

ggedetp Iqvk Ingvat i igkig lafavItf

-tv ImarfIxxxxxxxxxxxxxxxx--------xxx--------avInffavaVTIIVVA VPEGLPLAvTLSLAFAMK-KLmqer 
aLVR-HLSACETMGSASC I CTDKTG-

TLTTNHMVVe

-kiwasgaa

$x-x x x x x k x x x$

xxxxxmsetfak-

$-\mathrm{v}$

legvfhc---xxsevv-

$r \times x \times g-r h-$

timgtpte--tai lef-----xx-x----

vekrari--eht

gagk Ikvepfnsvkk-tmav

$-v-x x x x x x x x----x x x x x x k-$

-gas

$\mathrm{e}$

$-\mathrm{vv} \mid \operatorname{srcs}$ I v Idgtgx

vek I tdakakrv-asa i daf--ace----al rtLCLAYQdv

dggggdxpgegyt I I AVFG I KDPLRPGVREAVATCHAag-INV

RMVTGDN I NTAKA I ARECG I-

$-\mid \mathrm{td}--\mathrm{d}-$

-x-----iai egpefr

nkdpdqmre 
i i pk i--qVMA--RSLPLDKHTLVTNLr

--- gm-xne-

-VVAVTGDGTNDAPALHEADIGLAM--

xiagtev

AKENADV I I Md-DNFST I I nvAKWGRSVY I I QKFV

$-0$

fql-tvn-v-valmvnf i sasftg s--ap Itivql Iwv

nl imdt I ga la lateppn-da $-m m k r p p v---g-r g d n f i t k v m$ w-rnivgqsi $y--q|v v| g v|| \mid$

$-r g k s \mid x-x x-----x x x---x x$

ds----IIntfvfntfvfcqvfn-evnsremx-kinvf----x---gixsswi fsavvgvt agfavimv--el--|gtfantvhl

--------sgk|w|----ts--v | igsvg I vig-

ailkci

pvesgx

$x X X$

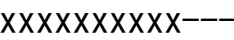

$x x x---x x x$

>osal I_IIB02 NP_001046064.1

m 

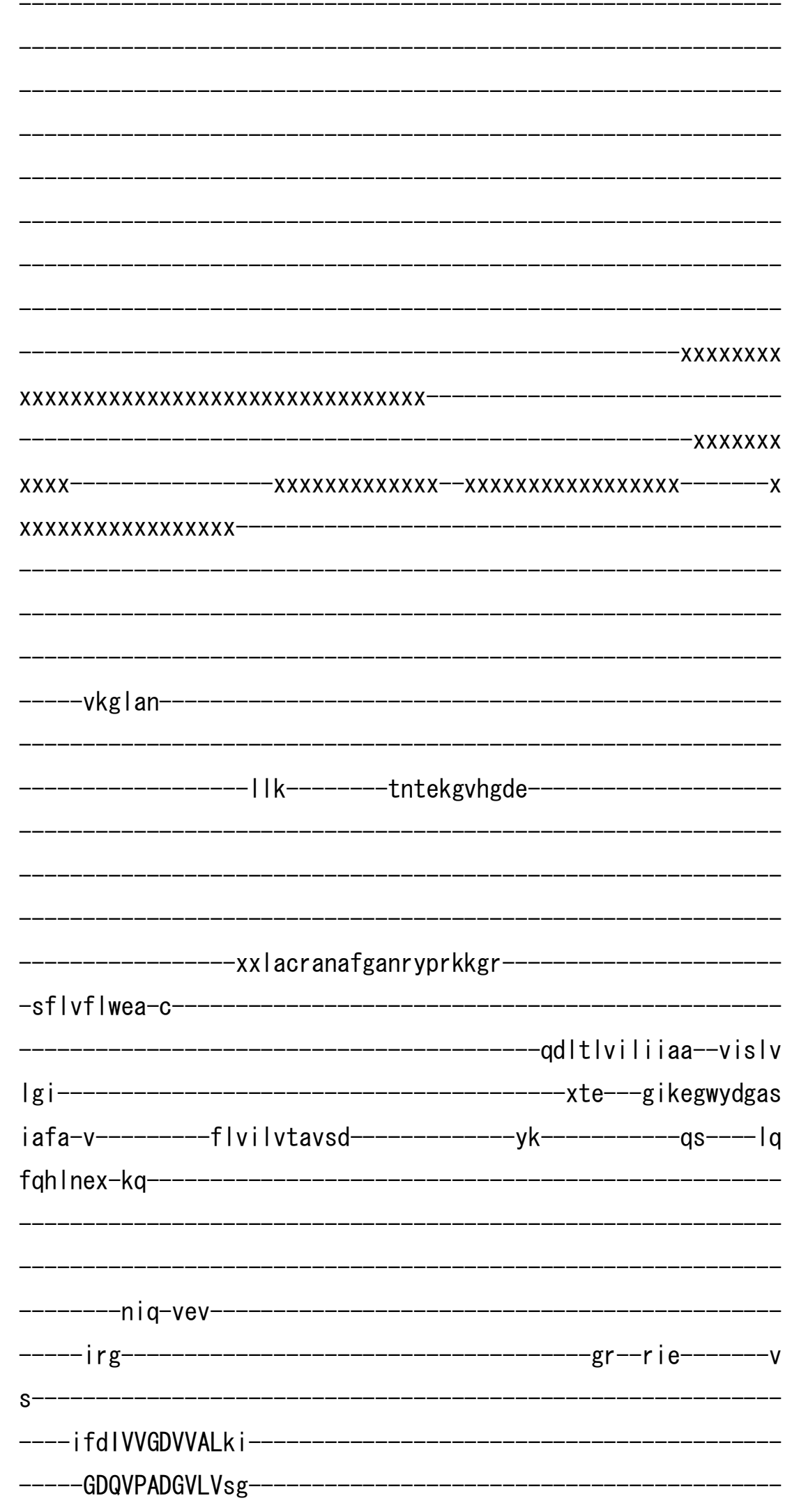
-h--s I a IDESSMTGESK IVVKdh-

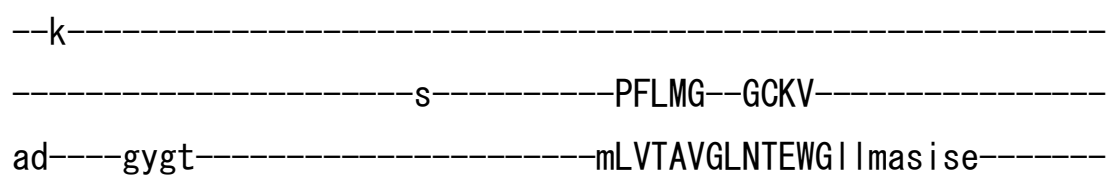

-dnneetp lqvr Ingvatf igivg I svaamv I

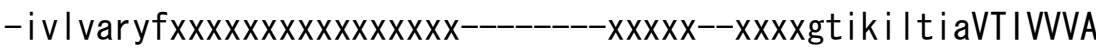
VPEGLPLAvTLTLAYSMQ-KMmadk-

-aLVR-RLSACETMGSATTI ICSDKTG

TLTLNQMTVV -rsvvggikl

$-k-x x$

xxxxxIspvvss

leg i aqn---xxgsvf-

exxxgxi

e i tgspte--kai l sw----xx-x----

Ihmkfae--eks

-kss i ihvspfnsekk-ragv

$-a-x x x--x x x----x x x x x x k-$

$\mathrm{e}$

-ivlalctnwldvngx 
shemtpdkanqf-kky i eem--aee----s I rcVAFAYRn I

-xxxxxpneeer i nwexpdne l a I I I VGMKDPCRPGVRNAVDLCKNag-VKV

RMVTGDNLQTARA IALECG I

$-1 \mathrm{t}-\mathrm{d}-$

$-x x x x x x v$ i i egkvfr

aysdaerea-

vadq i--sVMG--RSSPSDKLLLVKALk

$---\mathrm{kk}--\mathrm{gn}-$

-VVAVTGDGTNDAPALHEADIGLAM--

-xiggtev

AKESSD I I ILd-DNFASVVkVVRWGRSVYAN I QKF I

Q

fq I-tvn-v-aal i invvaa i ssg--_-_-_---_--_--_-_----n--vp I navq I I wv

nl imdt I ga la lateppt-dq---_--_-_-_-_-_----Imkrppv---o-rkep I vtn im

w-rnlfiqavf--qvtvlItInf-

$-\operatorname{rgrdl} x$

hl----tqdt I dhan-----kvfn-efnsrkpx-elnif---x---gvxrnhIflavvsit vvlqvi i i--ef--lgk----------ftstvr l-

-swk |w|----vs--vgigfvswp la

fagkfi

pvprtx $-x x x-$ 
g

>osal I_IIB03 NP_001049307.1

$\mathrm{m}$

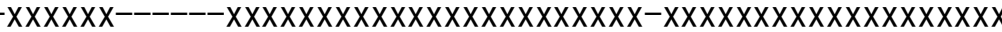

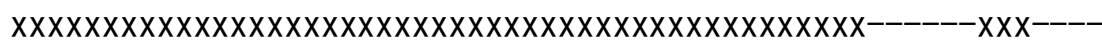
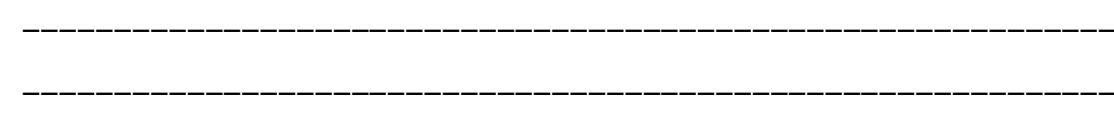

$-x x x x x x x x x$

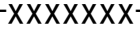

kshggveaiaa

$-k \mid c$ tspedg $\mid$ pksr

-xxqavreelfginrfaetesr

-sfwvfvwea-I 


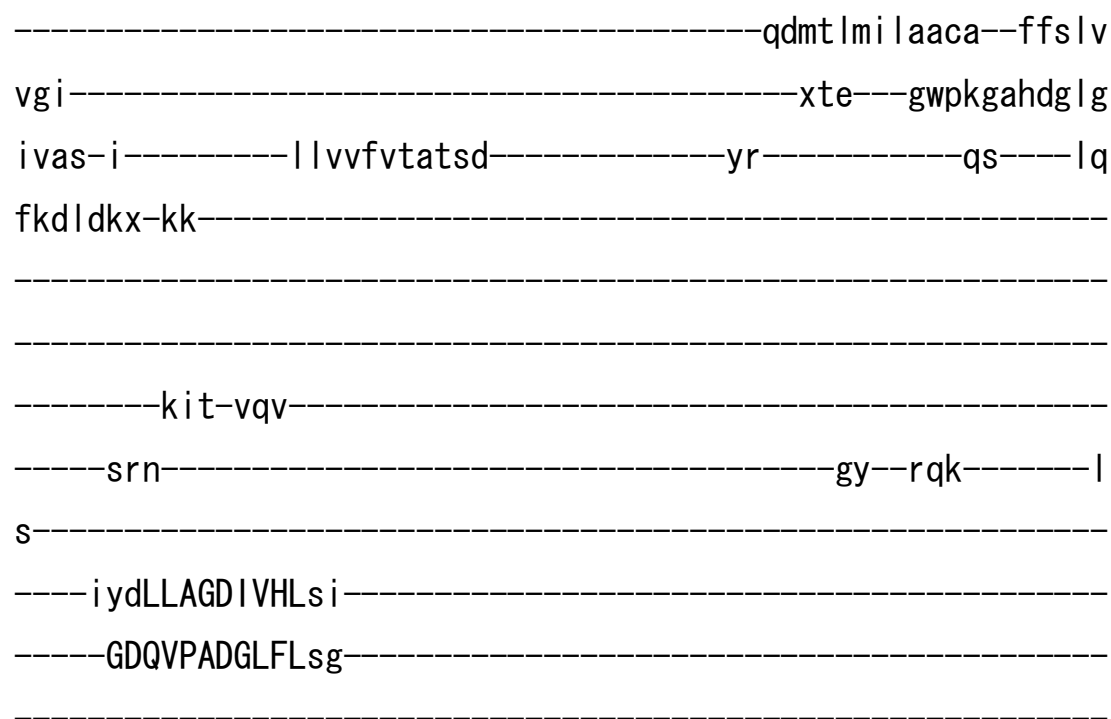

-f--s I I INESSLTGESEPVAVna-

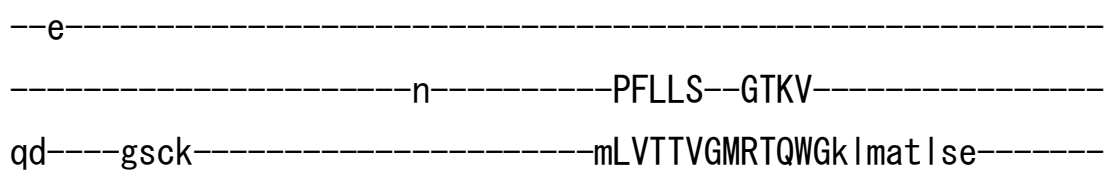

-ggddetp lqvk Ingvati igkigl ifavvtf -av|teg | fxxx-xxxxxxxxxxxx--------xxx--------e I leffaiaVTIVVVA VPEGLPLAvTLSLAFAMK-KMmndk-

aLVR-HLAACETMGSATTICSDKTG

-TLTTNHMTVv---_-_-_-------kac i cgk i

$x-x \times x \times x S x \times x$

xxxxxIpesamt

sqs ifnn---xxgdvv

fxxxg-sr 
-e i gtpte--tai lef-----xx-x

lggdf I a--vrk

-ast I vkvepfnsakk-rmgv

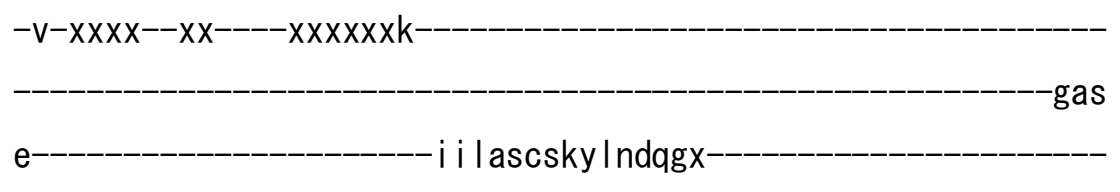

vvp I ddatvahI-nat insf--ane----al rtLCLAYVdv

-gdgf sandq i-pedgytc I GIVG IKDPVRPGVKESVA ICRSag-IMV RMVTGDN I NTAKA I ARECGI -

Ite--g------

------ia a egpdfr-

tksaee Ine-

I i pk i--qVMA--RSSPLDKHTLVKHLr

--- tt-xde-

-VVAVTGDGTNDAPALHEADIGLAM--

-xiagtev

-AKESADV I I Ld-DNFST IVtvAKWGRSVY I I I QKFV- 
fql-tvn-v-val i vnfssacltg s--ap I tavq I Iwv

nmimdt I galalateppn-deImkrtpv---g-rkgnf i snim w-rni lgqafy--qfiviwylqt

- egkw|x-xx-----xxx---xx

dl-----v|ntl ifncfvfcqvfn-evssremx-rinvf----x---gi ixdnnvfvavlgst vifqfi i v---qf--|gd-----------fanttp I-

-t lkqwf----nc--ifigfigmpia

aavkli

$-p v d f$

>osalI_IIB04 NP_001050661.1

m 


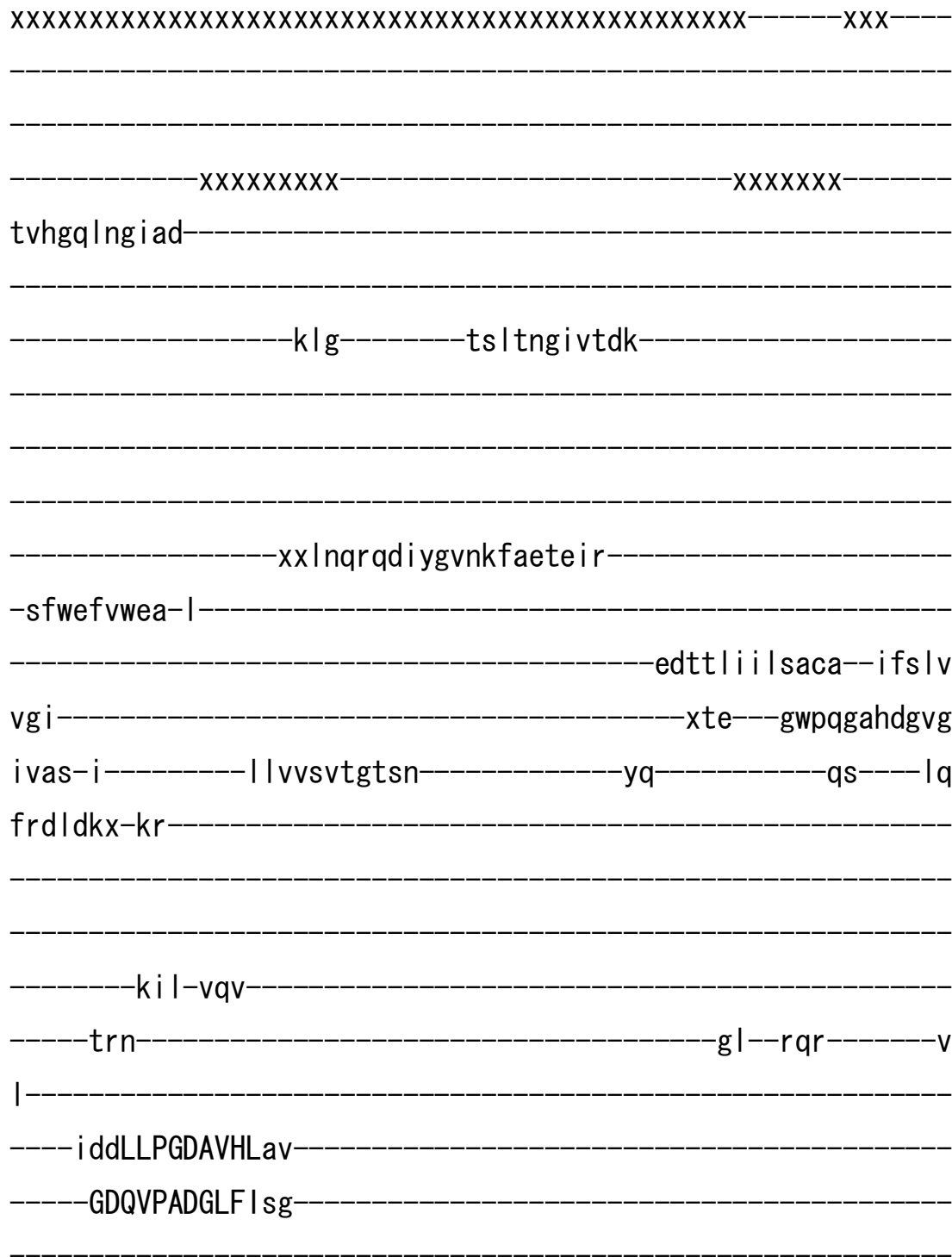

$f--$ sv IVDESSLTGESEPVFVne-

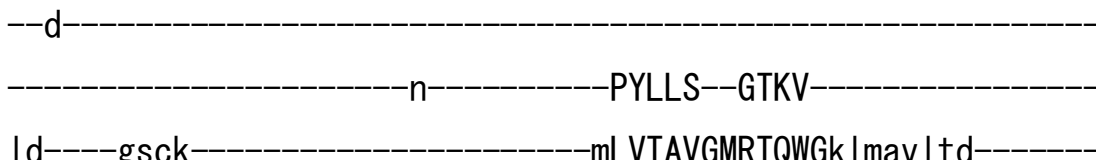

Id----gsck--_-_-_-_-_-_-_LVTAVGMRTQWGk I mav I td

-ggddetp Iqtr Ingvantigkig Iffav Itf

-ivlsgg i i xxx-xxxxxxxxxxxx--------xxx--------e i IdhfavaVTIVVVA VPEGLPLAvTLSLAFAMK-KMmndk- 
-aLVR-QLAACETMGSATVICSDKTG-

TLTTNRMTVV

-kacicgnt

$x-x x x x x q-x x$

\section{xxxxxfpevave}

les ifnn---xxgevv-

$-t x x x g-k y$

qi l gtpte--tal lef-----xx-x----

$\mid$ dgdcke--kq $\mid$

gskivkvepfnstkk-rmst

$-i-x x x x--x x----x x x x x x k$

e-

i v l aacdkf idergx

i vp Iddktssk I-nd i i kaf--sse-----a I r tLCLAYRem-

-eegfstqeq i-p I qgytc I G IVG IKDPVRPGVRQSVATCRSag-ISV

RMI TGDNIDTAKA I ARECG I

- tk-- d-

-x-----iaiegaefr

eksaee Ihd- 
I i pkm--qVLA--RSSPLDKHTLVKHLr

--- ta-xne-

-VVAVTGDGTNDAPALREADI GLAM--

-xiagtev

AKESADVVILd-DNFST IVtvAKWGRSVYVNI QKFV

$-Q$

fq I-tvn-v-val I vnftsacftg--_-_-_-_-_-_-_-_-_---d--ap I tavq I wv

nmimdt I ga la lateppn-nn---_-_-_-_-_-_----Imkkapv---g-rkgkf i tnvm

w-rni vgqs I y--qfavmwy lqt

-qgkhlx-xx------xxx---xx

di----vInt i i fntfvfcqvfn-e i ssremx-dinvl---x---gmxgns i f I gv l tgt iffafi Iv--qf--|gd-----------fanttp I-

--------taqqw I----is--i I fgf I gmp ia-

aaikli

avephx-

$-x x x-$

$-x x-$

$x x x$

>osalI_IIB05 NP_001053795. 1

$\mathrm{m}$ 

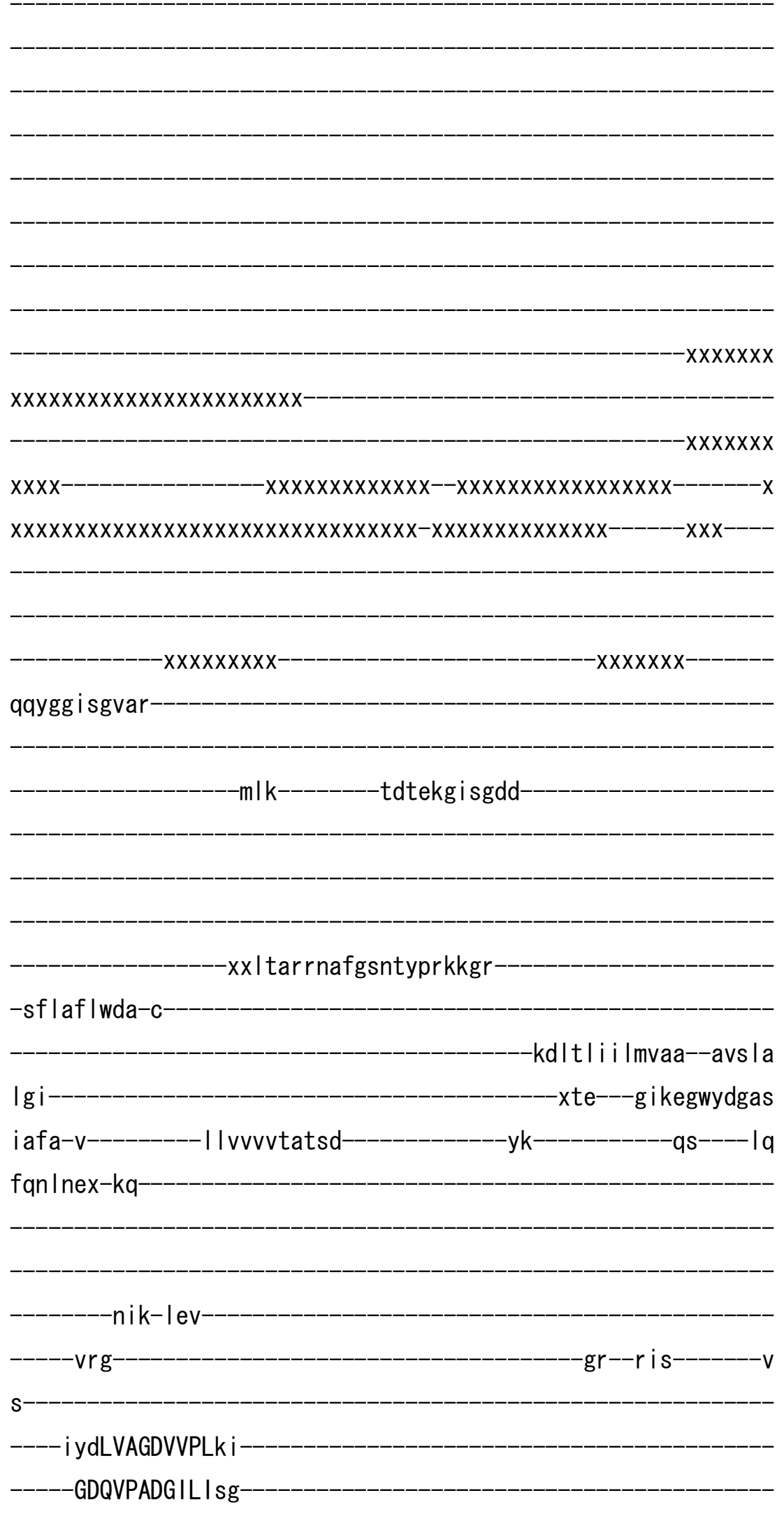
-h--s I sVDESSMTGESK IVHKdq-

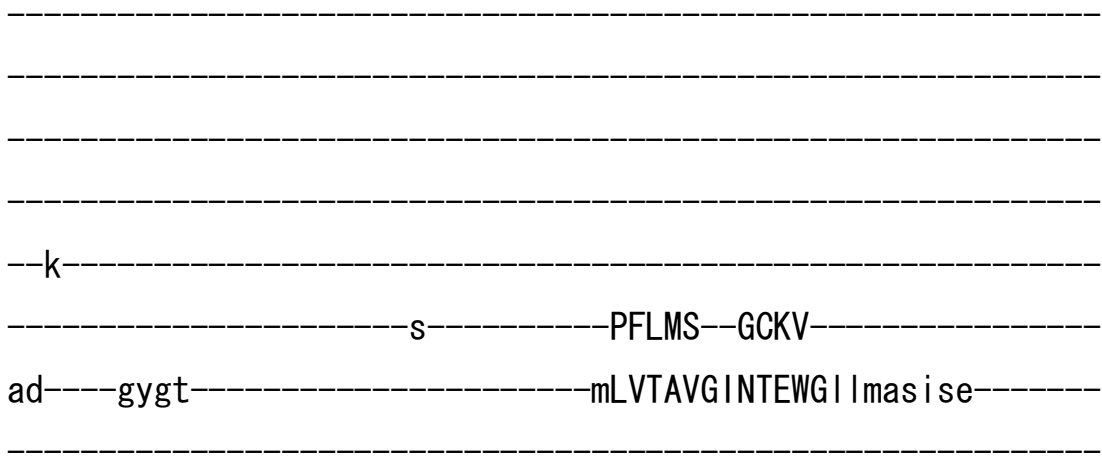

-dsgeetp I qvr Ingvatf i gmvg | sva lav |

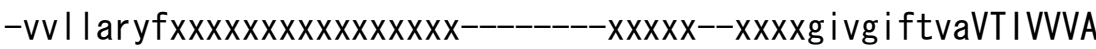
VPEGLPLAvTLTLAFSMR-KMmrdk-

aLVR-RLSACETMGSATTICSDKTG

TLTLNQMTVV

eayfggkkm-

$d-x x$

$x x x x \mid$ sasiss

veg i aqn---xxgs if-

exxxgxdp

evtgspte--kai I sw-----xx-x----

Igmrfnd--trt

-kss i lhvfpfnsekk-rggv

$-a-x x x x x x x x----x x x x x x k$

- i i ldscksw l aadgx 
khsmtpek i sef-kkf i edm--aas----s I rcVAFAYRty

-xxxxxpsedr radw i xpedd I imLGIVG IKDPCRPGVKDSVRLCAAag-IKV

RMVTGDNLQTARA I ALECGI

- s-- d-

xxxxxxvi i egkafr

alsdleree-

aaek i--sVMG--RSSPNDKLLLVKALr

$---k r--g h-$

-VVAVTGDGTNDAPALHEADIGLSM--

xiggtev

-AKESSD I I ILd-DNFASVVrVVRWGRSVYAN I QKF I $-Q$

fql-tvn-v-aal i i nvvaavssg-----_-_--_-----------n--vp I navq I w wv

nl imdt I ga la lateppt-dh----_-_-_-_-_----- Imqrppv---g-rrep I i tnvm

w-rnl i imalf--qvivlltlnf-

-rgts $\mid x-x x-----x x x x x x x x$

dk----vkntfi fntfv lcqvfn-efnarkpx-elnif---x---gi xgnh I fma i vait

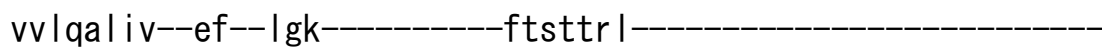

--------twq $\mid$ w |-----vs--i g laffswp la-

fvgkli 
V

>osalI_IIB06 NP_001055932.1

$$
\mathrm{m}
$$
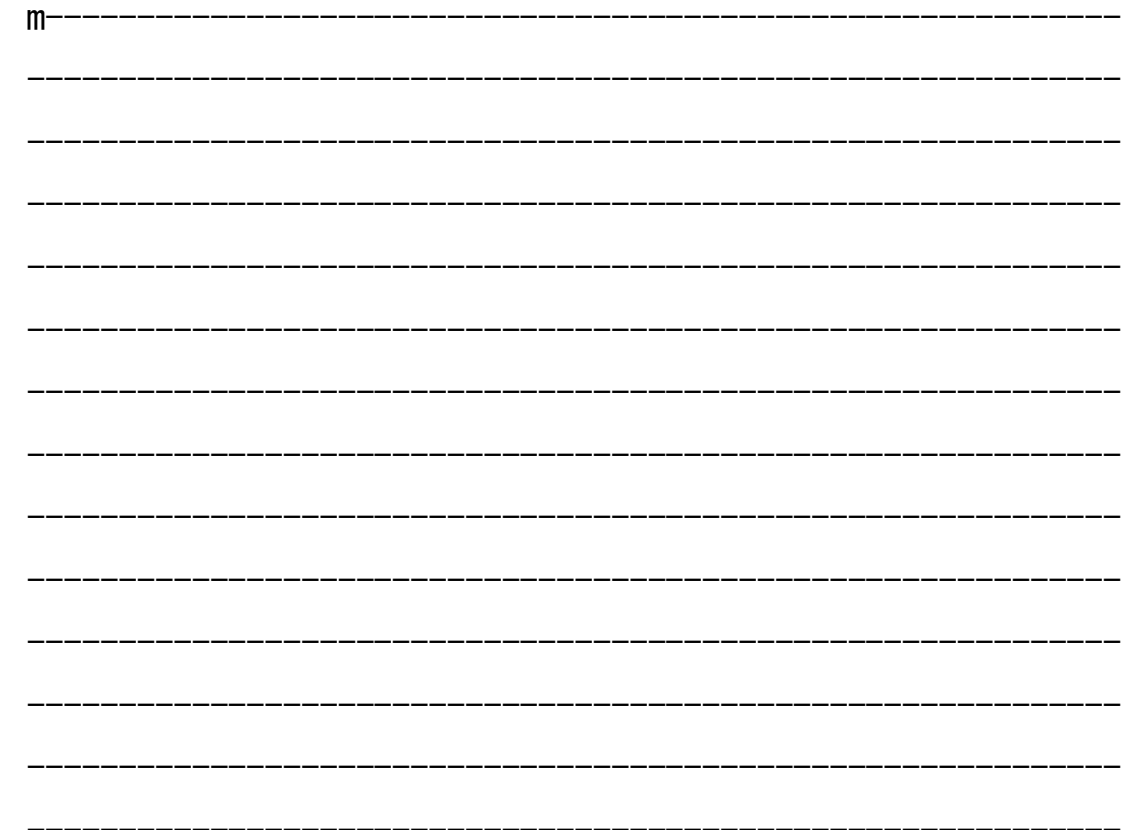

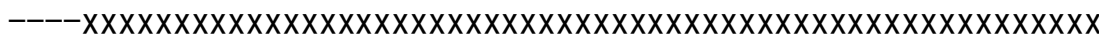
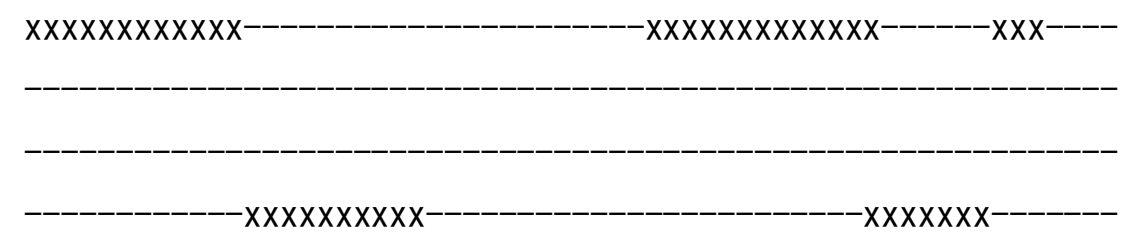

rmhggingisr

$$
\text { -kik--_-----as l edgaketd- }
$$

-sfwmfvwda-I 


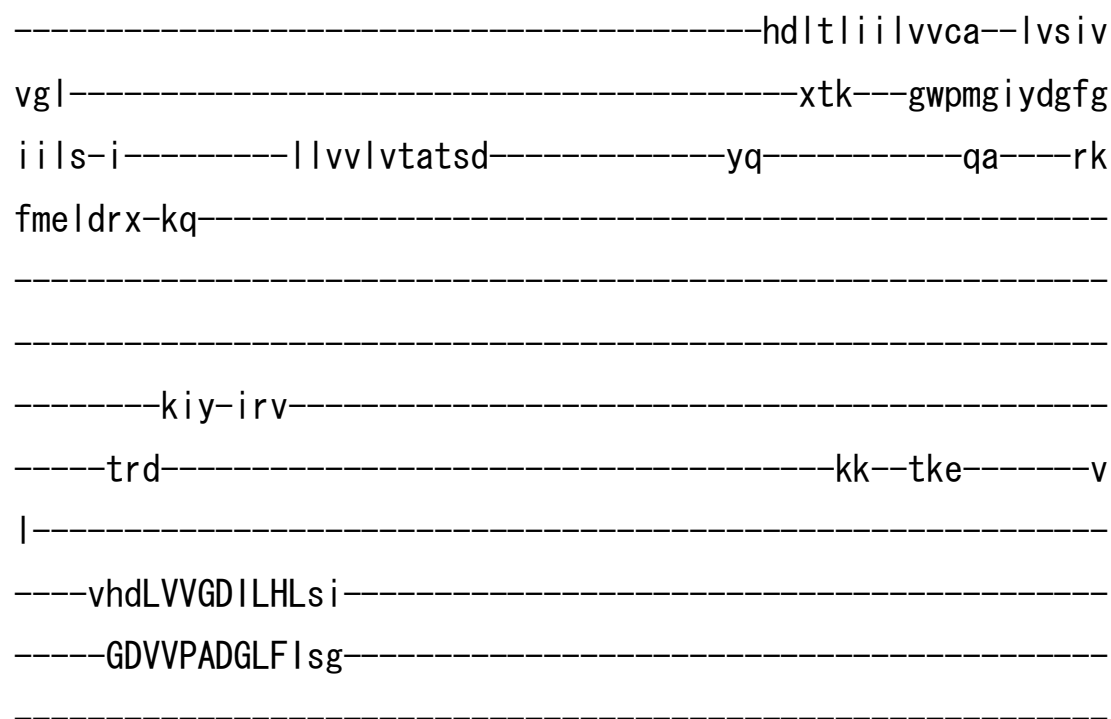

d--c ImIDESSLSGESEPVN Is--

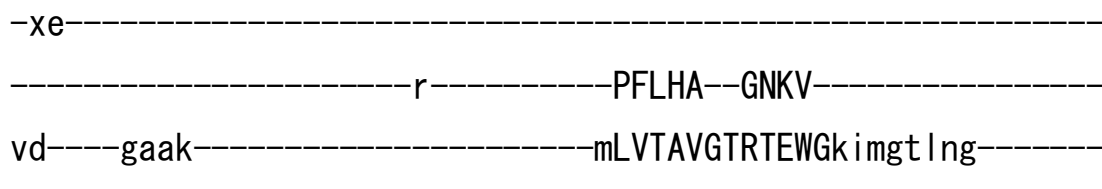

-dgvdetp Iqvk Ingvati igqig IvfavItf - Iv|larfl xxx-xxxxxxxxxxxx---------xxx---------t i vnyfa i aVTIIVVA VPEGLPLAvTLSLAFAMK-KLmhdk-

aLVR-HLAACETMGSASCI CTDKTG

$-T L T T N H M I V d-$ $-k v w i g d-v$

$x x x x x k x x x$

xxxxx i servma

i gg i fvn---xxsevv

$-k x x x g-k n-$ 
- ti lg late--tal lef-----xx-x

leeh $\mid y d--d y n$

$-k$ Itrikvdpfnsvkk-kmsv

$-t-x x x x--x x----x x x x x x k$

-gas

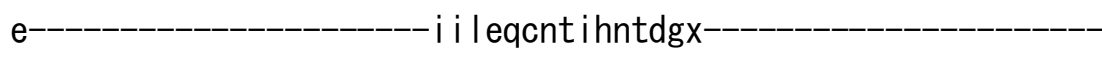

i vp I semqkhnv-Ini insf--ase----al rtLCI AFKdm-

-defpndqpxsddgyt I IAVFG IKDPVRPGVKDAVRTCMAag-IRV

RMVTGDN I NTAKA I AKECG I

te--d-

------iai egqq In

-nkssde lke-

I l pk i--qVIA--RSLPMDKYKLVTSLk

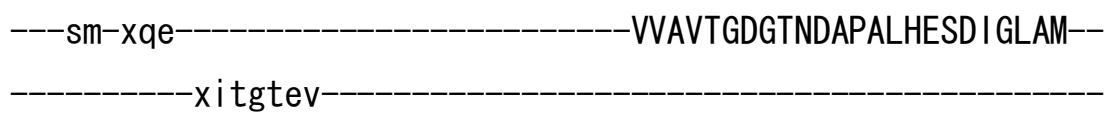

-AKESADV I IMd-DNFET I VnvARWGRAVYLN I QKFV------Q 
fql-tvn-i-val i vnfvsaci i -s--ap I tavq I Iwv

nmimdt I gala lateppn-de -mmkrppv---r-rgdnfitrim w-rni I gqg | y--q | | v lat Imv

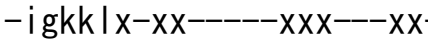

dk----tintl ifnsfvfcqvfn-e incremx-kinvl----x---gi xrnwifvgi l tat vifqvi iv--ef--|gt-----------fantvp |

sge|w|----|s--vvigsismi is

- vilkci

pvefnx

$-x x-$

$-x x x x x x x x x---$

$-x x x---x x x$

>osalI_IIB08 NP_001065710. 1

$\mathrm{m}$ 
kmhggvdgisk-

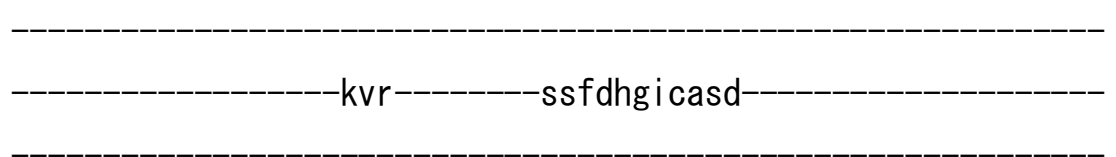

Idtrqni ygvnryaekpsr

-sfwmfvwda-f-

qdmt I i i Imvca-- I I sva

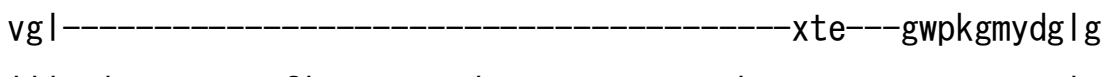

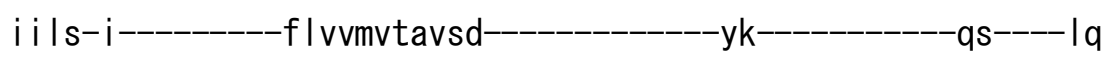

fkeldnx-kk-

$-y--s$ I I IDESSLSGESDPVYVs--

$-x d$

$-k$ if-ihv

$\operatorname{trd}$

-gr--rqk-------i

$\mathrm{s}^{-}$

----i ydLVVGD IVHLsi

GDQVPADGLYIhg

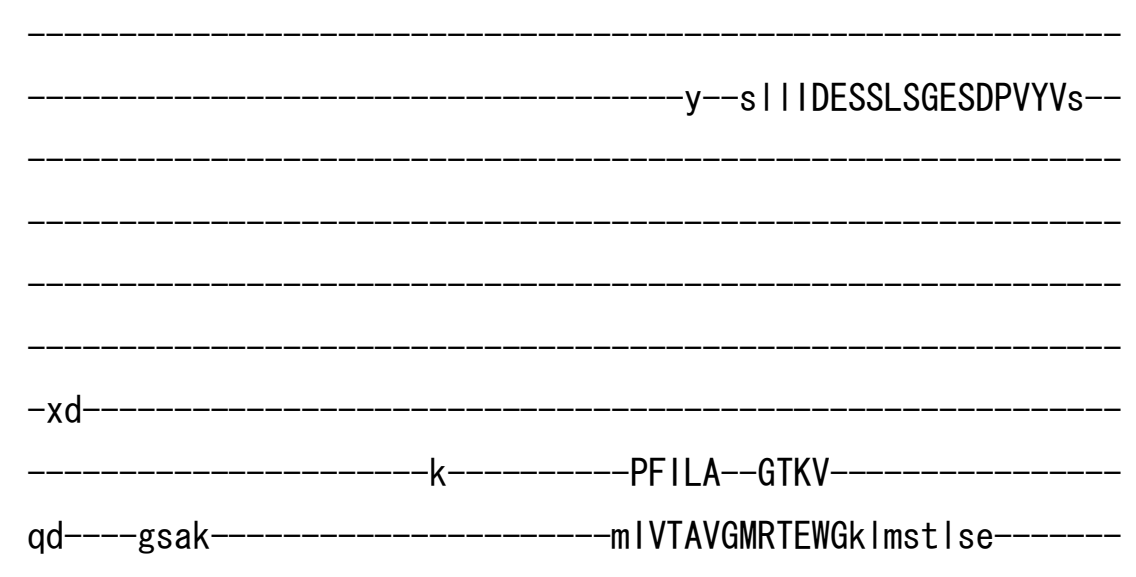

-ggedetp lqvk Ingvatvigkig I fai Itf

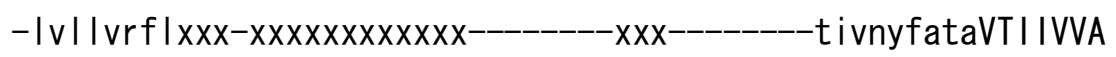
VPEGLPLAvTLSLAFAMK-KLmndk- 
-aLVR-HLSACETMGSAGT I CTDKTG-

$-T L T T N Y M V V d-$

-kiwisevsk

$\operatorname{xxxxxtxxx}$

xxxxxvssrtIs

Iqg i fen---xxaevv-

$-k x x x g-k q$

tvlgtpte--railef-----xx-x----

legvhda--eys

-actkvkvepfnsvkk-kmav

$-1-x x x x--x x----x x x x x x k$

-gas

e-----_--_-_-_-_--_--_- i i l qmcdmmvdgdgx-

a ip I seaqrkni-Idt insf--asd----al rtLCLAYKev

-ddd i ddnadxptsgft I IAIFG I KDPVRPGVKDAVKTCMSag-I TV

RMVTGDN I NTAKA I AKECG I-

- te--d-

-x-----va i egpefh

skspeemrd- 
I ipn i--qVMA--RSLPLDKHTLVTNLr

--- gm-xde-

-VVSVTGDGTNDAPALHEADIGLAM--

xiagtev

-AKESADV I VLd-DNFTT I I nvARWGRAVY I I QKFV

$-0$

fql-tvn-i-valvinfvsaci tg-_-_-_-___-__-__-_-_-_-ap I tavq I I wv nmimdt I ga l a lateppn-de------------------mmkrppv---r-kgesf i tkvm w-rnimgqs $|y--q| f v \mid$ ga $\mid m f$

-gges $\mid x-x x-----x x x---x x$

ks-----i intl ifnsfvfcqvfn-e insremx-kinvf----x---gixsnwifiaviaat vafqvvi i--ef--| gt--_-_-_-_--_fastvp |--_-_-_-_-_-_-_-_-_-_-_-_-_-_

--------nwqhw |----- |s--vg | gs i s l i vg--_-_-_-_-_-_-_-_-_-_-_-_

-vilkci

-pvgsgx-

$-x x-$

$-\mathrm{XXXXXXXXXX---}$

$\mathrm{xxx}---\mathrm{xxX}$

>osalI_IIB09 NP_001066105.1

m 


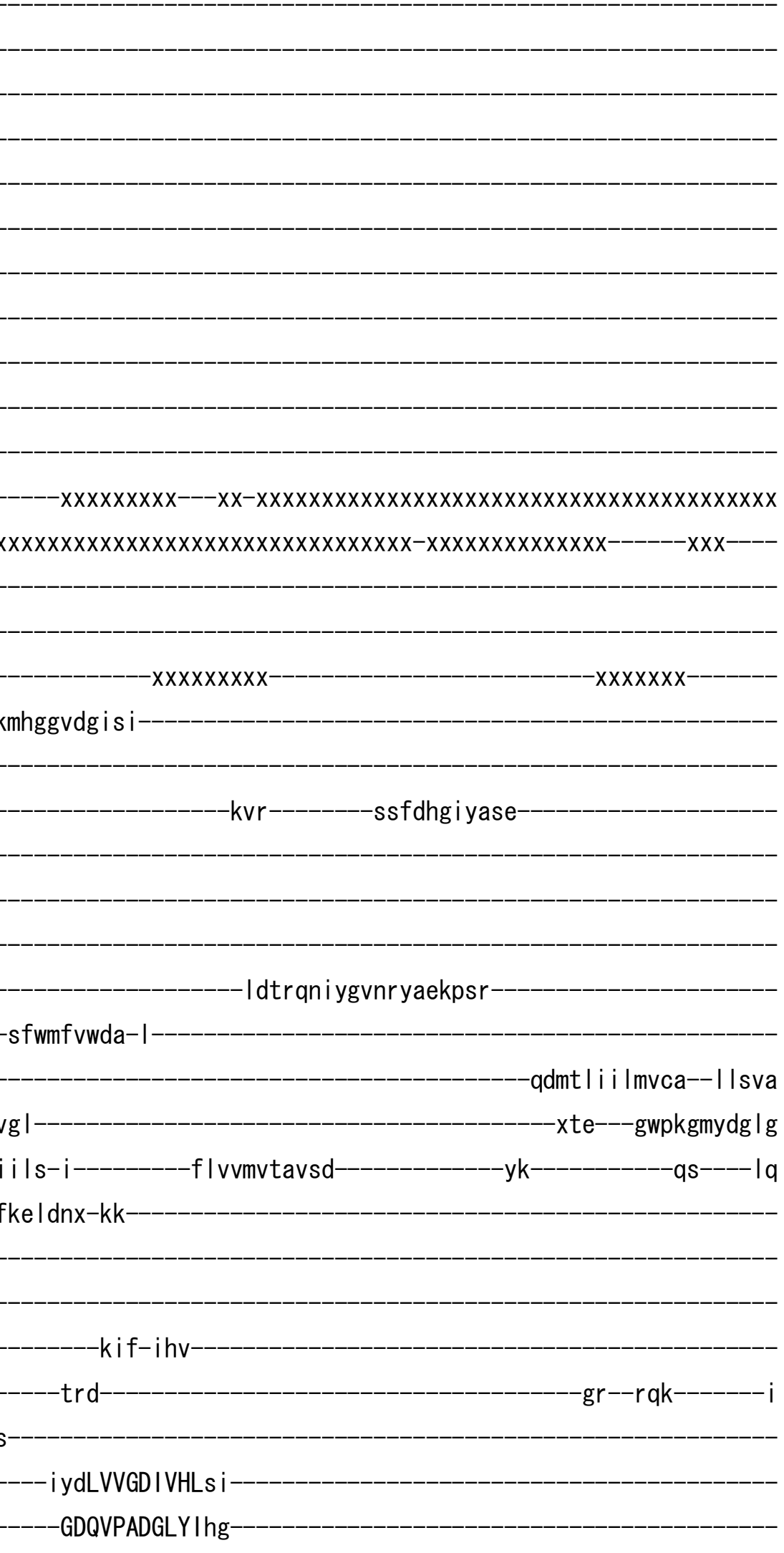


$-\mathrm{y}--\mathrm{s}$ I I IDESSLSGESDPVYS--

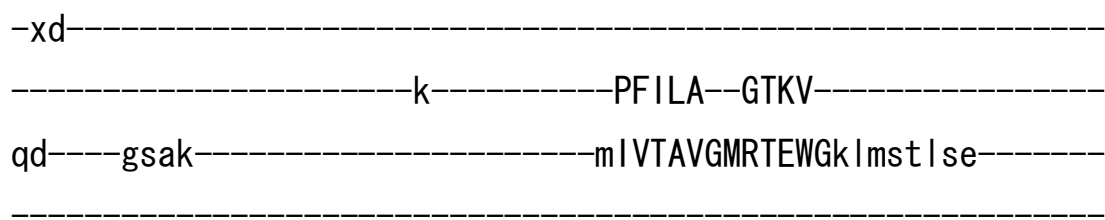

-ggedetp IqvkIngvati igkigIvfailtf

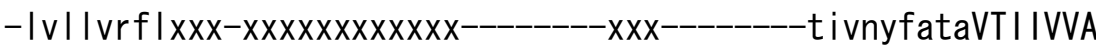
VPEGLPLAvTLSLAFAMK-KLmndk-

aLVR-HLSACETMGSAGT ICTDKTG

$-T L T T N H M V V d-$

-kiwisevsk

$-x x x x \operatorname{txx}$

xxxxxvsst Is

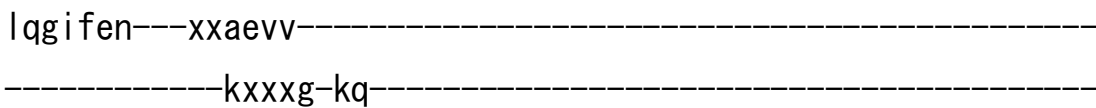

tvlgtpte--railef-----xx-x----

lkgdhda--eyr

actkvkvepfnsvkk-kmav

$-1-x x x x--x x----x x x x x x k$ 
a ip I seaqrkni-Idt insf--asd-----al rtLCLAYKev

-ddd i ddnadxptsgft I I AIFG I KDPVRPGVKDAVKTCMSag-I TV

RMVTGDN I NTAKAI AKECGI

Ite--d-

------vai egpefh-

sksteemrd-

I i In i--qVMA--RSLPLDKHTLVTNLr

$---g m-x d e-$

-VVSVTGDGTNDAPALHEADIGLAM--

$-x$ iagtev

AKESADV I VLd-DNFTTI I nvARWGRAVYIN I QKFV Q

fql-tvn-i-valvinfvsac i ig--_-_-_-_-_-_-_-_----s--ap I tavq I I wv nmimdt I ga la lateppn-de------_---_-------mmkrppv---r-kgesf i tkfm w-rnimgqs ly--qlfvlgalmf

-gger $\mid x-x x-----x x x---x x$

ks----i int l i fnsfvfcqvfn-e insremx-kinvf---x---gi ixsnw i f i av i aat vafqvv i i--ef--|gt-----------fastvp I-

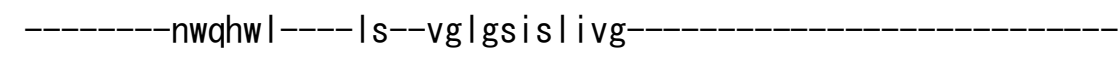

$-v i l k c i$ 
>dme II_I IB01 NP_726564. 3

$\mathrm{m}$

$-x x x x x x x x x x$

aengg ihe lck

$-k$ l y -tspnegl sgsk

xxeehr retfgsnv ippkppk

-tfIt|vwea-I 


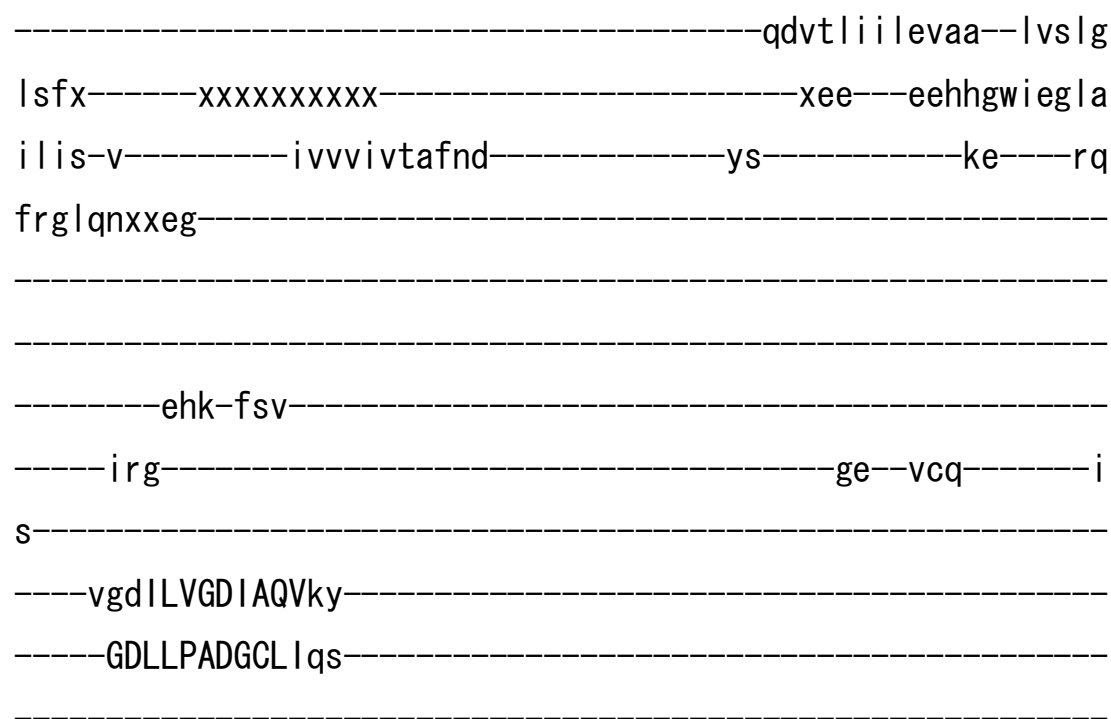

-n--dlkVDESSLTGESDHVKKgp-

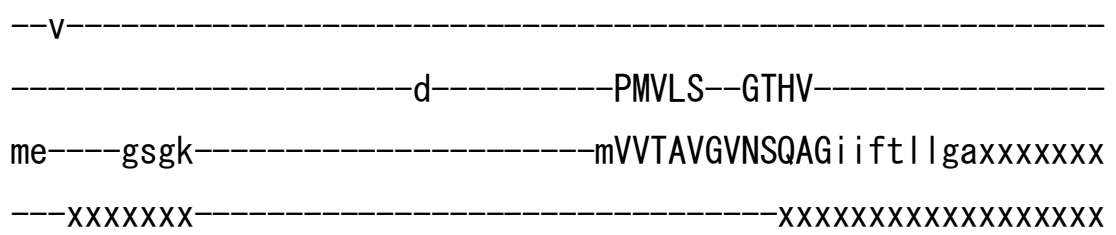
$x x x x x x x x x x x x x x x x x x x x x x x x x x x x x x$ hkkeksv lqak|tk la iqigyagst $i a v \mid$ tv

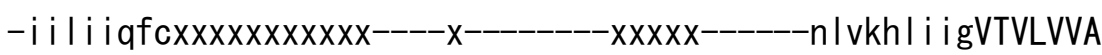
VPEGLPLAvTLSLAYSVK-KMmkdn-

nLVR-HLDACETMGNATAI CSDKTG

TLTTNRMTV qsy ice $-k \mid$ cka $\operatorname{gxxxg} \mid p$ 
iqvgnkte--cal Igf-----xx-x-

-I gvkyqs--irdexx

-edkftrvytfnsvrk-smgt

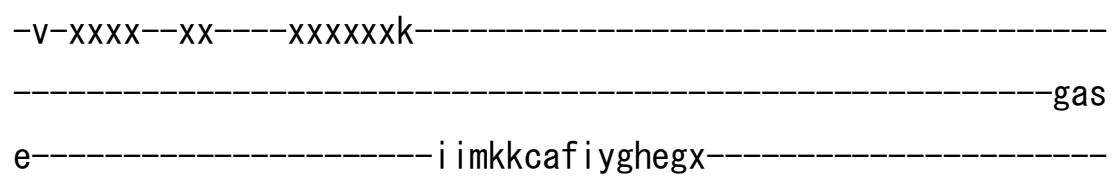

lekftrdmqer I xrev i epm--acd-----g | rt ISVAYRdf

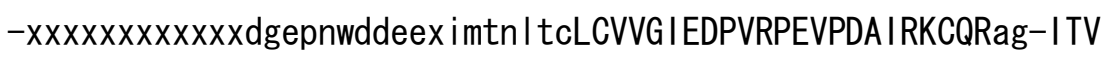

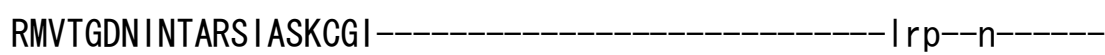

$-x x x---1$ i legkefnx

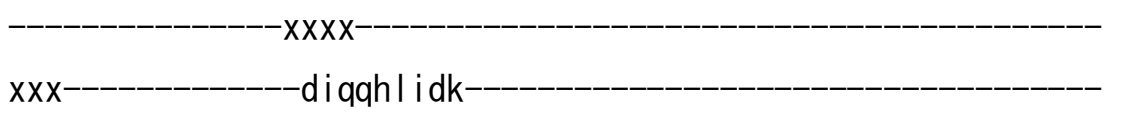

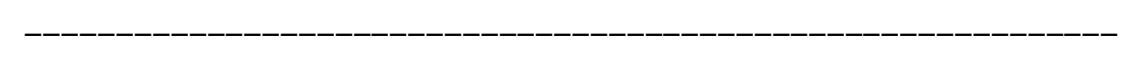

vwpk I--rVLA--RSSPTDKYTLVKGI i

---dsxxse--------xxx--_-_-_-_-_----VVAVTGDGTNDGPALKKADVGFAM--

-AKEASDI ILTd-DNFSS IVkaVMWGRNVYDSI AKFL------Q 


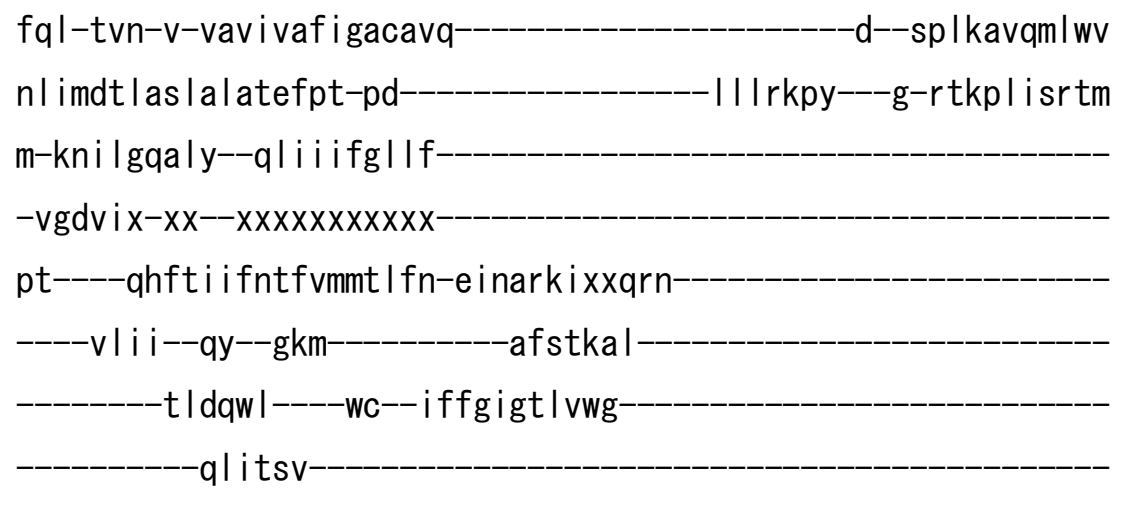

$-p t r k \mid x$

$-x x x-$

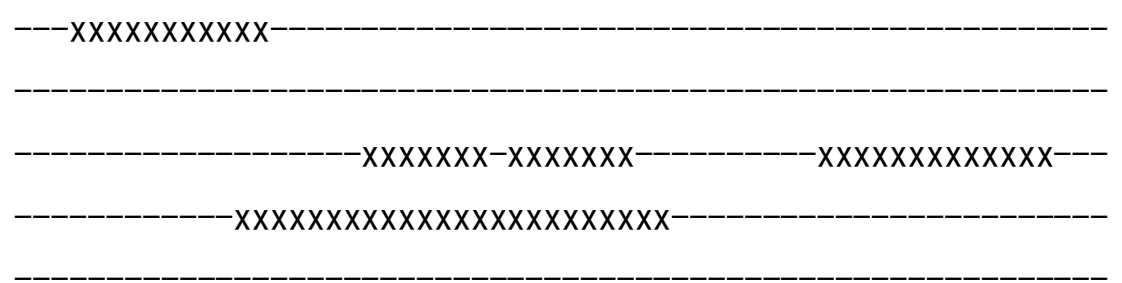

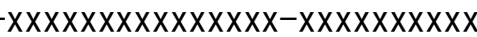

$-x x x x x x--x x x x x x x x x x x$

V

>osal I_IIB10 NP_001067159.1

m- 


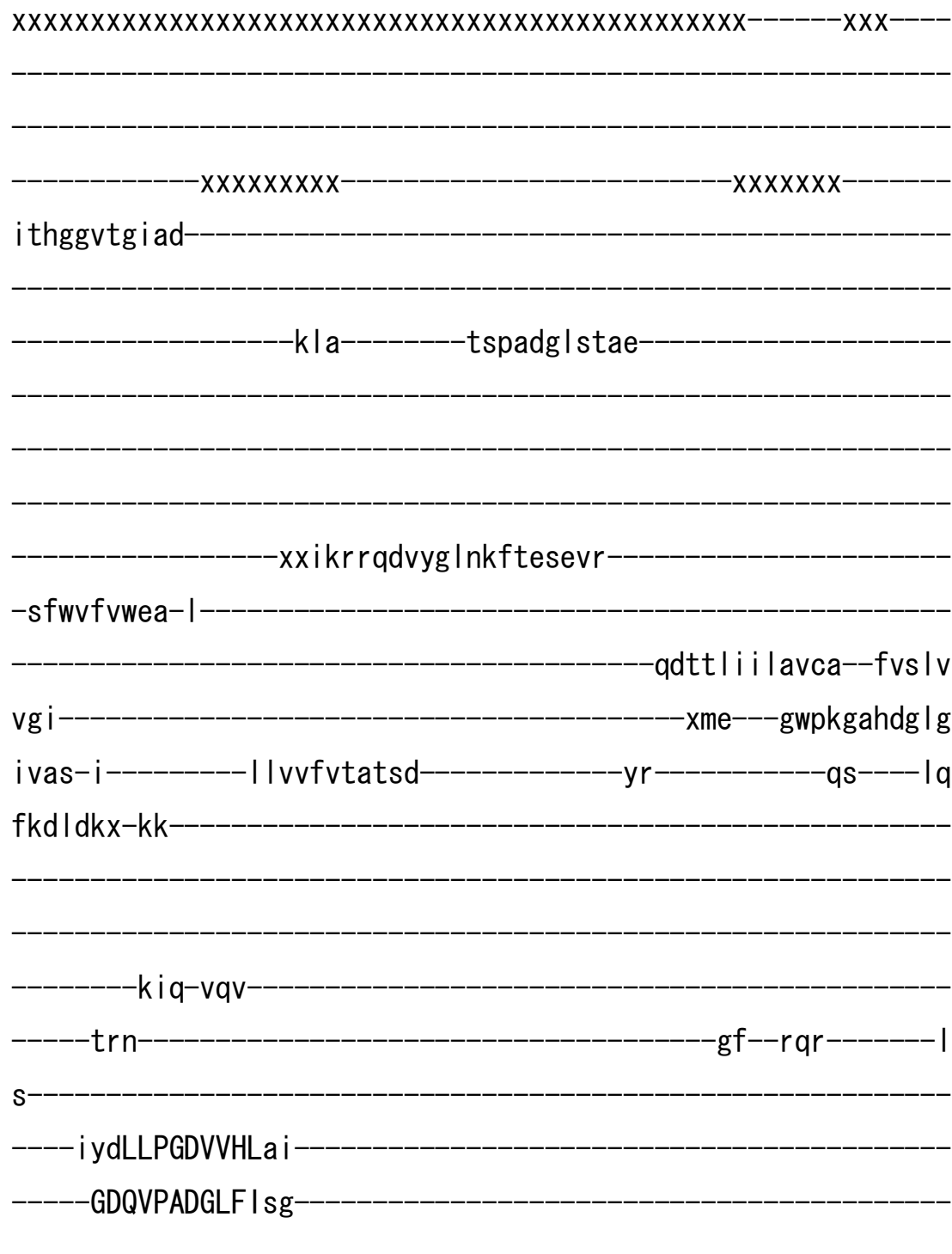

---s I I INESSLTGESEPVVVne-

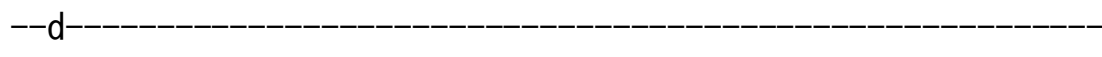

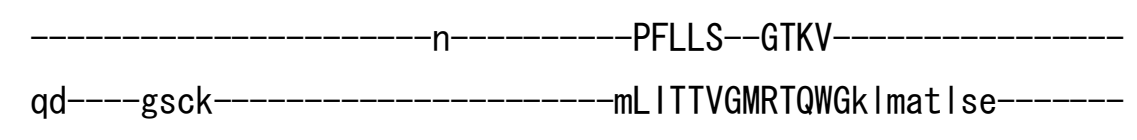

-ggddetp I qvk Ingvat i igkig I ffavitf

-iv I sqg I i xxx-xxxxxxxxxxxxx---------xxx---_-----eml ehfa i aVTIVVVA VPEGLPLAvTLSLAFAMK-KMmndk- 
-aLVR-HLAACETMGSATTI ICSDKTG-

TLTTNHMTV

-kacicgni

$-x-x x x x x k x x x$

xxxxx Ipetvvk

lesifnn---xxgevv-

ixxxg-ky

qi lgtpte--tal lef-----xx-x----

- Iggnfka--krd-

etk i vkmepfnstkk-rmcv

$-v-x x x x--x x----x x x x x x k$

-gas

e-

iv laacdkfmdetgx-

vvp Idkttadk I-ng i i esf--ane----a I rtLCLGYRem-

-eegfsveeq i-p l qgytc I G IVG IKDPVRPGVRESVATCRSag-I MV

RMVTGDN I NTAKAI ARECG I-

$-\mid$ te--d-

------ la i egpefr

eks Idellk- 
I ipk i--qVMA--RSSPLDKHTLVKHLr

$---\mathrm{tt}-\mathrm{xne}-$

-VVAVTGDGTNDAPALHEADIGLAM--

-xiagtev-

-AKESADVI I Ld-DNFST IVtvAKWGRSVYVN I QKFV

$-Q$

fql-tvn-v-val Ivnfssacftg-

-n--ap I tavq I Iwv

nmimdt I ga la lateppn-dd-

-Imkrepv---g-rtgkfitnvm

w-rni l gqsfy--qfi ivmwy lqt

$-q g k s m x-x x-----x x x---x x$

ev----vInti i fnsfvfcqvfn-e i ssremx-kinvl----x---gi xknyvflgvltst vvfafimv--qf--Igefantipl

-tr lqw i----as--v l I g I gmp i s

ai ikII

pvgss

>rnolI_IIC01 NP_036637.1

m- 
dhk Is Ide Igr

$-k y q$ -vdlskg| tnar

-aqdi I ardgpna I tppptt

\section{-pewvkfcrq-I}

fggfsi | |wiga--| lcf|

ayg $x$ xxxxxde---psndn ly lg i v

laav-v ivtgcfsyyqe -ak------------ss----k i mdsfkn--mv-

$$
-p q q-a \mid v-
$$

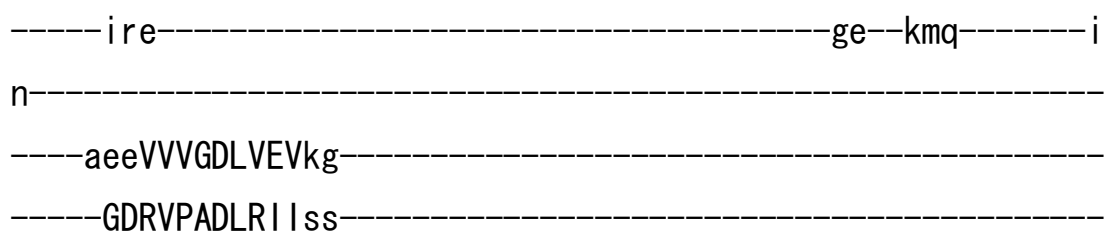

GDRVPADLRI Iss 
-h--gckVDNSSLTGESEPQTRsp-

$-X X X X X X X$

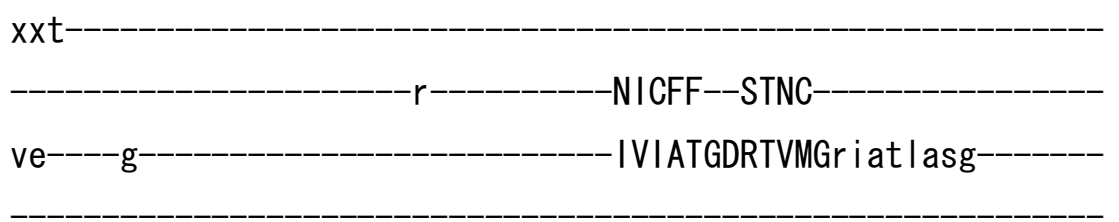

- levgqtp i ame i ehf iql i tgvavf Igvsff $-v|s|$ ilgy -swleavifIIGIIVAN

VPEGLLATVTVCLTLTAK-RMarkn-

cLVK-NLEAVETLGSTST ICSDKTG

TLTQNRMTVa

-hmwfdnqih-

$-x x x x x^{--e x x-}$

xxxxxdkrsptwx

-al

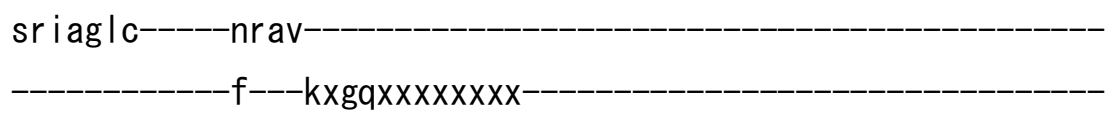

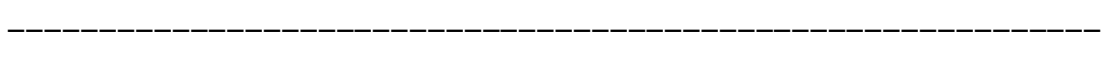

-dtagdase--sal lkc

ielscgs--vrkmxx

-rnpkvae ipfnstnkxqls i

$-x \times x \times x x------x \times x \times x \times x---$

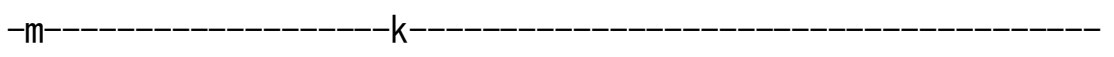

-gap

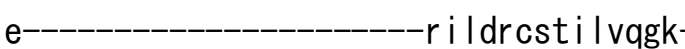


e ip I dkemqdaf-qnayme l--gg |----gervLGFCQLn I

------xxxxxxxgfkfdtde Inxptek I cfVGLMSM IDPPRAAVPDAVGKCRSag-IKV IMVTGDHPITAKAIAKGVGI

isexxe-

$-X X X X X$

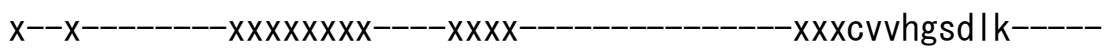

dmtseqlde

i I rdhxx i VFA--RTSPQQKL I IVEGCq

$---r q--g a$

IVAVTGDGVNDSPALKKAD I GI AM--

$-x$ isgsdv

SKQAADM I LLd-DNFAS IVtgVEEGRL IFDNLKKS I

A

yt I-tsn-i-pe itpfIIfi iani--_-_-_-_-_-_-_-_-_---p--|plgtvti l c i

d I gtdmvpa i s l ayeaae-sd-_-_-_-_-_-_-_-_-_-_imkrqpr---nxqtdk I vner I

i xmaygq igmi--qal ggfftyf $x x x x x x x x x x x x x x x x x x x x x x x x x x x x x x x x x x x x x x$ xearkvx

ef----tchtaffas i vvvqwad-I i icktrxxsvfaq--_-_---gm-knk i I ifg I lee tal aaf I s--ycxxmgv-----------al rmyp I

$-k v t w w f----c a--f p y s$ Il ifiyd

evrkli 
y

>rnolI_IIC02 NP_036638.1

m-

$-x$

$-x x x x x x x x x x x x x x x x$

$\operatorname{xx} x x x x x x x x x x x x$

ehkmsveevcr

-kyn---------tdcvgg|thsk-

-aqe i l ardgpna I tppptt

-pewvkfcrq-I 


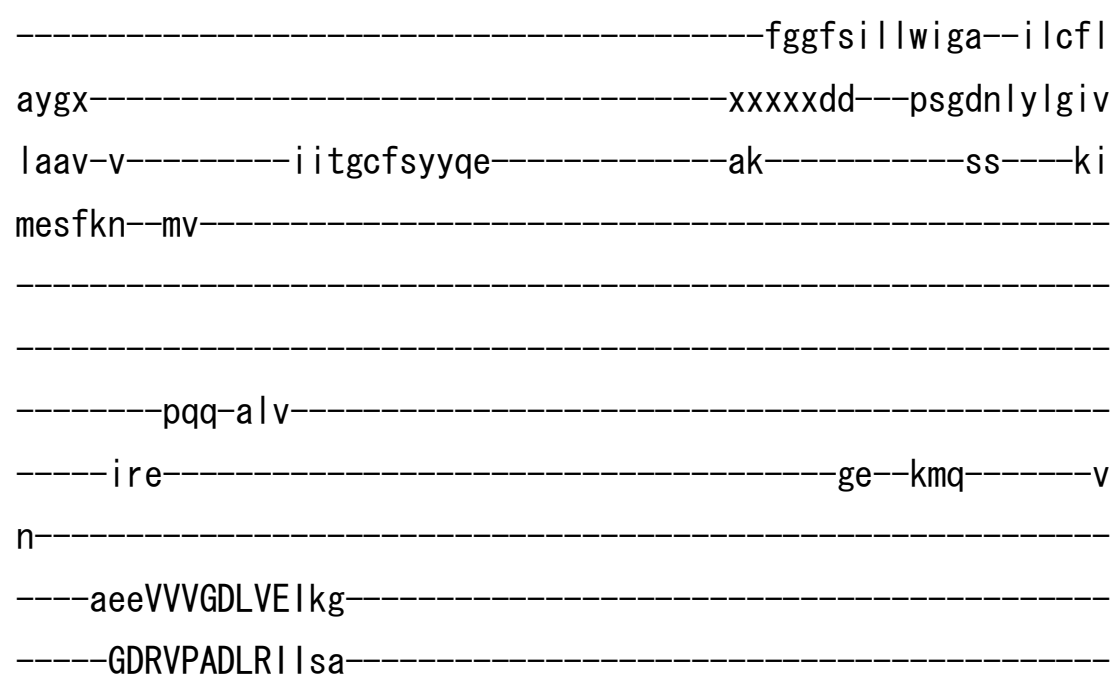

-h--gckVDNSSLTGESEPQTRsp-

$-x x x x x x x$

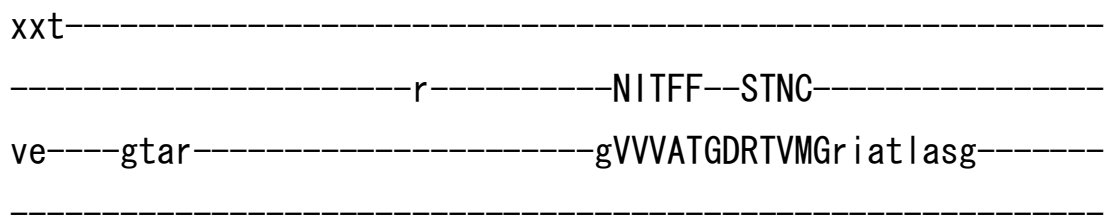

- levgktpiaieiehfiql itgvavflgvsff

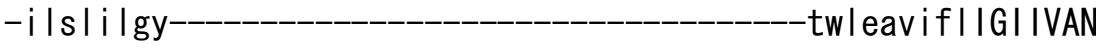

VPEGLLATVTVCLTLTAK-RMarkn-

cLVK-NLEAVETLGSTSTI CSDKTG

TLTQNRMTVa -hmwfdnqih-

$-x \times x x x--e x x-$

xxxxxdksshtwx

$-a \mid$

shiaglc-----nrav-

$f---k x g q x x x x x x x x$ 
-dvagdase--sal lkc-

- ielssgs--vk Imxx-

rnkkvae ipfnstnkxqlsi

$-\mathrm{xx} x \times x \times-----x \times x \times x \times x x---$

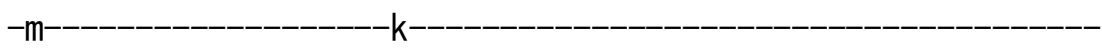

-gap

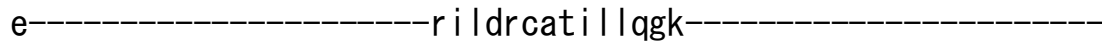

eqp I deemkeaf-qnay | e |--gg |----gervLGFCHYy I

-xxxxxxxgfafdcddvnxttdn I cfVGLMSMIDPPRAAVPDAVGKCRSag-IKV

IMVTGDHPITAKAIAKGVGI-

isexxe-

$-x x x x x$

x---x---------xxxxxxxx----xxxx----------------xxxcvihgtdlk-

dftseqide-

i I qnhxx i VFA--RTSPQQKL I I VEGCq

---rq--ga----_-_-_-_-_-_-_-_-_----IVAVTGDGVNDSPALKKAD I GVAM--

xiagsdv 


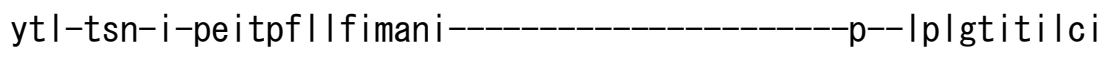
dl gtdmvpa i l ayeaae-sd--_--_-_-_-_-_-_--imkrqpr---nxrtdk I vner I i xmaygqi igmi--qal ggffsyf $x x x x x x x x x x x x x x x x x x x x x x x x x x x x x x x x x x x x x x x$ xeqrkvx--------------------------------------------------------ef----tchtaffvs i vvvqwad-I i icktrxxsvfqq-------gm-knk i l i fg I fee

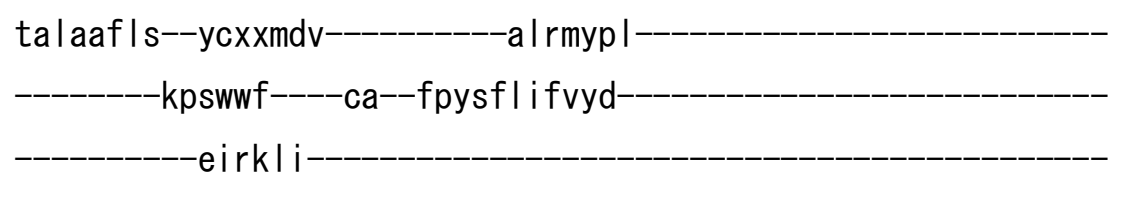

y

>rnoll_IIC03 NP_074039.1

m- 
dhk |t Ide Isa

-kys

-vdltk

- - -gckVDNSSLTGESEPQSRcp-

$-x x x x x x x$

$x x t$

$r$

-NI IFF--STNC-

ve----gtar

gVVIATGDHTVMGrias Itsg

- Itmgktp iate i ehf ihi i tavavf I gvtff

$-f|s|$ ilgy -twI davifI IGI IVAN

VPEGLLATVTVCLTLTAK-RMarkn- 
cLVK-NLEAVETLGSTST I CSDKTG-

TLTQNRMTVa

-hlwfdktvy

$-x x x x x--e x x x$

xxxxxpkssdtwx

$-\mathrm{yl}$

ar iag $\mid \mathrm{C}^{-----n r a d}$

f---kxhqxxxxxxxx-

tttgdase--sal lkf

i eqsysp--vsemxx

-knpkvae ipfnstnkxqms i

$-X X X X X$

$-x x x x x x x---$

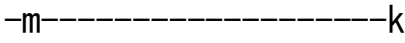

gap

$\mathrm{e}$

- i IdfcssfI Ingq

eypmdeemktdf-qnay i el--gg l----gervLGFCFLn I

-------xxxxxxgfafntee I nxpmen I cfAGL I SMIDPPRTAVPDAVSKCRSag-IKV

IMVTGDHPITAKAIAKSVGI

i sexxe-

$-x x x x x x$

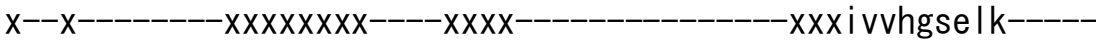

-dmdsgq Idn 
i I ksyxxiVFA--RTSPQQKLI IVEGCq

$---r \mid--g a$

-IVAVTGDGVNDSPALKKADIGI AM--

xitgsdv

SKQAADM ILLd-DNFAS IVtgVEEGRL IFDNLKKSI

$-\mathrm{A}$

ytl-tsn-i-peitpflIfivlsi-

- -- - plgtitilci

dlgtdmvpai s layetpe-sdimkr $|p r---n x k t d n| v n d r$ I

i xmaygqi gmi--qalagfftyf $x x x x x x x x x x x x x x x x x x x x x x x x x x x x x x x x x x x x x x$ xeqrkvx

ef----tcqtaffi i ivivqwad-l i icktrxxs Ifkq-

-gm-knkvlifgllee

ti laacls--yixxmdv-al rmyp I

$-k$ inwwf----ca--l pysvl if i yd

evrkli

irrrpx

$\mathrm{XXXXXXX}$

y

>rnoll_IIC04 NP_598201.1

m- 
dhr I snteleq

$-k y g$ -tni igg I ssvr

-ate I Iardgpnt I tppkqt

-pe i ikflkq-m-

-vggfsil lwiga--alcwi

afvx-xxxxxs----as I dnvy Igai

$|v| v-v$ i Itgi fayyqe -ak------------st----ni masfsk--mi$-p q q-a \mid v-$

s-----ird-----------------
GDQIPADIRLVfs 
$-X X X X X X X$

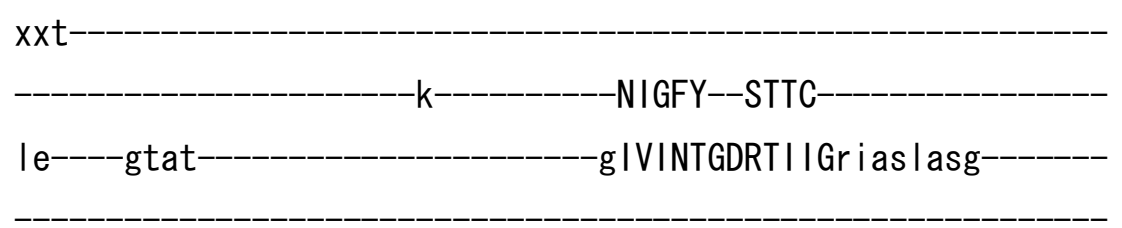

-vgsektpiai e i ehfvhi vagvavsig i iff

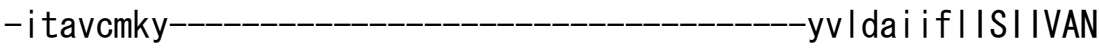
VPEGLLATvTVTLSLTAK-RMakkn-

CLVK-NLEAVETLGSTSI I CSDKTG-TLTQNRMTVahIwfdnqif-

$$
-x x x x=-e x x-
$$

xxxxxdqssgtwx $-s \mid$

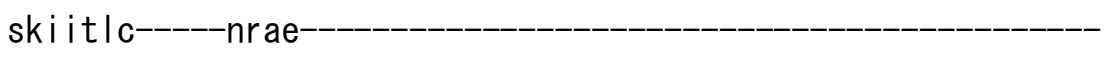

$$
\begin{aligned}
& f---r \times g q x \times x \times x \times x x
\end{aligned}
$$

$$
\text { tvvgdase--tal lkf- }
$$

- sev i I gd--vmg i xx

-rnhkvae ipfnstnkxqls i
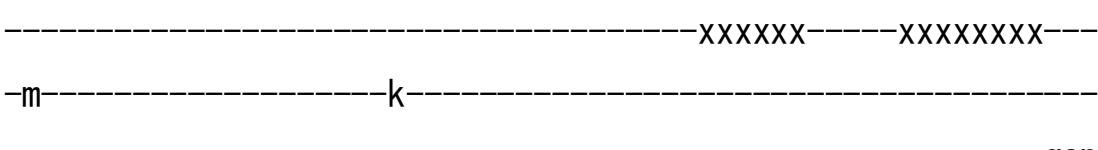

e

r i lekcstiminga 
eqp I dkssadsf-htayme |--gg |----gervLGFCHLy |

------xxxxxxxsy ifdvdsvnxptsnfcfVGLLSMIDPPRSTVPDAVSKCRSag-IKV IMVTGDHPITAKAIAKSVGI

i saxxe-

$-X X X X X$

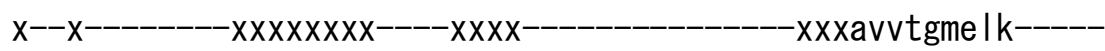
-dmtpeq Ide

I I tnyxx iVFA--RTSPQQKL I IVEGCq

$---r q--d a-$

IVAVTGDGVNDSPALKKADI G I AM--

-xiagsda-

AKNAADMVLLd-DNFASIVtgVEEGRL IFDNLKKT I

$-A$

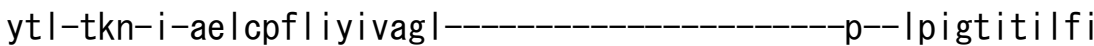

d I gtd i ips i a l ayekae-sd--_-_-_-_-_-_-_-_-_imnrkpr---hxkkdr I vntq I

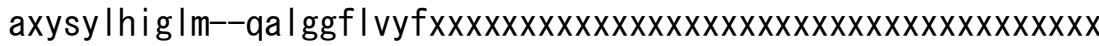
xyarkyx

ew----tgstaffva imiqq i ad-I i irktrxxs i fqq-------g I xrnkvi iwvg i asq vival ils--ygxxsvp---_-------al sftm I

-rvqywf----va--vphai l iwvyd

emrk|f 
y

>rnoll_IIC05 XP_341835.2

m-

$-\mathrm{XXXXXXXXXXXXXXXXXXXXXXXXXXXXXXXXXXX------XX----}$
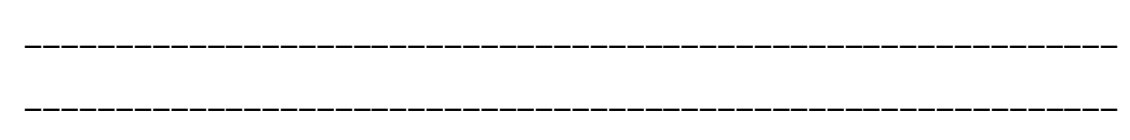

$x x x x x x x x x x x x x$

dhql svseleq

kyq--------tsatkg|kas $\mid$

-aae I I rdgpna I rpprgt

-peyvkfarq-1 
agg lqc Imwvaa--a icli

afax xxxxxdl----ttddn ly la $\mid a$

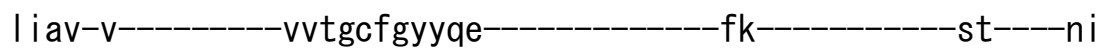

iasfkn--Iv-

q--gckVDNSSLTGESEPQTRsp-

$-x x x x x x x$

$x x t$

-pqq-atv-

ird

$-g d--k f q$ $-\mathrm{i}$

$n-$

----adqLVVGDLVEMkg

GDRVPADIRILsa

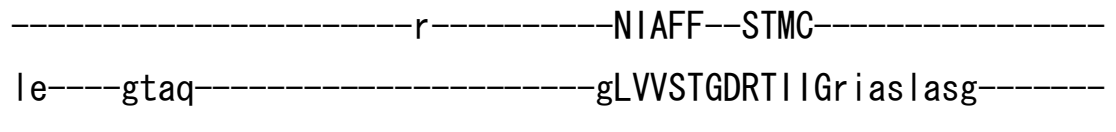

-venektp i a i i ehfvdi iag la i I fgatff

-vvamcigytfI ramvffMA IVVAY

VPEGLLATVTVCLSLTAK-RLaskn-

cVVK-NLEAVETLGSTSVICSDKTG

TLTQNRMTVs -hlwfdnhih

$-x x x x x--e x x-$

$x x x x x d q s s e t w x$

crvltlc-----nraa-

f----kxgqxxxxxxxx 
selt I gn--amgyxx

rfpkvce ipfnstnkxqlsi

$-x \times x \times x x-----x \times x \times x \times x x---$

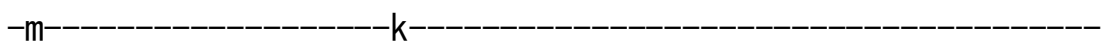

-gap

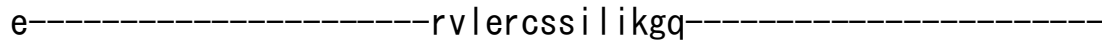

e $|p|$ deqwreaf-qtay $\mid$ s|--gg|----gervLGFCQLy |

-xxxxxxxgytfdveamnxpssg I cfAGLVSMIDPPRATVPDAVLKCRTag-IRV

IMVTGDHPI TAKA I AASVG I--_-_-_-_-_-_-_-_-_-_-_-_-_-_-_ i sexxe---_-_-

$-X X X X X X$

x---x-------- $x x x x x x x x-----x x x x---------------x x x c v$ ingmq $\mid \mathrm{k}$

dmdpse I ve-

al rthxxmVFA--RTSPQQKLVIVESCq

---r l--ga---_-_-_-_-_-_-_-_-_-_---IVAVTGDGVNDSPALKKAD I GVAM--

xiagsda

AKNAADM ILLd-DNFAS IVtgVEQGRL IFDNLKKS I 
yt l-tkn-i-peltpyl iyitvsv-_-_-_-_-_-_-_-_-_-_-----Iplgcitilfi elctdi fpsvs l ayekae-sd-_-_-_-_-_-_-_-_-_imh |rpr---nxr rdr I vnep I

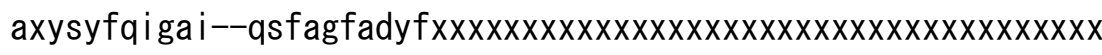
xgar lyx-

qy----tcytvff i s i emcqi ad-vl irktrxxsafqq--------gfxrnr i lvi aivfq vcigcflc--ycxxmpn-_-_-_-_-_-_ifnfmp i-_-_-_-_-_-_-_-_-_-_-_-_-_-_-_ rfaww I----vp--mpfg I I ifvydeirklg-

$\mathrm{y}$

>dme II_IIC01 NP_732572.1

m- 


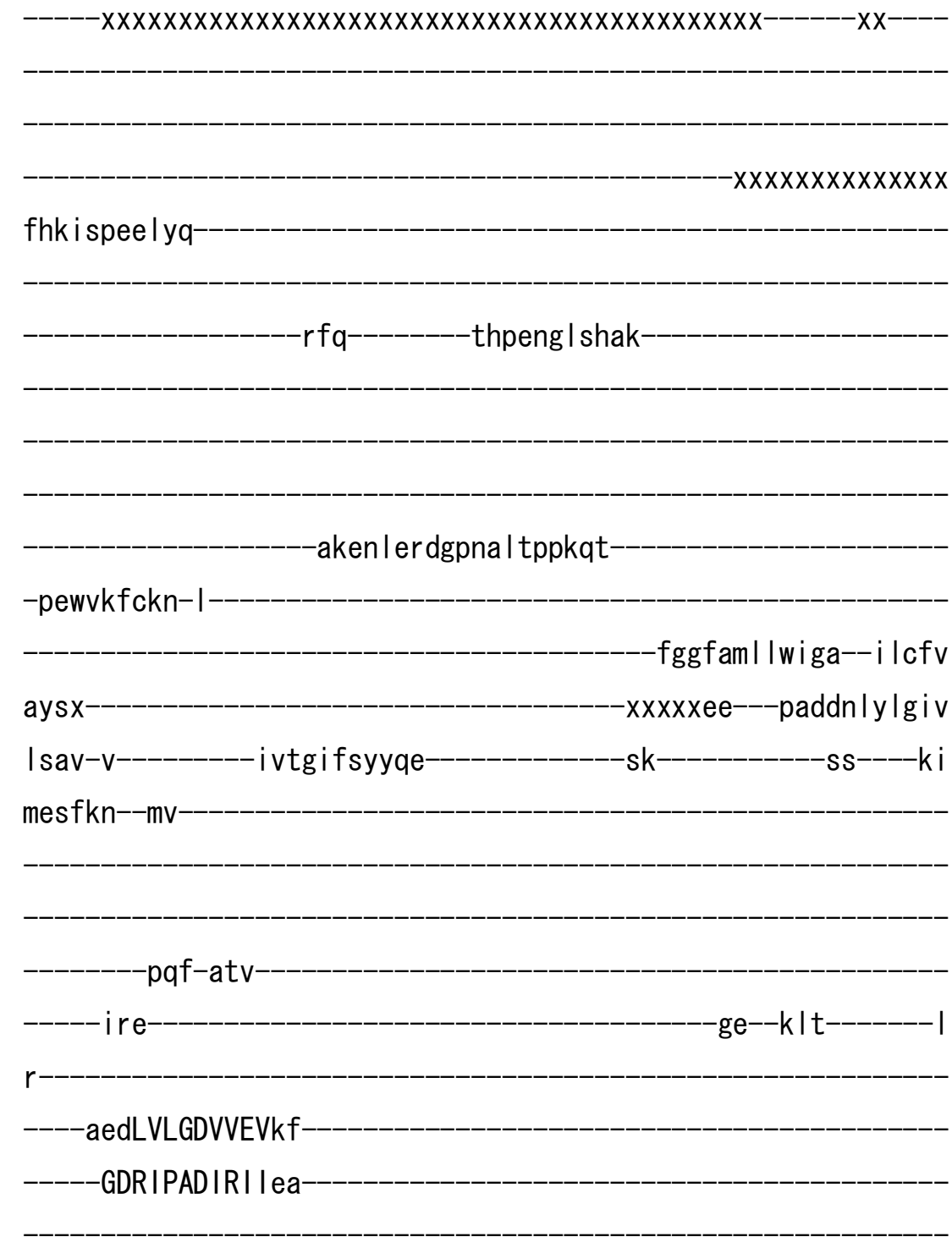

r--nfkVDNSSLTGESEPQSRga-

$-x \times X X X X$

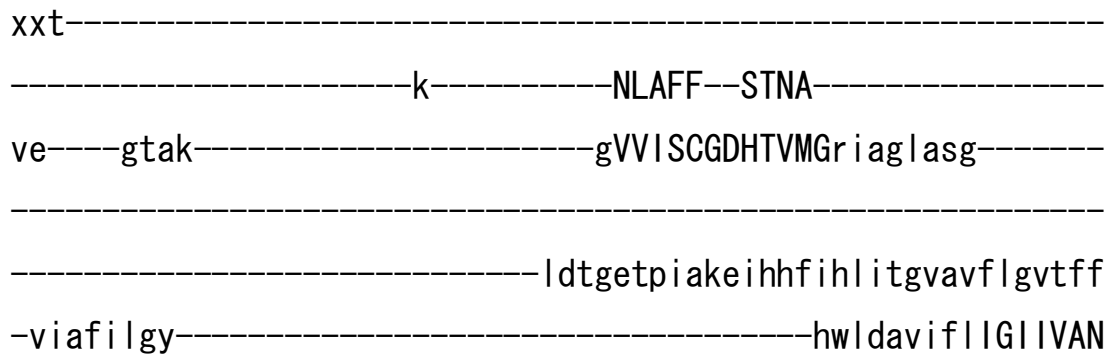

VPEGLLATVTVCLTLTAK-RMaskn- 
cLVK-NLEAVETLGSTST ICSDKTG-

TLTQNRMTVa

-hmwfdnq i

$-x x x x x--e x x-$

xxxxxdrtspgfx

$-a \mid$

sriatlc-----nrae-

f---kxgqxxxxxxxx

evsgdase--aal Ikc

mel a l gd--vmn ixx-

rnkkiaevpfnstnkxqvsi

$-\mathrm{XXXXXX-----XXXXXXXX---}$

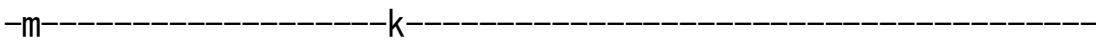

-gap

$\mathrm{e}$

-rilercstifingk

ekv I deemkeaf-nnayme |--gg|----gervLGFCDFm I

-xxxxxxxgfkfntdd inxp i dn I r fVGLMSM IDPPRAAVPDAVAKCRSag-IKV

IMVTGDHPITAKAIAKSVG

i sexxe

$-x x x x x x$

X--X--------- $x x x x x x x x-----x x x x----------------x x x a v$ vhgae $\mid r-----$

-dvssdq I de 
i I ryhxx iVFA--RTSPQQKL I IVEGCq-

$---r m--g a$

-IVAVTGDGVNDSPALKKADIGVAM--

$-x i a g s d v$

SKQAADM ILLd-DNFAS IVtgVEEGRL IFDNLKKSI

$-A$

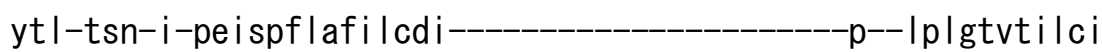

d l gtdmvpa i s layehae-ad------_--_--_--_---imkrppr---dxfndk I vnsr I

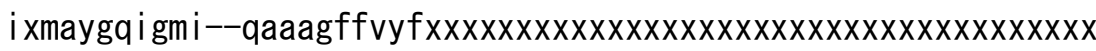

xrdrktx

ey-----tchtaff i s i vvvqwad-। i icktrxxs i fqq-------gm-rnwalnfglvfe tvlaaf Is--ycxxmek--_-_-_-_--_g|rmyp |-_-_-_-_-_-_-_-_-_-_-_-_-_-_

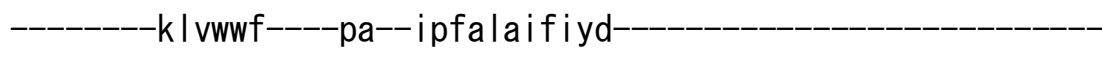

etrrfy

y

>drell_IIC01 NP_571761.1

$\mathrm{m}-$ 
dhk IsIdeltr

$-k y n$ -tdltrglsgtr

ake i lardgpna I tppptt

-pewvkfckq-I

fggfst I |wiga--i lcf I

aygx-xxxxxee---pandn ly lgiv

Isav-v -mitgcfsyyqe ak---_--_-----ss----ki mdsfkn--Iv-

pqq-alv

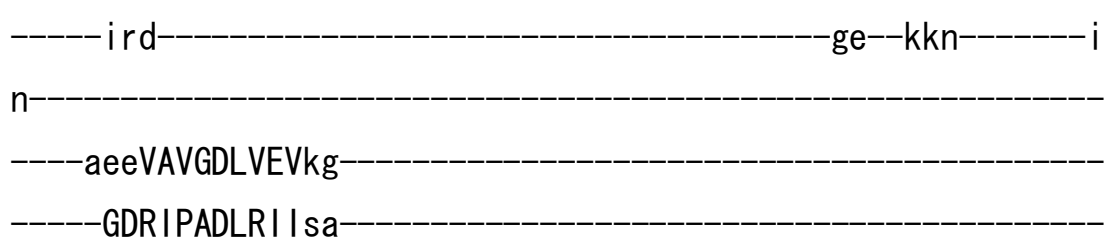

GDR IPADLR I I sa 
-h--gckVDNSSLTGESEPQTRtp-

$-X X X X X X X$

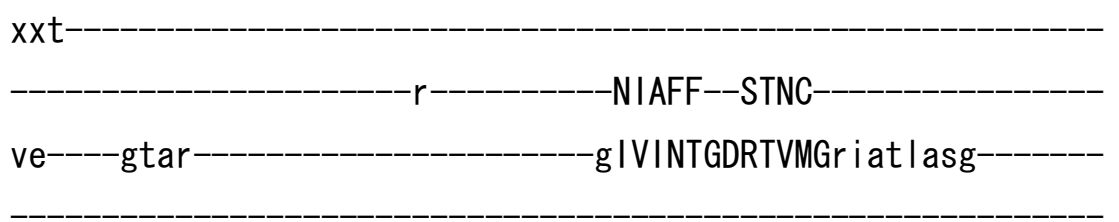

- levgrtpisieiehfihi itgvavfIgvsff

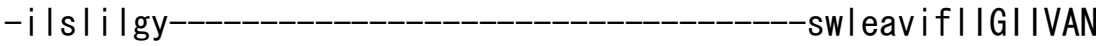

VPEGLLATVTVCLTLTAK-RMakkn-

cLVK-NLEAVETLGSTST ICSDKTG

TLTQNRMTVa

-hmwfdnqih

$-x x x x x^{--}-e x x-$

xxxxxdrssatwx

al

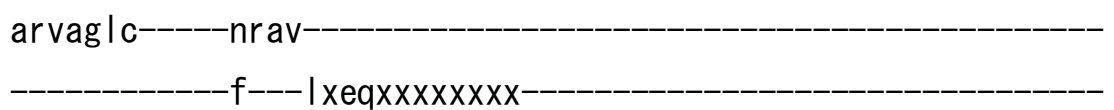

dvagdase--sal lkc

ielccgs--vkemxx-

-kynki se ipfnstnkxqlsi

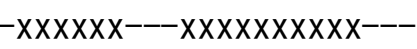

$-m$

$-k$

e-

ri ldrcssi l iggk 
eqp I ddemkdaf-qnay l e l--gg |----gervLGFCHFn I

------xxxxxxxdfqfdteevnxpten I cf I GLMSM I DPPRAAVPDAVGKCRSag-IKV IMVTGDHPITAKAIAKGVGI

isexxe-

$-X X X X X$

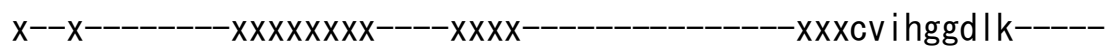

dl speqldd-

vlkhhxxi VFA--RTSPQQKLI IVEGCq

$---r q--g a-$

- IVAVTGDGVNDSPALKKADIGVAM--

$-x i a g s d v$

SKQAADM I LLd-DNFASIVtgVEEGRL IFDNLKKS I

$-A$

yt I-tsn-i-pe itpfIIfi iani--_-_-_-_-_-_-_-_-_---p--|plgtvti l c i

d I gtdmvpa i s l ayeaae-rd--_-_-_-_-_-_-_-_-_imkrqpr---nxktdk I vner I

i xiaygqi igmi--qalagfftyf $x x x x x x x x x x x x x x x x x x x x x x x x x x x x x x x x x x x x x x$ xeqrkix

ef----tchtaffts ivivawad-I i icktrxxsvfqq--_-_--gm-knk i I ifg I fee ta l aaf I s--ycxxmdv-----------al rmyp I

$-k p n w w f----c a--f p y s$ I I ifiyd

eirkli

irrspx

$-x x x x x x x x$ 
$y$

>drell_IIC02 NP_571762.1

$$
\text { m- }
$$

dhk ItIdelsr

-kyg---_----tdmikg I ssfr-

-akev I drdgpna I tppptt

-pqwvkfckq-I 
fggfat I lwfga--flcfl

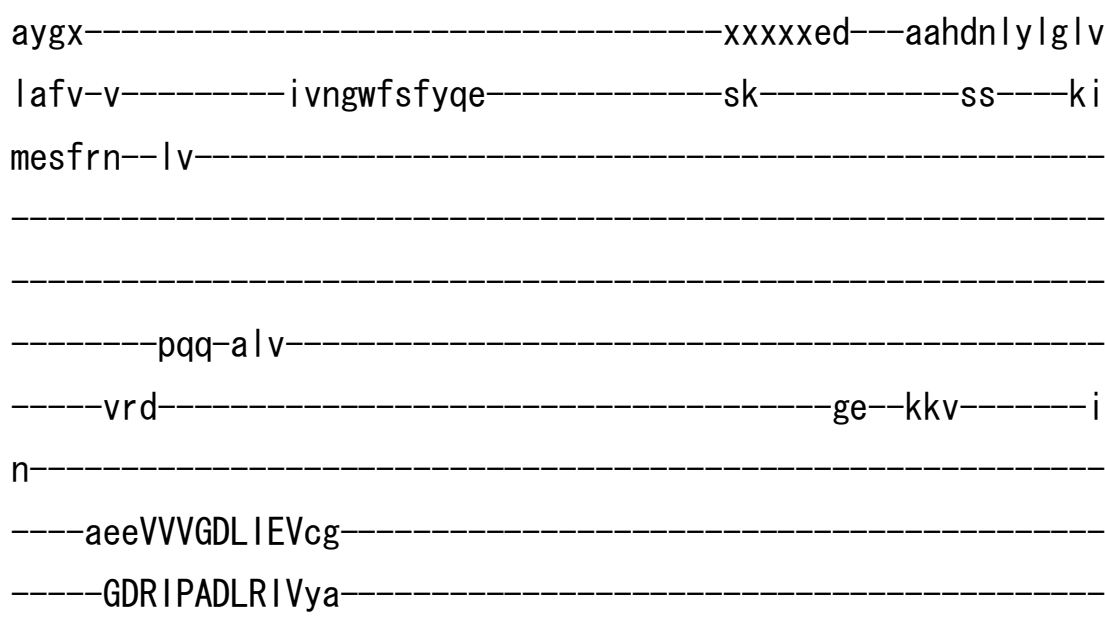

xx--------ckVDNSSLTGESEPQSRsp-

$-x x x x x x x$

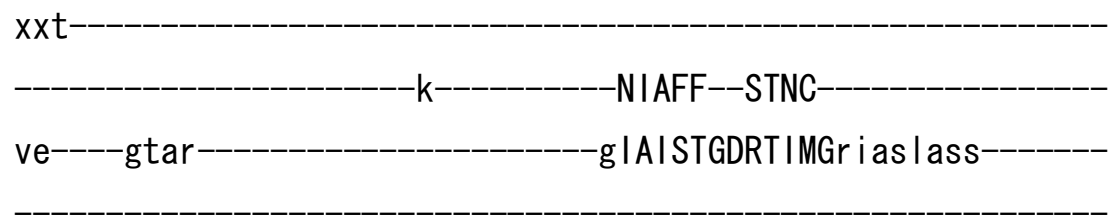

- leggatpiare i ehf ihi i savs if Igvtff

$-v|s|$ i lgy-aw i eavvf I IGIIVAN

VPEGLPATVTVCLTLTAK-RMakkn-

-cLVK-NLEAVETLGSTTT ICSDKTG

TLTQNRMTVa -hmwfdsqih-

$-x x x x x--e x x-$

$x x x x x d r$ ssptwx al arvag lc-----nrav f---rxeqxxxxxxxx 
-etagdase--sal lkc-

- ielccgs--vi emxx-

-kyrkiceipfnstnkxqlsv

$-x \times x \times x x-----x \times x \times x \times x x---$

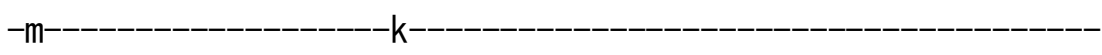

-gap

e-----------------------r i ldrcst i l ingk-------------------------

eqpmddenkdsf-qsayve I--gg |----gervLGFCQFn I

-xxxxxxxgfafdpedvnxpten I cfLGLMSMIDPPRAAVPDAVAKCRSag-IKV

IMVTGDHPITAKAIAKGVGI-

isexxe-

$-x X X X X X$

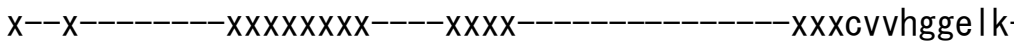

-nmndsd I de

i I ryhxx iVFA--RTSPQQKL I IVEGCq

$---r q--g a-$

-IVAVTGDGVNDSPALKKADIGVAM--

xisgsdv 
yt I-tsk-i-pemspf Imfvvvg -p--Iplgtvtilfi

dl gtd I i pa i syayenae-nd-_-_-_-_-_-_-_-_-_imkrqpr---nxqkdr I vner I i xmaygq i gmi--qavagffty i $x x x x x x x x x x x x x x x x x x x x x x x x x x x x x x x x x x x x x x$ xegrkix-

es----tchtaffi i ivvvqwad-II ivktrxxsi lqq -gm-knkvl ifaffee gal aaf I s--ycxxmd i-_---_-_----avrmyp I--_-_-_-_-_-_-_-_-_-_-_-_-_-_-_

$y$

>drell_IIC03 NP_571764.1

$\mathrm{m}-$

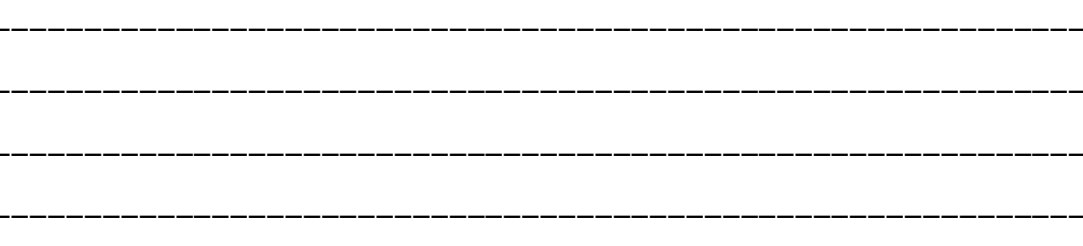




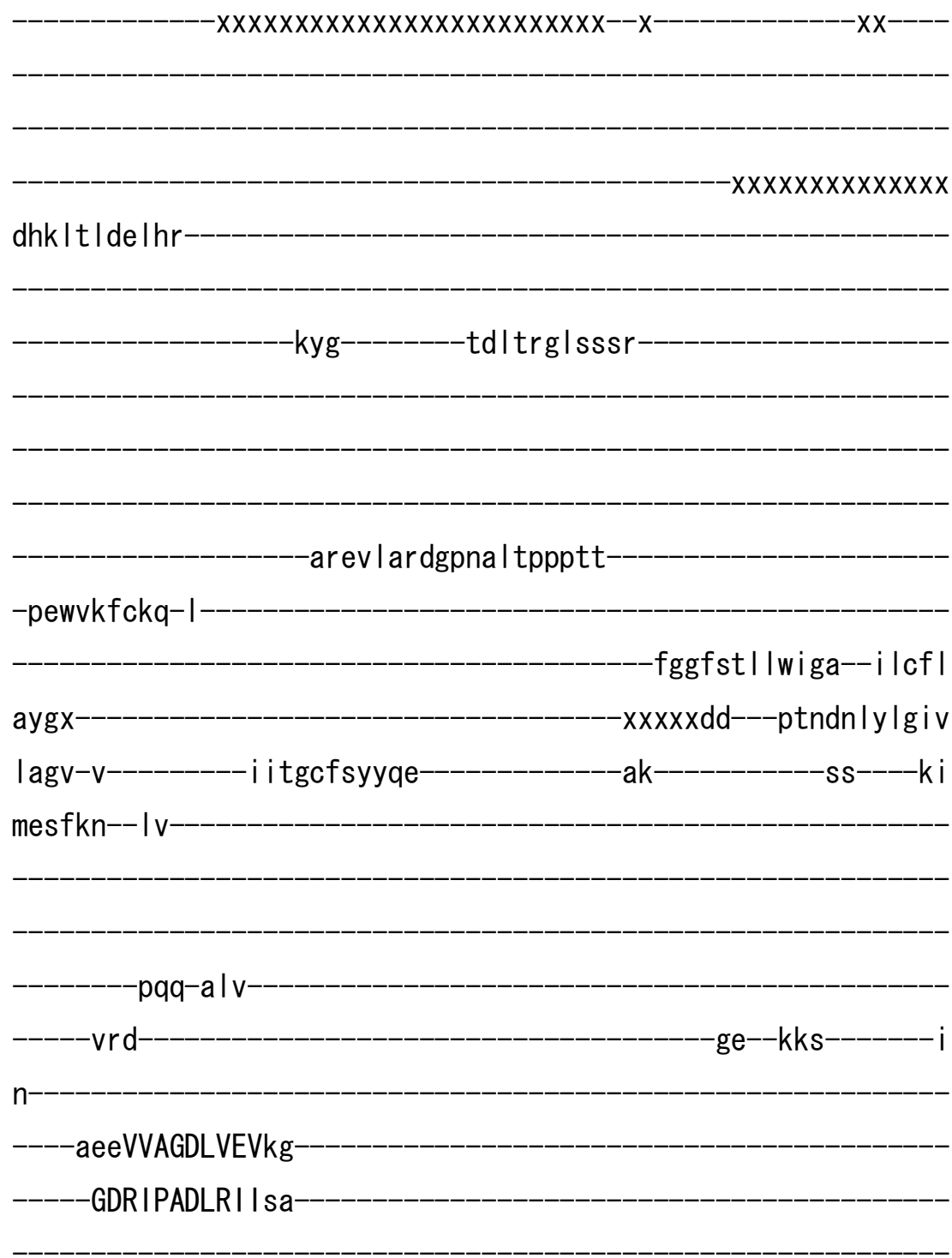

-h--gckVDNSSLTGESEPQTRtp-

$-x x x x x x x$

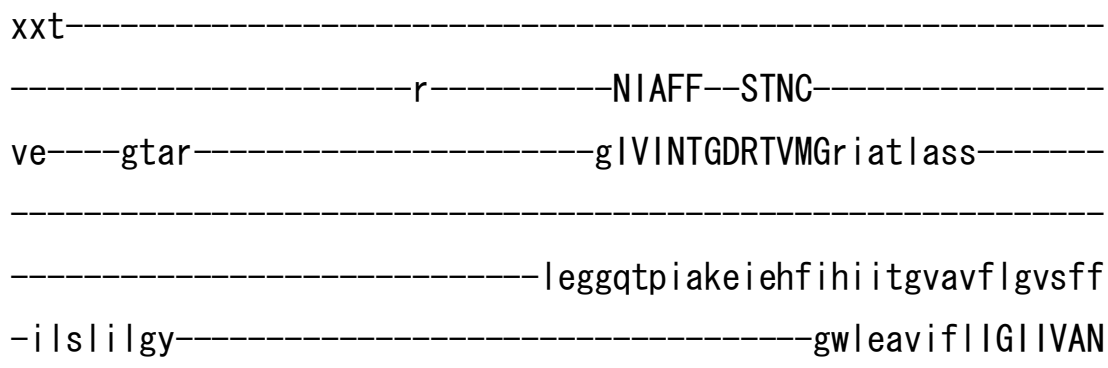

VPEGLLATVTVCLTLTAK-RMakkn- 
cLVK-NLEAVETLGSTSTI ICSDKTG-

TLTQNRMTVa

-hmwfdsqih

$-x x x x x--e x x-$

xxxxxdrssptwx

$-a \mid$

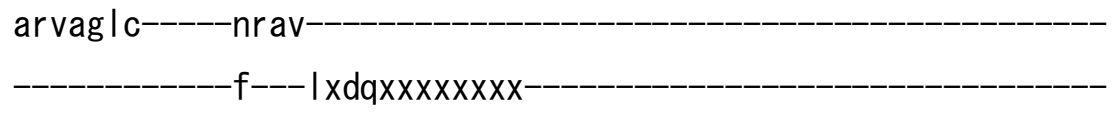

dtagdase--sal Ikc

i elccgs--vnemxx

-kytkiae ipfnstnkxqls i

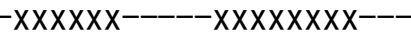

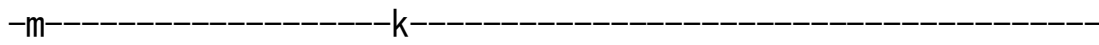

-gap

$\mathrm{e}$ -ri idrcsti l iqgk

qqp I deemkdaf-qnayve |--gg |----gervLGFCHFc I

------xxxxxxxgfafdteevnxpten I cfVGLMSMIDPPRAAVPDAVGKCRSag-IKV

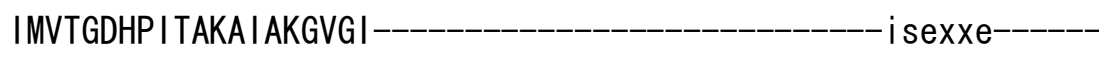

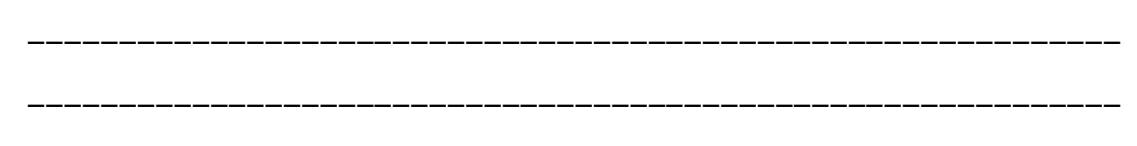

$-x x x x x x$

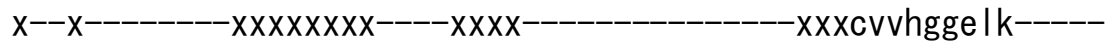

smseee Idd- 
i I khhxxiVFA--RTSPQQKLI IVEGCq

---rq--ga--_-_-_-_-_-_-_-_-_-_-_-_-IVAVTGDGVNDSPALKKAD I GVAM--

SKQAADMILLd-DNFASIVtgVEEGRL IFDNLKKSI------A

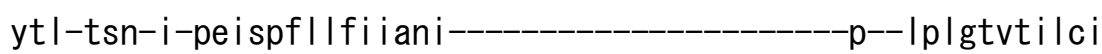
d I gtdmvpa i s layesae-sd--_-_-_-_-_-_-_-_imkrqpr---nxktdk I vner I i xmaygq i gmm--qavagffsyf $x x x x x x x x x x x x x x x x x x x x x x x x x x x x x x x x x x x x x x$ xerrkix

ef----tchtaffas i vi vqwad-I i icktrxxs i vqq-------gm-rnk i I i fg I fee ta l aaf I s--ycxxmdv--_-_--_---al rmyp I--_-_-_-_-_-_-_-_-_-_-_-_-_ --_--_---kpcwwf----ca--fpys I I i fvyd--_-_-_-_-_-_-_-_-_-_-_-_-_-_Irrspx----------xxxxxxxx-

$\mathrm{y}$

>drell_IIC04 NP_835200.1

$\mathrm{m}-$ 
dhk I tmeelsr

$$
\text { -kyg---------td l tkg I pvsr }
$$

-amev Imrdgpnal tppv it

$$
\begin{aligned}
& \text {-pewvrfcrq-I } \\
& \text { fggfat I I wiga--flcyf } \\
& \text { afsx- } \\
& \text {-xxxxx-e---pvndn ly l giv } \\
& \text { Itfv-v } \\
& \text {-tvngcfsysqe } \\
& \text { ak------------sc----ri } \\
& \text { mdsfkn--Iv } \\
& -p q k-a \mid v
\end{aligned}
$$

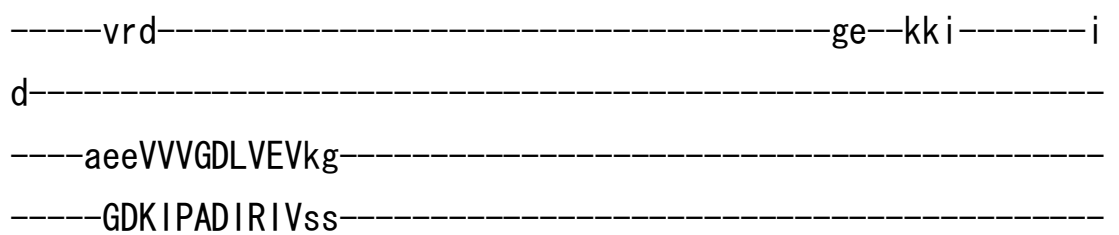


-h--gckVDNSSLTGESEPQIRta-

$-X X X X X X X$

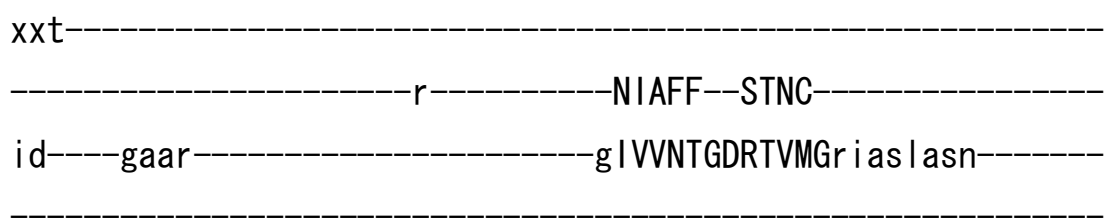

- leggqtp Igre i ehf ihi i tgvavf Igttf I

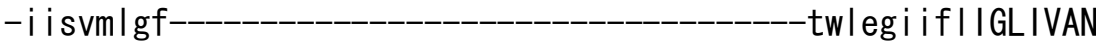

VPEGLPCTVTVALTLTAK-HMakkn-

cLVK-NLEAVETLGSTST ICSDKTG

TLTQNRMTVa

-hmwfdnqih

$-x x x x--e x x-$

xxxxxdrssatwx

$-a \mid$

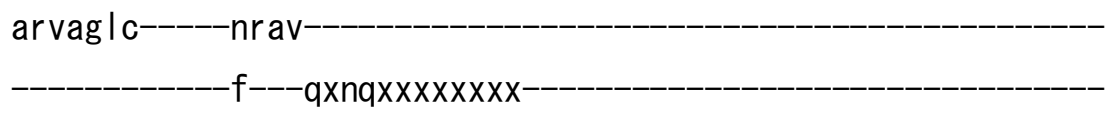

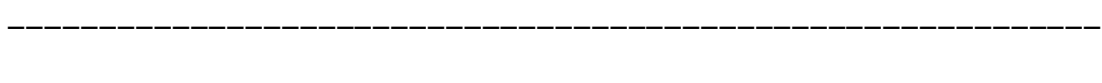

-etagdase--sal I kc

ielccgs--vtemxx

-nypkvae ipfnsiskxqlsi

$-\mathrm{x} x \times x \times x-----x \times x \times x \times x x---$

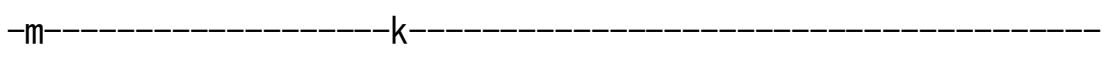

-gap

e----_--_--_-_-_-_-_---r i ldrcst i l i egk 
ehp I ddemkedf-qnayvq I--gg |----gervLGFCHFg I

-xxxxxxxgfafdteemnxpten I cfVGLMSMI IPPRAAVPDAVAKCRSag-IKV IMVTGDHPITAKA IAKGVGI

i sexxe-

$-X X X X X X$

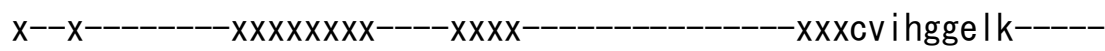

nmtdeqldd

vlqhhxxi VFA--RTSPQQKL I IVEGCq

$---r q--g a-$

IVAVTGDGVNDSPALKKADIGVAM--

$-x i a g s d v$

SKQAADM I LLd-DNFAS IVtgVEEGRL IFDNLKKS I

$-C$

yt I-stk-i-pemspf Imfv lag i--_-_-_-_-_-_-_-_-_---p-- |p I gtvti l c i

d I gtdmvpa i sfayenae-sd--_-_-_-_-_-_-_-_--imkrqpr---nxatdr I vner I

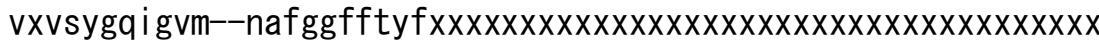
xearkvx

ey----tchtayfts i vimqwtt-I I vcksr xxs lakq---_----gm-knrvItfs I fee ta i aaf I s--ycxxmd i-----------avrmyp I--------------------------- kpmwwf----ca--fpymil ifiydevrkhf- 
$y$

>drell_IIC05 NP_571765.1

m

dhkItleelnr

-kyg---_--_--tdlnrglttar

-aae i l ardgpna I tppptt

-pewvkfckq-m 


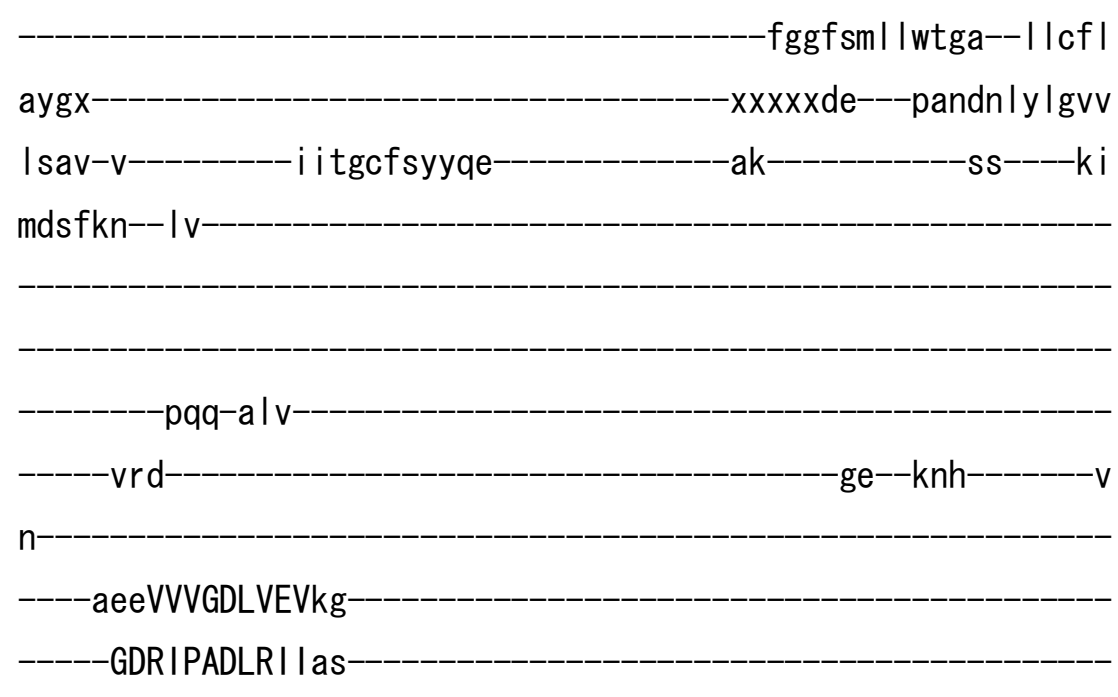

-h--gckVDNSSLTGESEPQTRsp-

$-x x x x x x x$

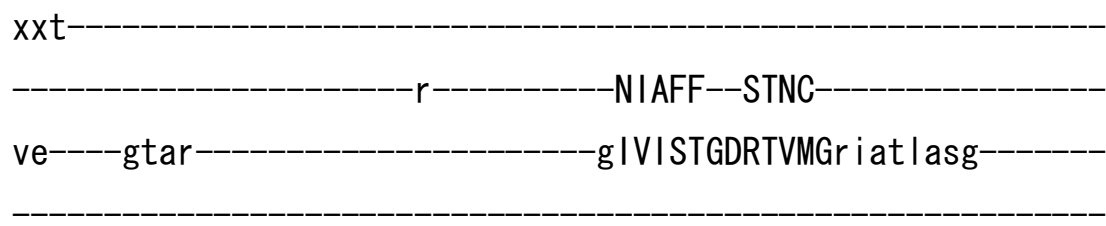

- levgrtpisieiehfihi i tgvavf Igvsff

$-\mathrm{vls}$ lalgy-swleavif I IGIIVAN

VPEGLLATVTVCLTLTAK-RMakkn-

cLVK-NLEAVETLGSTST I CSDKTG

TLTQNRMTVa -hmwfdnqih-

$-x x x x x--e x x-$

xxxxxdrssatwx

$-s \mid$

arvag lc-----nrav

f--- | xeqxxxxxxxx 
-dvagdase--sal lkc-

i elccgs--vkdmxx

-kytkvae ipfnstnkxqlsv

$-x \times x \times x x----x \times x \times x \times x \times x---$

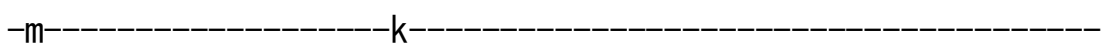

-gap

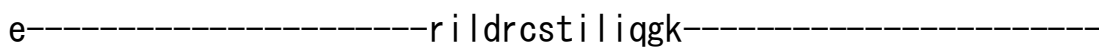

vqa I ddemkeaf-qnay | e |--gg |----gervLGFCDFc I

-xxxxxxxgfpfdtedvnxpten I cfVGLMSMIDPPRAAVPDAVGKCRSag-IKV

IMVTGDHPITAKAIAKGVGI-

isexxe-

$-x x x x x$

x---x---------xxxxxxxx----Xxxx----------------xxxcvvhggdlk-

dI sceqldd-

i I khhxx iVFA--RTSPQQKL I IVEGCq

---rq--ga----_-_-_-_-_-_-_-_-_----IVAVTGDGVNDSPALKKAD I GVAM--

-xiagsdv 


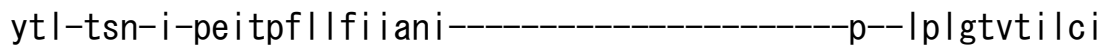

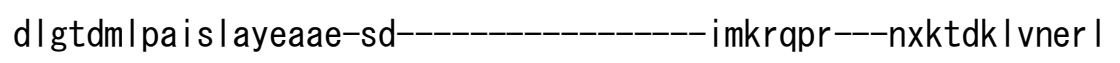
i $x$ i aygqigmi--qal agfftyf $x x x x x x x x x x x x x x x x x x x x x x x x x x x x x x x x x x x x x$

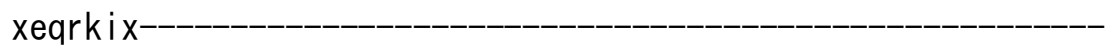
ef----tchtaffas i vvvqwad-I i icktrxxsvfqq-------gm-knk i l i fg I fee

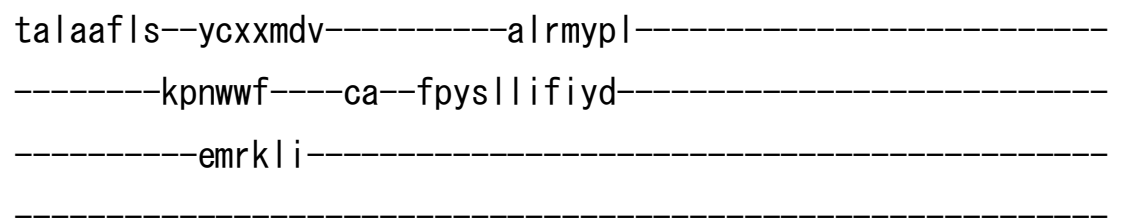

y

>drell_IIC06 NP_571758.1

m- 
$x X X X X X X X X X X X X X$

dhk It Idelst

-ryg

-vdlarglthkr

-ame i l ardgpna I tppptt

-pewvkfckq-I

-fggfsil |wiga--i lcf I

ays $\mathrm{x}$

xxxxxde---pvndn ly I gvv

Isav-v-

- i tgcfsyyqe

$-a k-$

$-s s----r i$

mdsfkn--mv-

s--gckVDNSSLTGESEPQTRsp-

$-p q q-a \mid v$

ird

ge--kla

$-\mathrm{i}$

n-

----aeeVVQGDLVEIkg

GDRVPADLR I Iss

$-x x x x x x x$

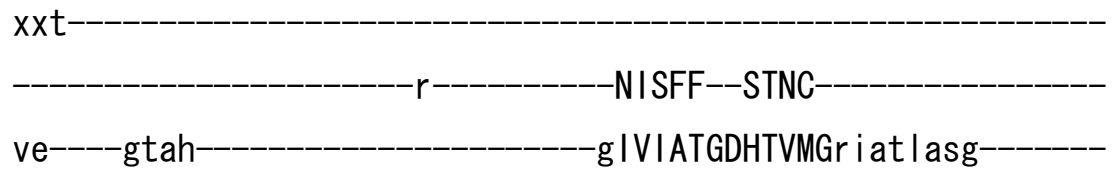

- levgqtp inme i ehf ihi i tgvaif I gmsff

-ils i i gytwl eavifIIGIIVAN

VPEGLLATVTVCLTLTAK-RMarkn- 
cLVK-NLEAVETLGSTST ICSDKTG-

TLTQNRMTVa

-hmwfdnqih

$-x x x x x^{--}-e x x-$

xxxxxdkssptwx

$-s \mid$

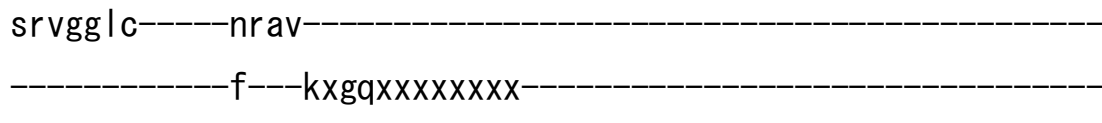

dtagdase--sal Ikc

vei Isgn--vet I xx

-nnrkvae ipfnstnkxqls i

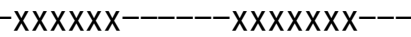

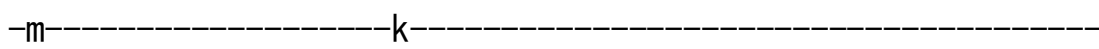

-gap

$\mathrm{e}$

-rildrcstiminge

efp idddwmdaf-qgayme |--gg |----gervLGFCHLf I

-xxxxxxxgfefdcddvnxpvnq I cfLGLISMIDPPRAAVPDAVGKCRSag-IKV

IMVTGDHPITAKAIAKGVGI-

i sexxe-

$-x x x x x x$

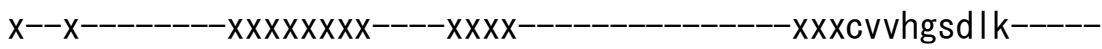

dmsaey Idd- 
i I rnhxx i VFA--RTSPQQKL I IVEGCq

---rq--ga--_-_-_-_-_-_-_-_-_-_-_-_--IVAVTGDGVNDSPALKKAD IGVAM--

SKQAADM ILLd-DNFASIVtgVEEGRL IFDNLKKS I------A

yt I-tsn-i-pe i tpf I If i i asv--_-_-_-_-_-_-_-_-_--- Ip I gtvt i I c i d I gtdmvpa i s layesae-sd-_-_-_-_-_-_-_-_-_imkrqpr---nxktdk I vner I i xmaygqi gmi--qal ggfftyf $x x x x x x x x x x x x x x x x x x x x x x x x x x x x x x x x x x x x x x$ xeqrkix

ef----tchtsffvs i vvvqwad-। i icktrxxsvfqq-------gm-knr i I i fg I fae tal aaf I s--ycxxmdv-_-_-_-_-_-_al rmyp |-_-_-_-_-_-_-_-_-_-_-_-_-_-kimwwf----ca--fpys I l ifvydeirkfi Irrnpx $-x x x x x x x x$

$\mathrm{y}$

>drell_IIC07 NP_571760. 2

$\mathrm{y}-$ 
ehkmsveever

-kfq---------tdivqgI tnak-

-ardf I ardgpnal tppptt

-pewvkfcrq-I

-fggfsi I lwiga--i lcf I

ayax -xxxxxdd---pagdn ly lgiv

Isav-v

- i itgcfsyfae

ak-----------ss----ki

mesfkn--mv-

$-p q q-a \mid v-$

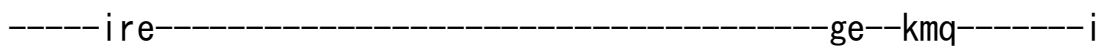

n-

----aeeVVAGDLVEVkg

GDR IPADLRI Isa 
-h--gckVDNSSLTGESEPQTRsp-

$-X X X X X X X$

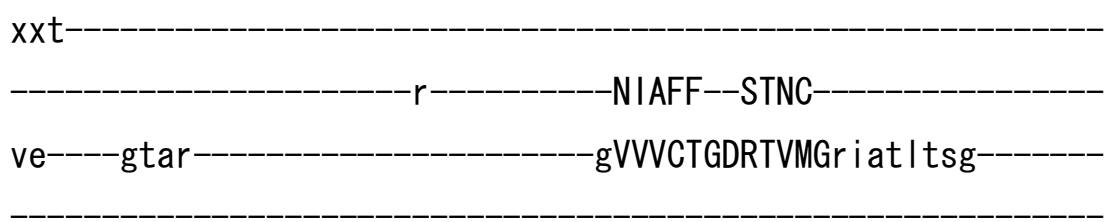

letgktpiakeiehfihi i tgvavflgvsff

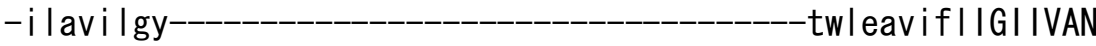

VPEGLLATVTVCLTLTAK-RMarkn-

cLVK-NLEAVETLGSTST ICSDKTG

TLTQNRMTVa

-hmwfdnqih-

$-x x x x--e x x-$

xxxxxdkssvtwx

-al

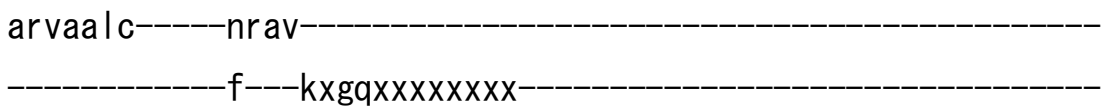

dvagdase--sal lkc

ielscgs--vkvmxx

$-k n k k v a e$ ipfnstnkxqlsi

$-\mathrm{x} x \times x \times x------x \times x \times x \times x x---$

$-m$

k

e

ri ldrcstiml qgk 
eqpmdeemkeaf-qnay | el--gg |----gervLGFCHV Im-

$-x x x x x x x g f a f d t d d v n x q t d n$ I cfVGLMSM IDPPRAAVPDAVGKCRSag-IKV IMVTGDHPITAKAIAKGVGI

isexxe-

$-X X X X X$

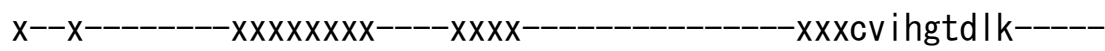

-dysqeqide

vI rnhxxiVFA--RTSPQQKLI IVEGCq

$---r q--g a$

- IVAVTGDGVNDSPALKKADIGVAM--

$-x i s g s d v$

SKQAADM I LLd-DNFAS IVtgVEEGRL IFDNLKKS I

A

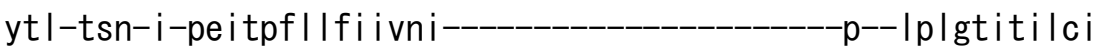

d I gtdmvpa i s l ayeaae-sd-_-_-_-_-_-_-_-_-_-_imkrqpr---nxmr dk I vner I

i $x$ i aygq i gmi--qa Iggffayf $x x x x x x x x x x x x x x x x x x x x x x x x x x x x x x x x x x x x x x$ xeqrkix

ef----tchtaffvs i vvvqwad-vi icktrxxsvfqq--_-_---gm-knk i I ifg I fee tal aaf I s--ycxxmdv----------al rmyp |

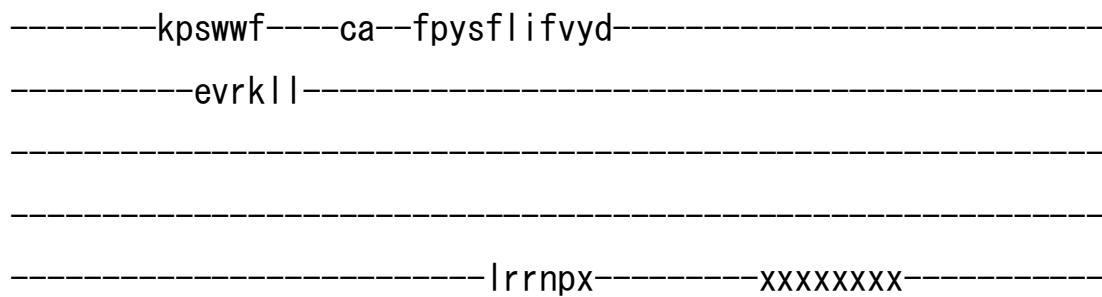


$y$

>drell_IIC08 NP_571759. 2

m

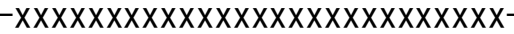

$-x x x x x x x x x x x x x x$

ehkms i eevcr

-kyn----_-_--td i vqgI tnar

-aaey l ardgpna I tppptt

-pewvkfcrq-I 


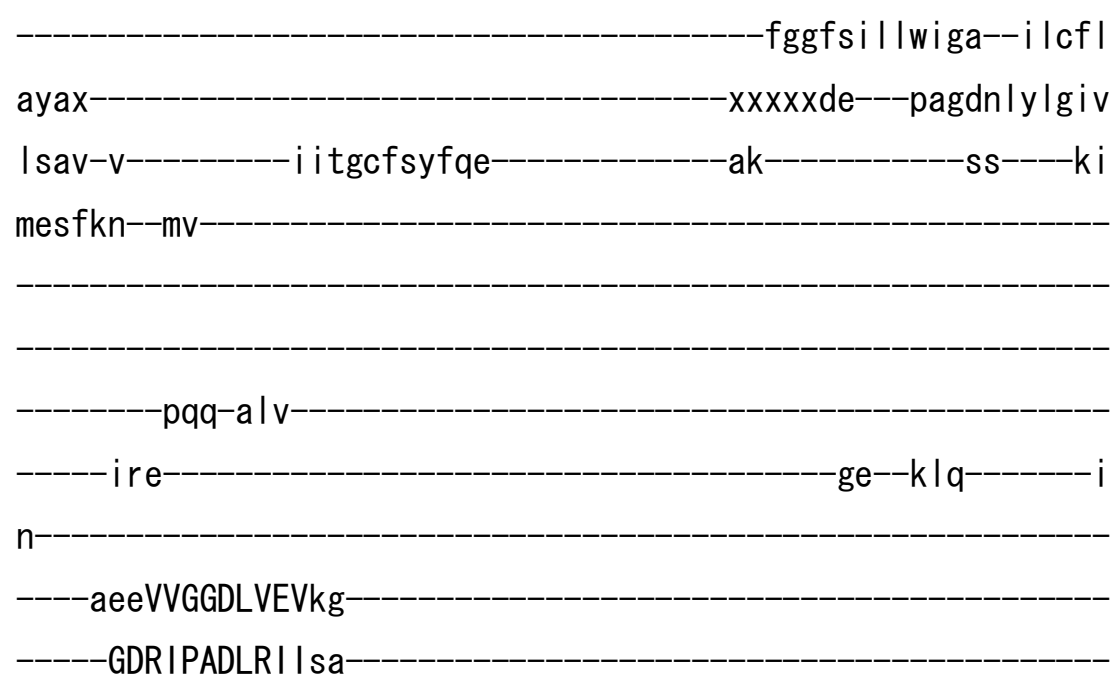

-h--gckVDNSSLTGESEPQTRsp-

$-\mathrm{XXXXXXX}$

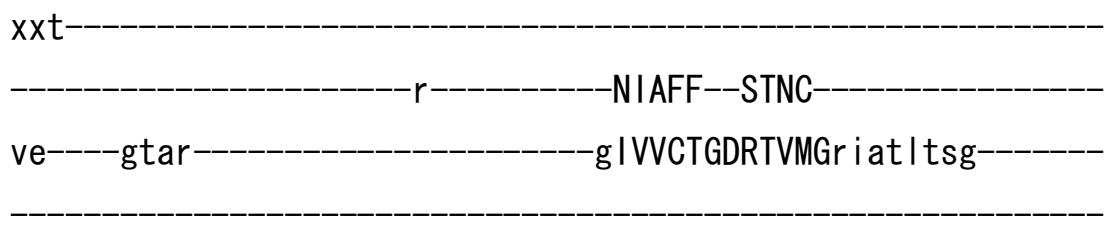

- letgktpiake i ehf ihi i tgvavf I gvtff

-i ls i i gy-swleavif I IGIIVAN

VPEGLLATVTVCLTLTAK-RMarkn-

cLVK-NLEAVETLGSTST I CSDKTG

TLTQNRMTVa -hmwfdnqih-

$-x x x x x--e x x-$

xxxxxdkssgtwx -al

arvaalc-----nrav

$f---k x g q x x \times x \times x \times x$ 
-dvagdase--sal lkc-

i elscgs--vkamxx

-knkkvae ipfnstnkxqlsv

$-x \times x \times x x-----x \times x \times x \times x x---$

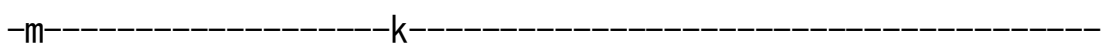

-gap

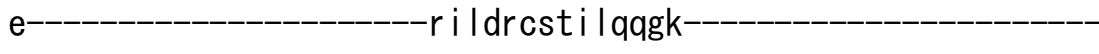

eqpmdee Ikeaf-qnay le l--gg |----gervLGFCHLvm

-xxxxxxxgfafdtdd inxqtdn I cfVGLMSMIDPPRAAVPDAVGKCRSag-IKV

IMVTGDHPITAKA IAKGVGI

i sexxe-

$-x X X X X X$

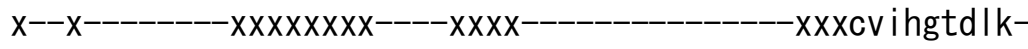

dl sqdqmde-

vlknhxxiVFA--RTSPQQKLI IVEGCq

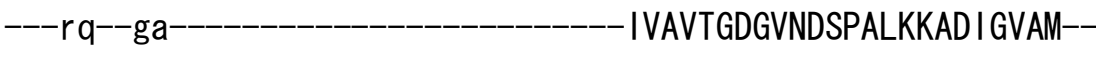
-xisgsdv 


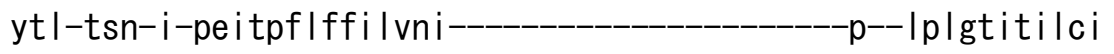
d l gtdmvpa i l ayeaae-sd--_--_-_-_-_-_-_--imkrqpr---nx I rdk I vner I ix i aygq igmi--qal ggffsyf $x x x x x x x x x x x x x x x x x x x x x x x x x x x x x x x x x x x x x x x$

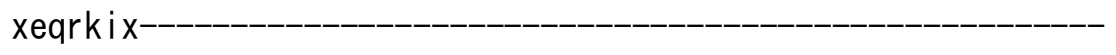
ef----tchtaffvs i vvvqwad-vi icktrxxsvfqq-------gm-knk i l i fg I fee

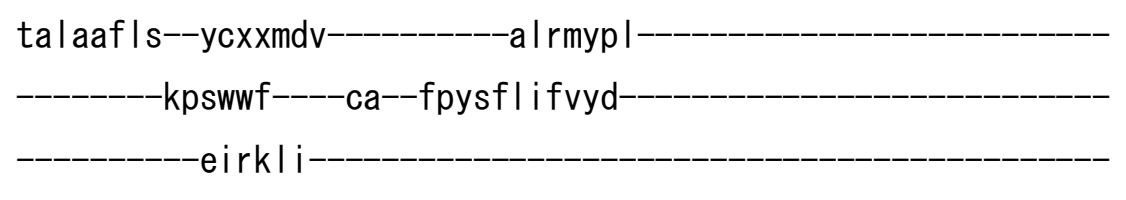

y

>ggal I_IIC01 NP_990852.1

a- 
$\mathrm{XXXXXXXXXXXXX}$

dhk Is Ide Ihr

$-k y g$ -tdlsrglttar

-aae i l ardgpnt I tppptt

-pewvkfcrq-I

fggfs | | |wigs--||cf|

aygx xxxxxge---pntdn ly lgvv

laav-v - i tgcfsyyqe

ak

$-s s^{----k i}$

mesfkn--mv

h--gckVDNSSLTGESEPQTRsp-

$-x x x x x x x$

xxt

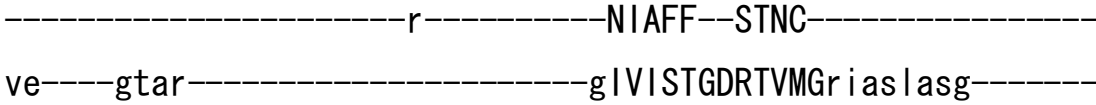

- leggktp i ame i ehf ihl i tgvavf I gvsff

-ilsliley twleavifIIGIIVAN

VPEGLLATVTVCLTLTAK-RMarkn- 
cLVK-NLEAVETLGSTSTI ICSDKTG-

TLTQNRMTVa

-hmwfdnqih

$-x x x x x--e x x-$

$x x x x x d k s s a t w x$

$-a \mid$

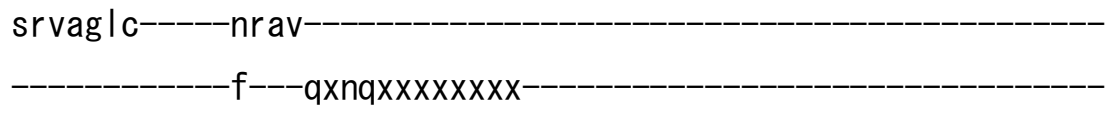

avagdase--sal Ikc

i elccgs--vkemxx

-rypkvve ipfnstnkxqls i

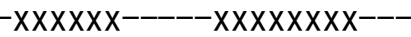

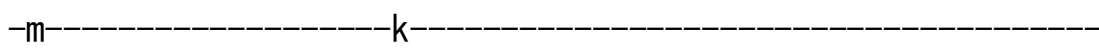

-gap

$\mathrm{e}$ - r i ldrcds i l ingk

vqp I deemkdsf-qnay | e |--gg |----gervLGFCHLa I

------xxxxxxxgfafdtdevnxpvek I cfVGLMSMIDPPRAAVPDAVGKCRSag-IKV IMVTGDHPITAKAIAKGVGI

i sexxe

$-x x x x x x$

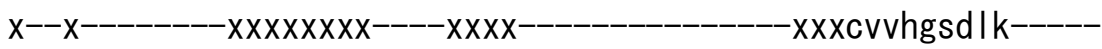

dmtseq Idd- 
i I Ihhxx iVFA--RTSPQQKL I IVEGCq-

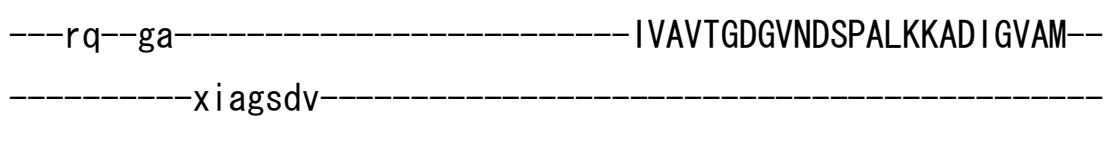

SKQAADMILLd-DNFASIVtgVEEGRL IFDNLKKSI------A

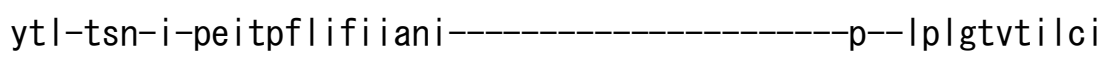

d I gtdmvpa i s layeqae-sd--_-_-_-_-_-_-_-_imkrqpr---nxktdk I vner I

i xmaygq i gmi--qalggfftyf $x x x x x x x x x x x x x x x x x x x x x x x x x x x x x x x x x x x-$

$-$

>ggalI_IIC02 NP_001026080.1

$\mathrm{m}$ 
dhk Istsqlee

$-k y g$ -ts idkg I ssar

-aae i lardgpns I tppkat

-pe ivkflkq-m-

-vggfsi I lwiga--vfswi

sfgx -xxxxxae---safdn l y Igvv

lalv-v i Itgi fayyqe -ak------------st----ni masfsk--mi $-p q q-a \mid v$

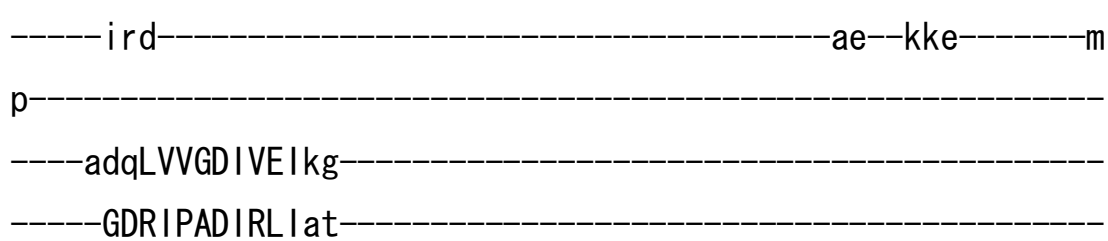
GDRIPADIRL I at 
---gckVDNSSLTGESEPQPRsc-

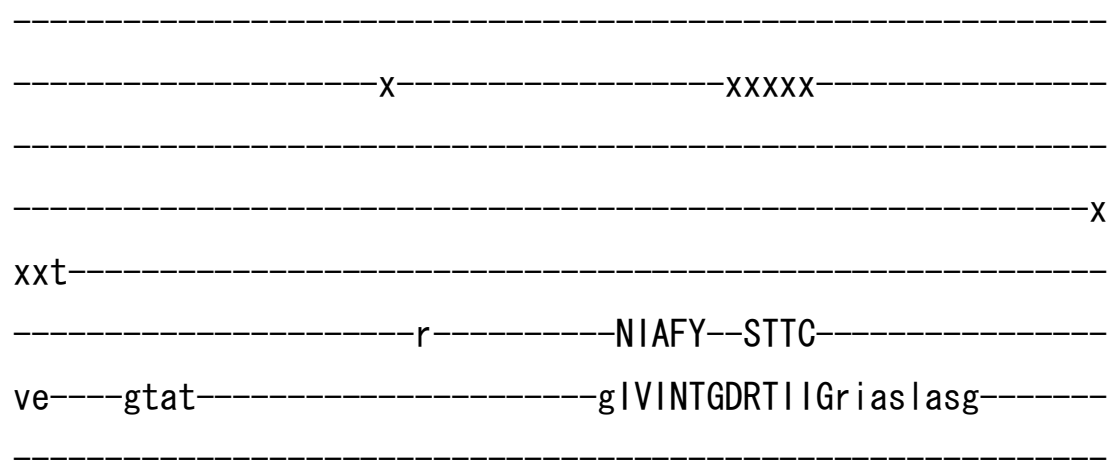

-vgnektp ia i e i ehfvy l vagva i s igv Iff

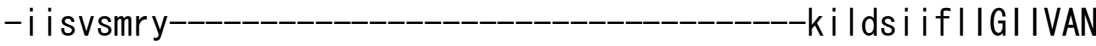
VPEGLLATVTVSLSLTAK-RMakkn-

CLVK-NLEAVETLGSTSI ICSDKTG

TLTQNRMTVa -hIwfdnqiy

$-x x x x x^{--}-e x x-$

xxxxxdqsspswx

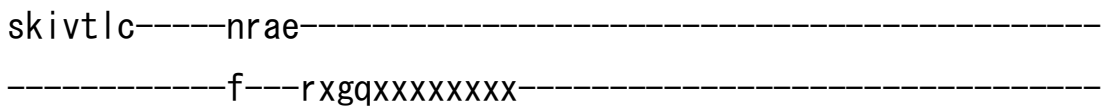

-vvvgdase--tal Ikf

aevv I gd--vmn ixx

-rnkkvae ipfnstnkxqlsi

$-\mathrm{x} x \times x \times x-----x \times x \times x \times x \times---$

$-m$

$-k$

e-

ri lercstimingk 
eep | dsekaeaf-qtayme |--gg |----gervLGFCHLy |

------xxxxxxxtypfdtdsmnxptsn I cfVGLLSMIDPPRSTVPDAVSKCRSag-IKV

IMVTGDHPITAKAIAKSVGI

i saxxe-

$-x x x x x x$

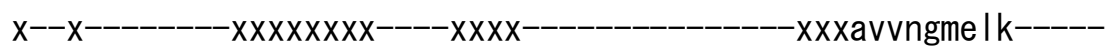

-dms I qe Ide

i I cdhxx i VFA--RTSPQQKL I IVEGCq

$---r q--g a-$

-VVAVTGDGVNDSPALKKADI G I AM--

-xiagsda-

AKNAADMVLLd-DNFAS IVtgVEEGRL IFDNLKKT I

A

yt I-tkn-i-ae lcpfl i y i i as i--_-_-_-_-_-_-_-_-_--o--mp igt i t i I f i

d I gtd i ipsva l ayekae-sd-_-_-_-_-_-_-_-_-_-_imnrrpr---nxkkdr I vneq I

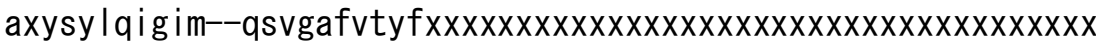
xyartyx

qw----tgytaffvs it iqqvad-l i irktrxxs ifqq--_----gl xrnkvi iwvg ifsq igial ilt--ygxxhvt-----------alnftp I

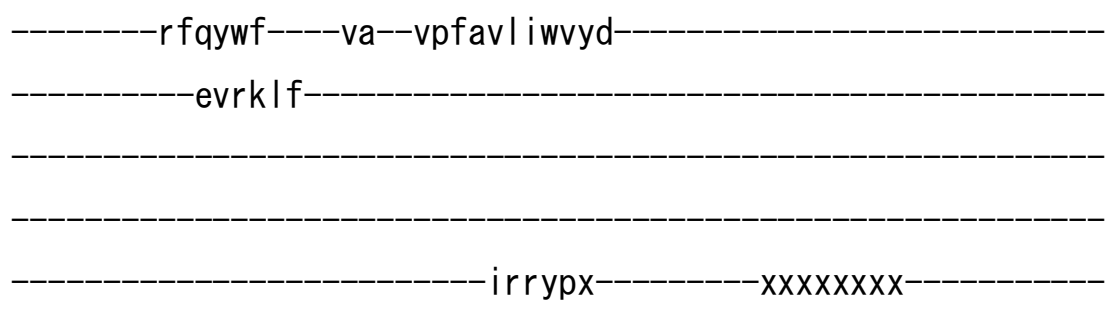


y

>mmul I_IIC01 NP_659149.1

m-

dhk Is Ide Ihr

-kyg-_--_---_td lsrgltpar-

aae i lardgpna I tppptt

-pewvkfcrq-I 


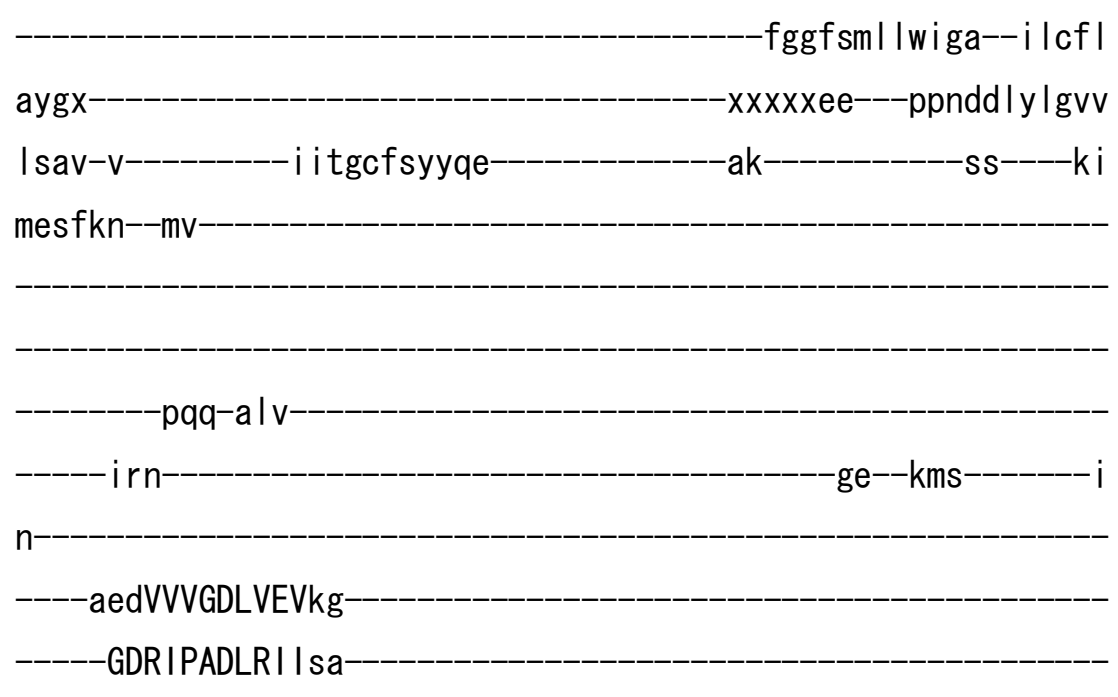

-n--gckVDNSSLTGESEPQTRsp-

$-\mathrm{XXXXXX}$

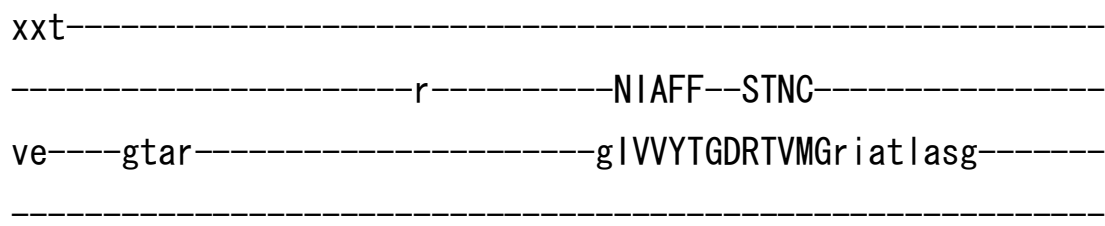

- leggatpiaee i ehfihl i tgvavf Igvsff

-ilsliley -twleavifIIGIIVAN

VPEGLLATVTVCLTLTAK-RMarkn-

cLVK-NLEAVETLGSTST I CSDKTG

TLTQNRMTVa -hmwfdnqih-

$-x x x x x--e x x-$

xxxxxdktsatwx

sriaglc-----nrav-

f----qxnqxxxxxxxx 
-avagdase--sal lkc-

- i evccgs--vmemxx

-kyskive ipfnstnkxqlsi

$-x \times x \times x x-----x \times x \times x \times x x---$

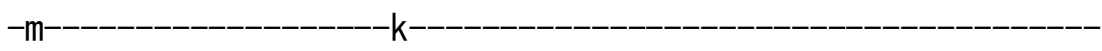

-gap

e-----------------------r i Idrcss i I l hgk-------------------------

eqp I dee | kdaf-qnay | e |--gg |----gervLGFCHL I I

-xxxxxxxgfafdtddvnxpvdn I cfVGL I SMIDPPRAAVPDAVGKCRSag-IKV

IMVTGDHPITAKAIAKGVGI-

isexxe-

$-x X X X X X$

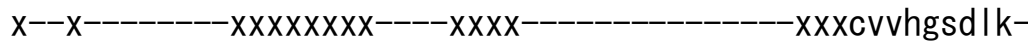

dmtsee Idd-

i I ryhxx iVFA--RTSPQQKL I IVEGCq ---rq--ga--_--_-_---_---_-_-_------IVAVTGDGVNDSPALKKAD I GVAM-- 
ytl-tsn-i-peitpfl ifi i iani---_-_-_-_-_-_-_-_-_ptvtilci

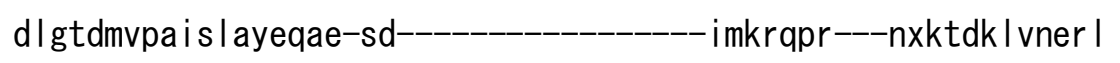
i xmaygqi gmi--qal ggfftyf $x x x x x x x x x x x x x x x x x x x x x x x x x x x x x x x x x x x x x x$ xeqrkix----------------------------------------------------ef----tchtaffvs i vvvqwad-Ivicktrxxsvfqq--------gm-knk i I i fg I fee

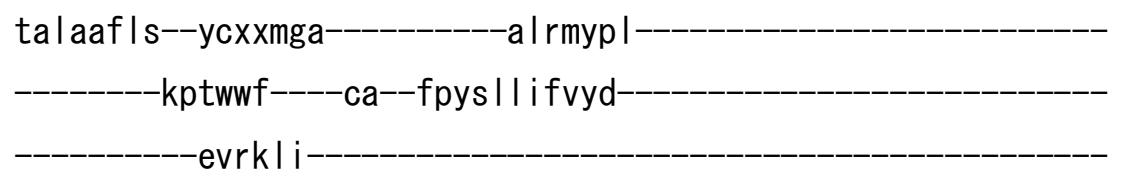
$-i r r r p x-$ $-x x x x x x x-$

\section{y} >mmul I_IIC02 NP_848492.1

$\mathrm{m}-$ 
$x X X X X X X X X X X X X X$

dhk I I de Igr

$-k y q$

-vdlskgltnar

aqd i l ar dgpna I tppptt

-pewvkfcrq-I

fggfsil |wiga--||cf|

ayg $\mathrm{x}$

-xxxxxde---psndn ly lg i v

laav-v-

ivtgcfsyyqe

$-a k-$

$-s s^{----k i}$

mdsfkn--mv-

-h--gckVDNSSLTGESEPQTRsp-

$-x x x x x x x$

$x x t$

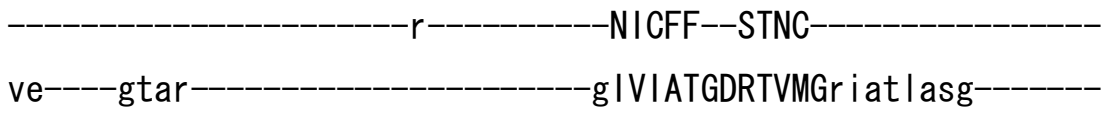

- levgqtp i ame i ehf iql i tgvavf I gvsff

$-v|s|$ ilgy

-swleavifIIGIIVAN

VPEGLLATvTVCLTLTAK-RMarkn- 
cLVK-NLEAVETLGSTST I CSDKTG-

TLTQNRMTVa

-hmwfdnqih

$-x x x x x^{--}-e x x-$

xxxxxdkrsptwx

$-a \mid$

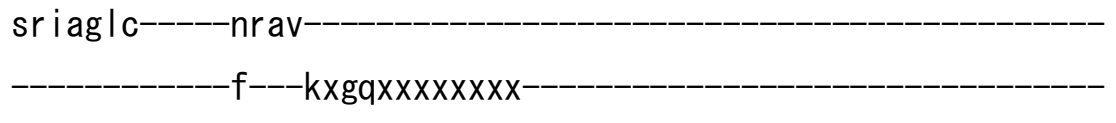

dtagdase--sal Ikc

i el scgs--vrkmxx

-rnpkvae ipfnstnkxql s i

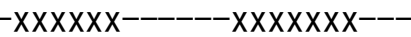

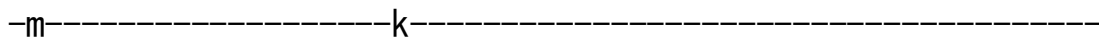

-gap

$\mathrm{e}$ -ri Idrcst i l vagk

e ip I dkemqdaf-qnayme I--gg |----gervLGFCQLn I -xxxxxxxgfkfdtde I nxptek I cfVGLMSMIDPPRAAVPDAVGKCRSag-IKV IMVTGDHPITAKAI AKGVGI-

i sexxe-

$-x x x x x x$

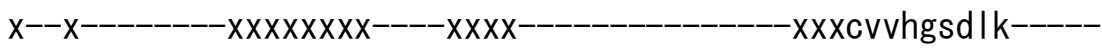

-dmtseq I de 
i I rdhxx iVFA--RTSPQQKL I IVEGCq

---rq--ga--_-_-_--_-_-_-_-_-_-_-_-_IVAVTGDGVNDSPALKKAD I G I AM--

SKQAADMILLd-DNFASIVtgVEEGRL IFDNLKKSI------A

yt I-tsn-i-peitpfIIfi iani---_-_-_-_-_-_-_-_-_---p--Iplgtvti lc $\mathrm{i}$

d I gtdmvpa i s layeaae-sd--_-_-_-_-_-_-_-_imkrqpr---nxqtdk I vner I

i xmaygq i gmi--qa Iggfftyf $x x x x x x x x x x x x x x x x x x x x x x x x x x x x x x x x x x x x x x$ xeqrkvx

ef-----tchtaffas i vvvqwad-I i icktr xxsvfqq-------gm-knk i l i fg I lee tal aaf I s--ycxxmgv---_---_---al rmyp I--_-_-_-_-_-_-_-_-_-_-_-_-

---------kvtwwf-----ca--fpys I I if i yd------------------------------

evrkli

Irrypx

$-x x x x x x x-$

y

>mmulI_IIC03 NP_659170.1

$\mathrm{m}-$ 
$-x$

$x X X X X X X X X X X X X X X X$

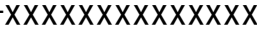

ehkmsveevcr

-kyn---------tdcvqg|thsk

-aqe i lardgpna I tppptt

-pewvkfcrq-I

fggfsi I Iwiga--i lcf I

aygxxxxxxdd---psgdn ly I g i v

laav-v

i i tgcfsyyqe

-ak------------ss----k i

mesfkn--mv-

$-p q q-a \mid v-$

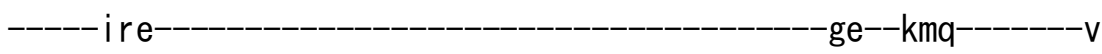

n-

----aeeVVVGDLVEIkg

GDRVPADLRI I sa 
-h--gckVDNSSLTGESEPQTRsp-

$-X X X X X X X$

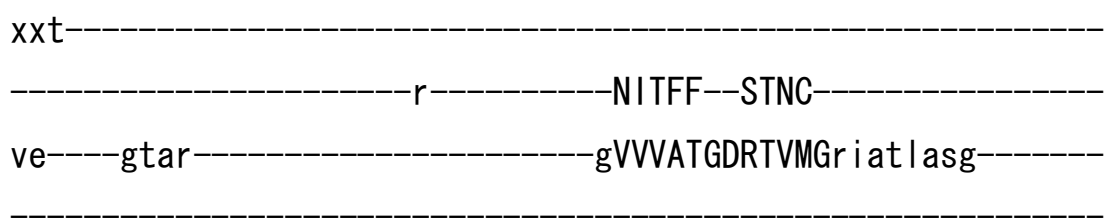

- levgktpiaieiehfiql itgvavflgvsff

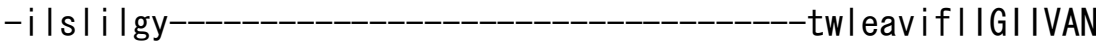

VPEGLLATVTVCLTLTAK-RMarkn-

cLVK-NLEAVETLGSTST ICSDKTG

TLTQNRMTVa

-hmwfdnqih-

$-x x x x--e x x-$

xxxxxdksshtwx

al

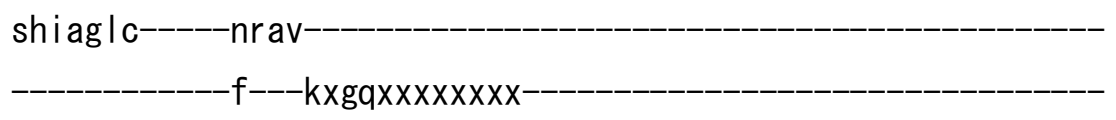

dvagdase--sal Ikc

ielssgs--vk|mxx

-rnkkvae ipfnstnkxqlsi

$-\mathrm{x} x \times x \times x-----x \times x \times x \times x x---$

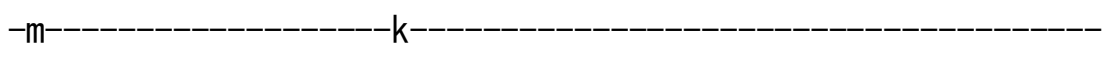

-gap

e----_-_-_-_-_-_-_-_---ri I drcat i I lagk 
eqp I deemkeaf-qnay | e |--gg |----gervLGFCHYy |

------xxxxxxxgfafdcddvnxttdn I cfVGLMSMIDPPRAAVPDAVGKCRSag-IKV IMVTGDHPITAKA IAKGVGI

i sexxe-

$-X X X X X$

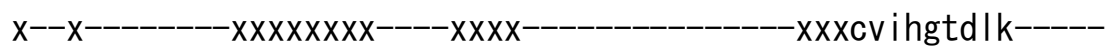

dftseqide

i I qnhxx iVFA--RTSPQQKLI IVEGCq

$---r q--g a$

IVAVTGDGVNDSPALKKADIGVAM--

$-x i a g s d v$

SKQAADM I LLd-DNFAS IVtgVEEGRL IFDNLKKS I

A

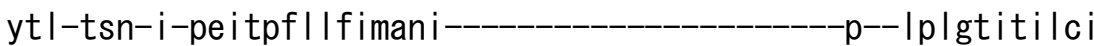

d I gtdmvpa i s l ayeaae-sd-_-_-_-_-_-_-_-_-_-_imkrqpr---nxrtdk I vner I

i xmaygq i gmi--qal ggffsyf $x x x x x x x x x x x x x x x x x x x x x x x x x x x x x x x x x x x x x x$ xearkvx

ef----tchtaffvs i vvvqwad-I i icktrxxsvfqq--------gm-knk i I i fg I fee tal aaf I s--ycxxmdv-----------al rmyp I

---------kpswwf-----ca--fpysfl i fvyd-

-eirkli

Ir $r$ pxxxxxxxxxxxxxxxxxxxxxxxxxxxxxxx

$x x x x x x x x x x x x x x x x$ 
>mmulI_IIC04 NP_619593.1

$\mathrm{m}$

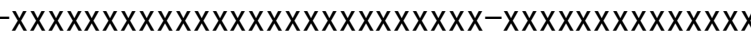

$-x x x-x x x x x x x x x x$

dhr I sntdleq

-kyg--------tni iqglss ir

aae I l ardgpna I tppkqt

-pe i ikflkq-m- 
-vggfsil Iwiga--alcwi

ayvx -xxxxxt----as Idnvy Igai

Iv Iv-v----------i I tg i fayyqe--------------ak-----------st----ni

masfsk--mi

$-x x$

-kVDNSSLTGESEPQARst-

$-x x x x x x x$

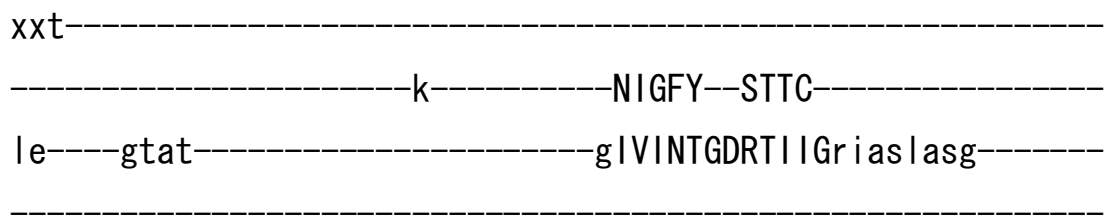

-vgsektpiai i i ehfvhivaavavsvgviff

-i tavcmky-yvIdai ifIISIIVAN

VPEGLLATVTVTLSLTAK-RMakkn-

-cLVK-NLEAVETLGSTSI ICSDKTG

-TLTQNRMTVahlwfdnqif-

$-x x x x x--e x x-$

xxxxxdqssgtwx $-\mathrm{s} \mid$

skiitlc nrae

f---rxgqxxxxxxxx 
sevi I gd--vmd i xx-

rnhkvae ipfnstnkxqls i

$x X X X X X-----X X X X X X X X---$

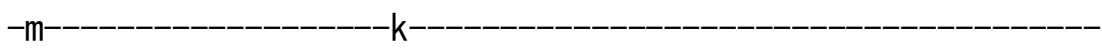

-gap

e----------------------r i l ekcst imingq

eqp I dkssadaf-htayme I--gg |----gervLGFCHLy I

-xxxxxxxsytfdvds inxptsn I cfVGLLSMIDPPRSTVPDAVSKCRSag-IKV IMVTGDHPITAKAIAKSVGI-

i saxxe-

$-X X X X X X$

x--x-------- $x x x x x x x x-----x x x x---------------x x x a v v t g m e l k$

-dmtpeqlde

I I inyxx iVFA--RTSPQQKL I IVEGCq

$---r q--d a$

-VVAVTGDGVNDSPALKKADIG I AM--

xiagsda 
yt I-tkn-i-aelcpfl iyivag I -p--lpigtitilfi

dlgtdi i ps i a layekae-sd--_-_-_-_-_-_-_--imnrkpr---hxkkdr I vnkq I

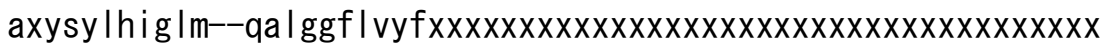
xyqrkyx

ew----tgstaffvaimvqqiad-li irktrxxs ifqqglxrnkviwvgi isq i ivalvls--ygxxsvt----------alsftml-

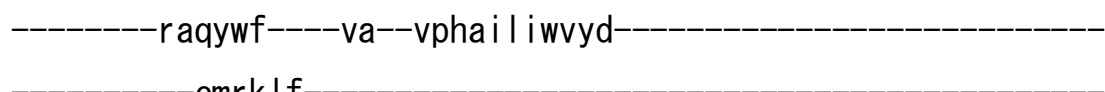
emrk|f-

irlypx-

$-X X X X X X X$

y

>ddi II_IIC01 XP_642777.1

$\mathrm{m}$

$X X X X X X X X X X X X X X X X X X X X X X X$

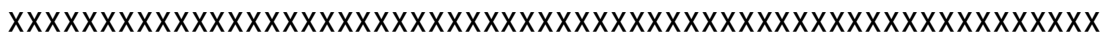

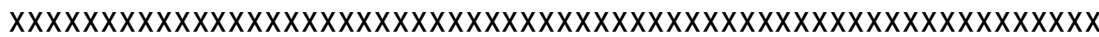




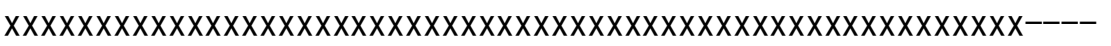

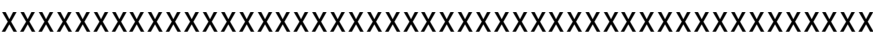

eheltleqise

$-k f s x x x x x x x-p s|s s g| t q s \mid$

-ane I I irngkni I kppkev

- pwyvqlgkc-f-

tnffmi l levag--alcfl

aygx

dr---nqrvnlylacv

Iyai-v--_-_-_-_-_iftcl|tfsqe-_-_-_-_-_-_-_rq-_-_-_-_-_-_tg----ni

mksfkn--II-

-n--gmkVDNSSLTGESDAQSCtv-
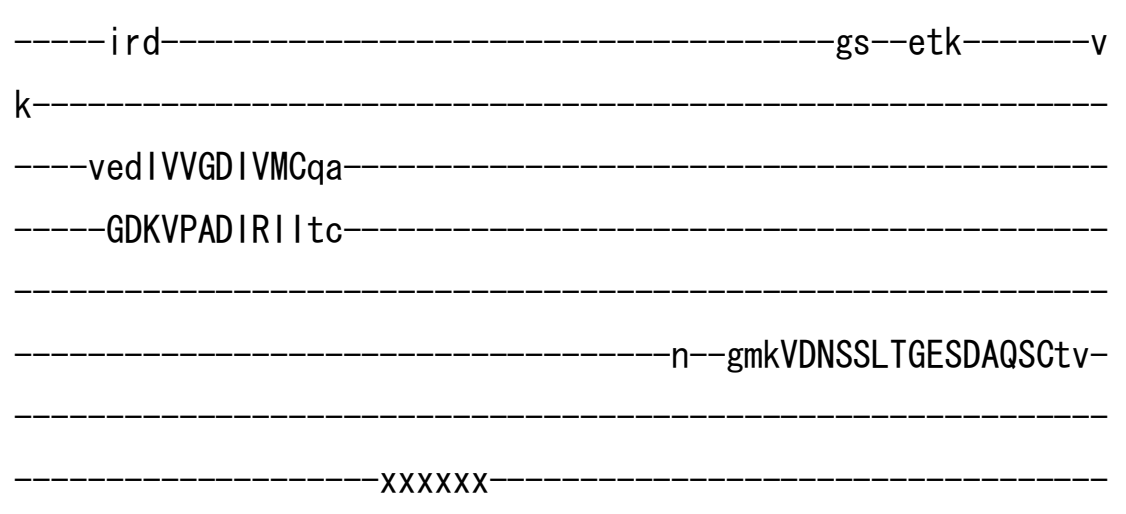

$-x$

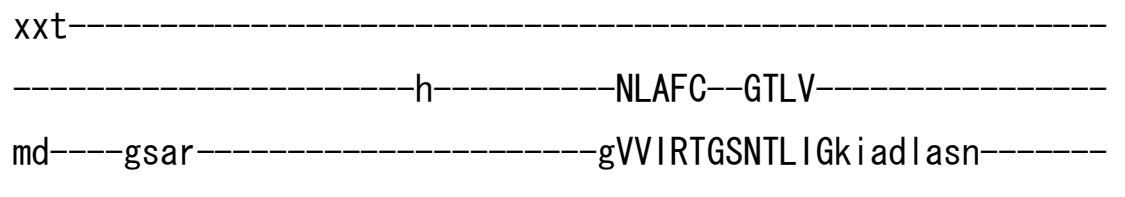

-teqtettlqieikrfvhfitalgi tmgliff

- i i gfavg i--_-_-_-_-_-_-_-_-_-_-_-_-_-_-_-_--_ds iqn I invLGL IVAN

VPEGLPST i TACLTVTAR-RLsrrn- 
-vYSK-KLESIETLGSITL I ASDKTG-

TLTQNRMTVs

-hmwydnt i v

$-x x x x--g x x x$

xxxxxdigtptcx

$-a \mid$

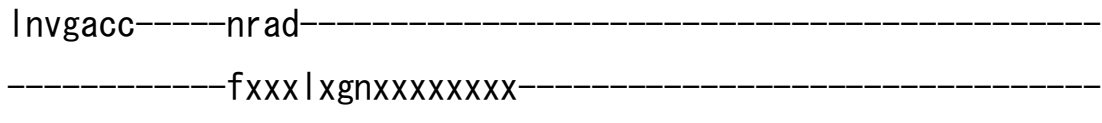

| i |gdase--sai |r |

cht i ed---ieitxx

-knpkvfe ipfnstnkxqlsi

$-X X X X X X X X X X X X X X X X X X X---$

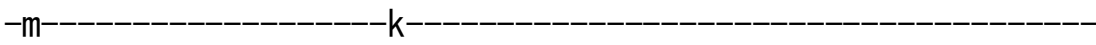

gap

e------_--_-_--_---_---- i i fekcsk Imingk-

ele idekikndf-iqayeal--gsm----gercLGFAQLI I

-xxxxxxxndmydaqt I nxpmtg I tfVGLCSLLDPPRENVPFAVHQCKTag-IKV

IMVTGDHPITAKAIAKKVGI-

i s--xp

$-x x x x x x$

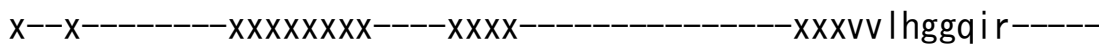

eltdadwdr 
vI-skxx iVFA--RTSPQQKSQIVENAq

$---k r--k e-$

-VVAVTGDGVNDSPALKKADIGVAM--

xivgsdv

AKETAD I ILLd-DNFAS IVag I EEGR I IFDNLKKS I

$-A$

yt I-sha-v-pevapf I Ini i sg i---_-_-_-_-_-_-_-_--_a i tsf I i l c i

d I gtemapa i s layetge-kd-_-_-_-_-_-_-_-_-_imsrkpr---vxgkdh I vttn I

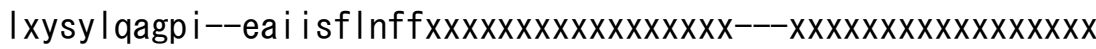
xqveal $x$

q------aqtay fmt I vtcqffn-l i tnrtrxxp I wsh-------ki ag i cafvv--ytxxvht--_-_-_-_--_i i esasv-_-_-_-_-_-_-_-_-_-_-_-_-_-_-_

$-p g \mid$ fwa----yp-- | pmifc I fswn-

eirkri

aydrpx

$\operatorname{xxxxxxxx}$

f

>ddi II_IIC02 XP_647420.1

m- 


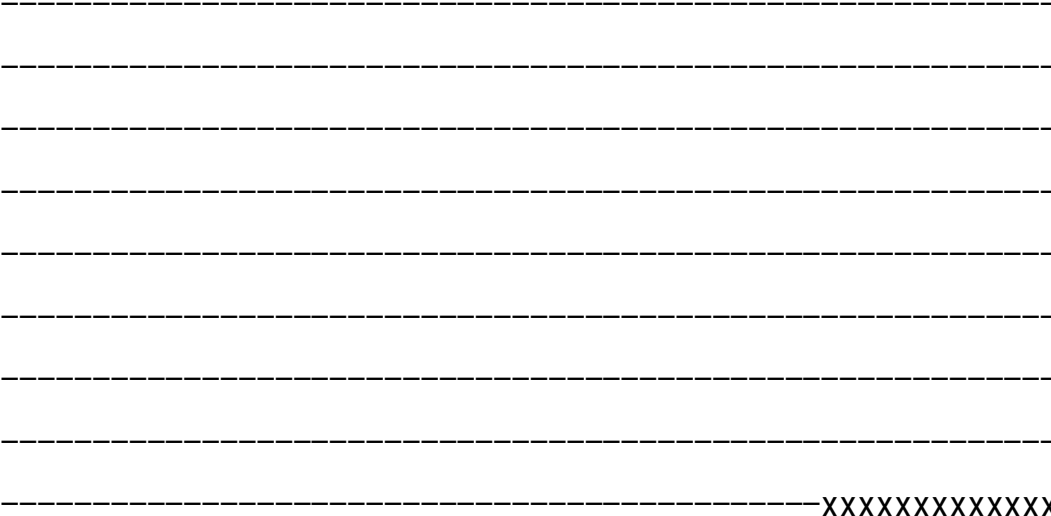

$-x x x x x x x x x x x x x$

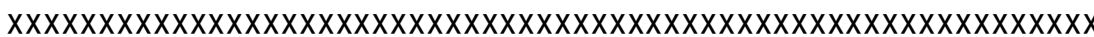

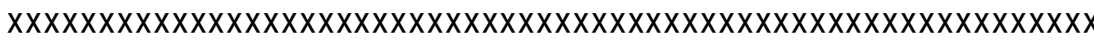

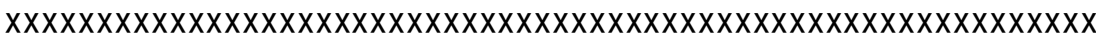

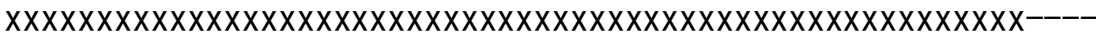

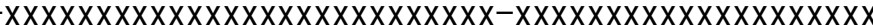

kepmsaidlaq

$x X X X X X X X X X$

$x x x x x x x x x x x x x x x x x x x a s e r$ le idgknal tpskpv

-pkwvkylke-f-

Iglfpimlevgg--ilsi i

afgx -xte---tgkdnly lgi i

I wi v-v----------fI tctfsy i qn--------------sk------------st----gv megfkk--la-

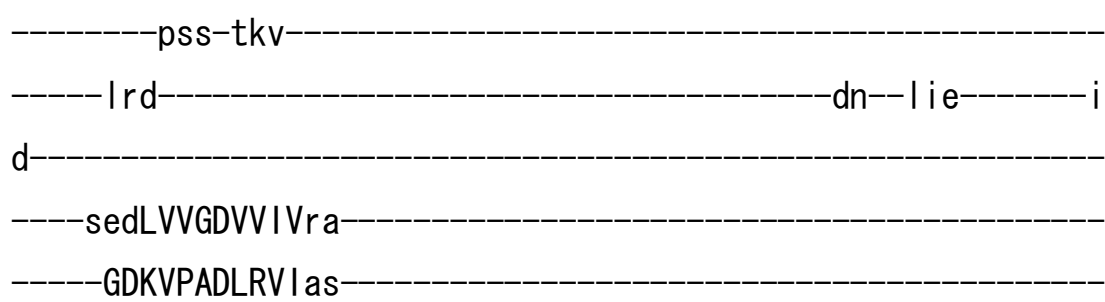


-h--hfkVDNASLTGETEPQTRsp-

$-X X X X X X X$

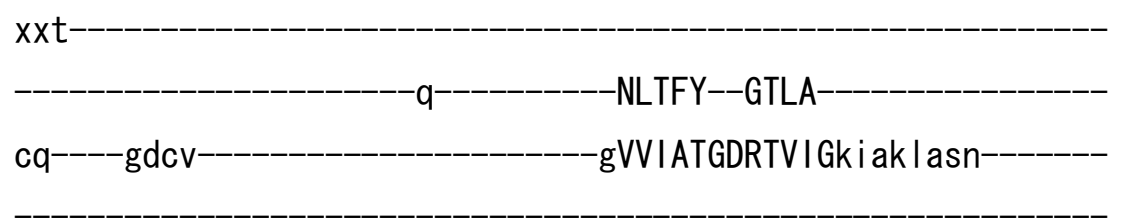

-skpnstpmkeeiekfiki isivafs Igaif।

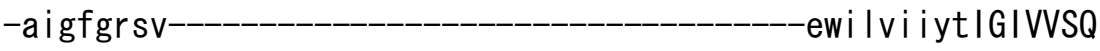

VPEALLPTITVTLNLTAK-RMsrkn-

-vLVK-NLLTVETLGSTTTIASDKTG

$-T L T Q N I M T V$

hlwydgtiy

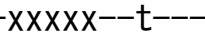

xxxxxnaqattfx

$-k \mid$

yqvaal c-----nrtv

$f x x-s x n q x x x x x x x x$

kc igdase--sal lkf

-ceqv-en--veqyxx

-rfpkyfe ipfnsvnkxqmsv

$-\mathrm{x} x \times x \times x-------X \times x \times x \times 1---$

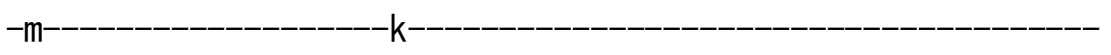

-gap

e-----_---_-_--_--_----r i i kmonr i l i ege 
eqe I dekh I qnf-qssyeh I--agk----gervLGLAYLp I

------xxxxxxxny i fdmeeknxptkd I vfVGLTALMDPPRPGVPEAIRTCKEag-IRV MMVTGDHPLTGTAIAKQVGI-_-_--_-_-_-_-_-_-_-_-_---_--i exxe--_-_-

$-x x x x x x$

x---x--------- xxxxxxxx-----xxxx-----------------xxxi a i tgsm Id

dl tseqwdk-

i I-s I xx ICFC--RTSPEQKLQIVAHLq

---kr--ge--------------------------IVAVTGDGVNDSPALKKADLGCAM--

-xitgsdv-

-AKEAAS I VLLd-DNFASI I agVEEGRM IFDKLKKSI------C

yt I-ssn-i-pea ipffcffv lam--_-_-_-_-_-_-_-_-_-_--p--val sg i I i lc i

dl gtdl i pvi syayegse-td--_-_-_-_-_-_-_--- Imkrkpr---nxkkdk I vs I r I

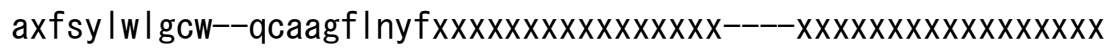
xkqiqix

n-----eaqtayf i a i vi srvga-cfcaktrxxs i fqq--_-_---gf-gnmvfnfgvcsm la i al f i v--hvxxvrt-----------ffgct i v---------------------------sykywl----ip--ipfavflvasn-

elr $|w|$ 


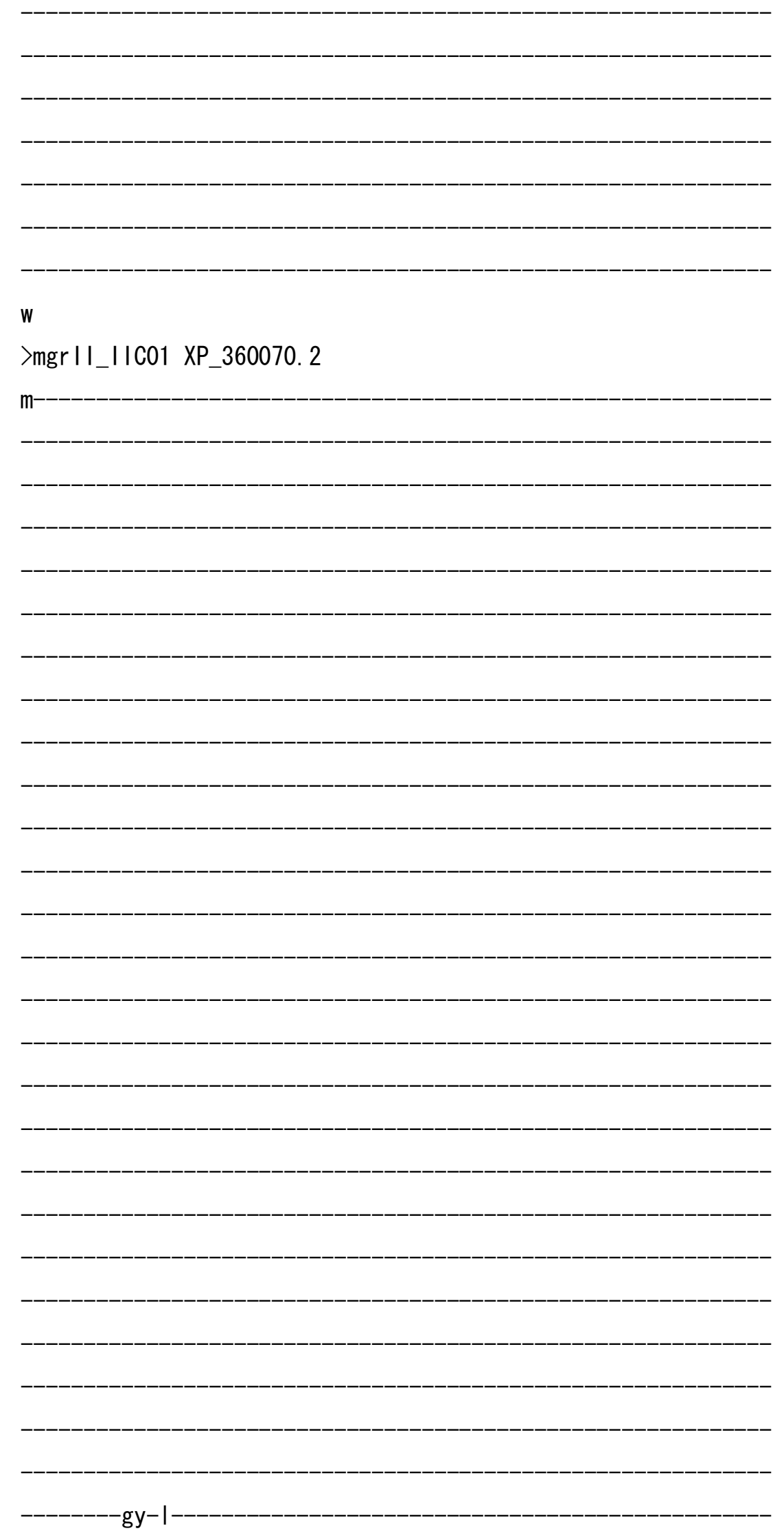




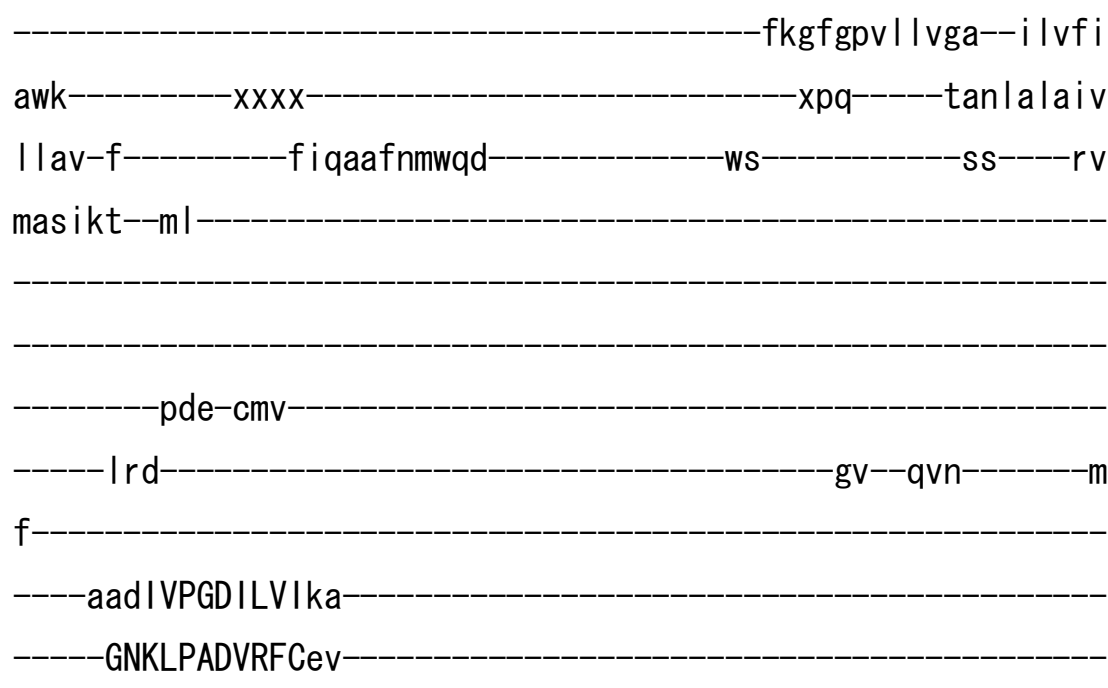

xxdakFDRSILTGESLPLPAtv-
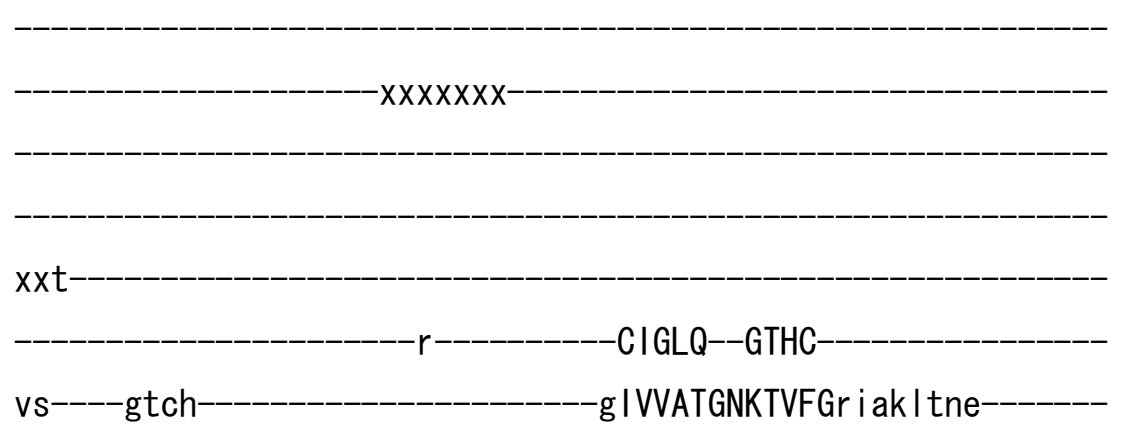

-pkkgmttlerevInfvwi icsimvImvvlvi

- i I watw I rxxxxxxx--_-_-_-_-_-_-_-_-_-_-_-_---svsg I i vscVSVAVAF

IPEGLPVAI TASLTISAN-MMrknk-

-vLCK-SLKTVETLGAVSVICSDKTG

TLTGNKMVVt----------------dcal ggath-

$-x x x x x x \mid x x x$

$x x x x x$ seec lagxxxx-

$-q \mid$

rtisal cxxxxdaat

$r x x \mid x n r$ 


\section{ringdatd--qai Irf}

-aetmgs--amatxx

cwqtrfdlafnsknkxmi rvxxxxxxxxxx

$-d x x x x x x x x x x x x x x x x x x x$

-gap

d

vI Igrctkyvgssgx

tInI spaardev-er i knew--sar----grrvLLLARKv I

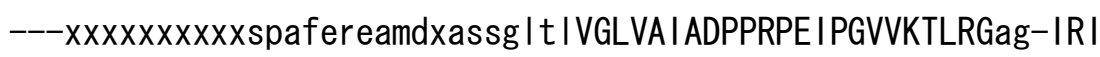
FMVTGDFALTAQA I ARECGI

itnxxs-

$-x x x x x x$

$x x x x x x x x x x x-x x x x x x x x----x x x x----------------x x i$ v i sgadma

-gm I snqwde

I aaeyxxvVFA--RTTPEQKLRIVRELq

---sr--hh-

- IVGMTGDGVNDAPALRAADVG IAI--

gggsdi

-AAEAADMVLL--ESFGSVVeaVRYGRTTFDNLKKT I 
>mgr II_IIC02 XP_365182. 2

m- 


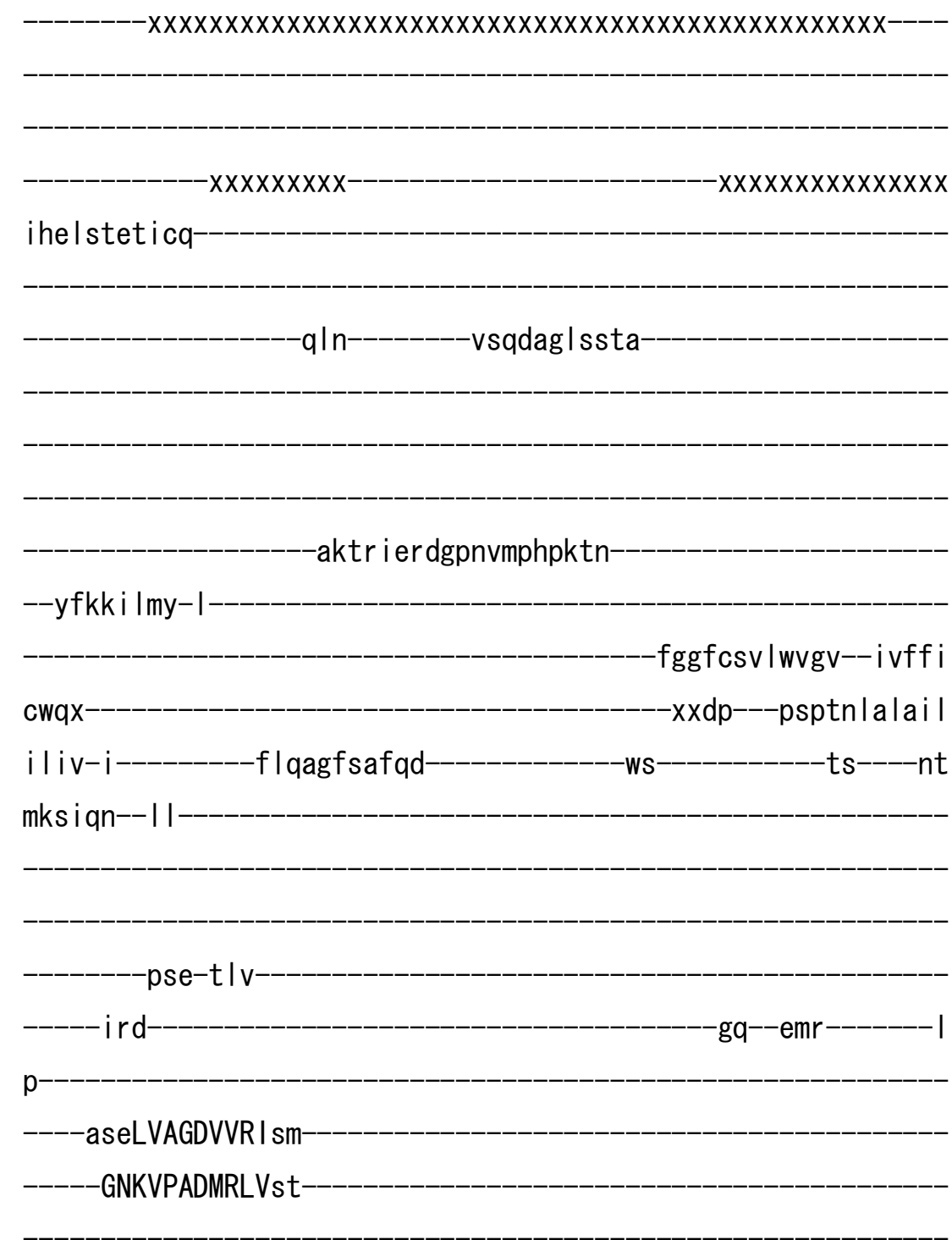

-xxd i rFDRAMLTGESDEVEGav-
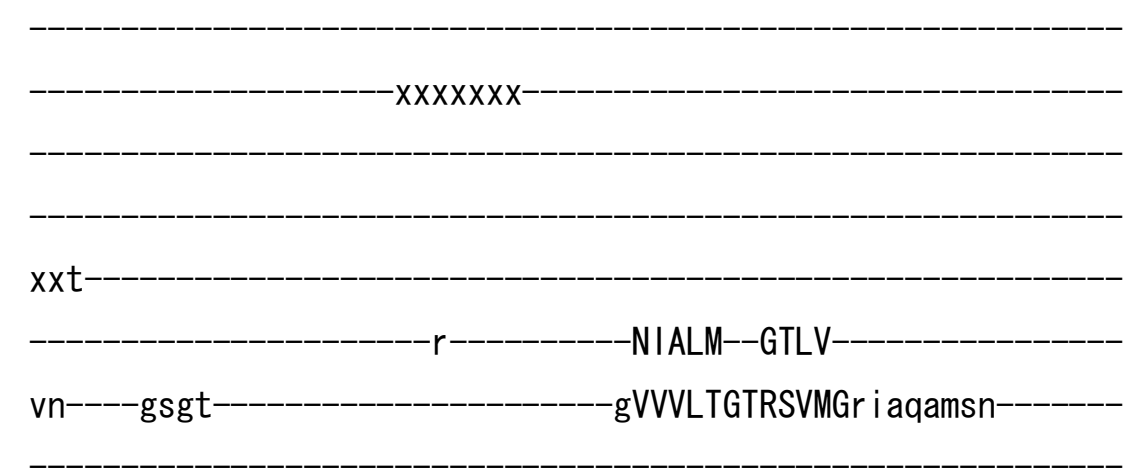

-vseket I iqre ivrfvki ivcftialasl ia

-i awgaw I rxxxxxxx---_-_-_-_-_-_-_-_-_-_-_-_-_-_-svvam I nnvMGCVVAF

IPEGMPI AvSLTLMIVAR-RMkasn- 
iLPK-GLATVETLGCVNVVCSDKTG-

TLTQNKMTVs svafvdses

VXXX

xxxxxqeerpmaxxx-

$-k \mid$

heaavlcxxxxxeptt

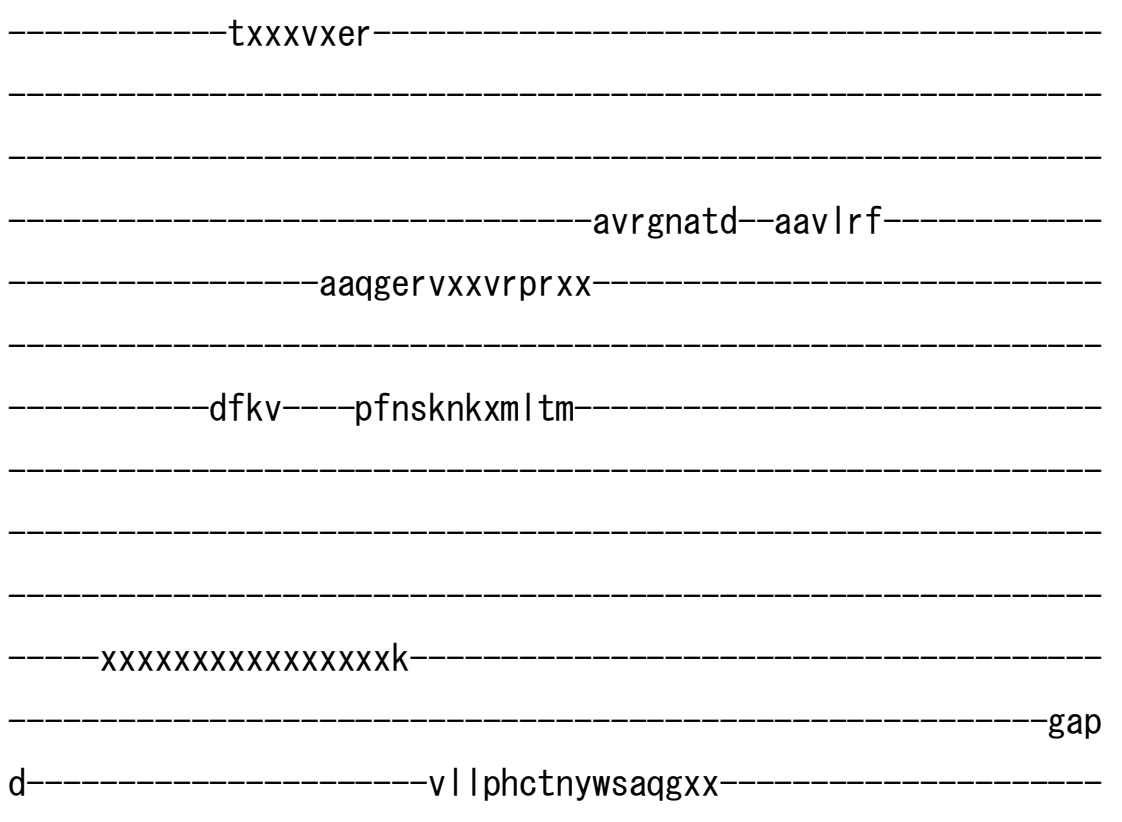

-_-_-_-_-_-_-_-_-_-_-_-_-_-_-_-_-_-_-_-_-_-_-_-_-_-_-_-_-_-_-_-_-_-_-_-

vrp I dgearat I-kgfqdkm--srr----aerv ILLCETtf

-----xxxxxxxxnafsdevasexiktg I tv I ALLGI IDPPRPETAHT ISECRRag-IRF

FMVTGDYGLTASA I ARN I GL------------------------------frgxxe------

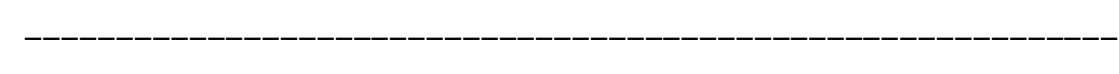

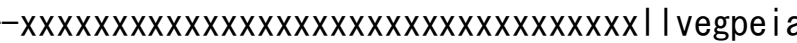

$-\mathrm{t}$ stddwd 
vvceyxxvVFA--RTTPEQKLR IVTELq-

$---a r--h n-$

-VVAVTGDGVNDAPAMRKADVGVAV--

isgsdv

-AIEAADLVLM--DKFDS I I da I RLGRLVFQNLQKV I

$S$

yll xags-y-se iapvi Invffgt $-p--|p| s a f \mid m i$ i i cvftdIflcls I imekae-fd-_-_-_-_-_-_-_-_-_l la ippr---dxrrdhl inar i yxqay Iftgtm--ett i ahsmffxxxxxxx-----_-_-_-_-_---_------xxxxxxx $x f \mid$ yegxxxxxxxxxxxxxxxx-

va----tgqsvyfvt I vm I qwgn-i lavrnr--rms i vxxxxxx-kqxrnpw Ip I smv Is laiavfvt--qtxxiqn-I fatatv

--------piefwf----ip--iplalail ladegrkII

$-v r \mid f p x x x x x x x x$

W

>cellIIIIC01 NP_506269.1

m- 


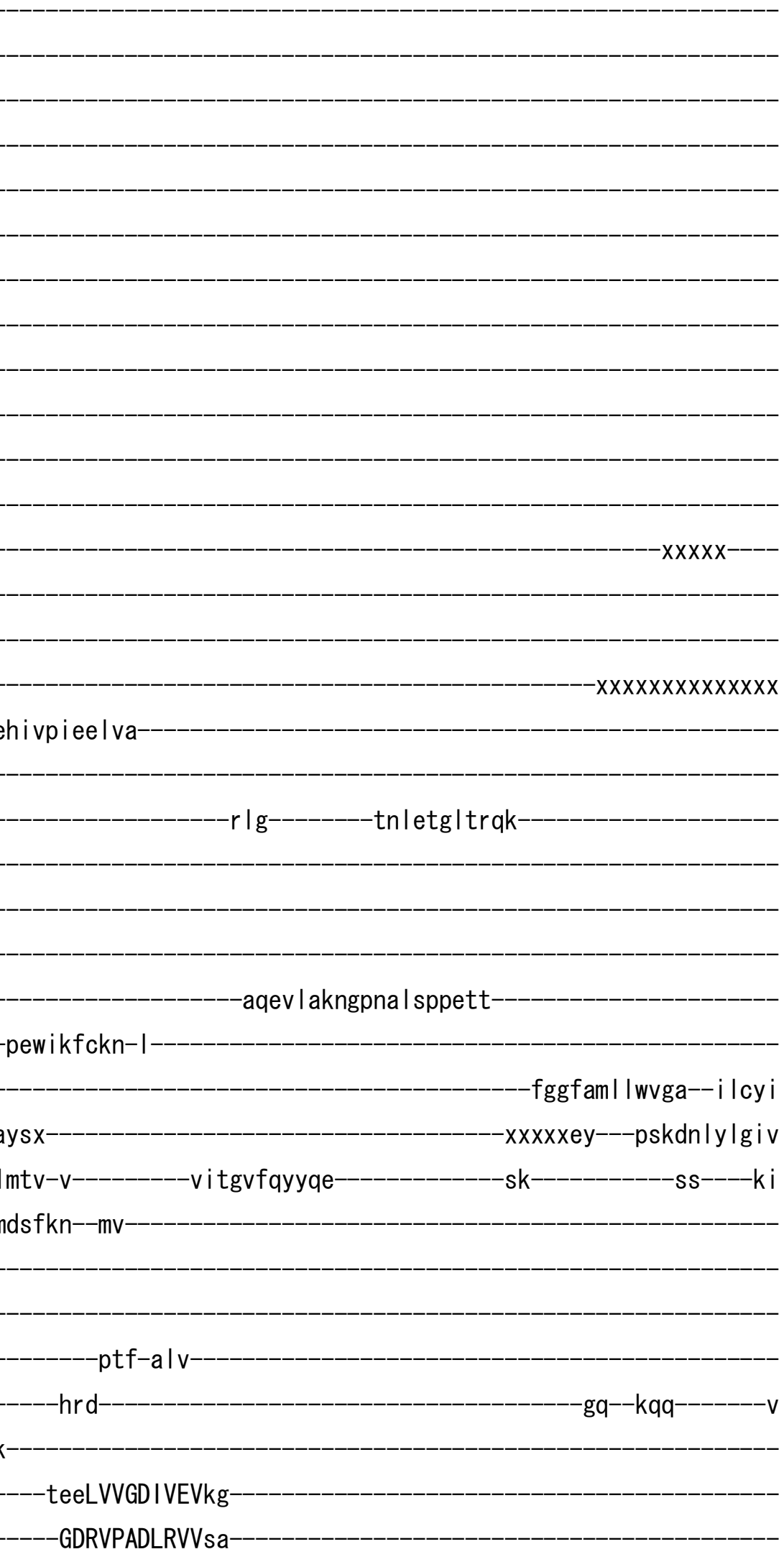


f--gfkVDNSSLTGESEPQSRsp-

$-X X X X X X X$

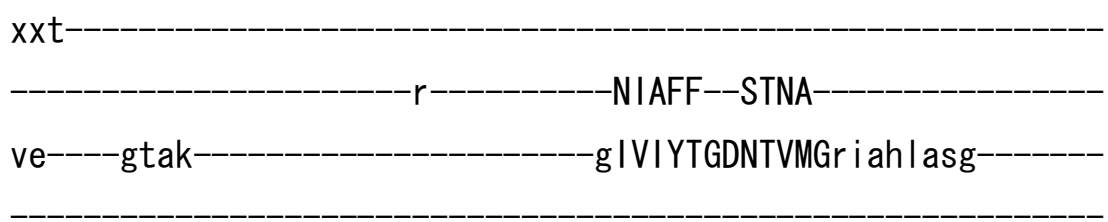

- Idtgmtp i are i ehf ihl i tgvavf I g isff

-i iafi igy -hwltavvfIIGIIVAN

VPEGL I ATVTVCLTLTAK-RMaskn-

cLVK-NLEAVETLGSTST I CSDKTG

TLTQNRMTVa

-hmwydetih

$-x x x x x--e x x-$

$x x x x-k r \operatorname{tgas} f x$

al

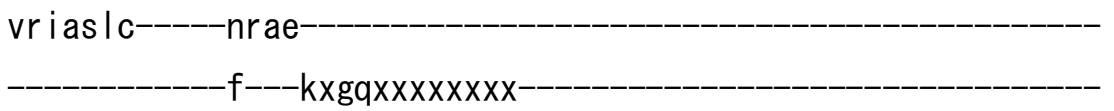

dctgdase--ial lkf

tel tqgn--viavxx

-knkkiae ipfnstnkxqvs i

$-x x x x x x$

$-x \times x x---$

$-m-$

-

$\mathrm{e}$

rildvcstifingk 
ese | tdk | redf-ntay l el--ggm----gervLGFCDFv |

-xxxxxxxgfkfdveevnxp I kn I rfVGLMSMIDPPRAAVPDAVAKCRSag-IKV VMVTGDHPITAKAIAKSVGI

isdxxe-

$-X X X X X$

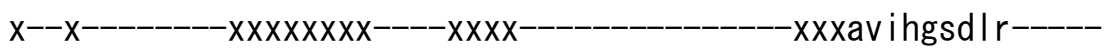

emsedqlae

i ikyhxx iVFA--RTSPQQKLMIVEGFq

$---k q--g q$

IVAVTGDGVNDSPALKKADIGVAM--

$-x i a g s d v$

SKQAADM ILLd-DNFAS IVvgVEEGRL IFDNLKKS I

$-A$

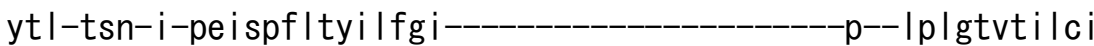

d I gtdmvpa i s l ayeeae-sd-_-_-_-_-_-_-_-_-_-_imkrqpr---dxi i dk I vner I

i $x$ laygq igmi--qasagfftyf $x x x x x x x x x x x x x x x x x x x x x x x x x x x x x x x x x x x x x x$ xanrkix

ey----tcqtayfvs i vvvqwad-I i i sktrxxs I vqq--_-_---gm-snwt I nfg Ivfe

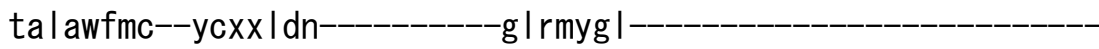

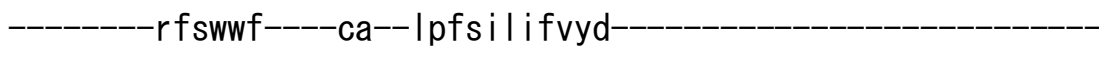
eirrfl 
y

>hsalI_IIC01 NP_000692. 2

m-

dhk Is Idelhr

-kyg---_-_--_tdlsrgltsar-

-aae i l ardgpna I tppptt

-pewikfcrq-I 


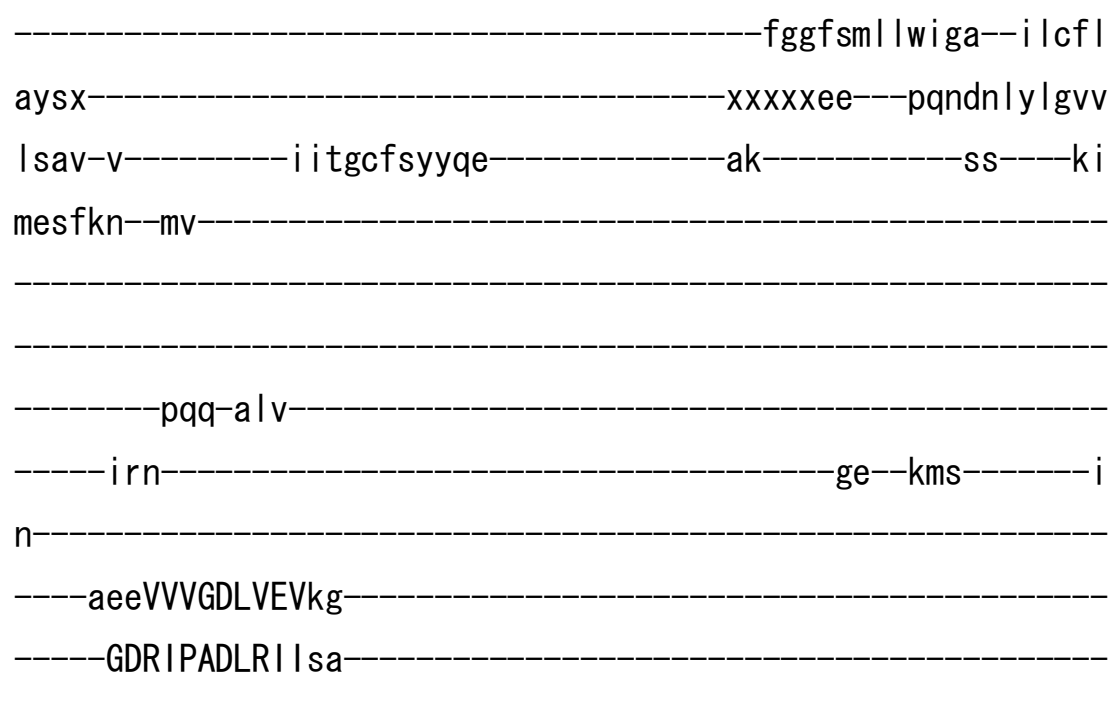

-n--gckVDNSSLTGESEPQTRsp-

$-\mathrm{XXXXXX}$

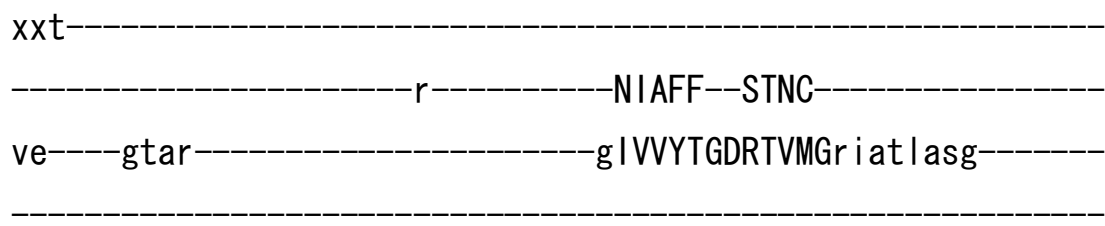

- leggatp i aae i ehf ihi i tgvavf I gvsff

-ilsliley-twleavifIIGIIVAN

VPEGLLATVTVCLTLTAK-RMarkn-

cLVK-NLEAVETLGSTST I CSDKTG

TLTQNRMTVa -hmwfdnqih

$-x x x x x--e x x-$

xxxxxdktsatwx

sriaglc-----nrav-

$-f---q x n q x x x x x x x x$ 
-avagdase--sal l kc-

-ielccgs--vkemxx-

ryakive ipfnstnkxqlsi

$-x \times x \times x x-----x \times x \times x \times x x---$

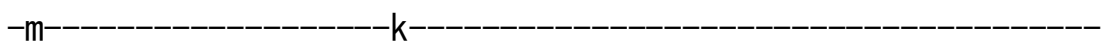

-gap

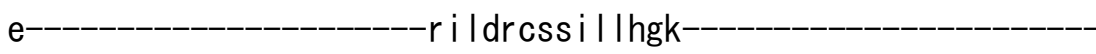

eqp I dee | kdaf-qnay | e |--gg |----gervLGFCHLf I

-xxxxxxxgfqfdtddvnxp i dn I cfVGL I SMIDPPRAAVPDAVGKCRSag-IKV

IMVTGDHPITAKAIAKGVGI-

isexxe-

$-x x x x x$

x---x---------xxxxxxxx----Xxxx----------------xxxcvvhgsdlk-

dmtseqldd-

i I kyhxx iVFA--RTSPQQKL I IVEGCq ---rq--ga--_--_-_---_---_-_-_------IVAVTGDGVNDSPALKKAD I GVAM-- 
ytl-tsn-i-peitpfl ifi i iani---_-_-_-_-_-_-_-_-_ptvtilci

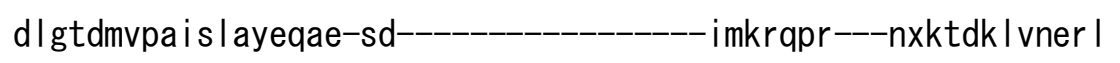
i xmaygqi gmi--qal ggfftyf $x x x x x x x x x x x x x x x x x x x x x x x x x x x x x x x x x x x x x x$ xeqrkix----------------------------------------------------ef----tchtaffvs i vvvqwad-Ivicktrxxsvfqq--------gm-knk i I i fg I fee

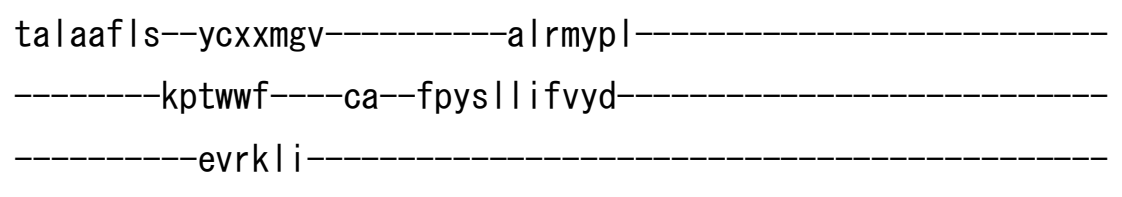
$-i r r r p x-$ $-x x x x x x x-$

y $>$ cell I_IIC02 NP_497034. 1

m- 
$X X X X X X X X X X X X X X$

dhe ip Idalfk

$-r y t$

-tsekng i seae

-atnr Inr dgpna I tppkqt

-skwiklags-i

-fggfnf I I wcaa--sasav

gygx

-xxxxxxxee---vpkdnmyma i i

latv-v

tvtglfdfyqn

$-r k-$

- sg----nl

mdsfan--mi

xg I kVDNSSLTGESEPQTRnm-

$-x x x x x x x$

xxt

k---------NLCMF--STSV-

le----gsge-_-_-_-_-_-_-_-_-_-_-_-_g | | IGTGDRTFVGr i aal ttq

-vdpgptp lake inhf ik i is i vaftvg iaff

- i lalvyey -pplkaivffMGIVVAN

VPEGIVPTVTVSLTLTAV-KMrkkf 
cLVK-KLQAVETLGSTSTI ICSDKTG-

TLTQNRMTVt

-hlwfdgkik

$x x x x x x x n x x x$

xxxxxyekedsyx

Ircat|c-----srsh

$f---r x p e x x x x x x x x$

vvngdase--vaimry

cemi rgdxxvdefxx

impkige ipfnstnkxqlsi

$-x x x x x$

$x \times x x x---$

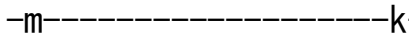

gap

$\mathrm{e}$

-ki lk lcstyyqnge-

tknvskkfekef-qqayet I--gsy----gervLGFCDLem-

xxxxxxxgfkfnmeepnxp i kn I r fLGL ISM IDPPRPGVPEAVRVCQNag-IRV VMVTGDHPITARA I ANQVHI

i eexxq

$-x x x x x x$

$\mathrm{xxxX}$

$-x x x x x x x x x x x x x x x x$

xxxvvihgeqlt

tmspkt Ika- 
vvtnyxx iVFA--RTSPAQKLQIVEAFq

--- sv--gn-

-IVGVTGDGVNDAPALRKADI G I AM--

xitgtdv

SKQAADM ILLn-DNFASIVtgVEEGRL IFDNLKKS I

$-A$

yt I-tsn-i-peitpfmsyv $|f g|$

$-p--\mid p m s$ i iai Imi

dlgtd I wpai sfayevpe-sd

imqrapr---nx Iydk | vnkr I

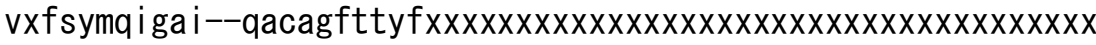

xesrkax-

es----ccygtffftivvtawsd-I fasktrxxs I vmq

gm-enhvIntsvift

cflaifvl--ntxxvnev lgvagf

--------rleigf----la--Ipfaffig lyd-

evrryf

irtypx

$\mathrm{XXXXXXXX}$

$\mathrm{y}$

>cellI_IIC03 NP_505083.1

m- 
t--dffLETSSITGEAEPLEFhs-

$-X X X X X X X X X$

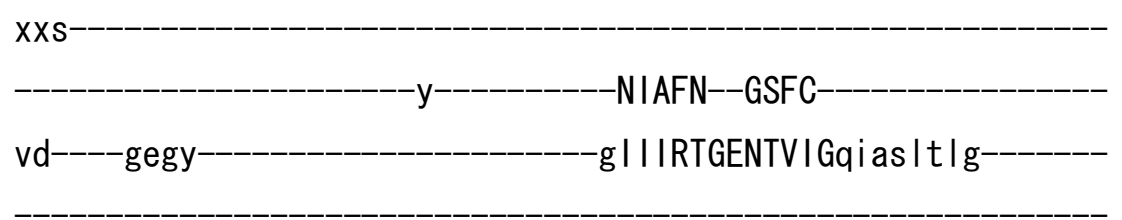

-qkdkkckfete ierfvqf i tvmai tmatvif

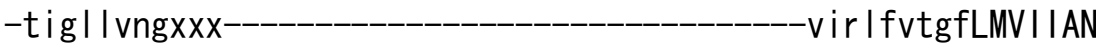

VPQGLPTTVTTELTI I AR-RMakkn-

vFLK-KLEKIDSVGATTLI ASDKTG

TLTKNCMTVt

dl wynnsyn

$x x x x x x x g x x x$

xxxxxnaint igxxxxxxx-

$-\mathrm{di}$

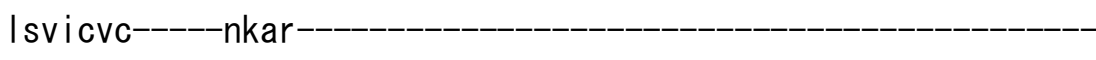

ixxx|xtkxxxxxxxxxxxxxxxxxxxxxxxx

I isgnpse--val Iry

asgmxxamevxx

sfhvvfevpfnsvrkxhlil

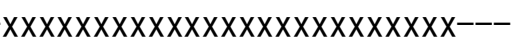

$-i$ $k$

d

$-v l$ ikscst in idgv 


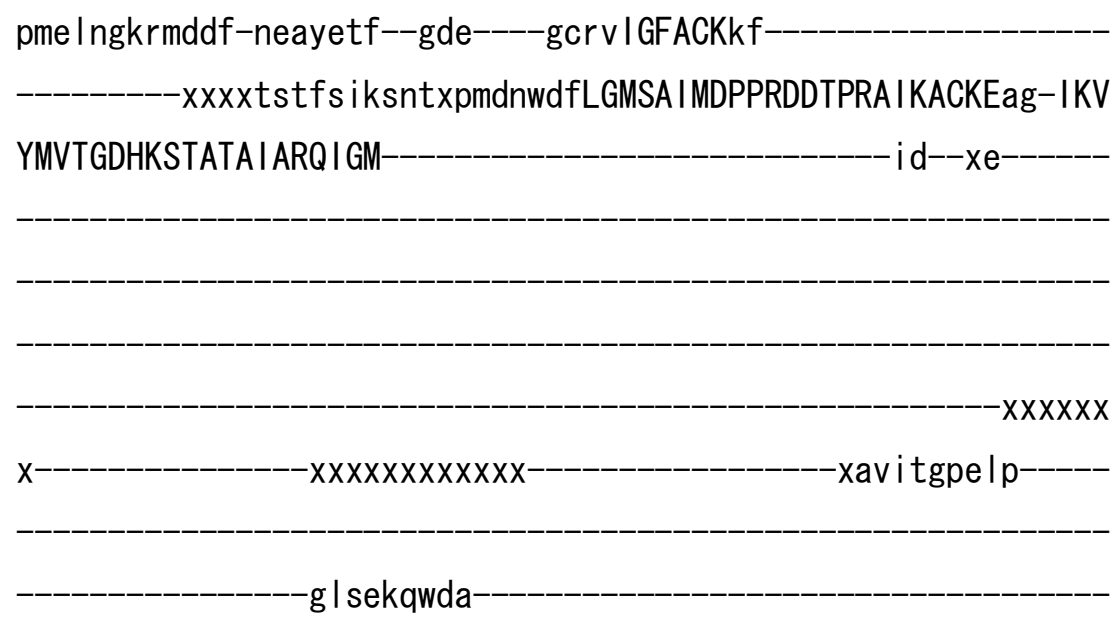

-g l sekqwda-

I I-ehxx iVFA--RTTPEHKLM IVTESq-

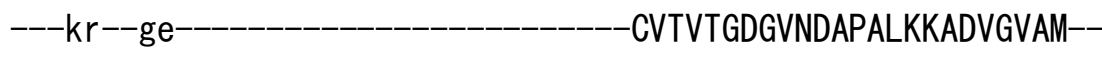

$-x$ lagsdv

-AKQAAD I ILLd-DNFSS IVag IEEGRLLFDNLRKT I------A

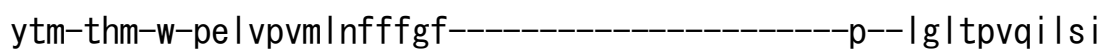

dl i td i ppavs l ayegpe-ad--_-_-_-_-_-_-_--_im l qppr----k-reth I vtkg I

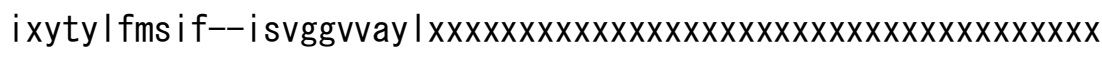
xeqqvfx

aa----qaaaafh i avvvgqawh-I wmc Itr--rvs i fx-------hgxan i va i lavi id Ill iclft--fvxxvqy--_-_-_-_---vfgsqpp--_-_-_-_-_-_-_-_-_-_-_-_-_-_

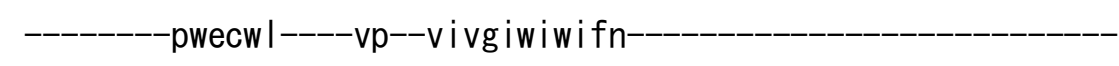

- elrkfg- 
W

>hsall_IIC02 NP_000693.1

$$
\text { m }
$$

dhk Is Ide Igr

kyq -vdlskg| tnqr

-aqdv I ardgpna I tppptt

-pewvkfcrq-I 


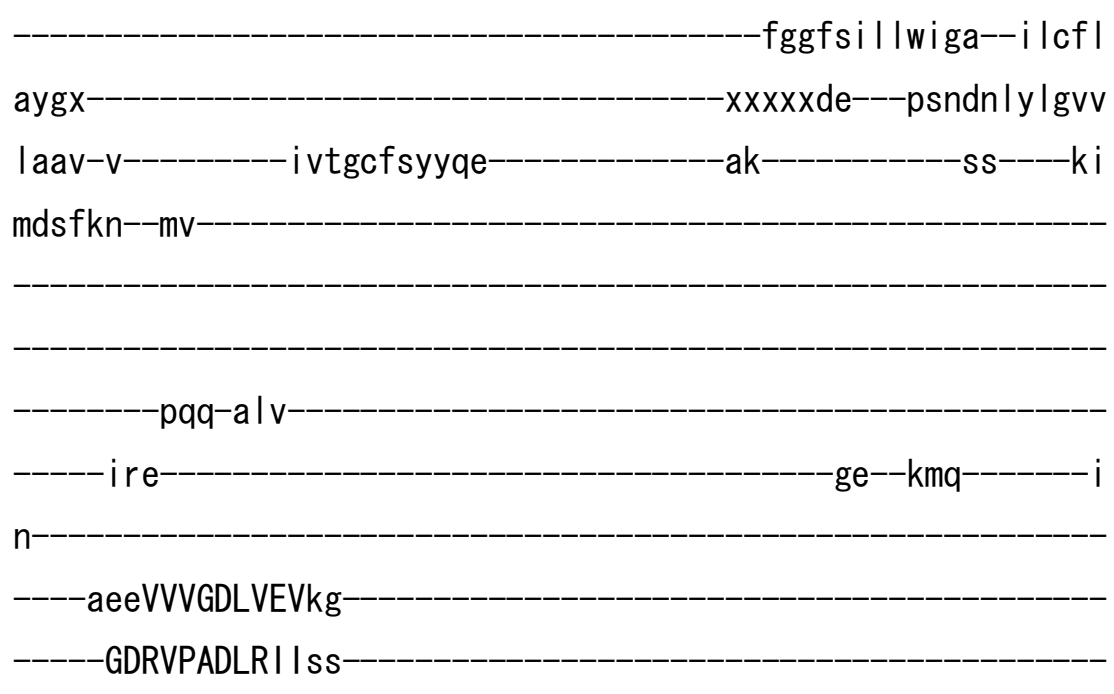

-h--gckVDNSSLTGESEPQTRsp-

$-\mathrm{XXXXXX}$

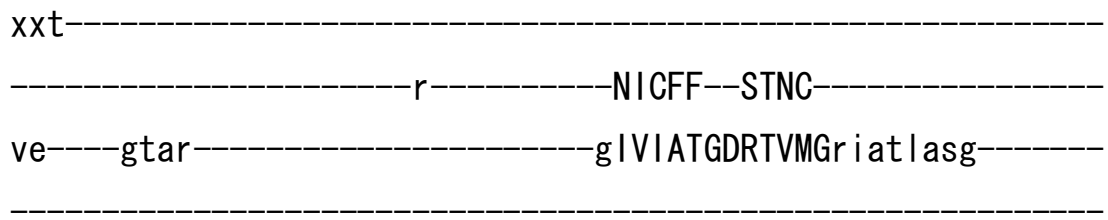

- levgr tp i ame i ehf iql i tgvavf Igvsff

$-v|s|$ ilgy-swleavif I IGIIVAN

VPEGLLATVTVCLTLTAK-RMarkn-

cLVK-NLEAVETLGSTST I CSDKTG

TLTQNRMTVa -hmwfdnqih-

$-x x x x x--e x x-$

xxxxxdkrsptwx

sriaglc-----nrav

$f---k x g q x x x x x x x x$ 
-dtagdase--sal lkc-

- ielscgs--vrkmxx-

rnpkvae ipfnstnkxqlsi

$\mathrm{XXXXXX}$

$-x X X X X X X--$

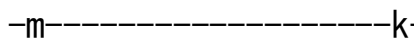

-gap

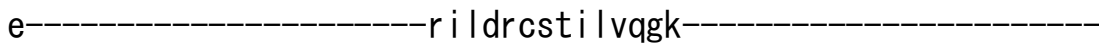

e i p I dkemqdaf-qnayme I--gg I----gervLGFCQLn I

xxxxxxxgfkfdtde Inxptek I cfVGLMSMIDPPRAAVPDAVGKCRSag-IKV

IMVTGDHPITAKAIAKGVGI-

isexxe-

$-x X X X X X$

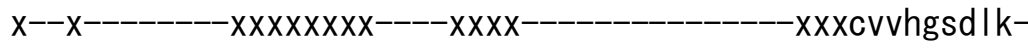

dmtseqlde-

i I knhxx i VFA--RTSPQQKL I IVEGCq ---rq--ga--_--_----_---_--_-------IVAVTGDGVNDSPALKKAD I G I AM-- 


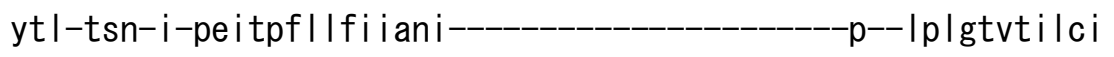

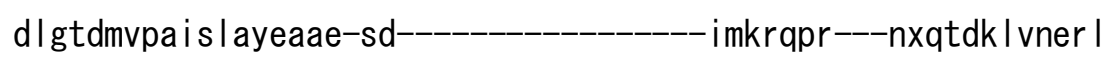
i xmaygqi gmi--qal ggfftyf $x x x x x x x x x x x x x x x x x x x x x x x x x x x x x x x x x x x x x x$

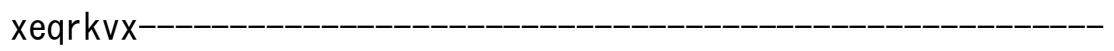
ef----tchtaffas i vvvqwad-I i icktrxxsvfqq-------gm-knk i I i fg I lee

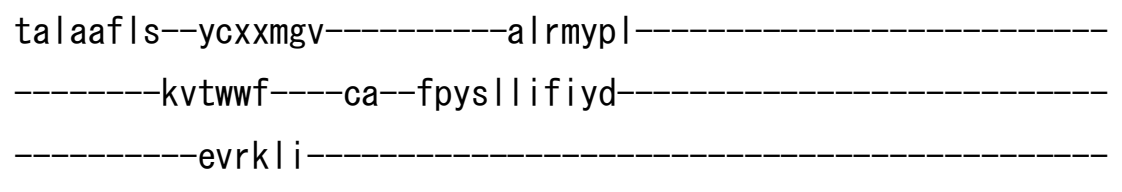
- Irrypx$-x x x x x x x$

y >hsall_IIC03 NP_689509.1

$\mathrm{m}-$ 


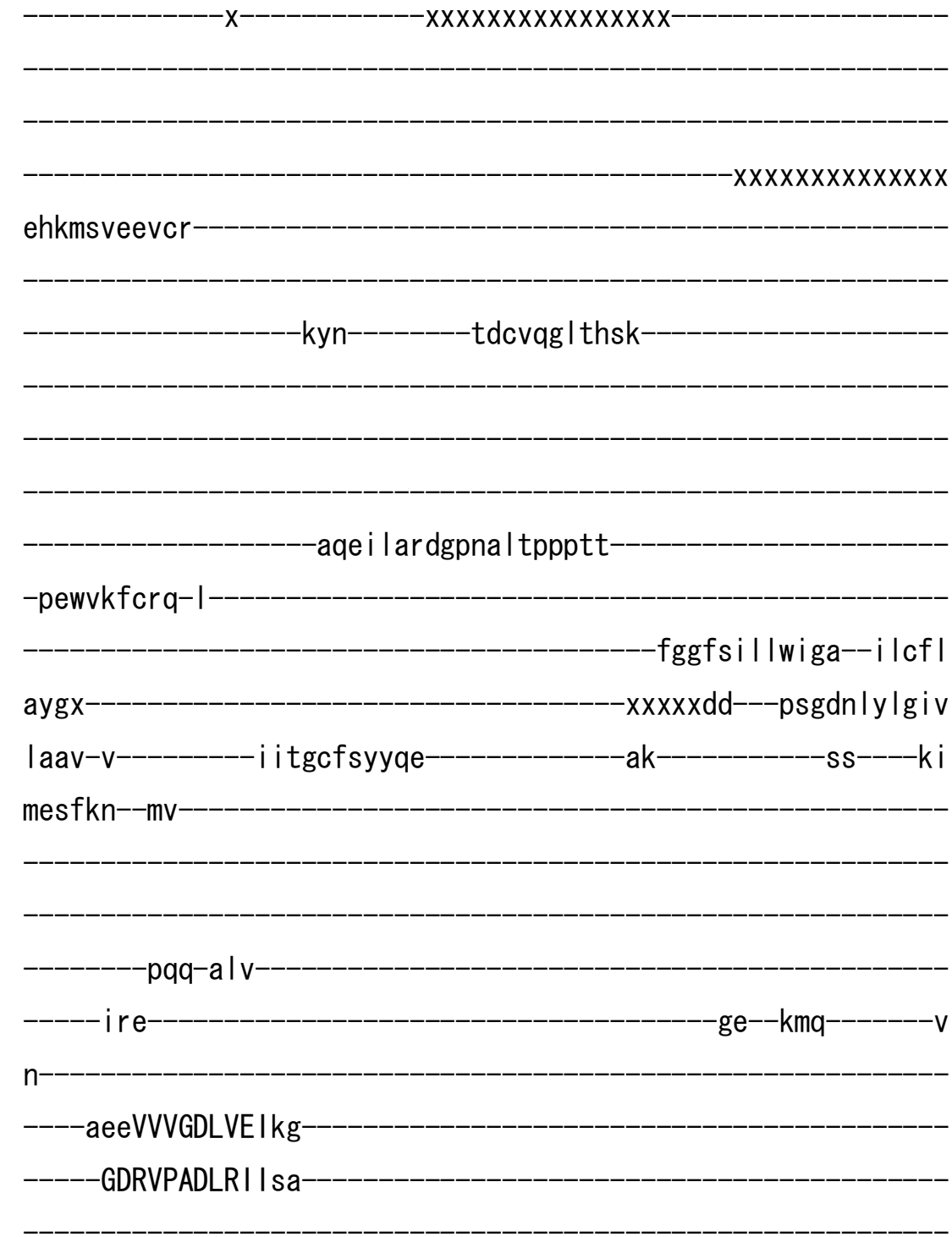

-h--gckVDNSSLTGESEPQTRsp-

$-x x x x x x x$

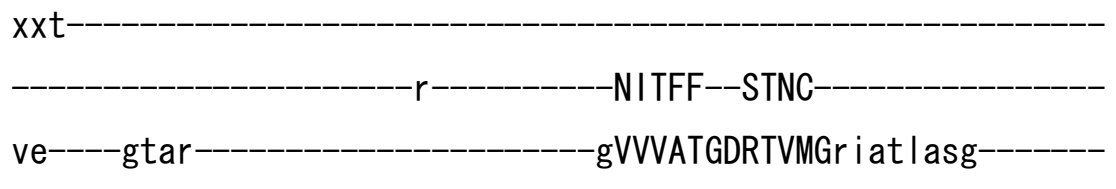

- levgktp i a i e i ehf iql i tgvavf Igvsff

-ilslilgy -twl eavifIIGIIVAN

VPEGLLATVTVCLTLTAK-RMarkn- 
cLVK-NLEAVETLGSTST ICSDKTG-

TLTQNRMTVa

-hmwfdnqih

$-x x x x x--e x x-$

xxxxxdksshtwx

$-a \mid$

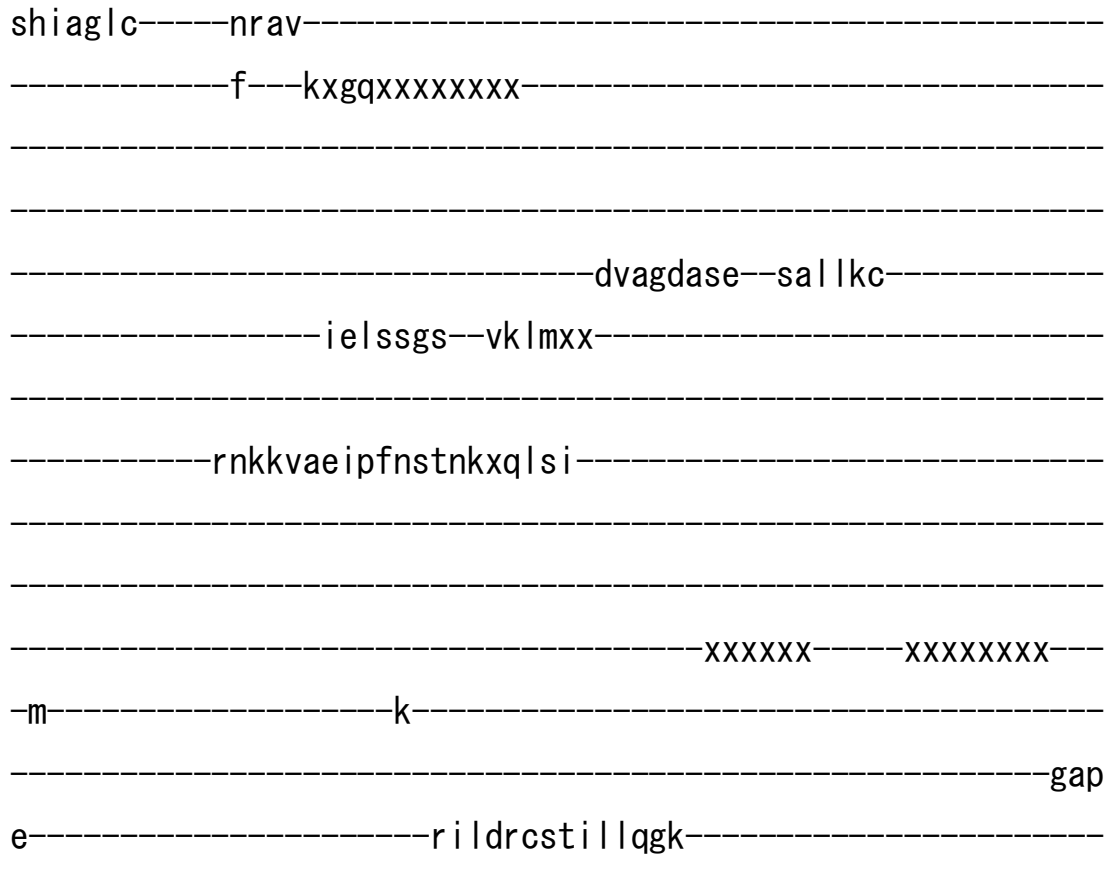

eqp I deemkeaf-qnay le I--gg|----gervLGFCHYy I

-xxxxxxxgfafdcddvnxttdn I cfVGLMSMIDPPRAAVPDAVGKCRSag-IKV

IMVTGDHPITAKAIAKGVGI

i sexxe

$-x x x x x x$

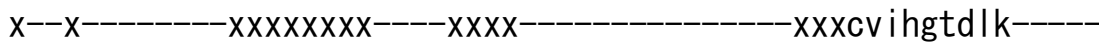

-dftseqide 
i I qnhxx iVFA--RTSPQQKL I IVEGCq

---rq--ga--_-_-_-_-_-_-_-_-_-_-_-_-IVAVTGDGVNDSPALKKAD I GVAM--

SKQAADM ILLd-DNFASIVtgVEEGRL IFDNLKKSI------A

yt I-tsn-i-peitpfIIfimani---_-_-_-_-_-_-_-_----p--Iplgtitilc $i$

d I gtdmvpa i s layeaae-sd--_-_-_-_-_-_-_-_imkrqpr---nxrtdk I vner I

i xmaygq i gmi--qal ggffsyf $x x x x x x x x x x x x x x x x x x x x x x x x x x x x x x x x x x x x x x x$ xeqrkvx

ef-----tchtaffvs i vvvqwad-I i icktrxxsvfqq-------gm-knk i I i fg I fee tal aaf I s--ycxxmdv---_--_----al rmyp I-_-_-_-_-_-_-_-_-_-_-_-_-_

---------kpswwf-----ca--fpysfl ifvyd-----------------------------

eirkli

Irrnpx

$-x x x x x x x$

y

>cellI_IIC04 NP_504328.1

$\mathrm{m}-$ 
$\operatorname{XXXXXXXX}$

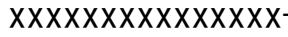

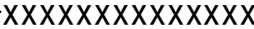

ehh Idmrql se

-kfsxxxxxxxxpkvsrg|skqv

aaer l esdgrna I sppkv i

-snwk I f Irq-f

-kn I I i Imfgaa--als Iv

ay ix

-dp---sdltnlcvg if

i ivi-i

fvmcivsffee

-kk---_-_-_----gv-----ev

vrafqs--Im-

$-p \mid s-c q v$

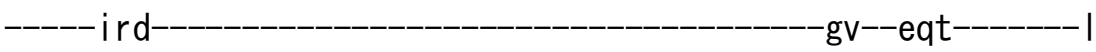

n-

----peeLVVGIVVVrs

GCKVPADIRV I ac 
t--dfyLETSS I TGEAEPLEFns-

$X X X X X X X X$

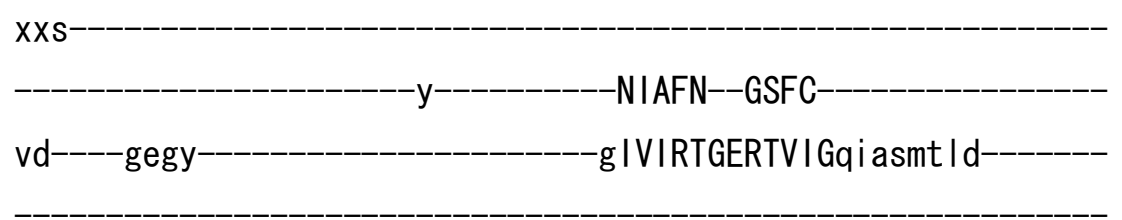

-qkeqkckfqve igrfvkf i tvma i imavi if -svg I g i nkxxx---------------------------------viryfvtgfLMVI I AN

VPQGLPTTVTTELTI I AR-RMakkn-

vFLK-KLEKIDSVGATTL I ASDKTG

TLTKNCMTVt

-d l wynnsyn

$-x x x x x x x r x x x$

xxxxxitsnpaqxxxxxxx-

$-d l$

I svvcve-----nkar

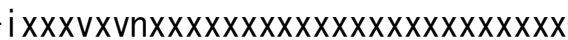

tivgnpse--val Iry

asamxxake Ixx

-sfqvifeipfnsvrkxhlil

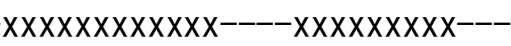

$-\mathrm{i}$

k

e

-vl ikncstmninge 
skeld lkrmedf-neayeaf--gde----gcrvIGFAQKkf -xxxxstvfs IksntxpmedwdfLGMSA IMDPPRDDTPKAIKACKAag-IKV YMVTGDHKSTATAIARQI GMid--xe------

$-X X X X X$

x--------------- $x \times x \times x \times x \times x \times x x------------------x a v$ i tgse $\mid \mathrm{p}------$

I I-qhxx iVFA--RTTPEHKLMIVTESq

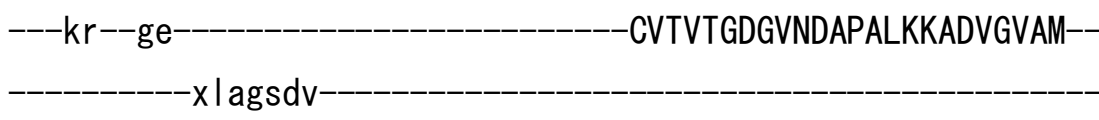

AKQAAD I ILLd-DNFSS IVag I EEGRLLFDNLRKT I------A

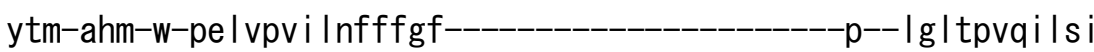

dl i sd i ppavs l ayegpe-ad-_-_-_-_-_-_-_-_-_-_ im l qppr---k-kdth I vtkg |

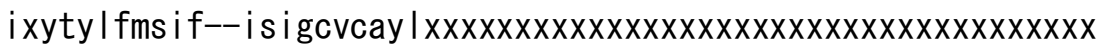
xeqqmyx

an-----qaaaafh i s I v Igqafh-I wmc Itr--rvs i fx-------hgxgn i va i favi id II licift--fvxxvky-----------ifgsqpp-

- pwqcwl----vp--ivvgvw i wi fn-

elrk Ig 
W

>hsalI_IIC04 NP_653300.1

$$
\mathrm{m}
$$

$-\mathrm{XXXXXXXXXXXXXXXXXXXXXXXXXXXXXXXXX------XX----}$

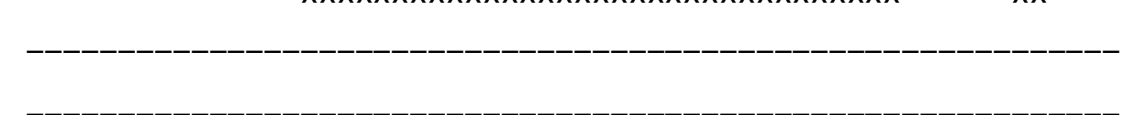

$\operatorname{xx} x x x x x x x x x x x x$

dhkItleelst

-kys---------vdltkghshqr

-ake i I trggpntvtppptt

-pewvkfckq-I 


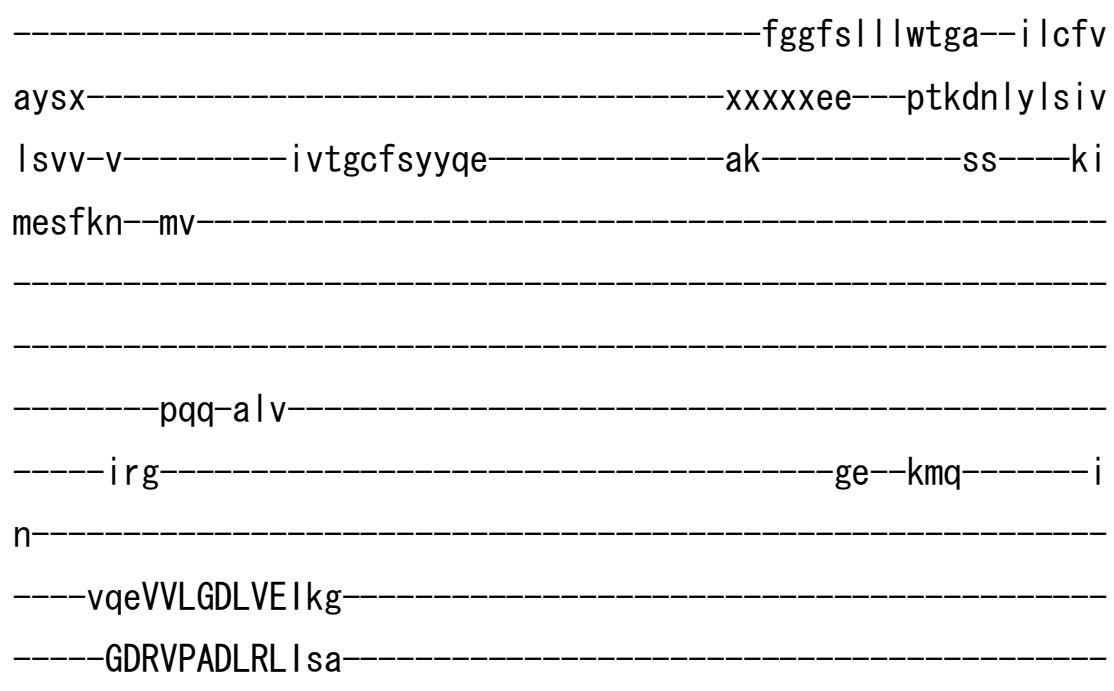

q--gckVDNSSLTGESEPQSRsp-

$-x x x x x x x$

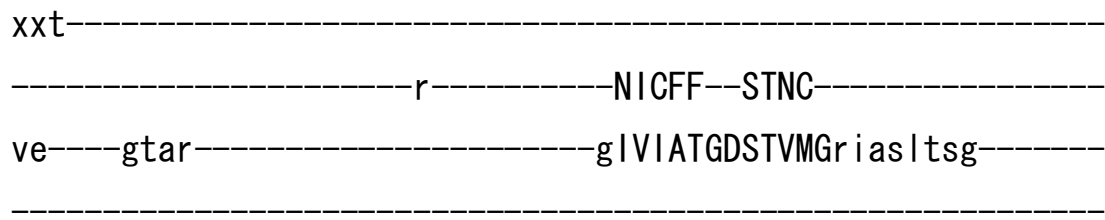

- lavgqtpiaae i ehfihl i tvvavf I gvtff -als I I gygwlea i f I IGIIVAN

VPEGLLATVTVCLTLTAK-RMarkn-

cLVK-NLEAVETLGSTST I CSDKTG

TLTQNRMTVa -hmwfdmtvy-

$-x x x x x--e x x-$

xxxxxtkssdtwx $-m \mid$ ar i ag |c-----nrad f---kxnqxxxxxxxx 
-attgdase--sal lkf-

i eqsyss--vaemxx

-knpkvae ipfnstnkxqms i

$\mathrm{XXXXXX}$

$-x \times x X X X X--$

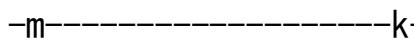

-gap

e

-rilefcstfIIngq

eysmndemkeaf-qnay | el--gg|----gervLGFCFLn I

xxxxxxgfpfntde inxpmdn I cfVGL I SMIDPPRAAVPDAVSKCRSag-IKV

IMVTGDHPITAKAIAKGVGI-

i sexxe-

$-x x x x x x$

x---x-------- $x x x x x x x x----x x x x--------------x x x$ i v vhgae $\mid k-$

diqskqldq-

i I qnhxx i VFA--RTSPQQKL I IVEGCq

---r|--ga-

-VVAVTGDGVNDSPALKKAD I G I AM--

xi sgsdv 
ytl-tsn-i-peitpflmfi i I gi

- --lplgtitilci

d I gtdmvpa i s layesae-sd--_-_-_-_-_-_-_-_imkr lpr---nxktdn I vnhr I

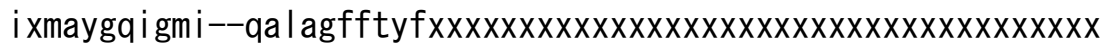
xeqrkvx-

ef----tcqtaffvtivvvqwad-li isktrxxs I fqq gm-rnkvlifgilee

tI laaf Is--ytxxmdv al rmyp |

$-k i$ tww -ca--ipysilifvyd

-eirkll

$\mathrm{y}$

>hsal I_IIC05 NP_001667. 4

m- 
$X X X X X X X X X X X X$

dhk Isnrelee

$-k y g$ -tdi imglsstr

aae I l ardgpns I tppkqt

-pe ivkflkq-m-

-vggfsi I lwvga--fl cw i

aygx

-xxxxxks---as Innvy Igcv

$|\mathrm{g}| \mathrm{v}-\mathrm{v}-$

i Itg i fayyqe

$-a k-$

st----ni

mssfnk--mi

q--gcrVDNSSLTGESEPQPRss-

$-p q q-a \mid v$

ird

se--kkt $-\mathrm{i}$

$\mathrm{p}$

----seqLVVGD IVEVkg

GDQIPAD IRVLss

$-x x x x x x x$

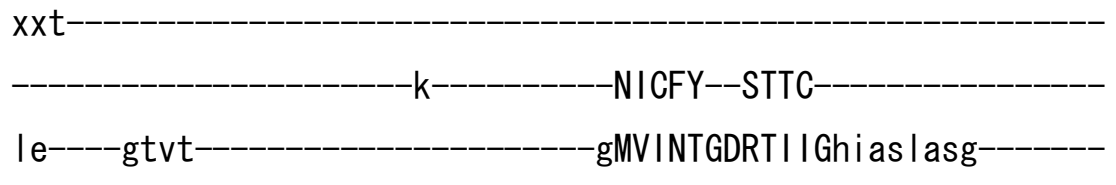

-vgnektp i a i e i ehfvhi vagvavs i g I Iff

-i iavs Iky qvIds i ifI IGIIVAN

VPEGLLATVTVTLSLTAK-RMakkn- 
cLVK-NLEAVETLGSTSI I CSDKTG-

TLTQNRMTVa

-hlwfdnqif

$-x x x x x--e x x-$

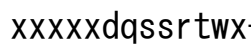

$-s \mid$

ski itlc-----nrae-

f--- kxgqxxxxxxxx

avigdase--tal lkf

sev i I gd--vme ixx

-rnrkvae ipfnstnkxqlsi

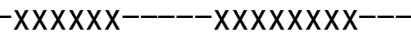

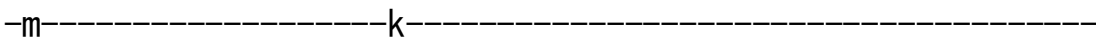

-gap

$\mathrm{e}$

-ri lekcstiminge

ehp I dkstaktf-htayme I--gg|----gervLGFCHLy I

-xxxxxxxtysfdi damnxptsn I cfVGLLSMIDPPRSTVPDAVTKCRSag-IKV

IMVTGDHPITAKAIAKSVGI

i saxxe

$-x x x x x x$

x---x--------- $x x x x x x x x-----x x x x----------------x x x a v v$ tgme $\mid k-----$

dmsseq I de 
i l anyxx i VFA--RTSPQQKL I IVEGCq

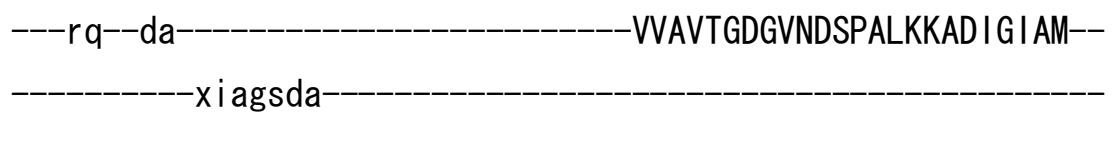

-AKNAADMVLLd-DNFAS IVtgVEEGRL IFDNLKKTI------A

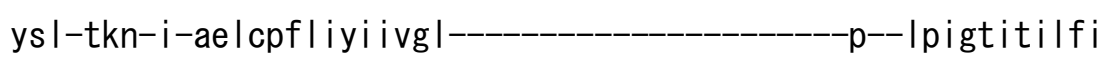
dlgtdi i ps i a layekae-sd-_-_-_-_-_-_-_-_imnrkpr---hxnkdr I vnqp I

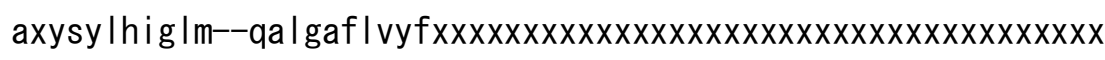
xyareyx

ew----tgytaffvg i I vqqi ad-I i irktrxxs i fqq--------gl xrnkvi wvg itsq i i igl i ls--ygxxsvt-_-_-_-_----al sftml-_-_-_-_-_-_-_-_-_-_-_-_-_-_-_ ---------raqywf-----va--vphai I i wvyd----------------------------evrklfir lypx$-x x x x x x x x-$

$\mathrm{y}$ >hsal I_IIC06 NP_000695. 2

$\mathrm{m}-$ 
dhql svaeleq

$-k y q$ -tsatkg | sas |

-aae I I I dgpna I rpprgt

-peyvkfarq-I

agg l qc Imwvaa--a ic l i

afax-xxxxxd |---ttddn I y la i a

l iav-v -fk-----_-----st----ni iasfkn--Iv

-pqq-atv

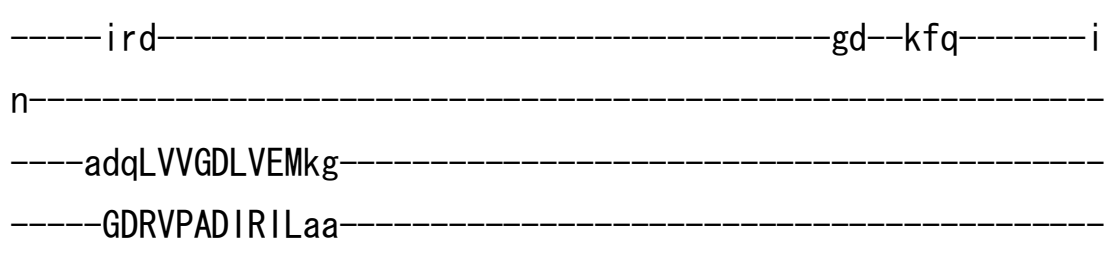


q--gckVDNSSLTGESEPQTRsp-

$-X X X X X X X$

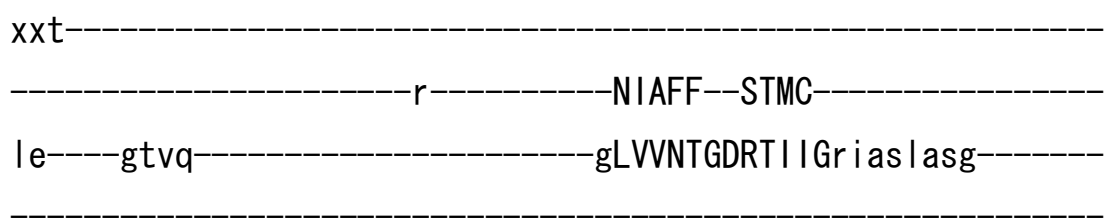

-venektp i a i e i ehfvdi i ag la i I fgatff

- i vamc i gy-

-tfI ramvffMAIVVAY

VPEGLLATvTVCLSLTAK-RLaskn-

cVVK-NLEAVETLGSTSV I CSDKTG

TLTQNRMTVs

-hIwfdnhih

$-x x x x x--e x x-$

$x x x x x d q s s e t w x$

-al

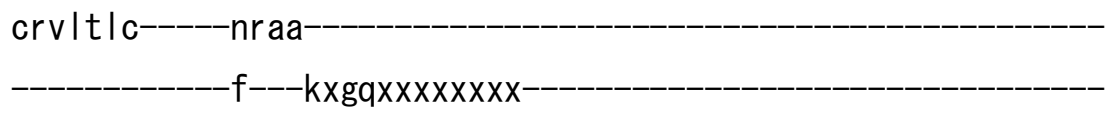

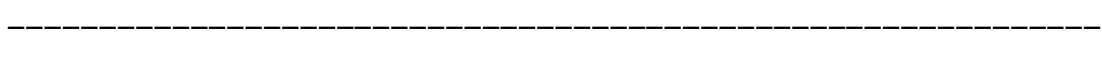

i vigdase--tal Ikf

selt I gn--amgyxx

-rfpkvce ipfnstnkxqls i

$-\mathrm{x} x \times x \times x-----x \times x \times x \times x x---$

$-m$

$-k$

e-

-rvlercssilikga 
e $|p|$ deqwreaf-qtay $\mid$ s|--gg |----gervLGFCQLy |

-----xxxxxxxgyafdveamnxpssg I cfAGLVSMIDPPRATVPDAVLKCRTag-IRV IMVTGDHPITAKAIAASVGI

i sexxe-

$-X X X X X X$

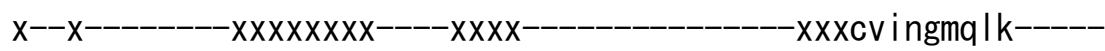

dmdpse I ve-

al r thxxmVFA--RTSPQQKLVIVESCq

$---r \mid--g a-$

IVAVTGDGVNDSPALKKADIGVAM--

-xiagsda-

AKNAADM I LLd-DNFAS IVtgVEQGRL IFDNLKKS I

A

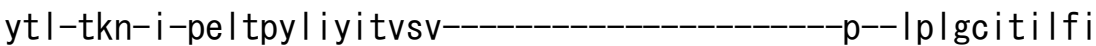

elctd ifpsvs l ayekae-sd-_-_-_-_-_-_-_-_-_imh | rpr---nxkrdr I vnep I

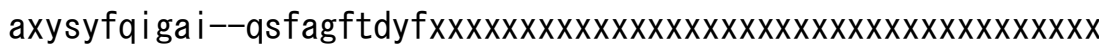
xgarlyx

qy----tcytvff i s i evcqiad-vl irktrxxsafqq--------gfxrnk i Ivi a ivfq vcigcflac--ycxxmpn-----------ifnfmp i

--------rfqwwl----vp--Ipyg i I ifvyd

eirklg 
y

>cnelI_IID01 XP_566813.1

$$
\text { m- }
$$

$-x X X X X X X X X X X X X X X X X X X$

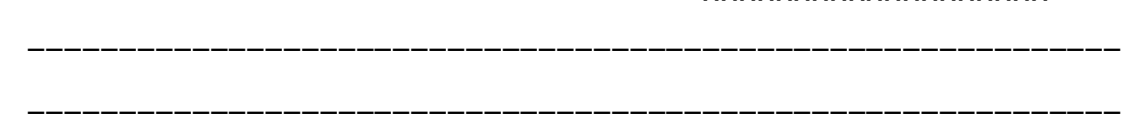

$X X X X X X X X X X X X X X$

phtalsgkile

-al g--------sdaasg | sdee-

-vsr r l qqygpnr I kppkrp

-si lki iarq-v 
-gnamt I i l i aam--ats I-

-gtmdw i sggv

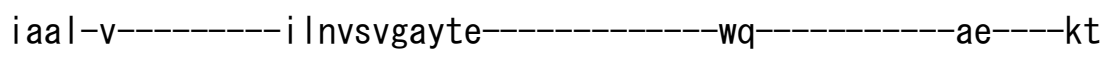

vasles--vg-

$-a p q-a t v$

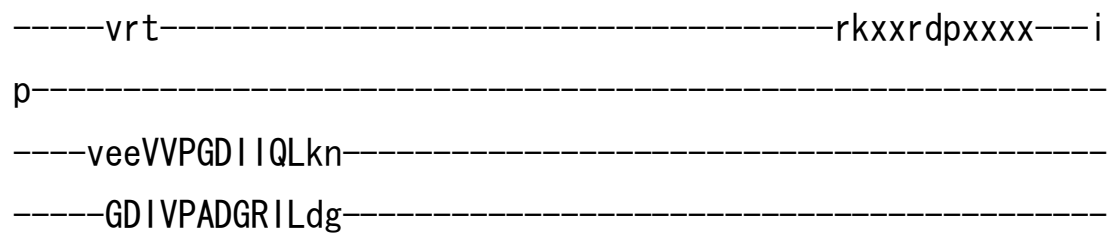

-hxxn l eADEAFLTGESLPVAKqt-

$-x x x x x x x x x x$

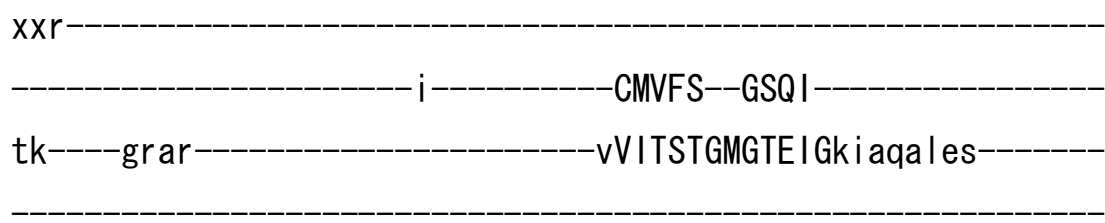

-xxxxxxxxxxxxxxxxxxxxgveettp I qmk Ink I ayf I laca I v i av i vv

-astgfnd--_-_-_-_-_-_-_-_-_-_-_-_-_-_-_-_-_-_--vp I s i atyaVAAAVS I

LPASL IAVvSLTLARAST-DLasrh-

-aLVR-RMDAIEALAGVENVCSDKTG

TLTVGRMVVr kvwvpaldw-

$-x x x x x x x \mid x x x$

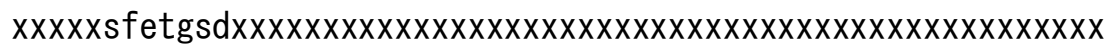

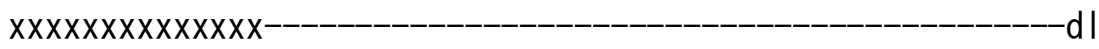

alcislc-----nqat

- Ixxxvxqdxxx 
-eangdpte--tal qva----xxxxxxxx

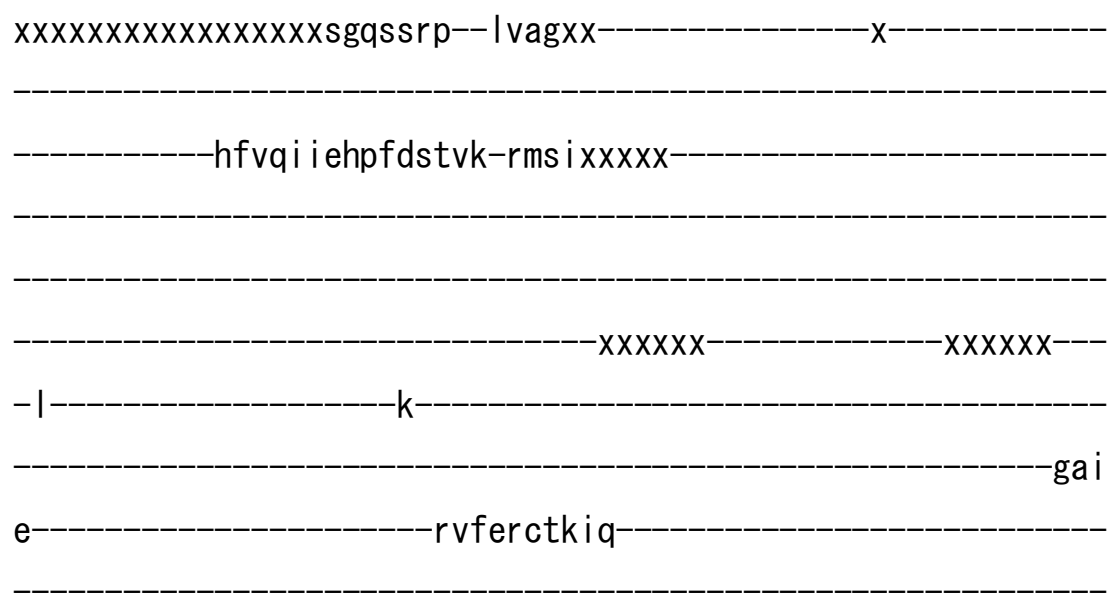

gqp i teehkkdi -mi kvdal---aaq----g | rvLALCGKr I

-xxxxvdevkstprdxfeadfhfLGLAGIFDPPRKESAGAVADCFRag-ITP

RMLTGDHPATATAIALNIGI-

$-1$

xxxxxxxsvmtgqqfd

s I sede idq

Ipe Ip--IVVA--RCAPETKVRMVDAIh

---rr--gq---_-_-_-_-_-_-_-_-_-_---STVMTGDGVNDSPALKRADVGVGM--

gtgsdv-

-AKQSAR I VLSd-DNFST I I ra I RKGRSVFKNLSKFL

$-\mathrm{L}$ 
V

>anilI_IID01 EAA64748.1

$\mathrm{m}$ 
$X X X X X X X X X X X X X X X X X X X X X$

ayc $|p f d v v| k$

-elg---------tnvdegl tkde-

aar r lqqygpnq I degegv

- svvki

-Ivlilam--avsf-

giqswi eggv

i sav-i----------i In ivvgffqe--------------ya------------ae-----kt

meslhs--Is

- -- -nfeTDEALLTGESLPVQKec-

$-x x x x x x x x x x$

$\mathrm{xxr}$

1

-NLAYS--SSTV-

tr----grar

gVVVNTGMATE I Gs i aaa I ra

$x x x x x x x x$

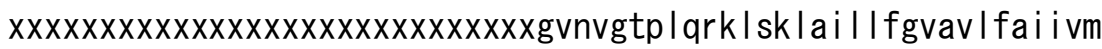
-aanlfs -ndnevi l yaVGTGLSM

IPACLVVVITITMAVGTK-RMvern- 
v IVR-KLDSLEALGAVTN I CSDKTG-

TLTQGKMVVk-

-kawipsrgt

$x x x x x x x x x x n x x x$

$x x x x x$ tp $\mid$ spvdxxxxxxxxxxxxxxxxxxxxxxxxxxx

$-d f$

Idvasma-----nl sh

$-v x x x \operatorname{exg} d x$

-rargepte--ia i evf-----xxxxxxxx

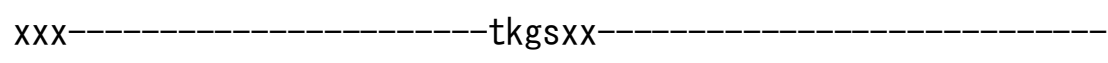

-vwhqkaefpfdstvk-rmsvxxxxx

$-x \times x x x x x x x x---$

-t-------------------k

-gav

$\mathrm{e}$

- r i vdacttavwdnsxx

pvalndemr sqi-| qnmee |--ak |----g| rvLALAHRpy

-xxxxxt l egsd Inrdx i ekn I cfLGL IGLYDPPRPETAGSI TACYKag-I IV

HMVTGDHPGTAKAIAQQVGI

Ipaxxs-

$-x x x x x x x x x m v m t a a q f$

r I sdee idd- 
Ipt I p--IVIA--RCAPQTKVRMINALh-

$---r r--g r$

FAAMTGDGVNDSPSLKHADVGI AM--

-xqagsdv

AKDASD I ILTd-DNFAS ILnaVEEGRR IFDN I QKFV

$-\mathrm{L}$

hI I-sen-i-aqact I I ig l afkdxxx--_-_-_-_-_-_-_-_xxfp I apve i I w imi tsgmpdmg I gmevaa-pd--_--_-_-_-_-_-_-_-_imdrppq------skqg i f twe i

i-vdi I vygfw--taal c lsafsxxxxxxx---_-_-_-_-_-_-_-_-_-----xxxxx

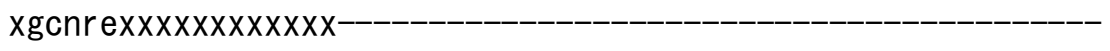

atxxxx | twfalf lawemvn|r|-sffrmqpxxkkyftxxxx---dvxrnkf I fws imag

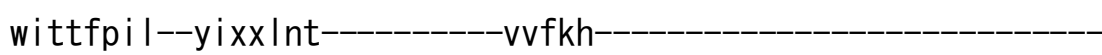

---------tg i swe-----wg--i i fveaa I ffax--------------------------

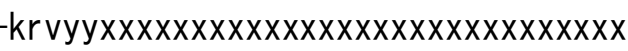

$x x x x x x x x x x x x x x$

g

>anilI_IID02 EAA58171.1

$\mathrm{m}-$ 
aht Ispde i Ir

-elk--------vnseeg|taae-

akkr le I fgpne l eggegv

-slaki i irq-i

-anamm I v l i i am--avsf-

gi eswi eggv

igav-i----------glnivvgvyqd--------------ya------------ae-----kt

mds $|r n--| s-$

spt-gva

--_---trd--_-_-_-_-_-_-_-_-_-_-_-_-_-_-_-_-_---gk--tnt--_-_-_- $\mathrm{i}$

$\mathrm{p}$

----ate IVPGDMIELkv

GDTVPADVRLVda 
-m--nfeTDEALLTGESLPVQKev-

$x x x x x x x x x x x x x$

-NIAYS--SSTV-

tr----grargVVIGTGMKTE I Ga i aaa Iha-

$-x x x x x x x x$

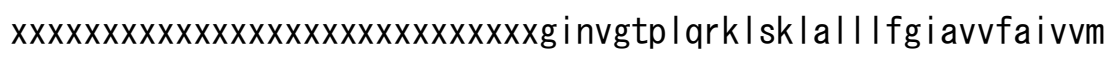
-ganemr -ndkev i i yaVATGLAM

IPACLVVVITITMAVGTK-QMverh-

-v IVR-RLDSLEALGAVTN I CSDKTG

TLTQGKMVAK

-rawips I gt

$-x x x x x x x x x x d x x x$

$x x x x x \mid p d p p v k x x x x x x x x x x x x x x-x x x x x x x x x$

dy

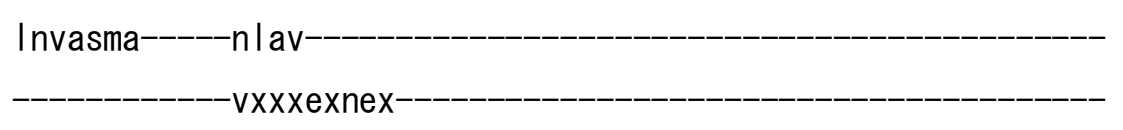

qargeptd--ia i qvf-----xxxxxxxx xxx--_-_-_-_-_-_-_-_-_-_-_-_tkgexx--_-_-_-_-_-_-_-_-_-_-_-_-_-_ -iwrqkaeypfdstvk-kmsvxxxxx

$-x x x x x x x x x x x---$

$-t$ $-\mathrm{k}$ e -rvidsctti I wtrnxx- 
p ipmsed iksqi-l qnmeal--ake----g | rvLCLASRef

-xxxxanseevpprexvekd I vfCGLVGLYDPPRPETAGAIEECYRag-ISV

HMVTGDHPGTARA I AAQVGI

ipaxxd-

$x x x x x x x x x m v m t a s q f d$

$k \mid t d e e i d d$

Ipt | p--IVIA--RCAPTTKVRMIDALh

$---r r--g r$

YAAMTGDGVNDSPSLKRADVG I AM--

xeagsdv

-AKDASELVLTd-DNFASI Ing I EEGRR IFDN I QKFV $-\mathrm{L}$

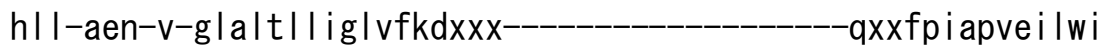
im i tsgapdmg I gmevaa-pd-------_--_--------vmdrppq------skqg i f twe i

i-vdm I vygvw--maal c l tafsxxxxxxx--_-_-_-_-_-_-_-_-_-_---- $x x x x x$ xgcnanxxxxxxxxxxxx--_-_-_-_-_-_-_-_-_-_-_-_-_-_-_-_-_-_-_-_-_-_-_ atxxxxmtwfalf l aweminmrr-sffrmqpxxkkyftxxxx---dvxrnkf I fcgvm Ig

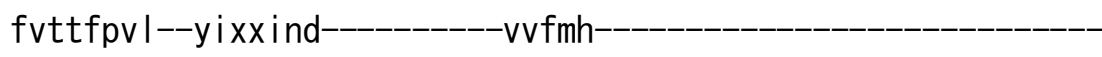

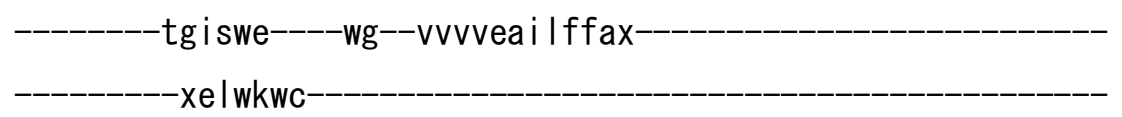

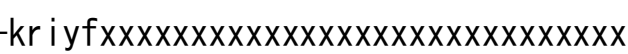


V

>anilI_IID03 EAA61850.1

$$
\text { m }
$$

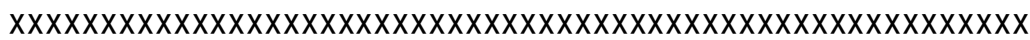

$x x x x x x x x x x x x x x x x x x x x x x x x x x x x x x x x x x x x x x x x x x x x x x x x x x x x x x x x--$

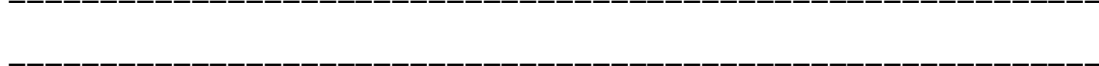

$x x x x x x x x x x x$

ahilspsslsa

$-11 k$

-vd l qhg I snee-

-assr lardgpnrvremeg I

-svwk i I Irq-v 
sns It l i lvivm--gvsf-

gindy i eggv

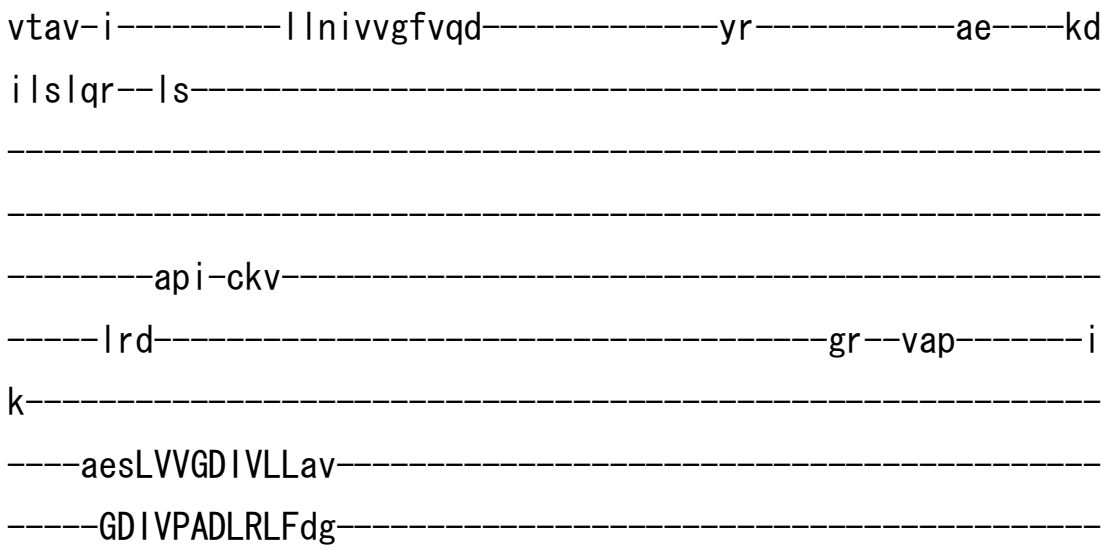

-m--nasMDEALLTGESLPVAKtp-

$-x x x x x x x x x x x$

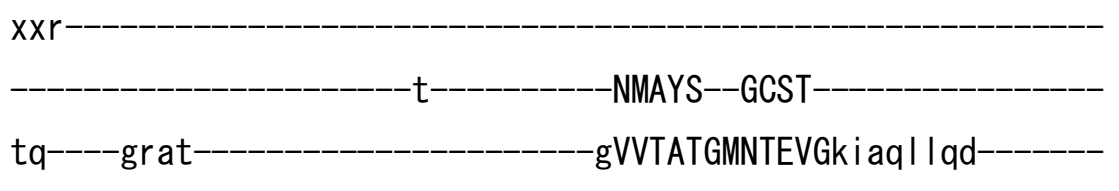

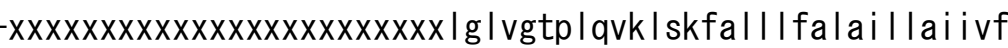

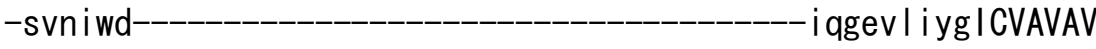
VPESL I AVITITVAVGTK-AMakgn-

- IVR-KLQCLEAVGGVTNICSDKTG

TLTQGKMVAr tawipdtgi

$x x x x x x x x x x x x x x x x x x x x x x--e x x x$

xxxxxdasn-

$-\mathrm{tf}$

itals|c-----nlst

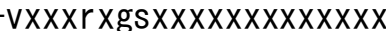


-tavgepte--val rvf----xxxxxxxx

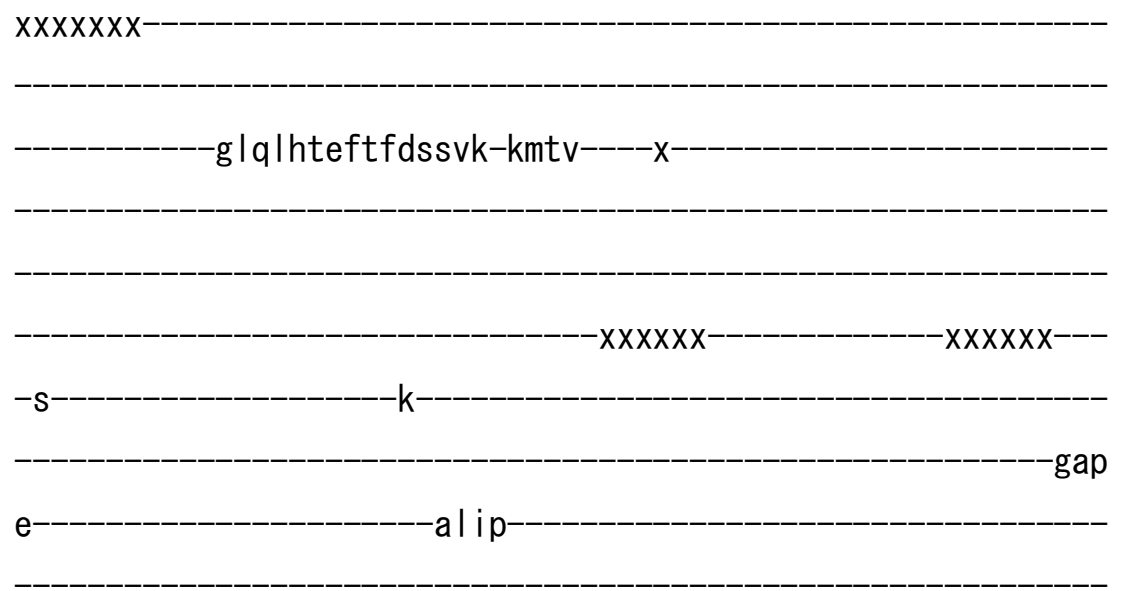

sIdi i deekevi-qdtaer I--age----g | rvLCIAYKks

-xxddesq i ssrnxaesn I rfAGLIGLYDPPRLETAAAVRKCQMag-ITV

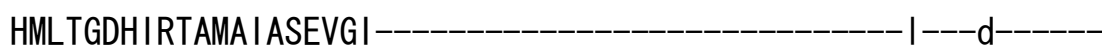

$-x x x x x x x x x \mid$ vmtakefd

r lsdadidk

i eq Ip--IVIA--RCSPTTKVRMVEAMh-

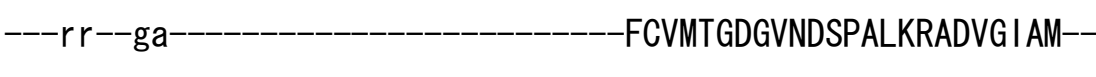
-xkngsdv 


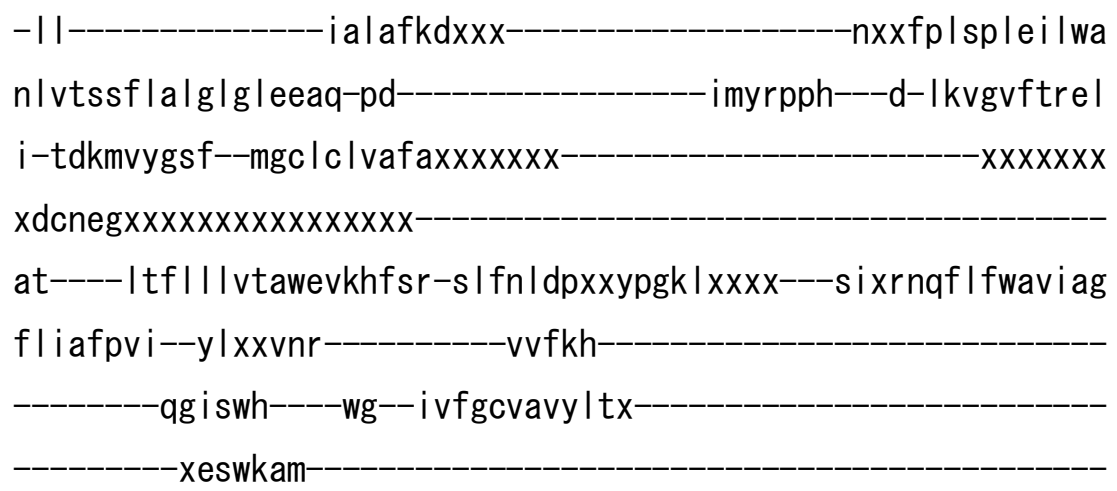


$\mathrm{XXXXXXXX}$

\section{pfllpteevaa}

$-\mathrm{t} \ln$ tdvdkg|tsaq

-vqk I qeeyppne I dagga i

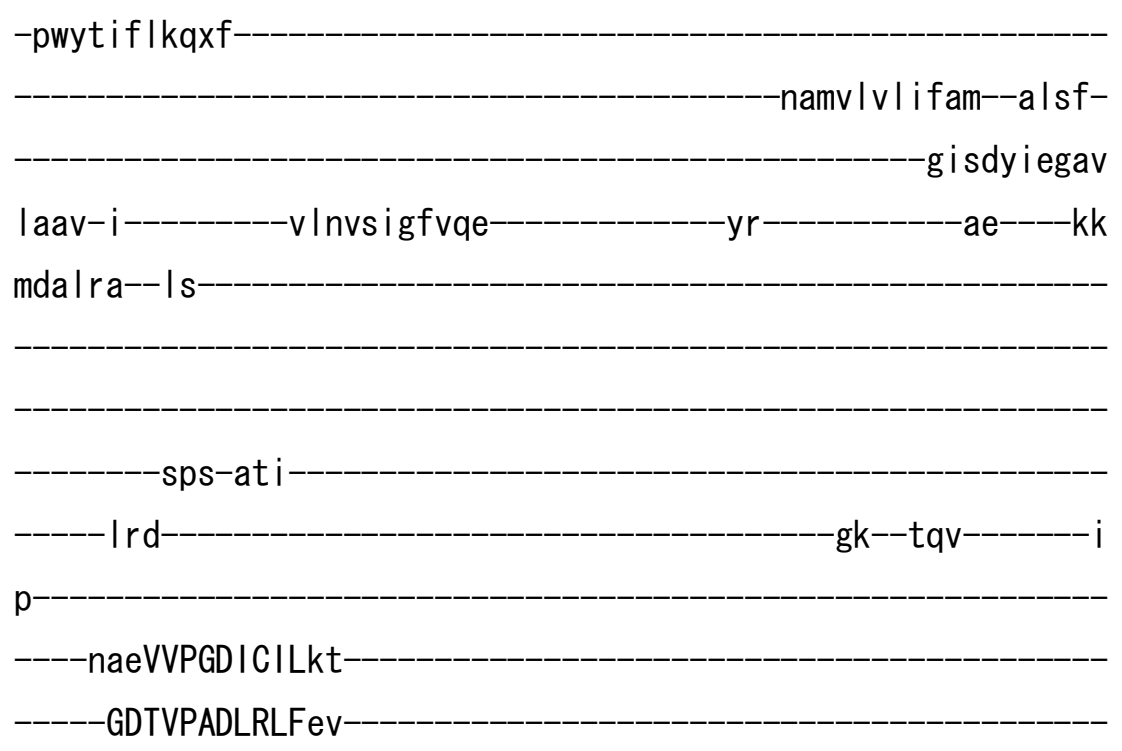

-m--n I sCDEQSLTGESMPVDK i v-

$-X X X X X X X X X X X X X X$

$x x x x x x$

xxr

$$
-\mathrm{V}
$$$$
\text { -NIAYA--TTVV }
$$

rk----grgr

g I VVETGMQTEVGk i aastrk

$x x x x x$

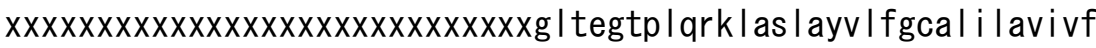
-gvnrfq -isheiviyaISTGIAI

IPESLVAVITITMVQATR-VMrkan- 
vVVR-DLSALEALGGVTN I CSDKTG-

TLTQGAM IVk-

$-k v w \mid$ patr i

$-x x x x x x x x x x d x x x$

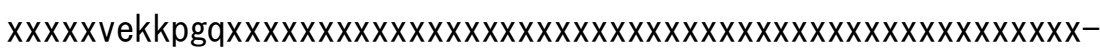

$-m f$

I lagalc-----nlat-

$-v x x x e x k q x x$

qttgepte--ialqvf-----xxxxxxxx

$-k v l e x x$

-gwkqvsefpfdstik-rmsvxxxxx

$\mathrm{XXXXXXXXXXXXX---}$

$-\mathrm{t}$

$-k$

gav

$e^{-}$

- r i idl csh l gqgdd

I qemndaaket i-l eqmndf--asq----gqrvLAIAYRkw

$-\mathrm{XxXxx}$

$x x x x x x x x x x x x x p e k s d e n v r s x i$ esg I I ILGLVGIYDPPRKETTPAIRECSSag-IKV HMLTGDHPATAAA I AKEVGI Ipfxxg

$-x x x x x x x x x a v q k a t d f d$

$-k m t d a e i d a$ 
Ipe Ip--IVIA--RCAPDTKTRMIEALr

$---r r--s a$

-FMAMTGDGVNDAPSLSNADVG I AM--

gsgsdv

-AKSASK IVLTd-DKFNS IVaa I REGRRMFEN I QKFV

$-\mathrm{L}$

hII-tsn-v-gevi I I iaglgfadxxx-_-_-_-_-_-_-_-_-_xxfp i sp I q i I wi

nmltssfpafg I grekan-ae-_-_-_-_-_-_-_-_-_-_imrkpph---s-tkrgvftnq i

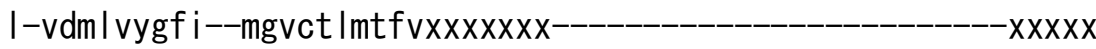

$x d e n t t x x x x x x x x x x x x x x x x$

ae----| tw I i I isawefks Irr-smfr Inpxxtskfpxxx----dixenrfI fwsvvia

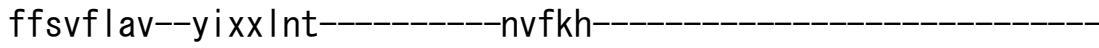

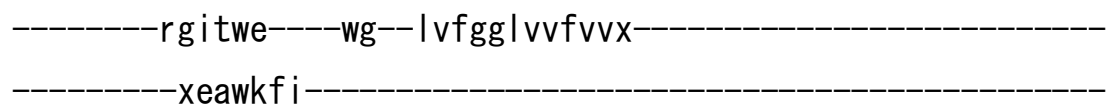

$-k r k f n x x x x x x x x x x x x x x x x x x x x x x x x x x x x x x$

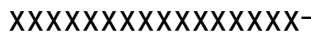

$\mathrm{XXXXX}$

d

>mgr II_IID02 XP_360418.1

$\mathrm{m}-$ 
ahal eteqvie

elq--------anpedg | tpae

akkr leefgrnefgeqegv

-kpwni l iaq-i

anamt I v I i lam--aasf-

gi es i i egcv

vaav-i---------- I Initvgfqqe-------------- Ik------------aa----kt

mds Irs-- Is-

spt-aqa

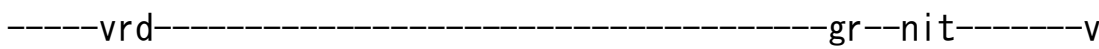

$\mathrm{p}$

----tve IVPGDMVELkt

GDTIPADVRI I ea 
-v--nfeTNEALLTGESLPVRKea-

$X X X X X X X X X$

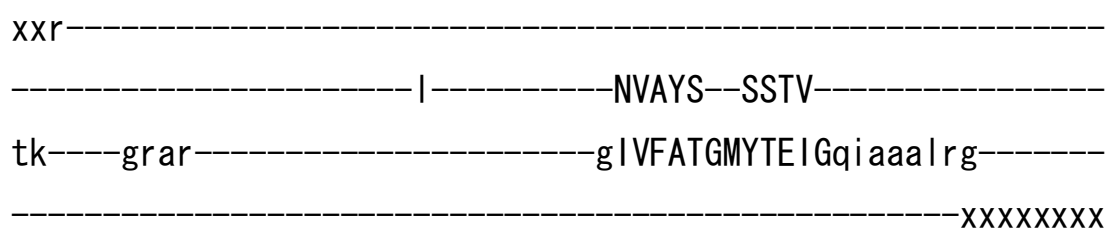

$x x x x x x x x x x x x x x x x x x x x x x x x x x x x x$ gvnvgtp |qrklaklavv|fgta i ica i i l -gandfd tsqevvi yaVATGVAM

LPASLVVVITITMAAGTK-RMvqrh-

-v IVR-KLKSLEALGGVTN ICSDKTG

$-T L T Q G N M I V r$

-kaw ipghgt

$-x x x x x x x x x x n x x x$

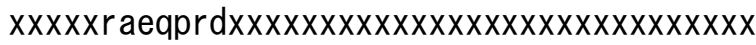

dy

Inlasla-----nlat

$-v x x x k x g e x$

-hargdpte--ia iqvf-----xxxxxxxx

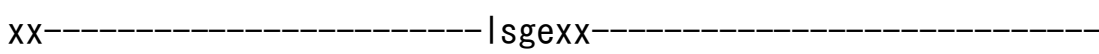

rwnqvaefpfdsdvk-kmsvxxxxx

$-x x x x x x x x---$

$-t$

$-k$

gat

e

-rv i pscrk i qmgde 
i ipftdeherdi-tanlevl--ak|----g|rvLSLASKrn-

-xxxvdnhdn I drkxfehe I vfQGL I G I YDPPRPESAPSVKKFHQag-VSV

HMLTGDHPETARA IALEVGI

Ipsxxg------

$-x x x x x x x x x m v m a a s d f d$

kl sddd idr

Ipr $|p--|$ VVA--RCAPQTKVRMIEALh

$---r r--n r$

FVAMTGDGVNDSPSLKRADVG I AM--

-xesgsdv

-AKEASD I ILTd-DNFASILnaVEEGRRMFDN I QKFV

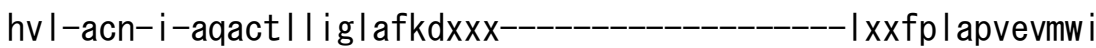
imi tsg I pdma I gfe i aa-pd----_-_-_-_-_-_-_---vmdrpph----d-mkag i ftwe I

v-admfayg | w--taa I c laaf I xxxxxxx--_-_-_-_-_-_-_-_-_-_-- xxxxx xnennsxxxxxxxxxxxx

atxxxx | twfalf I awem |hmrr-sffrmqpxxtky | txxxx---dvxrnk I Iffsivag fvtifpll--yixxint-----------vvfkh-

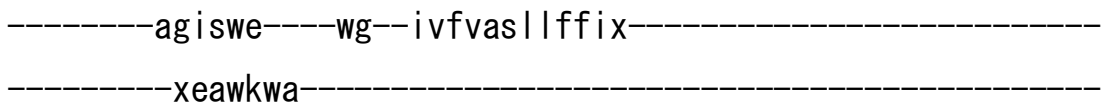

krvwi $x x x x x x x x x x x x x x x x x x x x x x x x x x x x x x$

$X X X X X X X X X X X X X X-X X X X X X X X X X X X X X X X X$ 


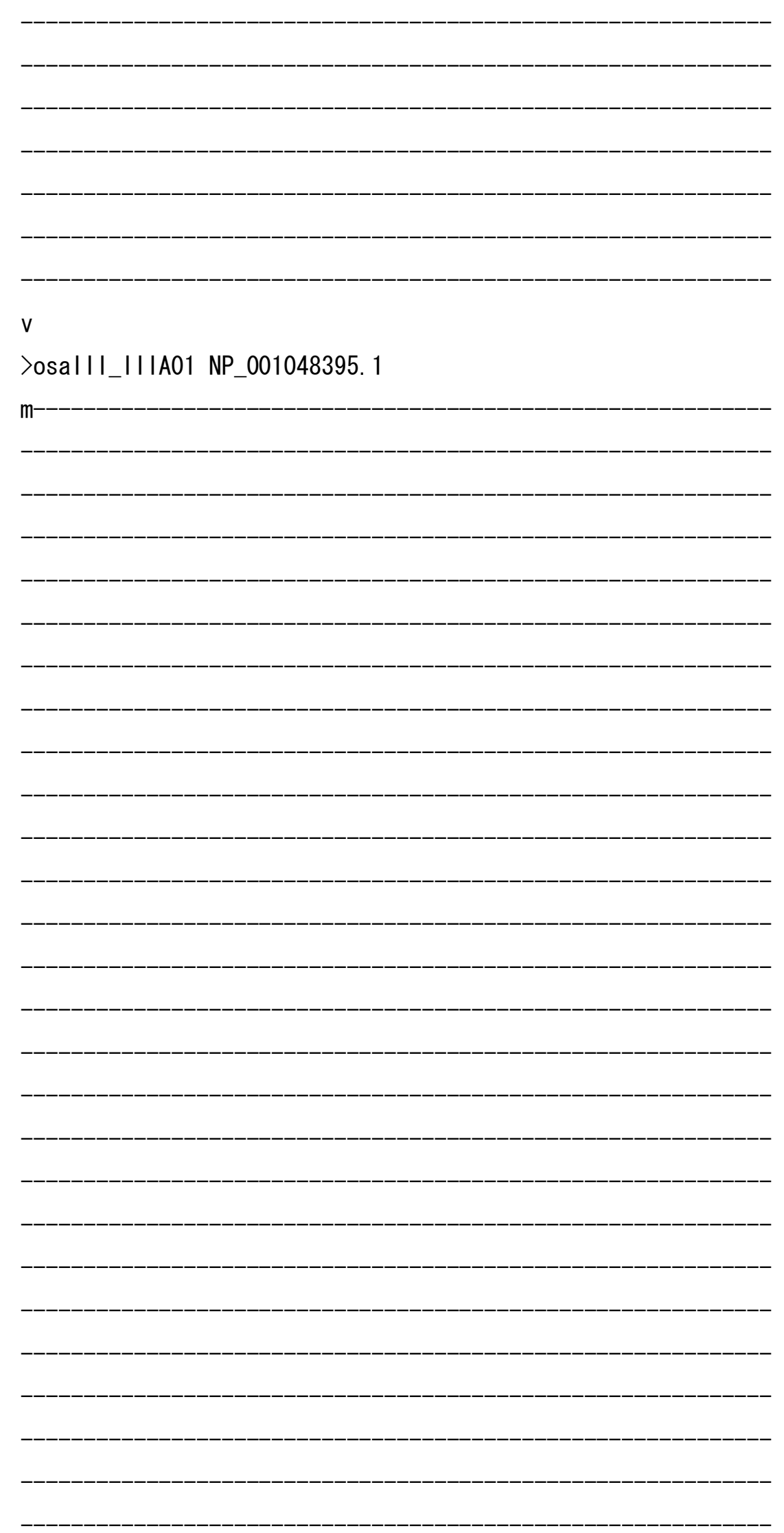




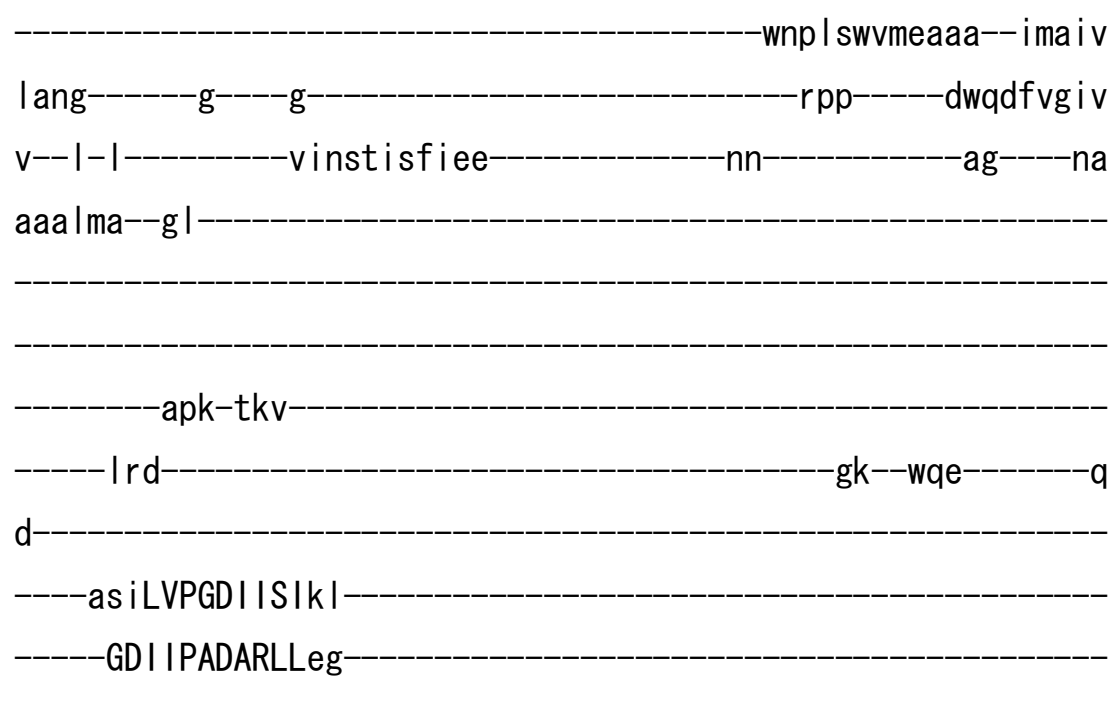

d--p I kVDQAALTGESMPVNKhx-

-QGVFS--GSTV-

kq----ge i e---_-_-_-_-_-_-_-_-_aVVIATGVHTFFGkaah I vds

-tnnighfqlvI taignfc i i sigvgmi i e i

-ivmypiqhr -ayrdg i dn ILVLLIGG

IPIAMPTV I SVTMA IGSH-RLsqqg-

-alTK-RMTAIEEMAGMDVLCSDKTG

TLTLNKLTVdkt I i-----------evygrg I dk-

v l lyaar------asr- 
lad--pkeara

gikevhf Ipfnpvek-rtai

-t-----yidg-n-gewhri isk-

-gap

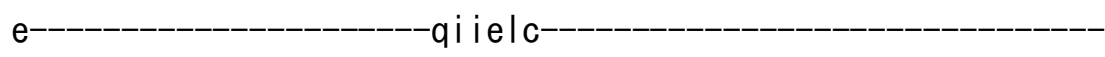

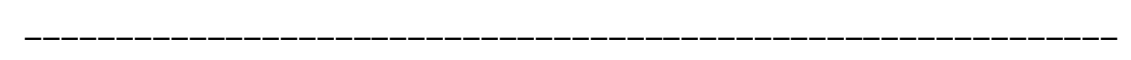

--kmskdaekkv-ht I i dqy--adr----g I rsLGVSYQkv

-pekskesegepwqfVGLLPLFDPPRHDSAETIRRALHI g-VNV

KMI TGDQLA I GKETARRLGM-

-gtnmypstt I lgd-kss

emsg I p ide-

I i eka--dGFA--GVFPEHKYEI VKRLq-

---dr--kh--_-_-_-_ ICGMTGDGVNDAPALKKAD I G I AV-$-x \times x x x x-$ 


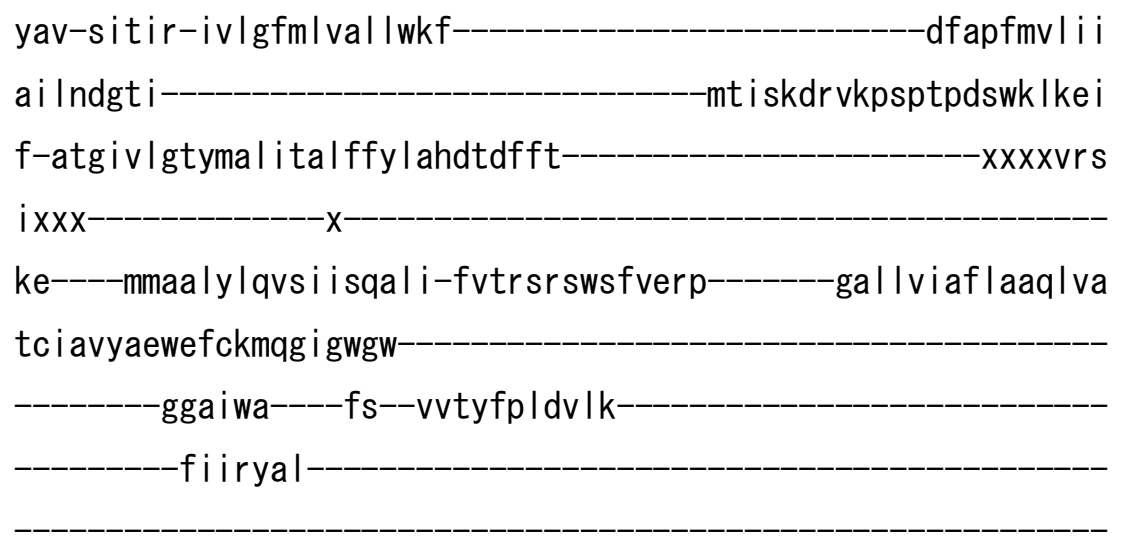

-sgrawnn-innktafvnkndygkgereaqwat

aqrt Ihg Inqsstxxx-

- fndktgyre | se iaeqaakraevar I xxxxxxxxxxxxxxxxxxxxx $x X X X X-X X X X$

>osalII_IIIA02 NP_001048647.1

m- 


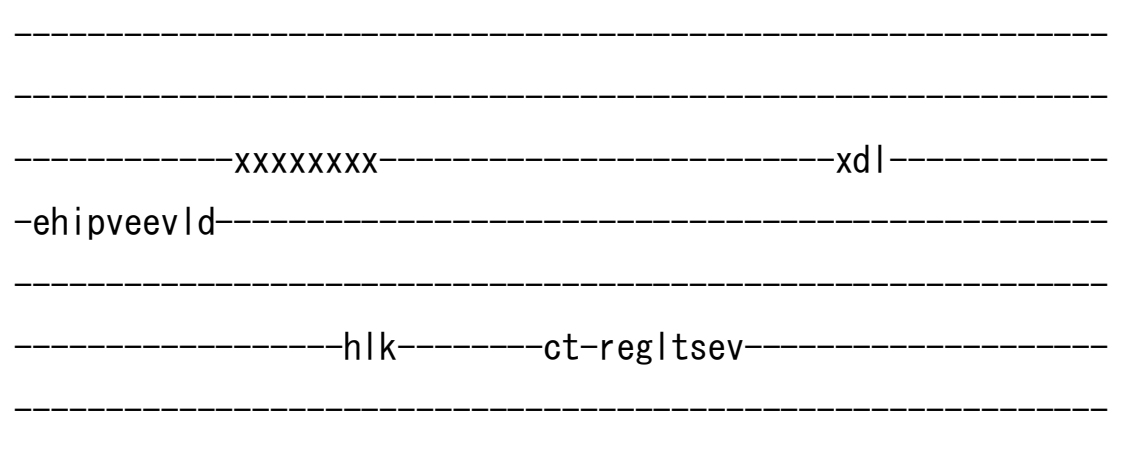

-aqqr ihsfgynk le-ekqe

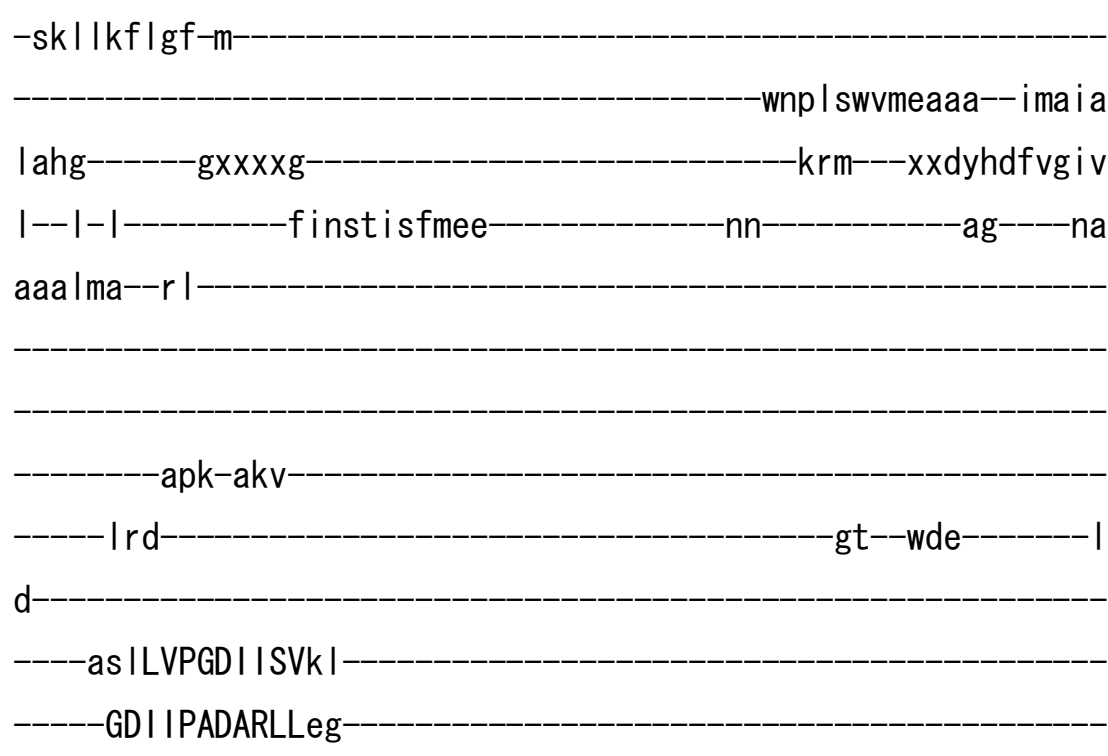

d--p IkIDQSALTGESLPVTKhx-

$$
-x
$$

-DGIYS--GSTC

kq----ge i e--------_-_--_--_-_-_--aVVIATGIHTFFGkaah I ves

-tthvghfqkv I ts i gnfc i cs i aagmvi e I

-I vmyavher

-kyrqivdnILVLLIGG

IPIAMPTV I SVTMA IGSH-KLaqqg- 
-al TK-RMTAIEEMAGMDVLCSDKTG-

TLTLNKLSVdkn l ievfekg i ek

vv Imaar-----asr

lenqdaid--faivsm

lpd--pkeara

-giqevhf Ipfnptdk-rtal

-t-----y lda-e-gkmhrvsk

gap

$e^{-}$

qi Inla

--snkce i erkv-hhv i gnf--aer----g I r sLAVAYQev

-pegtkespggpwqfVGLLPLFDPPRHDSAET IRRALD I g-VSV

KM I TGDQLAI GKETGRRLGM-

gtnmypsss I Igdrkdg

-diavlpvde 
I i eqa--dGFA--GVFPEHKYE IVQRLq-

$---a r--k h-$

- ICGMTGDGVNDAPALKKAD I G I AV--

$x x x x x$

ARSASD I VLTe-PGLSVI I saVLTSRAIFQRMKNYT

dfppfIvlvi

yav-sitvr-ivlgflllacfwkf

-mt i skdkvkpspypdswk I te i

ailndgti

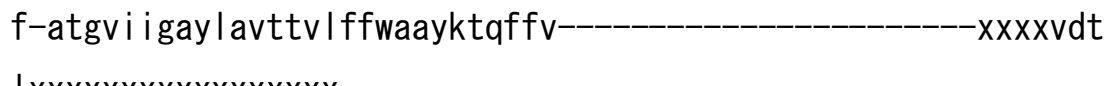

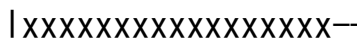

ek---- | asavy lqvst i sqal i-fvtrsrgwsflerp--_----g I I Imaafviaql ia

tvlaaiatwevasirgigwrw-

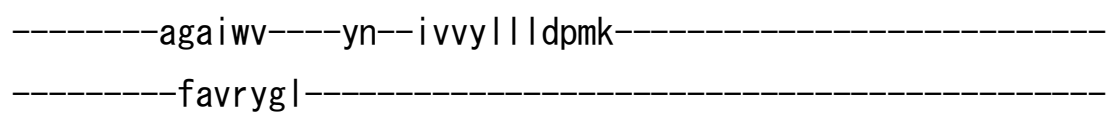

-sgkawn Iv idnkvaftnrkdfgrearvvawah

eqrt Ihg Iqsaas

-rekaaste Inqmaeearrraeitr Ixxxxxxxxxxxxxxxxxxxxxx

$x x x x x x x x x x$

V

>osalII_IIIA03 NP_001049185. 1

$\mathrm{m}$ 
$\operatorname{xxxxxxxxxxxx}$

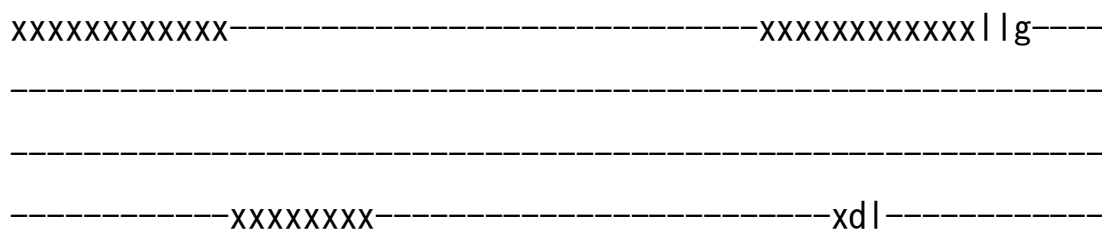

-enlpledvfe

-qln---------ts-qsgl ssad-

-aaer I I fganr le-ekre

-nki ikflsf-m-

-wnp I swvmeaaa--vma I v

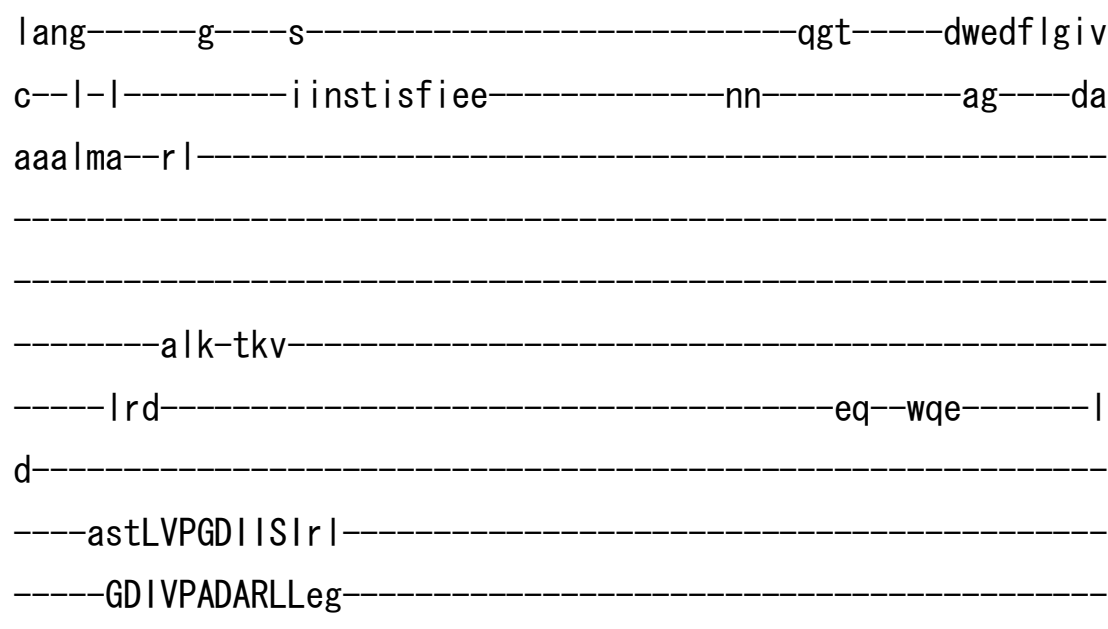


d--pIkIDQSALTGESLPVTKrx-

$$
-x
$$

-DIVFT--GSTC-

kh----ge i eaVVIATG I IHSFFGkaah I vds

-tevvghfqkvIts i gnfc ics i a iga ivev -i imfpiqhr syrdg i nnvLVLLIGG

IPIAMPTV ISVTLAIGSH-HLsqqg-

-a ITK-RMTAIEEMAGMDVLCCDKTG

-TLTLNHLTVdknl ievfsremdr

i i l laar-----asr-

lad--pkears

-sitevhf Ipfnpvdk-rtai

-t-----yvds-d-gnwfrvsk

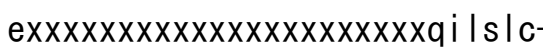


--ynkdd i sekv-ql i i drf--aer----g I rsLAVAYQev

-pekskhghggpwvfCGLLPLFDPPRHDSADT IRRALD I g-VCV

KMI TGDHLAI AKETGRRLGM-

-gtnmypsas I fgrhgdg

ggaavpvee

I veka--dGFA--GVFPEHKYE IVRMIq

---ggxxh---_-------_----_---_------VCGMTGDGVNDAPALKKAD I G I AV--

$-x x x x x$

-ARGAADIVLTe-PGLSVIVsaVLTSRAIFQRMKNYT $x x x x x x$ I

yav-sitir-ivigfvl las iwey---_-_-_-_-_-_-_-_-_-_-_-_--_dfppfmvl i i

a i Indgt i--_-_-_-_-_-_-_-_-_-_-_-_-_-_mt i skdrvkpspspdswk Ine i

f-aagvvigtylal vtv I fywtvtrttffe---_-_-_-_-_-_-_-_-_-_-------vrs

Ixxx--_-_-_-_-_---X--_-_-_-_-_-_-_-_-_-_-_-_-_-_-_-_-_-_-_-_-_-

de----i ssamy lqvs i i sqal $i$-fvtrsqg I sf lerp--_-_---ga l I i caf i laq I va

tl iavyat i sfasi saigwgw

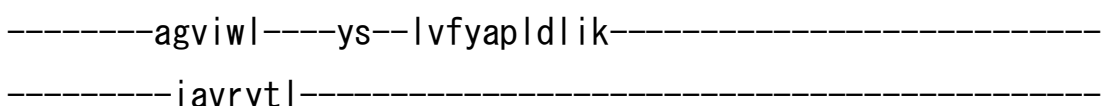

-sgeawn I I fdrkaafasrrdyggner rpetrw

prshhhhhqqrraxxx 
x|---| ssgwrptriaerakrraeiarr

>osalII_IIIA04 NP_001050947.1

m-

$-{ }^{-}-----x x k g g$

$-x x x x x x x x$

$-x d 1$

-enipieevfa

$-n \mid k$

cc-rqgltsee-

-aq | r lq I fgpnk le-ekee

$-s k f|k f| g f-m$ 
-wnp I swvmeaaa--imaia

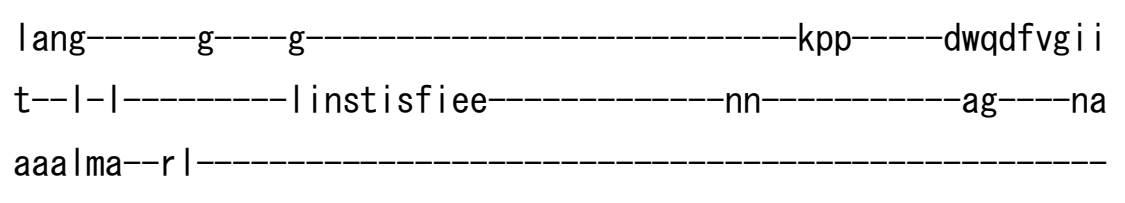

$-d--p \mid k I D Q S A L T G E S L P A T K g x-$

$--a p k-a k v-$

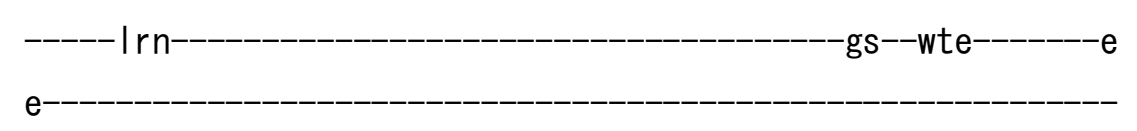

---aa iLVPGD I ISIk I-

- GDI IPADARLLeg-

$-D G V Y S--G S T V-$

kq----ge i e-----------------------aVVIATGVHTFFGkaah I vds-

-tnqvghfqkvltaignfcicsiavgmfvei

-i vmyp i ghr--_-_-_--_-_-_-_-_-_-_-_-_-_-_-_-_--pyrpg i dn ILVLLIGG

IPI AMPTV I SVTMA IGSH-RLsqqg-

-aITK-RMTA IEEMAGMDVLCSDKTG-

-TLTLNKLTVdkn l i---_--_-----e i fergvtq-

vi Imaar------asr-- 
lad--pkeara

giqevhf Ipfnptdk-rtal

-t-----y ids-d-gkmyrvsk

-gap

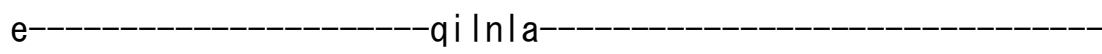

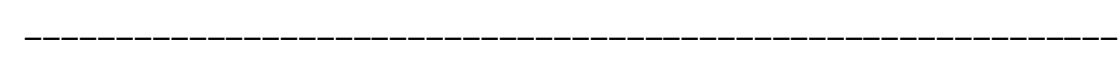

--hnktqi ierrv-havidkf--aer----g I rsLAVAYQev

-pdgrkespggpwr fVALLPLFDPPRHDSAET IRRALN I g-VNV

KMI TGDQLA I GKETGRRLGM-

-gtnmypssal I gankde

svaalpvdd-

I i eka--dGFA--GVFPEHKYE IVKRLq

---ar--kh--_-_-_-_-_-_-_-_-_-_-_ICGMTGDGVNDAPALKKAD I G I AV-$-x x x x x x-$ 


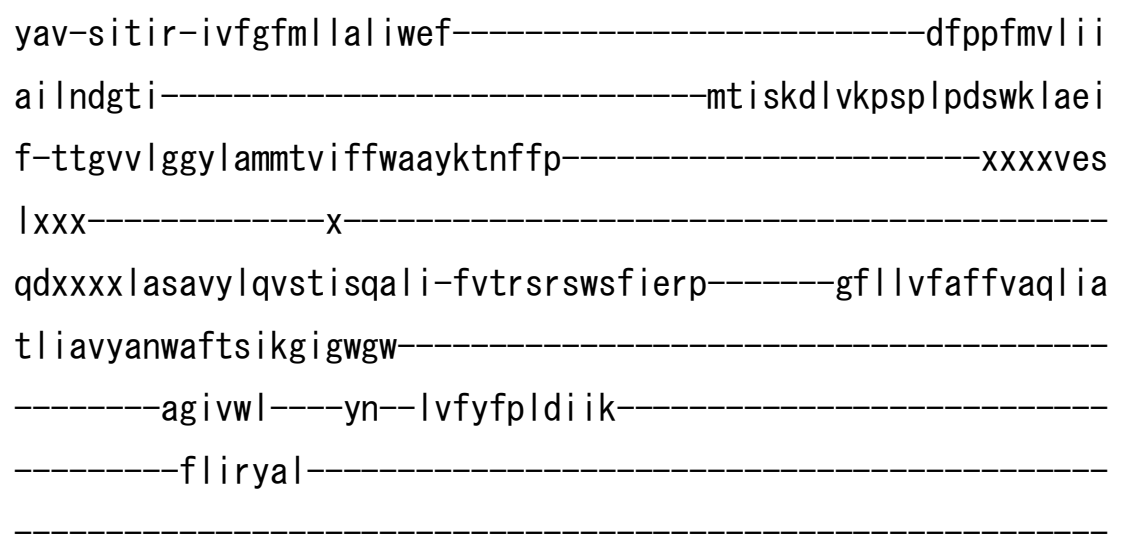

-sgkawd I v i eqr i aftrkkdfgkeere I kwah ahrt Ihg I qppdax-

-pfpektgyse Inqmaeeakrrae iar I xxxxxxxxxxxxxxxxxxxx $x x x x x-x x x x$

V

>osalII_IIIA05 NP_001054118.1

m- 


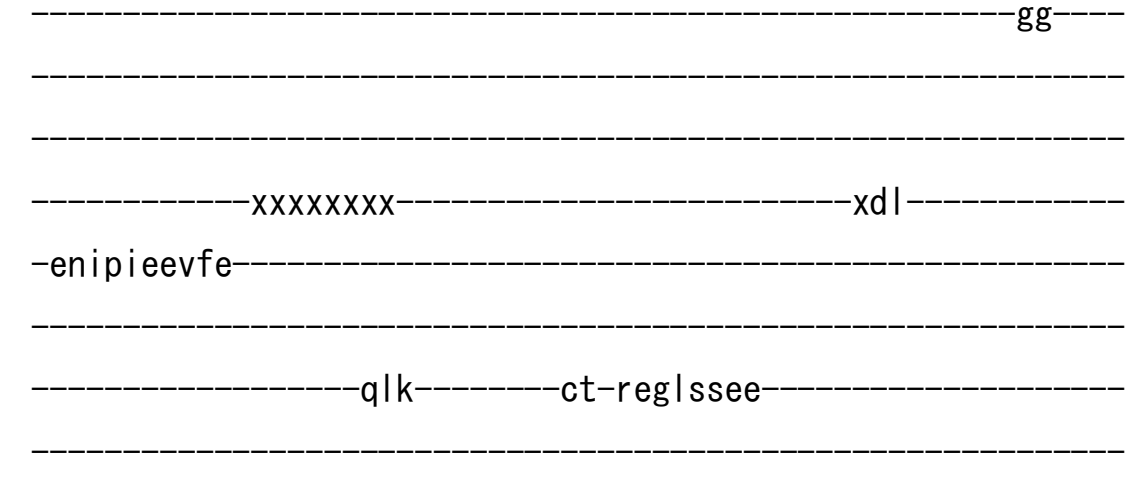

-gnrr i emfgpnk le-ekke

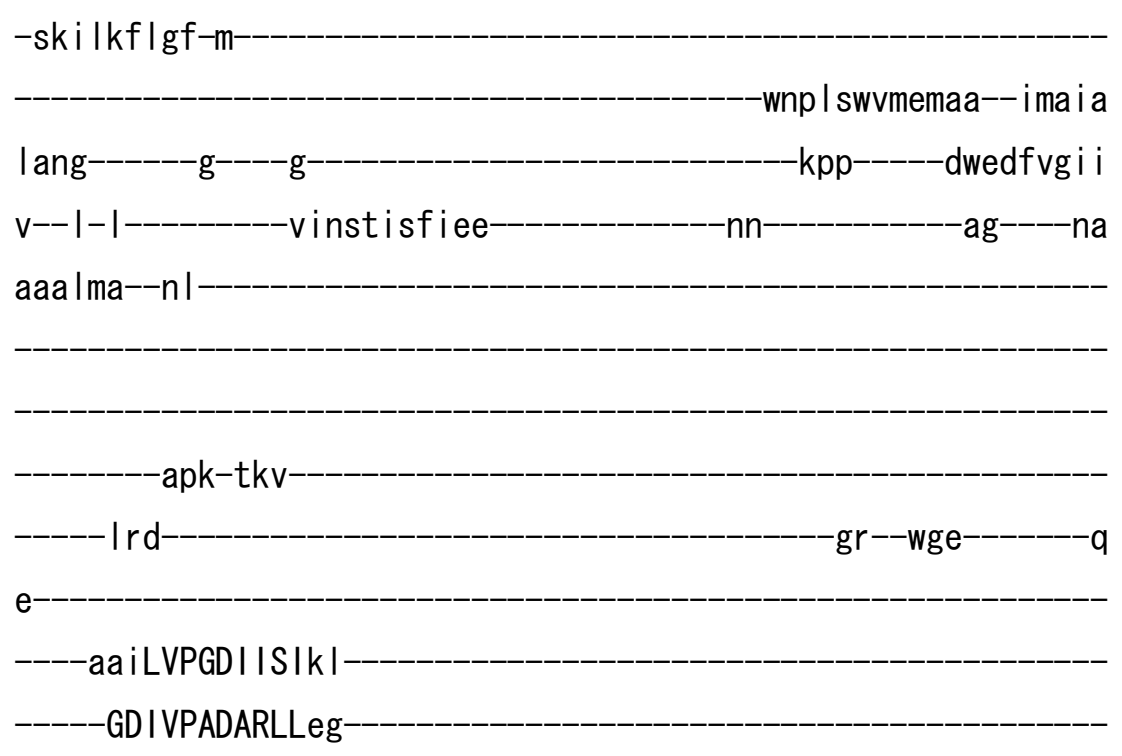

d--p I kIDQSALTGESLPVTKn--

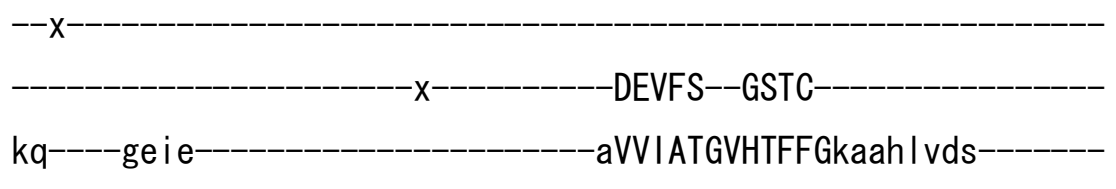

-tnqvghfqtvI ta i gnfc ics i avg i vie i

-ivmfpiqhr ayrsgienILVLLIGG

IPI AMPTV I SVTMA IGSH-KLsqqg- 
-al TK-RMTAIEEMAGMDVLCSDKTG-

-TLTLNKLSVdkn I v evftkgvdk

v I I aar-----asr-

tenqda i d--aamvgm

lad--pkeara

girevhf Ipfnpvdk-rtal

-t------y i da-d-gnwhrask

gap

$e^{-}$

qilt lc

--nckedvkrkv-hav i dky--aer----g | rsLAVARQev

-pekskesaggpwqfVGLLPLFDPPRHDSAET IRKALH I g-VNV

KM I TGDQLAI GKETGRRLGM-

gtnmypssa I I gankda

slealpvde 
I i eka--dGFA--GVFPEHKYE IVKRLq-

---ek--kh-

- IVGMTGDGVNDAPALKKAD I G IAV--

$-x x x x x$

ARSASD I VLTe-PGLSVI I saVLTSRCIFQRMKNYT

dfspfmvli i

yav-sitir-ivlgfllialiwky

-mt i skdrvkpsp I pdswk Ike i

ailndgti-

$-x x x x v r s$

f-atgivlgsylalmtviffwamhktdfft-

ixxx--_-_-_-_-_--_X--_-_-_-_-_-_-_-_-_-_-_-_-_-_-_-_-_-_-_-_-

he----mmsa ly lqvsi vsqal i-fvtrsrswsf ierp---_----g I I vtafm I aq I va tflavyanwgfar ikg i gwgw

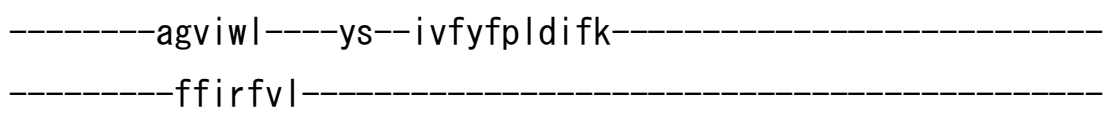

sgrawdnl l enk i afttkkdygreer eaqwat

aqrt Ihg I qppevxxx-

-x| fndkssyre I se iaeqakrrae iar I xxxxxxxxxxxxxxxxxxxxx

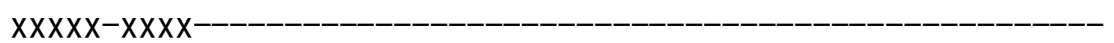

v

>osalII_IIIA06 NP_001055186.1

I 
$-x x x x x x x x h v g$

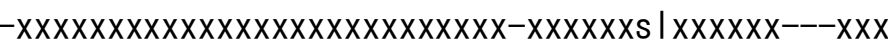

xesipigevfa

-vlk--------ss-pqg|tsad-

-gngr l e i fgrnk l e-ekke-

-skl Ikflgf-m-

-wnp I swvmeaaa--imaia

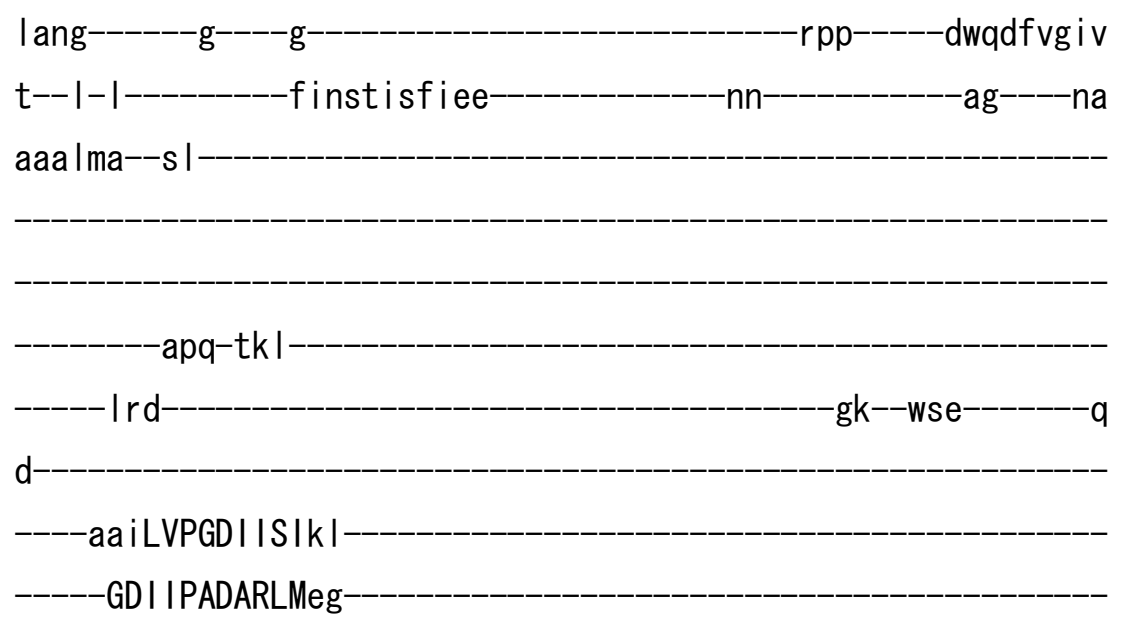


d--p IkIDQSALTGESLPVNKmx-

$-x$

-DSIYS--GSTC-

$\mathrm{kq}^{----}$ge i eaVVIATGVHTFFGkaah I vds

-tnnvghfqkvl ta ignfc ics i aagml i e i

- i vmyp iqhr-qyrdg i dn ILVLLIGG

IPIAMPTV I SVTMA IGSH-RLsqqg-

-al TK-RMTAIEEMAGMDVLCSDKTG-

-TLTLNKLTVdknmi epfvkdl dk

\section{ivlyaar-----asr-}

-tenqda id--as i vgm-

lad--pseara

-giqevhfmpfnpvdk-rtai

-t------y idtxd-gswhr i sk-

$\mathrm{e}$

-qi ielc 
--r I rddvsrrv-ha i i dkf--adr----g I r sLAVARQkv

-pegskdapgtpwqfLAVLPLFDPPRHDSSET I RRALN I g-VNV

KMI TGDQLAI GKETGRRLGM-

-gtnmypsss | ----kdg

dtgg I pvde

I i eka--dGFA--GVFPEHKYE IVRRLq

---er--kh-

ICGMTGDGVNDAPALKKAD I G I AV--

$-x x x x x$

-ARGASD I VLTe-PGLSVI I saVLTSRA IFQRMKNYT

yav-sitir-vvlgfll|aliwrf-

dfapfmvli i

ailndgti

-mt i skdrvkpsp I pdawr Iqe i

f-atgivlgtylalatvlffwavrdtdfftxxxxxxxxxxxxxxxxxxxxxxxxxxxyhp ixxx--------------

ee---- Imaavy lqvs i i sqal i-fvtrarswffverp--_-_--g I I vgaf I iaq Ima

tl i avyanwpfakmkg i gwsw

-gmviwl----fs--ivtffpldifk-

fairyfl

sgkawnnafdnktafane Idygkskreaqwai

aqrs Ihg lqqaetxxx 
Ifddnkdy l e I se i aeqakr rae i ar I xxxxxxxxxxxxxxxxxxxxxx

$x x x x x-x x x x$

V

>osalII_II IA07 NP_001056980.1

$\mathrm{m}$

$-x \times s \mid s$

$-x x x x x x x x$

$-x d 1$

-skapvaevfq

$-k \mid k$

cd-rkg I tgae

-gesr I I lygpnk le-ekke

$-s k|l k f| g f-m$ 


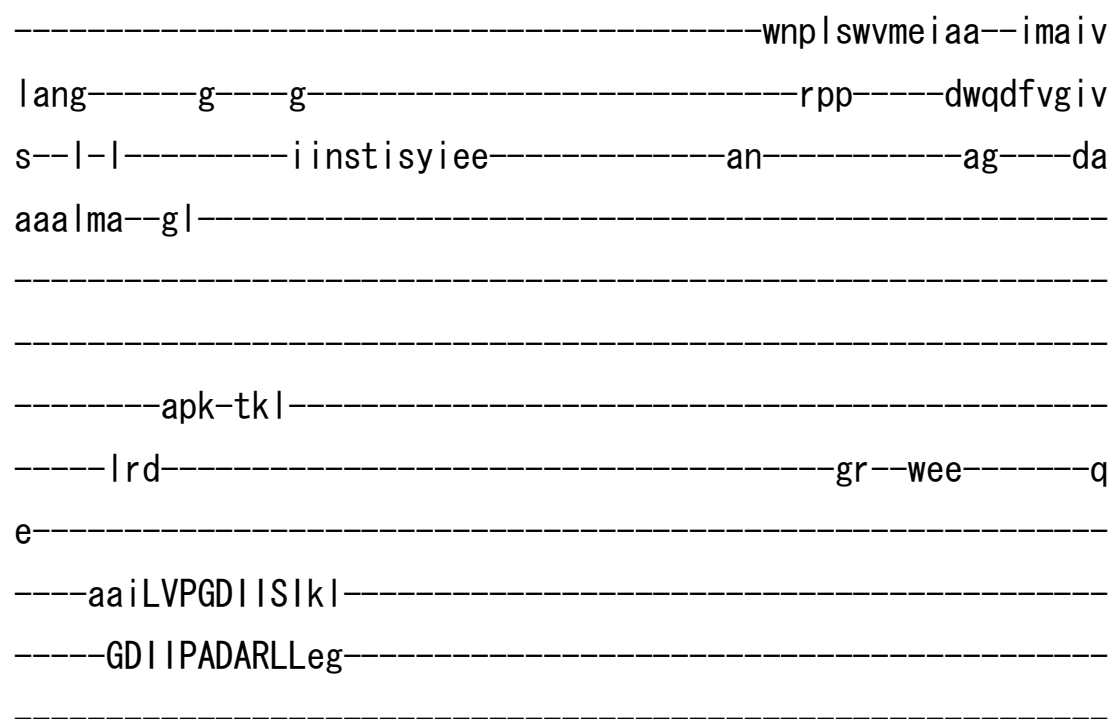

d--pIkIDQSALTGESLPVNKhx-

- QEVFS--GSTV

kq----ge i e----_--_-_--_--_----aVVIATGVRTFFGkaah I vds

-tnnvghfqqv I ta ignfc i i s i gagmavev

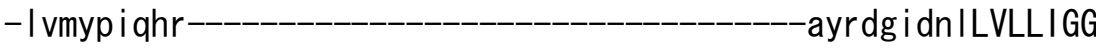

IPI AMPTVI SVTMA I GSH-RLsqgg-

-a ITK-RMTA IEEMAGMDVLCSDKTG

TLTLNKLTVdkt I evcskgvdk-

$-d m$

v l lyaar------asr- 
Idd--pkeara

giqevhf Ipfnpvdk-rtai

-t-----y idg-n-gdwhrvsk

-gap

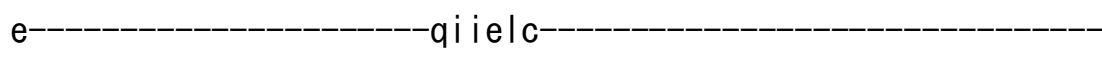

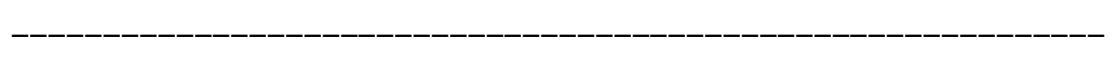

--nmaadaekkv-hal idsy--adr----g I rsLGVSYQqv

-pekskdsggdpwqf I GLLPLFDPPRHDSAET IRRALH I g-VNV

KMI TGDQLA I AKETGRRLGM-

-gtnmypstt I lgd-kns

qving I pide-

I i era--dGFA--GVFPEHKYEI VKRLq-

---em--sh-

-ICGMTGDGVNDAPALKKAD I G I AV--

$-x x x x x x$

-ARSASD IVLTe-PGLSVIVsaVLTSRAIFQRMKNYT$-1$ 
a i Indgt i----------------------------mt i skdrvkpsptpdcwk Ine i

f-I tgvv I gtymal vtv| ffy lahdtnfft-------------------------xxxxvts

ixxx---------------X--------------------------------------------

re-----Imaa ly lqvsi i sqal i-fvtrsrswsfverp--_--_--gf I I faffaaqmva

tai avyarwdfcr i gg i gwrw

--_--_---ggavwq----fs---vvty |p | dv lk-_-_-_-_-_-_-_-_-_-_-_-_-_-_-_

fi iryal-

$\operatorname{tg}$

-gkagdsaqk--

k

>osallI_IIIA08 NP_001059093.1

$\mathrm{m}-$ 


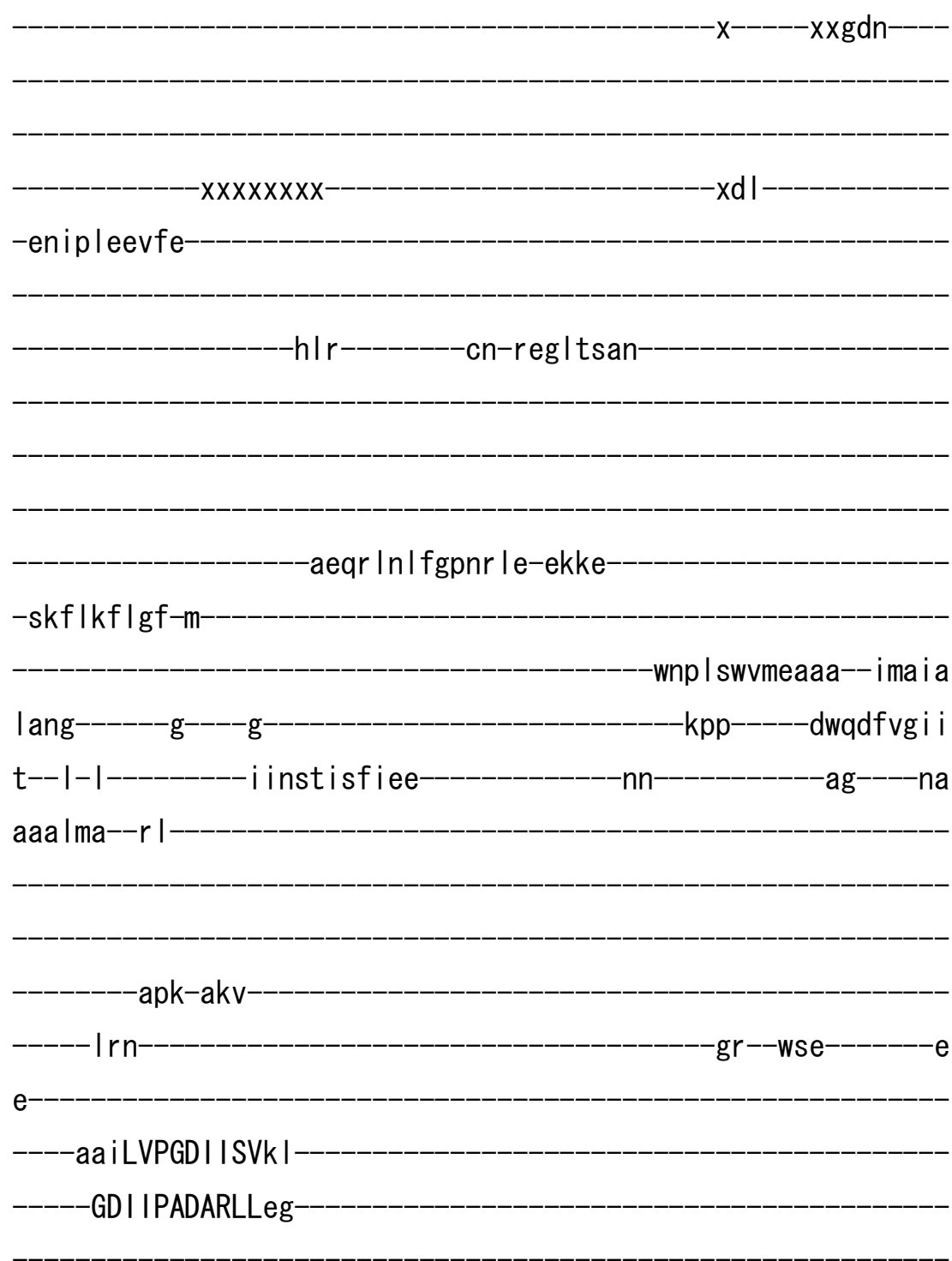

d--p IkIDQSALTGESLPVTKgx-
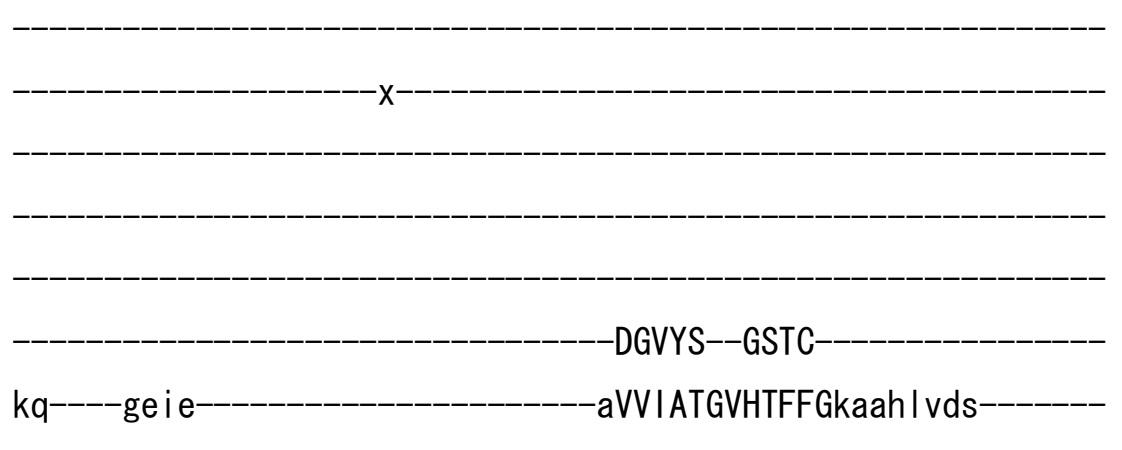

-tnqvghfqkv I ta i gnfc ics i a i gmvve i

-i vmyp i qhr-_-_-_-_-_-_-_-_-_-_-_-_-_-_-_-_-_-_dyrpg i dn I LVLLIGG

IPIAMPTV I SVTMA IGSH-RLaqqg 
-al TK-RMTAIEEMAGMDVLCSDKTG-

$-T L T L N K L T V d k s$ I i evfargvdq

$-d t$

vi Imaar-----asr-

tenqda id--at i vgm-

lad--pkeara-

-giqevhf Ipfnptdk-rtal

-t------y idg-e-gkmhrvsk-

gap

e-

$-q i \ln l a$

--hnkte ierrv-ravidkf--aer----g I rsLGVAYQqv

-pdgrkespggpwqfVGLLPLFDPPRHDSAET IRRALN I g-VNV

KMI TGDQLAI GKETARRLGM-

gtnmypssa I I gadkde

sivalpvde 
I i eka--dGFA--GVFPEHKYE IVKRLq-

$---a r--k h-$

- ICGMTGDGVNDAPALKKAD I G IAV--

$x x x x x$

ARSASD I VLTe-PGLSVI I saVLTSRAIFQRMKNYT

dfppfmvl i i

yav-sitir-ivlgfmllaliwkf-mt i skdrvkpspqpdswk I se i

a i Indgt i -xxxxves

f-atgvv I gsy lammtviffwvayktdffp-

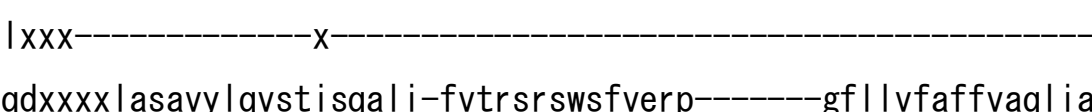
tl i avyanwgfasikg i gwgw

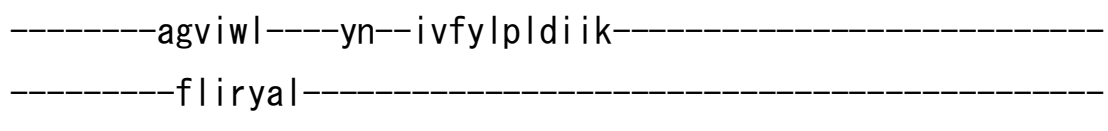

-sgrawd Iv l eqr i aftrkkdfgtqenq I kwat

aqrting I qpaatxx

-vfrdmtsynd Inq laeearrrae iar I xxxxxxxxxxxxxxxxxxxxxx

$x x x x x-x x x x-$

v

>osalII_IIIA10 NP_001067382. 1

m- 
x-----xxegn-

$-x x x x x x x x$

$x d 1$

-enipleevfe

-nl r--------cs-reglttqq-

aqqr l e i fgpnk l e-ekee

-skflkflgf-m-

-wnp I swvmeaaa--imaia

lang-------g-----g-------------------_--------kpp------dwqdfvg i i

aaa $|m a--r|$

-apk-akv-

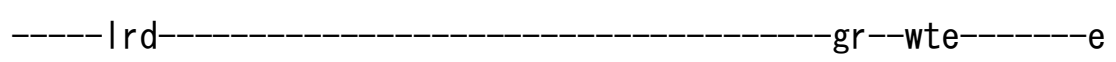

e

----aa iLVPGD IVSIk I

GDI IPADARLLeg 
d--p I kIDQSALTGESLPVTKgx-

$-x$

-DGVYS--GSTV-

$\mathrm{kq}^{----}$ge i e-

a IVIATGVHTFFGkaah I vds

-tnqvghfqkv I ta i gnfc i cs i avgmfve i

-ivmyp i ghr ayrpg idnILVLLIGG

IPIAMPTV I SVTMA IGSH-RLsqqg-

-al TK-RMTAIEEMAGMDVLCSDKTG

-TLTLNKLTVdkn l idvfergita

v i Imaar------asr-

tenqda id--ta i vgm-

lad--pkear a

-giqevhf Ipfnptdk-rtal

-t-----y idg-d-gkmyrvsk-

$\mathrm{e}$

-qi lhla 
--hnkpe i errv-hav i dkf--aer----g I rsLAVAYQev

-pegtkespggpwhfVGLMPLFDPPRHDSAET I RRALN I g-VNV

KMI TGDQLA I GKETGRRLGM-

gtnmypssal I gqnkde

s i aalpvdd-

I i eka--dGFA--GVFPEHKYE IVKRLq

---ar--kh-

ICGMTGDGVNDAPALKKAD I G I AV--

$-x x x x x$

-ARSASD I VLTe-PGLSVI I saVLTSRA IFQRMKNYT

yav-sitir-ivlgfmllaliwkf

dfppfmvli i

ailndgti

-mt i skdrvkpsp I pdswk I ae i

f-ttgvv I ggy l ammtvi ffwaayktdffp--_-_-_-_-_-_-_-_-_-_-_-- xxxxves

I $x x x$ $-x$

qdxxxx lasavy lqvsti sqal $i-f v t r s r$ swsfverpgfl Ivfafivaqlia

tl iavyadwafts ikgi gwgw

-ag i vwl----yn--l i fyfp Idi ik-

fliryal

sgkawd lv i eqr i aftrkkdfgkeere I kwah

aqrt Ihg I qppdax 
-mf sekagyne Inqmaeeakrrae i ar Ixxxxxxxxxxxxxxxxxxxx $x x x x x-x x x x$

V

>mgr III_IIIA01 XP_359783.2

$$
\mathrm{m}
$$

$-x x x x x x x x x x x x x x x$

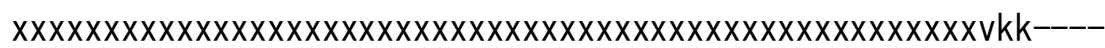

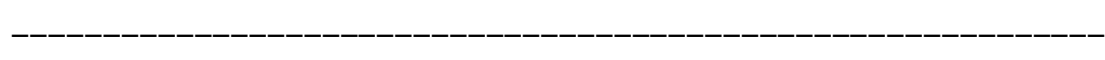

$-a x x x x x x x x x x x x$

xs Isdfntpae-

$-w \ln$ tnxsaglds le-

-verrrkysgwne Itxeken

$--m l l k f i g f-f$ 


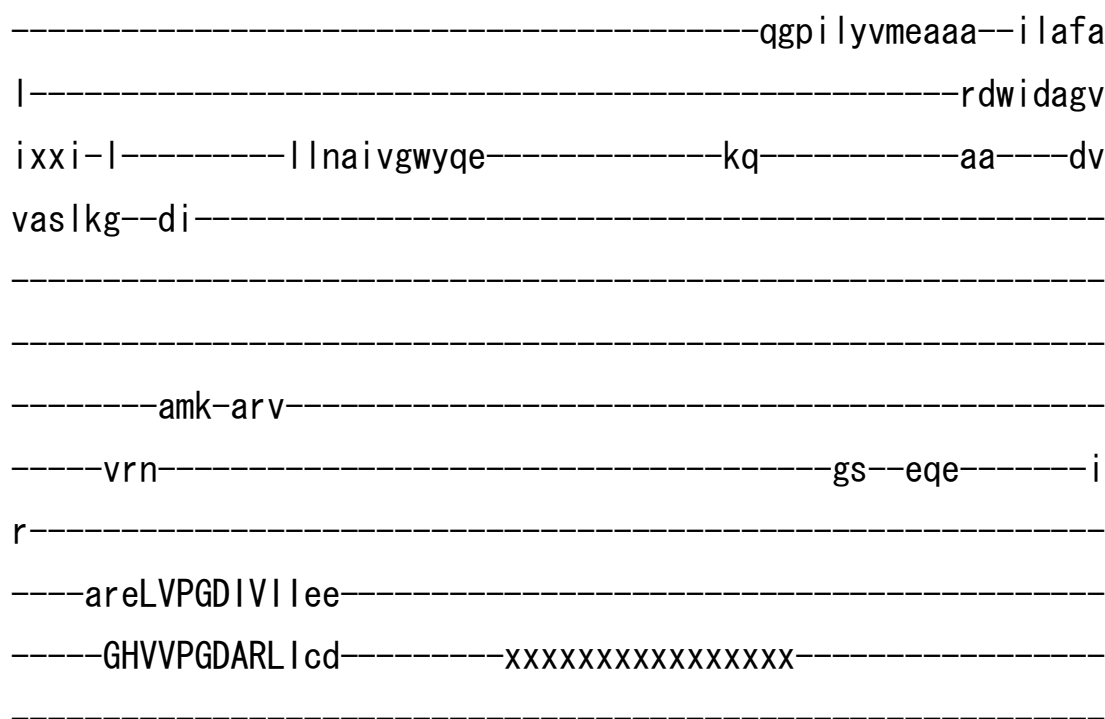

$-x x x x x x x x x x x x x x x x x x x x x x x x x x a--i v a I D Q S A I T G E S L A V D K y x-$
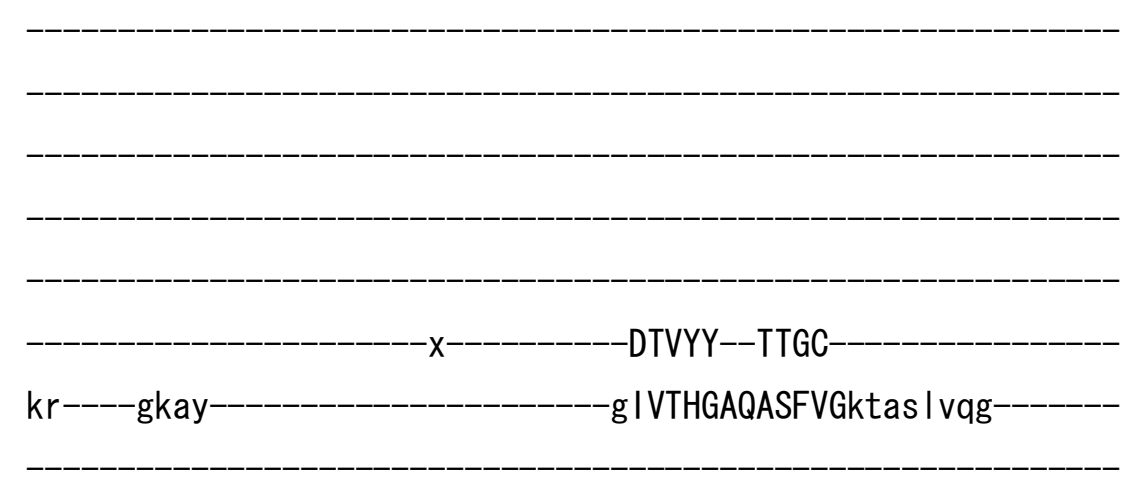

-aqdaghfka imns igsal I v Ivvvf i laaw -iggfyrh laxxxxxx------------------_---------ssvn I I hyvL ILLI IG VPVGLPVVtTTTLAVGAA-YLakek-

-aIVQ-KLTAIESLAGVDILCSDKTG

TLTANQLSVr epfvmegvdxx

mavaala-----sshx- 
iks Idp id--kitilt

xxxxpka--ke i i se

gwttekftpfdpvsk-ritsxxxxxxxxxxx

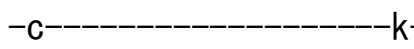

k-

-gap

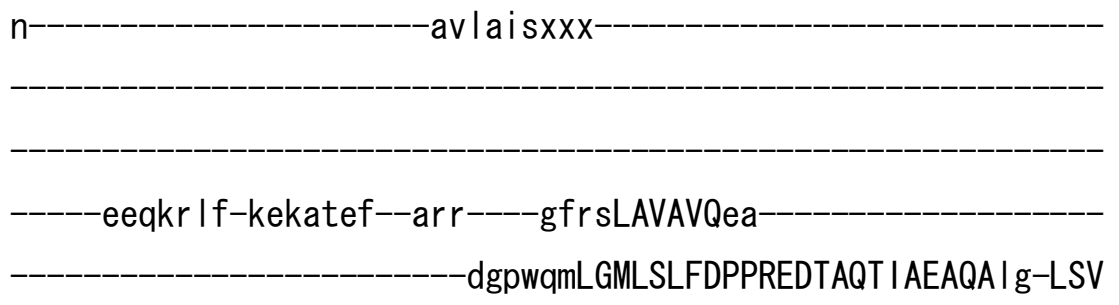

KMLTGDAIAI AKETCRMLAM-

gtkvynsdk I

xxx--------------dmagsaind-

Icera--dGFA--EVFPEHKYQVVEMLq

--- qr--gh-

-LTAMTGDGVNDAPSLKKSDCG I AVxx

$\mathrm{XXXX}$ 


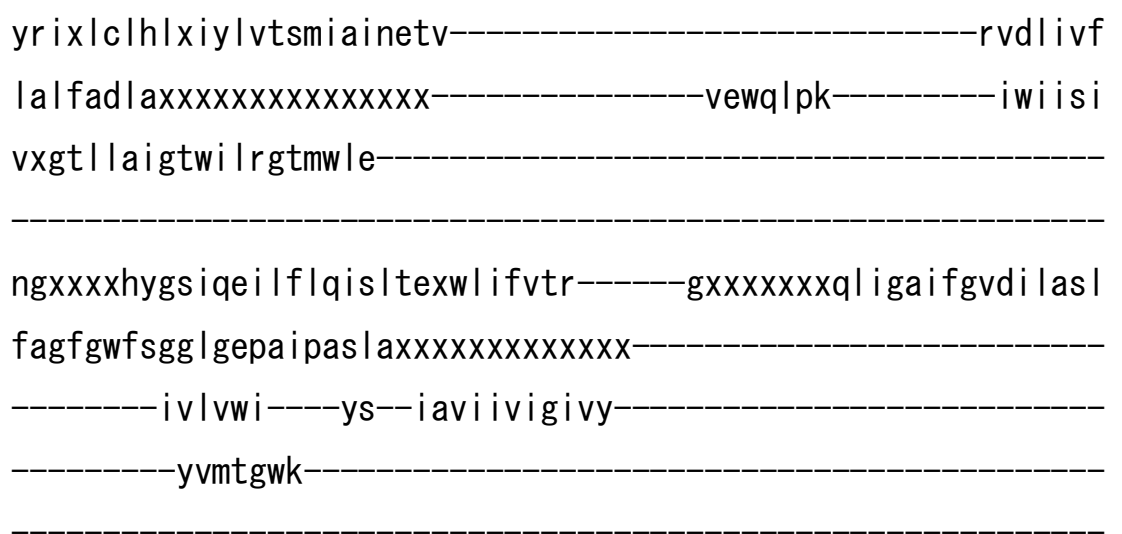

r Idd I grkkrsaqdtmmen i I th I skvavehe

idgrgghryy latxxx-

-xeee

e

>cnelII_IIIA01 XP_571736.1

m- 


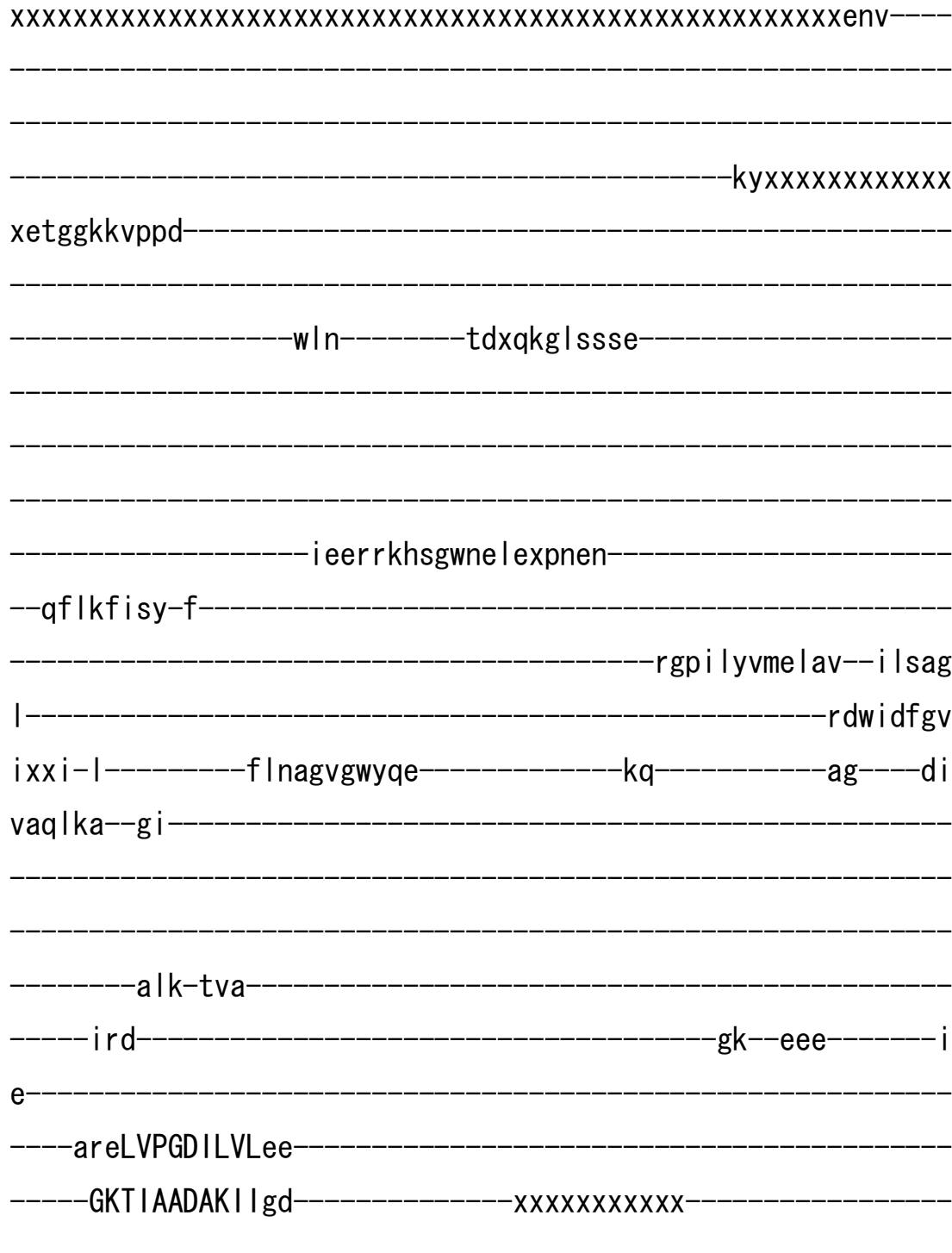

$-x x x x x x x x x x x x x x x x x x x x x x x x x x g x x \mid c s V D Q S A I T G E S L A V D K f x-$

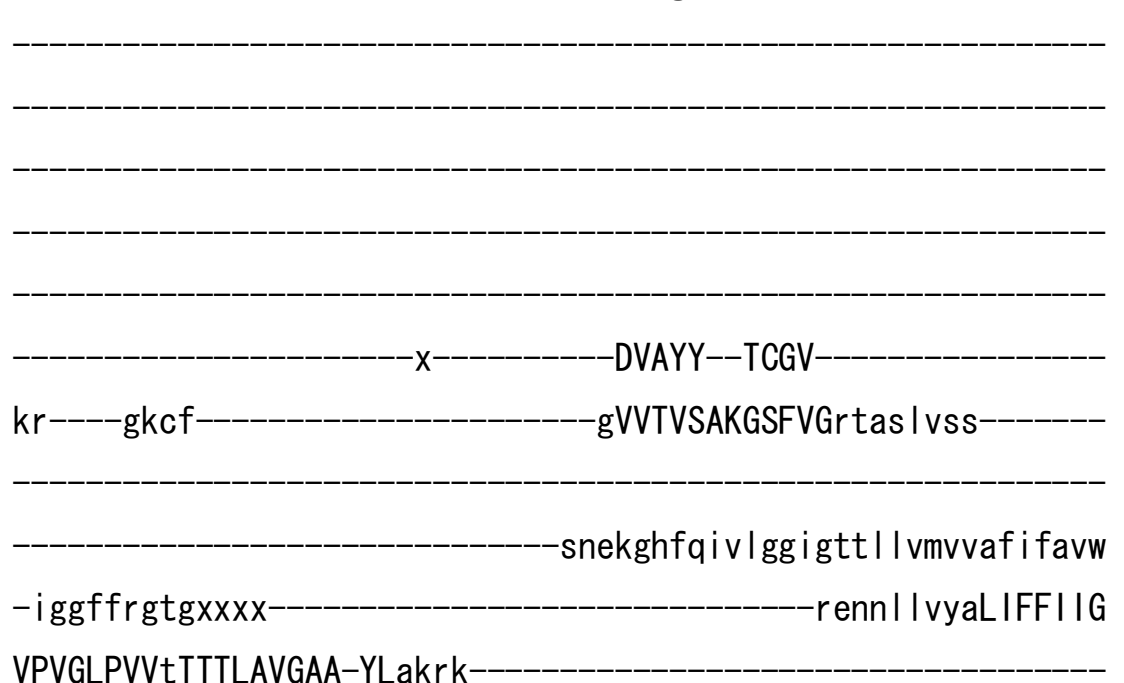

VPVGLPVVtTTTLAVGAA-YLakrk- 
aIVQ-KLTAIESLAGVDILCSDKTG

TLTANKLSLn-

epy i apdvdxx-

$-w f$

mavav la-----sshx

v I gldpid--kvtivg

xxxxpka--qem lkg

gwkthkftpfdpvsk-ritaxxxxxxxxxxx

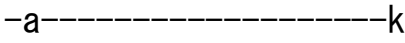

-gap

$\mathrm{n}$

-ailk|tx-

---fdpdtvsay-raqsqqf--asr----gfrsLGVAVKed

gkdwe I LGMLCMFDPPR I DTAKT I GEAHD I g-IQV

KMLTGDAVAI AKETCKQLGL

-ktnvydsek I

$\mathrm{xxx}$

gmagsdird- 
fveaa--dGFA--EVFPEHKYQVVNLLq-

---er--gh-

LTAMTGDGVNDAPSLKKADCG I AVxx

$x x x$

ARTAADVVFLd-EGLSTI I ta IKVARQIFHRMKAY I

$-1$

yrixlcvhlxvy ImIsililneti-_-_-_-_-_-_-_-_-_-_-_-_-_-_rvdlvvf

lai fadvaxxxxxxxxxxxxxxx---_-_-_-_-_-_---vewql pk---_------vwi ist

ixg I I laagtwi irat Iwid-

ngxxxxnfgstqe i Ifleval texwvifitr laqepgtxxxxxxxq I vaavigvdalat $\mathrm{i}$

falf gwi sgdaphg $-x x x x x x x$

--------vvkiwc----fs--fgvvi i illvy

- ImInsir

-wIds igrksrskknek lenf Itdlar Itivhe

tdhngsyyrfastxxx

xeggdngkkddkkdeaksgdtkkqegnaxxxxxxxxxxxxxxxxxxxxx

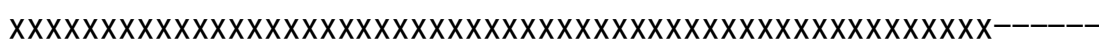

$\mathrm{n}$

>cnelII_IIIA02 XP_568571.1

$\mathrm{m}-$ 
$-X X X X X X X X X X X X$

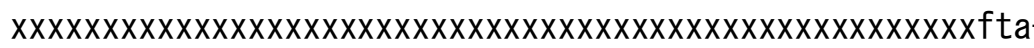

$x x x x x x x x$

$x d i$

-ehvvmeevyq

I q ct-dag| teae

-atdr i g i fgpnk le-ekse-

$-n v|l q f| s f-m$

-wnp I swvmegaa-- I vaia

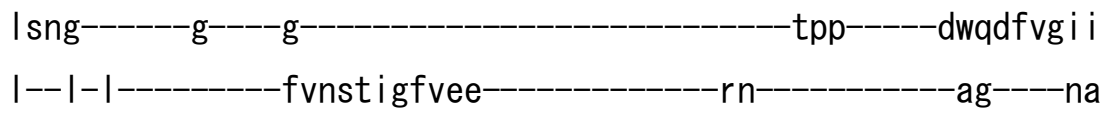

vka|md--s |

-apk-arv

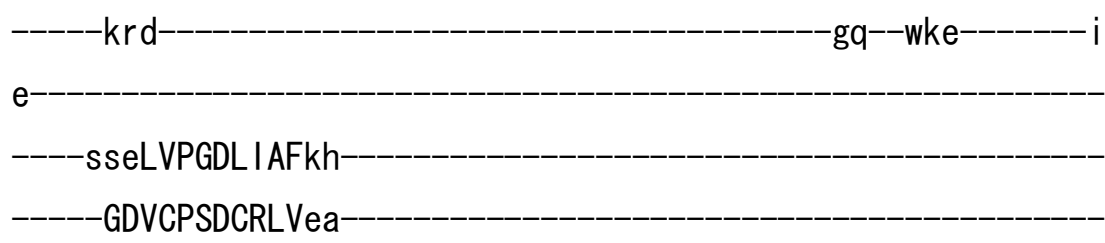


i--dvsMDQAALTGESLPVGKhx-

$$
\text { -DECFS--GSTC- }
$$

$\mathrm{kq}^{----}$geae-

-xndqugh lqqularigtfclvsigifvlle i

-lilyadfry -pyrrgIdniLVLLIGG

\section{IPIAMPTVISVTLAVGAQ-QLakhk-}

a IVT-RI TAIEELAGVTILCSDKTG

TLTTNKLT Idkenv

$-k c y s k w d v$

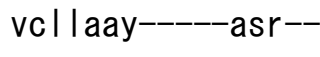

tenqda id--gcvvgt

lpd--pqqara

gik I ldfkpfnpvdk-rte i

-t-----yrdexdxgk Ikratk-

gmt g-

-i i ieic- 
xxnktne ledq I-eadveef--ar r----g I raLAVAFEdv

agddpsaegng fe I VGLLS IFDPPRSDTKKT I DDAMA I g-VKV

KMVTGDQLAI AKETGRRLGL

gdhmypakv lkegpea

gskhanlde-

mimda--dGFA--GVFPEHKFEIVKRIq

$---\mathrm{nl}--\mathrm{gh}-$

-LCAMTGDGANDAPALSRANVG I AVxx

$x x x x$

ARGAAD IVLTe-PGLST IVha I YGSRVIFQRMRNYA

\section{$-1$}

yac-avt ir-ivlcfa imafawrf--_-_-_-_-_-_-_-_-_-_-_-_-_-_ffppfmvl i i

av Indgt i--_-_-_-_-_-_-_-_-_-_-_-_-_-_--mt I s I drv I psttpdswd I aev

f-sfgvaygvy l sast $i$ a lyatmenttffe--_-_-_-_-_-_-_-_-_-_-_--_xxxvep

|--_-_-_-_-_-_-_-_-_-_-_-_-_-_-_-_-_-_-_-_-_-_-_-_-_-_-_-_-_-_-_-

kgxxxxghmv i l lqvai i sqal i-fvtrshgpswterp---_----sva Im lafc laq I vs

si i aayadwsfsquhsvsggw-

ig i vwi----wn--i vwyfp I dg ik

fimkktv-

i aa I gr rkarkagpavadaa Ihrapsrhes Iy

snrtnfItraanrxxx 
-gak i smsqne larfss i qaqqsgaal txxxxxxx

a

>anilII_IIIA01 EAA65724. 1

$\mathrm{m}$

$-\mathrm{X}$

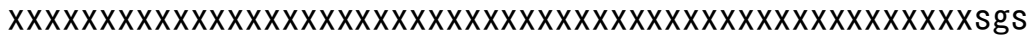

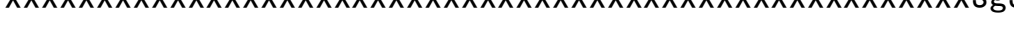

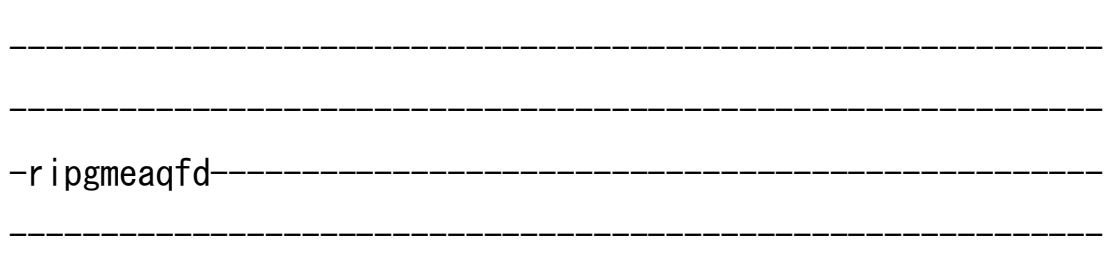

tdxttgltsve-

-aaqr rkkygpnq Ikxeken

$--m|k k f| s f-f$ 
-vgpvqfvmegaa--i lai$x \mid r d w v d f g v$

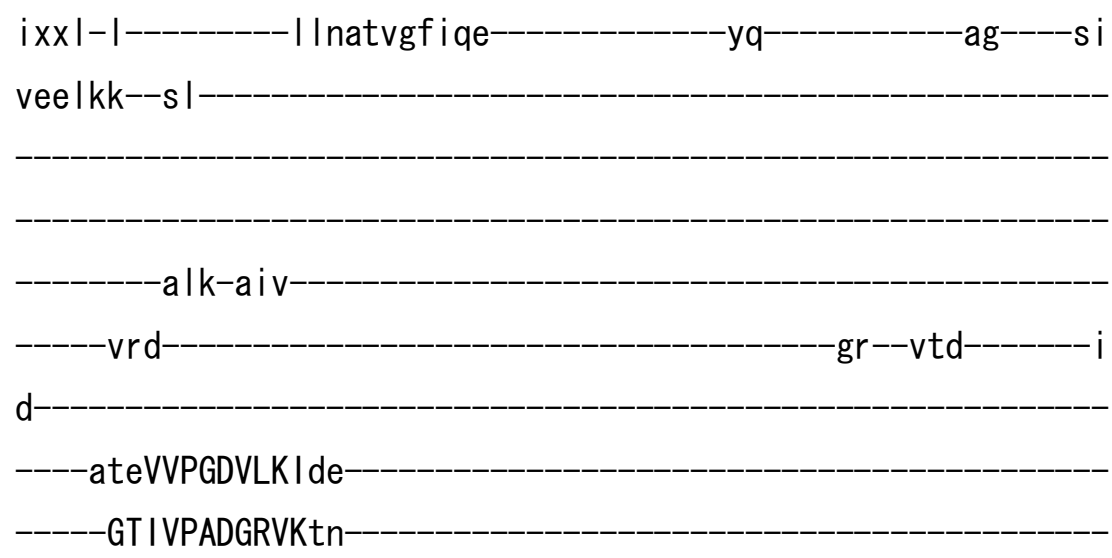

$-x \mid$ I qIDQSSVTGESLAVNKc--

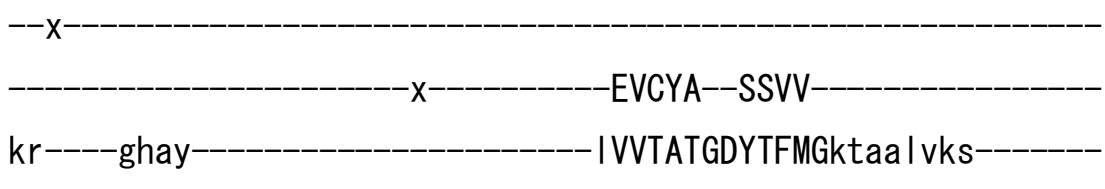

-xssnsghftevInrigat IIvIvvIt I ivvw

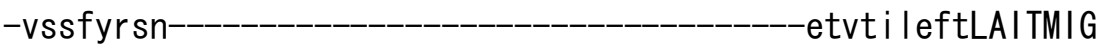
VPVGLPAVvTTTMAVGAA-YLakrq- IVQ-RLSAIESLAGVEVLCSDKTG

TLTKNKLTLs dpytvagvdxx

mItacla -asrx 
xxxxpra--kealsh

ykiqqfhpfdpvskxvtavxxxxxxxxxx

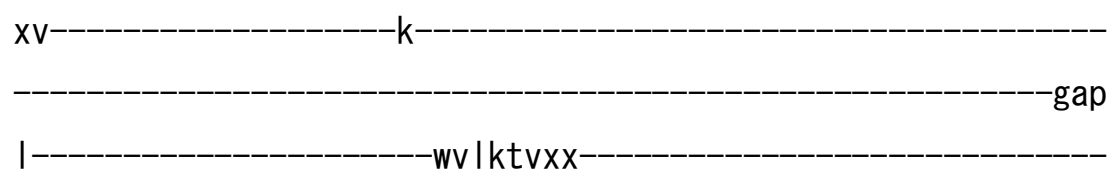

xxq i pesvekgy-sdkmdef--aqr----gfrsLGVARKpa

-ggewe i LG I VPCSDPPRDDTAAT INEAKT I g-LS I

KMLTGDAVPI ARETSRELGL

gtnvynsdk I

$\mathrm{XXX}$

$\mathrm{xxx}--------------d|\operatorname{tgse}| \mathrm{yn}$

yveaa--dGFA--EVWPQHKYNVVDILq

---qr--gy--_-_-_-_-_-_-_-_-_-_-_-_-_LVAMTGDGVNDAPSLKKADTG I AVxx

$\mathrm{xXXX}$

-ARSAAD I VFLa-PGLSA I I daLKTSRQ I FHRMHAYV

$-1$ 


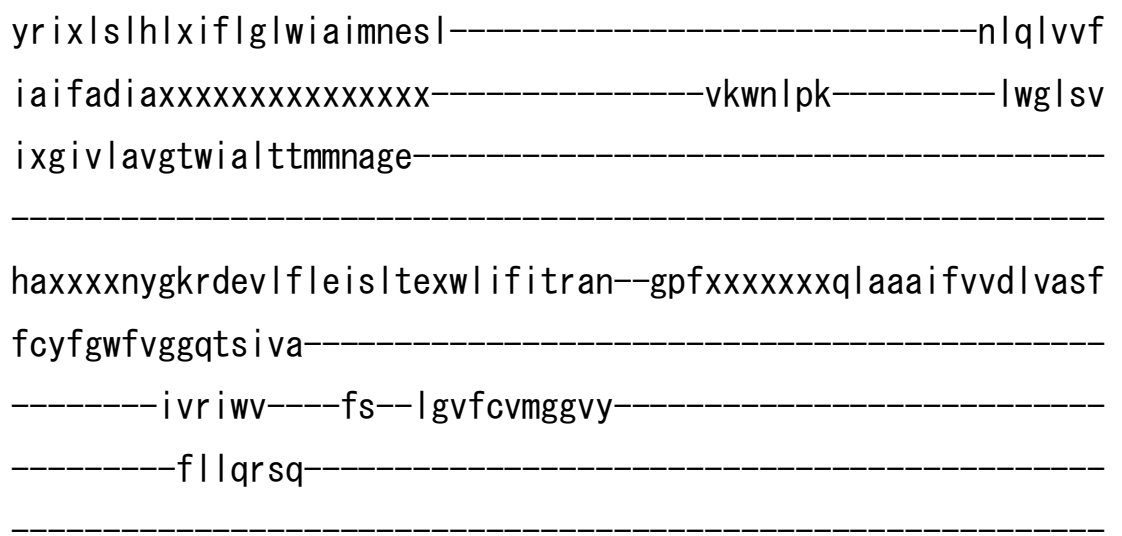

-tfdd imhfnf I qkrdsvsqrv I dd I vva I qrr seqheqssrtaer $x x x$

$-x$ I wkmdk I rkeraq

C

>anilII_IIIA02 EAA60937. 1

m- 


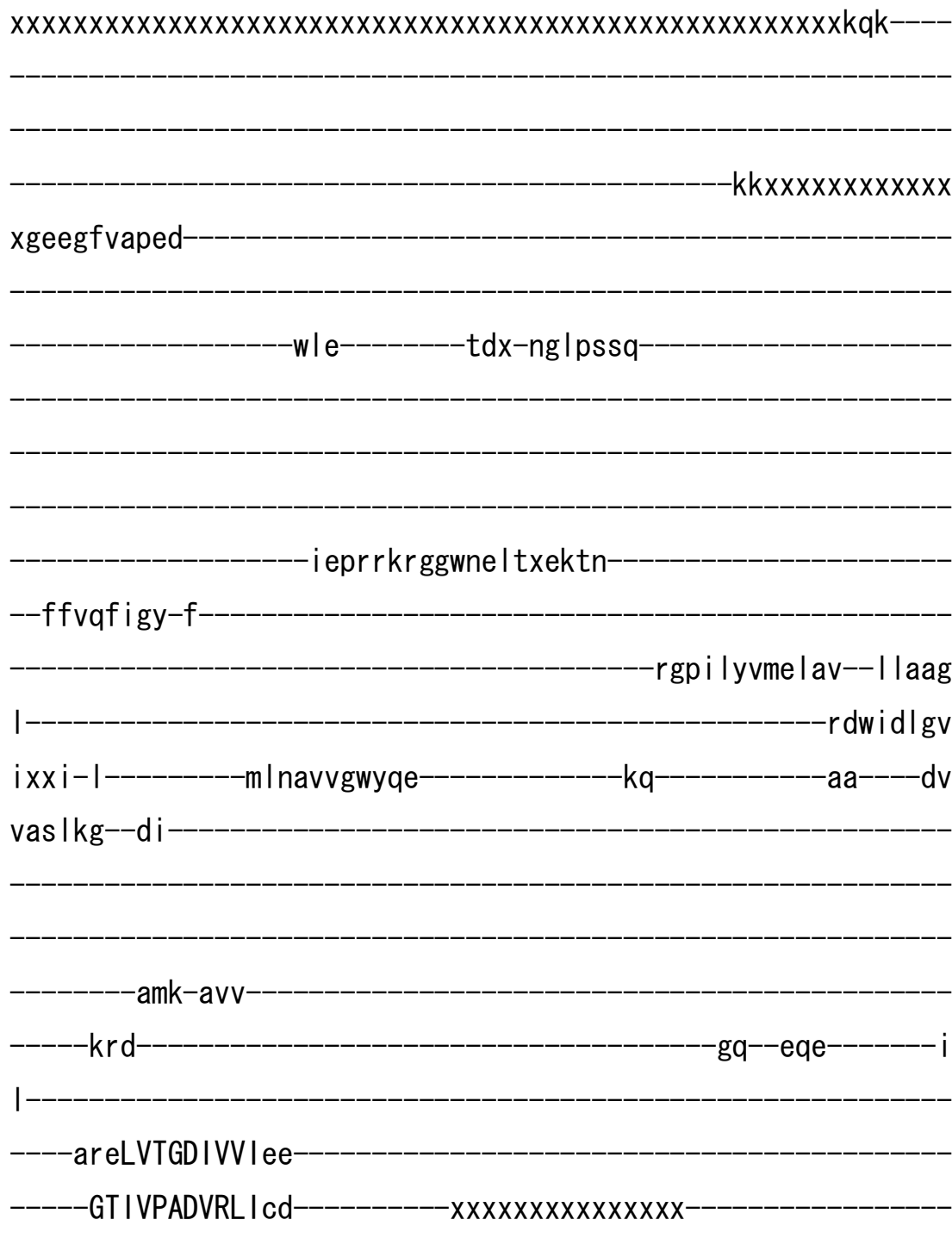

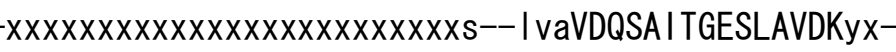

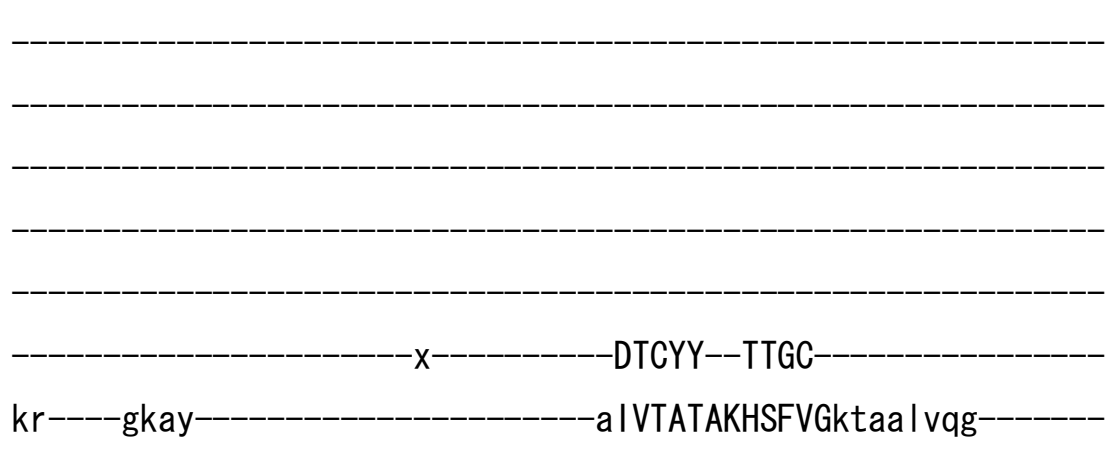

-aqdqghfkavmdni gts I I I vmfwi laaw

-i ggfyrh I kxxxxxx--_-_-_-_-_-_-_-_-_-_-_-_-_-_sdnt I I hwtL ILLI IG VPVGLPVVtTTTLAVGAA-YLaeqk- 
aIVQ-KLTAIESLAGVDILCSDKTG

TLTANQLSIr

-epyvnegvdxx

mavaa i a------snhx

-vknldpid--kvti It

xxxxpka--rei lar

nwvtekytpfdpvsk-rittxxxxxxxxxxx

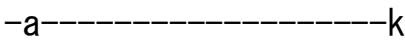

gap

k

a i l amsxxx

-peeaqkf-rekasef--arr-----gfrsLGVAVQke gepwq I LGMYPMFDPPREDTAHT I AEAQH I g-LSV

KMLTGDALA I AKETCKMLAL-

stkvydser I

$\mathrm{xxx}$ glagsaqhd 


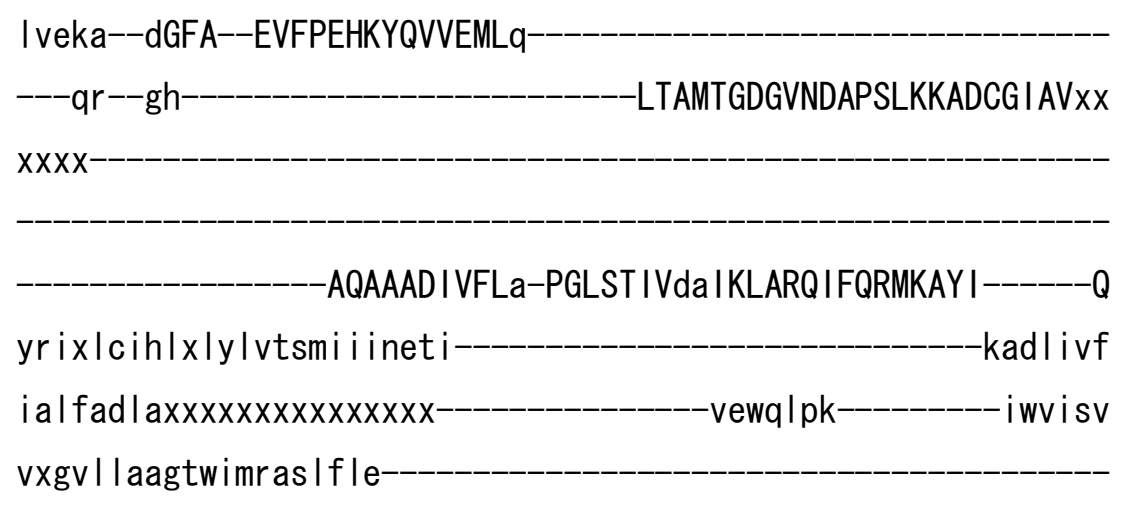




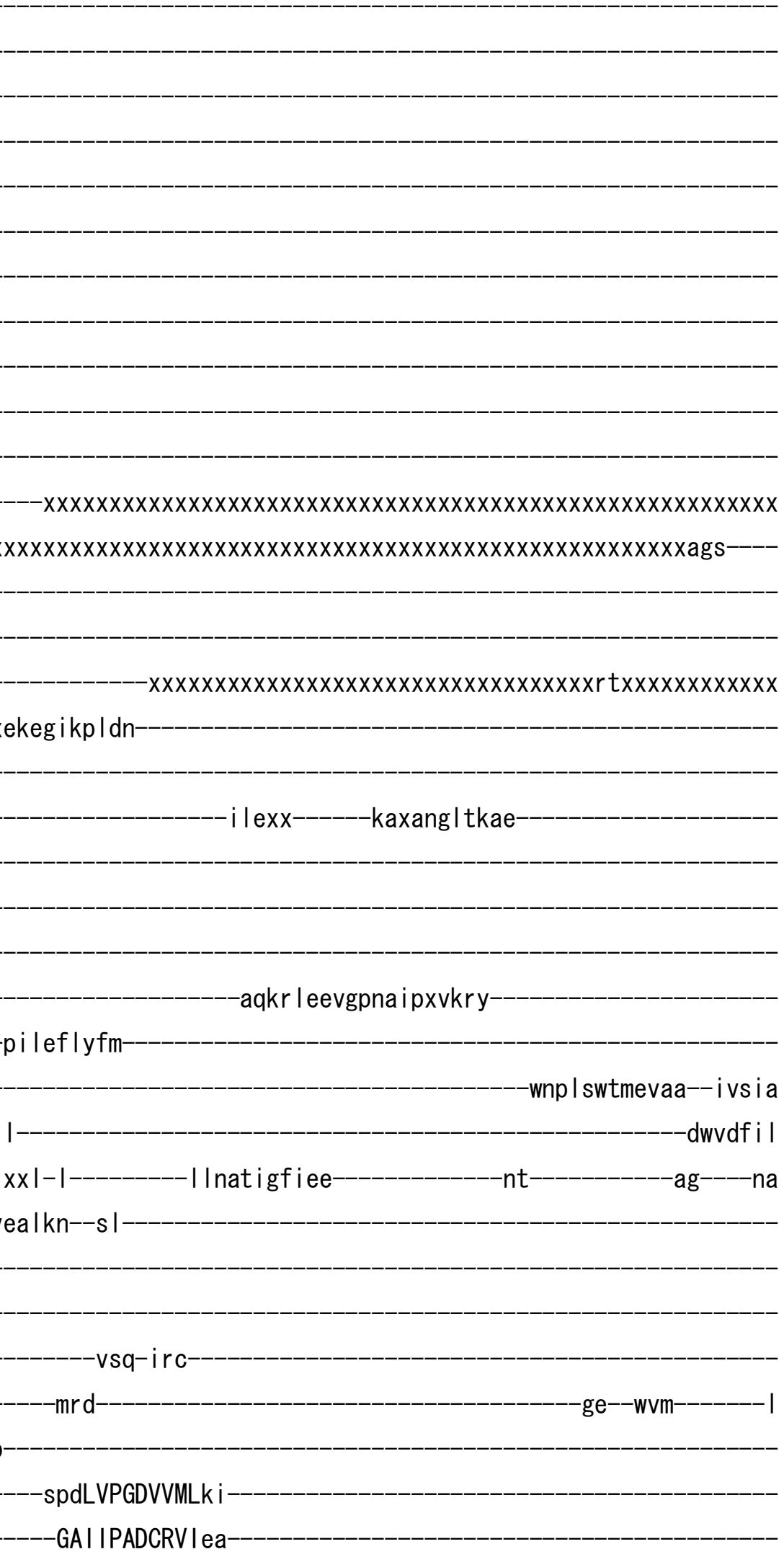


e--qvkIDQSSLTGESLPVTKk--

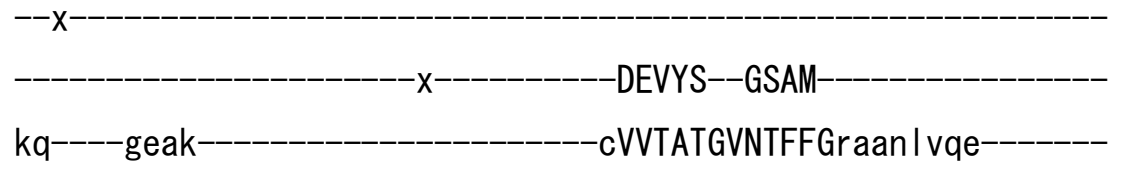

-teghgh Iqvi IrnigIfcisfiaiwvIvel

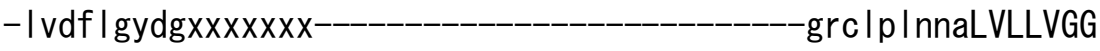
IPIAMPTVISVTMA IGAT-QLskkk--

-a IVS-RLASIEELAAMDILCSDKTG

-TLTLNILTVde - p pvgdtpk

ed

ivfhaf I-----acsx-

gedqda id--ka i sny

crdxxpnvdys

gne i vkhypfnpedkxamg I

-v----------xaxgkqfktak-

q

- i i r reaxxx 
-kqvgeav-eke i en I--adr----gyraLGVSVSyd apdfkvwhfEGL IPLFDPPRHDTEDT IKRALEmg-VSV

KMI TGDQLA I AKETARRLGM-

ggnlftipylennd I

gi sege

v i ema--dGFA--EMWPEHKYKVVDQLq-

---kr--kh--_----_-_-_-_---_-_----_VVGMTGDGVNDAPALKKAQI G I AV-$-x X X X X X$

ARSVSD IVLTs-SGLSVI I da I ISSRK IFQRMRNYV

ysv-aatvr-icttfgiltvawnf-kfptiatvi i

a i Indgtm -Iti skdrvrarnepdqwn I fev

f-tmalcygfylvgstivffai ihdgtwfhxxxxx-

rixxxxelrgliylqvsisglatxfvsrsqgfsyferpxxxxxxxfvmsqivatfigvyg frgyphdsfsdnpdypvhgtnxxxxxxxxxxxxx-

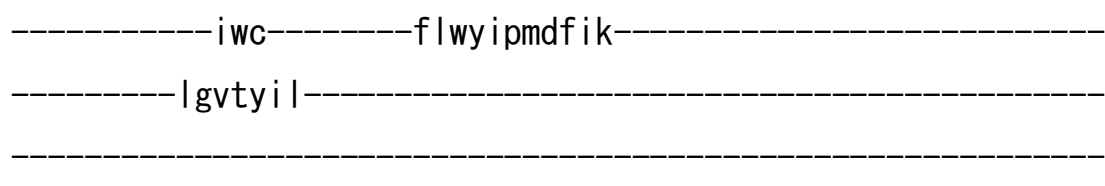

rgki ep inkdal rki ygwfgke ipkeatqvsh

kvaeqqakrdalhxxx 
xhhksvvtdnk

V

>athIII_IIIA01 NP_179486. 1

m

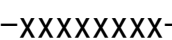

$-x d 1$

-ekipieevfq

qlk

ct-reglttqe-

-gedr i v i fgpnk l e-ekke

-skilkflgf-m- 


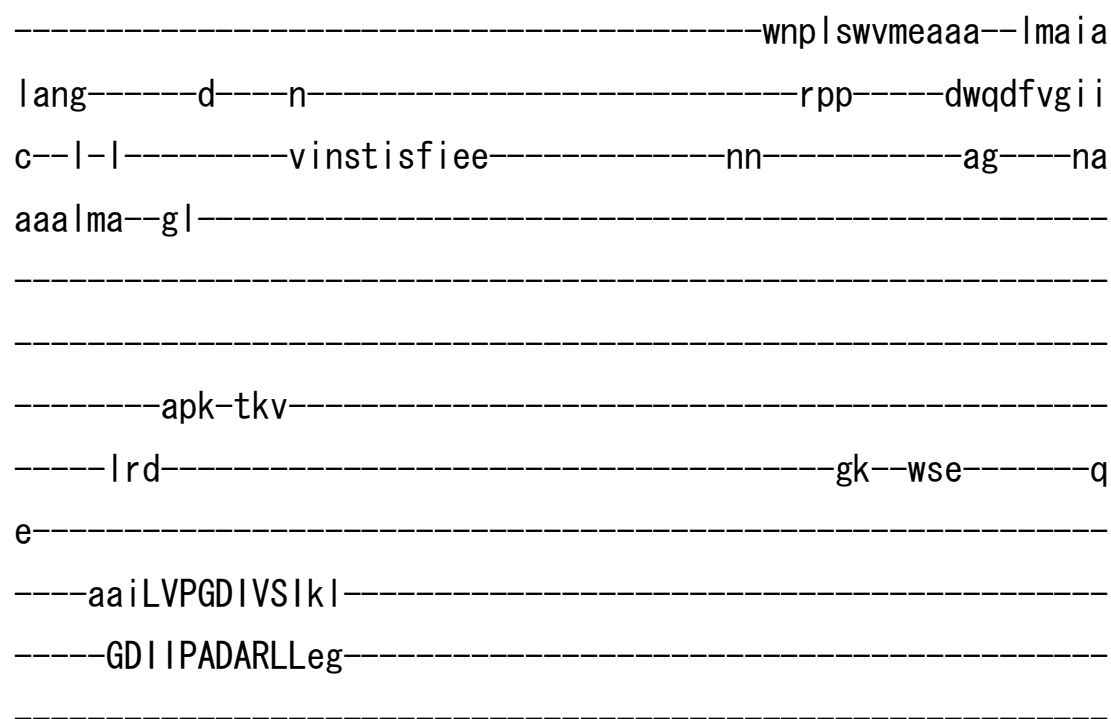

d--p lkVDQSALTGESLPVTKhx-

-QEVFS--GSTC-

kq----ge i e----_---_---_-_-----aVVIATGVHTFFGkaah lvds

-tnqughfakvltsignfcicsiaigiaie i

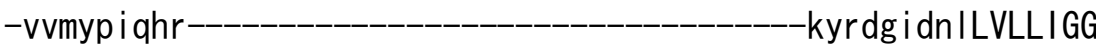

IPI AMPTVISVTMA IGSH-RLsqqg-

-a ITK-RMTAIEEMAGMDVLCSDKTG

TLTLNKLSVdknIV -evfckgvek -

v I I faam-----asr- 
lad--pkeara

gi revhf Ipfnpvdk-rtal

-t-----y ids-d-gnwhrvsk-

-gap

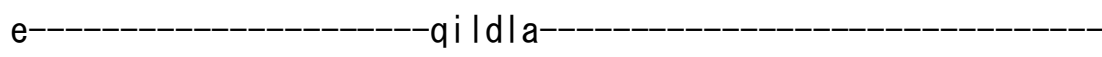

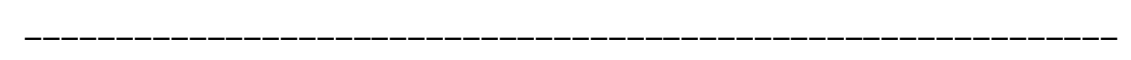

--narpd I rkkv-I sc i dky--aer----g | rsLAVARQvv

-pektkespggpwefVGLLPLFDPPRHDSAETIRRALN I g-VNV

KMI TGDQLA I GKETGRRLGM-

-gtnmypsaal l gtdkds

nias ipvee-

I i eka--dGFA--GVFPEHKYE IVKKLq

---er--kh---_--_--_-_-_-_-_-_-_-_--_IVGMTGDGVNDAPALKKAD I G I AV--

-ARGASD I VLTe-PGLSVI I saVLTSRA IFQRMKNYT $-1$ 


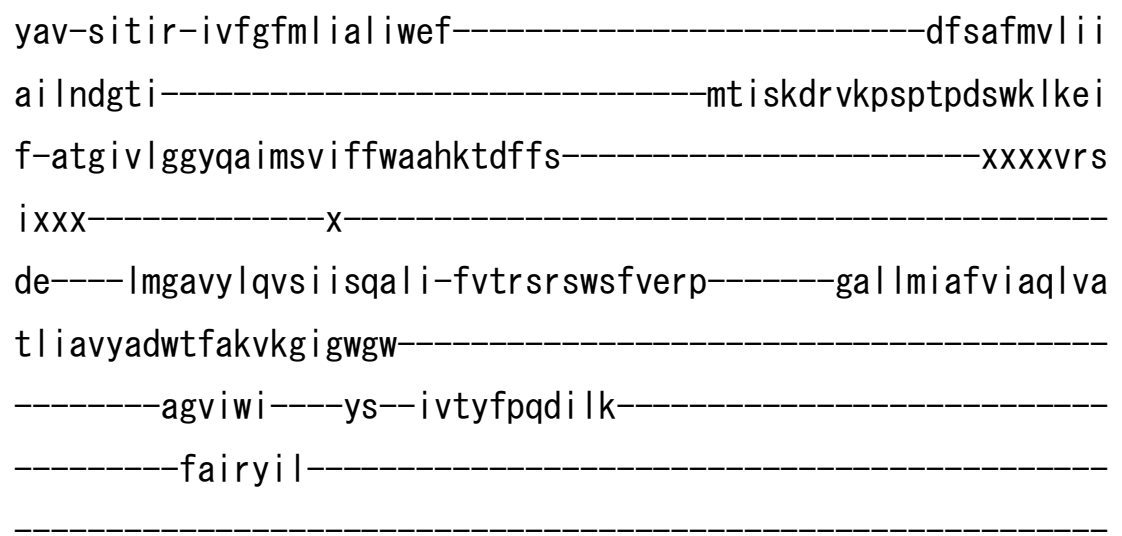

-sgkawas I fdnrtafttkkdyg i gereaqwaq

aqrt Ihg I qpkedxx-

-ifpekgsyrel se iaeqakrrae iar Ixxxxxxxxxxxxxxxxxxxx $x x x x x-x x x x$

V

>athIII_IIIA02 NP_194748.1

m- 


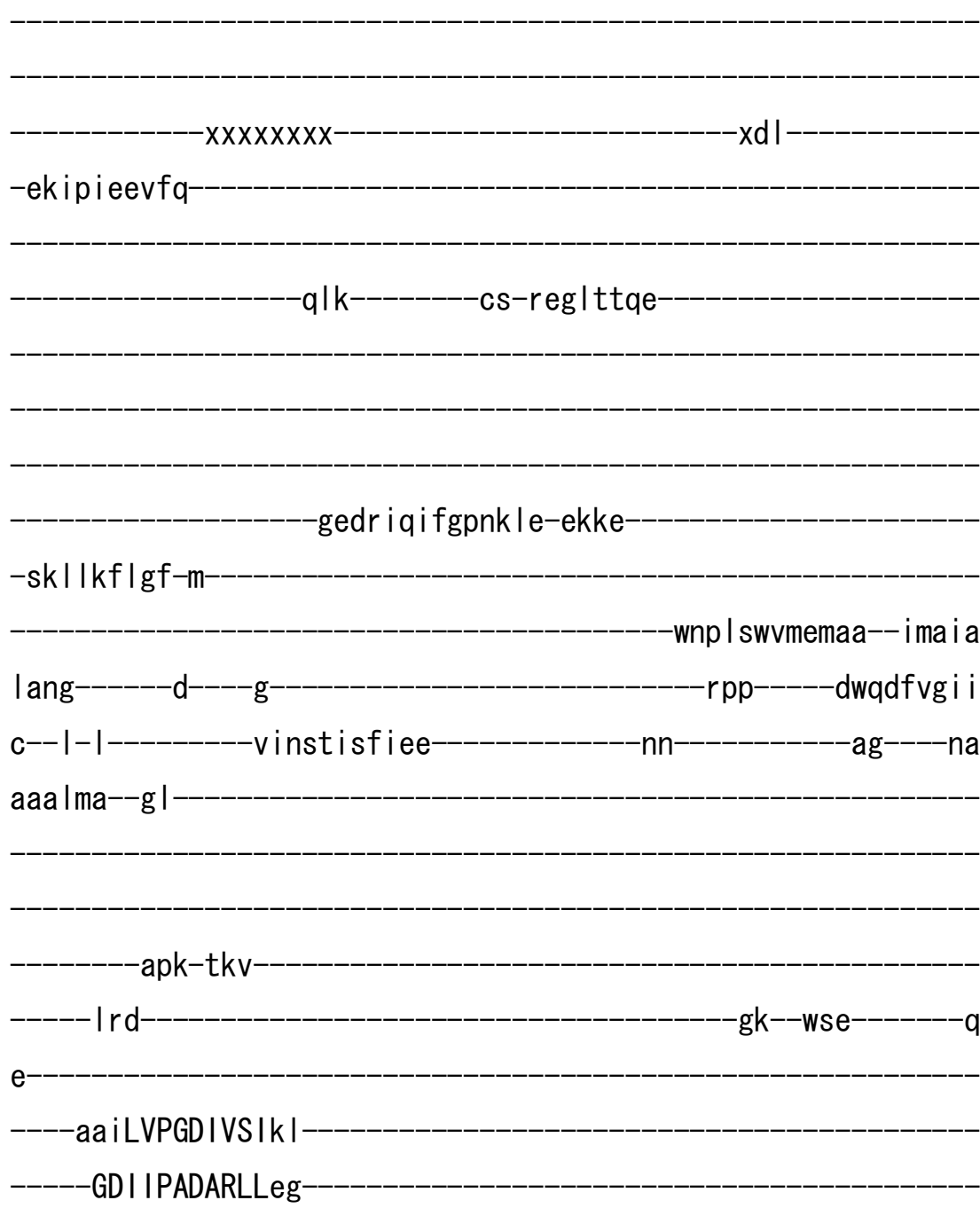

d--p I kVDQSALTGESLPVTKhx-

$$
x
$$

QEVFS--GSTC-

kq----ge i e----_---_-_-_---_-------aVVIATGVHTFFGkaah I vds

-tnqvghfqkvl tai gnfcics i a i gmvi i i

-ivmyp iqr r-

-kyrdgidnILVLLIGG

IPI AMPTVISVTMA IGSH-RLsqqg- 
-al TK-RMTAIEEMAGMDVLCSDKTG-

$-T L T L N K L S V d k n$ I evfckgvek

$-d q$

v I Ifaam-----asr

venqdaid--aamvgm

lad--pkeara-

girevhf Ipfnpvdk-rtal

-t-----yidg-s-gnwhrvsk

gap

$e^{-}$

qilela

--kasnd l skkv- I s i idky--aer----g I r sLAVARQvv

-pektkespgapwefVGLLPLFDPPRHDSAET IRRALN I g-VNV

KM I TGDQLAIGKETGRRLGM-

gtnmypssal I gthkda

nlas ipvee 
I i eka--dGFA--GVFPEHKYEIVKKLq-

---er--kh-

-IVGMTGDGVNDAPALKKAD I G I AV--

$x x x x x$

ARGASD I VLTe-PGLSVI I saVLTSRAIFQRMKNYT

dfsafmvl i i

yav-sitir-ivfgfmlialiwef-

-mt i skdrvkpsptpdswk Ike i

ai Indgti

$-x x x x v r s$

f-atgvv Iggyqa imtviffwaahktdffs-

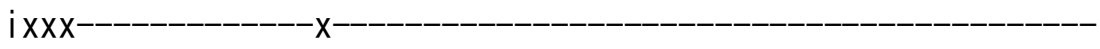

he-----Imgavy lqvsi i sqal i-fvtrsrswsfverp--------gal Imi af I i aql ia tl iavyanwefakirg i gwgw

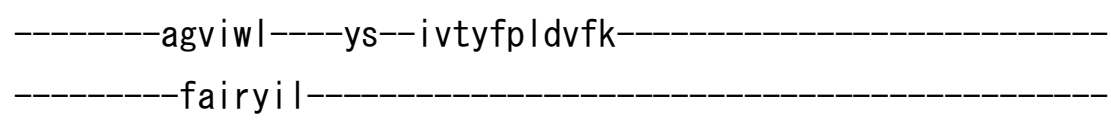

-sgkaw InI fenktaftmkkdygkeer eaqwa I

aqrt Ihg I qpkeaxx-

--ifpekgsyrelse iaeqakrrae iar Ixxxxxxxxxxxxxxxxxxxxx

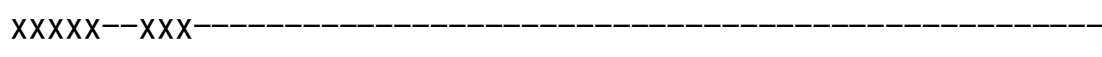

v

>athIII_IIIA03 NP_200545.1

m- 
$x d \mid$

-ekipieevfq

qlk cs-regl sgae

genr l q i fgpnk l e-ekke

-skl|kfIgf-m-

-wnp I swvmeaaa-- ima i a

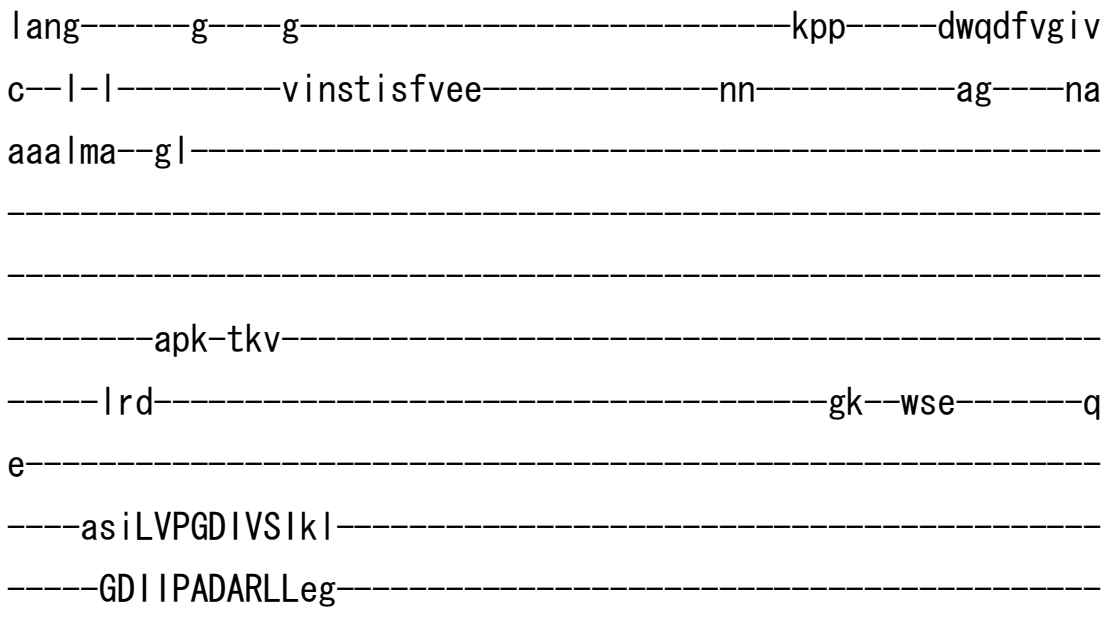


d--p I kVDQSALTGESLPATKgx-

$-x$

-EEVFS--GSTC-

$\mathrm{kq}^{----}$ge i eaVVIATGVHTFFGkaah I vds

tnqvghfqkvltaignfcicsiavgiaie i -vvmyp i arr-_-_-_-_-_-_-_-_-_-_-_-_-_-_-_-_hy dg i dn I LVLL IGG

IPIAMPTVISVTMAIGSH-KLsqqg-

-al TK-RMTAIEEMAGMDVLCSDKTG

TLTLNKLSVdknl ievyckgvek

- de

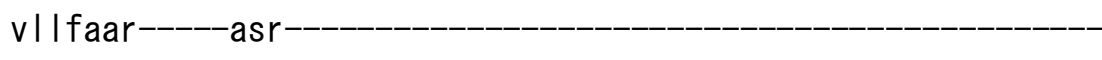

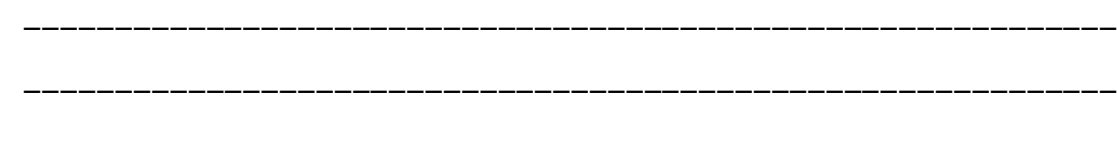

-venqda i d--aamvgm-

lad--pkeara

gireihfIpfnpvdk-rtal

-t-----fids-n-gnwhrvsk-

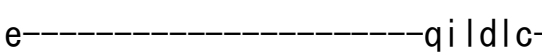


--narad I rkrv-hst i dky--aer----g|rsLAVSRQtv

pektkessgspwefVGVLPLFDPPRHDSAETIRRALDI g-VNV

KMI TGDQLAI AKETGRRLGM-

gsnmypsss I l gkhkde

amah i pved

I i eka--dGFA--GVFPEHKYE IVKKLq

$---\mathrm{er}--\mathrm{kh}-$

ICGMTGDGVNDAPALKKAD I G I AV--

$-x x x x x$

ARGASD I VLTe-PGLSVI I saVLTSRA IFQRMKNYT

yav-sitir-ivfgfmlialiwkf

dfspfmvli i

ailndgti

-mt i skdrvkpsptpdswk lke i

f-atgvv I ggyma imtvvffwaayktdffp--_-_-_-_-_-_-_-_-_-_----xxxxvrd

Ixxx--_-_-_-_-_---X-_-_-_-_-_-_-_-_-_-_-_-_-_-_-_-_-_-_-_-_-_-_

he----mmsa ly lqvs i vsqal i-fvtrsrswsfterp--_-_---gyf I I i afwvaql ia

tai avygnwefar ikg i gwgw

---------agviwl----ys--i vfyfp Idimk-

fairyil

agtawkni idnrtafttkqnyg i eer eaqwah

aqr I Ihg lantetxx- 


\section{-vvperggyre l se ianqakrrae iar I xxxxxxxxxxxxxxxxxxxxx}

\section{$x x x x x--x x x-$}

v

>athIII_IIIA04 NP_190378.2

m

-envpi ieevfe-

$-n \mid r$ cs-keg Ittqa-ader la I fghnk l e-ekke-

-skflkflgf-m- 
-wnp I swvmeaaa--imaia

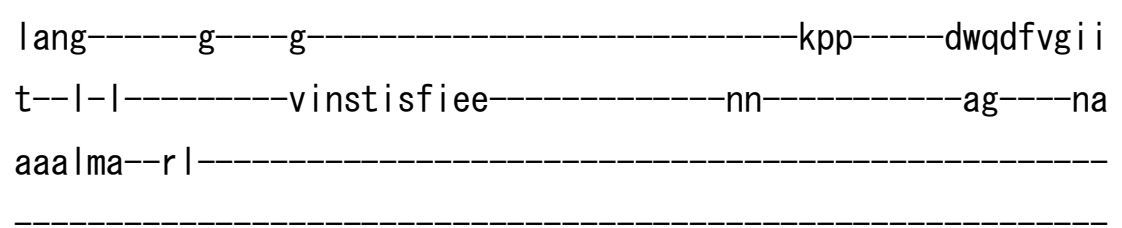

$--a p k-a k v-$

d-------_ I d--_-_-_-_-_-_-_-_-_-_-_-_-_-_-_-_-_-_-_-_-_-_-_-_-_-_-_-_-_-_-_-_-_-_

aaiLVPGDI|S|k|

GDIVPADARLLeg

$-d--p$ IkIDQSALTGESLPVTKsx-

-DGVYS--GSTC-

kq----ge i e---_--_-_-_-_-_-_-_-_aVVIATGVHTFFGkaah I vdt

-tnq i ghfqqv I ta ignfcics i avgml i e i

-vvmypiahr-ayrpgidnILVLLIGG

IPIAMPTVISVTMA IGSH-RLsqqg-

-alTK-RMTAIEEMAGMDVLCSDKTG-

TLTLNKLTVdknl i-evfmkgvda-

$-d t$

vv Imaar------asr-- 
lad--pkdara

giqevhf Ipfnptdk-rtal

-t-----y idn-e-gnthrvsk-

-gap

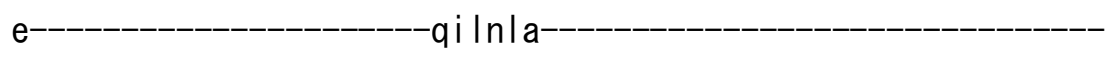

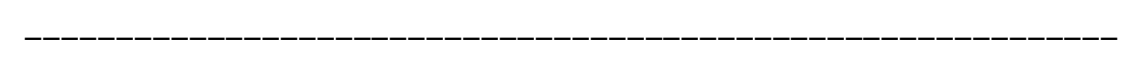

--hnkse i errv-hav idkf--aer----g I rsLAVAYQdv

-pegrkdsaggpwafVGLMPLFDPPRHDSAETIRRALN I g-VSV

KMI TGDQLA I GKETGRRLGM-

-gtnmypssal I gqnkde

sivalpvde-

I i eka--dGFA--GVFPEHKYEI VKRLq-

---ar--kh---_--_-_-_-_-_-_-_-_-_ ICGMTGDGVNDAPALKKAD I G I AV-- 


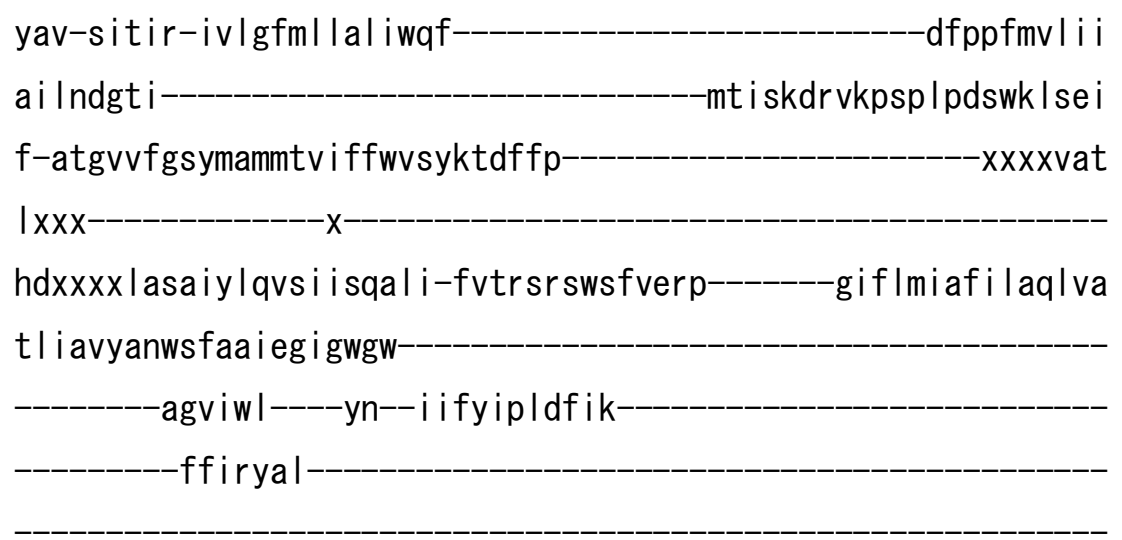

-sgrawd I v i eqrvaftrqkdfgkeqre I qwah aqrt Ihg l qapdtx

-mftdrthvse Inqmaeeakr rae iar I xxxxxxxxxxxxxxxxxxxxx $x x x x x-x x x x$

V

>athIII_IIIA05 NP_180028.1

$\mathrm{m}$ 


\section{-eevfe}

-elk

ct-kgg I tane

-ashr I dvfgpnk l e-ekke

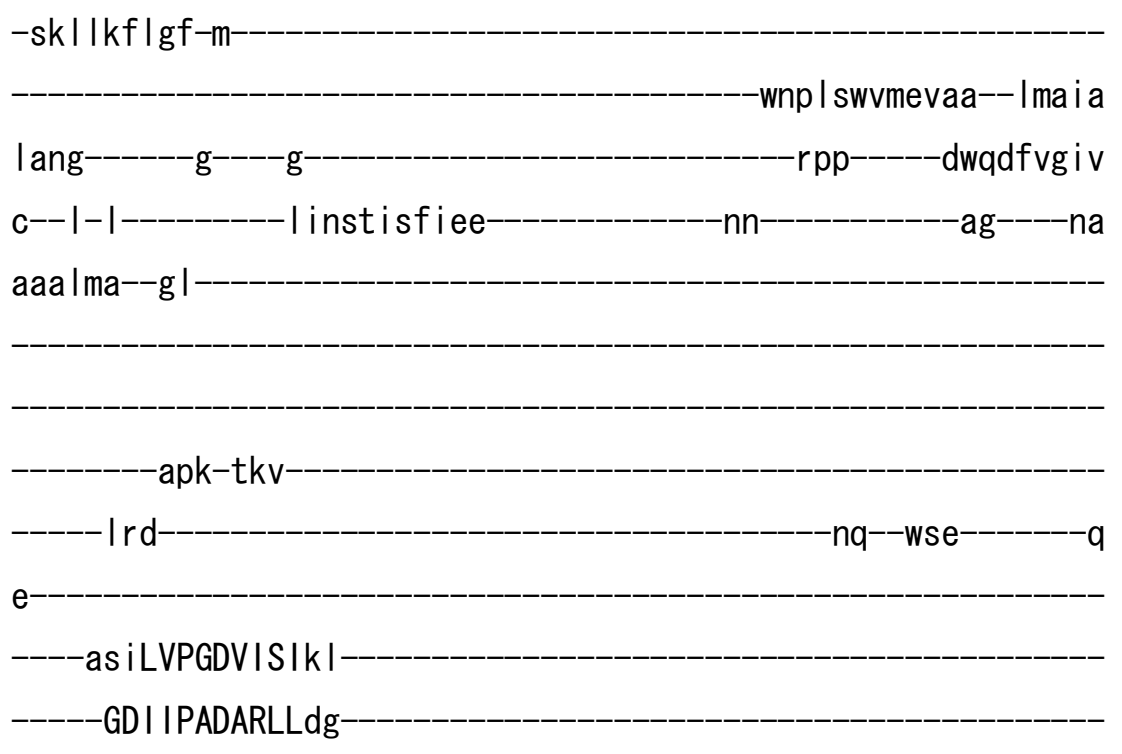

d--p IkIDQSSLTGESIPVTKn--

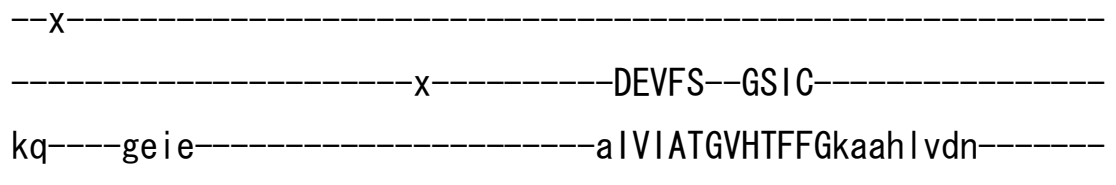

-tnq i ghfqkvI ts ignfcics i al g i ive I

-I vmyp iqr r

ryrdg idn ILVLLIGG

IPI AMPSV I SVTMATGSH-RL fqqg- 
-al TK-RMTAIEEMAGMDVLCCDKTG-

TLTLNKLTVdkn I v evfakgvgk

vf I laar-----asr-

i enqdaid--aai vgm

lad--pkeara

gvrevhffpfnpvdk-rtal

-t-----yvds-d-gnwhrask

gap

$e^{-}$

qi Inlc-

--nckedvrrkv-hgvidkf--aer----g I r sLAVARQev

l ekkkdapggpwq I VGLLPLFDPPRHDSAET I RRALN I g-VNV

KM I TGDQLAIGKETGRRLGM-

gtnmypssal I gqvkds

slgalpvde 
I i eka--dGFA--GVFPEHKYE IVHRLq-

--- qr--nh-

ICGMTGDGVNDAPALKKADIGI AVxx

$x x x x$

ARGASD I VLTe-PGLSVI I saVLTSRAIFQRMKNYT

dfspfmvl i i

yav-sitir-ivfgfmfialiwgf-mt i skdrmkpspqpdswk Irdi

ai Indgt i $-x x x x v r p$

f-stgvv Iggyqa Imtvvffwvmkdsdffs-

Ixxx---_-_-_-_--_-_-_-_-_-_-_-_-_-_-_-_-_-_-_-_-_-_-_-_

eq----mmaa ly lqvs i i sqa I i-fvtrsrswsyaecp-------g I I I gafviaq I va tfi avyanwsfar i egagwgw

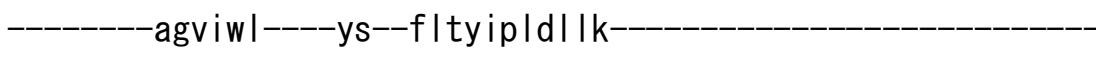
fgiryvl

-sgkaw Inl lenktafttkkdygkeer eaqwaa

aqrt Ihg I qpaekxx

-ifneknsyse I sqiaeqakrraevvr I xxxxxxxxxxxxxxxxxxxxx

$x x x x x-x x x x$

V

>athIII_IIIA06 NP_178762.1

m- 
-xxdis

$-x x x x x x x x$

$x d \mid$

-ekipvdevfq

qlk

cs-reg|ssee

-grnr lq i fgank le-ekve

-nkflkflgf-m

-wnp I swvmeaaa-- ima i v

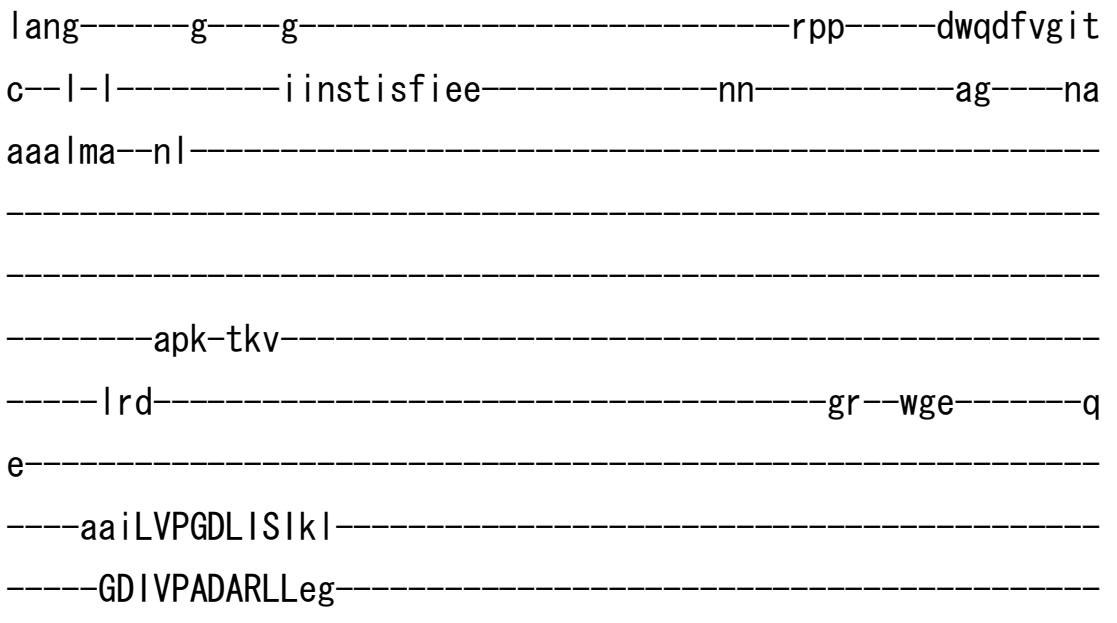


d--pIkIDQSALTGESLPATKhx-

$-x$

-DEVFS--GSTC-

$\mathrm{kq}^{----}$ge i eaVVIATGVHTFFGkaah I vds

-tnnvghfqkvl ta ignfc ics i g i gml i e i

- i imyp iqhr-kyrdg i dn ILVLLIGG

IPIAMPTV I SVTMA IGSH-RLsqqg-

-alTK-RMTA IEEMAGMDVLCSDKTG-

-TLTLNKLTVdkn l ievfskdvdk

villsar-----asr-

-venqda i d--ts i vnm

Igd--pkear a

gi tevhf Ipfnpvek-rtai

-t-----y i dt-n-gewhrcsk-

e

-qi ielc- 
--d lkgetkrra-he i i dkf--aer----g I rsLGVARQrv

-pekdkesagtpwefVGLLPLFDPPRHDSAET IRRALD I g-VNV

KMI TGDQLA I GKETGRRLGM-

-gtnmypsss I I-enkdd

ttggvpvde

I i eka--dGFA--GVFPEHKYEIVRKLq

---er--kh-

IVGMTGDGVNDAPALKKAD I G I AV--

$-x x x x x$

-ARSASD IVLTe-PGLSVIVsaVLTSRA IFQRMKNYT

yav-sitir-ivlgfmlval iwef-

dfspfmvli i

ailndgti

-mt i skdrvkpsp i pdswk lke i

f-atgvv I gtyma I vtvvffw lahdttffs----_-_-_-_-_-_-_-_-_----xxxxvrs

I $x x x$

ee----| iavly lqvsi isqal i-fvtrsrswsfverp-

gll l iaffvaqlia

tl iatyahwefar ikgcgwgw

--------cgviwi----ys--ivtyipldi lk

-fitryt I

sgkawnnmi enr tafttkkdygrgereaqwal

aqrt Ihg Ikppes 
-mfedtatyte I se iaeqakkraevar I xxxxxxxxxxxxxxxxxxxx

$x x x x x-x x x x$

V

>athIII_IIIA07 NP_191592. 1

m

$-x x$ iea

$-x x x x x x x x-$

$-x d 1$

-envpveevfq

$-\mathrm{hlk}$

ct-kegltsne

-vqer I I fgynk le-ekke

-skilkflgf-m- 


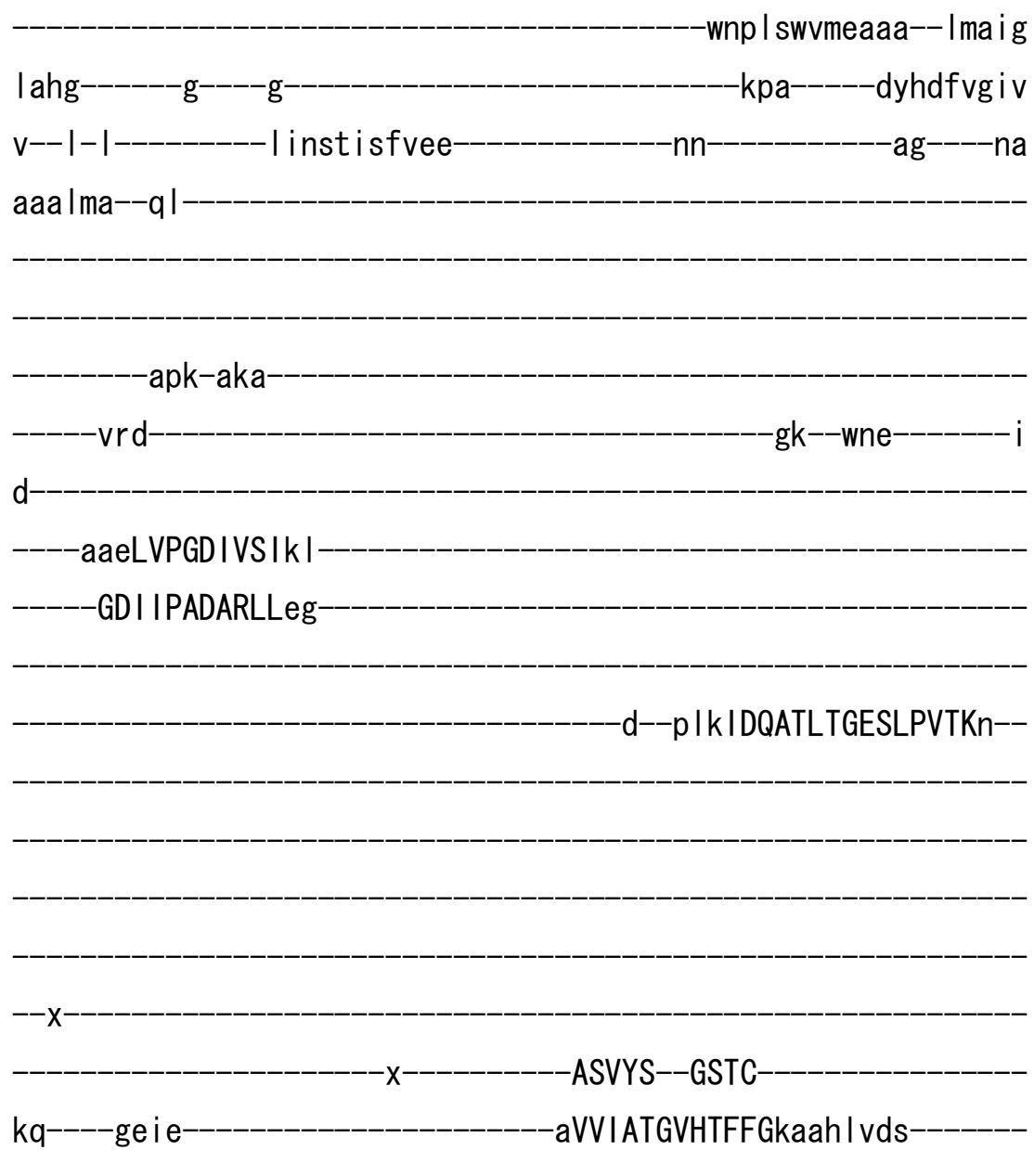

tthvghfqkvltaignfcics iavgmai i i

-vvi yg l qkr---------------------------------gyrvg i dn I LVLLIGG

IPIAMPTV ISVTMA IGAH-RLaqgg-

-alTK-RMTAIEEMAGMDVLCSDKTG

-TLTLNKLSVdknl i-----------evfkrgidr-

$-\mathrm{dm}$

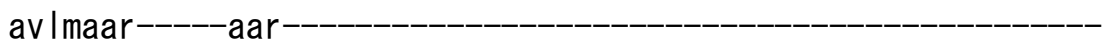




\section{lenqda id--tai vsm}

Isd--pkeara

gikelhflpfspanr-rtal

-t-----yldg-e-gkmhrvsk

-gap

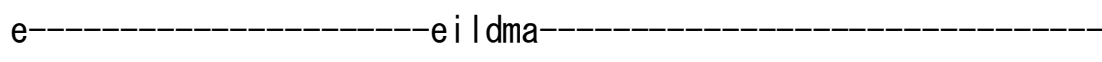

--hnk l e i kekv-hat idkf--aer----g I rsLGLAYQev

-pdgdvkgeggpwdfVALLPLFDPPRHDSAQT IERALH I g-VSV

KMI TGDQLA I AKETGRRLGM-

-gtnmypsss | Isdn-

-ntegvsvde-

I i ena--dGFA--GVFPEHKYE I VKRLq

---sr--kh-

-ICGMTGDGVNDAPALKKAD I G I AV--

$-x x x x x x$

-ARGASD I VLTe-PGLSVI I saVLTSRAIFQRMKNYT $-1$ 


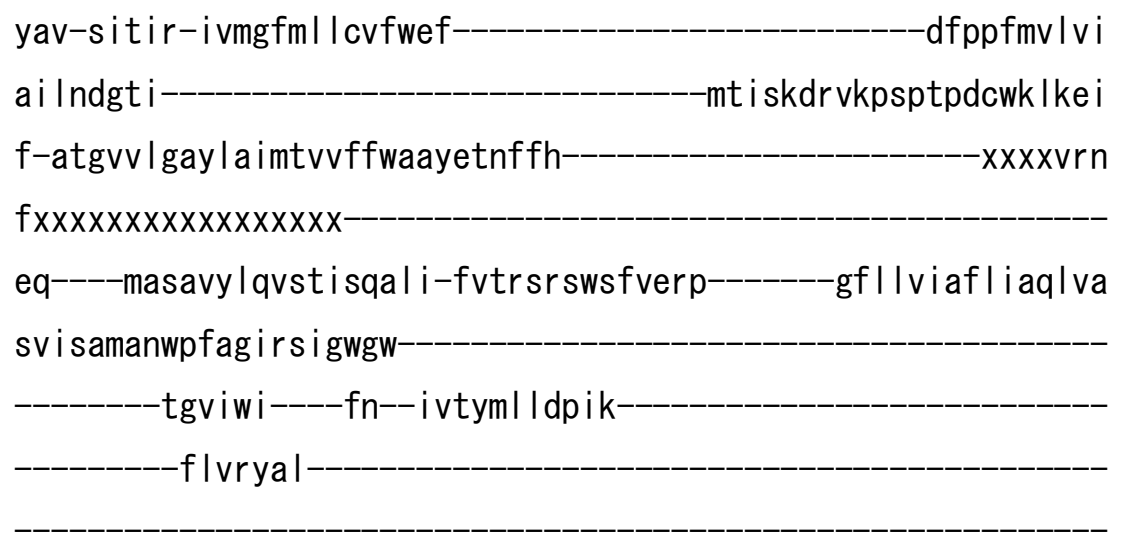

-sgkswdrmvegrtal tgkknfgqeermaawat

ekrtahg l etgqk

-pvyernsate Innmaeeakrrae iarmxxxxxxxxxxxxxxxxxxxxx $x x x x x x x x x x-$

i

>athIII_IIIA08 NP_189850.1

m- 


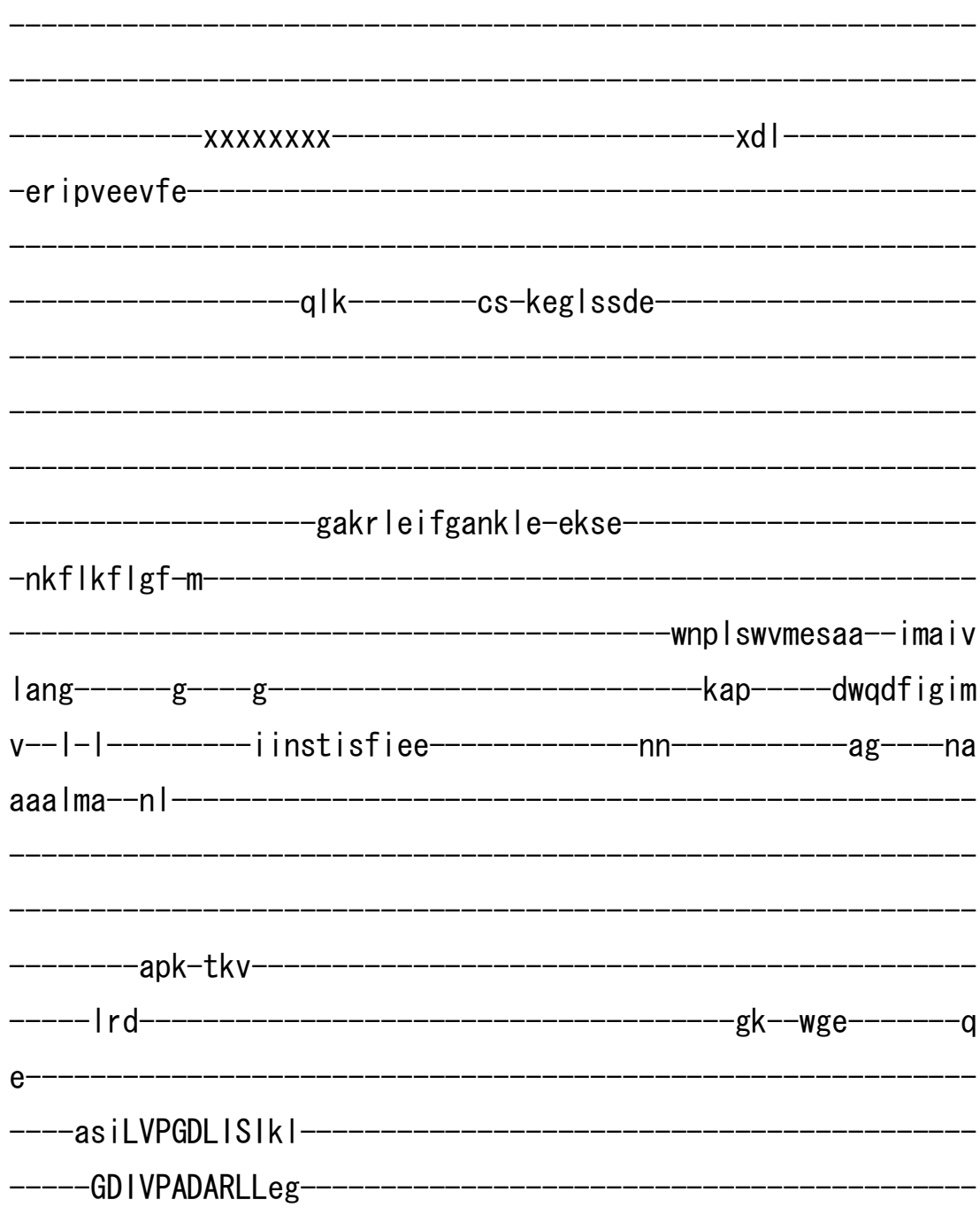

d--p IkIDQSALTGESLPTTKhx-

$$
-x
$$

-DEVFS--GSTC

kq----ge i e----_---_-_-_---_-------aVVIATGVHTFFGkaah I vds

-tnnvghfqkvltsignfcicsig Igml ie i

-I imypiahrtyrdgidnILVLLIGG

IPI AMPTV I SVTMA IGSH-RLsqqg- 
-al TK-RMTAIEEMAGMDVLCSDKTG-

TLTLNKLSVdks I i evfpknmds

vv Imaar-----asr

i enqda id--as i vgm

lgd--pkeara

gi tevhf Ipfnpvdk-rtai

-t------yide-s-gdwhrssk

gap

$e^{-}$

qi ielc-

--n l qgetkrka-hevidgf--aer----g I r sLGVAQQtv

-pektkesdgspwefVGLLPLFDPPRHDSAET IRRALE I g-VNV

KMI TGDQLA I GIETGRRLGM-

gtnmypsts I I gnskde

slvgipide 
I i eka--dGFA--GVFPEHKYEIVKKLq-

---er--kh-

- ICGMTGDGVNDAPALKKAD I G I AV--

$x x x x x$

ARSASD I VLTe-PGLSVI I saVLTSRAIFQRMKNYT

dfapfmvl i i

yav-sitir-ivlgfmlvaliwrf-mt i skdrvkpspvpdswk Ine i

ailndgt i $-x x x x v r s$

f-atgvv IgtymalttvIffw lahdtdffs $-$

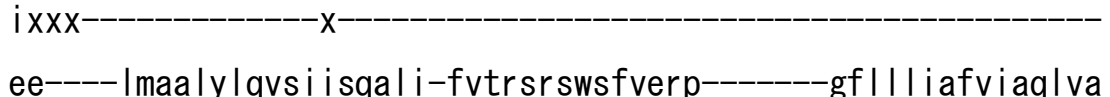
tl i avyanwgfar i vgcgwgw-

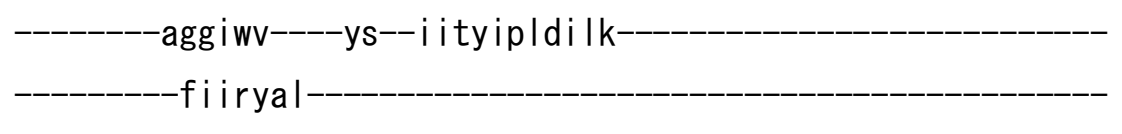

-tgkawdnminqktafttkkdygkgereaqwa I aqr I I hg I pppea

-mfndnkn--e I se i aeqakrraevar I xxxxxxxxxxxxxxxxxxxxx

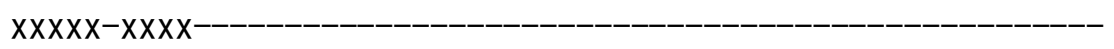

V

>athIII_IIIA09 NP_178181.1

$\mathrm{m}-$ 
$-x x----x x d s s$

$x x x x x x x x$

$-x d I$

-ekipieevlt

$-q \mid r$

ct-regItsde

gqtr l e i fgpnk le-ekke

$-n k v|k f| g f-m$

-wnp I swvme l aa--i ma i a

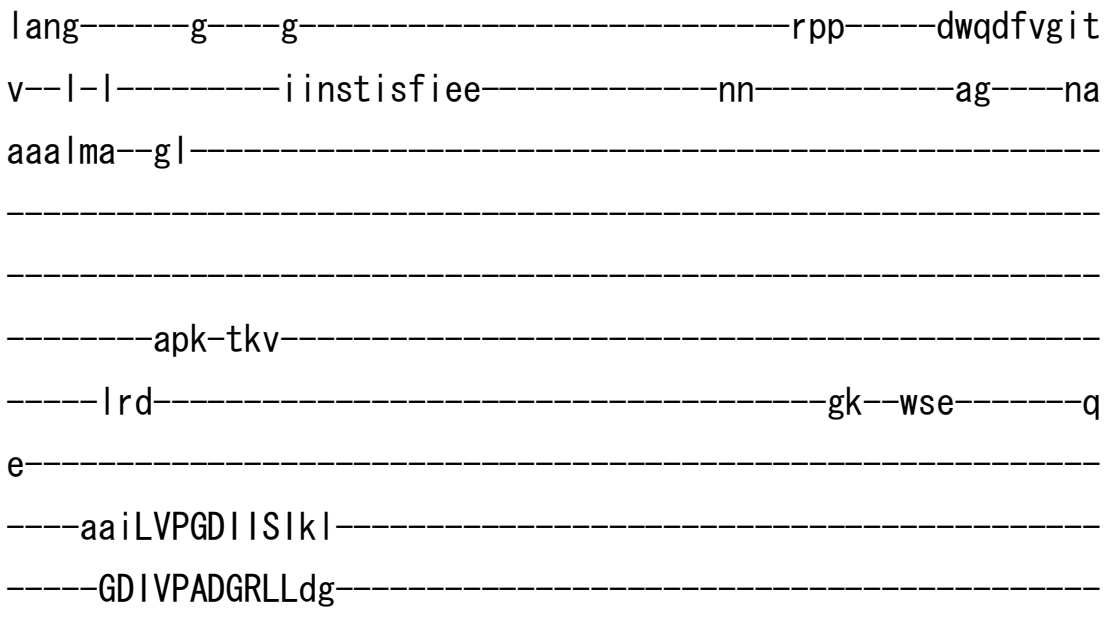


d--pIkIDQSALTGESLPVTKhx-

$x$

-QEVYS--GSTC-

$\mathrm{kq}^{----}$geleaVVI ATGVHTFFGkaah I vds

-tnqeghfqkvl ta ignfc ics i a igml i e i -vvmyp i qkr ayrdg i dn ILVLLIGG

IPIAMPTV I SVTMA IGSH-RLsqqg-

-alTK-RMTAIEEMAGMDVLCSDKTG-

-TLTLNKLTVdksmvevfvkdl dk

dq

I | vnaar------asr-

-venqda id--ac i vgm-

lgd--preare-

gi tevhffpfnpvdk-rtai

-t------y i da-n-gnwhrvsk-

e

-qi ielc 
--n I redaskra-hd i i dkf--adr----g | rsLAVGRQtv

sekdknspgepwqfLGLLPLFDPPRHDSAET I RRALD I g-VNV

KMI TGDQLA I GKETGRRLGM-

gtnmypssal I gqdkde

sias Ipvde-

I i eka--dGFA--GVFPEHKYEIVKRLq

$---\mathrm{em}--\mathrm{kh}-$

ICGMTGDGVNDAPALKRAD I G I AV--

$-x x x x x$

-ARSASD IVLTe-PGLSVIVsaVLTSRA IFQRMKNYT

yav-sitir-ivmgfml lal iwkf

dfspfmvliv

ailndgti

-mt i skdrvkpsp I pdswk Ike i

f-atgvv l gty lavmtvvffwaaestdffs---_-_-_-_-_-_-_-_-_-_---Xxxxvrs

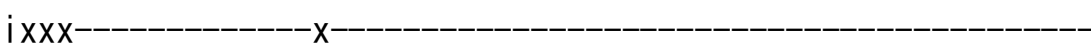

he-----I taavy lqvs i vsqal i-fvtrsrswsyverp--_-_---gfwl i saffmaql ia tl iavyanwnfarirgigwgw

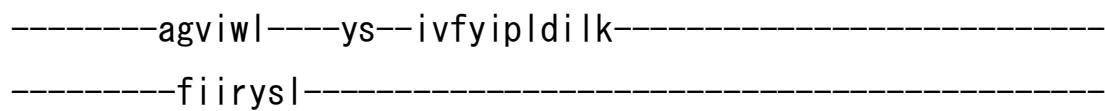

sgrawdnv i enktaftskkdygkgereaqwaq

aqrt Ihg I qpaqtxx- 
-mfndkstyre I se iadqakr raevar I Xxxxxxxxxxxxxxxxxxxxxx

$x x x x x-x x x x$

।

>athIII_IIIA10 NP_173169.2

$$
\text { m }
$$

$-x x x x x-x x|| d$

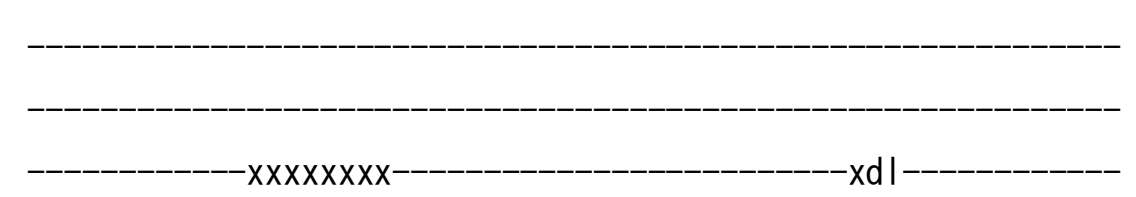

-gilpleevfe

$$
\text { -y | r--_-_---_ts-pqg | | sgd- }
$$

-aeer Ik i fgpnr le-ekqe

$-n r f v k f l g f-m$ 


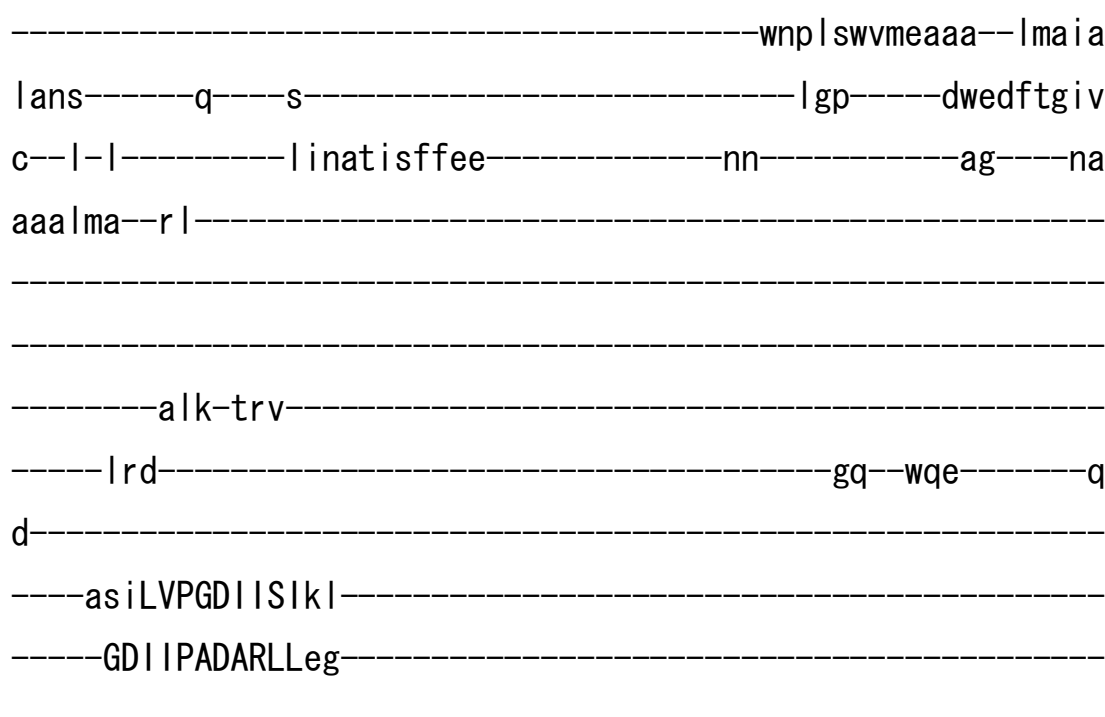

---p IkIDQSVLTGESLPVTKk--

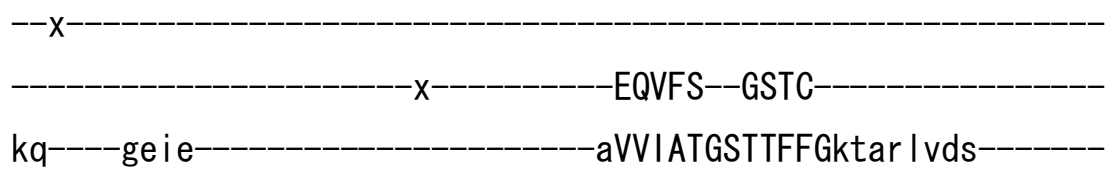

-tdvtghfqqults ignfcicsiavgmvle i

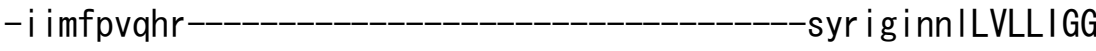

IPIAMPTVISVTLAIGSH-RLsqqg-

-al TK-RMTAIEEMAGMDVLCCDKTG

TLTLNSLTVdknl i -evfvdymdk

$-d t$

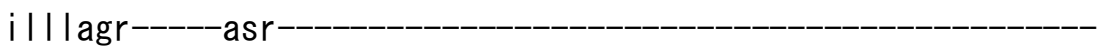


lad--preara

-nireihfIpfnpvdk-rtai

-t-----y ids-d-gkwyratk

-gap

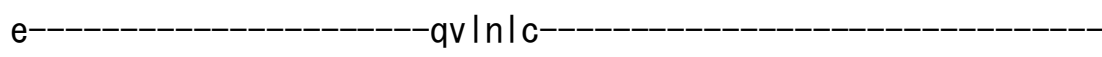

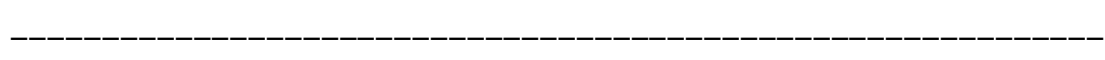

--qqkne i aqrv-ya i idrf--aek----g I rsLAVAYQe i

-peksnnspggpwr fCGLLPLFDPPRHDSGETILRALSI g-VCV

KMI TGDQLA I AKETGRRLGM-

-gtnmypsss I I ghnnd

eheaipvde-

I i ema--dGFA--GVFPEHKYEI VKILq-

$---\mathrm{em}--\mathrm{kh}-$

-VVGMTGDGVNDAPALKKADIG I AV--

$-x x x x x x$ 


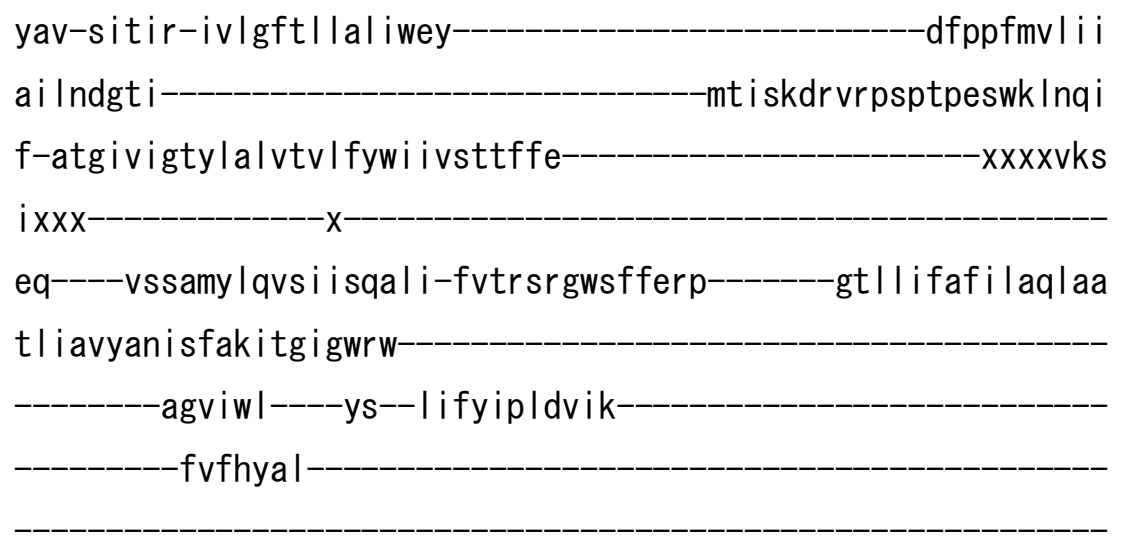

-sgeawn I v ldrktaftykkdygkddgspnvt i sqrsrsaeel-

rgsrsraswiaeqtrrrae iar Ixxxxxxxxxxxxxxxxxxxxxx $x X X X X X X X$

V

>athIII_IIIA11 NP_201073.1

m- 


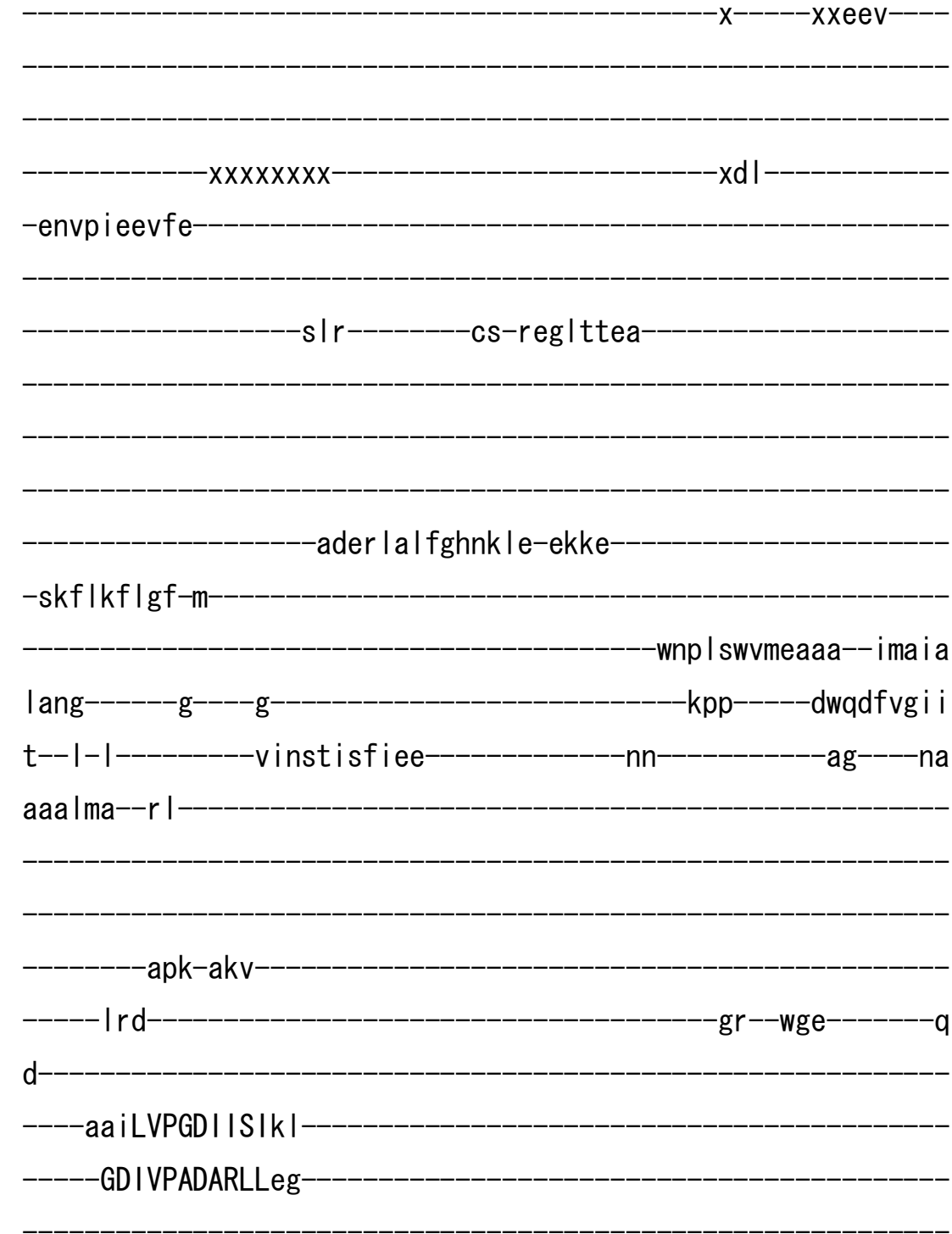

d--p IkIDQSSLTGESLPVTKgx-
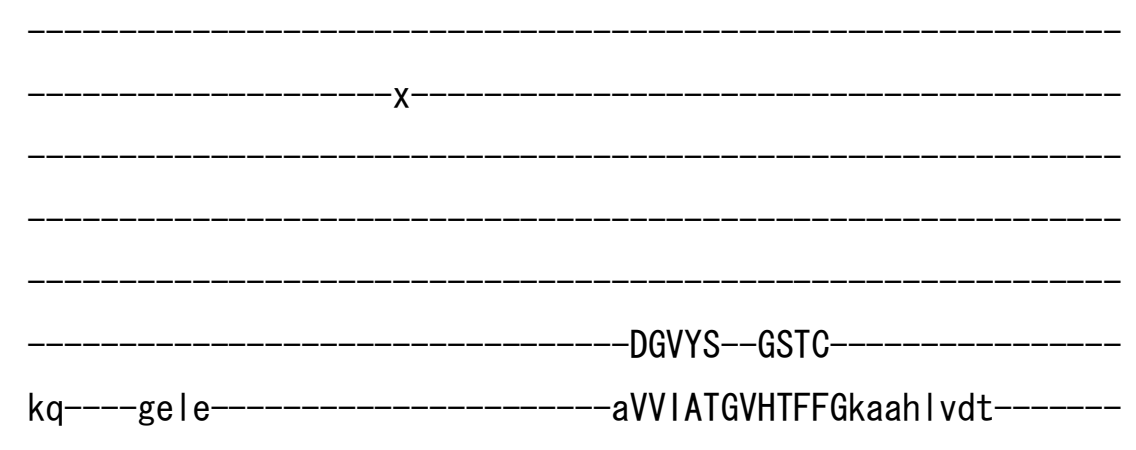

-tnhvghfqqv I tai gnfc ics i avgmi i i i

-vvmyp i qhr--_-_-_-_-_-_-_-_-_-_-_-_-_-_-_-_ayrpg i dn I LVLL I GG

IPI AMPTV I SVTMA IGSH-RLsqqg- 
-al TK-RMTAIEEMAGMDVLCSDKTG-

TLTLNKLTVdkn l ievftkgvda-

$-d t$

vv Imaaq-----asr

lenqda id--aa i vgm-

lad--pkeara

gvrevhf Ipfnptdk-rtal

-t-----yids-d-gkmhrvsk-

gap

$e^{-}$

qi $\ln l a$

--hnrae i errv-havidkf--aer----g I r sLAVAYQev

-pegtkesaggpwqfMGLMPLFDPPRHDSAET IRRALN I g-VNV

KM I TGDQLAIGKETGRRLGM-

gtnmypssal I gqhkde

sigalpidd- 
I i eka--dGFA--GVFPEHKYEIVKRLq-

$---a r--k h-$

- ICGMTGDGVNDAPALKKAD I G I AV--

$x x x x x$

ARSASD I VLTe-PGLSVI I saVLTSRAIFQRMKNYT

dfppfmvli i

yav-sitir-ivlgfmllaliwkf-mt i skdrvkpsp I pdswk I se i

a i Indgt i $-x x x x v s t$

f-atgvvfgsymammtviffwaayktdffp$-$

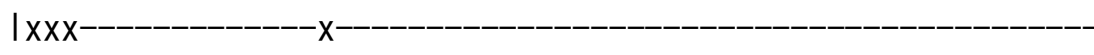

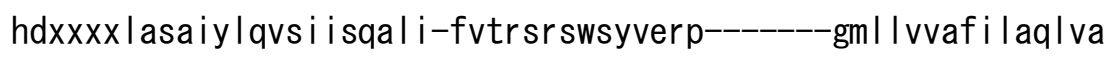
tl i avyanwsfaa i eg i gwgw

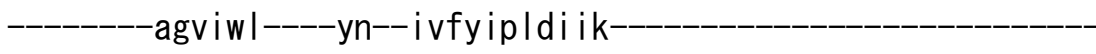
fliryal

-sgrawd I v i eqrvaftrqkdfgkeqre l qwah

aqrt Ihg I qapdax

-mfperthfnel sqmaeeakr rae iar Ixxxxxxxxxxxxxxxxxxxx xxxxx-xxxx------------------------------------------------

$\mathrm{v}$

>ddi IV_IV01 XP_643540.1

$\mathrm{m}$ 
$-x x x x x x x x x x x x x$ snnsstn

---cytIfInd-

$-x x x x x x x$

-gkkfpknfirttkyt

$-\mathrm{il}----$

-sfipknlfeqf

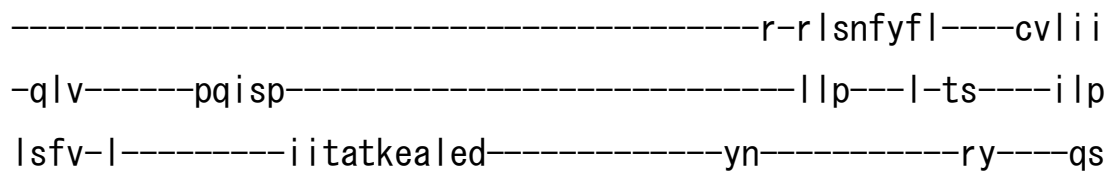

dkknn lepyt----------ivr

dkknnlepyt-ivis

d-ak I-

$$
-\mathrm{x}------\mathrm{t}-
$$

s

----sqd ICVGDI IRI qn-

GQQIPADLVLI st- 
-shdeg-I---cyVETSNLDGETNLKVRka-

|gdtnk | staedis I | rgs i vyetpner Iyrfngr i vixx

$x$

eniihs

Inh

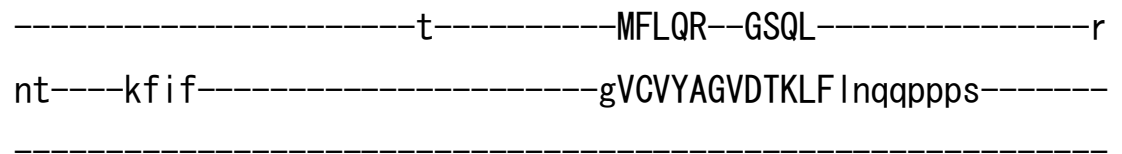

-kfstvek IInkli Ifvfifqlivcllca -vasafyqe i vvedm------- |y | g-------------xxxs | s i ygvrnfftyF ILFNTM IPISLWVTIEMVKVGQAK-FMefdsym-

$-x x x x x x x x x x x x e e k e v e k g$

cKAK-TSNLNEDLGRIQHIFSDKTG

-TL TEN IMRFc----_--_--_-----kcs i gsd iy

$x x x x x x x x x$

$-X X X X X--X X X X$

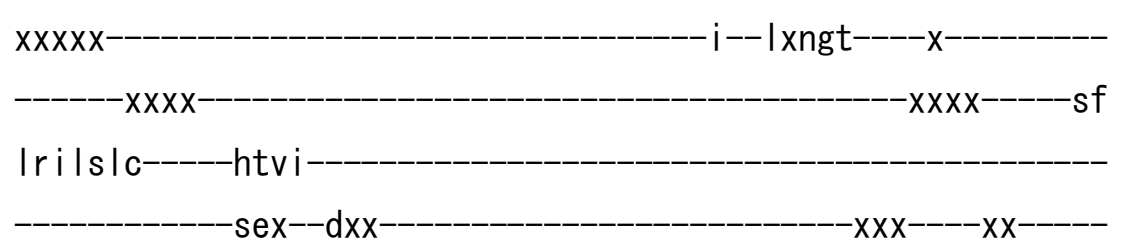

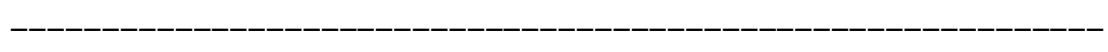

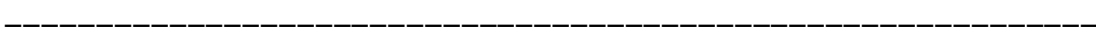

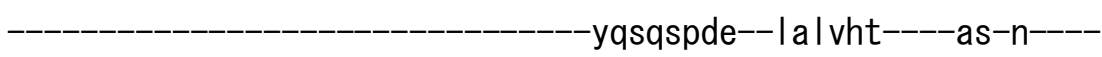

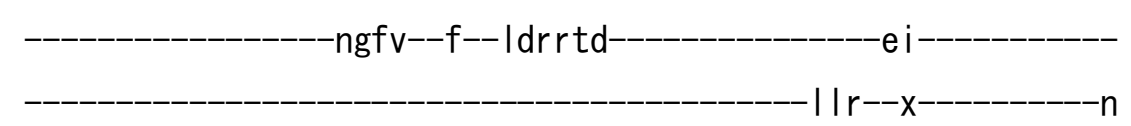

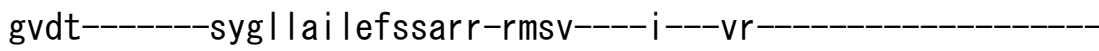

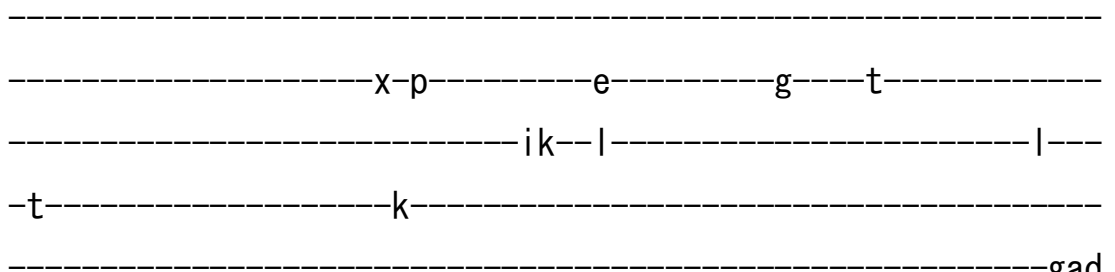

$\mathrm{m}$

-s i scr I I xxk

$-x$ 
xxx--xx--det-Inf I knf--srd----gyrtLMVAERd I--tveeyedwkqqffq--as------ts i enr eek i eavce I-i ekd I s IVGTTA IEDKLQNQVPET ISYLLNag-LH I WVLTGDKQETAVNIGYSCRLfdpamel i----fv------ntes---xx-------ecgxxxx-

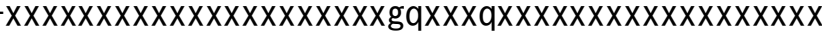
$X X X X X X X X X X X X X X X X X X$ xxeyg I vidght Itf al ndhkekfIr

I gracksv ICC--RTTPLQKALVVRVVk ---qs--ek--_-_-_-_---k--_-_-_-_-_--ISLA I GDGANDVSM I QEAHVGI GI-fgkegta

AARASDYCIHqfSHLKRLL--CVHGRYSYIRVSGL I------Q ysf-yknms-ft I c I Iwfsfss If--_-_-_-_-_-_-_-_-_-_--tgqt i fdswi itfy ni Ifts I ppffyg I fekd idee-_-_-_-_-_-_-_-_--s imenpn l yks iqqsq i I skks f-fvwn I I I whs I vtf-fgvk-I If-_-_-_-_-_-_-_xxxxxxxxxxv--_-_-_ ag--iwt I gt I vstas i I tvnvr-ma i etk I wny i s lv---_----gmi i s laayf im l v |

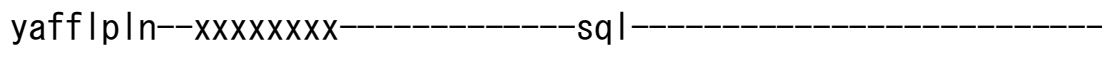
---------etgsyy-----ft-- i i vi i i va i fpd----------------------------

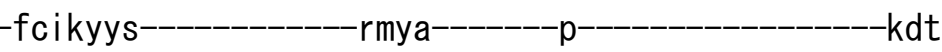
$-x \times x x x x-$ 
>ddi IV_IV02 XP_639280.1

m-

$-x x x x x x x x x x x x h n \mid d d g g$

---irtiyand-

-xxxnkkypsnr i sntkyt $-\mathrm{i}$

-tfipknImeqf 


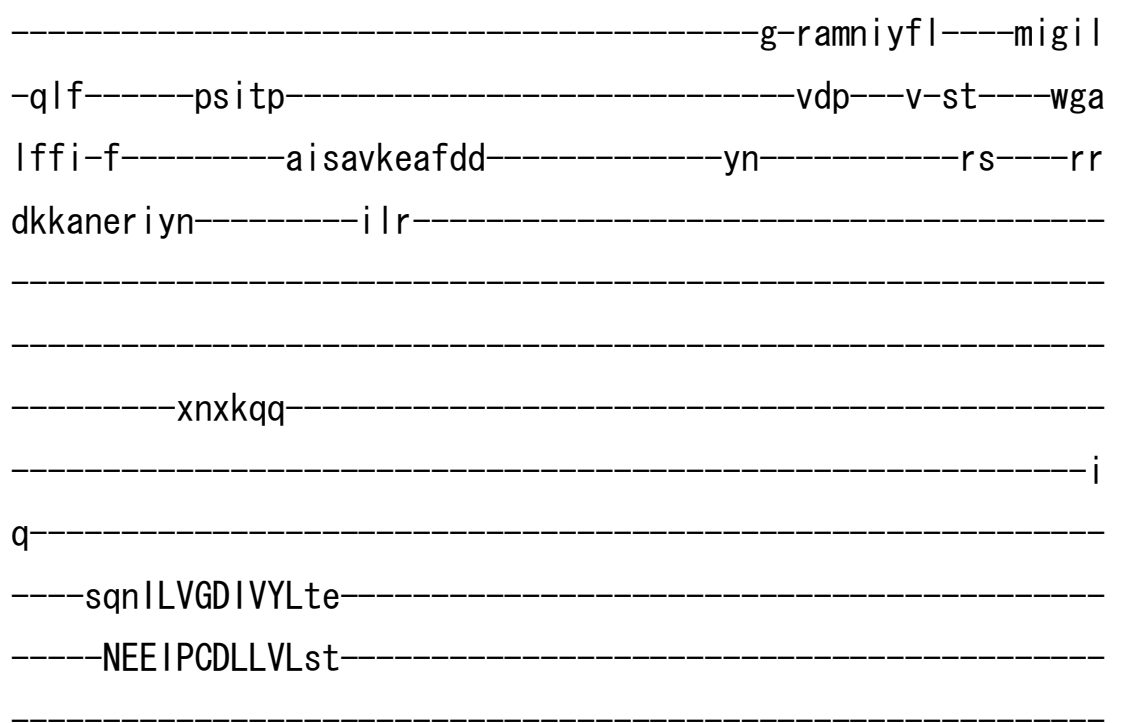

-sdk i t-n--x I yVQTSNLDGETDLKIKys-

-iketsgle Isq Iksfagv lecpvpnae inkfdsr I smxx

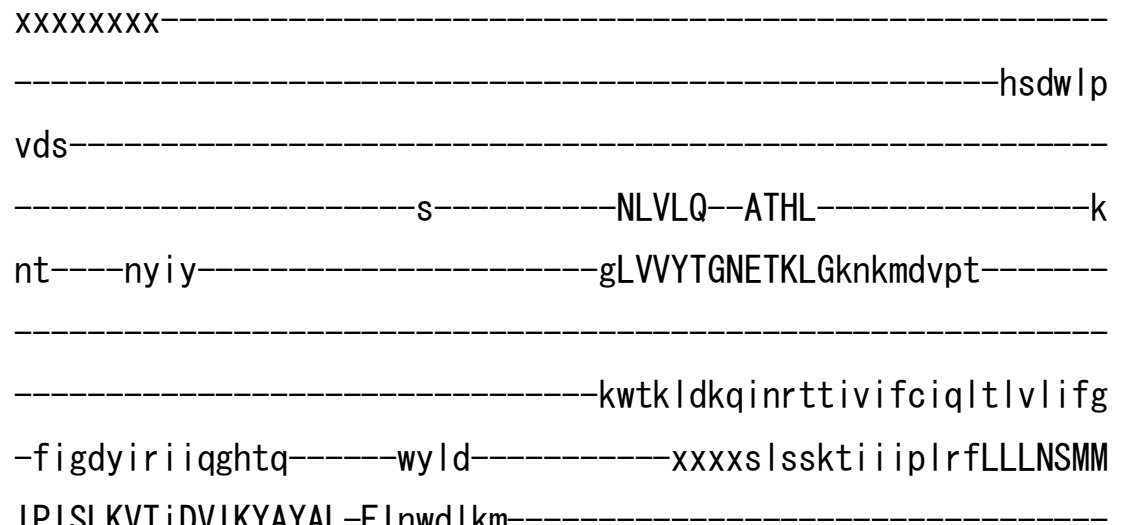

-ynsdidep-

aTAN-STALSEDLGQIEYIFTDKTG

TLTENVMLFs-

kcsing i vy-

$-x x x x x x x x x x x x x x x x x x x x$

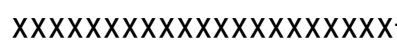

$-x x x x x x x x x x x$

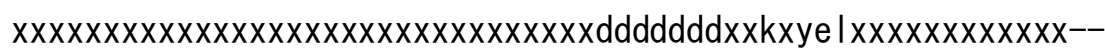

$-e f$

frslslc-----htiv

$-p m x x x d x x x x x x x x x$ 


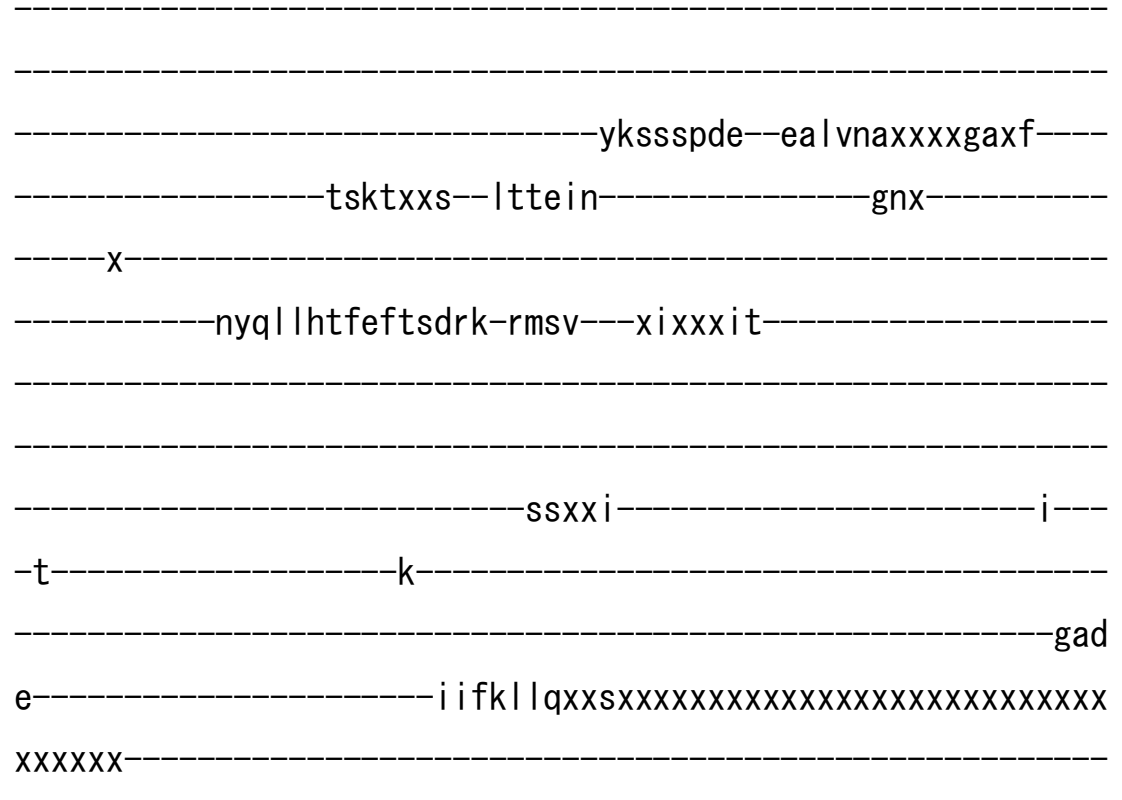

xxxxxxxxxn ly-skqi def--ash----g I r tLCLAQR i i--qkedyerwyqshyq--x at-----taiqdrs I I Ineaye I-lerd Ih ILGI TA IEDKLQEDVPRT IECLRQaq-IKI WMLTGDKYSTAIQIANSCNL-ietnqnin---$-x x x x n n n n x x x x x x x x x x n n n x x x x$

$x x x x x x x x x x y n x x x s x x x x x x x x x x x x x x x x x x$ $x x x x x x x x x x x x x x x x x x x x x x x x x x x x x x x x x x x x x x x x x x x x x x x$ ass i i i eghv $|s|$ $-v 1$ -mfsaseflk-

I sq I vgs I ICC--RVTPSQKAQVVKMIk-

---dt--gk -ITLAI GDGGNDVSMI QEANVGVGI-sgregla 
ysf-yksmf-Icfiqi lyqffsgf-

agtsffntfs Itsy

ni Iftg I pvi gf-i fdkd lpes--_-_-_-_-_-_-_--_i i rrnpy lysvgqdssafnvkv

i-sqwi i ral tqs i fvfxft I gxyvf-_-_-_-_-__-_-_-_-_-_-_xxxxxs--_-_-_-

id--yns i smisftsi ifiqs It-Iffeshtitwinhi---_---l iwgt ipiyl iclvv

Invipsldxx svithlv

-esgsvw----fs--i l imtl la iapi-

-itigyll

$-x x x x p x x$

ne i

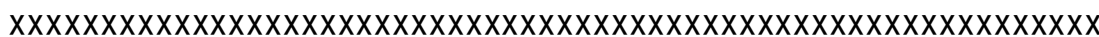

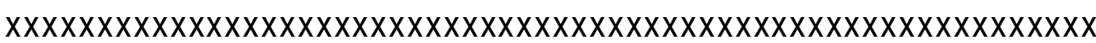

$x x x x x x x x x x x x x x x x x x x x x x x x x x x x x x x x x x x x x x$ s $n n n d n n n x x x x x x x x x x x x x x x x x$ $x x x x x x x x x x x x x x x x$

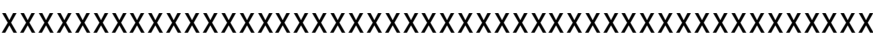
$x x x x x x x x x x x x x x x x x x x x x x x x x x x x x x x x x x x x x x x x x x x x$

>ddi IV_IV03 XP_637508. 1

m- 
$X X X X X X X X X X X X X$

xxxpktvyinn-

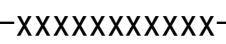

-nnnknnnnnnk

cimv

$x w \mid d-$

-vsxxxxgps

tinlxixx--------I inaikegyed

$\mathrm{fr}$

$-r y----q s$

dkrtnsqick-

-sgessxg--xcfIETSNLDGESNLKYKqs-

xxxxxxxxxxxxxxnnnnnnnni cnfkfneks i i ecegan InInkfdgs iql xx

\section{$x x x x x x x$}

-niek Ip

$\mathrm{ti}$

e-

$-Q L L I R--G T R L x x x$

ny i y

gLVVYVGHETKYMIntmstpt

-krsklektmeriliylfivql Ilclfst

-Img I yfd Infhkgs-------wy I d-xxxnfpmttftrfftfLVLFSTM

IPISLYVT iEVIRFLQVL-SInkdkkm- 
$-x x x x x x x x x x x x x x x x x x x x x x x x x x x x x x x x$ tkefeet aEAR-TSNLNEELGQVEYIFSDKTG

TLTKNEMVFk-icsingkiy

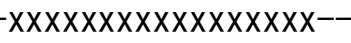

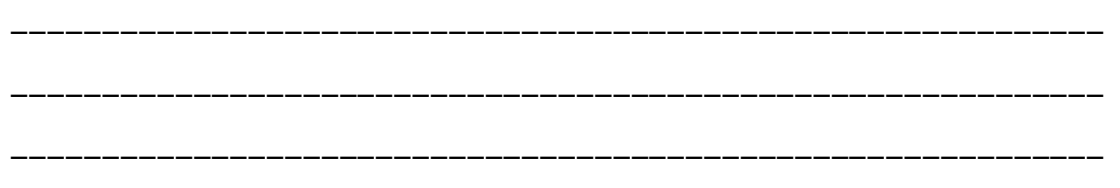

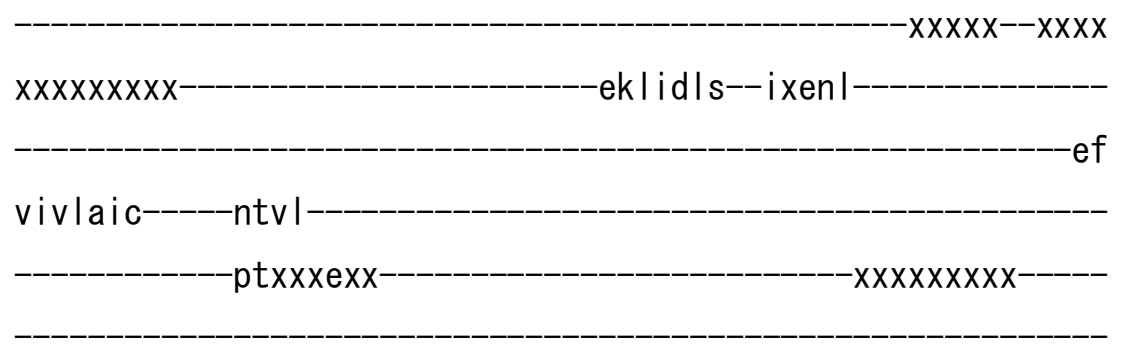

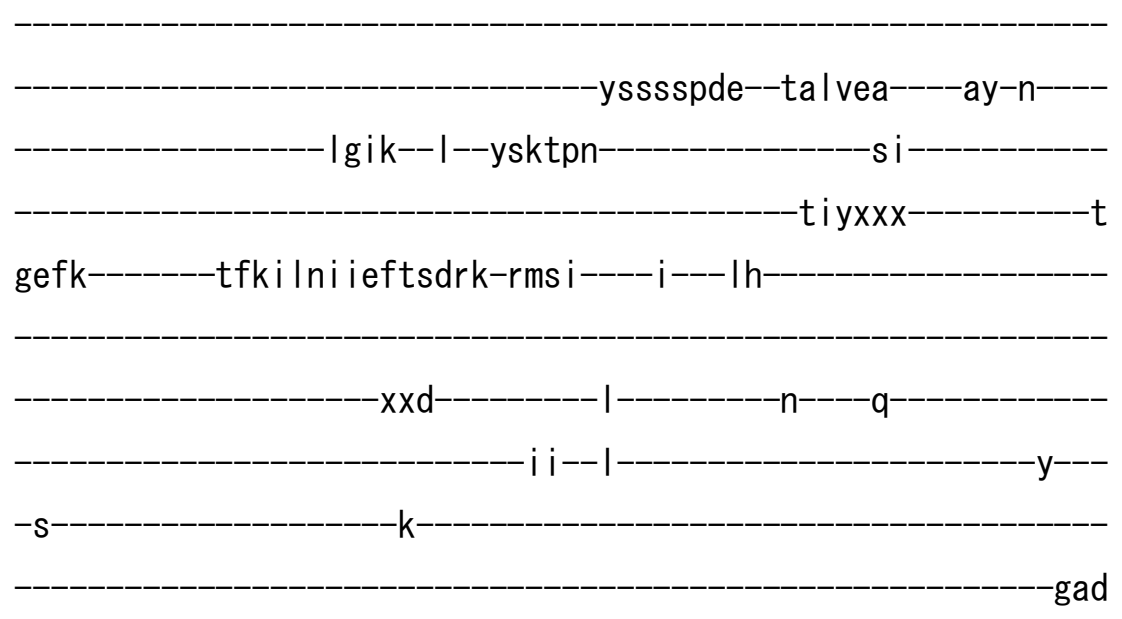

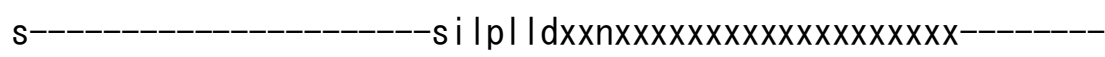

$x x x x x x x$

xxx--xx--kns-kes |k |f--ssn----g|rtLCI TKRI|--sddey l ewnk | yke--as------ Ismierdikme I iskq-i etk I I ILGVTAIEDKLQDNVSTTISTLIKag-IKI YILTGDKVETAITIGLSCSL-_-_-_-_-_-_-_-_-_-_-_-_-_-_-_Ikd lq I I i-_-_

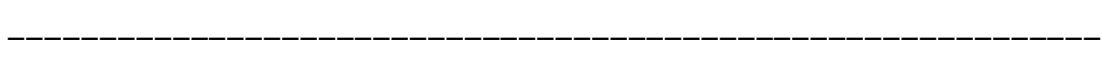


I sm I cks i VCC--RVTPFQKSEVVR IVk-

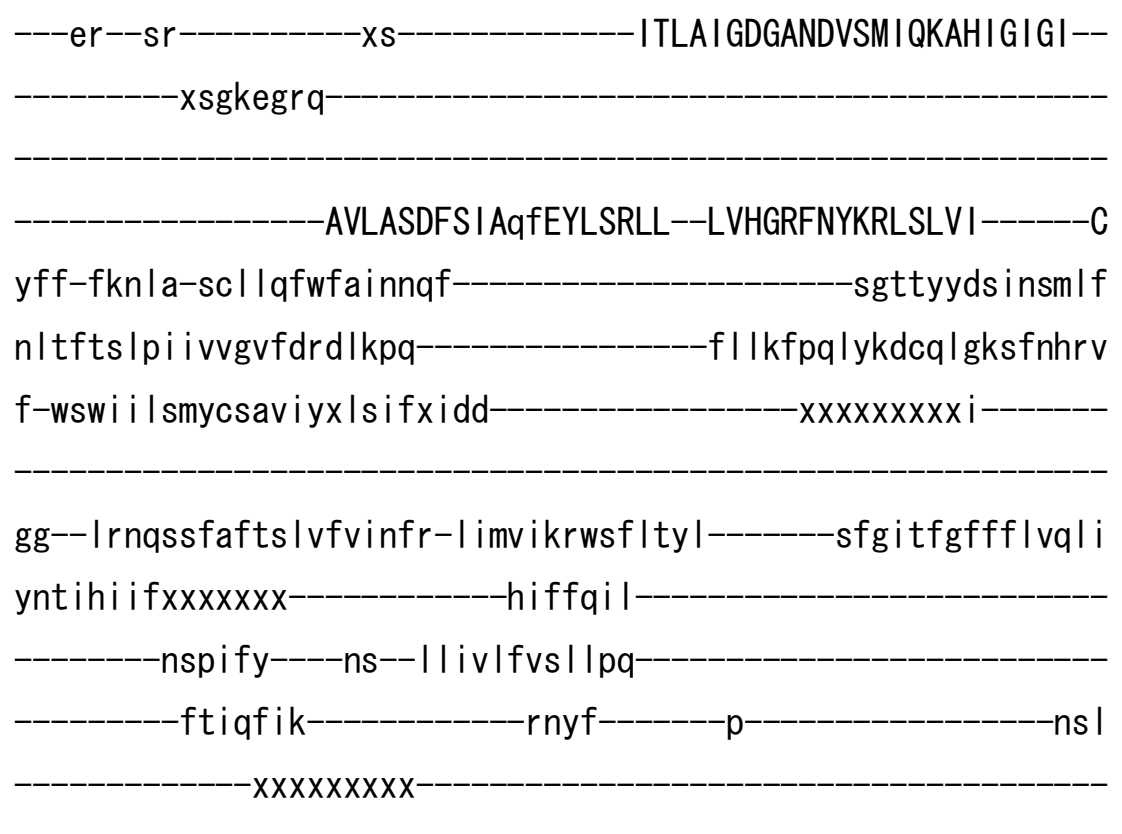

-xxxxxxxxxxndnnnnnkxxxx-

>ddi IV_IV04 XP_645920. 1

m- 
XXXXXXXXXXXXXXXXXXXXXXXXXXXXXXXXXXXXXXXXXXXXXXXXX

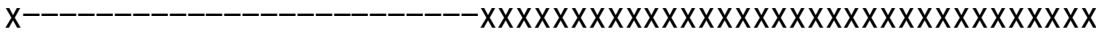

XXXXXXXXXXXXXXXXXXXXXXXXXXXXXXXXXXXXXXX i ssgaar--------

$-x \times X X X X X X X X X X X X X X X X X X$

$x x x x x x x x$

---srnifing

$-x x x x x$

-pfkfihnkisttkyt

$-p w---$

-sfipknlyeqf

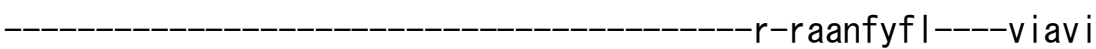

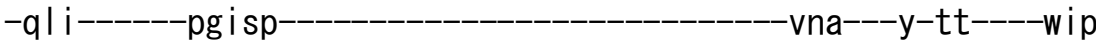

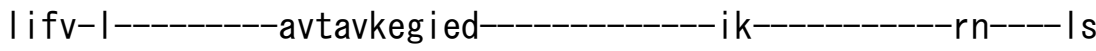

dktvnn I dcr--_---_--i I r

$n-g k f$

$-x------i$

$-\mathrm{V}$

p

----wkqVKVGDI CQVnk

GERFPADLVVLns 
-seghg----xcy IETSNLDGETNLKQRqa-

-xpqtfe i Irseed lahfrgn i ecehpnnv i yvynga i qmxx

dsqkhp

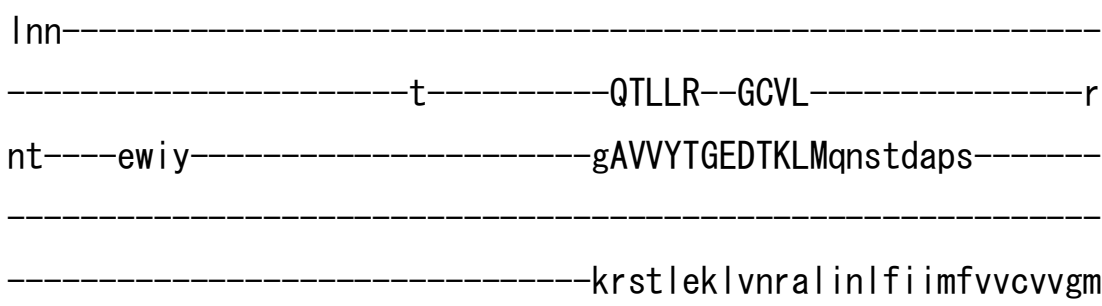

-ivsvi I tstni dkq---_---wy I d--_-_-_-_-_--xxxkdvrkav In I fsfMI AFAVM

IPISLYVSIELVKVAQAV-YVgwdvkm-

-ydpetntp

aRTR-TSNLSEELGQIEYIFSDKTG

TLTRNQMDF I

kcsvgkmvy

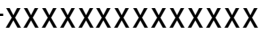

$-x X X X X--x X X X$

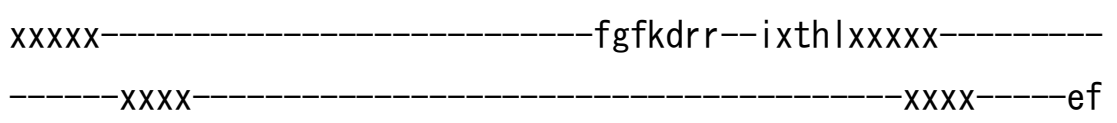

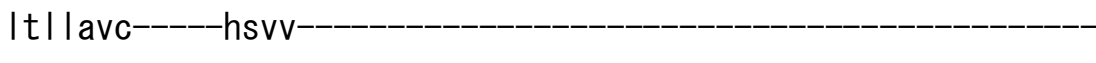

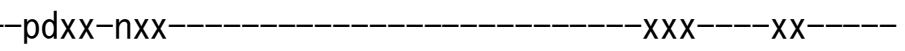

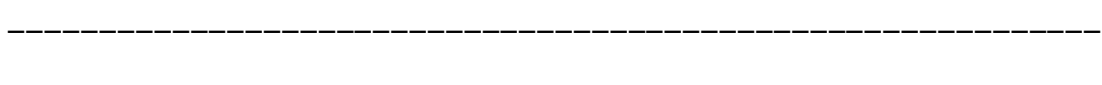

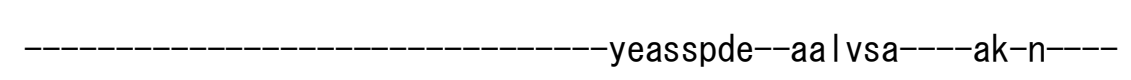

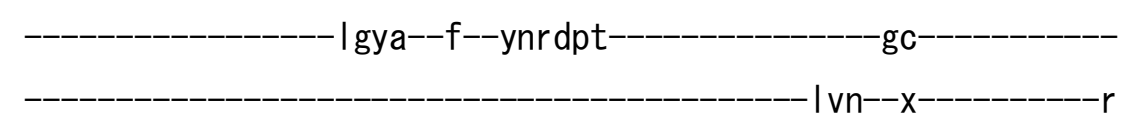

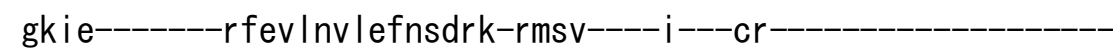

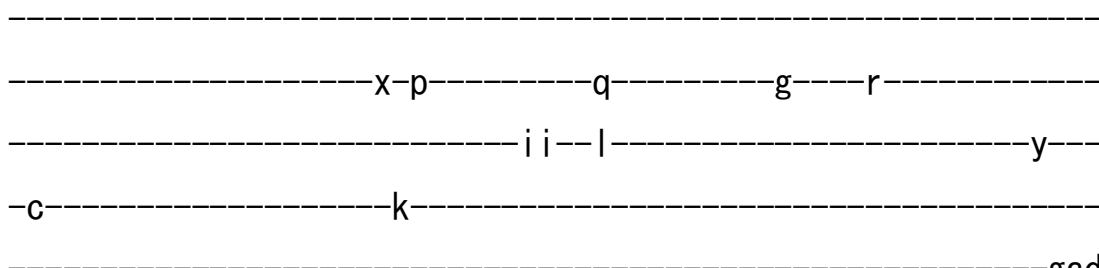

-gad

$\mathrm{t}$

$-t v|p| \mid r--k$

$-x$ 
xxx--xx--s it-lef l qdf--aad----g|rtLCLAYTy |--eeedyqqwne l yke---aa-----i s i qdrdmkvdkvse I-i ern I s I GSTA IEDKLQEGVPQA I ANL IKan-IK I WVLTGDKQETAINIGFSCHLItsdmr i i----

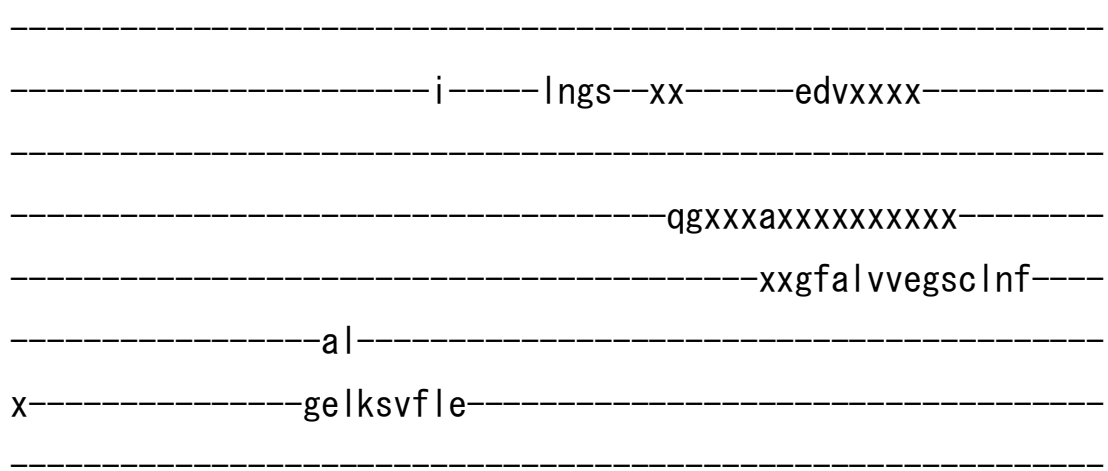

laancksv ICC--RTTPLQKAQVVKMVr

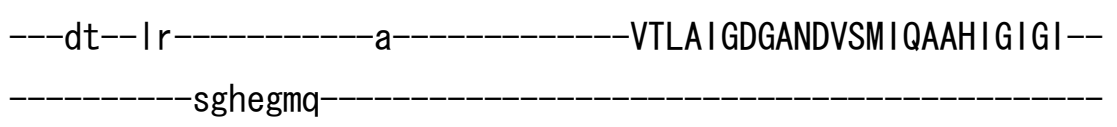

-AVMASDYSI AqfSFLYRLL--VVHGRWDYKRNSKLM------L

ycf-yknmv-famtqfwfgi i ynsf saqtmfdsws is if nvvftg I pi ivcaifdqdvsaessqkypq lyasgqkdsefnIrv I-wvwi veawihsvvif-fgvy-gly xxxxxxxxxxxt-

Id--I wamgqnifi Ivvitvnfk-lafetrywtwithf -siwasi I iwfawvav laaipgigxxxxxxxx -yvayk if --------aspsfw----|s--iav lpt i c lapd-

-viykyiq $-r d v k$ $-y n y$

$-x x x x x x x x x x x x x x x x x x x x x x x x$ 
>ddi IV_IV05 XP_643182. 1

m

xxxxxxxxxxxxxrttypni

---yfntfknn

$x x x x x x x x x x x$

$-k s$ I fsdne isttkyt

ry

-nfifknlfeqf 


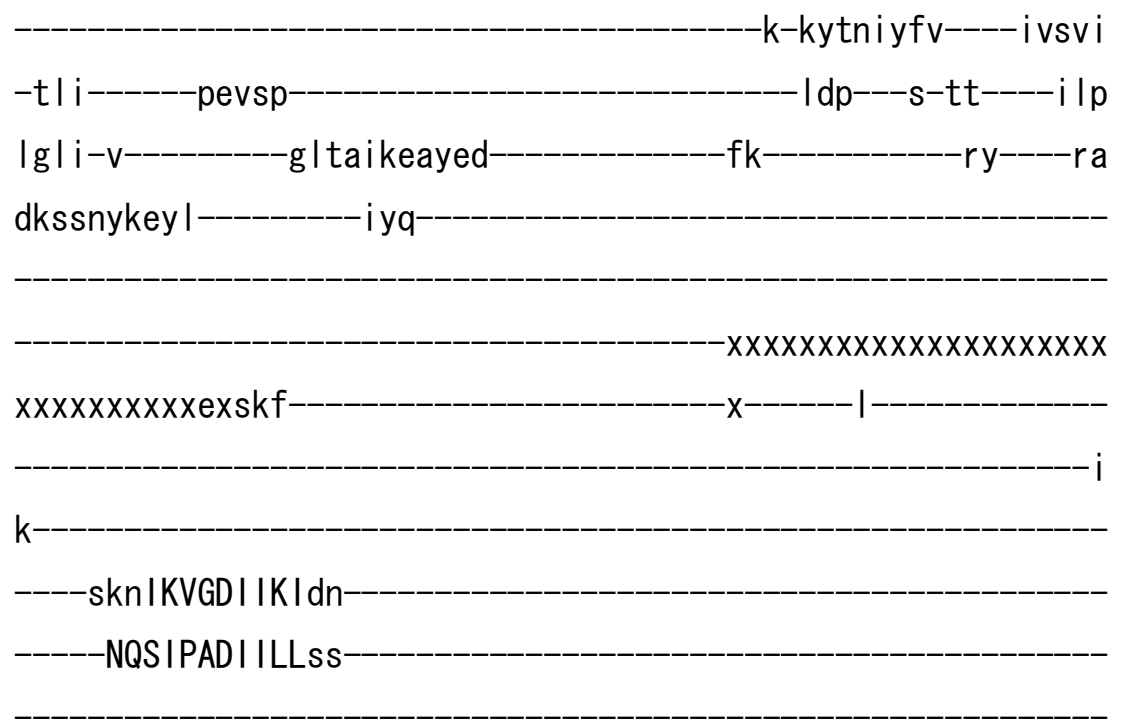

-ss I gdxv---cfVETSELDGESNLKIFks-

$-x x x x x x x x x x x x x n$ Inesqqeqqqqf $i k|k g n i q c e f p n n n| y k f k g i$ InI xx

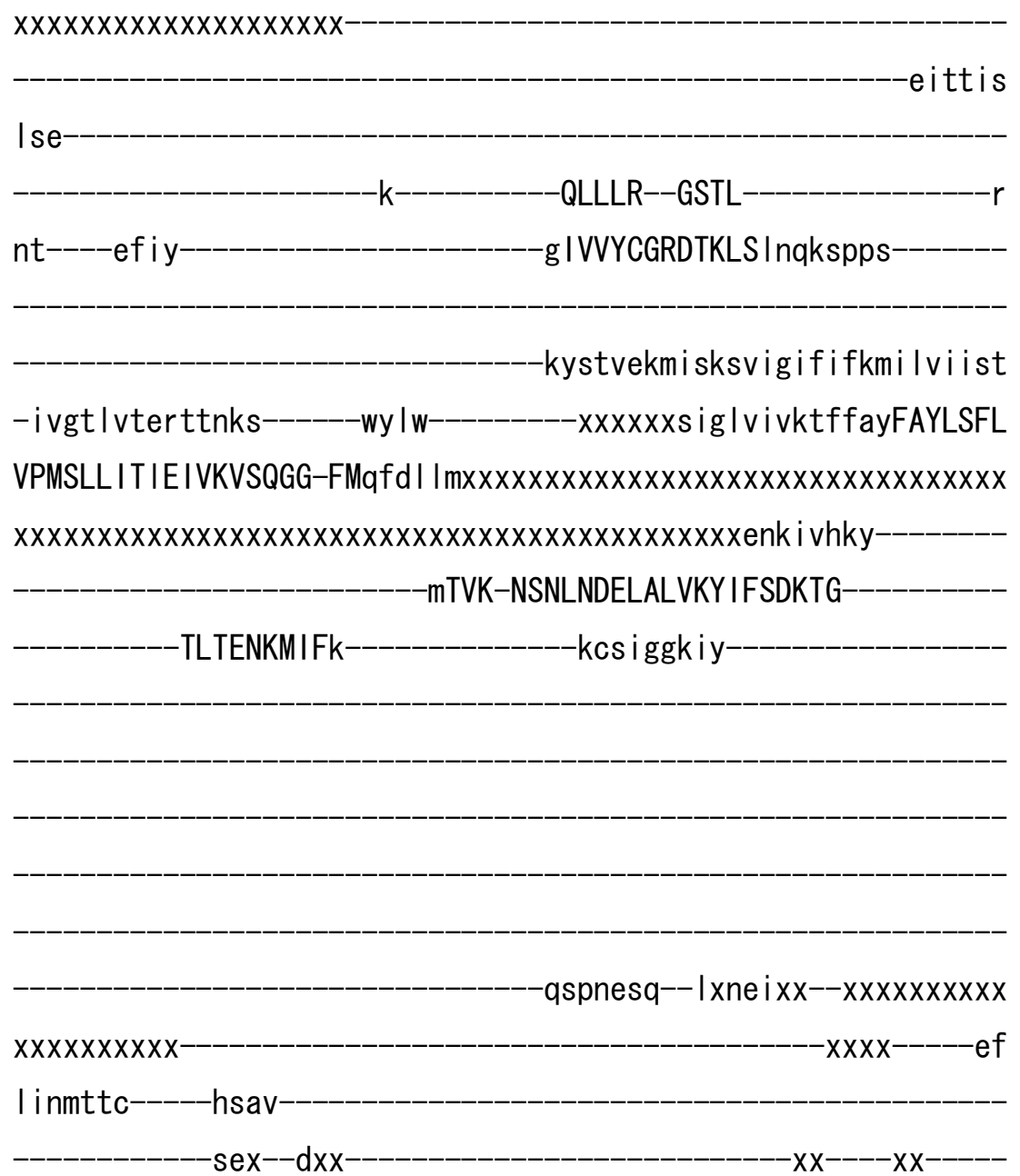




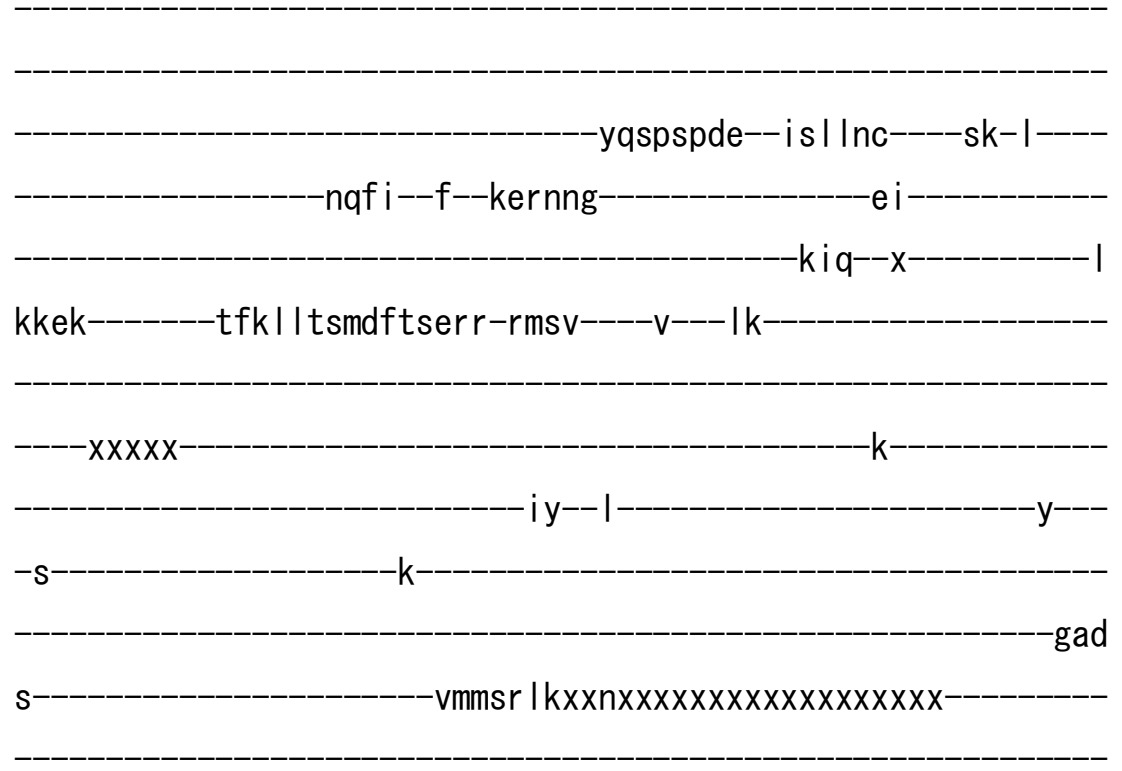

xxx--xx--ekt-tqhi kef--sne----g| r tL ILAMKe i--e i dqfnqwfekyns--i I------ns i enr eeq i ee Innq- I end I I I IGCTA IEDKLQDGVPET IEFLLNsn-IKV WI I TGDKQETAIN I GYSCKL--_-_-_-_-_-_-_-_-_-_-_-_-_-_-_- I shsnq I f-_-_i i-----nsns-----------rdnxxxx$x x x x x x x x x x$ i exxxnxxxxxxxxxxxxxxxx--xxnys I vidges I if if lefenkfIs-

iakkchsv ICC--RVTPIQKSLIVKMVk

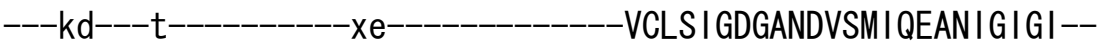
fgnegsq 


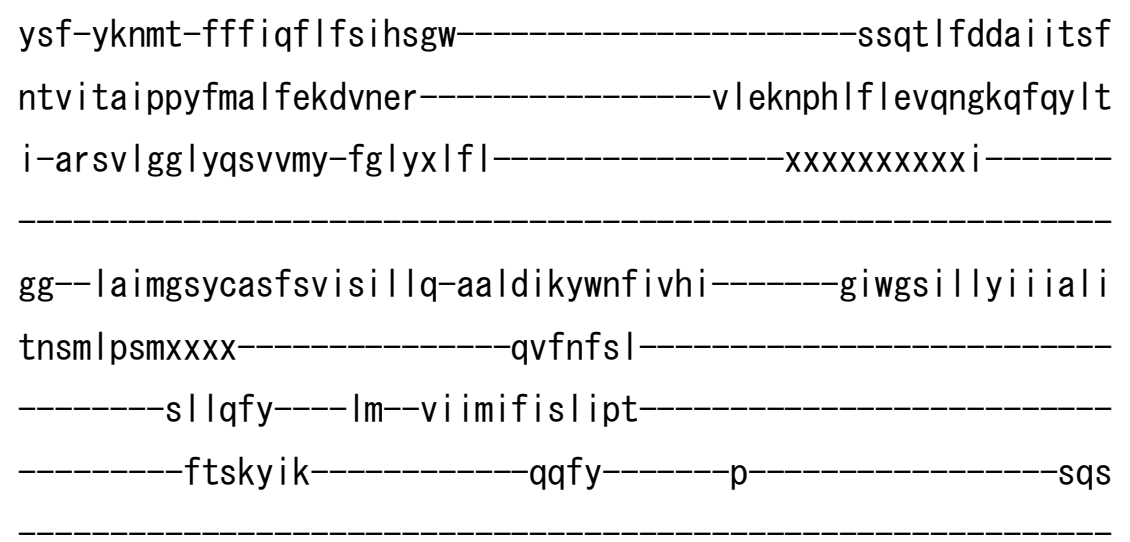

>ddi IV_IV06 XP_640653. 1

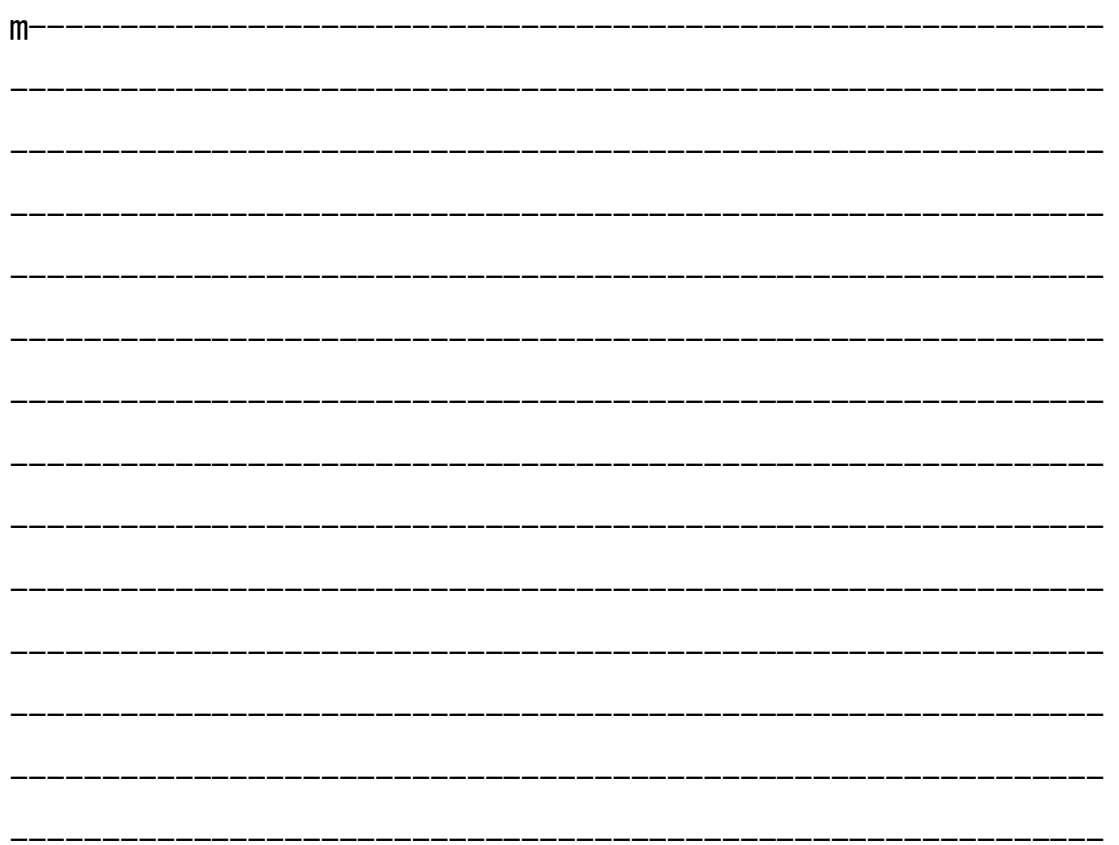


---prtvhign-

$-x X X X X$

-khgygkne i atsrys

si----

-sripri ivsql

$-i-r$ linvyfi----iiivl

-sfi-------dgvsp---_-_-_-_-_-_-_-_-_-_-_-_---tgk---f-tt-----lgp

wl it-i----------t i svl re i i ed---------------lk------------rq----kq

deavnygtse---_--_---vfr--_-_-_-_-_-_-_-_-_-_-_-_-_-_-_-_-_-_-_-

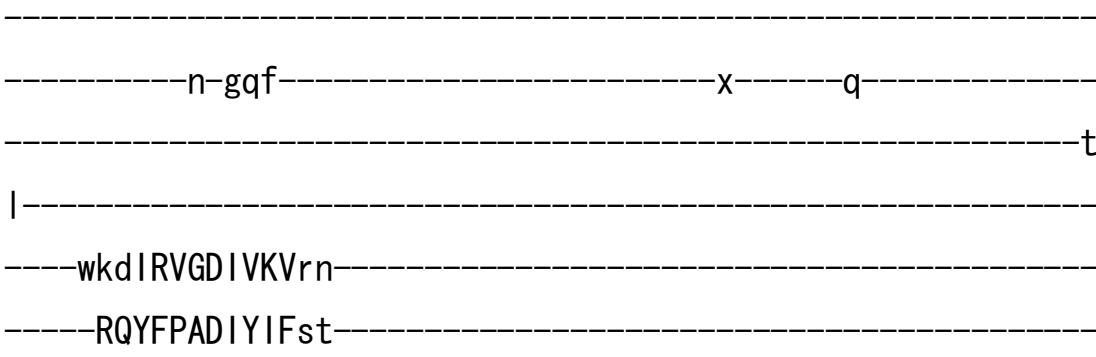

-sepen-v---cwFESKNLDGETNLKQRqa-

-xket i y lkdnae I I snfcg I i ecnsptkd I I I lkgsfff--

ddnyvi

Itp

$-Q L L L R--G T K L$

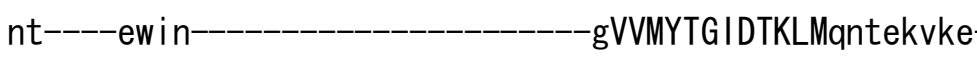

-kkshiedItnhf i if ifflqi I lcggsa

-iangvwstsnhdv-

-wy I I -xxxtgiveggksf I tfLVLYNN I

IPISFYAT IEVVRF IQTC-F I nndvem 
-yheetdtp

-aLVK-TANLNEELGQIEYVFTDKTG-

-TLTQNAMTFk

-kcs i ggyvy

$x x x x x x x x x x x x x x x x x x x x$

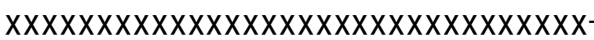

$-x x x x x--x x x x$

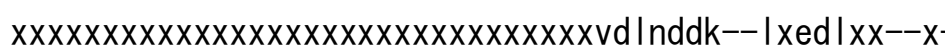

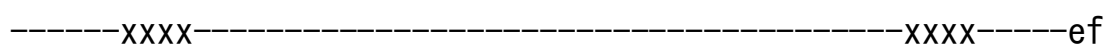

fyvlsic-----hsvi-

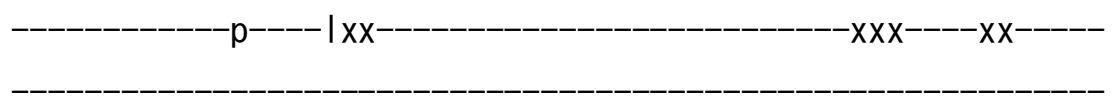

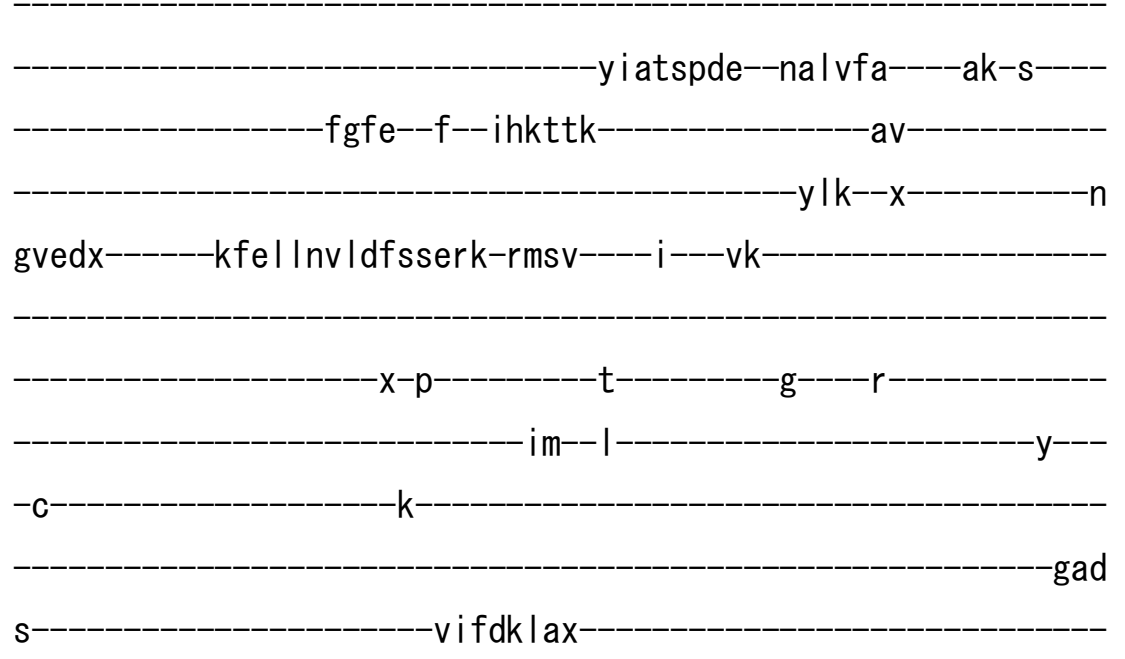

$\mathrm{xxx}--\mathrm{xx}--\mathrm{dts}-\mathrm{i}$ thi iqdf--gyq----g|rtLCVASTq I--dervyqqwaqqyhax $x$ I txxxxxpsq lekdke i ervae I-i etdfq ILGVTGVEDKLQEGVPET IQLLTEag-IKM

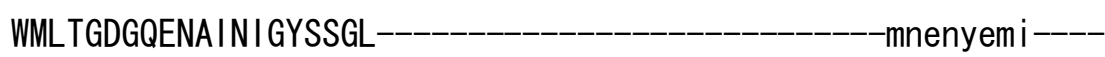

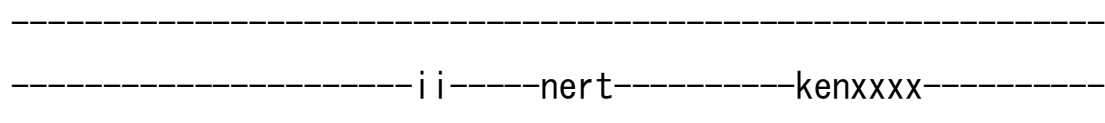

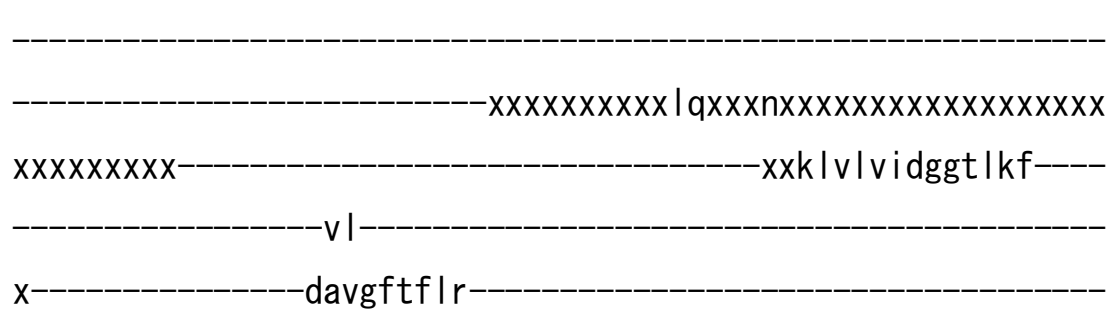


l akmcdtv ICC--RVSAHQKSKIVKLVr

---kt--fa--_--_-----p---_-_-_-_---KTLA I GDGANDVMM I QCAHVG I GI--

-A IYAADYSISGFQHLGRLL--MVHGHHSYRRMSKL I------C

y if-ykn iv-Iyfcaf I faifsgw--_-_-_-_-_-_-_-_-_---sgqt I fetyn I tay svvyt I ip I i vycv l ekdvner-_-_-_-_-_-_-_-_--t i yqhpq l ykeg i qhkyfnhft f-l qwi angfyhgfvaf-al vyxtvv---------_-------- xxxxxxxxxt--------

qe--lyafg i ivyscvml ivt lk-lalethhwtwinhl---_----amwgs I vvff i iwnvi

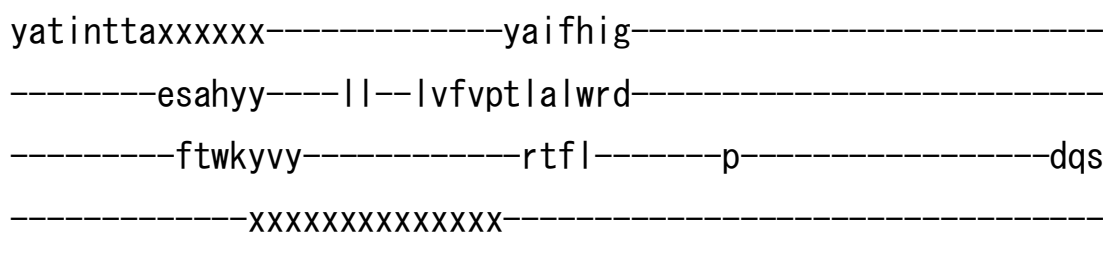

xxxxxxxxxxiksyrpnnxxxxxxxxxxxxxxxx $x x x x x x x x x x x-$

$-$

>ddi IV_IV07 XP_645972. 1

$\mathrm{m}-$ 


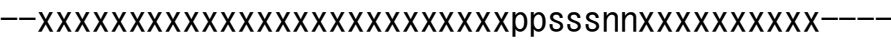

$-x x x x x x x x x x x x x x x x x x x x-$

$-\mathrm{x}$

$---y s p \mid$ I isd

xiylsrtiilel

-e-eqpkkyfyxx--nials

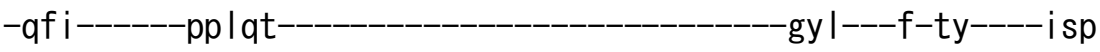

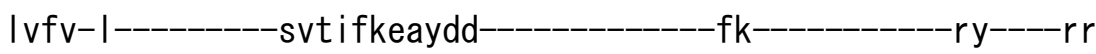

dkeansqhys----------k It

$k-n g f$

- - ------ $s$

$\mathrm{p}$

----ssd IKVGDF I KVet

-NQRVPCDMIFLrt 
-tekng-s---sfIRTDQLDGETDWKLRrs-

vni tqk Is I ded I vnI ras i yaekpkkd i ynf i gnftnxx

sgetes

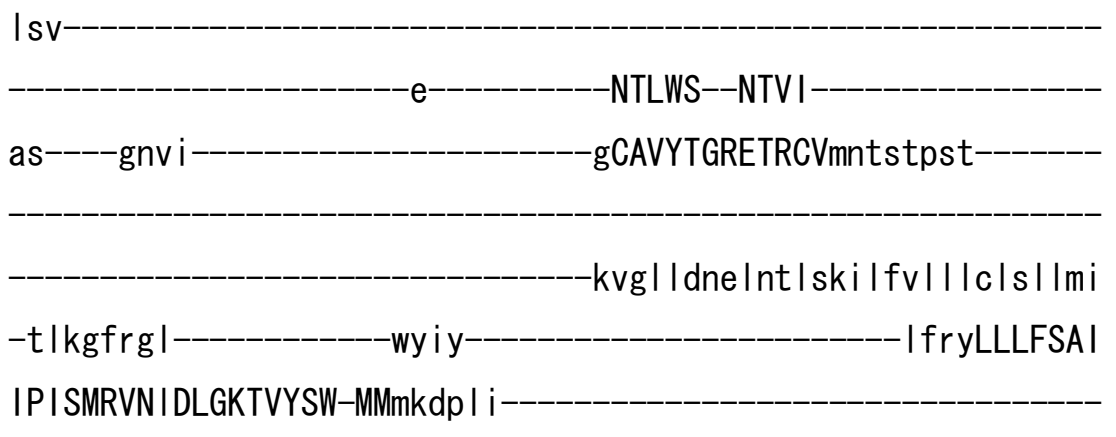

$-p g$

-tVVR-SSTIPEELGRVEYLLTDKTG

TLTQNDMVFk

-klhlgsvsf

$-X X X X X X X X X X X X X$

$-X X------X X X$

$x x x x x x x x x x x x x-----------x x x x x x x x f s r k-i k$

ea

itaialc-----hnvt

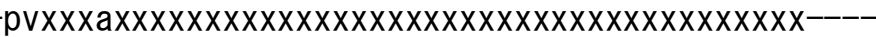

$x X X X X X X X X X X X X X X X X X X X X$

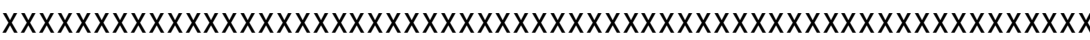

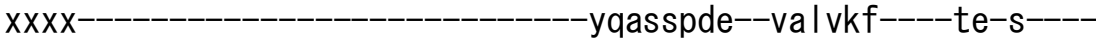

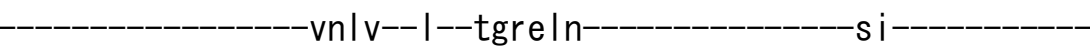

$\mathrm{tl}$

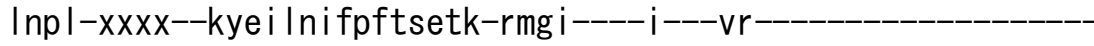

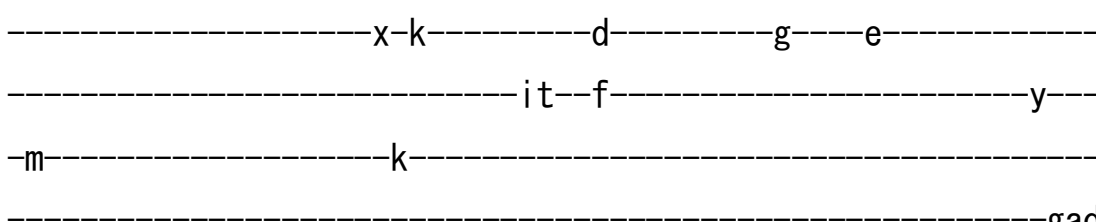

-gad

a

imak I v--xax 
-dw I-deecgnm--are----g | rtLAFGKRsm--seeeyhr fvatfna--ak-----tt i vdr aakvqea i at-i ekd I e I I ALTGVEDKLQEKVKPTLEMLRNad-VKV WMLTGDK IETATCIAISTKL-vsrtqs If----

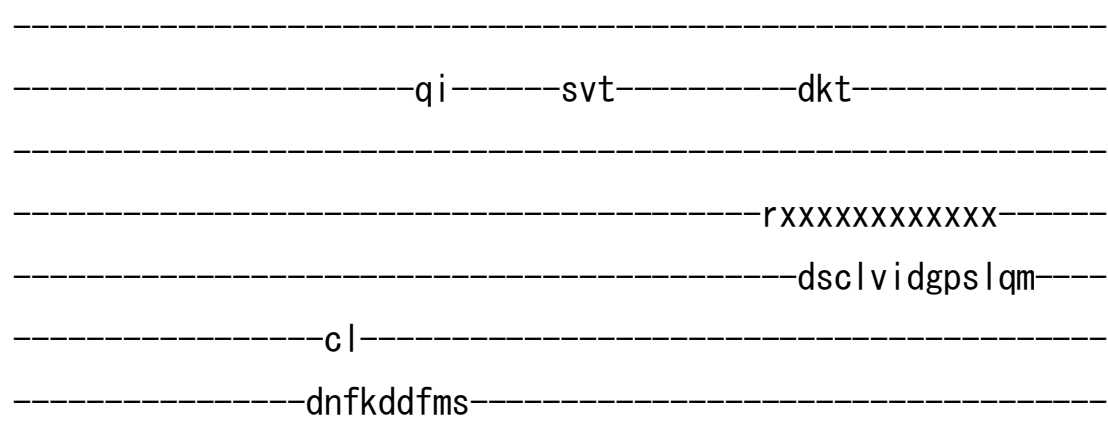

i atkapsvVCC--RCTPTQKADIVRL I

---ek--tk-----------x-----_--_----RTCA I GDGGNDVSM I QAADVGVGI--

fvi-hrgli-isfiqcvfsaiyyf aa i s i yngm I lvgy at |ytnapvfs|-v|dedvpmeivfrype lyhe lakgrs I sykt

f-fiwv lksvyqgga im-IIsi-IIf$-\mathrm{e}^{--------}$

ss--InnivsitftslilcelIn-vateintwhrymig-slivtl Isyvitmmi pql laf elsfi- swefv----wk--vmvit I vscfp|-yi iqfiq$-k k \mid d-$ 
>ddi IV_IV08 XP_643516.1

m-

-xxxxxxxrrkkarp

---sypi ieir

-nfifknl feqf 


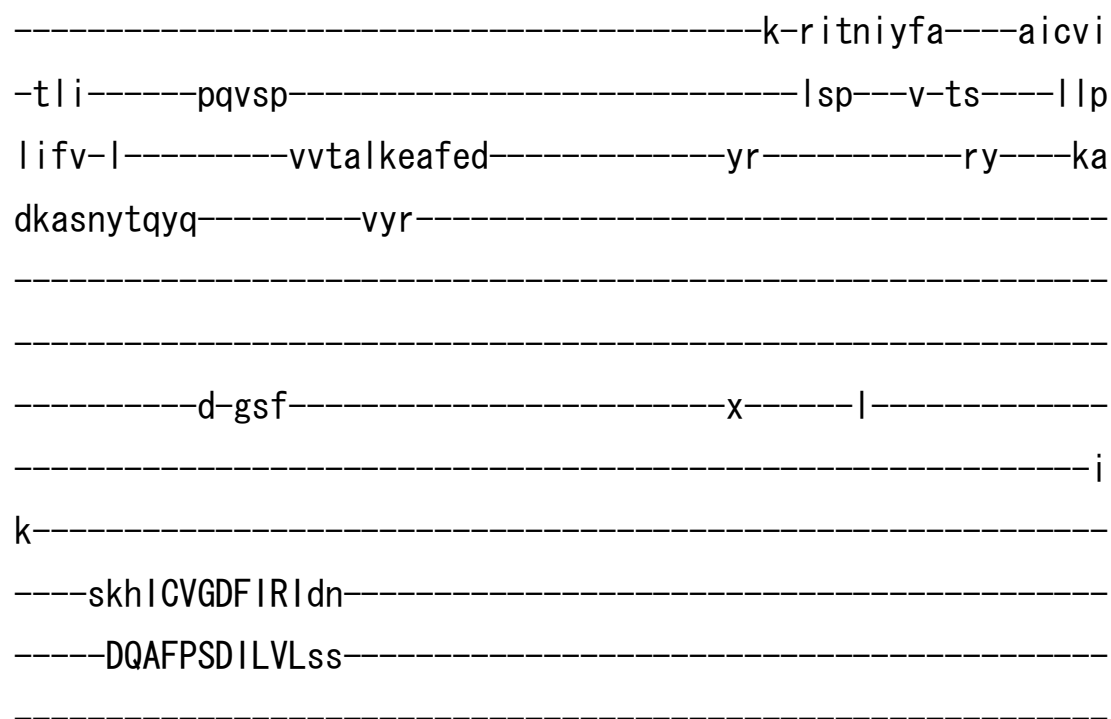

-nledg-i---cyVETSQLDGETNLKLFka-

-aketns I tqeq I I I nan i ece I pnnn lykfkgkft Ixx

-dnstfs

Ise-

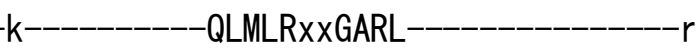

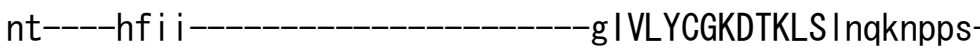

-kfstietr Igrsvigifcfkvvlvi iat -vlss I fefntards-------wy|r------------xxxxs I gft i vknfvsyFAILSFL IPMSLMVT I EVVKVSQAK-YMewdvkm----_-_-_-_-_-_-_-_-_-_-_---- Xxxx $x x x x x x x x x x x x x x x x x x x x x x x x x x x x x x x x x x x x x x x x x x x x x x x x k$ ski Inky -mSVK-NSNLNDELAL IKYIFSDKTG

TLTENRMLFskcsingtcf-

dgamnqq-- $\mid x \operatorname{dev} x x--x$ $\mathrm{XXX}$ $-x \times x x-----e f$

| Inms |c-----haav

$-s d x--n x x$ $-x x x----x x-----$ 


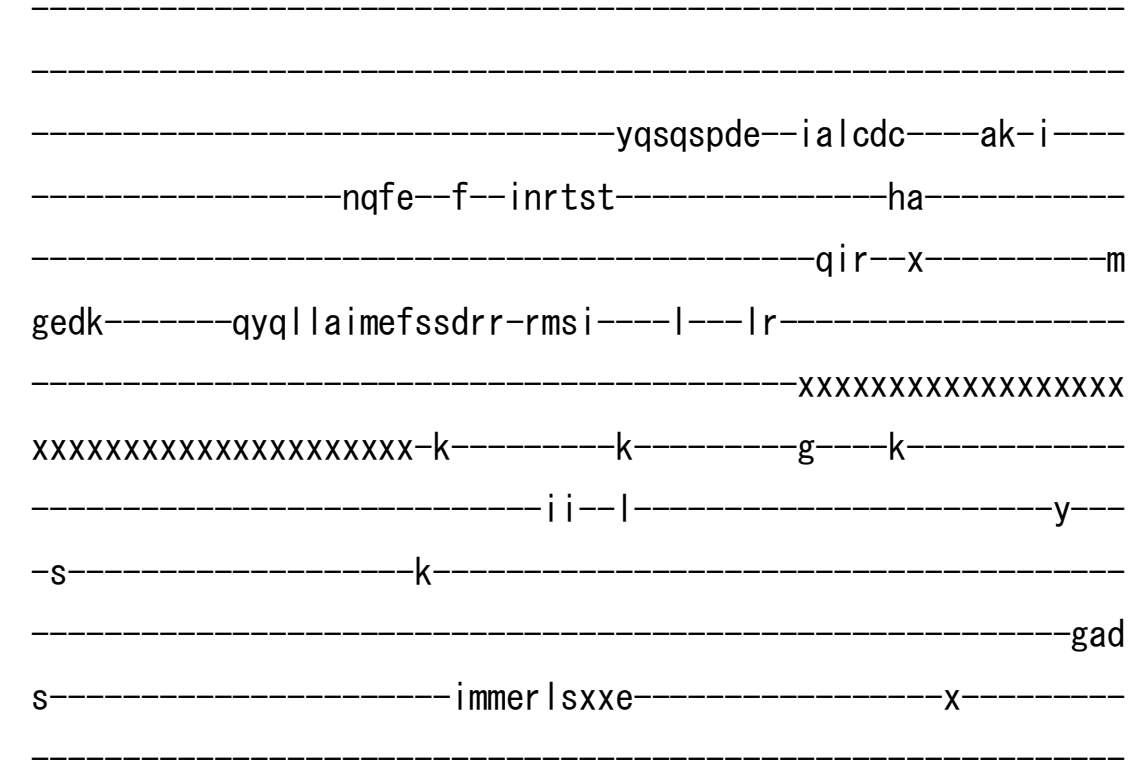

xxx--xx--eqt-keh i sqf--sre----g|rtL ILAKRe i--sqeeysnwsqqyhe--as-----t I i ihdreaemer Indq-i ergfe IVGCTAIEDKLQDGVPET IDYLLKan-IKV WI ITGDKQETAINIGYSCKL-Ivpe ipi i----

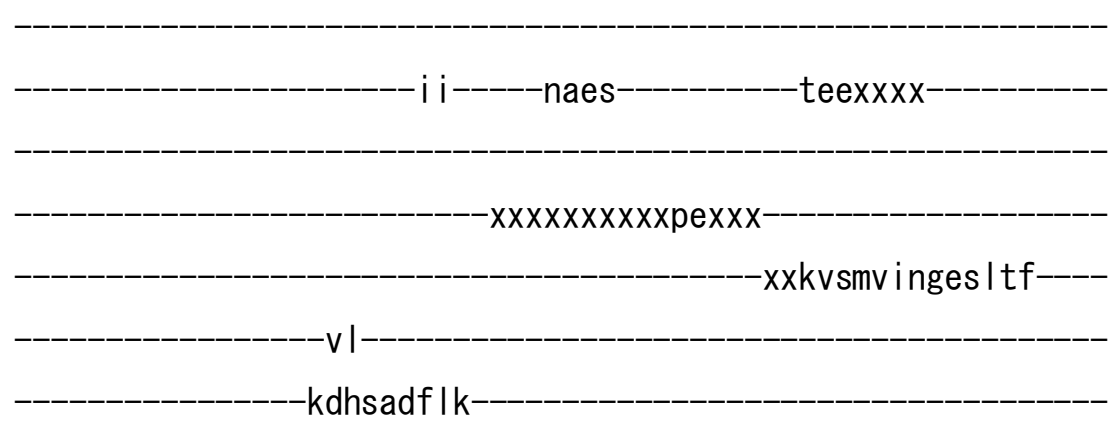

i aakchsvVAC--RVTPLQKALIVRLVk

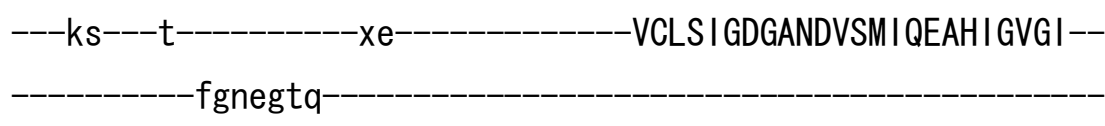

-AARASDYALL $r$ fRHLARL I--TVHGRYSMVRNSLCI------K 


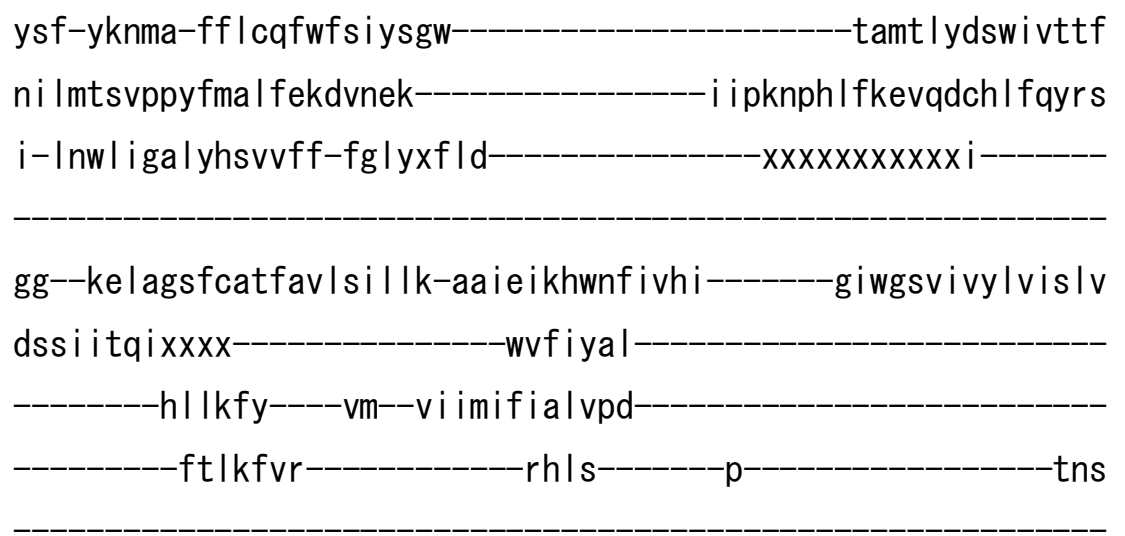

>ddi IV_IV09 XP_643097. 1

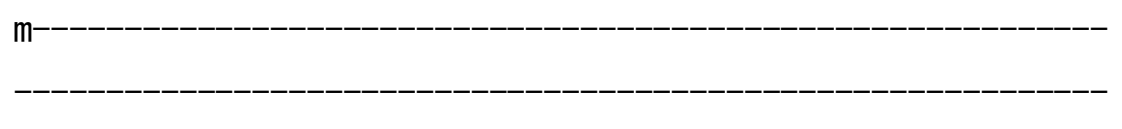

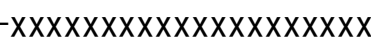

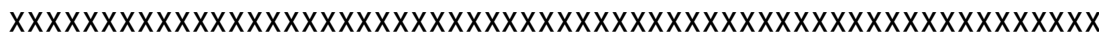
XXXXXXXXXXXXXXXXXXXXXXXXXXXXXXXXXXXXXXXXXXXXXXXXXXXXXXXXXXX XXXXXXXXXXXXXXXXXXXXXXXXXXXXXXXXXXXXXXXXXXXXXXXXXXXXXXXXXXX 


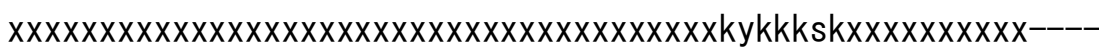

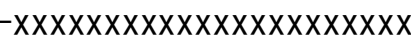

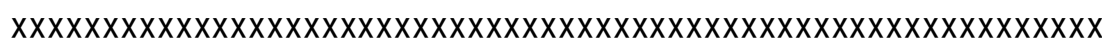

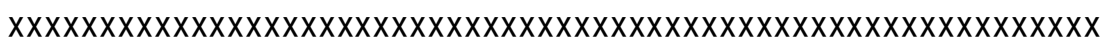

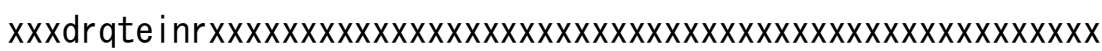

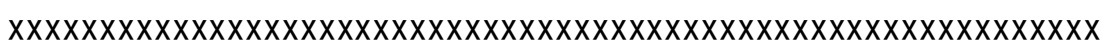
$x x x x x x x x x x x x x x x x x x x x x x x x x x x x x x x x x x x x x x x x x x x$

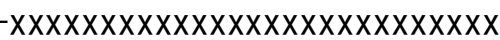

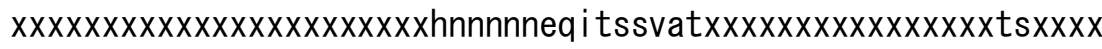
xtfipkvifyqf

srlanlytxxxxi lcmf

$\mathrm{xfsp}$ $-\operatorname{vgxxxx|tp}$

Il vv-i-avtsfkelaed $-r h----k q$

dke ingrets-

$-x x x x x x x x x x x x x x x x x x x x x x x x x x x x x x x x x x x x x x x x$

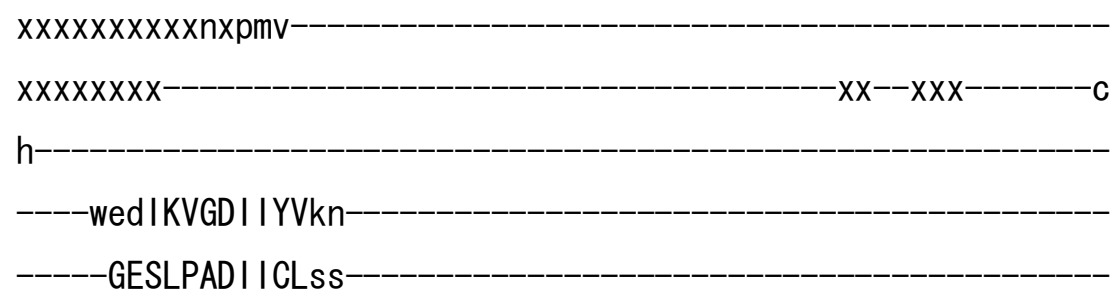

-srsdg-r---ayLETANLDGETNLKAKss-

i skcqwikgakd lddfsckvdyegpnnd i ysfdgv I tixx

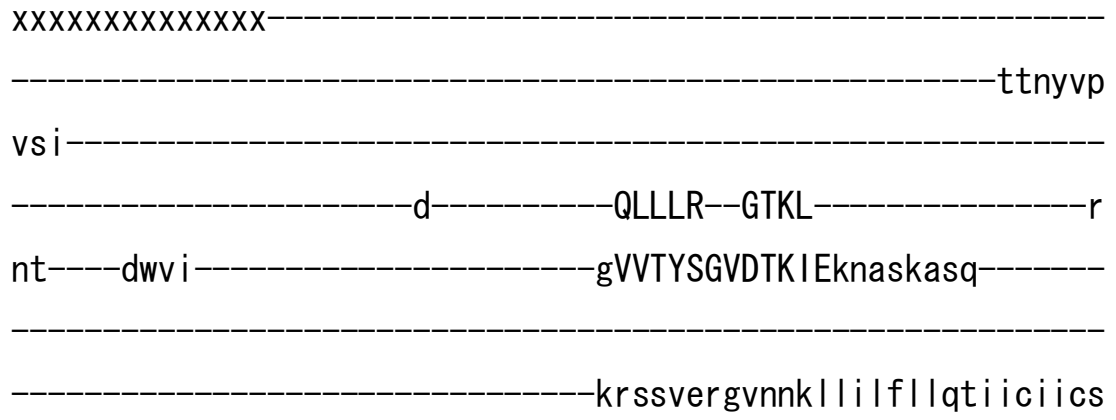

-i ghnrwh l eddkda-----xxwy i d----------_----xdpeqtedf i yvsyVILYNTL IPLSMYVSmEMIRVSNAH-F Idsd lem 
-ydestdtp

aQAR-NTN INEELGQIQYLFSDKTG

TLTCNEMVFn

rcti ggavy

$x X X X X X X X X X X X X X X X X X X$

\section{$x X X X X X X X X X X X X X X X X X X$}

$x x x x x x x x x x x x x x x|n p| g i p--v x p e i x x x x x$
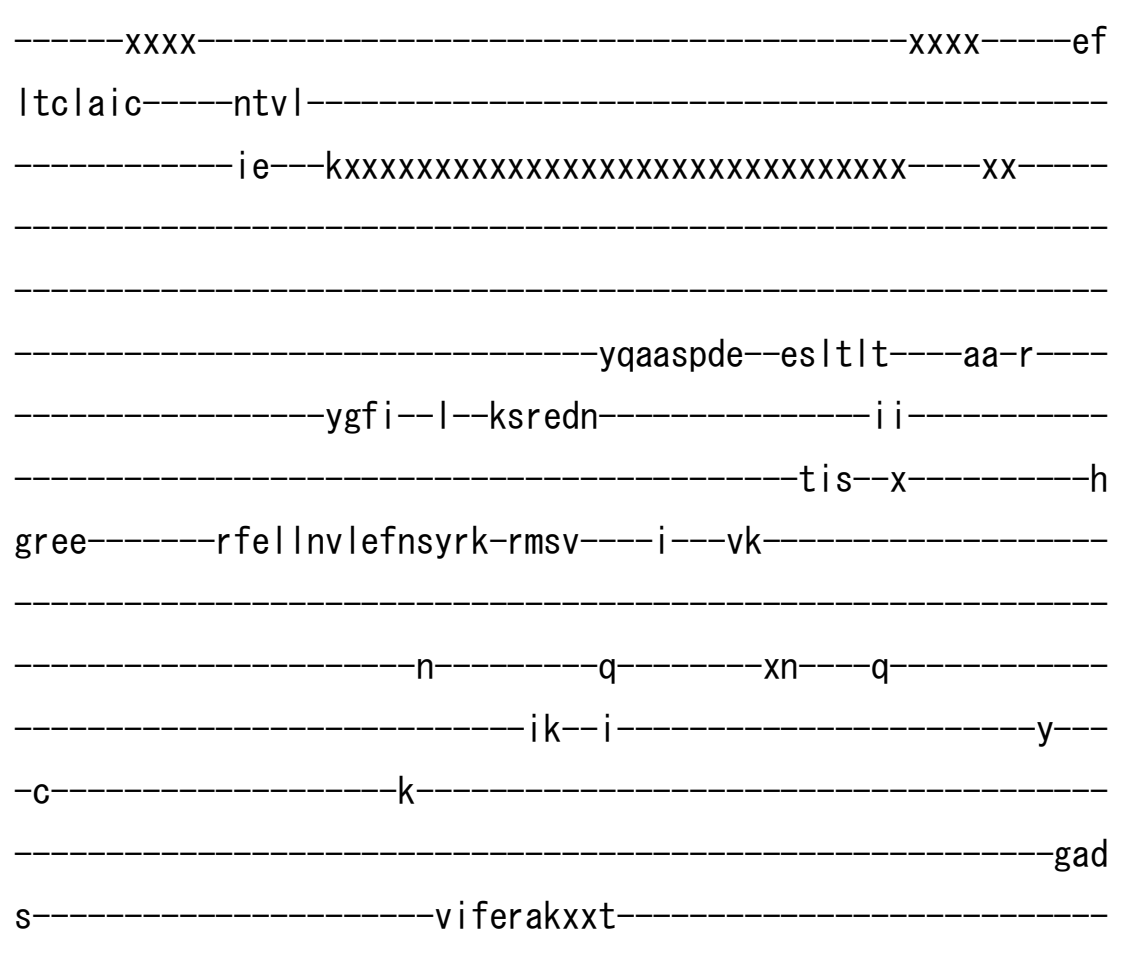

xxx--xxxxqst-ekh I sef---acs----g | r tLCMSVRt i --eheey i awnk i hqe--as-----is I vkkaekvdaace I-i ekd I I I GSTGIEDRLQDHVPET I SALREag-IKV

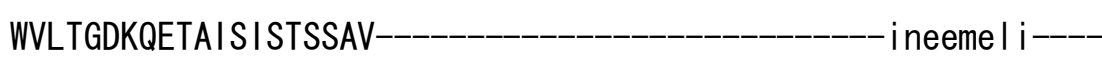

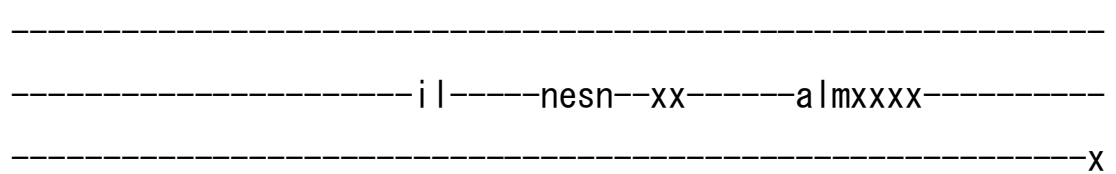

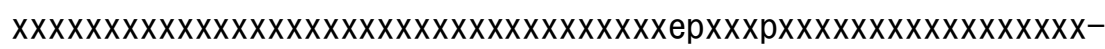

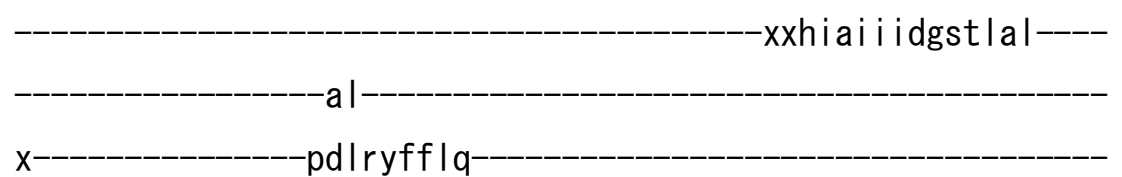


vaktcesvVCC--RCSPSQKAKVVNLVa-

---erxx If---------xxxa-------------ITMS I GDGANDVPMI QKAHVGVGI--

sgregmq

AVLASDFA I AnfSMLRRL I--LVHGNRSYKRMTKL I------L

ysf-skn i a-I s i sqfwfgffsgf-_-_-_-_-_-_-_-_-_-_-_-_sgqmi y fdf I ft l y nal fts I pv i f I gtfdqdtked-_-_-_-_-_-_-_---d I Inkpy lyrvcqsnspfstwk

f-i wwvf i gmwqsat if-fvtfxvmn---------_-----------xxxxxxxxt--------

|g--|ws i gtaay i y lvvtvn |q-isfi trywtrqt i |-------avs i svvstf $\mid$ fvm $\mid$ ysvlgryvxxxxx--------------hi ifely-

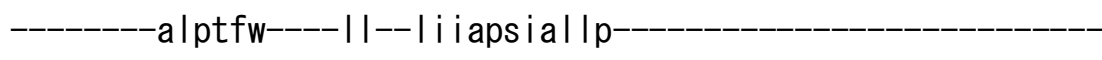

$-y$ i ivs In-

>athIV_IV01 NP_568146. 1

$\mathrm{m}$ 
$x x x x x x x x x x x x x x x x x x x$

$x x x x x x x x x x x x x x x x x x x x x x x x x x x x x x x x x x x x x x x x$ ke $i$ ded

---arliyind

$-x x x x x-$

rfeftgnsiktakys

$-\mathrm{vf----}$

-tfiprnlfeqf

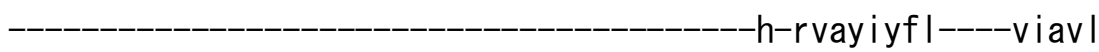

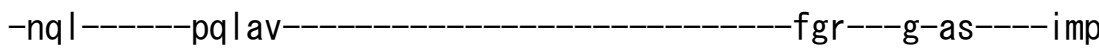

lafv-I--_-_-_---Ivsaikdayed-_-_-_-_-_-_-_fr-_-_-_-_---_rh----rs

drvennrlal---_------vfe---_-_-_-_-_-_-_-_-_-_-_-_-_-_-_-_-_-_

-

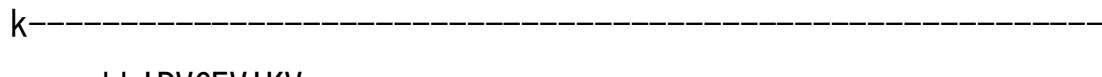

----wkhIRVGEVIKVqs

NQTLPCDMVLLat 
-sdptg-v---vyVQTTNLDGESNLKTRya-

-kqet I lkaadmesfngf i kcekpnrni ygfqanme i--

dgrrls

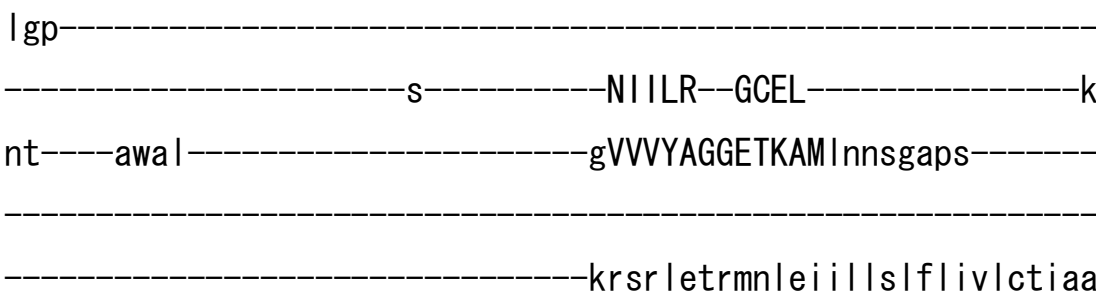

-ataavw IrthrddI--xxxxfyrrxxxxxxxxxxxxxxxgwgwe ifftffmaVIVYQIM

IPISLYISmELVRIGQAY-FMtnddqm-

-ydessdss

fQCR-ALN I NEDLGQ I KYLFSDKTG

TLTDNKMEFq

-cac i egvdy

$-X X X X$

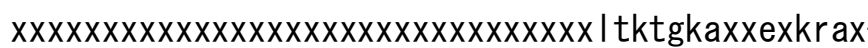

ef

fls laac-----ntiv

$-p$ i $x x x t x x x x x x x x x$

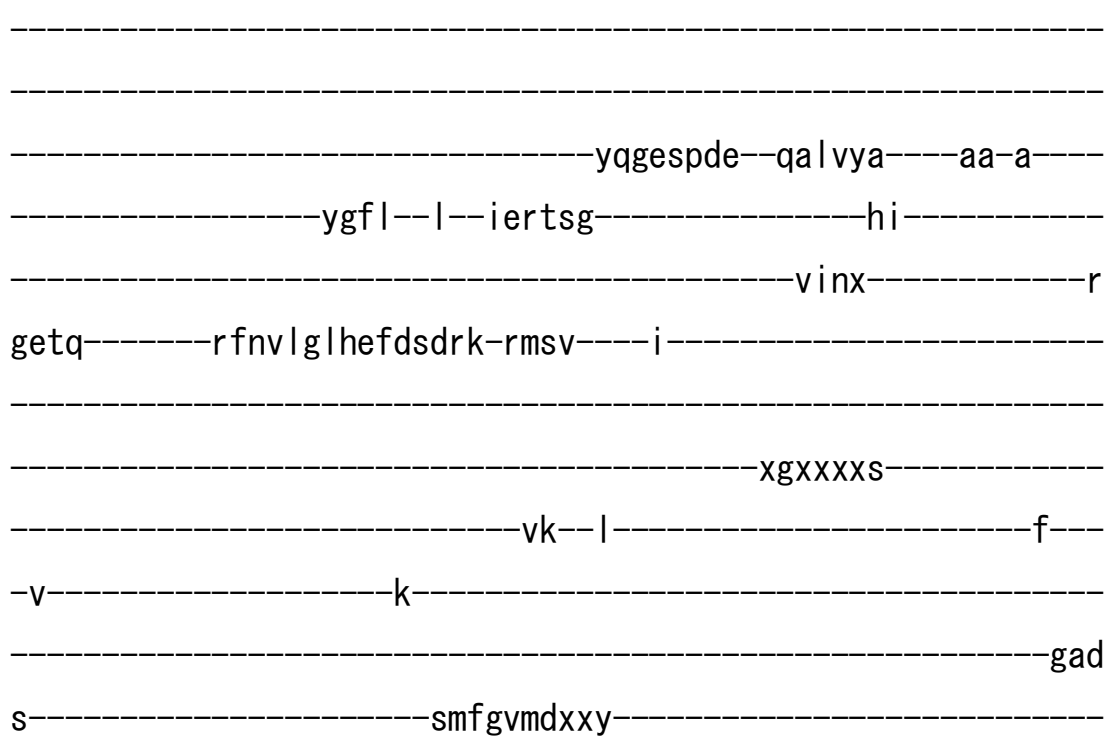




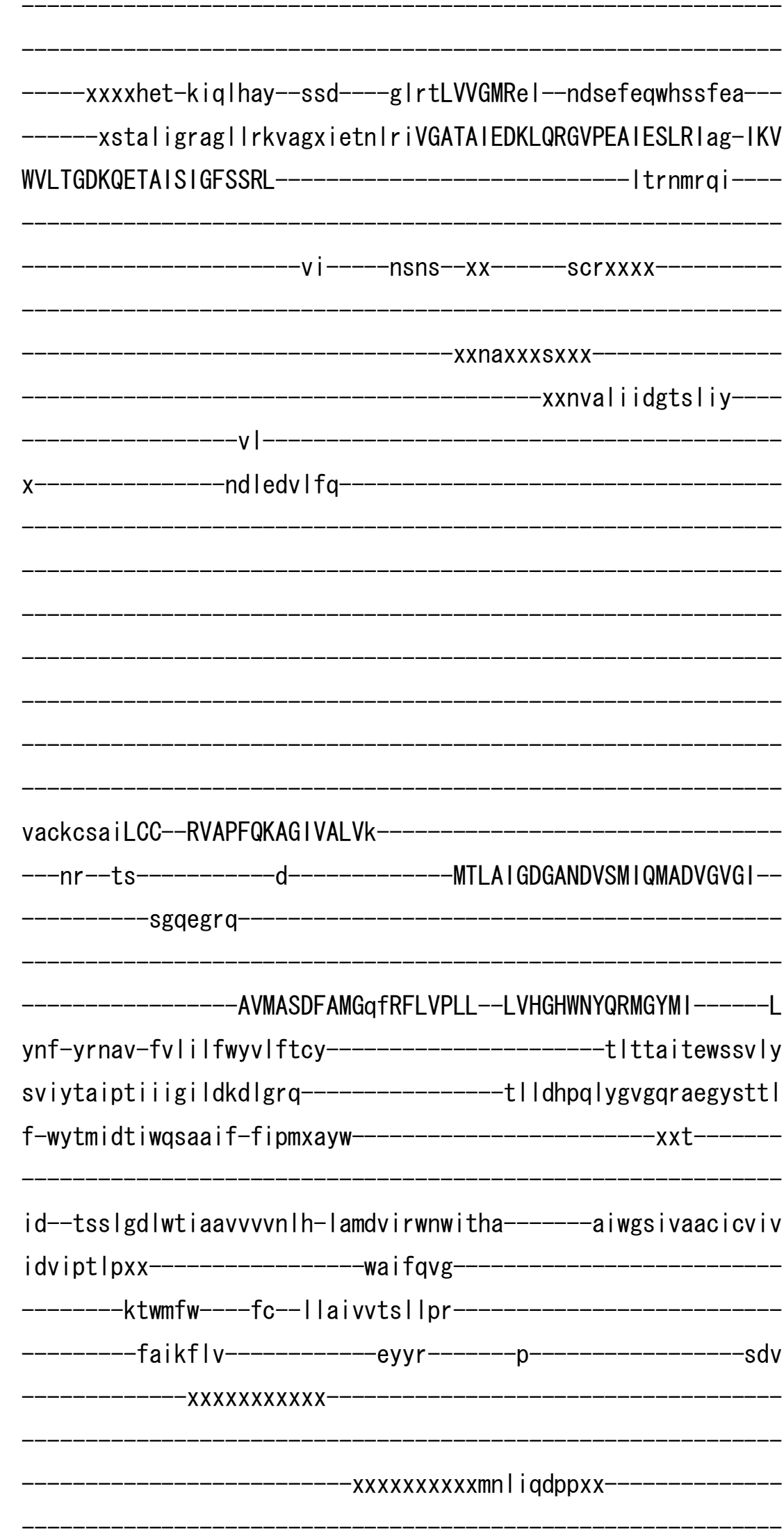




\section{>athIV_IV02 NP_568633. 1}

m-

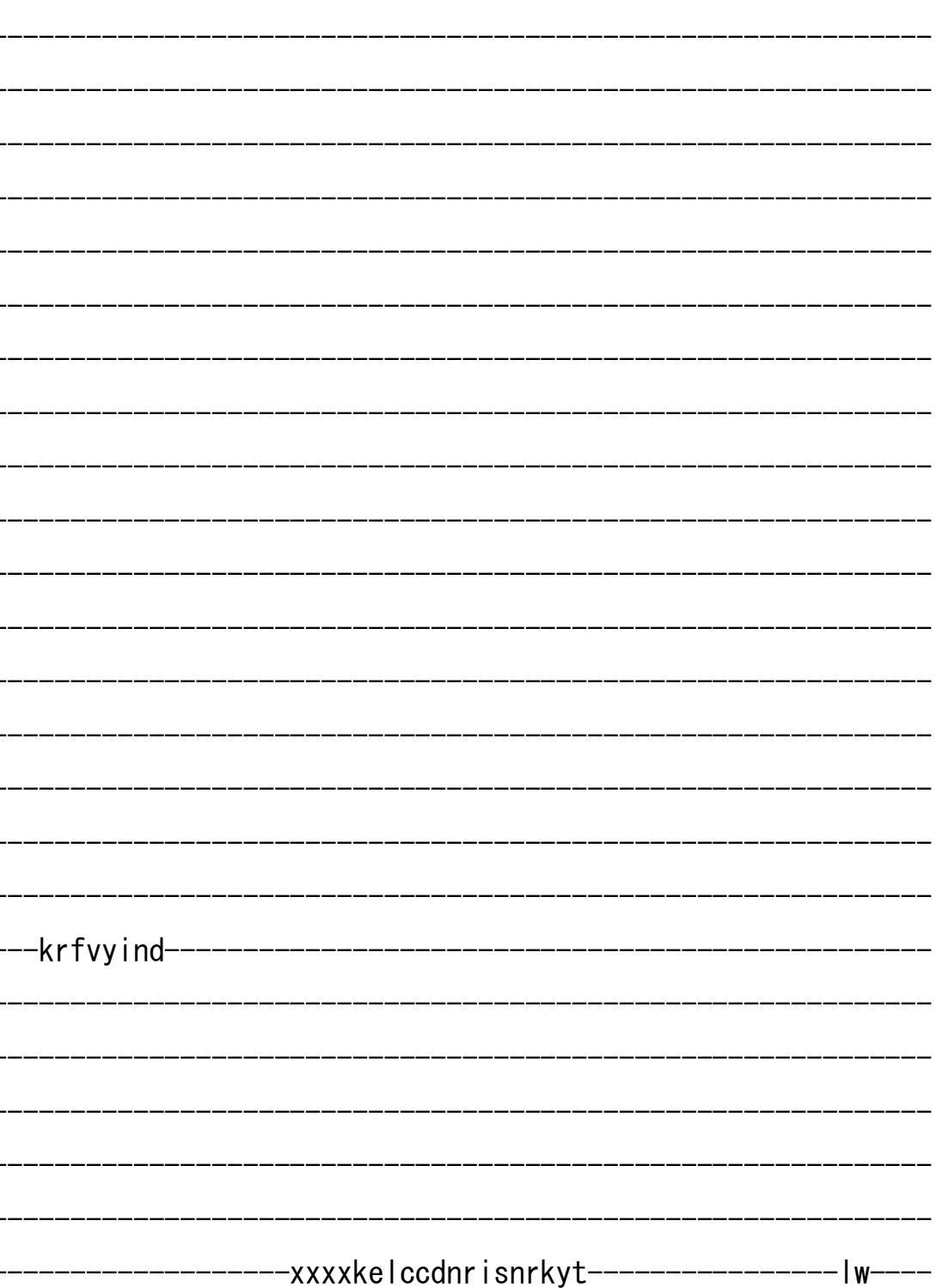

-nfIpkn I weqf 


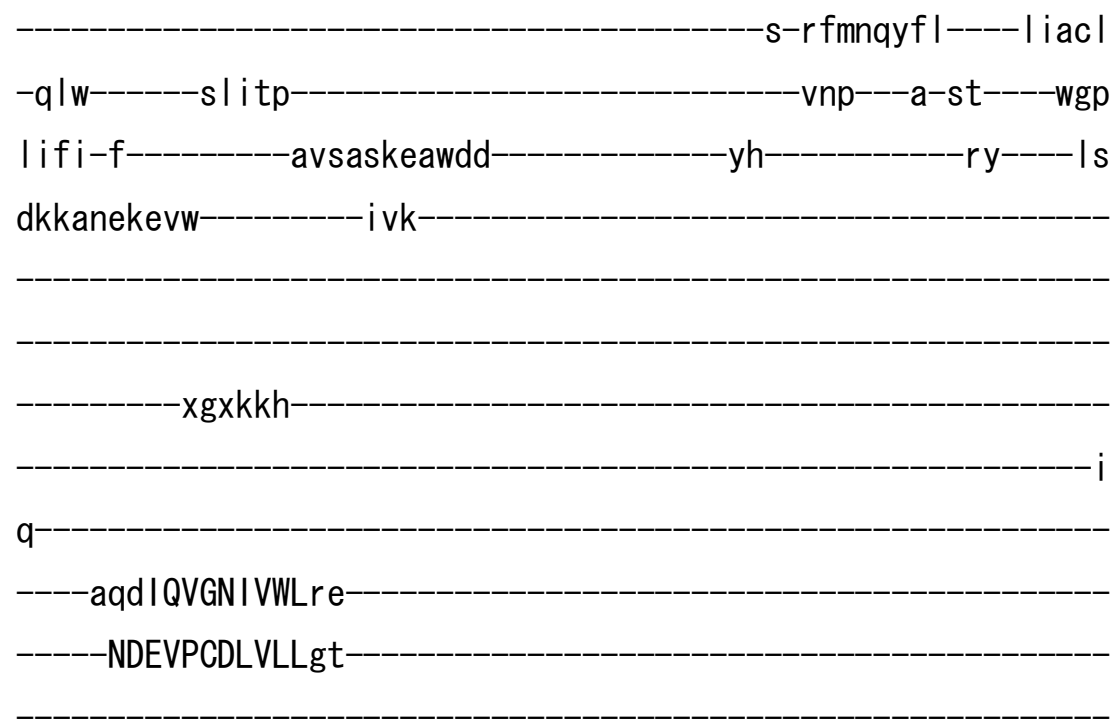

-sdpqg-v---cyVETAALDGETDLKTRvi-

-psacvgidle l Ihkmkgv i ecpvpdkdi rrfdanmr I xx

$x x x$

dndvcs

$\mathrm{ti}$

NTLLQ--SCYL----------------r

nt-----ewac-----------------------gVSVYTGNQTKLGms rg i aep

-k I tamdamidk I tgai fvfqi vvv IvIg

-i agnvwkdtearkq-------wyvq------------xxxxapwye I I vip I r fELLCSIM

IPISIKVSIDLVKGLYAK-FI ewdvem-

idqetgta-

SYAA-NTA ISEDLGQVEYILTDKTG

TLTDNKMIFr

rcciggify-

-xxxxxda lkdaq--|xna ixxxxxxxx-

$-r f$

$\mid$ tvma ic-----ntv|

$p v x x x a x x$ 


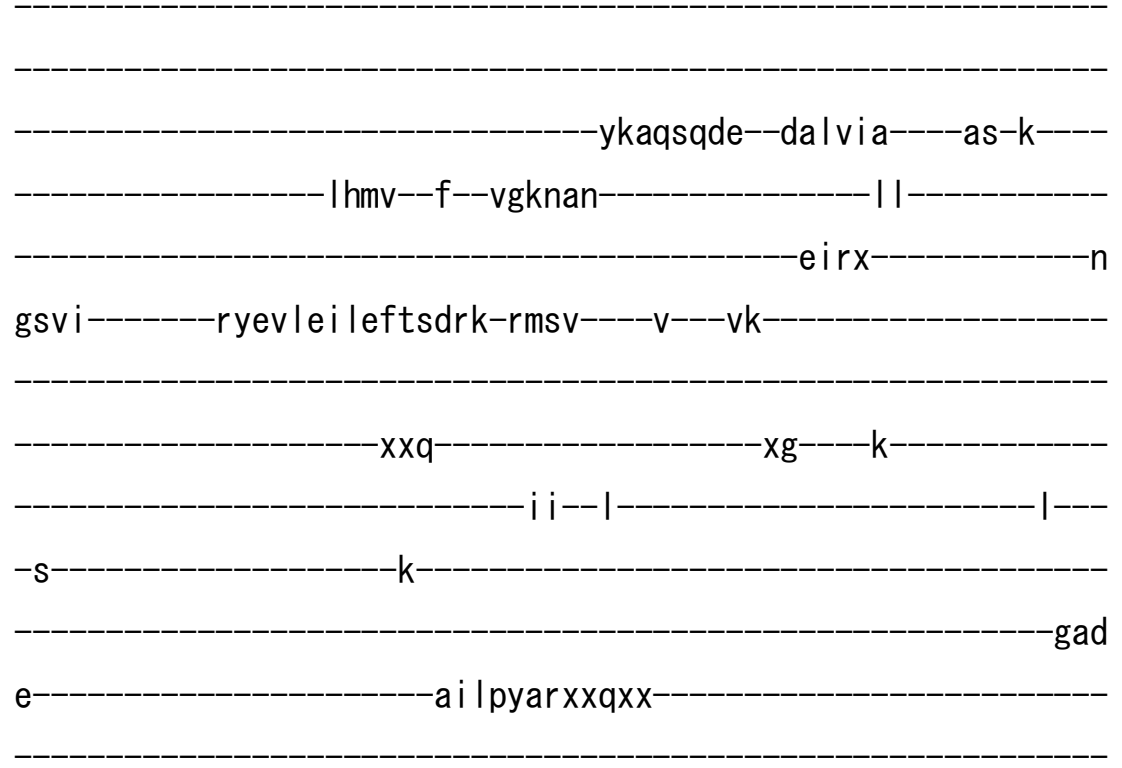

-rti-gdavehy--sql----g |rtLCLAWRe I--eeney l ewsvkfke--as------s I I vdr ewr i aevcqr- I ehd I y i LGVTA IEDRLQDGVPET IETLRKag-INF WMLTGDKQNTAIQI ALSCNFispepkgq

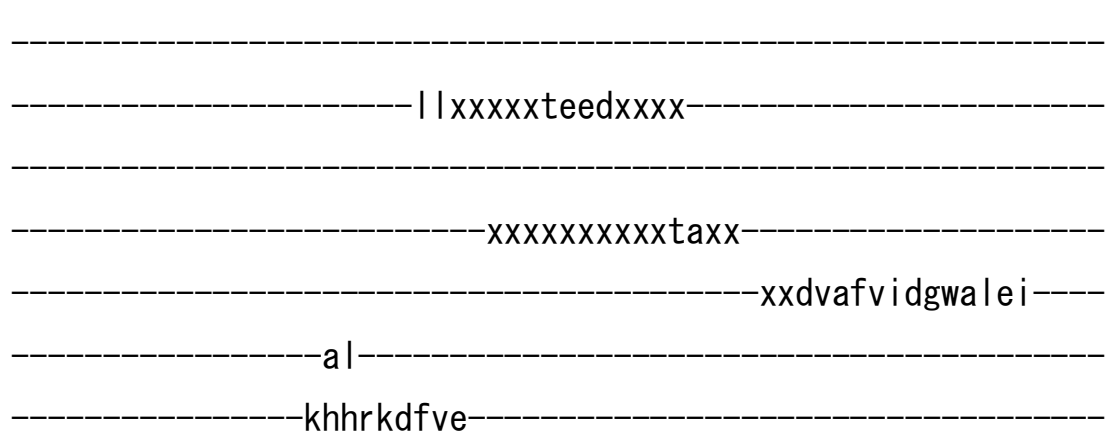

$-k h h r k d f v e-$

la i srta ICC--RVTPSQKAQLVEILk

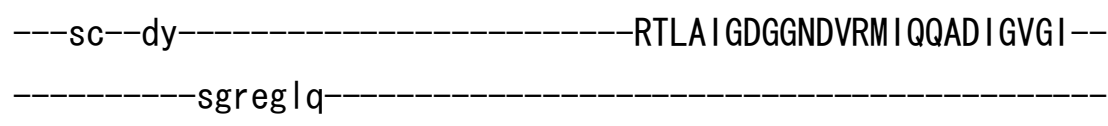

-AARAADYSI Gr fRFLKRLI I--LVHGRYSYNRTAFLS------Q 
ysf-yks II-icfiqiffsfisgv-_-_-_-_-_-_-_-_-_-_-_-_sgts I fnsvs Imay nvfytsvpv I vs-vidkd I sea-_-_-_-_-_-_-_-_-_svmqhpq i I fycqagr I I npst f-agwfgrs I fhai ivfxitihxyay-

ekxxmee I gmval sgc iw lqafv-vaqetnsftv lqh I siwgn Ivgfyainf I

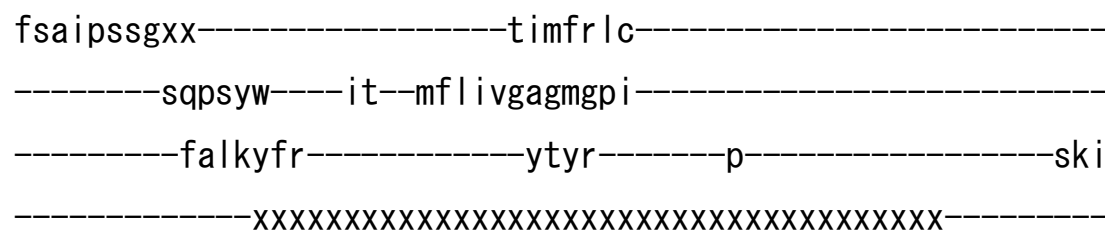

$-x x x x x x x x x x \mid$ sdspnatxxxxxxxxxxxxxxxx $\operatorname{xx} x \operatorname{xx} x \operatorname{xx} x \operatorname{xx} x \operatorname{xx} x \mathrm{x}$

>athIV_IV03 NP_176191. 1

$\mathrm{m}$ 
$---y r t v y c n d-$

$-x x x x x$

-pvrfkgns isttkyn

-vf---ー

-tflpkg|feqf

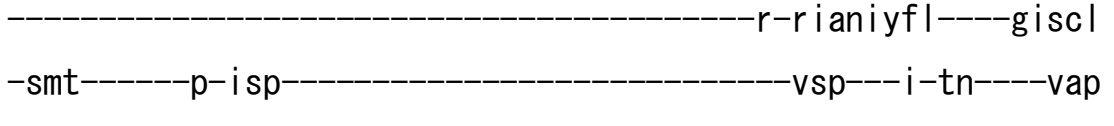

I smv-|--_-_-_-_| vs l ikeafed-_-_-_-_-_-_--wk-_-_-_-_-_--rf-_---an

dms innstve---------i lq

tnsdg-i---cyVETANLDGETNLKIRka-

lertwdy I vpekayefkge i qceqpnns I ytftgn I vv--

qkqt Ip

$\mathrm{Isp}$

-QLLLR--GCSL----------------r

$d$

gAVVFTGHETKVMmnamnaps

nt----eyiv

krstlekkldkli itifcrlvtmoliga

-igcs ivtdredk-

$y \mid g-$

xxxxxxxxxng Imi gfftfft IVTLFSSI

IPISLYVS IEMIKFIQSTXFInrdInm 
-yhaetntp

aSAR-TSNLNEELGQVEYIFSDKTG-

TLTRNLMEFf

kcs iggvsy-

$-x x x x x x x x x x x x x x$

$-x x x x x--x x x x$

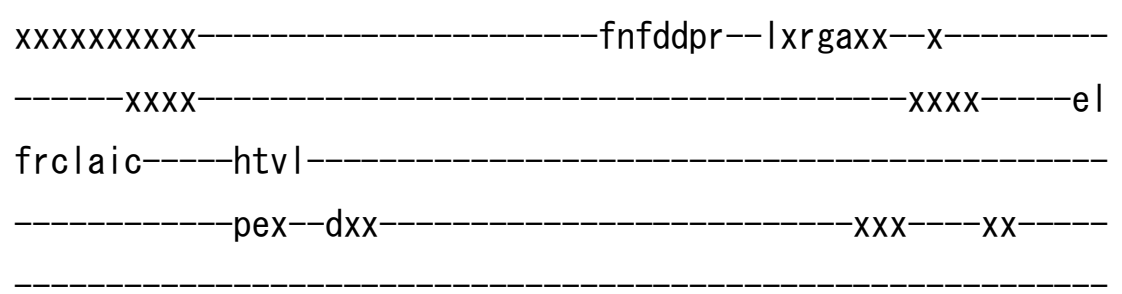

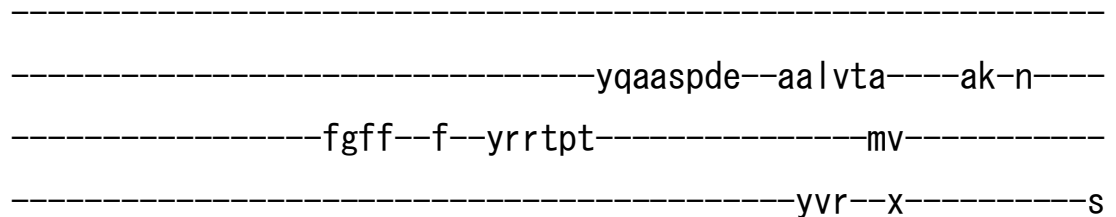

hvekxxxxxxxaye i Invlefnstrk-rqsv-----v---cr

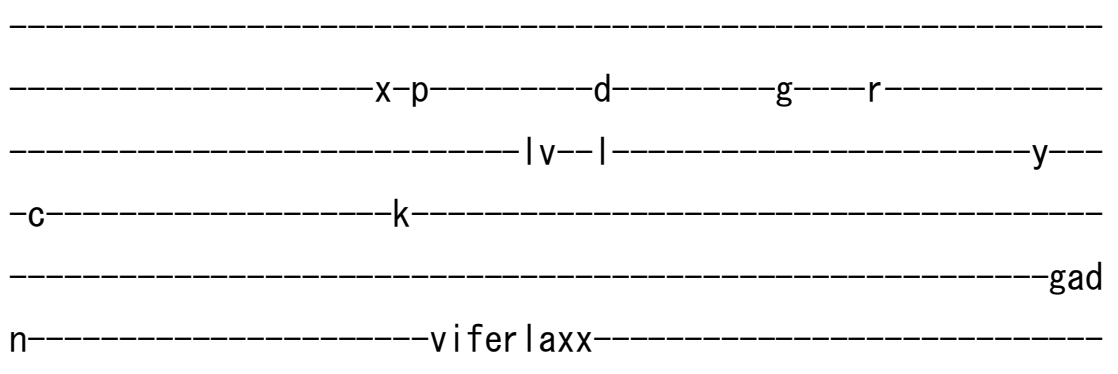

xxx--xx--kvt-rehlehf--gss-----g | r tLCLAYKdI--npetydswnekfiq ${ }^{---}$ ak-----sal rdrekk I devae I-i ekd I i I IGSTAIEDKLQEGVPTCIETLSRag-IK I WVLTGDKMETAINI AYACNL-_-_-_-_-_-_-_-_-_-_-_-_-_-_-_-_innemkqf-_-_-

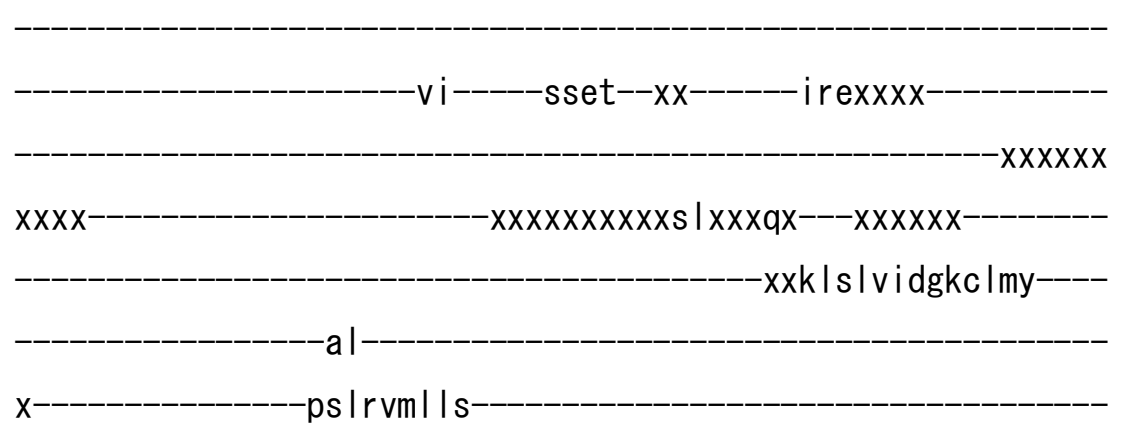




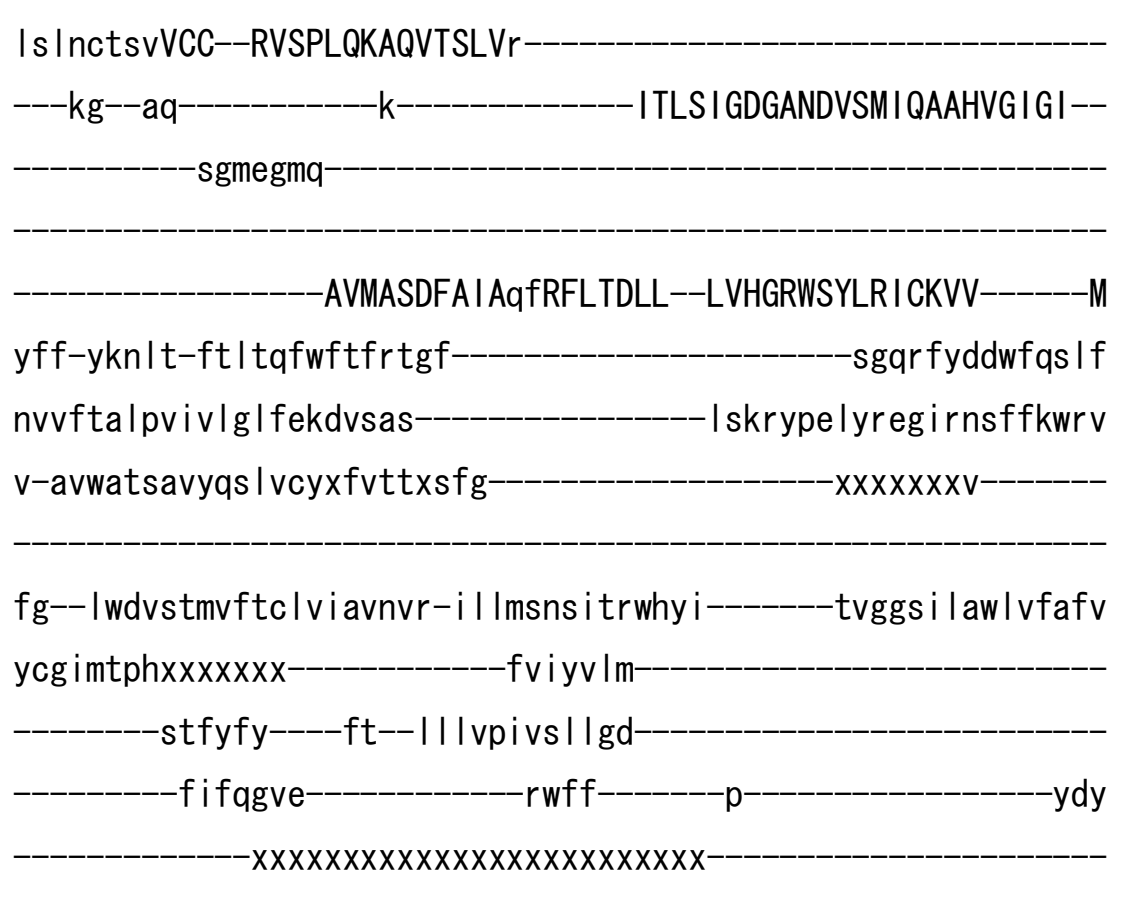

$-x x x x x x x x x x s q|p r e| s x x x x x x x x x x x x x x x x$ xxxxxx-------------------------------------------------------$-x x x x x x x x x x x x x x x x x x x x x x x x x-$

>athIV_IV04 NP_173193. 1

$\mathrm{m}-$ 
-tffpkclyeqf

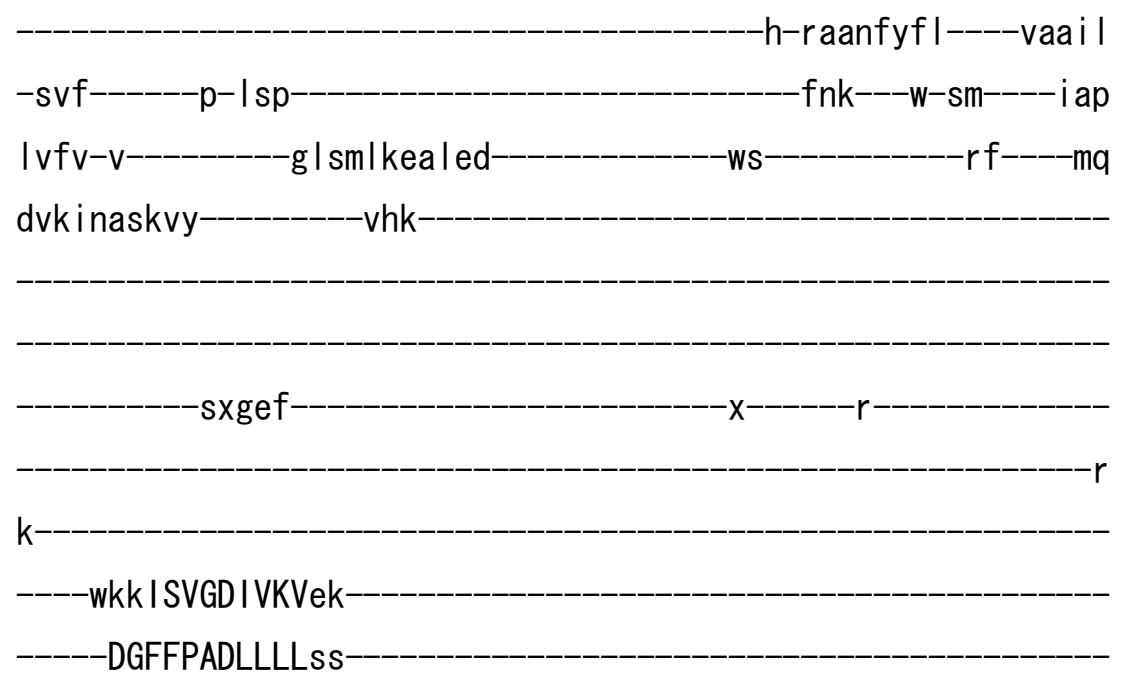


-syedg-i---cyVETMNLDGETNLKVKrs-

- evt I s Idydsfkdftg i ircedpnps I ytfvgn ley--

erqifp

$\mathrm{Idp}$

S----------QILLR--DSKL---------------r

nt----pyvy-

gVVVFTGHDTKVMqnstksps

-krsriektmdy i iyt I Ivlli I isciss

-sgfawetkfhmpkw------wy |r-----xxxxxxxxxxxnnpvyagfvhl i taLLLYGYL

IPISLYVS iEVVKVLQAS-FInkdIhm-

-ydsesgvp

aHAR-TSNLNEELGQVDT ILSDKTG

TLTCNQMDF I---------------kcs i agtsy

$X X X X X X X X X X X X X X X$

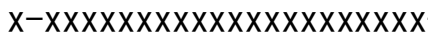

$-X X X X X--X X X X$

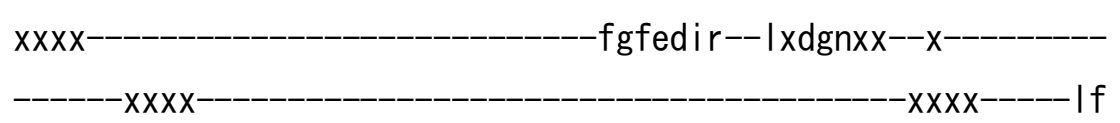

frilaic-----htai

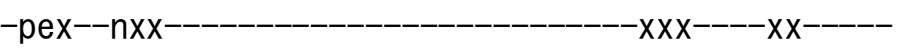

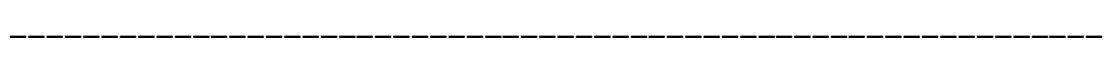

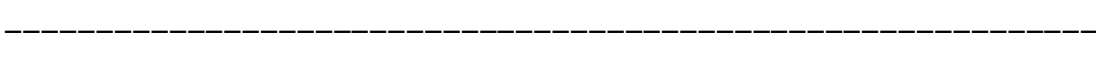

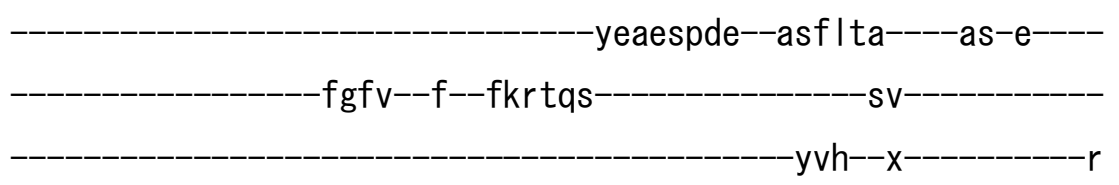

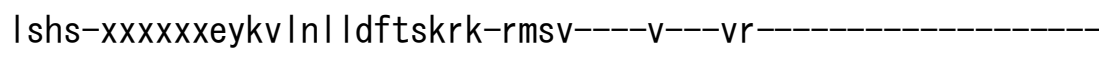

-

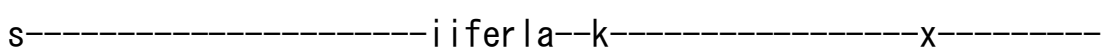


xxx--xx--gpt-tkh I ney--gea----g | r tLALSYRk |--deeeysawnaefhk--ak----xs i gsdrde I l er i sdm-i ekd I i IVGATAVEDKLQKGVPQC IDKLAQag-LKL WVLTGDKMETAINIGYSCSL

ci------tvvn--xx-

$-x x x x x x$

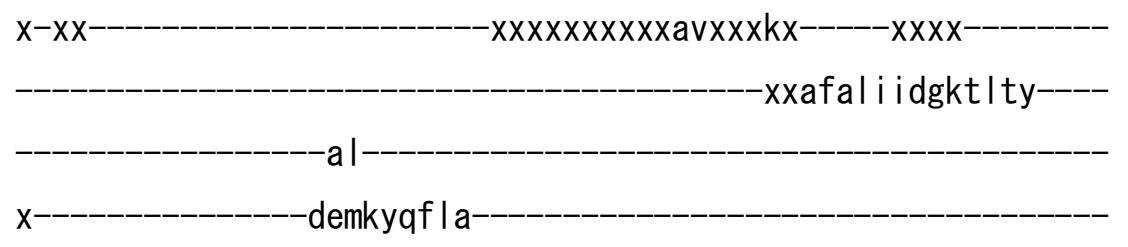

lavdcasv ICC--RVSPKQKALVFPLFp

---yaxxtg-----------k----_--_--- ITLA I GDGANDVGM I QEAD I GVGI--

-AVMASDFSI AqfRFLERLL--VVHGHWCYKR I AQM I------C

yff-yknia-fg|t I fyfeaftgf--_-_-_-_-_-_-_-_-_-_---sgqsvyndyy IIIf

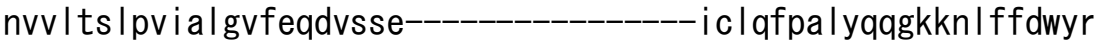
i-I gwmgngvyss Ivif-fInixi i ad--mdavgttmftc $\mathrm{i}$ i wavnvq-ialtvshftwighv--_----l i iwgs i $\mathrm{g}$ | wy I fva I ygmmpps I xxxxxx---------------i I ve i la -papi yw----ia--tflvtvttvlpy-

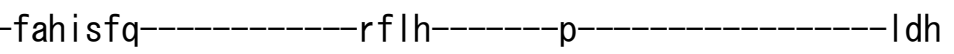
$-x x x x x x x x x x x x x x x x x x x x x x x x x x$ 
>athIV_IV05 NP_177414. 1

m

-argrirs

$-x x$

$-x x x x x x x x x x x x x x x x x x x x y-$

$-x x x x x x x x x$

---srtvfena

$-x x x x x x$

-xp | ryrsnyvsttryn $-1 \mathrm{i}$

-tffpks lyeqf 


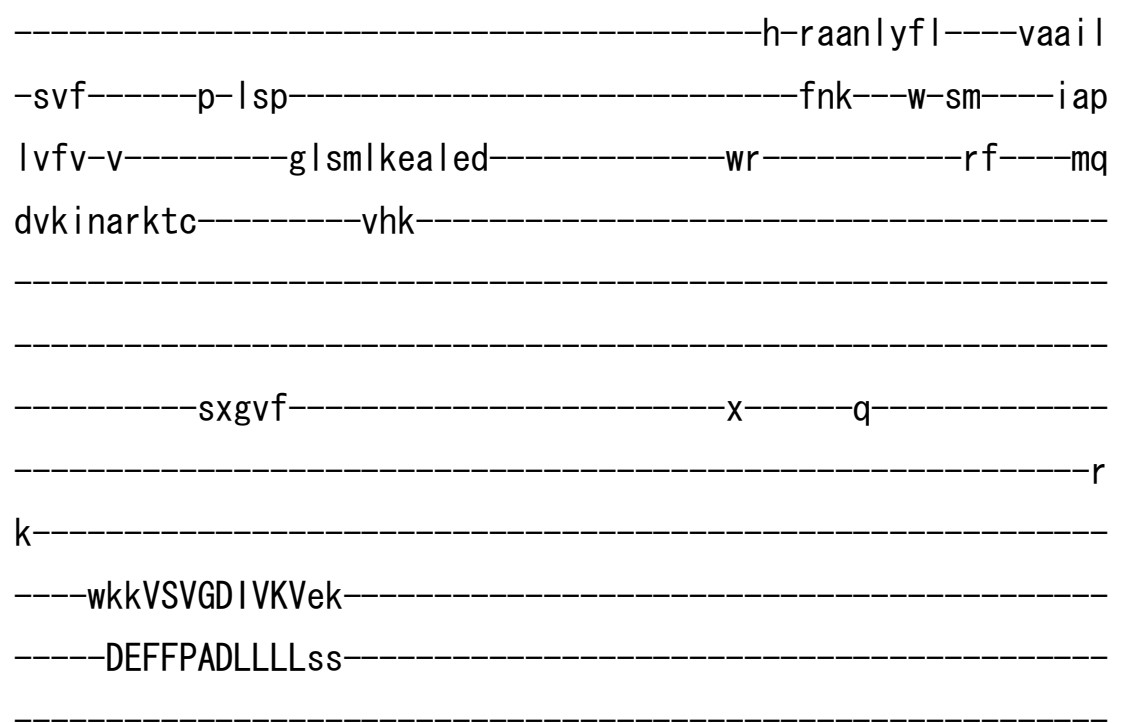

-syedg-i---cyVETMNLDGETNLKVKrs-

- evs $|p| d d d e s f k n f m a t i r c e d p n p n \mid y t f v g n l$ ef--

erqtfp

Idp

-s----------QILLR--DSKL---------------r

nt----tyvy gVVVFTGFDTKVMqnstksps

-krsriertmdy i iyt IIvlli Iisciss -sgfawetefhmpkm-------wy | r-----xxxxxxxxxxxnp i yagvvhl i taLLLYGYL IPISLYVS iEVVKVWQAS-FInqd I hm-

-yddesgvp

aNAR-TSNLNEELGQVHT ILSDKTG

TLTCNQMDF I -kcs i agtsy

$x X X X X X X X X X X X X X X-X X X$

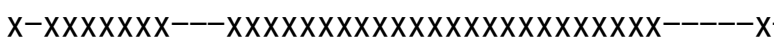

$-x \times x x x--x x x x$

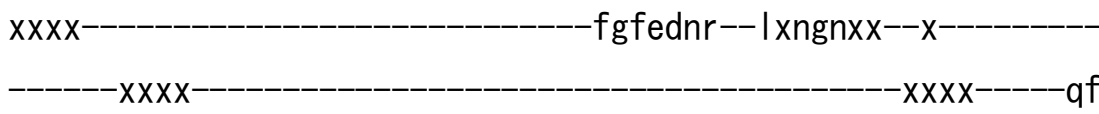

frilaic-----htai-

pex--nxx- 


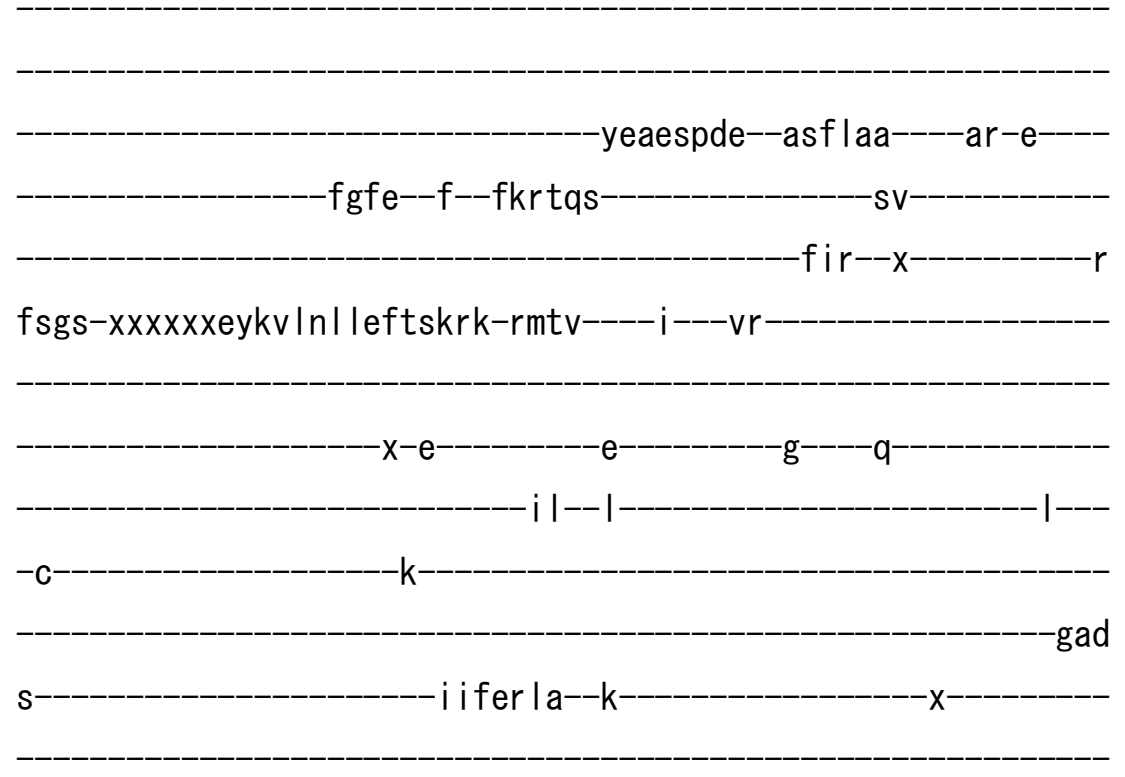

xxx--xx--gpt-trhl tey--gea----g | rtLALAYRk |--dedeyaawnsef I k--ak----xs i gsdrde I l etgadm-i eke I i I IGATAVEDKLQKGVPQCIDKLAQag-LKL WVLTGDKMETAINIGFACSL-

Irqgmrqi----

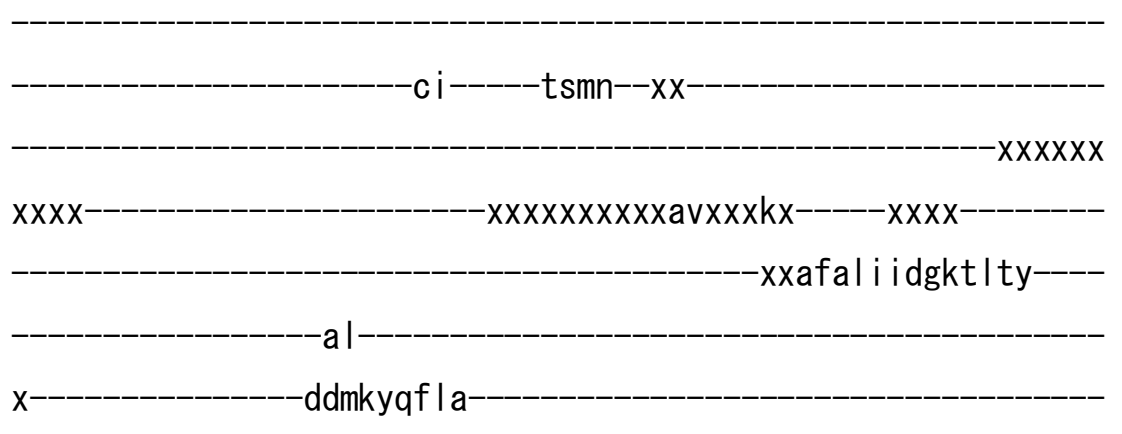

I avdcasv ICC--RVSPKQKALVVRLVk

---eg--tg------------k-----_-------TTLA I GDGANDV GM I QEAD I GVGI--




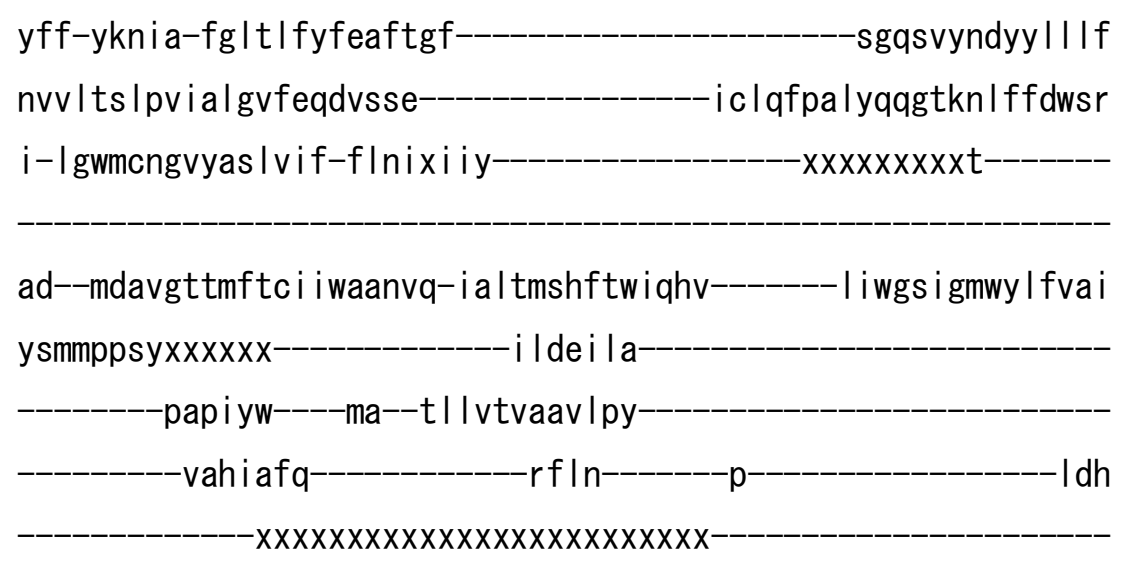

-xxxxxxxxxxarvdakirxxxxxxxxxxxxxxxx $x x x x x x x x x x x$

>athIV_IV06 NP_175830.1

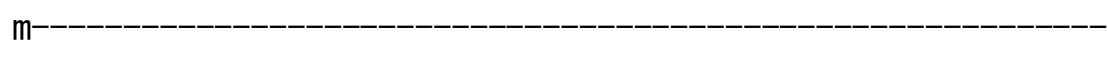




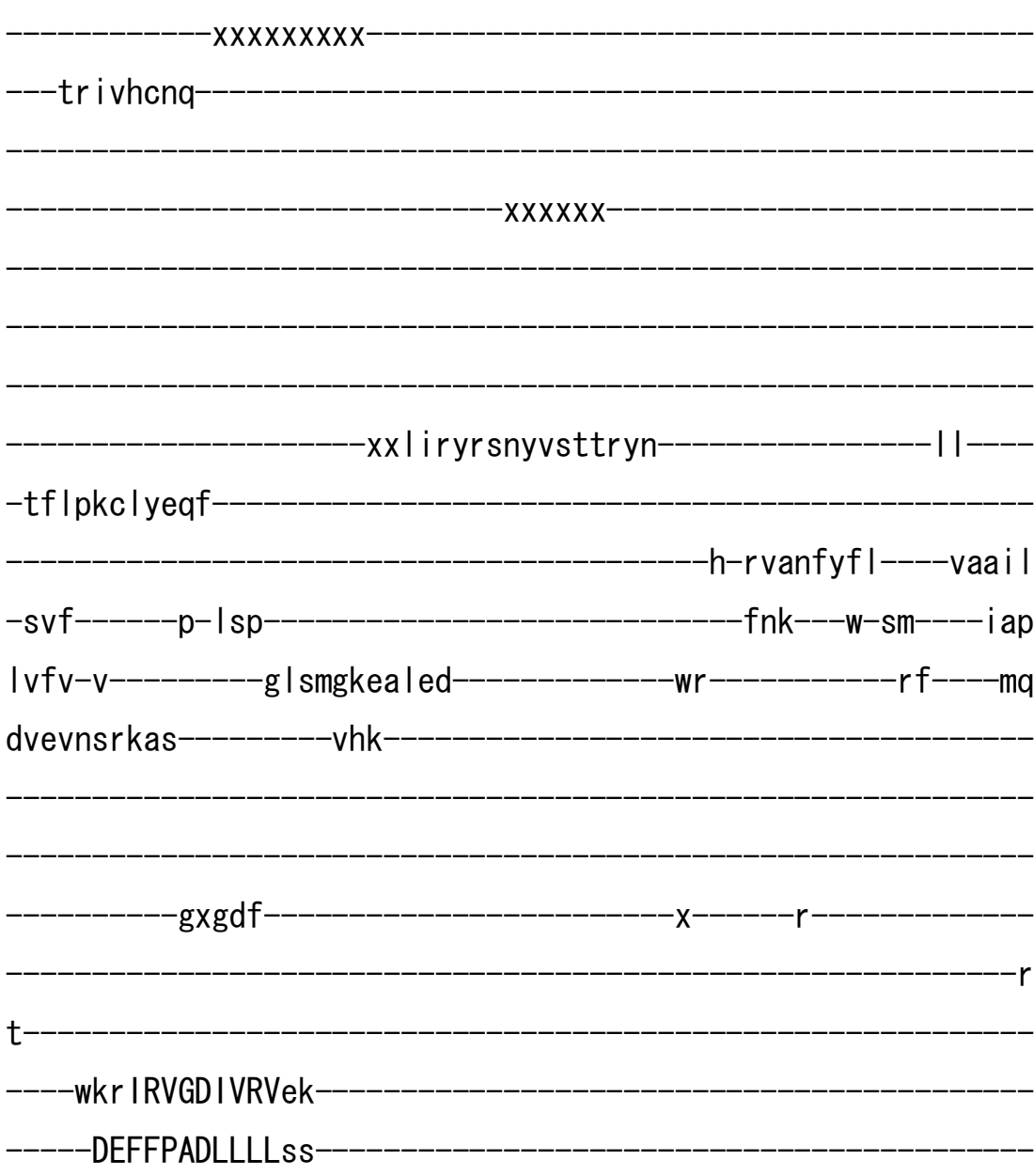

-syedg-i---cyVETMNLDGETNLKVKrc-

- dat l a lekdesfanfsgtikcedpnpn l ytfvgn lec--

dgqvyp

Idp

QILLR--DSKL

nt----ayvy

gVVVFTGHDTKVMqnstksps

-krsr i ekrmdy i iyt I fall Itvsfiss

- | gfavmtk | Imaew------wy | r-----xxxxxxxxxxxxnp l yawvvh I i taLLLYGYL IPISLYVS iEVVKVLQAH-FInqd Iq I 
-ydsesgtp

aQAR-TSNLNEELGQVDT ILSDKTG-

TLTCNQMDF I

-kcs iagtsy-

$-x x x x x x x x x x x x x x x-x x x$

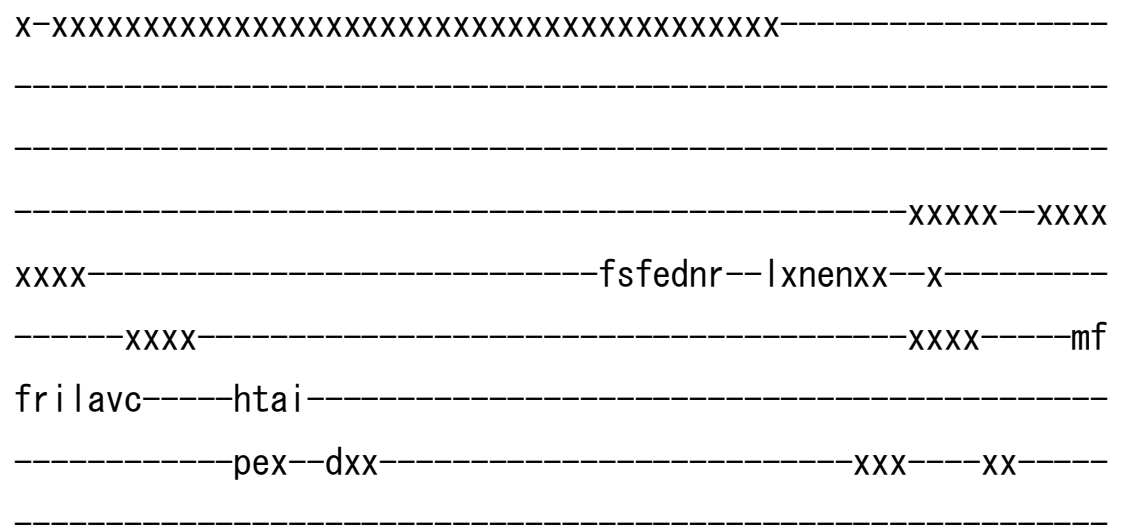

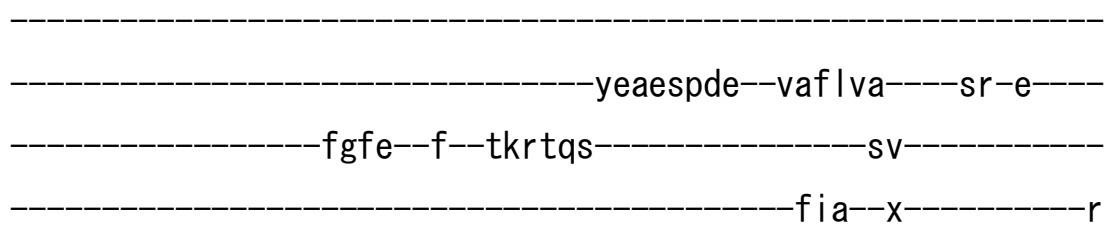

fsss-xxxxxxeyki Inl Idftskrk-rmsa----i---vr

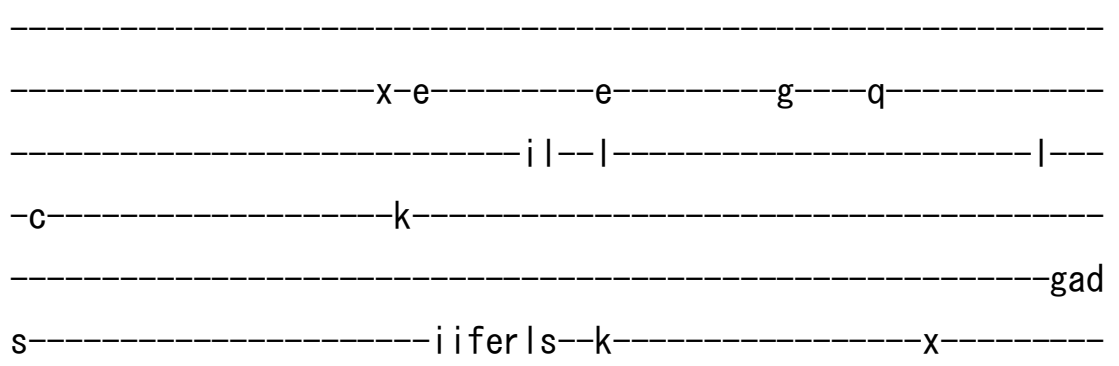

xxx--xx--gat-skh Invy--gea----g | rtLALGYRk I--deteyaawnsefhk--ak----xsvgadrdem l ekvsdm-meke I i IVGATAVEDKLQKGVPQCIDNLAQag-LK I WVLTGDKMETAIN I GYACSL--_-_-_-_-_-_-_-_-_-_-_-_-_-_--_ I rqgmkqi --_--

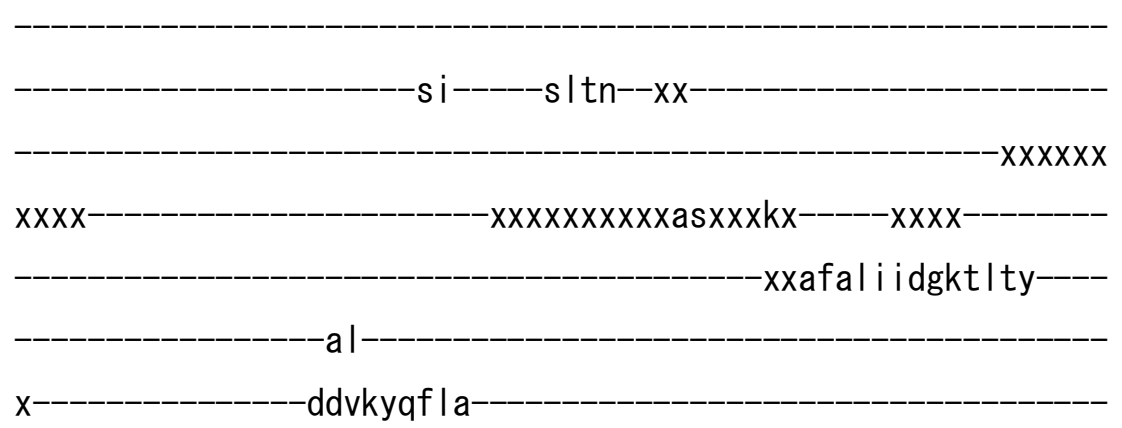


lavdcasv ICC--RVSPKQKALVTRLAk

---eg--tg------------k-----_-_-----TTLA I GDGANDVGM I QEAD I GVGI--

-AVMASDFSIAqfRFLERLL--VVHGHWCYKRIAQM I------C yff-yknit-fglt I fyfecftgf--_-_-_-_-_-_-_-_-_-_-_--sgqs i yndsy II If nvv Its I pvi s I gvfeqdvpsd--_--_-_-_-_-_-_--vc l qfpa l yqqgpkn I ffdwyr

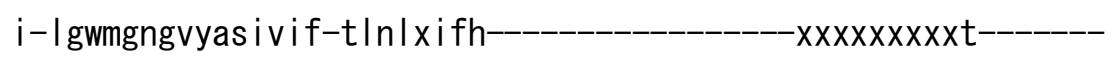
ad--mnamgtamftc i i wavnvq-ial tmshftwighv----_---mi wgs i gawyvfla l ygm I pvk I xxxxxx-_-_-_-_-_-_---mlve i la--_-_-_-_-_-_-_-_-_-_-_-_-_-

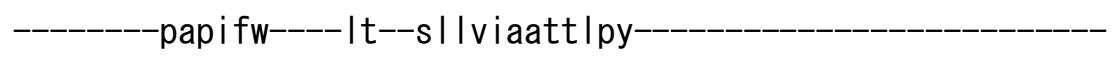
--------- I fhi syq------------rsvn-------p---------------- Idh

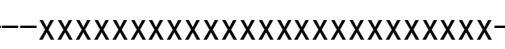
-xxxxxxxxxxarvdakirxxxxxxxxxxxxxxxxx $\operatorname{xx} x \operatorname{xx} x \operatorname{xx} x \operatorname{xx} x x x-$

>athIV_IV07 NP_188006. 1

m- 
grrirs

$-x x-$

$-x x x x x x x x x x x x x x x x x x x x-$

$x x x x x x x x x$

---trivhenq

$-x x x x x$

xv|rytsnyvsttryn

li----

-tfIpkclyeqf

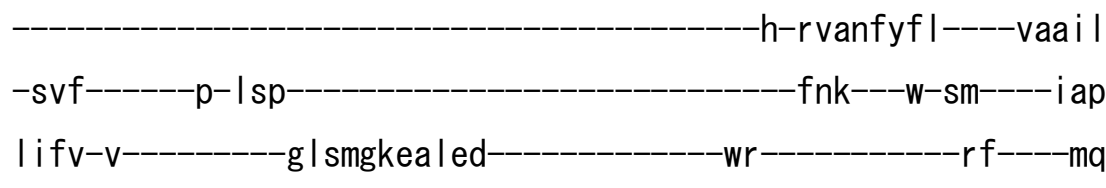

dvkvnsrkat----------vhr

gxgdf-

$-x------r$

k

----wkkLRVGDVVKVek

DQFFPADLLLLss 
-syedg-i---cyVETMNLDGETNLKVKrc-

Idvt Iplerddtfqsfsgtikcedpnpn lytfvgnley--

dgqvyp

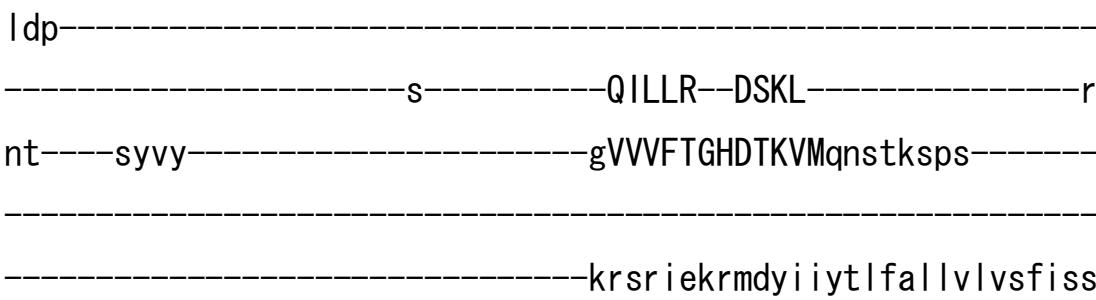

- I gfavmtkmhmgdw-------wy | r-----xxxxxxxxxxxnnfhawvvh I i taVLLYGYL

IPISLYVS IELVKVLQAT-FInqd I qm-

-ydsesgtp

aQAR-TSNLNEELGQVDT ILSDKTG

-TLTCNQMDF I---------------kcs i agtsy

$x x x x x x x x x x x x x x x-x x x$

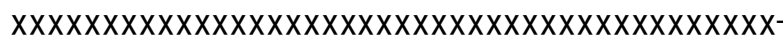

$-x x x x x--x x x x$

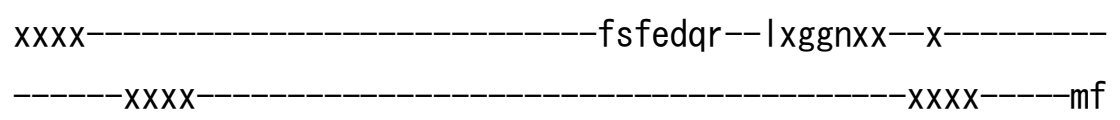

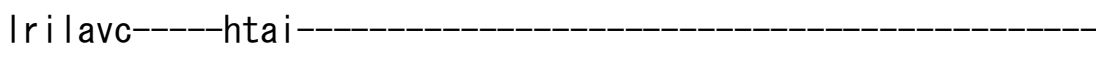

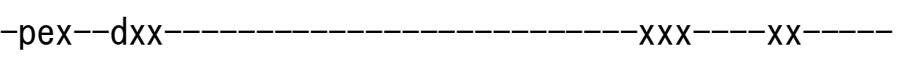

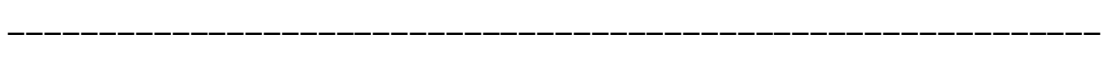

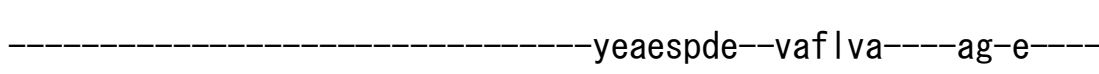

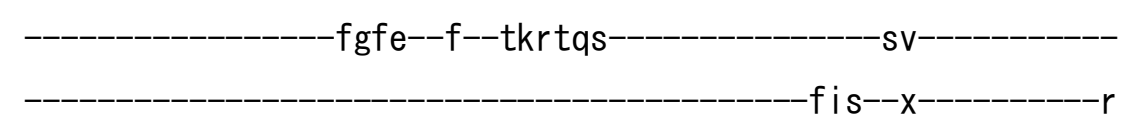

--hs-xxxxxxeykfInvIdftskrk-rmsv----i---vr-------------------

-------------------------------------------------------------

-gad

s

- i ifer ls--k

$-x$ 
xxx--xx--eat-skh Ingy--gea----g |rtLALSYRk I--deteys i wnsefhk--ak----xsvgadrdem l ekvsdm-meke I i IVGATAVEDKLQKGVPQC IDKLAQag-LK I WVLTGDKMETAINIGYACSL

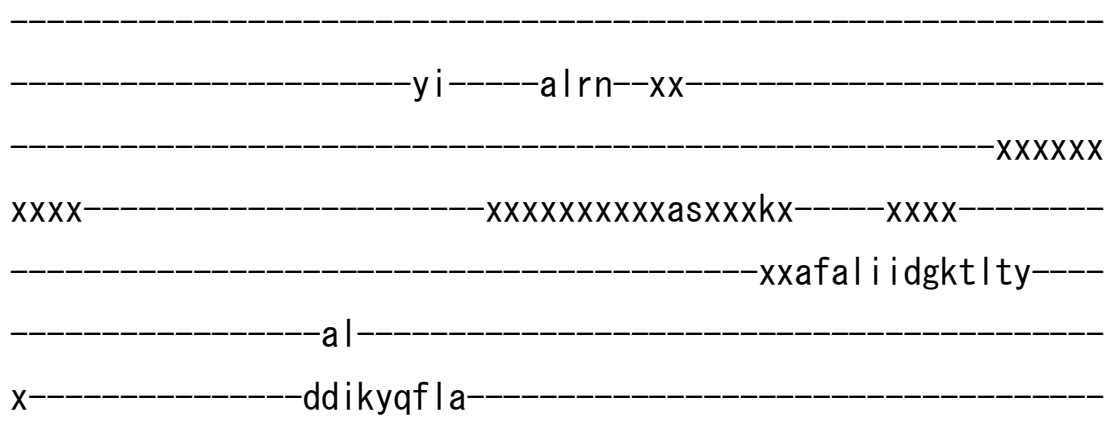

lavdcasv ICC--RVSPKQKALVTRLAk

---eg--tg---_--------k----_--_----TTLA I GDGANDVGM I QEAD I GVGI--

-AVMASDFSI AqfRFLERLL--VVHGHWCYKRIAQMI------C yff-yknit-fg|t I fyfeaftgf-_-_-_-_-_-_-_-_-_-_-_-sgqa i yndsy II If nvi I ts I pvia l gvfeqdvsse---_-_-_-_-_-_-_--vc l qfpa lyqqgpkn I ffdwyr i-igwmangvyasvvif-sInixifh-_-_-_-_-_-_-_-_--_xxxxxxxxt---_----

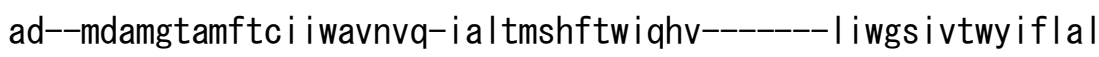
fgml ppkvxxxxxx---_-_-_-_-_---ml set la--_-_-_-_-_-_-_-_-_-_-_-_-_-

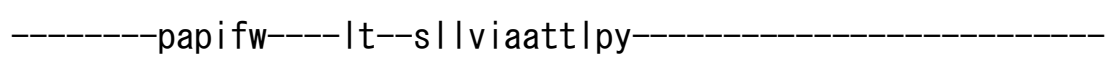
- 
>athIV_IV08 NP_189425. 1

m-

- -----ger rk---------xx

$-x x x x x x x x x x x x x x x x x x x x x-$

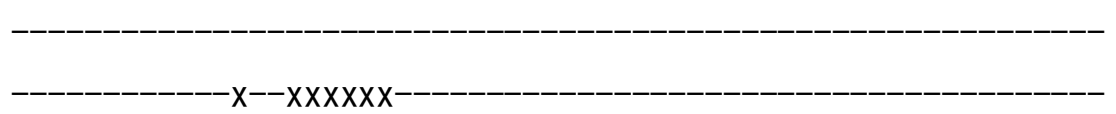

---srvvfand

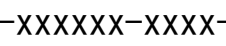

-yrgnyvsttkyt

-nf ipks I feqf 


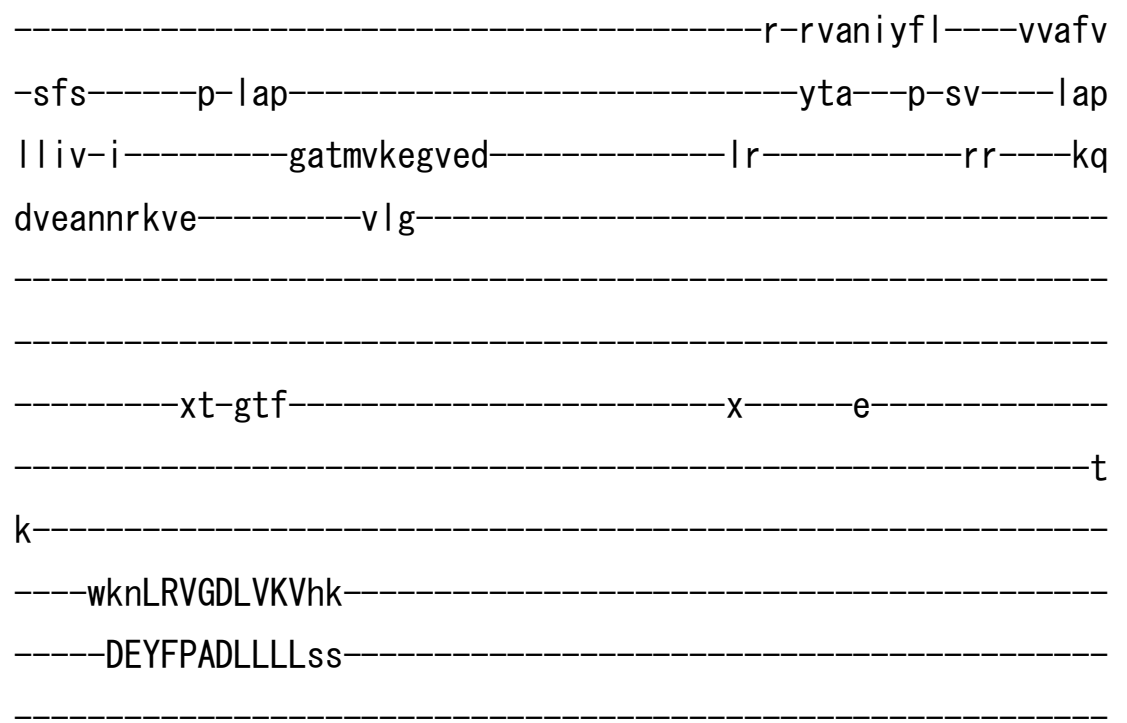

-syedg-i---cyVETMNLDGETNLKLKha-

- leitsdees iknfrgmikcedpnehlysfvgt lyf--

egkqyp

$\mathrm{Isp}$

QILLR--DSKL----------------k

nt-----dyvygVVVFTGHDTKVMqnatdpps

krskiekkmdqi iyilfsiliviaftgs -vffg iatrrdmsdn-xxxxxwy | r-----xxxxxxxxxxxravaaaffhf I taLMLYGYL IPISLYVS iEVVKVLQSI-FInqdqem-

-yheetdrp aRAR-TSNLNEELGQVDT ILSDKTG

TLTCNSMEF -kcs i agtay

$x \times X X X X X X X X X X X X X X X X X$

$X X X X X X X$

$-x \times x x x--x x x x$

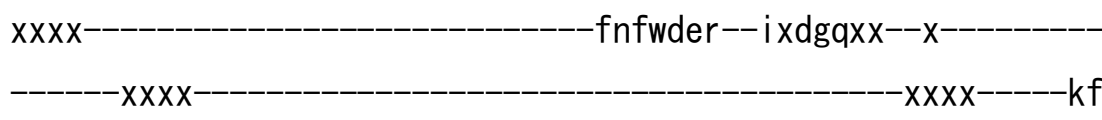

frvlaic-----htai

$p d x--n x x-$

$-\mathrm{xxx}----\mathrm{xx}-----$ 


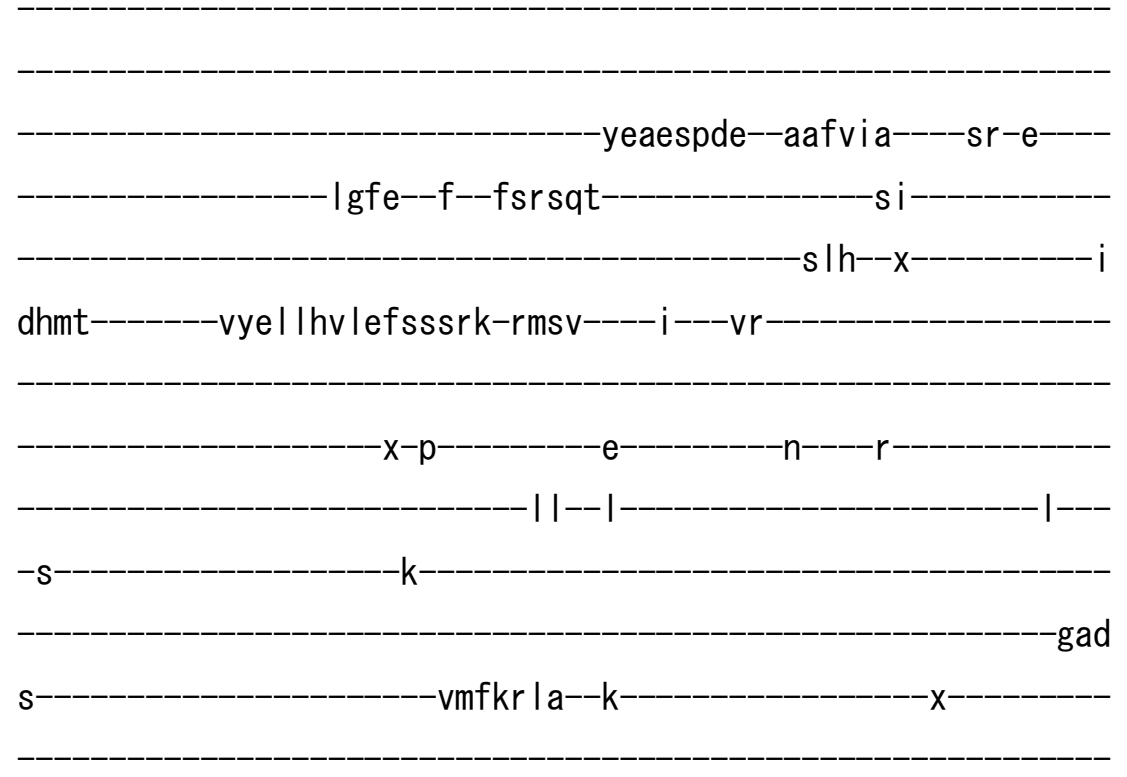

xxx--xx--ret-keh i kky--aea----g | r tLVI TYRe i--dedey i vweeef In--ak----x I vtedrdal i daaadk-i ekd I i ILGSTAVEDKLQKGVPDCIEKLSQag-VK I

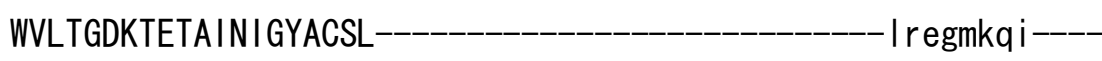

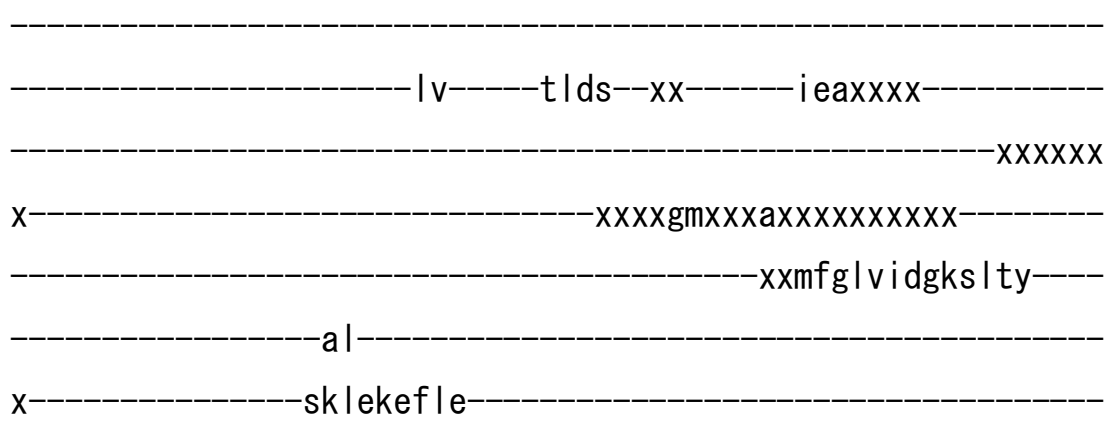

la ircnsv ICC--RSSPKQKALVTRLVk

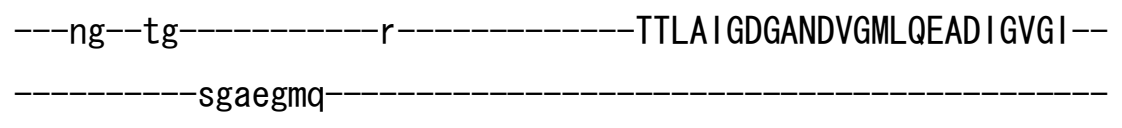

-AVMASDFA I AqfRFLERLL--LVHGHWCYRRI TLMI------C 


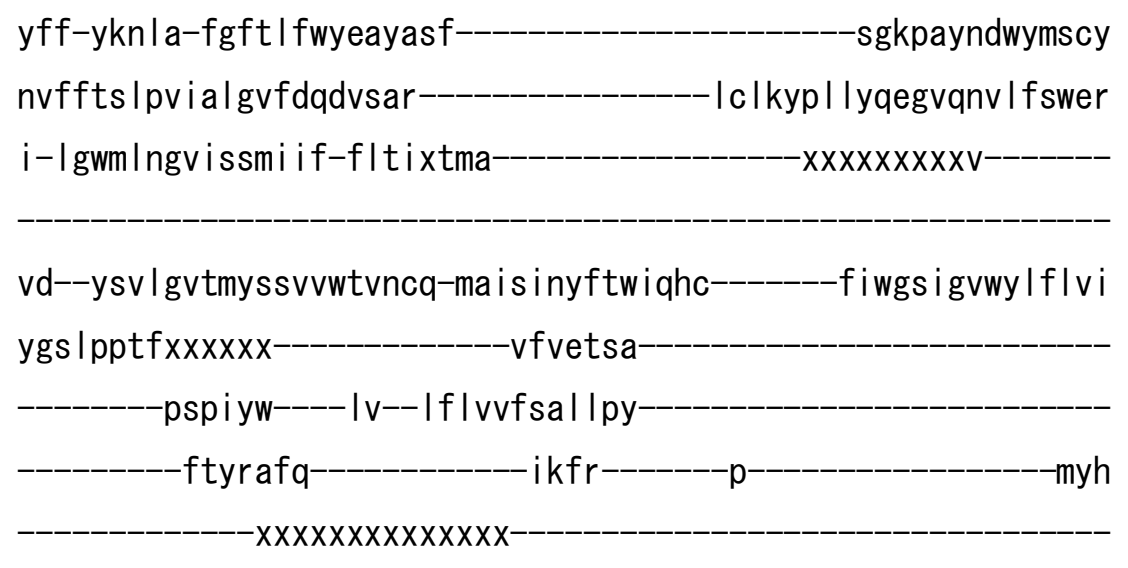

>athIV_IV09 NP_177038. 1

m- 


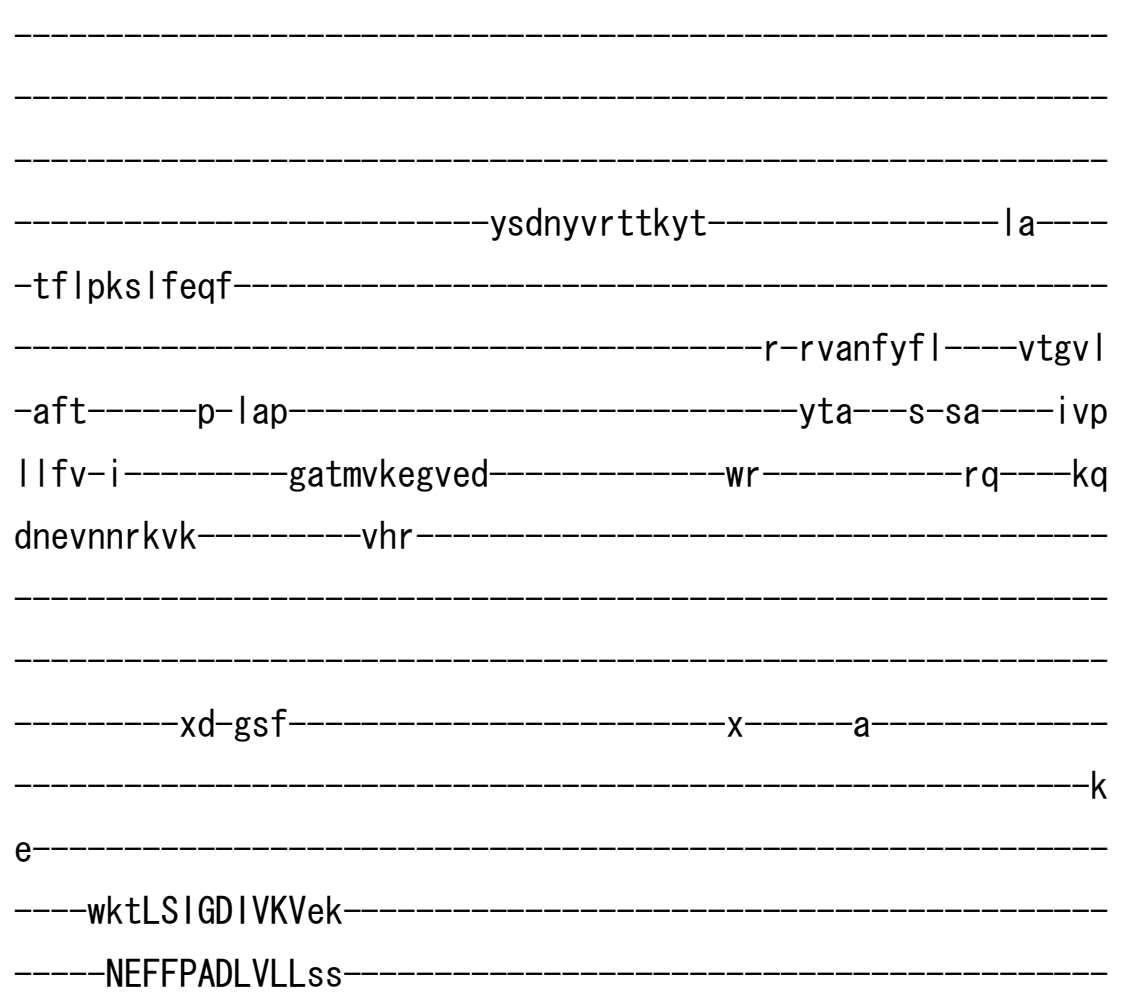

-syeda-i---cyVETMNLDGETNLKVKqg-

- evtss |rdefnfkgfeafvkcedpnan lysfvgtme |--

-kgakyp

$\mathrm{Isp}$

$-Q L L L R--D S K L-$

nt----df if

gAVIFTGHDTKVI qustdpps

-krsmi ekkmdk i i y Imf fmv i tmaf i gs

-vifgvttrddlkdg--xxxxwy I r----xxxxxxxxxxxxrapvaai yhf I taVMLYSYF IPISLYVSiEIVKVLQSI-FInqdihm- 
-yyeeadkp

aRAR-TSNLNEELGQVDT ILSDKTG

TLTCNSMEF

-kcsvagtay

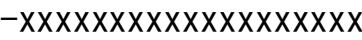
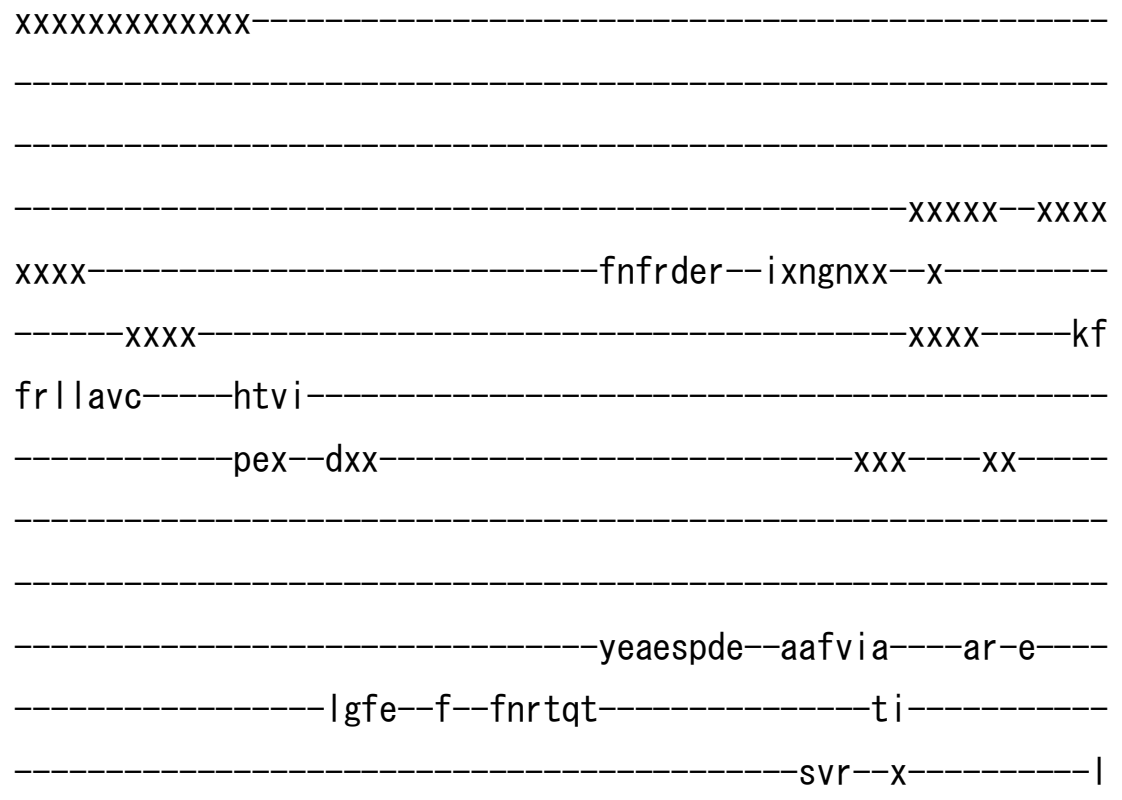

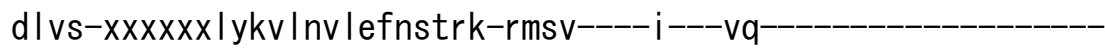

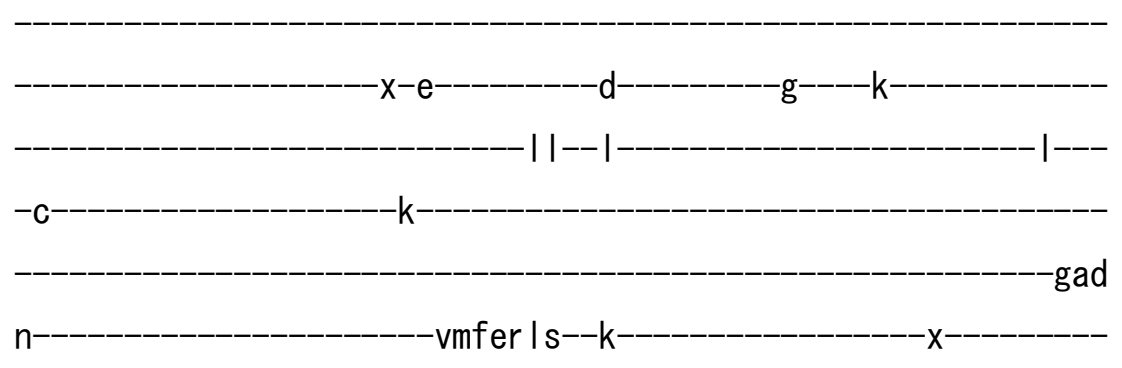

xxx--xx--eet-rdhvney--ada----g | rtLILAYRe I--dekeykvfner i se---ak----xsvsadres I i eevtek-i ekd I i ILGATAVEDKLQNGVPDCIDKLAQag-IK I WVLTGDKMETAIN I GFACSL-_-_-_-_-_-_-_-_-_-_-_-_-_-_---_ I rqdmkq i---_

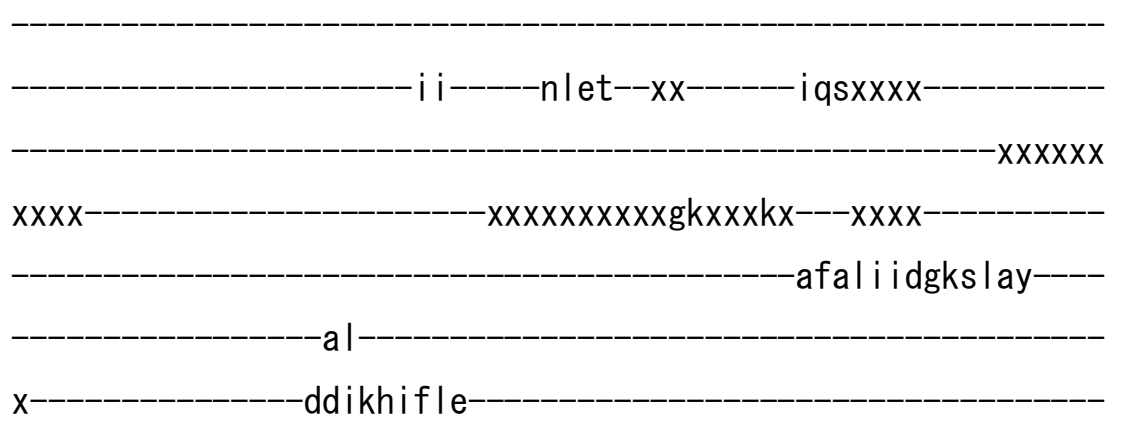


I avscasvICC--RSSPKQKALVTRLVk-

---sg--ng--_--_--_---k--_-_-_-_-_--_TTLA I GDGANDVGMLQEAD I GVGI --

-AVMSSD I A I AqfRYLERLL--LVHGHWCYRR ISTMI------C

yff-yknit-fgft Ifl yetyttf-_-_-_-_-_-_-_-_-_-_-_--sstpayndwf I I I nvffss I pvi a I gvfdqdvsar--_--_-_-_-_-_---yc l kfp I I yqegvqnv I fswr r i-I gwmfngfysav i if-flckxs lq------------------xxxxxxxxxt--------

pg--re i I ggtmytc i vwvvn la-ma la i syft l iqhi--------vi wss i vvwyffitv

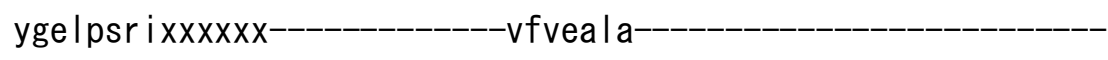

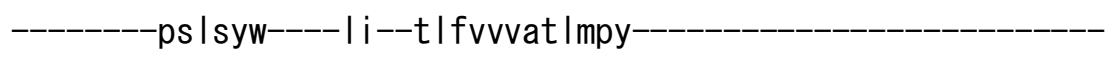

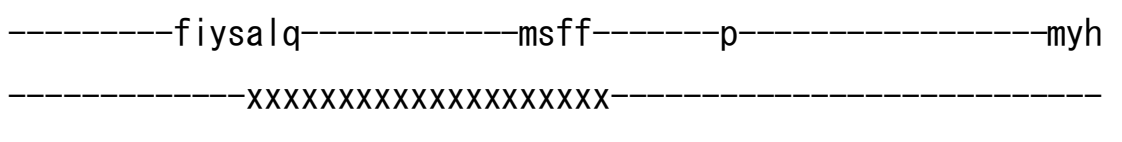
-xxxxxxxxxxvgftarlexxxxxxxxxxxxx--$-$ >osalV_IV01 NP_001042736. 1 $\mathrm{m}-$ 


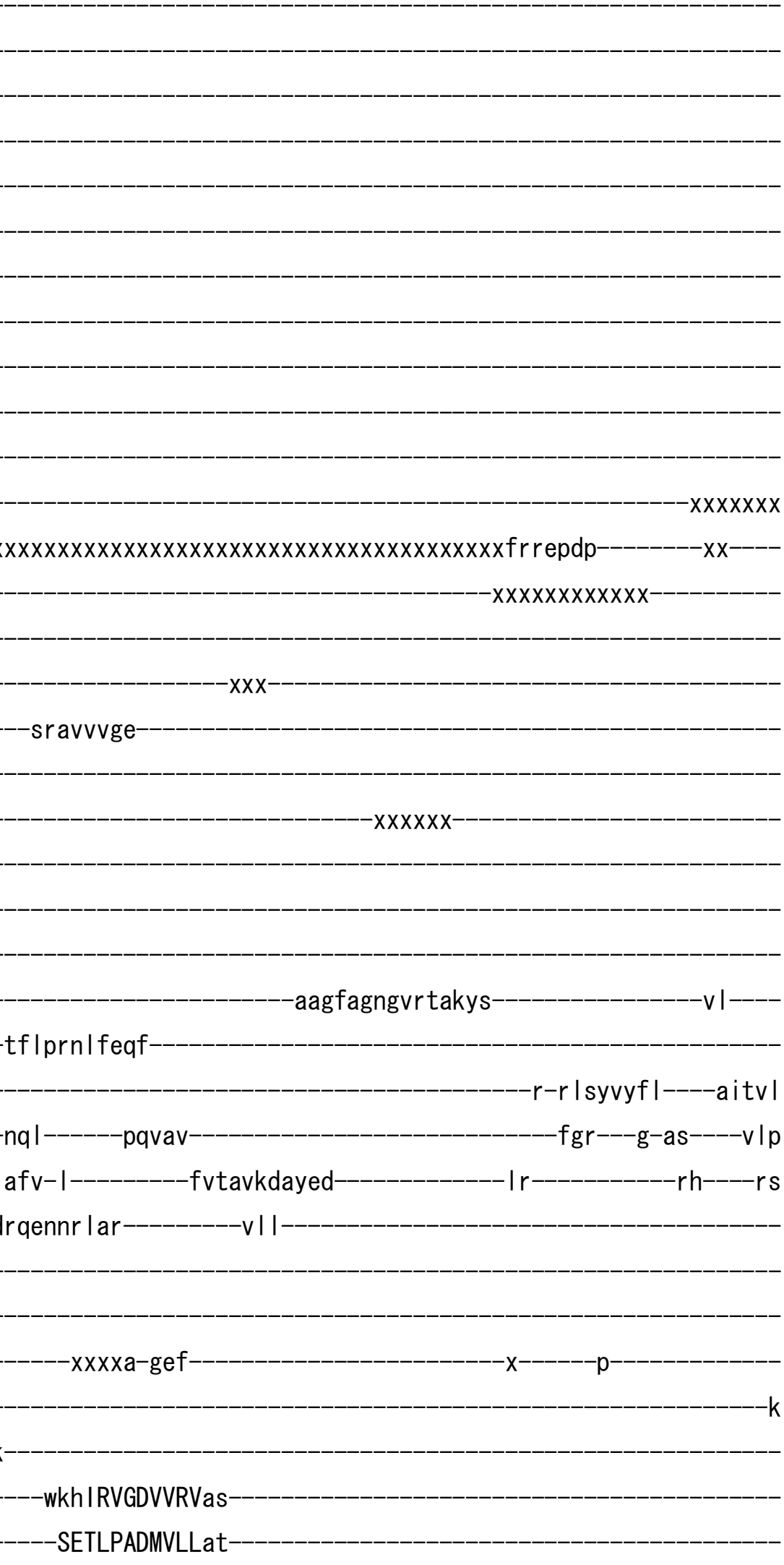


-sdpsg-v---ahVQTVNLDGETNLKTRya-

kqetal r fsqdggi ggv lhcerpnrni ygfqan le i--

dgkrvs

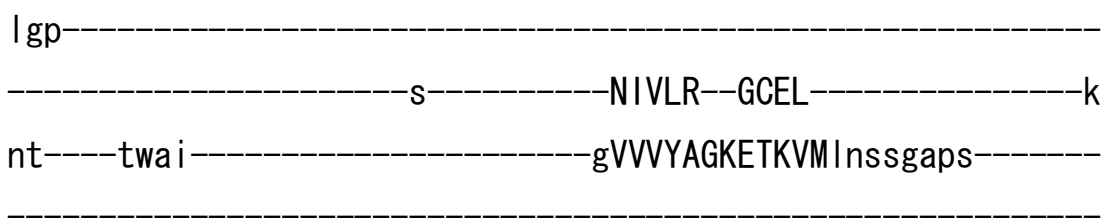

-krsr letqInretvi I siml igmcttas

-vlag i wi Inhrgd l--xxxxffrexxxxx---xxxxxxxgmgmq i f itf ImaVIVYQVI

IPISLYISmELVRLGQAY-FMgadrd I

-ydessrsk-

-fQCR-ALN I NEDLGQ I KYVFSDKTG-

TLTENKMEFq

-cas irgvdy

C

$-\operatorname{sgxxxcxx}$

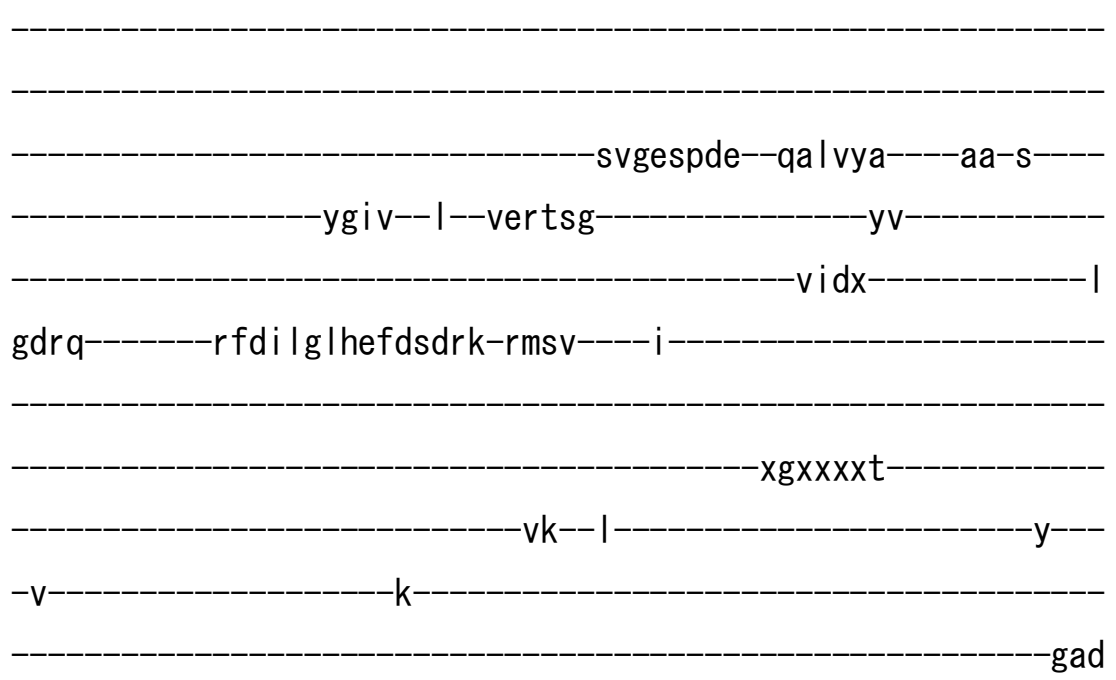

s

-s|fgitkxx|x- 


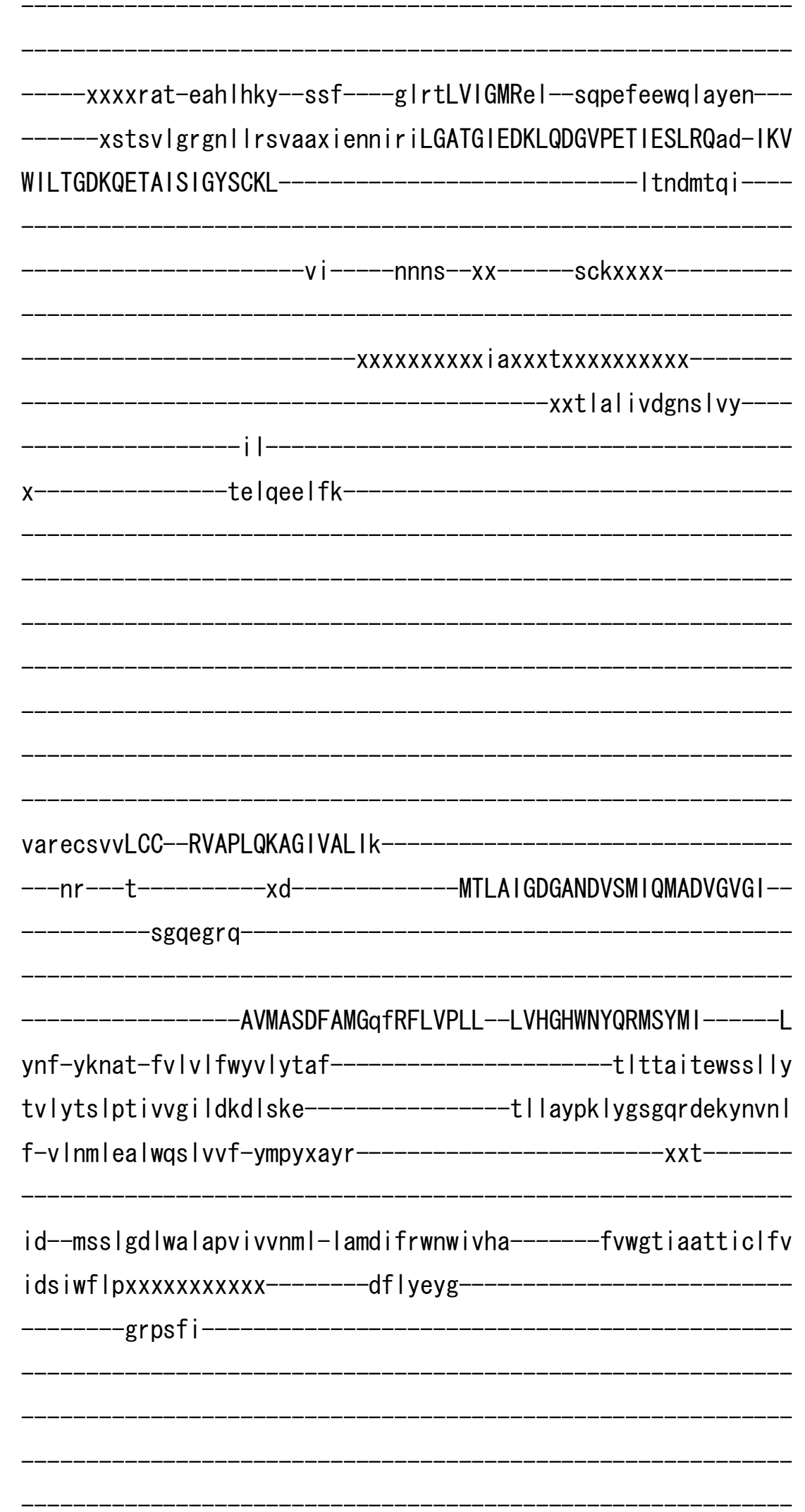




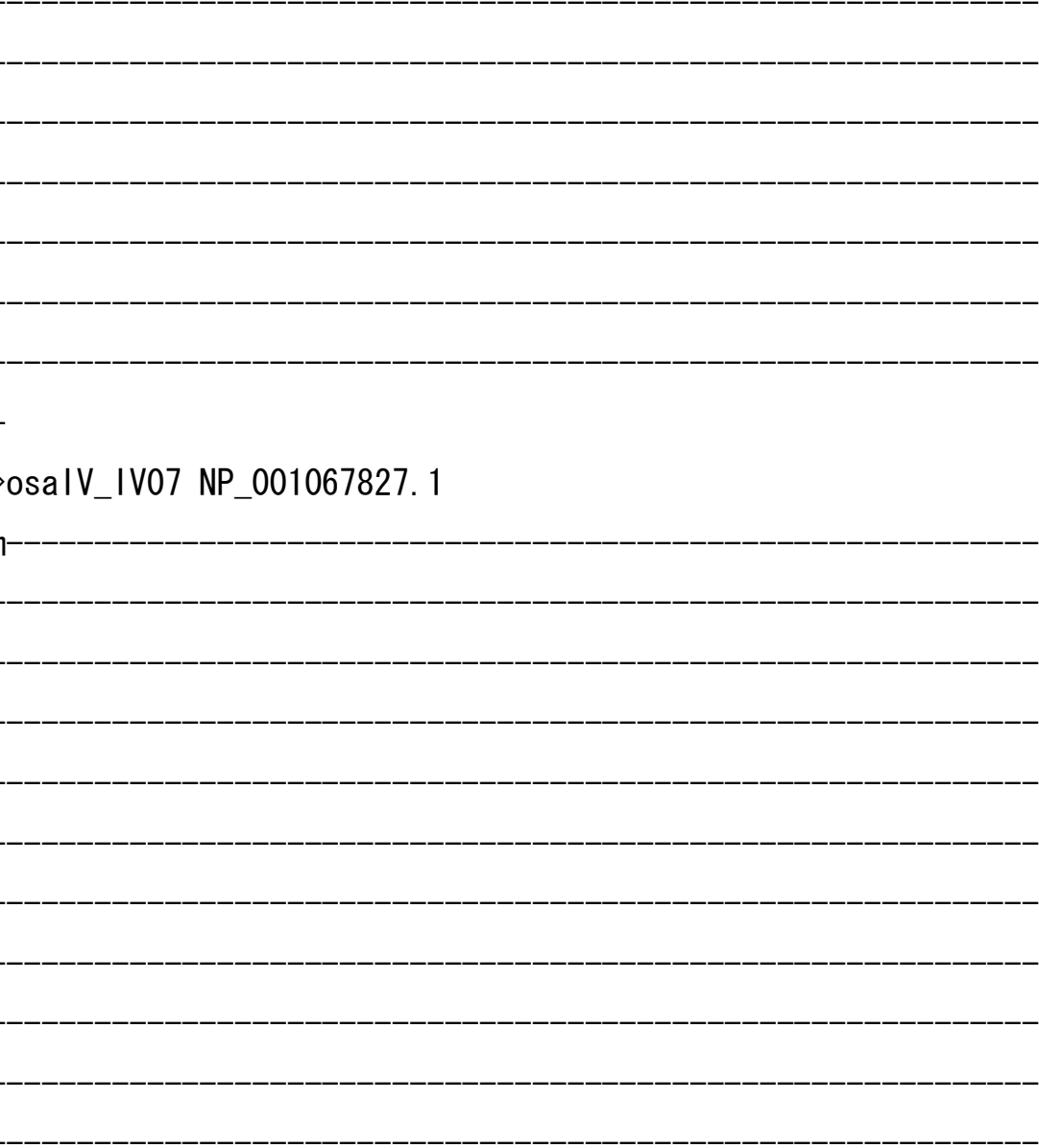

$x x x x x x x x x x x x$

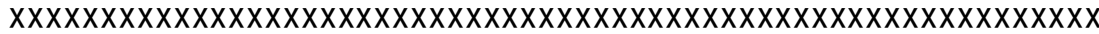

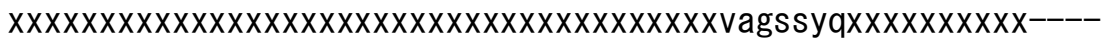
$-x x x x x x x x x x x x x x x x x x x x x x$ $\mathrm{xxxxxxxx-1-10}$ $-X X X X X X X X X X X X X X X X X X X X X X X X X X X X X X X X X X X X X X X X X X X X X X X X$ xxxrrr|r|rg $x x x x x x x x x x x$ $-x x x x x x x x x x p n d y c d n r$ i sntkyt $-11$

$-n f I p k n \mid w e q f$ 


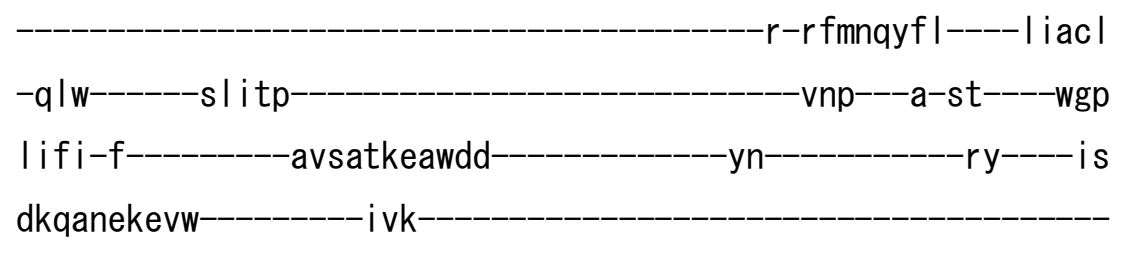

$-x x x x x x$

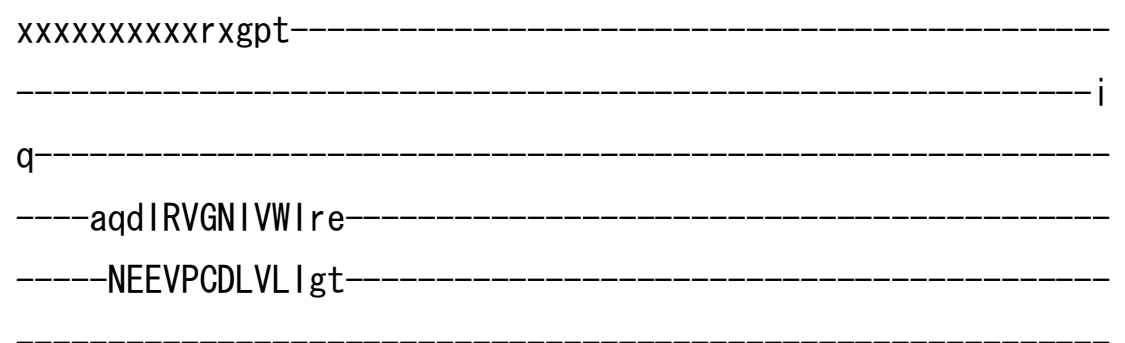

-sdsqg-i---chVETAALDGE IDLKTRvi-

$-p \mid$ tcvg Idseq I hk i kgv i ecpnpdkd i r r I dan i I xx

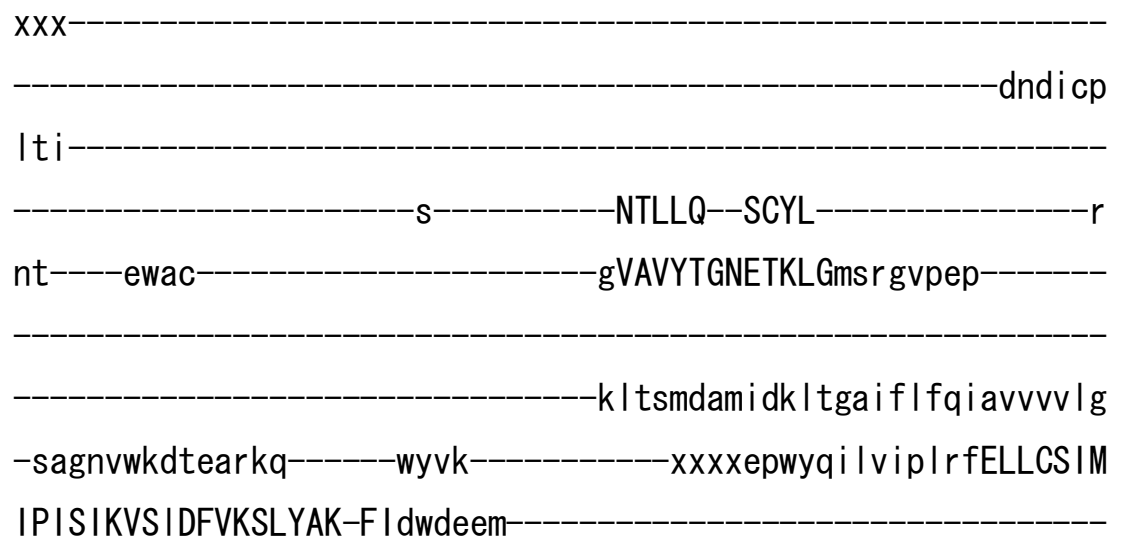

IPISIKVSIDFVKSLYAK-FIdwdeem-

-ydhetdtp

aHAA-NTAI SEDLGQVEY ILTDKTG-

-TLTENKMIFr-rcciggtfy

$-x x x x d a|r d v e--| x n a v x x x x x x x$ $-k f$

Itvmt |c-----ntvi-

$-p i x x x s x x-$ 


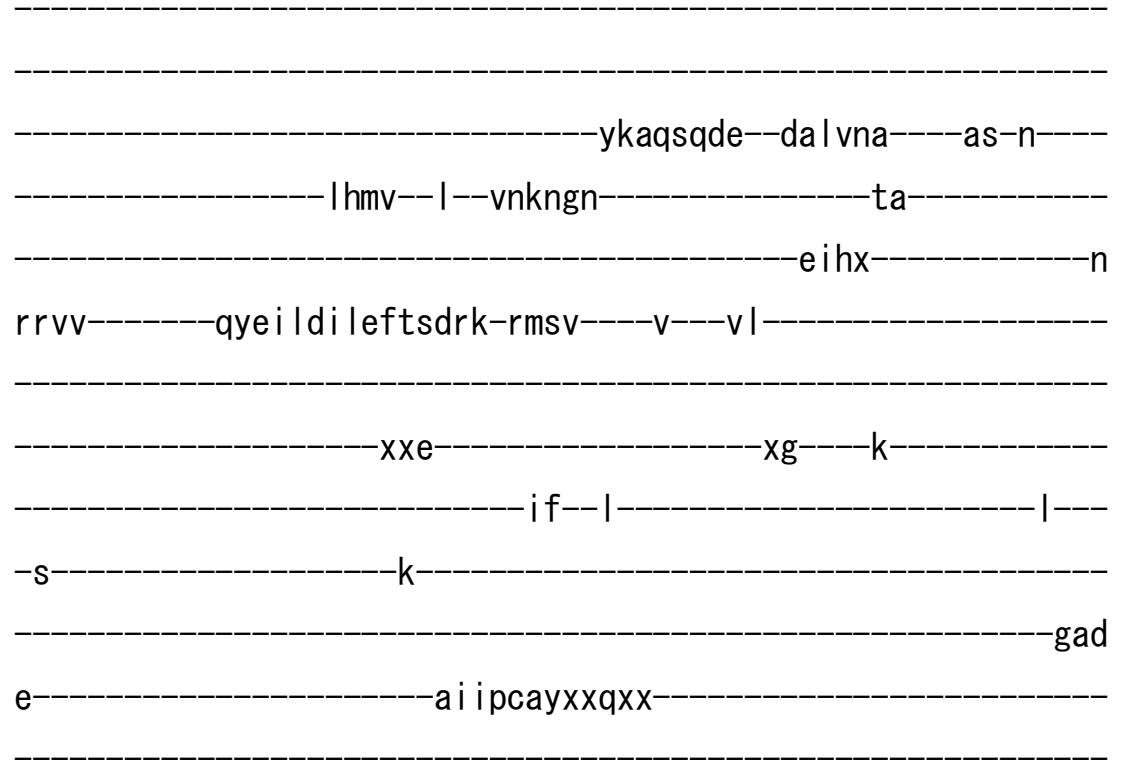

-ktf-vdavdky--aq l----g | rtLCLGWRe I--eseey l ewsr sfke--an-----sal idr ewkvaevcqk- I ehs I e iLGVSA IEDRLQAGVPET IE ILRQsg-INF WMLTGDKQSTAIQIALLCNLissepkgq-

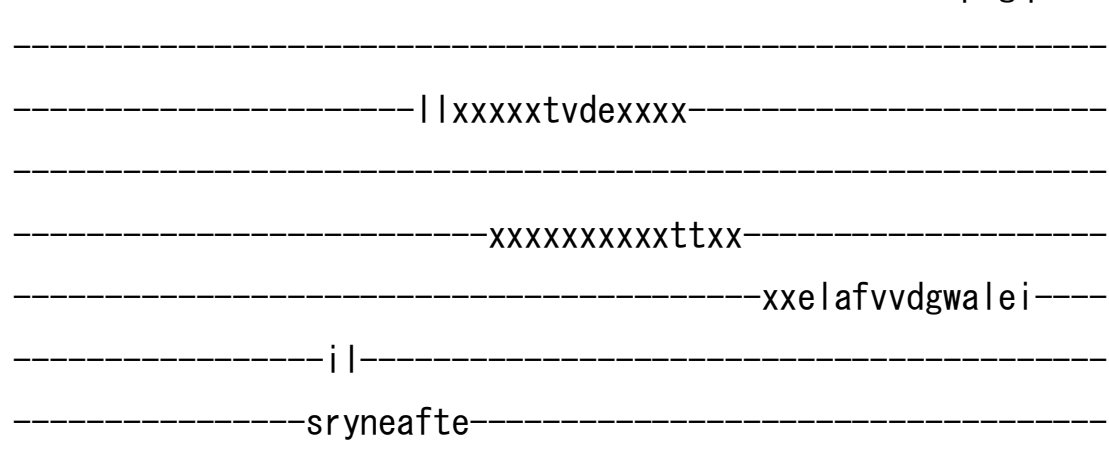

laal skta ICC--RVTPSQKAQLVKLLk

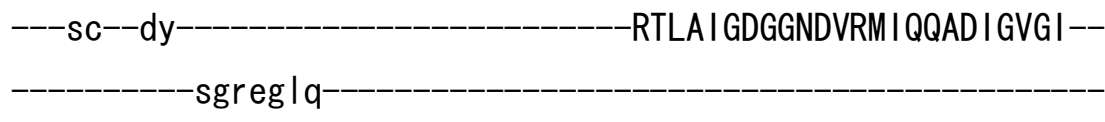

-AARAADYSVGKfRFLKRL I--LVHGRYSYNRTAFLS------Q 
ysf-yksII-icfiqilfsflsgi agts I fnsvs Imay nvfyts ipvltt-vldkdlsek-tvmqnpe i I lycqagr I Inpst

f-agwfgrs lyhai vvfxitvhxyan

ekxxmee I smva Isgs iw lqafv-vt l emnsftfvqf I

aiwgnf i afyi inff

issipsagxx timfrlc

rqptyw----vt-- I I I s sgvgmgpv-

$-l a l k y f r$

-ytyr $-p$ sai

$-x x \operatorname{xx} x \operatorname{xx} x \operatorname{xx} x \operatorname{xx} x \operatorname{xx} x x x$

>athIV_IV10 NP_189189. 1

$\mathrm{m}$

- 


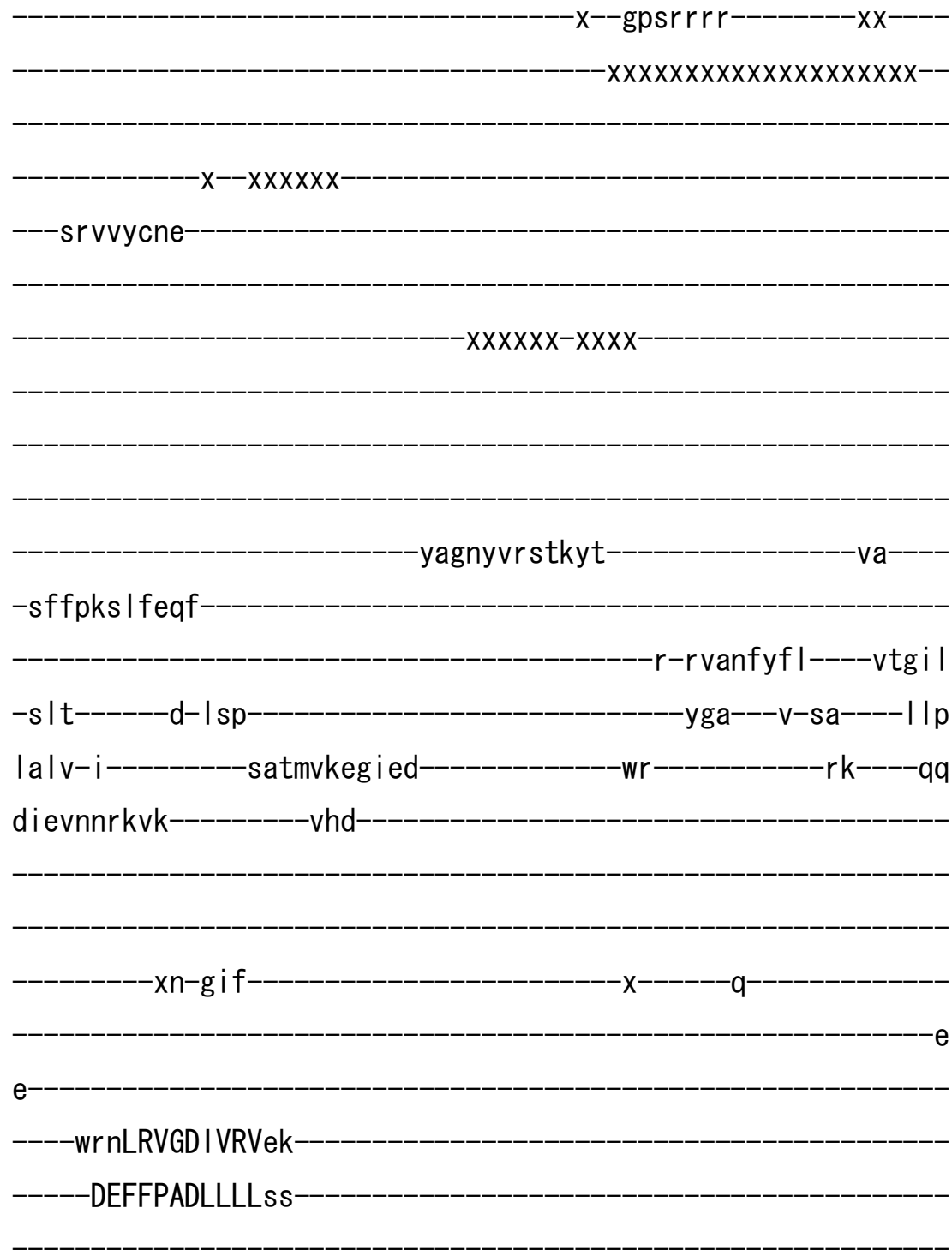

-syeds-v---cyVETMNLDGETNLKVKqg-

-xeatss I Inqdsdfkdfrgvvrcedpnvn lyvfvgt la l--

eeerfp

Is i

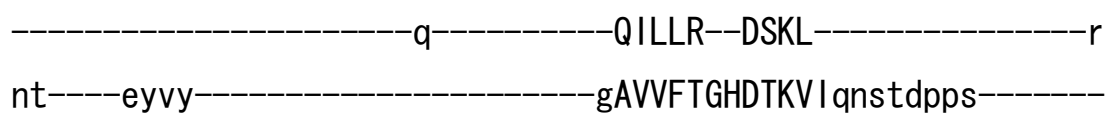

-krsr iertmdk i i y Imfg I vf Imsfvgs

-i i fgvetredkvkn-xxxxxwy lk-----xxxxxxxxxxxr apmaai yhfftaTMLYSYF IPISLYVSiEIVKVLQSI-FInrdihm 
-yyeetdkp

aQAR-TSNLNEELGMVDT ILSDKTG

TLTCNSMEF $\mathrm{i}$

-kcs i agkay

$-X X X X X X X X X X X X X X X X X X X$

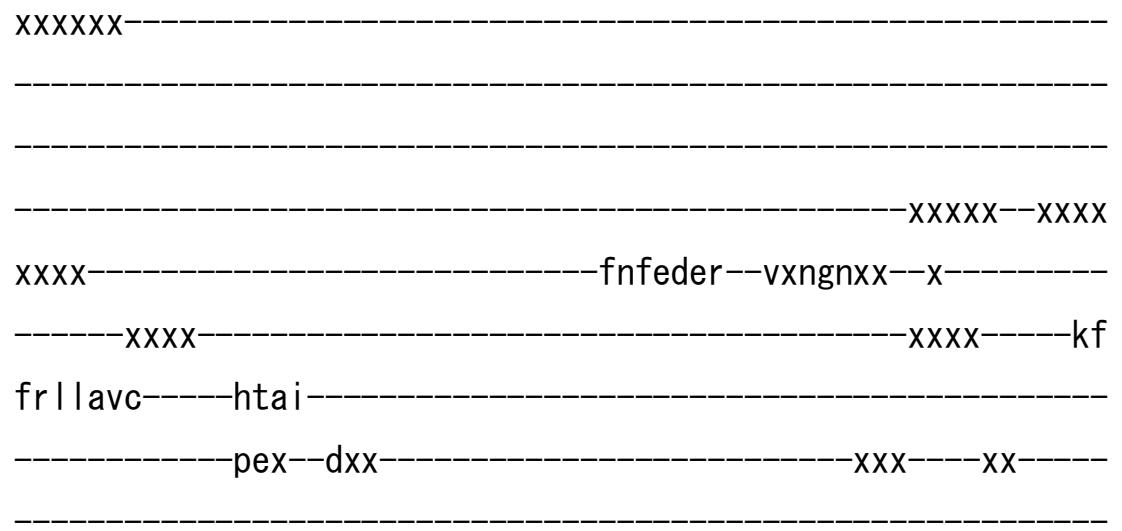

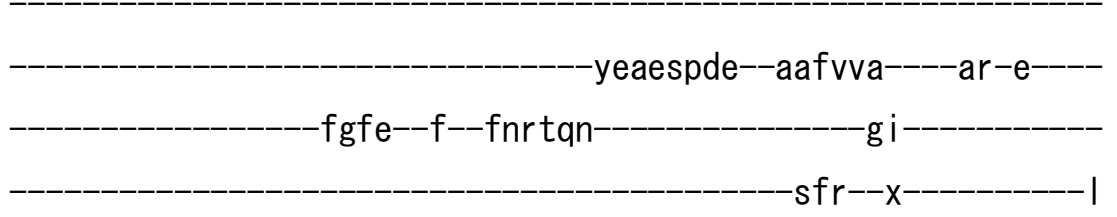

dlvs-xxxxxxvyr I Inv lefnstrk-rmsv----i----vr---_-_-_-_-_-_-_-_--

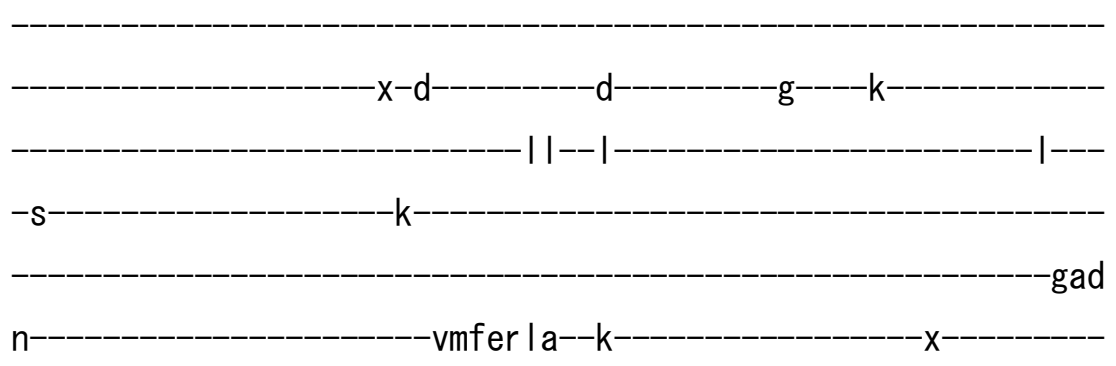

xxx--xx--akt-qehvnqy--ada----g | r tLVLAYRev--deney i efnksfne--ak----xsvsedreal ide itdk-merd I i ILGATAVEDKLQNGVPECIDKLAQag-IK I WVLTGDKMETAINIGFASSL-_-_-_-_-_-_-_-_-_-_-_-_-_-_---_ I rqemkq i---_

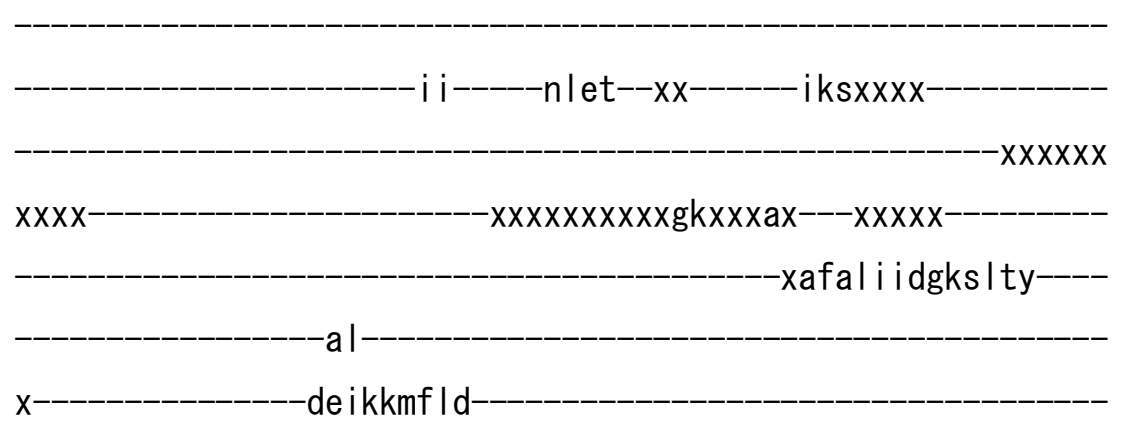




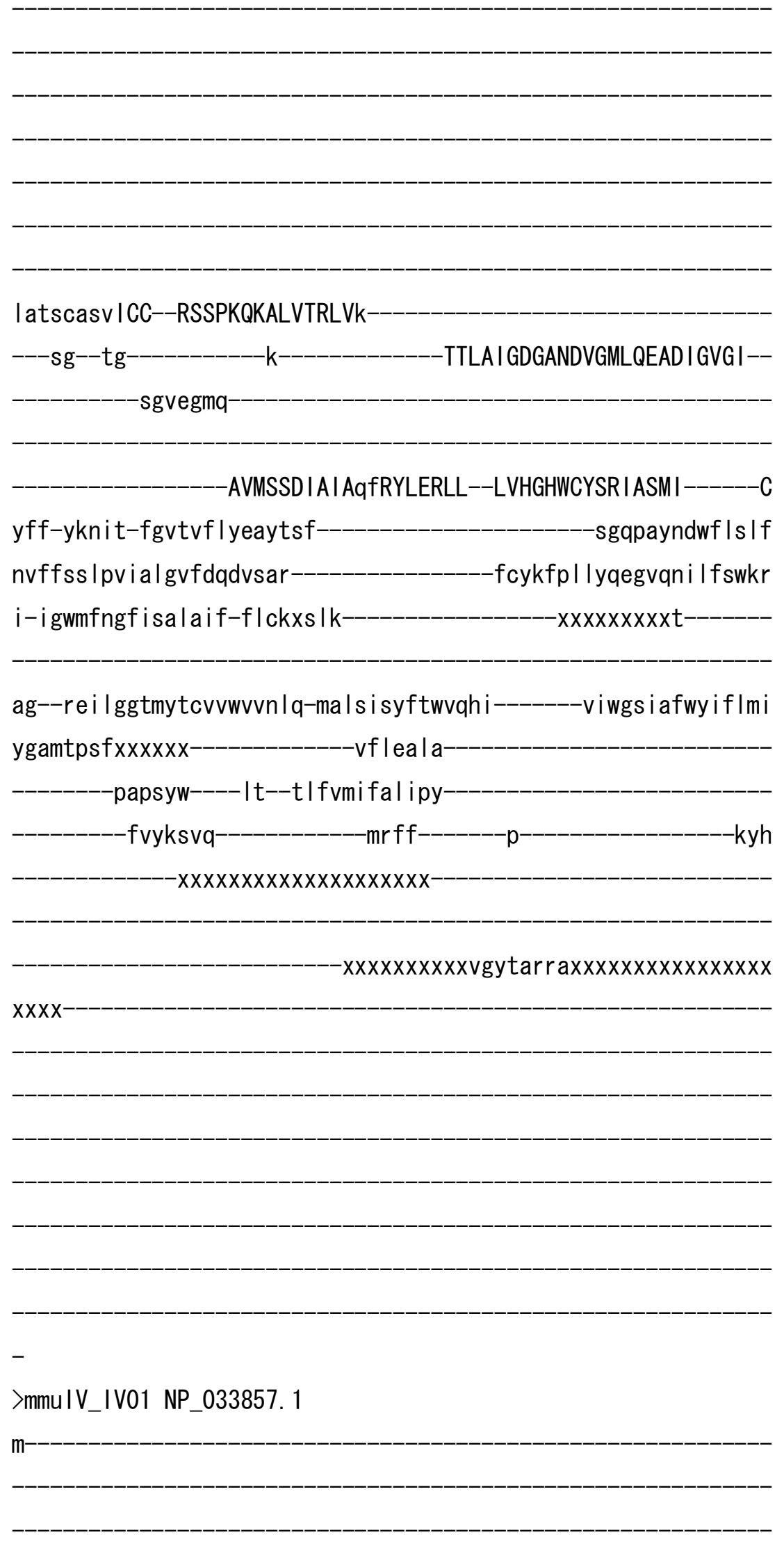




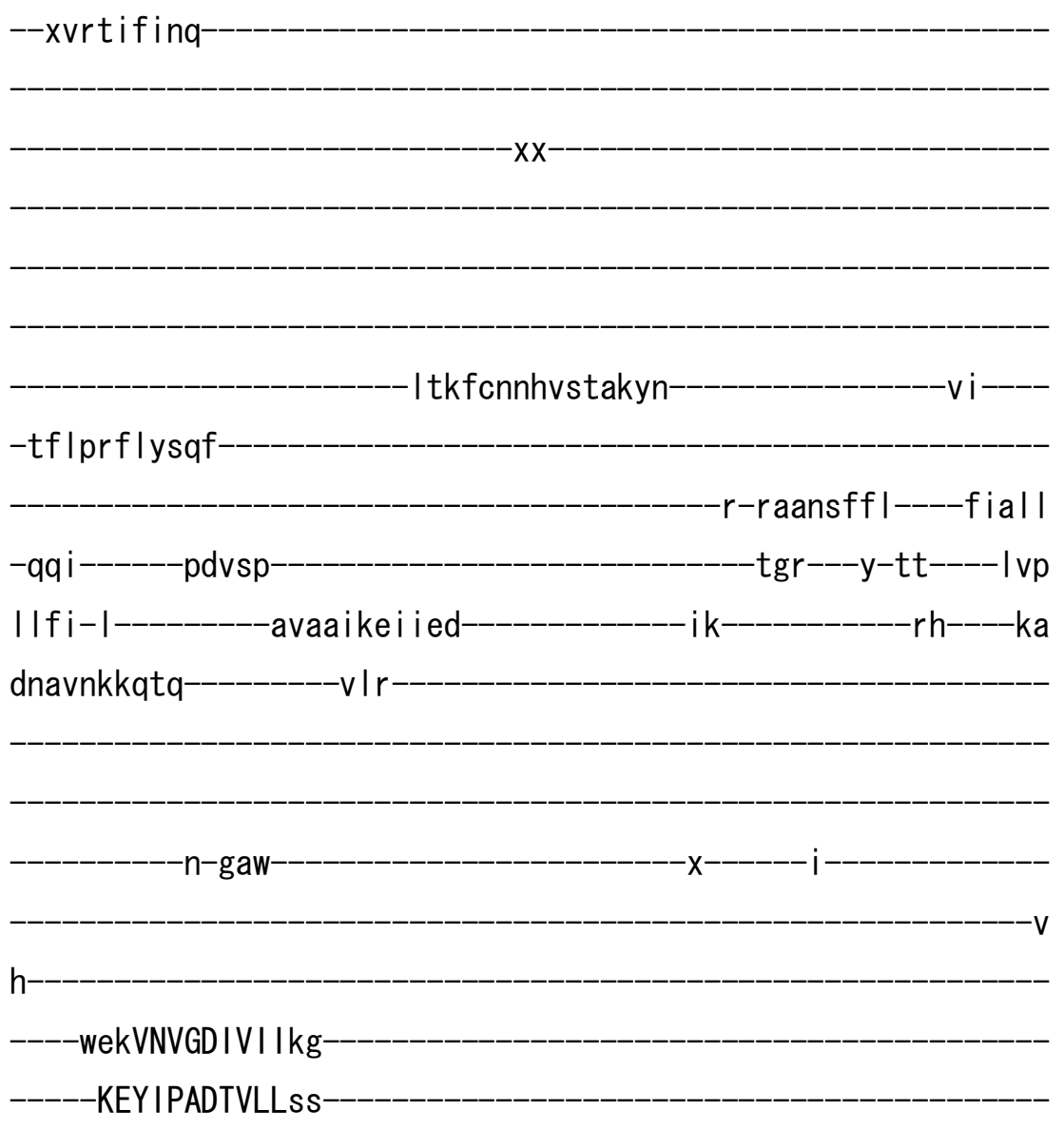


-sepqa-m---cy IETSNLDGETNLKIRgg-

- patsdikdids Imr isgr i ecespnrhlydfvgnir Ix-

-ghgtvp

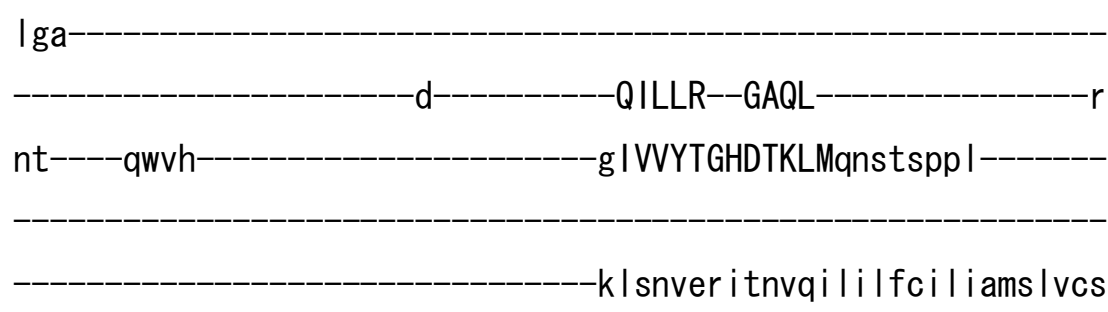

-vgsa i wnr rhsgkd-------wy Ih--_-_-_--_---xxxggasnfg I nf I tf I ILFNNL

IPISLLVT IEVVKFTQAY-F InwdIdm-

-hyeptdta

aMAR-TSNLNEELGQVKYIFSDKTG-

- TLTCNVMQFk

$-k c t$ iagvay

$-x-$

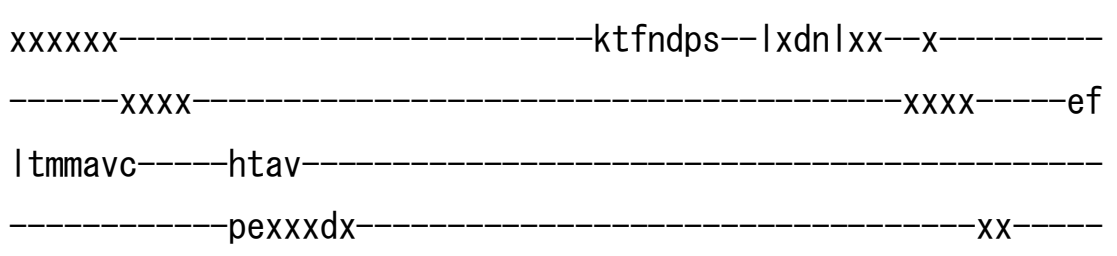

---_--_-_-_-_-_-_-_-_-_-_-_-_-_-_-_-_-_-_-_-_-_-_-_-_-_-_-_-_-_-_-_-

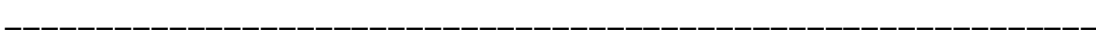

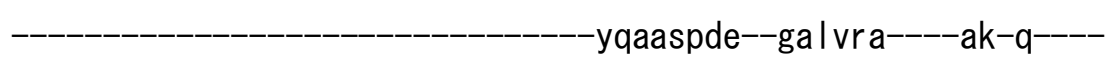

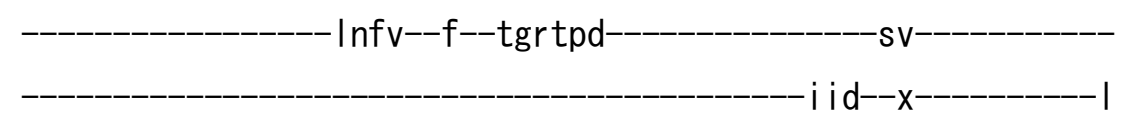

gqee--------ryel Inv leftsark-rmsv-----v---vr------_---_----_-_--

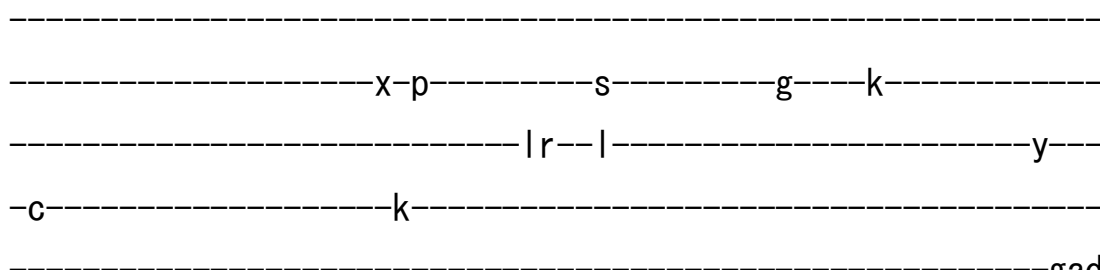

- gad

t----------------------viyer la-

$-x$ 
xxx--xx--e it-Ikhl eqf--ate-----g|rtLCFAVAe i--sesdfeewr avyhr--as-----tsvqnr I I k leesye I-i ekn I q ILGATAIEDKLQDQVPET IETLMKad-IK I WILTGDKQETAINIGHSCRL-Ikrnmgmi----

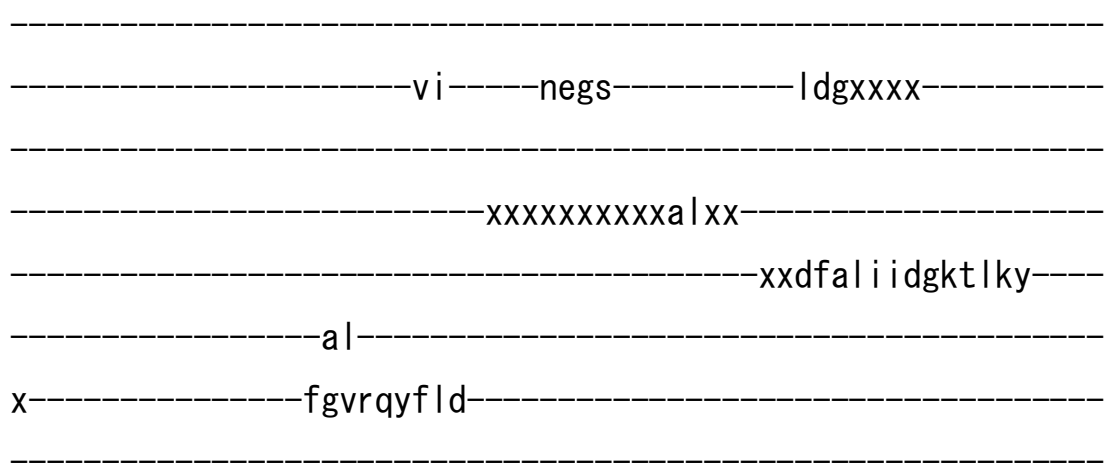

la l sckav ICC--RVSPLQKSEVVEMVk

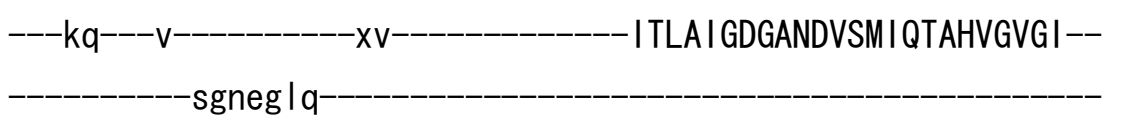

AANSSDYSIAqfKYLKNLL--MVHGAWNYNRVSKCI------L

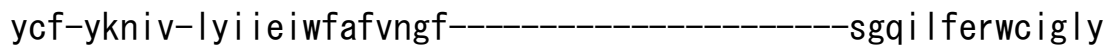
nvmf tampp I I I g i ferscrke--_-_-_-_-_-_---nm I kype lyktsqna Idfntkv f-wvhclng I fhsvi If-wfp|xalq-_-_-_-_-_-_-_-_-_-_xxxxxxxxt--_-_-_sd--y I I gnfvytfvvitvclk-ag letsywtwfshi---_----ai wgs i a lwvvffg i yss I wpavxxxxxxx-------------geaam I f-

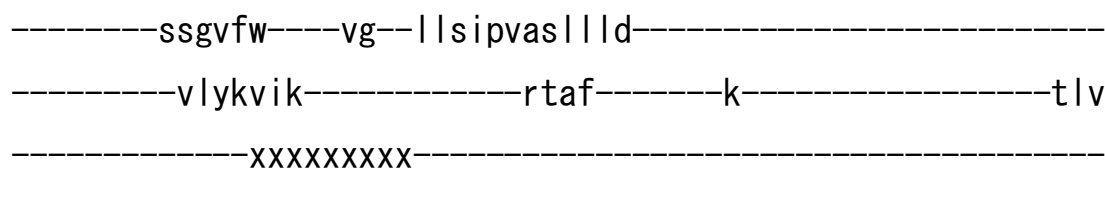

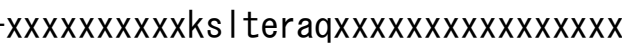
$\operatorname{xxx} x \operatorname{xx} x \operatorname{xx} x x x x x x x$ 
>athIV_IV11 NP_172780. 1

$\mathrm{m}$

-

$-x x x x x x x x x x x x x x x x x x x x y-$

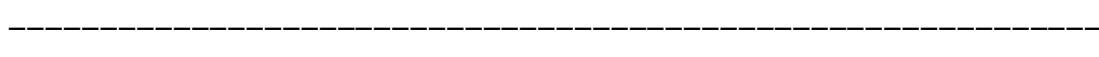

$-x--x x x x x$

---srvvycne-

$-x x x x x x-x x x x$

-yvgnyvrstkyt la

-sf i pks I feqf 


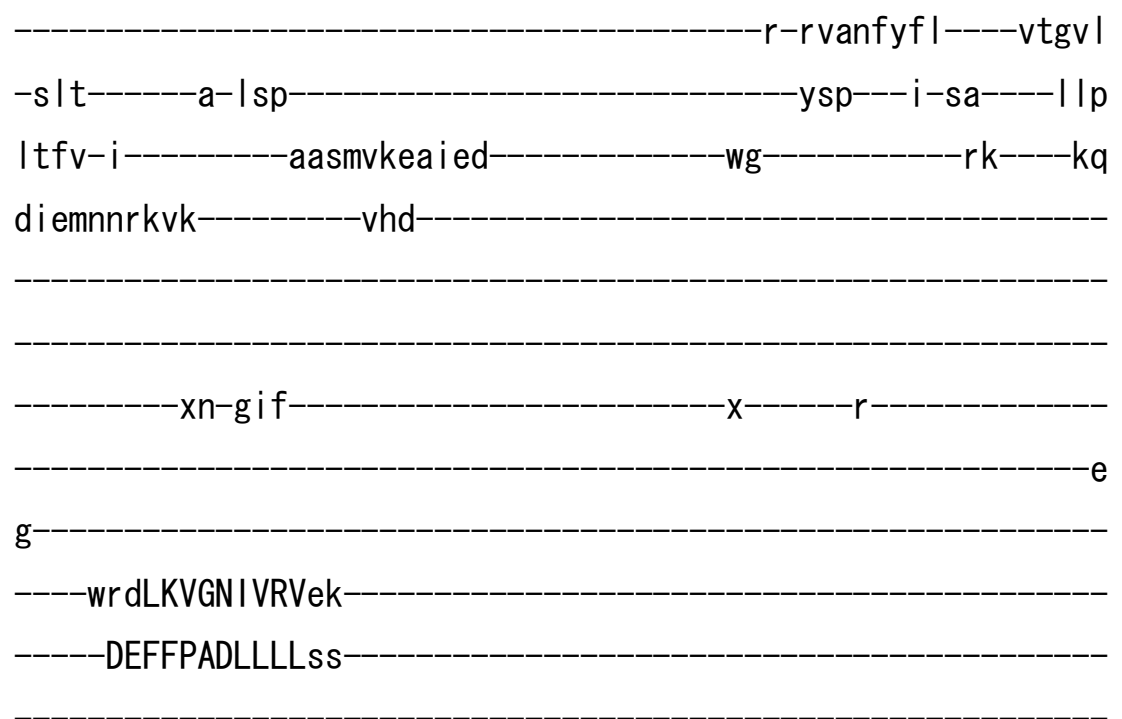

-syeds-i---cyVETMNLDGETNLKVKqg-

-xeatssalhedsdfkelkavvkcedpnad lytfvgt Ihf--

eeqr Ip

Isi

$\mathrm{t}$

-QLLLR--DSKL

nt----eyiy

gVVVFTGHDTKVI qnstdpps

krsrierkmdk i iy Imfgvvf Imsfigs

-ivfg i etredrvrnxxxxxxwy |r----xxxxxxxxxxxxrapmaavyhfftaVMLYSYF

IPISLYVSiEIVKVLQSL-FInndi Im-

-yyeendkp-

aHAR-TSNLNEELGMVDT ILSDKTG

TLTCNSMEF i

-kcs i agtay

$\operatorname{xx} X X X X X X X X X X X X X X X X X$

$\mathrm{XXXXXX}$

$-x \times x x x--x x x x$

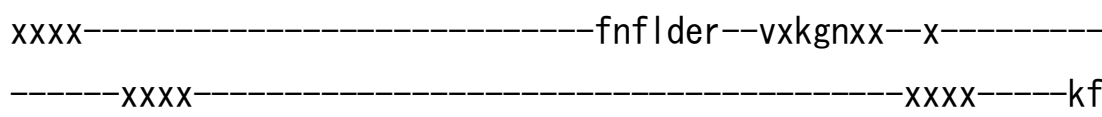

frl lavc-----hta i

pex--dxx

$-x x x----x x-----$ 


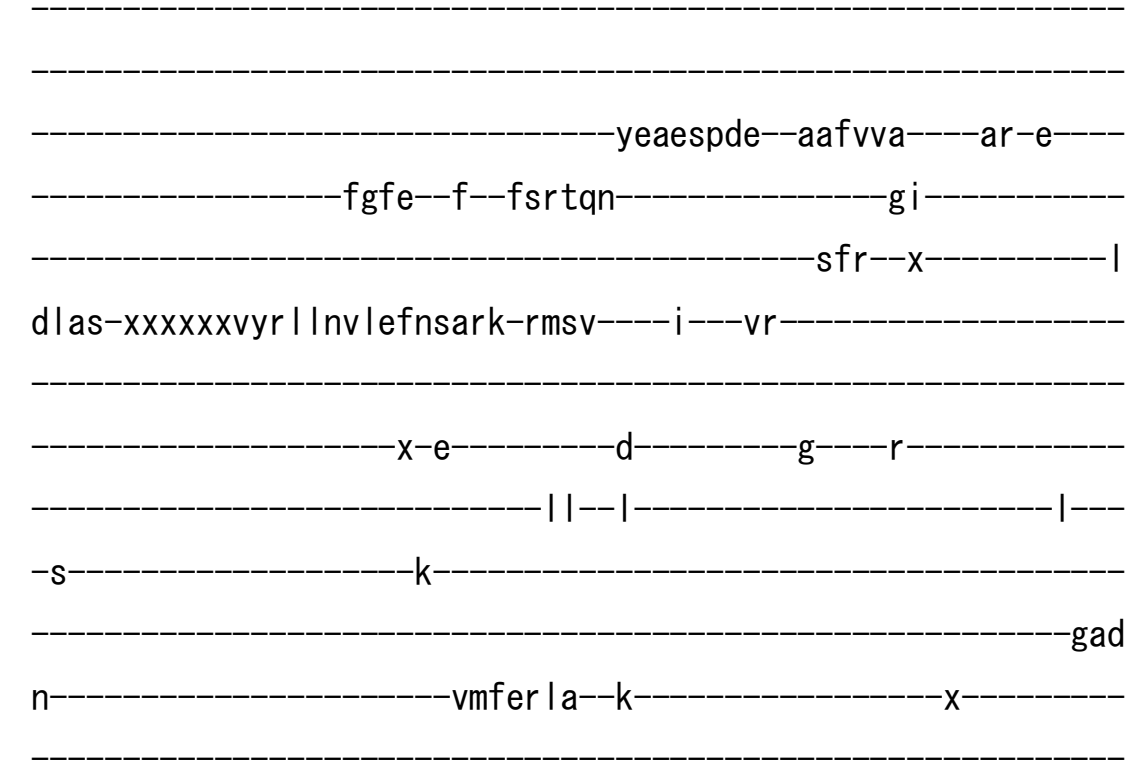

xxx--xx--ekt-rehvney--ada----g | rtL ILAYRev--deney i efsknfne--ak----xsvtadres I i de i teq-merd I i ILGATAVEDKLQNGVPDCIDKLAQag-IK I

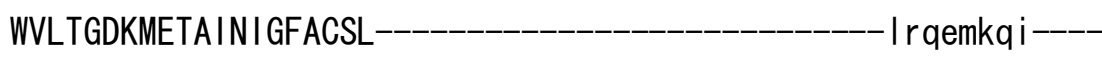

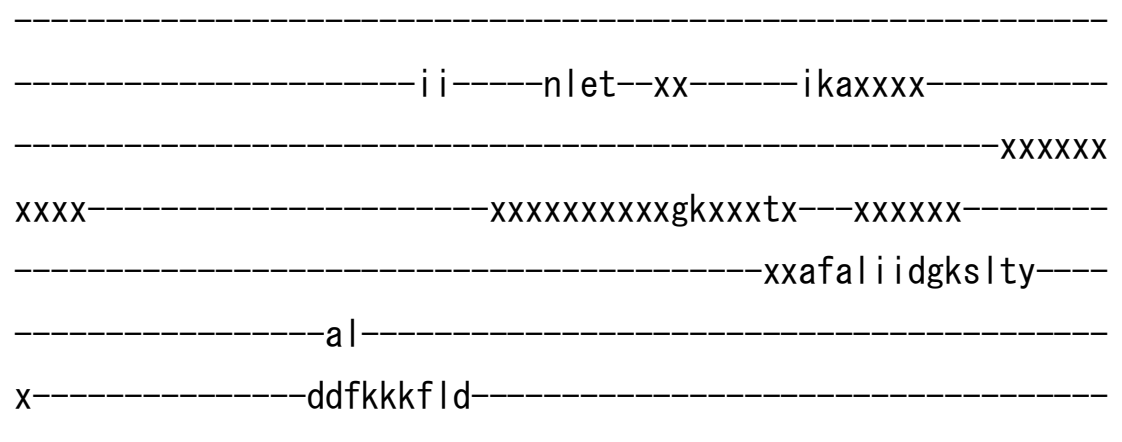

latgcasv ICC--RSSPKQKALVTRLVk-

---sg--tg-----------k-------------TTLA I GDGANDVGMLQEAD I GVG I--




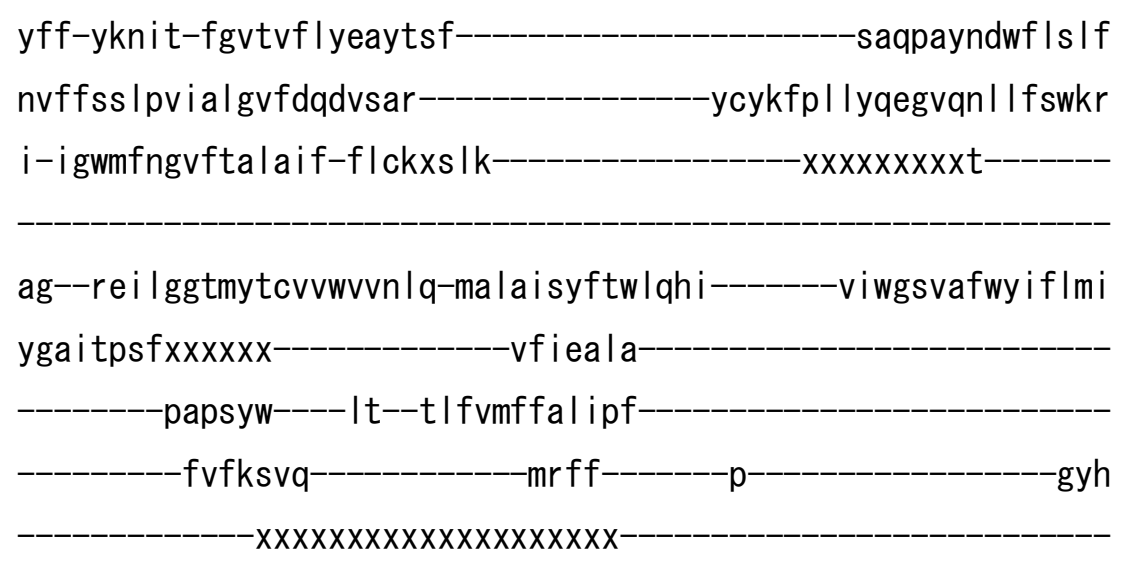

$-x x x x x x x x x x v g f t a r r a x x x x x x x x x x x x x x x x x$

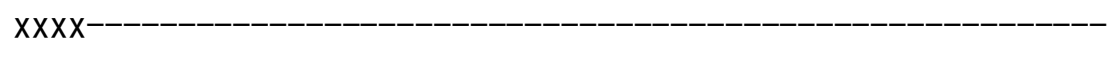

$-$

>athIV_IV12 NP_173938.1

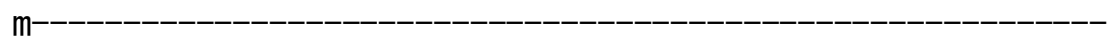




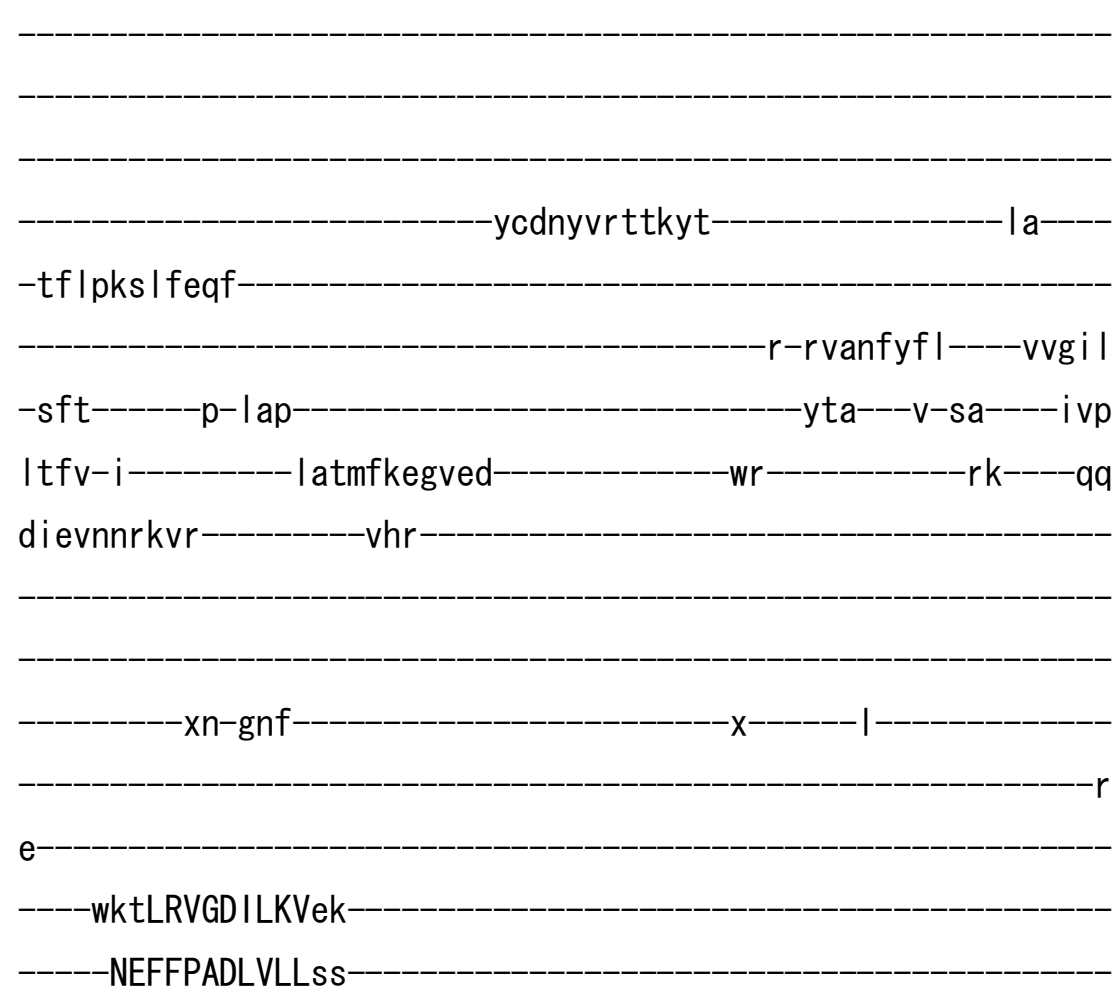

-syeda-v---cyVETMNLDGETNLKLKqg-

- levt I I I ree Infrdfeafikcedpnan Iysfvgtmd|--

-kgekyp

$\mathrm{sp}$

- QLLLR--GSKL-

nt----dy iy gVVIFTGPDTKVVqnstdpps

-krsmi erkmdk i i y Imf Imvfs laffgs

-vI fg i wtrddfang--xxxxwy lk----xxxxxxxxxxxxrapmaai yhf I taLMLNSYF IPISLYVSiEIVKVLQSI-FInqdihm 
-yyeeadkp

aHAR-TSNLNEELGQVGT ILSDKTG

TLTCNSMEF

-kcs i agtay

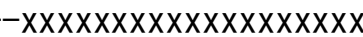
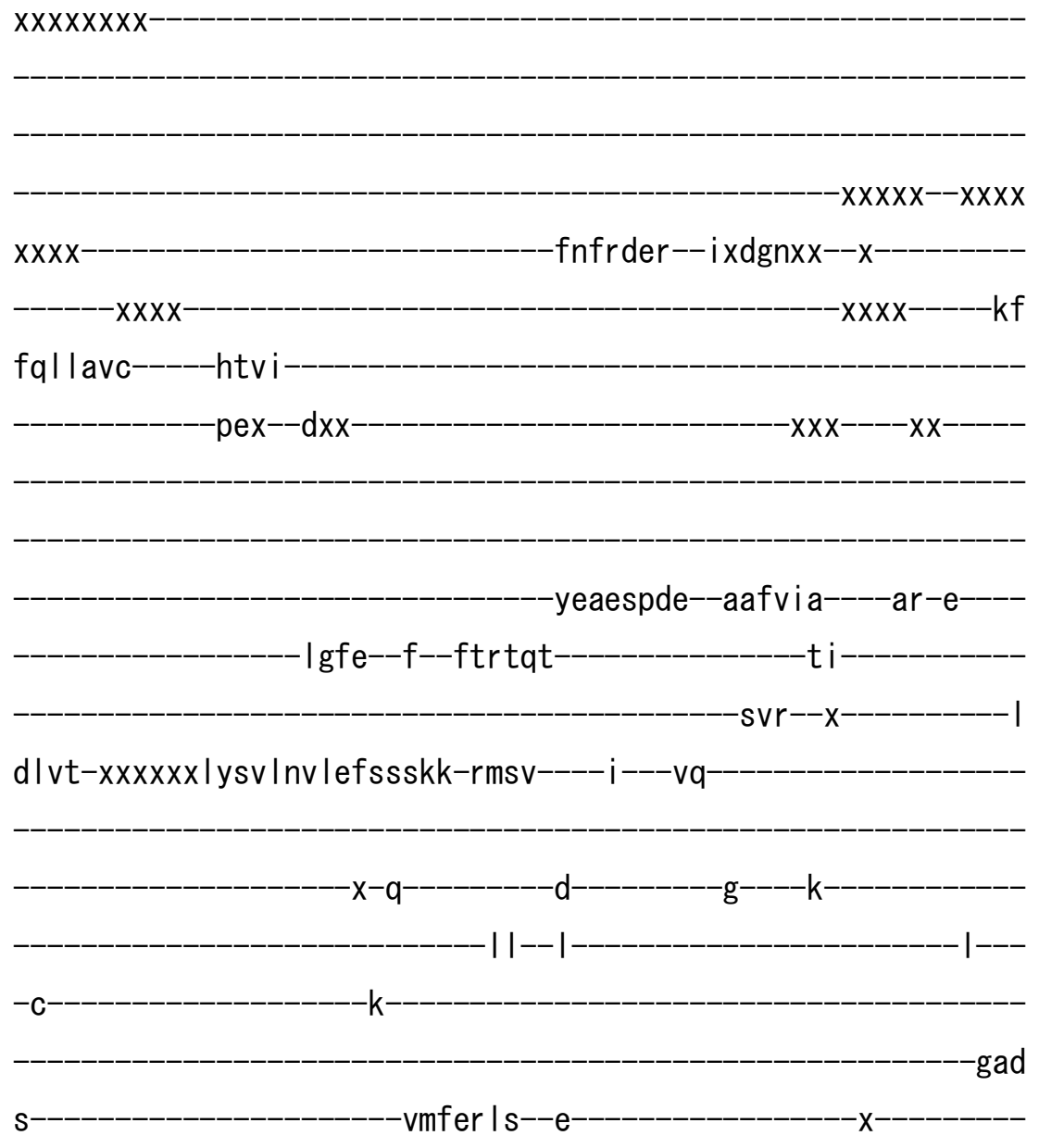

xxx--xx--ket-rdhvney--ada----g | rtLILAYRe I--deneyevfter i se---ak----xsvsadreal i devtek-i ekn I v ILGATAVEDKLQNGVPDC INKLAQag-IK I WVLTGDKMETAINIGFACSL-_-_-_-_-_-_-_-_-_-_-_-_-_-_-_-_ I r dmkq i--_-

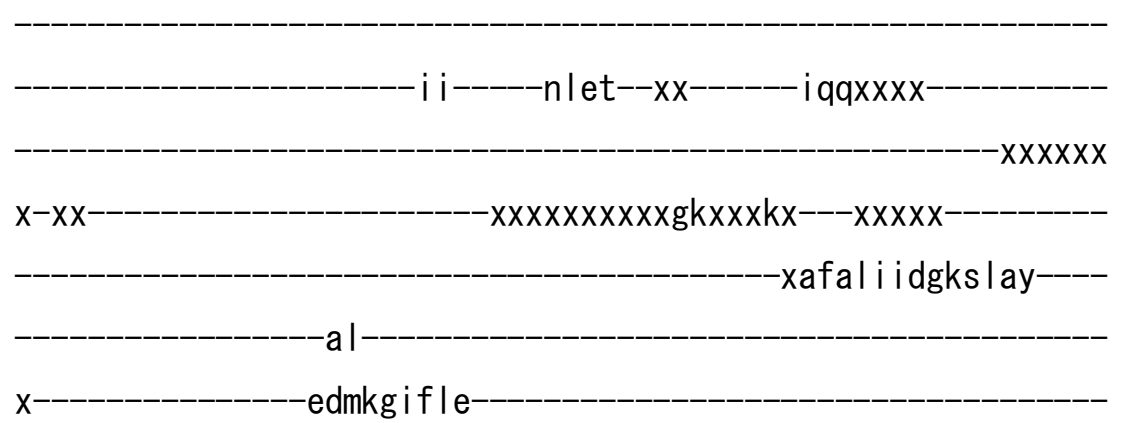


I a i gcasv ICC--RSSPKQKALVTRLVk

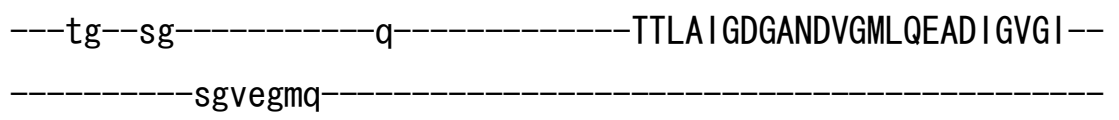

-AVMSSD I A I AqfRYLERLL--LVHGHWCYRR I SKMI------C

yff-yknit-fgft I I yeaytsf-_-_-_-_-_-_-_-_-_-_-_-_satpayndwy I s I y svffts I pviclg ifdqdvsap--_-_-_-_-_-_-_-_fc lkfpv lyqegvqn I I fswr i-Iswmfhgfcsa i i if-flckxsle---------_-------- xxxxxxxxxt--------

ag--rdi I ggtmytcvvwvvs |q-mv I t i syft I iqhv--------vvwgsvvi iwy If Imv

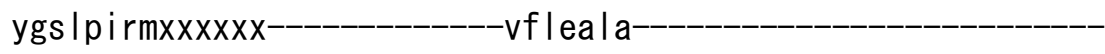
---------papsyw----it---t I fvvl stmmpy----------------------------

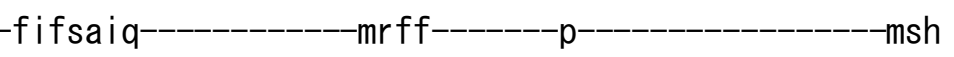
$-x x x x x x x x x x x x x x x x x x x x x-$ $-x x x x x x x x x x v m r$ shqpex-

>mmulV_IV02 NP_056618. 1

m- 


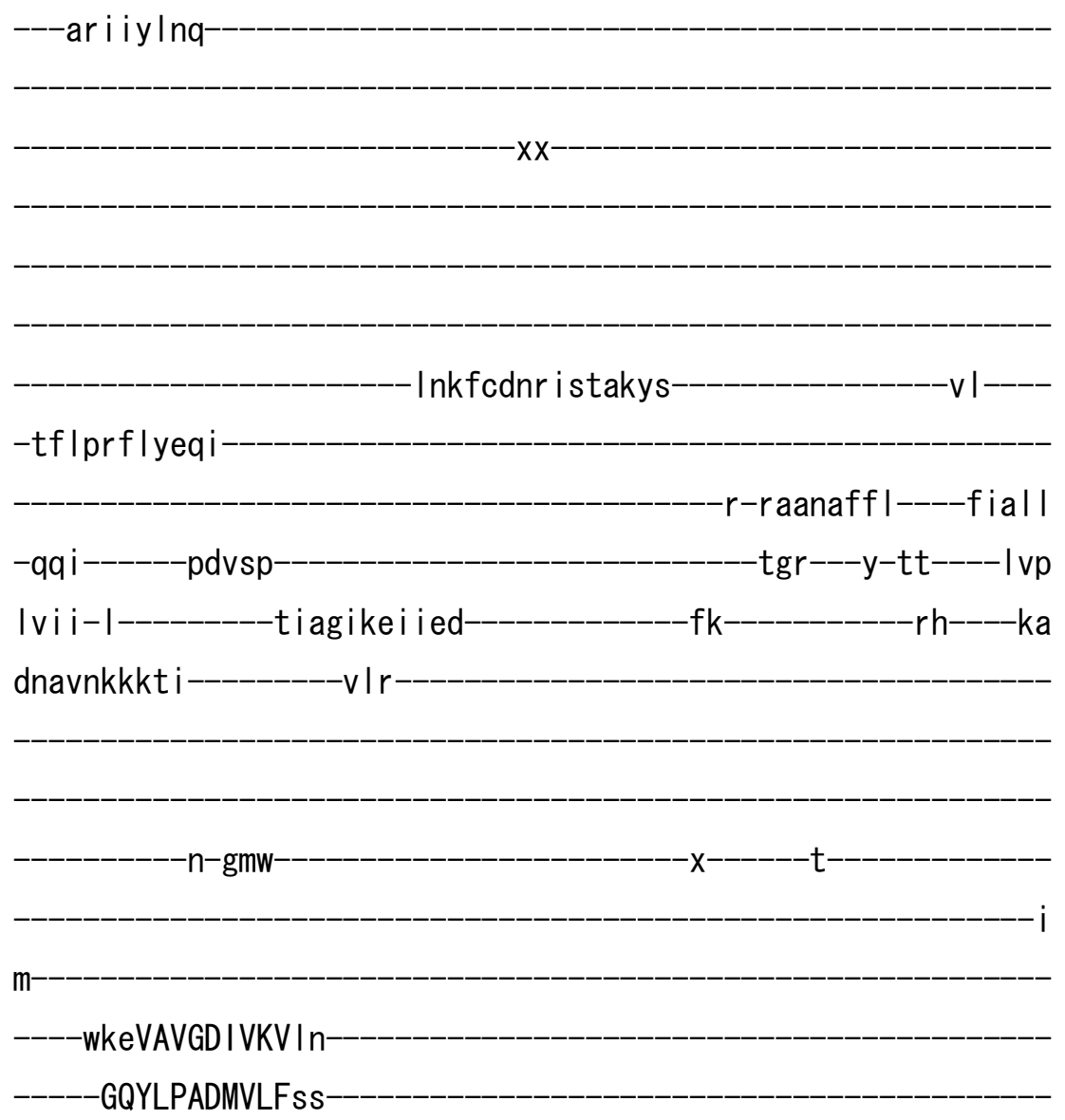


-sepqg-m---cyVETANLDGETNLKIRgg-

- shttdmqtrdv Imk I sgr i ecegpnrh lydftgn IhI X-

-gkssva

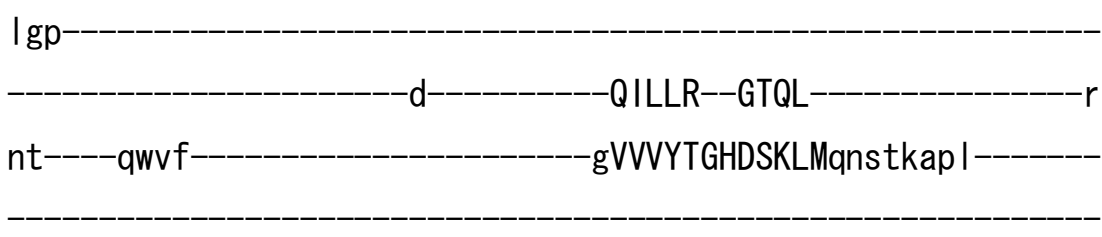

-krsnvekvtnvqi lvIfgi I lvmalvss -vga I fwngshggks-------wy i k--_--_--_-_--xxxtnsdnfgyn I I tf I ILYNNL IPISLLVTIEVVKYTQAL-FInwdmdm-

-yyi endtp

aMAR-TSNLNEELGQVKYLFSDKTG-

-TLTCN I MNFk---------------kcs i agvty

$x x x x x x x x x$

$-x x x x x--x x x x$

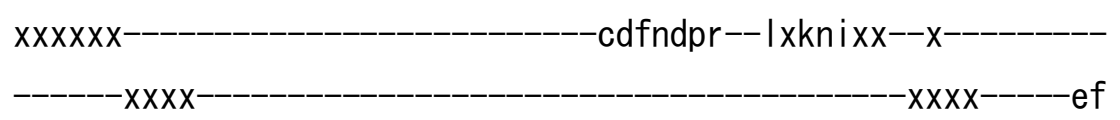

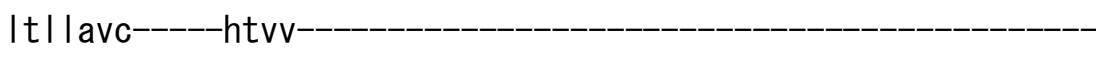

$-p e x x x d x$

$-\mathrm{xx}------$

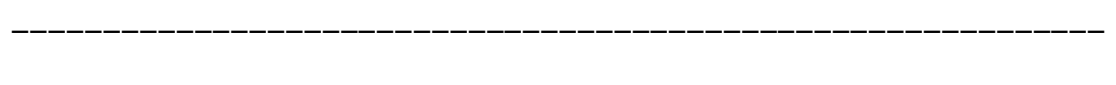

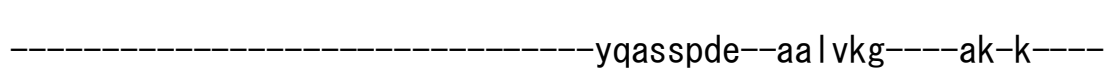

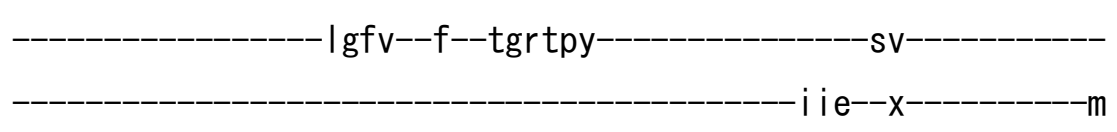

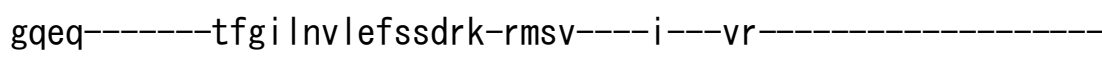

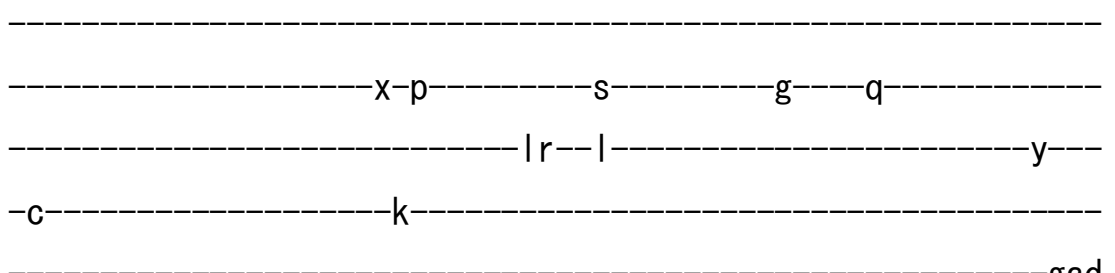

n-------_--_--_-_--_----vifer Is-

$-x$ 
>mmu IV_IV03 NP_001001488. 2

$$
\text { m- }
$$

$-X X X X X X X$

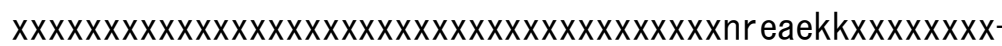

---twqvkand-

$-\mathrm{XXXXXXXXXXX-10}$

-xxxxxxxeskyasnaiktykyn $-g f$

-tf Ipmn I feqf 


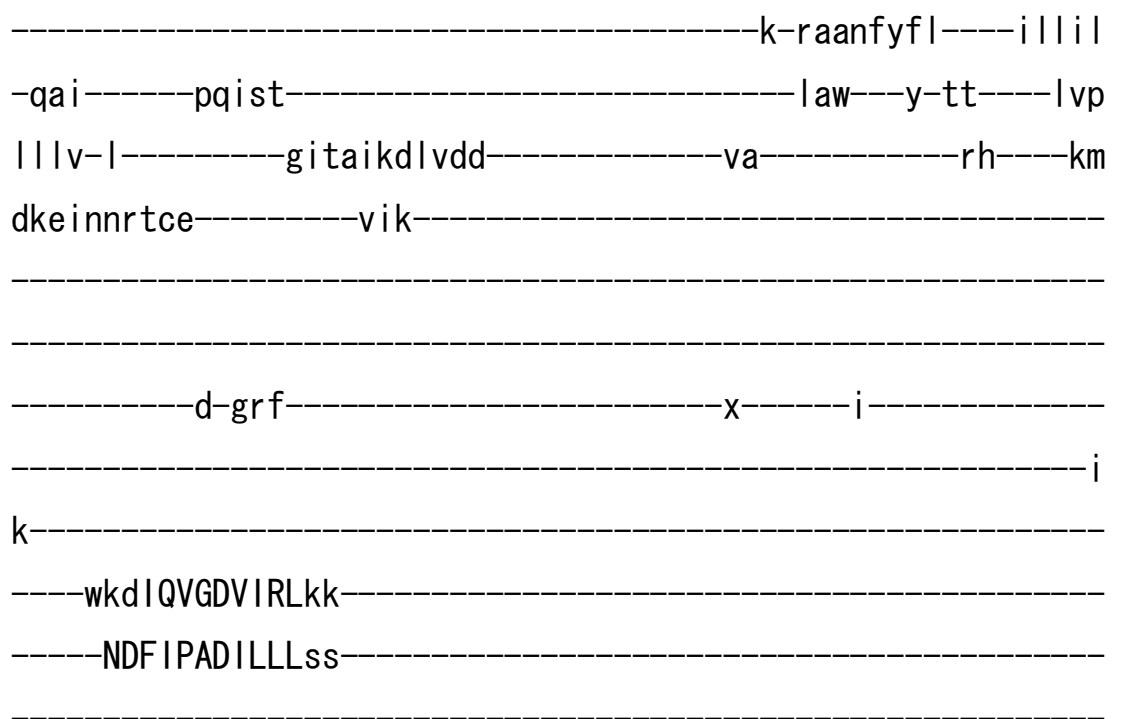

-sepns-I---cyVETAELDGETNLKFKma-

-xe i tdqy lq i edn l atfdgf i eceepnnr I dkftgt I fw--

knqsfp

Ida

$d-$

KILLR--GCVI

gLVIFAGADTKIMknsgktrf

nt-----dvch-

krtkidy Imnymvyt i fivl i Ivsagla

-i ghayweaqvgnys-------wy ly-----------xxxxxtpsyrgf I nfwgy I IVLNTM

VPISLYVSVEVIRLGQSH-F I nwd I qm

yyaekdtp

aKAR-TTTLNEQLGQIHYIFSDKTG

TLTQN IMTFk -kccingt iy

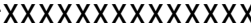

$-x \times x x x--x x x x$

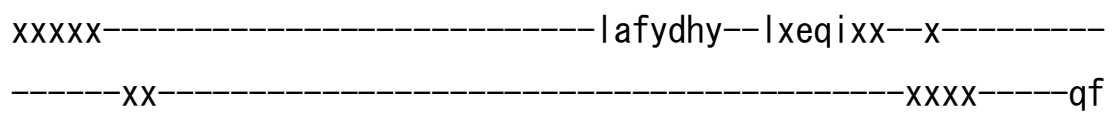

ffllsic------htvm

$-v----d x x$

$-x \times x----x x-$ 


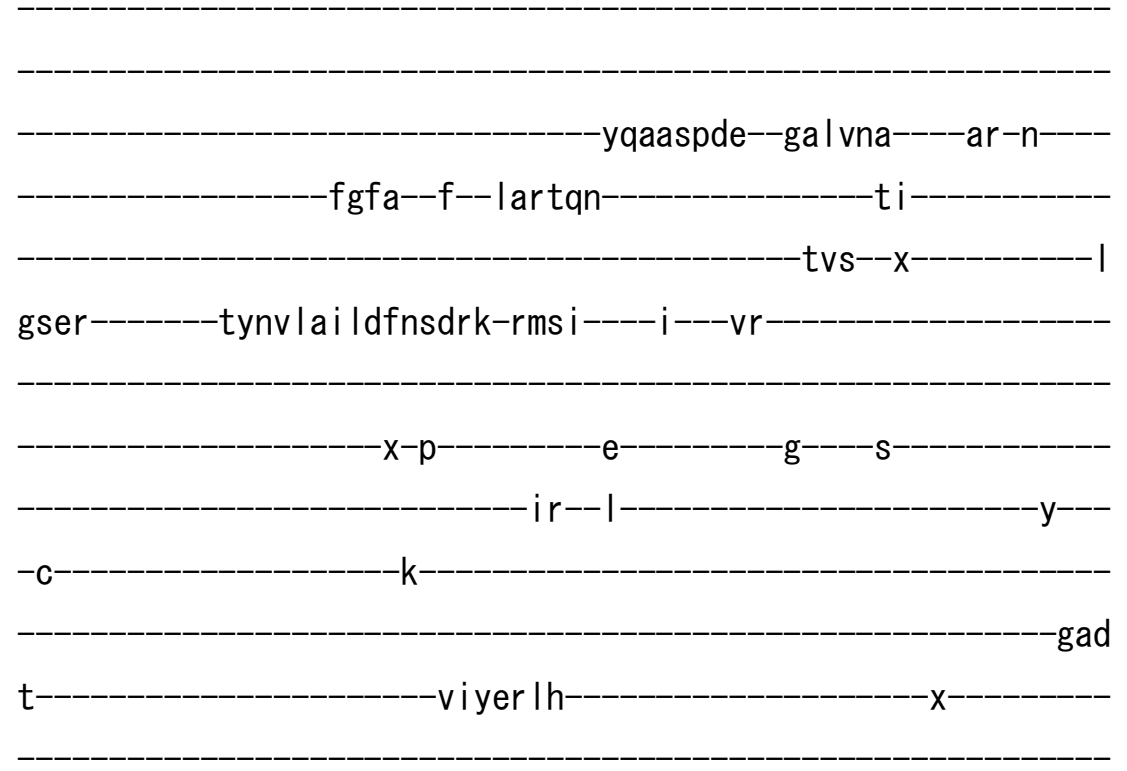

xxx--xx--qet-qdal dif--ase-----t I rtLCLCYKe i--eekeftewnnkfma--as-----vassnrdea I dkvyee-i ekd I i ILGATA IEDKLQDGVPET ISKLAKad-IK I WVLTGDKKETAENIGFACEL- tedttic----

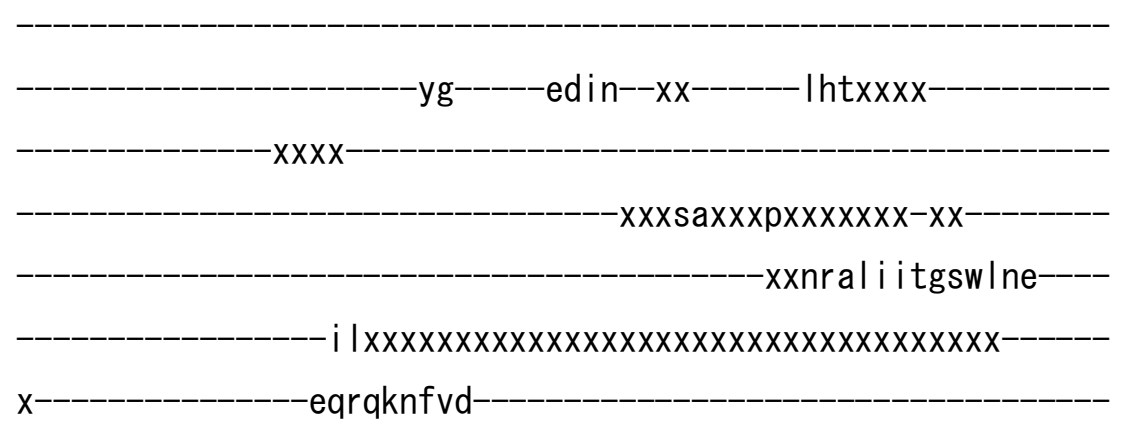
I acecsav ICC--RVTPKQKAMVVDLVk

---ry--kk--_-_-_-_---a--_--_-_-_----ITLA I GDGANDVNM I KTAHI GVGI--




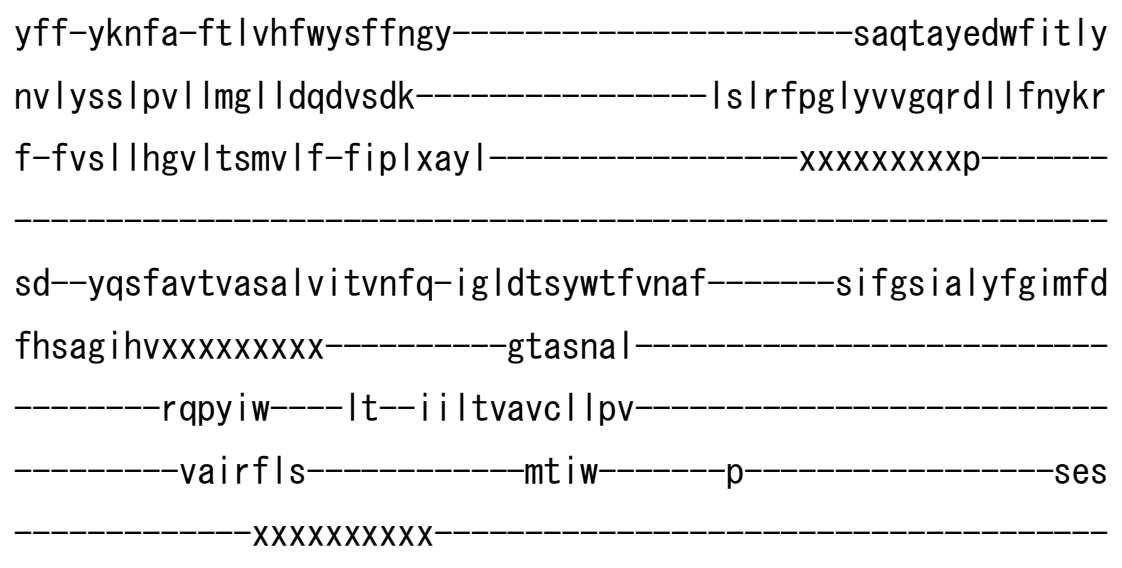

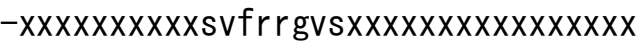

\section{$\operatorname{xXXXXXXXXXXX}$}

$x \operatorname{xx} x x x x x x x x x x x x x x x x x x$

>mmulV_IV05 NP_080370. 2

$\mathrm{m}$ 


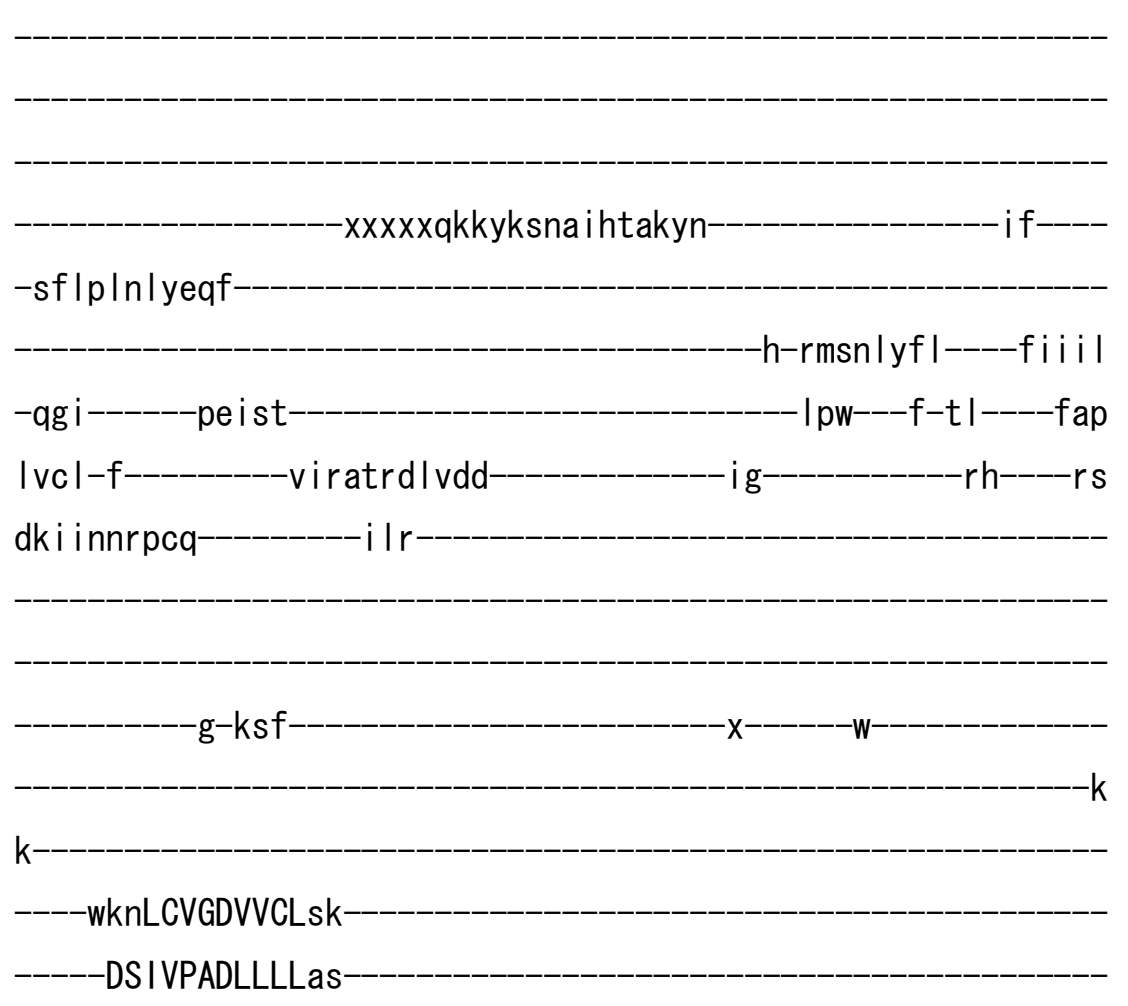

-tepss-I---cyVETADIDGETNLKFRqa-xtvthhe Itspkkmasfagtvtceepnsrmhhfvgs l ew--

-nsrkyp

Idi

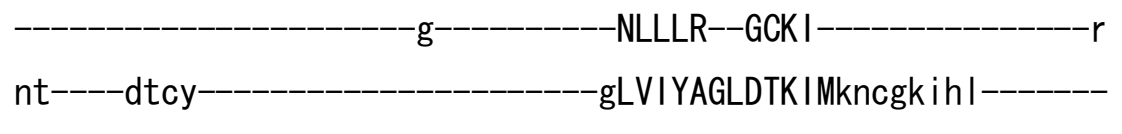

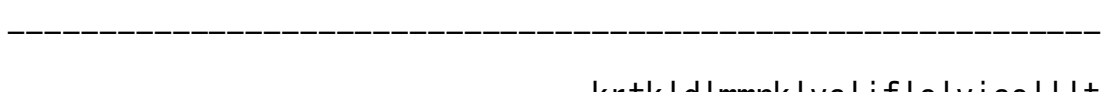

-vgftfmvkqfkakh-------yyms----_------xxxxxsdamesff i fwgfL ILLSVM

VPMAMF I I aEF I YLGNSI-F I nwd I nm- 
aKAR-STSLNDQLGQVQYIFSDKTG
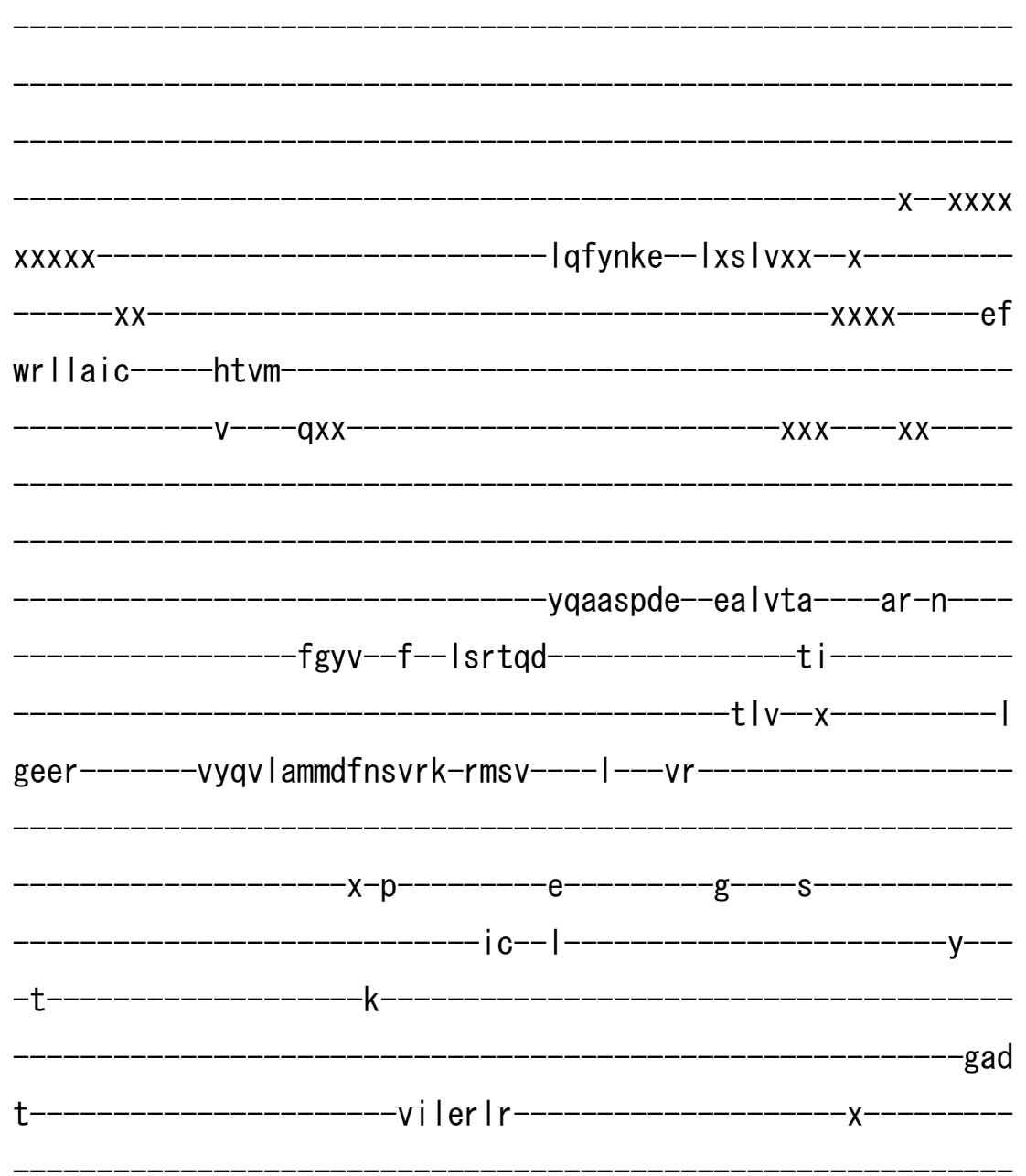

xxx--xx--att-eev l aaf--aeq----t I rtLCLAYKdv--eedaykewepehqe--aa----- I I q qnr aqa I hqvynk-meqn I q ILGATA IEDKLQDGVPET IKCLKKgn-IK I WVLTGDKPETAVN I GFACQL--_-_-_-_-_-_-_-_-_-_-_-_-_--- I senmi i l--_-

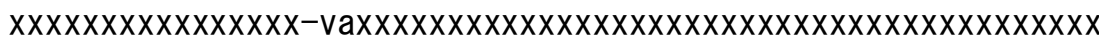
$x---------------v q r e r a f v d-$ 


$$
\begin{aligned}
& \text { laskcqav ICC--RVTPKQKALVVALVk- } \\
& \text {----ky---qq-----------v---_-_-_-_-_VTLA I GDGANDVNM I KT AD I GVGL-- } \\
& \text { AVQNSDYVLAqfCYLQRLL--LVHGRWSYMRVCKFL------R } \\
& \text { yff-yktva-smmaq iwfs I vngf-_-_-_-_-_-_-_-_-_-_-_--_saqp I yegwf la I } f \\
& \text { nl lyst I pv l y i g I feqdvtae--_-_-_-_-_-_-_--ks I kmpe l ymagqkge I fnys } \mathrm{i} \\
& \text { f-mqai thgt itsminf-fvtvxvss---------------------xxxxxxxs-------- } \\
& \text { hd--yqs I gv I vai ss I I svt l e-vm I vvkywt I | fvg---_-_--avv I s I ssyv Imts I }
\end{aligned}
$$

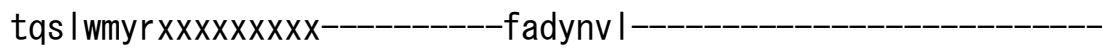

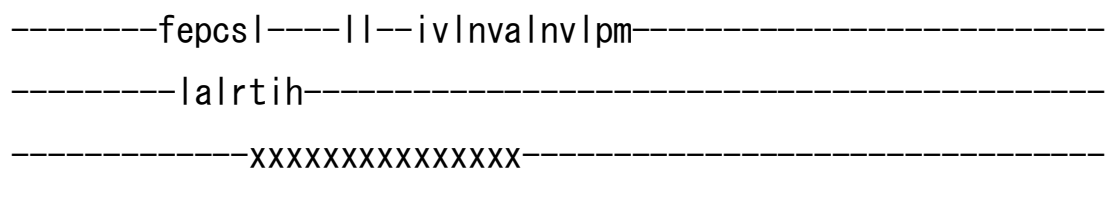

xxxxxxxxxxyh|rrgipxxxxxxxxxxxxxxxx

xxxxxxxxxxxxxxxx---10-10

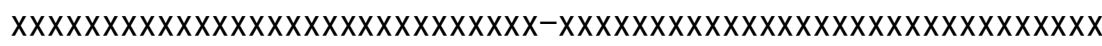
$x x x x x x x x x x x x x x x x x x x x x x x x x x x x x x x x x x x x x x x x x x x-----------------$

$-$

>mmulV_IV06 XP_001005370.1

$\mathrm{m}-$ 
xxxkkk I lev

\section{-kfqyadnr ihtskyn-}

- $\mathrm{l}$----

-tfipinlfeql

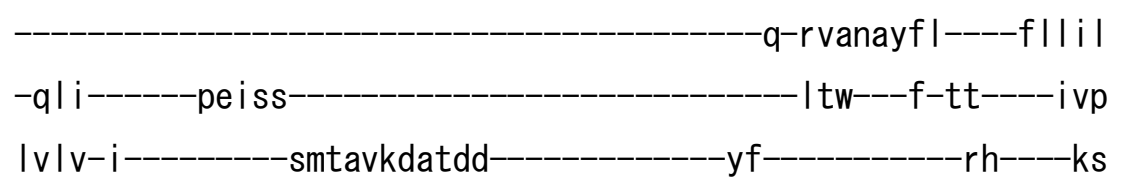

dnqvnnrqsk---_------vli-_-_-_-_-_-_-_-_-_-_-_-_-_-_-_-_-_-_-_-_-

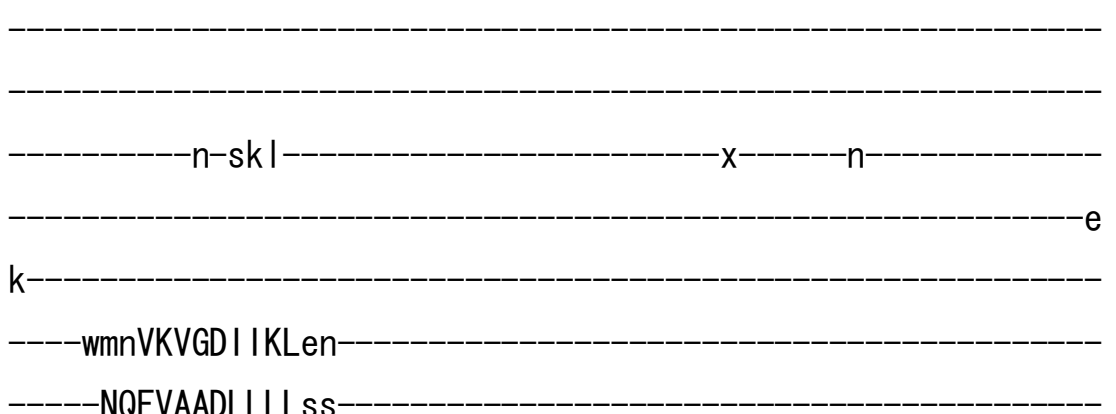

NQFVAADLLLLss 
-sephg-I---cyVETAELDGETNLKVRqa-

-xpvtse I gad i ss I aefdg i vrceapnnk I drfsgv I sw--

$-k d s k h a$

Isn

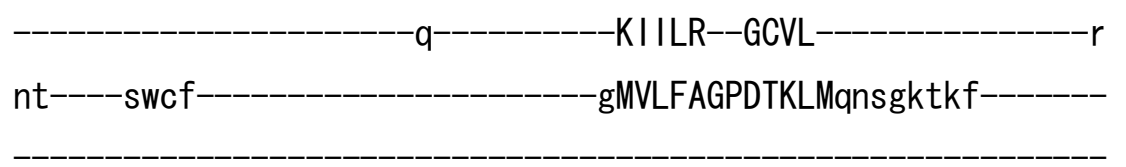

-krtsidr Imnt IvIwifgf Ivclgi i la

-vgssi l esevgdqf-----xtppf--_-_-----xxxxxxsf I fsgf I tfwsyVI ILNTL

VPISLYVSvEVIRLGHSY-F Inwdrkm-

-yyaskamp

aEAR-TTTLNEELGQIEY IFSDKTG

TLTQN IMTFk

-kcs ingrvy

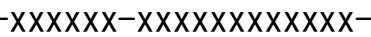

$-x X X X X--X X X X$

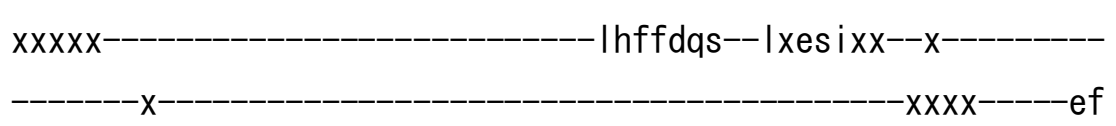

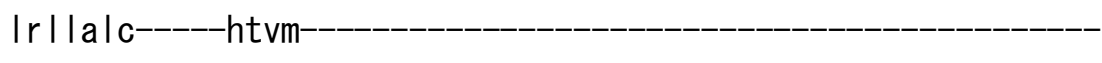

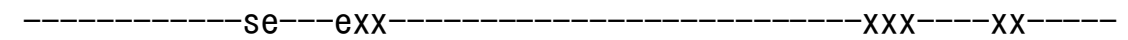

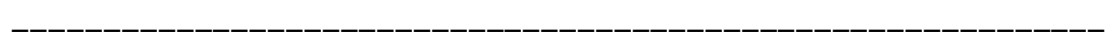

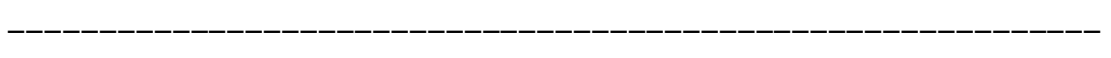

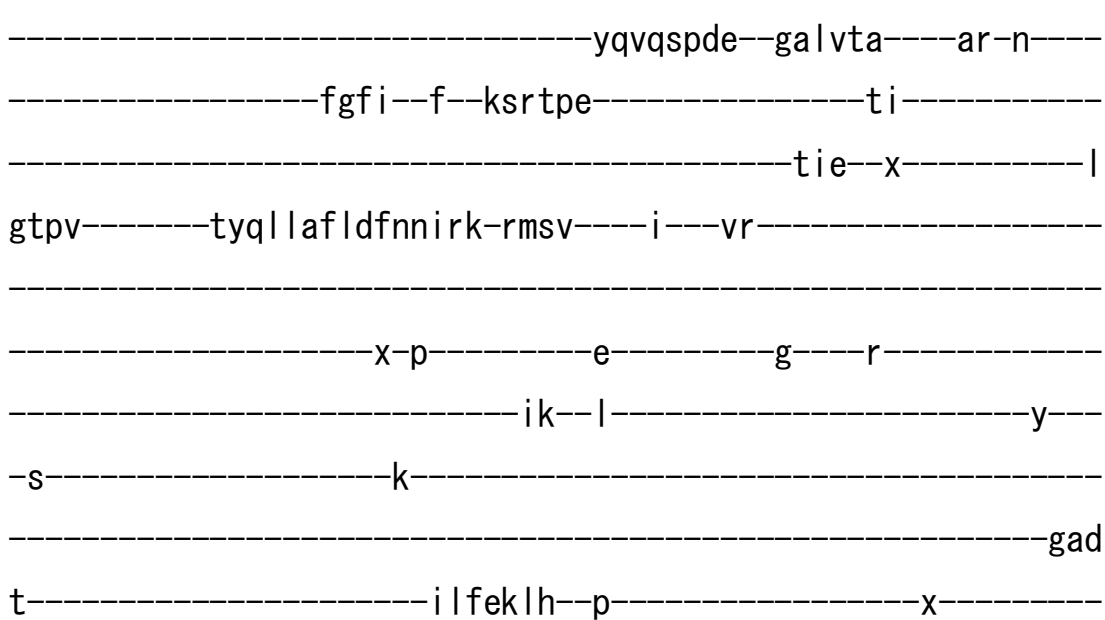




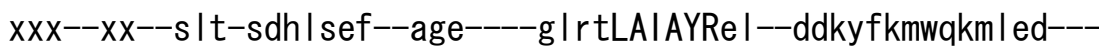
an-----sat l erder i sg I yee-i erd Im I LGATAVEDKLQEGVIET I TSLSLan-IK I WILTGDKQETAIN I GYACNV-_-_-_-_-_-_-_-_-_-_-_-_-_-_ I tdamda |--_-

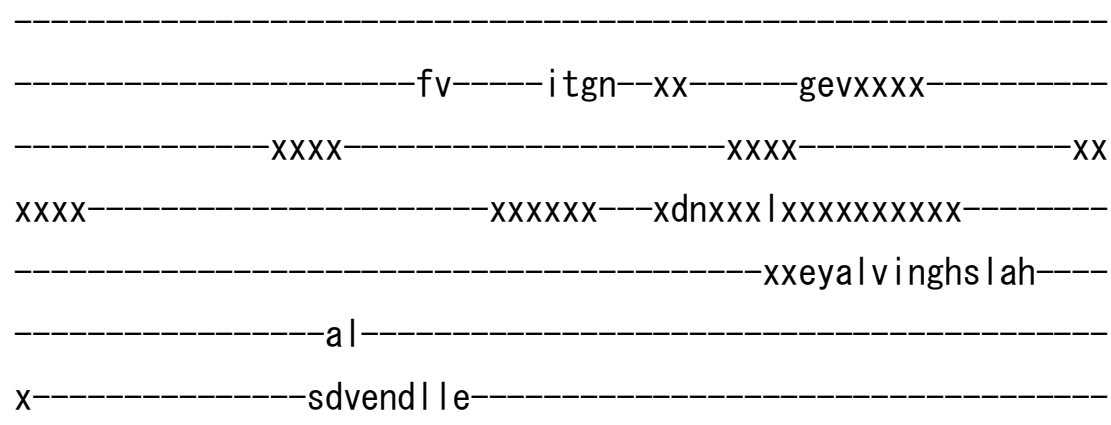

lacvcktvVCC--RVTPLQKAQVVELVk

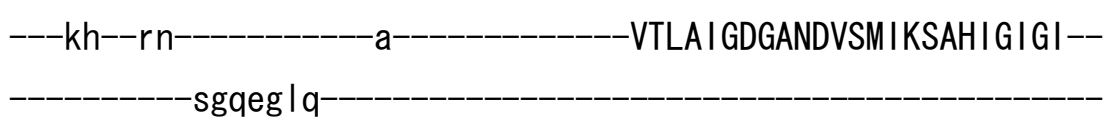

AVLASDYALAqfRYLQRLL--LVHGRWSYYRMCKFL------C yff-yknfa-ft I vhfwfaffcgf-_-_-_-_-_-__-_-_-_-_-_-_aqtvydqwf it If n i vyts I pv l amgmfdqd ineq-----------------nsmdypq l yepgq I n I fnkr r f-ficvahg i ytslalf-fipyxafy-_-_-_-_-_-_-_-_--_xxxxxxxxxi---_--ad--Iqsfavtvats Ivivvsiq-ialdtsywtvvnhv--_-_---fiwgsvatyfsi I la mhsdgvfgxxxxxxxxxgnarhs I

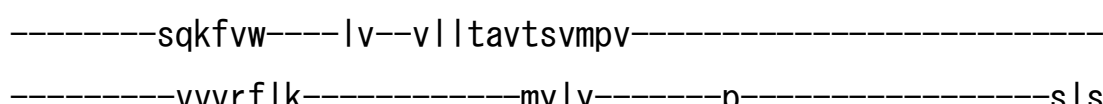

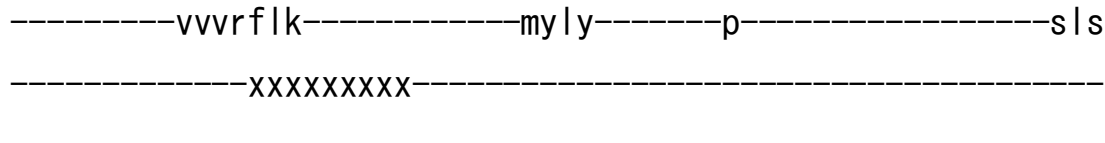




\section{>mmuIV_IV07 NP_056546. 2}

$\mathrm{m}$

$-x x x x x x q p v r h k k x x x$

$-x x x x x x x x x x x x x x x x x x x x-$

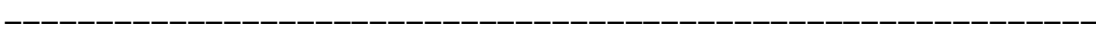

---prtvwlgh

-xxxxdqryprnvinnqkyn-

-tflpgv|fsqf 


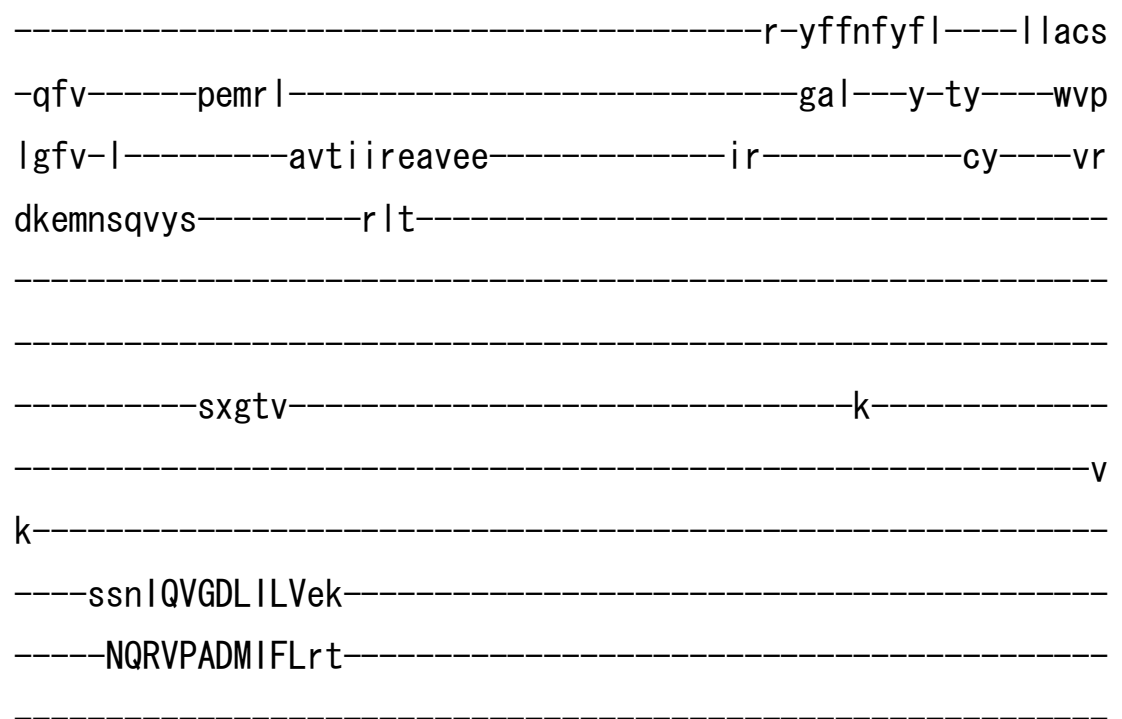

-sekng-s---cfLRTDQLDGETDWKLRIp-

-vactqr Iptaad I I qi rsyvyaeepni dihnf I gtftrxx

$x x$

-ppises

Isi

-NTLWA--GTVI-

as----gtvv

gVVLYTGRELRSVmntsdprs

kiglfdlevncltki Ifgalvvvs Ivmv

-alqhfagr-

- wy $\mid q-$

- i irfLLLFSN I

IPISLRVNIDMGK IVYSW-VIrrdsk i

$-\mathrm{pg}$

tVVR-SST IPEQLGRISYLLTDKTG

TLTQNEMVFk rlhlgtvay-

$-x x x x x x x x x x x x x x$

$x X------X X X$

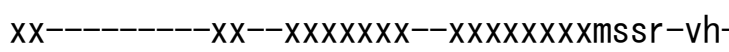

vkaial c-----hnvt

$-p v x x x$ 


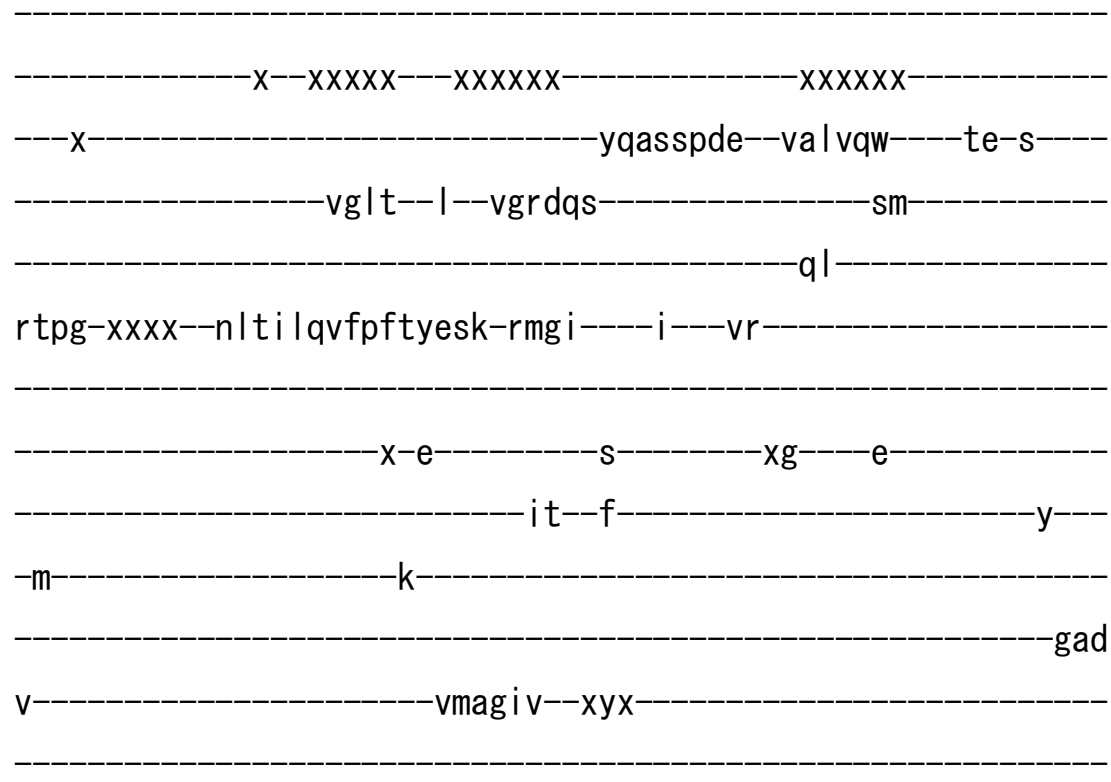

-dw I-eeecgnm--are----g | rvLVVAKKs I--teeqyqdfearyvq--ak-----I svhdrs I kvatvi es- I ememe ILCLTGVEDQLQADVRPTLETLRNag-IKV WMLTGDKLETATCTAKNAHL-vtrnqdih----

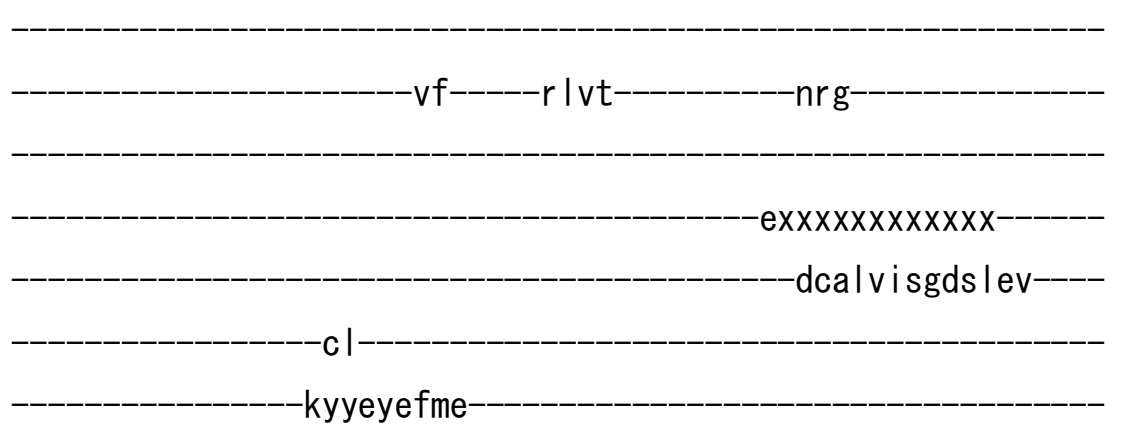

l acqcpavVCC--RCAPTQKAQIVRLLq

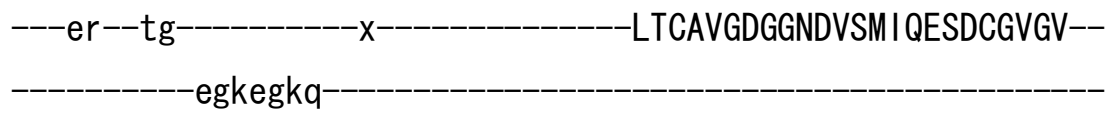

-ASLAADFSI TqfKHLGRLL--MVHGRNSYKRSAALS------Q 
fvi-hrslc-istmqavfssvfyfasvplyqgf I i igy stiytmfpvfsI-vIdkdvkse -vamlype lykd I lkgrp I sykt

f-I iwv I i siyggstim-ygal-IIf e

se--fvhivaisftsliltel Im-valtiqtwhw Imtv aellslacyiasIvf Ihef- $i-$ dvyfi -at |sfl----wk--vsvit|vsc|p|$-y v|k y| r$ $-p$ psy skl---ts >mmulV_IV08 NP_056620. 2

$\mathrm{m}$ 


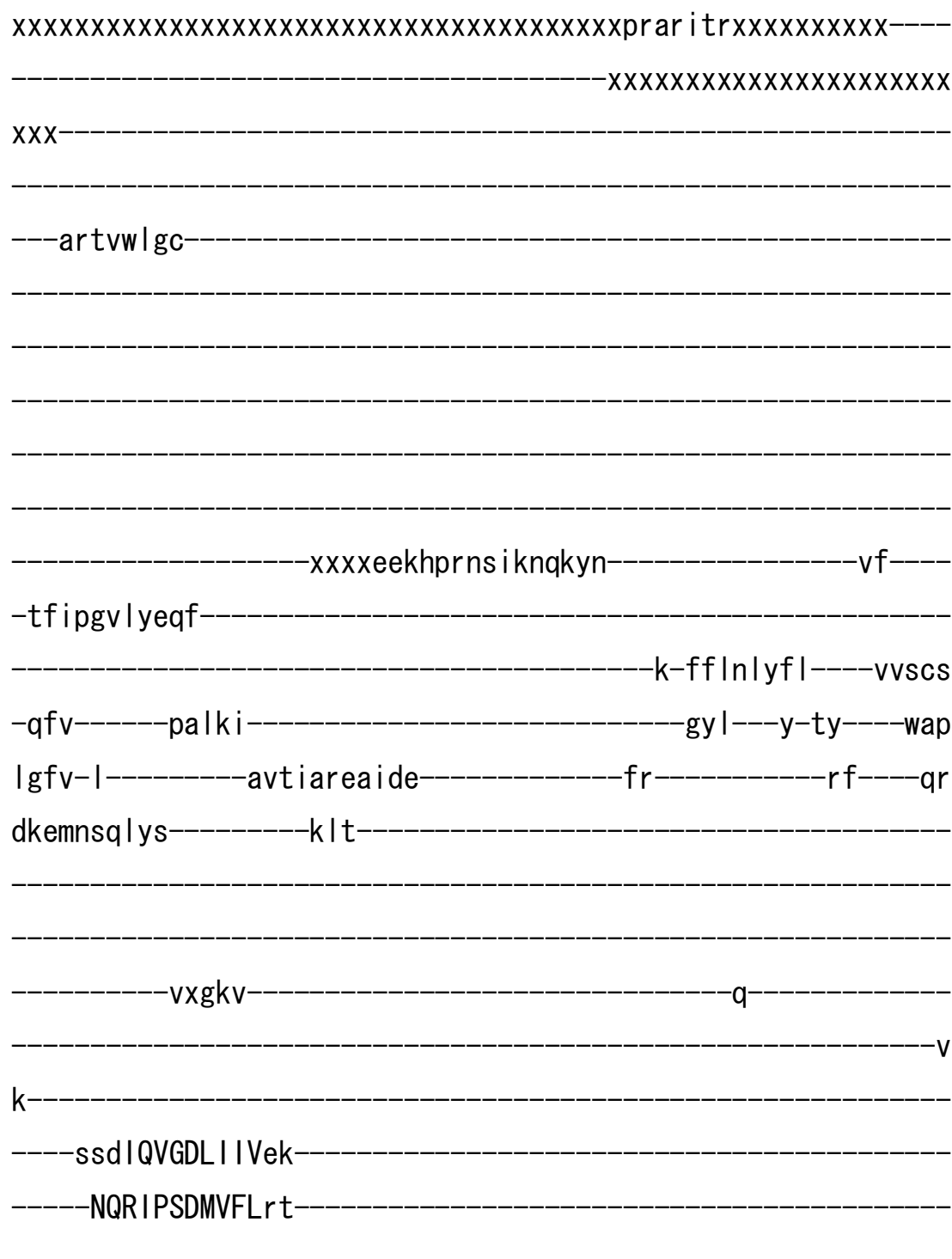

-sekag-s---cfIRTDQLDGETDWKLKva-

-vsctar Ipa Igd I fs i sayvyaqkpq I d ihsfegtftrxx

$x x$

-ppihes

Isi

e---------NTLWA--STIV-

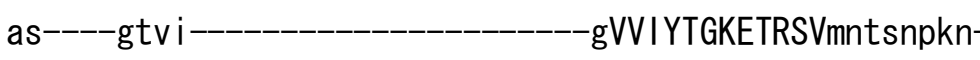

-kvg I Id le InqI tka If Ialvv Isvvmv

-t l qgfagp--_-_-_-_-_--wyrn--_-_-_-_-_-_-_-_-_-_-_-_ f frfLLLFSY I

IPISLRVN I DMGKAAYGW-MImkden i 
-tVVR-TSTIPEELGRLVYLLTDKTG

-TLTQNEMVFk-

rlhlgtvsy

$x X X X X X X X X X X X X X$

$-x X------x X X$

$x x-x x x x x x x x x x--x x x x x x x--x x x x x x x x v s s r-i h-$

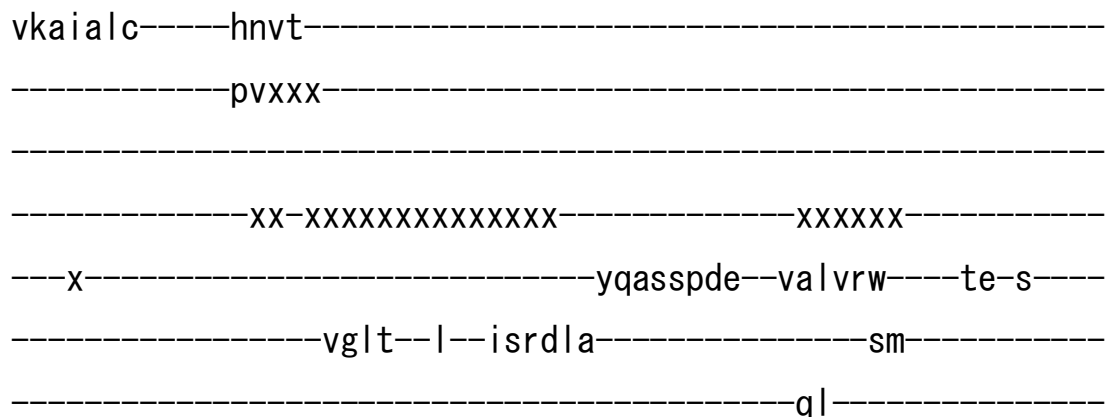

ktps-xxxx--tyci lqmfpftsesk-rmg i----i---vr

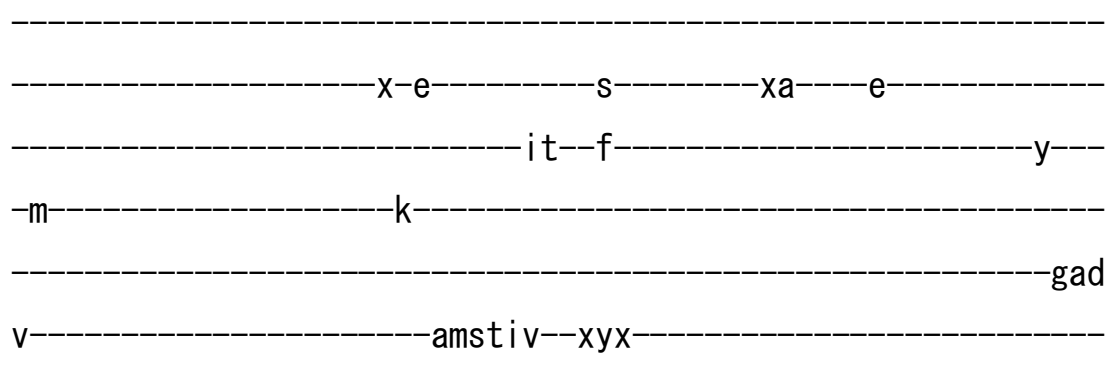

-dw I-eeecgnm--are----g | rtLVVAKRt I--teegyqdfesrysq--ak------ s i hdra l kvaavves- - ereme ILCLTGVEDQLQADVRPTLEMLRNag-IK I

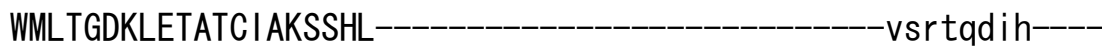

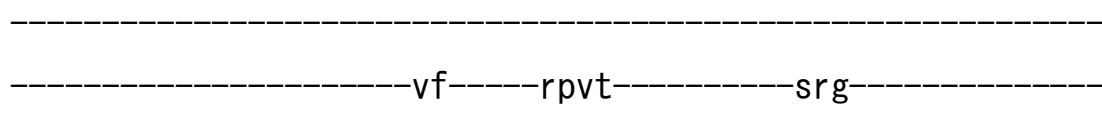
$\mathrm{cl}$

ryyehe I ve- 
I acqcpavVCC--RCSPTQKAHIVTLLr

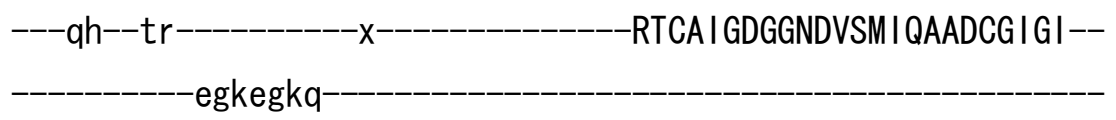

-ASLAADFSI TqfRHI GRLL--MVHGRNSYKRSAALG------Q

fvm-hrgli-istmqavfssvfyf-_-_-_-_-_-_-_-_-_-_-_--_asvp I yqgf Imvgy at i ytmfpvfs I-vI dqdvkpe--_-_-_-_-_-_---ma i lype lykd I tkgrs I sfkt

f-I i wv I i s yqgg i Im-ygal-I I f-_-_-_-_-_-_-_-_-_-_-_-_-_-_e--_-_-_-

de--fvhvvaisftal i Itel Im-valt irtwhw Imvv--------aef I s I gcyvas laf

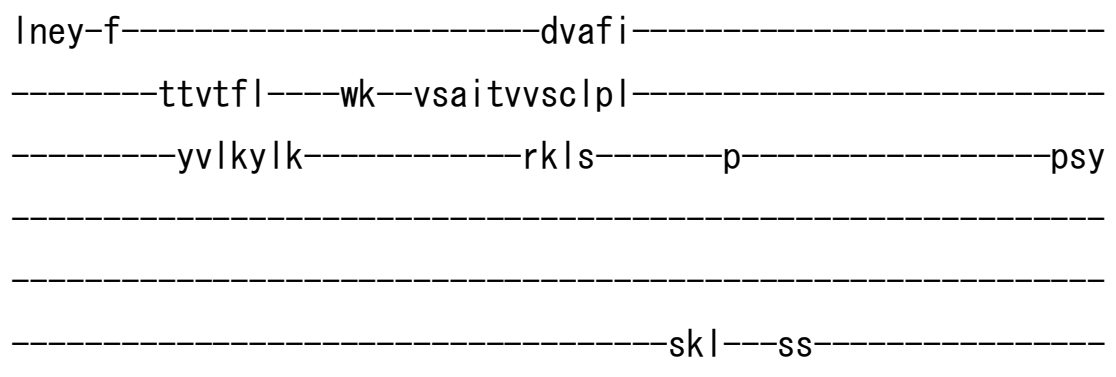

$-$

>mmulV_IV09 NP_033858. 1

$\mathrm{m}-$ 
-sflpknlfeqf

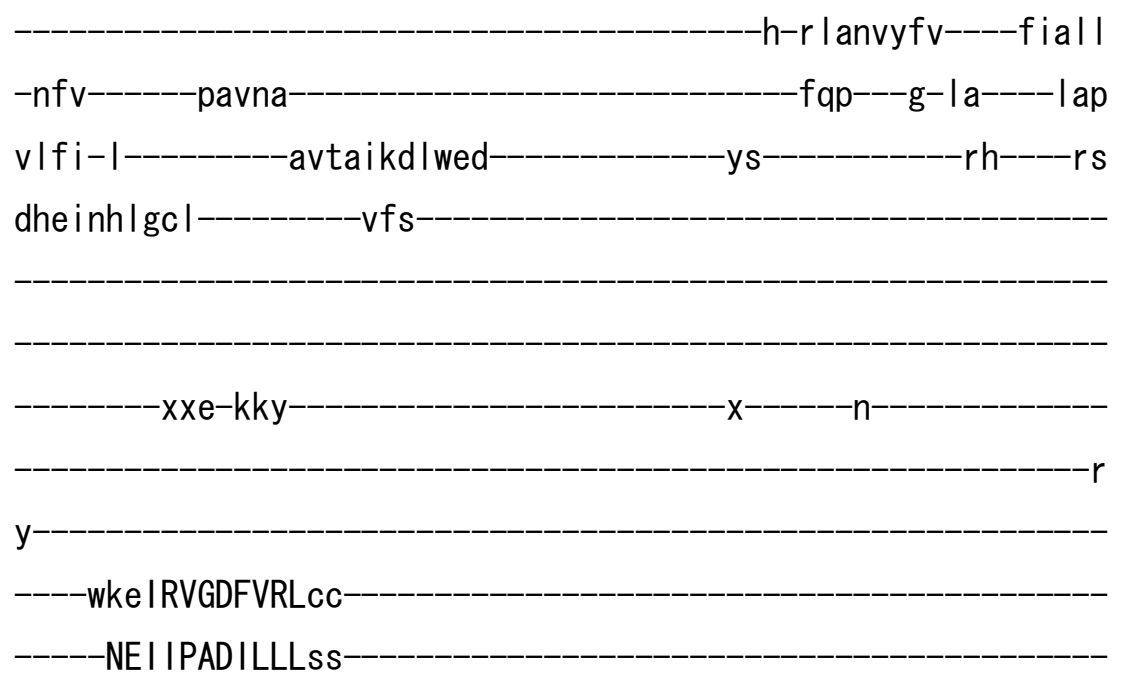


-sdpdg-I---chIETANLDGETNLKRRqv-

-vrgfselvsefnp Itftsvi ecekpnnd I srfrgy imhx-

ngekag

Ihk

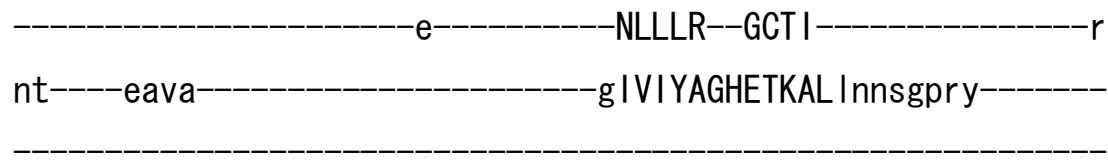

-krsqlerqmncdv Iwcv| I Ivcis Ifsa -vghg I wvr ryqekk----xxfdvp---------xxxxxxxspataavysfftmI IVLQVL IPISLYVSiEIVKVCQVY-FInqdie I

-ydeetdsq

IQCR-ALN I TEDLGQ IKYIFSDKTG

TLTENKMVFr

-rctvsgiey

$x x x x x x x x x x x x x x x x x x x$

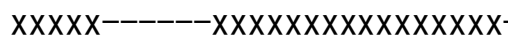

$-x x x x x--x x x x$

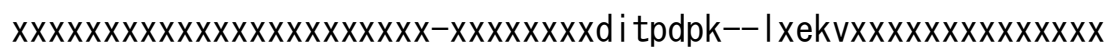

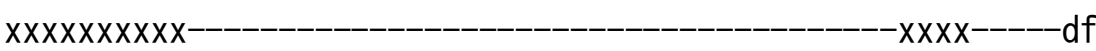

fialtic-----ntvv---------- $x x x x x x x x x x x x x x x x x x x x x x x x x x x x x x x x x x x x$

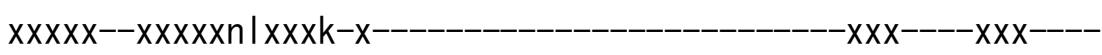
$-x x x x x x----------------$

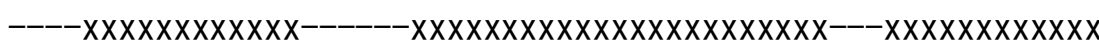
$x x x x x x x x x-------------------x x y e a e s p d e--a a l v y a----a r-a----$

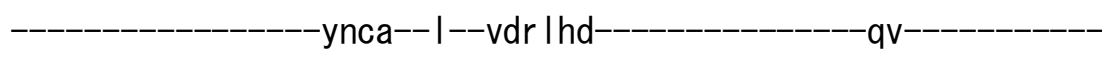
- svexxx----------1

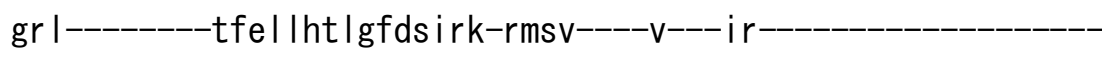

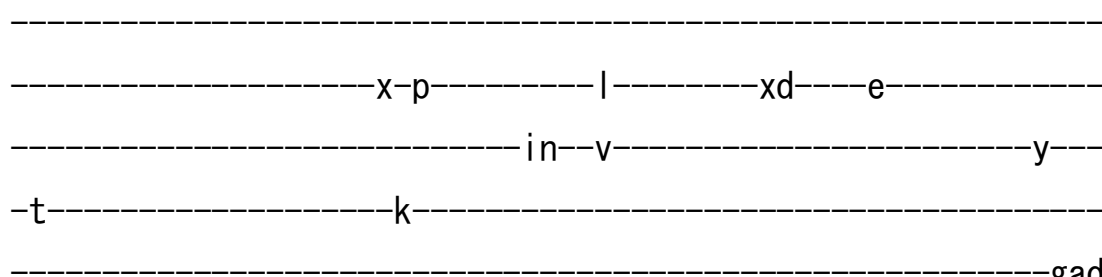

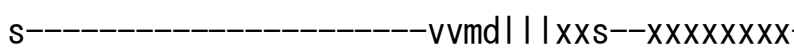


xxx--xx--skt-qny |n ly--ave----g | rtLCI AKRv |--skeeyacw I qsh i e--ae-----asvesree I I fqsavr-l etn I h LGATG IEDRLQEGVPET I AKLRQag-LQ I WVLTGDKQETAINIAYACKL-

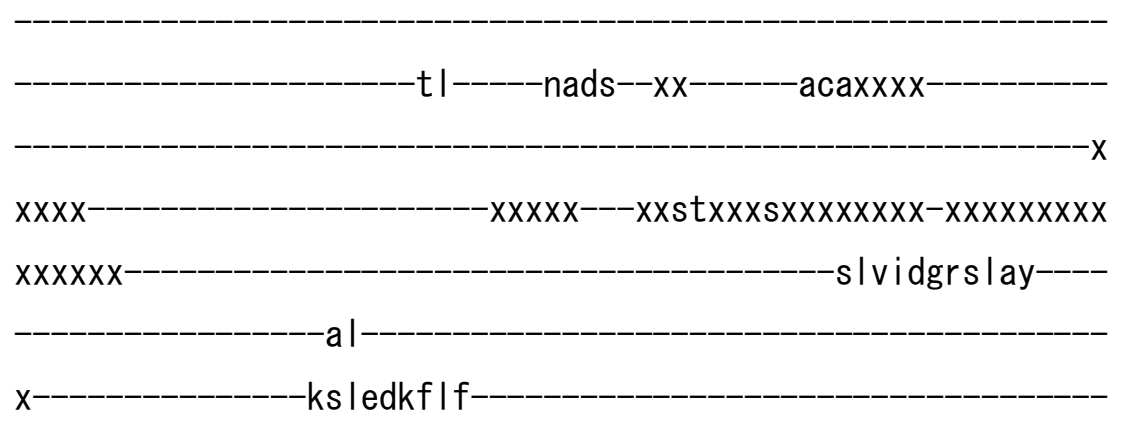

lakqcrsvLCC--RSTPLQKSMVVKLVr

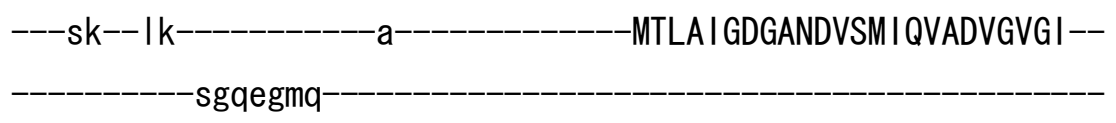

-AVMASDFAVPr FRYLERLL--IVHGHWCYSRLANMV------L

yff-ykntm-fvg I I fwfafycgf-_-_-_-_-_-_-_-_-_-_-_-_sasami dqwy I iff

nl Ifss I pq I vtgv I dkdvpad--_-_-_-_-_-_---m I I repq l yksgqnmeeyrpra

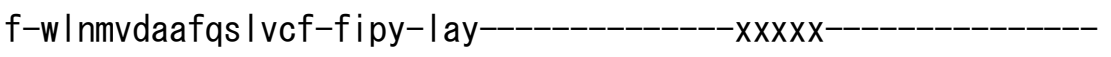

-d--vftwgtpvtaialftfI|h-Ig i etktwtw|nw|--_-----acgfstf Iffsval i yntscatc--xxxxxxx----------wtmqt I l--------------------------

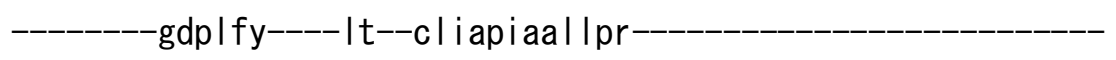

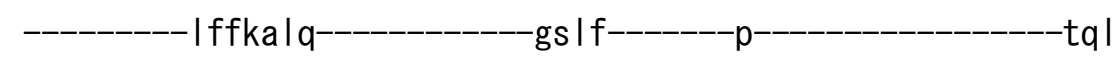

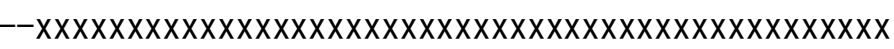

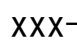




\section{>mmulV_IV10 NP_795973. 2}

m

$-x x x x x x x x x x x x x x x x x x x x x x x$ settp |

$-\mathrm{xx}----$

$-\mathrm{xxxx}$

$-x x x x x x x$

---qrvvypnn-

$x x x x x x x x x x x-$

rrypgns icttkyt $-11$

-tfIpqn I feqf 


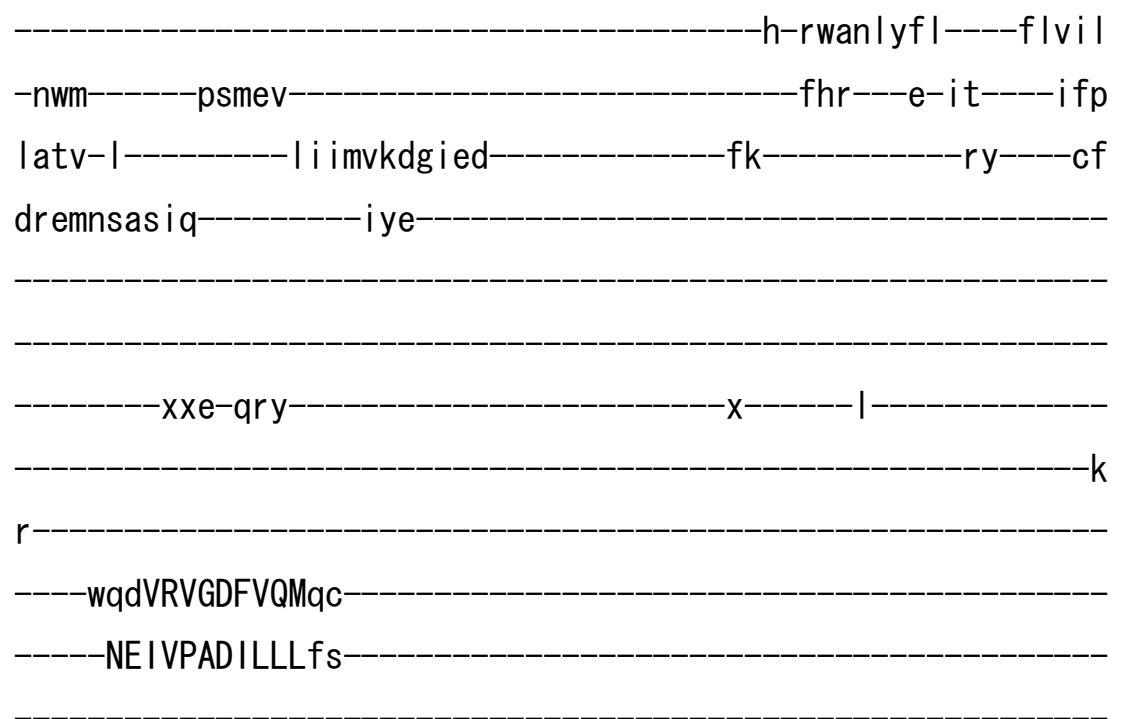

-sdpsg-v---chLETANLDGETNLKQRrv-

-vkgfsqpevqfqpehfhst i vcekpnnh I skfkgymehx-

dqtrtg

fgs-

e----_-----SLLLR--GCTI-------_---_---r

nt-----evaa--_-_-_-_-_-_-_-_-_-_--_g IVI YAGHETKAM Innsgpry

-krskierrintdiffcig IIfImc Iiga

-vghs I wngtfkehp------xfdvp---------xxxxxxxs I a I ggfymf I tm I ILLQVL

IPISLYVS iELVKLGQVF-LLhnd Id I

-ydeetdls

iQCR-ALN I TEDLGQIQY IFSDKTG

TLTENKMVFr

rctivgsey

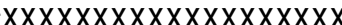

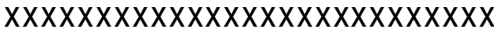

$-x x x x x--x x x x$

$x x x x x x x x x x x x x x x x x x x x x x x x x x x x x x x x x x x d v t p d k n--\mid x s k v x x x x x x x x x x-x x x x$

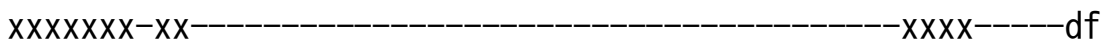

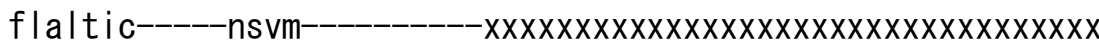

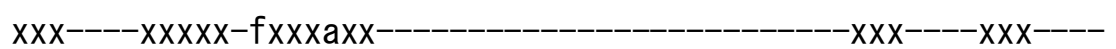




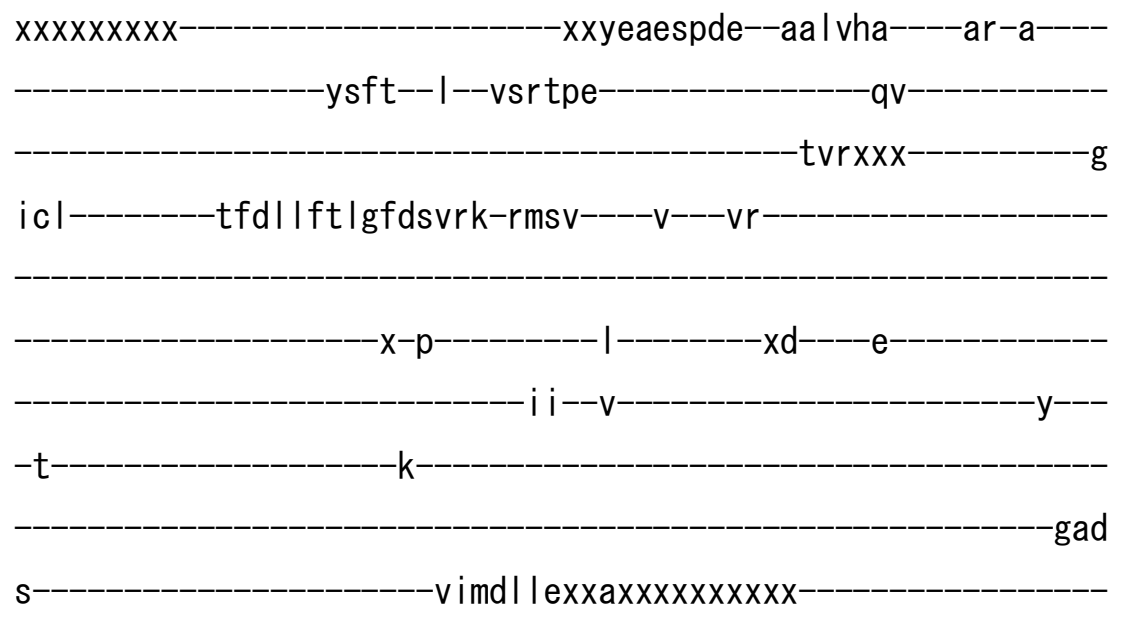

xxx--xx--art-qkhld ly--ard----g I rtLCIAKKvv--deedfarwasfrre--ae------as I dnree I Imetaqh- I enh I t I LGATG IEDRLQEGVPDT I AALREag-IQL WVLTGDKQETAVNIAYSCKLIdqtdtvy----

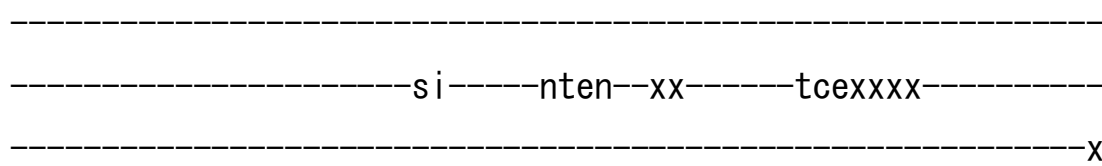

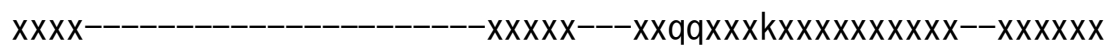

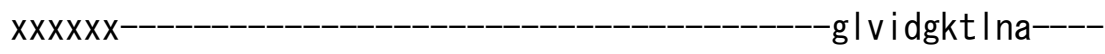
if x---------------gk lenkfle-

I tqycrsvLCC--RSTPLQKSM IVKLVr

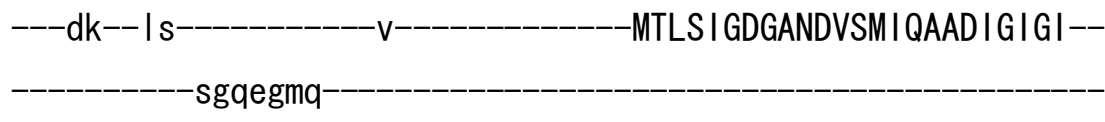

-AVMSSDFAIArfSHLKKLL--LVHGHWCYSRLARMV------V 
yyf-yknvc-yvnl I fwyqffcgf sgstmi dywqmi ff

nIffts Ipp i ifgvldkdvsae

-tl lalpe lyksgansecynlpt

f-wvsmadafyqs I icf-fipy-Ity $x x x x x$

-d--vftfgtpint is Itti I Ih-qamemktwtv Ihg I $-v \mid$ Igsf Imyfuvsli ynatcvtc-- $x x x x x x x$ -wvmerql -sdptfy----|i--c| Itpvval |pr$-y f|| s \mid q$ -gtyg $-k$ sli $-x x x x x x x x x x x x x x x x x x$

XXXXXXXXXXpasasasaxxXXXXXXXXXXXXXX

$x x x x x x x x x x x x x x x x x$

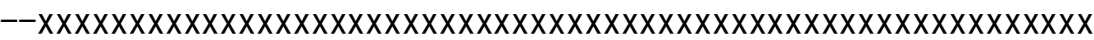
$x x x x x x x x x x x x x x x x x x x x x x$

$-$

>mmulV_IV12 NP_056619. 1

$\mathrm{m}$ 
---srtiyvgh-

$-x \times x \times x x x x \times x x$

-pqrypdnr ivsskyt $-\mathrm{fw}----$

-nf ipknlfeqf

$-r-r i a n f y f I----i$ iflv

-qli---_---id-tp---_-_-_-_-_-_-_-_-_-_-_-_--tsp----v-ts----g $\mid p$

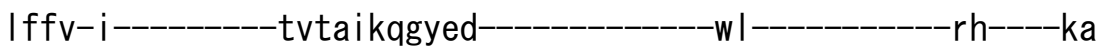
dnamnqcpvh----------fiq

-nradg-t---chVTTASLDGESSHKTHya-

-vqdtkgfhteadvds I hat i eceqpqpd I ykf fvgr invxx $\mathrm{xxx}$

-dpvvrp

Igs

-NLLLR--GATL---------------k

nt----ekif

gVAIYTGMETKMAInyqsksq

-krsaveksmntfli ivylcilvskal int

-v lkyvwqsepfrde------xwyne--------xxxxxxxxn If I raftdf I afMVLFNY I

IPVSMYVTVEMQKFLGSY-FI twdedm- 
pLVN-TSDLNEELGQVEYIFTDKTG

TLTENNMAFk-

eccieghvy

$-x x x x x x x x x x \mid$ pdssg i $x x$ i xsspxxxxx

$-x x x x$

fraiclc-----htvq-

$-x X X X X$

$x x x x x x x x x x x-p d-x x s x x-$

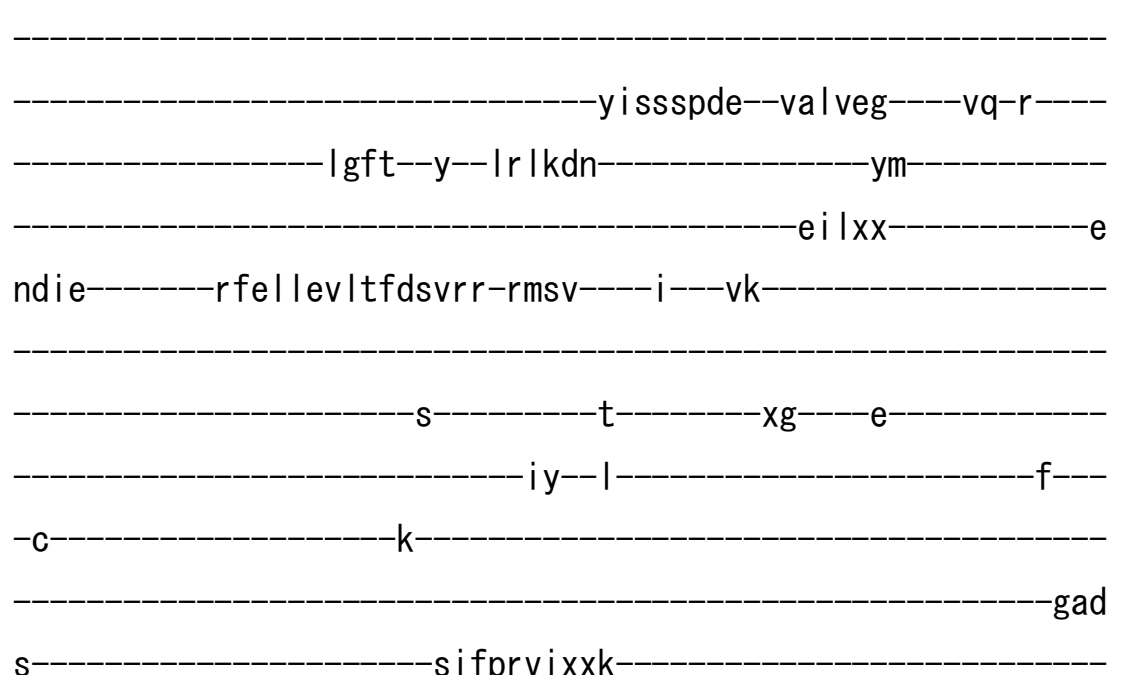

sifprvixxk

-xdqv-rsrvern--ave-----g I r tLCVAYKr I--epeqyedacr I lqs---

ak-----va I qdrekk I aeayeq-i ekd I v ILGATAVEDRLQEKAADT IEALQKag-IKV WVLTGDKMETASATCYACKL---_-_-_-_-_-_-_-_-_-_-_-_-_frrstql I---_

el-----ttkk--xx-------eqsxxxx-

$x x x x x x x x x x \operatorname{cs} x x x \operatorname{tx} x x x x x x x x x$

$-x x d y g \mid$ i idgaa $|s|$

im

$-x x x x x x x$

$x-x$

gnyre Ifle 
icrncsavLCC--RMAPLQKAQIVKLIk-

---fs--ke-----------xp-------------I ITLA I GDGANDVSM I LEAHVG I GV-igkegrq

-AARNSDYAIPkFKHLKKML--LVHGHFYYIRISELV------Q

yff-yknvc-fi fpqf l yqffcgf-_-_-_-_-_-_-_-_-_-_-_-_sqqt I ydtay It ly nisfts I p i I lys I meghvg id-_-_-_-_-_-_-_-_ I krdpt I yrdiaknal I rwrv f-iywtf I gvfdalvff-fgayx ife---------_---------xxxxxxxxxm-------

fg--nwtfgt Ivftvmv|tvt Ik-laldthywtwinhf---_----viwgs I I fy iafs I I wggv i wpf--xxxxxxx-----------yvf i sml----------------------------

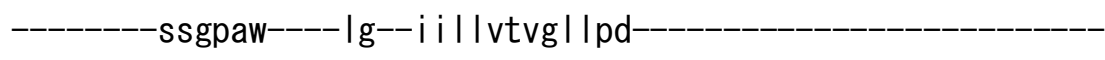

---------vlkkvlc-----------rqlw------p-------------- tat

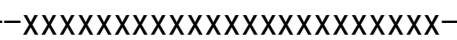

$-x x x x x x x x x x$ aqcsspsgxxxxxxxxxxxxxxxx

$\operatorname{xx} x \operatorname{xx} x \operatorname{xx} x \operatorname{xx} x \operatorname{xx} x \operatorname{xx} x \operatorname{xx} x \mathrm{x}$

$-$

>mmulV_IV13 NP_083846. 2 
---trtiyian

-nfvpkn I feqf

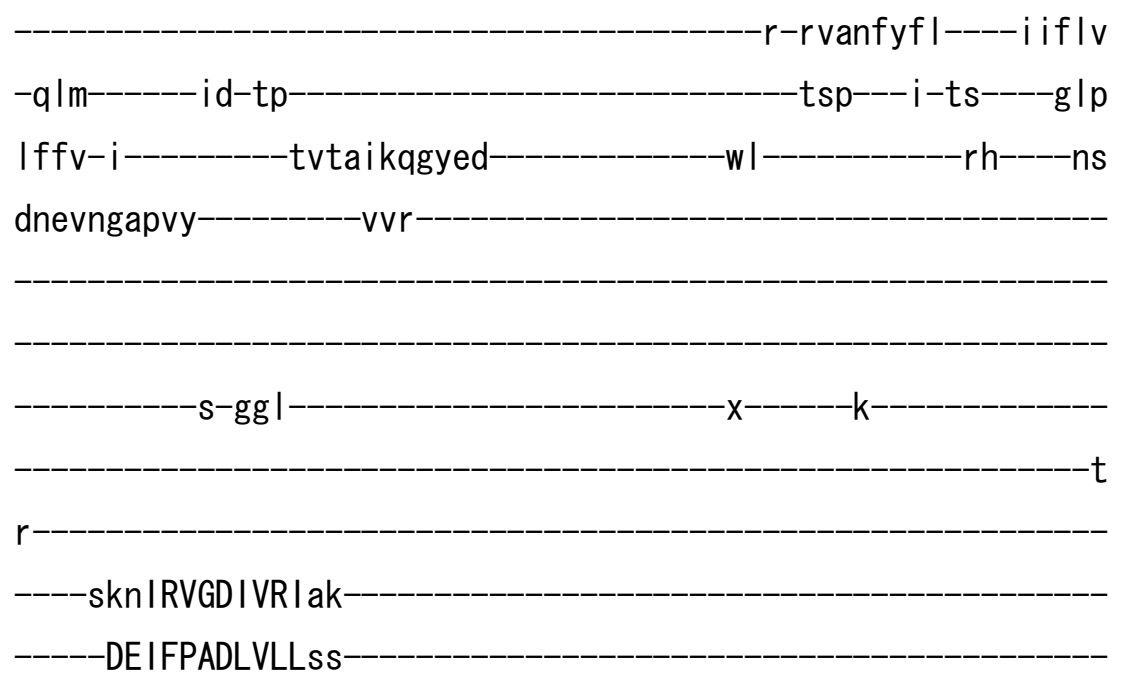


$-d r$ Idg-s---chVTTASLDGETNLKTHvs-

-vpetav lqtvan Ids I i av i ecqqpead I yr fmgrm i ixx

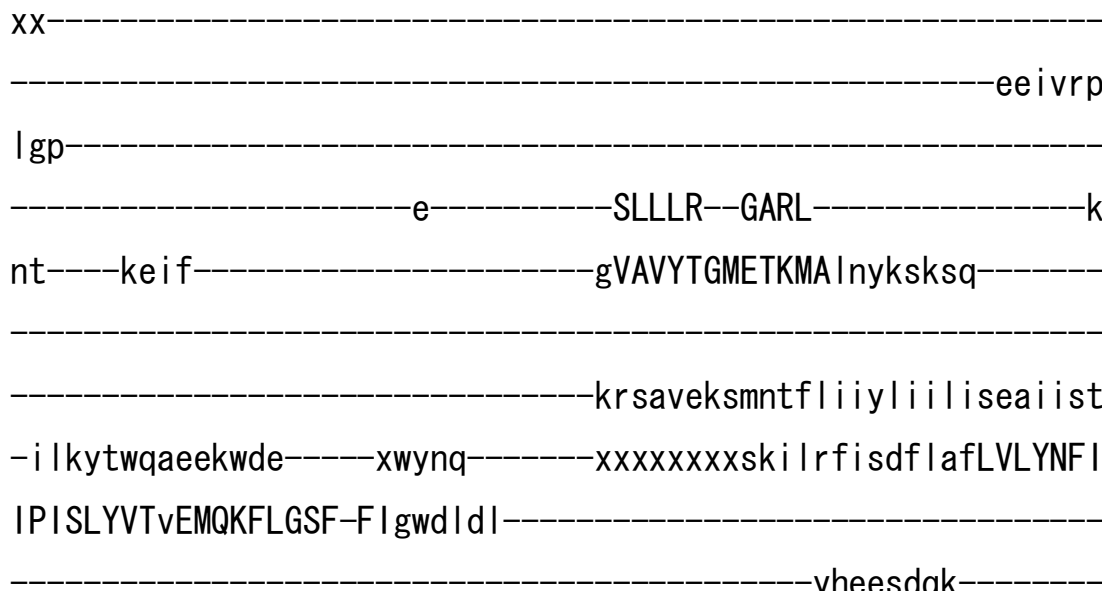

aQVN-TSDLNEELGQVEYVFTDKTG

TLTENEMQFr

-ecsinglky

$-x x x x x x--x x x x$

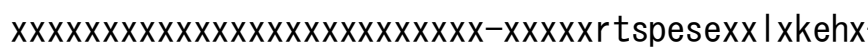

If

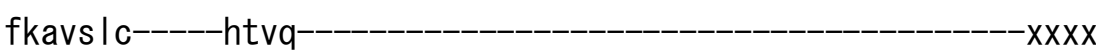

$x x x x x x x x x x x x n \mid x x x q x x$

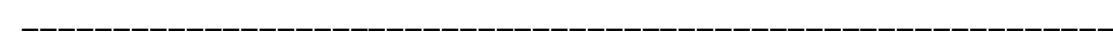

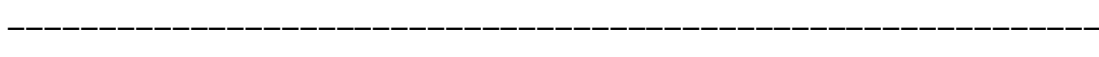

--_---_--_-_--_--_-_--_---_----yyasspde--kal vea----aa-r----

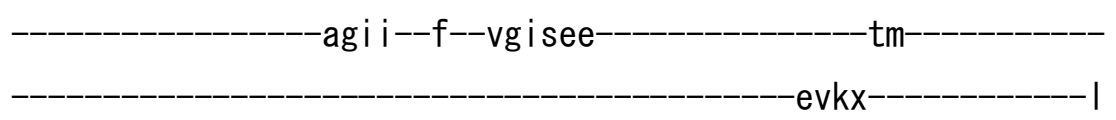

gr le-------ryk| Ihi lefdsdrr-rmsv----i---vq-

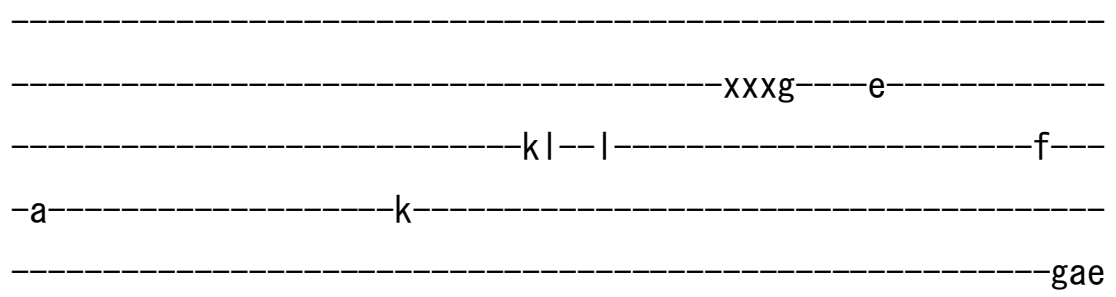

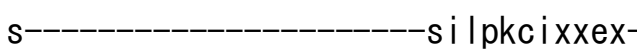


-akt-rihvdef--alk----g|rtLCIAYRqf--takeyedvdr r I fe--ar-----tal qhr eek l adafqy-i ekd I i ILGATAVEDRLQDKVRET IEALRMag-IKV WVLTGDKHETAVSVSLSCGH------_-_-_-_-_-_-_-_-_-----fhr tmn i I----

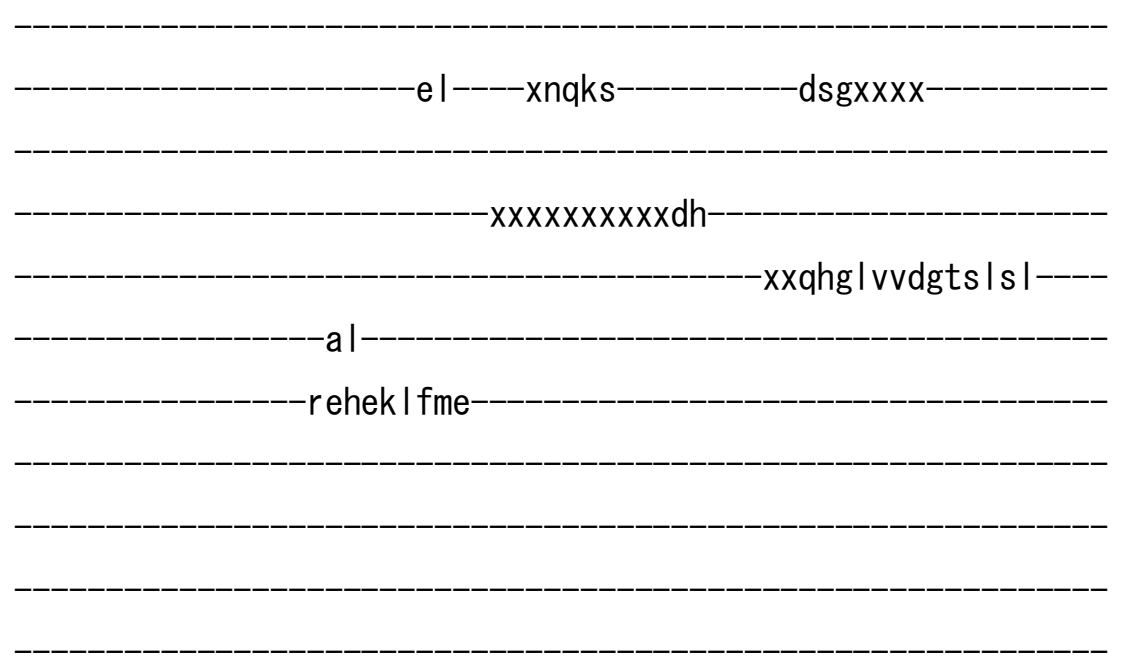

vcrncsavLCC--RMAPLQKAKVIRLIk

---i s--pe-----------xp----_--_-----ITLAVGDGANDVSM I QEAHVG IGI--

AARNSDYAI Ar FKFLSKLL--FVHGHFYYIRI ATLV------Q

yff-yknvc-fi tpqf I yqfyc If--_-_-_-_-_-_-_-_-_-_---sqqt I ydsvy It I y nicfts I pv I iys I veqh idph--_-_-_-_-_-_----v l qskpt l yrdi skng I I sika

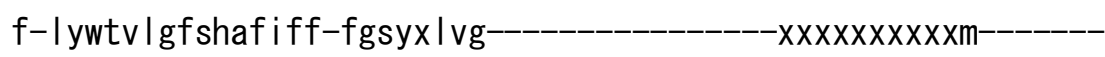

fg--nwtfgt Ivftvmvi tvtvk-mal ethfwtwinhl---_----vtwgs i i fyf i fs If

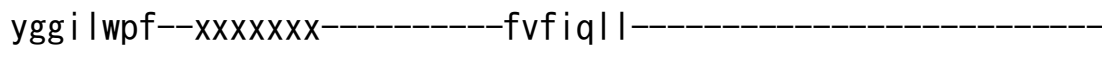

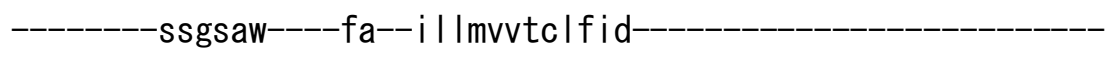

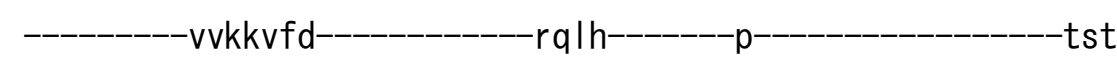

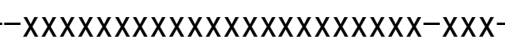


>mmuIV_IV14 NP_001032952. 1

m

$-x x x x x--x x x-$

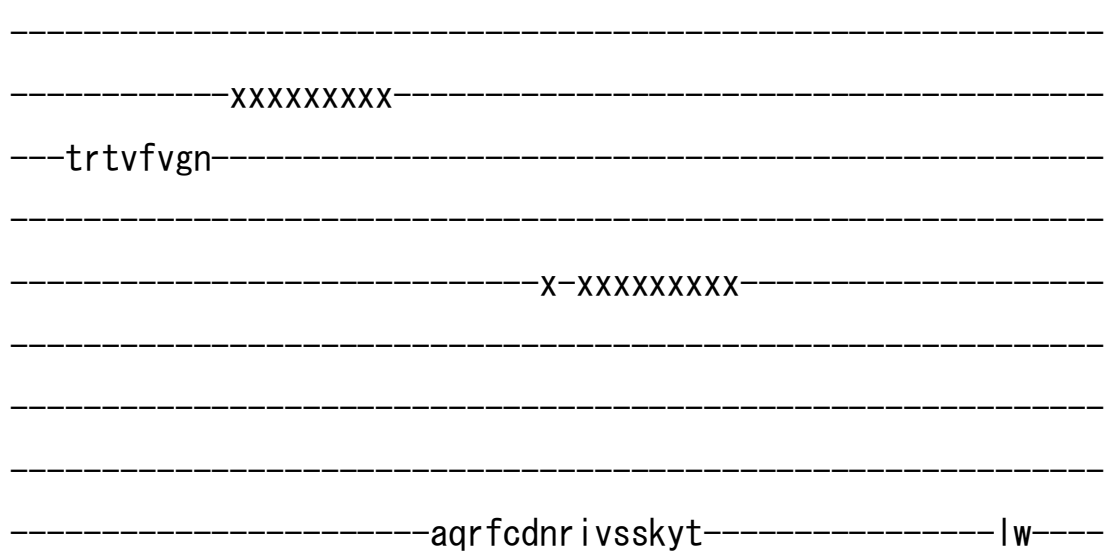

-nflpknlfeqf 


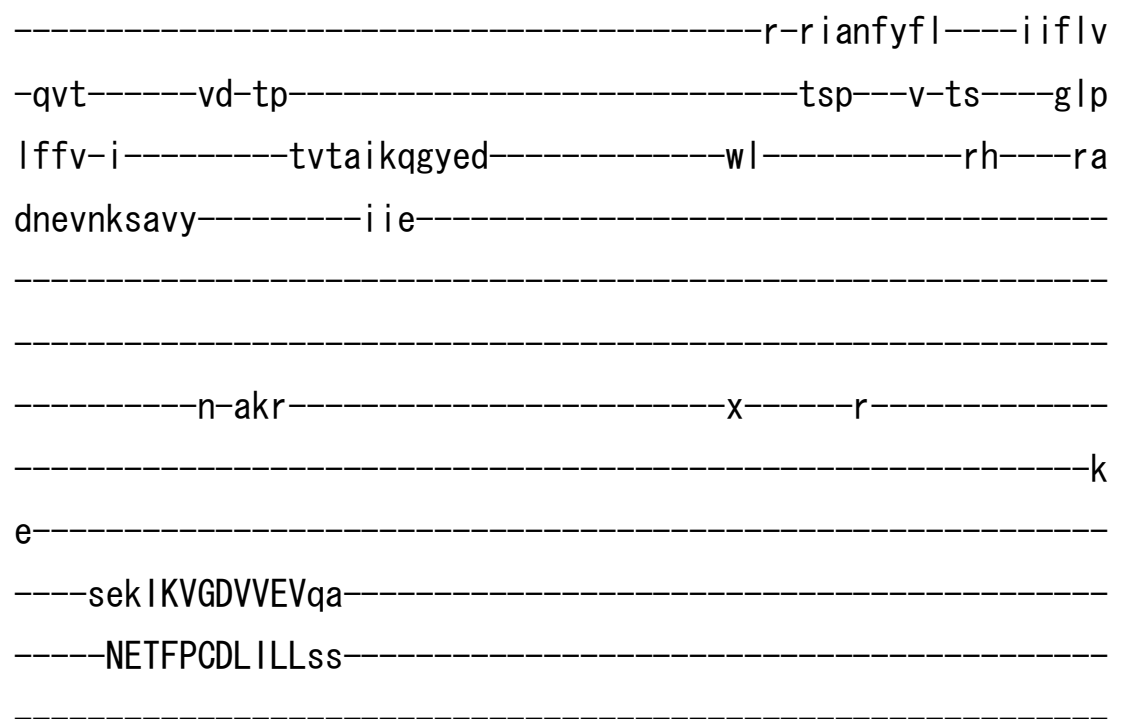

cttdg-t---cyVTTASLDGESNCKTHya-

-vrdt i a Ictaes i dn I rat i eceqpqpd I yrf fvgr i s i xx

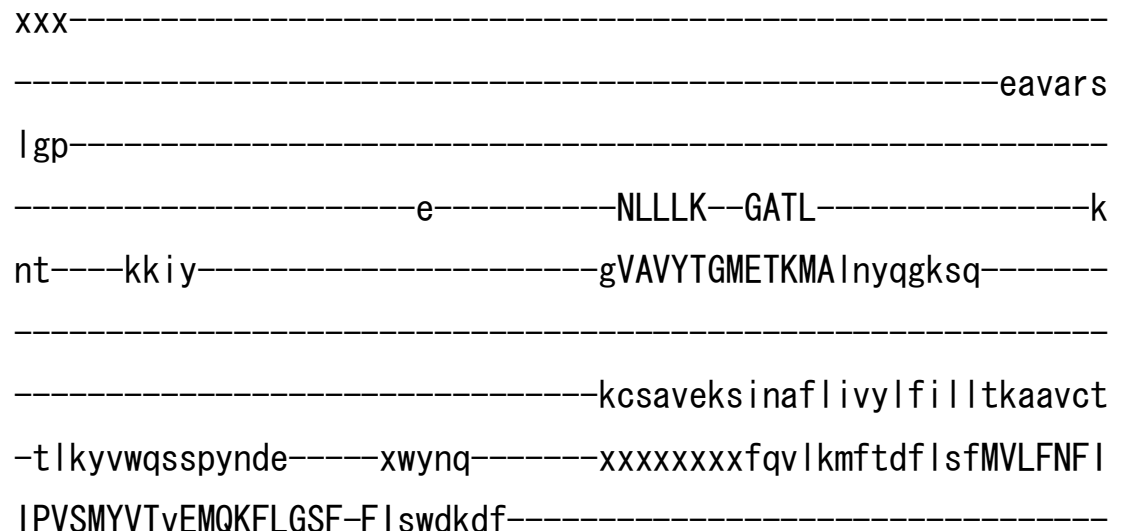

fdee ineg

aLVN-TSDLNEELGQVDYVFTDKTG

TLTENSMEF $\mathrm{i}$ ecc idghky-

$-x x x x x x x d g|s q t d x x|-a y f x x x x x$

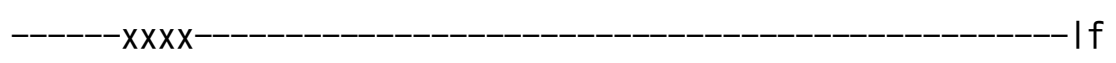

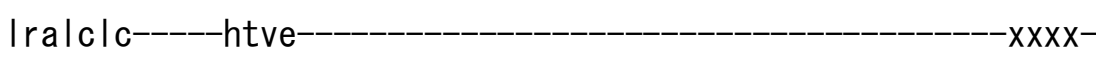

-----xxxxxxxegxxx--x- 


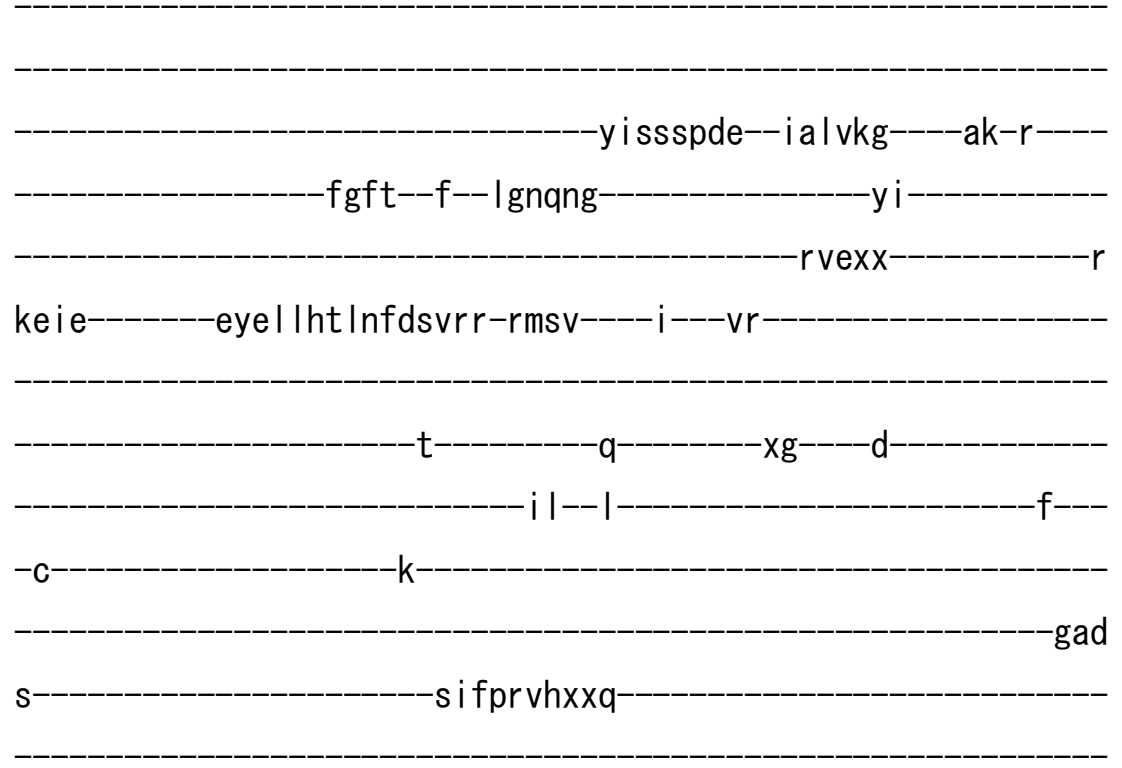

-xelt-kdhvern--amd----gyrtLCVAFKe i--ppddfer i naq l ve--ak-----ma l qdr eek l ekvfde-i etnmn I I GATAVEDKLQDQAAET IEALHAag-LKV WVLTGDKMETAKSTCYACRLfqtntell----

el-ttktxxxxxxx---edrxxxx $-x x x x x x x x x x e f x x x t x x x x x x x x x x$ $-x$ eyg $\mid$ i idgst $|s|$ i I $-X X X X X X$ x---------------nnyks if lq-

i cmkctavLCC--RMAPLQKAQIVRMVk

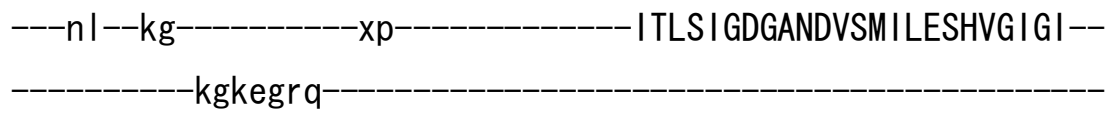


>ggalV_IV01 XP_420729. 2

$\mathrm{m}-$ 


\section{-tfiprflysqf}

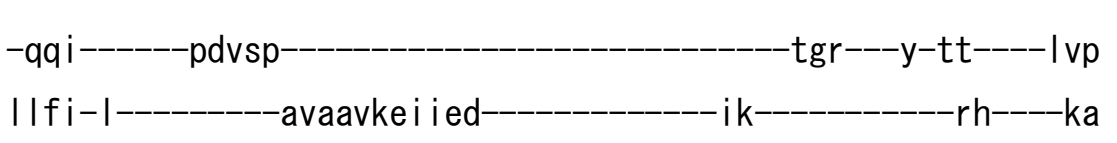

-sepqa-m---cy IETSNLDGETNLKIRqg-

|p|tsdikdies Imr Isgr i ecespnrh lydfvgnir Ix-

-ghgtvp

Igs

QILLR--GAQL

nt-----qwvh-

g IVYTGHDTKLManstspp I

-kmsnveritniqililfciliamslics

-i gsavwnr rhserd-------wy Id-_-_-_--_-_--Xxxggasnfg I nf I tf I ILFNNL

IPISLLVTIEVVKFIQAY-FInwdidm- 
-hyeptdta

aMAR-TSNLNEELGQVKYIFSDKTG

$-T L T C N V M Q F k$

-kctvagvay

$-x x x x x x x x x x x x x x$

$\mathrm{XXXX}$

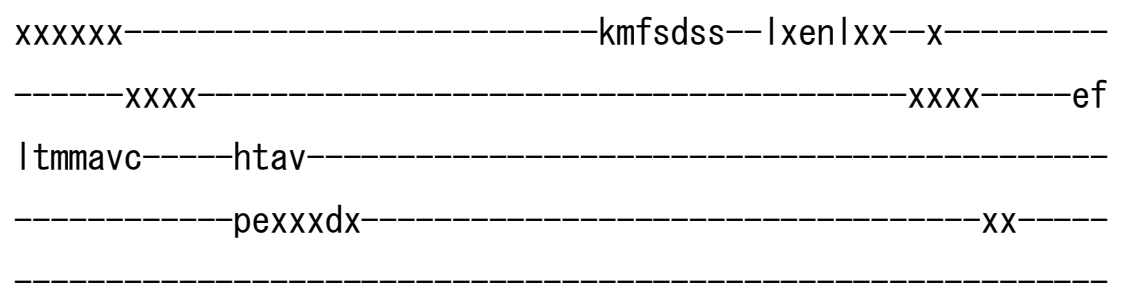

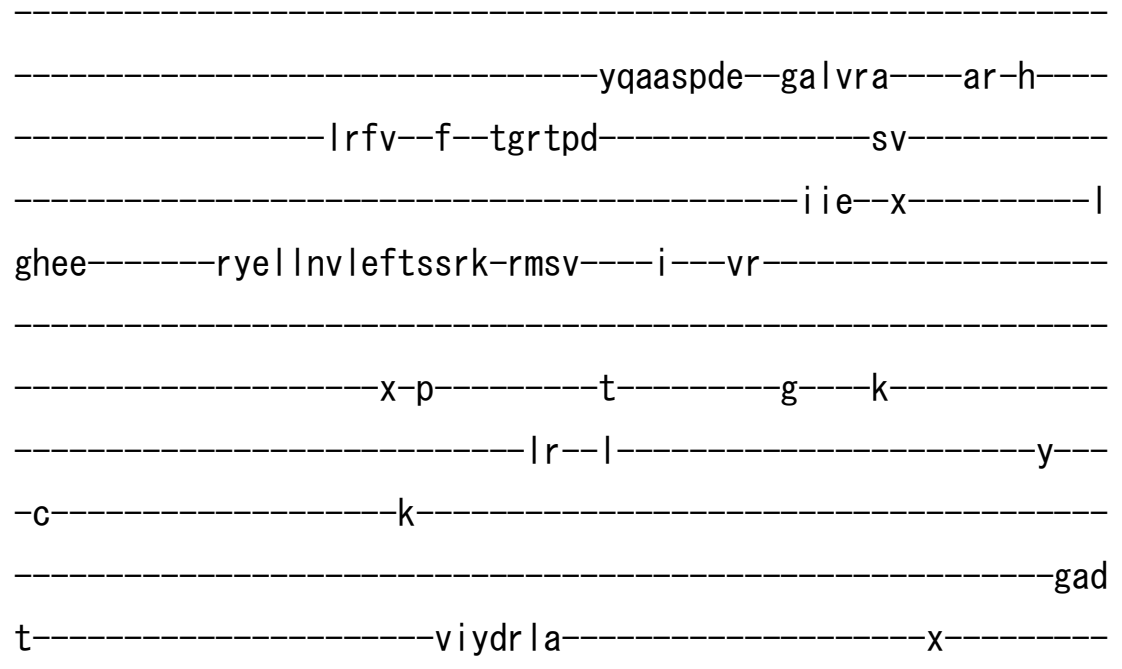

xxx--xx--e it-Ikhl eqf--ate----g|rtLCFAVAe i--sesdyqew I dvyhr--as-----ta iqnralk l eesye I-i ekn I q ILGATAIEDKLQDKVPETIETLMKad-IKI WILTGDKQETAIN I GHSCKL--_-_-_-_-_-_-_-_-_-_-_-_-_-_ I rknmg I i-_-_

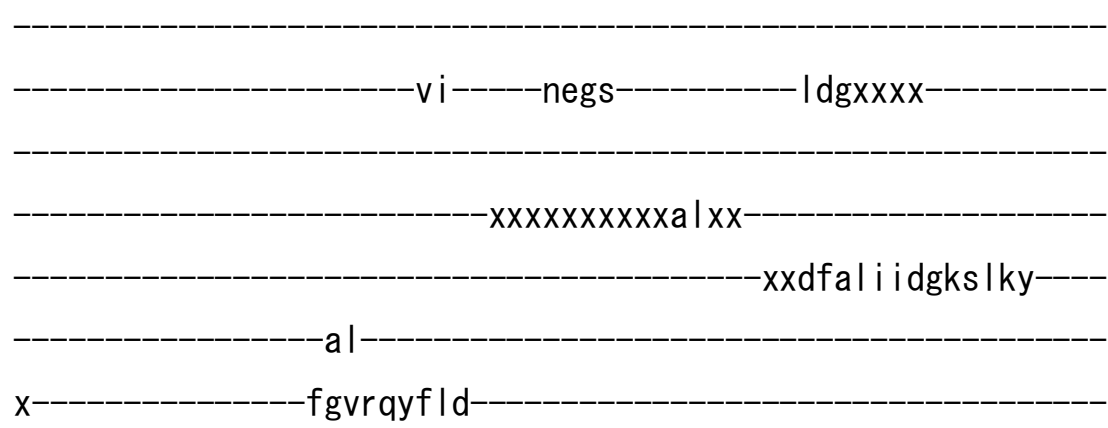


la l sckav ICC--RVSPLQKSEVVEMVk

----kq----v-----------xv-----_-_-----VTLAI GDGANDVSM I QTAHVGVGI--

-AANSSDYSIAqfKYLKNLL--LVHGAWNYNRVAKCI------L

ycf-ykn i v-Iy i i i iwfafvngf-_-_-_-_-_-_-_-_-_-_-_-_sgq i I ferwc ig ly nvmftampp I t I g i ferscrke------_-_-_-_----nm l kype l yktsqna I dfntkv f-wvhc Ing I fhsfi If-wfp I xalq-_-_-_-_-_-_-_-_xxxxxxxxt---_---

sd--y | I I gntvytfvv Itvc|k-ag letsywt I fshi-------ai wgs i a Iwvvffg i

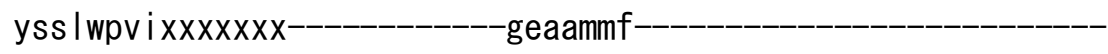
---------ssgvfw----mg-- I l c i pmtal I fd------------_-----------------------vvykvvk-------------ratf--------k------------------tIv $-x x x x x x x x x-$ $-x x x x x x x x x x k s \mid$ teraqxxxxxxxxxxxxxxxx

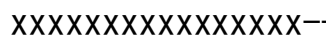

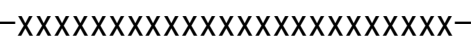

$-$

>ggalV_IV02 XP_417130. 2

m- 
---artiyvnq

$-x x$

-qskfrdnwvstakys

vV----

-tfiprflyeqi

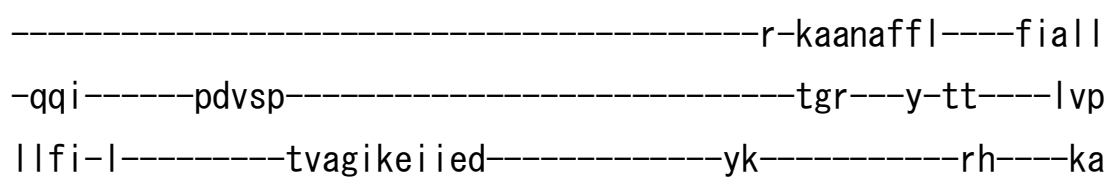

dsavnkkktv

$-v \mid r$

n-gmw-

$-x------d$

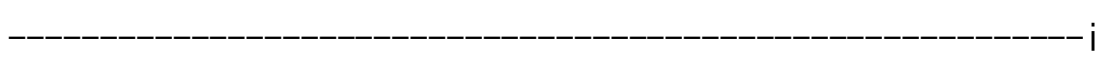

V

----wkeVAVGD IVKVtn

GQHLPADMI I Iss 
-sepqa-m---cy IETANLDGETNLKIRgg-

|s | tas |qsree Imkvsgr i ecegpnrhlydftgt |r |x-

-gqspvp

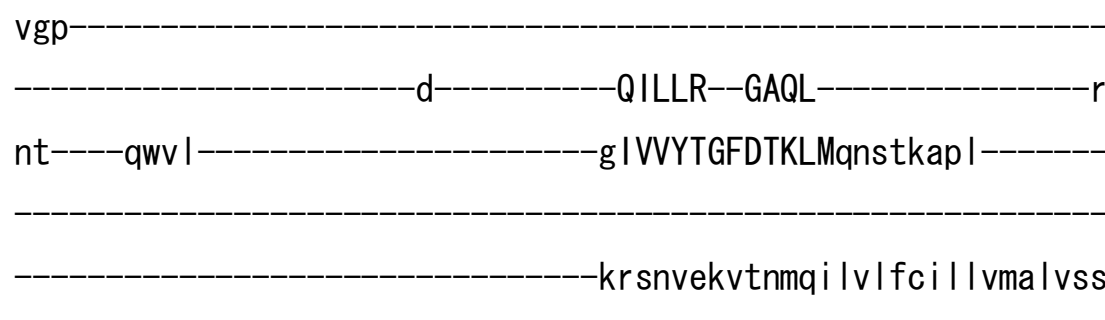

-vga I I wnr thgevv--_----wy I g--_-_-_-_-_--xxxm I svnfgyn I I tf I ILYNNL

IPISLLVT IEVVKFTQAL-FInwdmdm-

-yypetdtp

aMAR-TSNLNEELGQVKYLFSDKTG-

-TLTCN I MNFk--------------kcs i agvty

$x x x x x x x x x x$

$-x X X X X--x X X X$

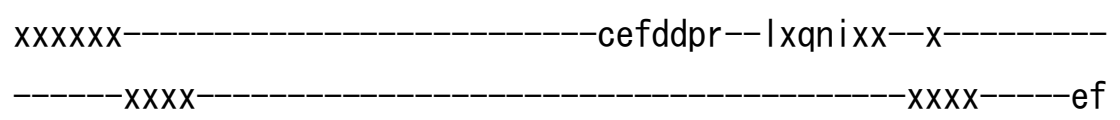

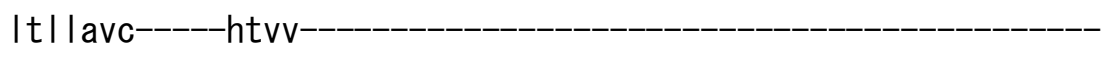

$-\operatorname{pexxxnx}$

$-\mathrm{xx}-----$

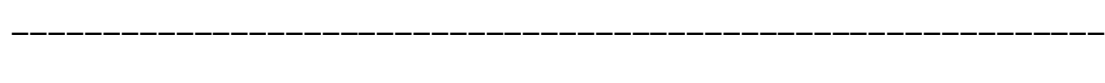

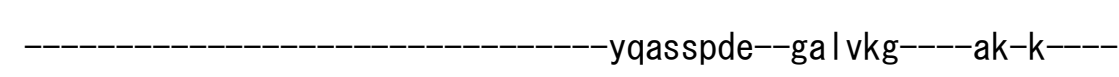

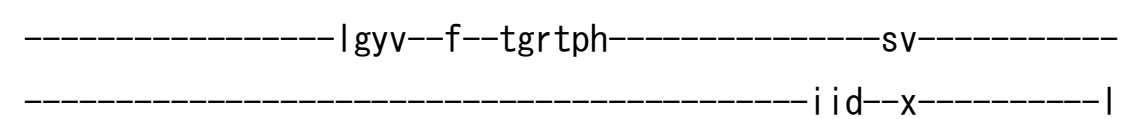

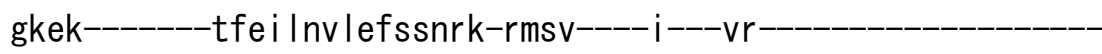

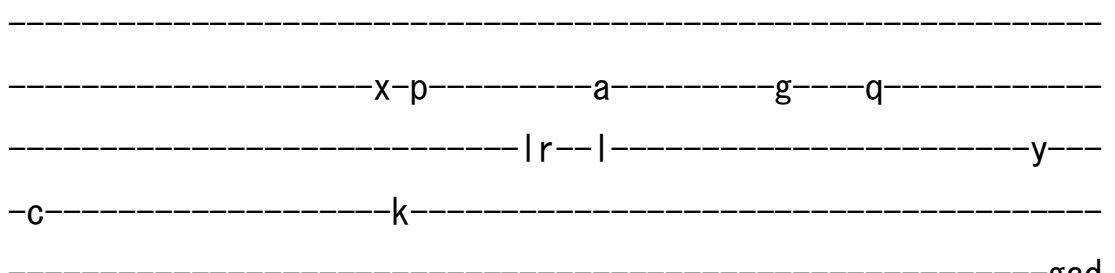

n---_--_-_-_-_-_-_-_-_--vifer Is-

$-x-$ 
xxx--xx--eqt-I chl eyf--ate----g | rtLCIAYAd I--sensyr ew I nvyne--as-----i I l kdrtqk l eecye i-i ekd I I ILGATA IEDRLQAGVPET I ATLMKae-IK I WILTGDKQETALNIGYSCRLi sqsms I i----

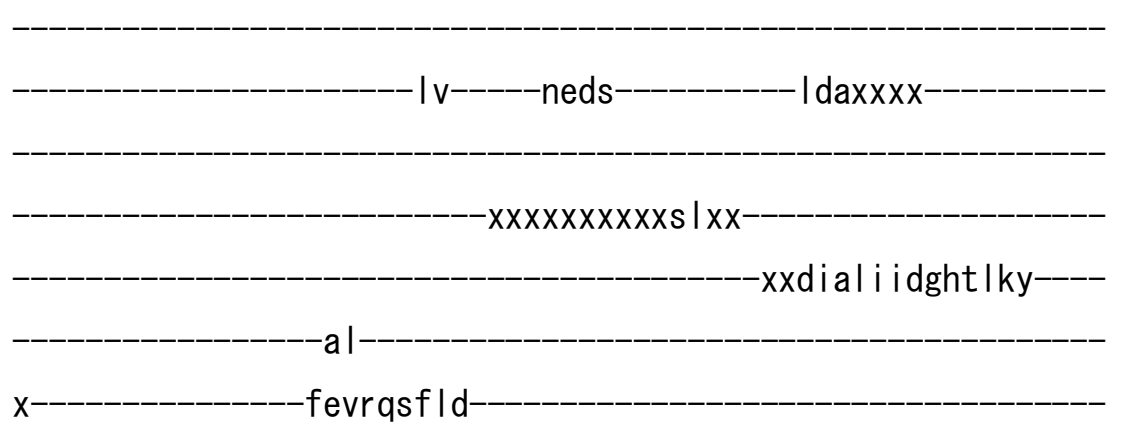

la l sckav ICC--RVSPLQKSE IVDMVk

---kh---v---_-------xa---_-_-_-----ITLAI GDGANDVGMI QTAHVGVGI--

-ATNCSDYAIAqfSYLEKLL--LVHGAWSYNRVTKC I------L

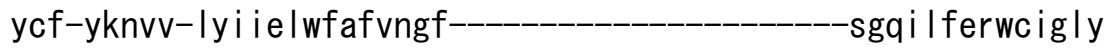
nviftalppft I g i fersctqd-_-_-_-_-_-_-_---sm I rfpqlyk i tanadgfntrv f-wghc inal ihs i i If-wfp|xvle-_-_-_-_-_-_-_-_-_-_xxxxxxxxg--_-_-_id--y I fvgn i vytyvvvtvc|k-ag lettawtrfsh |--_-_---avwgsm I I w I vffgv ysa iwptfxxxxxxx----------gqagmv I-

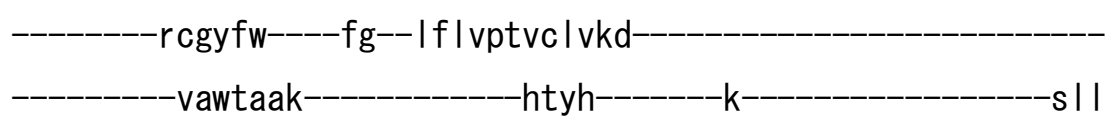

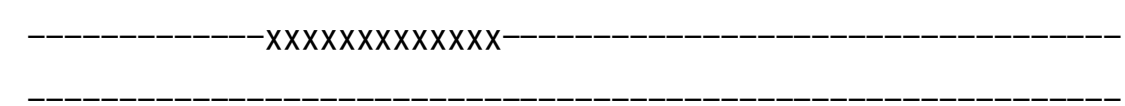

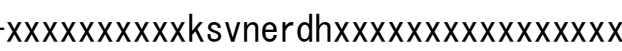
$\operatorname{xx} x \operatorname{xx} x \operatorname{xx} x \operatorname{xx} x \operatorname{xx} x \mathrm{x}$ 
>ani IV_IV01 EAA64843.1

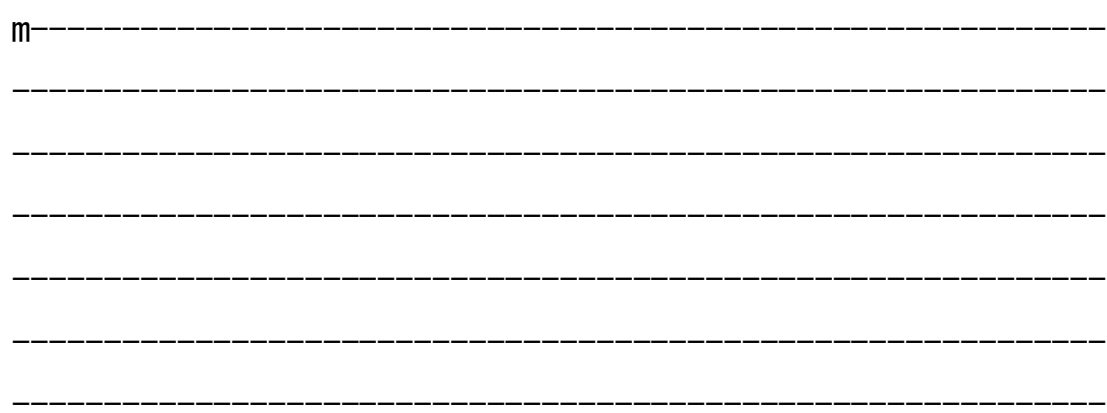

$-X X X X X X X X X X X X X X X X$

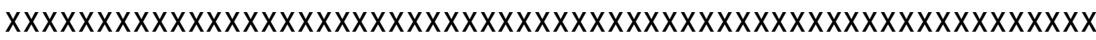

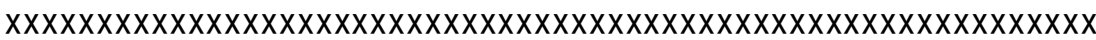

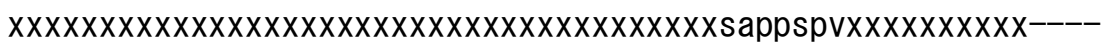
$-x x x x x x x x x x x x x x x x x x x x-$ -_-_-_-_-_-_-_-_-_-_-_-_-_-_-_-_-_-_-_-_-_-_-_-_-_-_-_-_-_-_-_-_-_

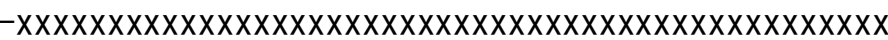
xxxkrkaaita

$-x x x x x x x x x x x x$

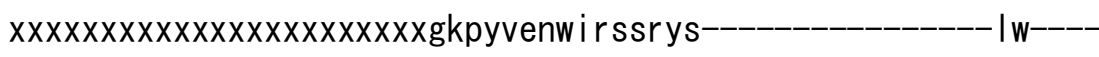
-sffprqffaqf 


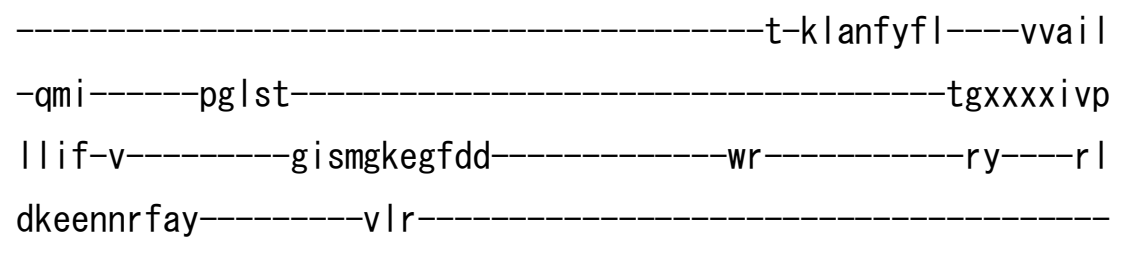

$-x x x x x x x x x x x$

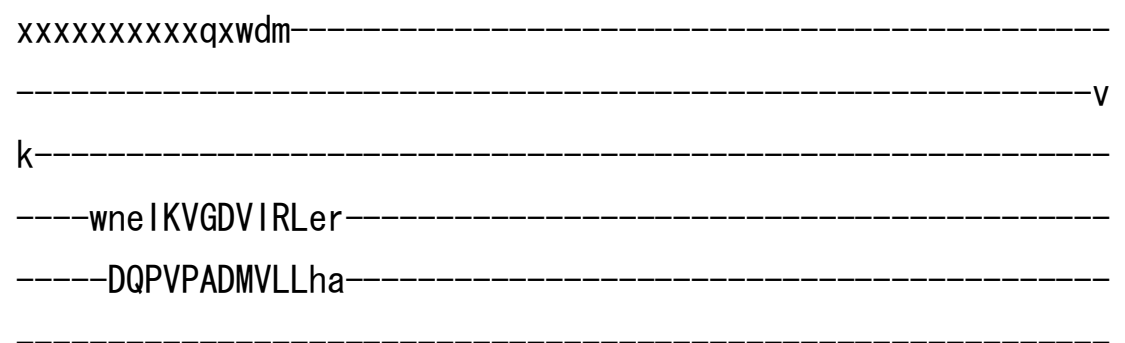

-dgpnc-t---ayVETMALDGETNLKTKqp-

-xhsvakvcgtvgg icsss ihfavedpn I l lykfdgnvtvx-

-agektp

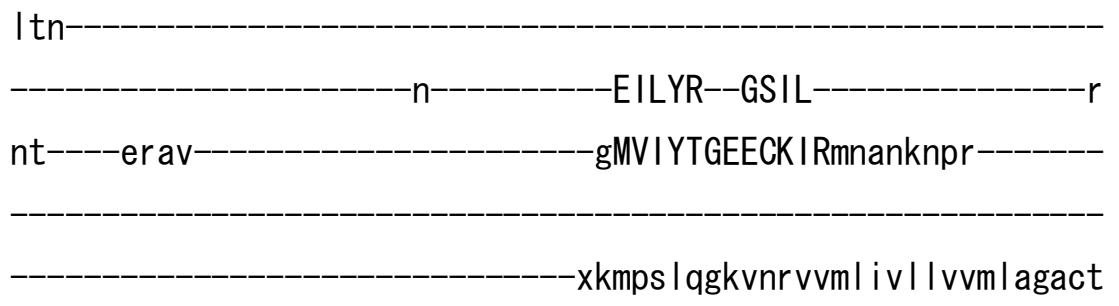

-i aykywshdvesha---_---wy l e---_-_-_-_-_-_--_danvsygpvftsf I I MFNTM

IPISLYVSmE IVKVAQML-MLnadidm-

-ydpesdtp

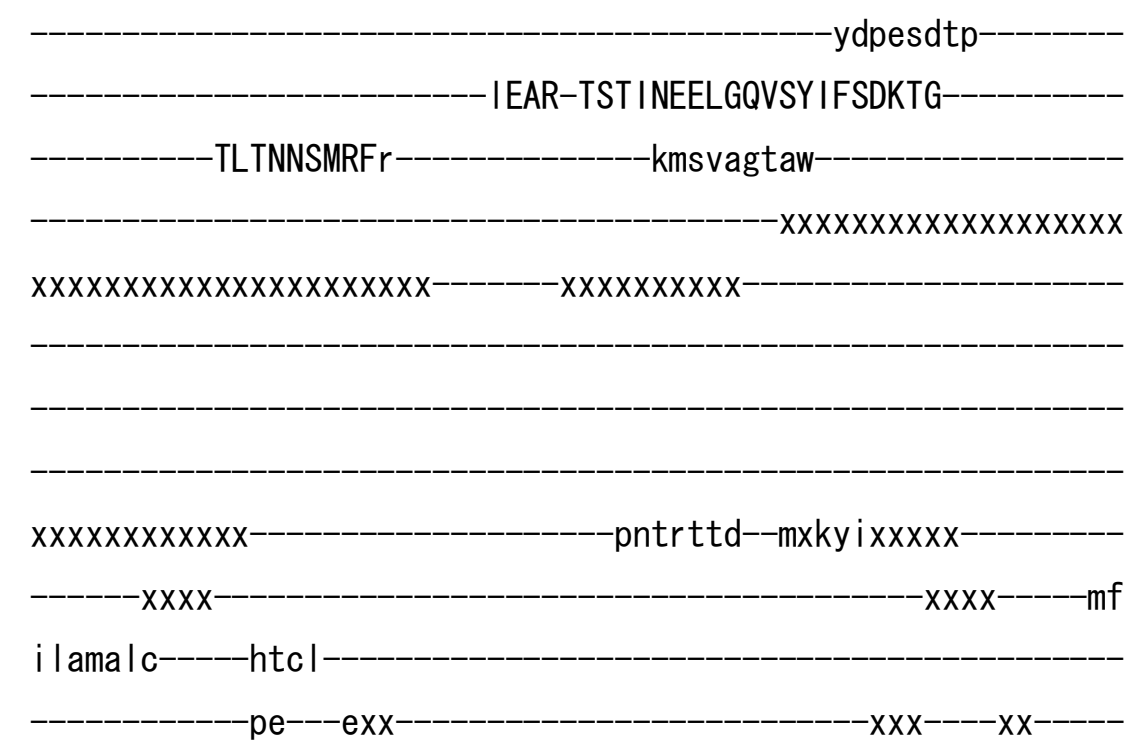



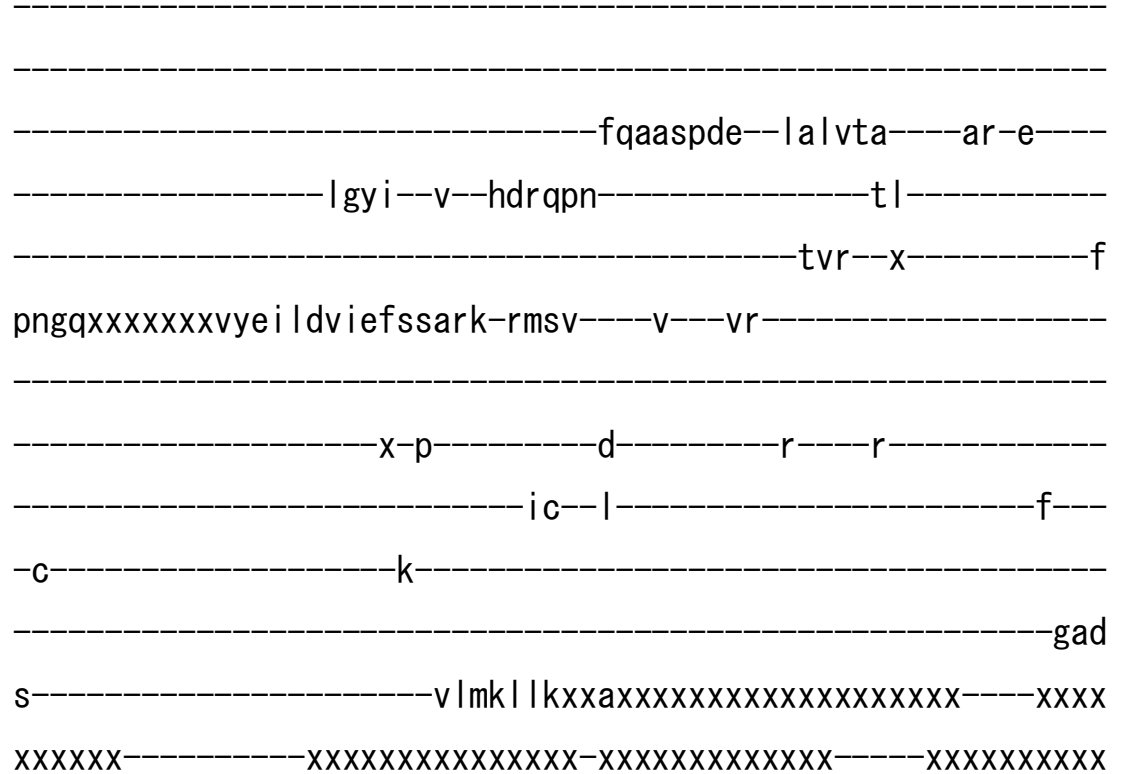

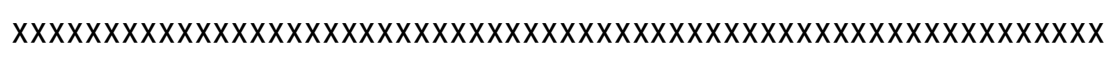
$x x x--x x--e r c-f q h|n d f--a t e----g| r$ tLLYAHRf I--deatyaewr cayqe--at-----ts I vnrqer i eqvgqq-i eqq I I TGATA IEDKLQKGVPET IDKLRRan-IKL WML TGDKRETAINVGHSCRL---_--_-_-_-_-_-_-_-_-_-_-_-_--vkdyss I v----

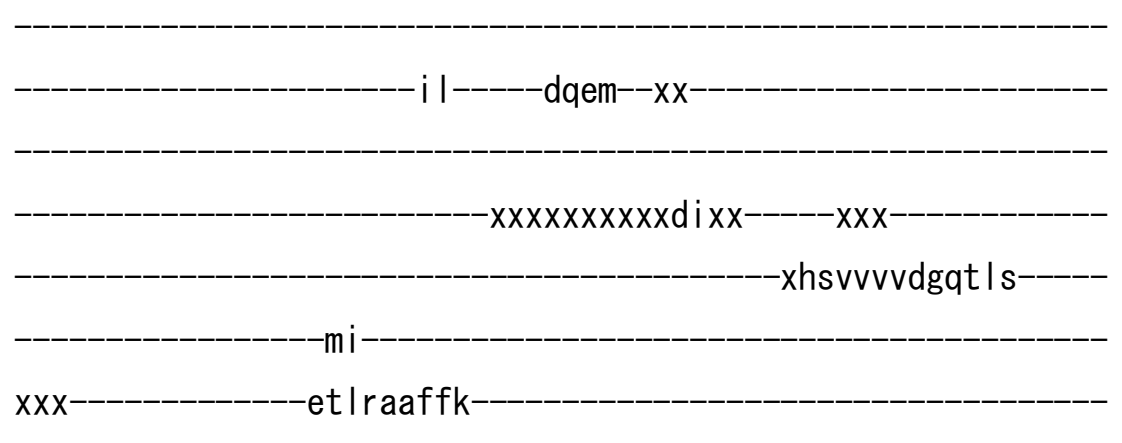

la i lvdsv ICC--RASPKQKAFLVKSIr

---rq--vq--_-_---_---xs---_-_-_-_-_-_-VTLA I GDGAND I AM I QEAHVG I G I--




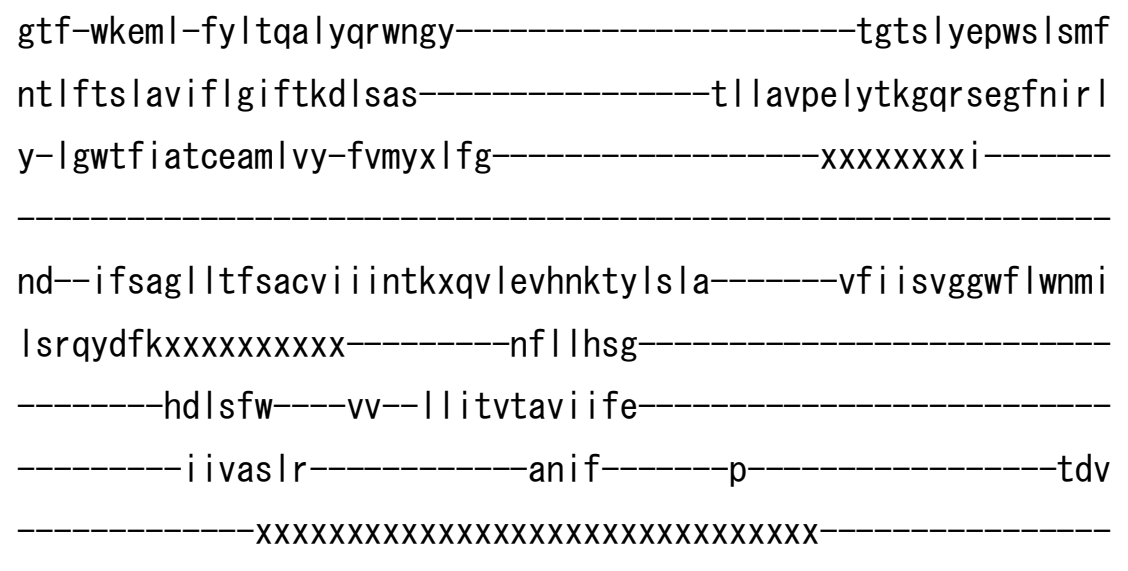

-xxxxxxxxxxeeaemear xxxxxxxxxxxxxxxx

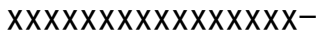

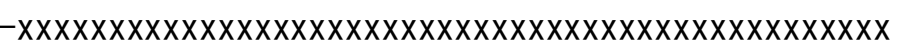
$x \times x \times x x$

$-$

>ggalV_IV04 XP_425888. 2

$\mathrm{m}-$ 


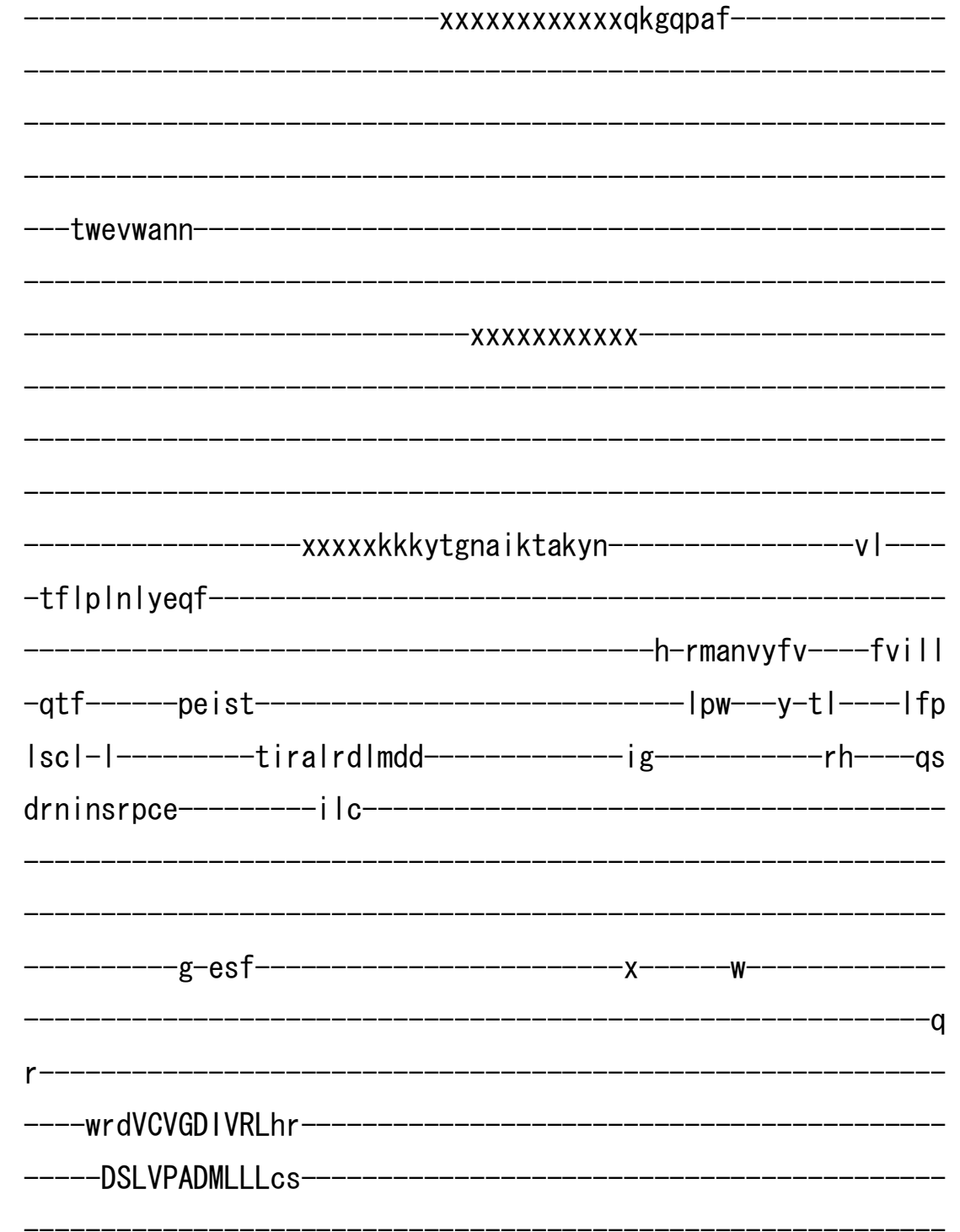

-sepss-I---cyVETSDIDGETNLKFRqa-

- I vthqe Itsegs I aafdgrvtceepnsrmhsftgv I qw--

-rgetyp

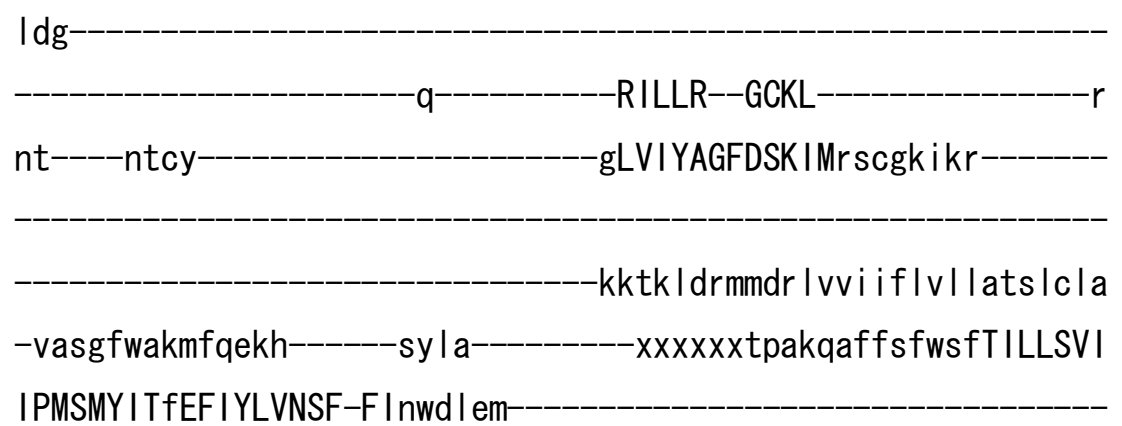


-yyavkdip

aKAR-STSLNDQLGQVEYIFSDKTG
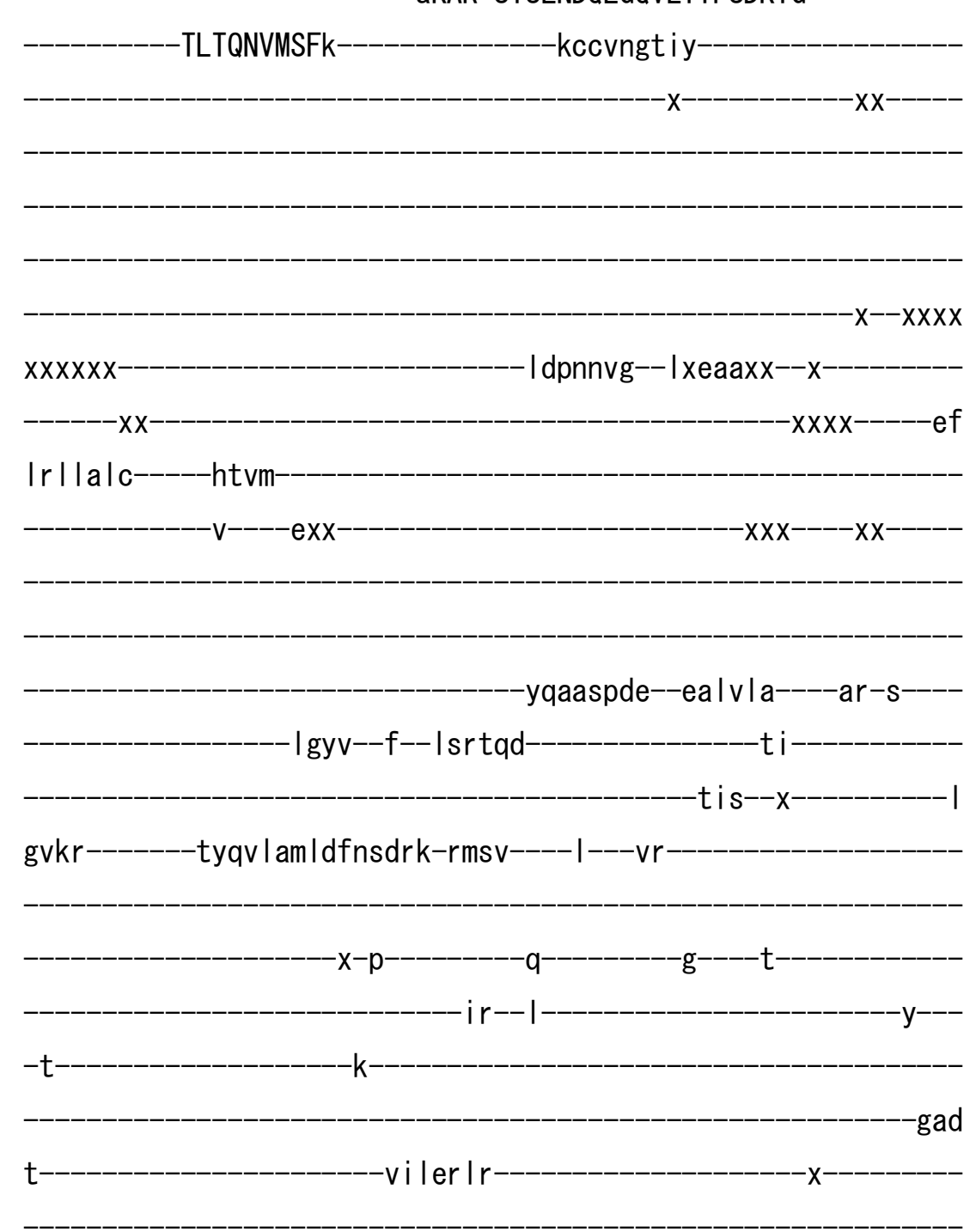

xxx--xx--dft-eral I If--aee-----t | rtLCLASKe I--seaeydewgr rhrv--an-----v I I qgrace I dr I yee-meqd I e ILGVTAIEDKLQEGVPET I QLLKLgn-IKV

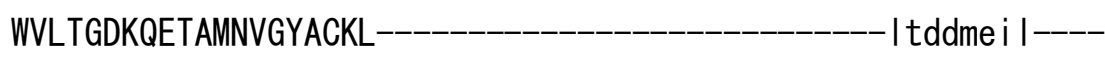

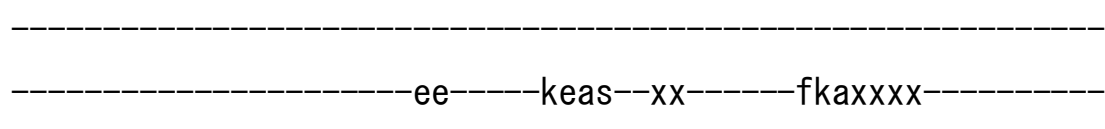

$x x x x x x x x x s q x x x \operatorname{exx} x$

$-x x k r a l v i s g d f$ Ivrxxxx

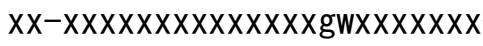
$-x x x x x x x x x x x x x x x x x-$ $x$

slvekafvd- 
latscqav ICC--RFTPRQKAL IVQLVk

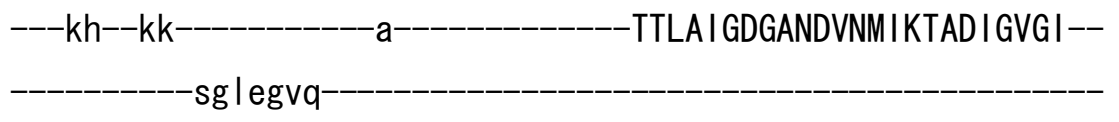

-AVQCSDYALAr fCYLQRLL--L I HGRWGYLRICKFL------R

yff-yktfa-g I l tqvwfafhngf-_-_-_-_-_-_-_-_-_-_-_--_taqp I yegwf Ia l y nvfytaypv I smg I l eqdvsak-_---_-_-_--_-_---ks I rfpe lyt i gqqdq I fnyr $\mathrm{i}$

f-svt I lhgvsts Itsf-yial xafe---------_------_----xxxxxxxv--------

gd--yesfsvtvatsal | sv Ime-i i Idtkfwtalsf |---_---mvtas | | |fc|fsf |

tqs i dafr xxxxxxxxx-----------datanal-----------------------------

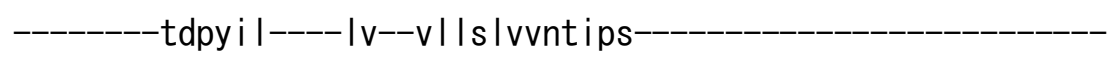

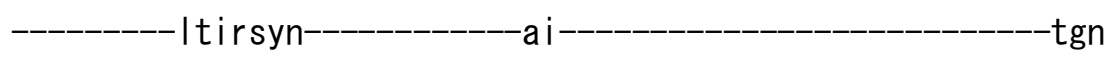

$-x x x x x x x x x x x x x x x x x x x x x x x x$

$-x x x x x x x x x x$ thiprgsfxxxxxxxxxxxxxxxxx

xxxxxxxxxxxxxxxx---_-_-_-_-_-_-_-_-_-_-_-_-_-_-_-_-_-_-_-_-

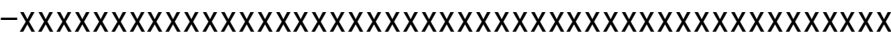

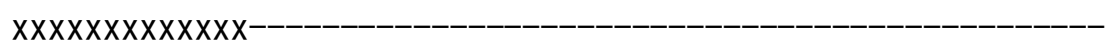

$-$

>ggalV_IV05 XP_429208. 2

$\mathrm{m}-$ 
xxxxxxxxkkklkee-

---erhlqann

$-x x x x x$

qfeyasnsiktskyn

$\mathrm{ff}$

-tfiplnIfeqf

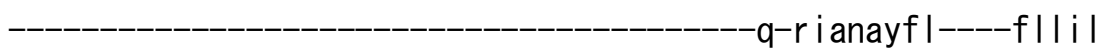

-ql i-------pqi ss------------------------------- law---f-tt-----vvp

Ivlv-I--_-_-_--avsgvkda i dd-_-_-_-_-_-_fn--_-_-_-_---rh----ks

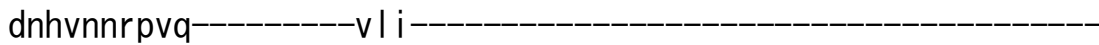

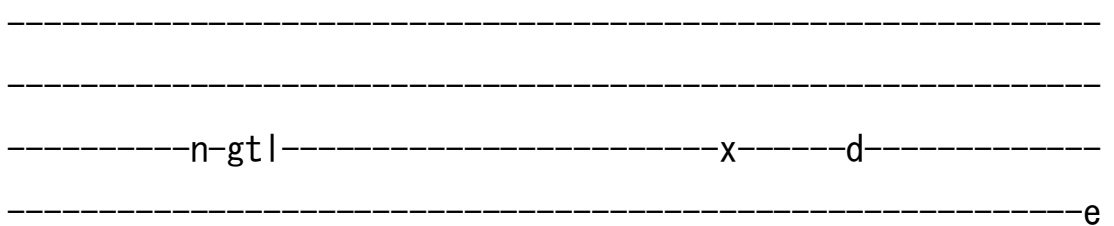

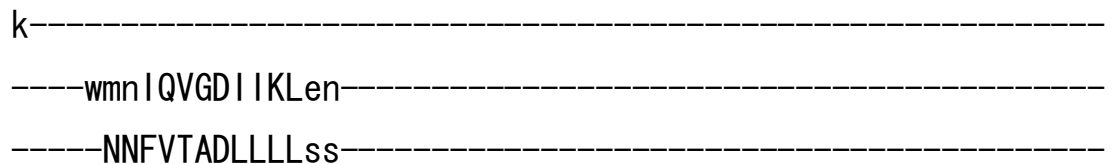


-sephs-I---vy IETAELDGETNLKVKqa-

-xtvtae I ged lqk I tdfngef i ceapnnk I dnftgt lal--

rgekya

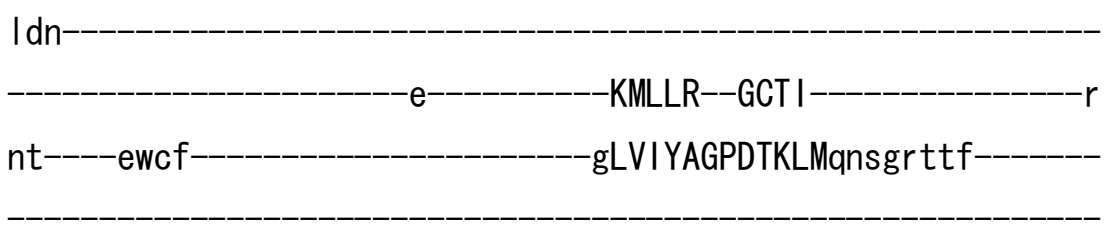

$-k r t s i d r$ Imnv I I Imvmmc I i I shwq ly

- I gnl i-----kgyhf------xvy |p--_-_-----xxxxxxsapfsaf ImfwsyVI ILNTV

VPISLYVSVEI IRLGNSF-YI dwdrkm-

$-y y p$ Indtp

aQAR-TTTLNEELGQIKYIFSDKTG

TLTQN IMCFn

-kcsingksy

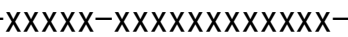

$-x x x x x--x x x x$

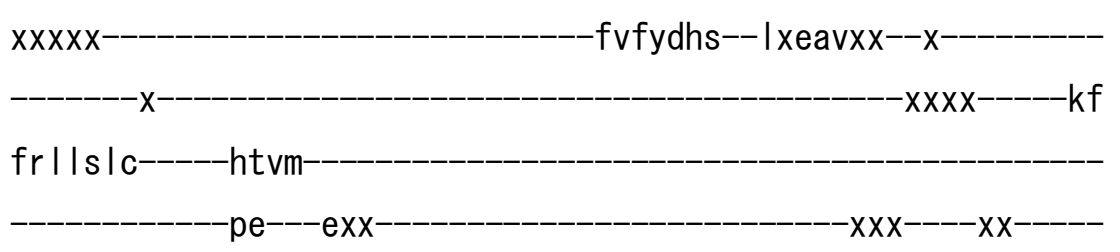

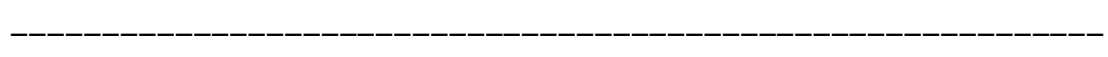
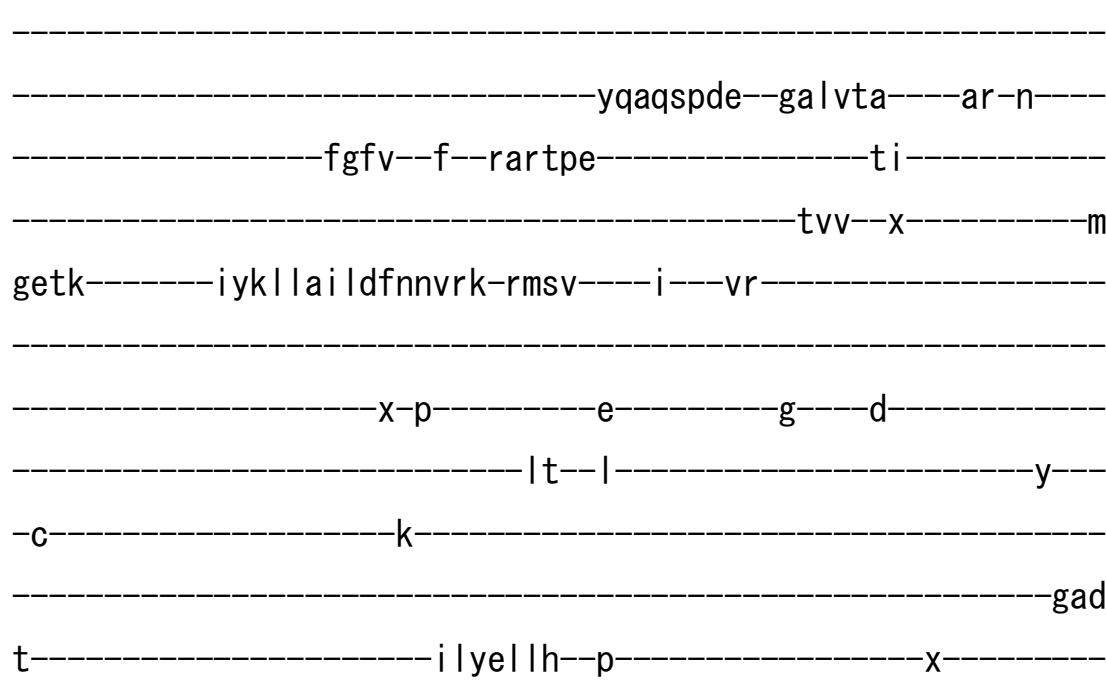
xxx--xx--eet-teh Inef--age----g|rtLVVAYKnI--deeyfadwikrhhe--as-----ta l egr edk I se i yee-i ekd Im I LGATA IEDKLQDGVPQT IETLGKas-IK I WVLTGDKQETAMN I GYSCNL- yddmadv----

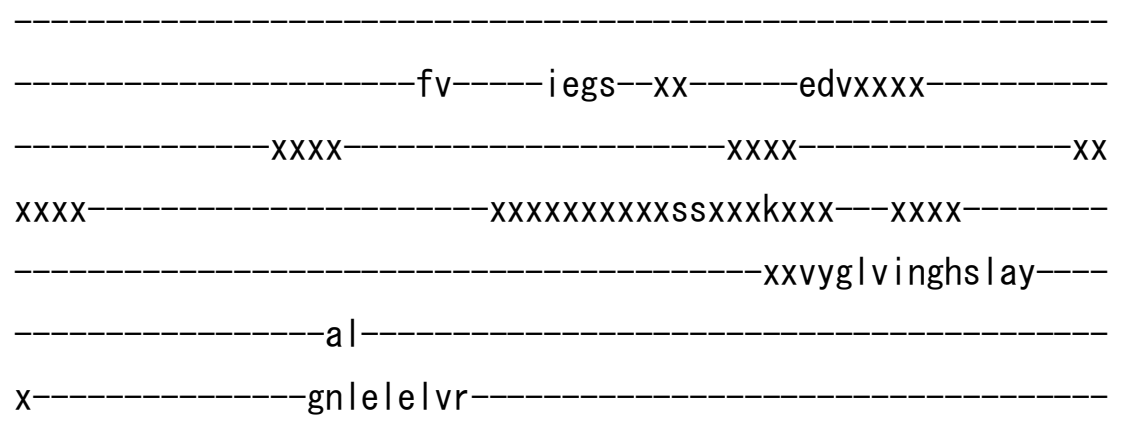

tacmckVvICC--RVTPLQKAQVVELVk----ky--kk-----------a----_--_---VTLAI GDGANDVSM I KTAHI GVGI--

-AVLSSDFSFAqfRYLQRLL--LVHGRWSYIRMCKFL------K yff-yknfa-ft I vhfwygffsgf-_-_-_-_-_-__-_-_-_-_-_saqtvydqwfit ly n Imyts I pv I gms I fdqdvddr-_-_-_-_-_-_-_-_-ws I I fpq l yvpgqqn I y fnk i v f-ikcm l qg i yss I i If-fi i pyxamy-_-_-_-_-_-_-_-_-_-_xxxxxxxxxi---_-_-ad--yqsfa Imaqtc I I ivvsvq-ig Idtsywtvvnqf-----_--fiwgs I svyfaitft

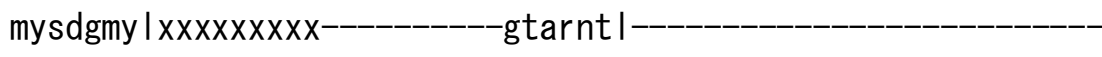

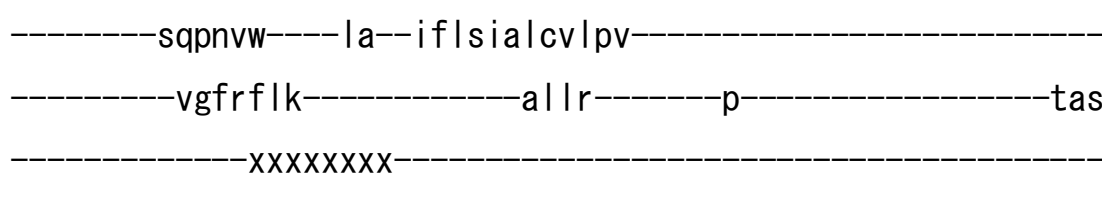
xxxxxxxxxxxkrr|rrtsxxxxxxxxxxxxxxxx $\operatorname{xx} x \operatorname{xx} x \operatorname{xx} x x x$ 
>ani IV_IV02 EAA58087. 1

m-

$-x x x x$

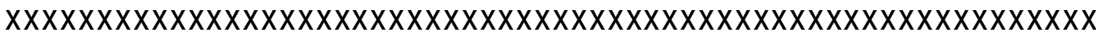

$\mathrm{X}$

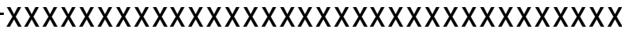

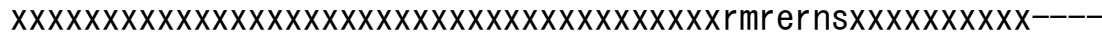

$-x x x x x x x x x x x x x x x x x x x x-$

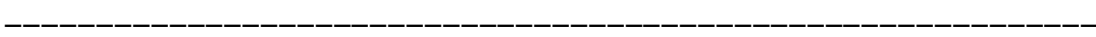

$x x x x x x x x x$

---dstvsedg

$x x x x x x x x x x x-$

$-\mathrm{XXXXXX}$

xxxxxxxxxxxxxxxxxxxxxxxhkfvdnhvstakyn----------------iv----

-tflpkflyeqf 


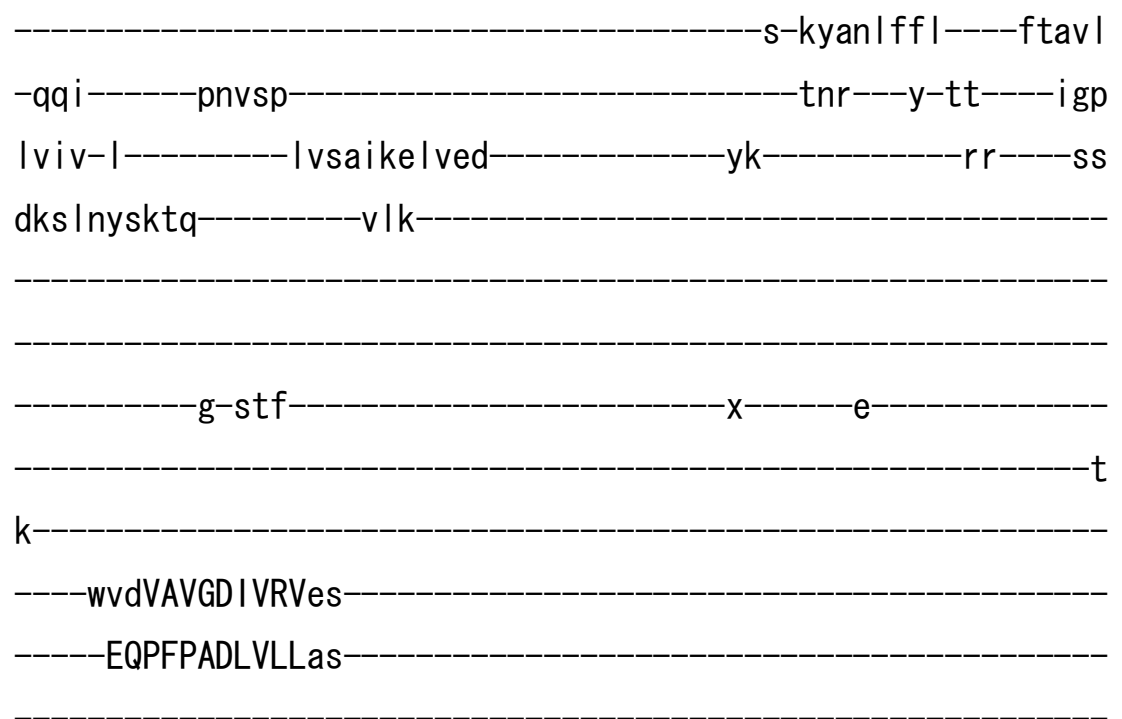

-sepeg-I---cy IETANLDGETNLKIKqa-

-ipetsh IvspadIsr Isgrirseqpnss I ytyeat I tmxx

$x x$

gerelp

lap

$d-$

- QLMLR--GATL

nt----pwih-

gVVVFTGHETKLMrnatatp i

-krtavermvniqi ImIvsi Ival svvss

-vgd I i i rqtekdk I-------ty Id------------xxxxnpgkqf i md i f tyWVLYSNL

VPISLFVT IEIVKYSQAF-LInsdIdi

$-y y d v t d t p$

aTCR-TSSLVEELGQIEYIFSDKTG

TLTCNMMEFk

ect igg i qy

XX

$-x x x x x--x x x x$

$x x x x x x$

vgvhdfk--kxrqnxx--x

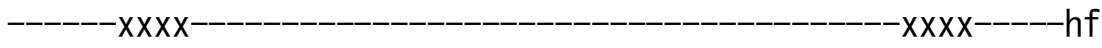

It | |atc-----htvi

pexx-exx-

$-\mathrm{XXX}-----\mathrm{XX}-$ 


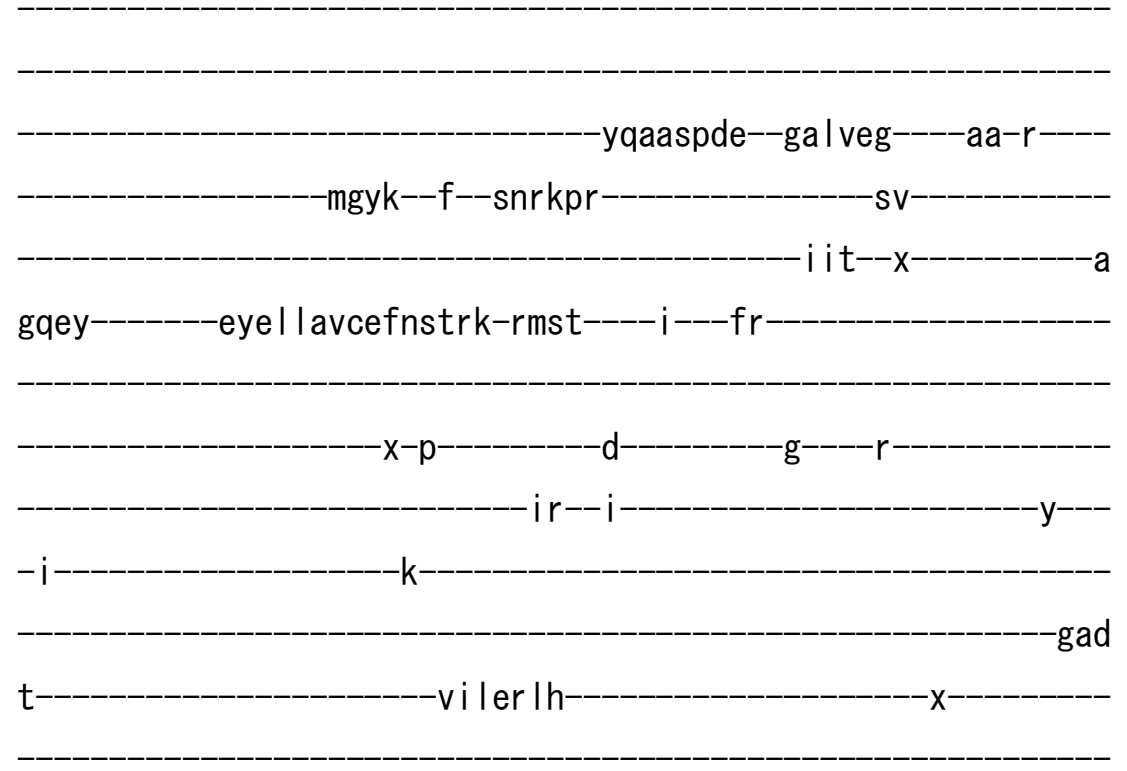

xxx--xx--egt-| qh l eey--asd----g | r tLCLAMRe i--pedefqqwyq i fdk--aa----xtvggnraee I dkaae I-i ekdfy ILGATAKEDRLQDGVPDT IHTLQTag-IK I

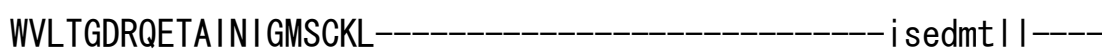

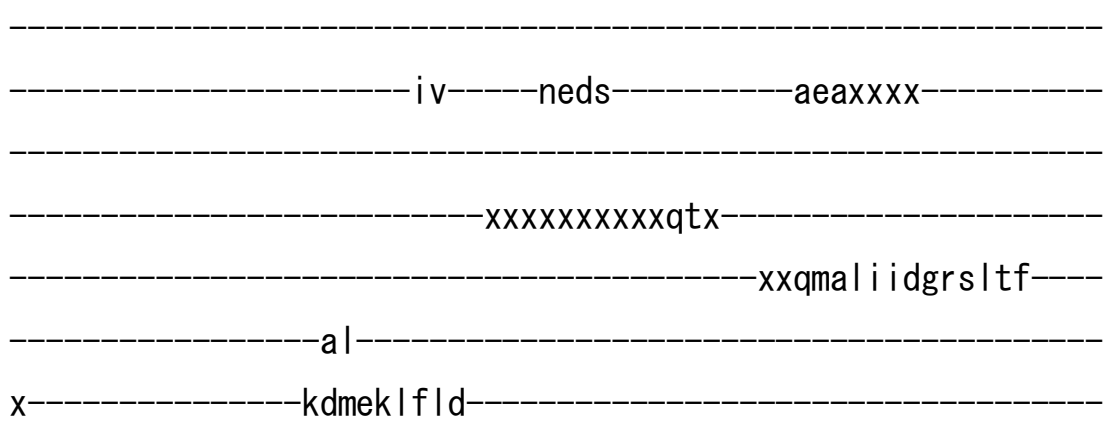

I av I ckavVCCxxRVSPLQKALVVKLVk

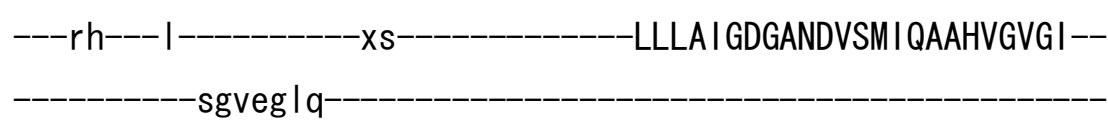

-AARSADVSI AqfRYLRKLL--LVHGAWSYHRISRVI------L 


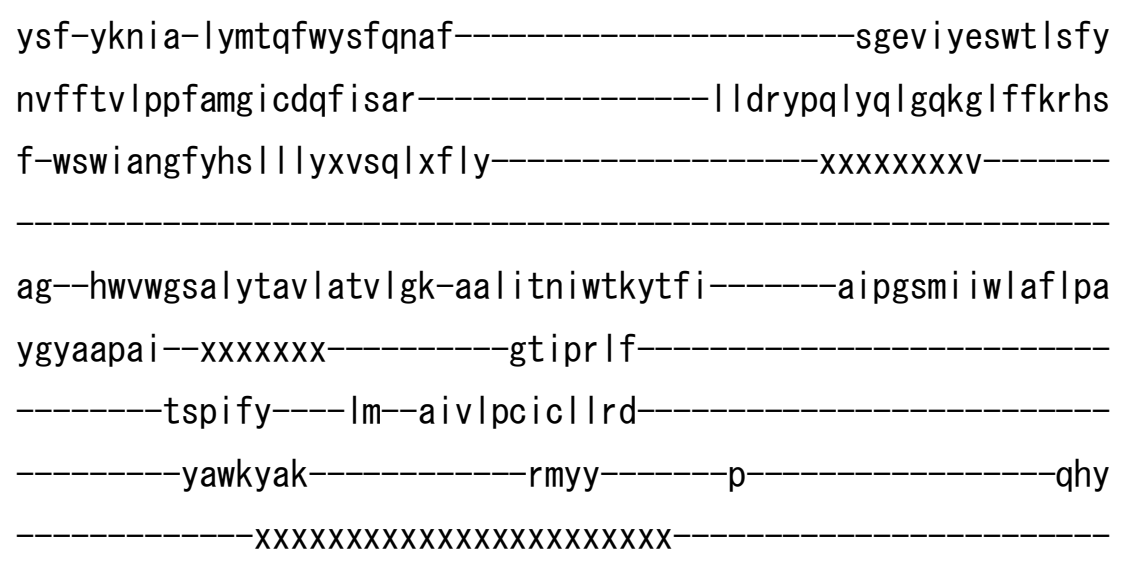

-xxxxxxxxxxmrkqrgyaxxxxxxxxxxxxxxxxx

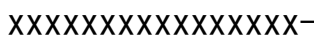

$-x x x-$

>ani IV_IV03 EAA58143. 1

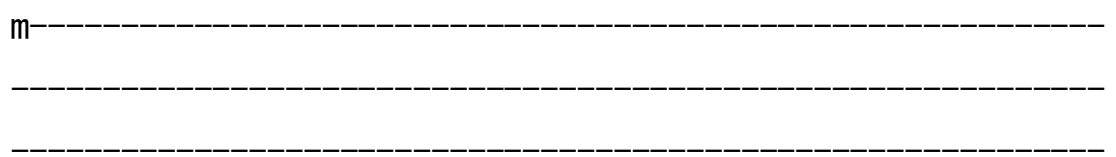




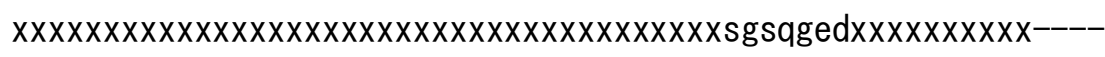

$-x x x x x x x x x x x x x x x x x x x x x x x-$

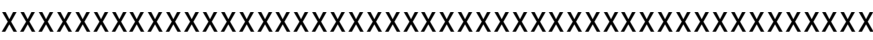

$\mathrm{xxxprdv|vgq}$

-xxrakyppnivsnakyt

-pw----

-sflprtlynef

s-fffniyfl-----|vals

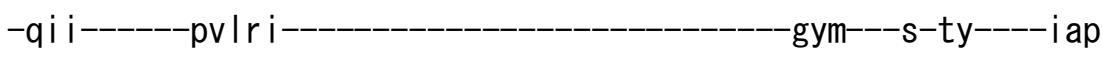

lafv-v-_-_-_-_--s i s lgkealdd-_-_-_-_-_-_-_vg-_-_-_-_----rr-----rr

daeanaeefs---------vla

$-x x x x x x x x x$

$x x x x x x x x x x v x v i \mathrm{i}$

$-k$

k

----srdLKVGDVLKVrk

-NQRLPADVVILks $x x x x x x x x x x x x x x x x x x x x x x x x x$

$-x x x x x x x x x x x x x x x x x x x t d i s s x s^{--x t f I R T D Q L D G E T D W K L R I p-~}$

svIsqal s ledftr lkitasgpdkrvnefvgni el xx $x x x x x x x x x x x x x x x x x x x x x x x x x$

-qnssap

$\mathrm{ti}$

-NTAWA--NTVL-

$-\mathrm{d}$ xaAIIYTGSQTRAAIstspsrs

-kvgl leyeinnItkilcaltItIsi ilv

-al egfaptndke-wyva-imiyLILFSTI

IPMSLRVNIDMAKSVYGR-F I erdkdi 
$-p d$

-tVVR-TSTIPEDLGRIEYLLSDKTG-

-TLTQNEMELk-

-kihvgtvsy-

$-x x x x x x x x x x x x x x$

$\mathrm{xx}$

$---x x x x x x x x x x x x x x x x x x x--x x x x x x x x i g s r-v r$

$-\mathrm{di}$

vlalalc-----hnvt-

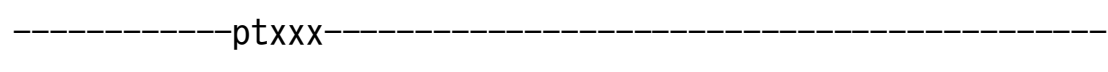

$-x x x x x x x$

---x-----------------------------yqasspde---i a i vry-----te-e----

$-v g \mid k--v--a y r d r$ r----------------ti------------

$\mathrm{vl}$

estdxxxxx-xrar i ldifpftsdsk-rmgi----i----ve-

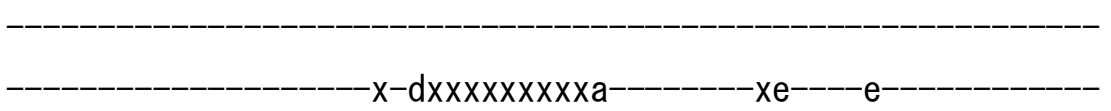

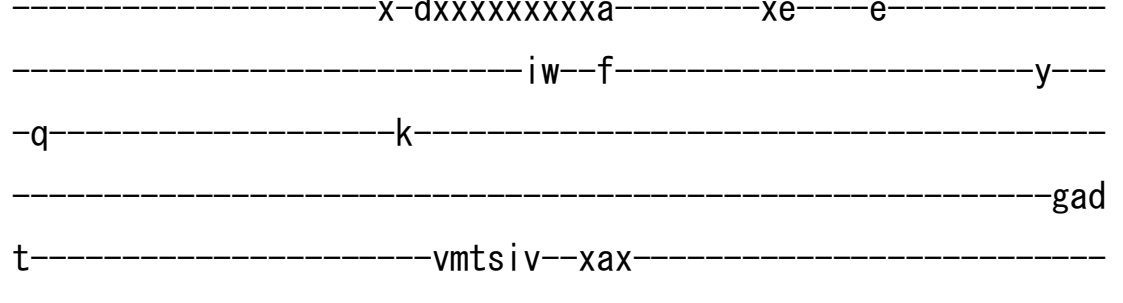

-dw I-deetanm--are----g|rtLVVGRKr I--sp l qyqefaskykq--as------ I s agrd i gmqkvvnex I ehd I e ILGVTGVEDRLQRDVKKPSLELLRNag-VK I WMLTGDKVETARCVAISAKL-_-_-_-_-_-_-_-_-_-_-_-_-_-_----vargqy ih---_ -_-_-_-_-_-_-_-_-_-_-_-_-_-_-_-_-_-_-_tv-_-_-_-_akvk-_-_-_-_-_-_dks-_-_-_-_-_-_-_-_-_

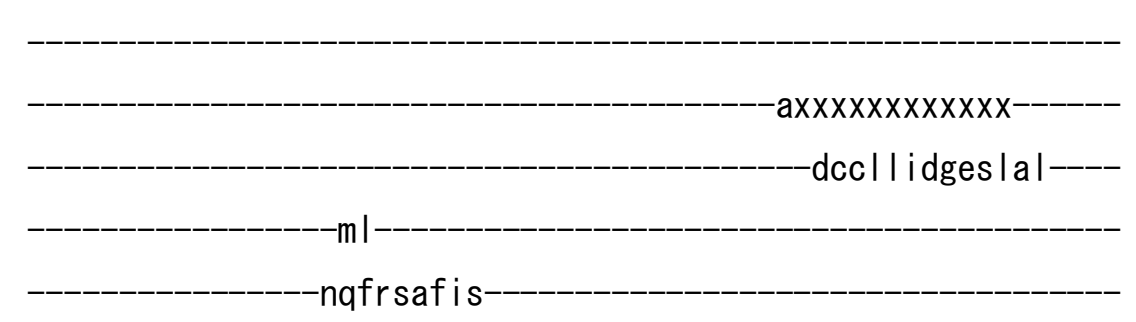


vav I I pav IAC--RCSPTQKAEVADL I r

---Ih--tk-----------x------_---_---RVCC I GDGGNDVSM I QAADVG I GI I--

-ASLAADFSI TqfHHI TKLL--VWHGRNSYKRSAKLA------Q

fim-hrg I i-i sacqtmys i ashf-_-_-_-_-_-_-_-_-_-_-_-_-_pkg I f i nw Imvgy atvytnapvfs I-v Idrdvdeq---_-_-_-_-_-_--_an lype lyke Iktgrs I syrs

f-ftwv I vsvyqgaviqx Isqi-IId-_-_-_-_-_-_-_-_-_-_-_-_-_-_-_-_-_-

is--gkr I i svsftal vmne I Im-va iavttwhpvmi f--_-_---cl I gtal vyaasvpf

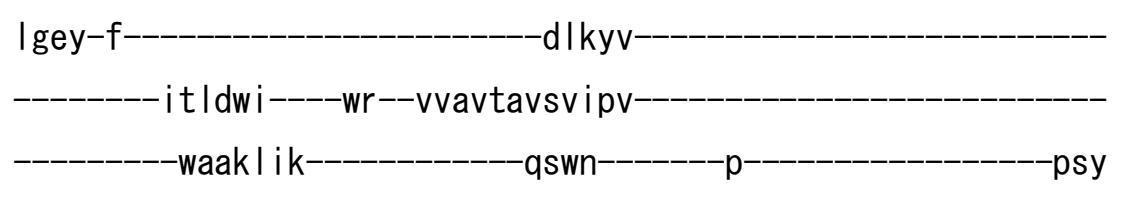

rkvrg

>ggalV_IV06 XP_417508. 2

$\mathrm{m}-$ 


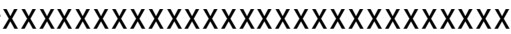

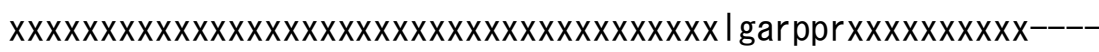
$-x x x x x x x x x x x x x x x x x x x x^{--}$

---prtvw Igh

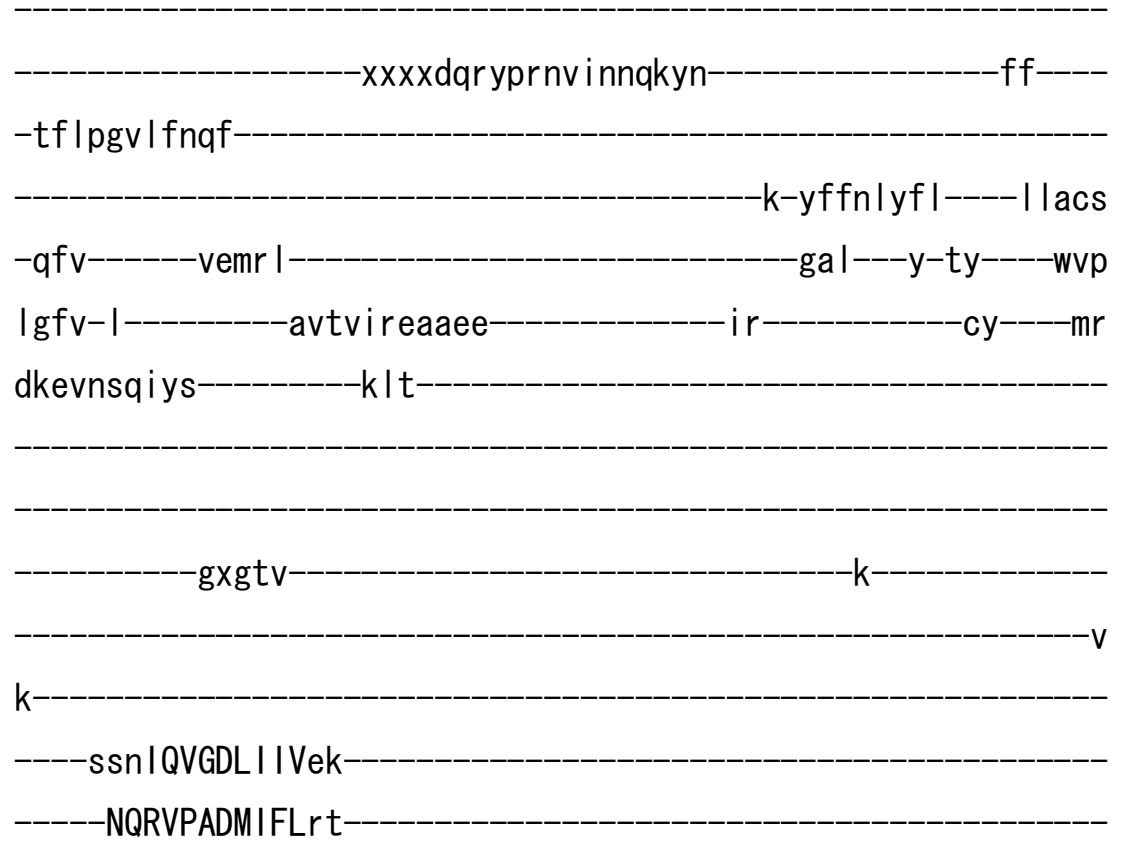


-sekng-s---cfLRTDQLDGETDWKLRIp-

-vtctar Iptasd I I qi r syvyaeepni dihnfvgtftrxx

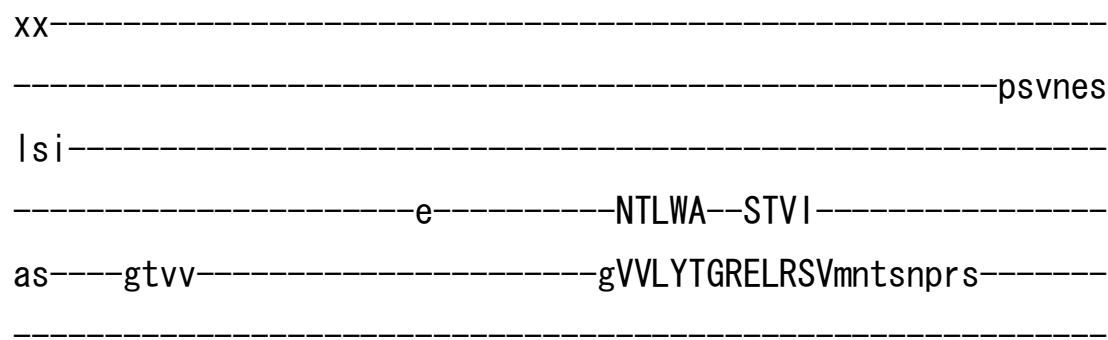

-kiglfdlevncltkilfgalvvvs Ivmv -a lqhfagr--_-_-_-_-_--wy lq--_-_-_-_-_-_-_-_-_-_-_i i r fLLLFSN I

IPISLRVNIDMGKIVYSW-VIrrdski

$-p g$

-tVVR-SSTIPEQLGRISYLLTDKTG

-TLTQNEMVFk--------------rlh I gtvay

$-x x x x x x x x x x x x x x$

$-x X------X X X$

$\mathrm{xx}----------x x--x x x x x x x--x x x x x x x x m s s r-v h-$

ea

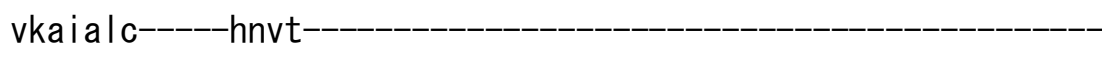
pvx $x x$

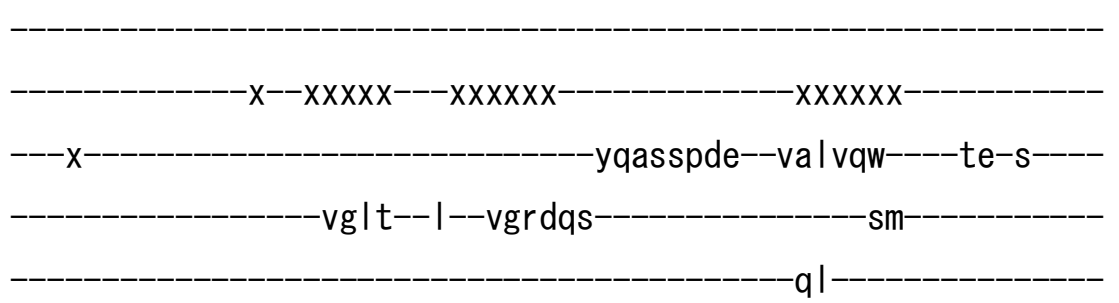

rtpg-xxxx--nft i lqi fpftyesk-rmgi-----i---vr---------------------

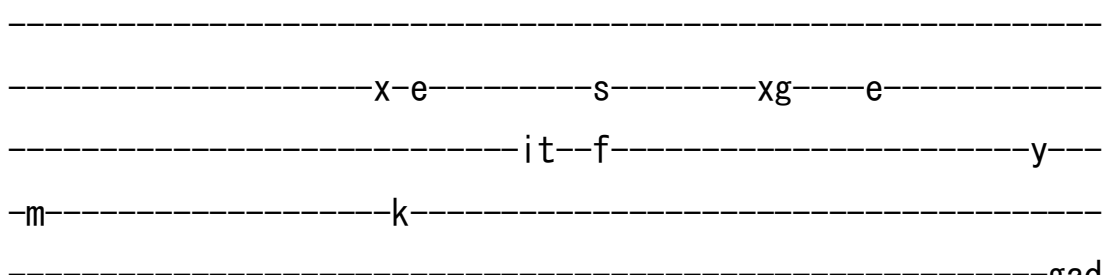

-gad

v------------------------vmag i v-- xyx 
-dw I-eeecgnm--are----g|rvLVVAKKs |--teeqyqdfearyvq--ak----- I svhdr s I kvatv i es-I ememe ILCLTGVEDQLQADVRPTLETLRNag-IKV WMLTGDKLETATCTAKNAHL-vtrtqdih----

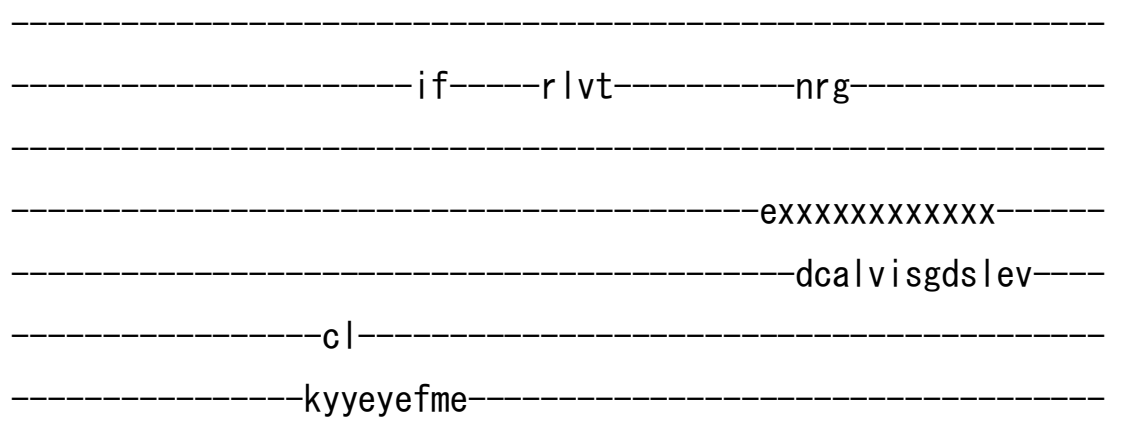

I acqcpavVCC--RCAPTQKAQIVRLLq ---er--tg-----------x-----_--_----_LTCAVGDGGNDVSM I QEADCGVGV--

-ASLAADFSI TqfKHLGRLL--MVHGRNSYKRSAALS------Q

fvi-hrslc-istmqavfssvfyf -asvp I yqgf I i igy stiytmfpvfsI-vldkdvkse-vam lype lykd I I kgrp I sykt

$f-I$ iwv l is iyqgs i im-ygal-IIf $-\mathrm{e}-------$ se--fvhivaisftsliltel Im-val tiqtwhw Imi v--_-_---ael| s I scy ias I vf Ihef-i dvyfi -at |sfl----wk--vtvit|vsc|p|$-y v|k y| r------------r r f s$ $-p s y$ -skl---ts 
>mgrIV_IV01 XP_369133. 1

m

Xxxxxxxxxxxxxxxxxxxxxxxxxxxxaprqvga

$-x x x x x x x x x$

---irfagsqd

$x x x x x x x x x x x-$

xghgyl snairtsryt

$-1 \mathrm{y}$

-dffpkqvwfqf 


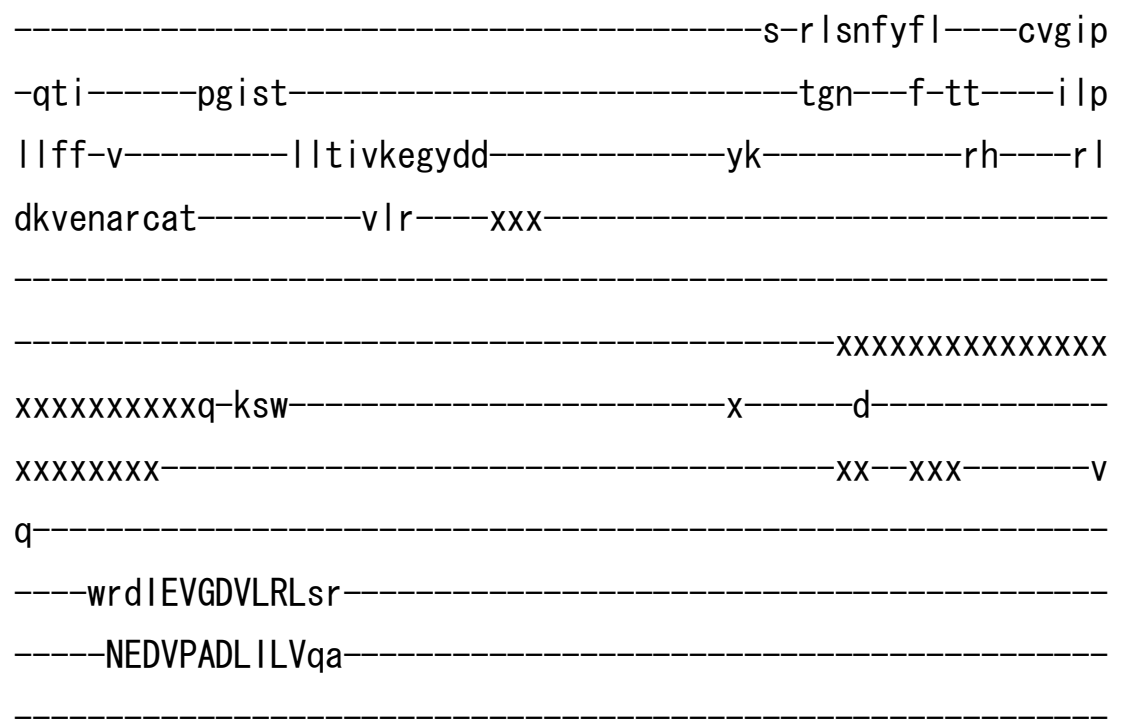

enedh----xayVETMALDGETNLKSKsv-

-panI tk las isdivqsg I e i vaedpnpd I hnfdgr I ti--

agdtap

$\mathrm{ti}$

n-----------EVLYR--GCTL----------------r

nt-----paav------------------------gVVINTGEECK IRmnanrhpk--------

-xkkpalekttnrivlt I vayvfsctagcs

- i gy I I war stenss-------wy I r----------------gagvavqe i vvgyA IHFNN I

IPLSLYVTIELTKLFQML-MLngdi em-

yheasdtp

aKCN-TNT ILENLGQVGYVFSDKTG

TLTENVMQFr

kms i ag i sw-

$\mathrm{XXXXXX}$

$\mathrm{XXXXXXXX}$

$X X X X X X X X X X X X X X X X X X X X X X X X X X X X X X X X X X X X X X X X X X X$

$-x X X X X--X X X X$

$x x x x x x x x x x x x x x x x x x x x x x x x x x x x x x x x x x p g \mid$ ssad-- $\mid$ xey $i x x x x x$

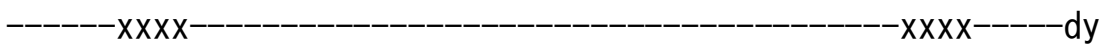

| |a|a|c-----htc|

- pe---yxx

$-\mathrm{xx}------\mathrm{xx}----$ 


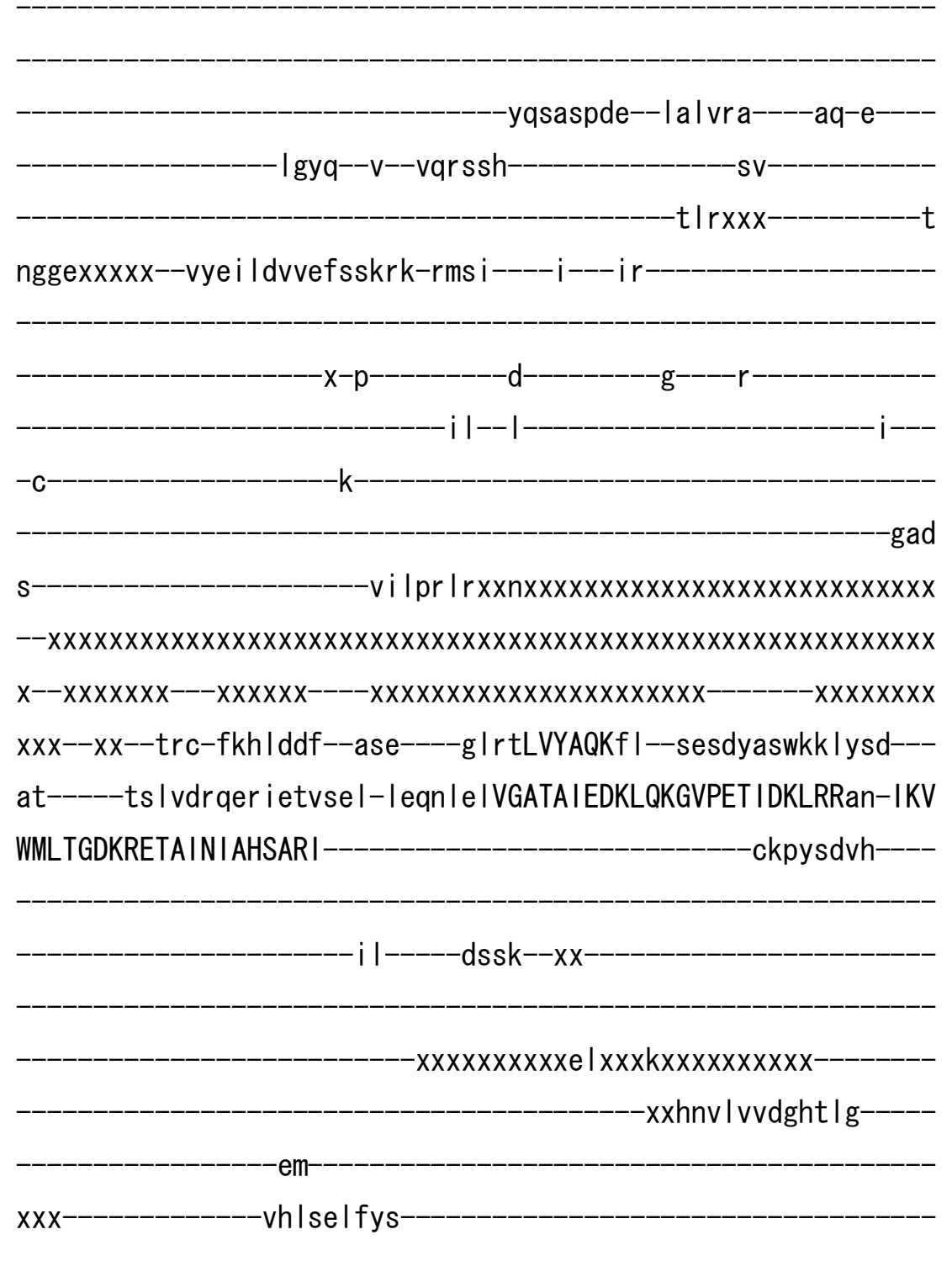

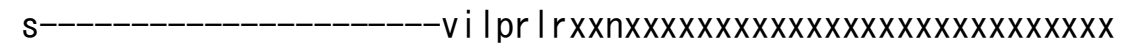

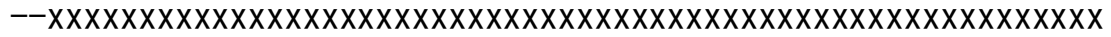

$x x x--x x--$ trc-fkhl ddf--ase-----g|rtLVYAQKf I--sesdyaswkk I ysd---

at-----ts I vdrqer i etvse I-I eqn I e IVGATA IEDKLQKGVPET IDKLRRan-IKV

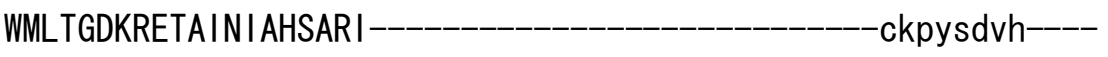

i l-----dssk--xx

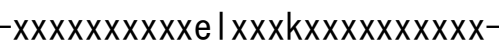

$-x x h n v|v v d g h t| g$

xxx-------------vhlselfys

I apvvdsv ICC--RASPAQKALLVRAVr

---qk-- $\mathrm{i} \mathrm{i}-----------x s x x x x x x x x x x x x x$ LTLSIGDGGNDLAMLAEAHVGVGI-sgreglq-

-AARVADYSI AqfRFLARLL--LVHGRWNYSRTARFV------L 


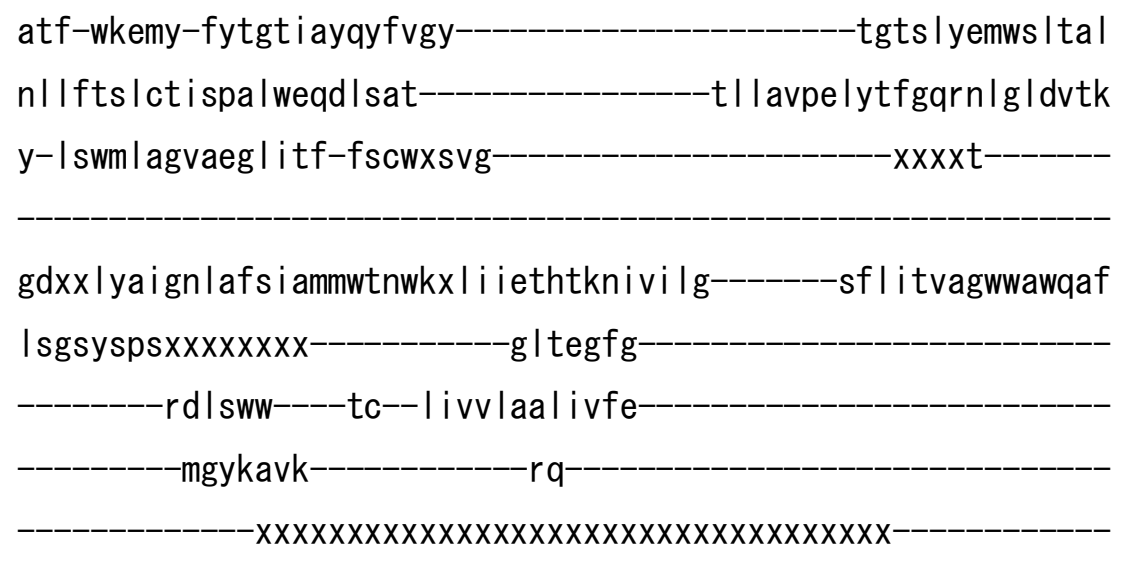

$-x x x x x x x x x x a q a d d \mid r r x x x x x x x x x x x x x x x x x$

\section{$x X X X X X-$}

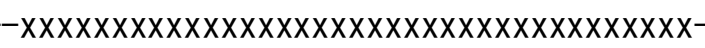

>ani IV_IV04 EAA60094. 1

m- 


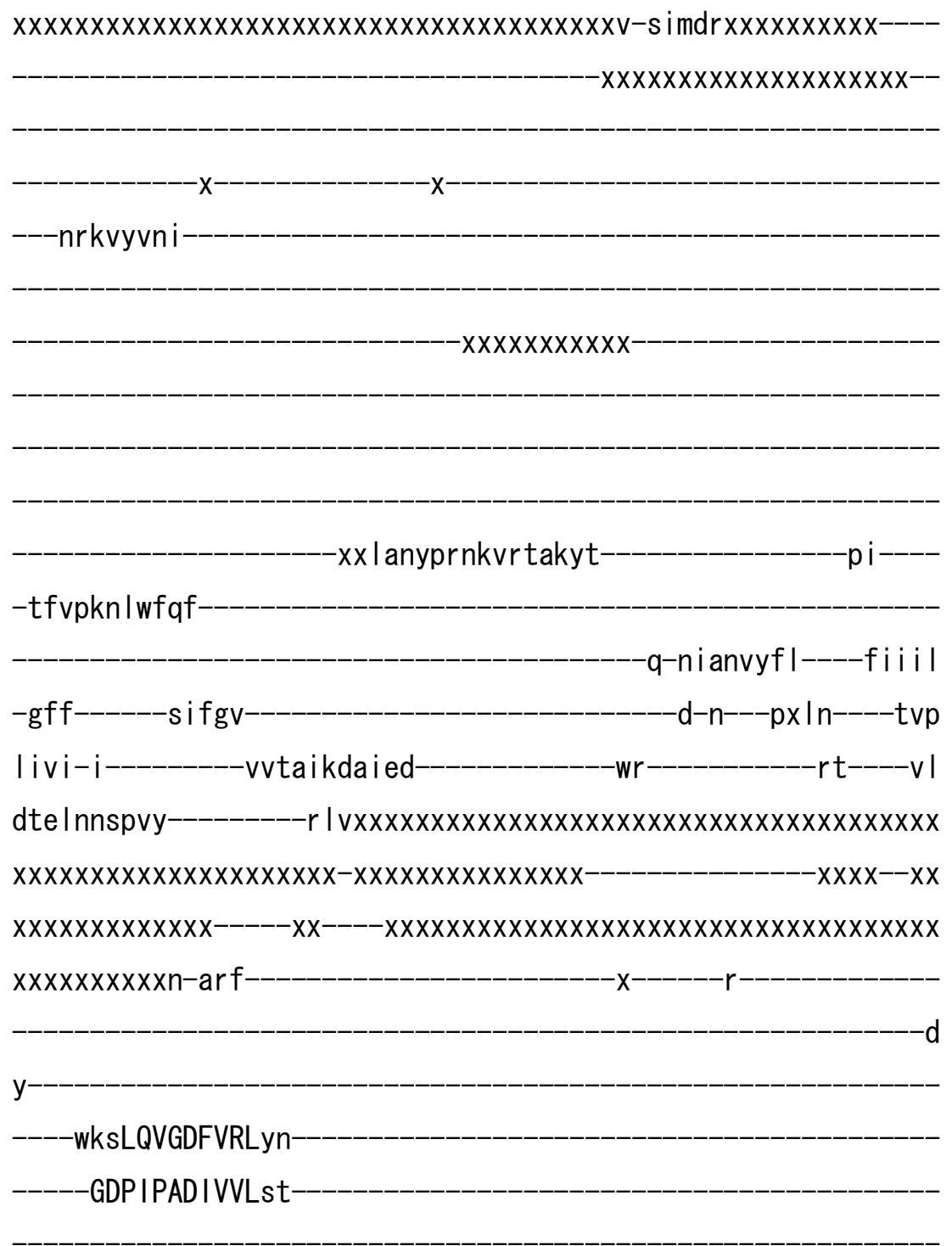

-sdpdg-a---cyVETKSLDGETNLKVRqa-

Ihcgrkvrhardcersef i i eseaphpn l yayngavrwxx

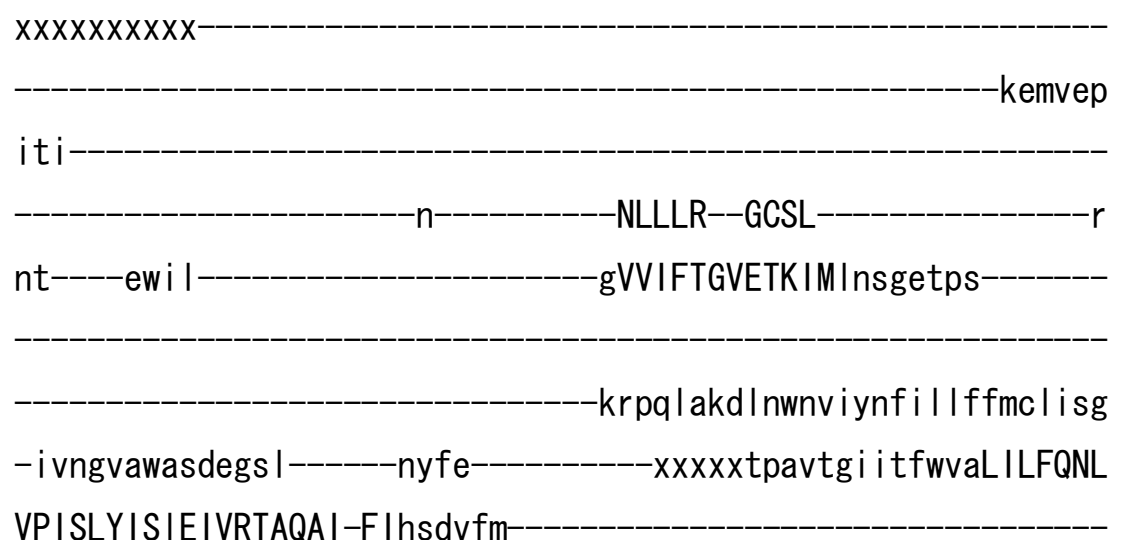

VPISLYISIEIVRTAQAI-FIhsdvfm- 
$-y y d k$ lg is

cIPK-SWNISDDVGQIEYIFSDKTG

TLTQNVMDFk

-kctvngvsy

$-x x x x x x x x x x x x x x$

$-x x x x x--x x x x$

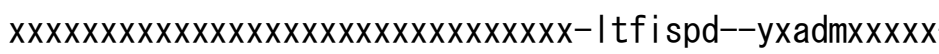

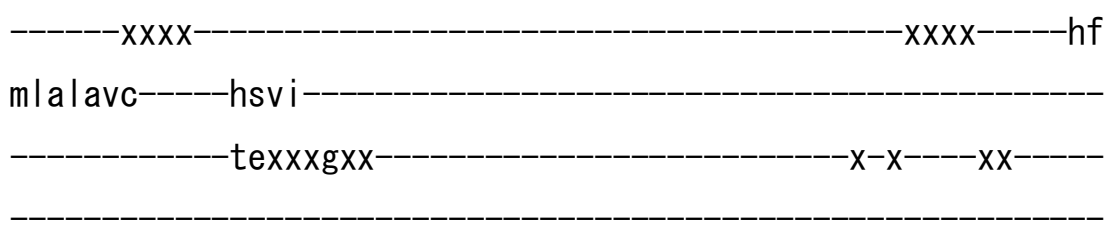

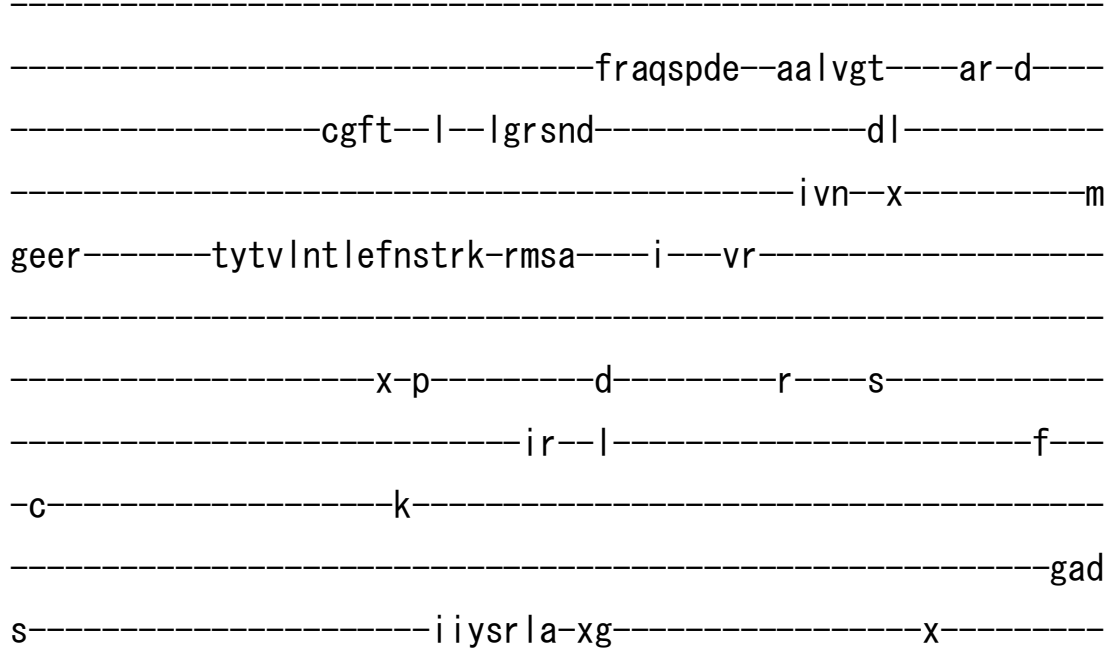

xxx--xx--kkt-aqh letf--are----g|rtLCVADRk |--seeeyr awskehdi---aa-----aa I tdreek I envasa-i eqd Im I IGGTAIEDKLQDGVPDT ISLLARag-IKL WVLTGDKVETAINIGFSCNL_-_-_-_-_-_-_-_-_-_-_-_-_-_-_--_ I tneme I i--_-

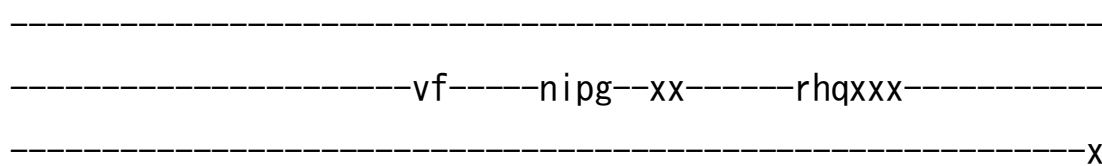
$x x x x$ $-x-x x x x x x x x d e x x x e x x--x x x x x x$

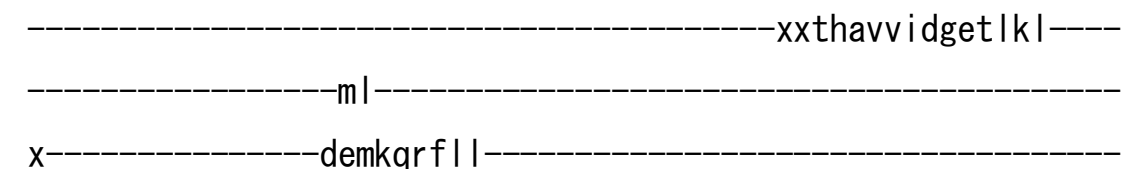


IckqcksvLCC--RVSPAQKAAVVKLVk

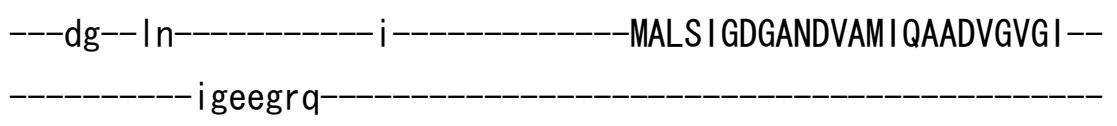

-AAMSADYA I GqfRFLQRL I--LVHGRYSYRRLGETT------A nff-ykn I v-wtfal fwys i yndf--_-_-_-_-_-_-_-_-_-_-dgsy I fdy ty i i I v nlafts I pvi Img i fdqdvddk--_-_-_-_-_-_-_--vs l avpq l ymr g i er l ewsqak f-w I hmadgfyqsvi ff-ympyx l ye-----------------xxxxxxxxxxxv-------sd--rnrmg i I vascav i asnty-i Imnsyrwdw Itv |--_-_--ina i sc I I i ffwtgv

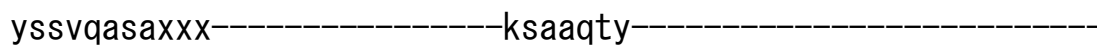

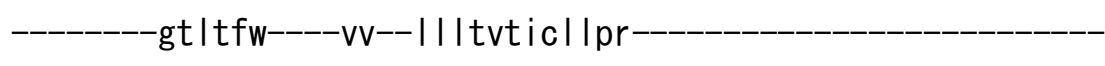
----------fvvksvq-------------kvff--------p------------------Idv - $X X X X X X X X X X X X X X X X X X X X X X X X X X X X X X X X X X X X X X X X X------$

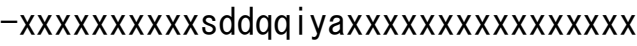
xxxxxxxxxxxxxxxx------------------------------------------

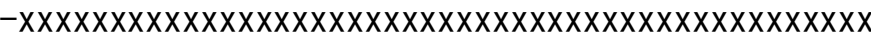

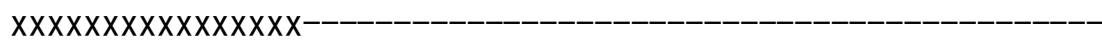
$-$ >ggalV_IV07 XP_416881.2 $\mathrm{m}-$ 
---tr|vrss|

-qfeppkffkra

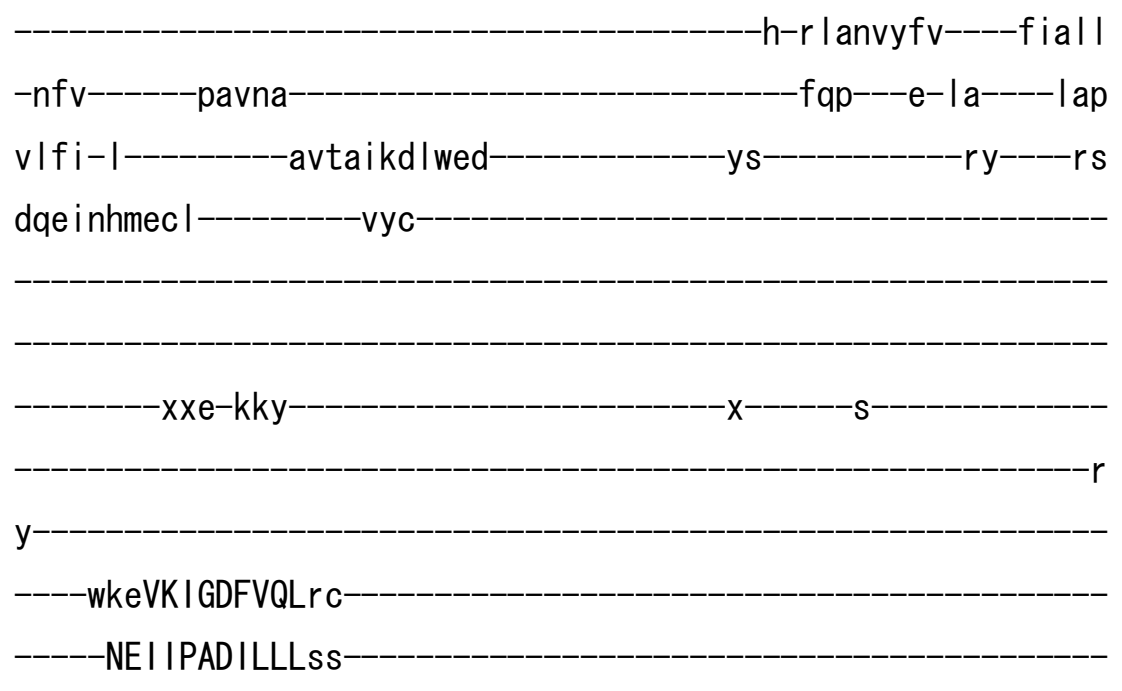


-sdpdg-I---chIETANLDGETNLKQRqv-

-vrrfleldsefdp Ikftsvi ecenpnndI srfrgsivhx-

sgkkdg

Ifk-

nt-----eevv-----------------------g I V I YAGHETKAL I nnngpry-------

-krsklerqmnadv Iwcilill imc Ifsa

-i ghg I wvwqygekk---xxxfdvp---------xxxxxxxspv l asvy I f I ta I IVFQVM

IPISLYVS IEIVKICQVY-FIhqdkdI

-ydeetdsq-

IQCR-ALN I TEDLGQVQF IFSDKTG

TLTENKMVFr

-rctvsgiey

$x x x x x x x x x x x x x x x x x x x$

$\operatorname{xx} x \times x------x x x x x x x x x x x x x x x$

$-x x x x x--x x x x$

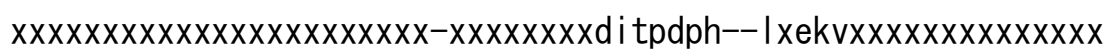

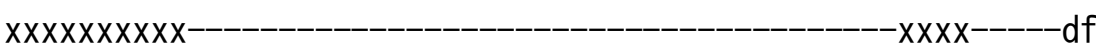

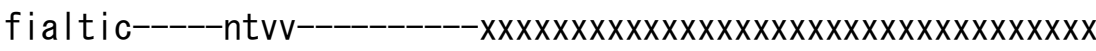
xxxxxxxxxxxxs|xxxk-x------------------------ $x x x----x x x---$

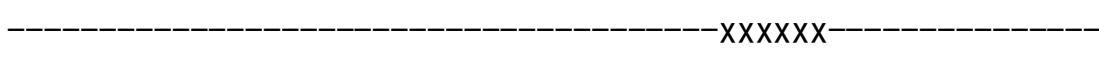

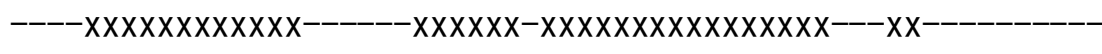

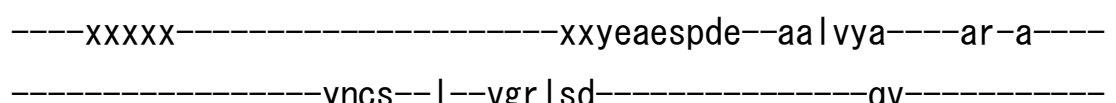

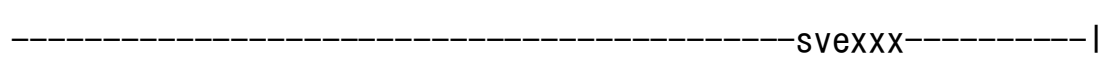

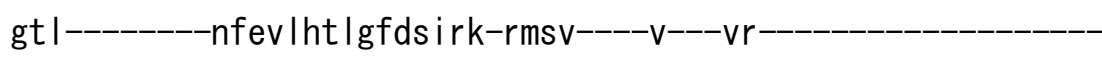

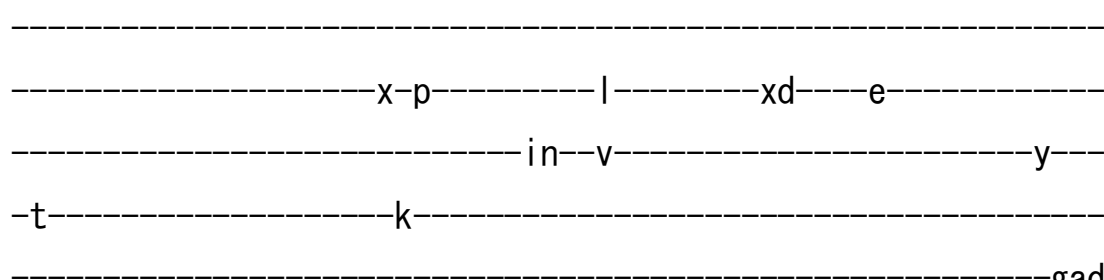

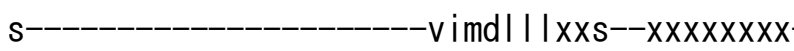


xxx--xx--tkt-qny Inly--avd----g|rtLCIAKRv|--skeeyacw lkshle--ae-----ss i enree I I q qsa I q-i ekn I h ILGATG IEDRLQDGVPET I ASLRKag-LQ I WVLTGDKQETAVNIAYACKLIdhdee i i----

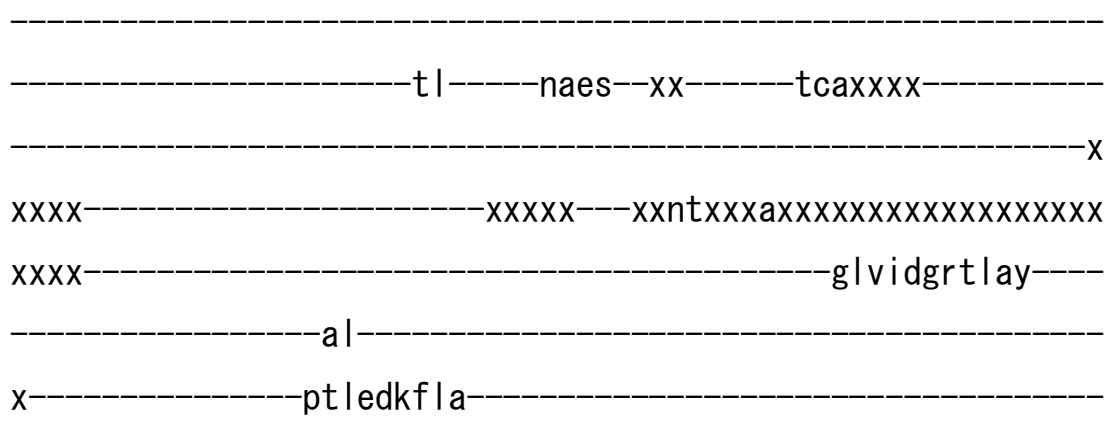

lakrcrsvLCC--RSTPLQKSMVVKLVr

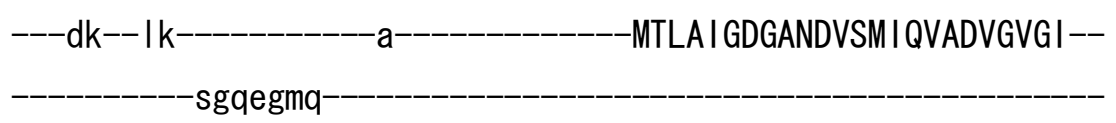

AVMASDFAIPr fRHLEKLL--LVHGHWCYSRLANMV------L yff-yknam-fval I fwyqfycgf-_-_-_-_-_-_-_-_-_-_-_-_sgssmvdqwy I iff nl Ifss I pq I i tgv I dkdvpae--_-_-_-_-_-_-_---v I I svpq I yksgqnmeeyqphm f-wmnm i damyqs I vcf-fi i py-fty-_-_-_-_-_-_-_--xxxxx--_-_-_-_-_-_-_-d--ifswgtpitttalfti i Ih-laietktwtf Ihws--_-----scifs il Ifffvalv ynascpvc---xxxxxxx-----------wtmer Im---------------------------

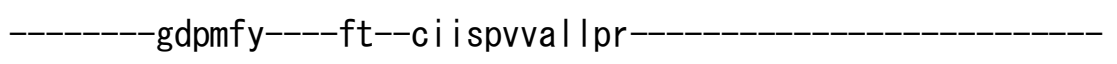

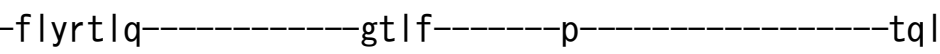
$-x x x x x x x x x x x x x x x x x x x x x x x x x x x x x x x x x x x x x x x x x x x x x x x x$ $x x x x x x x x$ xxxxxxxxxxxes I fqehtxxxxxxxxxxxxxxxx 
>mgr IV_IV02 XP_366691. 2

$$
\text { m- }
$$

$-X X X X X X X X X X X X$

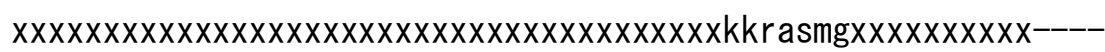
$-x x x x x x x x x x x x x x x x x x x x--$

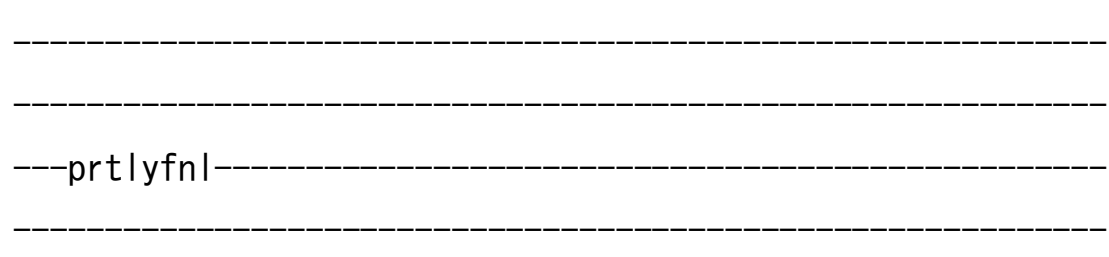

$-x x x x x x x x x x x-$

xxvdtyprnkirtakyt

$-\mathrm{pl}$

-sfipknlwlqf 
-h-nianiyfl----flvil

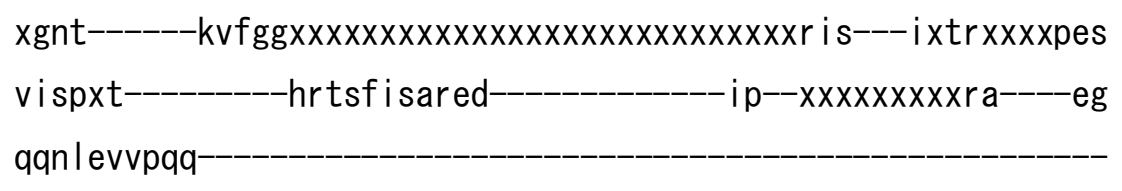

$x x x x x x x x x$

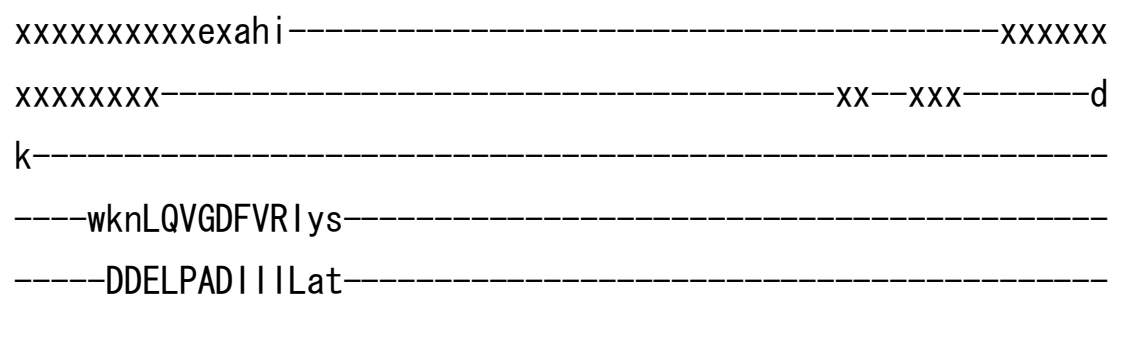

-sdpeg-a---cylETKNLDGETNLKFRqa-

- lkcgrnmknsrdceraqf i veseppqpn l ykynga i kwxx

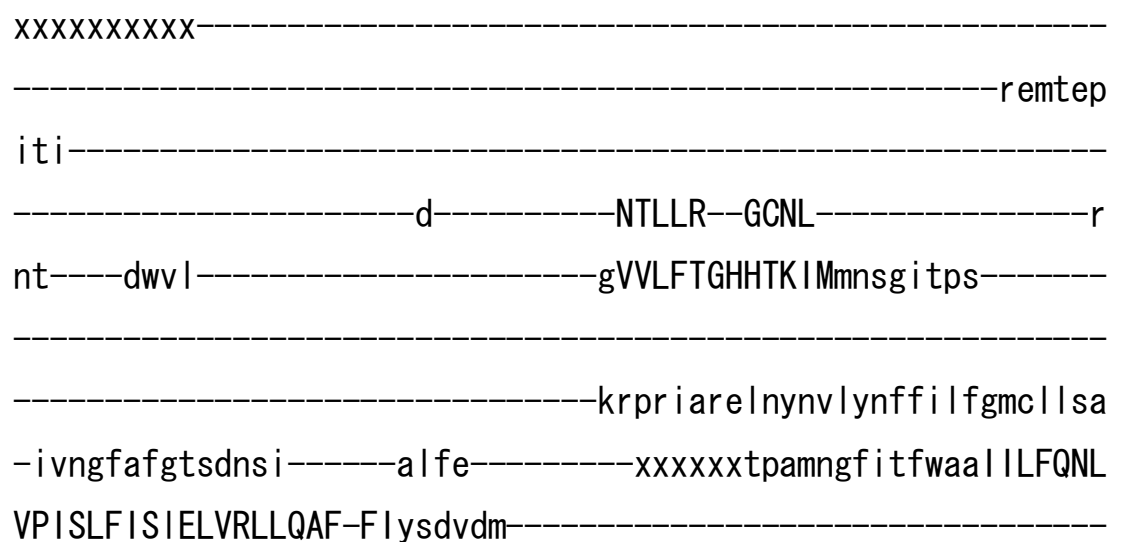

VPISLFISIELVRLLQAF-F Iysdvdm-

-yyep i dqp

cIPK-SWNI SDDLGQIEYIFSDKTG-

-TLTQNVMEFk-

kat ingqpy-

$-x x x x x x x x x x x x x x$

$-x x x x x--x x x x$

$x x x x x x x x x x x x x x x x x x x x x x x x x x x x x x x x-|q f i a p d--f x e d| x x x x x-$

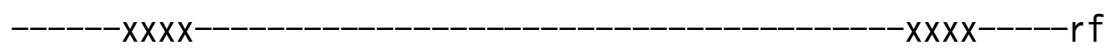

mlalalc-----htvi

- pexxxgxx-

$-x-x----x x-----$ 


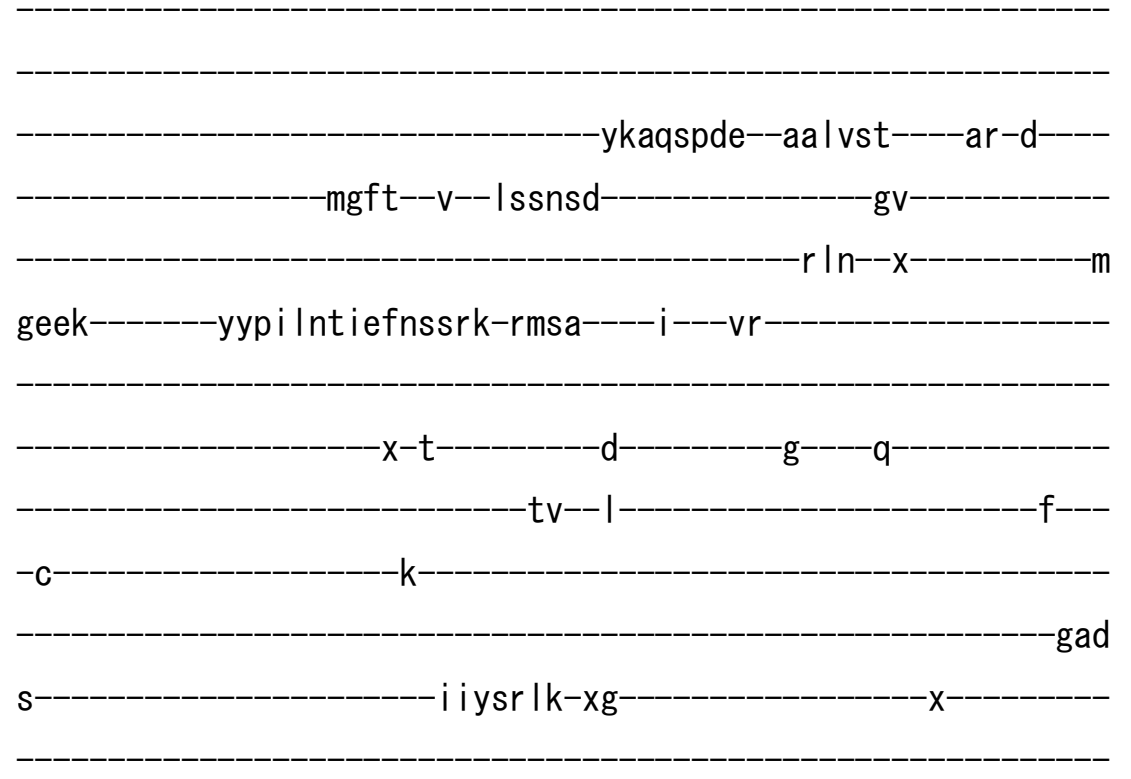

xxx--xx--tdt-aqh l emf--are----g | r tLCI AERv |--geqeyqawskeyav---aa-----aavenredkmea i adq-i eqd I I ILGGTAIEDRLQDGVPQTIAVLAEag-IKL WVLTGDKVETAINIGFSCNL-

- Inndme I I----

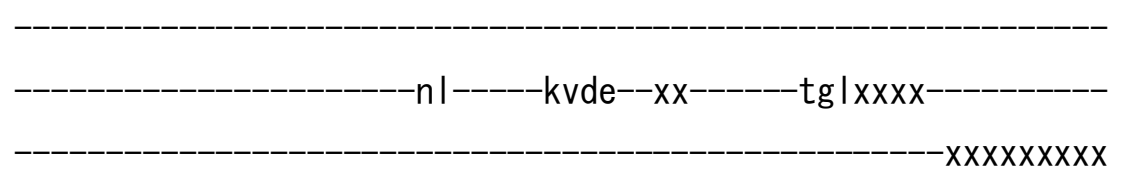

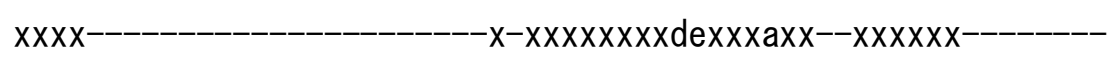
xxthavvidgft Irw-

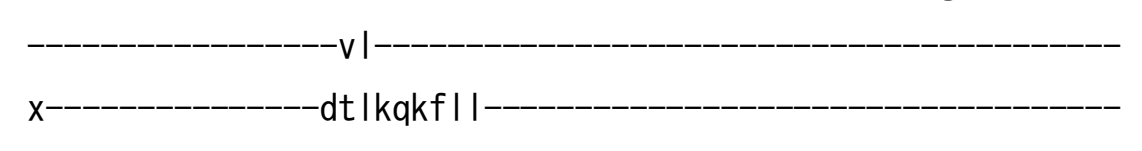

IckqcksvLCC--RVSPAQKAAVCAMVk

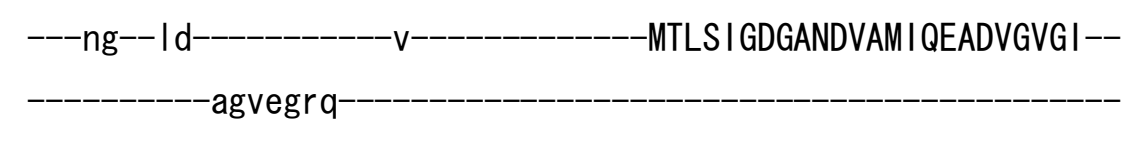


nff-ykn Iv-wamp If I fqiycdfdmty I fdyty i Imf

nl Iftsvpvi Imgvldqdvsdt -vs lavpqlyrrgier lewtqtk

f-wlym I dgtyqgvmsf-fipyxvvv$-x x x x x x x x x x x v$

td--rvrfgay iahpavvtinly-i I insyqwdw I iv lvvvlsdlfvffwtgv ftsntysqxxx eaapqiy

--------aqpsfw----av--fi i i tpvmcmfpr

-fsikalq--_-_-_-_-_--kvyw--_-_---p--_-_-_-_-_-_-----ydv

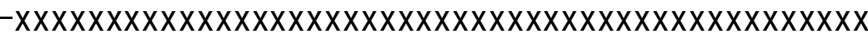

$\mathrm{xx}$ xxxxxxxxxxathtrtqtxxxxxxxxxxxxxxxx $x x x x x x x x x x x x x x x x$

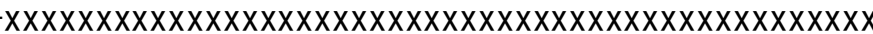

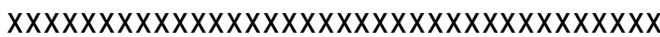

>mgr IV_IV03 XP_361592. 2

m 


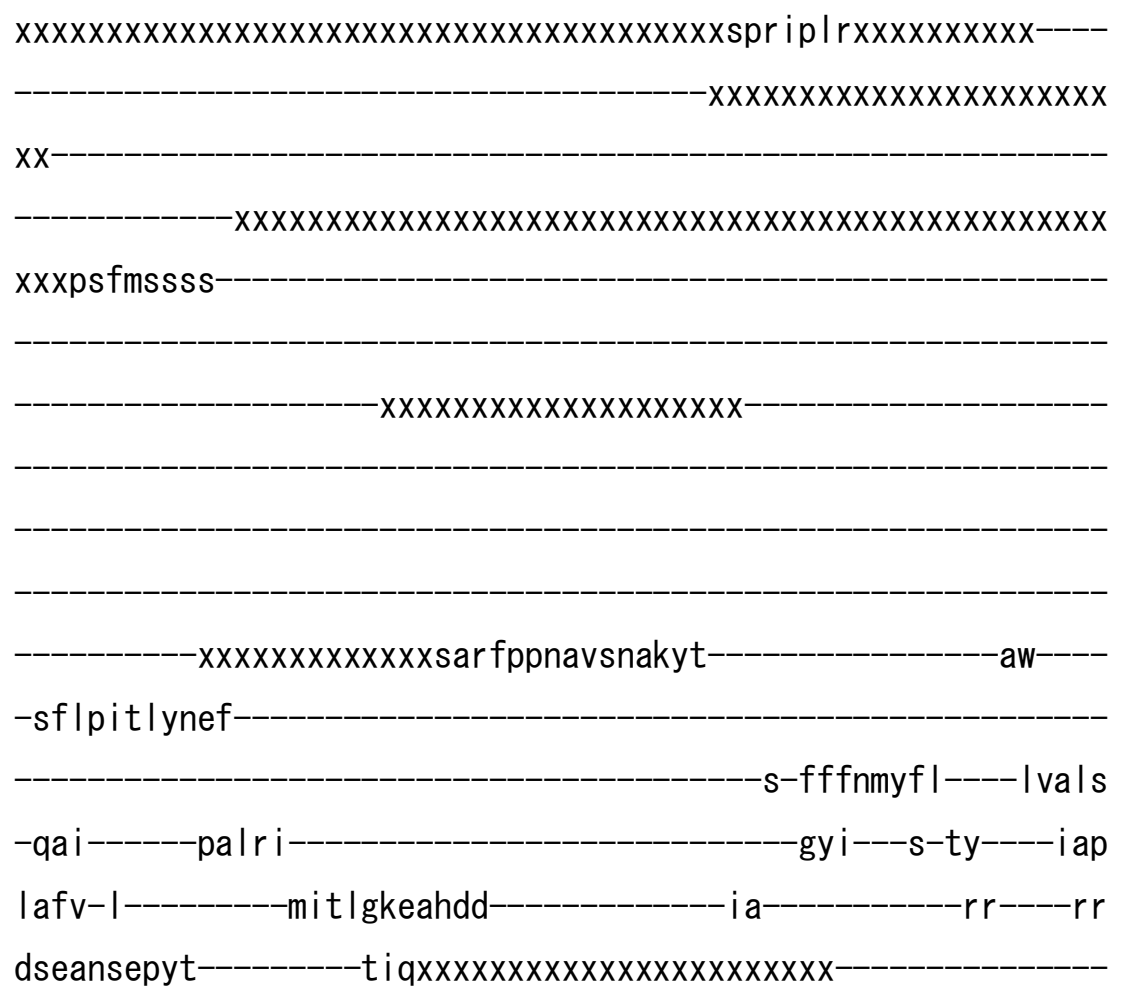

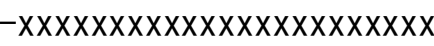

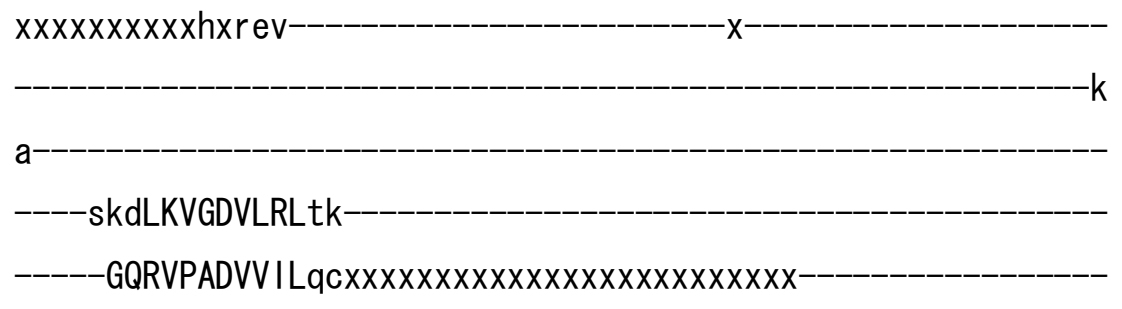

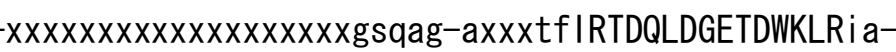

-sp I sqn latee Ivrfrvtagkpdkkvnefvgt l e Ixx $\operatorname{xx} x \operatorname{xx} x \operatorname{xx} x \operatorname{xx} x \operatorname{xx} x \mathrm{x} x$

l antap

$\mathrm{Iti}$

d-----------NTAWA--NTVI-----------------a

sr----avt I----------_-------------aVIVYTGPQTRSA I stspsrs-------

-ktgl leyeinsItkilcaltlalsi ilv

-afqgftntegnv---------wy ik--_-_-_-_-_-_-_-_-_-_-_-_imrfLVLFST I

VPISLRVN IDLGKSVYSW-F I ardpgm- 
aVVR-TSMIPEDLGRIEYLLSDKTG

-TLTQNEMEMk-

-kihigtvsy

$x x x x x x x x x x x x x x$

$x x x x x--x x x x$

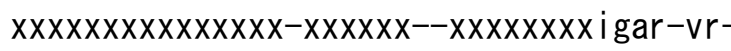

$-d v$

vlalalc-----hnvt

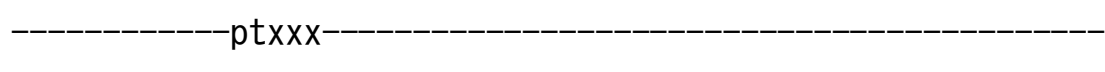

$-x x x x x x x$

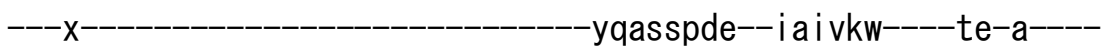

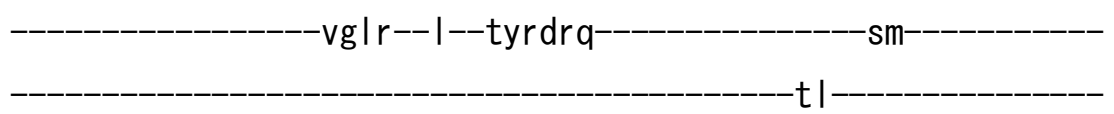

sfsexxxxx-xhf

-dw I-deetanm--are----g | rtLVVGRKr I--saeqyrefssryqe---

as------ l a ingr dagmqkvvshx l erd l e ILGVTGVEDKLQKDVKPSLELLRNag-IK I

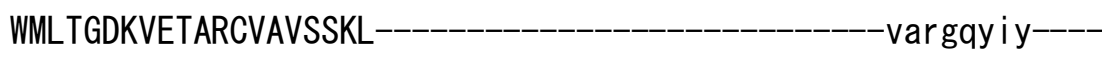

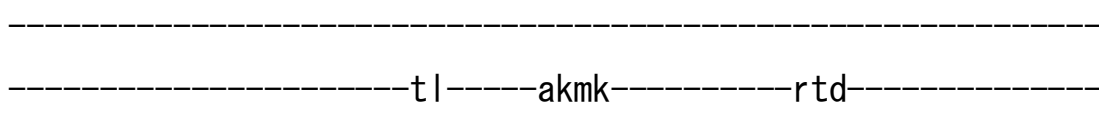

SXXXXXXXXXXXX

dac $\mid$ idges $|a|$

II

qyhr Idfvs- 
vavr I ptvVAC--RCSPTQKAEVARL Ik

$---d y--\operatorname{tr}$ $x-$ -RVCCI GDGGNDVSMI QAADVGVGI-vgkegra

-ASLAADFSIEqfHHLTKLL--VWHGRNSYKRSAKLA------Q

fvi-hrgli-iavcqtmysiamhf epdg I ykdw I lvgy atmytafpv $|s|-v \mid d k d v d e n$ I an lype lyke Itsgrs Isyrt

f-fvwv Ivs i yqgg i iq-glsq-IIt e-------

vd--gpkmvavsftv Iv Ine I Im-va i e i ttwhpvmi v-sivgtfIvymgsipf Isgy-fdl kfmit |rtmxxxxyr--kvqe Imvdf |r I

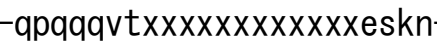
$-p x x x x x x x x x x x x x x x x x x p h$ npl

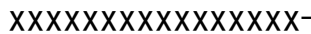

XXXXXXXXXXXXX

>ggalV_IV08 XP_414491.2

m- 


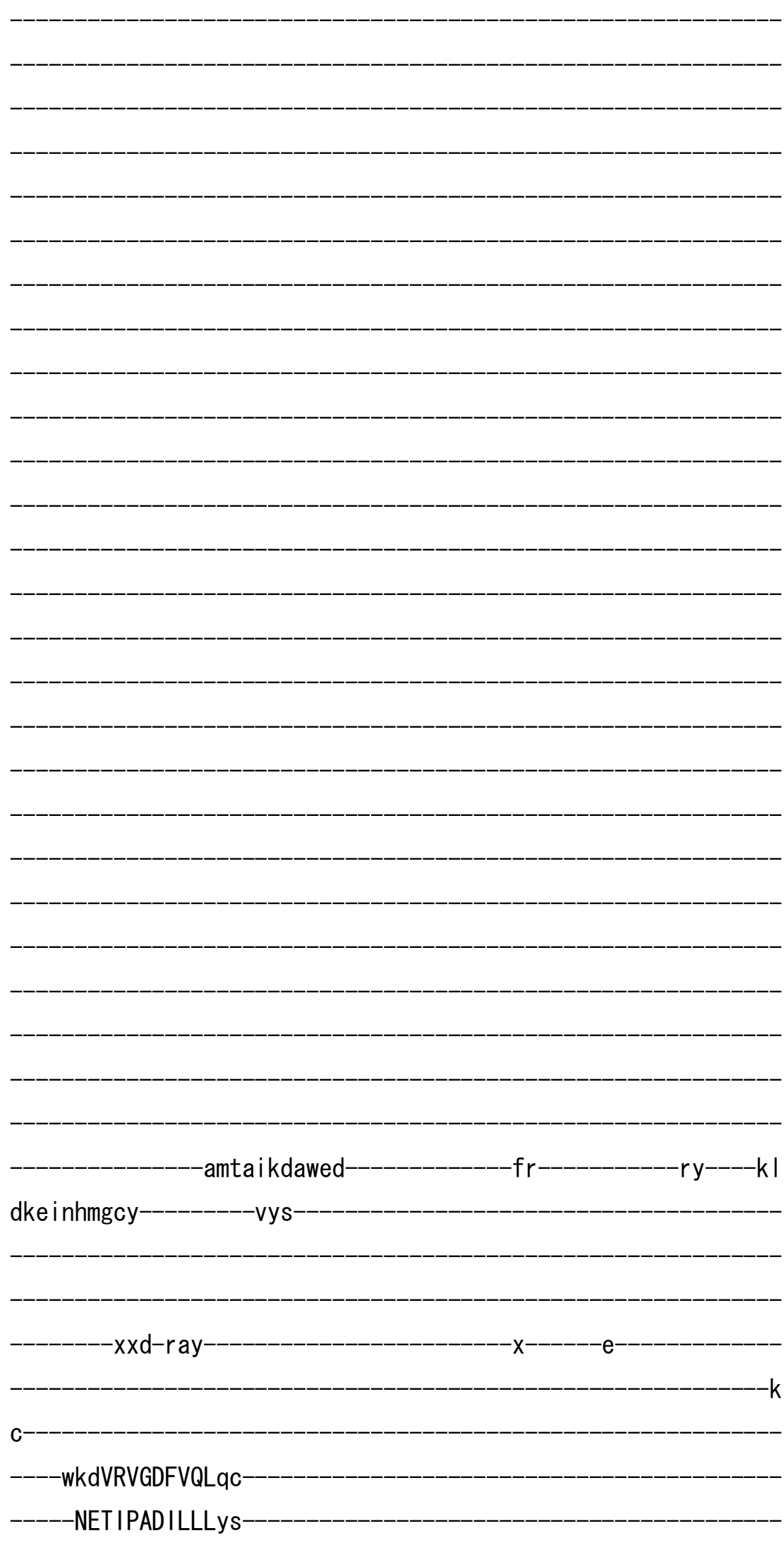


-sdqng-i---chLETANLDGETNLKQRrv-

-vmgfssqntsfepeffant i ice Ipnnd Inkfkgy

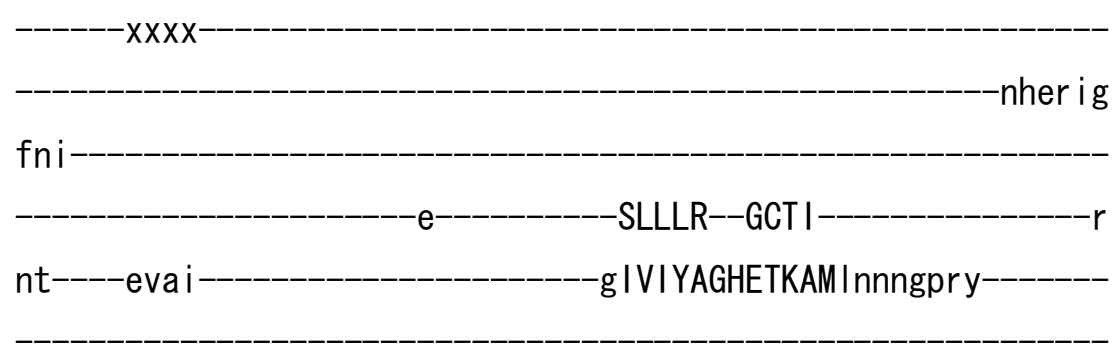

-krskierrmnvdiflcvg I Iftmclvgs -i ghg i wsgsfpehp------xydvp---------xxxxxxxspv I sgfymf I tm I ILLQVL IPISLYVS iELVKLGQVF-LIhndIdI

$-y$ deead I

i QCR-ALN I TEDLGQI QY IFSDKTG

TLTENKMVFr-------------rctvdghef

$-x x x x x x x x x x x x x x x x x x x$

$\operatorname{xx} x \operatorname{xx} x \operatorname{xx} x \operatorname{xx} x x x x x x x x x x y--x x y$

$-x x x x x--x x x x$

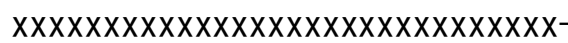

$-x x x x x x x x x x x x x x x x x x x x x x x x$

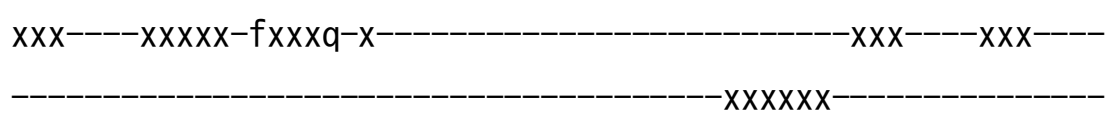

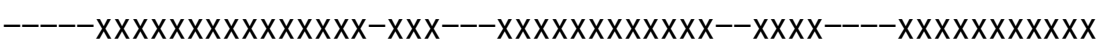

xxxxxxxxx--------------------- xxyeaespde--aal vya----aq-a----

--------------------ysft--1---vsrtpe------------------qv-------------

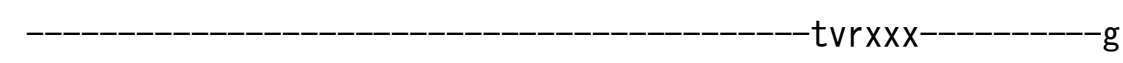

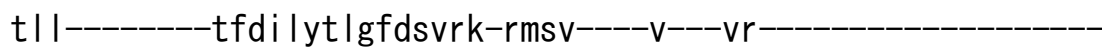

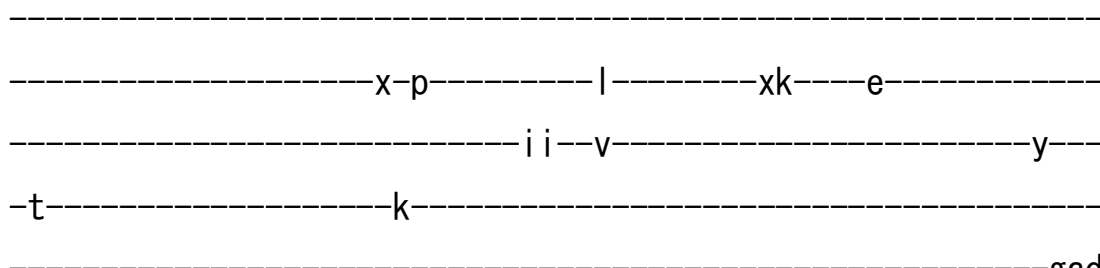

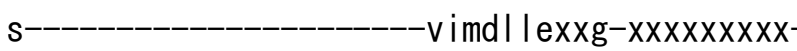


xxx--xx--dkt-qkh| dcy--ard----g | rtLCI AKKv |--seddfqkwanfrqe--ae-----aa i dnr de I I metagh- | etk I I LGATG I EDRLQDGVPDT I AALREag-IQ I

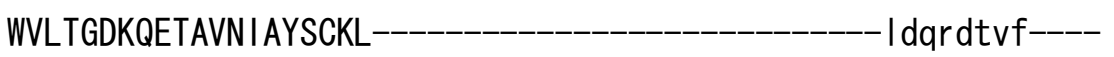

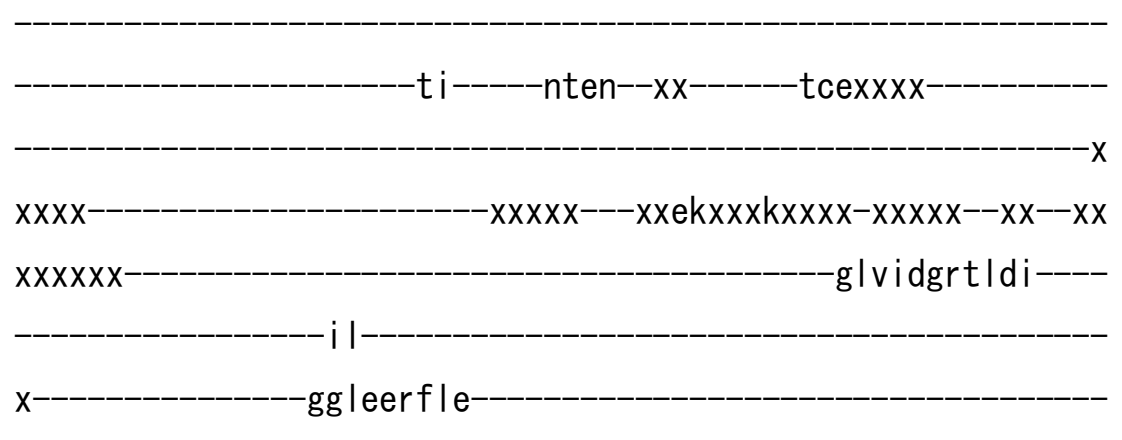

I ar I crsVLCC--RSTPLQKSMVVKLVr

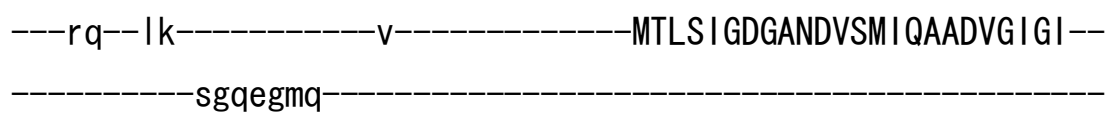

-AVMASDFA ISr fKHLKKLL--LVHGHWCYTRLAKMV------I

yff-yknvs-yvnl I fwyqffcgf--_-_-_-_-_-_-_--_-_----sgntm i dywqmiff

nIffts I pp I vfgv I drdvsae-_-_-_-_-_-_-_-_tI I s l pe lykngqnse i yk I st

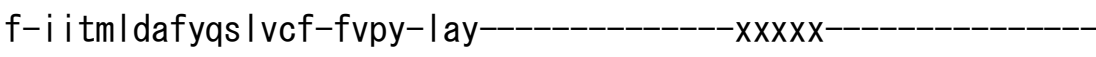

-d--vfsfgnpintvs I Iti I Ih-qa lemktwt I fhwi---_----ti i gsvvi y lvfs I i ynavcvac--xxxxxxx----------wimekql-------------------------

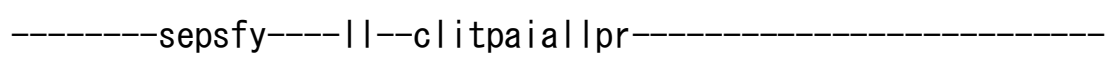

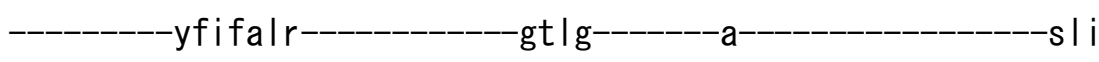

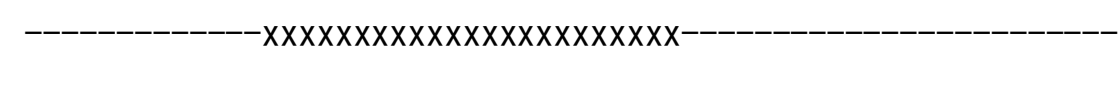




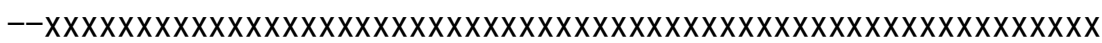

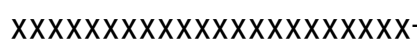

>ggalV_IV09 XP_420722. 2

m

$x x x x x x x x x x x x x-------x x x x x x x x x x x x x x x x x x x$ atkt $i g k$

---hrivipcl

$-x x x x x x x x x x x-$

$-k$ lymnnkirttkyt $-11$

-tfiprnlfeqf 


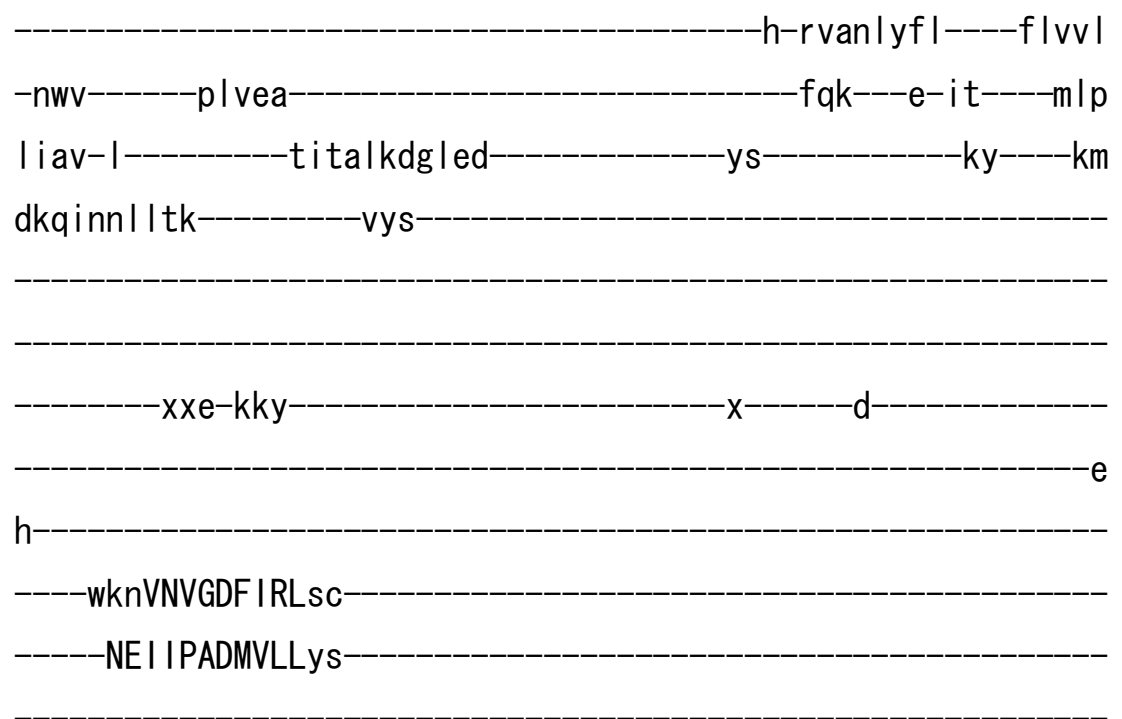

-sdldg-i---cylETAGLDGETNLKQRqv-

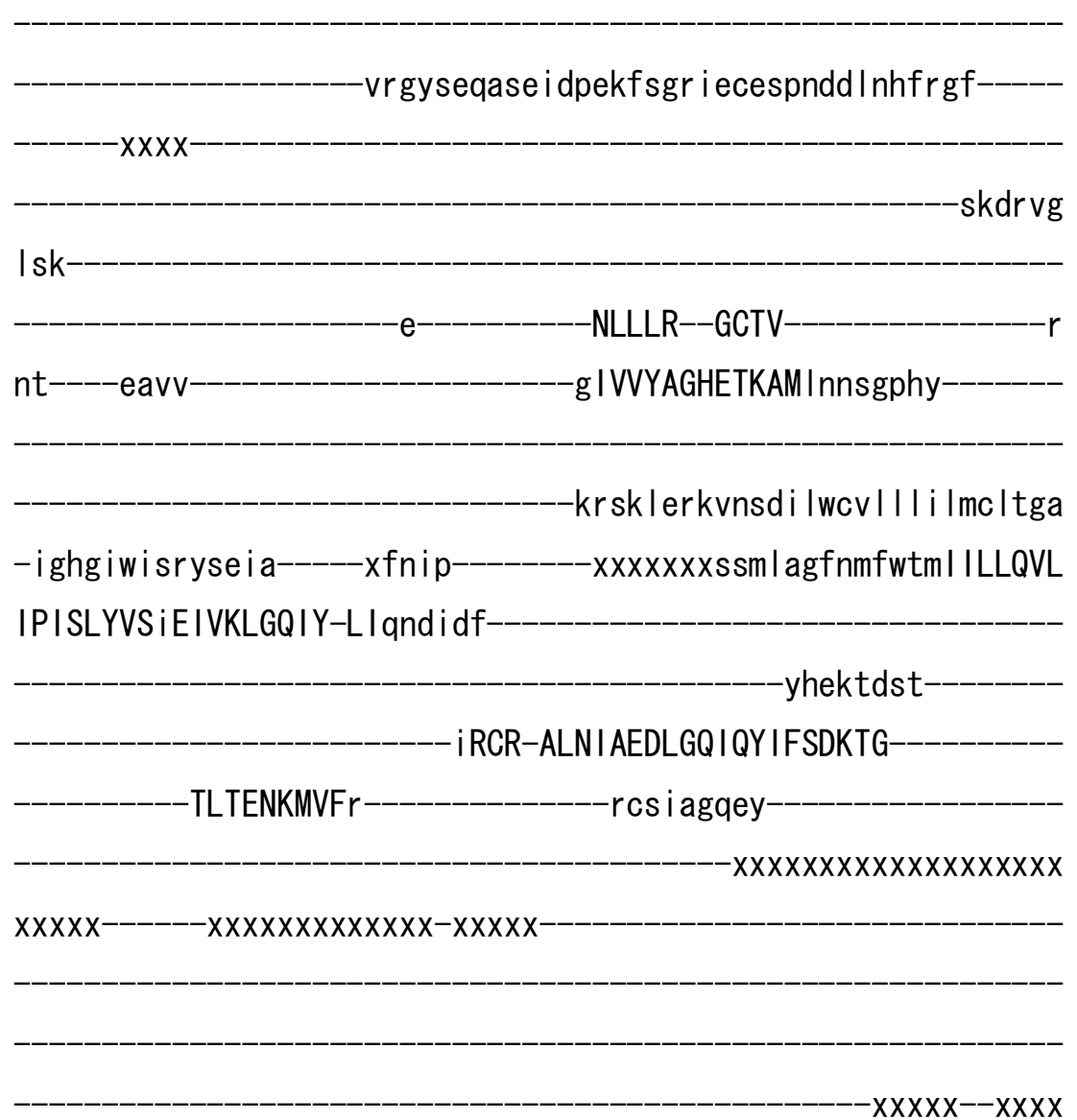

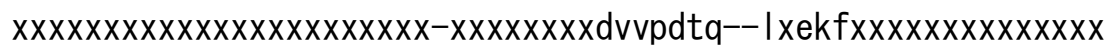
$x x x x x x x-x x-----------------------$ flala ic $-----n$ tvv--------- $x x x x x x x x x x x x x x x x x x x x x x x x x x x x x x x x x x x$

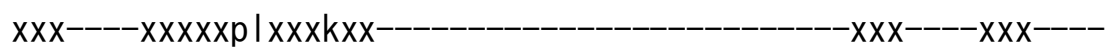




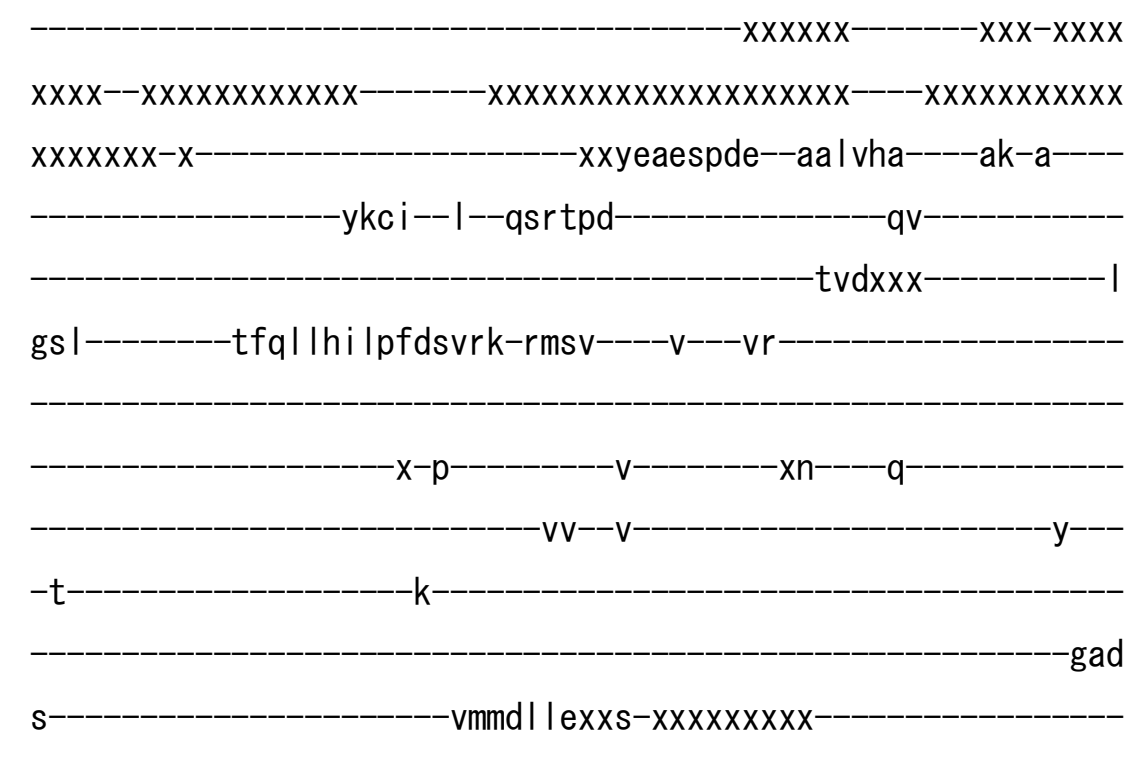

xxx--xx--ert-qkh I ddy--ark----g I r tLCI AKKvm--sdeeytew Innhf I--ae-----ts idnree I I lesair-l etk I t ILGATG IEDRLQDGVPDT I QALRKag-IK I WMLTGDKRETAVNIAYACKL- epddr if----

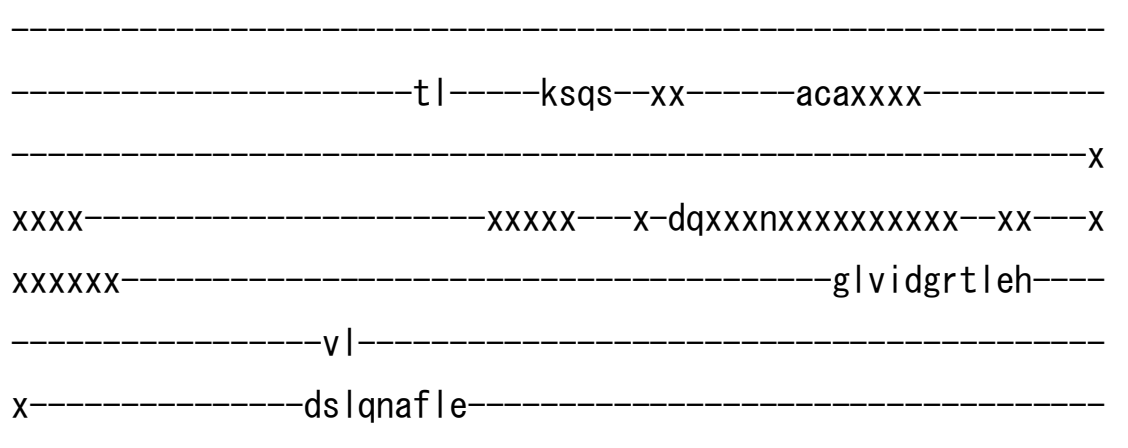

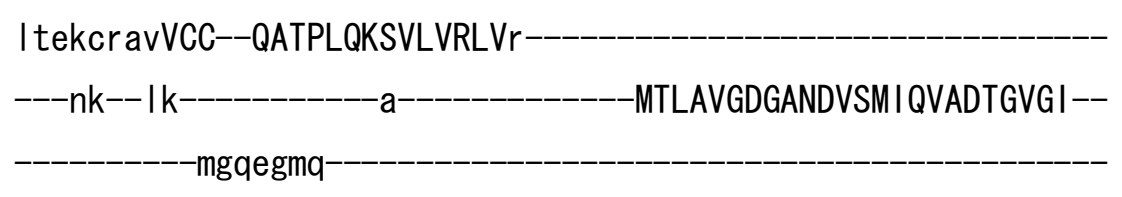

-AVMASDFA I SqfRHLRKLL--LVHGHWCYTRL TNM I------L 
yff-yknva-yvnl I fwyqffcgfsgtsmtdywi I iff

nl Iftsvpp i iygvldkdvsae i Imqi pq lyvmsqksvay Ipst

f-witl Idafyqs lacf-fvpy-fty $x x x x$

-d--ifsfgnpintaalf i i Ifh-I I iecksvtwihtv -vitgsilfyfifala fgatyrth--xxxxxxx rimekhm-

---------adpvfy-----|v--c|lttcval |pr-

-yfirala gt If SSV

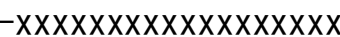

xxxxxxxxxxxcrme lqvtxxxxxxxxxxxxxxxx

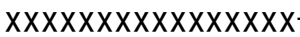

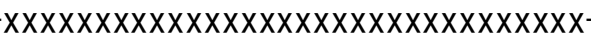

>mgr IV_IV04 XP_359925. 2

$\mathrm{m}$ 
-dfIprqlvfqf

-m-kianfyfl----i iai I

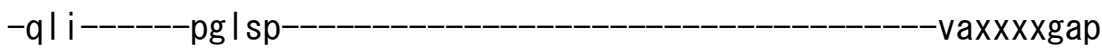

I i vf-v--_-_-_---afsmakegydd--_-_-_-_-_--yr-_-_-_----ry----k |

dlvenrssaw--_-_-_---vldxxxxxxxxxxxxxxxxxxxxxxxx---_-_-_-_-_-_-

$-x x x x x x x x x x x x x x x x x x x x x x x x x x x x x x x x x x x$ $x x x x x x x x x x n x w s h$

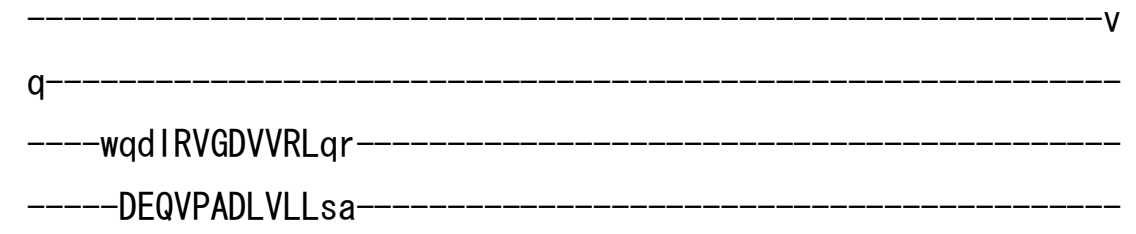

-sgtng-i---ay IDTMALDGETNLKSKqa-

-xp I l akrcgtvag i chcdatvi aedpn I d i ysfdgsvrv--

gdktmp

Itr

d----------QVVFR--GSTM-

-gLVINSGEECK IRmnahksvr

nt----reay-

-_-_-_-_-_-_-_-_-_-_-_-_-_-_-_-_-_-_-_-_-__-_-_-_-_-_-_-_-_-_

-xkhpamqavtnrivlII ivv Ivmlavg |k

-i gndefeaefgptn---_---wymf-_-_-_-_-_-_-_--_gvr I s i seqvfgf I ILLNTL

IPLSLYISIEAIKIGQLY-MLq-diem- 
-ydaesntp

- VAN-TTTILENLGQVSYIFSDKTG

TLTENVMRFa

$-k \mid$ s I ggyvw-

$x x x x x x x x x x x$

- telqt Ig--|xqh|xxxxx

$x \times x x$

$x \times x x-----h f$

i $\mid$ cma l c-----ntc l

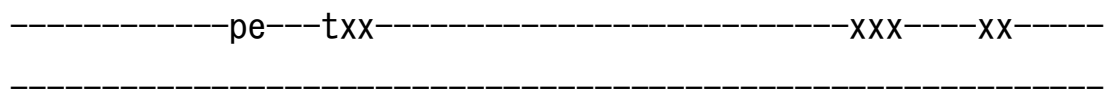

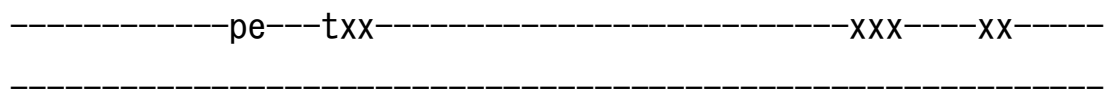

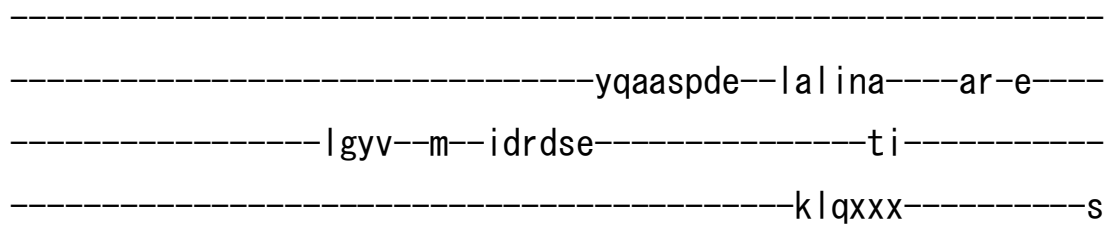

gsndxxxxx--tyti Idvieftsdrk-rmsv-----i--- Ig

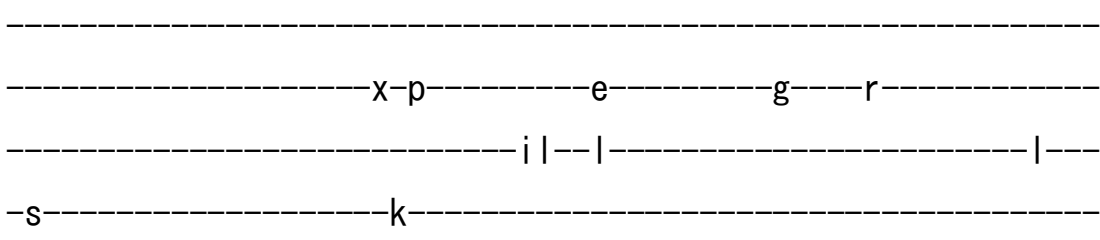

-gad

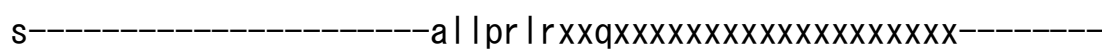

$-x x x x x x x x x x x x x x x x x x x x x x x x x x x x x x$

$x x x x x x x$

xxx--xx--eac-frhi edf--ase-----g|rtLLFSYRy i--eeadyvawkr eyhe---

ae------ts I vdrqar idsvaek-i e le le I AG I TA IEDKLQDGVPET IDKLRRan-IKV

WMLTGDKRETAINIGHSAKI-_-_-_-_-_-_-_-_-_-_-_-_-_-_---_cqpfsd if--_-

i I-----dasaxxxx-

xxxxxxxxxxevxx-

$-x x x$

$-x h s v v v i d g|t| t$

ev

xxx------_-_----kaskd I fyd- 
I I trvdav ICC--RASPAQKAVVVQCIr

---dk--vp-----------xs------_--_---LTLA I GDGANDVAM I QASHVG I GI --

-AARI SDYSIAqfRFLQRLL--LVHGRWNYVRTSKYI------L gtf-wke i v-fy Inqa I farynay-_-_-_-_-_-_-_-_-_-_-_-_tgts I fesas I tvf nal ft I I avv Imgvfeqd I sae--_-_-_-_-_-_-_--t I I afpe I yv I gqqdkg Infvk y-fgwmv I gaaeal vif-ftvf--------------------xxxxxxxxxxxxt-----------ifp i gnavftavvifinikx I I vemhhktwiv lg--_----gf I i ti tgwfvwn If

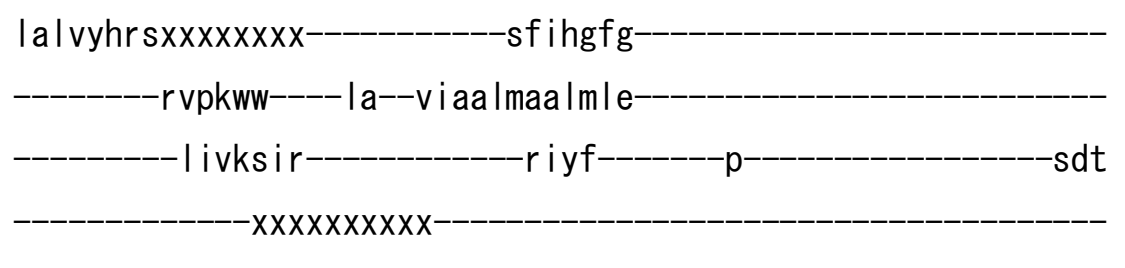
-xxxxxxxxxxhqaer aqexxxx--

$-$

>hsalV_IV01 NP_006086. 1

$\mathrm{m}-$ 


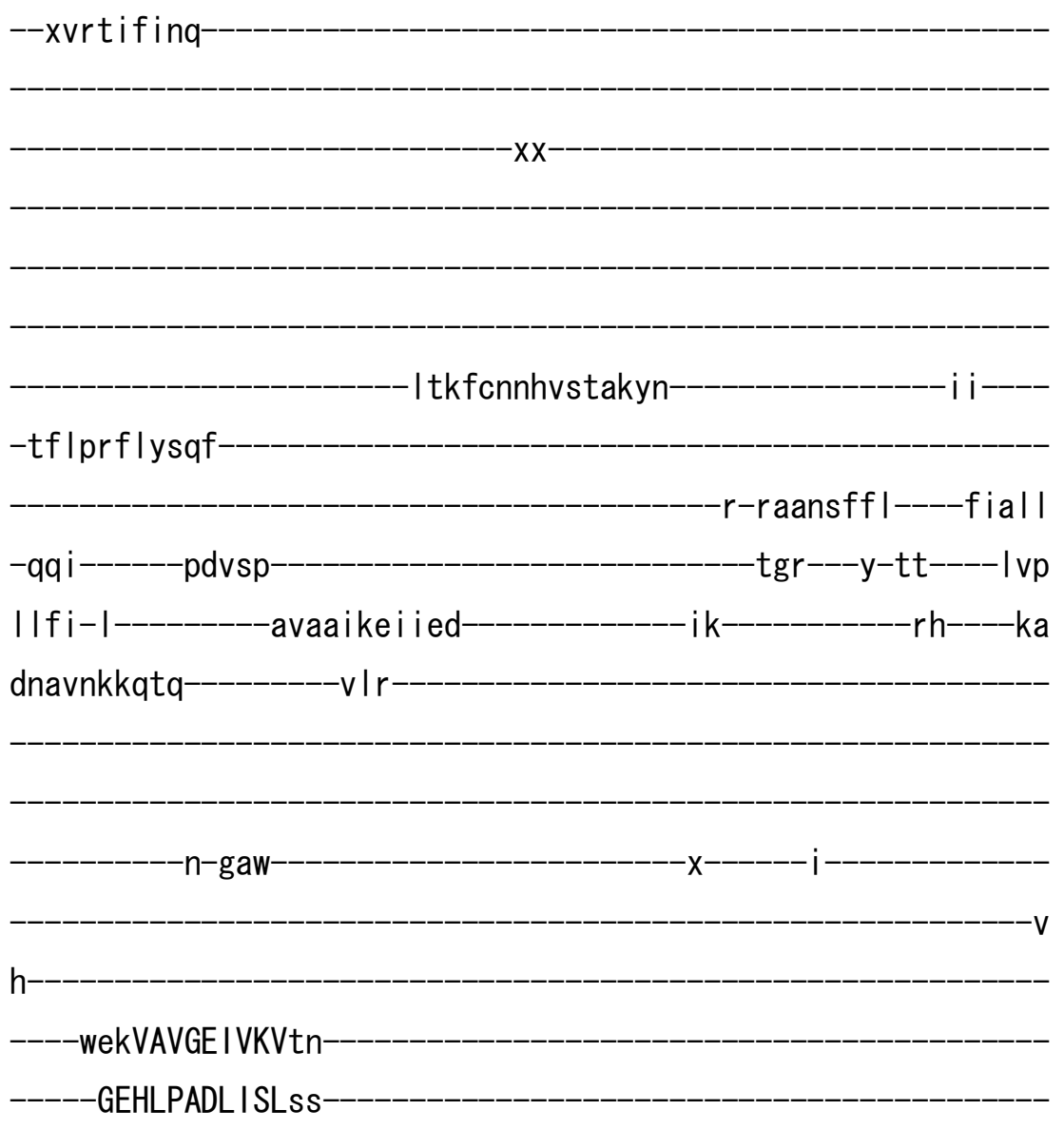


-sepqa-m---cy IETSNLDGETNLKIRgg-

- patsdikdvds Imr i sgr i ecespnrh I ydfvgni i Ix-

-ghgtvp

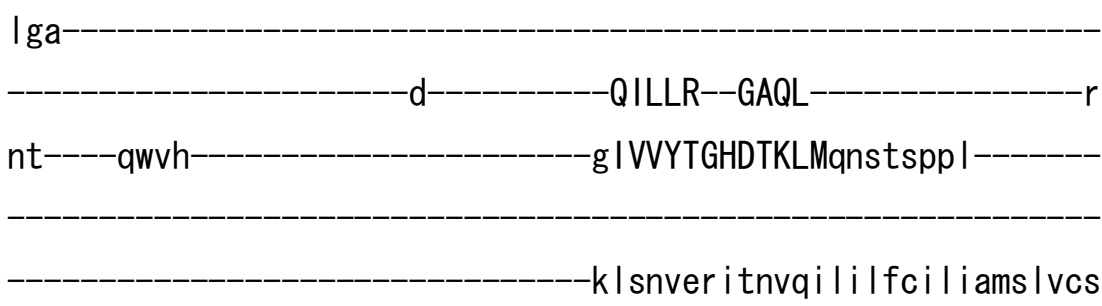

-vgsa i wnr rhsgkd-------wy In------------Xxxggasnfg Inf I tf I ILFNNL

IPISLLVT IEVVKFTQAY-F Inwd Idm-

-hyeptdta

aMAR-TSNLNEELGQVKYIFSDKTG

-TLTCNVMQFk

-kctiagvay-

$-x x x x x x x x x x x x x x$

$-X X X X$

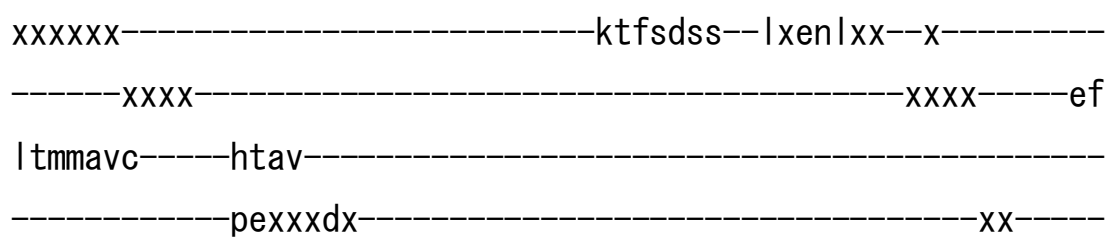

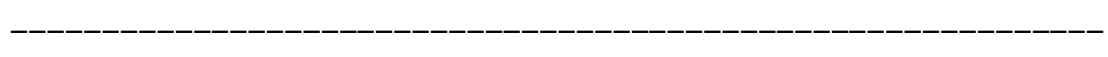

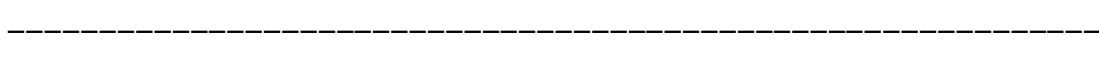

-_-_-_-_-_-_-_-_-_-_-_-_-_-_-_---yqaaspde--gal vra----ak-q----

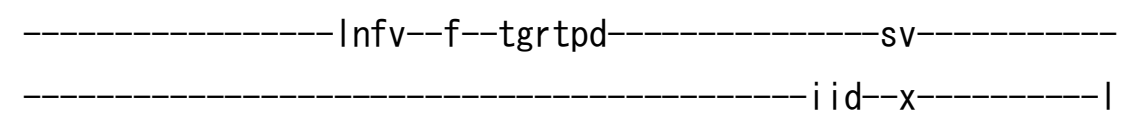

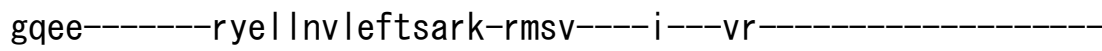

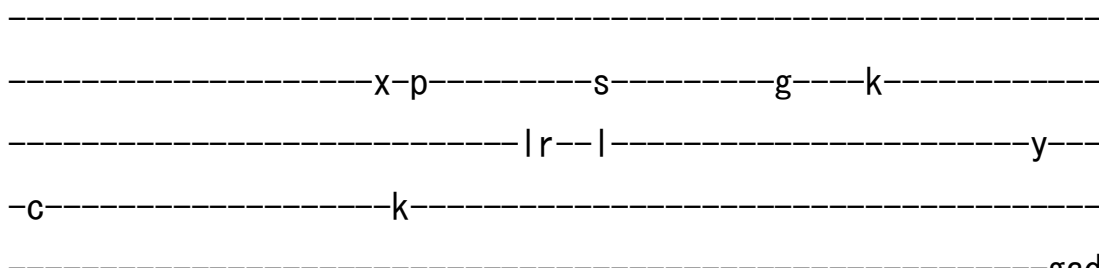

-gad

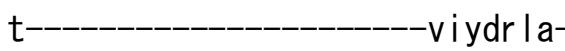

$-x$ 
xxx--xx--e it-Ikhl eqf--ate-----g|rtLCFAVAe i--sesdfqewr avyqr--as-----tsvqnr I I k leesye I-i ekn I q ILGATAIEDKLQDQVPET IETLMKad-IK I WILTGDKQETAINIGHSCKL-Ikknmgmi----

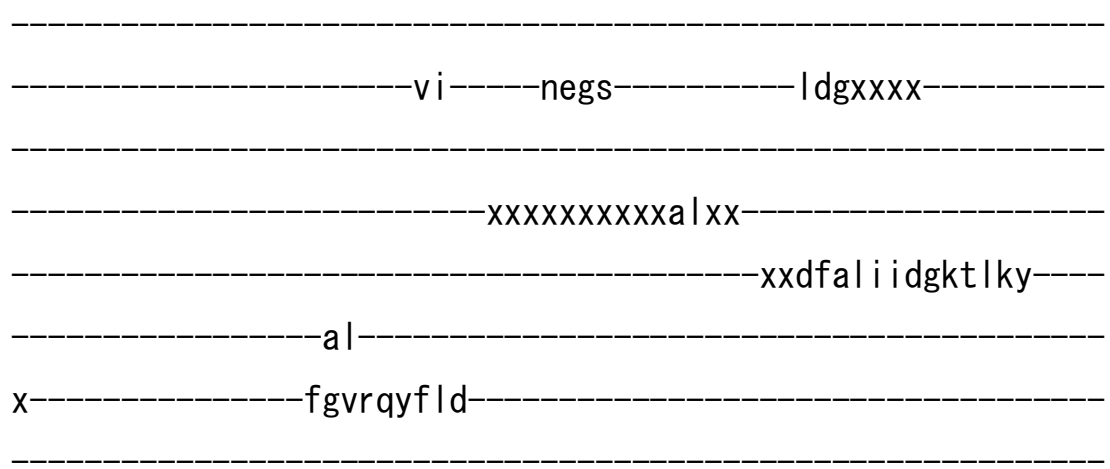

la l sckav ICC--RVSPLQKSEVVEMVk

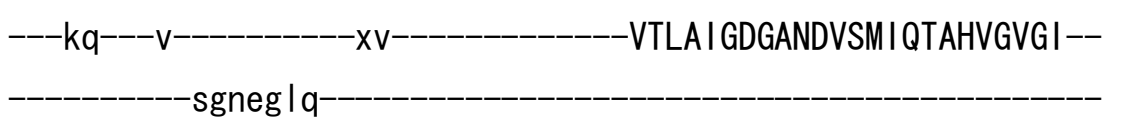

AANSSDYSI AqfKYLKNLL--MIHGAWNYNRVSKCI------L

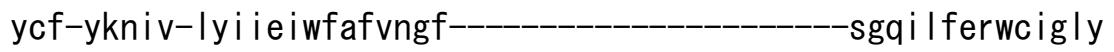
nvmf tampp I I I g i ferscrke--_-_-_-_-_-_---nm I kype lyktsqna Idfntkv f-wvhclng | fhsvi If-wfp| xalq-_-_-_-_-_-_-_-_--xxxxxxxxxt---_--sd--y I I gnfvytfvvitvclk-ag letsywtwfshi---_----ai wgs i a lwvvffg i

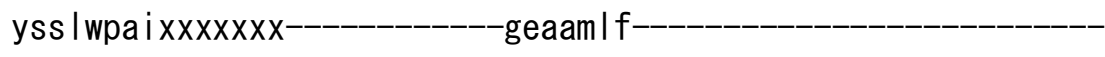

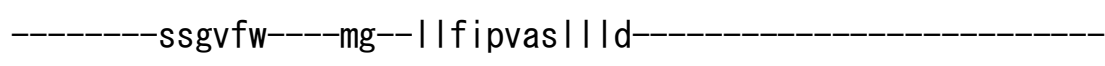

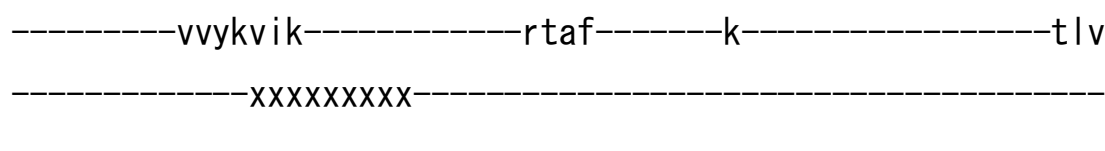


>hsalV_IV03 NP_005594. 1

$\mathrm{m}$

$-X X X X X X$

$x x x x x x x x x x x x x x x x x x x x x x x x x x x x x x x x x x x x x x x x x x x$ n

---twqvkand-

$x x x x x x x x x x x-$

xxxxxxxeskyannaiktykyn

af

-tfipmn I feqf 


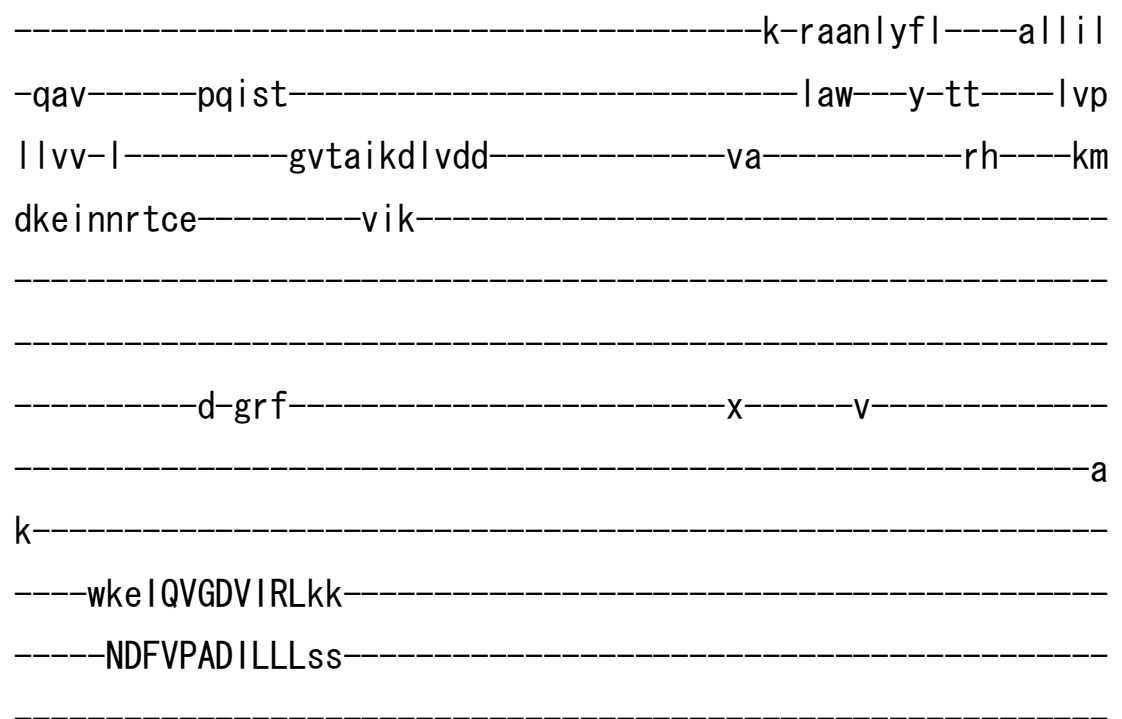

-sepns-I---cyVETAELDGETNLKFKms-

-xe i tdqy l qredt I atfdgf i eceepnnr I dkftgt I fw--

-rntsfp

Ida

$d-$

KILLR--GCVI

gLVIFAGADTKIMknsgktrf

nt-----dfch-

-krtkidy Imnymvyt i fvv I i I lsag la

-i ghayweaqvgnss-------wy l y-----------xxxxxtpsyrgf I i fwgy I IVLNTM

VPISLYVSVEVIRLGQSH-F I nwd I qm

yyaekdtp

aKAR-TTTLNEQLGQIHYIFSDKTG

TLTQN IMTFk -kcc ingq iy

$x x x x x x x x x x x x x x$

$-x \times x x x--x x x x$

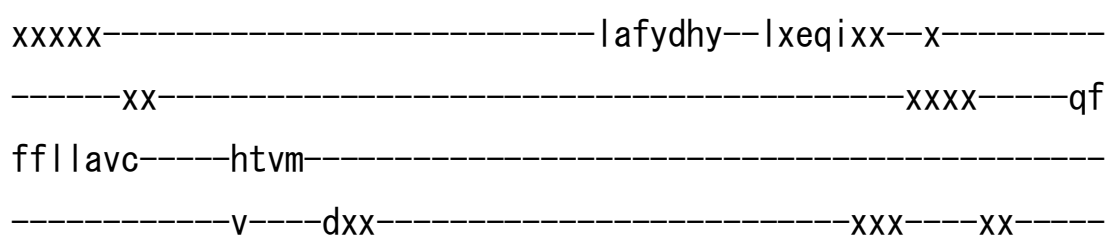




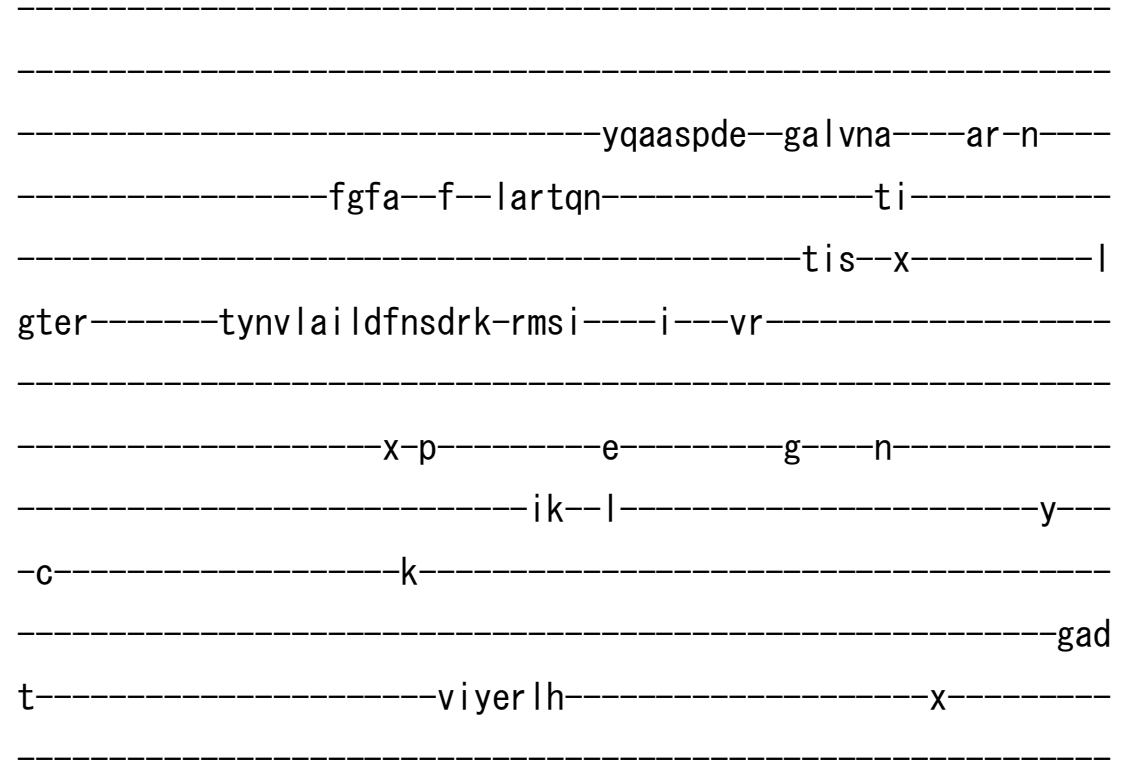

xxx--xx--qet-qdal d if--ane-----t I r tLCLCYKe i--eekeftewnkkfma--as-----vastnrdea I dkvyee-i ekd I i ILGATA IEDKLQDGVPET ISKLAKad-IK I WVLTGDKKETAENIGFACEL- tedttic----

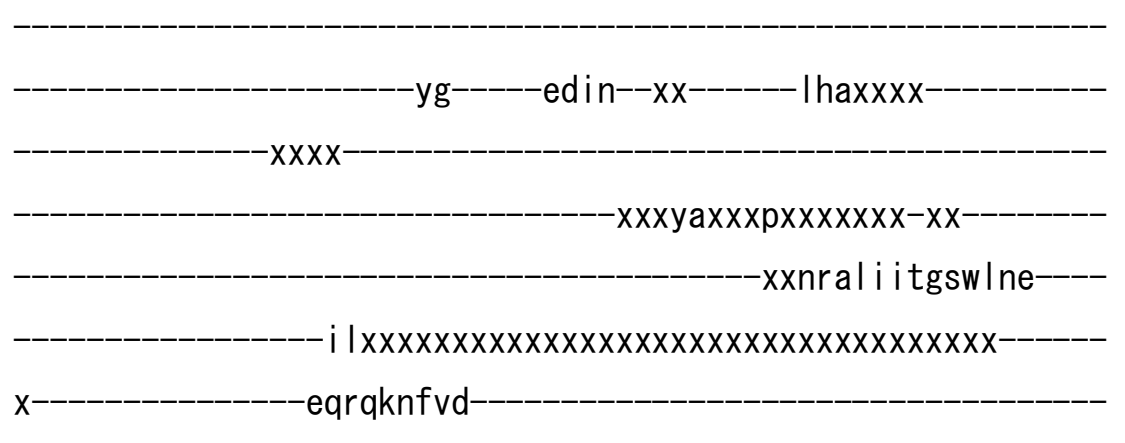
I acecsav ICC--RVTPKQKAMVVDLVk

---ry--kk--_-_-_-_---a--_--_-_-_----ITLA I GDGANDVNM I KTAHI GVGI--




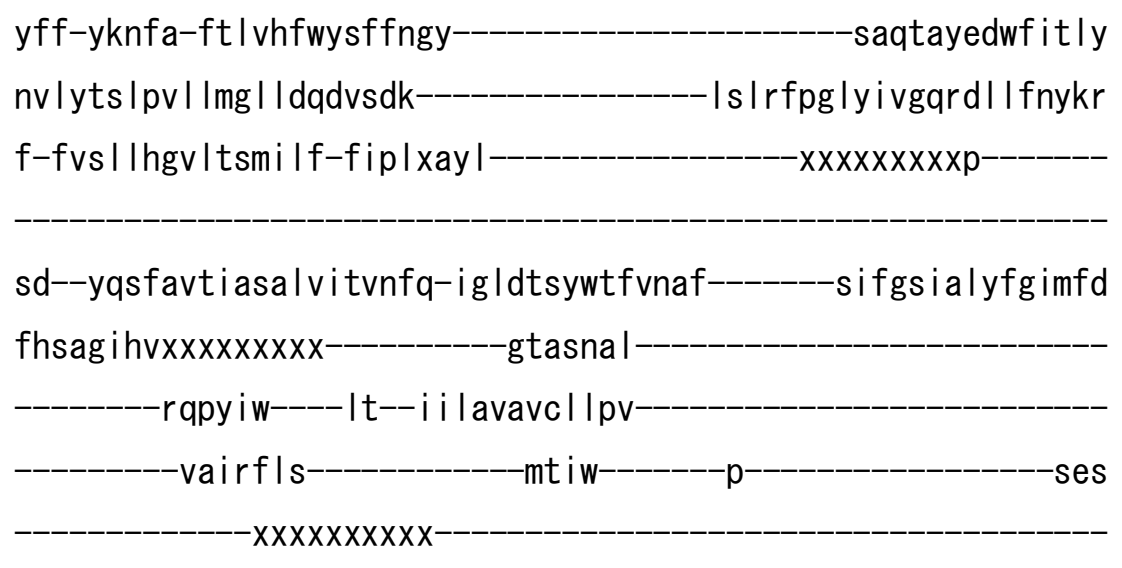

$-x x x x x x x x x x q v f r r g v s x x x x x x x x x x x x x x x x$

\section{$\operatorname{xXXXXXXXXXXX}$}

$x \operatorname{xx} x x x x x x x x x x x x x x x x x x$

>hsalV_IV04 NP_065185.1

m- 


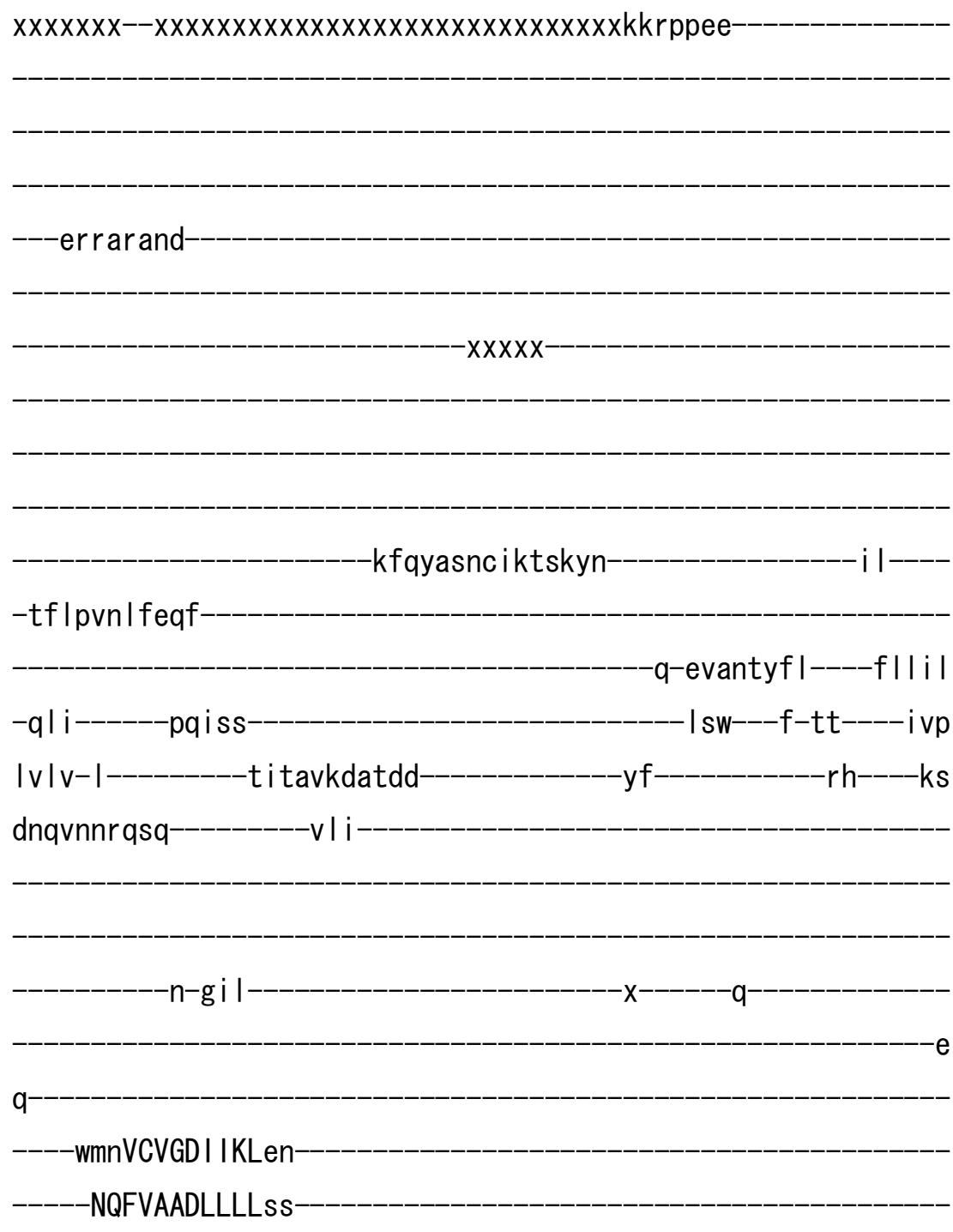

-sephg-I---cy IETAELDGETNMKVRqa-

-xpvtse I g-di sk lakfdgev i ceppnnk I dkfsgt l yw--

kenkfp

Isn-

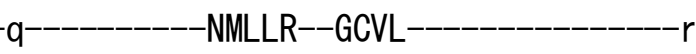

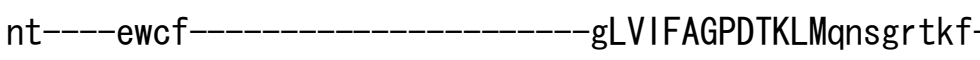

-krtsidr Imnt I I wi fgf I vomgvi la

-i gna i wehevgmrf------xvy Ip---_------xxxxxxsaffsgf I sfwsy I I ILNTV

VPISLYVSVEVIRLGHSY-F I nwdkkm- 
fcmkkrtp

aEAR-TTTLNEELGQVEYIFSDKTG

TLTQN IMVFn

-kcs inghsy

$X X X X X-X X X X X X X X X X X$

$-x x x x x--x x x x$
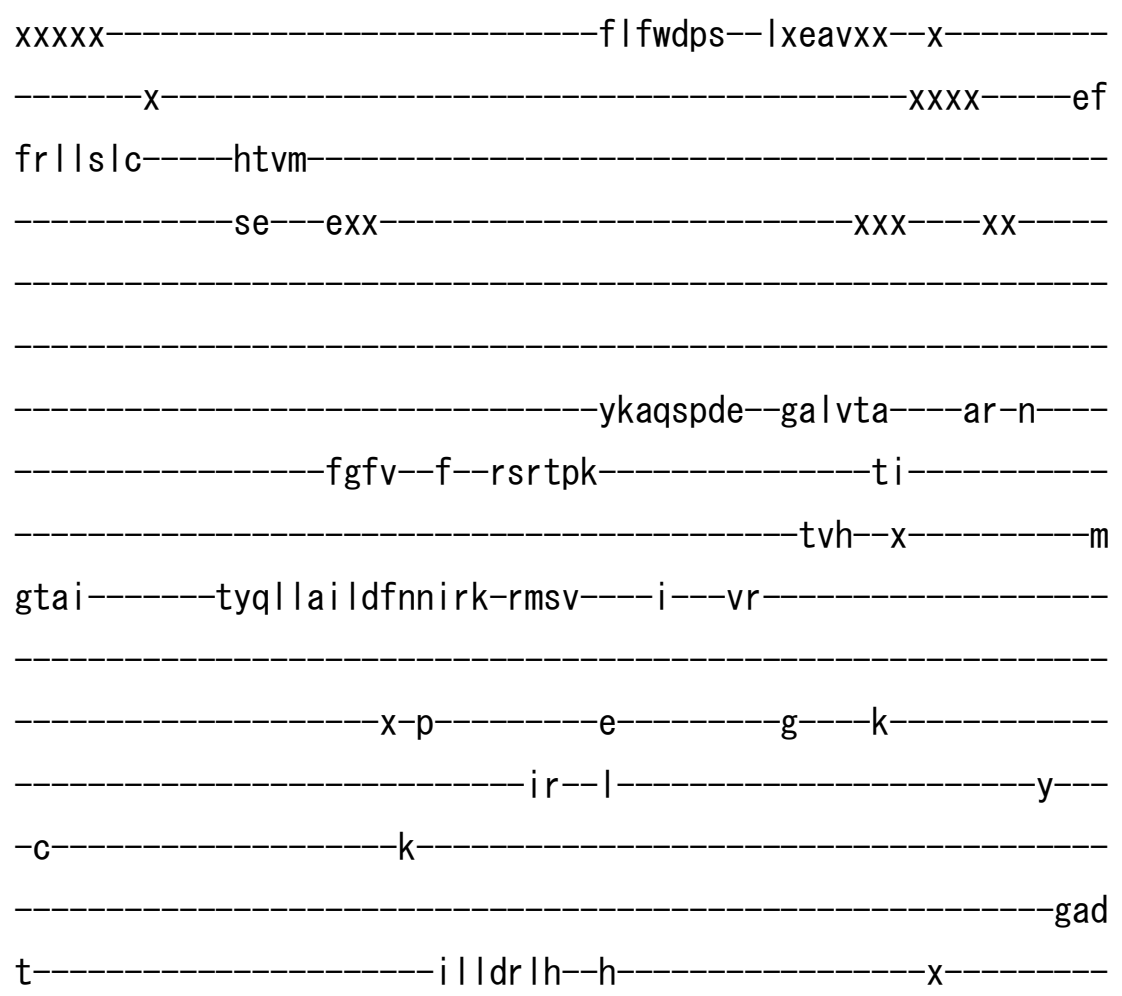

xxx--xx--ntt-mdh I ney--age----g $\mid r$ tLVLAYKd I--deeyyeewaer $r \mid q^{---}$ as----- laqdsredr l as i yee-vennmm I LGATAIEDKLQQGVPET I ALLTLan-IK I WVLTGDKQETAVN IGYSCKM-_-_-_-_-_-_-_-_-_-_-_-_-_-_-_--_I tddmtev-_--

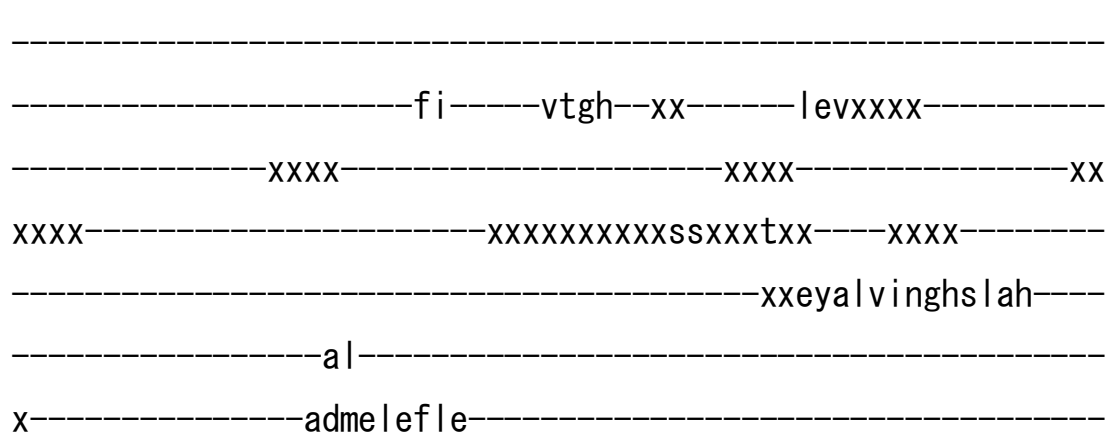


tacackav ICC--RVTPLQKAQVVELVk-

----ky--kk---_--------a----_--_--_--VTLAI GDGANDVSM I KTAHI GVGI--

-AVLASDYSFSqfKFLQRLL--LVHGRWSYLRMCKFL------C

yff-yknfa-ftmvhfwfgffcgf-_-_-_-_-_-_-_-_-_-_-_-_-_aqtvydayfitly ni vyts I pv l amgvfdqdvpeq--_-_-_-_-_-_-_-_ smeypk I yepgq In I I fnkre f-ficiagg i ytsv Imf-fi pyxvfa---------_--------xxxxxxxxx|------ad--yqsfavtvats I vivvsvq-ig I d tgywta inhf---_----fi iwgs I avyfa i I fa

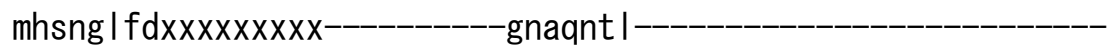

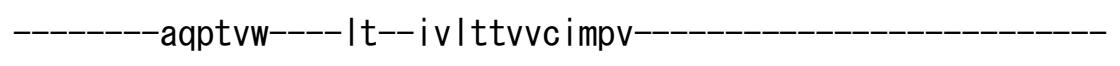

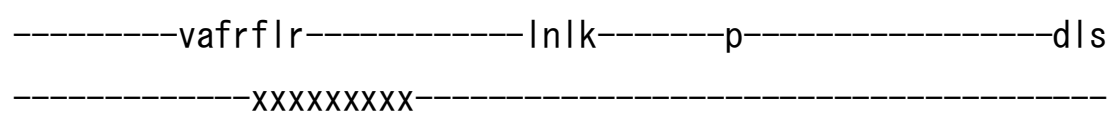
$-x x x x x x x x x x m r r v g r t g x x x x x x x x x x x x x x x x$ $x x x x x x x x x x x x x x x x-$ $-x x x x x---x x x x x x x x x x x x x x x x x x x x x x x x x x x x x x x-$ >hsalV_IV05 NP_620168. 1 $\mathrm{m}-$ 
- $X X X X X X X X X X X X X X X X X X X X X X X X X X X X X X X X X X X X X X X X X X X X X X X X X X X X X X X X X X X$

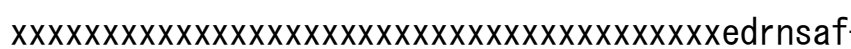

$-s f|p| n \mid$ yeqf

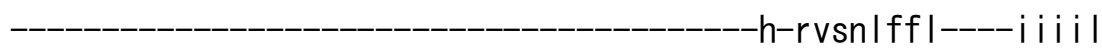

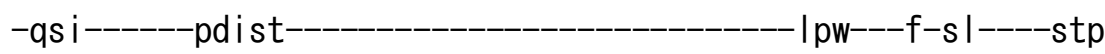

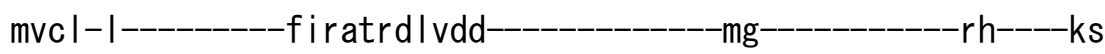
drainnrpcq----------i Im-

k

----wqdLCVGDVVCLrk-

-DNIVPADMLLLas 
-tepss-I---cyVETVDIDGETNLKFRqa-

-xmvthke lat ikkmasfagtvtceapnsrmhhfvgc l ew--

-ndkkys

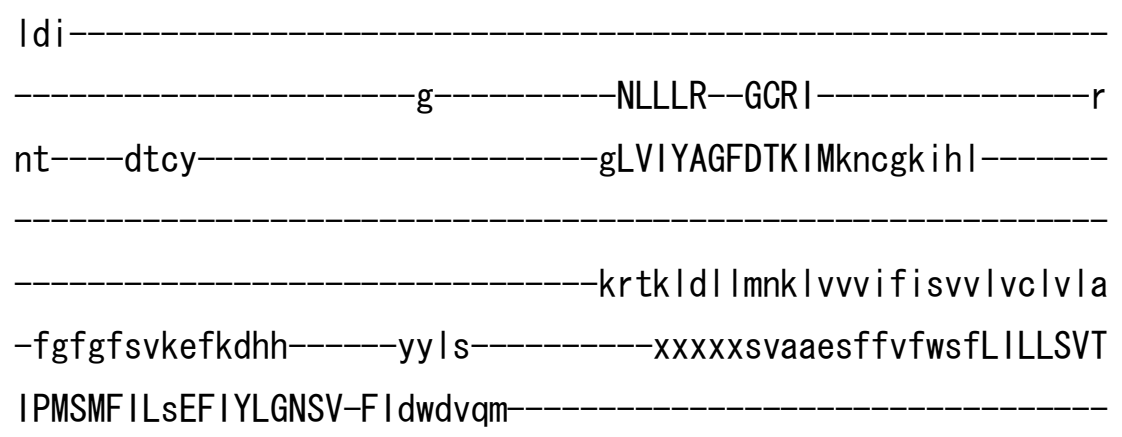

-yykpqdvp-

-aKAR-STSLNDHLGQVEYIFSDKTG

-TLTQNILTFn-

kccisgrvy-

$x x x x x x x x x x x x x x$

$-x--x x x x$

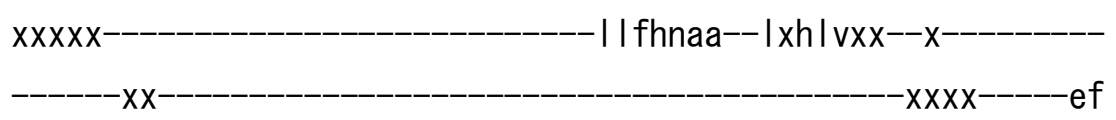

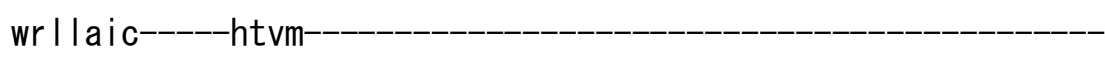

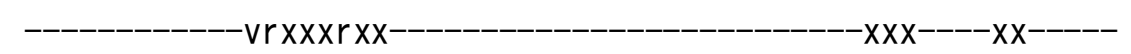

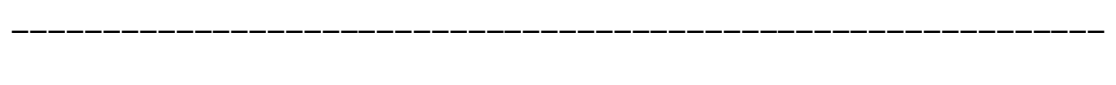

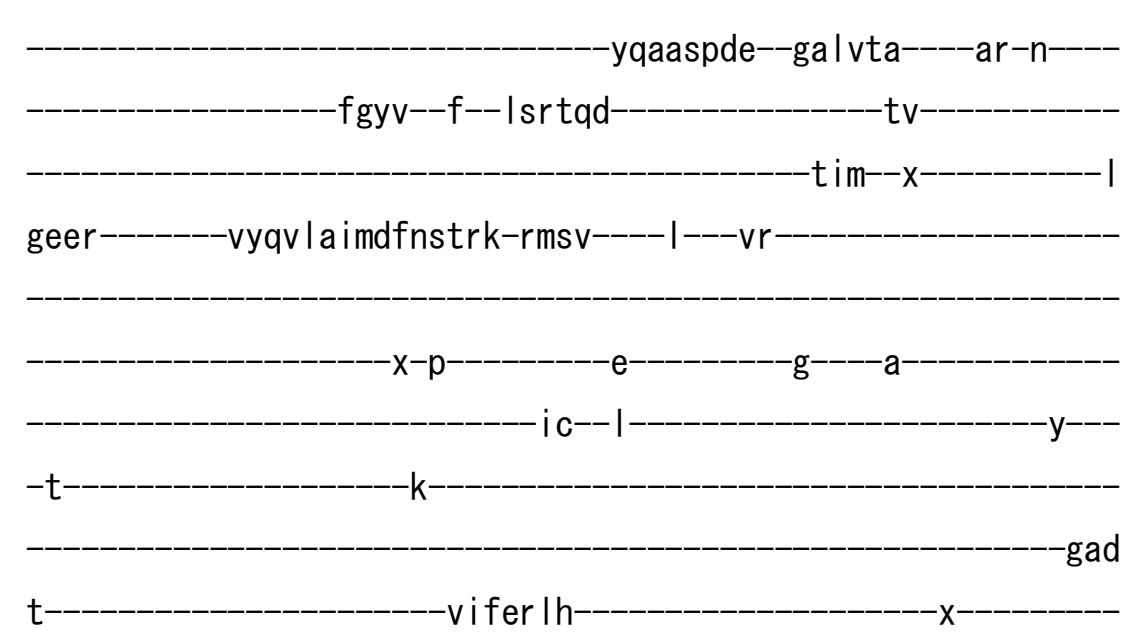


xxx--xx--fat-eea laaf--aqe----t I r tLCLAYRev--aedi yedwqqrhqe--as------ I I qnr aqa I q-----------q I LGATA IEDRLQDGVPET IKCLKKsn-IK I WVLTGDKQETAVNIGFACEL| senml i |----xxk la l vingdf I dkxxxx

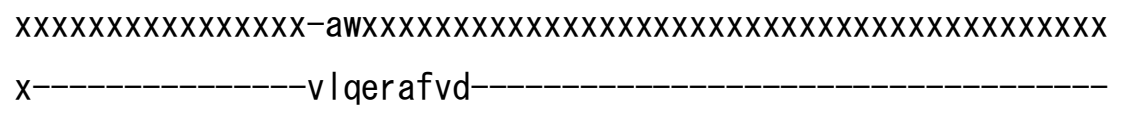

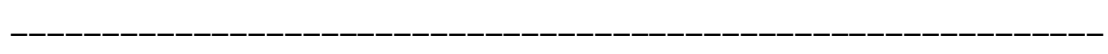

laskcqav ICC--RVTPKOKAL IVALVk

---ky--hq-----------v-------_------VTLA I GDGAND I NM I KTADVGVGL--

AVQNSDFVLGGFCFLQRLL--LVHGRWSYVRI CKFL------R

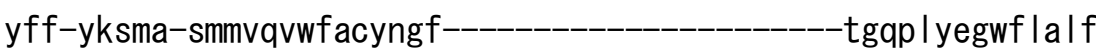
nl lyst I pv lyig I feqdvsae---_-_-_-_-_-_---qs l ekpe l yvvgqkde I fnywv f-vqa i ahgvtts I vnf-fmt I x i sr-_-_--_-_-_-_-_-_-_--xxxxxxxf-_-_---sd--hqsfavvvalsc I I si tme-vi I i ikywtalcva---_----ti I Is I gfyaimttt

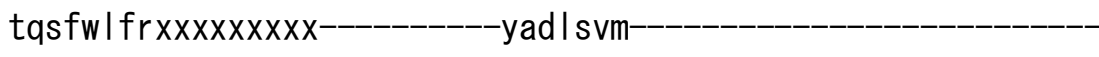

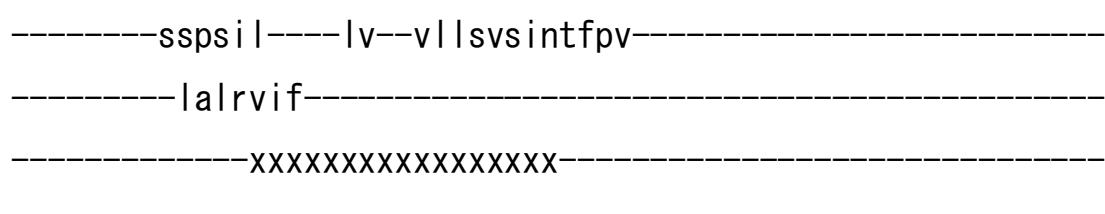
xxxxxxxxxxxphvhresrxxxxxxxxxxxxxxxx 
>hsalV_IV06 NP_079113. 2

$\mathrm{m}$

xxxekk|rev

---er i vkand-

$-\mathrm{xxxxx}$

-kfayadnr ihtskyni I

-tflpinlfeqf 


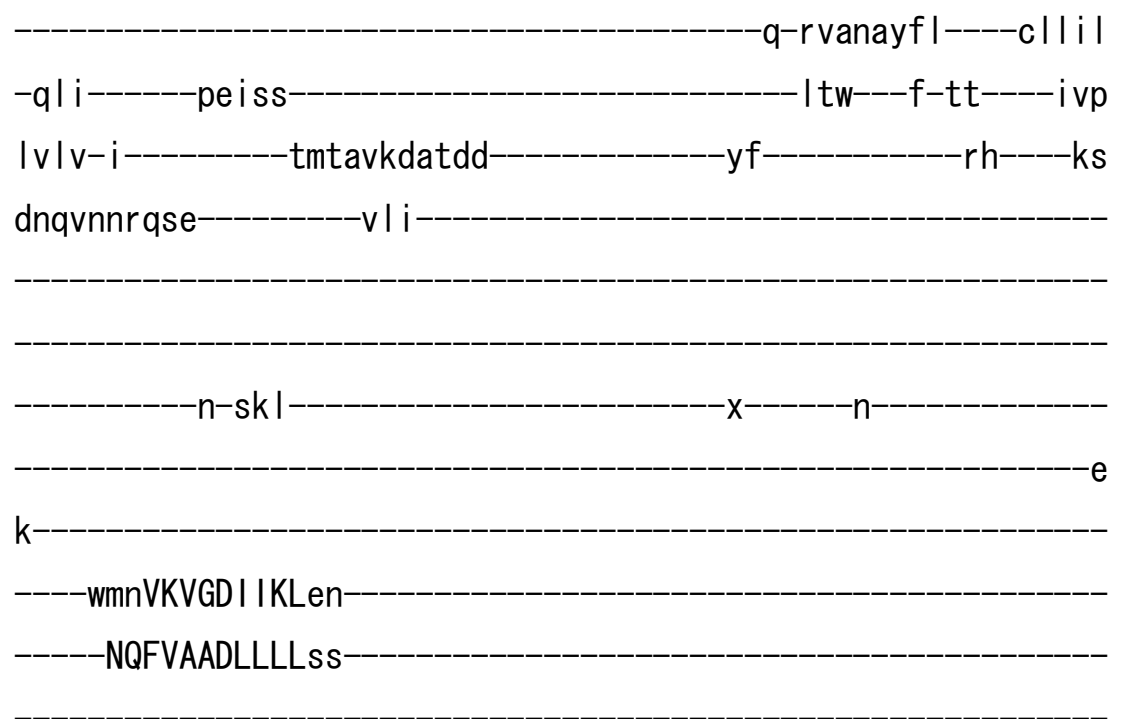

-sephg-I---cyVETAELDGETNLKVRha-

-xsvtse I gad i sr I agfdg i vvcevpnnk I dkfmg i I sw--

$-k d s k h s$

Inn-

e-

$-\mathrm{KI}$ ILR--GCIL

nt----swcf

gMVIFAGPDTKLMqnsgktkf

-krtsidr Imnt Ivlwifgfliclgi ila

-igns i wesqtgdqf------xtf If----------xxxxxxssvfsgf I tfwsy I I ILNTV

VPISLYVSVEVIRLGHSY-F I nwdrkm-

-yysrkaip

aVAR-TTTLNEELGQIEYIFSDKTG

TLTQN IMTFk rcsingriy-

$x x x x x-x x x x x x x x x x x x-$

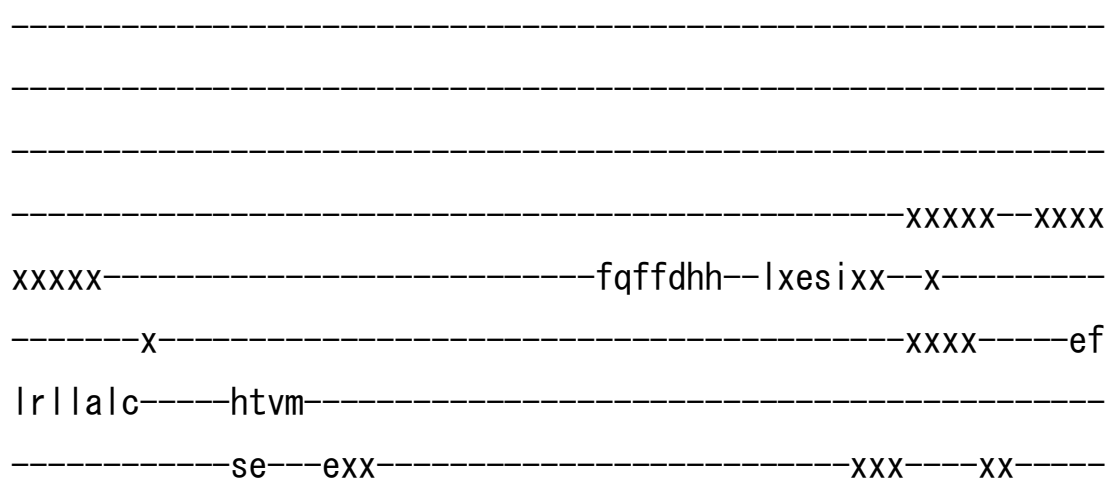




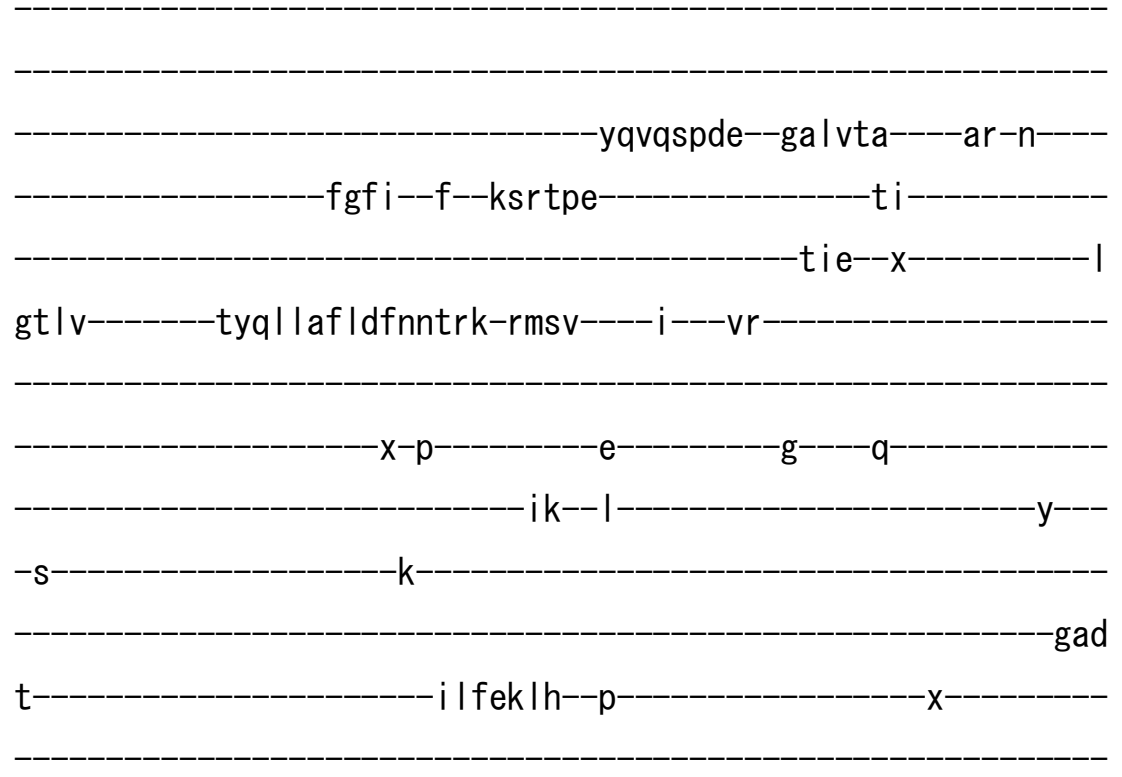

xxx--xx--s |t-sdh I sef--age----g | r tLAIAYRd I--ddkyfkewhkm l ed--an------aateerder i ag I yee-i erd ImI LGATAVEDKLQEGVIETVTSLSLan-IKI WVLTGDKQETAINIGYACNM-

Itddmndv----

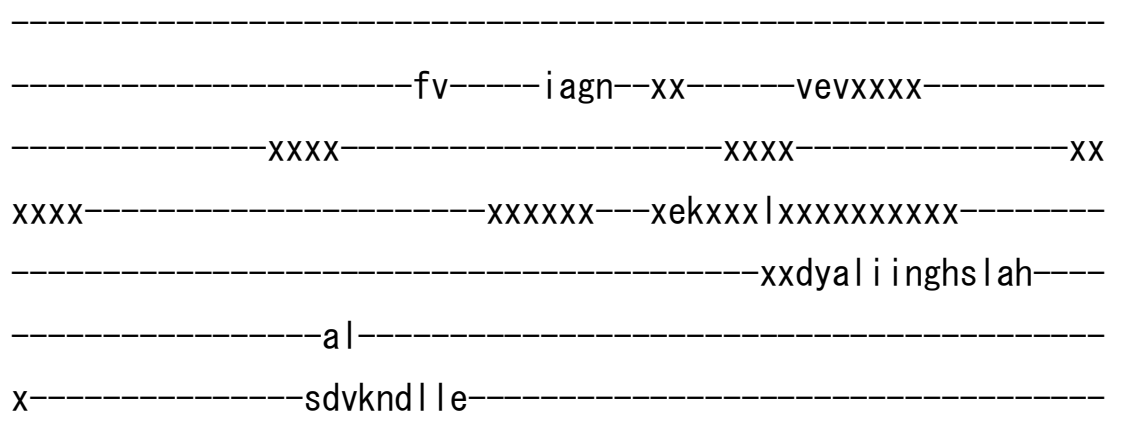

lacmcktv ICC--RVTPLQKAQVVELVk

---ky--rn-----------a---_-_-_-_--VTLA I GDGANDVSM I KSAH I GVG I--

-AVLASDYSFAqfRYLQRLL--LVHGRWSYFRMCKFL------C 
yff-yknfa-ft I vhfwfgffcgf-

saqtvydqwfit If

ni vyts I pv l amg i fdqdvsdq

nsvdepq lykpgq In I I fnkrk

f-ficvlhgiytsIvIf-fipyxafy

$x x x x x x x x i$

ad--yqsfavtmats I vivvsvq-ialdtsywtfinhv-

fiwgsiaiyfsilft

mhsng ifgxxxxxxxxx

gnarhs I

-tqkciw----Iv--i I Ittvasvmpv

-vafrflk--_-_-_-_----vdly

$-p$

$\mathrm{t}$ /s

$-X X X X X X X X$

xxxxxxxxxxxprtrrssxxxxxxxxxxxxxxxx

$\operatorname{xx} x \operatorname{xx} x x x x x x x x x x x$

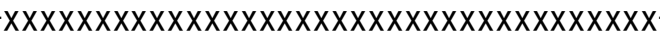

>hsalV_IV07 NP_006036.1

m- 
-- prtvw Igh-

-xxxxdqryprnvinnqkyn-

-tf Ipgv I fnqf

$-k-y f f n l y f I----I$ lacs

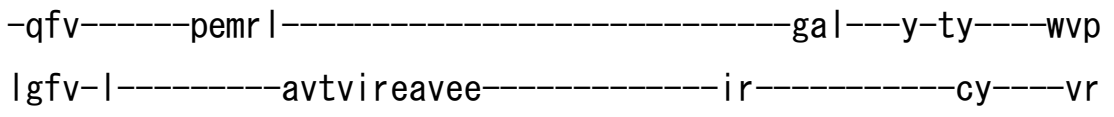

dkevnsqvys------_---rit-

-sekng-s---cfLRTDQLDGETDWKLRIp-

-vactqr I ptaad I I qi r syvyaeepni dihnfvgtftrxx

$\mathrm{XX}$

pp ises

Isi

e-

-NTLWA--GTVV-

as----gtvv-

gVVLYTGRELRSVmntsnprs

-kig Ifdlevnc Itki I fgalvvvs Ivmv

-alqhfagr

wy $\mid q-$

i irfLLLFSN I

IPISLRVNIDMGKIVYSW-VI rrdsk i 
-tVVR-SSTIPEQLGRISYLLTDKTG

-TLTQNEMIFk---------------rlhlgtvay

$-x X X X X X X X X X X X X$

$-X X------X X X$

$\mathrm{xx}----------x x--x x x x x x x--x x x x x x x x m s s r-v h-$

-ea

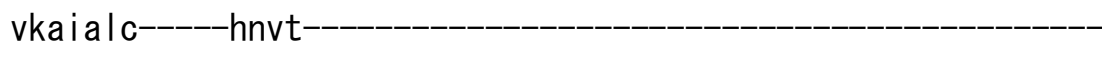
$p \vee x \times x$

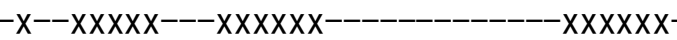

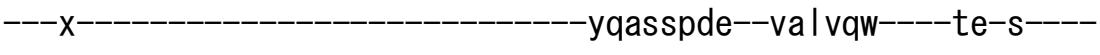

-vglt--|--vgrdqs----------------sm-

q 1

rtpg-xxxx--nfti lqifpftyesk-rmgi----i---vr

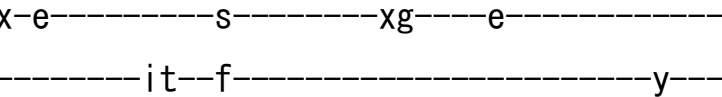

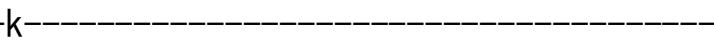

-gad

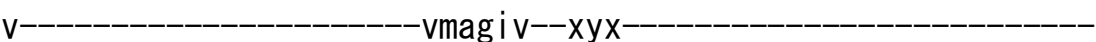

-dw I-eeecgnm--are----g | rvLVVAKKs I--aeeqyqdfearyvq---

ak-----I svhdrs I kvatvi es- I ememe ILCLTGVEDQLQADVRPTLETLRNag-IKV WMLTGDKLETATCTAKNAHL-_-_-_-_-_-_-_-_-_-_-_-_-_-_-_--vtrnqdih-_-_

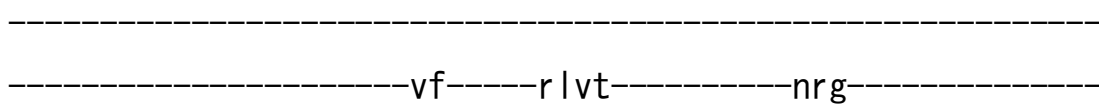

$\operatorname{exx} x x x x x x x x x x$

-dcalvi sgds l ev---cl

kyyeyefme 
I acqcpavVCC--RCAPTQKAQIVRLLq-

---er--tg--_--------x------_--_--_--LTCAVGDGGNDVSM I QESDCGVGV--

-ASLAADFSI TqfKHLGRLL--MVHGRNSYKRSAALS------Q

fvi-hrs Ic-is tmqavfssvfyf-_--_-_-_-_-_-_-_-_-_---asvp I yqgf I i igy st i ytmfpvfs I-vI dkdvkse---_--_-_-_-_-_---vam l ype lykd I I kgrp I sykt

f-l iwv I i i yqgst im-ygal-I If-_-_-_-_-_-_-_-_-_-_-_-_-_e--_-_-_

se--fvhivaisftsli tel Im-valt iqtwhw Imtv---_----ael I s lacyias I vf

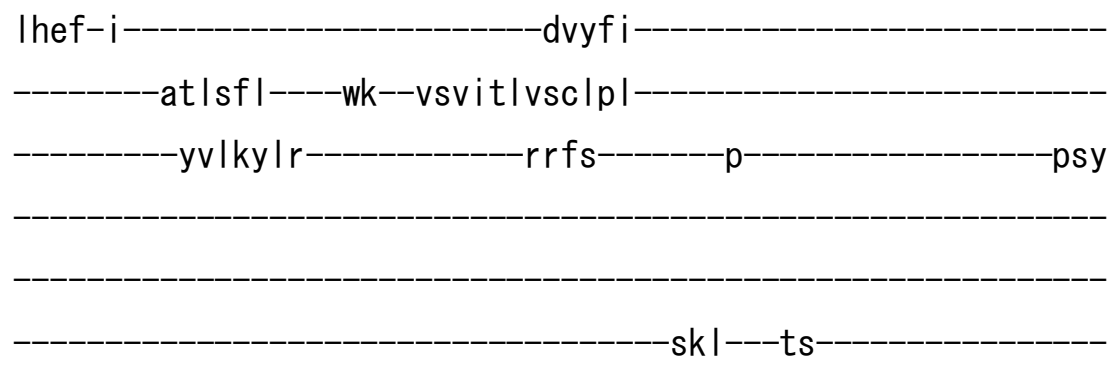

$-$

>hsalV_IV08 NP_940933. 3

$\mathrm{m}-$ 


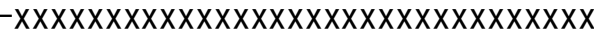

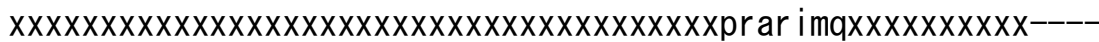

$-x x x x x x x x x x x x x x x x x x x x x x$

$\mathrm{xXX}$

$---\operatorname{artvw} \operatorname{gc}$

-xxxxeekhprnsiknqkyn

$-v f----$

-tfipgvlyeqf

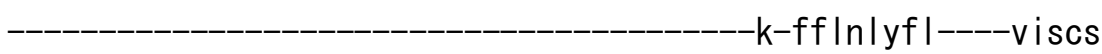

-qfv------palki----------------_------------gyl----y-ty----wap

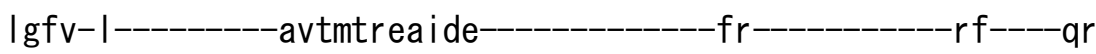

dkevnsqlys----------k lt

-vxgkv-

q

$\mathrm{k}-$

----ssd IQVGDL I I Vek

-NQRIPSDMVFLrt 
-sekag-s---cfIRTDQLDGETDWKLKva-

-vsctqq I pa I gd I fs i sayvyaqkpqmd ihsfegtftrxx

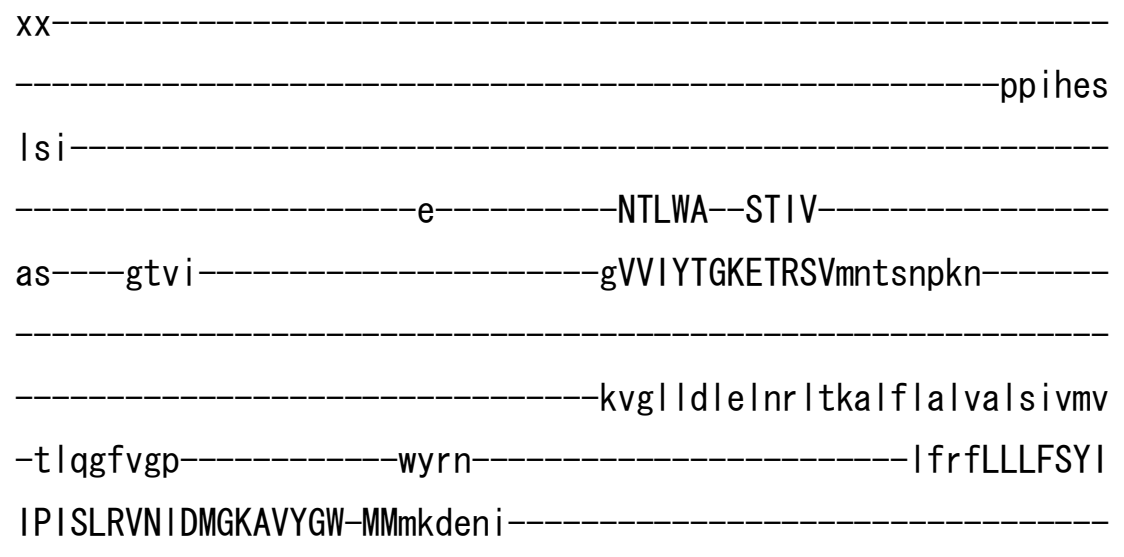

$-p g$

-tVVR-TST IPEELGRLVYLLTDKTG

-TLTQNEMIFk--------------r IhIgtvsy

$x x x x x x x x x x x x x x$

$-x X------x x x$

$x x-x x x x x x x x x x--x x x x x x x--x x x x x x x x v s s r-i h-$

-ea

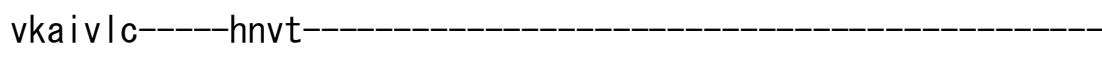

$-p \vee x x x$

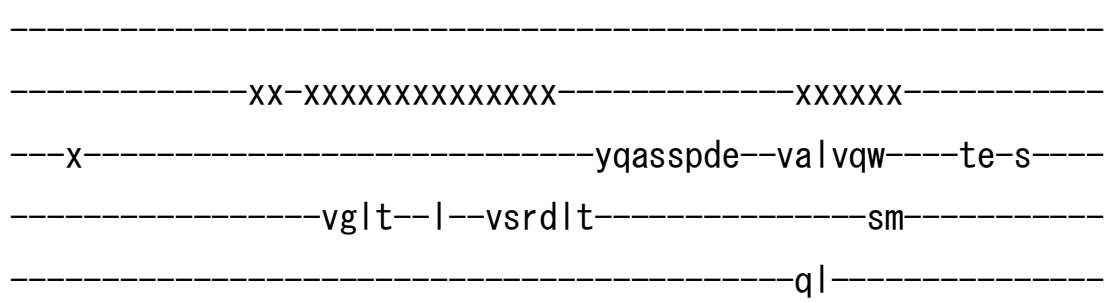

ktps-xxxx--sfci lqlfpftsesk-rmgv----_----vr--_-_-_-_-_-_-_-_-_-_

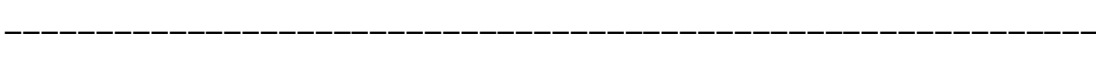

-m-------------------- k--------------------------------------

v-----------_------------amspi v-- xyx 
-dw I-eeecgnm--are----g|rtLVVAKKa I--teeqyqdfesrytq--ak----- I smhdr s I kvaavves- I ereme I LCLTGVEDQLQADVRPTLEMLRNag-IK I WMLTGDKLETATCI AKSSHL-vsrtqdih----

--ー-ー-ー-ー-ー--ー-ー-ー if

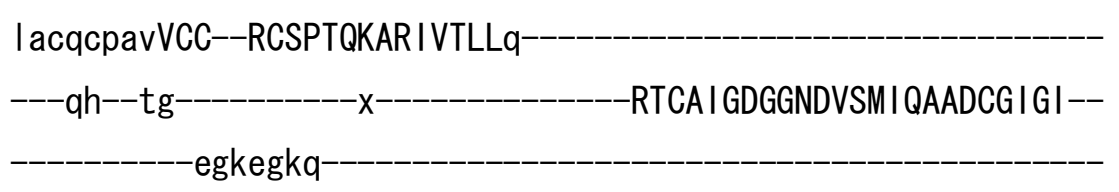

-ASLAADFSI TqfRH I GRLL--MVHGRNSYKRSAALG------Q

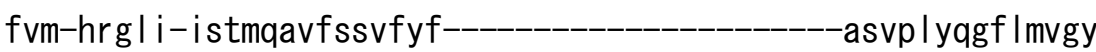

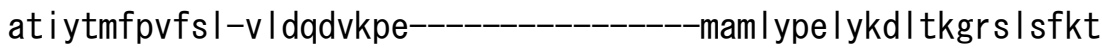
f-l iwv l i s i yqgg i Im-yga l-v l f-_-_-_-_-_-_-_-_-_-_-_-_-_--_e--_-_-_ se--fvhvvaisftal i Itel Im-valtvr twhw Imvv-------aef I I gcyvss laf

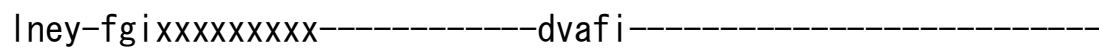

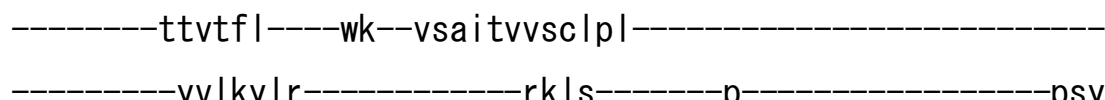
ckl---as 
>hsalV_IV09 NP_077816.1

m-

$-\operatorname{xxxxxxxxxxxxxx----xrrrregr}$

---trtvrsnI

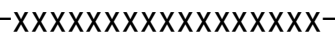

xxxxxxxaqh ladnr Ikttkyt $-11$

-sflpkn I feqf 


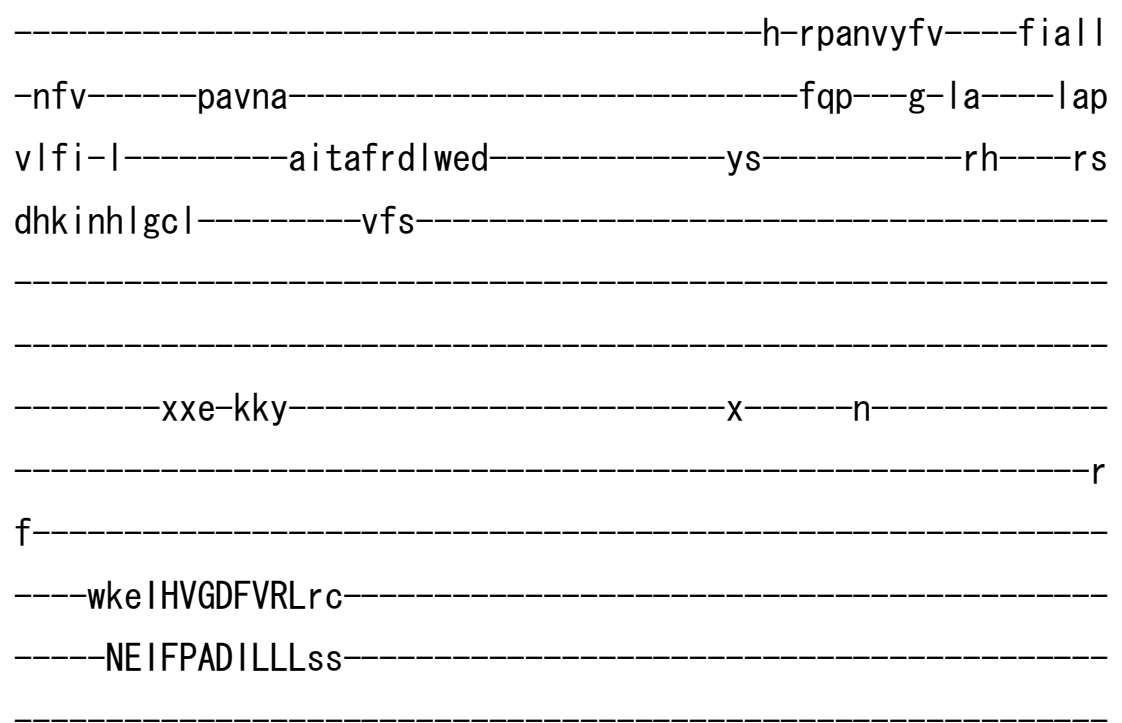

-sdpdg-|---chIETANLDGETNLKRRqv-

-vrgfselvsefnpltftsvi ecekpnnd Isrfrgci ihx-

-ngkkag

lyk

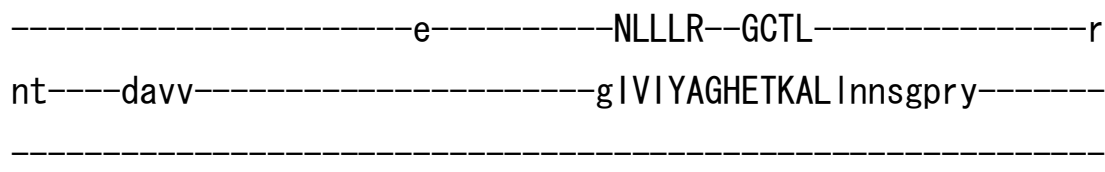

-krsk leramncdv Iwcv I I |vcms Ifsa

-vghg I w i wryqekk-----xxfyvp---------xxxxxxxspvtaavysf I tmI IVLQVL IPISLYVS iEIVKACQVY-FInqdmq I

-ydeetdsq

IQCR-ALN I TEDLGQIQY IFSDKTG

TLTENKMVFr -rctvsgvey

$x \times x X X X X X X X X X X X X X X X X$

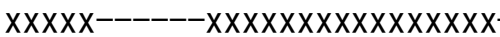

$-x x x x x--x x x x$

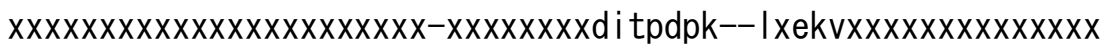

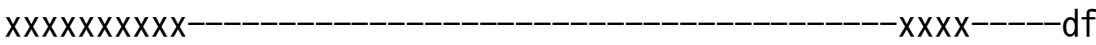

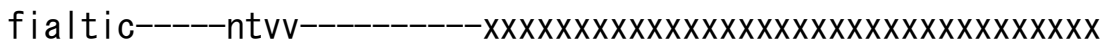

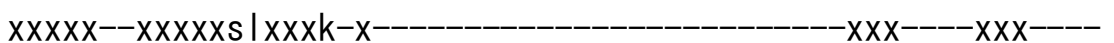




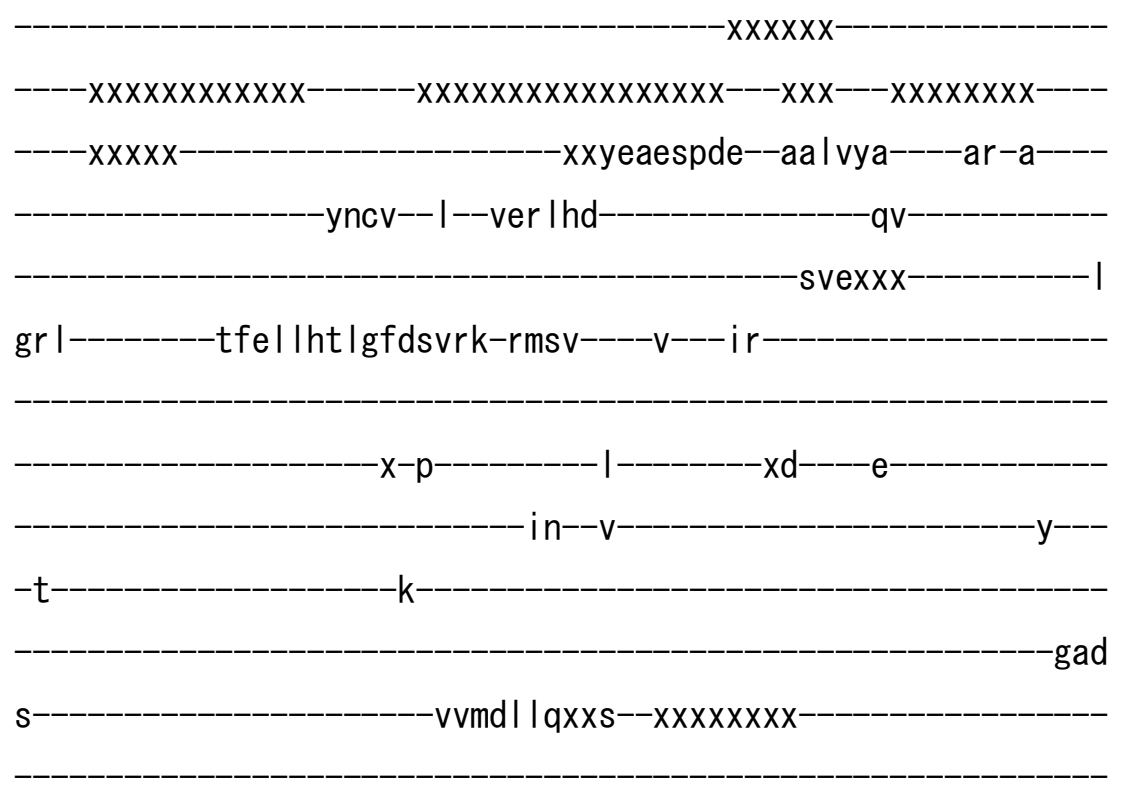

xxx--xx--skt-qny | nvy--aae----g|rtLCI AKRv |--skeeyacw I qsh le--ae-----ss l ensee I I fqsa i - l etn I hILGATG IEDRLQDGVPET ISKLRQag-LQ I

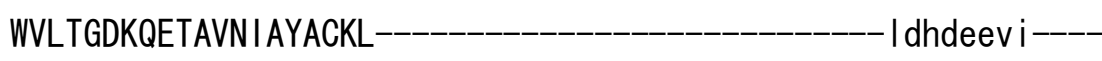
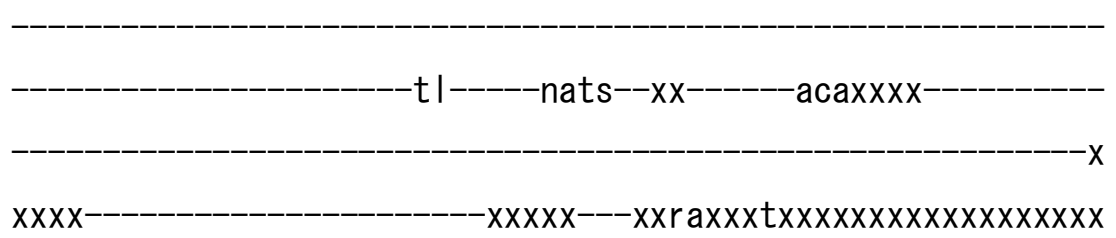

$$
\begin{aligned}
& \text { xxxxxx-----------------------------_---------s l vidgr s lay---- } \\
& \text { al } \\
& \text { x---------------knledkf If- }
\end{aligned}
$$

lakqcrsVLCC--RSTPLQKSMVVKLVr

---sk-- |k--_-_-_-_--_a--_-_-_-_-_--MTLA I GDGANDVSM I QVADVGVGI--


yff-ykntm-fvgl Ifwfqffcgfsastmi dqwy l iff

nl Ifss I pp I vtgv Idrdvpan--_-_-_-_-_-_--v I I tnpq lyksgqnmeeyrprt

f-wfnmadaafqs I vcf-sipy-lay---------------xxxxx----------------

-d--|ftwgtpivtial| If I|h-|gietktwtw|nwi--_-_--tcgfsv IIfftval i

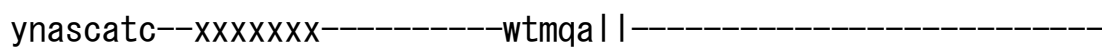

-gdpvfy----|t--c|mtpvaal Ipr--_-_-_-_-_-_-_-_-_-_-_-_-_

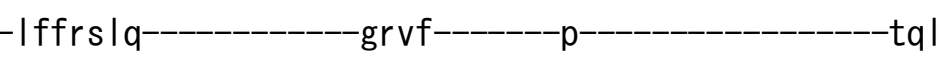

$-x x x x x x x x x x x x x x x x x x x x x x x x x x x x x x x x x x x x x x x x x x--x x x x$

$-x x x x x x x x x x p v c s \mid$ eas $x x x x x x x x x x x x x x x x x$

$x x x x x x x x x x x x x x x x$

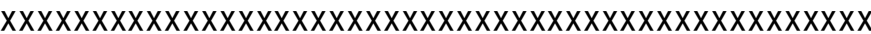

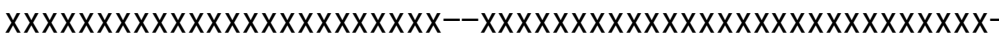

>ggalV_IV10 XP_416948. 2

$\mathrm{m}$ 
---srtiyvgh-

$-x x x x x x x x x x x-$

-pqrfpdnr ivsskyt $-\mathrm{fw}----$

-nfipknlfeqf

-_-_-_-_-_-_-_-_-_-_-_-_-_-_-_

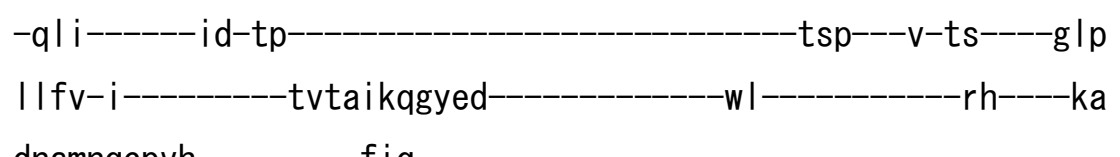

dnamnqcpvh----------fiq

$-k$

q

----srkLRVGD IVMVke-

DETFPCDLIFLss

-srgdg-t---cfVTTASLDGESSHKTHya-

-vqdtkafhseqe i da I hat i eceqpqpd I ykfvgr i nvxx

$x x x$

eptarp

Igs

-NLLLR--GATL-----------------k

nt----ekif

gVAIYTGMETKMAInyqsksq

-krsaveksmnvfl ivylciliskalint

-v l kyvwqsepfrde------xwynq--------xxxxxxxxnqf I qaftdf I afMVLFNY I

IPVSMYVTVEMQKFLGSY-FLtwdeem- 
pLVN-TSDLNEELGQIEYVFTDKTG

TLTENNMEFv

eccieghvy

$-x x x x x x x x x x$ Ihdctgixxixsspxxxxx

$-x \times x x$

fralclc-----htvq--_-_-_-_-_-_-_-_-_-_-_-_-_-_-_-_-_-_-_-- $x \times x x-$

$--x x x x x x x x x-q \mid x x x s x x-$

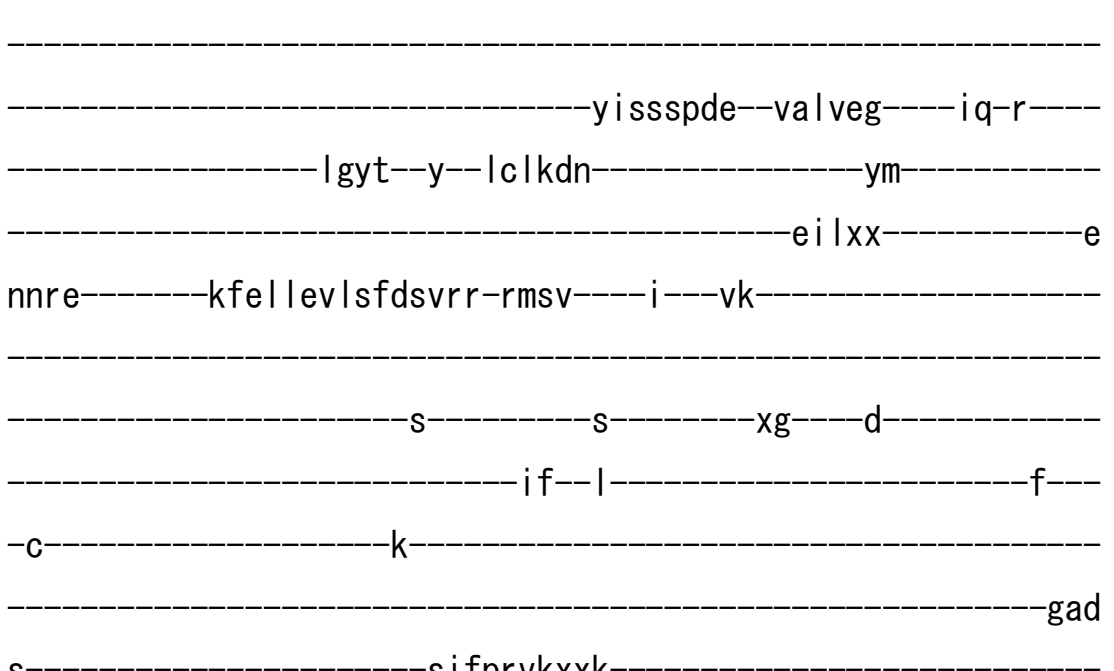

sifprvkxxk

-xdqi-rsrvern--ave----g|rtLCVAYKk I--taeeysnaqkm I qn---

ak----- la l qdrekk l aevyek-i erdf i ILGATAVEDRLQEKAADT IEALQKag-IKV WVLTGDKMETAAATCYACKL--_-_-_-_-_-_-_-_-_-_-_-_-_-_-_frntqi I--_-

el------ttkk--xx-------eqsxxxx-

xxxxxxxxxxhsxxxtxxxxxxxxxx-

$-x x d y g \mid$ i i dgaa $|s|---$

im

$-x x x x x x x$

$\mathrm{x}$

gnyre Ifle 
i crncsavLCC--RMAPLQKAQIVKL Ik-

$---\mid s--k e-$ $x p$

- ITLA I GDGANDVSM ILEAHVG I GI-igkegra

-AARNSDYAIPKFKHLKKML--LVHGHFYYVRI SELV------Q

yff-yknvc-fifpqf I yqffcgf sqqp I ydtay |t ly nisftsIpillysImeqhvsad tI kreps Iyrdvaknal I rwra f-iywtf Igvfdavvff-fgayx Ifd $x x x x x x x x x$

fg--nwtfgt IvftvIvftvtlk-laldthywtwinhf -miwgs I fyivfs|I wgg $\mathrm{i}$ i wpf--xxxxxxx $-y v f m q m I$

--------ssgpaw----| g-- i i l I itvs I lpd-

$-v|k k v| c-$ rqlw tat

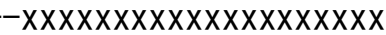

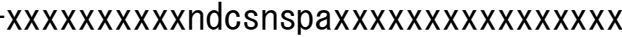

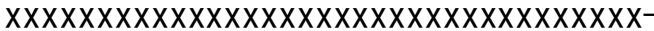

>ggalV_IV12 XP_420240. 2

$\mathrm{m}$ 
---trtvvvgh

-nf I pkn I feqf

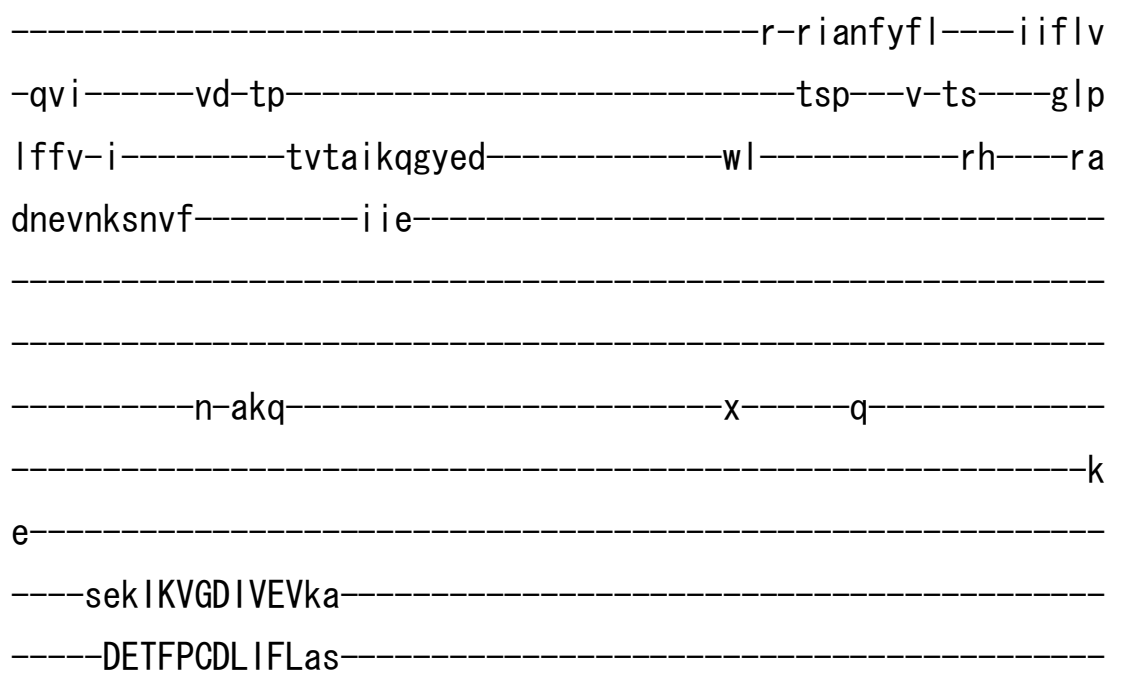


-sstdg-t---cyVTTASLDGESNFKTHya-

-vrdttv Ictdea ids I tat i eceqpqpd I ykfvgr i imxx

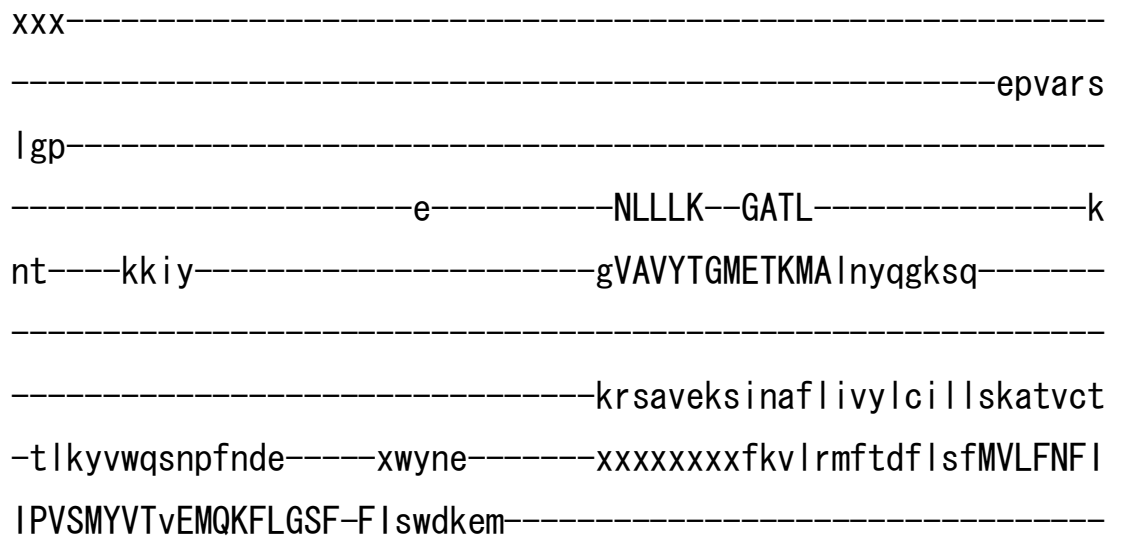

-ydeemeeg-

aLVN-TSDLNEELGQVEFVFTDKTG-

TLTENSMEF $\mathrm{i}$

ecc idghky-

$-x x x x x x d g f s q t d x x \mid-k y y x x x x x$

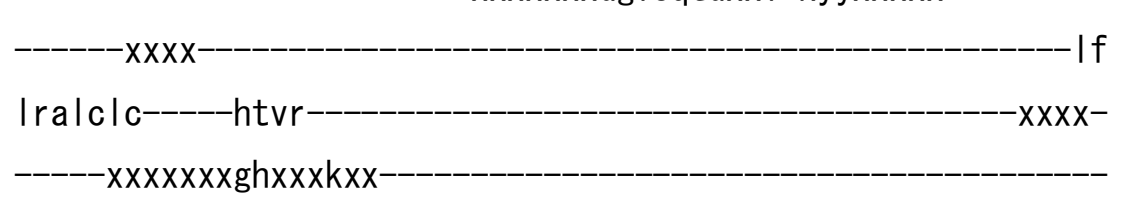

\section{If} $x-$

-

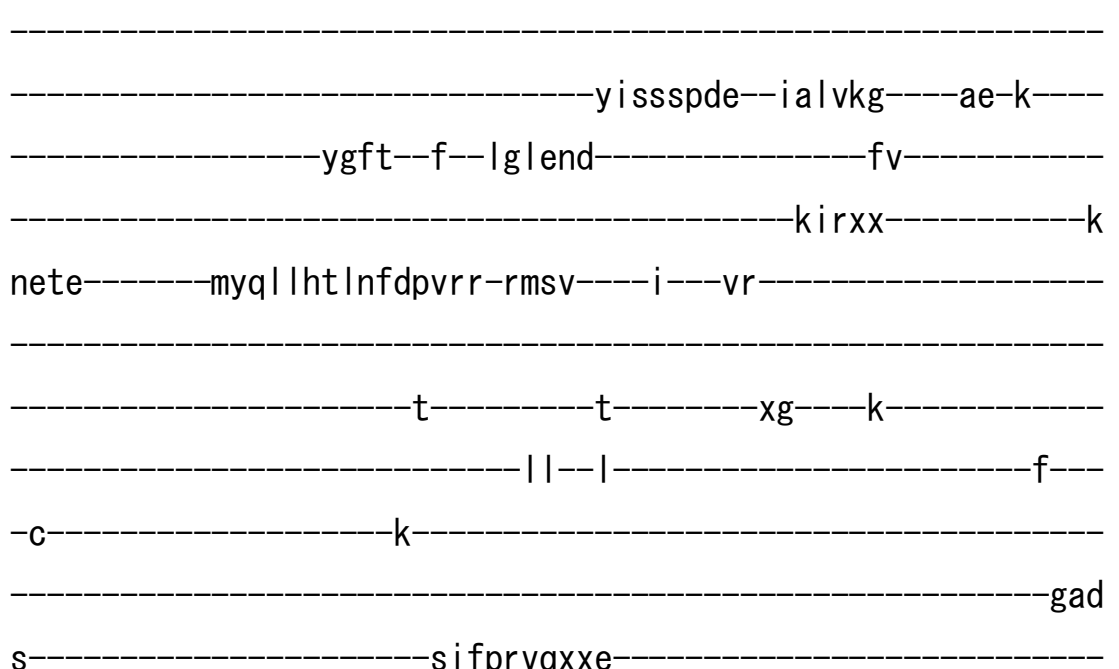


-xqqt-kvhvdrn--ald----gyr tLCVAFKe I--tqkeydr idrq Ine--ak-----mal qdreekmakvfed-teadmh I I GATAVEDRLQEQSAET I EALHAag-MKV WVLTGDKMETAKSTCYACRLfatstell----

el------tarvxxxxxxx---edrxxxx-

$-x x x x x x x x x x d v x x x r x x x x x x x x x x$

xeyg $\mid$ i idgst $|s|$

i I

$-X X X X X X$

x----_-----------shykniflq-

ic lkctavLCC--RMAPLQKAQIVRMVk

$---n t--k g-$

$\mathrm{xp}$

- ITLS I GDGANDVSMILEAHVG IGI--

-kgkegra

AARNSDYAVPkFKHLRKLL--LAHGHLYYVRI AHLV $-Q$

yff-ykn l c-fi l pqf I yqffcgf-_-_-_-_-_-_-_-_-_-_-_-_sqqp I ydaay I tmy nicfts I p i lays I l eqh in id---------------t I tsdpq I ymkvsdnam I qwrp f-I ywtf I gafeg I vff-fgvyx Ifq--_-_-_-_-_-_-_---xxxxxxxxxv---_--fg--nwtfgtivftvlvftvt|k-laldtrfwtwmnhf--_-----viwgs lafyvffsff wggv i wpf---xxxxxxx-----------fvfahml---------------------------

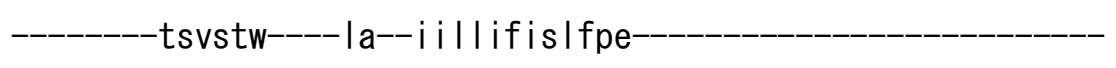

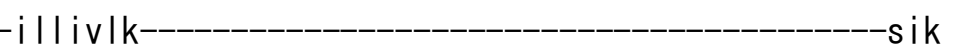




\section{>hsalV_IV10 XP_932686. 2}

m

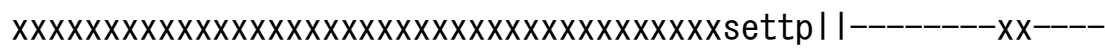
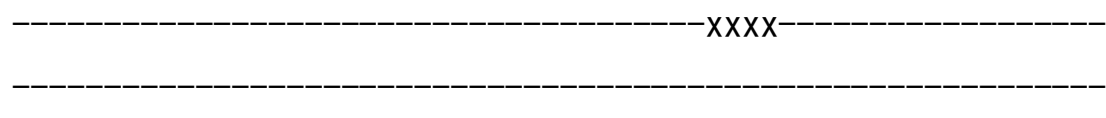

$-\mathrm{XXXXXX}$

---qrvvfpnn-

$-x x x x x x x x x x x$

rrypgnrtcttkyt

-tfiprnlfeqf 


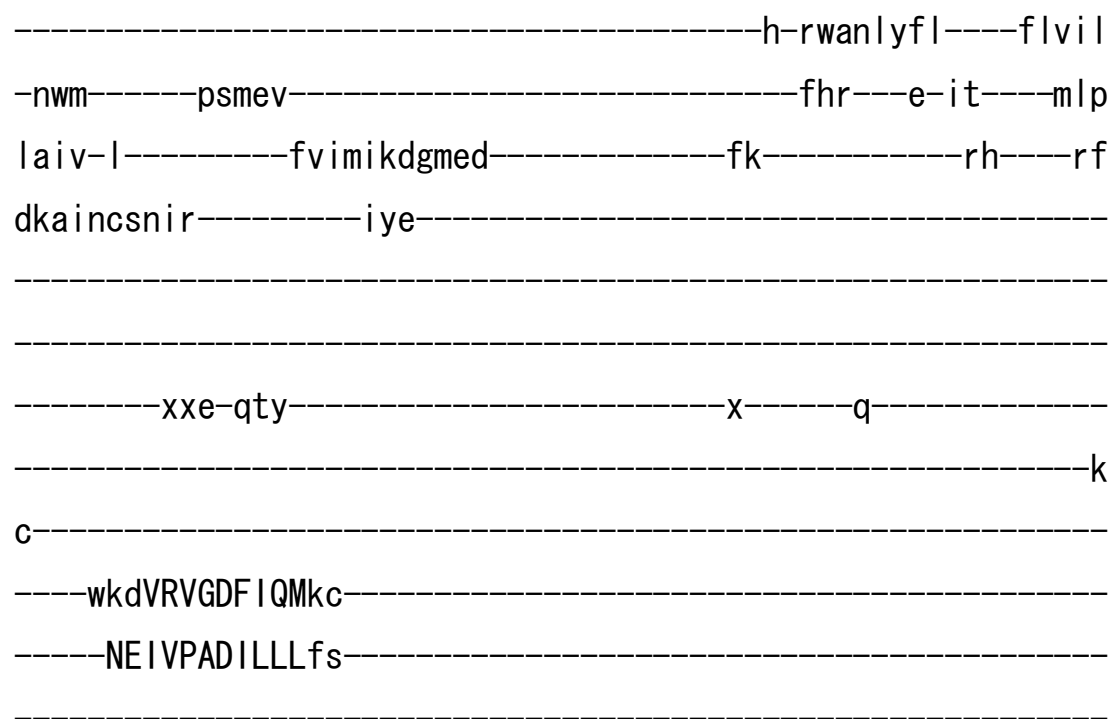

-sdpng-i---chLETASLDGETNLKQRcv-

-vkgfsqqevqfepe I fhnt i vcekpnnh Inkfkgymehx-

dqtrtg

$\mathrm{fgc}$

e----_-----SLLLR--GCTI---------_-----r

nt-----emav-------------------------g I VIYAGHETKAM Innsgpry

krskierrmnidiffcigililmcliga

-vghs i wngtfeehp------xfdvp---------xxxxxxxpsa I ggfymf I tmI ILLQVL

IPISLYVS iELVKLGQVF-FLsndIdI

-ydeetdls

iQCR-ALN I AEDLGQIQY IFSDKTG

TLTENKMVFr -rct imgsey

$\operatorname{XXXXXXXXXXXXXXXXXX}$

XXXXXXXXXXXXXXXXXXXXXXXXXX

$-x x x x x--x x x x$

$x x x x x x x x x x x x x x x x x x x x x x x x x x x x x x x x x x x d v t p d k n--\mid x t k v x x x x x x x x x x x x x x x$

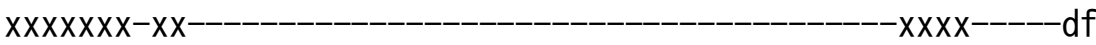

flaltic-----nsvm---_-_--- $x x x x x x x x x x x x x x x x x x x x x x x x x x x x x x x x x x x x$

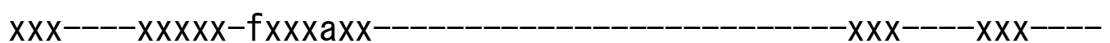


yy l-yknvc-yvn I I fwyqffcgf------------------------ssstmi dywami iff nlffts I pp I vfgv I dkdi sae-_-_-_-_-_-_-_-_-_ I la l pe lyksgqnsecyn I st f-wi smvdafyqs I icf-fi py-lay---------------xxxxx---------------d--vftfgtpintis Itti I Ih-qamemktwt i fhgv-------v| Igsf Imyf I vs I I

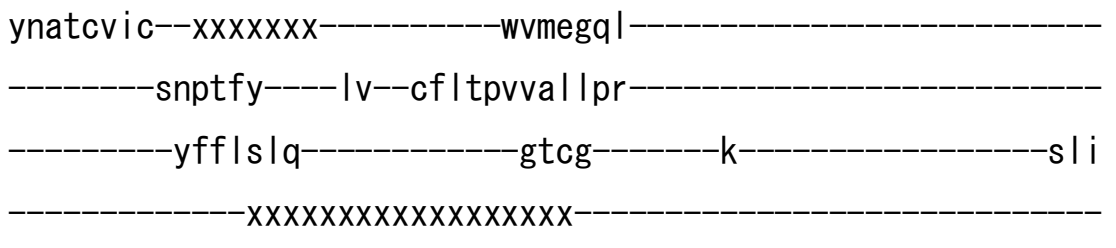
-xxxxxxxxxxpvpeva-------------xxxxxx xxxxxxxxxxxxxxx-------------------------------------------

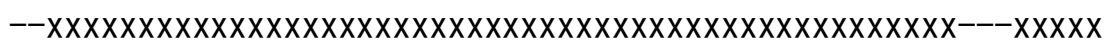
$x x x x x x x x x x x x x x x x x x x x x$

>hsalV_IV11 NP_065186. 2

m- 


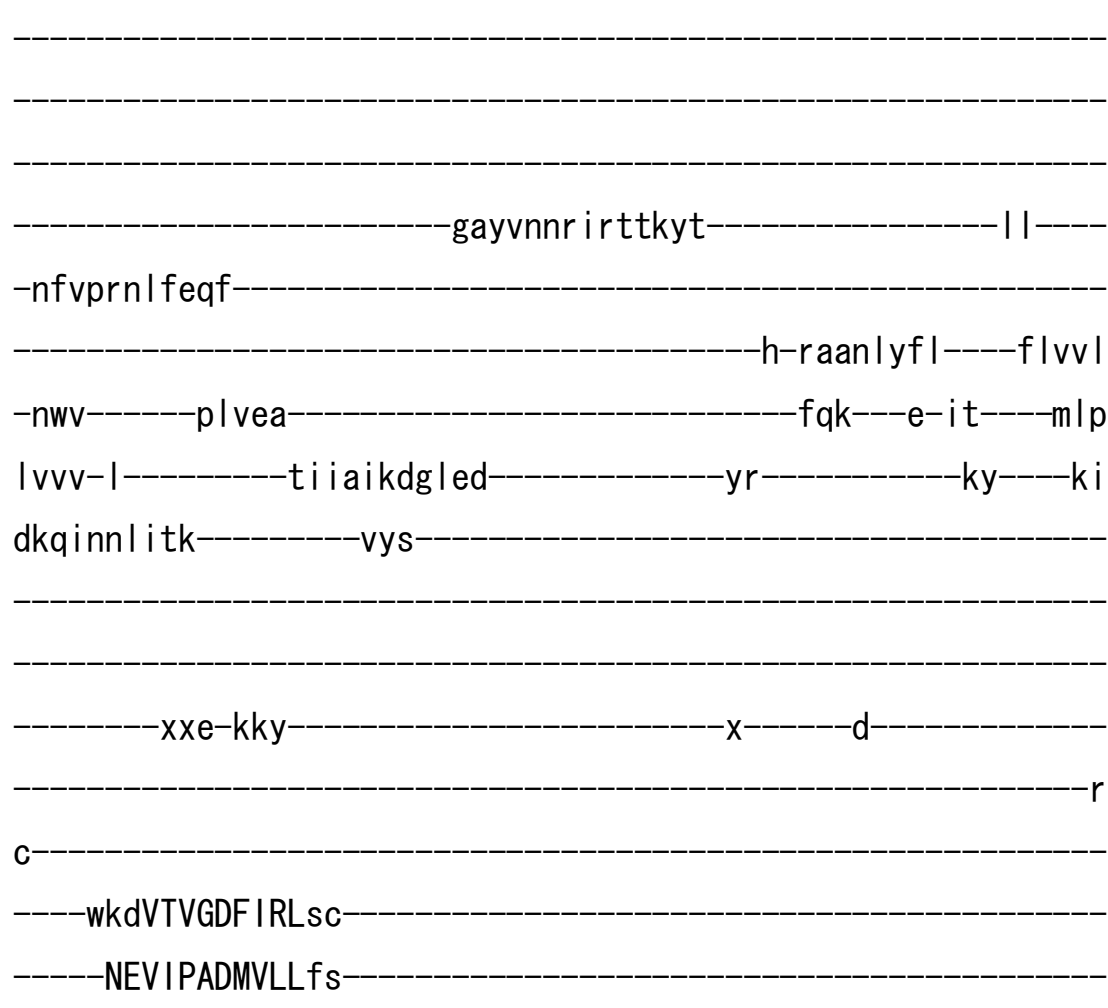

-tdpdg-i---chIETSGLDGESNLKQRqv-

-vrgyaeqdsevdpekfssri i ecespnnd I srfrgf

$-x \times x x$

-nkervg

Isk

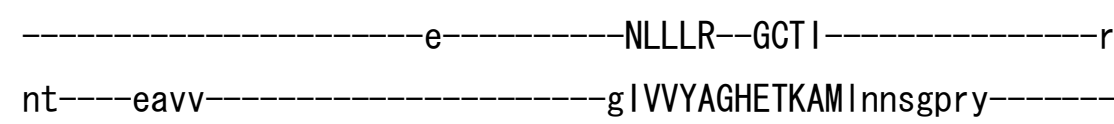

-krsk lerrantdv Iwcvm I I vimc I tga

-vghg i w I sryekmh------xfnvp---------xxxxxxxsp I lagfymfwtm I ILLQVL

IPISLYVS iEIVKLGQIY-FIqsdvdf 
-ynekmds i

- QQCR-ALN I AEDLGQIQYLFSDKTG

TLTENKMVFr

-rcsvagfdy

$-x x x x x x x x x x x x x x x x x x x$

$\operatorname{xx} x x x x x x x x x x--x x x x x x x x x x x x x x x y$

$-x x x x x--x x x x$

$x x x x x x x x x x x x x x x x x x x x x x x x-x x x x x x x x d v p p d t r--\mid x d k f x x x x x x x x x x x x x x x$

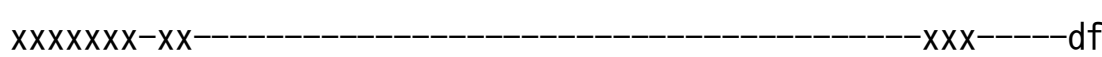

fial a i c-----ntvv----------- $x x x x x x x x x x x x x x x x x x x x x x x x x x x x x x x x x x x$

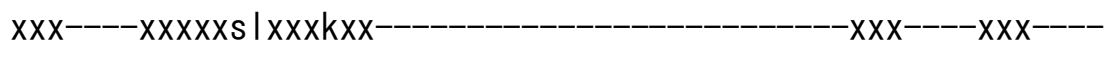
$-x x x x x x-------x x x x x x x x$

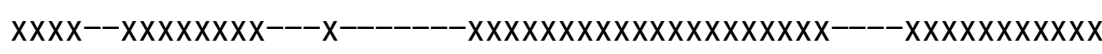

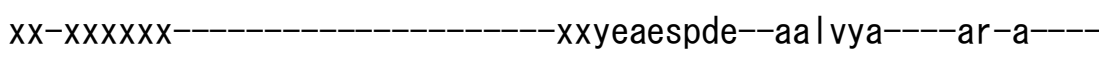

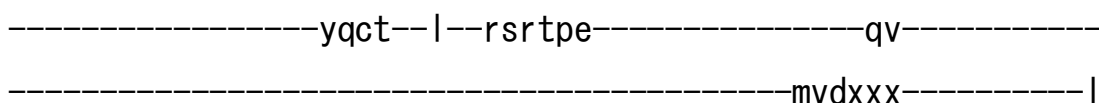
gp I---------tfql lhi Ipfdsvrk-rmsv-----v----vr

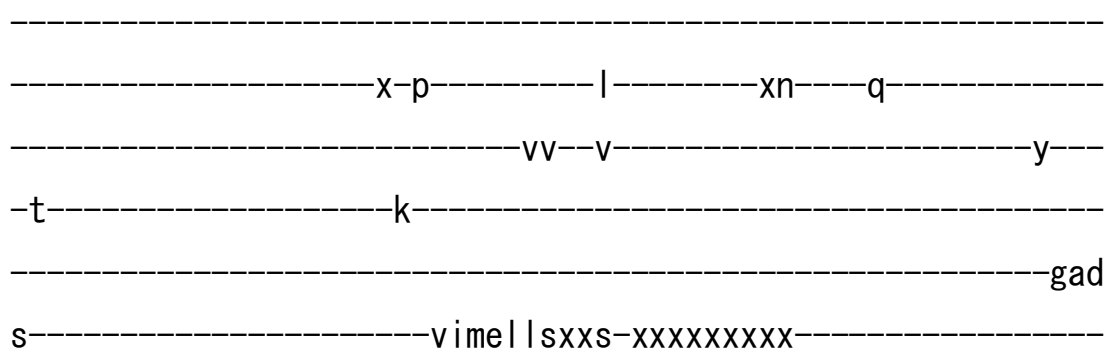

xxx--xx--ekt-qkh I ddy--akq----g | r tLCI AKKvm---sdteyaew I rnhf I--ae-----ts idnree I I lesamr- l enk I I ILGATG IEDRLQEGVPES IEALHKag-IK I

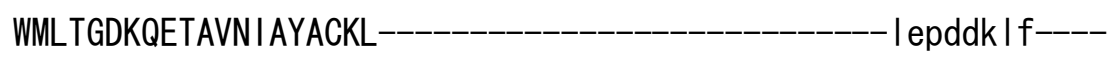

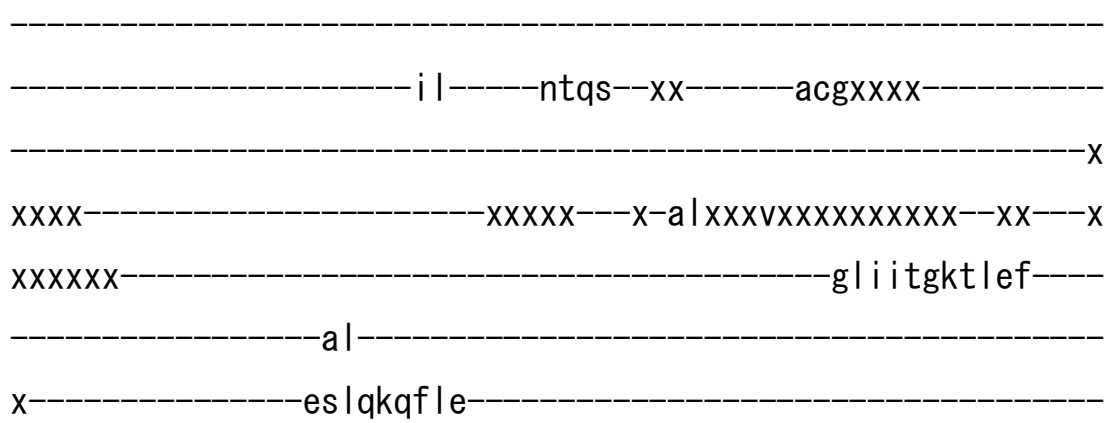


ItswcqavVCC--RATPLQKSEVVKLVr-

---sh-- I q------------v------------MTLA I GDGANDVSM I QVAD I G I GV--

sgqegmq

-AVMASDFAVSqfKHLSKLL--LVHGHWCYTRLSNMI------L

yff-yknva-yvn I I fwyqffcgf-_-_-_-_-_-_-_-_-_-_-_-_-_stsmtdywv I iff

nl I ftsappv i ygv l ekdvsae--_-_-_-_-_-_-_-_t Imq l pe lyrsgqkseay I pht

f-wit I I dafyqs I vcf-fvpy-fty---------_-----xxxxx----------------

-d--ifafgnp Intaalfiv| Ih-Iviesks I twihl I--_-_---vi igs i I syf I faiv

fgamcvtc---xxxxxxx-----------wimqehm-----------------------------

---------Idpvfy-----Iv--ciltts ial I pr----------------------------

---------fvyrvlq------------gs If--------p----------------spi

$-x \operatorname{xx} x \operatorname{xx} x x x x x x x x x x x$

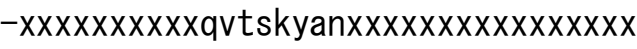

$\operatorname{xx} x \operatorname{xx} x \operatorname{xx} x \operatorname{xx} x x x x x-$

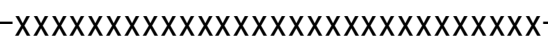

>hsalV_IV12 NP_056020.1

$\mathrm{m}-$ 
$-x x x x x x x x x-x x x-$

$-x x x x x x x x x$

---srtiyvgh

-nf ipkn I feqf

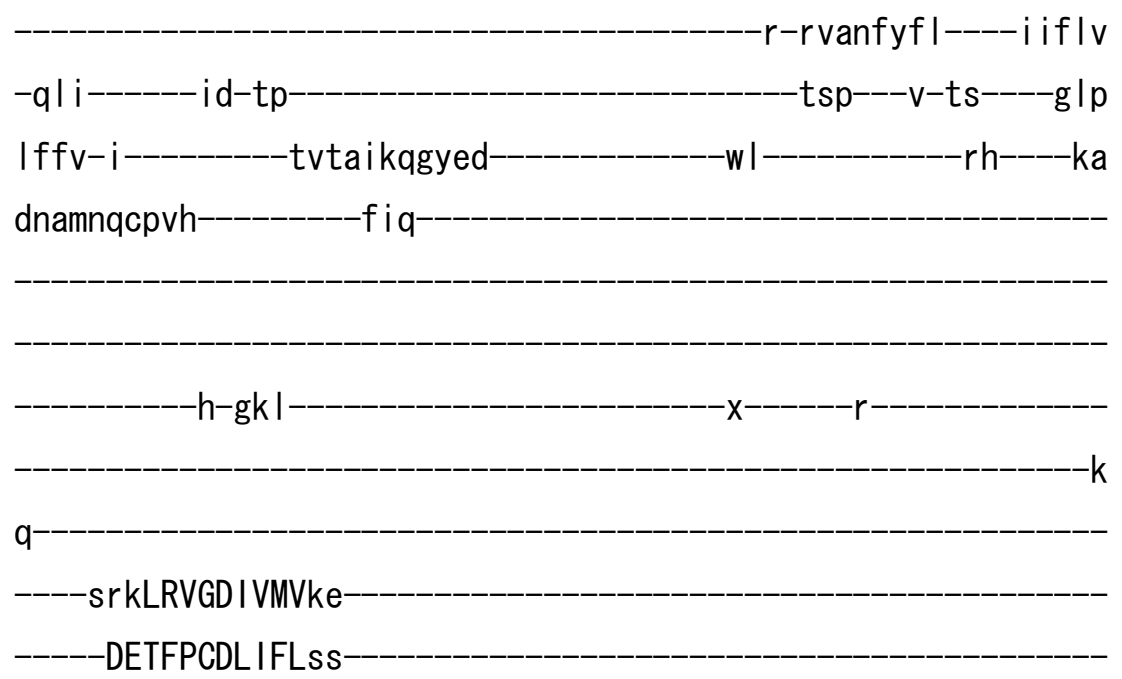


-nrgdg-t---chVTTASLDGESSHKTHya-

-vqdtkgfhteed i gg I hat i eceqpqpd I ykf vgr i nvxx

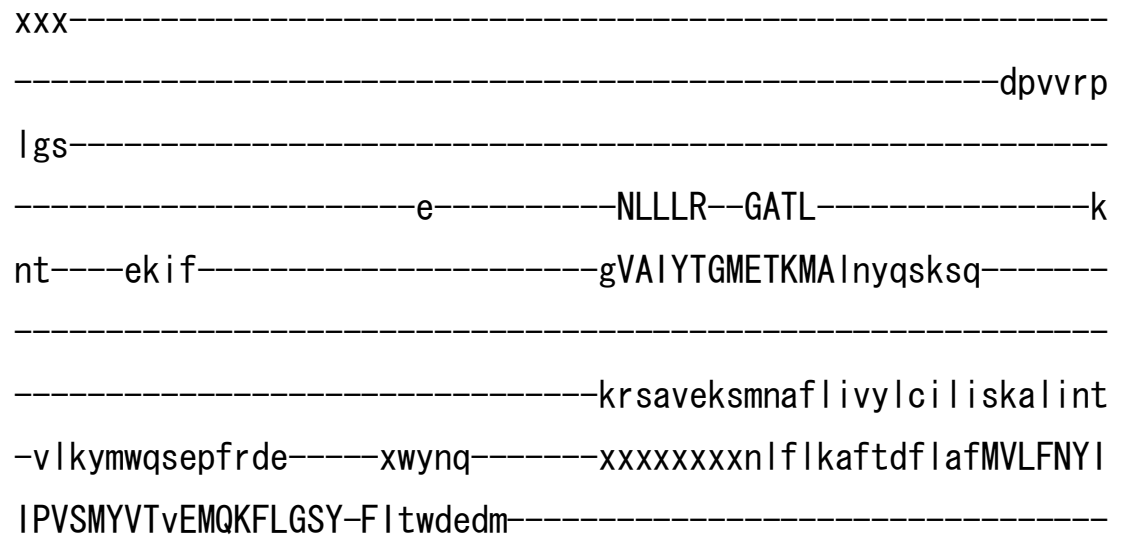

fdeetgeg

-pLVN-TSDLNEELGQVEYIFTDKTG

-TLTENNMEFk-

eccieghvy

$-x x x x x x x x x x \mid$ pessgixxixsspxxxxx If

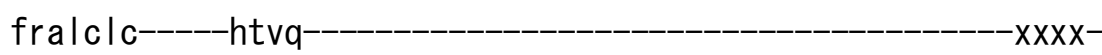

$--x x x x x x x x x-p d x x x s x x-$

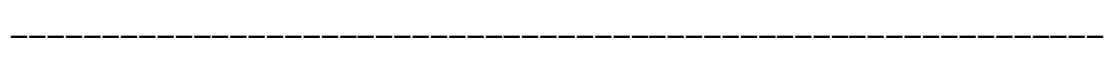

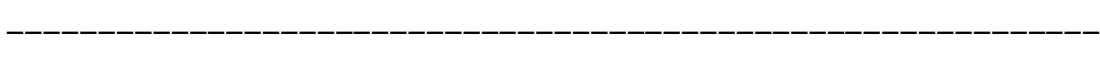

------------------- I gft--y-- I r I kdn----------------ym-----------

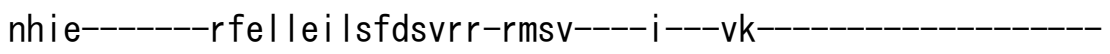
-----------------------------------------------------------s-s i fprvixxk- 
-xdqi-rarvern--ave----g | r tLCVAYKr I--iqeeyeg i ck I lqa--ak-----va l qdrekk l aeayeq-i ekd I I I LGATAVEDRLQEKAADT IEALQKag-IKV WVLTGDKMETAAATCYACKL-frrntqlI----

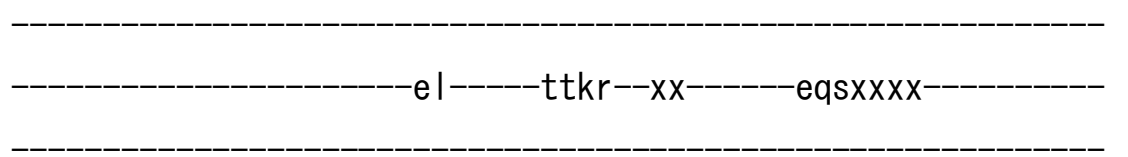

$x x x x x x x x x x h s x x x t x x x x x x x x x x$

$-x x d y g \mid$ i idgaa $|s|$ im$-X X X X X X$

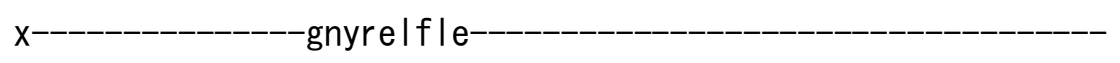
icrscsavLCC--RMAPLQKAQIVKL Ik ---fs--ke-----------xp-------------ITLAI GDGANDVSM I LEAHVG I GV--AARNSDYAIPK FKHLKKML--LVHGHFYYIRI SELV------Q yff-yknvc-fi fpqf I yqffcgf--_-_-_-_-_-_--_--_---sqqt l ydtay lt l y nisfts Ipi I lys Imeqhvg id--_-_-_-_-_-_-_--vlkrdpt Iyrdvaknal Irwrv f-iywt I Ig Ifdal vff-fgayxvfe---------_--------xxxxxxxxxi------fg--nwtfgt Ivftvmvftvt|k-laldthywtwinhf--------viwgs I I fyvvfs I I

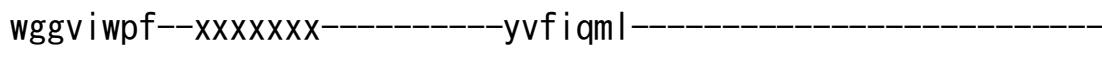

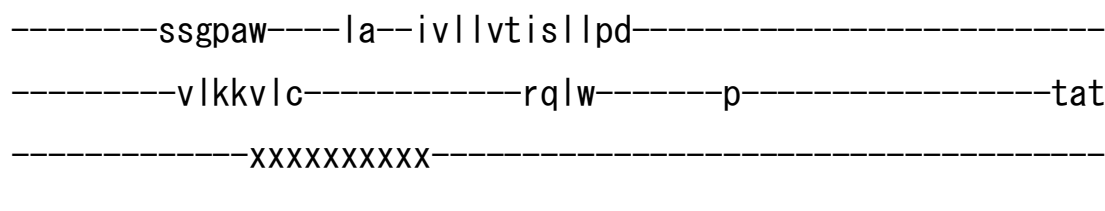


>spolV_IV01 NP_593720.1

$$
\text { m- }
$$

$X X X X X X X X X X X X X X X X X X X$

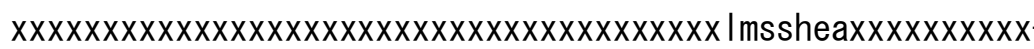

$x x x x x x x x x x x x x x x x x x x x x x x x x x x x x x x x x x x x x x x x x$ mssheax $x x x x x x x x x-$

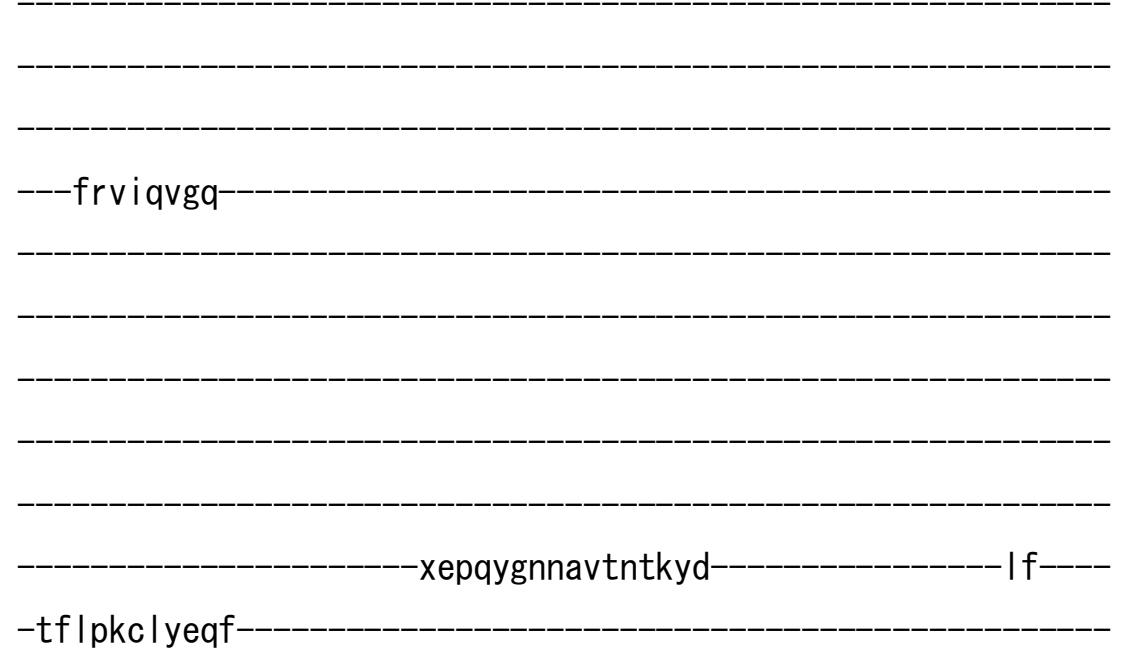

-tf Ipkclyeqf 


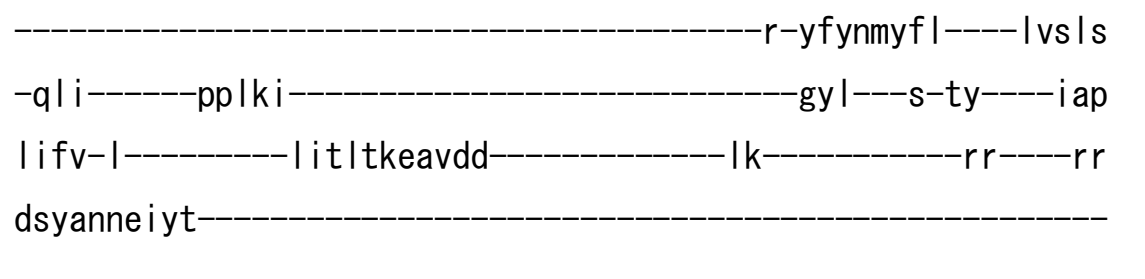

$-x x d x p c a-$

-tv--g-n--xafIRTDQLDGETDWKLRip-

csnqhteg i vhadap iksvhhfygtft I--

-nnqkrp

isv

$d$

-HTLWA--NTVL

as-----dgvy-

gVVVYTGKDTRQSmnsskakt

-kvgllekeinfyskilctfvlvIsigIt

-fshgiktd-

-wy is

-vfryLILFSSI

IPINLRVN I DLAK I VHSK-NTesdpn I

$-p g$

-vVVR-SSNIPEELGRIEYVLTDKTG-

-TLTQNEMEMk-

-klhvgtmgf

$x x x x x x x x x x x x x$

$-x x x x x x x x d s k t \mid v r$

$-n \mid$

vlalslc-----hnvt

$-p s x x x d x x x x$ 


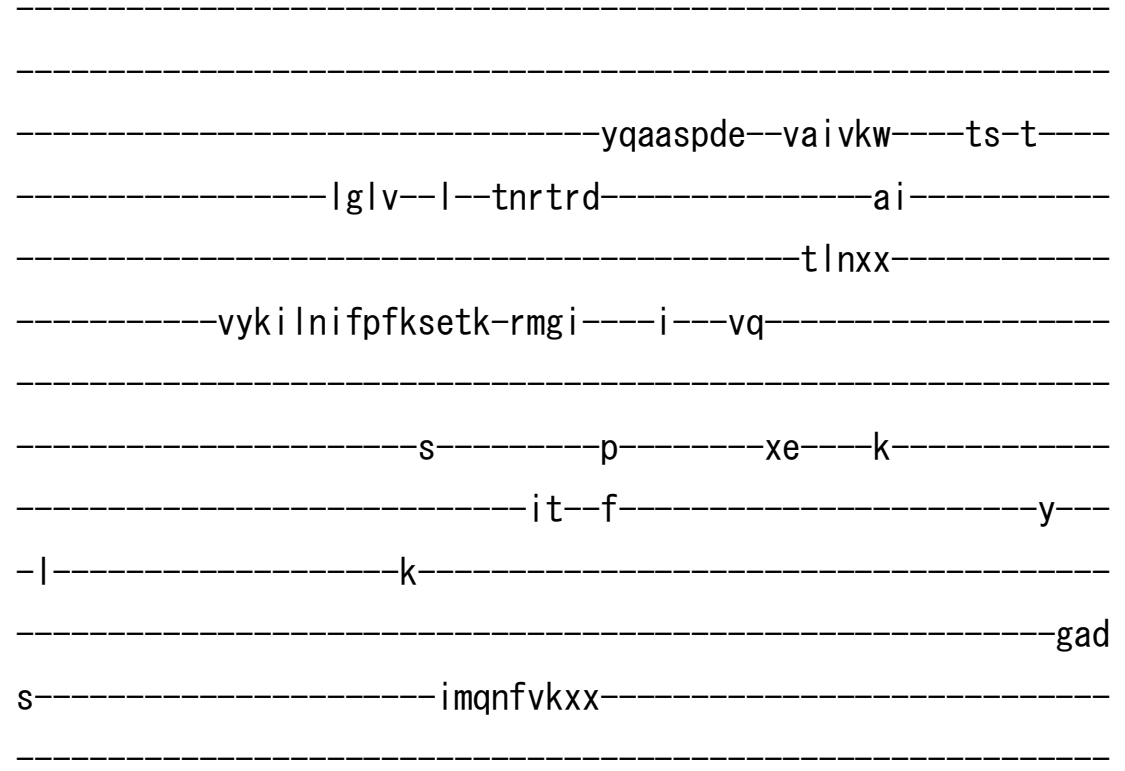

-fwl-eeecgnI--are----g |rtLVVAKKdI--saeeysafs l ahsd---as----xsfsnsrdkkmee i vsr x I endmd I LGL TGVEDKLQKDVKI TLELLRNag-IHV WMLTGDKVETARCIAISSRL-vsrgqyih----

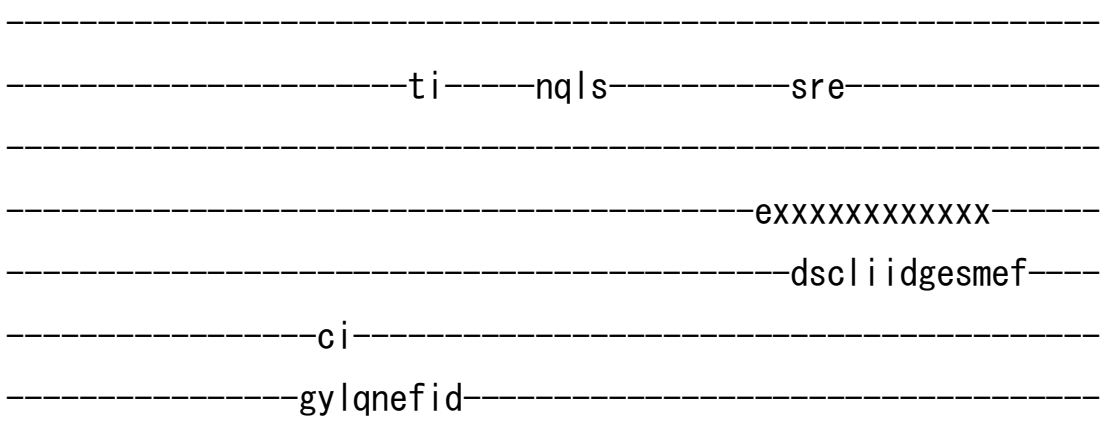

ivsdIssvVIC--RCTPTQKANMTRLIq

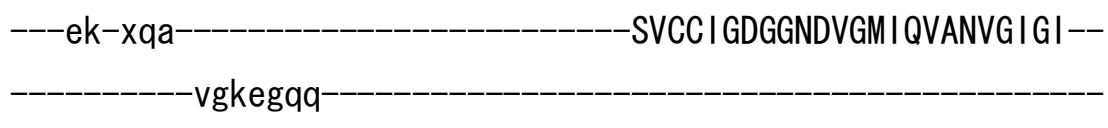

-ASLAADYSVKefSHVSRLL--LWHGRISYKQTSKLA------M 
fvi-hrgll-isvcqvvysvisaf ep i a I fqg I I lvgy stmytmIpvfsi-vydrdvsek -Ivf Ifpelykemreqkcfsykn

f-iscvl isvyqgl i iqxftfyxigf

ee--egkm lavcfscl i fne I im-valqintweqti vmsel It Immy i Isvpf Itny felkfI Iglkfy----wv--sal i If is I lpv-wcgkalk rklk $-p$ ssy

aklar

>hsalV_IV13 NP_055431. 1 


\section{$-X X X X X X X X X$}

---trtiyvan

$-x x x x x x x--x x-$

-pqkfidnr i isskyt

- vW----

-nfvpknlfeqf

-r-rvanfyfl----i iflv

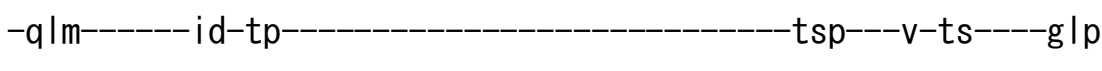

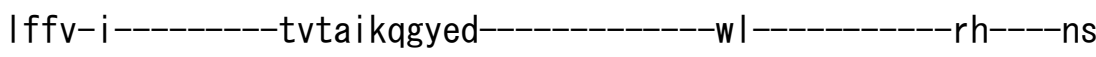

dnevngapvy----------vvr

$\mathrm{t}$

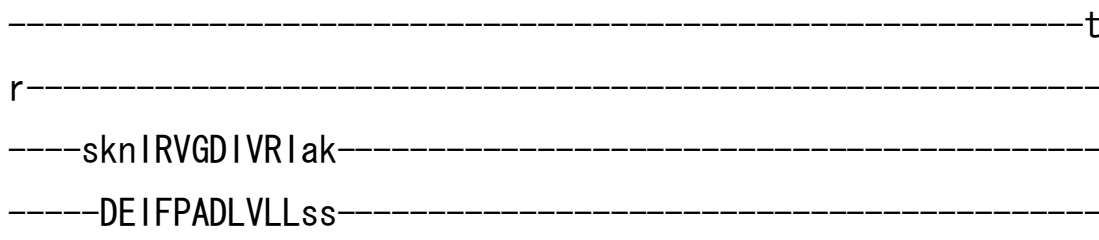

$-d r$ ldg-s---chVTTASLDGETNLKTHva-

-vpeta I lqtvan I dt I vav i ecqqpead I yr fmgrmi i xx

$$
\mathrm{xx}
$$

-eeivrp

Igp-

-e----------SLLLR--GARL----------------k

nt----ke i f----------_--_---------gVAVYTGMETKMA Inyksksq-------

-krsaveksmntfl i i y lvi l i seavist

-i I kytwqaeekwde------xwynq-------xxxxxxxxski I rf i sdf I afLVLYNF I

IPISLYVTVEMQKFLGSF-F I gwd I d I 
aQVN-TSDLNEELGQVEYVFTDKTG-

TLTENEMQFr

ecs ingmky-

$-x x x x x x--x x x x$

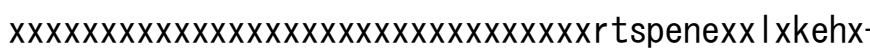

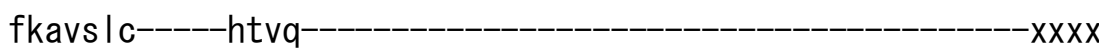

$x x x x x x x x x x x x n \mid x x x q x x-$

--yyasspde--kal vea-----aa-r-----

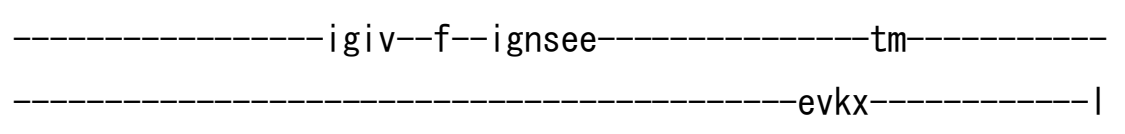

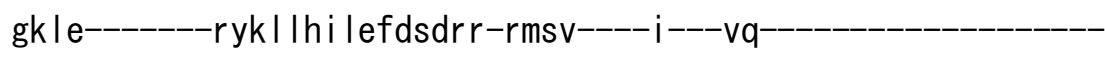

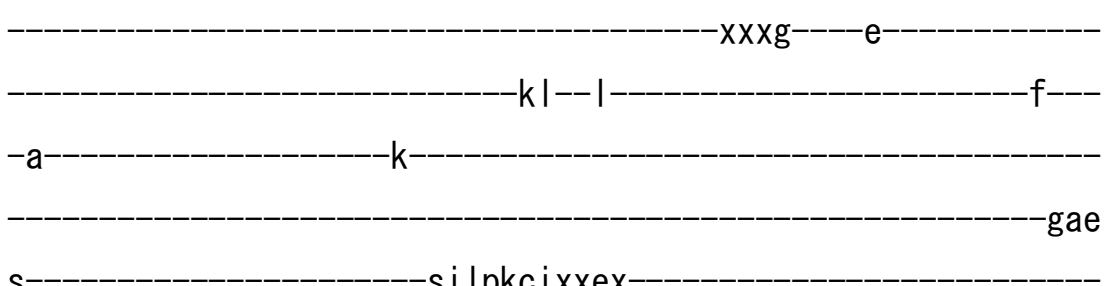

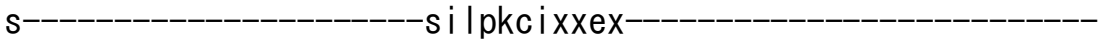

-ekt-rihvdef--alk----g|rtLCIAYRkf--tskeyee i dkr i fe---

ar-----ta l qqreek l aavfaf-i ekd I i ILGATAVEDRLQDKVRETIEALRMag-IKV WVLTGDKHETAVSVSLSCGH-_-_-_-_-_-_-_-_-_-_-_-_-_-_-_-_fhr tmn i l--_-

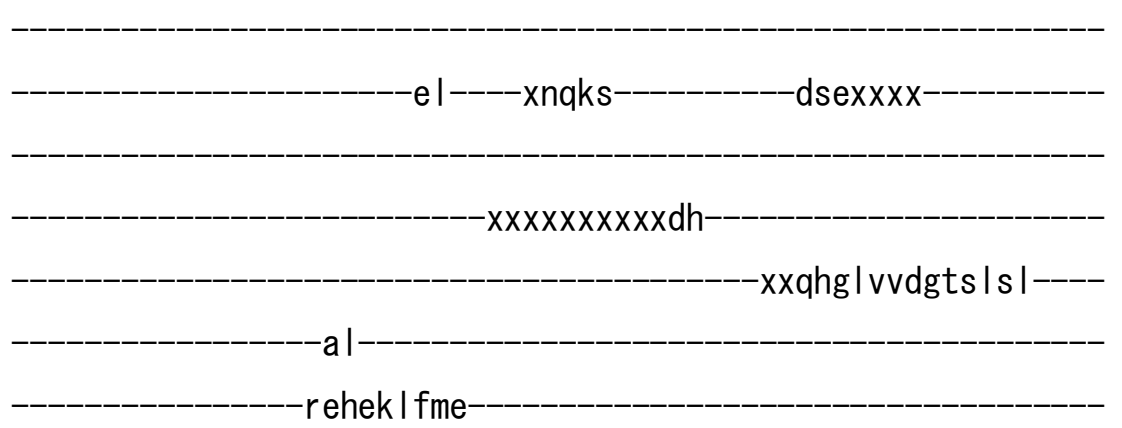


vcrncsavLCC--RMAPLQKAKVIRLIk

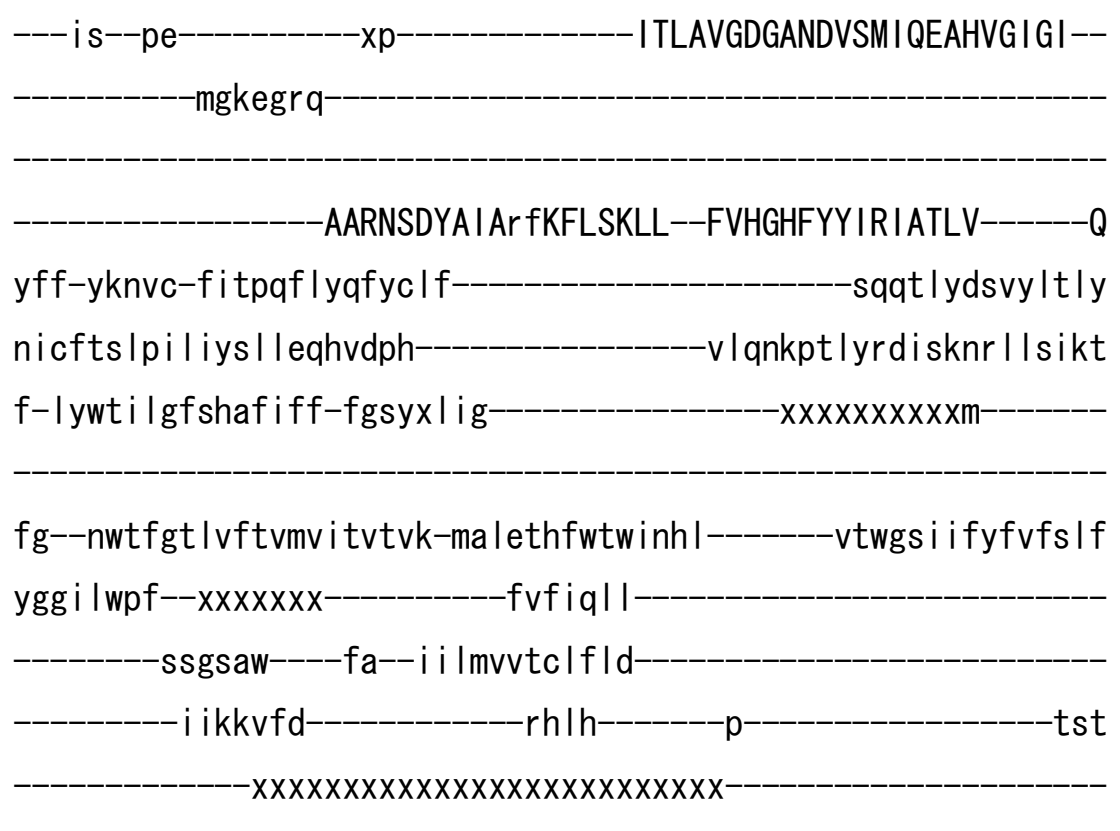

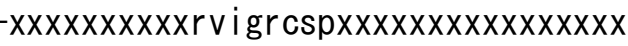

\section{$x x x x x x x x x x x x x x x$}

$-$

>spolV_IV02 NP_592849.1

$\mathrm{m}-$ 


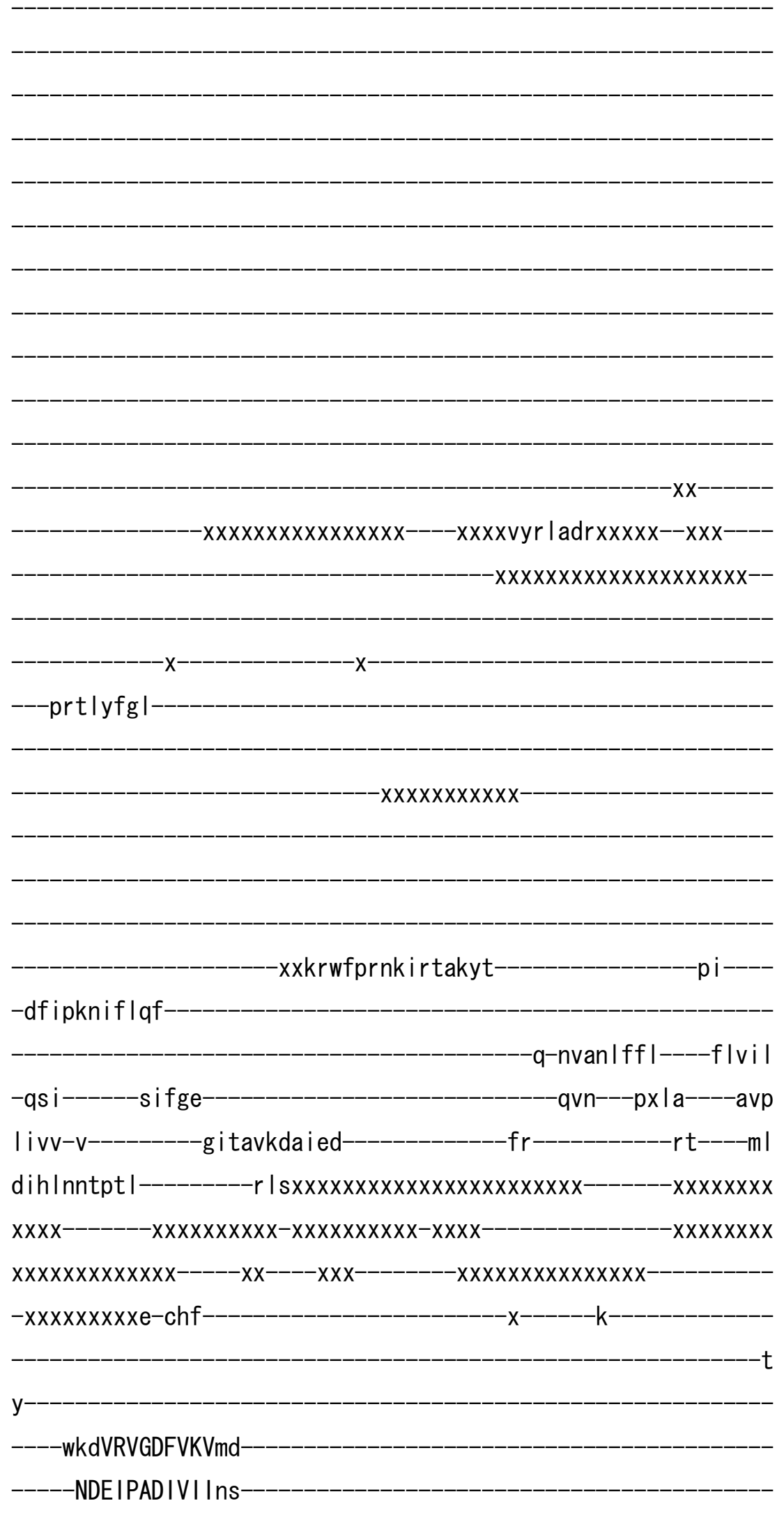


-sdpeg----xcy IETKNLDGETNLKMRha-

Itcgknvvdeascercrfwi esepphan I yeyngacksxx

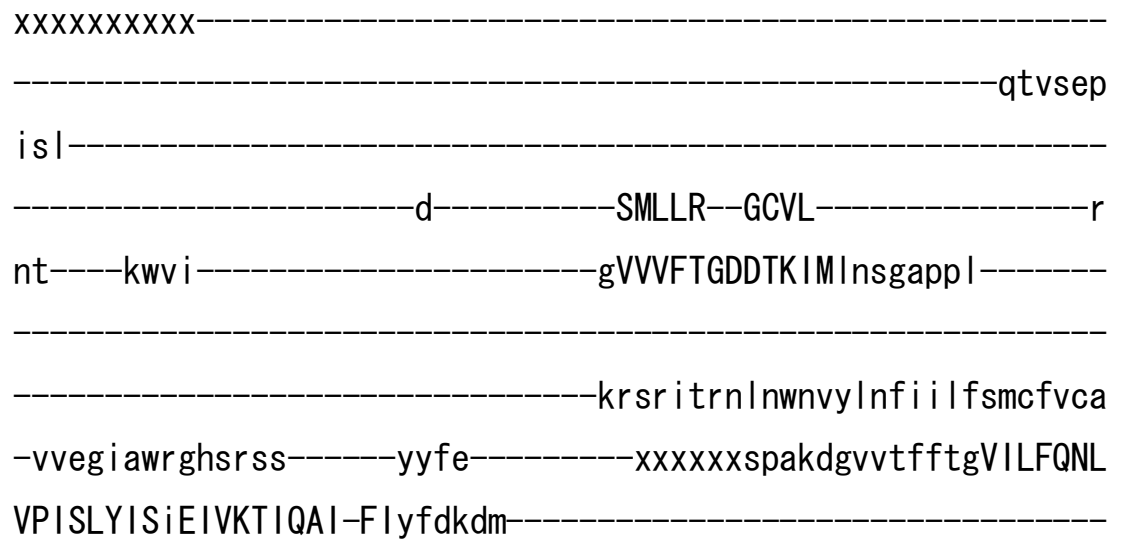

-yykklkya-

cTPK-SWN I SDDLGQVEYIFSDKTG-

-TLTQNVMEFk -

-kctingvay

$-x \times x \times X X X X X X X X X X----$

$-x x x x x--x x x x$

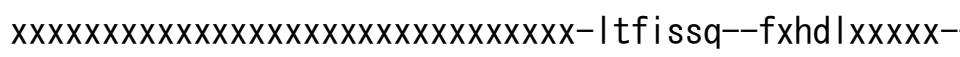

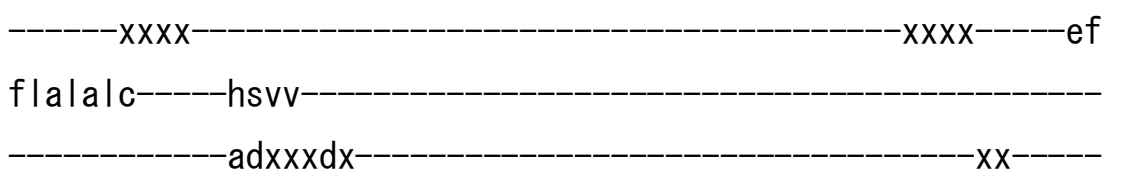

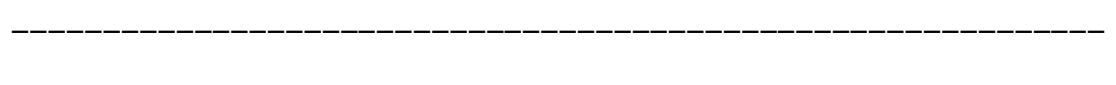

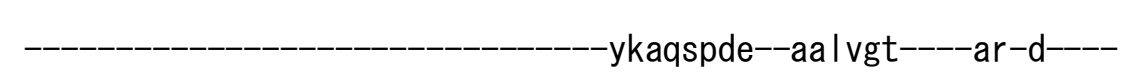

-------------------vgfv--f-- Idqr rd-----------------im------------

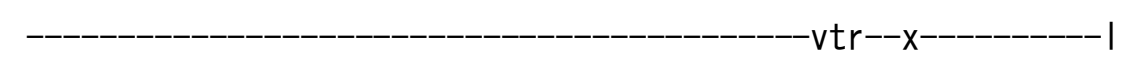

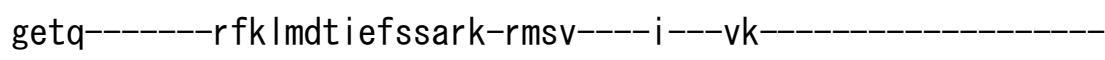

------------------------------------------------------------

s----------_----_------ i i fer l exxe 
xxx--xx--ktt-seh| r if--al e----g | r tLCI AKRe l--teeeyyewkekyd i--aa-----sa i enr eeq i eevad I-i esh I I I LGGTAIEDRLQEGVPDS I ALLAQag-IKL WVLTGDKMETAINIGFSCNLI dagmdmi----

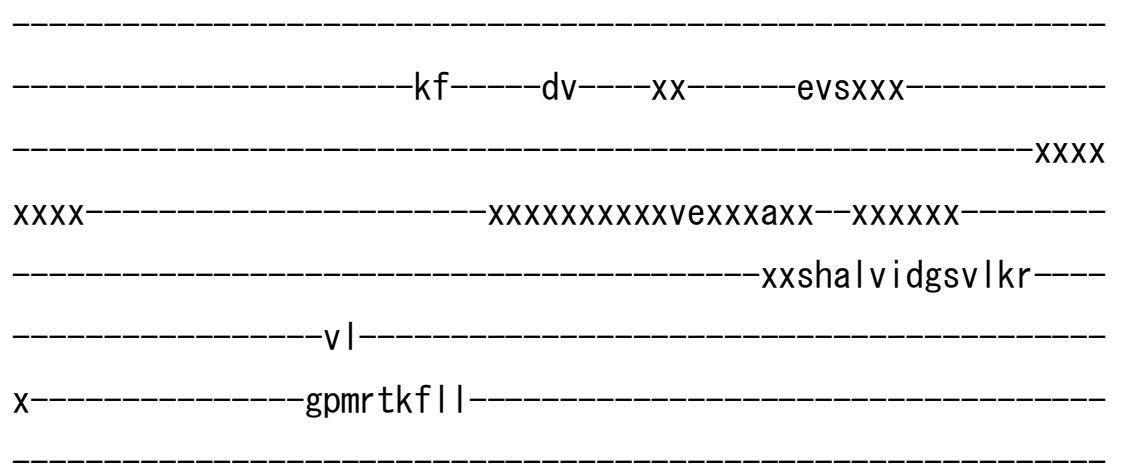

I ckrckavLCC--RVSPAQKADVVQLVr

---es-- l e------------v-------------MTLA I GDGANDVAM I QKAD I GVGI --

-AAMSADYAIGqfRFLSKLV--LVHGRWDYNRVAEMV------N

nff-yksvv-wtft I fwyq i ynnf---_--_--_--_--_-_-_---dany I fdytyvm I f nl i fss I pv i vmgvydqdvnad--_-_-_-_-_-_-_--Is I r ipqlykrg i lq Insark i f-igymldgfyqsvicf-ffsfxvin--_-_-_-_-_-_---xxxxxxxxxxxt----_--ma--vqd I gvyvaapt imvvdty-vi I nqsnwdvfs i g------- I wa I sc I tfwfwtgv ysqs lytyxxx -ksasrif-

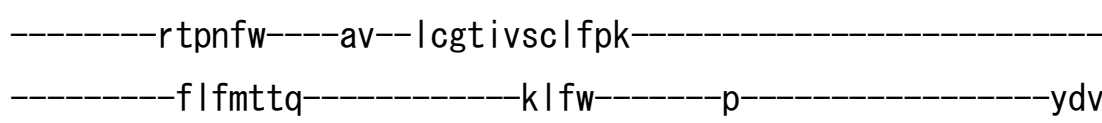
$-x x x x x x x x x x x x x x x x$ 
>hsalV_IV14 NP_775965. 2

$\mathrm{m}$

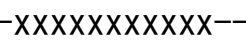

$-x \times x x x x x x x$

---trtvfvgn

$-x-x x x x x x x x x-$

-aqrfcdnr ivsskyt

I

-nflpkn I feqf 


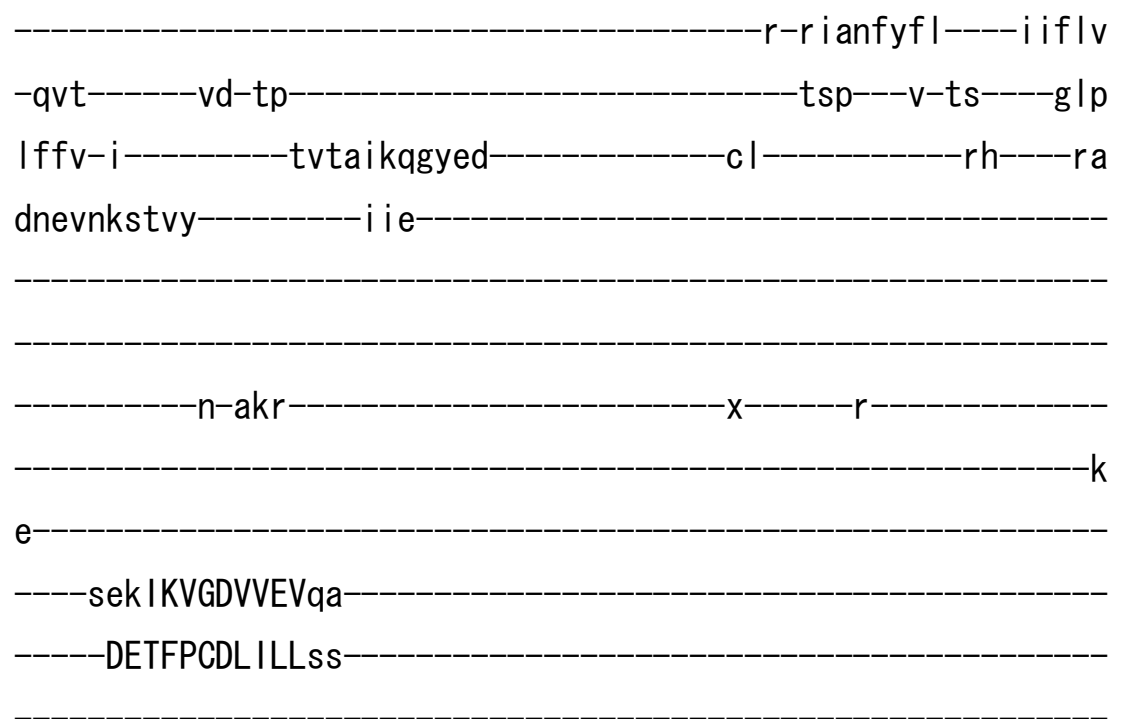

cttdg-t---cyVTTASLDGESNCKTHya-

-vrdt i a Ictaes idt I raa i eceqpqpd l ykfvgr inixx

$\mathrm{xxx}$

eavars

Igp-

-NLLLK--GATL-----------------k

nt----ekiy-

gVAVYTGMETKMA Inyqgksq-

-krsaveks inafl ivylfi II tkaavct

-t I kyvwqstpynde------xwynq--------xxxxxxxx I kv I kmftdf I sfMVLFNF I

IPVSMYVTVEMQKFLGSF-FI swdkdf

-ydee ineg-

aLVN-TSDLNEELGQVDYVFTDKTG

TLTENSMEF $\mathrm{i}$ ecc idghky-

$-x x x x x x x d g|s q t d x x|-$ tyfxxxxx

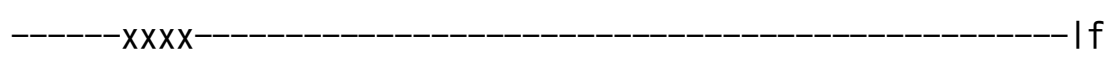

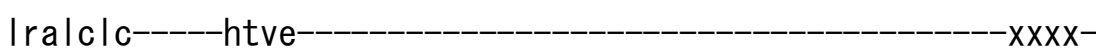

-----XXXXXXxesxXX--x- 


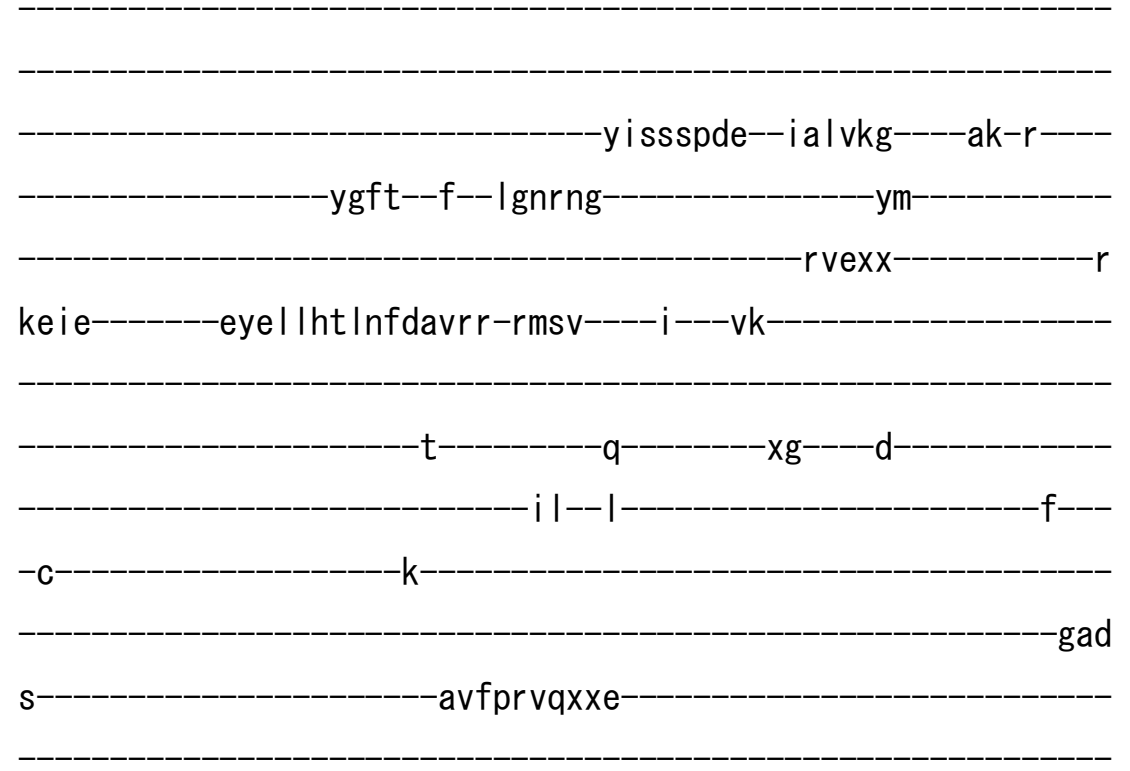

-xelt-kvhvern--amd----gyrtLCVAFKe i--apddyer inrql i e--ak-----ma l qdr eekmekvfdd-i etnmn I I GATAVEDKLQDQAAET IEALHAag-LKV WVLTGDKMETAKSTCYACRLfqtntell----

el-ttktxxxxxxx---edrxxxx $-x x x x x x x x x x e f x x x t x x x x x x x x x x$ $-x$ eyg $\mid$ i idgst $|s|$ i I $-X X X X X X$ x---------------nnyks if lq-

i cmkctavLCC--RMAPLQKAQIVRMVk

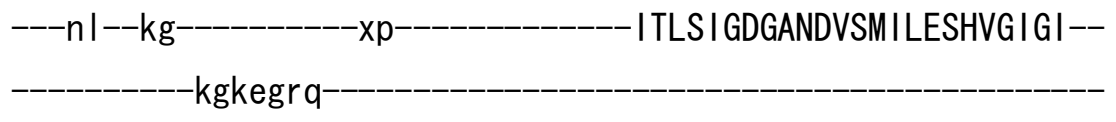


yff-yknlc-fi lpqflyqffcgf-sqqp I ydaay I tmy nicfts Ip i lays I leqhin id-_-_-_-_-_-_-_--t I tsdpr Iymk i sgnam I q I gp f-Iywtf l aafegtvff-fgtyx Ifq-_-_-_-_-_-_-_-_-_xxxxxxxxxv-_-_-_-_ yg--nwtfgtivftvlvftvtlk-laldtrfwtwinhf--------viwgs lafyvffsff wgg i iwpf--xxxxxxx---_-_-_--_fvfaqm I--_-_-_-_-_-_-_-_-_-_-_-ssvstw----la--i illifislfpe-

-illivlk $-n v r$

\section{$\mathrm{XXXX}$}

>spolV_IV03 NP_594759.1

m- 

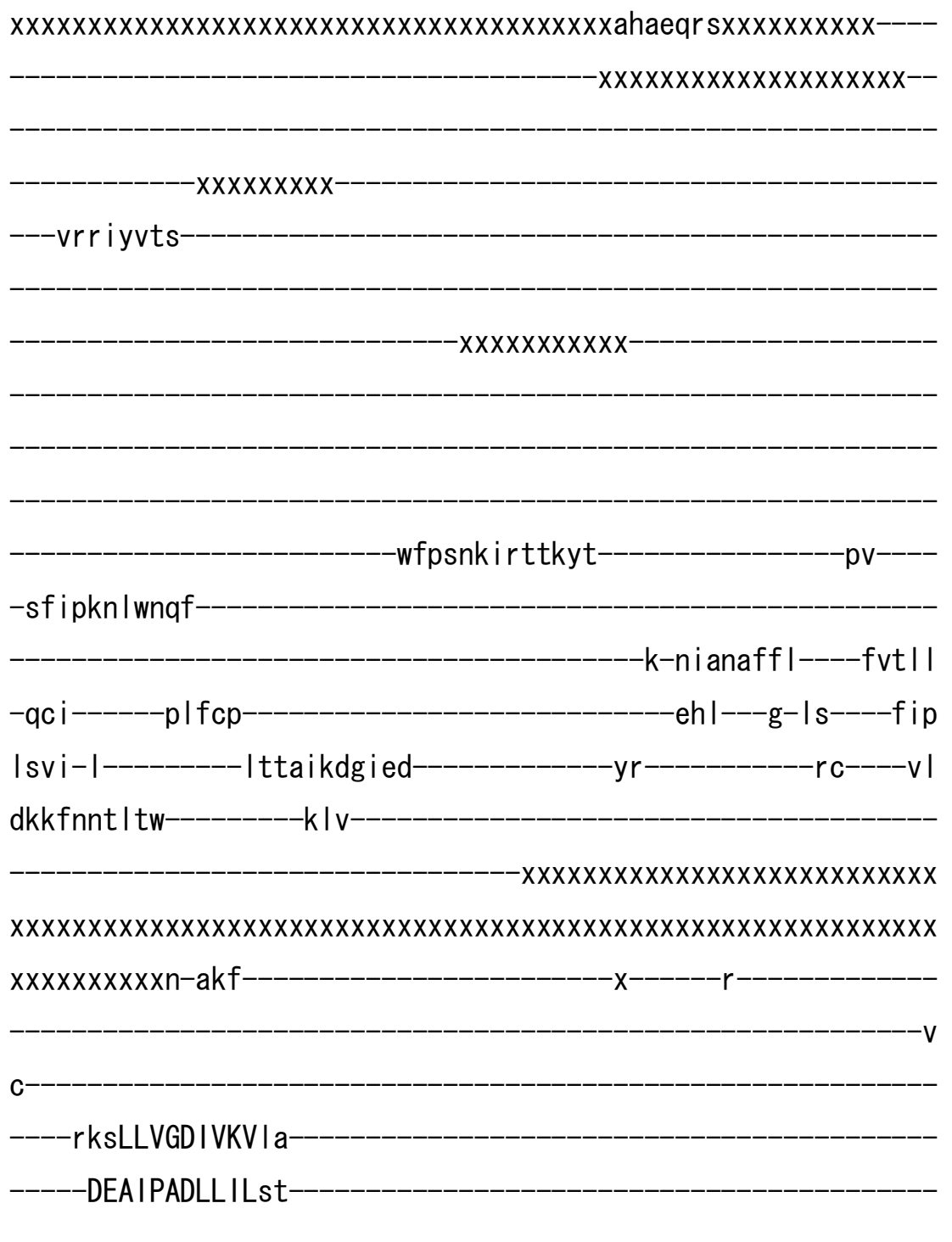

-ensng-v---cyVETKNLDGETNLKDKya-

Icstkcckseyrcsaasfwveceqphad l ys Ingvvkaxx

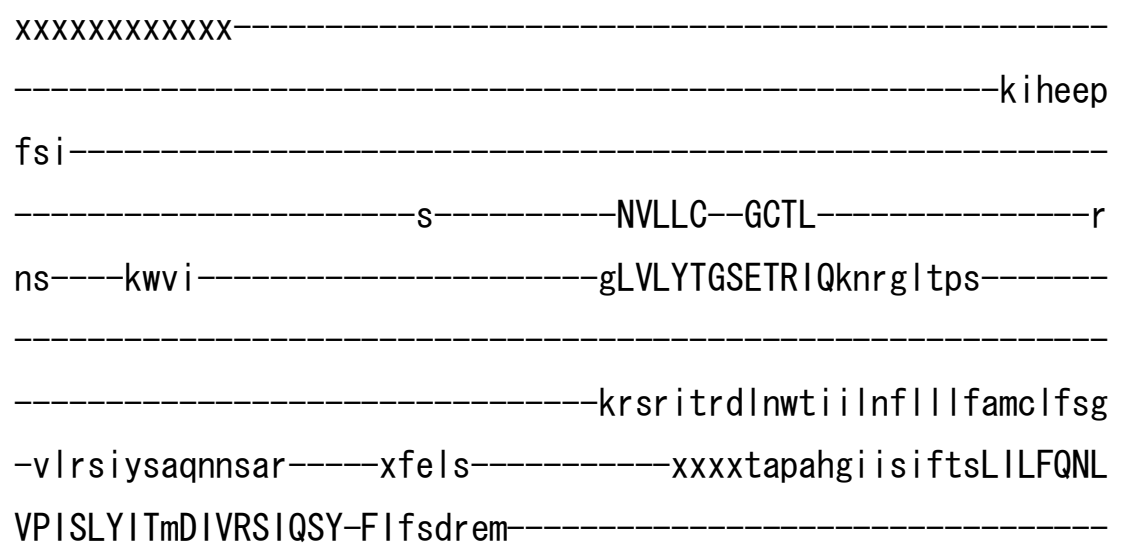


-ydek Idcp

cSPK-SWN ISDDLGQIEYIFSDKTG

TLTQN IMSFk

-kcsingiry

$x x x x x x x x x x x x x x x x x x x$

\section{$\mathrm{XXXXXXXXXXXXXXX-10}$}

$-x x x x x--x x x x$

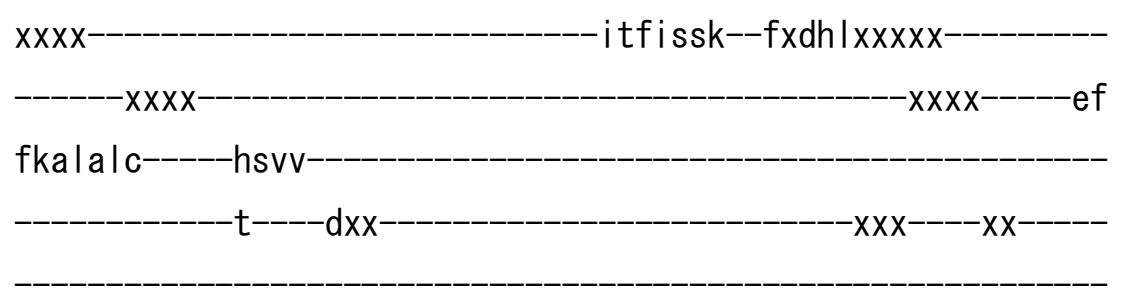

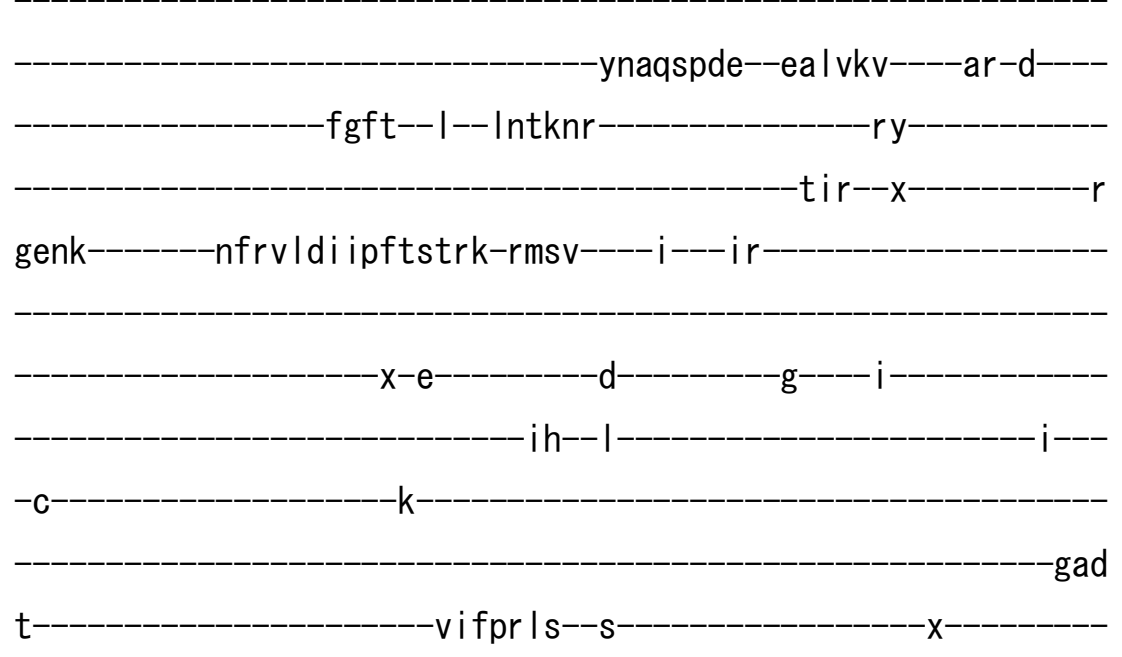

xxx--xx--ekt-kkhl asf--sse----gfrtLCI ARRt i--dkqdy l ewkvnfne--an-----sa i hernekvskvsem-i eqe I e ILGGTAIEDKLQENVPET I ALLA Iag-IKL WVLTGDKVETAINIGYSCNL-_-_-_-_-_-_-_-_-_-_-_-_-_-_-_-_ I dpnmt i f-_-_-

ri-----dansxxxxxxxxxxirnx-

$x x x x x x x x x x d e x x x f x x x x x x x x x x$

-khaividgdalnf

$\mathrm{vl}$

X---------------eqvsf If Im- 
IckqcktvLCC--RVSPSQKAAVVALVk

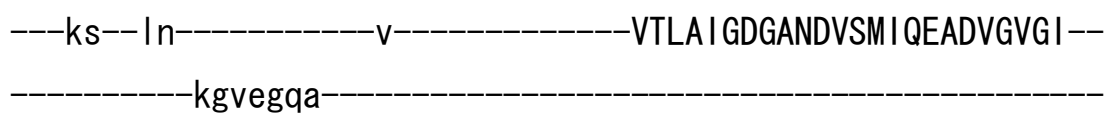

-ASMSADYA I GqfSFLGRLL--LVHGRWDYKRMSQM I------S

fff-yknvi-wtf i I fwyqfynef-_-_-_-_-_-_-_-_-_-_-_-_dgny i fdytyvm If nl Ifts I pvi i agcfdqdvdas-_--_-_-_-_-_-_---vsmknps I yqrg i I g l ewngkr f-wsym I dg i yqs I vcfxval fxfkf------------------xxxxxxxxxxi-------ec--iediglfissptifvinif-i Imnqer Inl isli--------twmfs igvfwiwtfi ysevgpsyxxx------------------ksasrtc------------------------------------qtfgfw-----cv--tvltialcl lpr------------_--------------

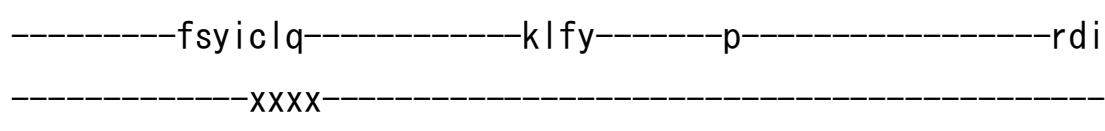

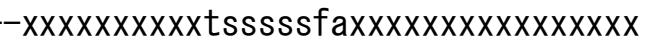

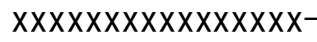

$-$

$>\tan I V \_I V 01$ XP_955173. 1

m- 


\section{$-x x x x x x x x x x x x x f k k p d e e$}

---irniklni

$-x x x x$

-pcsnrvkttkyt

- py----

-nf ifknlyeql

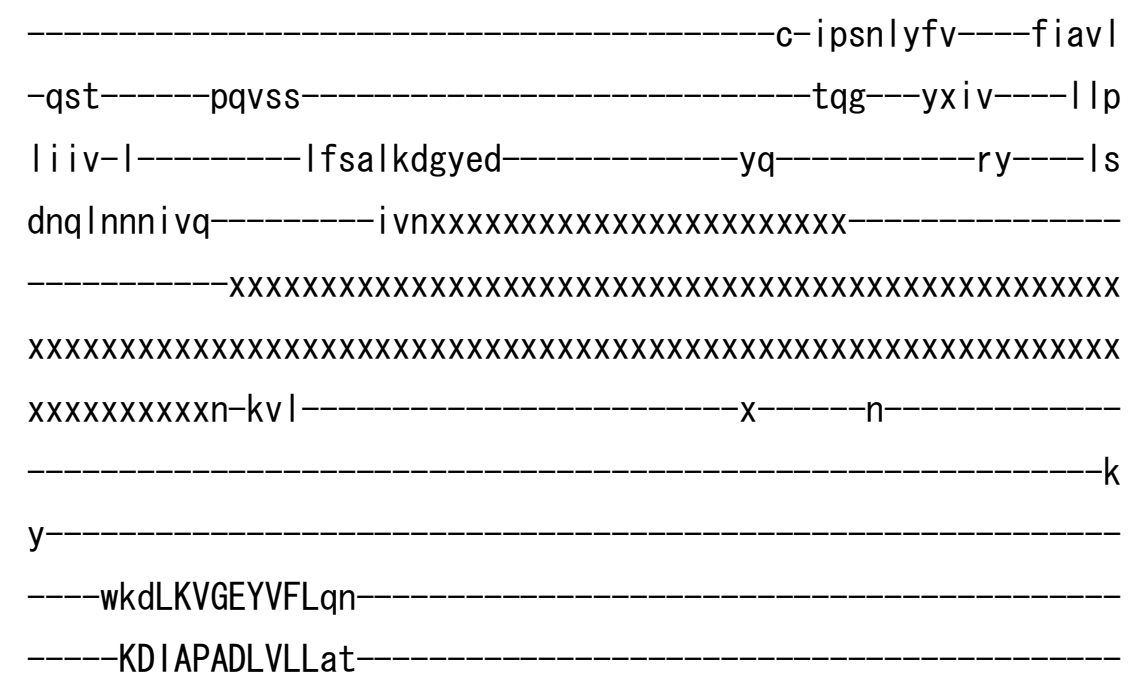


-seeng-f---ayVDASSLDGETNIKKKes-

xxxxxxe I gsdfehvi tevar I I ghfkcegpnkn I isfdgh I kyxx

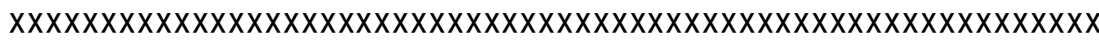

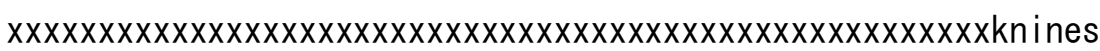
ket qxxxx------NLLLR--ECKLxxxqw i i gFVYYTGHDTKIYkn i skapy

-kvsnlqkkmmkttl I ici iqfi Iclvat -fynly ihtnkvyek-----xsyls-----------xxxxxsgfy i $\mathrm{f}$ i i yy I swMALTANF VPISAIVT I NAVKLIQGF-FI qfddsm-

-yche I kmn-

-aKAR-NTMLNEELGQIKYLFSDKTGxxxxxxxxxx xxxxxxxxxxTLTCNKMEFr $-k f$ s imghsy

$-x x x x x x x x x x x x x x x x x x-$
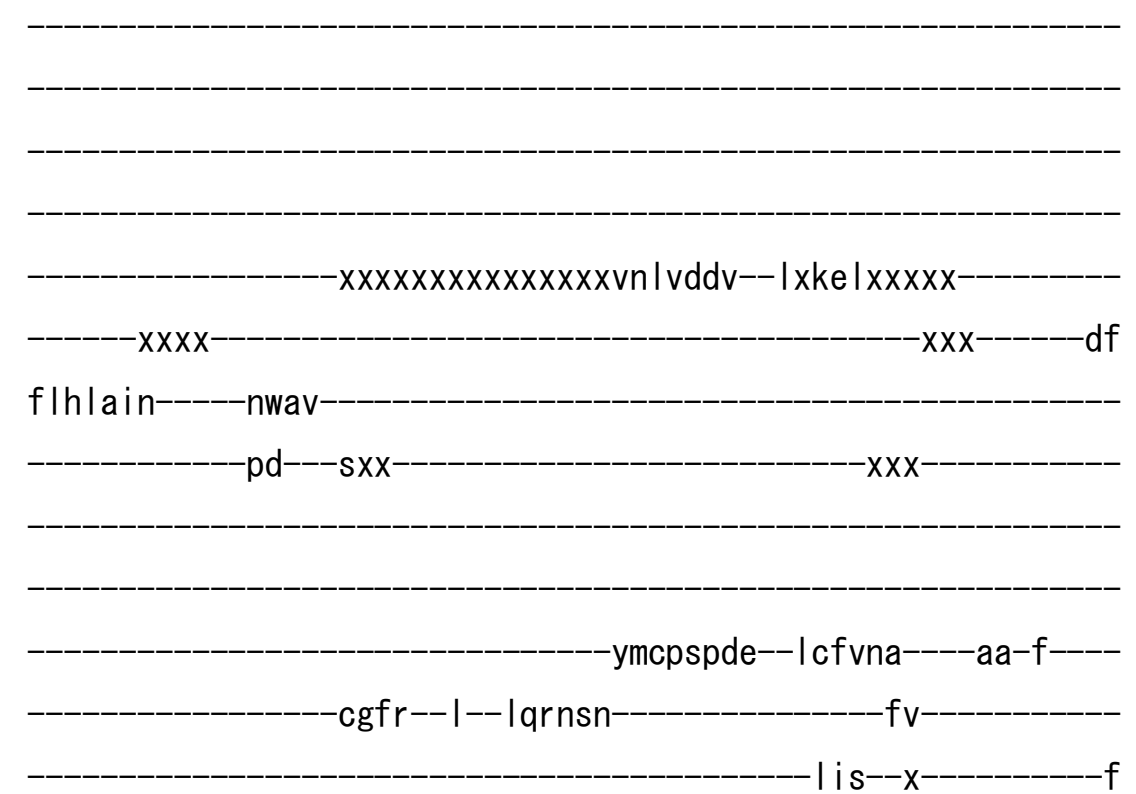
nq i y-------kiki i aqadfdykrk-cstt-----i---i sxxxxxxxxxxxxxxxxxxxx xxxxxxxxx------xxx------------- $x x x x x x x x x x------------------x x x$

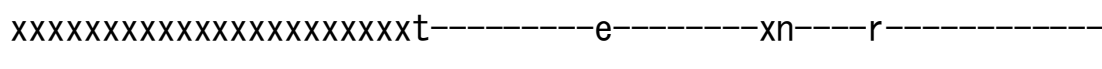

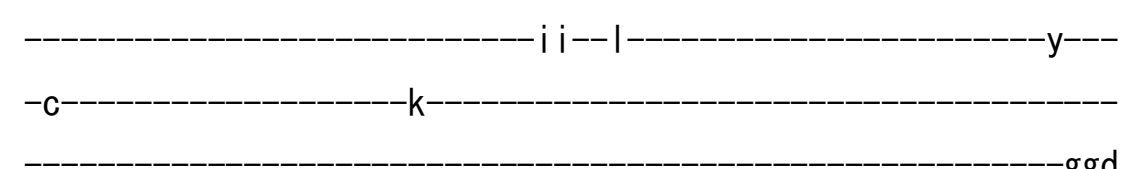
$n$ imikklkxxk 
x----xx--vvt-I rnmkky--svg----g | rtLVFAKRe i--e l kefnew l keynr---

ik----- I t i esrdek l aecvsk-l ecd l e I QGVTGIEDKLQTGVSECIEQLLMag-IR I

WMLTGDNLDTSIN IG I ATNL-

- vnmlsdri----

$-m \mid-----d s n t x x x x$

$-x x x x x x x x x x x x i d x x x n x$

xxhrclildsisiey

if $x x x x x x x x x x x x x x x x x x x x x x x x x x x x x x x x x x x x x x x x x$

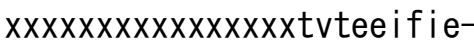

i lkrvhsv ICC--RMTPYLKGAVVTFVk

$---n k---1$

$\mathrm{xg}$

- ITLAVGDGANDCNMIQIAHVGIGI--

-kgregsq

-AFNASDFG I GefRFLSPL I--LHHGRLCYRNLSKCI $S$

ymf-yknvi-li i Iffyay i s If-_-_-_-_-_-_-_-_-_-_-_-_sgak i yys I fva i y nvvfts i pvg i f g i vdqdynr e---_-_-_-_-_-_---fsvkyphvyq I gq inhyfnvik f-sgwi Inai iqsav if-fmmt-vg|--_-_-_-_-_-_--_xxxxxxxxxxxxi--_-_-_ad--apt I giml Issvfi ivsck-Ivletwyftkitl|---_-_--shl is ifffi i tvcs fssspi iys $x x x x$ -gsafvlf-

-tsyrfw----iv--i I gt Im I smyrd-

-yfykvfk------_------ysfc-

$-q y y$

$-x x x x x x x x x x x x x x x x x$ 


\section{>tanIV_IV02 XP_951951.1}

m-

$-x x x x x x x x x x x x x x x x x x x x x x \mid k d r m t f x x x x x x x x x x$

---yksircis

xxxxxxpk I f Iyng I vnskyn $-f f$

-tfvplvlysqf 


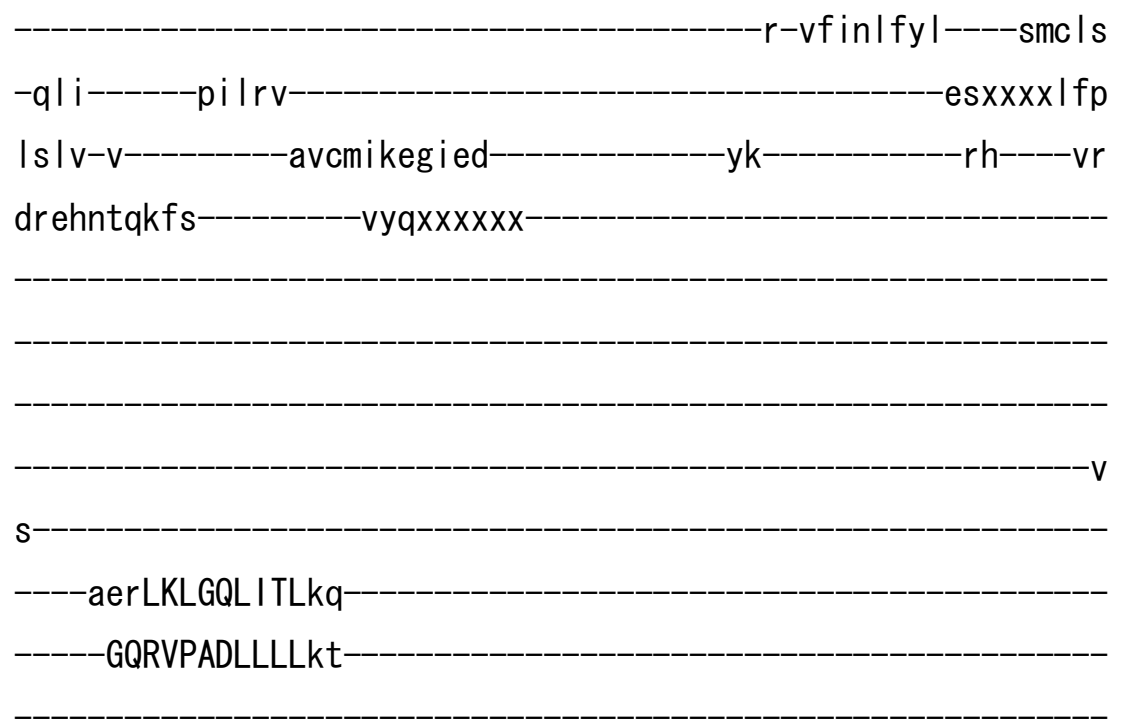

-sdpng-a---sfVRTDQLDGETDWKLKra-

-vattqamsn Ikdi I glysvatveeprddfynftgk i cf xx

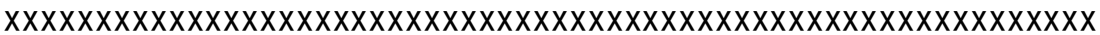

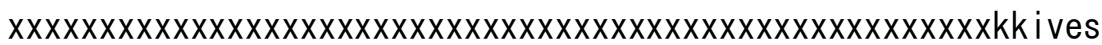
$\operatorname{lnv}$ $d-$ $-N I V W M--N S I V$

as----gtihgLTIYIGKDARACInskraki

-kyg I fekelnr IfivlilimlvmcvlmI -spsgfmsm-wyvt-flkyLLLFST I

VPLSLRISIEIAKYVYNR-GI vmdkk i

$-\mathrm{pn}$

-tMPR-TTMI I PEELGR I NYLLTDKTG

TLTQNVISLe -kly itrgyy

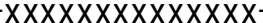

$-x x x x---x x x x$

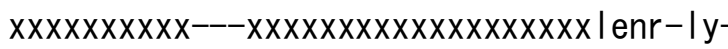

II s lavc------hnvr

$-p v x x x$ 


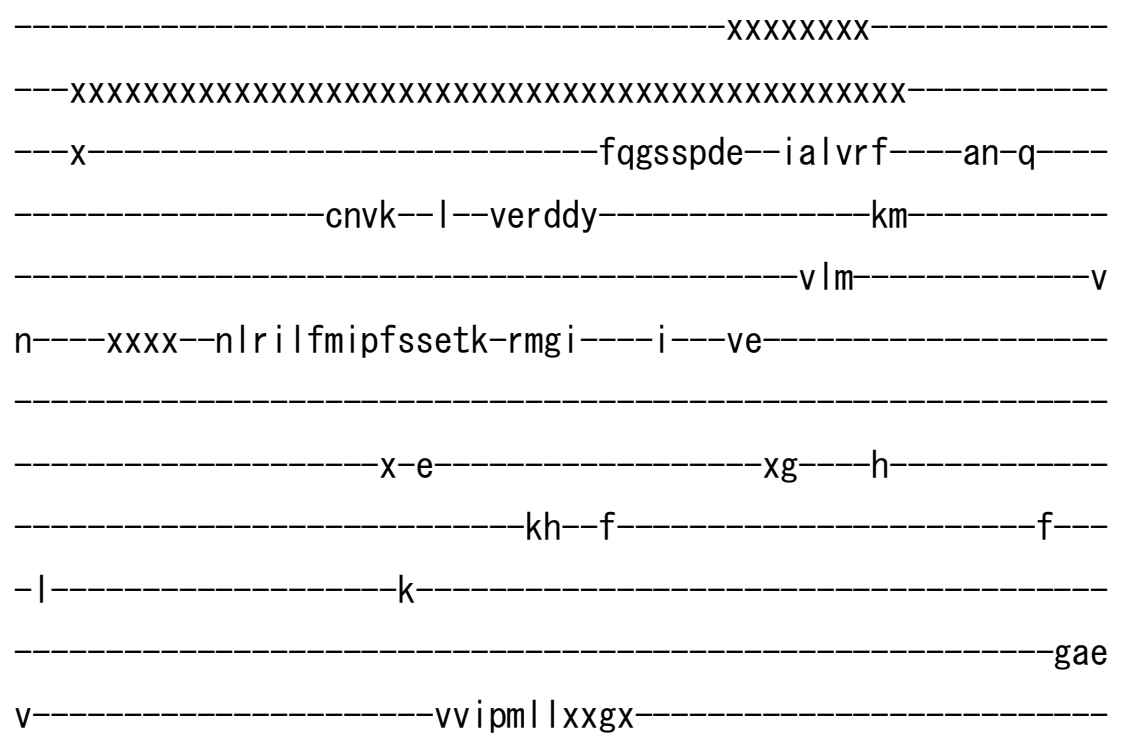

-vwl-seecdn I--arm----g|rtLVFAYRs i--sdseysnfvqkyne--an------ s l ydrvkki rkvtgt- | ehgm I IVGL TGVRDKLQRNVGSTLESLKNad-IK I WMLTGDK IDTAKCVAISSGMiqrnnsif---$-q i-----s v d k$

$x x q i x x x s x x x x x x x x x x x x x x x x x$

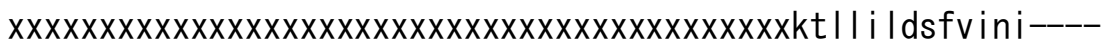
$\mathrm{si}$

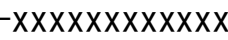
xxx-------------kalkr Ifik-

sI I lann iLFC--RCTPQMKADIVKLLk-

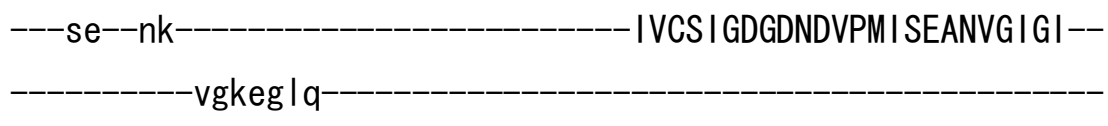

-ASLSSDYSLVefSHIKRLL--LWHGRNSYKRSSTMS------N 
fli-hrgvi-isvmqaifstiyyf-mp i affagw I lvgf tsyynmipifcl-vldeeisee dvm Ifpelyvelkkgrelnikt

f-I iwiwi sifagai Im-vgai-i If e-

ns--IvslisigctsIflleiln-liselhhwhrlti i glvctcivyfys I Iv frnnfdmef $i$ arksff-----wk--viai sm l gw lpi -yfikflt$-k|| k$ $-p-$ -pqy iklivt

>dme IV_IV01 NP_725290. 1

m- 


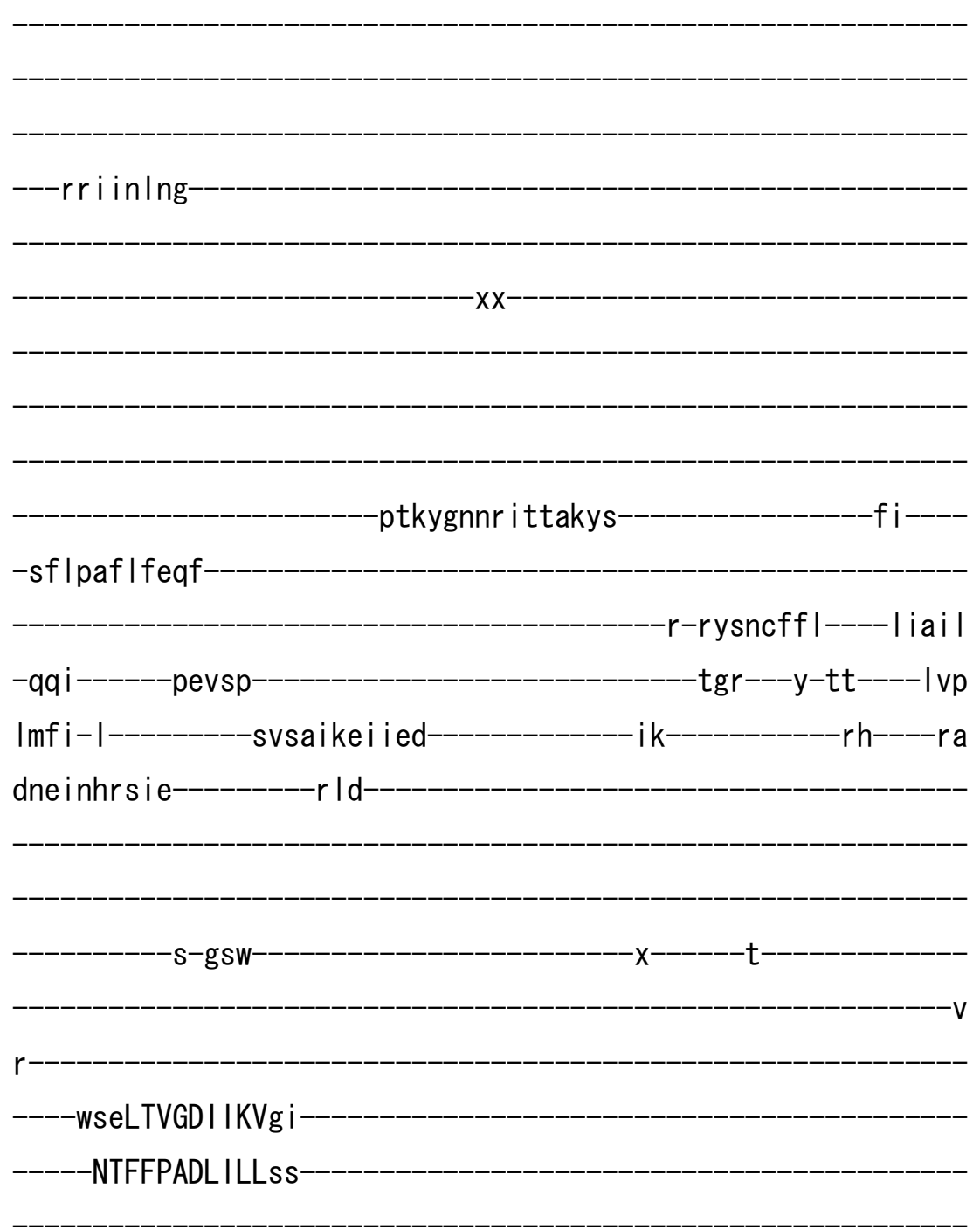

-sepqa-m---cfIETANLDGETNLKIRqa-

- patae | letkd lar legr i ece I pnrh l yefngv | rex-

-gkppaa

Ign-

-QVLQR--GAIL----------------r

d----------QVLQR--GAIL

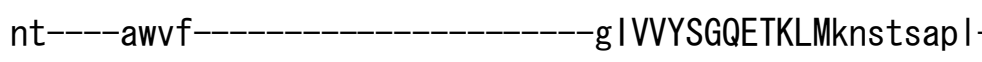

$-k r s t v d k$ Itntqi ImI fmi I is I c i isg

- Icn I fwtrehsetd-------wy I g--_-_-_-_-_---xxxfktks I gyn I I tfF ILYNNL

IPISLQVTIELVRFLQAI-FInydi em- 
aMAR-TSNLNEELGMVKYIFSDKTG-
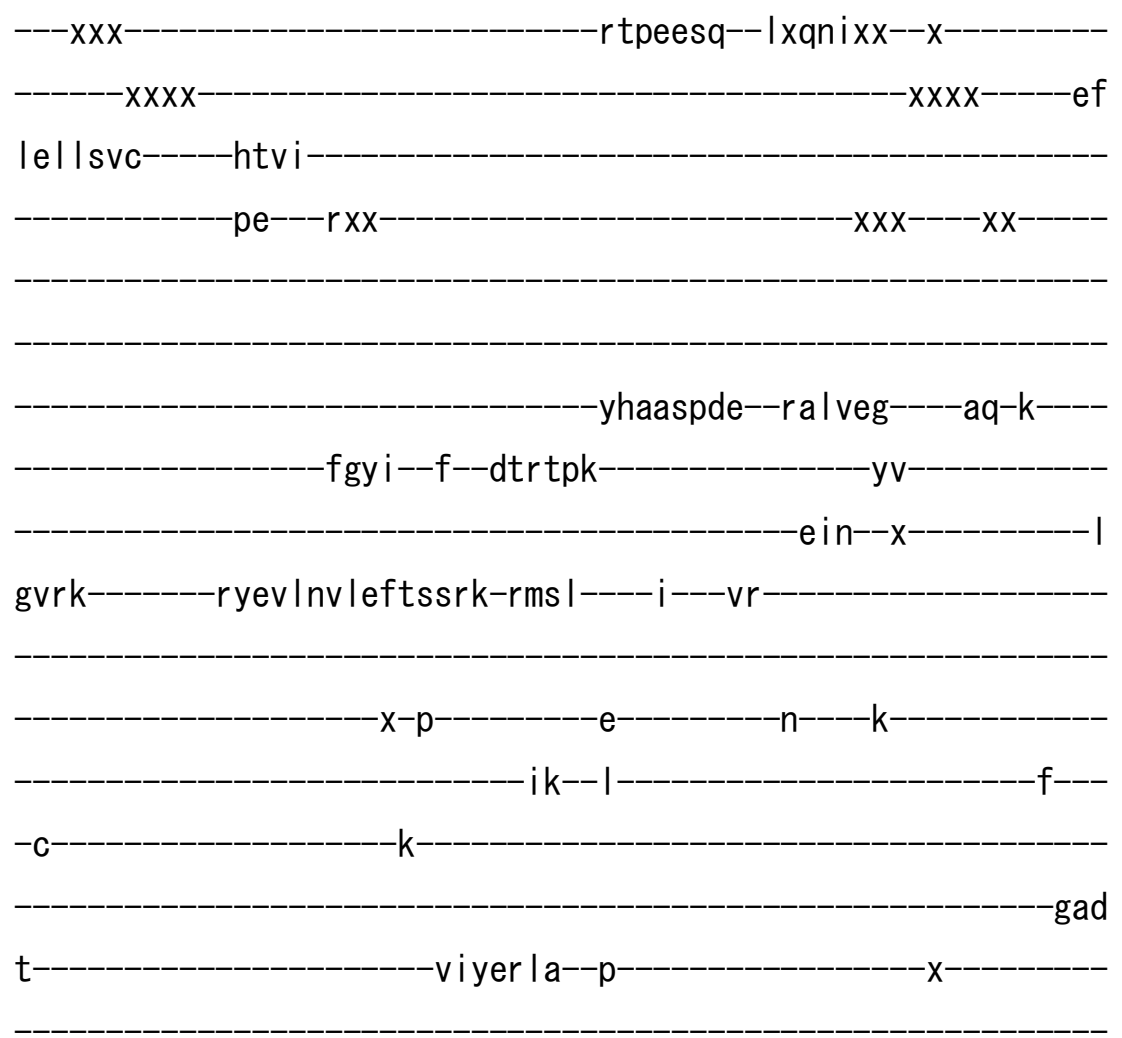

xxx--xx--eqt-I rhleef--asd----g | r tLCLAVAd i--rpdvyqewsqtfdk--as-----va l qnresk l edaan I-i enn I r ILGATAIEDRLQDGVPET I ASLLDag-IY I WVLTGDKQETAINIGYSCRL-_-_-_-_-_-_-_-_-_-_-_-_-_-_-_-_ i shsmd i i--_-

-


Ic i IcrvvICC--RVSPMQKAEVVEMVt

---qsx---_--_--_-----xa----_-_-_-_---VTLA I GDGANDVAM I QKANVG I G I--

AACASDYSIAqfRYLQRLL--LVHGAWNYARI SKL I------L
ysf-yknvc-Iyvi e I wfavysgw-_-_-_-_-_-_-_-_-_-_-_-_sgqi I ferwtig I y nv I ftamppfamg I fekfctae--_-_-_-_-_-_-_-_tmi rypm l yktsqnak I fnvkv f-wiwifnal lhsvfIf-wlplxayt------------------xxxxxxxxxt-------sd--y I Imgn I vytyvi vtvc|k-ag | i tnswtw | th |-------ai wgs i v Iwfgf। I i yshvwpmfxxxxxxx---_-_-_-_---_gmdiql I--_-_-_-_-_-_-_-_-_-_-_-_-_-_

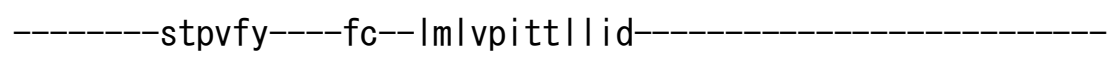
---------vick|vh------------ntvf-------k-------------t/t

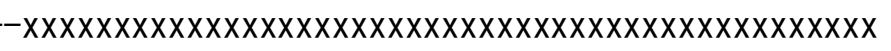

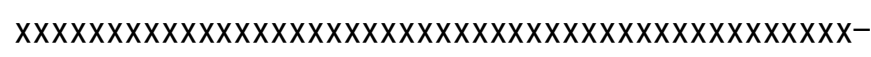
$-x x x x x x x x x x a t$ I hiftexxxxxxxxxxxxxxxx

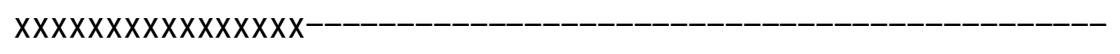
$-x x x x x x x x x x x x x x x x x x x x x x x$

$-$ >dmeIV_IV02 NP_723806.1 m- 


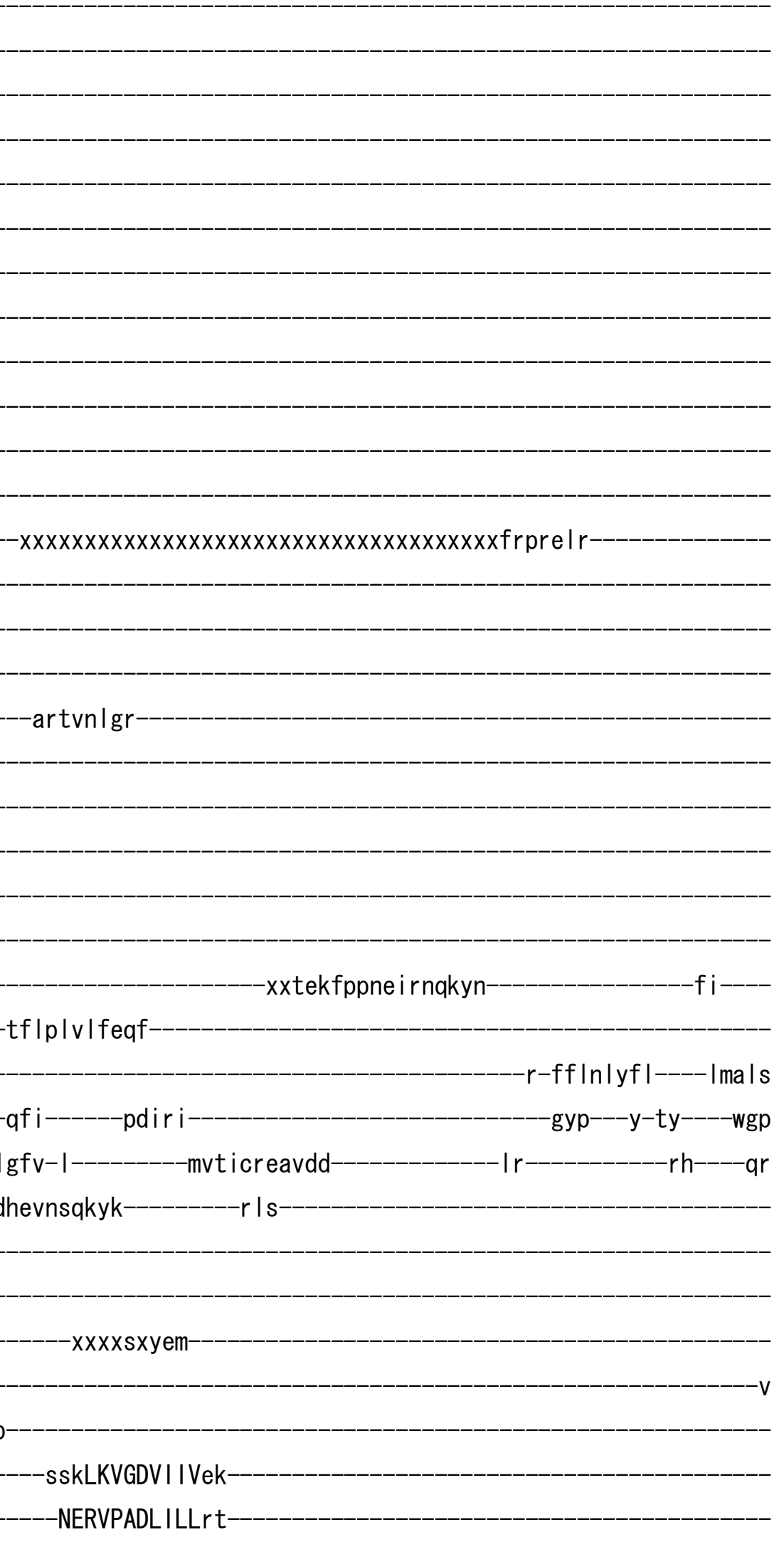


-sdrsg-s---vfVRTDQLDGETDWKPRIa-

-vpytak Isrdse I hs i dasfyvekpqnd ihsf i atf cmxx

gsedtg

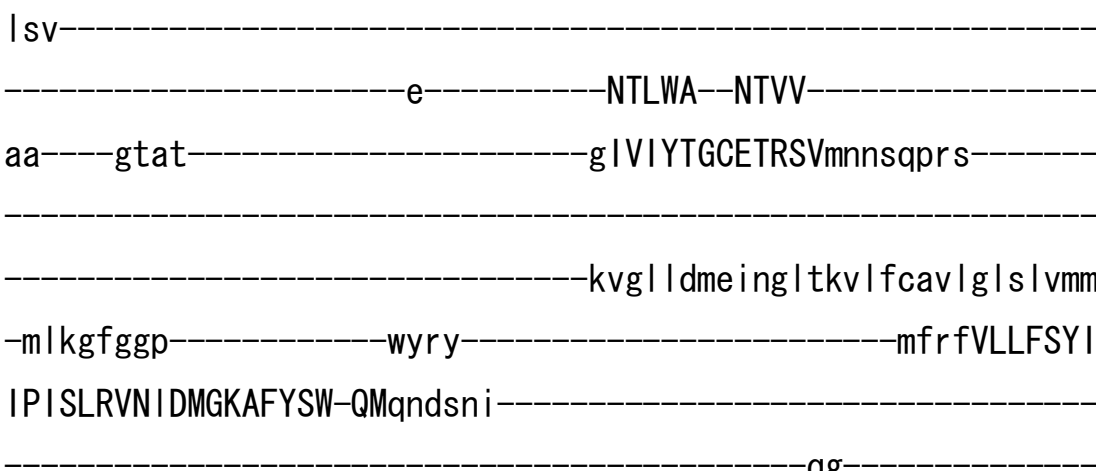

-tVVR-STTIPEELGRISYVLTDKTG

TLTQNEMVFk kihlgivsh-

$-x x x x x x x x x x x x x x$

$-X X------X X X$

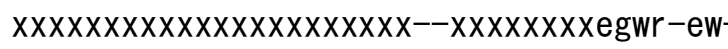

ea

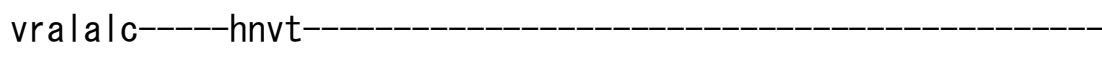
$-p \vee x x x$

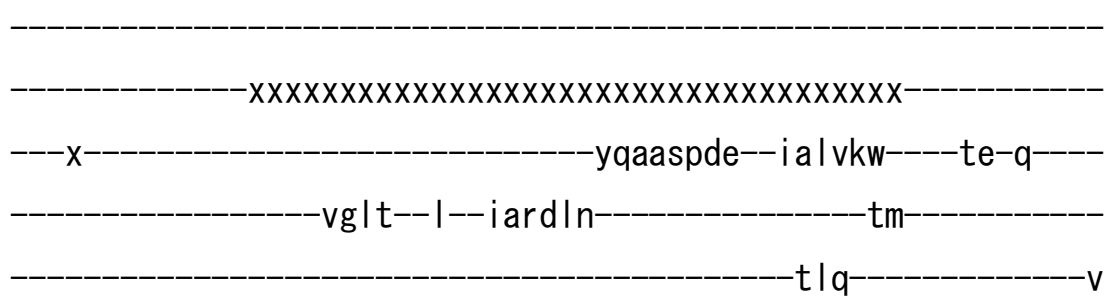

ktpsxxxxxxxhyqi lqlfpftsesk-rmgi--_-_----vr-_-_-_-_-_-_-_-_-_-_-

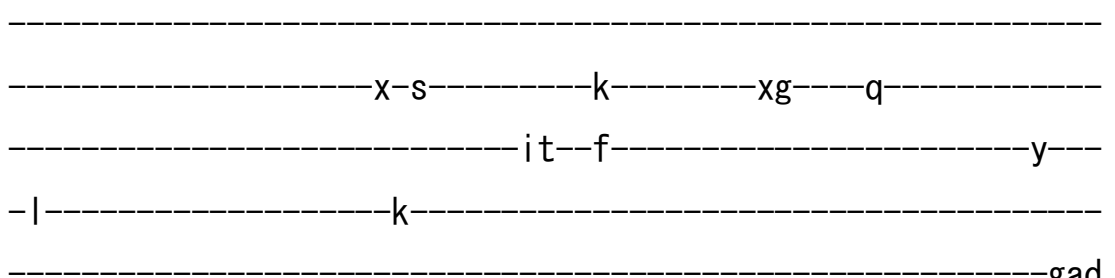

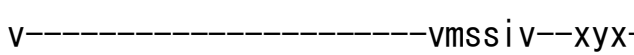


-dw I-seesgnm--are----g|rtLVVAKKv |--teeqysdfetryna--ar----- I s i tdrvakvaavtes- | ere I e ILCLTGVEDRLQENVRPTLELLRNag-VRV WMLTGDKLETACCIAKSSQL -igrnqg Ih----

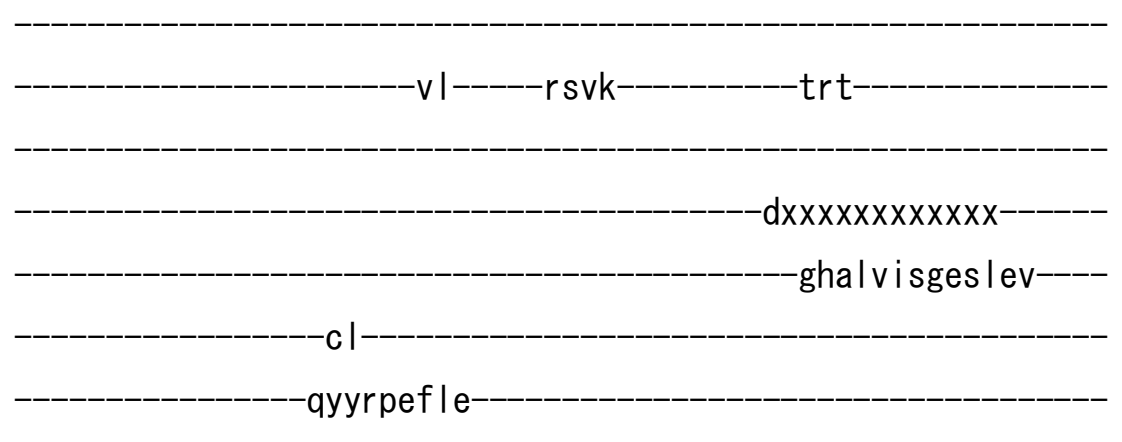

lataspavVCC--RCSPTQKAQVVAL I

---kh--tg-----------x-----_--_----RTCAVGDGGNDVSM I QQADAGVGI--

-ASLAGDFSIPqfSHI AKLL--LI IHGRRSYKRSAALS------Q

fvi-hrgli-itt l qavfsavfy l-_-_-_-_-_-_-_-_-_-_-_-_ssva l yqgf Imvgy st I ytmfpvfs I-v|dqditse--_-_-_-_-_-_-_-_tavtype lykd I skgr s I sykt f-f iwv l i s i yqggv im-ygal-i If-_-_-_-_-_-_-_-_-_-_-_-_-_---v--_-_-_ de--fihivaisfsal imtel im-valtvr twhr Imv|-------aelfs lalyl is lav lhey-fdwef $\mathrm{i}$ -wsydf I----wk--vs|it|vsc|p| yi ikflr---_--------kkcs 
>dme IV_IV03 NP_995666.1

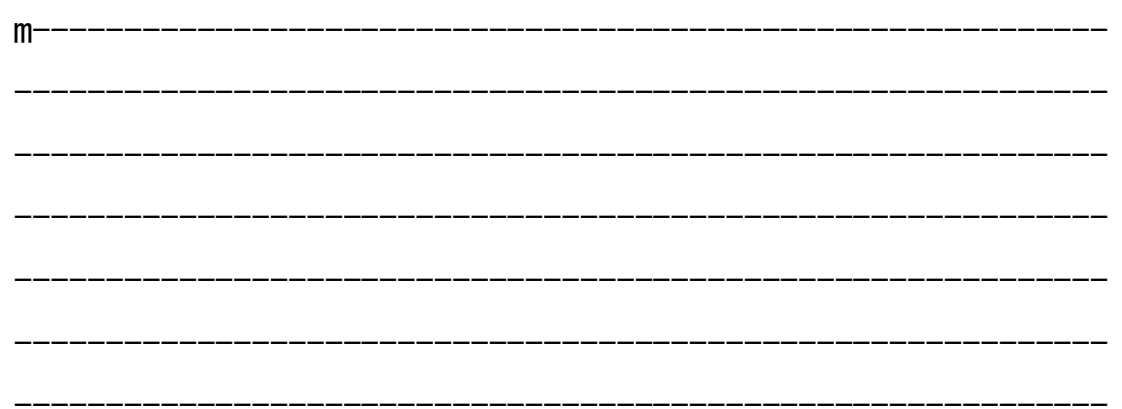

$-X X X X X X X X X X X X$

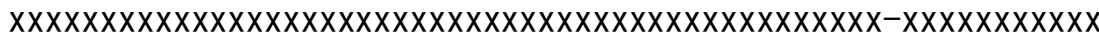

$\mathrm{X}$

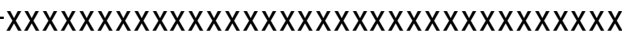

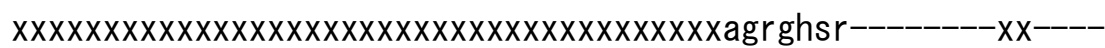

$-x x x x x x x x x x x x x x x x x x x x-$

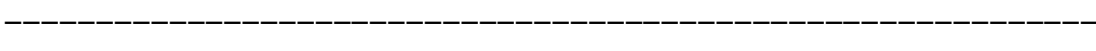

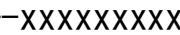

---ghsrqasr

$-x x x x x x x x x x x-$

$-x x x x x x x x x x x x$

$-x x x x x x x x$

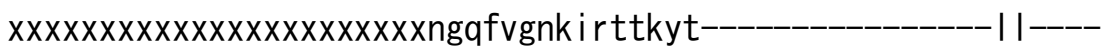

-sfipknl leqf 


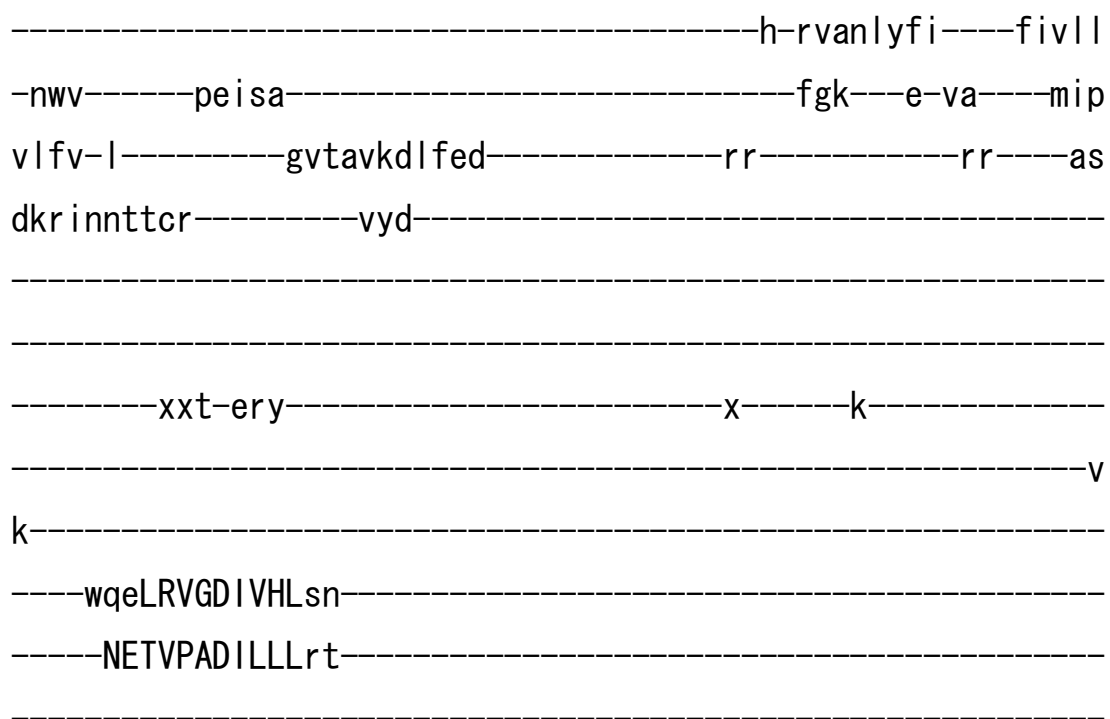

-sdpqg-v---cy IDTCDLDGETNLKRRev-

-vrgfeemqs i fvpskfvsrveadapttk lyrfhgal ihx-

tgervp

ist

-CLLLR--ESRL---------------k

nt----dy ie

g IVVYAGHETKSM Innsgpry

-krsqveqqmnidviwcvi i l l i lcvvga

-igcrmw I ssfthfp------xpy I p------------xxxxtanmesmw i fwty IVILQVM

IPLSLYVT iELCKILQVF-HIhnnvd I

fdaetnkq

tECR-AMN I TEELGQIQHIFTDKTG

TLTENKMIFr -rcvvngsdy

XXXXXXXXXXXXXXXXXXXXXXXXXXXXXxxxgtyltphxxrxqef

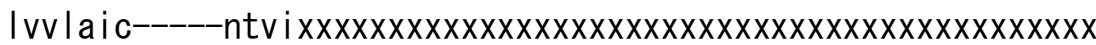
$x x x x x x x x x x x x q p x x x s x x$ 


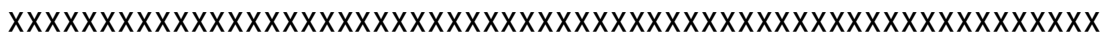

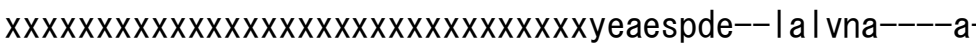

ysydxx|-- Inrspnqix

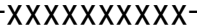

eye i lkv Ipfdssrk-cms i

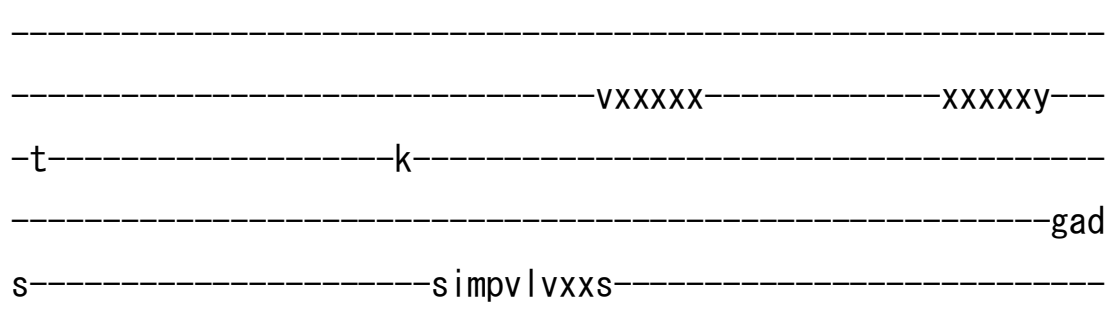

xxxxxxxxxeqt-qq | Iry--are-----g|r iLVMAKRt |--nsadytdwwarhqe---xems lenrerr I rdsfax I esn I t ILGATGIEDRLQDGVPETI ASLLSag-ISV WVLTGDKPETAINIAYSAKLftqqmel i----

$r \mid$ tars $r \operatorname{daxxxx}$

$-x x x x x x x x x x d k x x x t x x x x x x x x$ xxqral vvdgkt Itf il

xxx--_-_-_-_-_--sk I i lpf Ir

I skrcasvLCC--RSTPLQKAYLVKVVk

---ee---|-----------х |--------------RTLA I GDGANDVSM I QMADVGVGI--


yff-yknaa-fvfl i fwyqlycgf-

sgqvmmdqmy Im ly

nl ifts I pp lai gvydkrvaed-_-_-_-_-_-_-_ I I knpy lykngr I gvayrphd

f-wl i I dal yqs I vif-fval-cay---------------xxxxxx---------------

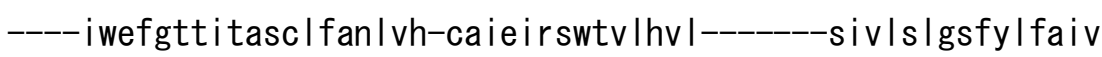
ydsvcmncxxxxxxx-------------wvifvcf--_-_-_-_-_-_-_-_-_-_-_-_-_-asavhw----|v--im|stvvav|pr

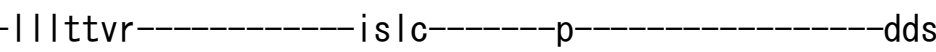
$-x x x x x x x x x x x x x x x x x x x x x x x x x$

xxxxxxxxxxygsknqi i xxxx

>dmeIV_IV04 NP_731669. 1

$\mathrm{m}$

$-x x x x x x x x x x x x$

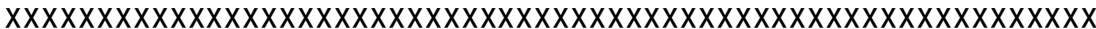
$\mathrm{X}$ 


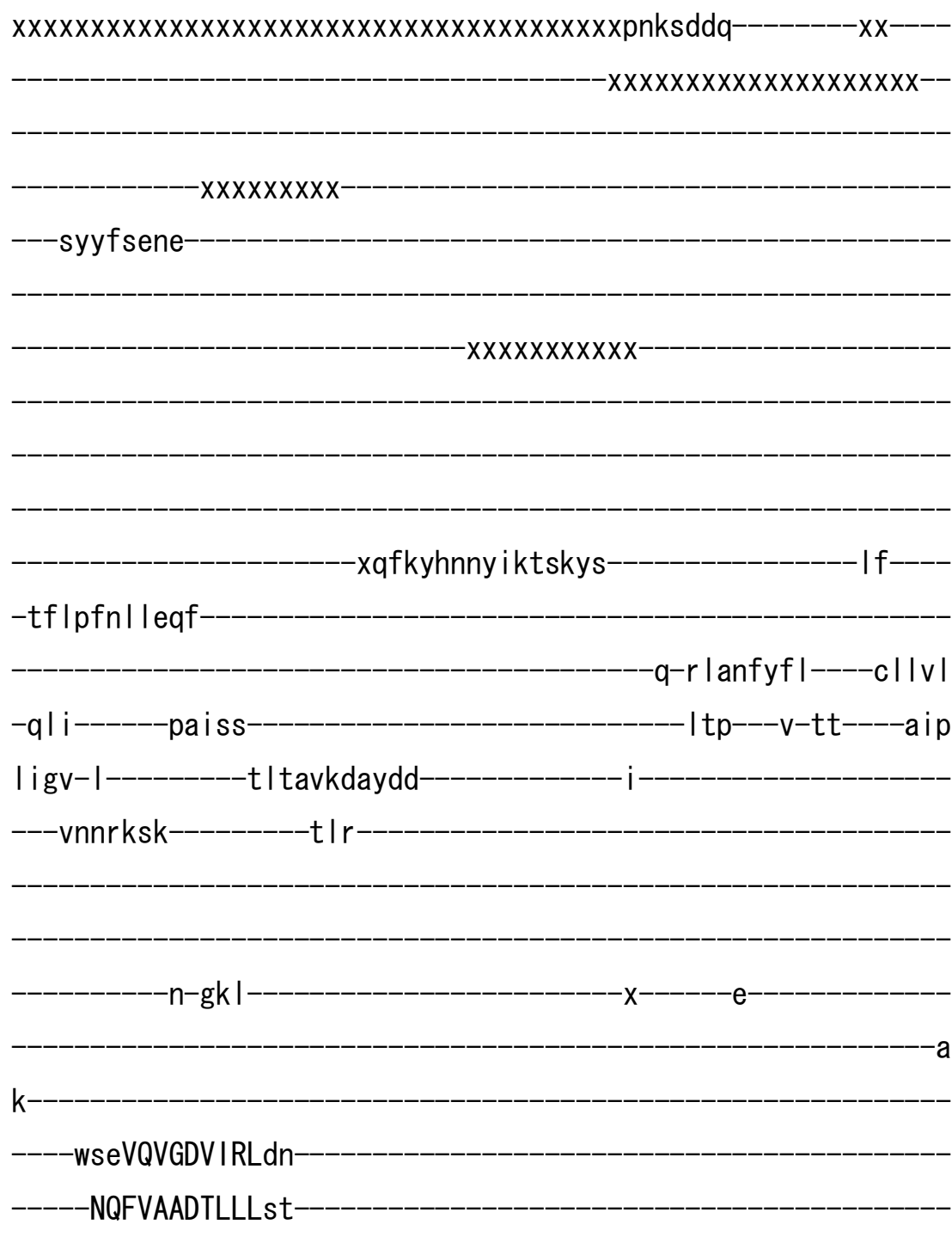

-sepng-I---cfIETAELDGETNLKAKqc-

-xtet i e I gdrhds I wnfnge i i cerpnn I Inkfdgt I iw--

-rgarfa

Idn-

KILLR--GCVL---------------r

e---_-_---KILLR--GCVL

gVVVFAGVTKLMansgktqf

-kstgvdr I Inf i i igivIfIvsicalfa

-i gca iweg I igqhf------x|y |p---xxxxxxxxxxxxgatvig I I vffsyAIVLNTV VPISLYVSVEVIRFVQSF-LI nwdeem- 
-yypttnty

-aKAR-TTTLNEELGQIQY IFSDKTG-

-TLTQN I MTFn-------_------kcs ingr sy

$x x x x x x x x x x x x x x x x x x x$

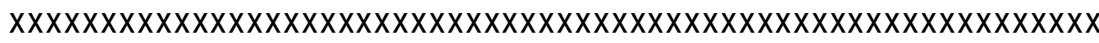

$X X X X X X X X X X X X X X X X X X X X X X X X X X X X X X X X X X X X X X X X X X X X X X X X X X X X X X X X X X X X X X$

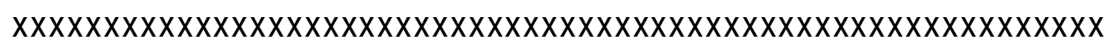

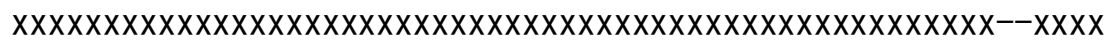

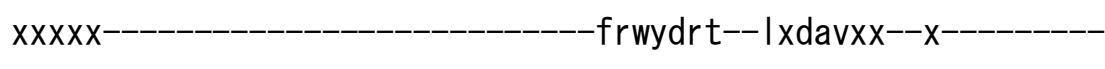

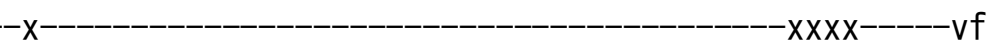

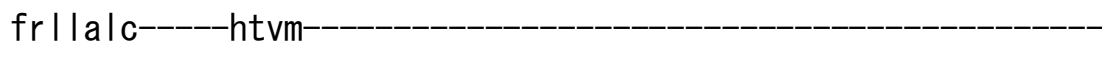

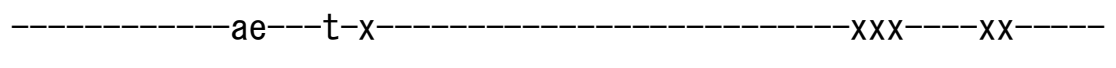

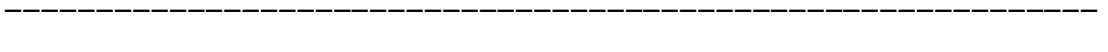

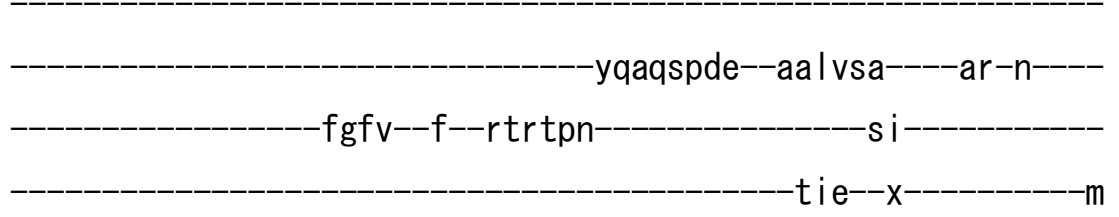

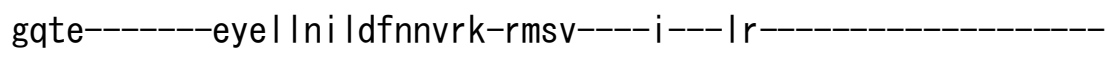
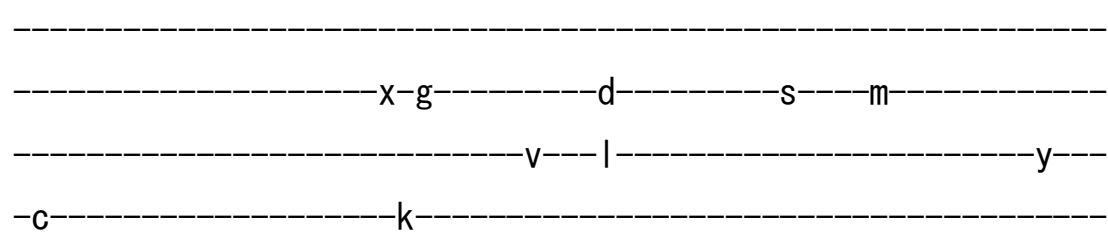

- gad

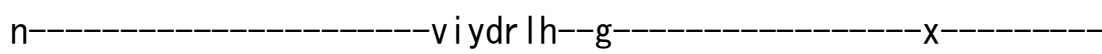

xxx--xx--art-qdh I nkf--age----g|rtLALAERr I--teqyyndwr srqqe--aa-----I smdsr eqk I nai yee-i esemq I VGVTAIEDKLQDGVPKSI ANLQNag-IK I

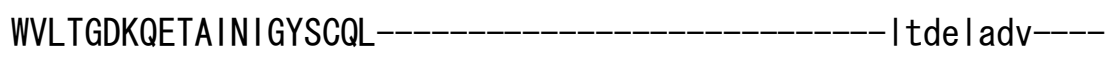

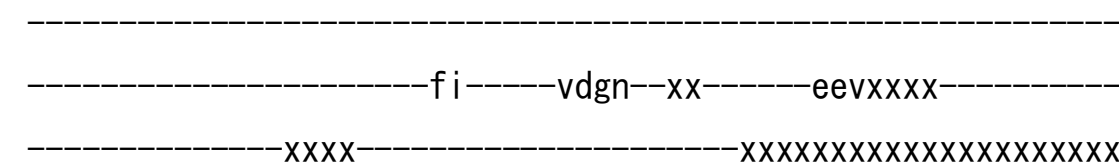

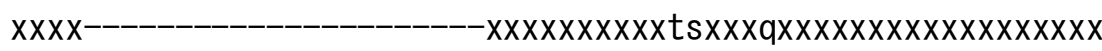

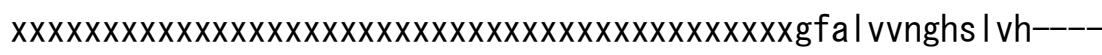
$-c 1$

x---------------pe lenkf Id- 


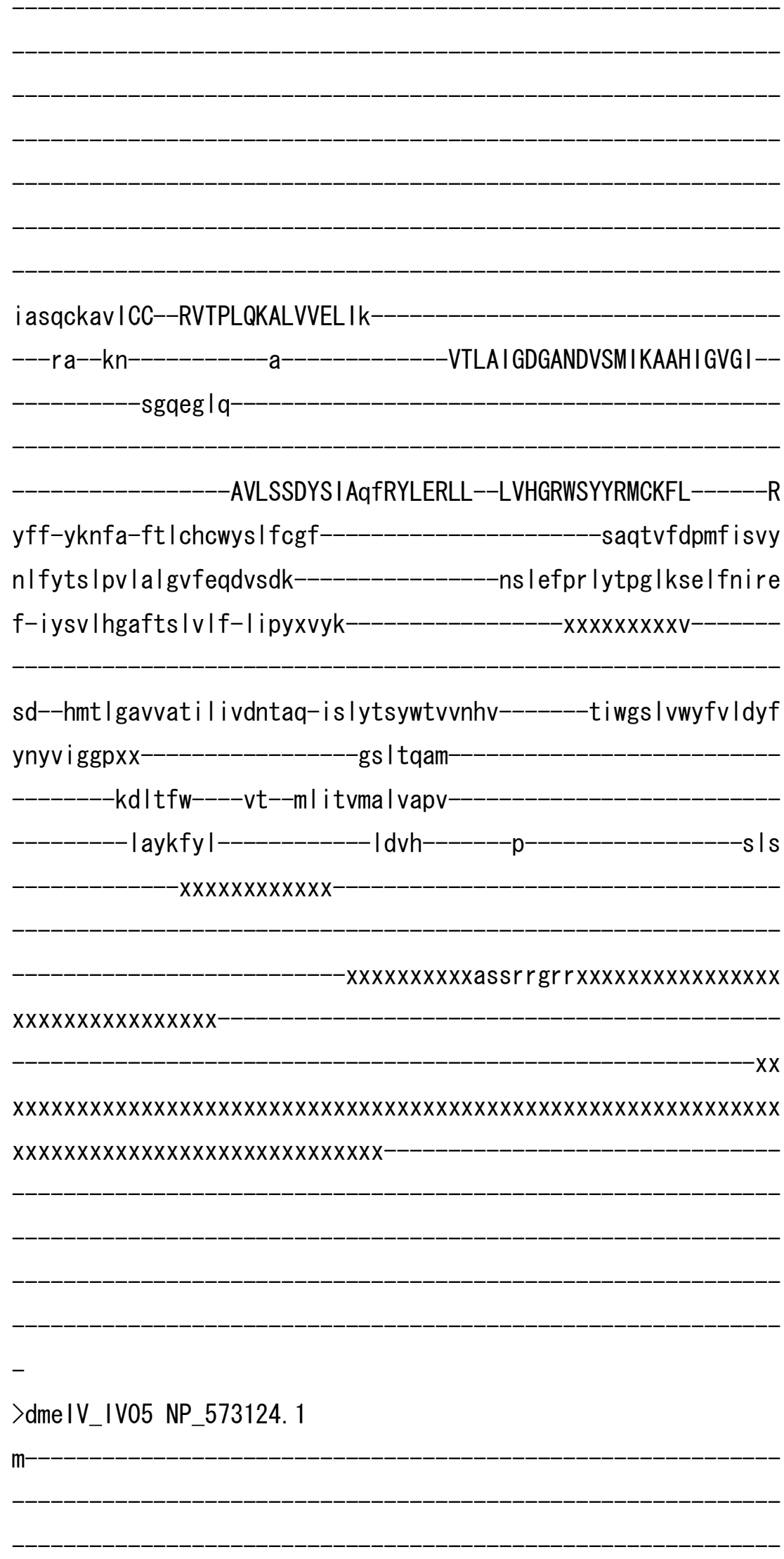




\section{xtfIpInfyeqf}

-rravyfyfxxxxivsff

xnet-

IIfv-m

$\mathrm{dk}$ Ivnt $-i s x x x|| p$

- i i talkegled-ys $-r s^{----k s}$

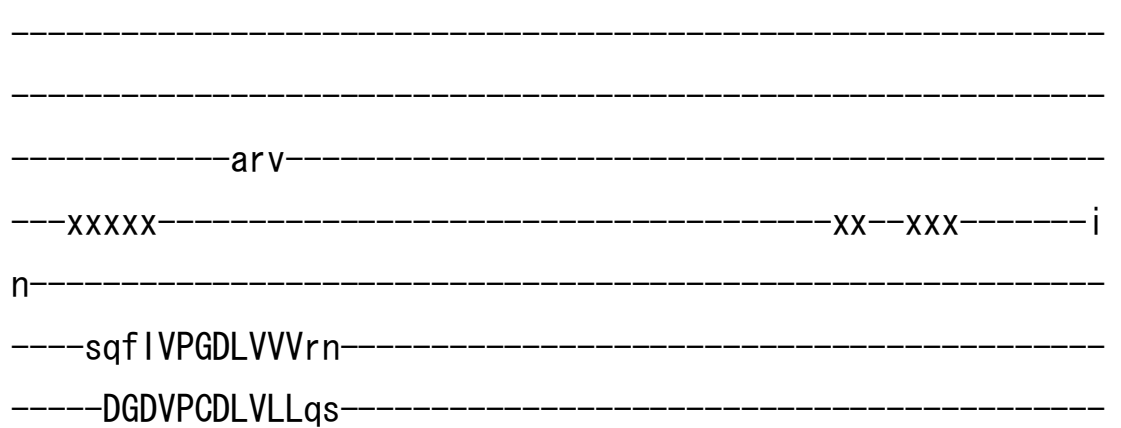
DGDVPCDLVLLqs 
-ssadr----xcfVNTANLDGETNLKTI Icv-

-ptny I l agdhe I qgkdc i vcepssad l yt fngr l e I xx

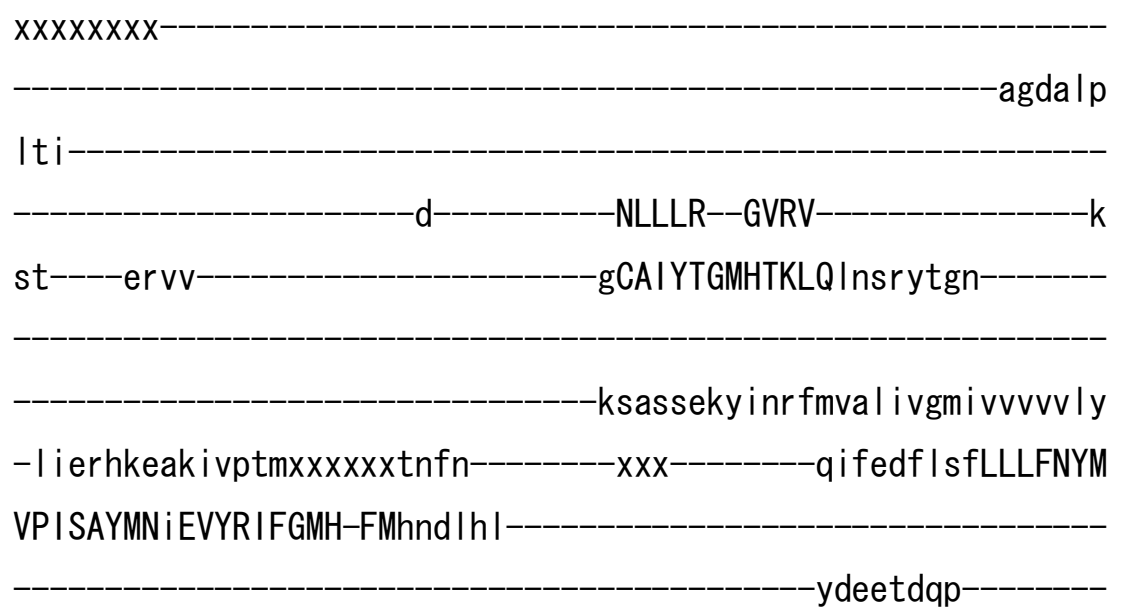

-CRVN-ASNLNEELGQVNILFSDKTG-

-TLTKNLMKFv-

ncyvpgtny-

xxxxxxxvsegtdexxexek|xxxxx

$-\mathrm{vl}$

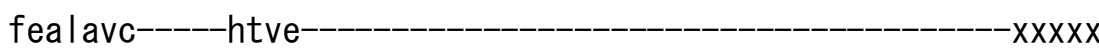

$x x x x x x x x x x x x e q x x x m x x x x x x x$

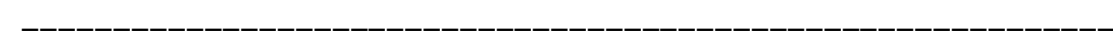

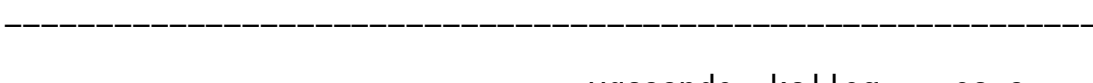

$----------------------------------y q a s s p d e--$
-
-

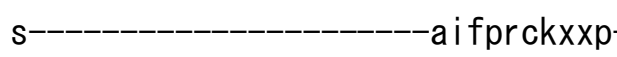


-xxeqt-daq i tky--aqn----g | r tMAVGRRm I--tdde I fhfee l yrk--an-----tq I snrne I i ascyet-vene I d LGATALEDALQEHVGETLEALQAag-LK I WVLTGDKVETAYNIGLACRHiprgskgh---$-\mathrm{i}$ inttxxxx

$x x x x x x x x x x d e x x$

-vlivdgttita II ehtprqfad-

la | rcravLCC--RLSPLQKSE IVTL Ik

$---r r---k-$ $-x y$ - TAA I GDGANDVSMIQEAHIGIGI-tgregkq

AARCADFSI ArfEMLRRLF--LVHGHYNSQRLAFLV $L$

fyc-ykn i i-itgcma l yqvyd ly--_-_-_-_-_-_-_-_-_-_--_satnvyns i y Iw If divy i sfsftv l a icdkdysee--_-_-_-_-_-_-_--t I I shpe l ykp I ahnrqasmgv f-s Iwi Ingfvqcfi if-fftyxm In-_-_-_-_-_-_-_-_--_xxxxxxxxxt--_-_-_as--fqtfgtml i ti i vi vgn Ik-I I |vahymtyrnfa--_-_---mi las i aafm| tty | ynlytsgexx dvynqf I-

--------sslpiw----|f--ti icsvacl|pdfvikvvn- 
>dme IV_IV06 NP_573125. 1

m-

$-x x x x x x x x x x x x x x d r \mid g a h r$

$-x x$

$-x x x x x x x x x x x x x x x x x x x x-$

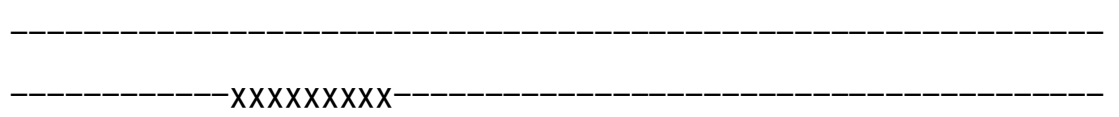

---wlqiqigg

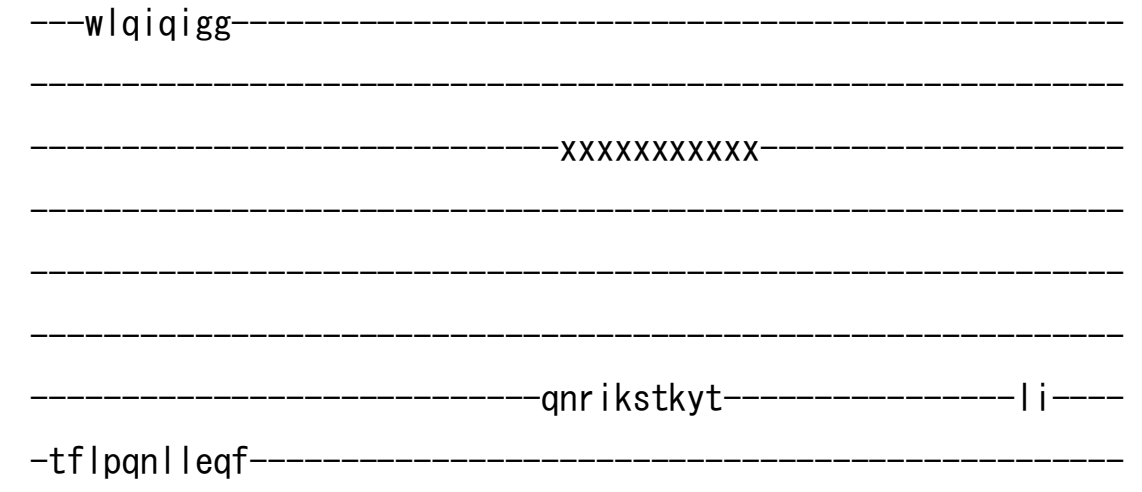




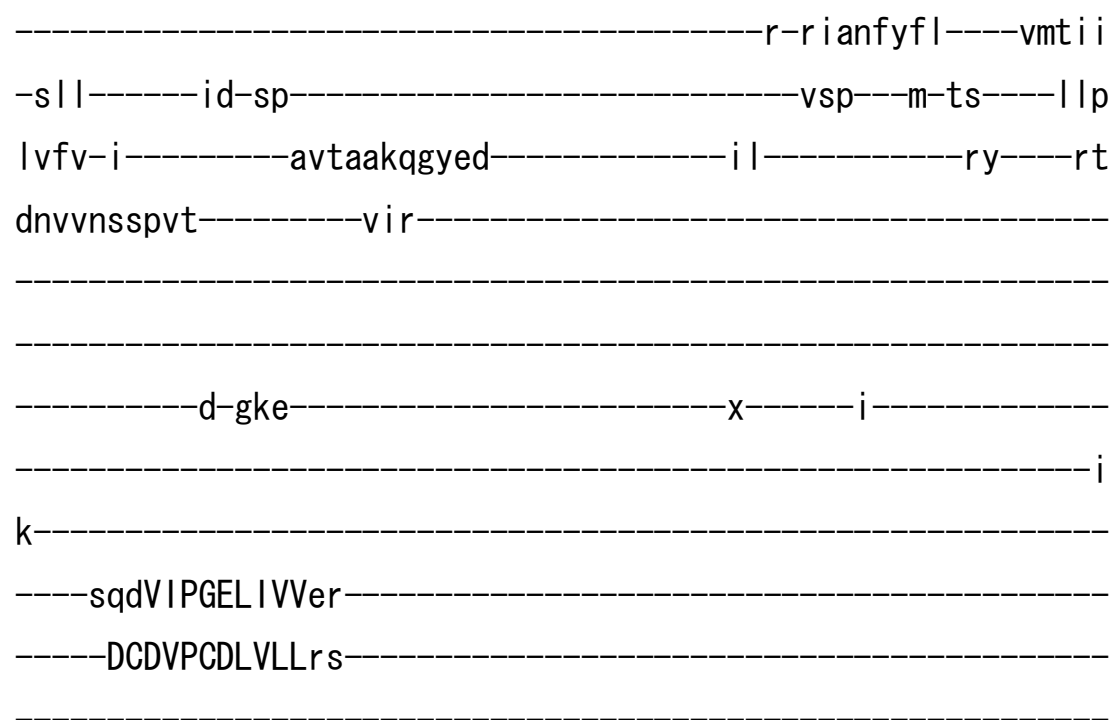

-tdphg-k---cfITTANLDGESNLKTLmv-

-prdlptvdl pemhk I gi i ecespttd lysfngk i I xx

$$
x
$$

egrvlp

Ist

-NVLLR--GSRV---------------k

nt----ecvi-_-_-_-_-_-_-_-_-_-_gCAVYTGM I SKLQInsr I trn-

-knassetyinrflivilvaliaivtlly

-fIkryne Ifvipk Ixxxxxxtdsy---------xxx---------qf I qdy I sfLILFNYL

IPISLYVT iELQRVIGSW-FMewd I I

-yenetdqp

cVVN-TSNLNEELGQINILFSDKTG

TLTKNEMNFa

qcs ingnkf

-xxxxxxxedeetkaxxdxnkfxxxxx

$-v f$

fqals i c-----htvq

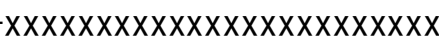

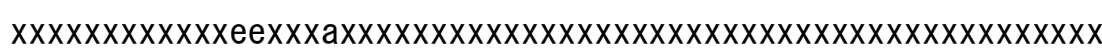




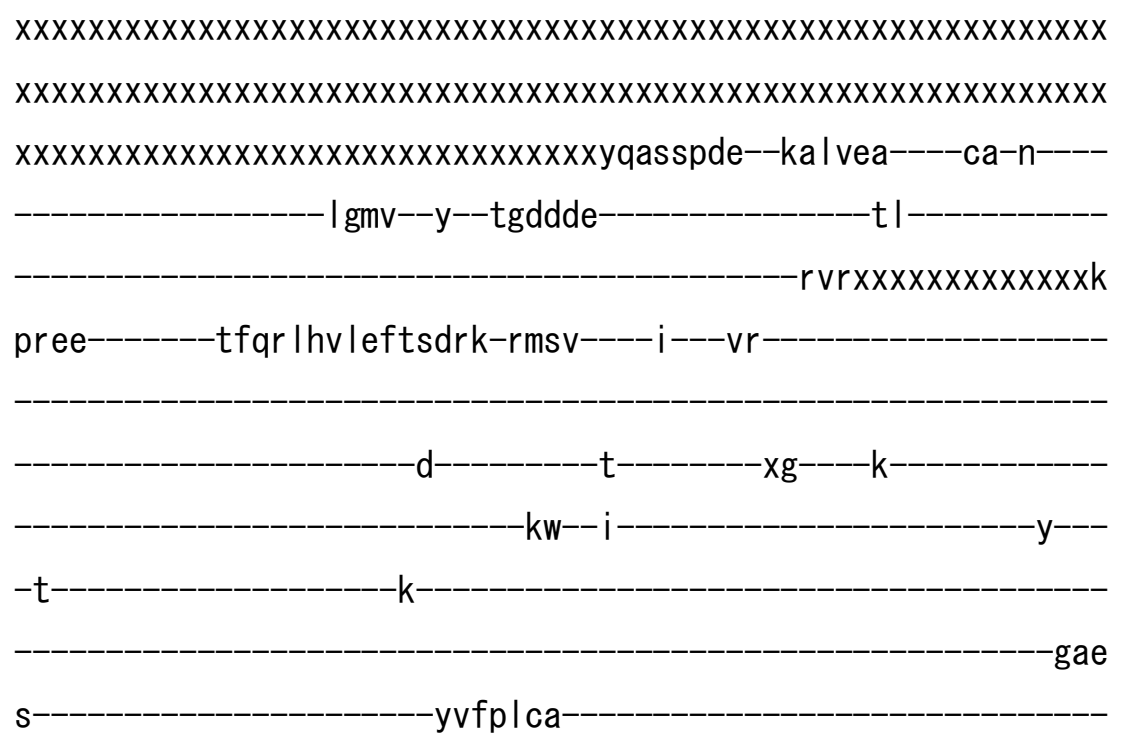

xxx--xxxxtkt-dahi sdf--ar I----g|rtLAI ARR I i--seeeyqdf I ve laq--an-----ss l enrkq I seecyak-i esn I d ILGATAVEDALQDDVADTLVSLQAag-IK I

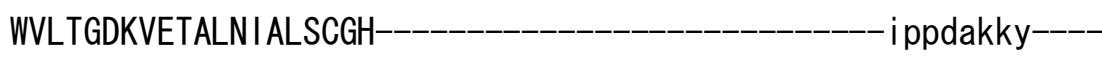
fi-----meckxxxx$-x x x x x x x x x$ ixxxi -xxecal lidgks Igv al aeassefrd-

vavkctavLCC--RLSPLQKSEVVSLIk

---ss--ne---_-------xy--_-_-_-_---NTAS I GDGANDVSM I QEAHVG IGI--




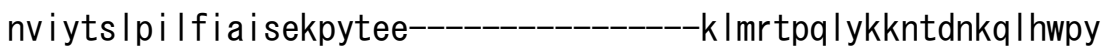

va--fscfgt I Imwtvvvvvn lk- |w lesmy I sfwy if -waynn I I-

-as I pvw----- |w--i i vtcvac I vpd-

-ytirmla $-r a \mid n-$

>pfalV_IV02 XP_001350596.1

m- 


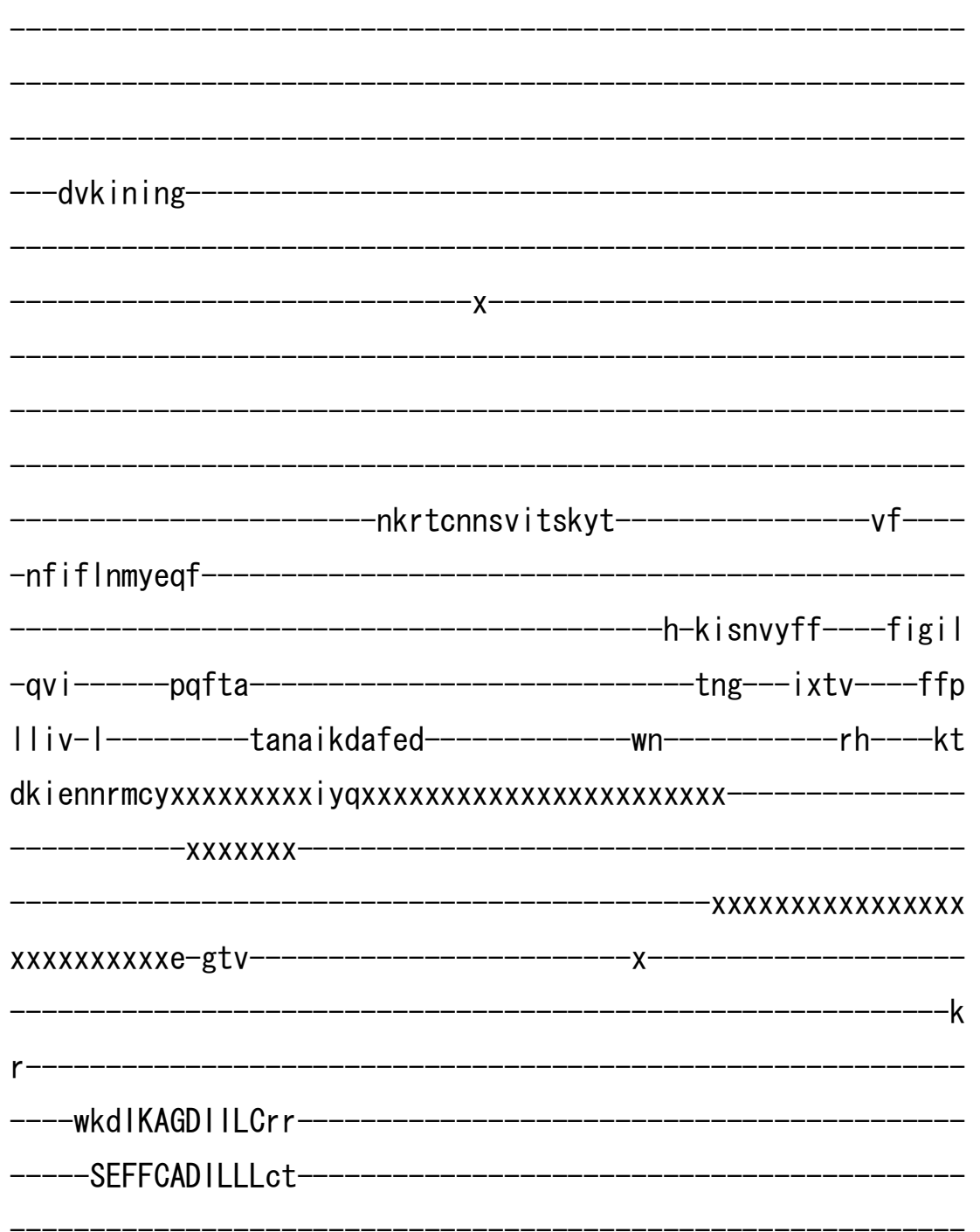

-shkng-i---afVETSSLDGETNLKVKea-

-xxxxxx i I gndrnsai dnvkn I kgf i I sdkpnkd I stmygt i y fxx

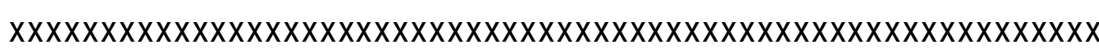

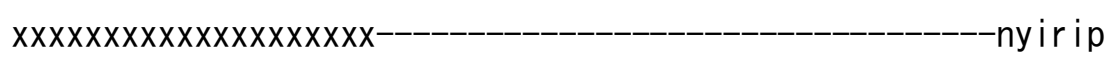
fde--_-_-_-_-_-_-_-_-_-_-_-_-_-_-_-_-_-_-_-_-_-_-_-_-_-_-_-_-_

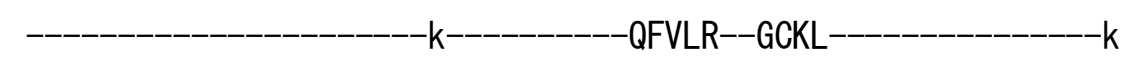

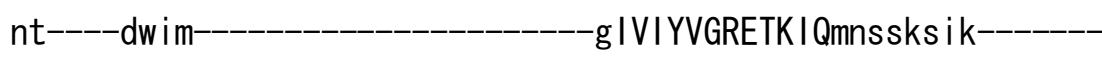

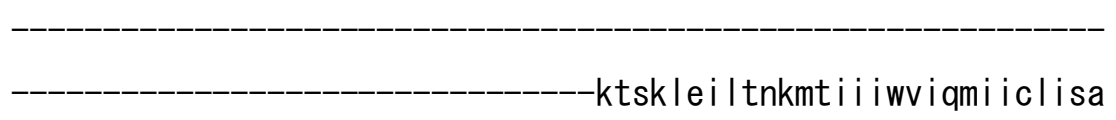
-yyna i i vsssrknn------xry l p---_-----xxxxxxxkpy i vg i i sffswVV I TGNF VPISLIVTmSFVKVVQAY-FIscdknm $x x x x x x x x x x x x x x x x x x x x x x x x x x x x x x x x x x x$ 


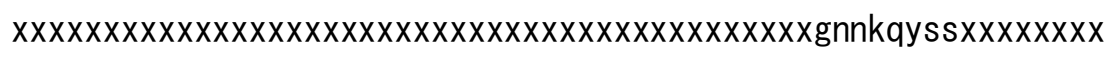
$x x x x x x x x x x x x x x x x x x x x x x x x x x a V P R-T S S L I E E L G Q I E Y I F S D K T G$
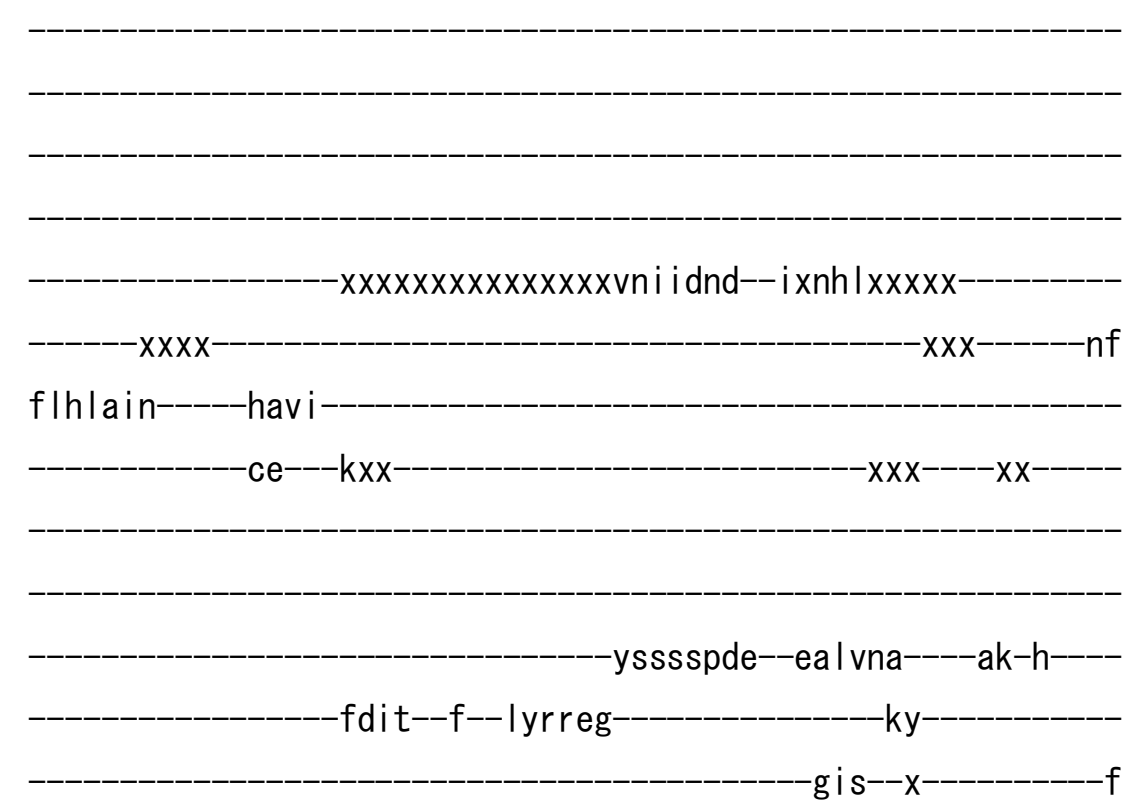

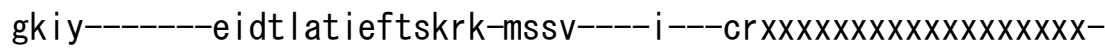

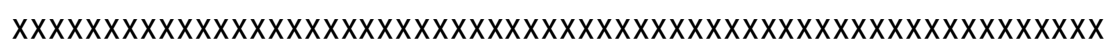

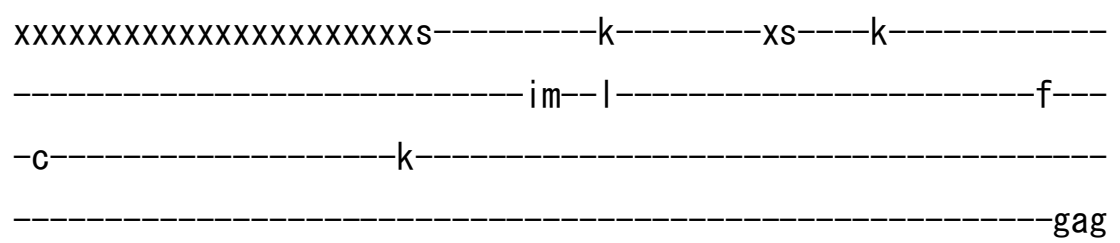

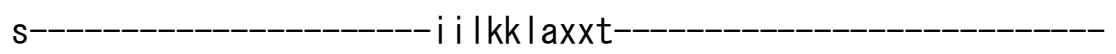

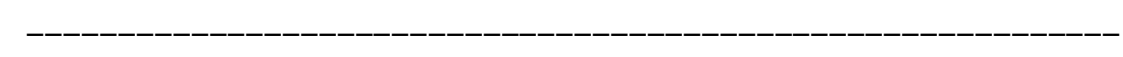
x----xx--e it-i ehmety--ade-----g | r tLCIAQRe I--seesfaewy l yke--as----- I s ikdreek l esvaey-i end I i IQG I TG IEDKLQEGVSST IEDLRMag-IH I WMLTGDK IETAMN I G I AANL-_-_-_-_-_-_-_-_-_-_-_-_-_-_-_-_i dnyseqf-_-_iy-----teeyxxxxxxxxxxiddxxxx-

$-x x x x x x x x x x x x d d x x x n x x x x x x x x x x x x x x x x x x x$

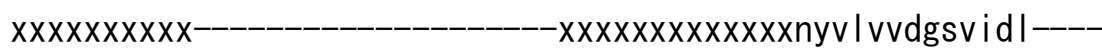
11 x---------------ekmerkffy- 


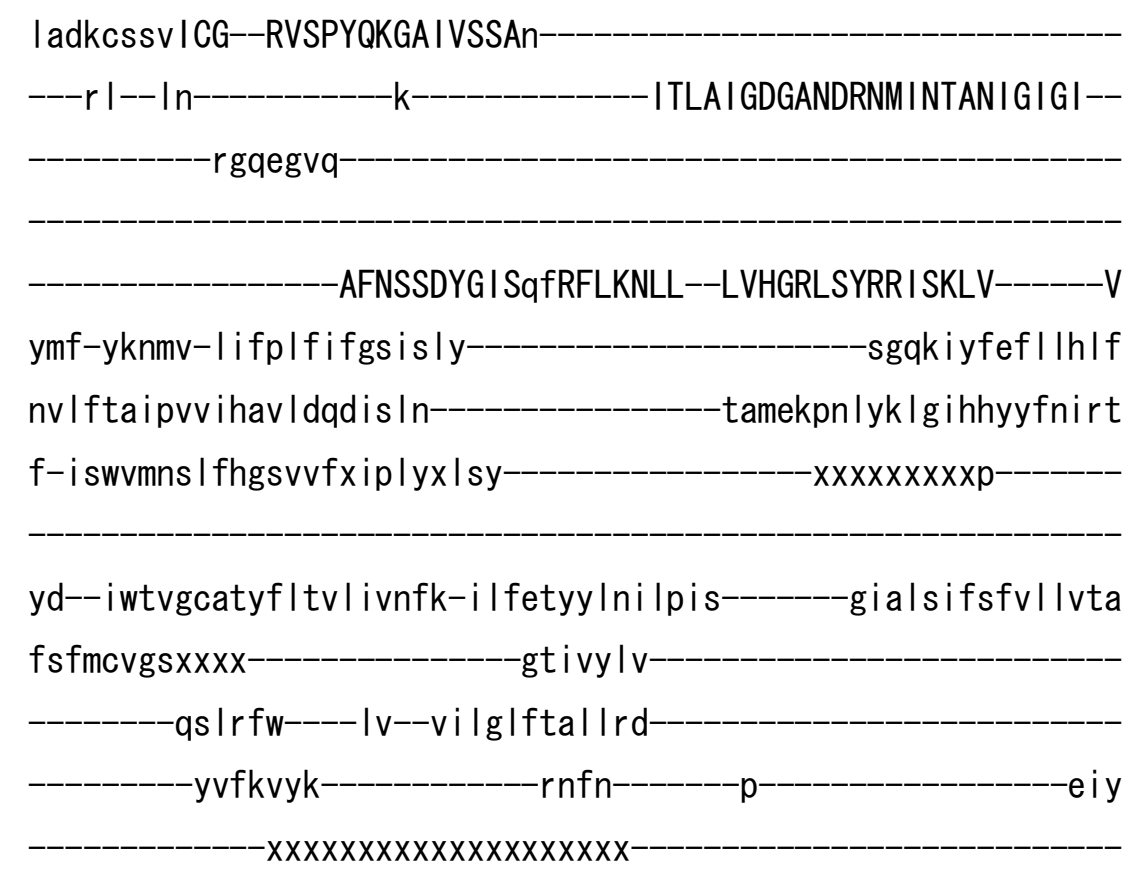




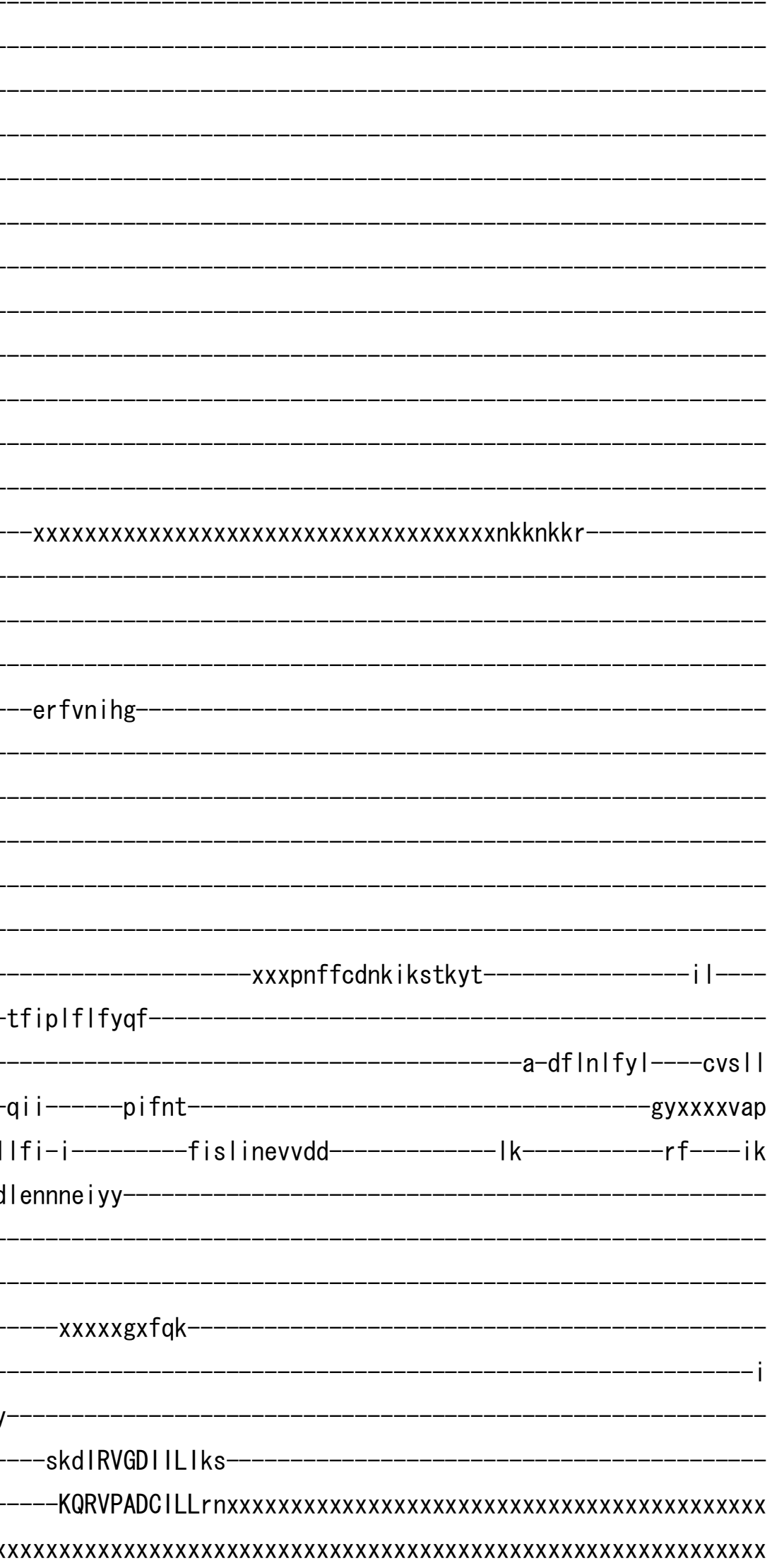


$x x x x x x x x x x x x x x x x x x x x x x x x x x x x x x n e t t n x t x x x$ tyVKTDKIDGETDWKIKyp-

in ifanlkr Ikdfftidi If i leqpknd i yk i egsfvixx

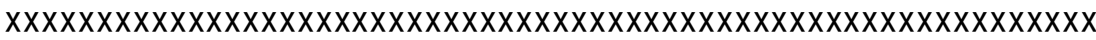

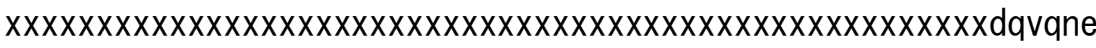

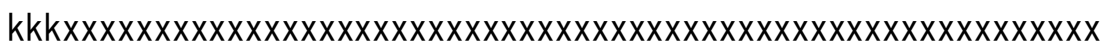
$x x x x x x x x x x x x x x x x x x x x x x x n x x x x x x x x x x$ NF ILF--NSVI-

ts-----sdvi--_-_-_-_-_-_-_-_-_-_-_-_LVIYTGSDTRVNmsta i sk i---_-_-_ $-k r g m i d e k|n l i t| f \mid f i$ ilt Ifsiymo $\mathrm{xvk} \mid \mathrm{nn} \mathrm{I}$ -wy $\ln$ fir fMLLFSSV

IPISLSVNINIAKIYYTL-LI qrdkev

es

- I IK-NSG I IENFGD IDY IFTDKTG-

TLTENVMVLkxxxxxxxxxxxxxxxkns iqgnnm-

$x x x x x x x x x x x x x x x x x x x x$

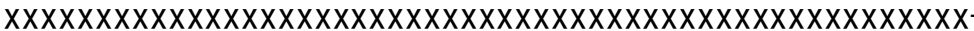

$-x X X X X--X X X X$

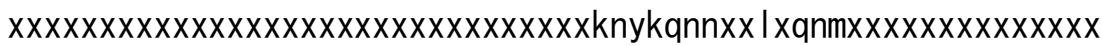
$x x x x x x x x x x x x x x x x x x x x x x x x x x x x x x x x x x x x x x x x x x x x x x x x x x x x x x x x x x x q t$ fl sf I i c-----nn i r---------------------------------------------

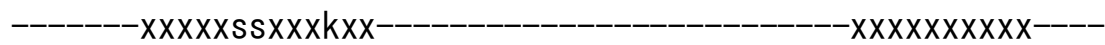

$x x x x x x x x x x x x x x x x x x x x x$ $\operatorname{Xx} X X X X X X X X X X X X X X X X X X X X X X X X X X X X X X X X X X X X X$

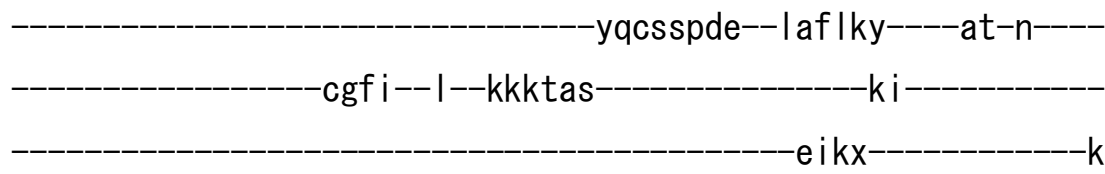
niml--------eydillhipfssetk-rmsi-----f---vr

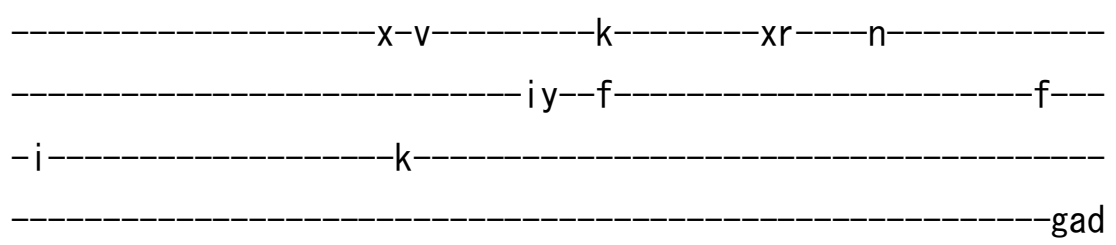

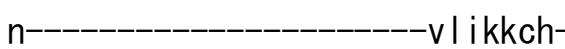


$x x x--x x x x y e e-s d h \mid s n i-$

-g I rvLVHGCLnv--eeqffhnfsal ynk---

-nkdvkgq l eni l ey-vekni kvLAI TGVEDKLQEGVGKTIEMLYNsg-IKV

WVLTGDKIETAICICKNANI-

$-k k k k h n i y---$

if------rhenxxxxxxxxxxfnsxxxx-

xxsyvlffdni i iqn

$\mathrm{i}$

ky ipnafvd-

faanaravVCC--RCSPIEKKEIAILIk-

---t i ----k-----------xk------_-------KILCI GDGGNDVAM I QSAD I G I GV-lgkegkq

-VVHDSDI IVSkFKNIKKL I--LYYGNNTFLQTSSLC $S$

fl i-hrgfi-Ity lqf i ysy iffs-_-_-_-_-_-_-_-_-_-_-_-_ipvs i fqgw l qi gy ttyyttapfls I-IIdikikkn---_-_-_-_-_-_--_ iy lype iyknkkhkrkIdlks f-f i i vw i s i fagtvvm-|gal-k|f-_-_-_-_-_-_-_-_-_-_-_-_n--_-_-_ dn--ynnl inisfssl ivle imn-ihleveswhp Imi s----_---an i csf i vy i fsmf i

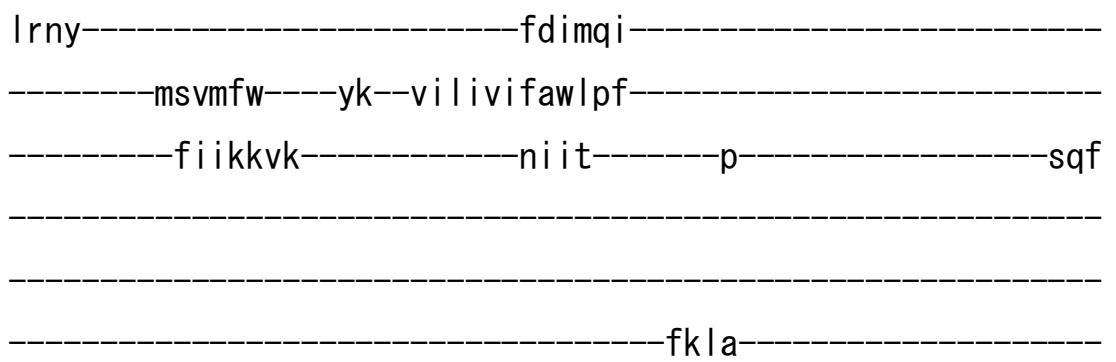




\section{>tpalV_IV01 XP_765339. 1}

m-

$-x x x x x x x x x x x x x x x x x x x x x x \mid$ rdritfxxxxxxxxxx

---yksircis

xxxxxxpk I f Iyng I vnskyn $-f f$

-tfvplvlysqf 


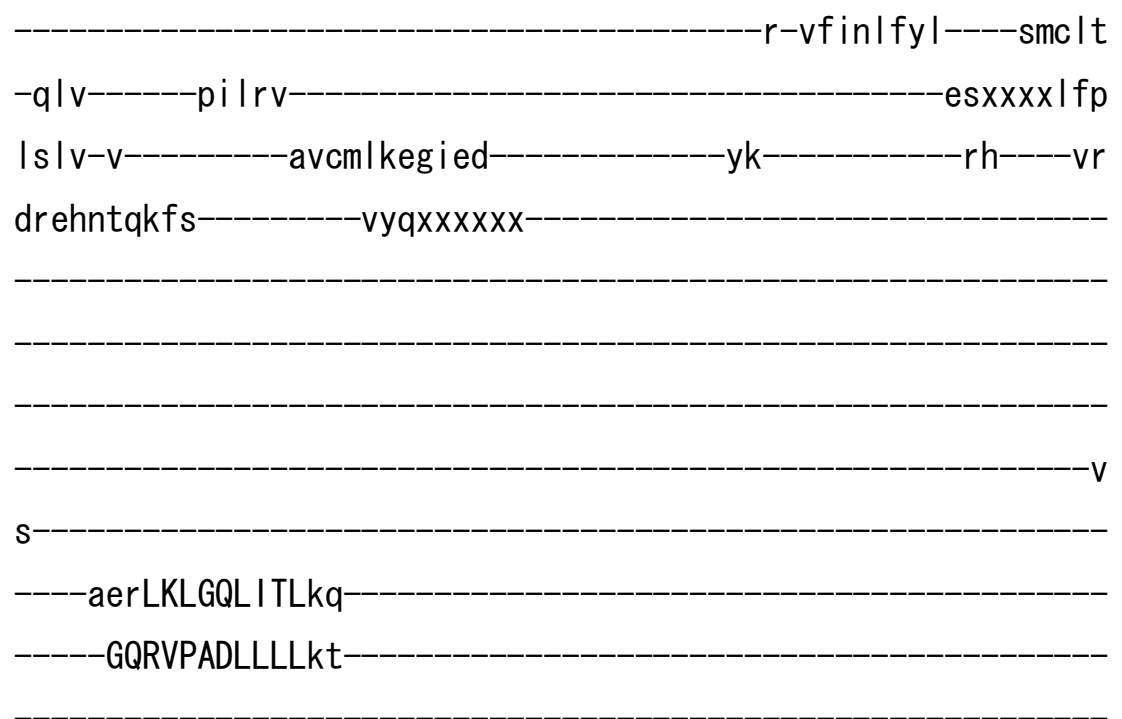

-sdpng-a---sfVRTDQLDGETDWKLKra-

-vattqamstvke i I l ysvatveepkddfynftgk i cf $x x$

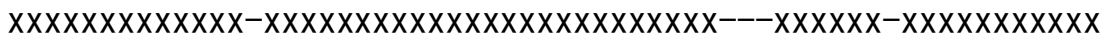

$x x x x x-----x x x x x x x------x x x x x x x x x x x-x x x x x x x x x x x x x x x x x x x k r$ ives

$\operatorname{lnv}$

d----------NIVWM--NSIV

as----gtih--_--_-_-_-_-_-_-_-_-_LTIYYIGKDARACInskrakm-

-kyg Ifekelnr Ifivli I im I vmcvmm I

-cpngfmsm--------------wyvt-------------------------fI kyLLLFSTV

VPLSLRISIEIAKYVYNR-AIVIdnki

$-\mathrm{pq}$

-tMPR-TTMI PEELGR INYLLTDKTG

TLTQNVISLe -klyitrgyy

$x x x x x x x x x x x x x x$

$-x x x x x--x x x x$

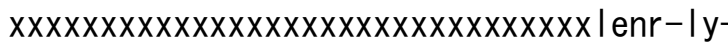

I I s lavc-----hnvr

pixxx 


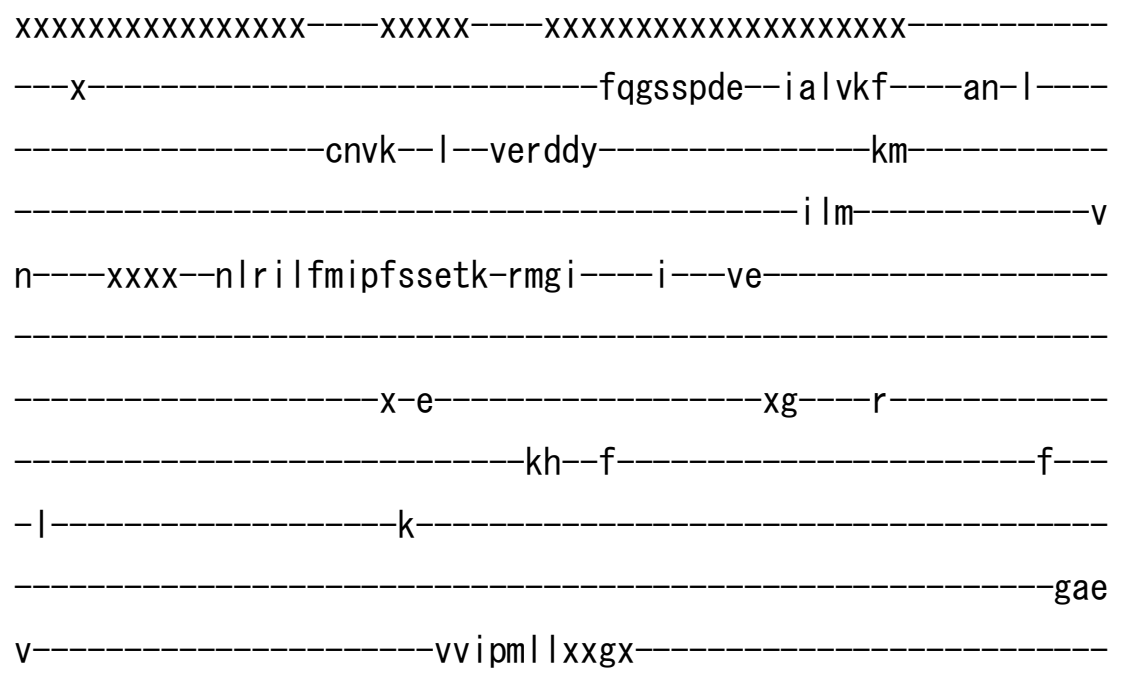

-vwl-seecdnI--arm----g | r tLVFAYRai --sdseydnfvqkyne--an------ s l ydrvkk i rkvtgt- | ehgm I I VGL TGVRDKLQRNVGSTLESLKNag-IK I WMLTGDK IDTAKCVAI SCGMiqknns if---qi-----svdk-

$x x y \mid x x x s x x x x x x x x x x x x x x x x x x$

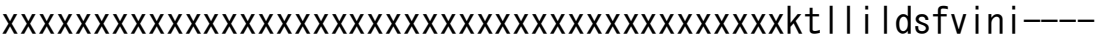
av $-\mathrm{XXXXXXXXXX}$ $\mathrm{xxx}--------------k t|r r| f v k-$

s I I annvLFC--RCTPQMKAD IVKLLk

--- se--nk-IVCSIGDGDNDVPMI SEANVG IGI-vgkegla 
fli-hrgvi-isvmqaifstiyyf-mp i affagw I lvgf tsyynmipifcl-vldee icee dvm Ifpelyvelkkgrelnikt

f-I iwiwi sifagai Im-igai-i If e-

ns--IvslisigctsIflleiln-IvselhhwhrIti i glvctcivyfys I Iv frnnfdmef $i$ arksff-----wk--vla i sm l gw lpi $-y$ vikylt$-k i l k$ $-p-$ -pqy iklivt

>tpalV_IV02 XP_763506. 1

m- 
---Irviklni

$-x x x x$

-vcsnrvkttkyt

-py----

-nfifknlyeql

-c-ipsnlyfv----fiavl

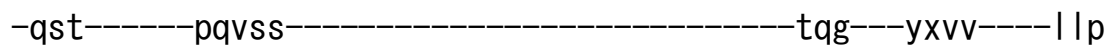

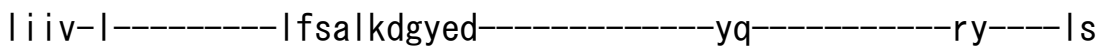

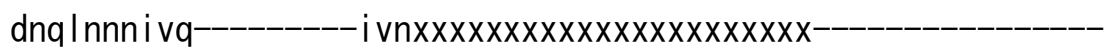

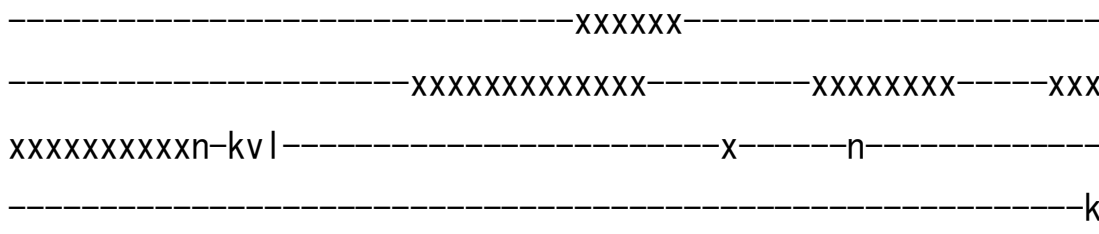

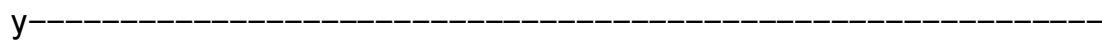

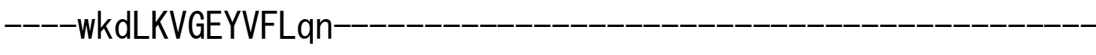

KDIAPADLVLLat

-seeng-f---afVDASSLDGETNIKKKesX

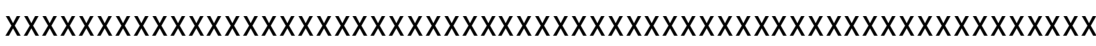

$x x x x x x x x x x x x x x x x x x x x$ e Igsdfehv i tevqr I Ighfkcegpnkn l isfdghlkyxx

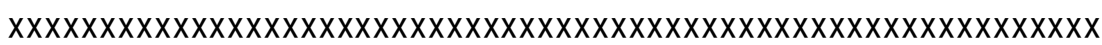

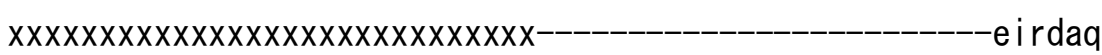

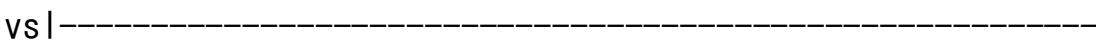

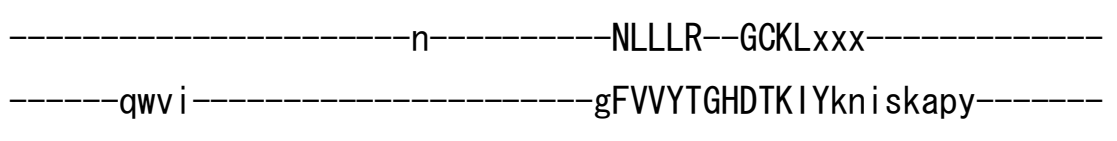
-kvsnlarkmmrttflicviqfvlcliat -fyn I ymhthkvyek-----xpy I s-----------xxxxxsg I y i vfvyyfswMALTANF VPISAIVT INAVKLIQGF-FIqIddsm- 
$-y c e e l k m n$

aKAR-NTMLNEELGQVKHLFSDKTG

xxxxTLTCNKMEFr

-kfs imghsy

$-x x x x x x x x x x x x x x x x x x-$

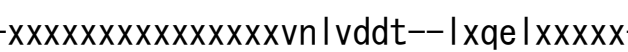

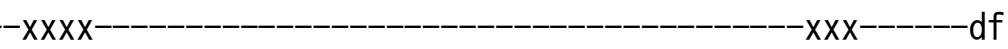

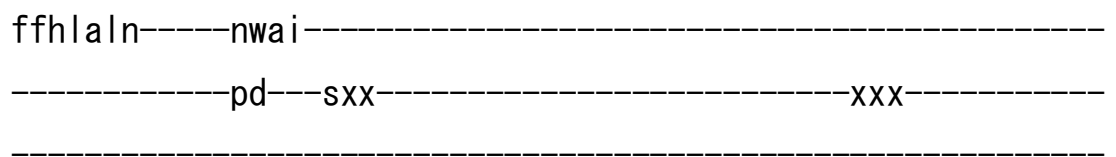

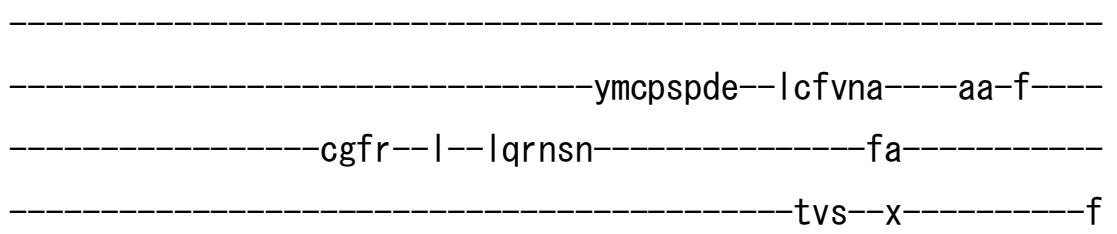

nk i y--------kvki i aqadfdyrrk-cstt-----i---vaxxxxxxxxxxxxxxxxxxxx

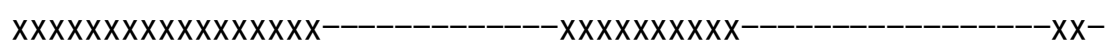

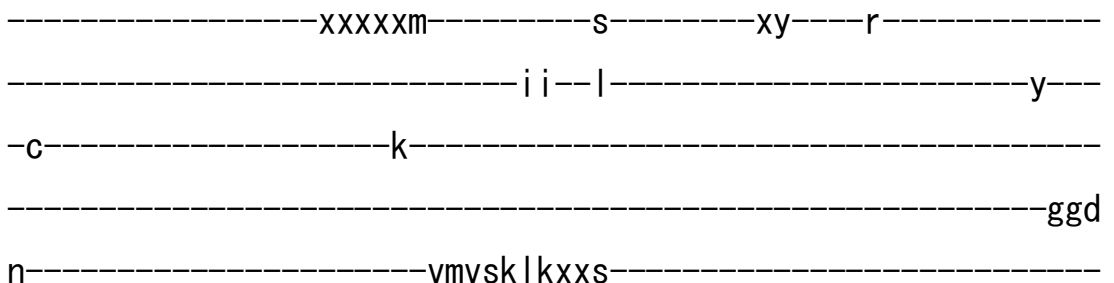

x----xx--svt-I rnmkny--svg----g | rtLVFAKRe I

-kgrdek I aecvgr- lecg l I QGVTG IEDKLQDGVSECIEKLLMag-IR I

WMLTGDNLDTS IN I G I ATNL_-_-_-_-_-_-_-_-_-_-_-_-_-_-_-vnm I sdr i-_-_

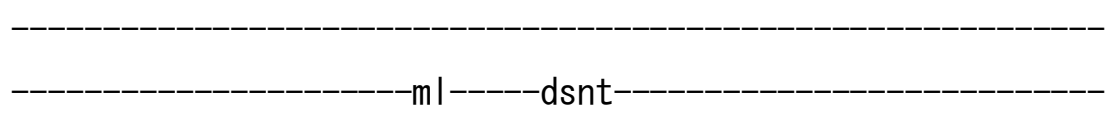

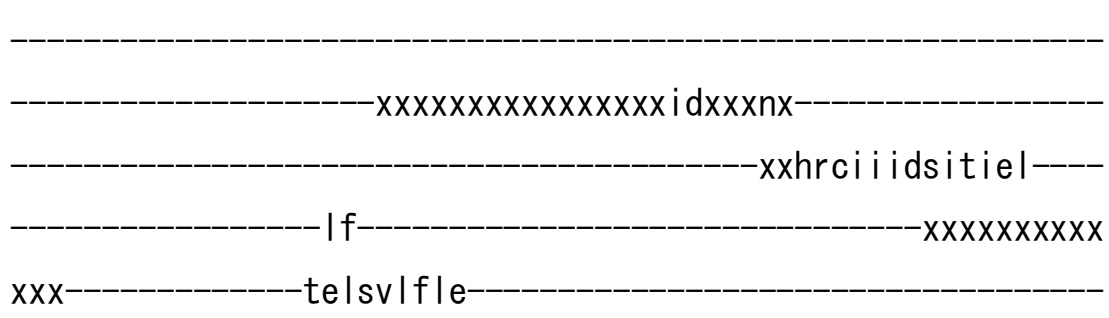


IvrrvhsvICC--RMTPYLKGAVVSFVk-

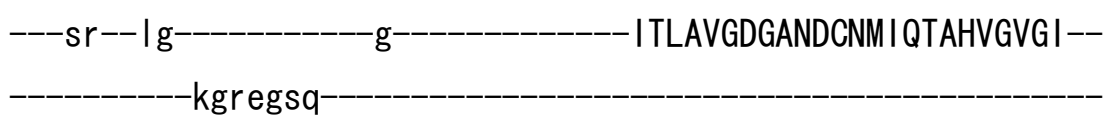

-AFNASDFGI GqfRFLSPLI--LHHGRCCYRNTSKCI------S

ymf-yknv i-I i ip Iffyay i s If-_-_-_-_-_-_-_-_-_-_-_-_sgqk i yys I fva i y nvvftsvpvg i f g i vdqdynks---_-_-_-_-_-_--- I sakyphvyq I gqrnyyynvvk

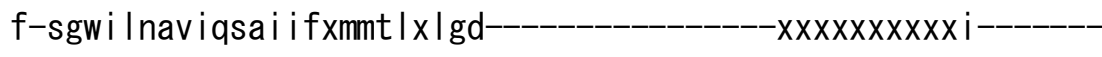

ad--apt I g iml I savf i ivsck-Iv letwyftkit| |----_---shl i s ifffivtvcs

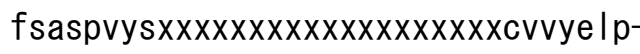

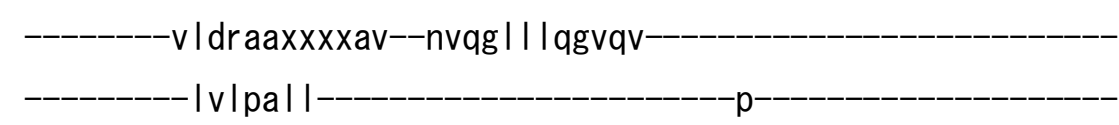
$-x x x x x x x x x x n p d t k p|| x x x x x x x x x x x x---$

$-$

>dreIV_IV01 XP_683800.1

$\mathrm{m}-$ 


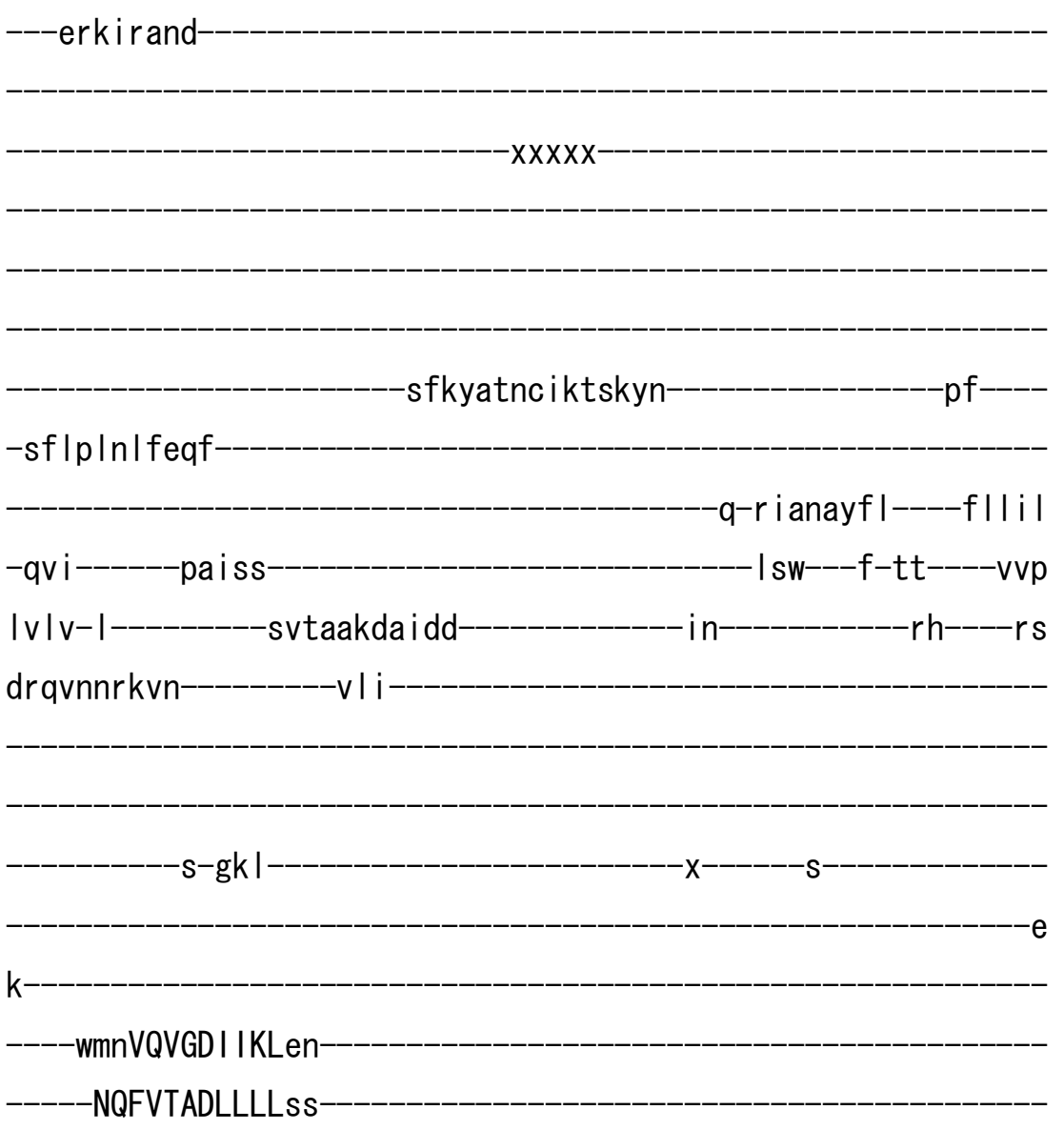


-sep In-I---vy IETAELDGETNLKVKqs-

-xtvtgdmghn l ea l aafngevcceppnnr Idrftgt Itf--

dtqkys

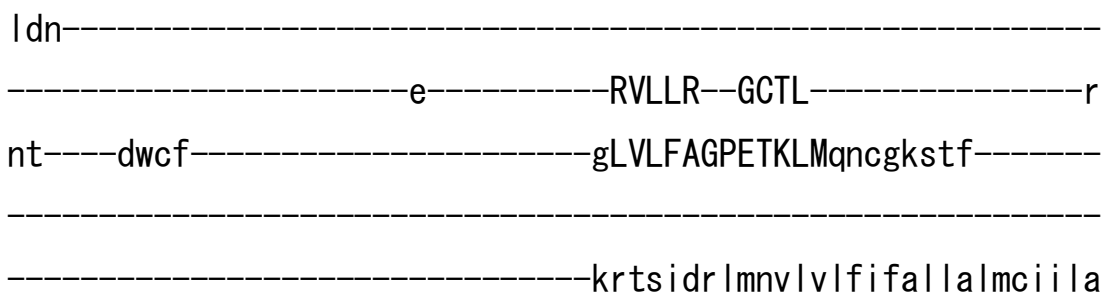

-vghg i wenytgskf---_--xvf Ip--_-_-_-_-_--xxxnaafsaf I tfwsy I I ILNTV

VPISLYVSmEVIRLGNSY-YInwdrnm-

-yhtrtdtp

aEAR-TTTLNEELGQIKYIFSDKTG-

TLTQN IMTFn

kcsingksy

$-X X X X X X X X X X X X X X X X X X$

$-x x x x x--x x x x$

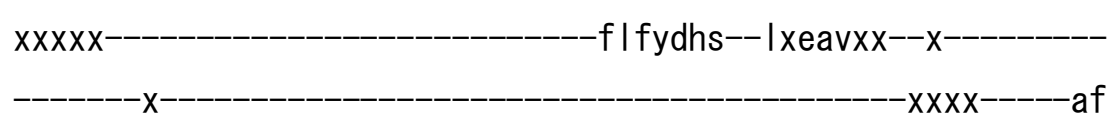

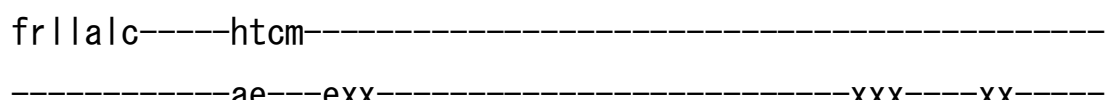

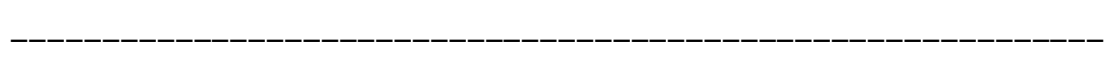

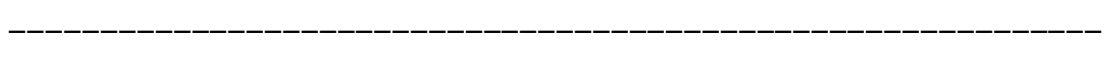

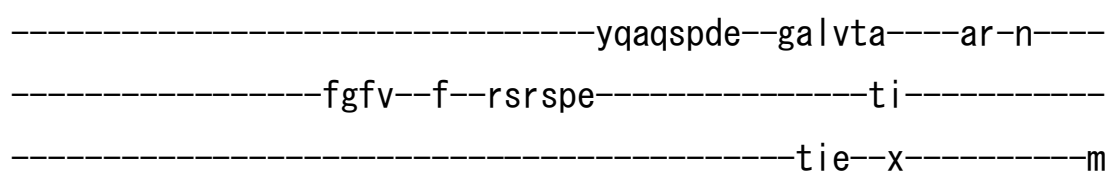

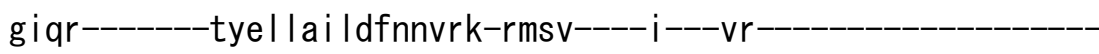

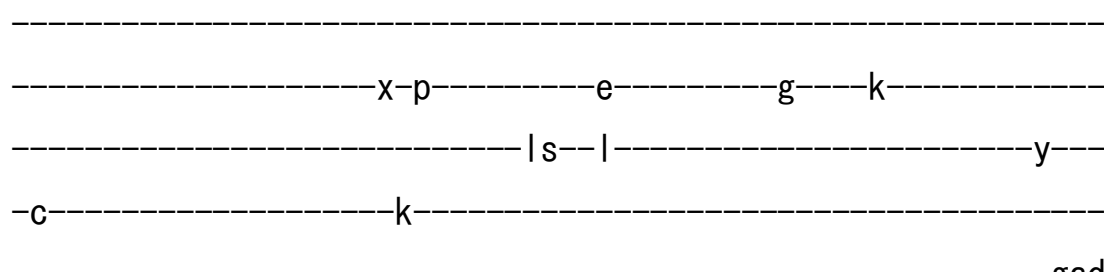

-gad

$\mathrm{t}$

-i iyer Ih--p-

$--x$ 
xxx--xx--evt-teh I nef--age----g | rtLVLAYKd I--dedyfaewkqrhhe--ss-----vamedreek I dkvyee-i ekdmm I I GATA IEDKLQDGVALT I ELLAKae-IK I WVLTGDKQETAENI GYSCNLIreemndv----

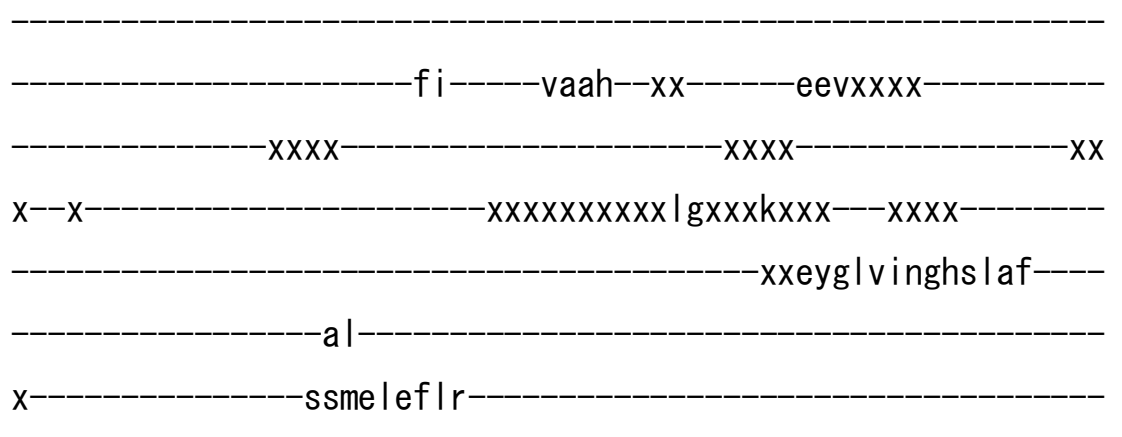

tacmcktvICC--RVTPLQKAQVVELVk

---ry--kk-----------a----_--_----VTLA I GDGANDVSM I KAAHI GVGI--

AVLSSDFSFAqfRFLQRLL--LVHGRWSYLRMCKFL------R yff-yknft-ftfvhfwyaffcgf-_-_-_-_-_-__-_-_-_-_-_saqtvydegfitly n I vyta I pv I gms I fdqdvnan-----------------ws I efpq l yvpgq I sqyfskra f-mmcalhscyss I I If-fvpyxtty-_-_-_-_-_-_-_-_-_-_xxxxxxxxxg--_-_-_ad--yqsfal i tqtc|tvtvcvq-|g|d| sywtvvnh|-----_--fvwgs I gmfff Itft

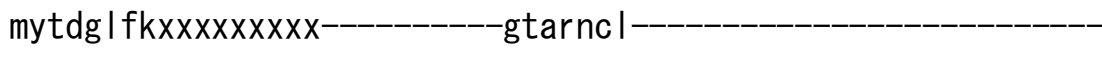

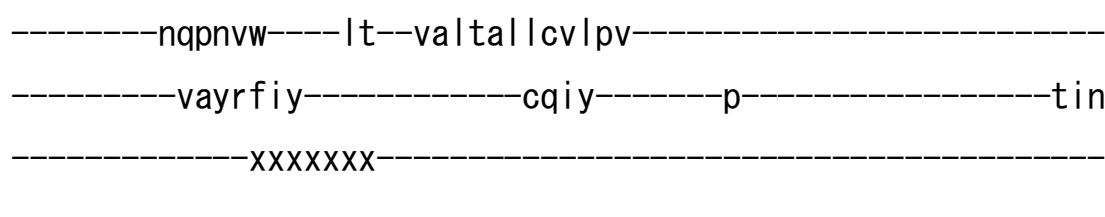




\section{>dreIV_IV02 XP_687715. 2}

m

$-x x x x x x x x x$ lepalke

---erhvrand-

$-\mathrm{XXXXX}$

-rfsyadnr iktakyn

$-v f$

-tflpinlfeqf 


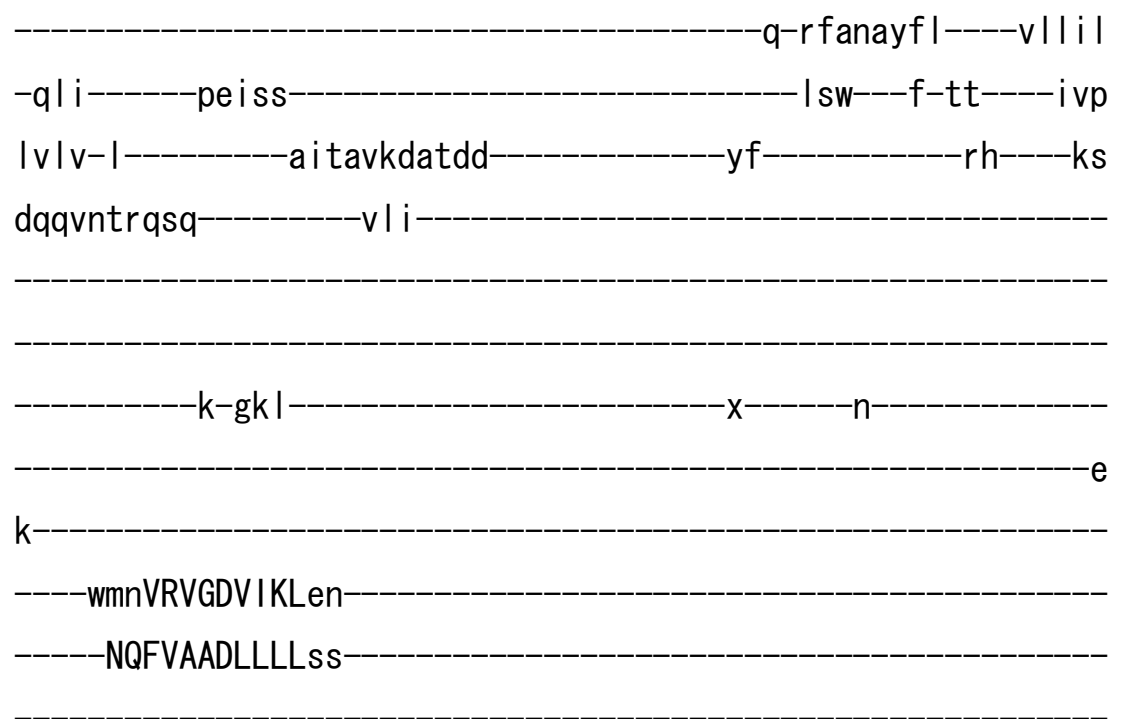

-sepyg-I---cy IETAELDGETNLKVRqa-

-xtvtsd I gddvak I adfngev i ceppnnk I dkf i gt l yw--

-kdnkyp

Idn-

e-

- KMLLR--GCVL

nt-----ewcf

gLVIFAGLQTKLMqnogrtkf

-krtsidkImnt I v Iwifgfl i cmg i i la

-ignt i weqsvgsdf------xay lq----------xxxxxxnavfsgf I tfwsy I I ILNTV

VPISLYVSVEVLRLGHSY-F Inwdr rm

-yysrkdtp-

aEAR-TTTLNEELGQVEF IFSDKTG

TLTQN IMVFn -kcs ingkty

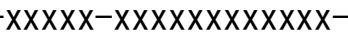

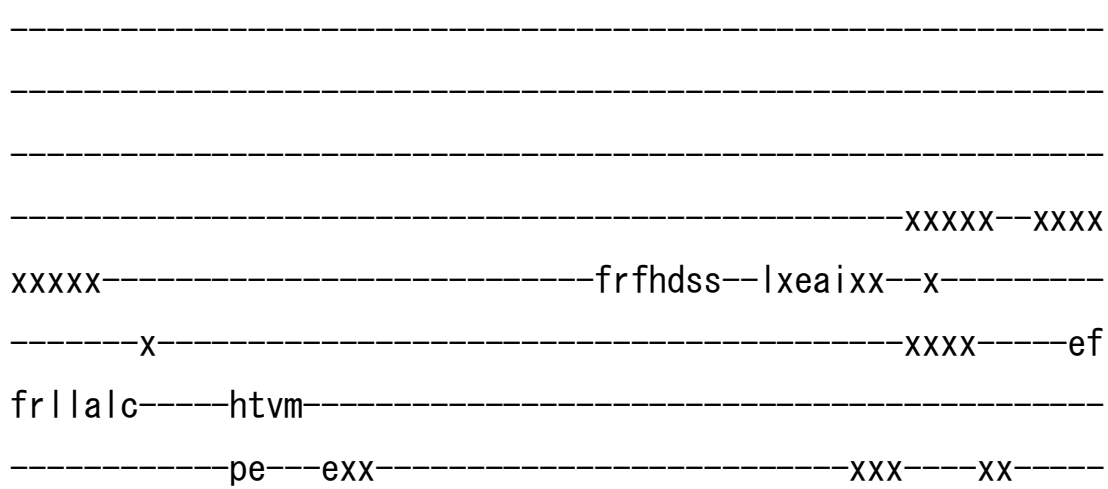




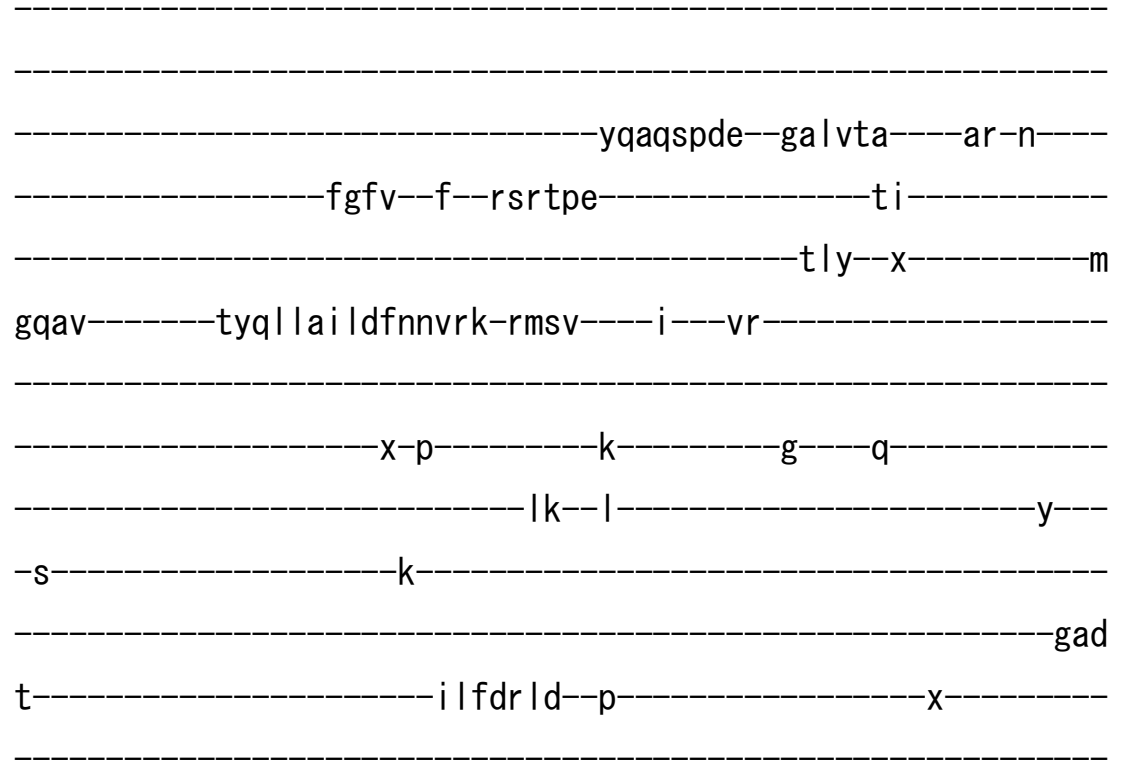

xxx--xx--ftt-seh I nef--age----g | rtLALAYKd I--dedvfdewtkk I If--as------tal dnreek I ga l yee-i eqgmm I LGATA IEDKLQEGVPET I ACLTLan-IKI WVLTGDKLETAMNI GYSCNMIrddmnev----
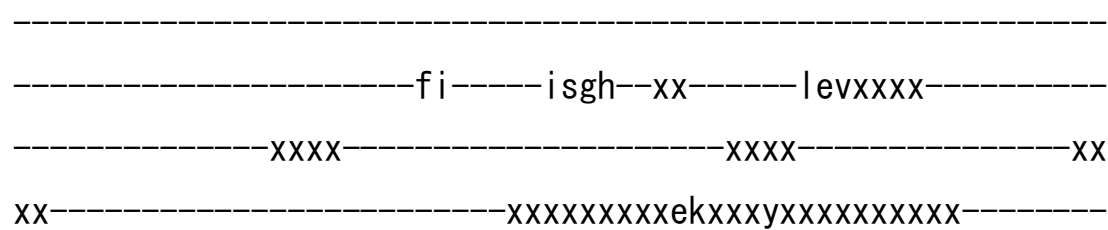
xxeyal $\mathrm{i}$ inghs 1 ah al

x-------------aeleqi lvd

vac IcksvICC--RVTPLQKALVVELIk

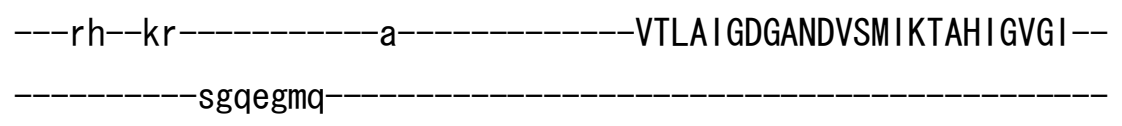

-AVLASDYSFAqfRYLQRLL--LVHGRWSYHRMCNFL------C 


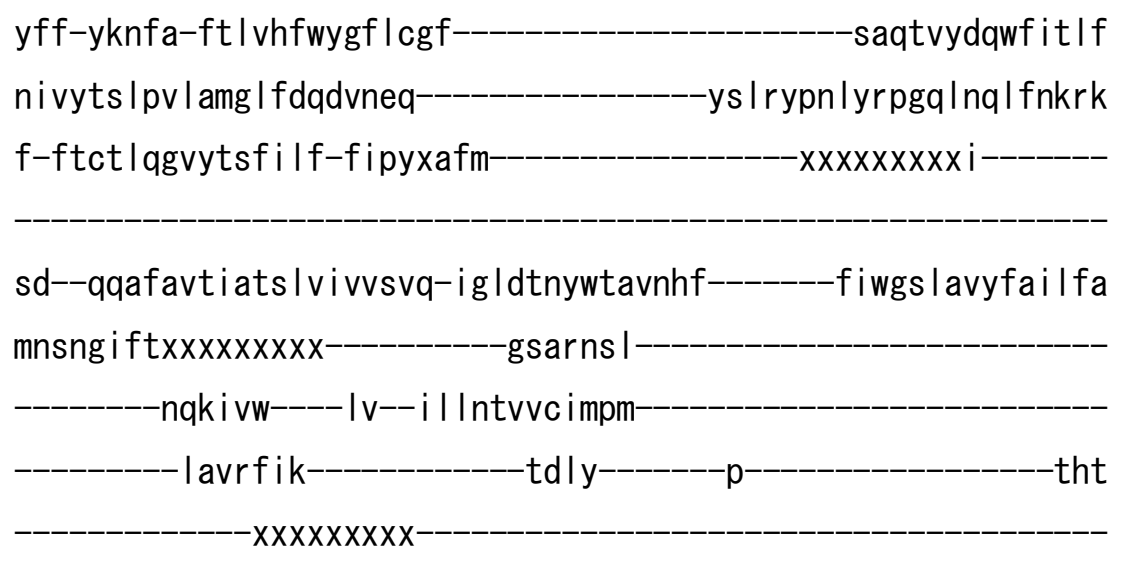

$-x x x x x x x x x x \mid r r v r r t s x x x x x x x x x x x x x x x x$

\section{$\operatorname{xxXXXXXXXXXXXXXX-10}$}

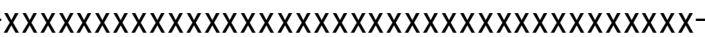

>drelV_IV03 XP_691382. 2

$\mathrm{m}-$ 
---srtvyigh-

\section{-nf ipknlfeqf}

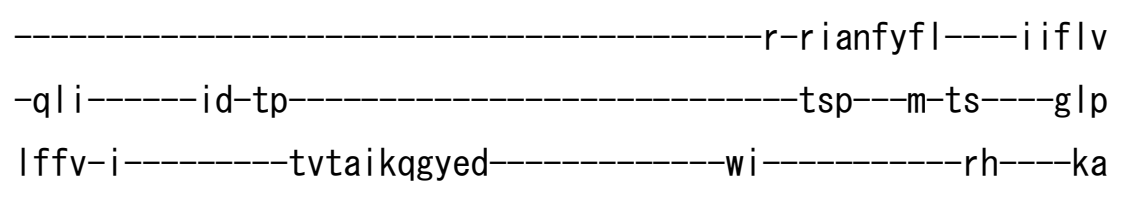

dnsvnqcpvhivq-

-sredg-t---cfVTTASLDGESSHKTYya-

-vqdtkafstaeevdt I hat i eceqpqpd I ykf vgr in i xx

$x x x$

ep iarp

Igs -NLLLR--GATL----_------------k

nt----eyihaVAIYTGMETKMA Inyqsksq

-krsaveksmnay livylcil i skal int

-vlkyvwqadpnrde------xwynqxxxxxxxxhvl iraftdf I afMVLFNY I

IPVSMYVTVEMQKFLGSY-FI I wdddm 
pLVN-TSDLNEELGQVEYVFTDKTG

TLTENNMELr eccvdghvy

$-x x x x x x x x x x$ I pgaagmxxixsspxxxxx

$-x x x x$

fralclc-----htvq--_-_-_-_-_-_-_-_-_-_-_-_-_-_-_-_-_-_-_-- $x \times x x-$

$--x x x x x x x x x x h q x x x t x x-$

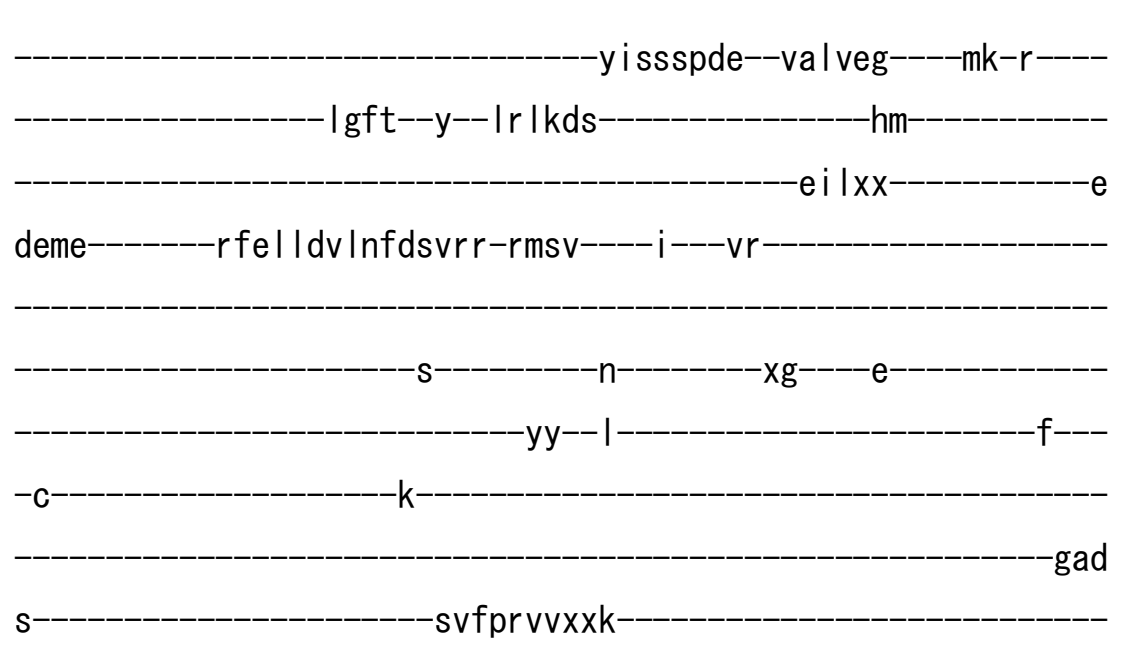

-xeqv-rarvehn--ave----g|rtLCVAYKr |--sqeeyeetcr I Its----

ak----- la l qerdkk I aeaydv-i ekdf i ILGATAVEDRLQDKAADT IESLHKag-IKV WVLTGDKMETAAATCYASKL-_-_-_-_-_-_-_-_-_-_-_-_-_-_---_fhrntqi I---_

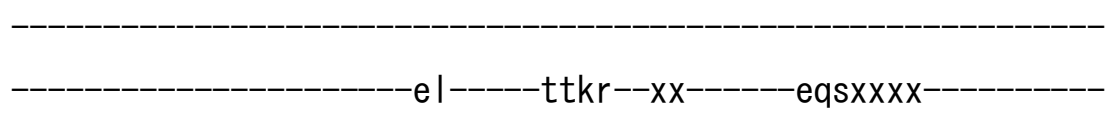

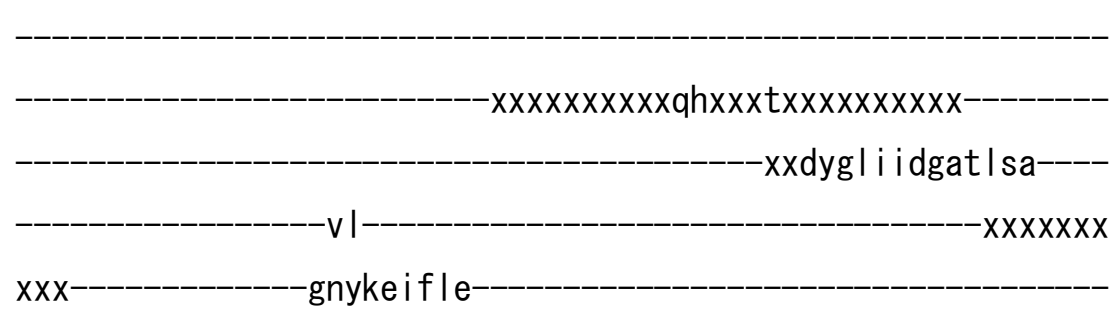


icrncsavLCC--RMAPLQKAQIVKLIk-

---as--ke-----------xp-------------ITLA I GDGANDVSMI LEAHVGI GI--

-mgkegrq-

-AARNSDYA I TKFKHLKKML--LVHGHYYYIRIAELV------Q

yff-yknvc-fi fpqf I yqffcgf-_-_-_-_-_-_-_-_-_-_-_-_sqqp I ydtay It l y

nisfts Ip i I lys Imeqhinmd-_-_-_-_-_-_-_-_-_ I krdps Iyrdiakns I I twpt

f-iywtf I gvfdavvff-fgafx| fd---------_----------xxxxxxxxxm--------

fg--nwtfgt Ivftvlvftvt Ik-laldthywtwinhf---_----viwgs I I fyvifs I I

wgg i iwpf--xxxxxxx----------yvfmqml--_-_-_-_-_-_-_-_-_-_-_-_

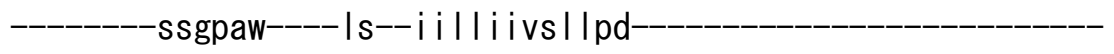

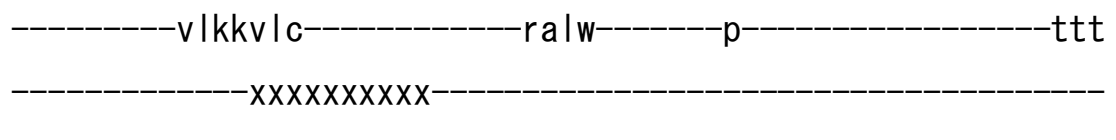

$-x x x x x x x x x x s q t s s s v s x$

>dreIV_IV04 XP_691903. 2

$\mathrm{m}$ 
$-x x x x x x x x x x x k k k k v e e$

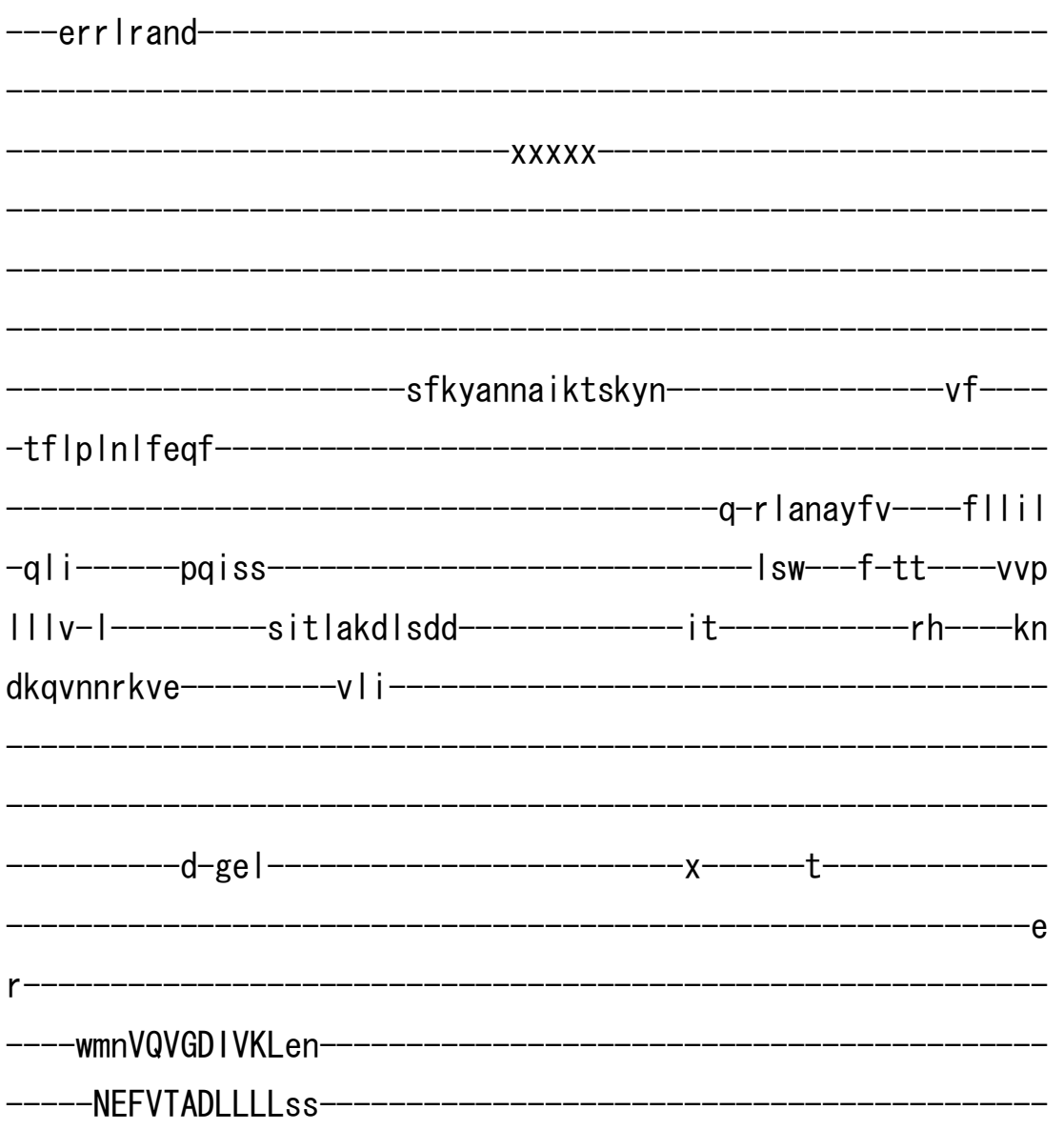


-sep In-I---iy IETAELDGETNLKVKqa-

-xt I tge I cnd iar l aafkgevrceppnnr I dkftgt I vv--

ggetfa

$\mid d n$

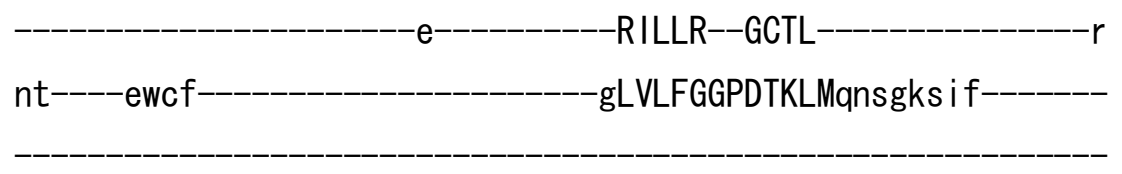

-krtsidhImnvIvIfifgflafmctils

-igna i weyqegnsf------xvf Ip----_------xxxxxnas I saf I tfwsyVI ILNTV

VPISLYVSVEILRLGNSY-F Idwdrkm-

-yhvksdtp-

aQAR-TTTLNEELGQIKYIFSDKTG

-TLTQN IMTFn

-rcsingksy

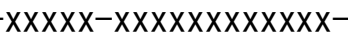

$-x X X X X--x X X X$

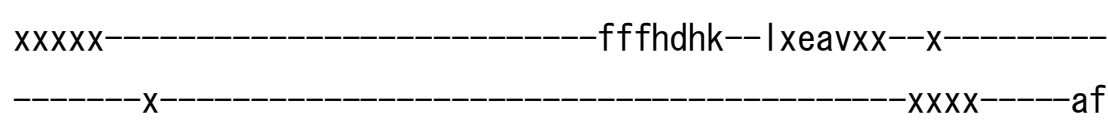

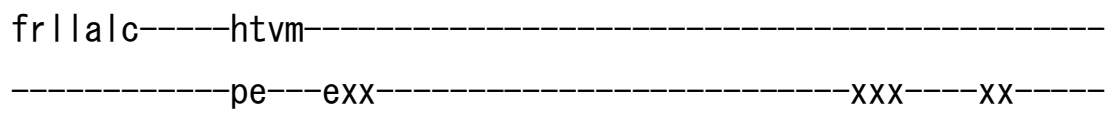

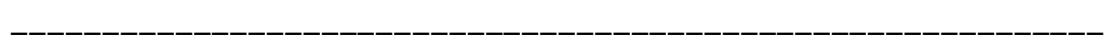

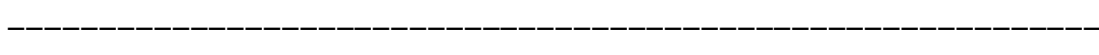

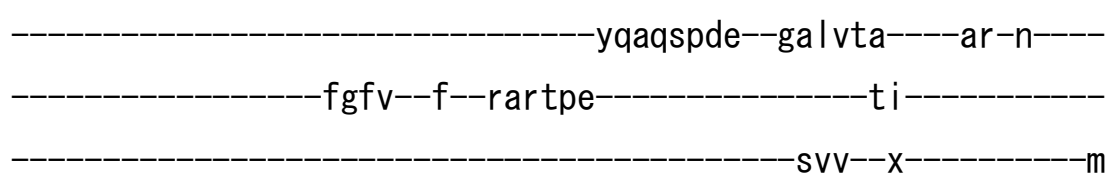

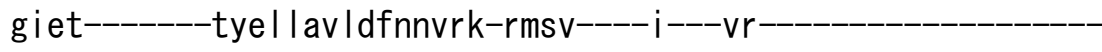

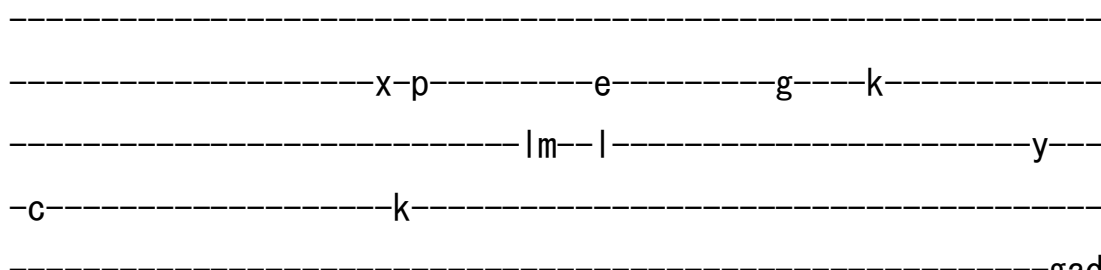

-gad

$\mathrm{t}$

- i i yer $I h--p$

$-\mathrm{x}$ 
xxx--xx--evt-teh I ney--age-----g |rtLALAYKd I--dedkfaewrrrhhe--as-----i a l edreek I da i yee-i ekd I i I IGASAVEDKLQDGVPQT IEQLAKad-IK I WVLTGDKQETAEN I GYSCNM - r reemtei----

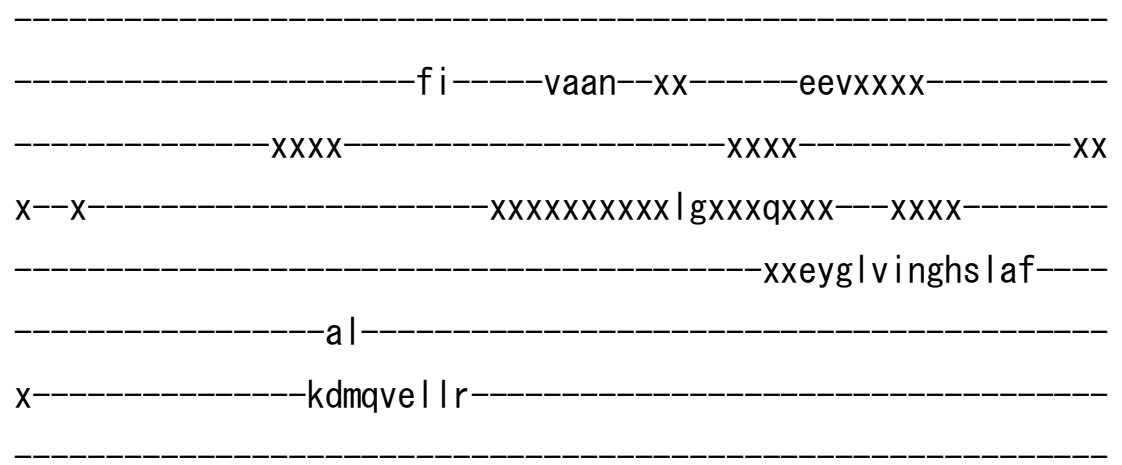

tacmcqtvICC--RVTPLQKAQVVELVk

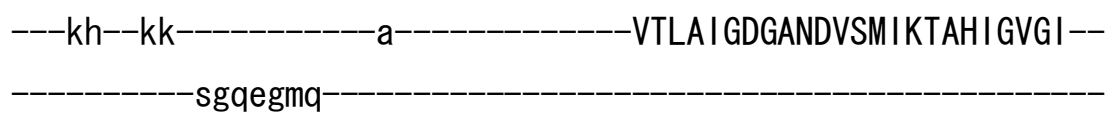

AVLSSDFSFAqfRYLQRLL--LVHGRWSYLRMCTFL------R yff-yknft-ftfvhfwyaffcgf-_-_-_-_-_-_-_-_-_-_-_-_saqtvydewyit ly n l vyta I pv I g i I fdqdvndr-_-_-_-_-_-_-_-_-wsfaypq l yapgqmnqy fskma

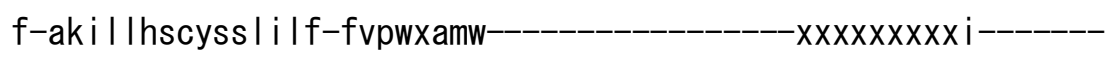
ad--yqsfa I laqtc I I iavsvq-Ig Idtyywtavnhf-----_--f I wgs I svyfavtft

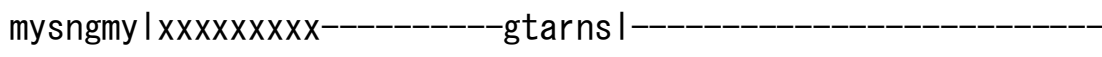
$--------n q p n v w----$
- 
>dreIV_IV05 XP_692383. 2

m

$-x x x x x x x x x x x x x x h r d q d r p$

---irkvvsn I

$x x x x x x x x x x x-$

nrqyegnaiktnkyr

- I

-sflpmn I feqf 


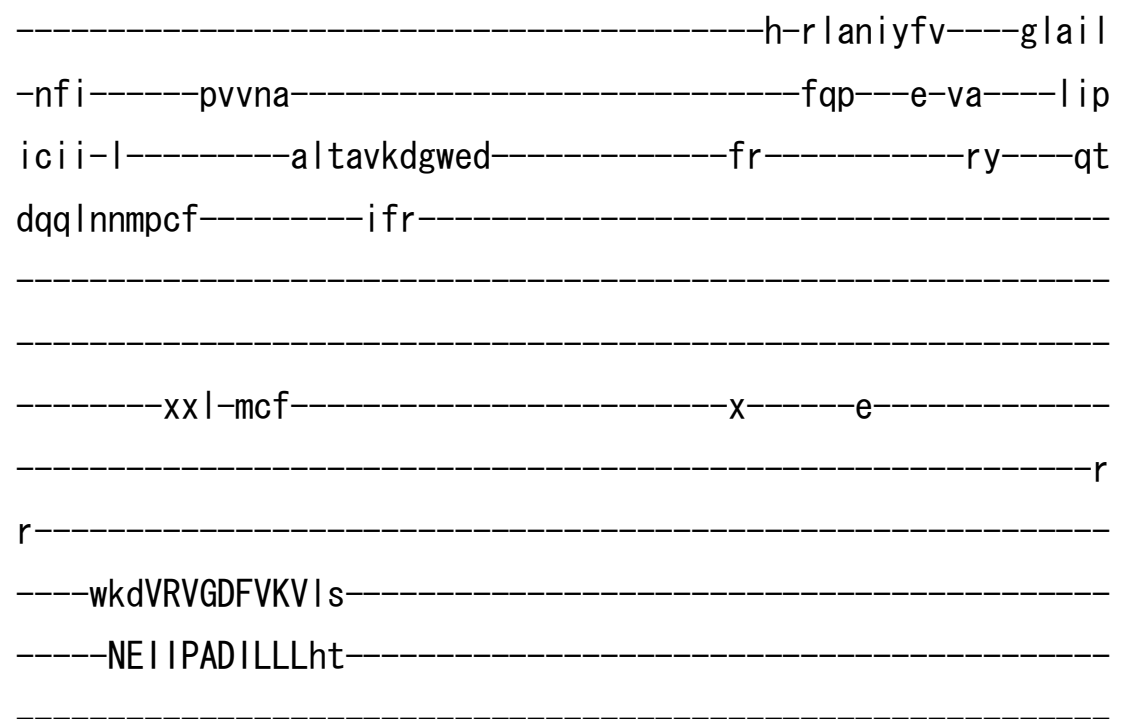

-sdpng-v---chMETANLDGETNLKQRkv-

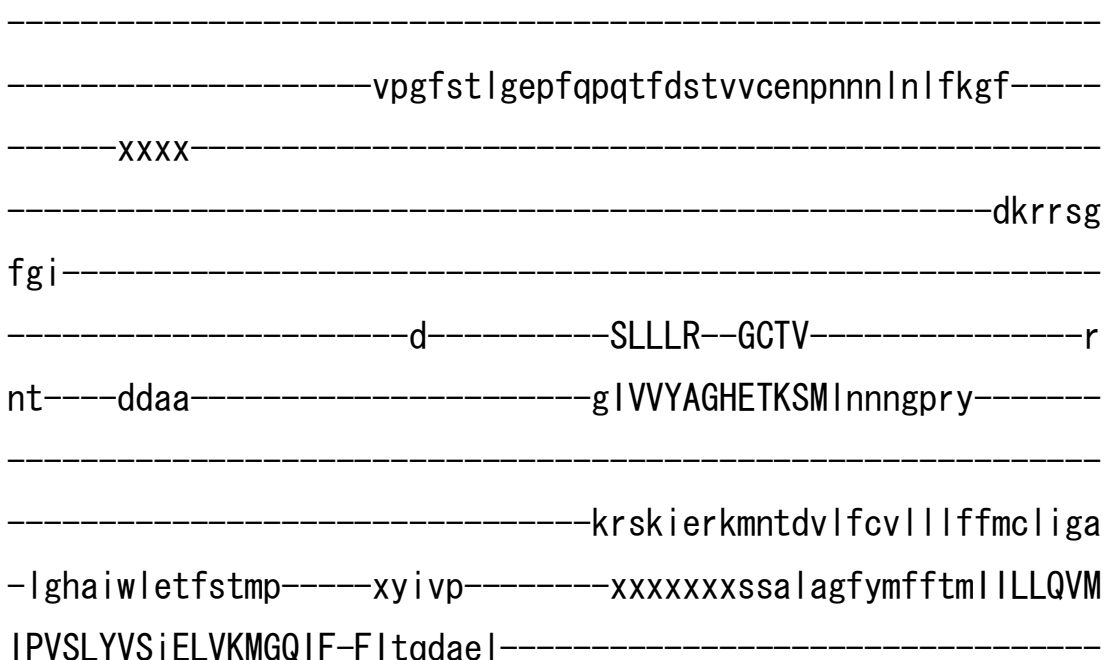

$-y d q e$ ldsr

-vQCR-ALN I TEDLGQIQYVFSDKTG

TLTENKMVFkrctimgtey-

XXXXXXXXXXXXXXXXXXX

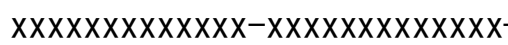

$X X X$

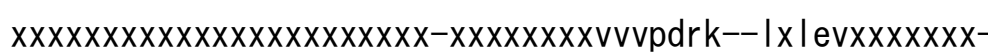

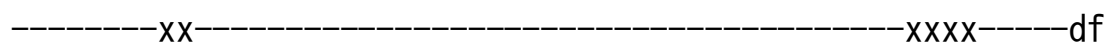

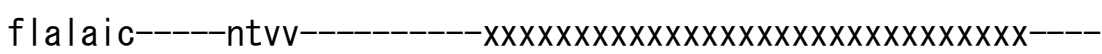
$-x x x x x h n x x x h x x-$ $-x x x----x x x----$ 


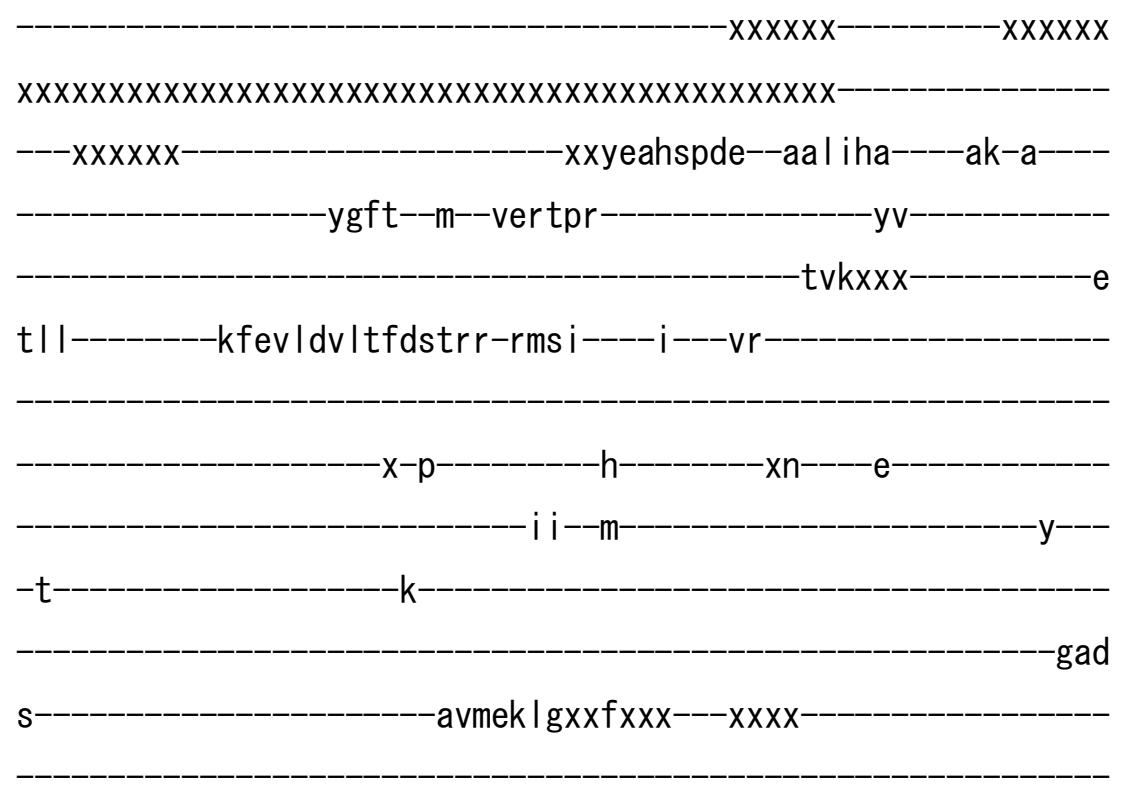

xxx--xx--vrt-qkd I dmy--ard----g | r tLCFAKKv i--seqefkawftsrqe--a I-----sa i dekeer I metand-i enn I I I LGATG IEDRLQENVPET I QALRRag-MQV WVLTGDKPETAVNIAYSCKLIdhed Ivf----

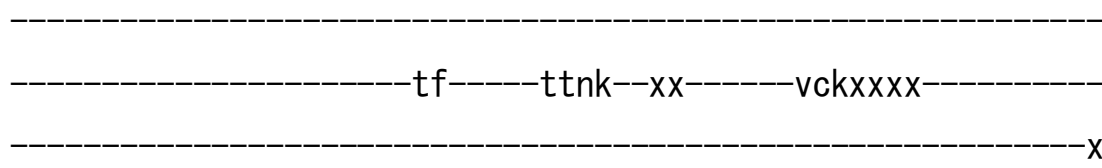

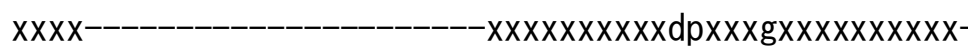
$-x x$ s i g vidgpt Inm al

x----_---------ed lveqfve-

IckhcravLCC--RVTPLQKSSVVKVIr --- qk-- $\mathrm{r}$------------v------_------MTLAVGDGANDVNM I QAADVG I GV-- 
yff-yknva-yvn I I fwyqffcgfsgtami dyw Imiff

nIfftsapp imfg imdkevsds

-tIIsIpelyrrgahsegyrrst

f-wi a i ldafyqs Ivcf-fipy-wty $x x x x x$

-g--ifsfgtpmntvs Ifti i lh-la i ikswtvvhwv imvgsvlvyfiltla ysaicisc-- $x x x x x x x$ -wimhkqm-adpmfy----|v--cv|ttvval Ipr -yt|rvlk-gt la $-p$ slh

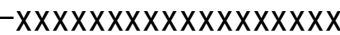

>dreIV_IV06 XP_693773.2

m 


\section{-tfiprflyeqi}

$-r-r a a n a f f I----f i a l m$

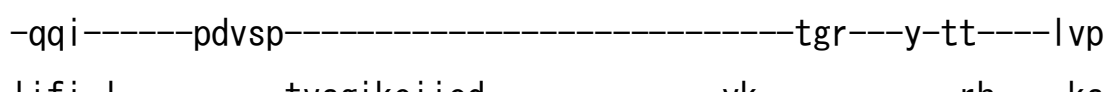

I if i-I-_-_-_-_-_tvagike i i ed--_-_-_-_-_--yk-_-_-_-_---rh----ka dntvnkkktt----------v|r

-sepqa-m---cyTETSNLDGETNLKIRqg-

|s |tasfqs |ed| ialsgr lecegpnrhlydftgt|r|x-

-nhnpap

Igp-

-QVLLR--GAQL----------------r

$-\mathrm{d}$

- g IVYTTGHDSKLMqnstkap I-

nt----qwvv

-krsnvervtnmqi Iv I fgi I lvmalvss

- i gaa i wnkqhtdea------xwy Is-xxxdis InfaynIItfIILYNNL

IPISLLVTIEVVKFTQAL-FInwdvem 
-yyaetdtp

aMAR-TSNLNEELGQVKYLFSDKTG

-TLTCNVMHFk-

kctiagity

$-x x x x x x x x x x$

$-x x x x--x x x x$
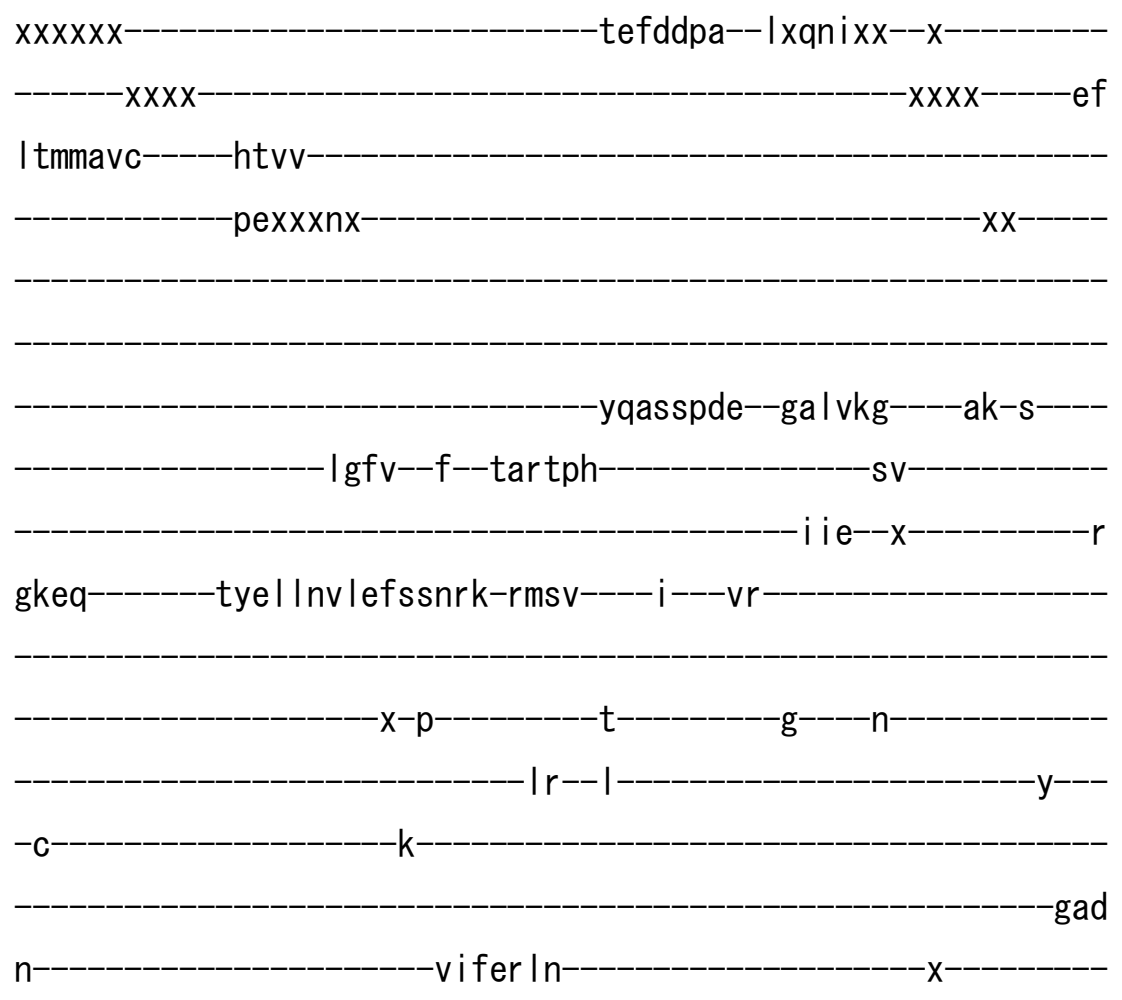

xxx--xx--e It-vah l eqf--ate----g | rtLCFAYVdI--eegay l ew l keynr--is-----tv I kdraqk l eecye I-i ekn I I ILGATA IEDRLQAGVPET I ATLMRad-IK I WVLTGDKQETAINIGYSCRL-_-_-_-_-_-_-_-_-_-_-_-_-_-_---_vshgms I i---_

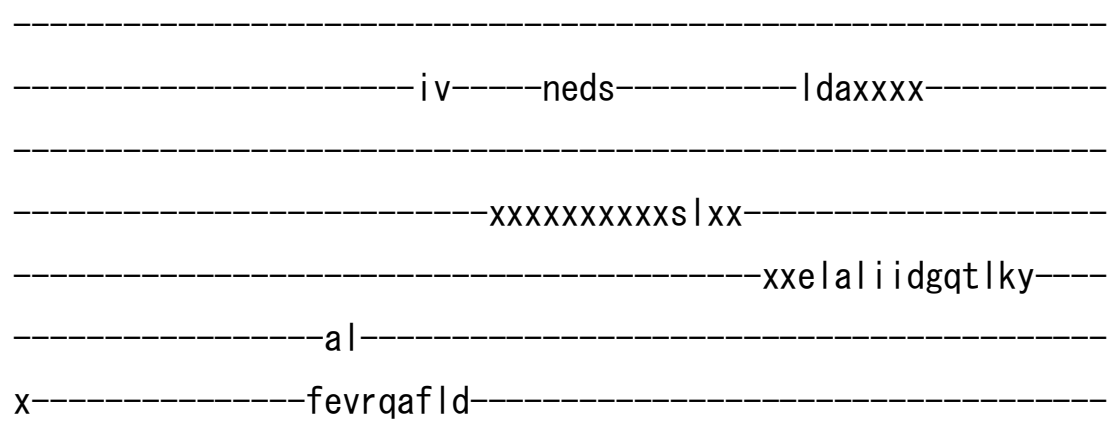


I a l sckav ICC--RVSPLQKSE IVDMVk

----kh----v-----------хa-----_-_----- ITLAI GDGANDVGM I QTAHVGVGI--

-ATNSSDYSIAqfSYLEKLL--LVHGAWSYNRVTKCI------L

ycf-yknvv-ly i i l Iwfafvngfsgqilferwcigly nv iftal ppft I g i fdrpcsqq--_-_-_-_-_-_---nmi rfpq lyr i tqnaegfntkv f-wghc inal ihs i i If-wfp I xal e------------------ xxxxxxxxxs-------vd--y I fvgn i vytyvvvtvc lk-agmettawtrfsh I--------avwgsmv I wm I ffav ysa iwpt i $x x x x x x x-------------g q i y-$

epashf---------tvtctchtvtra- pgfyg $\mid p-$

>dreIV_IV07 XP_694281.2

$\mathrm{m}-$ 
-xxstteng $\mathrm{i}$

-tffplnlyeqf

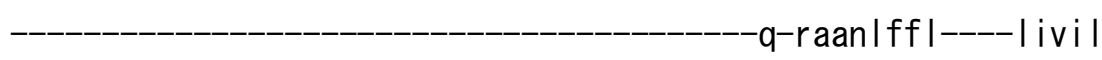

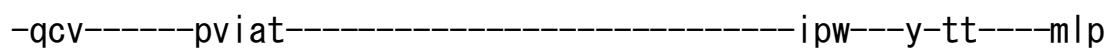

I I i v-I--_-_-_-_fvrgckdlatd-_-_-_-_-_-_--vg-_-_-_-_---rr----rs

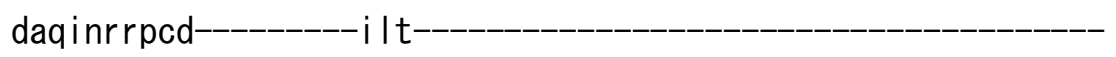

$-p-e g f$ $-\mathrm{x}------\mathrm{t}-$

k

-wkdVCVGD ILRVhk

-DQVIPADLLLLCs 
-tepys-I---cyVETADIDGETNLKFRqa-

-xxxxhte Ingdsteqn I aafda i vrceepngn I hsfrgefhw--

-kgerhl

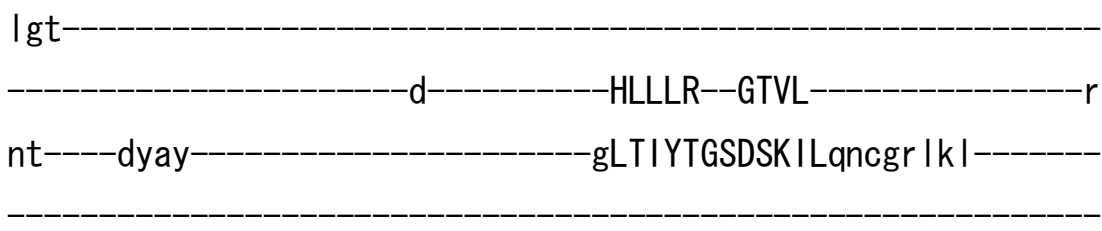

-kkthveillnktvIvilIfmIttall la

-i gag i feyr i spny-------dvvs----------xxxxxxspayqgf I tfwgy I ILLSPS

MPMSLYITfEVIHMVHCL-LI gwdvem-

$-y$ wedtncp

aHAR-TTTLNEELGQVGHLLSDKTG

$-T L T Q N R L L F r$

qcfiaghiy-

$-x X X X X X X X--x X X X$

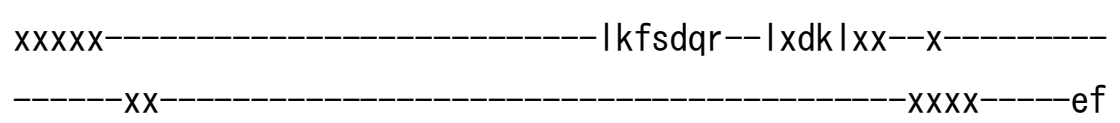

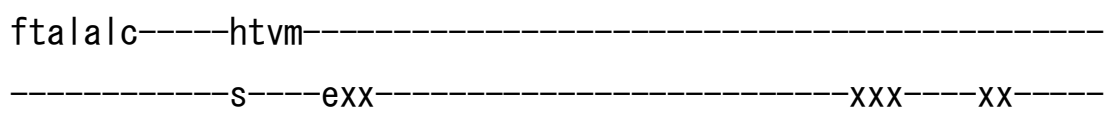

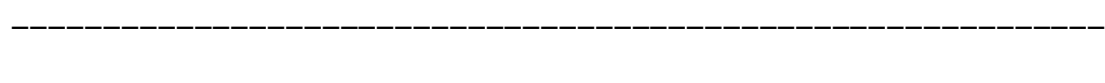

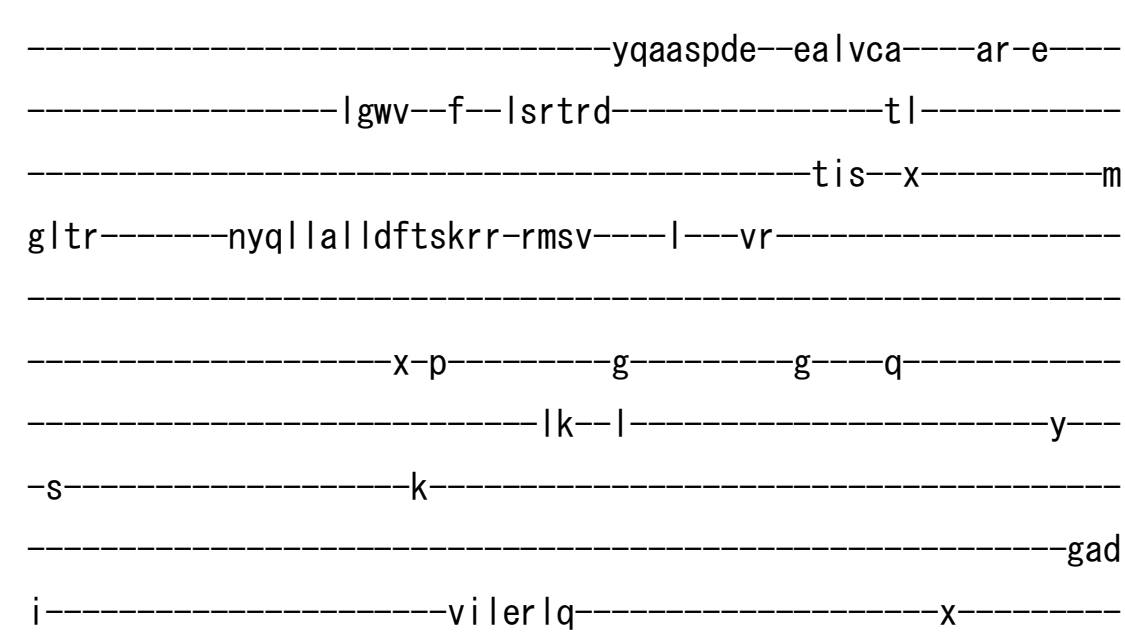


xxx--xx--est-drale lf--aqs----c| r tLCVAVRpv--pea I wtewsra InI---xgtatgnqet I I ee i ydxmekd Im I LGVTA I EDRLQEGVPET I ASLRRag-VKV WVLTGDKTETAVNVGYACKL$-m d p d t t \mid i----$

qg------eels---xx-------qscxxxx-

xxrteltk

x----------------sewgakfva-

I sgqcqsvLCC--RVTPAQKAEVVEMVr

---kh--sa--_-_-_-_---s--_-_-_-_-_--_I TMA I GDGANDVNM I KTAHI GVGL-cgvegsq

-AVLNADFALAqfSFLRKLL--LVHGHWSYYRISILL------R

yfl-yktta-falvhtwysfyngf saqpmyeswy is If tttyts I p i qctg i feqd i sar------------------sc I cwpe i ys i gqkkq I fnpsv I-ast I lysfyss i iff-fvpm-g i l--------------xxxxx---------------d--fqt lai ti etsv I fatt le-vi lctkywtkwnva--_-----avi fs laaff I st la Ihstr|ft--xxxxxxxxxx-------asq-nay-

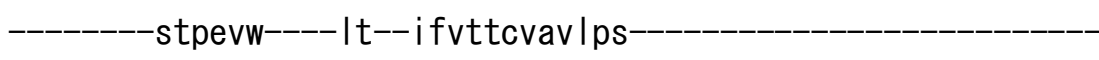

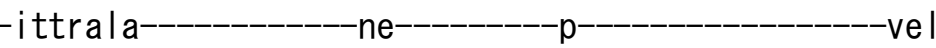
$-x x x x x x x x x x x x x x x x x x x x x$

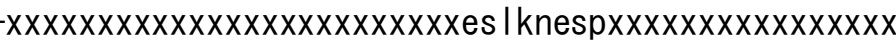
$X X X X X X X X X X X X X X X X$ 
$x X X X X X X X X X X X X X X$

$-x X X X X X X X$

$-X X X X X X X X X X X X X X X X X X X X X X X X X X X X X X X X X X X X X X X X X X X X X X X X X X X X$

$\mathrm{X}$

>dreIV_IV08 XP_695556. 2

$\mathrm{m}$

$-x x x x x x x x x x x x x x x x x x x x x k$ skkesk

---trtvhani

$x x x x x x x x x x x-$

-nrhyannk ikttkyt - |

-sflpknlfeqf 


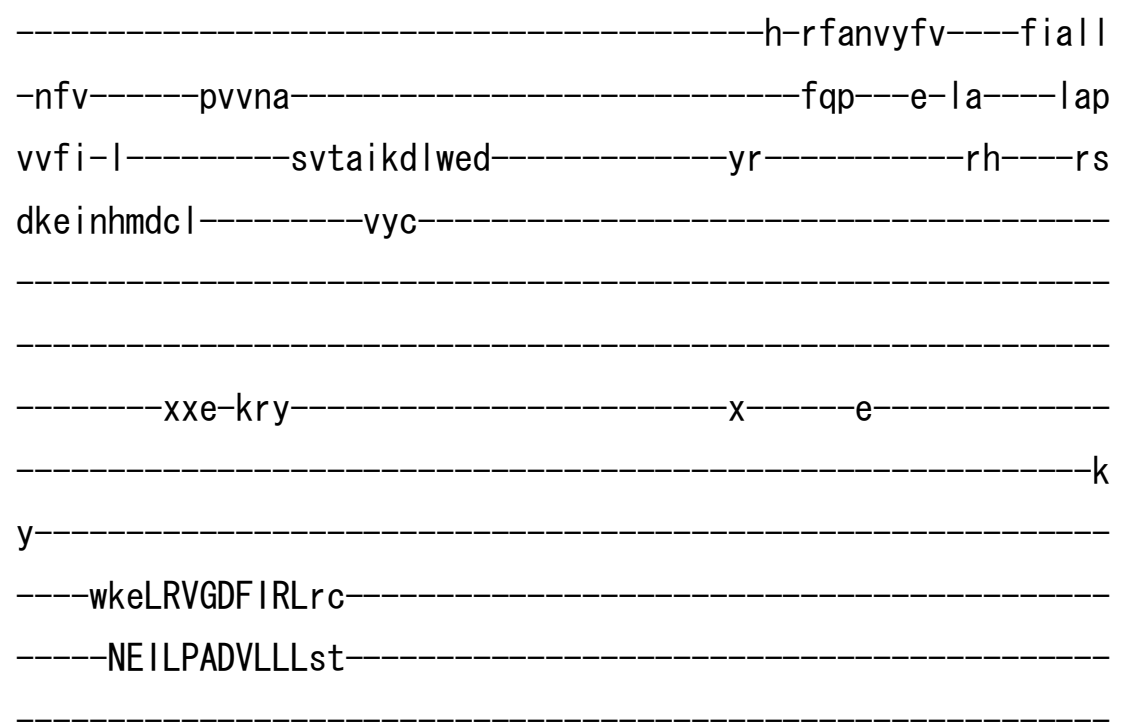

-sdpdr-I---chIETATLDGETNLKQRqv-

-vrsfidldcefdp I kynsvi ecekpnnd Inrfrgy i ihx-

sgrrdg

lyk

$d-$

- NLLLR--GCT I

$-r$

nt-----eeav------------------------g IVIYAGHETKAM I nnngpry

-krsklerqmnvdvfwcvi i I lvmc Ifsa

-ighg I wmfqygdkr-----xxfdv|---------xxxxxxxspvvsai y If I tm I IVFQVL

IPISLFVS iEIVKICQVY-FIhqd Ie I

-ydeetdsh

I QCR-ALN I TEDLGQMQY IFSDKTG

TLTENKMVFr -rctvagvey

$x \times X X X X X X X X X X X X X X X X X$

$\operatorname{XXXXX------~} \mathrm{X} X X X X X X X X X X X X X$

$-x x x x x--x x x x$

$x x x x x x x x x x x x x x x x x x x x x x x x x-x x x x x x x x d i$ tpdpq-- $x d k v x x x x x x x x x x x x x x x$

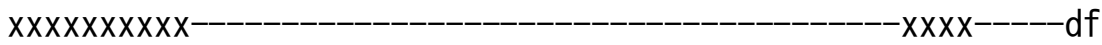

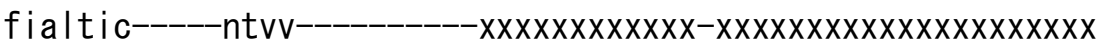

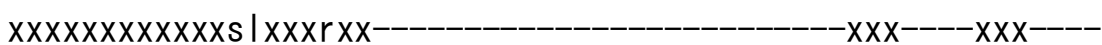




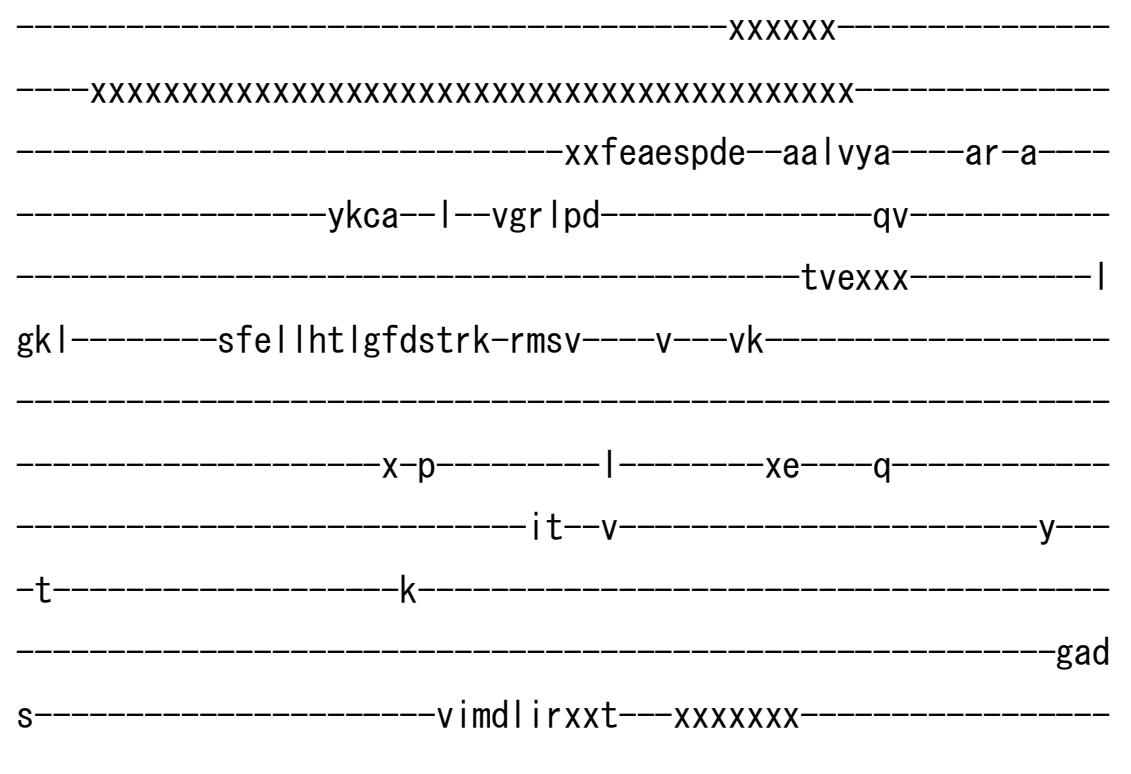

xxx--xx--ykt-qny I nly--aad----g | rtLCI AKKv |--skeeyasw I qrhl e--ae-----taiqrree I f fesal $r$-letn I qILGATGIEDRLQDGVPETIASLRKag-LQ I

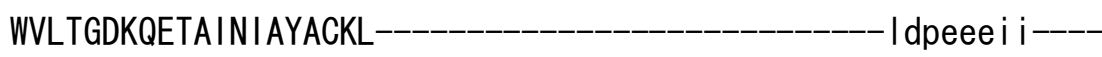

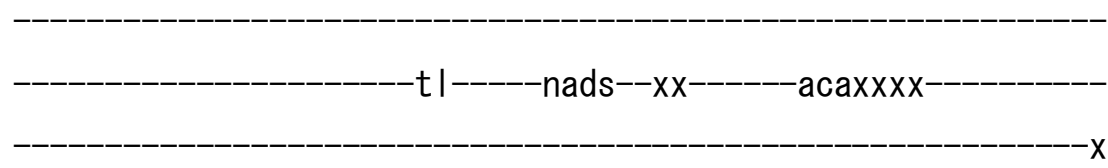

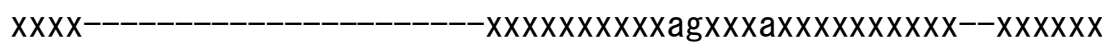

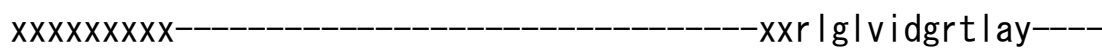
al

x---------------ks ledkf la-

varscrsvLCC--RSTPLQKSMVVKLVr ---nk--Ik------------v-----_-_--_--MTLA I GDGANDVSM I QVADVGVGI-- 
yff-yknam-fval i fwyqfycgfsgsamvdqwy l iff nImfsafpq I i tgt I dkdvsae--_-_-_-_-_-_--t I qe I pq l ymngqnseeykpym f-wmnm i dafyqs I icf-fipy-fay-_-_-_-_-_-_-_--xxxxx--_-_-_-_-_-_-_-_ -d--Iftwgtpittlalftil Ih-Ig i etktwtwmnwv-------sigfsmal fftvalc ynascptc---xxxxxxx---_--_----wtmar I I-gdp|fy----||--cvvtpvaal Ipr-

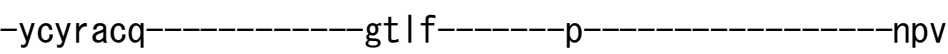
$-x x x x x x x x x x x x x x x x x x x x x x x x x x x x x x x x x x x x x x x x x x x x x x x x$ $x x x x x x$ xxxxxxxxxxekalaspi $x x x x x x x x x x x x x x x x$ $x x x x x x x x x x x x x x x x-$

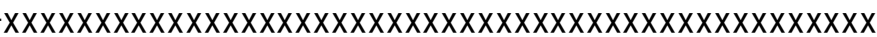
$x x x x x x x x x x x x x x x x x x x x x x$

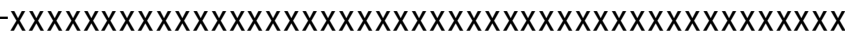

$\mathrm{X}$

>dreIV_IV09 XP_698351.2

m-

$-x X X X X X X X X X X$ $\mathrm{X}$ 


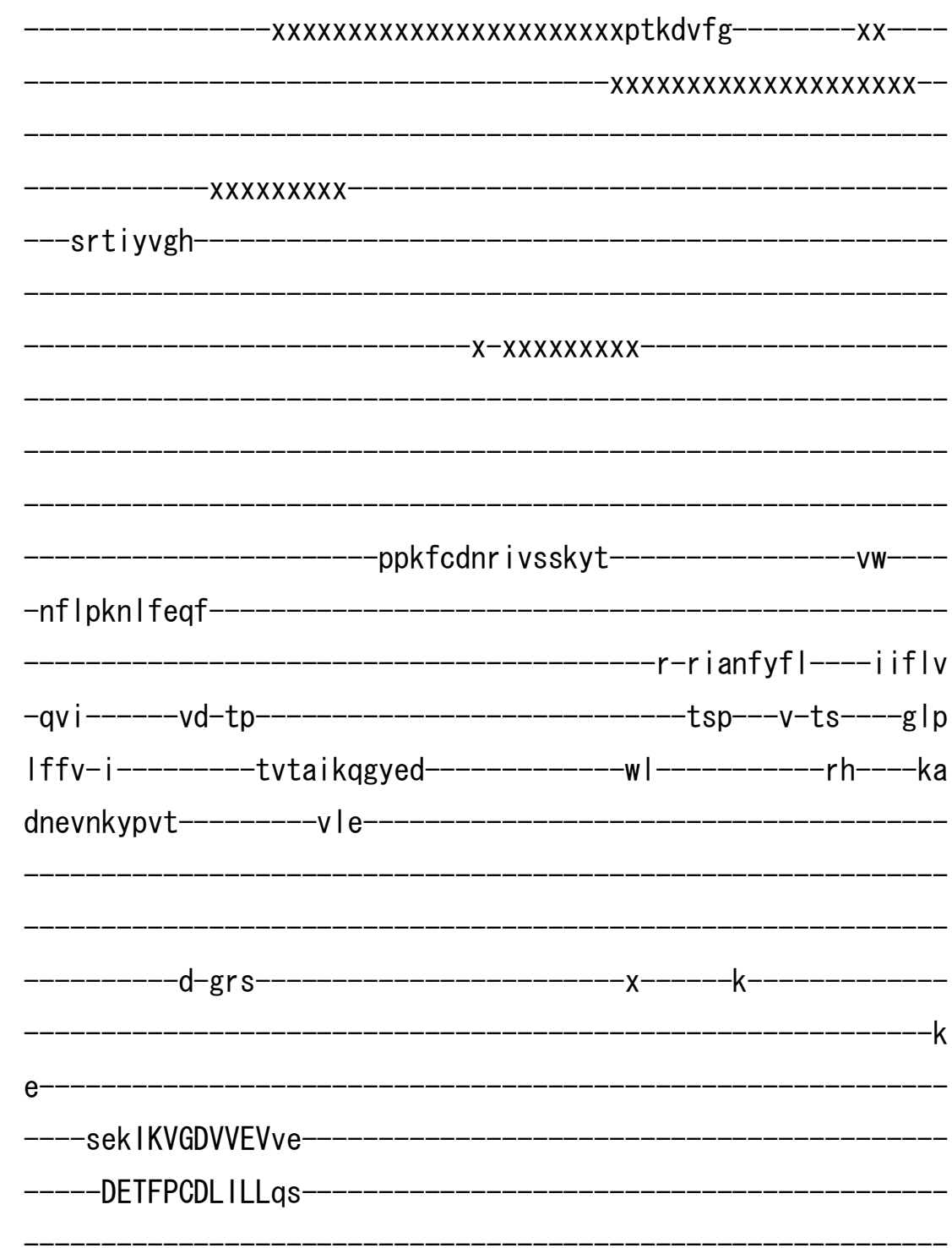

-sredg----xcfVTTASLDGESNHKTHyt-

--vpd i er-----d l qv I taa i eceqpqpd l ykfvgr

-eeavrs

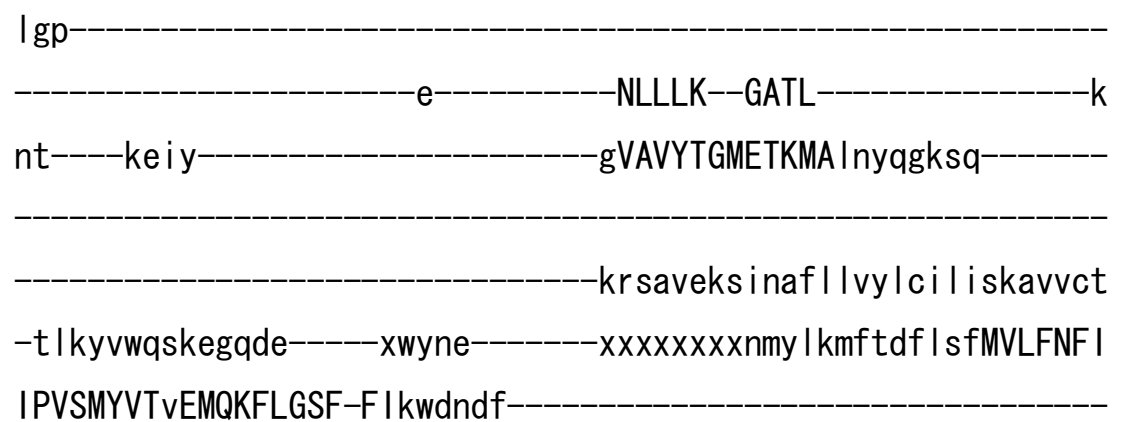


aLVN-TSDLNEELGQVEYVFTDKTG

TLTQNNMEF $\mathrm{i}$

eccidgfay

$-x x x x x x x x d g f t v t d x x|x k| q x x x x x$

$-x x x x$

Iralc|c-----htvq-

$-\mathrm{XXXX}$

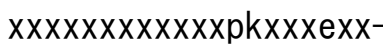

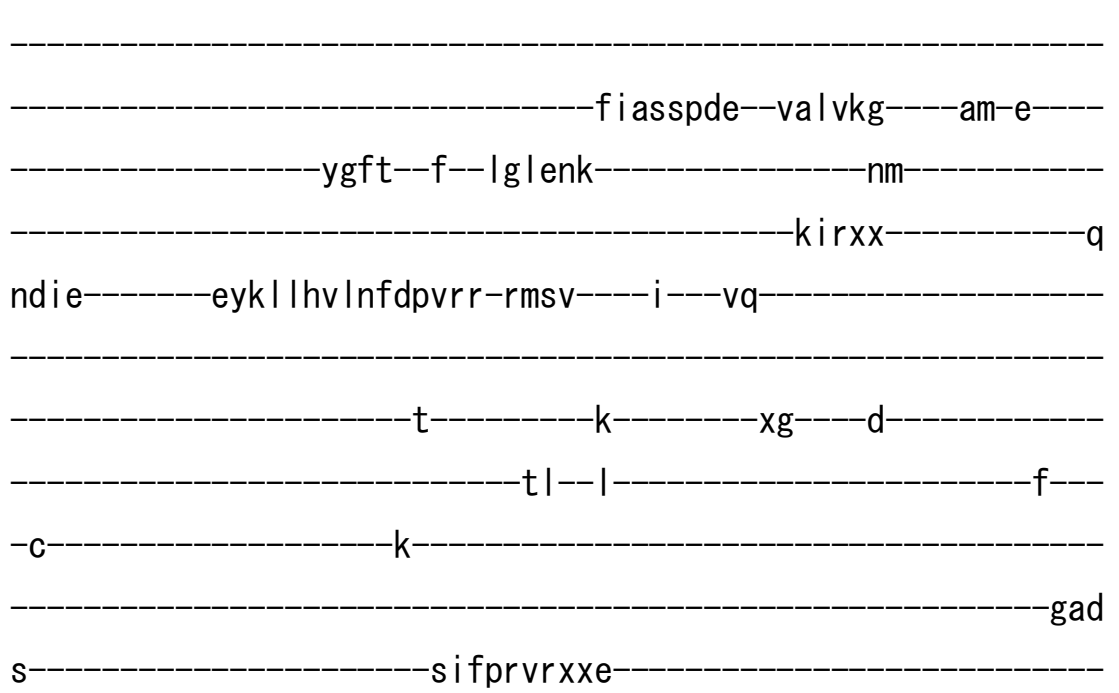

xdr i-r Ihvern--atd----gyrtLCVAYKq I--saeeyal adtg I re--ar----- | a l qdr eek I mamynq-vetgms I I GATAVEDRLQEEAAETMEALQGag-MK I WVLTGDKMETAKSTCYACRL--_-_-_-_-_-_-_-_-_-_-_-_-_-_-_fargte I I--_-

el-----tvrt---xxxxxxxxedrxxxx-

xxxxxxxxxxdaxxxkxxxxxxxxxx-

-xeygf i idgat | s I

vm-

$-x x x x x x x$

$\mathrm{x}$

slyrg If $\mid q$ 
icqnctavLCC--RMAPLQKAQIVKMVk

---nc--kg----------xp------------ ITLSI GDGANDVSMI LEAHVGI GI--

$-k g k e g r q-$

-AVRNSDYAIPK I KHLKKLL--LAHGHLYYVRIAHLV------Q

yff-ykn I c-fi I pqf I yqffcgy--_-_-_-_-_-_-_-_-_-_-_--sqqp I ydaay I tmy

nicftsmp i l ays I l eqh i a i e-_-_-_-_-_-_-_-_-_ I I dnat I yre i aknam I rwrp

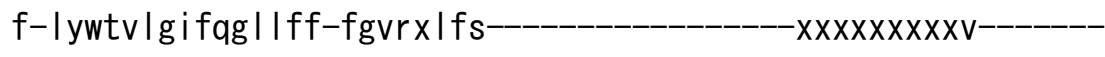

fg--nwsygt ivftvlvftvtlk-laldtrhwtwinhf--------viwgs lafyvffsff

wgg i i wpf---xxxxxxx--_-_-_-_--_fvfanm I-_-_-_-_-_-_-_-_-_-_-_-_-_-_-_

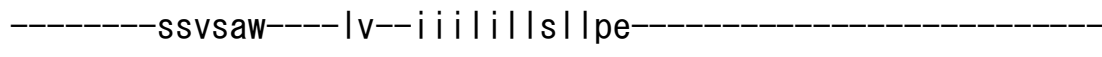

illavir

-xxxxxxxrqtaavtpxxxxxxxxxx

>cellV_IV01 NP_503858.1

$\mathrm{m}$ 


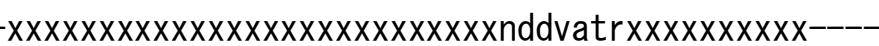

$-x \operatorname{xx} x x x x x x x x x x x x x x x x x-$

$x x x x x x x x x$

$---s r t v r i g y$

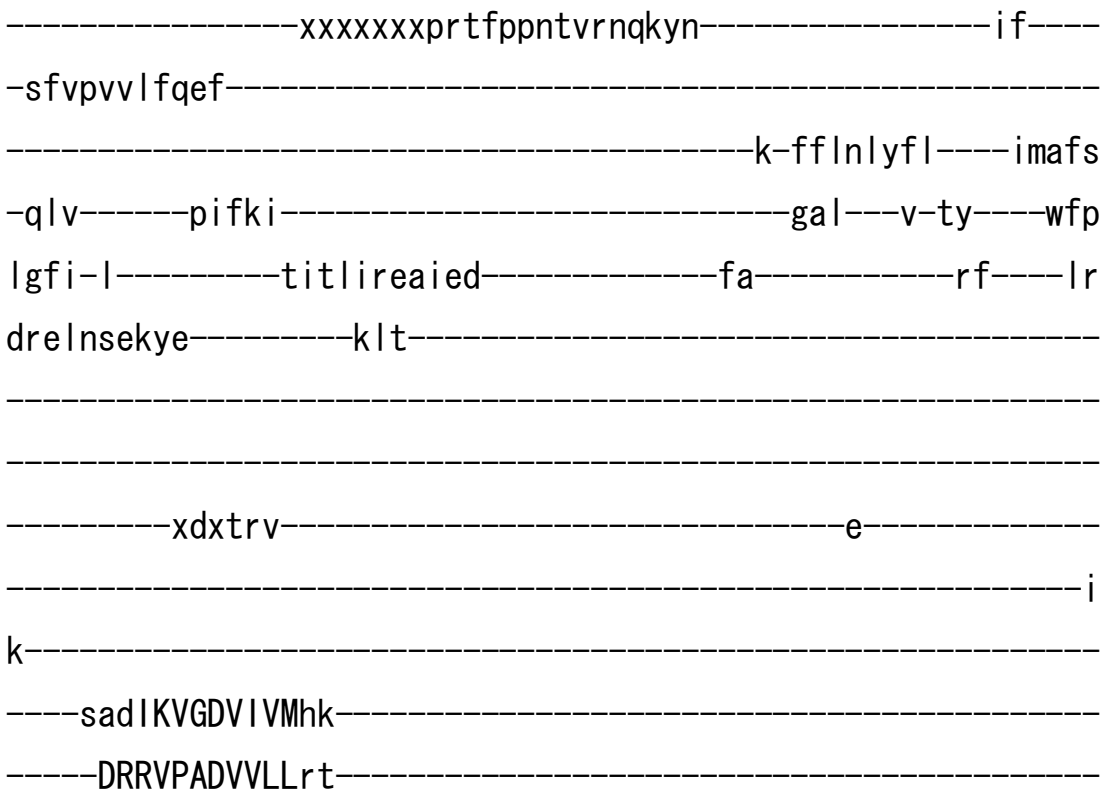


- sdkss-s---cfIRTDQLDGETDWKLKip-

ipftqh lanese ime Incevyaekpqkd i ysfvgtvkixx
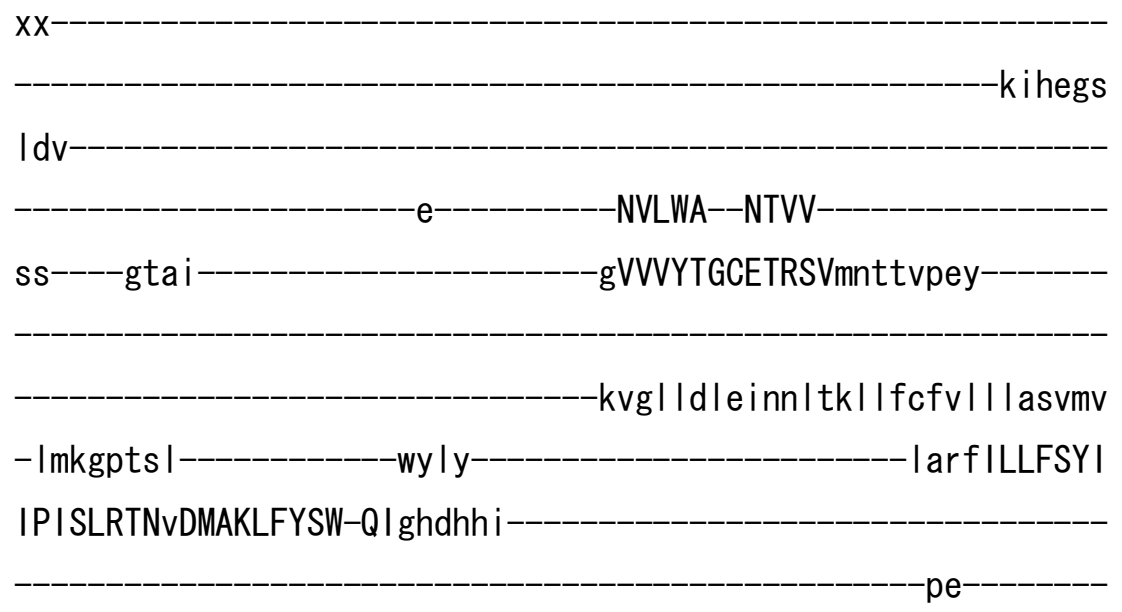

-tVR-SSTI IPEELGRISFLLSDKTG

-TLTKNEMHFk--------------kihlgtvaf

$x x x x x x x x x x x x x x$

$-x X------X X X$

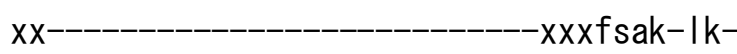

- na

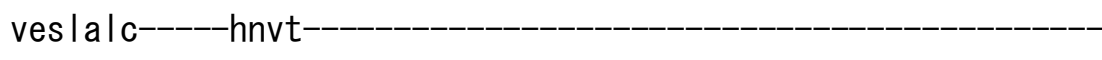

$-p \vee x x x$

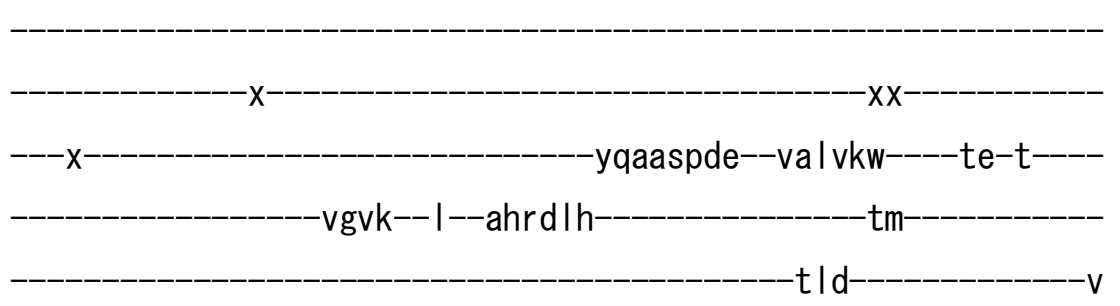

qpsn-xxxxxxqfqi Ihvfpftserk-rmgi----_i---vk--_-_-_-_-_-_-_-_-_-

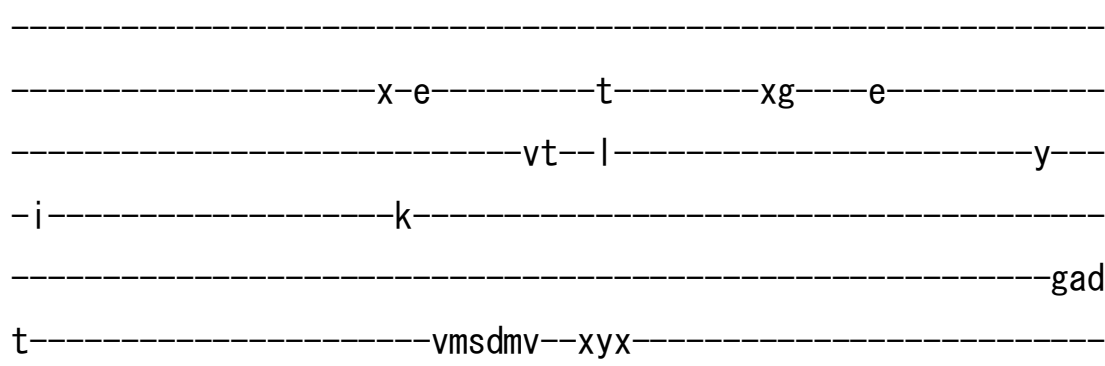


-dw I-deectnm--are----g | r tLVFAKK i I--srae l eafdqayh----aa----xms i tnr tanmanvvknx I erd I q I ICLTGVEDRLQDQVTTSLELLRNag-IK I WMLTGDKLETAICIAKSSGLfsksdhih----

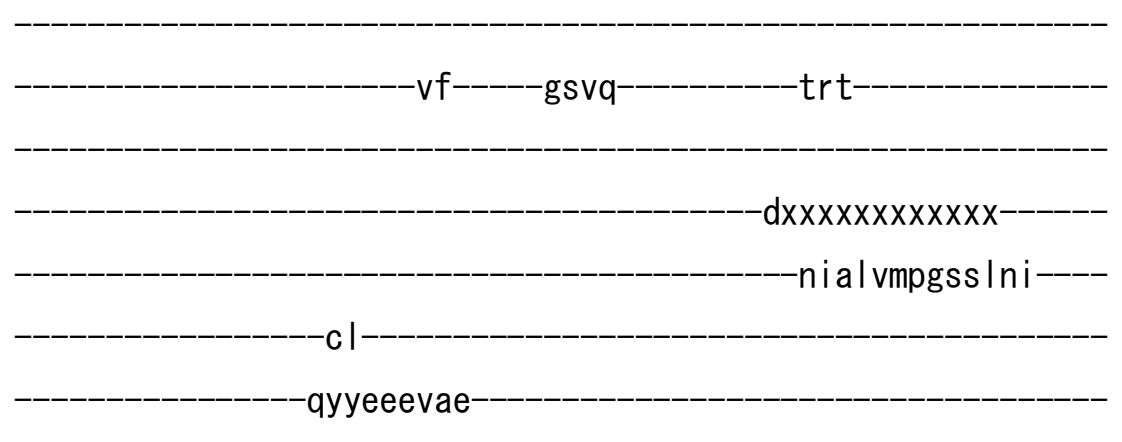

IvcastavVCC--RCSPEQKAQIVQLLr

---ky--ra---_-------x I---_--_-_--_RVAA I GDGGNDVSM I QAAHAG I GI--

-ASLAADFSI TqfSHI CRLL--LVHGRFSYKRSCTLA------Q

fim-hrgll-isivqaifscrfyf -vavs I yqgv Imvaf tscyt I |pvfs I-iadrdvtat---_-_-_--_--_---nal type lyke I vkgrt I sykt

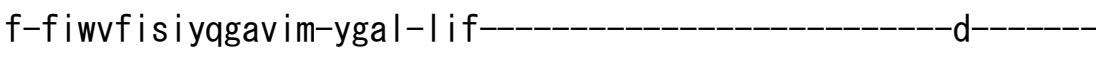
td--i Ihi vsisfsal iatel im-val tvhtwhw ImI v--_-_---aqal $\mid$ g I yvvs I it Idqf-f drkfv-

--_---_--fswvfi-----tk--ttai tvvss Ip I-_-_-_-_-_-_-_-_-_-_-_-_-_-_-




\section{>cel IV_IV02 NP_001021458.1}

m-

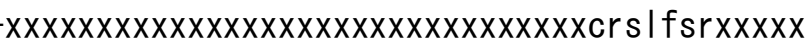

---srtvrvgy

xxxxxxxnvtftpntvenqkyn

-sfvpivlfqqf 


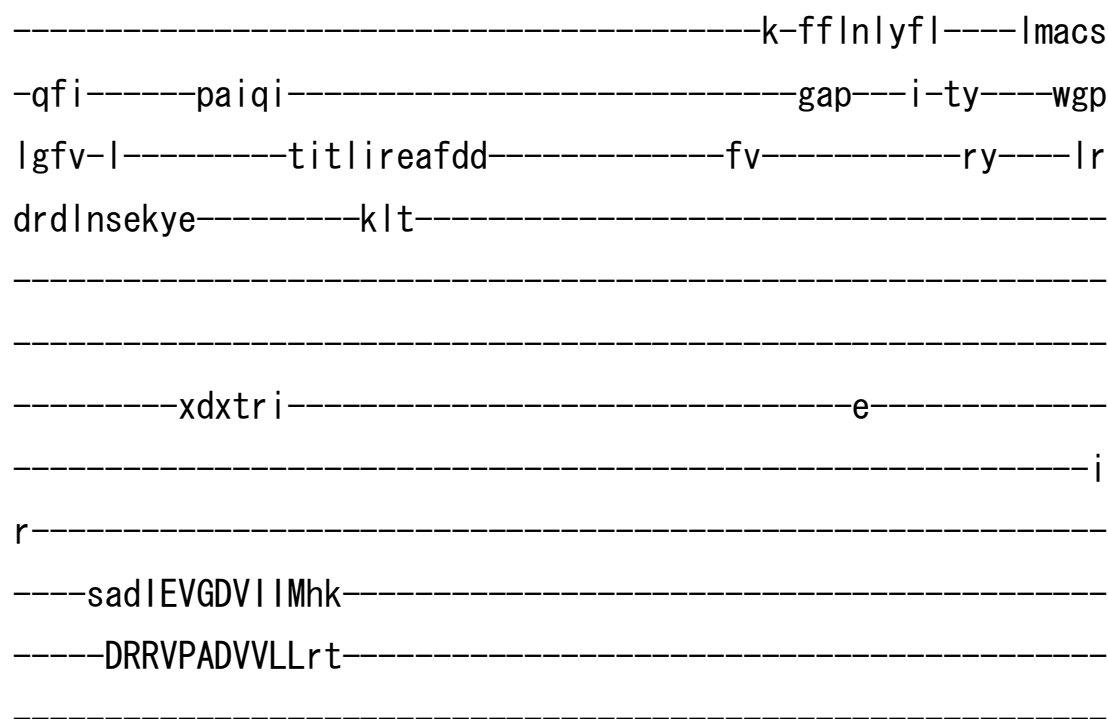

-tdksg-a---cfIRTDQLDGETDWKLRip-

-vphtqh I pnead ime Incevyaekpqkd i hafvgt I kixx

$x x$

-nvqdgs

Inv

NVLWA--NTVV-

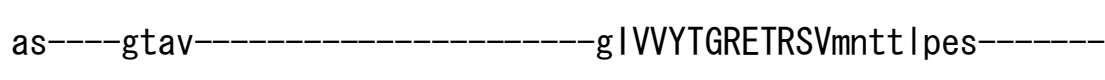

-kvg I I levnnItk I fcfv|v|ssvmv

-amkg Idn I-

-wyryImrfILLFSYI

IPISLRVN IDMAKLFYSW-QI grdkhi

tVIR-SSTIPEELGRISFLLSDKTG

TLTKNEMHFK -kihlgtvaf

$-x x x x x x x x x x x x x x$

$-x x------X X X$

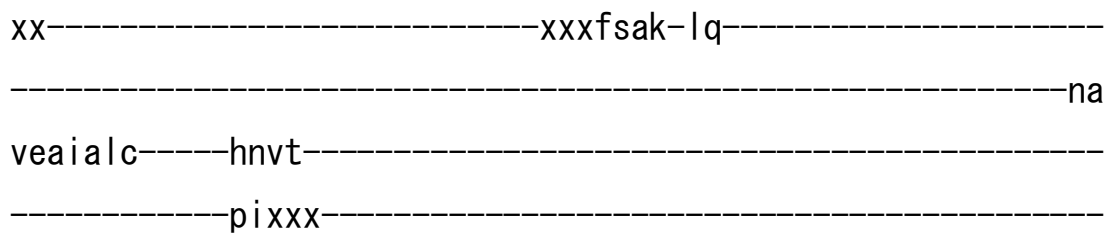




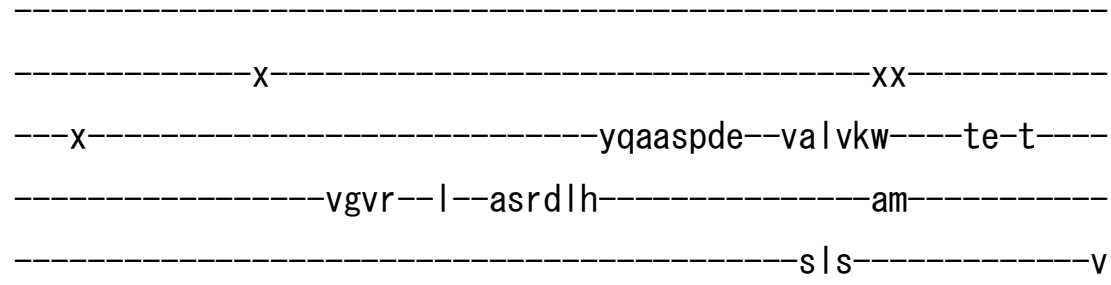

qlpn-xxxxxxqfai lyvfpftsetk-rmgi----i---vk---_-_-_-_-_-----_-_

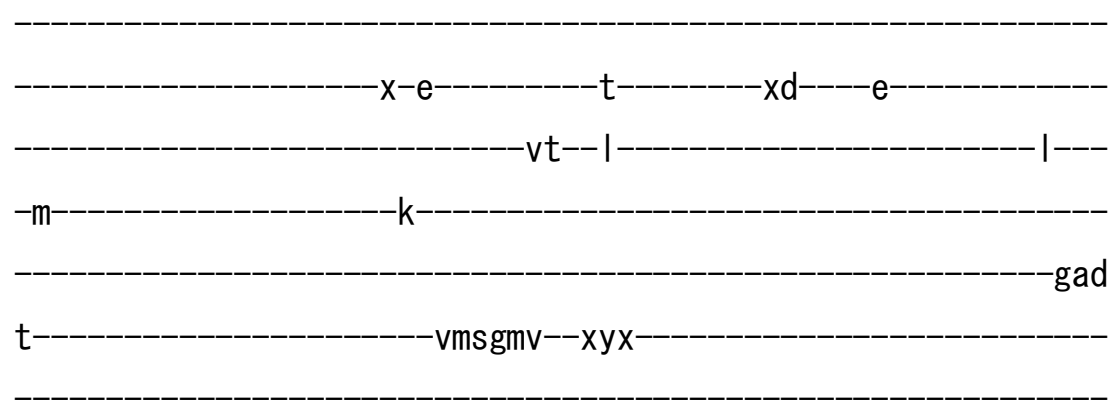

-dw |-deecsnm--are----g | rtLVVARKp |--sqae l eafdrayh----aa----xms i sdr sqnmanvvnrx I erd I q I LCL TGVEDRLQDQVTTSLELLRNag-IK I WMLTGDKLETAICI AKSSGL-fsrsdnih----

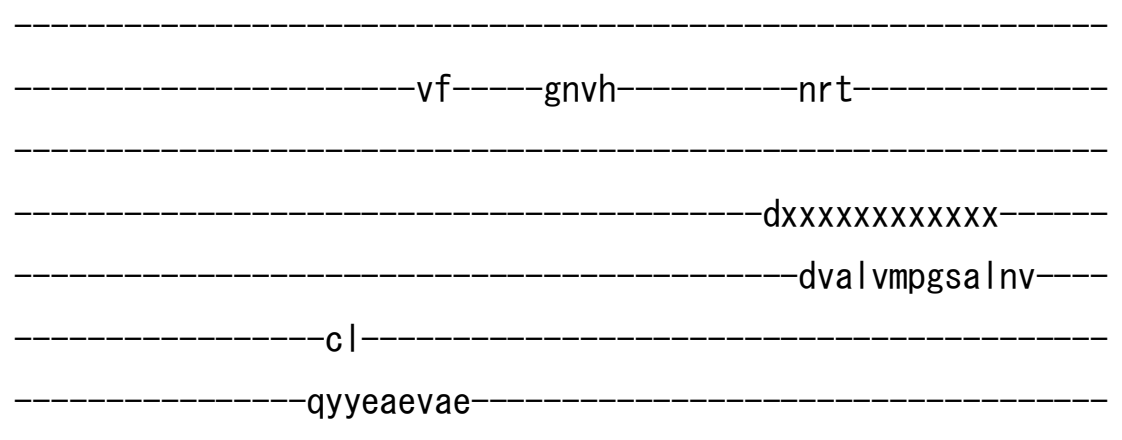

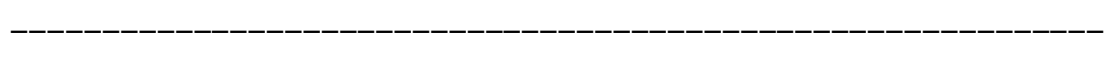

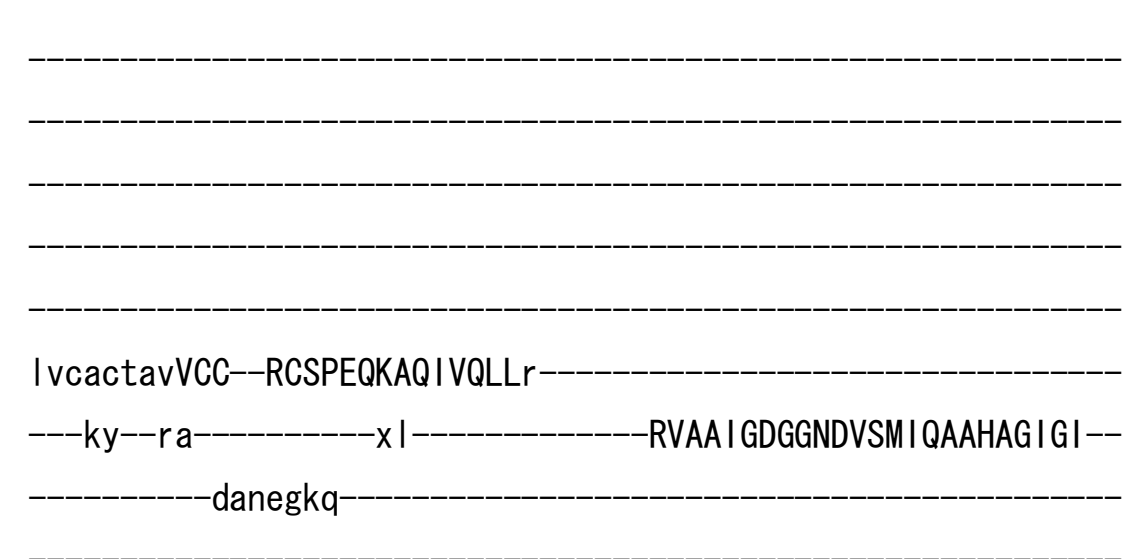

-ASLAADFSI TqfSHVCRLL--LVHGRFCYKRSCALS------Q 
fvm-hrgli-istmqaifscvfyf asvs I yqgv Imvay stcytm|pvfs|-vvdrdvtat nal type lyke I gkgrs I sykt

f-ciwv I is l yqgavim-ygal-Ivf $d-$

ad--fihvvsisfsal i vte I im-vamtvhtwhwaml I -aqals $|g| y m i s|i|$ fday-fdrafv- swvfi----sk--ttai tavsc $|p|$ yivkalr rkfs $-p-$ -psy akv----n>cel IV_IV03 NP_495244. 1

$\mathrm{m}-$ 


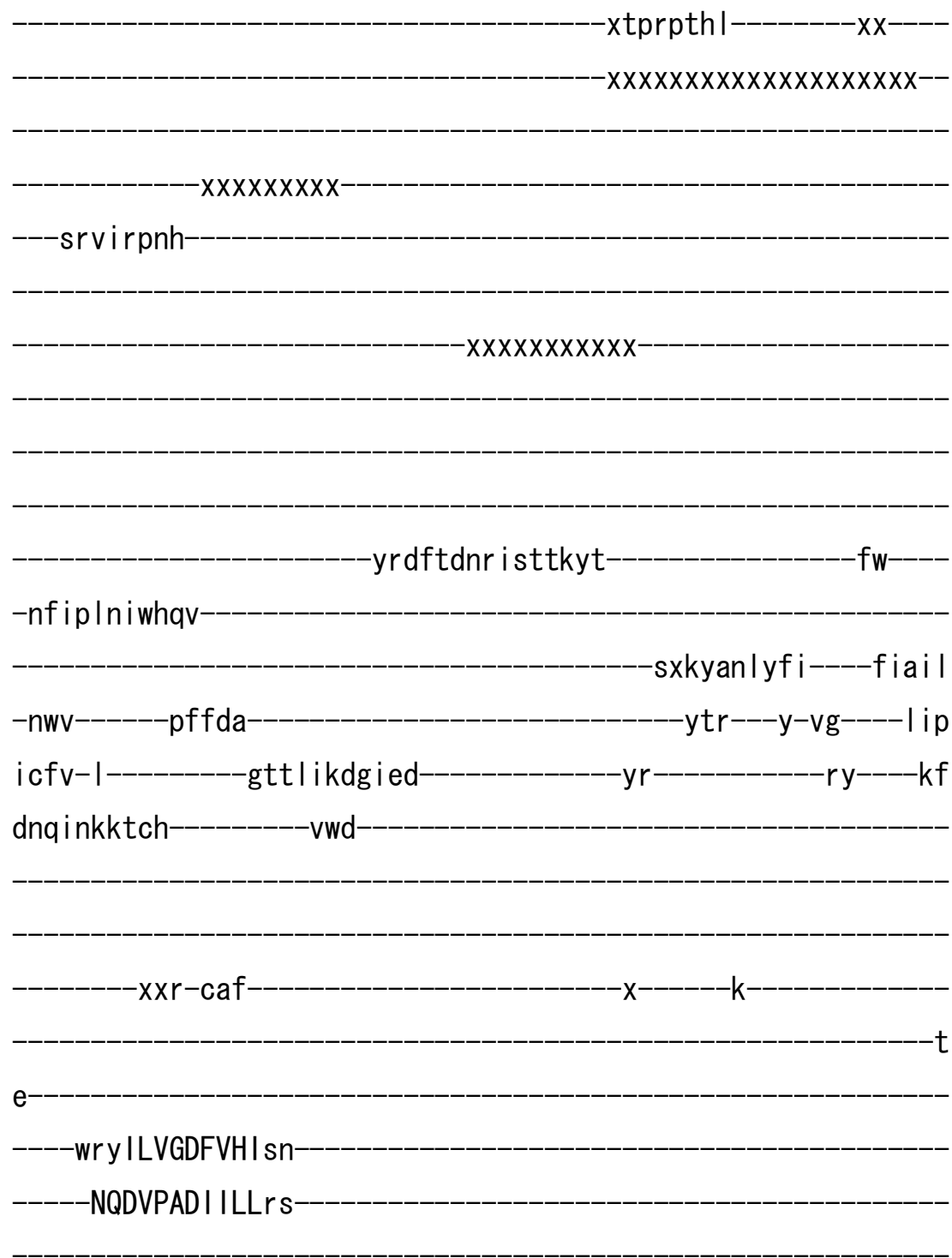

-ssesg-t---cy IETCNLDGETSLKQRmv-

-xxxv idyskkdstfkppdftgvvtcekpdks i yt i rakvef $x$ -

-pgqsdv

i ik

e-

-NMLLR--GSR I

$-k$

nt----tfve

g I VVYAGHDTKVMqnngraph-

ktsgiek Itnkf i iacfiml I Imv lyga

-itsavwvgqhpvde---xxxfi Isxxxrpf i egf i g i gafF INYQLL VPISLYITVEIIKALQIY-FIsndiqI 
-ydqksdra

iDCR-SLSIPEELGTVTHVLSDKTG

TLTENMMIFr

ncafdetdy

$x x x X X X X X X X X X X X X X X X X$

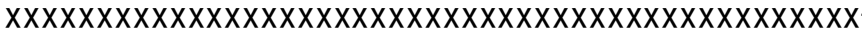

$x X X X X X X X X X$

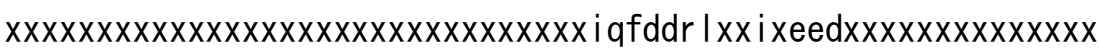

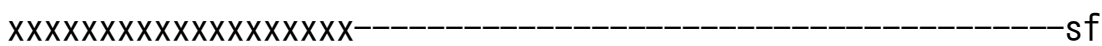

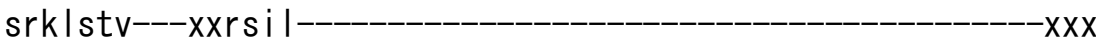

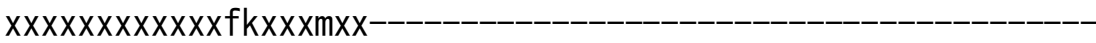

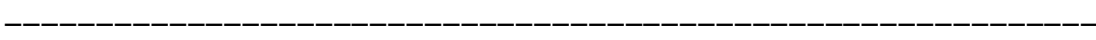

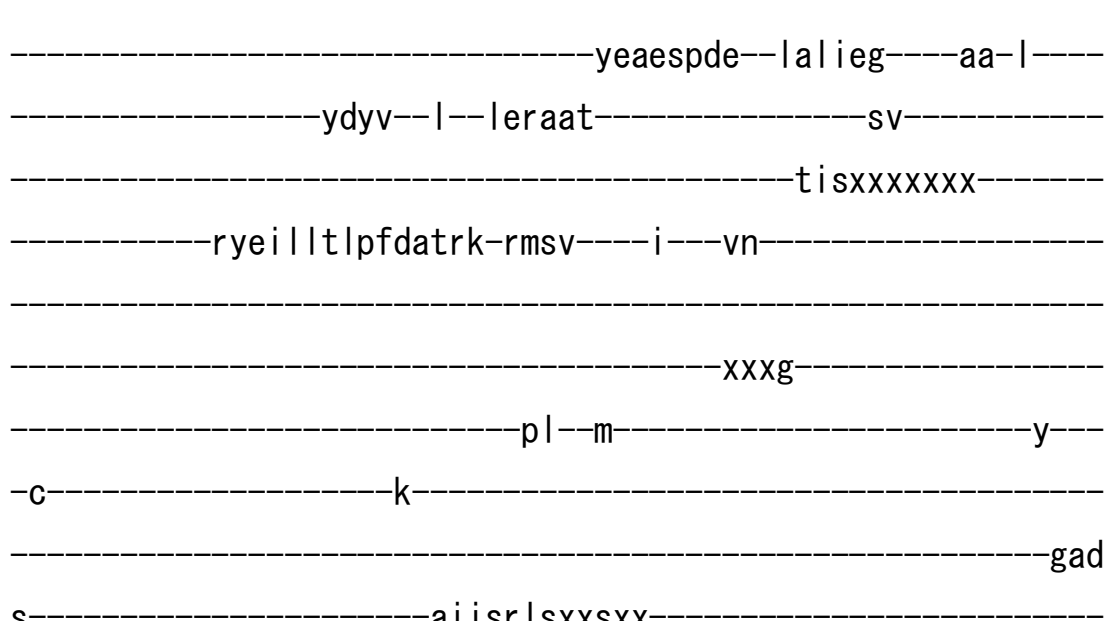

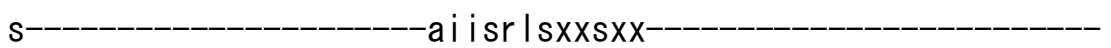

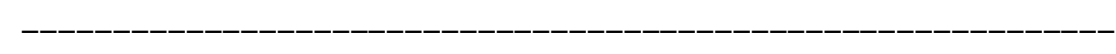

-----xxxxqd I-kdh I dny---akk----g I r tLCFAMKy i --skedfedf I dsyrf--Im----xdatser ekm I sekade- letn I I SGVTGIEDRLQDGVPDTLRALRDag-IQV

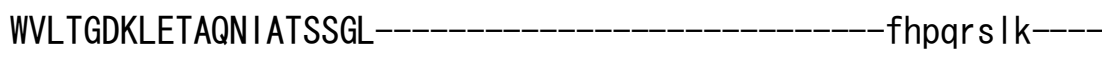

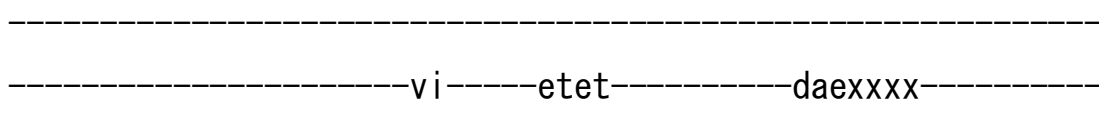
xxg Ini imspaair I

x---_-_-_-_-_---qdgnah Ime 
a lkkaktvLCY--RMTPSEKATIVNTVk

---kr--ik-NVLAI GDGANDVPMI QAAHVG I G I-agkeg la

AAMACDFA I Ar FKFLSRLL--LVHGHWSYYRLANTF $-\mathrm{L}$

yf I-yknan-avf $\mathrm{i}$ i fyyqfyngasgtn i vdp i wgv i y pi iftsvqpvvvgv Idqdyddq t Imnkpe lyvigrenqlytwkh f-frdvidg i yqaavi iy-yvay$x x x x x t$

ss-- | wemgfy i atss i I vnsgh- | a lqvrywhwq | valfsffilfnfaff faec I taaxxxxxxxxx -wmp i hamgdprfw----yy--qfitvival cprxxxxxxxxxxxxxxxxxxxxxxxxxxxx

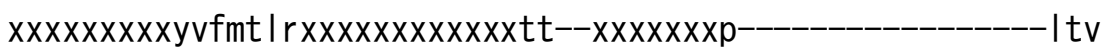

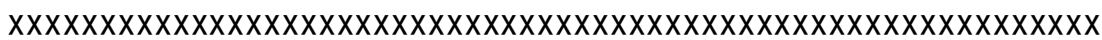

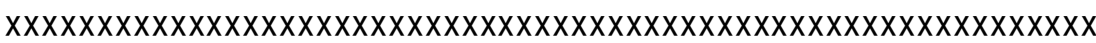

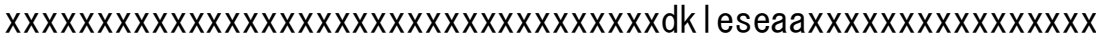
$x x x x x x x x x x x x$

$-$ >cel IV_IV04 NP_001022894.1 m 
- pteardn

---nrhihlgk-

$-x x x x x x x$

aqrfcsnr istckyn

$-g f$

-sflprflyeqf

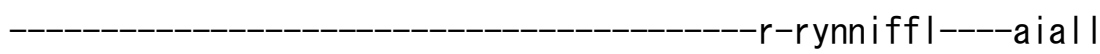

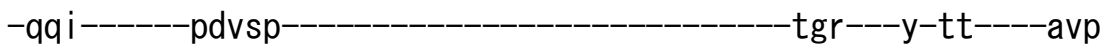

fl i i-l--_-_-_-_--svsalke i fed--_-_-_-_-_-_--vk--_-_-_-_-_-rr----rs

dnkvnafsve---------ilv

$-\mathrm{d}-\mathrm{ghw}-$

$-\mathrm{x}------\mathrm{e}-$

$-k$

q

----wkdVSVGDFIRIdn-

-DSLFPADLLLLas 
-seqqg-m---ay IETSNLDGETNLKIKqa-

-Iditstmtspek I sqfese itceppsrhvnefngni i i--

ngvarh

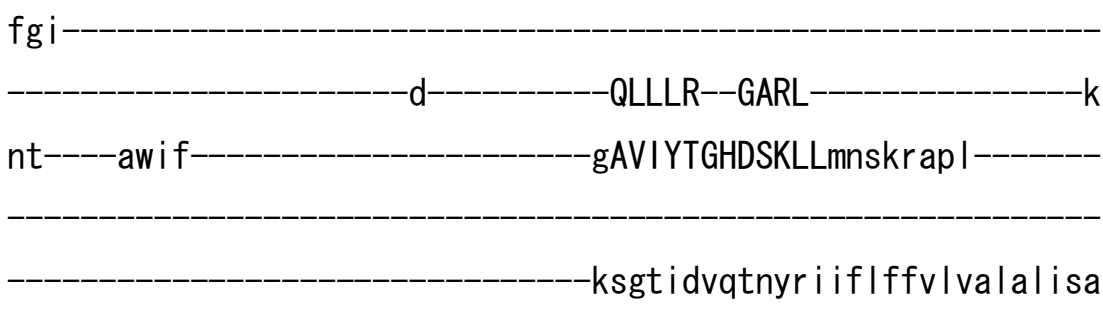

-tgse i wr gnn i pqa------wy I s------------xxxxdpkgsf I wgv I tfF ILYNNL

IPISLQVTIEVVRFFQAI-YInndi em-

$-y d v n s d s c$

a I AR-TSNLNEELGQVKF IMSDKTG

-TLTRNVMKFk----_--_-_-_---r I sigsrny

$x x x x x$

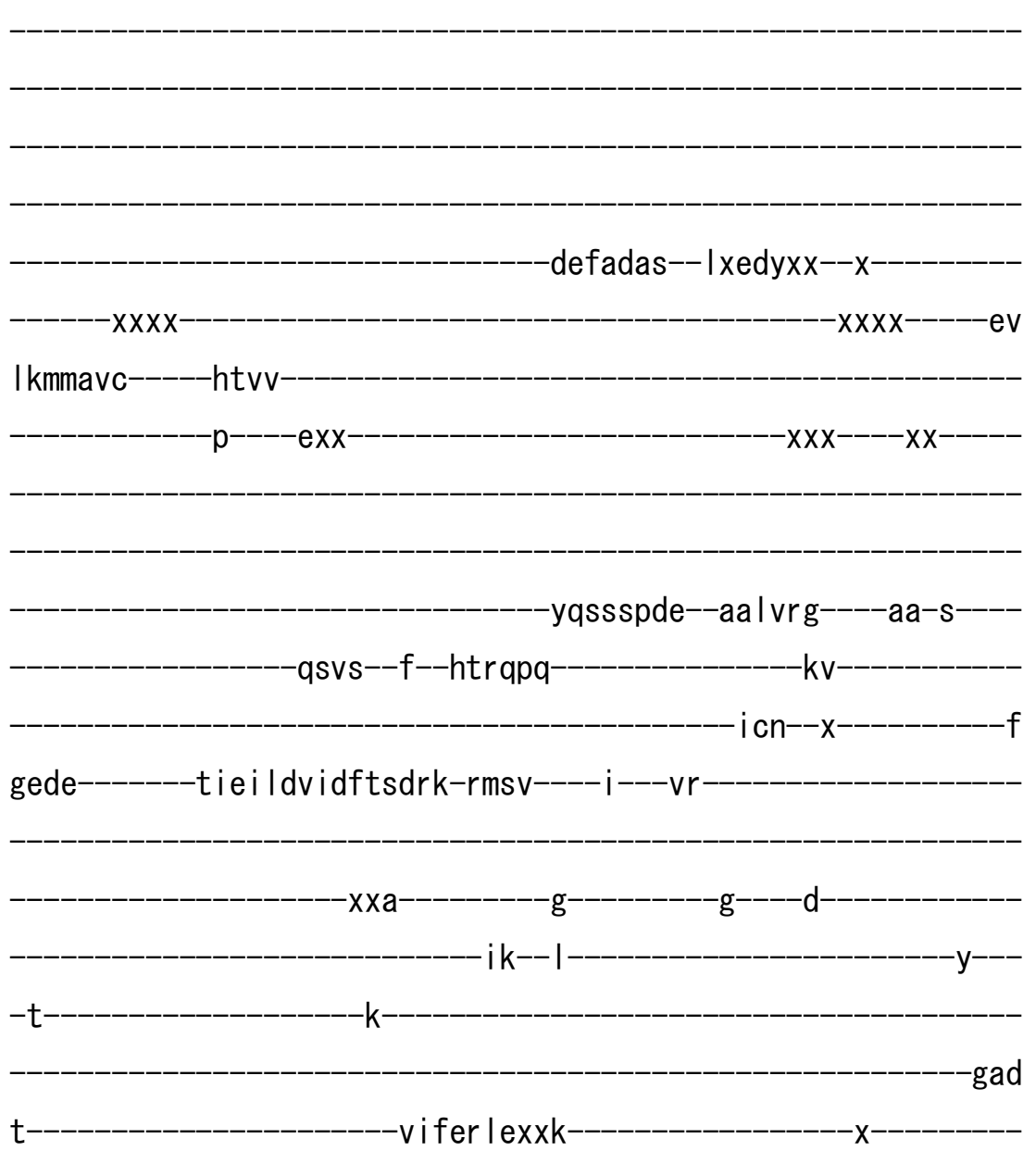


xxx--xx--eyc-tehl edy--asf----gyrtLCFSMRh I--teqeysqwapeykk--a i----- l a i dnrak | ladaaek- lernmi IVGATAIEDKLQEWVPET IQALMAad-IRV WMLTGDKRETAINI AHSCAL-chtntell----

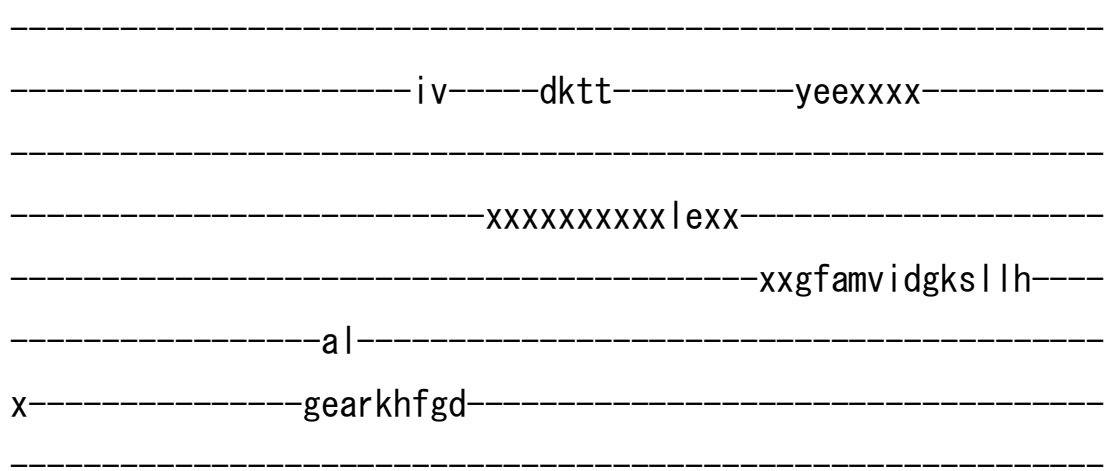

la | rchavVCC--RMSPMQKAEVVEMVr

---kl---a-----------xh----_-_-_---VVLA I GDGANDVAMI QAANVGVGI--

AASASDYA IPr FHFLRRLL--LVHGAWNHDRSVKV I------L ysf-ykn i c-ly i i e I wfamf saw-_-_-_-_-_-__-__-_-_-_-_sgqt i ferwt i gmf

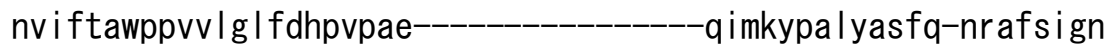

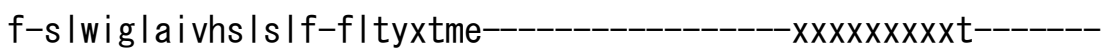
gg--wIm I gncaytfvvatvcfk-al lecdswtwpvvv-------ac i gs i g I wi vfviv ys I vfph i xxxxxxxx-_-_-_-_-_---gmaa imm--_-_-_-_-_-_-_-_-_-_-_-_-_-_

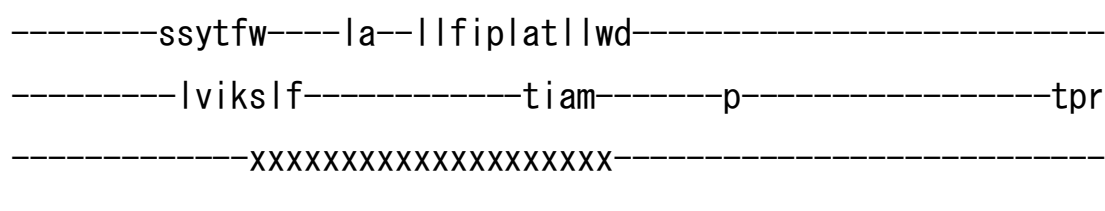
-xxxxxxxxxxr||tss|rxxxxxxxxxxxxxxxx $\operatorname{xx} x \operatorname{xx} x \operatorname{xx} x \operatorname{xx} x \operatorname{xx} x \mathrm{x}$ 
>celIV_IV06 NP_499363. 2

$\mathrm{m}$

$-1 p q q$

---r Ivvpnnq

$-x x x x x x x x x x x$

rkycgnr isttkys

$-\mathrm{v}$

-tfipknlfeql 


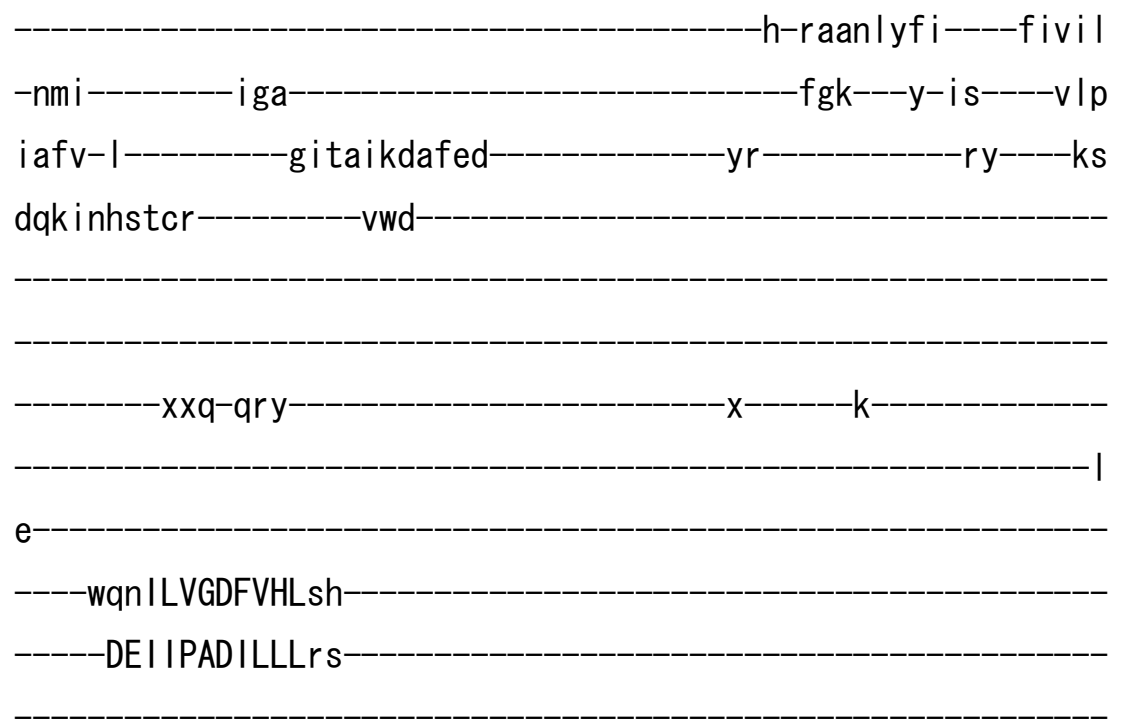

-sdvng-v---cyVETCNLDGESNLKQRqa-

-xxxxxkyhnanvp I eyspaefnyr i aceqpttdvykfegr l eaxx

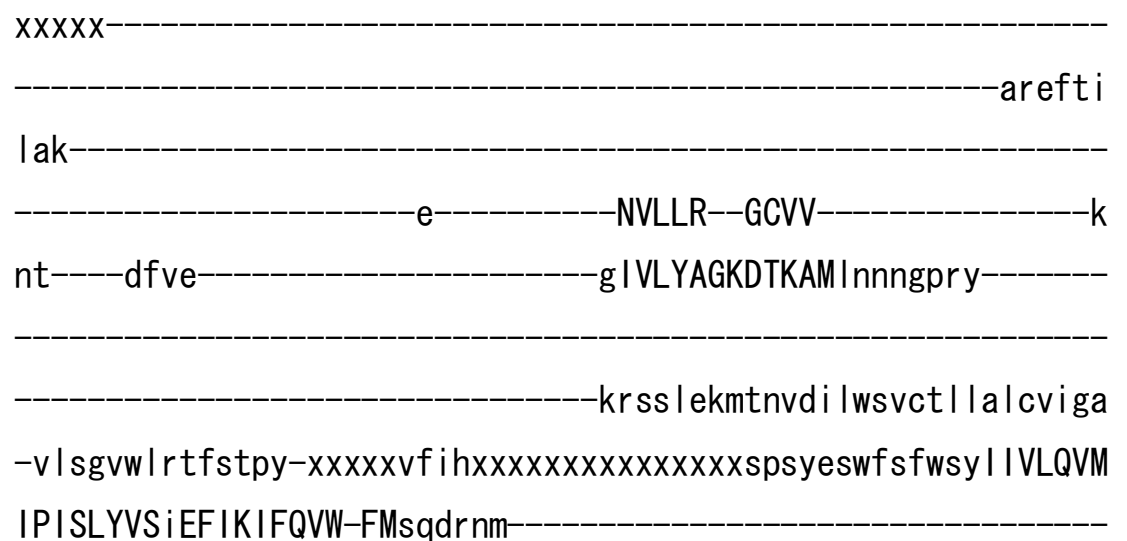

-yydkvdkr

IQCR-ALN I TEELGQ I QYVMSDKTG

TLTENQMVFr rcsvngndy-

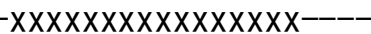

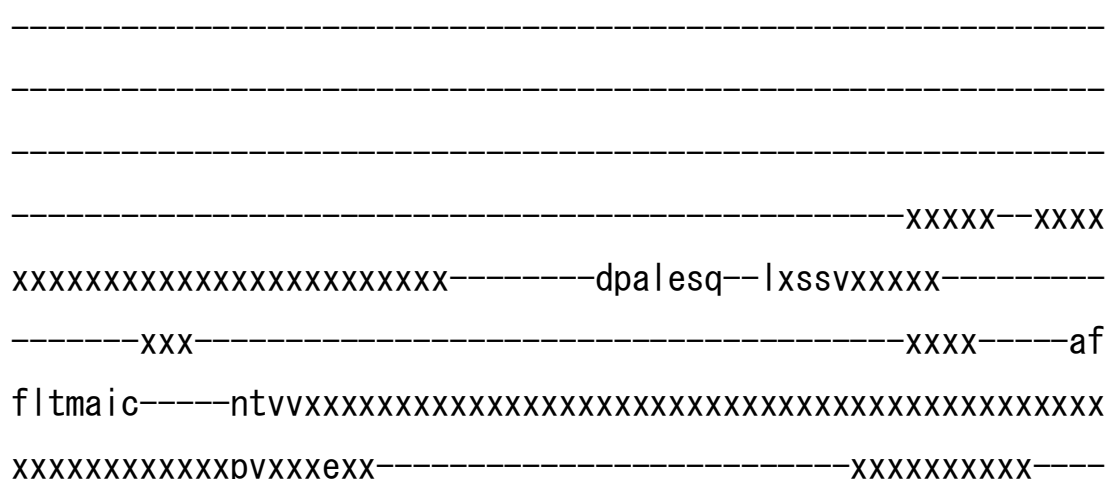




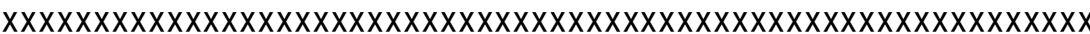
xxxxxxxxx---------------------Xxydsespde-- la l vea----ar-e----

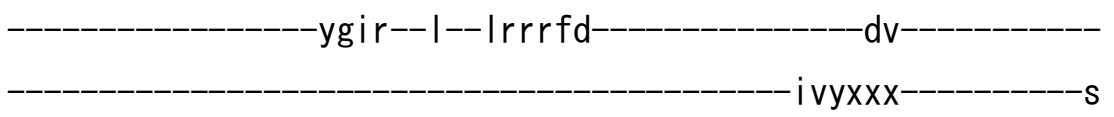

tssv-------kykv lht Ipfdadrk-rmsv----i---ir

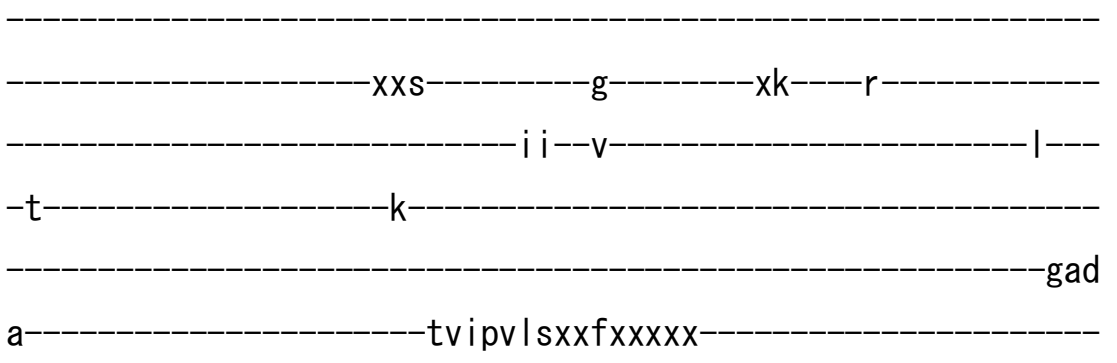

xxx--xx--fks-qeh I tqy--ake----g I rtLCLSMK i w--teeeyqgwkekhee---xe I dmmdketm I aest I xaeqd I e ILGVTA IEDRLQDGVPECIHSLREag-IRV WVLTGDKIETAVNIAYSSRLfspsmd I I----

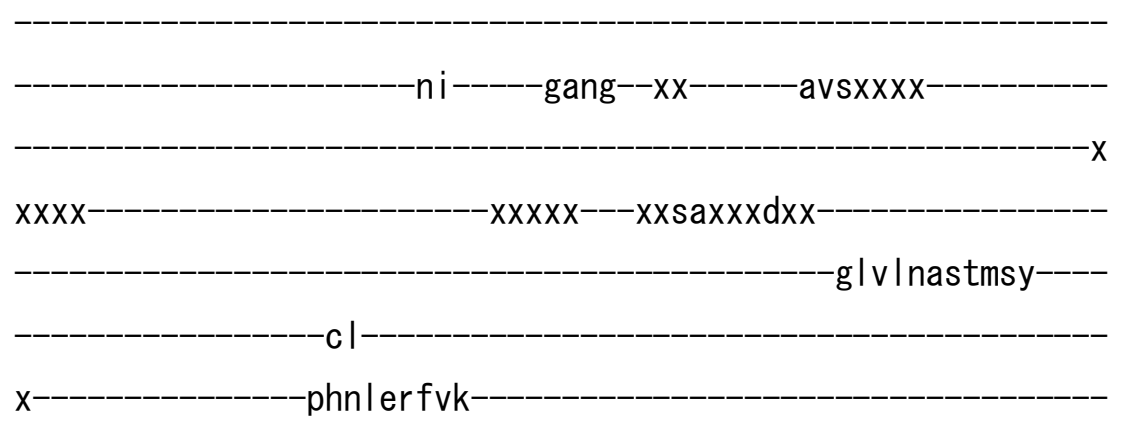

I rgcrsvLCC--RATPLQKAQLVNLAk-

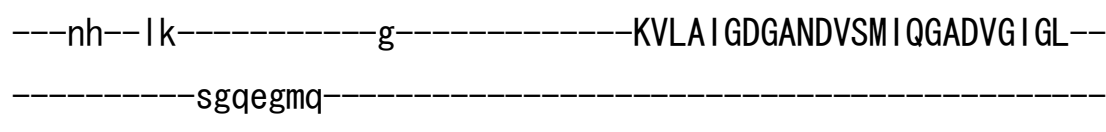


yff-yknam-Ivfvifwyqifngf-saqvp i dpvy Imvy

nl iftsvpp I Ifgc Idqdasae

- I I depr lyeqgr I gkryrwys

f-winm I davwqs I vvf-fi cy-fty

$x \times x n$

vd--mwtfgh I Ivtql i i vntfh-la I fvqywtwpmfw-

$-s m f|s v| l f f i c a l \mid$

yngfvtan $x x x x x x x x x x x$ -mvslksf-

-ss lefw----ma--l i i svvlc|tpr

$-y v \mid t t v v$ ntvs $-p-$ stt

xxxxxxxxxxfkkktesrxxxxxxxxxxxxxxxx

\section{$\operatorname{XXXXXXXXXXXXXXXX}$}

$x x x-$

>dreIV_IV10 XP_700358. 2

$\mathrm{m}$ 


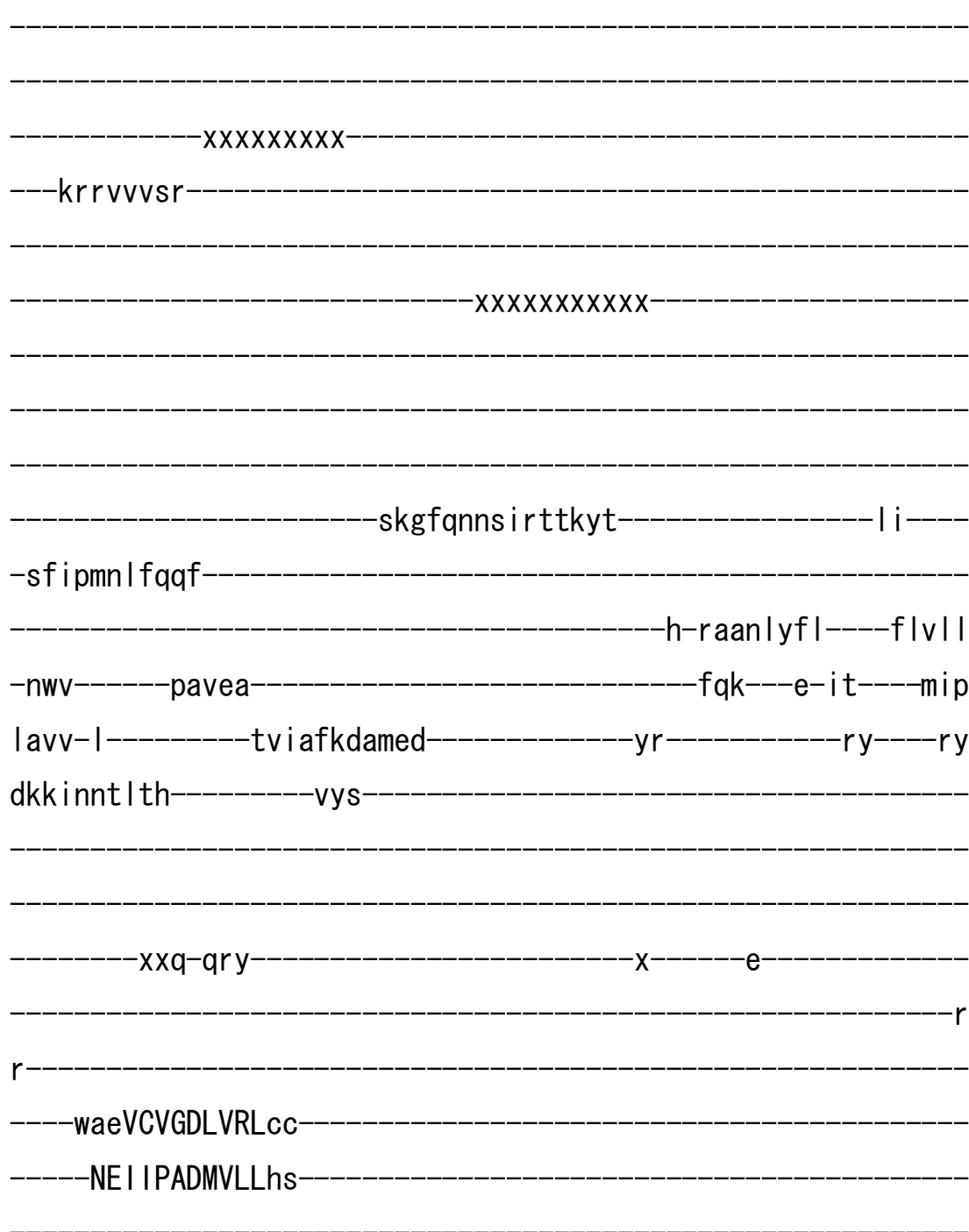

-sdsng-v---chIETANLDGETNLKQRqv-

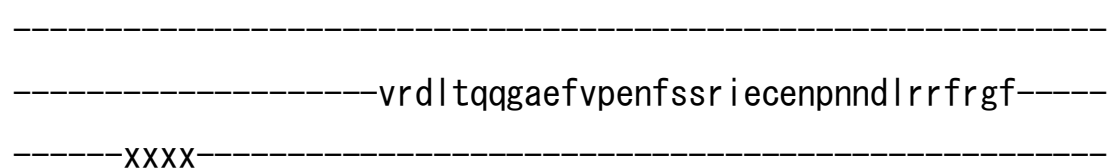

$-X X X X$

-gkvrvg

Ihs

-e----_------NLLLR--SCTV-

nt-----etvi-----------------------g I VVYAGHETKAMqnnsgpry

-krsklerr InIdv Iwsval I I Imcfi iaa

-ighg I I I se ledp i---_---fm i p--_-_-_-_-_---xxxhpa l aafymfwtm I IVLQVL

IPISLYVSiEIVKLGQIY-FI qnd IdI- 
-ynpvldtg

-vQCR-ALN I TEDLGQIQYLFSDKTG-

TLTENRMLFr-

-rctvagtey-

$-x x x x x x x x x x x x-----x x$

$x X X X X-X X X X X X X X X X X X X X X X X X X$

$-x x x x x--x x x x$

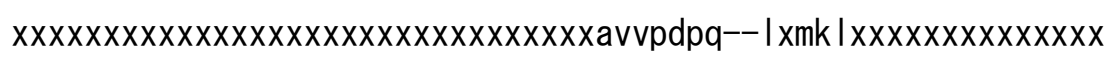

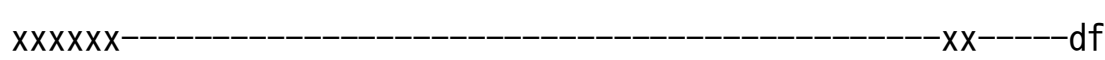

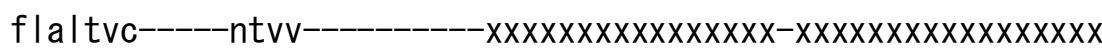

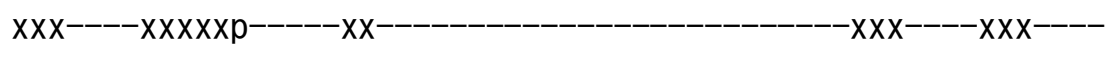

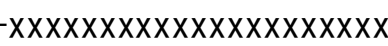

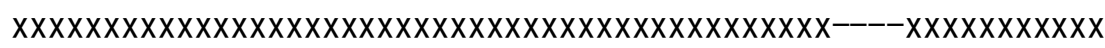

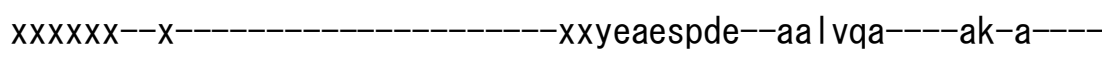

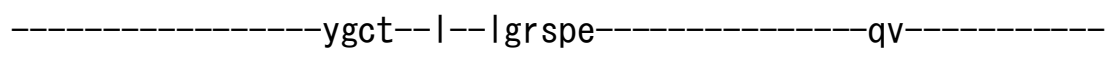

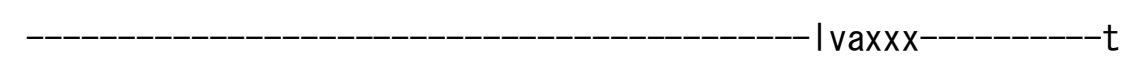

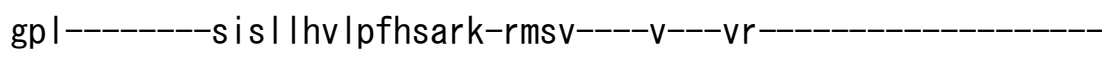
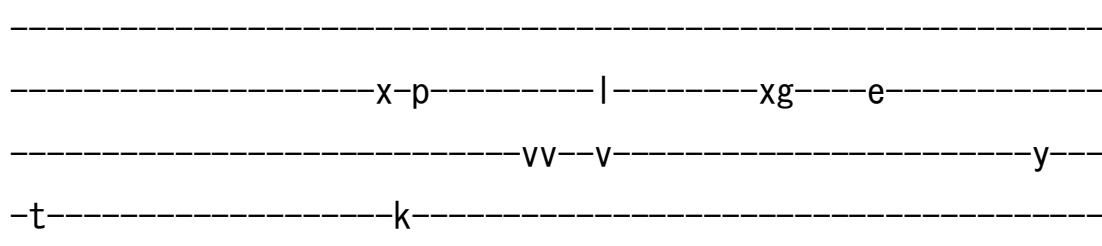

-gad

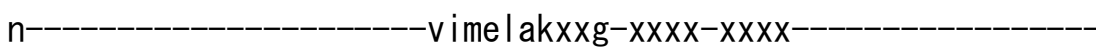

xxx--xx--elt-qrh|dqy--are----g|rtLCVAKKv |--geqeyeawmkrhey--ae-----ts i enree I I lesaer-letd I tILGATGIVDRLQEEVPETIEALQEag-IKV WVLTGDKQETAIN I AFACKL-_-_-_-_-_-_-_-_-_-_-_-_-_-_-_-_ I rptdh i l--_-

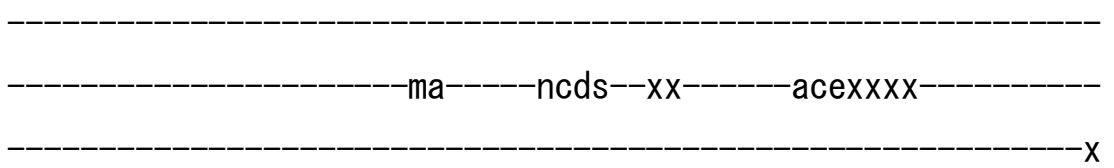

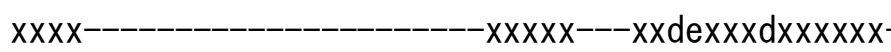
- vidgrt Idy---al

x---------------ke lqgafld- 
ItcccrsvICC--RSTPLQKSQVVRLVr

$---d k--l e-$

-MTLAI GDGANDVSMI QMADVGVGI-sgqegma

-AVMSSDFA I Sr fKFLRKL I --LVHGHWCYARLANM I L yff-yknvvxdvn I I fwyqffcgf-_-_-_-_-_-_-_-_-_-_-_-_-_sgstmtnswv I iff

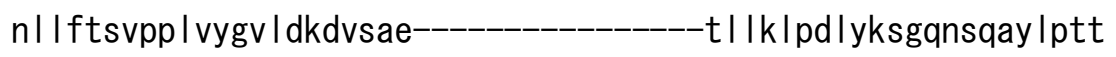

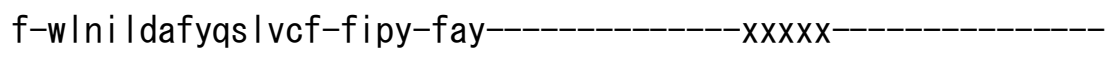
-g--vfsfaspingsall i illh-qviesrt I twihas-------II ifsg I fyyafv I I

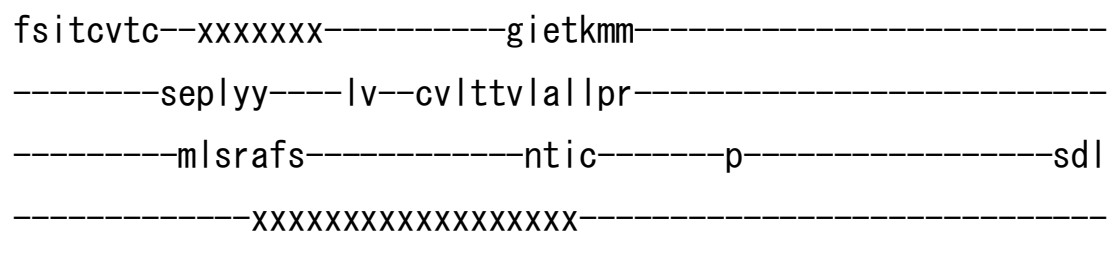

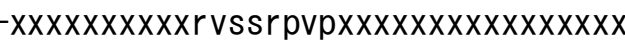
$x \times x \times x \times x$

$-$

>dreIV_IV12 XP_001331862.1

m- 


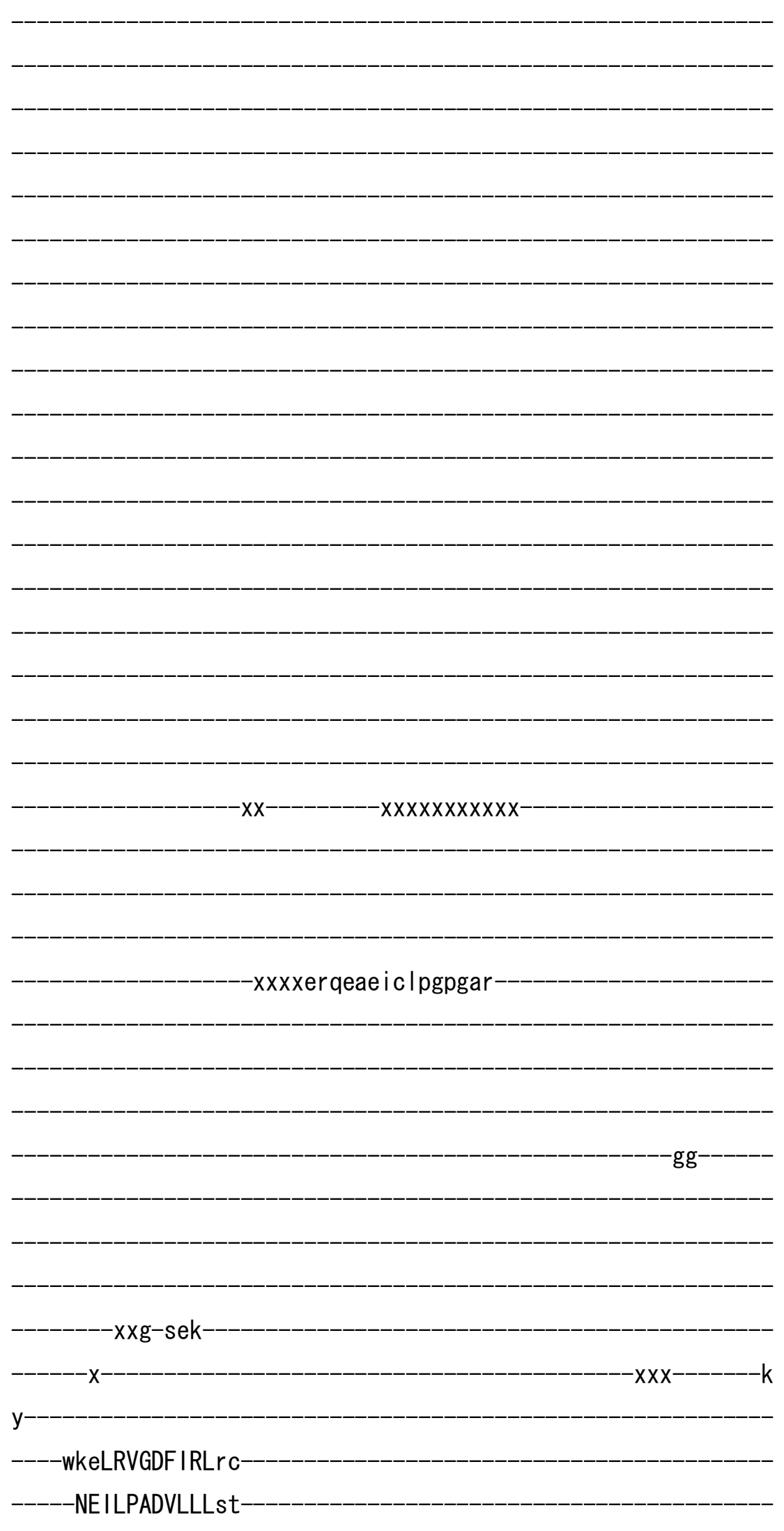


-sdpdr-I---chIETATLDGETNLKQRqv-

-vrsfidldcefdp I kynsvi ecekpnnd Inrfrgy i ihx-

sgrrdg

lyk

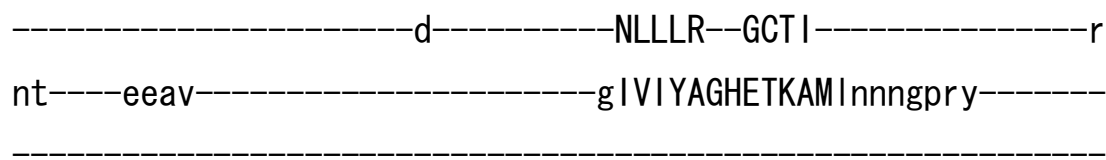

-krsk l erqmnvdvfwcvi i I l vmc Ifsa -i ghg I wmfaygdkr-----xxfdv |--_-_----xxxxxxxspvvsa i y I f I tm I IVFQVL IPISLFVSiEIVKICQVY-FIhqdIe I

-ydeetdsh

I QCR-ALN I TEDLGQMQY IFSDKTG

TLTENKMVFr

-rctvagvey

$x x x x x x x x x x x x x x x x x x x$

$x \times x x x------x x x x x x x x x x x x x x x$

$-x X X X X--X X X X$

$x x x x x x x x x x x x x x x x x x x x x x x x x-x x x x x x x x d i$ tpdpq-- $x d k v x x x x x x x x x x x x x x x$

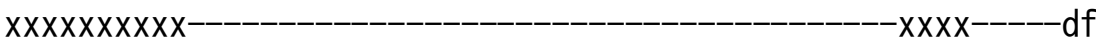

fialt i c-----ntvv---------- $x x x x x x x x x x x x-x x x x x x x x x x x x x x x x x x x x x$

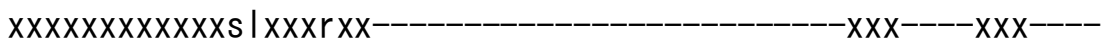
$-x x x x x x$

$---D X X X X X X X X X X X X X X X X X X X X X X X X X X X X X X X X X X X X X X X X X$

-xxfeaespde--aal vya----ar-a----

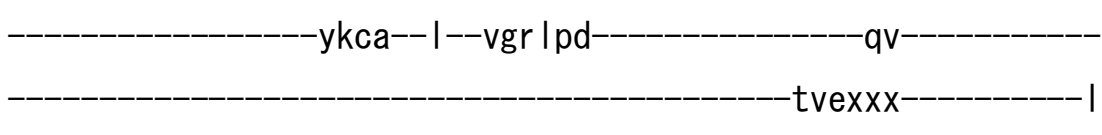

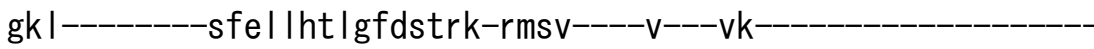

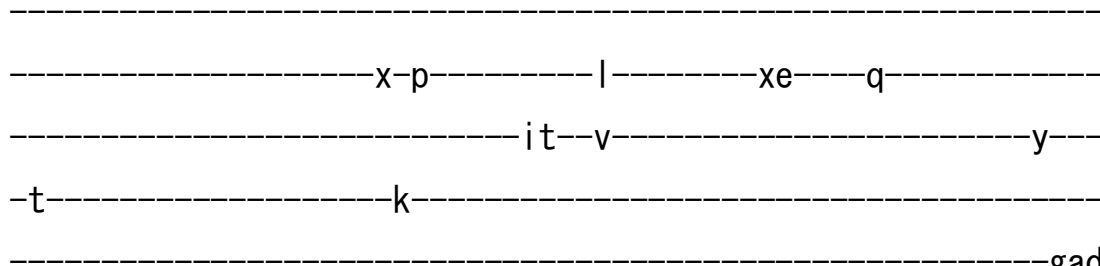

-gad s - vimdl irxxt--- xxxxxxx- 
xxx--xx--ykt-qny Inly--aad-----g|rtLCIAKKv |--skeeyasw larhle--ae-----ta iqr ree I I fesa | r- letn I q ILGATG IEDRLQDGVPET I ASLRKag-LQ I WVLTGDKQETAINIAYACKLIdpeee i i----

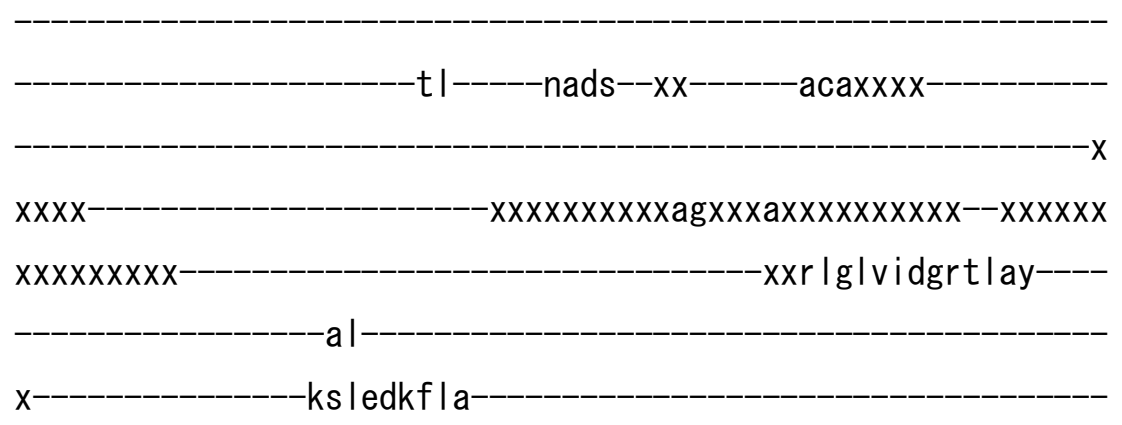

varscrsvLCC--RSTPLQKSMVVKLVr

---nk-- lk------------v----_--_----MTLA I GDGANDVSM I QVADVGVGI--

AVMASDFALPr fRYLQKLL--LVHGHWCYSRLANMI------L

yff-yknam-fval i fwyqfycgf--_-_-_-_-_-_-_--_--_---sgsamvdqwy I iff

nImfsafpq I itgt I dkdvsae--_-_-_-_-_-_-_--t I qe I pq l ymngqnseeykpym

f-wmnmi dafyqs I icf-fipy-fay-_-_-_-_-_-_-_xxxx--_-_-_-_-_-_-_

-d--Iftwgtpittlalftil Ih-Ig i etktwtwmnwv--------sigfsmal fftvalc ynascptc---xxxxxxx-----------wtmqr II----------------------------_------gdpl fy-----||--cvvtpvaal | pr-_-_-_-_-_-_-_-_-_-_-_-_-_-_

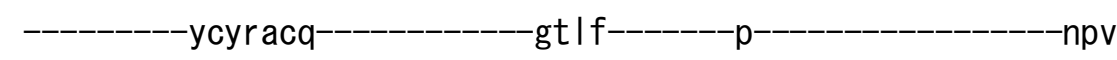

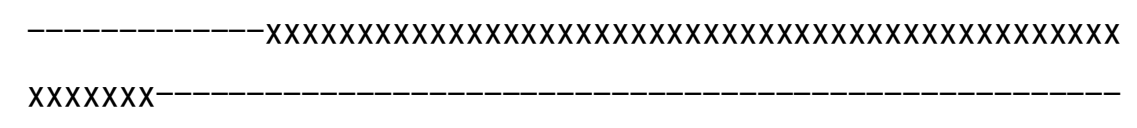
xxxxxxxxxxeks laspi $x x x x x x x x x x x x x x x x$ $\operatorname{xx} x \operatorname{xx} x \operatorname{xx} x x x x x x x x$ 


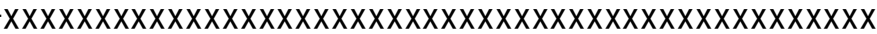

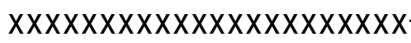

$-X X X X X X X X X X X X X X X X X X X X X X X X X X X X X X X X X X X X X X X X X X X X X X$

$\mathrm{X}$

>drelV_IV13 NP_001038619.1

$\mathrm{m}$

XXXXXXXXXXXXXXXXXXXxprarvsqxxxxxxxxxx----

$-x x x x x x x x x x x x x x x x x x x x x x$

$\mathrm{xxX}$

---artvw Igh

xxxxeekypknaiknqkyn iv----

-tfvpgvlyqqf 


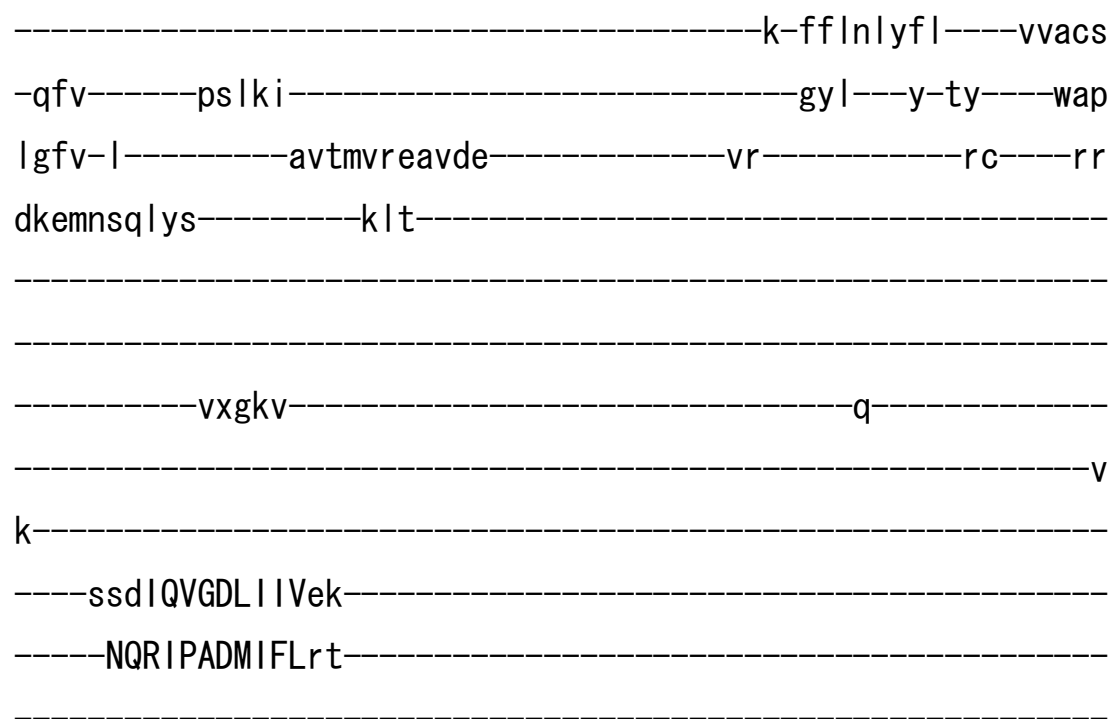

-sektg-s---cfIRTDQLDGETDWKLRig-

-vactar Ipalgd I fs i sayvyvqkpql dihsfegnftrxx

\section{XXXXXXXXXXXXXXXXXXXXXXXX}

-ppihes

Isi

e-NTLWA--STVV

as----gtvigVVIYTGKEMRSVmntsqskn-

-kvg I I le Inr I tka If I aqvv I svvmv

-al qgf I gpwfrnIfrfVVLFSYI

IPISLRVN IDMGKSAYGW-MImkden i

$-p g$

-tVR-TST IPEELGRLVYLLTDKTG

TLTQNEMVFkrlhlgtvsy

$-x X X X X X X X X X X X X$

$-X X$

$-\mathrm{XXX}$

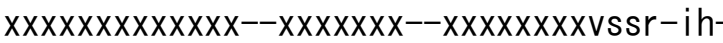

vka ial c-----hnvt

$p v x x x$ 


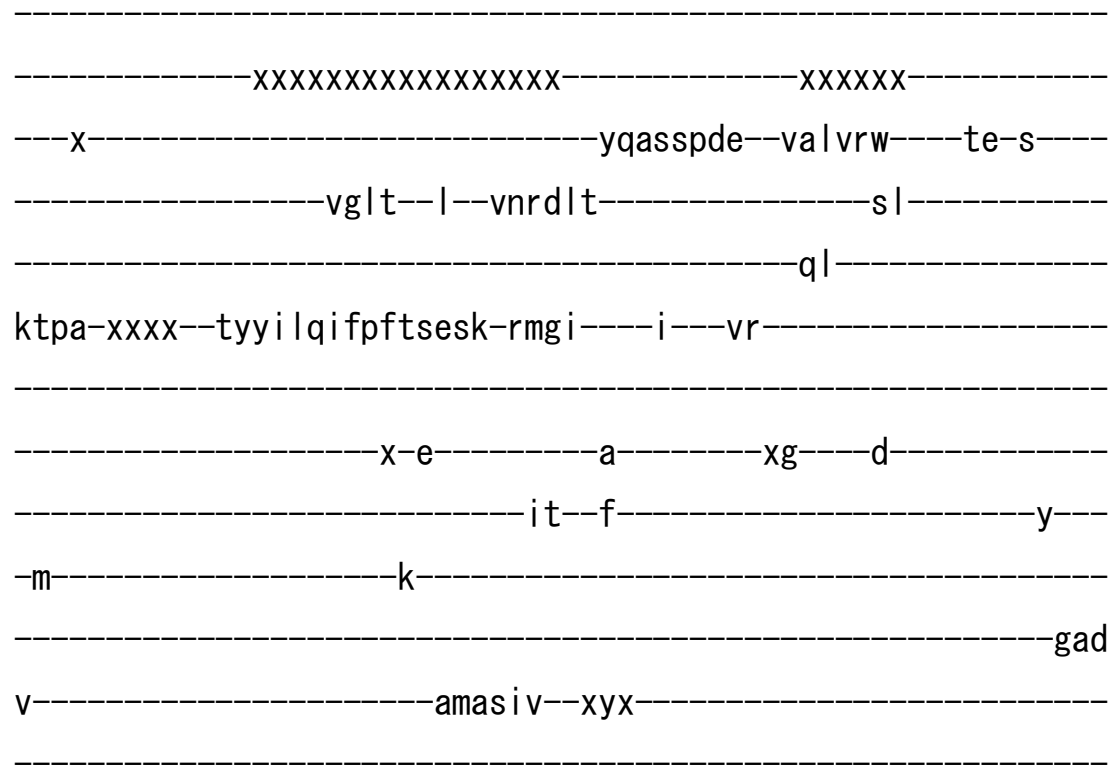

-dw |-eeecgnm--are----g $\mid$ rtLVVAKKs I--teeqyqdfenrynq--ak-----I s ihdrn l kvaavves-lereme ILCLTGVEDQLQADVRPTLELLRNag-IK I WMLTGDKLETATCIAKSSHL-vsrnqdih----

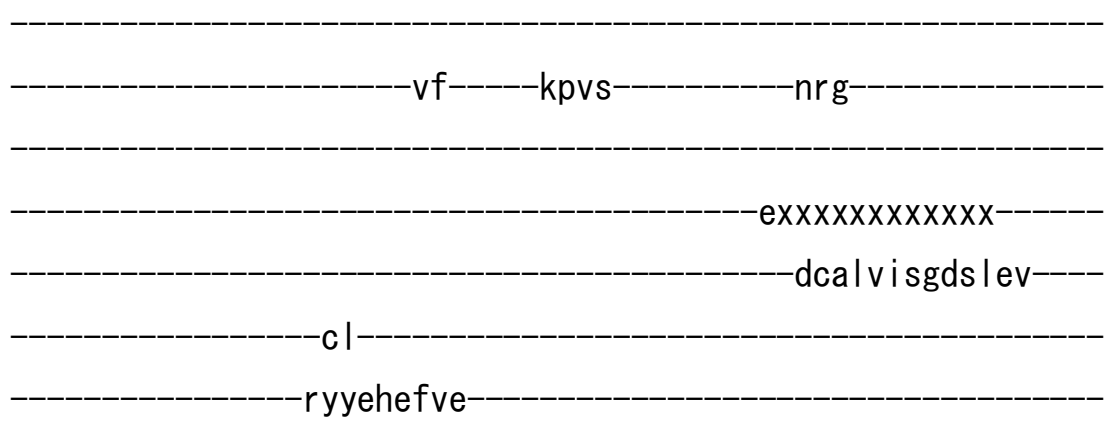

-ryyehefve

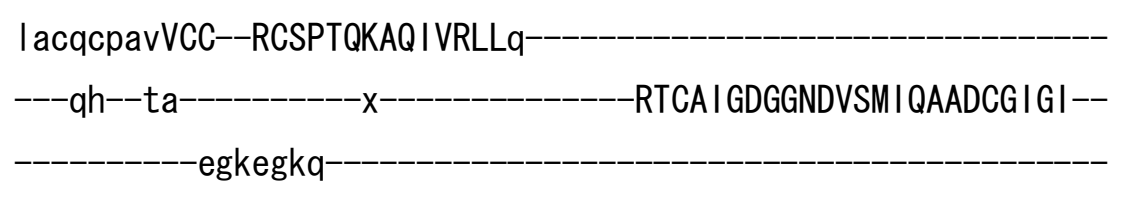

-ASLAADFSI TqfKHI GRLL--MVHGRNSYKRSAALG------Q 
fvm-hrgmi-istmqavfss i fyfasvp I yqgf I mvgy atiytmfpvfsI-vIdqdvkpe -mal lype lykdItkgrs Isfkt

f-I iwv I i s i yggg i Im-ygal-v|fd-

qe--fvhvvaisftaliltel Im-valtirtwhw Imvv aql is lacylaslaf Iney-fdlsfi ttrvfl-----wk--vcvit|vsc|p|$-y$ i ikylk rkfs $-p-$ -psy skl---ss

>cne IV_IV01 XP_566703. 1

m

$-x x x x x x x x x x x x$

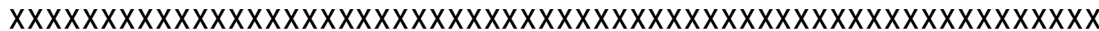
X 


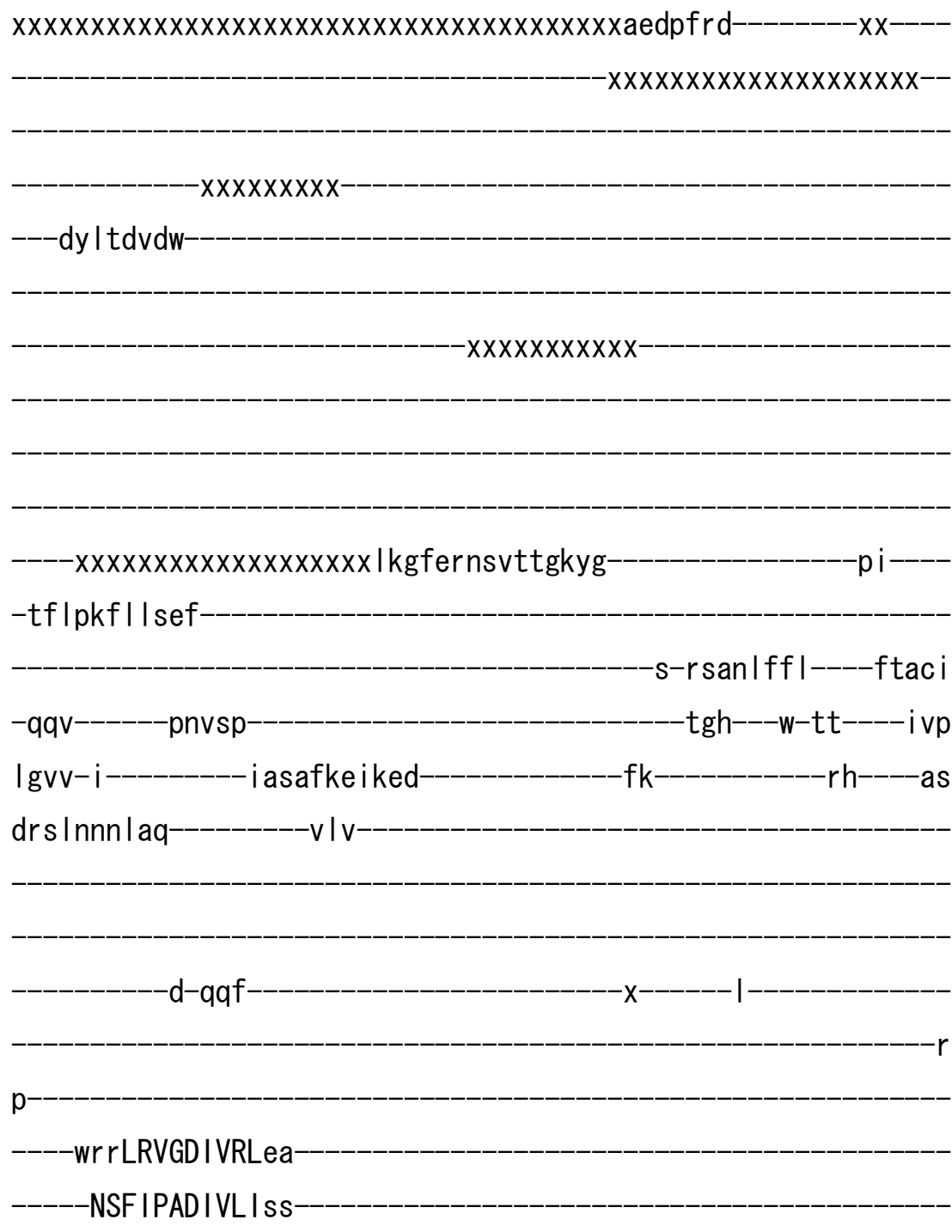

-sepeg-I---cyVETANLDGETNLKIKqa-

-hpstas Itnphsvs I I rghi I seppnss I ytydgtfh I xx $x x x x x$

-aptkip

vgp-

nt----gwvy---_-_-_-_-_-_-_-_-_---'gVIVNAGHETKLMrnateapv-------

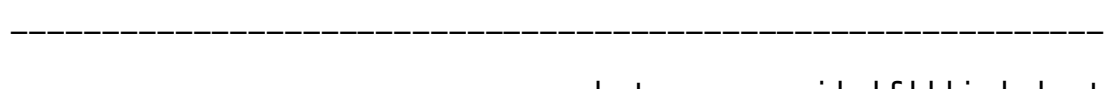

-igss i rtw I fdkna------wy I r----_-----xxxxxxnkarqfi i ed i I tf I ILYNNL

IPISL IMTmEVVKFQQAS-L Insd I dm- 
$-y y a p t d t p$

aLCR-TSSLVEELGQIAYIFSDKTG

TLTRNEMEFr

ect i fgtmy

$-x x x x x x x x x x x x x$

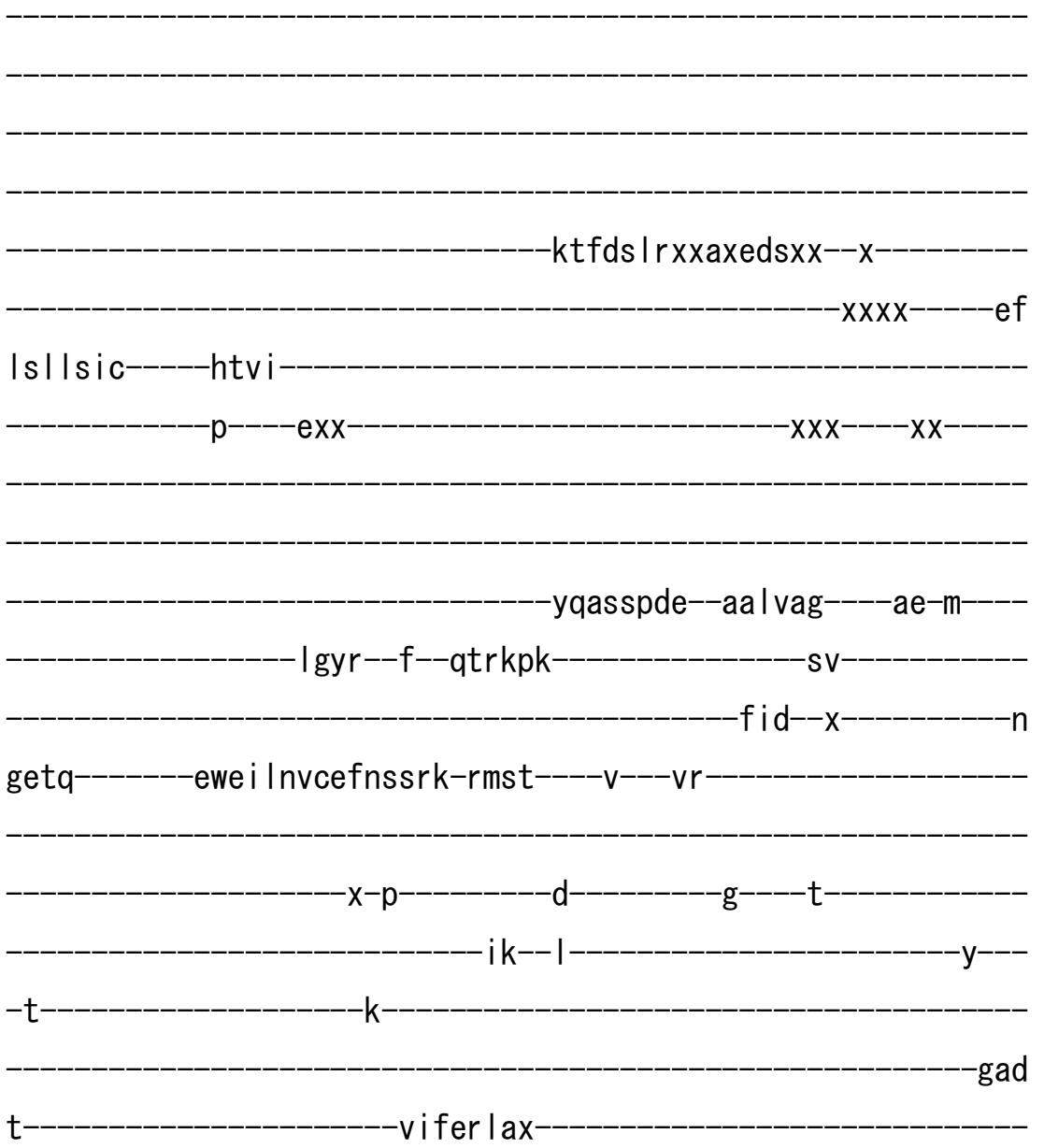

xxx--xx--ept-I vhl edy--ate----g | r tLCLAYRd i--seeeysswsa I ynn--aa-----sqmsgraea I dkaaev-i eqn I q I LGATAVEDKLQDGVPDA I HTLQQag-IK I WVLTGDRQETAIN I GLSCRL--_-_-_-_-_-_-_-_-_-_-_-_-_-_-_ i sesmn I v-_-_-
- 
I a imckav ICC--RVSPLQKALVVKLVk

---rs----t-----------xa------_-------PLLA I GDGANDVSM I QAAHVGVGI--

-AARSADI A I SqfRFLRKLL--LVHGSWSYQRLTKL I------L

ysf-yknit-fal t I fwyswfndy-_-_-_-_-_-_-_-_-_-_-_-_-_sgq i afegwsmsyy nvvfti I pp I vi g i fdqfvsar-_-_-_-_-_-_-_--m I drypq lyh I gqqnyfftp i $r$

f-fywvgnafyhsvIIfxfsvl-vfy-_-_-_-_-_-_-_-_-_xxxxxxxxxn--_-_-_-

sg--|wvwgtt | y lav | |tv |gk-aal i sdvwtkyt la-------aipgsfiftmialp |

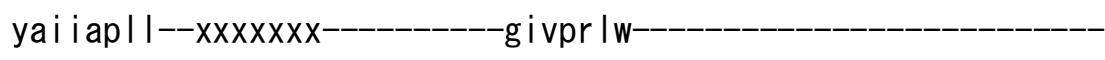

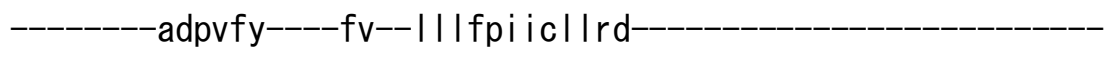

---------yvwkyyr-------------rtyh--------p-----------------asy

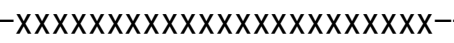

-xxxxxxxxxxmrrqrgfaxxxxxxxxxxxxxxxx

$\operatorname{xx} x \operatorname{xx} x \operatorname{xx} x \operatorname{xxy}$

$-$

>cneIV_IV02 XP_570157.1

m- 


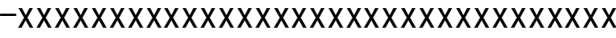

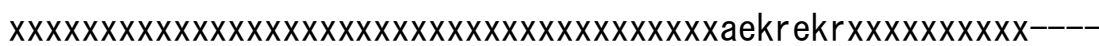

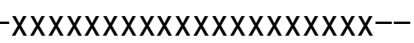

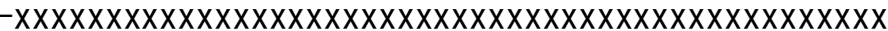
xxxrtvyvni

-tfipknl leqf

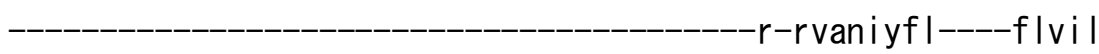

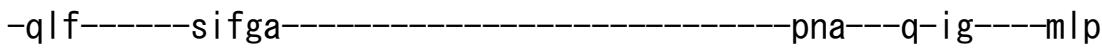

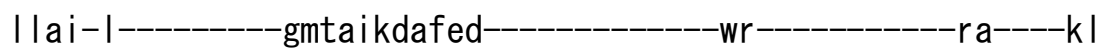
dnevnnsatt----------k /g

$x x x x x x x x x x x x x x x x x x x x x x x x x x x x x x x x x$

xxxxxxxxxxqxnqm------------------------ $x x x x x x x e^{-------} x x x x x x x$

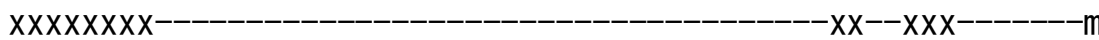

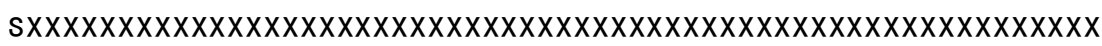
$x \times x \times w k k L E V G D F V L L r d$ 
-snada- |---cfVETKNLDGETNLKIRrs-

Ikatsaitseed I ehahfvvdseaphan I ysyngv I ryxx

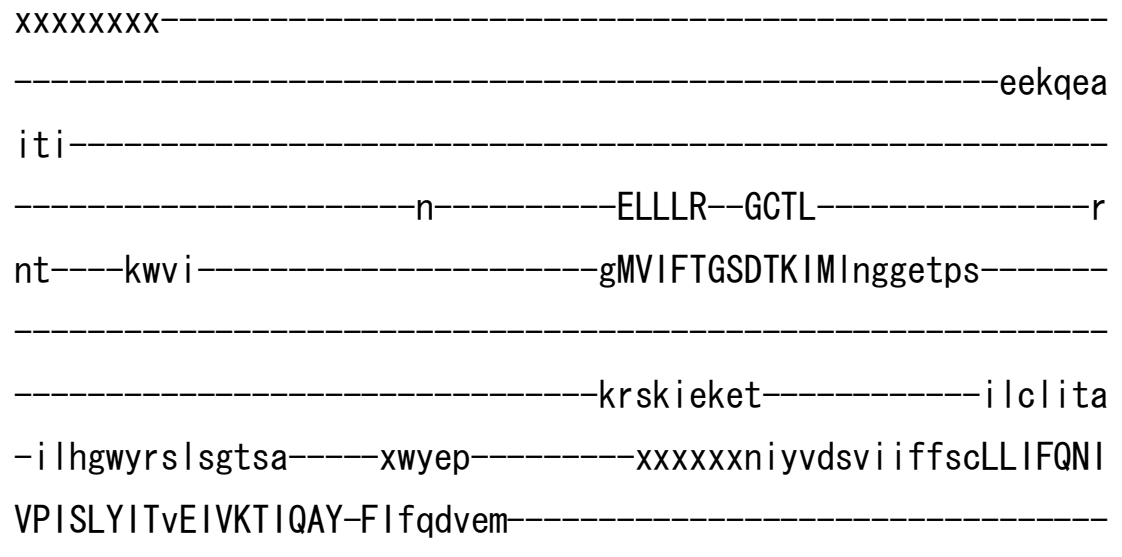

-yyepydtp-

cVPK-TWDI SDDLGQIEYIFSDKTG-

-TLTQN I MEFk---------------kcs ihgvpf

$X X X X X X X X X X X X X X----$

$-x x x x x--x x x x$

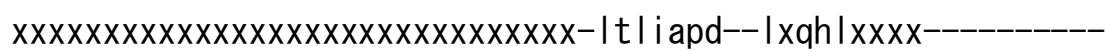

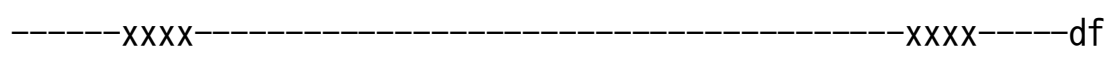

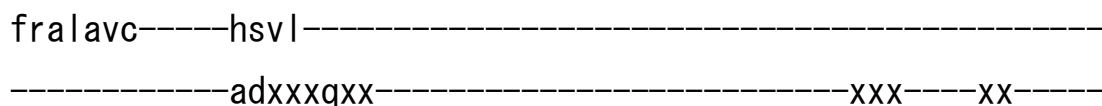

-

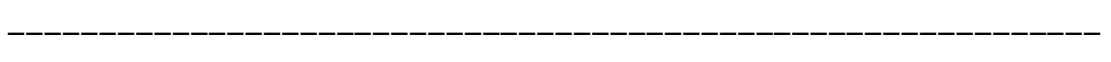

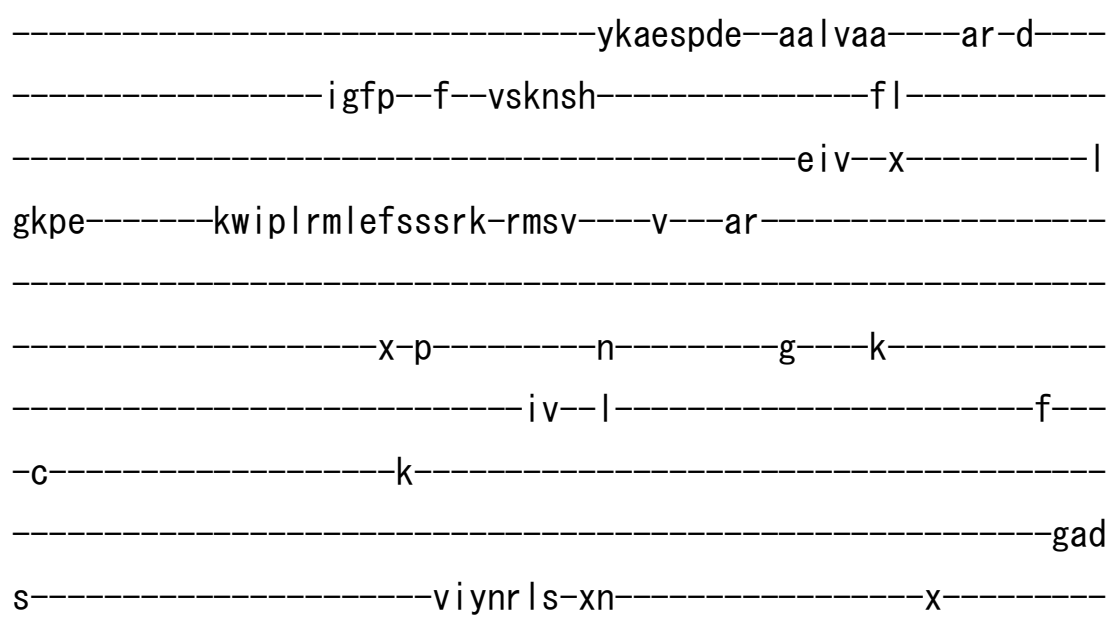


xxx--xx--dat-|kd letf--ang----g | rtLCIAYRn I--seeefsdwskkyda--as------aatvdrege i ekacd I-vehs I t i LGATALEDKLQEGVPDA I ATLHRag-IKL WILTGDKLQTAIE IGYSCNLIndmev

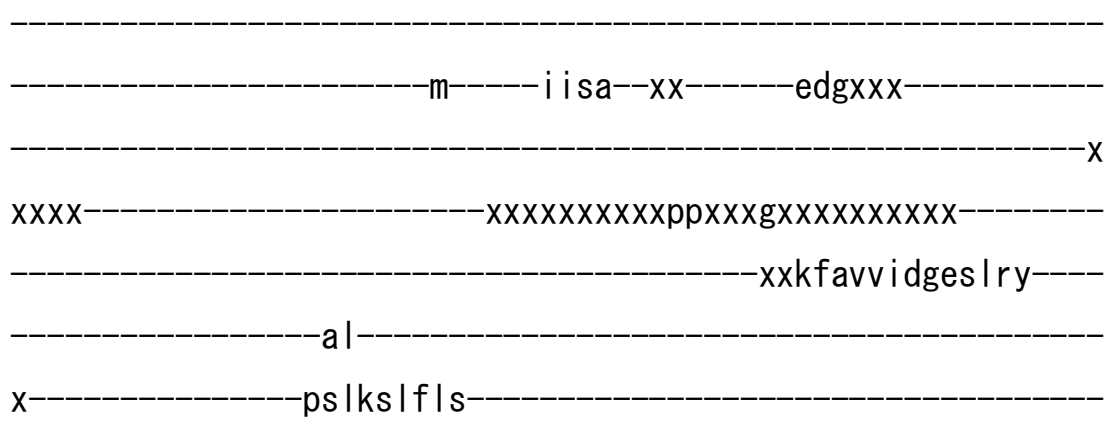

I gtqcaav ICC--RVSPSQKASTVRLVk

---eg--cn-----_------a-----_--_----MTLA I GDGANDVAM I QEAN I GVGL--

AAMSADYAFGqfRFLTRLL--LVHGRWSYVRVADMH------A

nff-ykn i i-ftvsmfwffifssf---_-_-_-_-_-_-_-_-_---daty I feyt I I Imy nIffts I pvgf I gafdqdvnpt--_-_-_-_-_-_-_--_aamvfpq I ykrg i as l eytrtr f-w lymfdg I yqsav if-fi i py-fay-_-_-_-_-_-_-_--xxxxxxxxxxxxt----_--_ ns-- I wd i gttvacagv I sangy-vs in i rywtvmtwi--------invast I I i y i y i pi ysavtalpxxxxxxxxx-

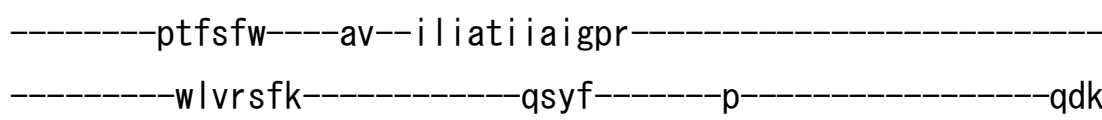
$-x x x x x x x x x x x x x x x x x x x x x x x x x x x x x x x x x x x x x x x x x x x x x x x x$

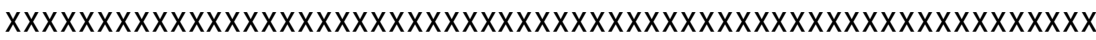

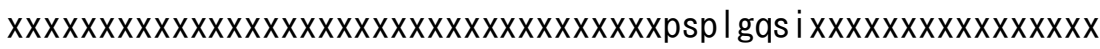
$x x x x x x x x x x x x x x x x$ 


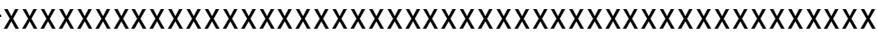

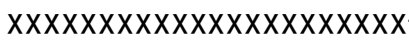

$-x x x x x x x x x x x x x x x x x x x x x x x x x x x x x x x x x x x x x x x x$

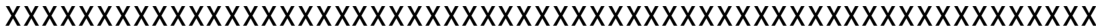

>cneIV_IV03 XP_572575. 1

$\mathrm{m}$

$-X X X X X X X$

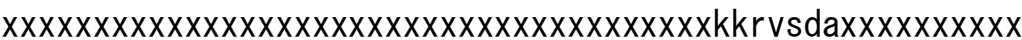

$-x x x x x x x x x x x x x x x x x x x x-$

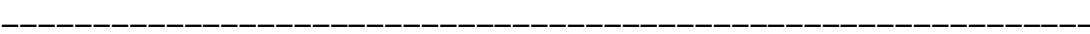

--- grtiplap

xxxkspypanvvrnqkys

$-\mathrm{i}$

-sflplvfyeqf 


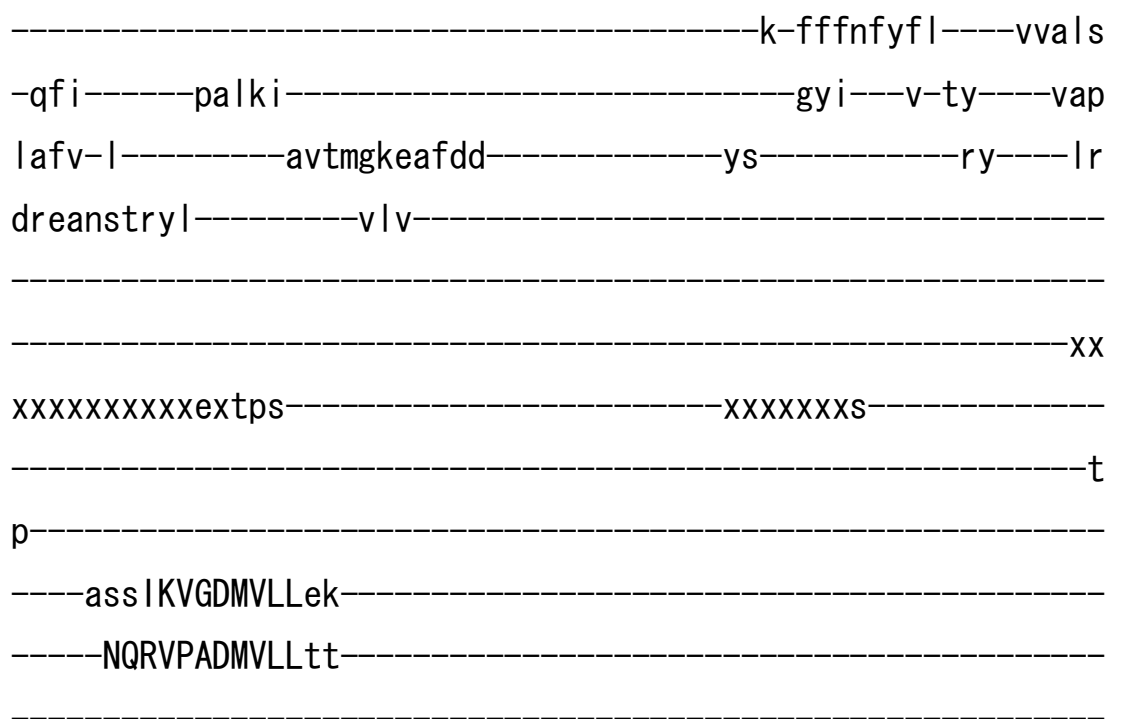

-seeeg-t---cfIRTDQLDGETDWKLKva-

-vgetakmgeafvgsaegs I yadpp ikd ihtfygvft I xx

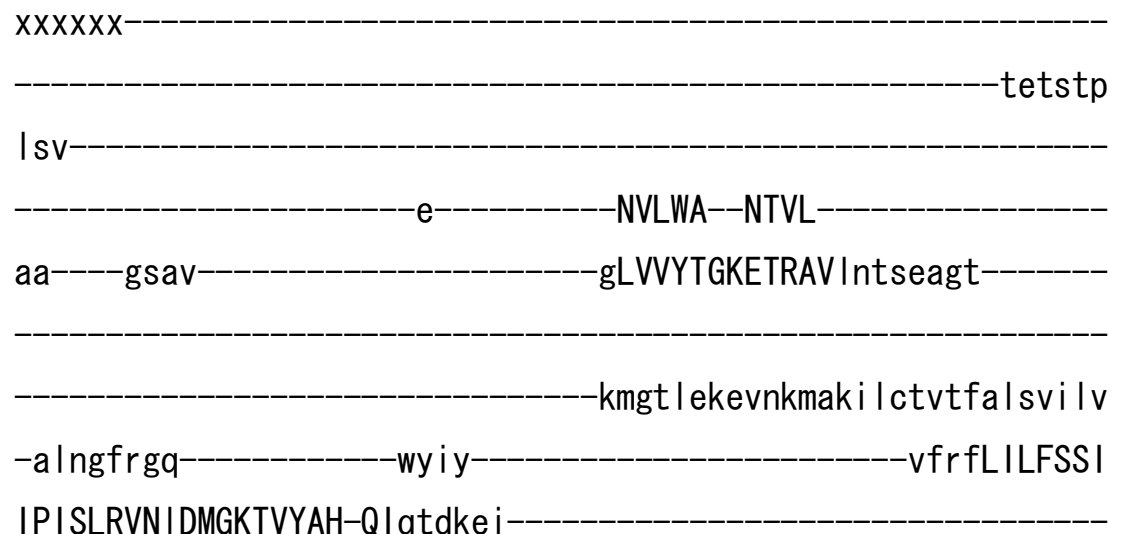

-pe-

tIVR-TSTLPEELGRVEYLLSDKTG

-TLTRNEMELk-klhmgt I vf-

$-X X X X X X X X X X X X X X$

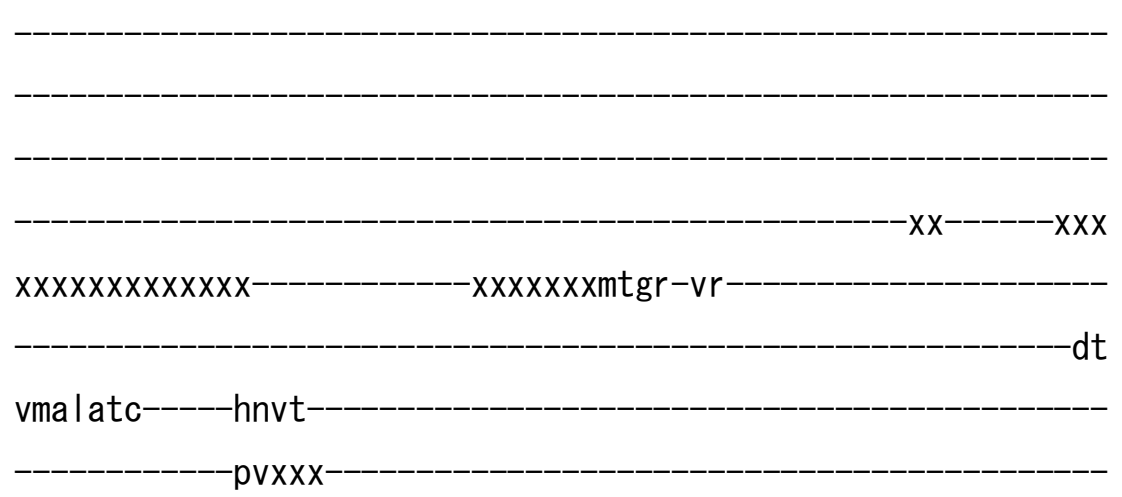




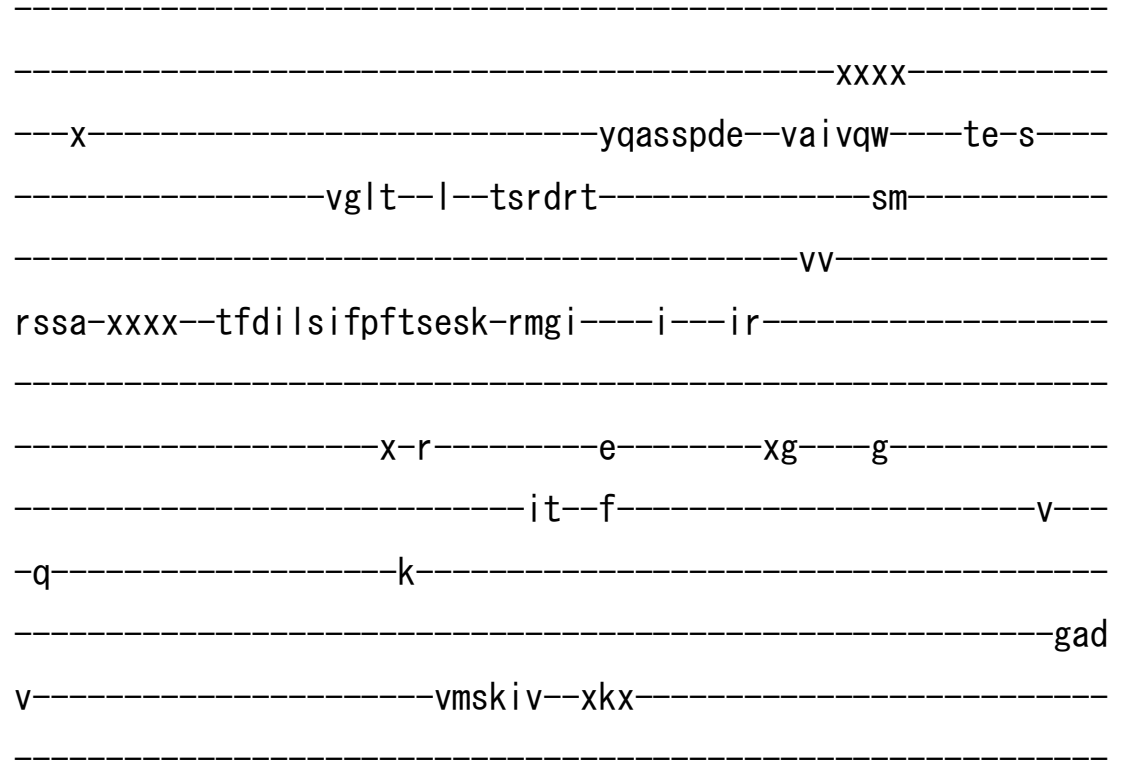

-dw |-eeetgnm--are----g | rtLVLARKk I--seeayaafdkayra--aq----x I psesrass i tsv i sqx I ete l e ILALTGVEDKLQEDVKSTLELLRNag-LK I WMLTGDKIETATNIAVSSKL-vargqyih----

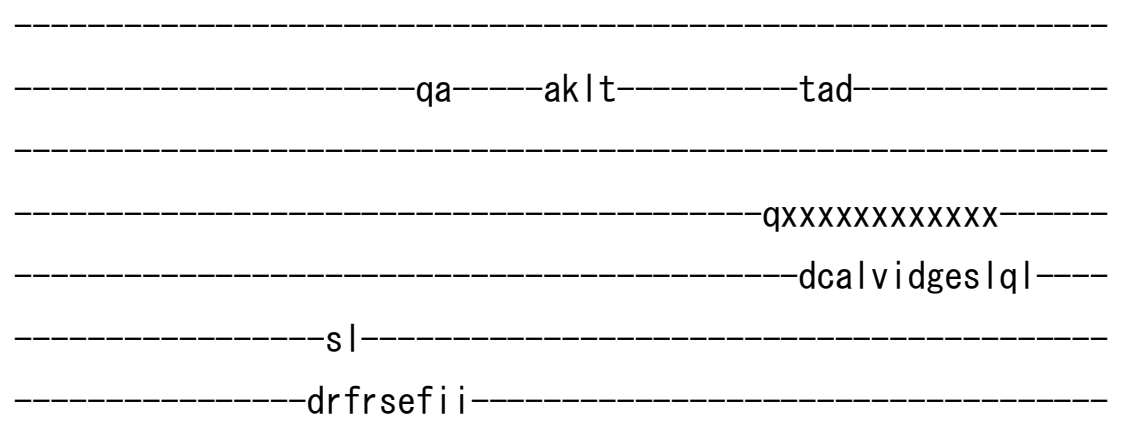

latq I p i vVAC--RCSPTQKADVAKL Ir

---ey--tk-----------x-------_-----TVCC I GDGGNDVSM I QAADVGVGI--

-ASLAADFS INafSYLTKLL--LWHGRNSYKRSAKLS------Q 
fvi-hrgli-iaviqavfssifff-

ap i a l yqgw l qvgy

at IytmapvfsI-vldkdvned

lal lypelykeltkgrs Isykt

f-ftwlti svyqgg i im-I|s|-IIf

e-

se--fl hiva isftalvinel im-valevttwhsymv I

se Igtalvyfgsmav

Ipey-f-

dlafv

- sstfv----yk--vtvivavssfp|

yvikaah-

qr In

$-p$

aay

-kkvag i

>cne IV_IV04 XP_568665. 1

m- 
---prsvfine

$x x x x x x x x x x x-$

-xxxxahhfatnqnvtskyt

-tfvpkn I feqf

$-x X x$

$x x x x x x x x x y d-k t f-$

$-x^{-------p}$

$x x x x x x x x$

$-x x--x x x$

$-k$

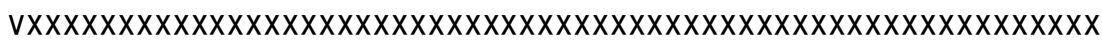
xxxxwedVKVGDFVKI ye

NEQFPAD I I I Cat

-seeed-v---ay IETKNLDGETNLKSRng-

-vpg I sh Intaeacanah I rid Idppesnmfr I ngavmnxx $x x x x x x$

qhpihp

vtl

TTMLR--GCVL----------------k

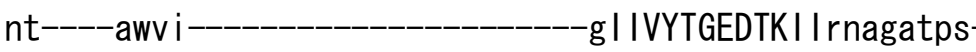

-krsmvekqmnpqvi in I vi laa i avvca

-i vdhvnevgwdeqq----xxwm I y--_-------xxxxxxnpn ing I vtfanaF I TFQN I VPISLYIS IEAVRTIQAA-FIywdrdi 
xxkkdgvttr

-tTAR-SWNLSDDLGQIEYIFSDKTG

TLTQNAMIFr

qcsvggkiy

$X X X X X X X X X X X X X X X X X X X X$

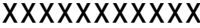

$-x x x x x--x x x x$

$x x x x x x x x x x x x x x x x x x x x x x x x x x x x x x x x$ kd leahd

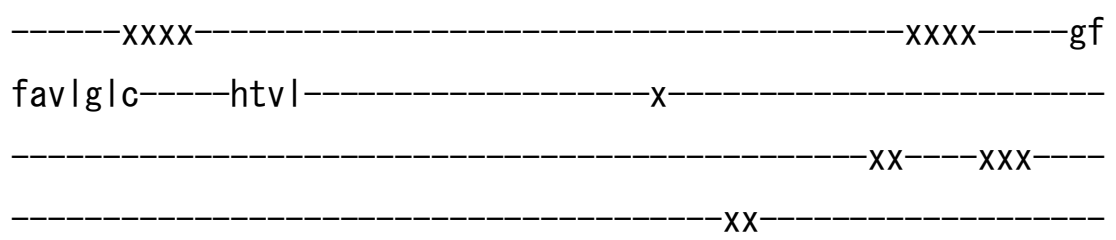

-xxykaqspde--aal vqs----aa-d----

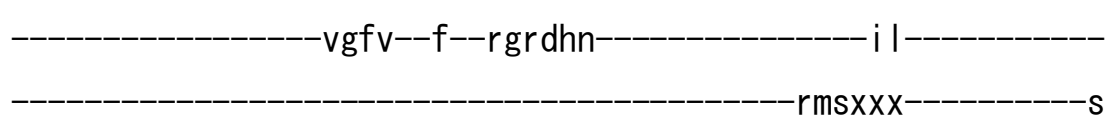

depd-------eyel Ihv lefnsark-rmsv----i----Ir

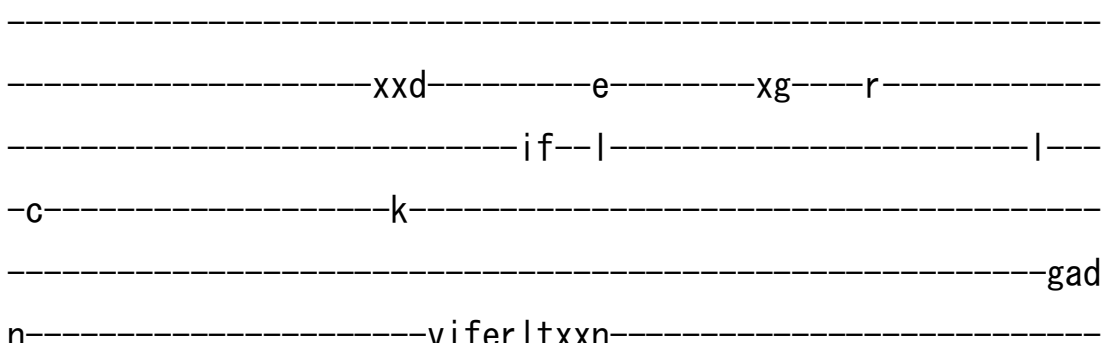

xxx---xxxekt-dqd l qyf--ase----g | r tLCLAYR i I--dpqvyerwakeyhn--at-----val qdreeq i esvsss-i erd I i ILGATA IEDKLQDGVPDT I SDLKRag-IKV

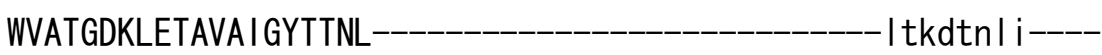

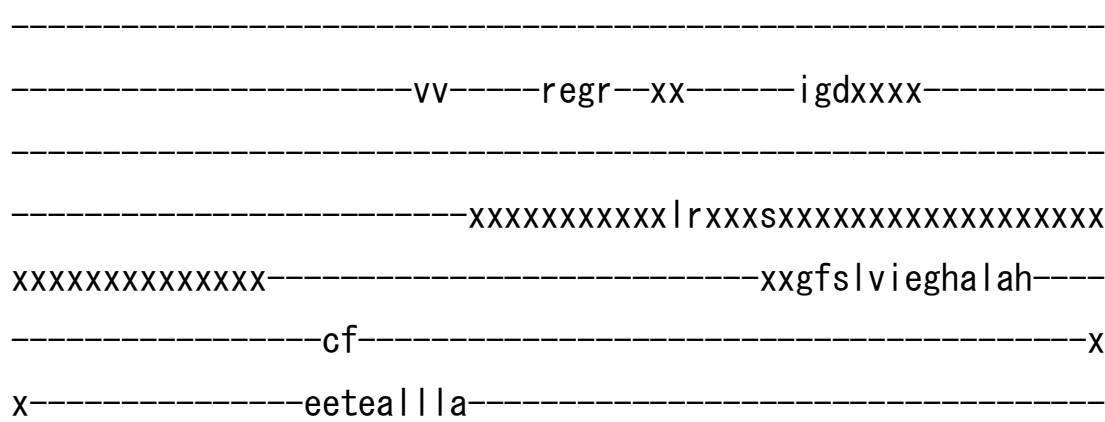


I strcntv ICC--RVSPLQKAQIVHLIk-

$---\mathrm{dn}--\lg$

-MCLAI GDGANDVSMIQAADVGVGI-sgeeg $\mid q$

AVNSSDYAIAqfRYLKRLL--LVHGHWSYFRNSSMI $-\mathrm{L}$ nff-ykn i i-gi gv I fwfmi ycgw-_-_-_-_-_-_-_-_-_-_--sttyvfayvy I I fw nvfwt I vpvi a ig I fdrnidde-_-_-_-_-_-_-_-_-_t Imal pe lyrasregkyfg Imr

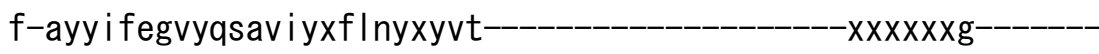

ydxxmyemsttqa i gavmvan If-sg In i dawtgwvwf---_----avwfgpf I iwvftav ysvippssxxxxxx-_-_-_-_-_-_-_-_gndvf If-_-_-_-_-_-_-_-_-_-_-_-_-_-_-_ ---------rsaayw-----fg--wpfvti ial l pr-----------------------------

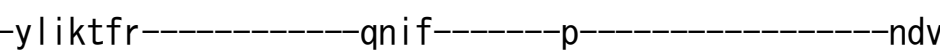

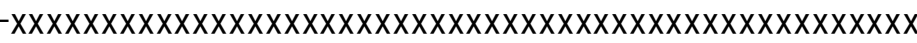

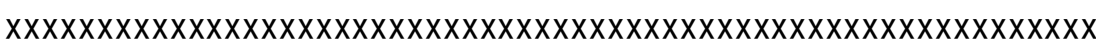

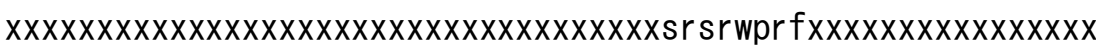
$x x x x x x x x x x x x x x x x$

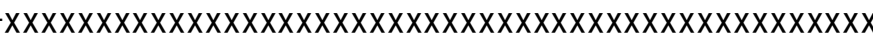

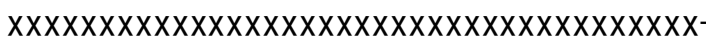
$-$

>ehi IV_IV01 XP_650935. 1

$\mathrm{m}-$ 


\section{-xxxxxxxxxxxxx|rkkpeq}

---prti i ipi

-xxxnkkfcsnkvennryt

$-\mathrm{yl}----$

-ny i pkc I fnqf

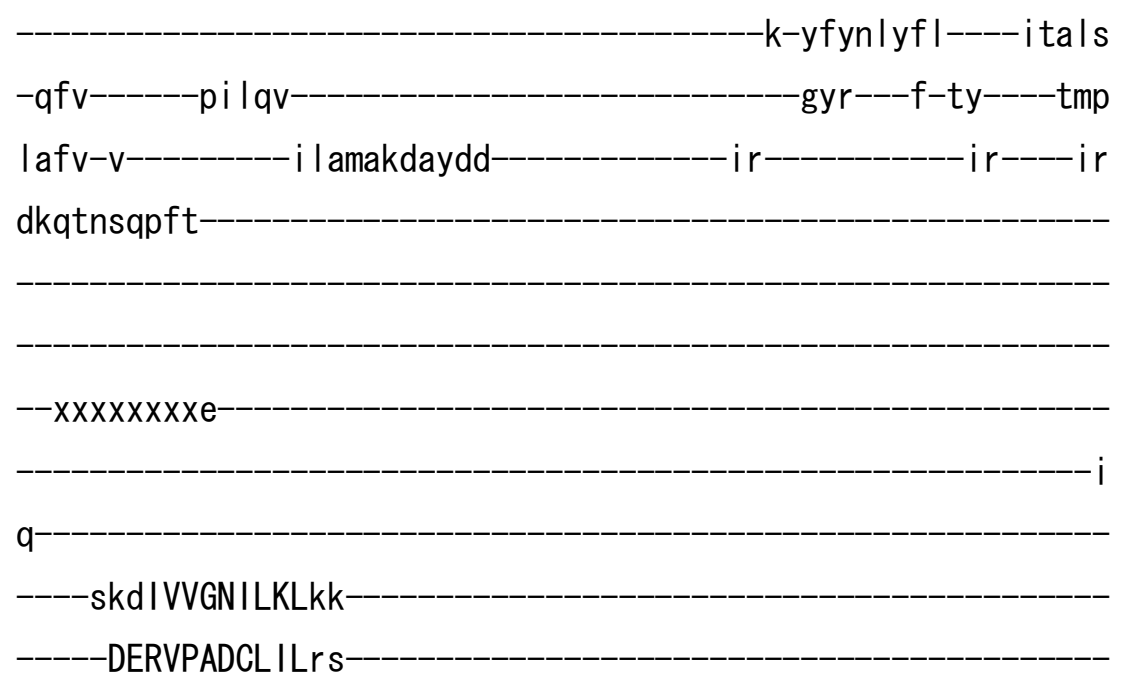


-teesg-s---ifIRTDQLDGETDWKLRra-

-i getakmd iqt i cnsqfe I nveaphadvys fagt i r i xx

dpkiys

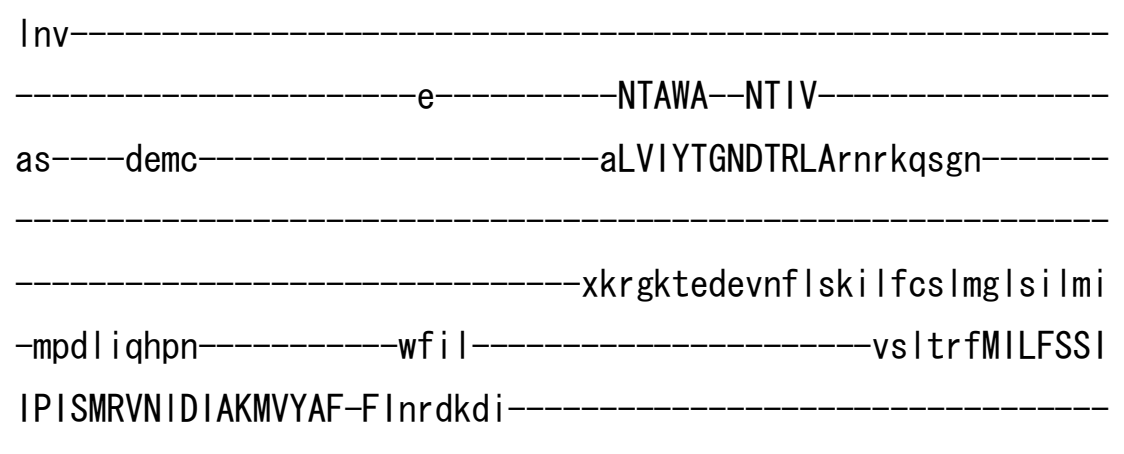

$-a g$

aEVR-NSTLPEELGRVDFVLSDKTG

-TLTKNEMTFq-

$-v$ lcmqset $i$

$-X X X X X X X X X X X X X$

$-x X$

$-x x x x--x x x x x x x x k n p k f i m$

$-r c$

vqamalc-----hnvt

$-p s x \times x$

$-x x x x$

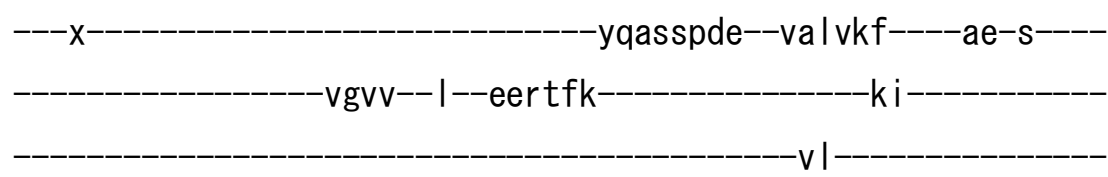

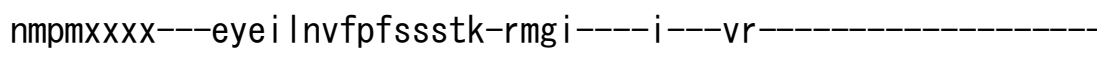

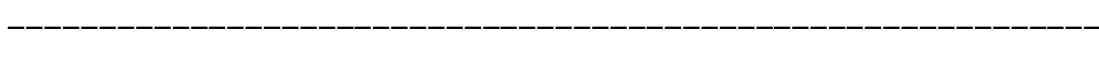

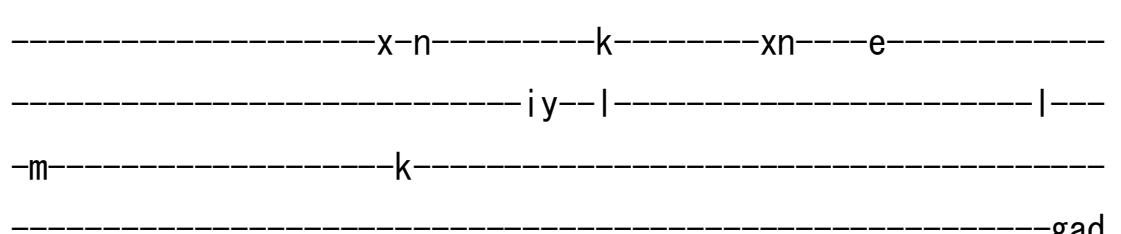

$\mathrm{n}$

- vmski i--xdx 
-ewl-seecnnI--are----g|rtLVFGSRkm--sqeeyqafnerydh--an-----t I mtgreeev I kvqes-i ehg I naMCI TGVEDELQEDVQKTLEMLKQan-VRV WMLTGDKVETATCIAKSTKL-vdidqe i $\mathrm{i}----$

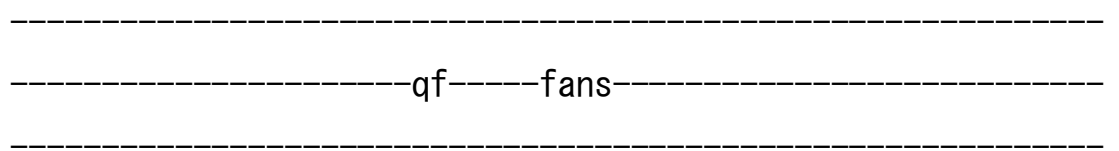

$-x d x x x x x x x x x x x x$

-xxtkal ivdgnt $|s|$ $-m \mid-$ kemskefiq

falkaqavVCC--RCLPSQKADIVRLVk

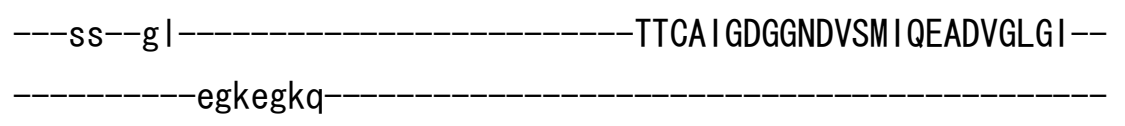

-ASMAADFSIKafSHMLKLL--LWHGRNSYIRTSDLA------L

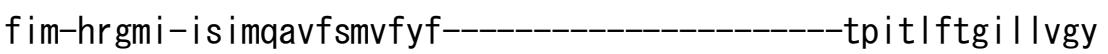
stfytmapvfs I-vlder i s lk--_-_-_-_-_-_-_-_dimqfpv lyaemqkgqn I sfkt f-sawmf i s i lqgsv im-ml am-i I f-_-_-_-_-_-_-_-_-_-_-_-_-_--_d--_-_-_ss--fvhivsisftvlilielln-igftirrwnwi ifa--------seassv Imyl isi iv Irpt-f epsfv$-f s$ lgfw----wr--vavitl insfp-

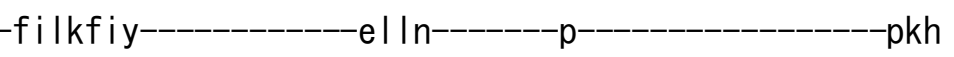


>ehi IV_IV02 XP_655890. 1

$\mathrm{m}$

-xxxxi i ip/vsxxxxxxxxxx

$-x x x x x x x x x x x x x x x x x x x x--$

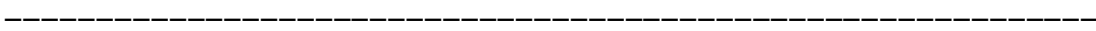

---prsvyrpe

-xxxvqkyttnkvsntrtt

-sf I pms I fnqf 


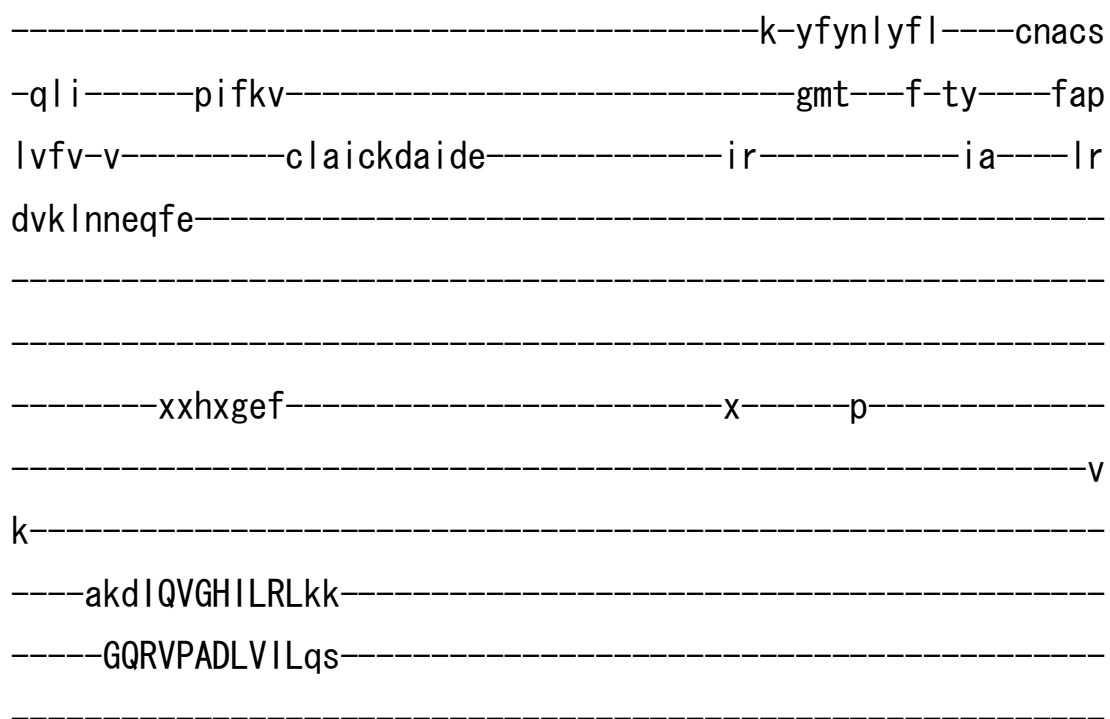

-sdedg----xcf IKTDQLDGETDWKFRrc-

-ikefag I d I s l ent i fc i dceapqna i ysftgr i rk--

endt ip

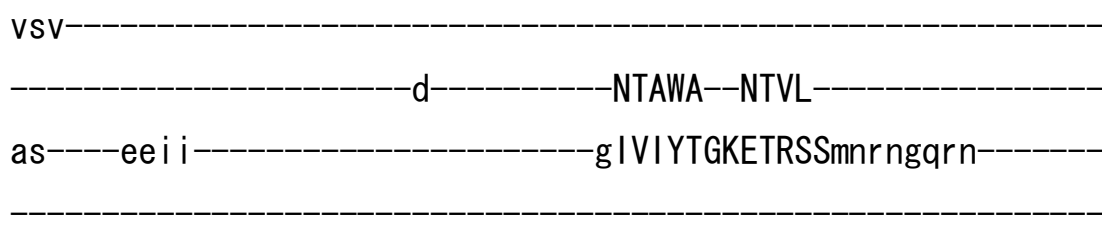

xkwgrvdma Insvskv I fc im I g I i Imv

-vpdlcrgifssfti-vvvrrfMILFSSI

IPISIRVNIDISKLIYSM-FI stdeki

eg-

aEVR-NSS IPEELGQVQFLLSDKTG

TLTKNEMSFk il s I gnqty

$x x x x x x x x x x x x x$

$x x$

$-x x x x--x x x x x x x x s t s k-I f$

ikala lc-----hnvt

$-p s x \times x$ 


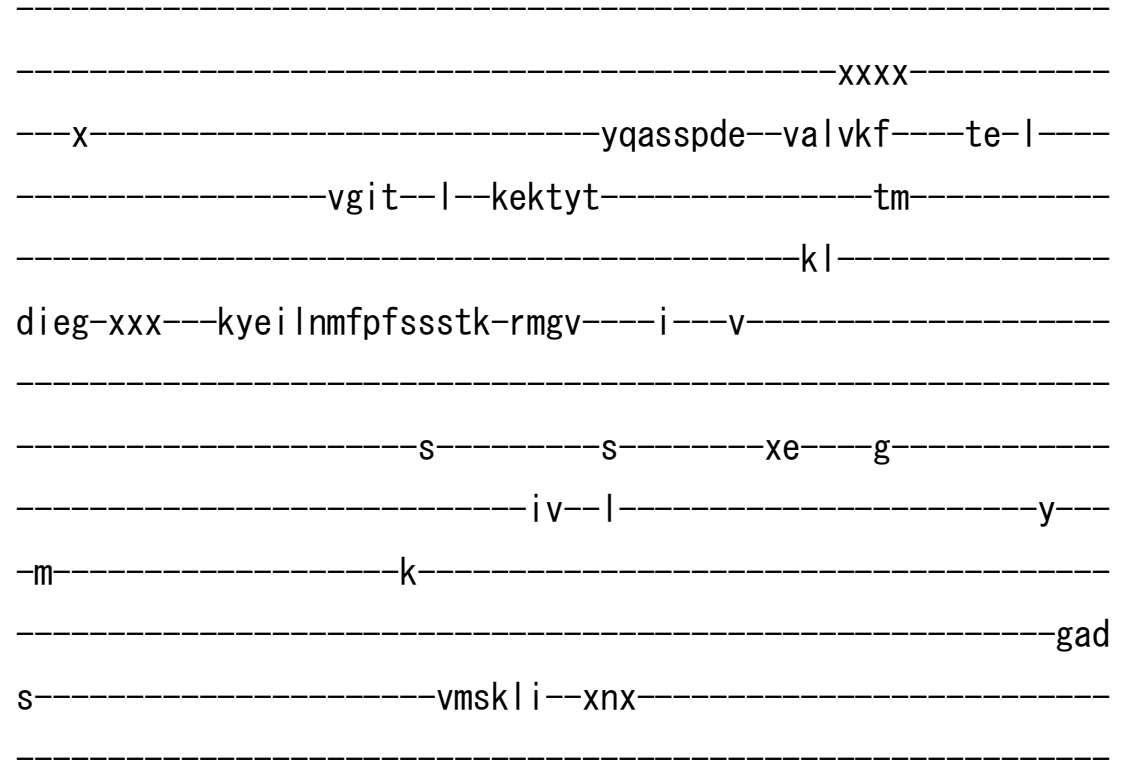

-ew I-geecgnI--akd----g |rtLVFGKKvi--skedye i fkkefna--as-----samtdrde l i tqs i ak-i enn I kvLCI TGVEDELQDDVQQSLEMLRHag-IRT WMLTGDKVETALCIAKSTRLi gvdqev ef------fans $\mathrm{vl}$

-keipdkfir

falqaksv ICC--RCMPTQKAE IVLLVk

---ks--gi-RTCAI GDGGNDVSMI QAADVGLGI-egkegkq 


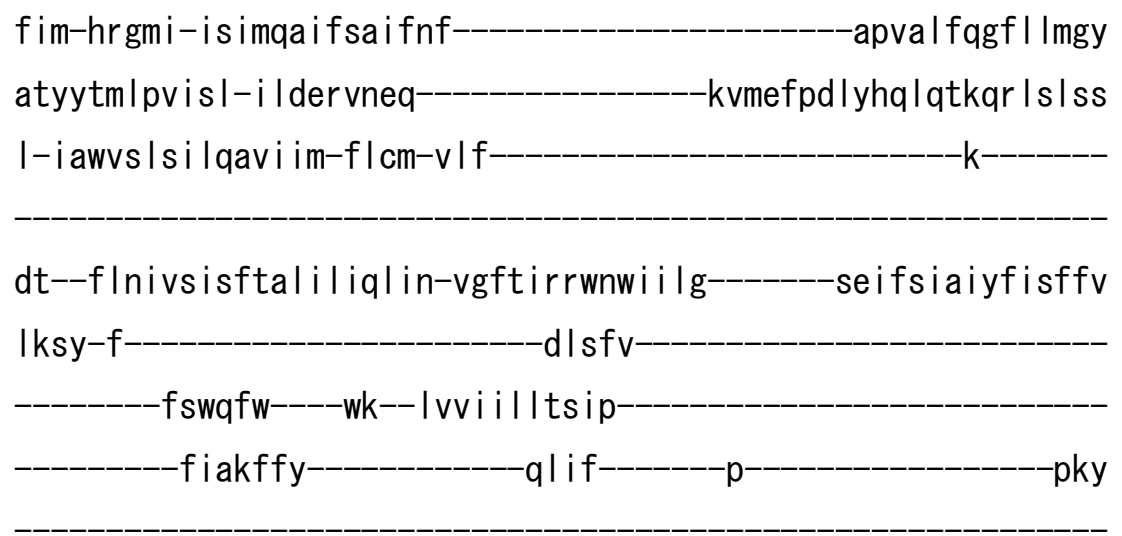

-mtvenrrcxxxxxxxxxxxxxxxx

\section{$\operatorname{XXXXXXXXXXXXXXXX-10-10}$}

$-x x x x x x x x x-----x x$

>ehi IV_IV08 XP_653689.1

m 


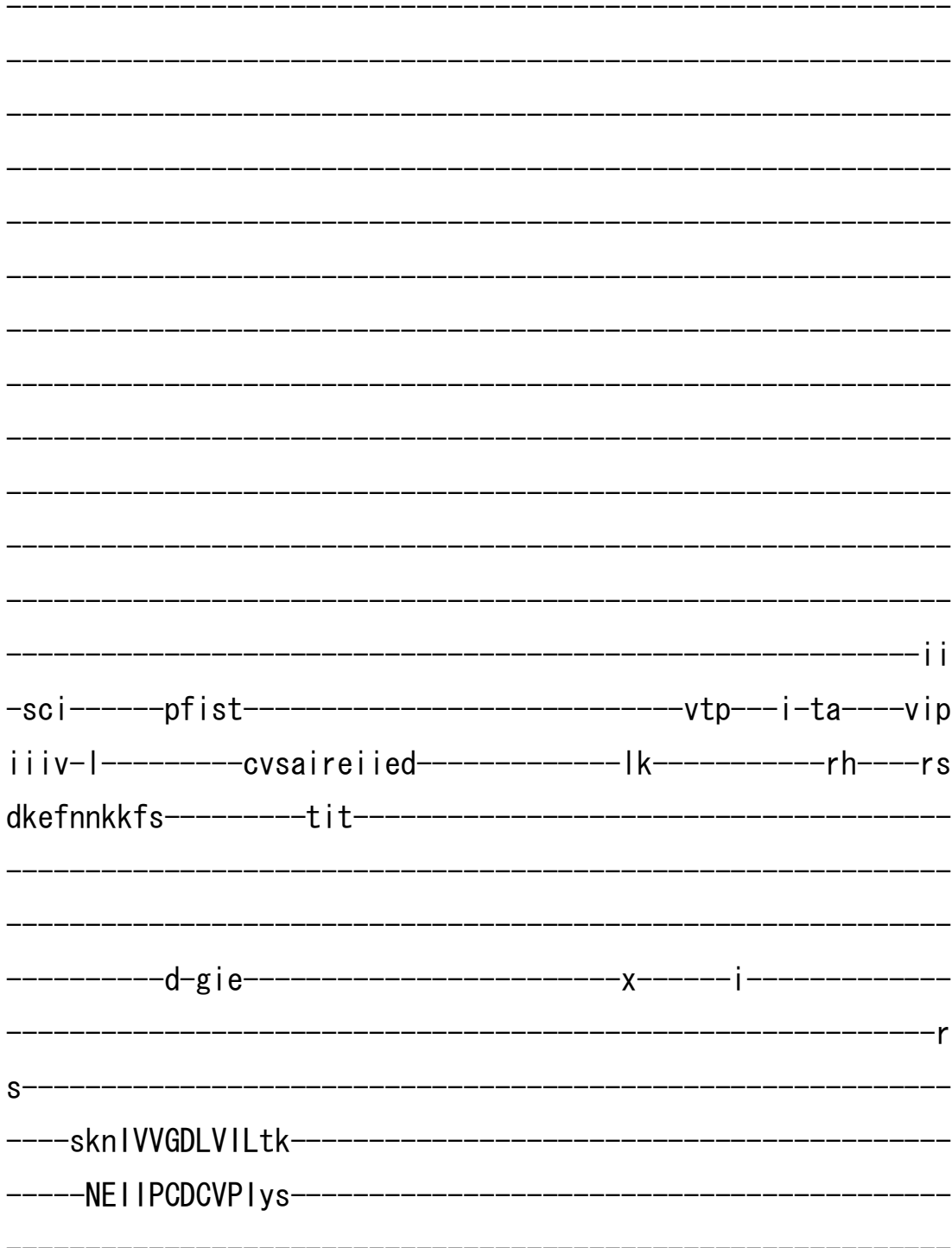

-s lpdg-i---ckVETAALDGETSLKTVyv-

-pkql skssenst Idfkgm lecgfptsnfndfkgcfkfx-

geni it

Ids

-NLLLQ--GSVL-----------------r

-k---------NLLLQ--GSVL

kt----dkvi-----------------------aVVCYTGKYTKQS Insskp in--------

-knst I dhr Inqfv Istvffql i Icsima

xfaswkyknviqdeg-----xwy Ih----_-----xxxxxxstttysikkffgyFNL ISYT

IPISVGVT IEVCRF IQGN-IMemdddf- 
-mKTN-NCSMIEEMGGVQY ILSDKTG

TLTENKMQFs

kcsiyge iy

$-x x v$ I sgs i qxx i xdnpxxx

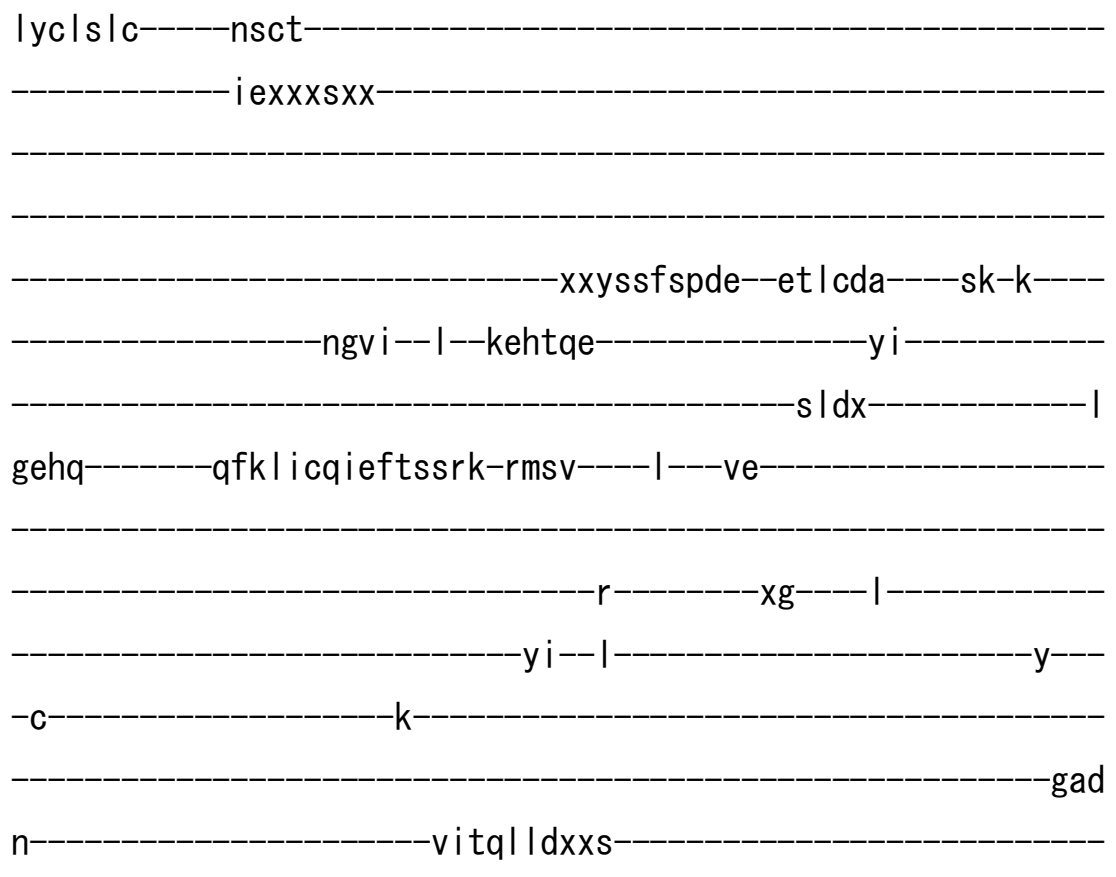

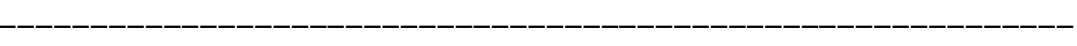

-----xxxxk i s-qi hannf---ase----g| rtLFI ARR i f--tpee i l qwkqkfds---

$-x d p$ I and i krqqme I yex I enh I e I GVTA IEDQLQEDVPST I SMLREag-LK I WV I TGDKMETAISVGLNCRL--_-_-_-_-_-_-_-_-_-_-_-_-_-_-_ f i sgqkmi

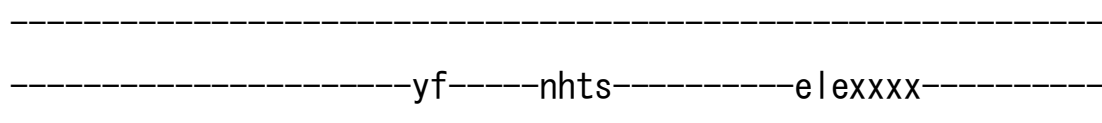

$-x x x x x x x x x x s k x x x d x x x x x$

xxt I givihenn idw---$\mathrm{fi}$

-khqkkfss 
la ikaesvICC--RVSPKQKADIALCVk

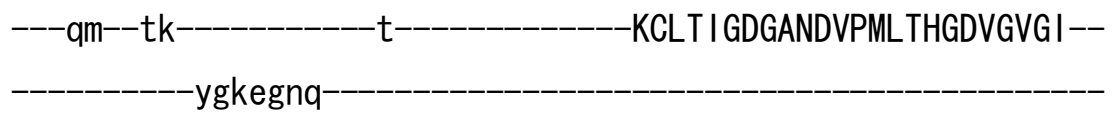

-AAI TADFA IRr fKHLTKL I--LFYGRNGKYQI TTLI------K

fcf-yknaa-ff Imd iwfat i snf--_-_-_-_-_-_-_-_-_-_--_tcq i i yddwvmtcf ntffts I ppaai a Ifdye I pwe--_-_-_-_-_-_-_-_tik lypkshretftdkqykiks f-vewy l ygg l qs i Iff-vi fy-f i i---------_-----xxxxx-xxxxxxv------ng--ftftvvtvttys Ifsiylt-mfvytkrvgnti i l---_----sf Igsi l ly i i lyt i

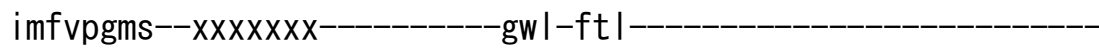

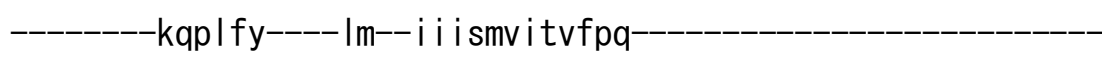

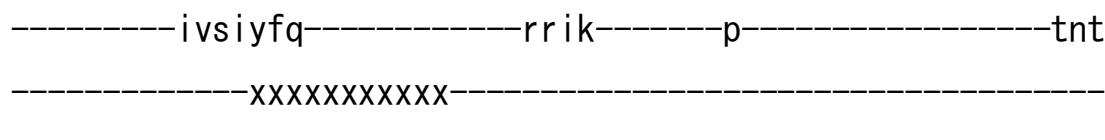
$-x x x x x x x x x x p s i d n q t x x x x x x x x x x x x x x x x$ $\operatorname{xx} x x x x x x x x x x x x x x-$ $-x x x-$ >ehi IV_IV09 XP_656739.1 m- 
xxxxxxxxpekse Ir

---yptihlfd

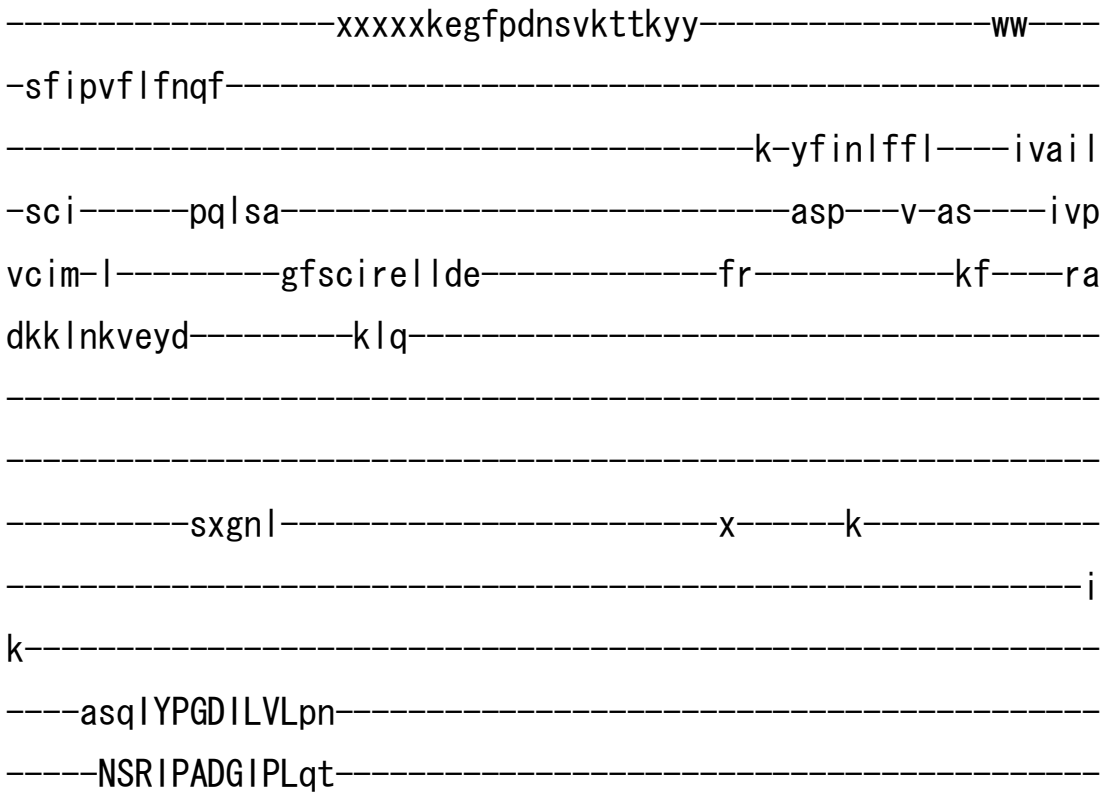


-tnedg----xvfIETSELDGESNLKQRIv-

-pshf i dksyed i ck I kgdf I ttapdpnfdhfvgtfe i--

esqsys

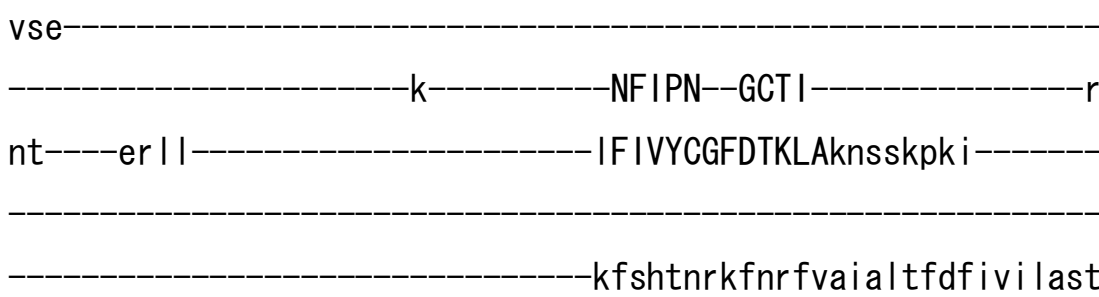

-vcyftysy i yrkge-----xwy lp-------------xxxnvwkqtv IhffsfLNMFSFL

VPVSCLVSIEFSKMFQML-FMsfdsdI

$-x x x x x x x$ igenetkg

cMPW-TGTLNDELGLVEYVLSDKTG

TLTENEMKF $\mathrm{i}$

kcs idgymf $x x x x x x x x x x x x x x x x x$

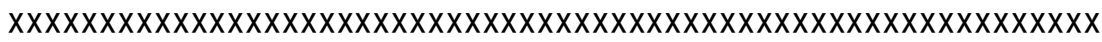

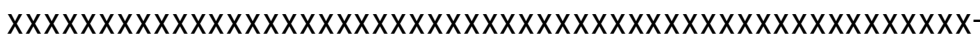

$-x x x x x--x x x x$

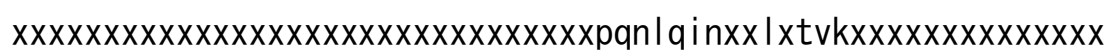

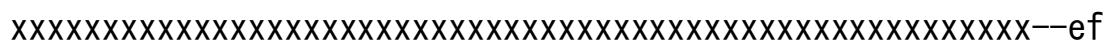

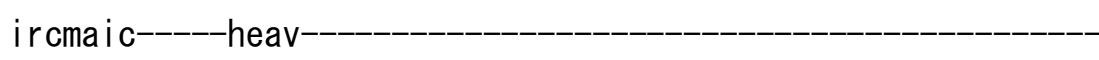
-snxxxkxx

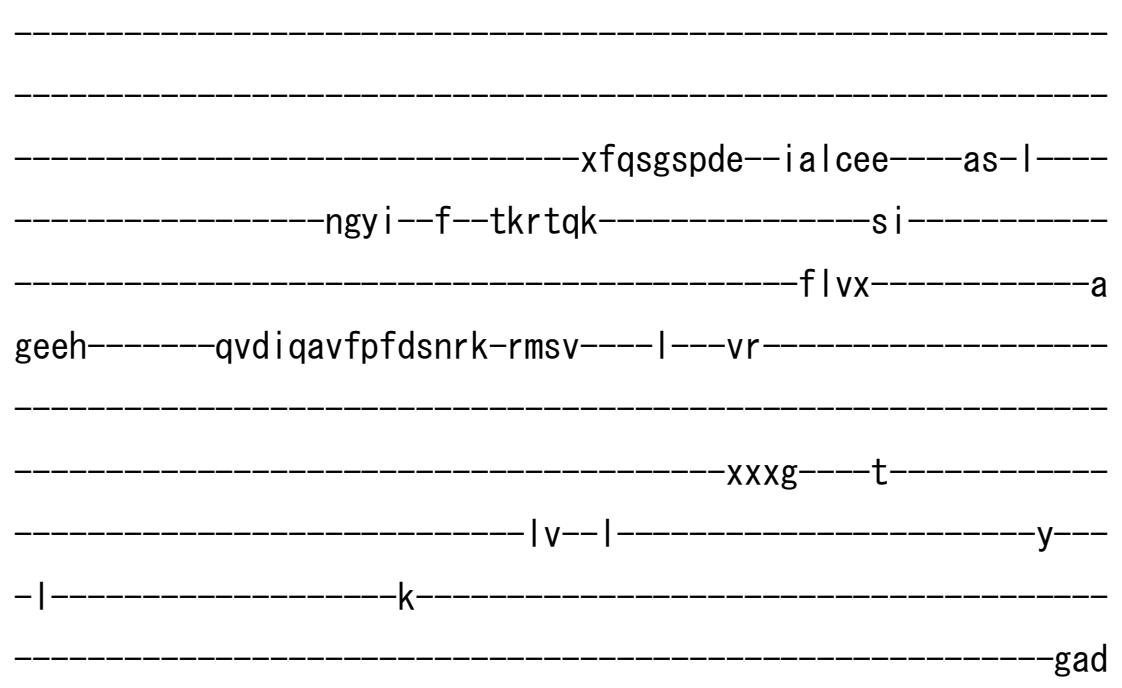
$\mathrm{m}$ $-t t|p| m k x x e x x$ 
-kea-qmdvnkf--are----gyrtLVLGYKi i--pdsvf i ewm i kydn--ak-----s I i vgrdeavnaa i se-i ekdft I LGVSAVEDKLQDGVPET I QSLRRgg-IR I WMI TGDKVETAIN IGSTCGL-tqqek i ih----

-mt------tdnc---xx-------vlkxxxx-

-xxdysvvfdfgvyna

vgklddfwn-

iv I gakav ICC--RVTPL IKGE I IKNAk-

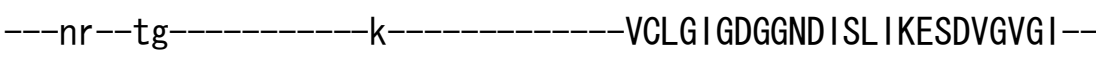
fgkegtq

-AAQTSDYAVRKFRHLQKLI--YFHGRQALRRNTLIV $-K$

I sf-yknva-fi Imqfwfgi iqnaf-_-_-_-_-_-_-_-_-_-_-_--sgqs l yed I i it If nmimtsmppvf i gafe id ipff---_-_-_-_-_-_----sardypeahke i l kgnnfts it

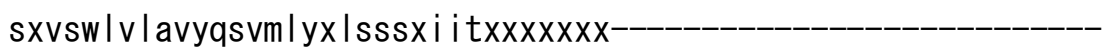
gkxxgygcfsilisitstltiltxmalsikswniflvl-_-_-_-gffvsfilslvcill vsfvpqms $x x x x x x x x x x x x x$

sqpsty----If--il|yvilaitp- IIrnfc- 
$\mathrm{X}$

>rnolV_IV02 XP_214553. 3

$\mathrm{m}$

$-X X X X X X$

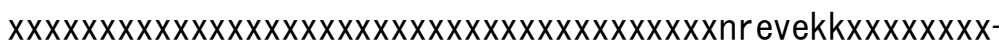

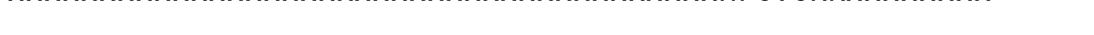

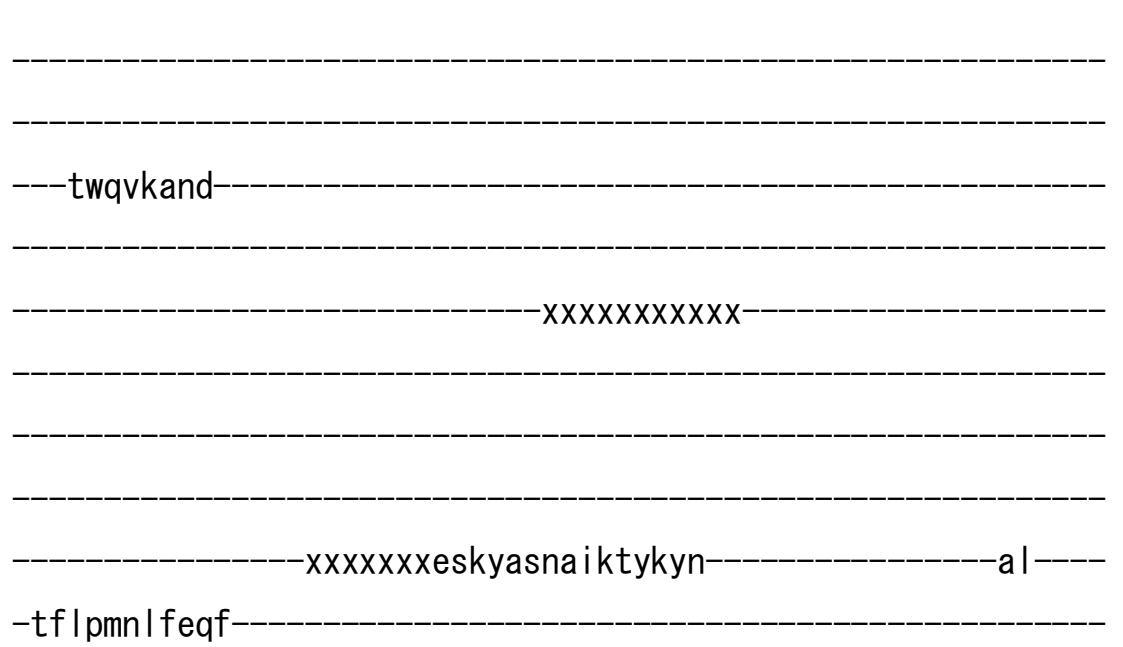

-tf I pmn I feqf 


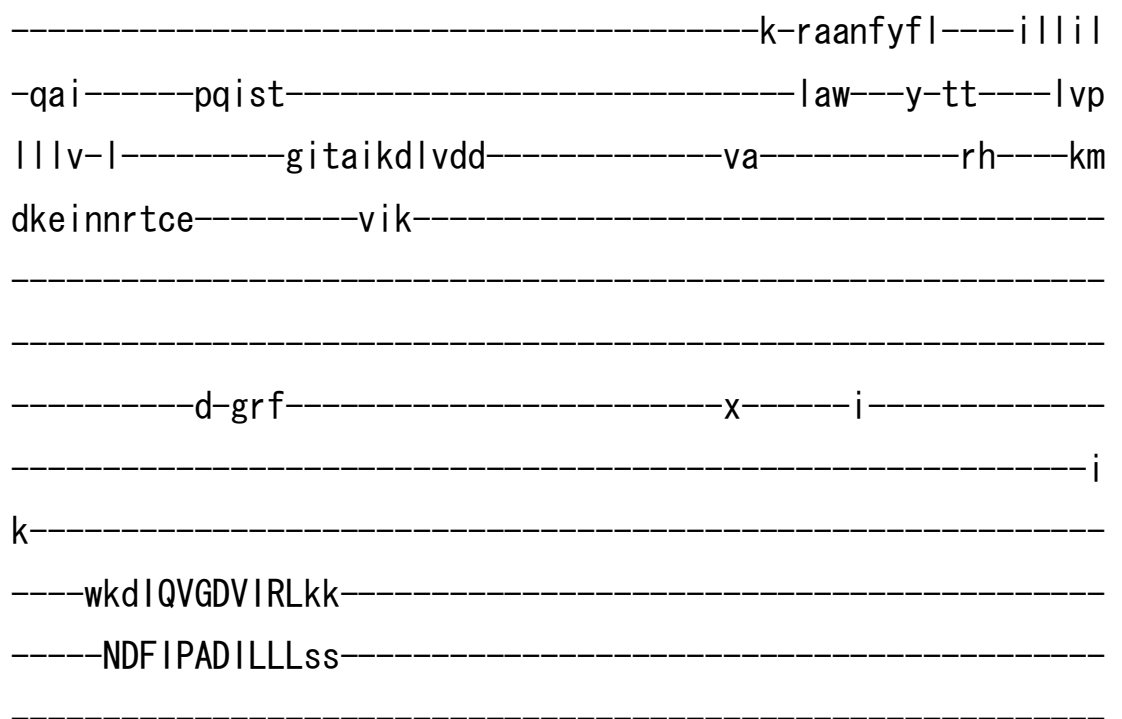

-sepns-I---cyVETAELDGETNLKFKma-

-xe i tdqy lq i edn l atfdgf i eceepnnr I dkftgt I fw--

-rnqsfp

Ida

$d-$

KILLR--GCVI

gLVIFAGADTKIMknsgktrf

nt-----dvch-

-krtk idy Imnymvyt i i i v l I Ivsag la

-i ghayweaq i gnys-------wy ly-----------xxxxxtpsyrgf I nfwgy I IVLNTM

VPISLYVSVEVIRLGQSH-F I nwd I qm

yyaekdtp

aKSR-TTTLNEQLGQIHYIFSDKTG

TLTQN IMTFk -kccingt iy

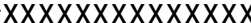

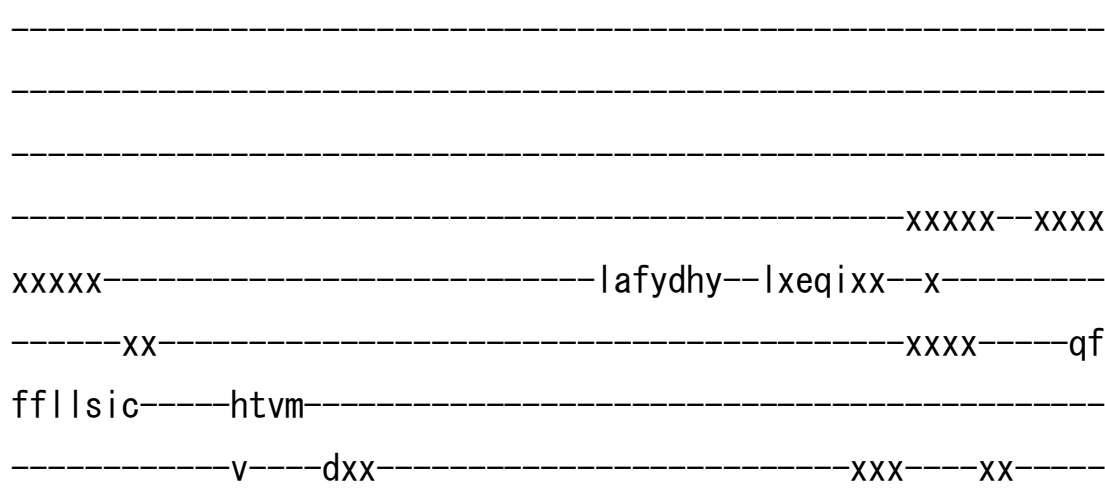




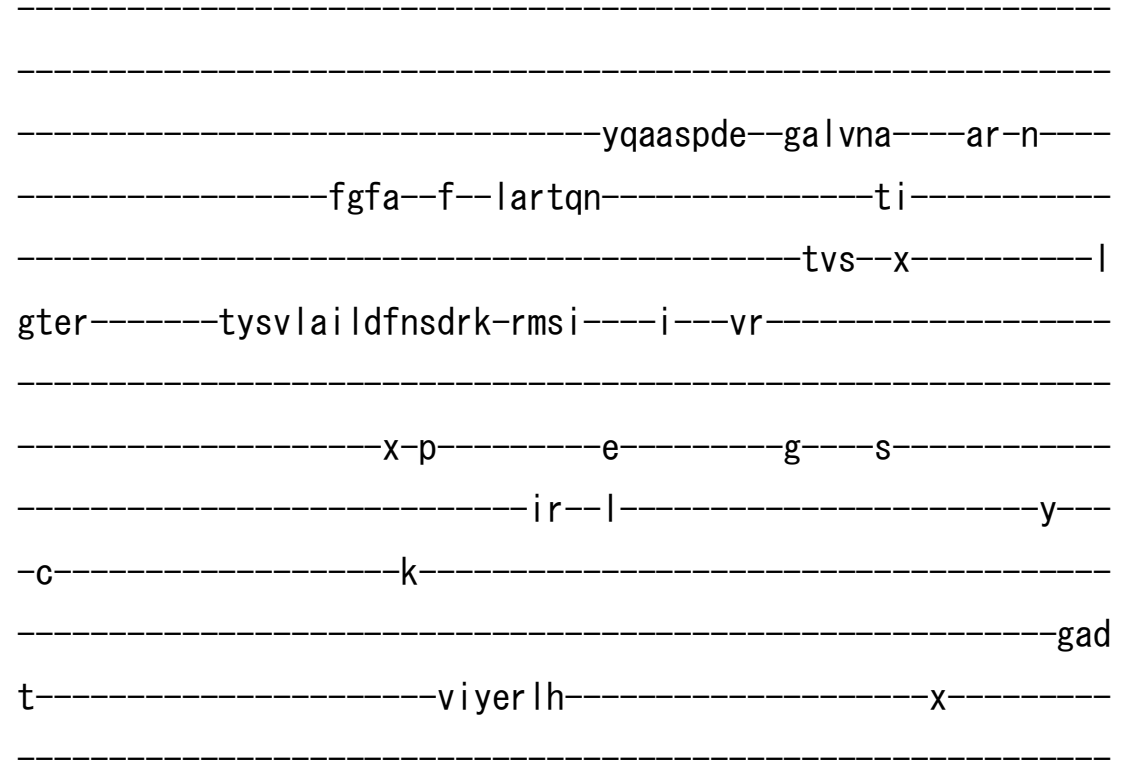

xxx--xx--qet-qdal dif--ase-----t I rtLCLCYKe i--eekefaewnkkfma--as-----vassnrdea I dkvyee-i erd I i ILGATAIEDKLQDGVPET ISKLAKad-IK I WVLTGDKKETAENIGFACEL- tedttic----
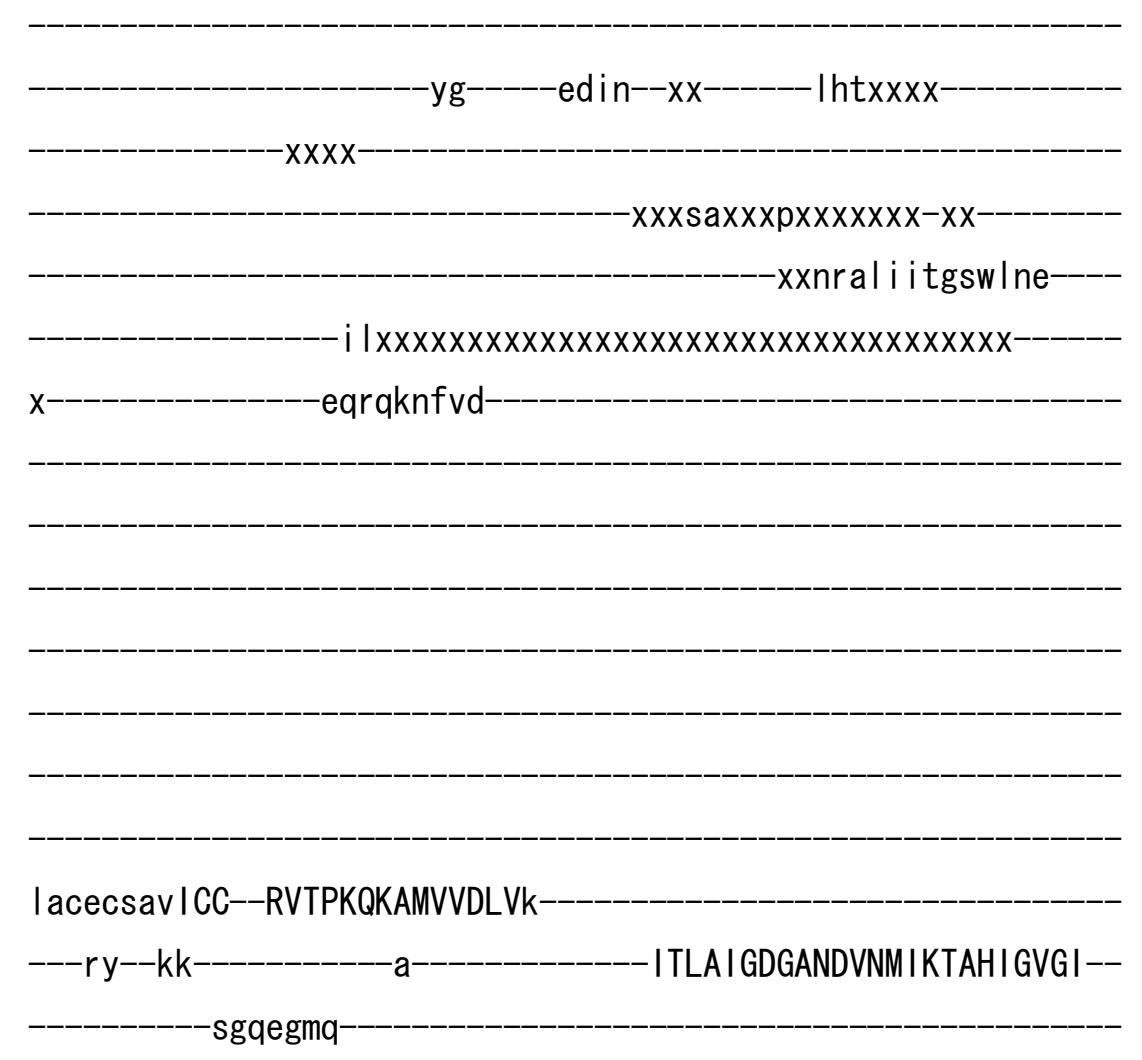

-AVMSSDYSFAqfRYLQRLL--LVHGRWSYIRMCKFL-------R 


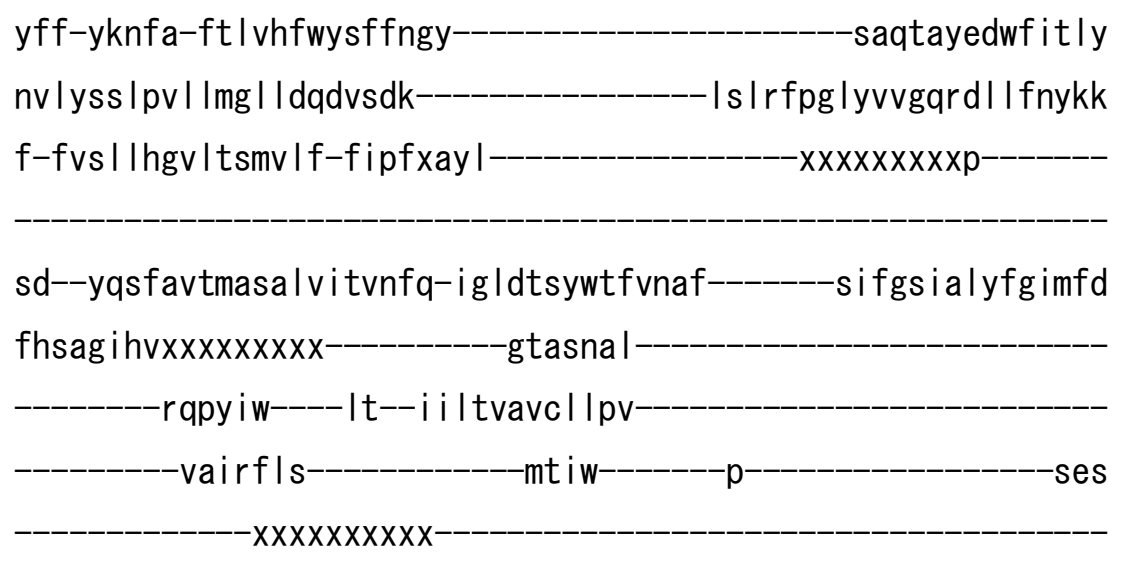

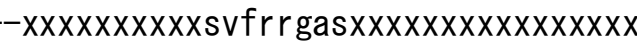

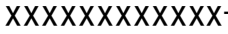

$\operatorname{xx} x x x x x x x x x x x x x x x x x x x$

>rnolV_IV03 XP_001062555. 1

m- 
-tfipvnlfeqf

-q-evantyfl----fIIil

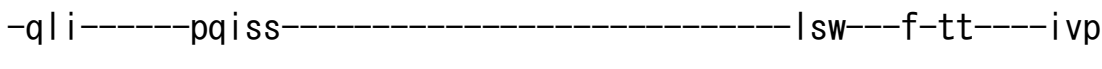

I v lv-I----------t i tavkdatdd--------------yf------------rh-----ks

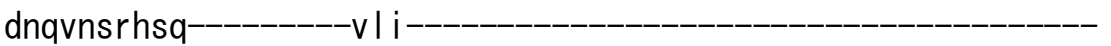

-sephg-|---cy IETSELDGETNMKVRqa-

-xpvtse I g-di sq lakfdgev i ceppnnk I dkfsgal yw--

$-k g n k f p$

Isn

$-q$ $-N M L L R--G C V L$

nt----ewcfgLVIFAGPDTKLMqnsgrtkf

-krts idr Imnt I v I w i fgf I vomgvi I a

-i gna i wehevgtrf------xvylp-xxxxxxsaffsgf I sfwsy I I ILNTV VPISLYVSVEVIRLGHSY-F I nwdkkm 
fcmkkrtp

aEAR-TTTLNEELGQVEYIFSDKTG

TLTQN IMVFn

kcsinghsy-

$X X X X X-X X X X X X X X X X X$

$-x x x x x--x x x x$
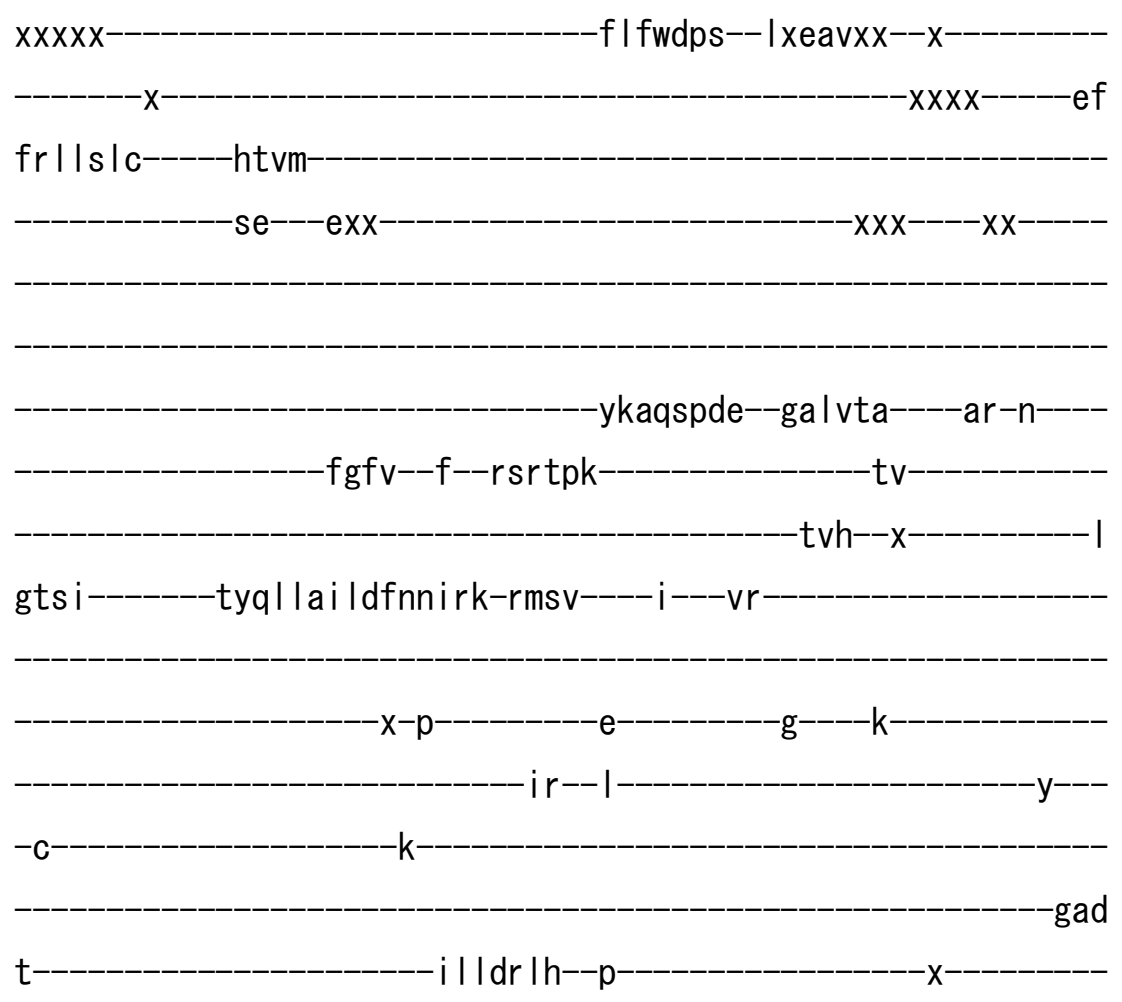

xxx--xx--nst-tdh Iney--agd----g $\mid r$ tLVLAYKd I--deeyyeewar r r lq--as----- laqdsredr l as i yee-vesdmm I LGATAIEDKLQQGVPET I ALLTLan-IK I WVLTGDKQETAVN IGYSCKM-_-_-_-_-_-_-_-_-_-_-_-_-_-_-_--_I tddmtev-_--

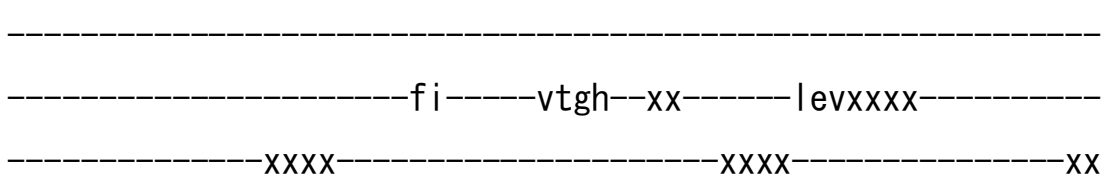

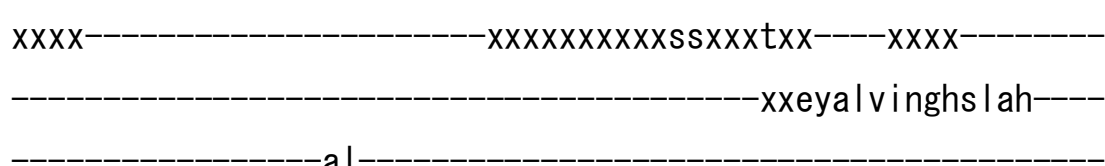
x---------------adme l ef I e 
tacackav ICC--RVTPLQKAQVVELVk-

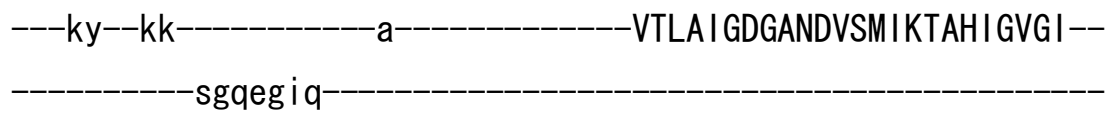

AVLASDYSFSqfKFLQRLL--LVHGRWSYLRMCKFL------C

yff-yknfa-ftmvhfwfgffcgf-_-_-_-_-_-_-_-_-_-_-_--_saqtvydayfitly

ni vyts I pv l amgvfdqdvpeq--_-_-_-_-_-_-_-_ smeypk I yepgq I n I f fnkre

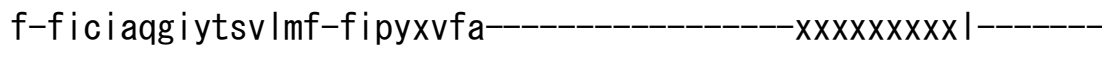

ad--yqsfavtvats I vivvsvq-ig Idtgywta inhf-_-_-_--fi iwgs I avyfai I fa

mhsng I fdxxxxxxxxx---_---_---gnaqnt I-_-_-_-_-_-_-_-_-_-_-_-_-_-

---------aqptvw-----|t--i $v \mid$ ttavc i mpv-----------------------------

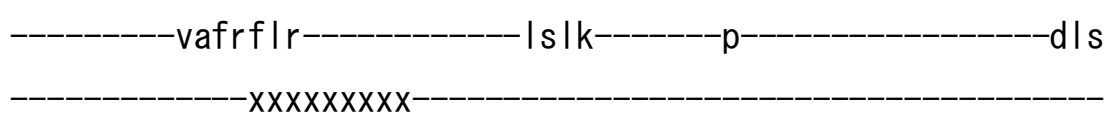

xxxxxxxxxxmrrvgrtgxxxxxxxxxxxxxxxx

xxxxxxxxxxxxxxxx---_-_-_-_-_-_-_-_-_-_-_-_-_-_-_-_-_-_

$-x x x x x---x x x x x x x x x x x x x x x x x x x x x x x x x x x x x x x-$

>rnolV_IV04 XP_001069520.1

m- 


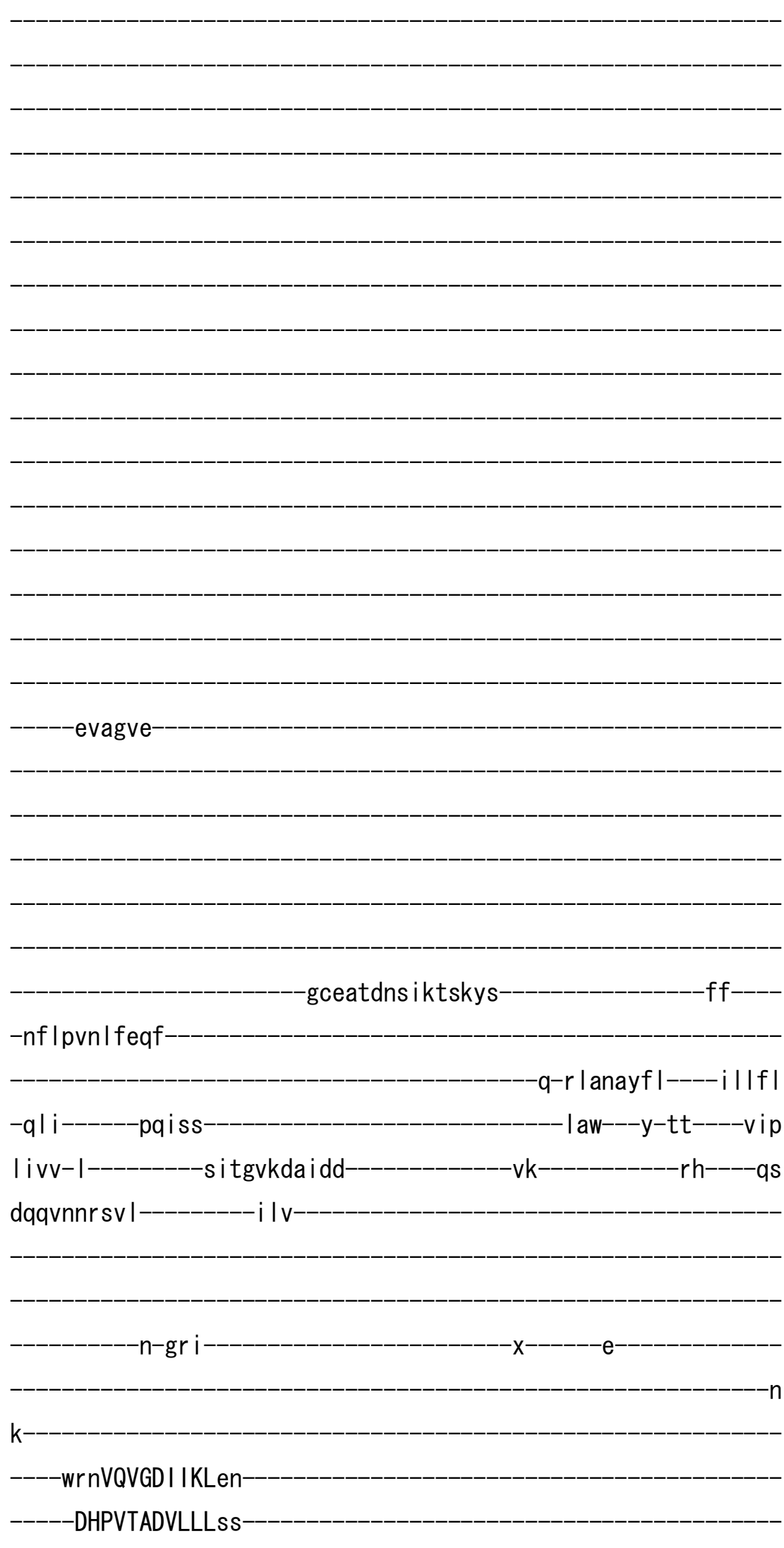


-sepyg-|---ty IETADLDGETNLKVKqa-

-xsatsdmedn le I I safngevrceppnnk | drfsgt I sy--

lgdtyf

Idy

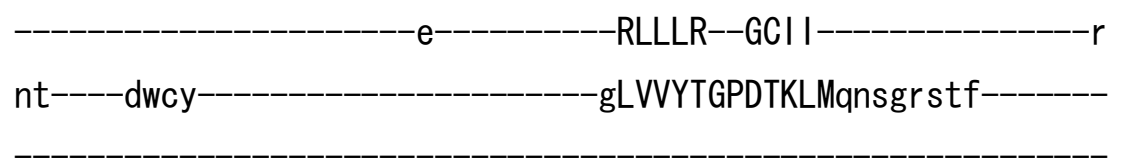

-krthidh Imnv Iv Iwi fmI Iggmcfl| s

-ighg i wesnrgyhf------xaf I p--_-------xxxxxxssaassa l a fwsyF IVLNTM

VPISLYVSVEI IRLGNSY-YInwdrkm-

-fyapkntp

-aQAR-TTTLNEELGQVEYVFSDKTG-

-TLTENVMIFn

-kcs ingkty

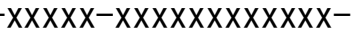

$-x x x x x--x x x x$

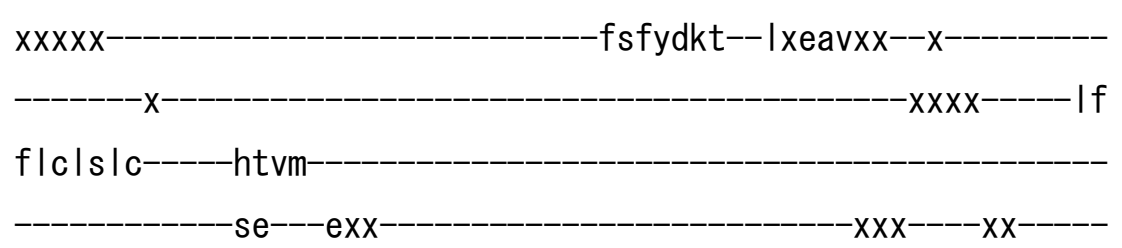

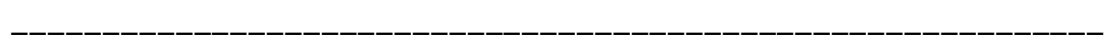

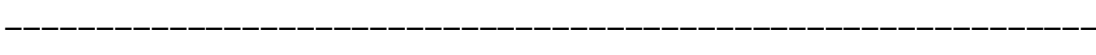

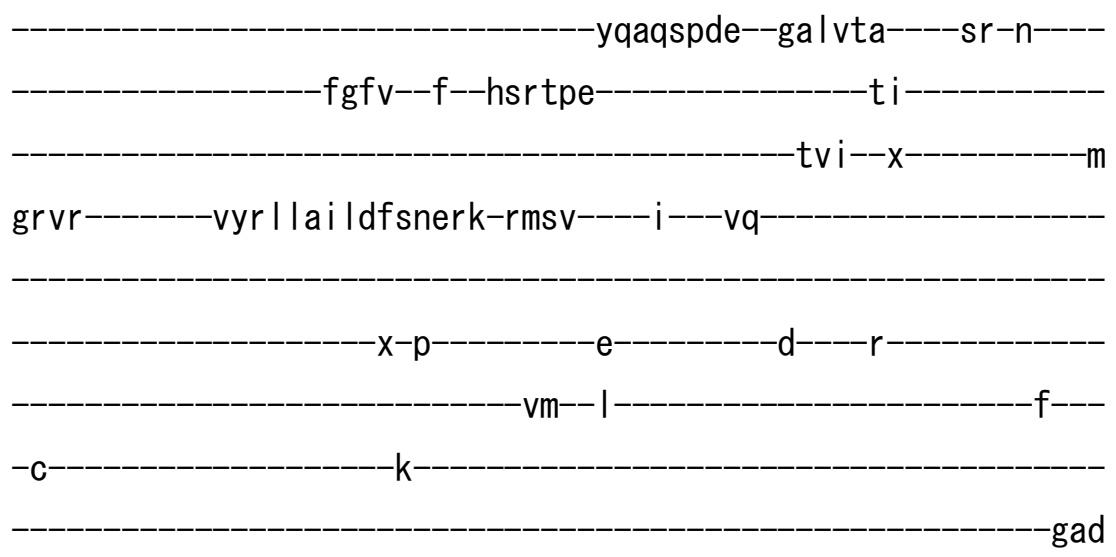

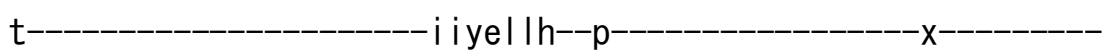


xxx--xx--dvt-mdq Iddf--ase-----g|rtLMVAYRe I--dkaffqtwikkhge--aw----- I t l enr ekk I a l vyee-i erd I v ILGATA IEDKLQSGVPET IVTLNKak-IK I WVLTGDKQETAVN I AYSCRI fkdemdav----

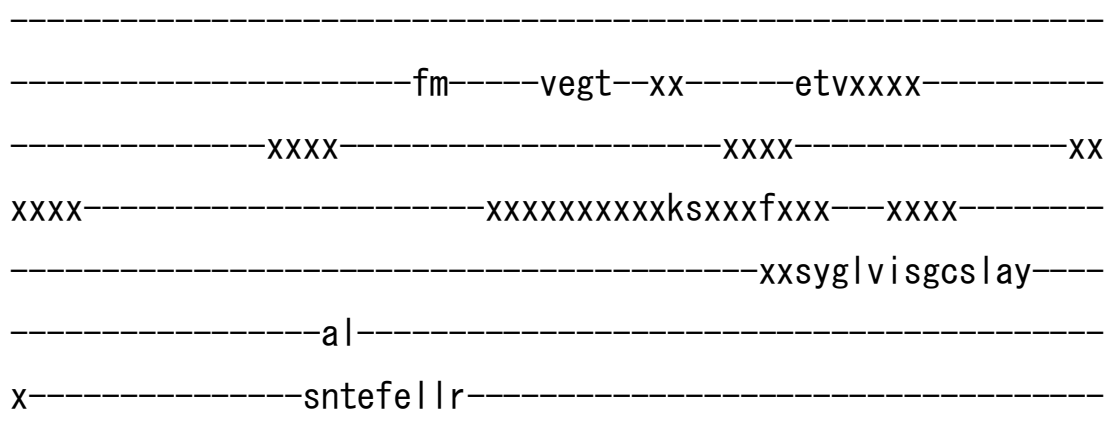

tacmckgVVCC--RMTPLQKAQVVDLVk ---ry--kk--_--_-_----v--_-_-_-_-_-_VTLA I GDGAND I GM I KAAHI GVGI --ATLSSDFSFCqfRYLQRLL--LVHGRWSYNRMCKFL------S yff-yknfa-ft I vhfwyaffngf--_-_-_-_--_-_---_-----saqtvydiwfitfy nl iyts Ip i Ig I I fekdvnet-_-_-_-_-_-_-_---ws I cype l yepgqhn I y fnkke f-ikcl lhg i yssfvlf-fvpmxtvf-_-_-_-_-_-_-_-_-- xxxxxxxxxi---_-_-_ sd--fqsfs I lvatt I iwvmtmq-ialsttywtminha--_-_-_-ftwgs I g lyfci If I $|c s d g| c \mid x x x x x x x x x----------$ gvarng $\mid$

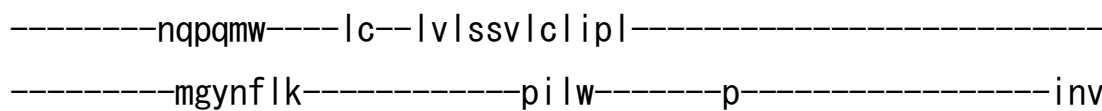
$-x x-$ 
>rnolV_IV06 XP_234937. 3

m-

xxxxxxxxxedkdtef

---twevkand-

$-x x x x x x x x x x x-$

-xxxxxqkkyksnaihtakyn

$-v f$

-sflpInlyeqf 


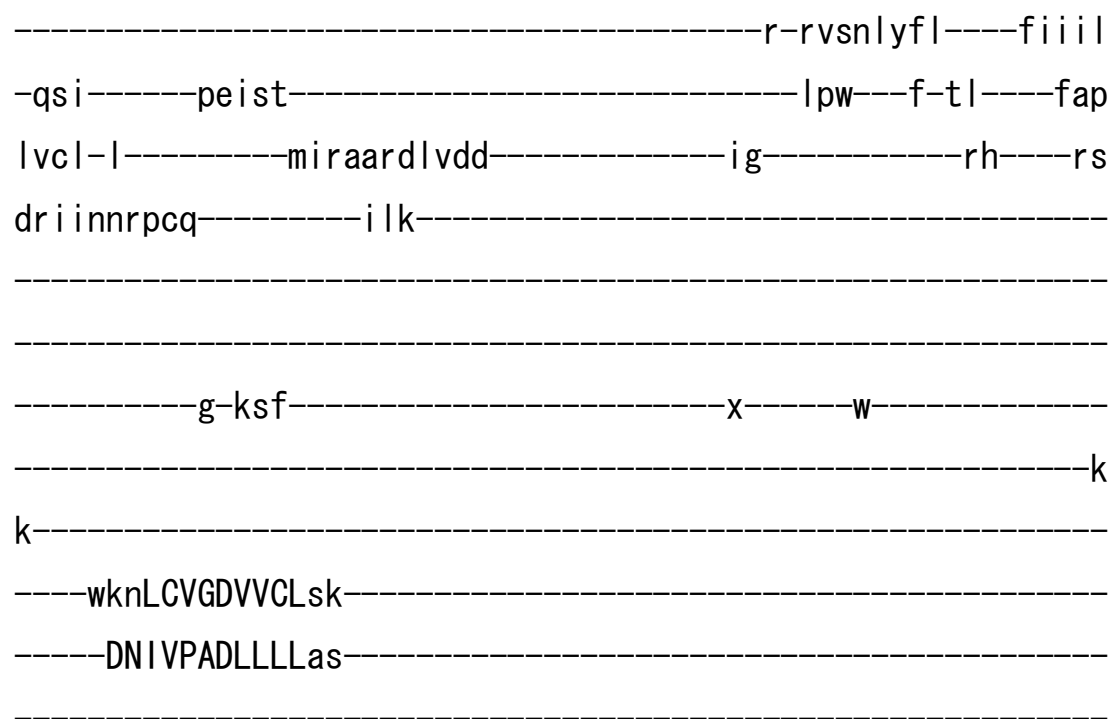

-tepss-I---cyLETADIDGETNLKFRqa-

-xmvthhe I tspkkmasfag i vtceepnsrmhhfvgs I ew--

-nsrkyp

Idi

g----------NLLLR--GCKI----------------r

nt-----dtcy----------------------gLVI YAGLDTK I Mkncgk i I

-krtkIdImmnkIvilifmsIviasmfIt

- I gfafmvkefkakh-------hyms-----------xxxxxtdamdsff i fwgfL ILLSVM

VPMAMF I I aEFIYLGNSI-F Inwd I sm

-yyep I dip

aKAR-STSLNDHLGQVQYIFSDKTG

TLTQN IMTFk -kccingcty

$x \times X X X X X X X X X X X X$

$-x--x x x x$

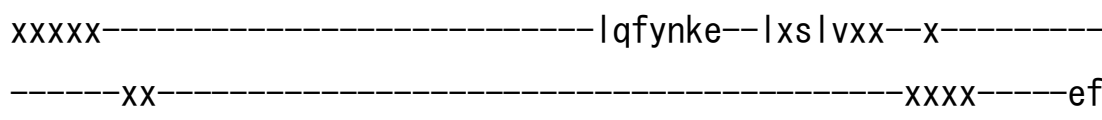

wr I la ic------htvm

$-v----q x x$

$-\mathrm{xxX}-----\mathrm{xX}-----$ 


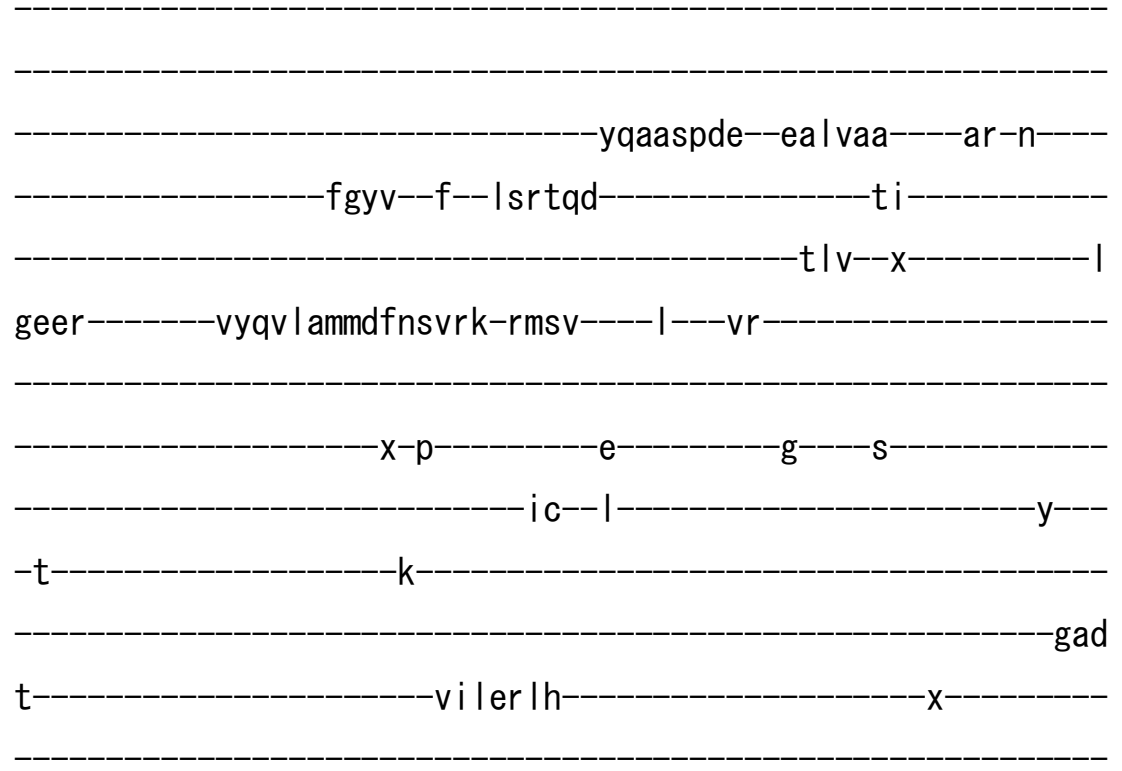

xxx--xx--att-eev l aaf--aeq----t I rtLCLAYKdv--aedaykewepehqe--aa----- I I I qnraqa I hqvynk- I eqn I q ILGVTA IEDKLQDGVPET IRCLKKgn-IKM WVLTGDKPETAVNIGFACQL-

| senms i |----

$$
\text { -ed-----kdik--xx------- lenxxxx--------- }
$$

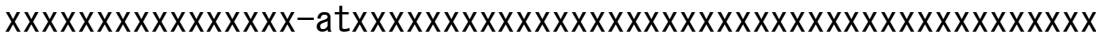
x------_-------errerafvd

laskcqav ICC--RVTPKQKALVVALVk

---ky---qq------------v------_--_----VTLA I GDGANDVNM I KTAD I GVGL--

-AVQNSDYVLAqfCYLQRLL--LVHGRWSYMRICKFL------R 
yff-yktva-smmaq iwfs Ifngfsaqp I yegwf la If

nllyst Ipvlyigl feqdvtae -ks I kmpe lytagqkde I fnys i

f-vqaiahgtitsl inf-fvtixvsy

$-x x x x x x s$

pd--yqsfgvIvaiss I I sitl le-vi lvvkywt I Ifvgtvvvs|ssyvifts | tes IIIfrxxxxxxxxx fadynvi

--_------repss I-----|v--iv Invt Int I pm--_-_-_-_-_-_-_-_-_-_-_-_-_ lavri ir $-x \times x X X X X X X X X X X X$

$-x x x x x x x x x x m r \mid r r g m p x x x x x x x x x x x x x x x x$ $x x x x x x x x x x x x x x x x$

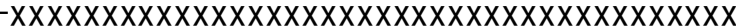

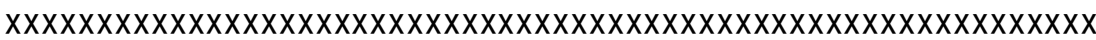

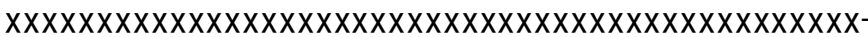

$-$

>rnolV_IV07 XP_001055184.1

$\mathrm{m}$ 


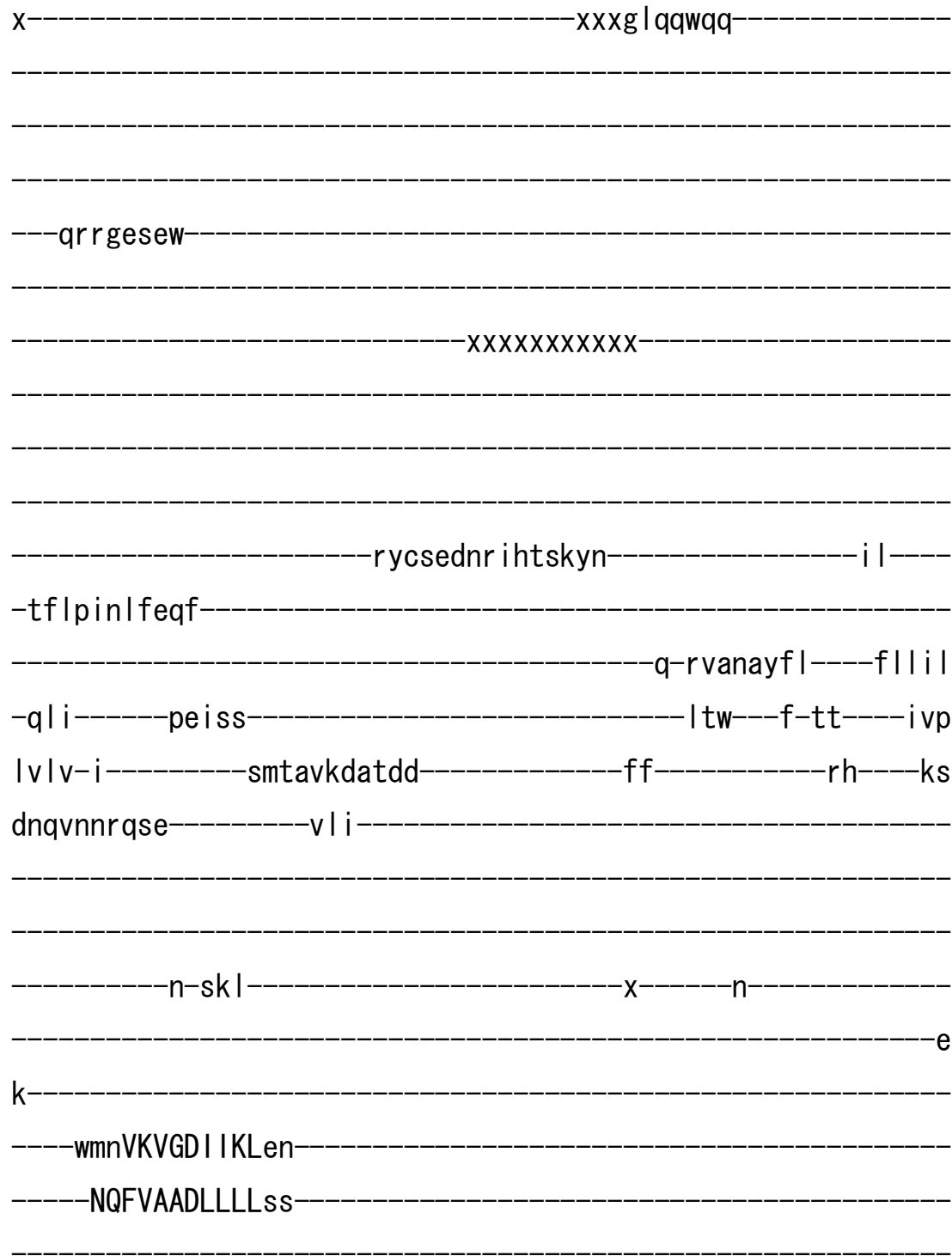

-sephg-I---cyVETAELDGETNLKVRqa-

-xpvtse I gad i ss I akfdg i vi ceapnnk I drfsgv I sw--

-kdskht

Isn-

q----------KI ILR--GCVL-

nt----swcf-_-_-_-_-_-_-_-_-_-_-_-_-gMVLFAGPDTKLMqnsgktkf-

-krtsidr Imnt I viwifgflvclgi i la

-vgns i wesevgnaf------xts If--_-_------xxxxxxss I fsgf I tfwsyVI ILNTL

VPISLYVSVEVIRLGHSY-F I nwdrkm 
aEAR-TTTLNEELGQIEYIFSDKTG
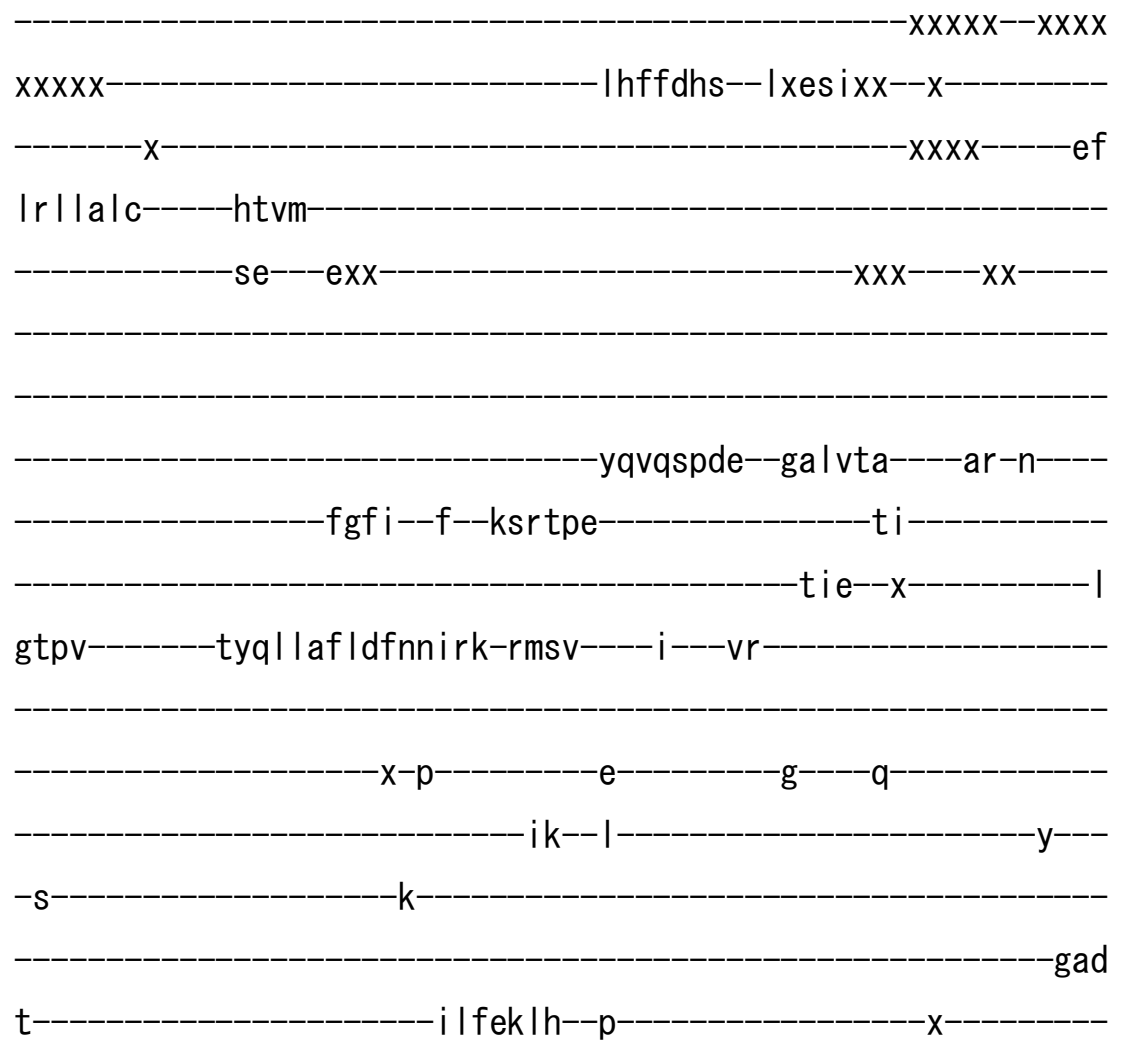

xxx--xx--s It-sdh I sef--age----g | rtLAIAYRe |--ddkyfkmwqkm led--an------sa i aerder i sg I yee-i erd ImILGATAVEDKLQEGVIET I TSLSLan-IK I WILTGDKQETAINIGYACNV-_-_-_-_-_-_-_-_-_-_-_-_-_-_-_--_I tdamdav-_-_-

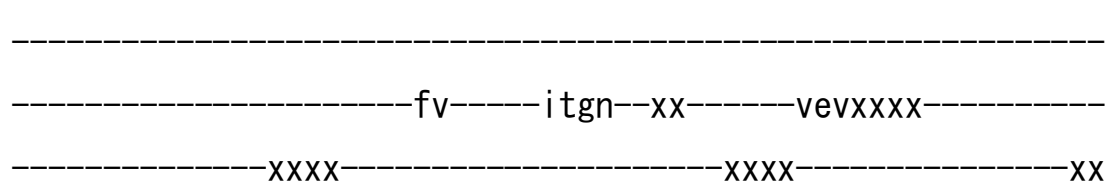

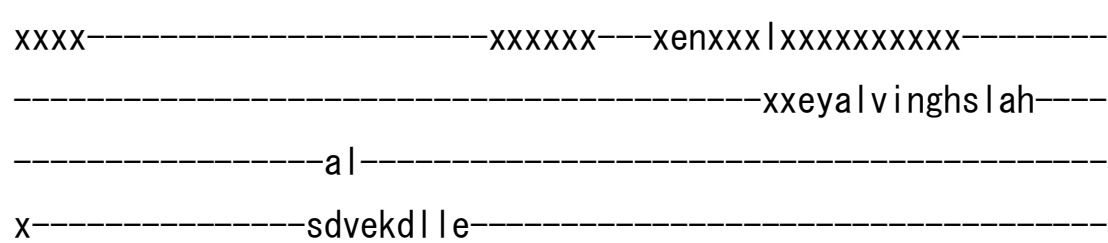




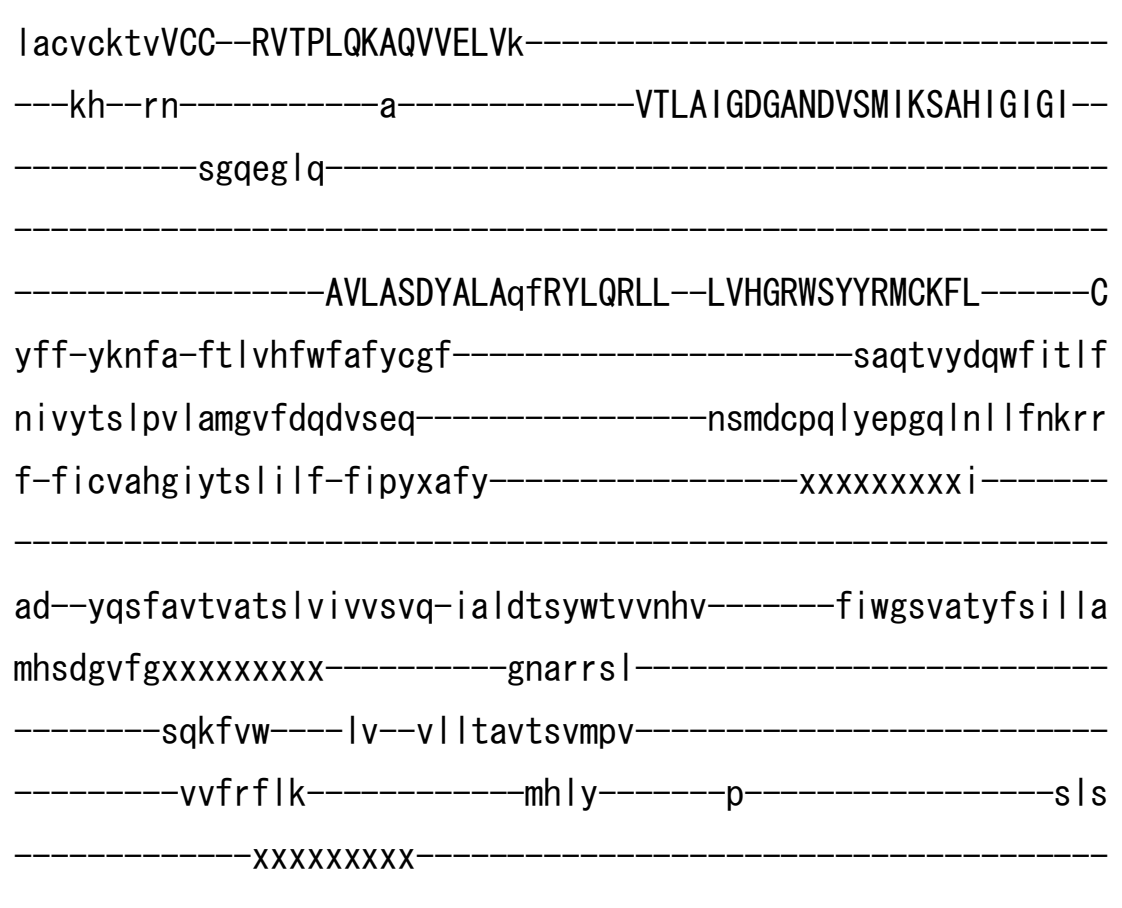

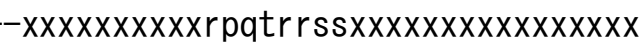

\section{$\operatorname{xxx} x \operatorname{xx} x \operatorname{xx} x x x x x x x-$}

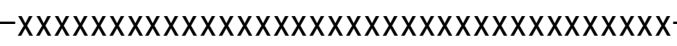

$-$

>rnolV_IV08 XP_225706. 3

$\mathrm{m}-$ 


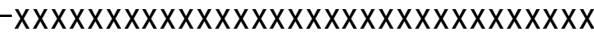

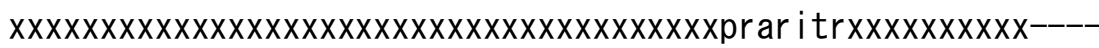

$-x x x x x x x x x x x x x x x x x x x x x x$

$\mathrm{XXX}$

$---\operatorname{artvw} \operatorname{gc}$

xxxxeekhprns iknqkyn

-vf----

-tfipgvlyeqf

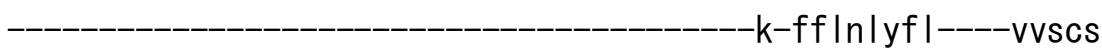

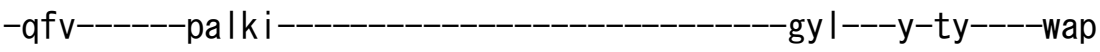

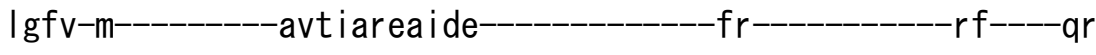

dkemnsq lys----------k lt

$\mathrm{k}-$

----ssd IQVGDL I I Vek

NQRIPSDMVFLrt 
-sekag-s---cfIRTDQLDGETDWKLKva-

-vsctar I pa I gd I fs i sayvyaqkpq I dihsfegtftrxx

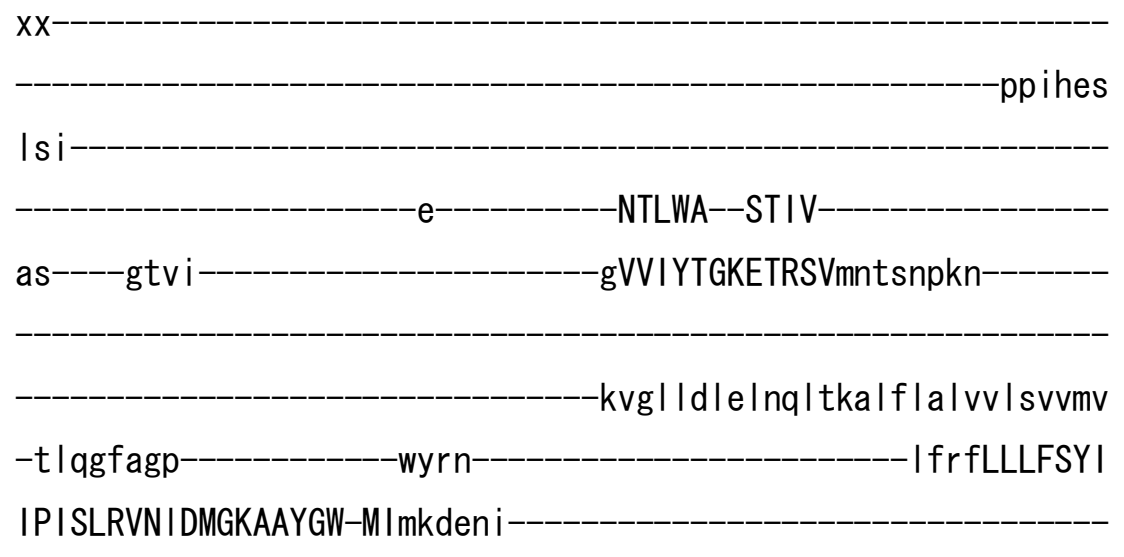

$-p g$

-tVVR-TST IPEELGRLVYLLTDKTG

-TLTQNEMVFk--------------r Ih I gtvsy

$x x x x x x x x x x x x x x$

$-x X------X X X$

$x x-x x x x x x x x x x--x x x x x x x--x x x x x x x x v s s r-i h-$

ea

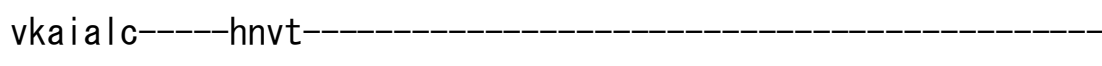

$-p v x x x$

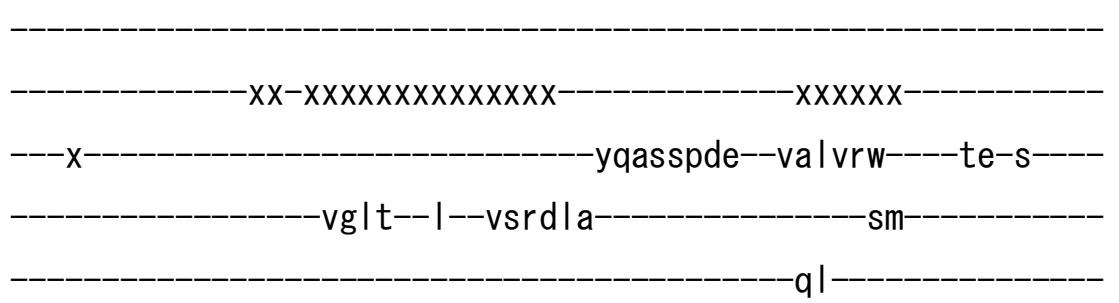

ktps-xxxx--tyc i l qmfpftsesk-rmg i-----i---vr---------------------

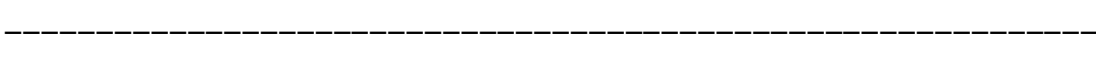

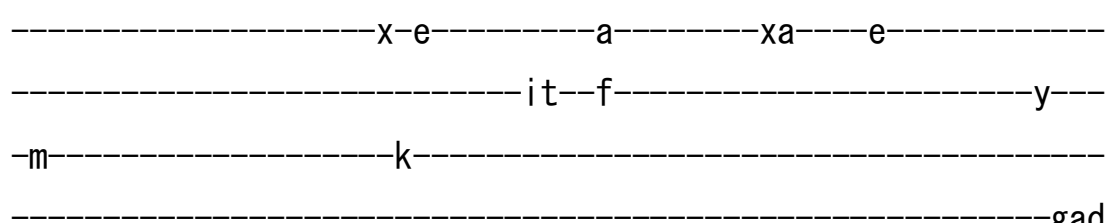

-gad

v------------------------amsti $v--x y x$ 
-dw I-eeecgnm--are----g|rtLVVAKRt |--teeqyqdfesrysq--ak----- I s ihdrt I kvaavves-I ereme ILCLTGVEDQLQADVRPTLEMLRNag-IK I WMLTGDKLETATCI AKSSHL-vsrtqdih----

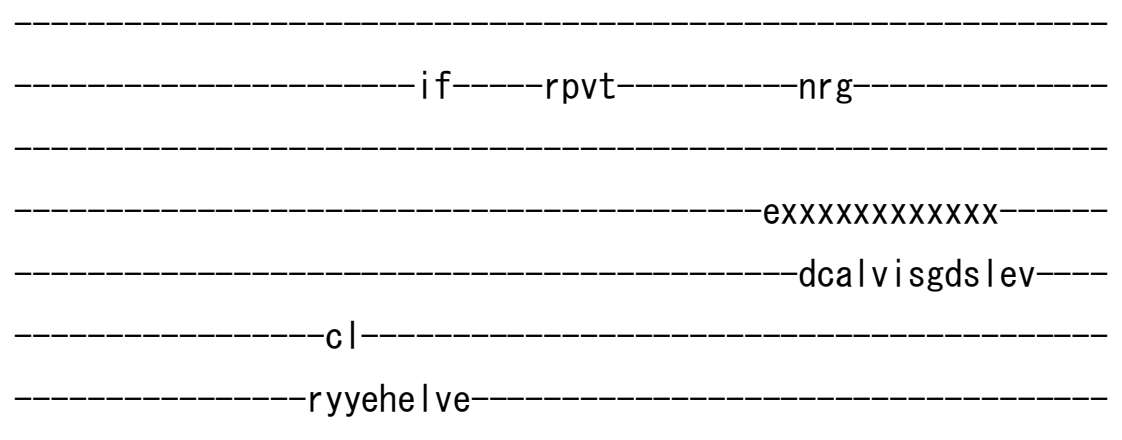
I acqcpavVCC--RCSPTQKAHIVTLLr

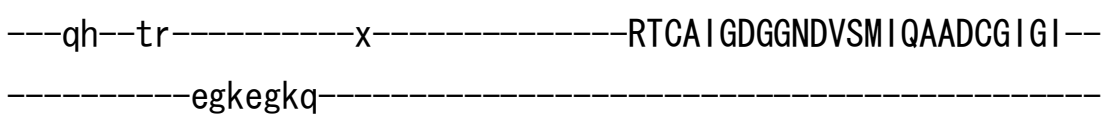

-ASLAADFSI TqfRH I GRLL--MVHGRNSYKRSAALG------Q

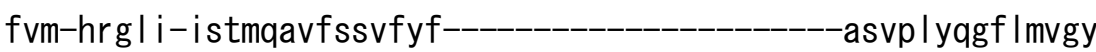
at i ytmfpvfs I-vldqdvkpe--_-_-_-_-_-_-_--ma i lype lykd I tkgrs I sfkt f-I iwv l i s i yqgg i Im-yga |-I I f-_-_-_-_-_-_-_-_-_-_-_-_-_--_e--_-_-_ ae--fvhvvaisftal i Ite I Im-valti twhw Imvv--_-----aef I s gcyvas laf Iney-fgi xxxxxxxxx---_-_-_-_-_-_dvafi--_-_-_-_-_-_-_-_-_-_-_-_-_-

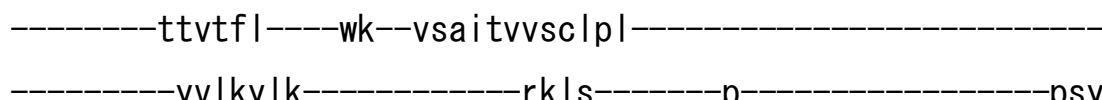
skl---ss 
>rnolV_IV09 XP_344902. 3

m-

-XXXXXXXXXXXXXXXXXXXXrrrwegr

---trtvrsnl

$-x x x x x x x x x x x x x x x x x$

xxxxxxxiqh ladnr lkttkyt - I

-sflpkn I feqf 


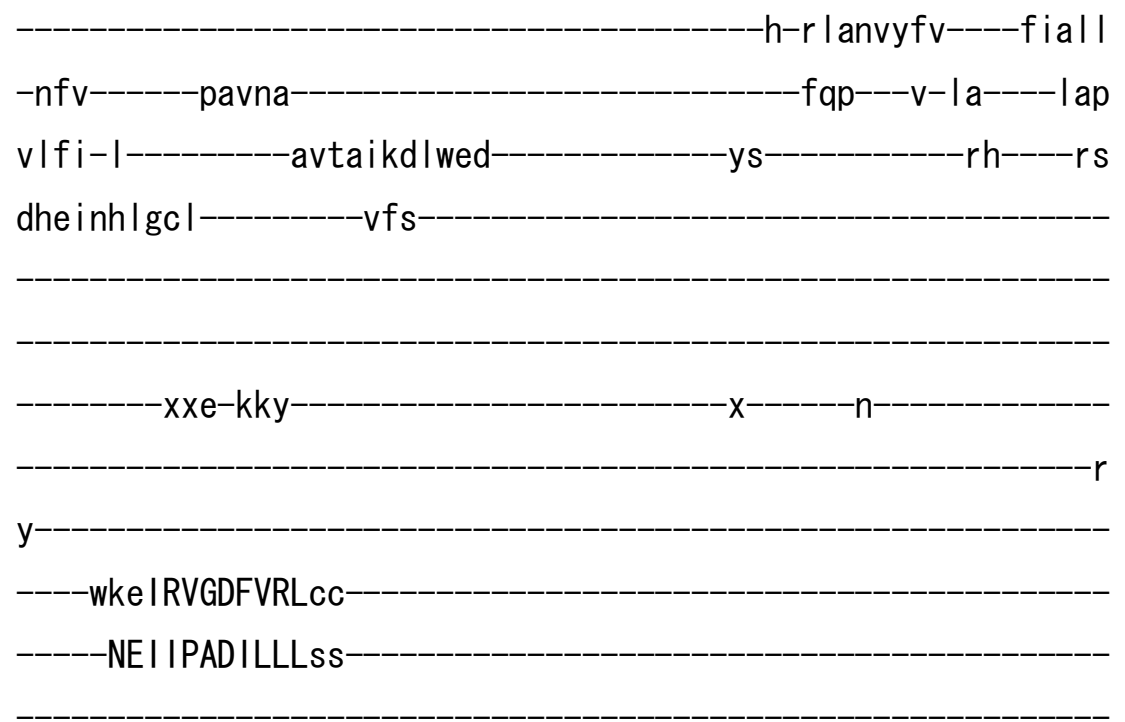

-sdpdg-|---chIETANLDGETNLKRRqv-

-vrgfselvsefnpItftsvi ecekpnndIsrfrgci ihx-

-ngekag

lhk

NLLLR--GCT I

gIVIYAGHETKAL Innsgpry

-krsqleramncdv IwcvIII vcis Ifsa

-vghg I wvr ryqekk-----xxfdvp---------xxxxxxxspataavysf I tmI IVLQVL

IPISLYVS iEIVKVCQVY-FInqdie I

ydeetdsq

IQCR-ALN I TEDLGQIKY IFSDKTG

TLTENKMVFr

-rctvsgiey

$\operatorname{xx} X X X X X X X X X X X X X X X X X$

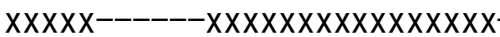

$-x x x x x--x x x x$

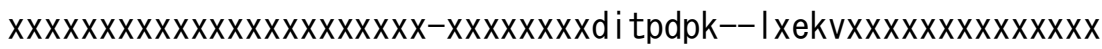

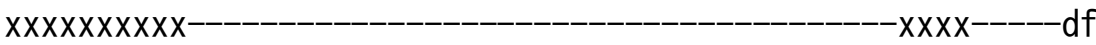

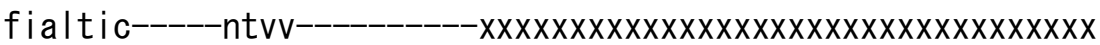

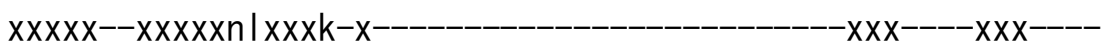




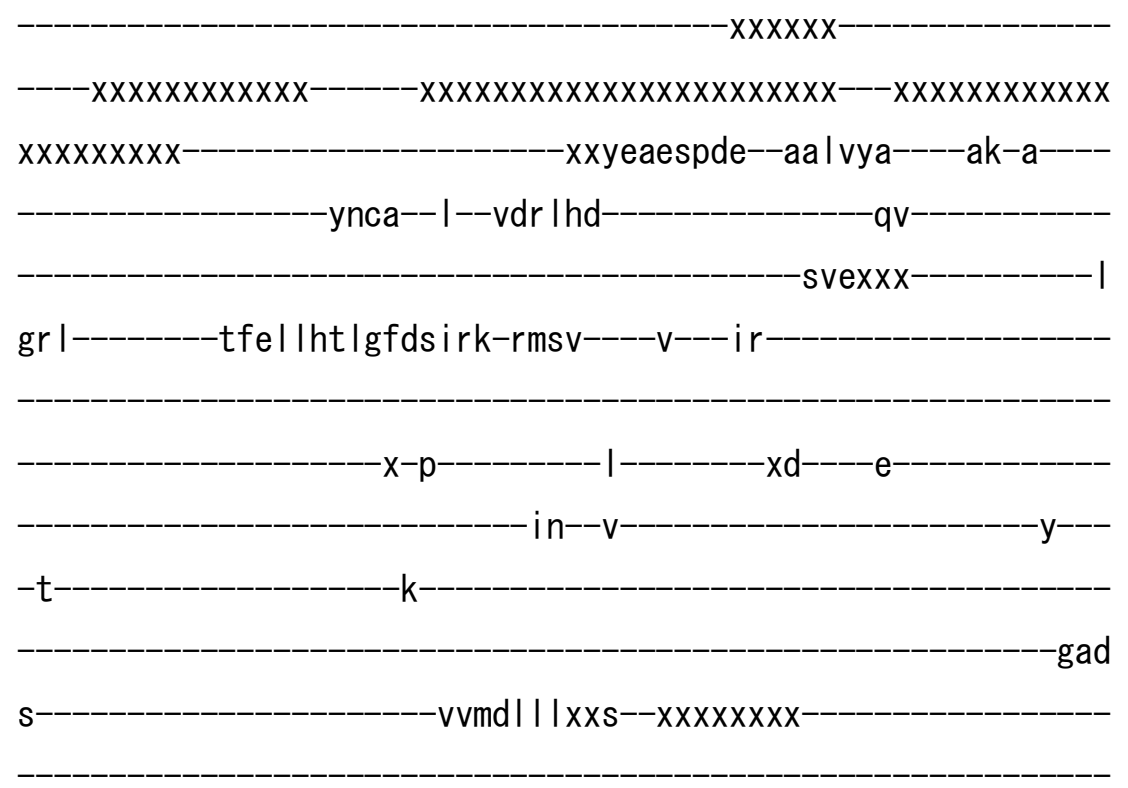

xxx--xx--skt-qny | n ly--ave----g|rtLCI AKRv |--skeeyacw I qsh i e--ae-----tsvesree I I fqsavr-l etn I hILGATG IEDRLQDGVPET I AKLRQag-LQ I WVLTGDKQETAINIAYACKL- dhgeevi----
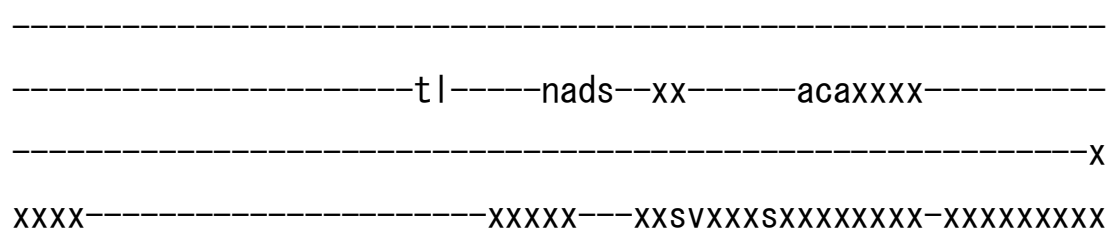

$$
\begin{aligned}
& \text { xxxxxx----------------------------_---------s l vidgr s lay---- } \\
& \text { al } \\
& \text { x------_---------ks ledkfIf- }
\end{aligned}
$$

lakqcrsvLCC--RSTPLQKSMVVKLVr

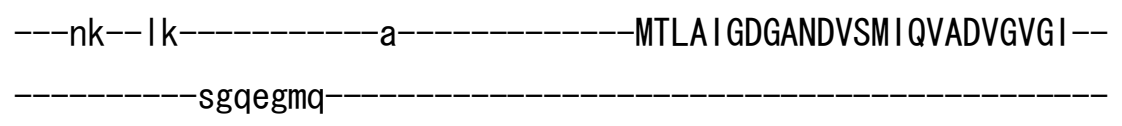


yff-ykntm-fvg I Ifwfafycgfsasami dqwy l iff

nl Ifss I pq I vtgv I dkdvpad -mI I rkpq lyksgqnmeeyspra f-w Inmvdaafqs I vcf-f i py-lay $x x x x x$

-d--vftwgtpvta ialftf I |h-|g i etktwtw Inw I acgfstfiffsvali yntscatc---xxxxxxx--_-_-_-_--wtmpt I I-gdp I fy----|t--c l i apvaal Ipr - ffkalq-_-_-_-_-_---gs I f $-p-$ tql

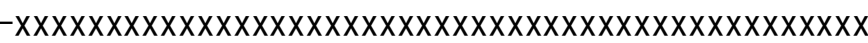
$x x x$ Xxxxxxxxxx|acspeas $x x x x x x x x x x x x x x x x$ $x x x x x x x x x x x x x x x x x$

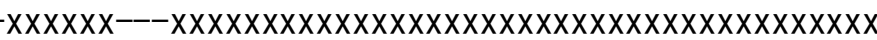
XXXXXXXXXXXXXXXXXXXXXXXXXXXXXXXXXXXXXXXXXXXXXXXXXX

$-$

>rnolV_IV10 XP_220314. 4

$\mathrm{m}$

$-x X X X X X X X X X X X X X X X X X X x$ $\mathrm{X}$ 


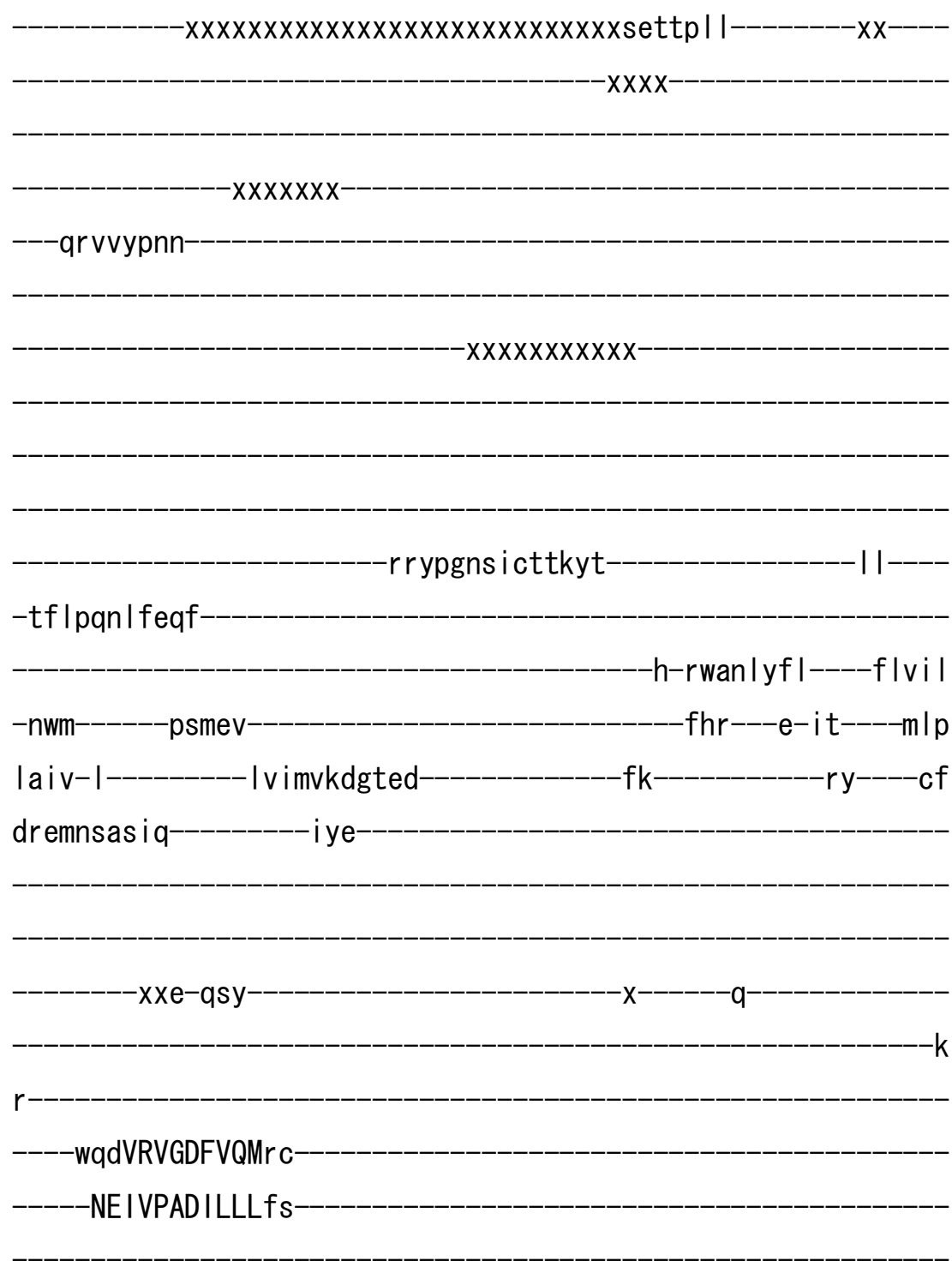

-sdpsg-v---chLETANLDGETNLKQRrv-

-vkgfsqqevqfapehfhst i vcekpnnh I skfkgymehx-

detrtg

fgs

$-\mathrm{e}^{-----------S L L L R--G C T I---------------r}$

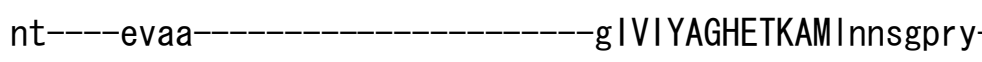

-krskierrintdiffcig I f Imcliga

-vghs I wngtfkehp------xfdvp---------xxxxxxxspa I ggfymf I tm I ILLQVL

IPISLYVS iELVKLGQVF-LLhndIdI 
-ydeetdls

i QCR-ALNI TEDLGQIQY IFSDKTG-

TLTENKMVFr

-rctivgney

$-x x x x x x x x x x x x x x x x x x x$

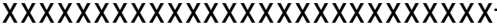

$-x x x x x--x x x x$

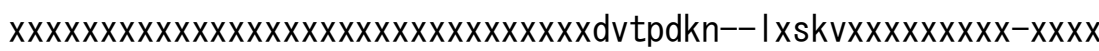

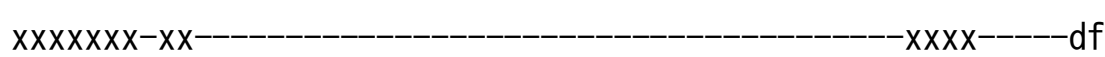

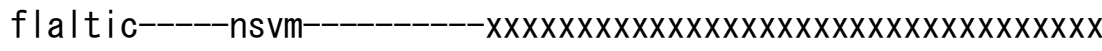

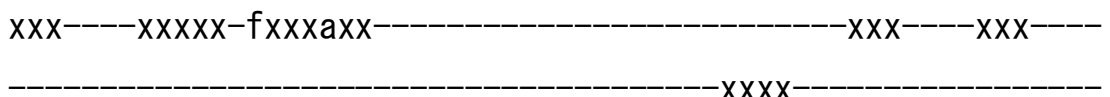

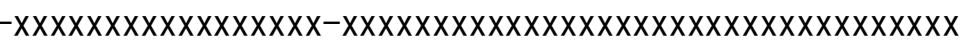

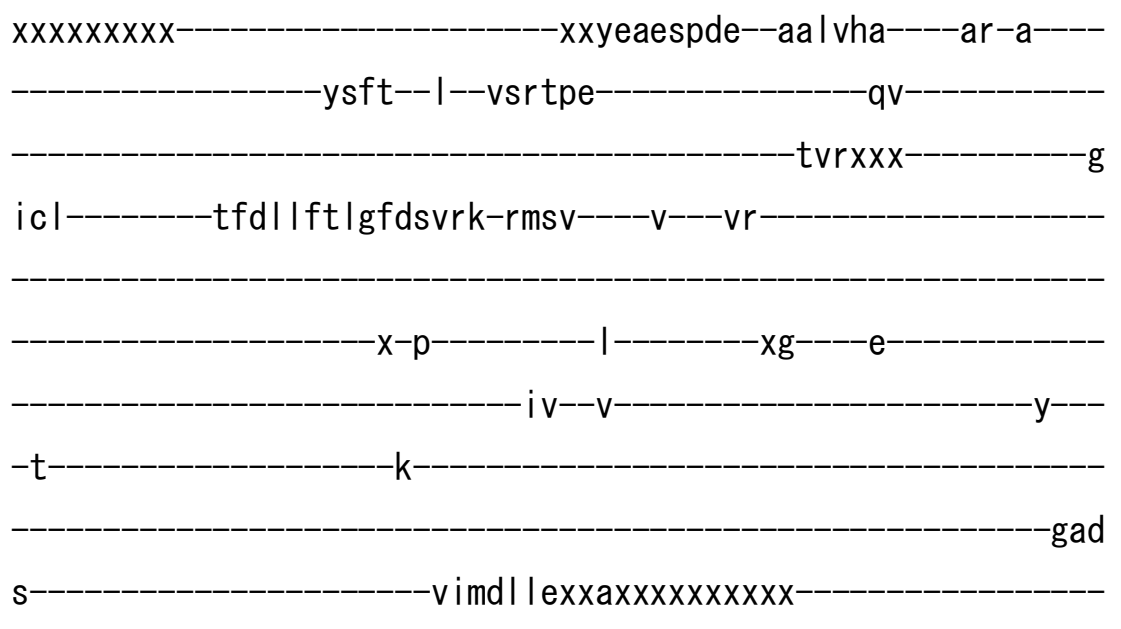

xxx--xx--art-qkh|d ly--ard----g | r tLCIAKKvv--needfarwasfrre--ae-----as I dnree I Imetaqh- I enr I t ILGATG IEDRLQEGVPDT I AALREag-IQL WVLTGDKQETAVNVAHSCKL-_-_-_-_-_-_-_-_-_-_-_-_-_-_---I dqadtvy---_

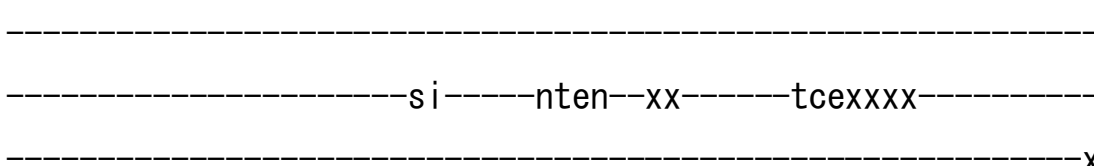

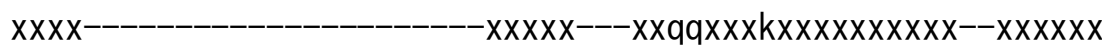

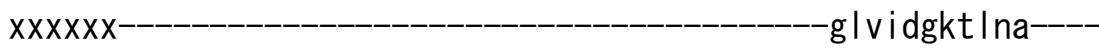
if

x--------------gklenkfle 
I tqycrsvLCC--RSTPLQKSM I VKLVr-

---dk-- | s-----_------v---_-_-_-_---MTLS I GDGANDVSM I QAAD I G I G I--

-AVMSSDFA I ArfSHLKKLL--LVHGHWCYSRLARMV------V yyf-yknvc-yvn I I fwyqffcgf-_-_-_-_-_-_-_-_-_-_-_-_-sgstmi dywam iff nlffts I pp i vfg i Idkdvsae-_-_-_-_-_-_-_-_t I a l pe lyksgqnsecyn I pt f-wvsmadafyqs I icf-fipy-Ity--------------Xxxxx---------------d--vftfgtpintis Itti I |h-qamemktwtv Ihg |----_---v| Igsf Imyfvvs I i

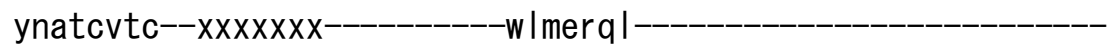
---------sdpmfy-----|t---c|l tpvval | pr---------------------------

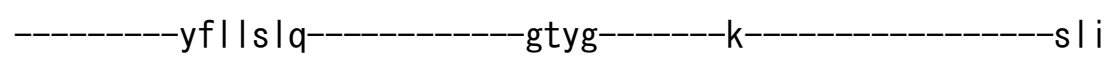
$--x x x x x x x x x x x x x x x x x x-$ $-x x x x x x x x x x p a s a s a--x x x x x x x x x x x x x x x x$ $x x x x x x x x x x x x x x x x-$

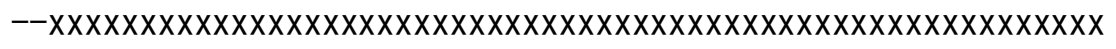

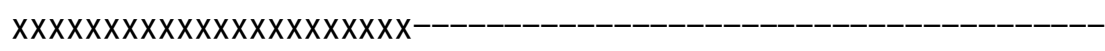

$-$ >rnolV_IV11 XP_341210.3

$\mathrm{m}-$ 


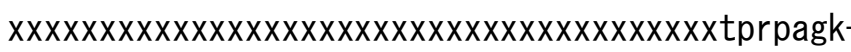

---hrvviphl

-nfvprnlfeqf

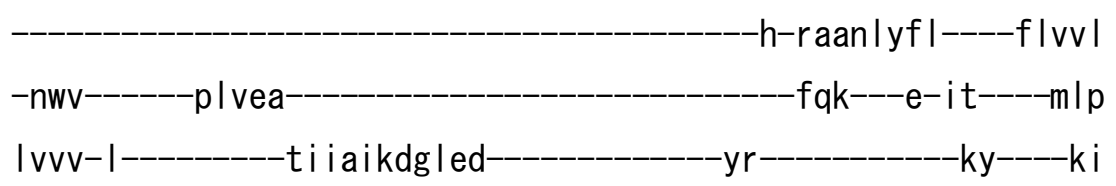

dkqinnlitkvys

$-x x e-k k y$ $-\mathrm{x}-------\mathrm{d}$

C

c-

----wknVTVGDF IRLsc

NE I IPADMVLLfs 
-tdpdg-i---chIETSGLDGESNLKQRqv-

-vrgyaeqdsevdpekfssr i ecespnnd I srfrgf

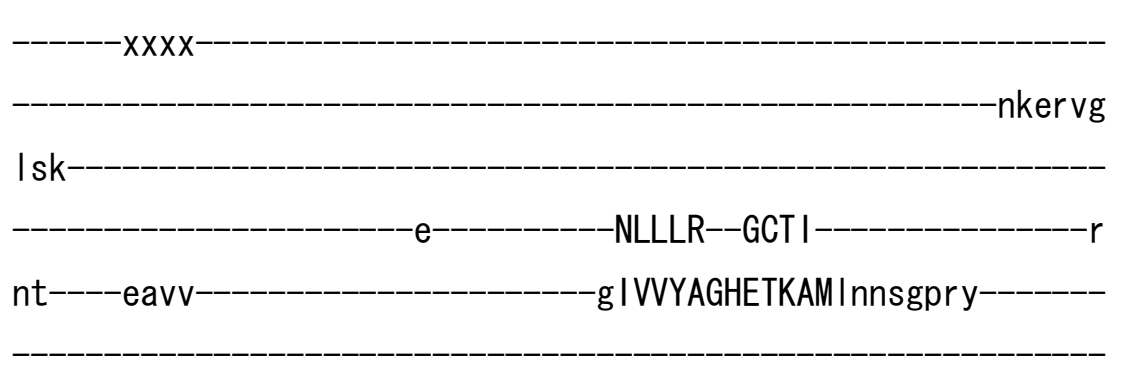

-krsklerrantdv IwcvIII ivmc Itga

- Ighg iw I sryenm|------xfnip---------xxxxxxxspv I tgfyvfwtmI ILLQVL

IPISLYVSIEIVKLGQIY-FIqsdvef-

-ynekmdst

i QCR-ALN I TEDLGQI QYLFSDKTG-

TLTENKMVFr

-rcsvagfdy

$-x x x x x x x x x x x x x x x x x x x$

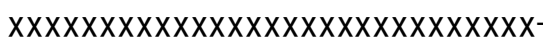

$-x x x x x--x x x x$

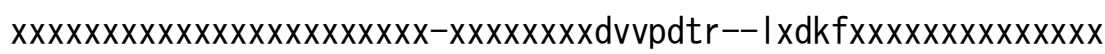

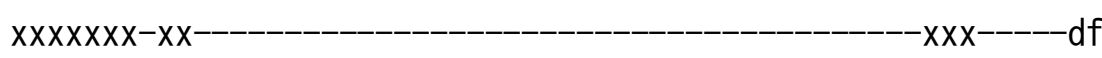

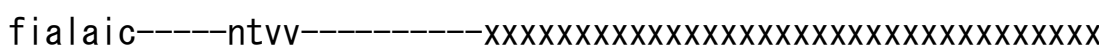

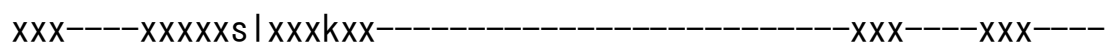

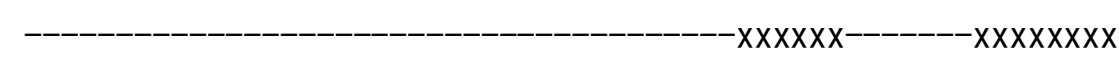

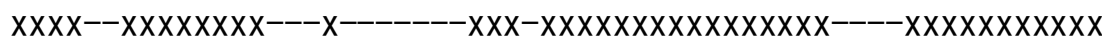
xxxxxxxxx--------------------- xxyeaespde--aal vya----ar-a----

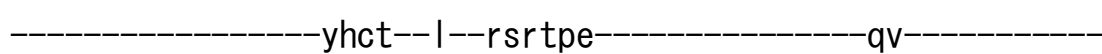

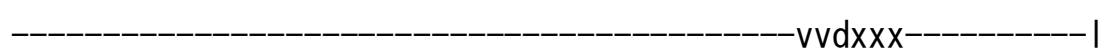

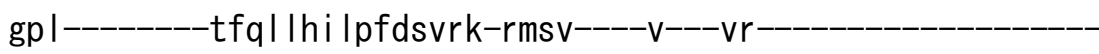
-

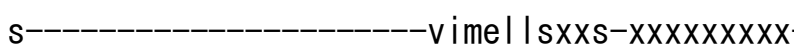


xxx--xx--ert-qrhIdey--akr----g|rtLCVAKKvm--sdteyaew I rnhf I--ae-----ts i dnr ee I I vesamr- I enk I t ILGATG IEDRLQEGVPESIEALHKag-IK I

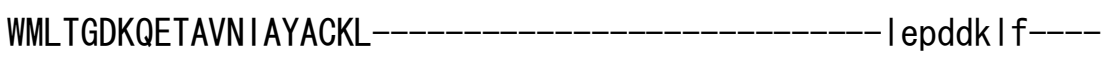

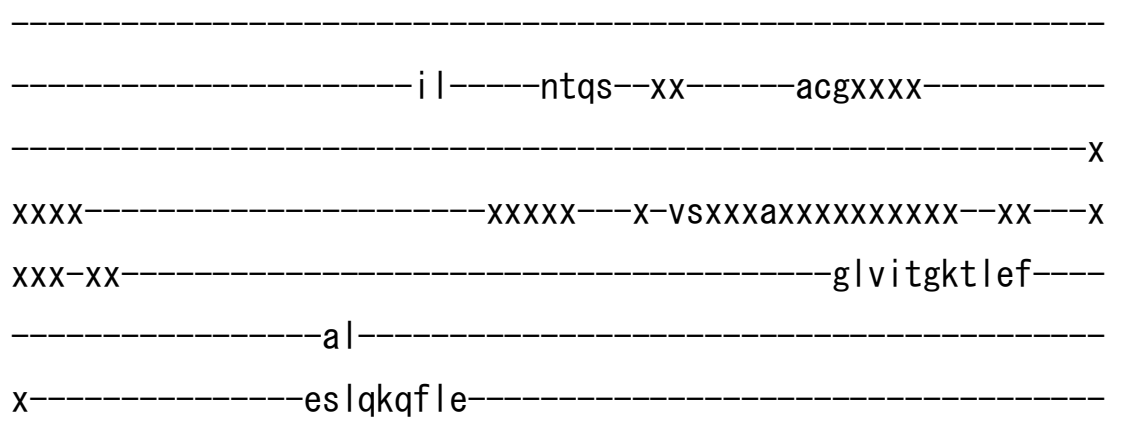

I tawcqav ICC--RATPLQKSEVVKLVr

---nh--- I h------------v----_--_----MTLA I GDGANDVSM I QVAD I G I GV--

AVMASDFAISqfRHLSKLL--LVHGHWCYTRLSNMI------L

yff-yknva-yvn I I fwyqffcgf--_-_-_-_-_-_-_-_-_-_---sgtsmtdywv l iff

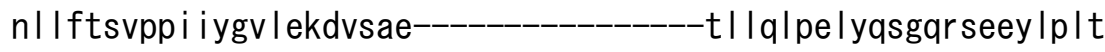
f-wit I I dafyqs I vcf-fvpy-fty-_-_-_-_-_-_-_--_xxxx--_-_-_-_-_-_-_-_ -d--iftfgnpIntaalli i IIh-Iviesks I twihml--------vivgsi I syfffala

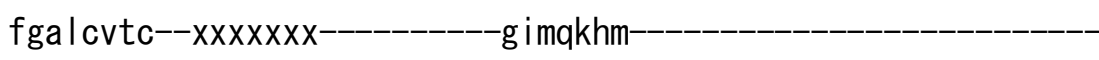
---------Idpvfy-----Iv--cvlttcval Ipr---------------------------

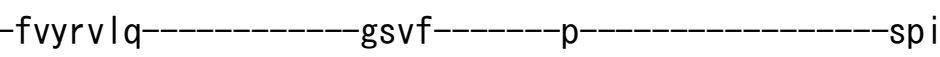
$-x x x x x x x x x x x x x x x x x x-$ 
$>$ rnolV_IV12 XP_225014. 4

$\mathrm{S}$

$X X X X X X X X X X$

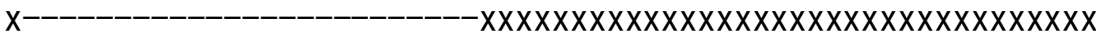

XXXXXXXXXXXXXXXXXXXXXXXXXXXXXXXXXXXXX| apaaqg--------

$-x X X X X X X X X X X X X X X-X X X-$

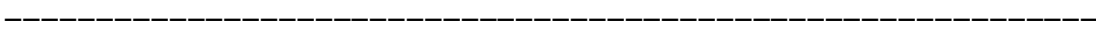

$-\mathrm{XXXXXXXX}$

---srtiyvgh

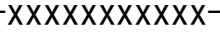

-parypdnr i vsskyt

fw-

-nfipkn I feqf 


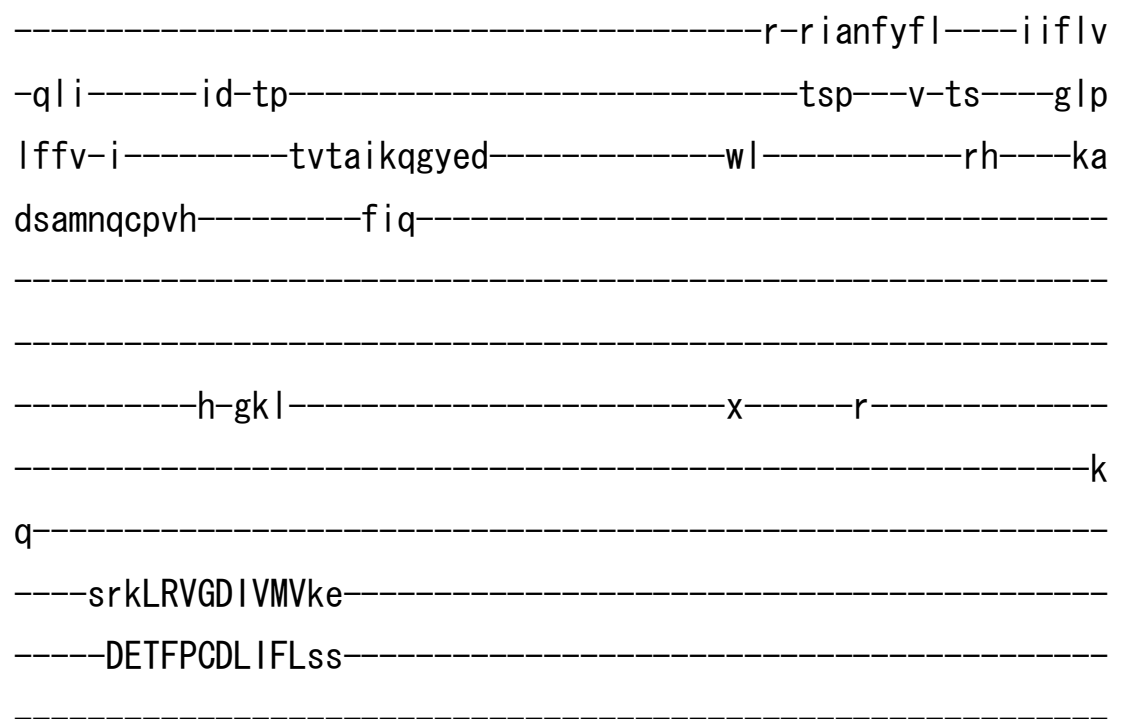

-nradg-t----chVTTASLDGESSHKTHya-

-vqdtkgfhteadvds I hat i eceqpqpd I ykfvgr invxx

$x x x$

dpvvrp

Igs

-NLLLR--GATL-----------------k

nt----ek if

gVAIYTGMETKMAInyqsksq-

krsaveksmntflivylcilvskalint

-v lkyvwqsepfrde------xwynq--------xxxxxxxxn I f I raftdf I afMVLFNY I

IPVSMYVTVEMQKFLGSY-FI twdedm-

fdeemgeg-

pLVN-TSDLNEELGQVEYIFTDKTG

TLTENNMAFk eccieghvy

xxxxxxxxxx Ipdssg i xxixsspxxxxx $-x x x x-$ If

fraiclc-----htvq $-x x x x$

$x x x x x x x x x x x-p d-x x s x x$ 


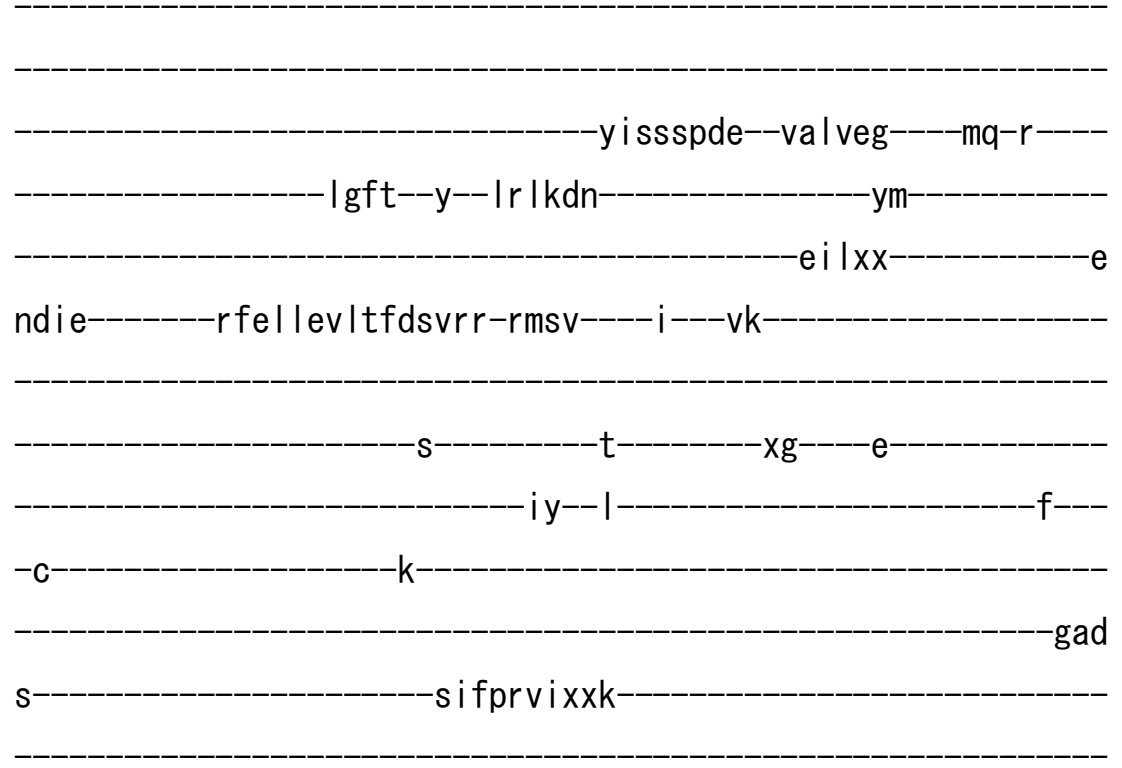

xdqv-rsrvern--ave-----g | rtLCVAYKr I--epeqyedacr I l qd--ak-----va I qdr ekk I aeayeq-i ekd I i ILGATAVEDRLQEKAADT IEALQKag-IKV

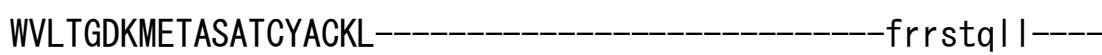

el------ttkk--xx-------eqsxxxx$-x x x x x x x x x x \operatorname{cs} x x x t x x x x x x x x x x$ $-x x d y g \mid$ i idgaa $|s|$ im$-X X X X X X$ x-x------------- gnyre Ifle-

icrncsavLCC--RMAPLQKAQIVKLIk

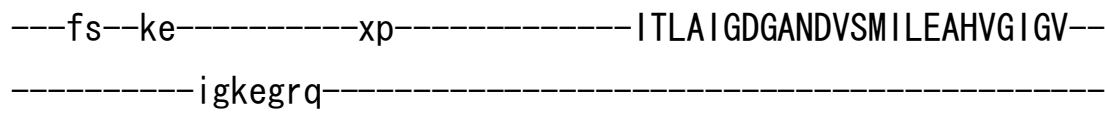

-AARNSDYAIPkFKHLKKML--LVHGHFYYIRI SELV------Q 


\section{nisftsIpillysImeqhvgid -vlkrdps Iyrdiaknal I rwr I}

>rnolV_IV13 XP_342229. 3

$\mathrm{m}$ 
---trtiyian

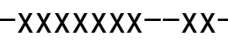

-pqkfidnr i isskyt

- vw----

-nfvpkn I feqf

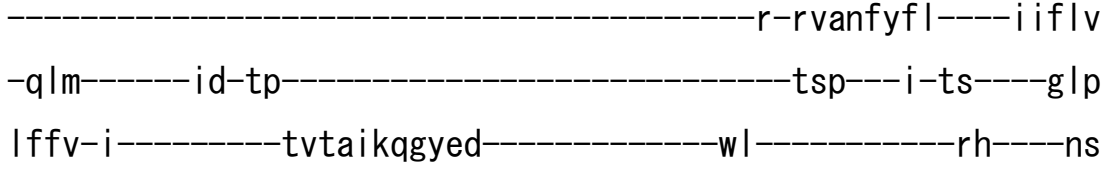

dnevngapvy----------vvr

t

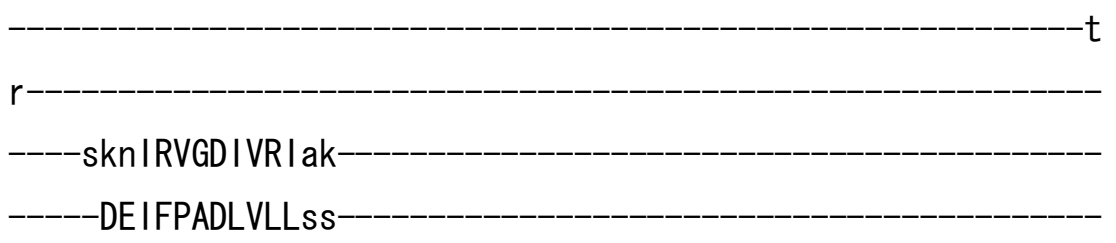

$-d r$ ldg-s---chVTTASLDGETNLKTHvs-

-vpetav Iqtvan Ids I i av i ecqqpead I yr fmgrmi i xx $\mathrm{xx}$

-eeivrp

$\operatorname{lgp}$

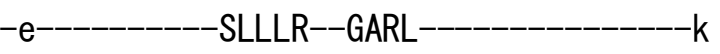

nt----ke i f----------_--_---------gVAVYTGMETKMA Inyksksq-------

-krsaveksmntfli iyli il iseai ist

-i I kytwqaeekwde------xwynq-------xxxxxxxxski I rf i sdf I afLVLYNF I

IPISLYVTVEMQKFLGSF-F I gwd I d I 
aQVN-TSDLNEELGQVEYVFTDKTG-

TLTENEMQFr

ecsinglky-

$-x x x x x x--x x x x$

$x x x x x x x x x x x x x x x x x x x x x x x x x x x x-x x x x x$ tspese $x x \mid x k e h x$

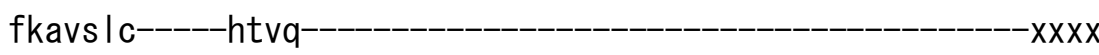

$x x x x x x x x x x x x n \mid x x x q x x-$

--yyasspde--kal vea-----aa-r----

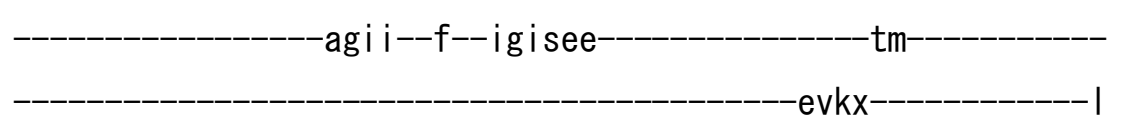

grve--------ryk I lhi lefdsdrr-rmsv-----i---vq---------------------

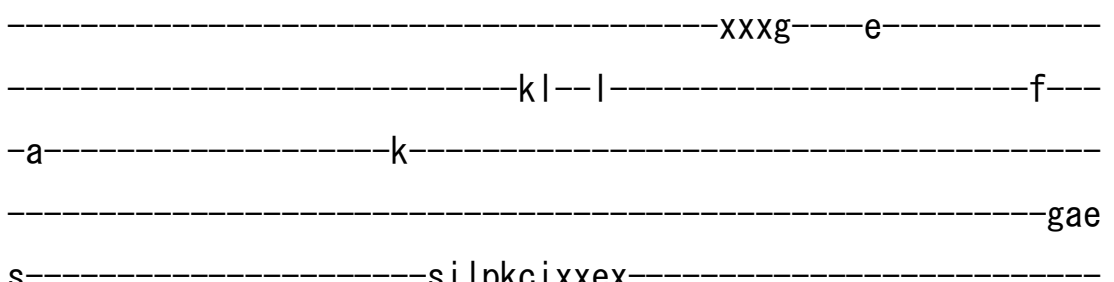

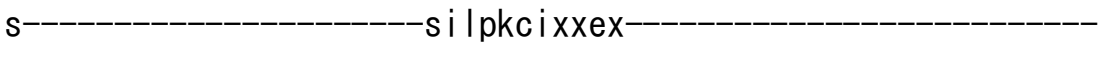

-akt-rihvdef--alk----g|rtLCIAYRqf--taeeyedvnr r Ife---

ar-----ts I qrqeek I agvfqy-i ekd I v ILGATAVEDRLQDKVRET IEALRMag-IKV WVLTGDKHETAVSVSLSCGH-_-_-_-_-_-_-_-_-_-_-_-_-_-_-_-_fhr tmn i I--_-

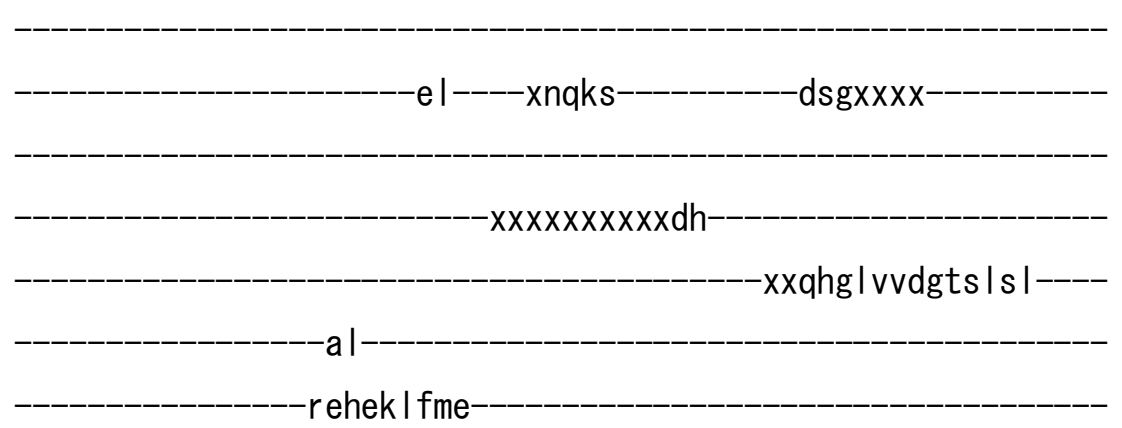


vcrncsavLCC--RMAPLQKAKVIRL Ik

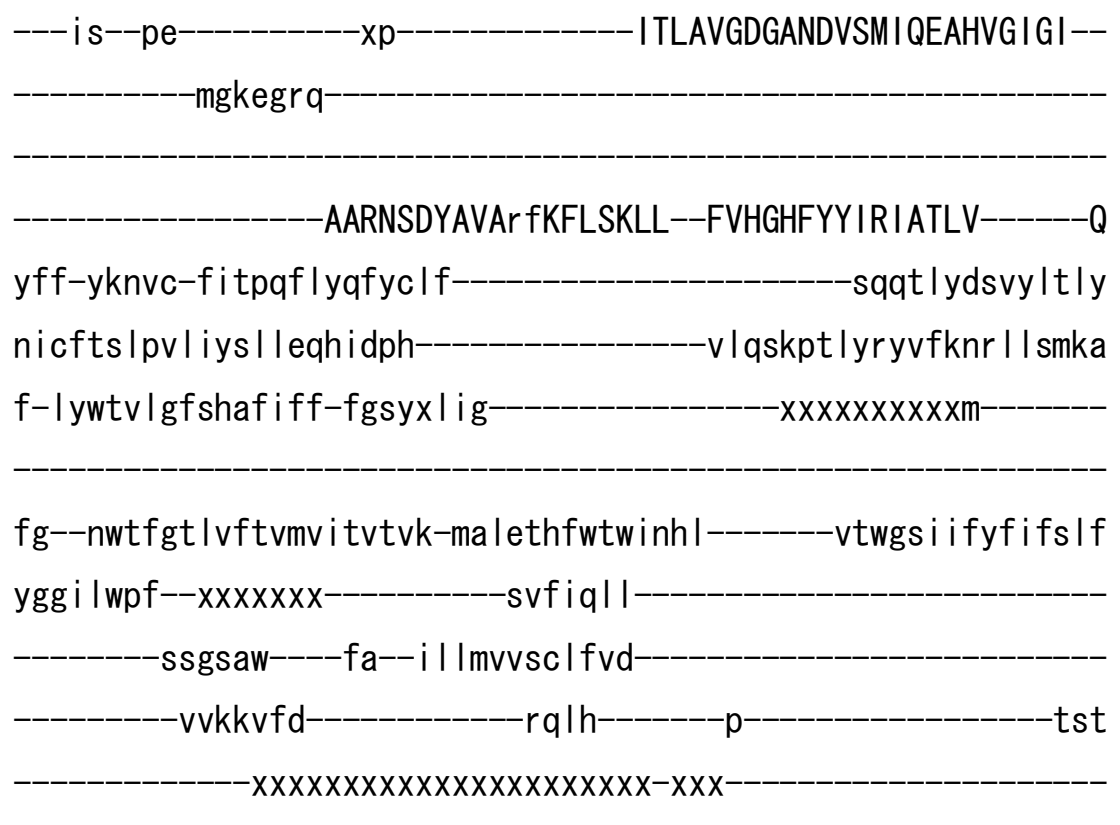

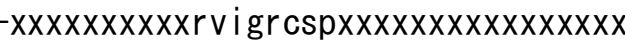

\section{$x x x x x x x x x x x x x x x$}

$-$

>osaV_V101 NP_001055495. 1

$\mathrm{m}-$ 
$x x x x x x x x x x x x x x x x x x x x x x s r \mid d f w$

flalyalw Ivvvvpaldftda

$\mathrm{p}$

Ivv Igal sashv laf I ftawsvdfrafvgyskarh I stpqspqssxxxxxxxxxvxx----

$x x x x x x x x x x x x x x x x x x x--$

qgnasev I g i egdrapaytenxxxxxxxxxxxxxarfi iyssqednffk

Iryptkepxxx

xxxxtgygtea

-xintavdkwgrni feypqpx

fqk Im

$-x x x x x e p f f v f q--v f c v g$

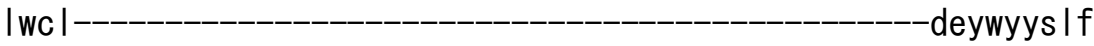

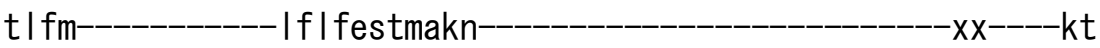

Itelrrvkvd-

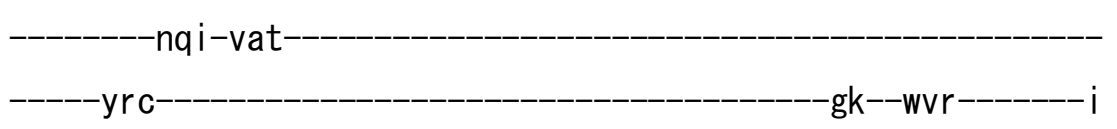

$\mathrm{p}$

---- gteLLPGD IVSI $g r---x$

$-x x x e D R S V P A D M L L L a g$ 


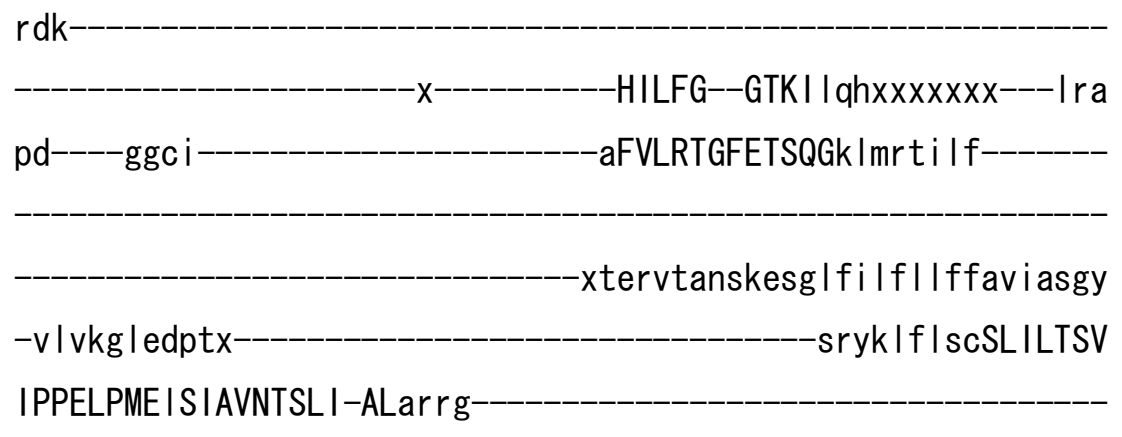

\section{¡FCT-EPFRIPFAGKVDICCFDKTG}

-TLTSDDMEFx$-x x x x$ ledde

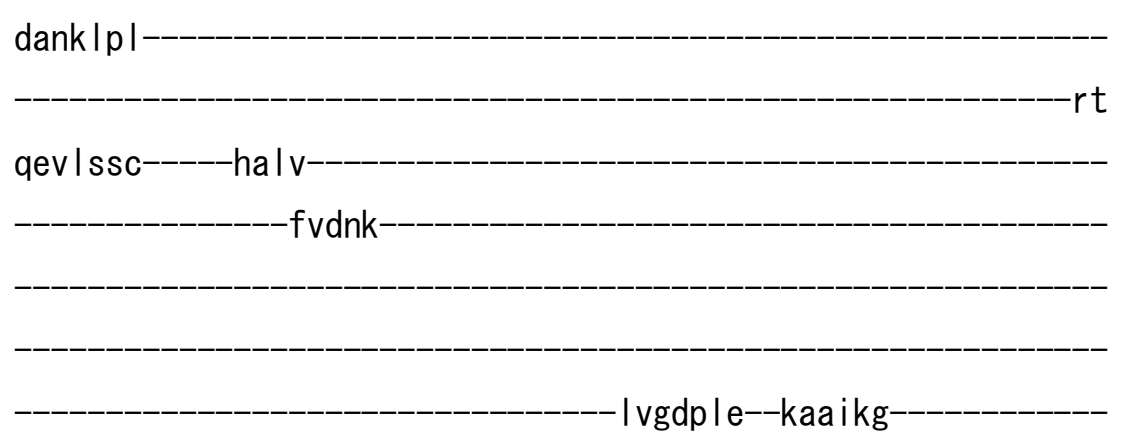

idwixxs--xxxais

-xxxxxxxxkivhryhfashlk-rmsvxxxx-

$-x x x x x x+--$

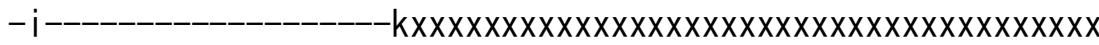

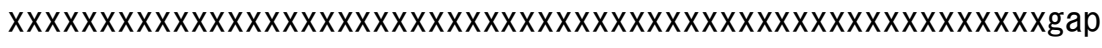

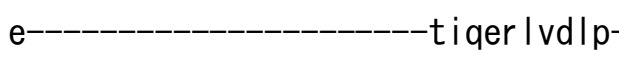


-agy-vetykky--trq----gsrvLALAYK I I

-xdmpvnear s l erdxvesd I tfAGFAVFNCPIRSDSGAVLQELEQss-HDL

VMITGDQALTACHVAGQVHI -cs--kpvl-ilt

rtk-----_-_--_----------g

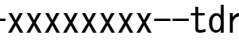

apysaee

vaavxgshd

lcisgdcfe

$-m \mid q--r-t d a v i q$

vipyv--kVFA--RVAPEQKELVLTTFk

--- tv--gr-

-VTLMCGDGTNDVGALKQAHVGIALXX

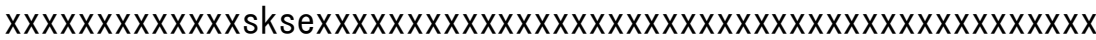

xxxxx---------------------------------------------------- $\mathrm{xxxx}$

$x x x x x x x x x x x x x x x x x$ ASMASPFTAKh-ASVAPTLd I IRQGRSTLVTTLQMF-------K

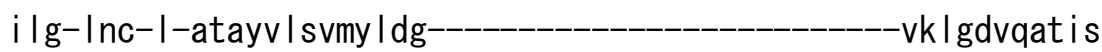

gvftaaff-Ifi sharp I-qt--_-_-_-_-_-_-_-_-- I saerph--_-_---xni fcayv

$f-\mid$ s i Igqfamxxffl i savneatkympeexxxxx-

se----fhpn I vntvsymvnmmi-qvatfavnymghpfnq-----si tenkpfkya lyaa

vafftvitxxmfrdlndymk lep I

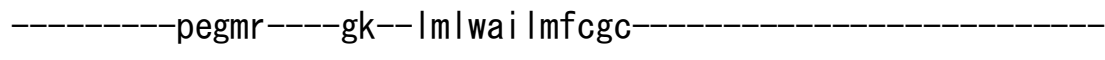

ygwer i I 
e

>aniV_V101 EAA63717. 1

$\mathrm{m}$

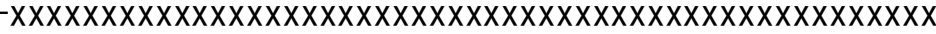

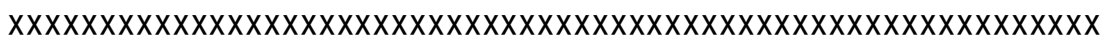

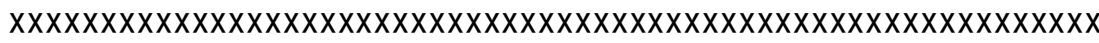
$X X X X X X X X X X X X X X X X X X X X X X X X X X X X X X X X X X X X X X X X X X X X X X X X X X X X X X V$ $X$ ip $\mid$

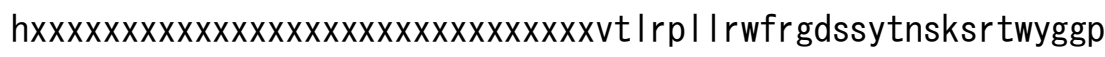
gsmp I ssmngarhprndpqsgnnywrpd i epqgnhgvvttvhgsp $x x x x x x x x p x x x x x x$ $X X X X X X X X X X X X X X X X X X X X X X X X X X X X X X X X X X----X X X X X X X X X X X X X X X X X X X X X X$

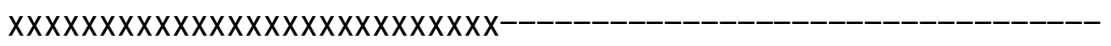
-_-_-_-_-_-_peansgsae i cr l qrdt I ggvxxxxxxxxxxxxxxfyperkcfap I syv Idaepkpaxxx

-xxxxeglttka-xi ervqhhygdntfdipvpx $-f i e l w$ 


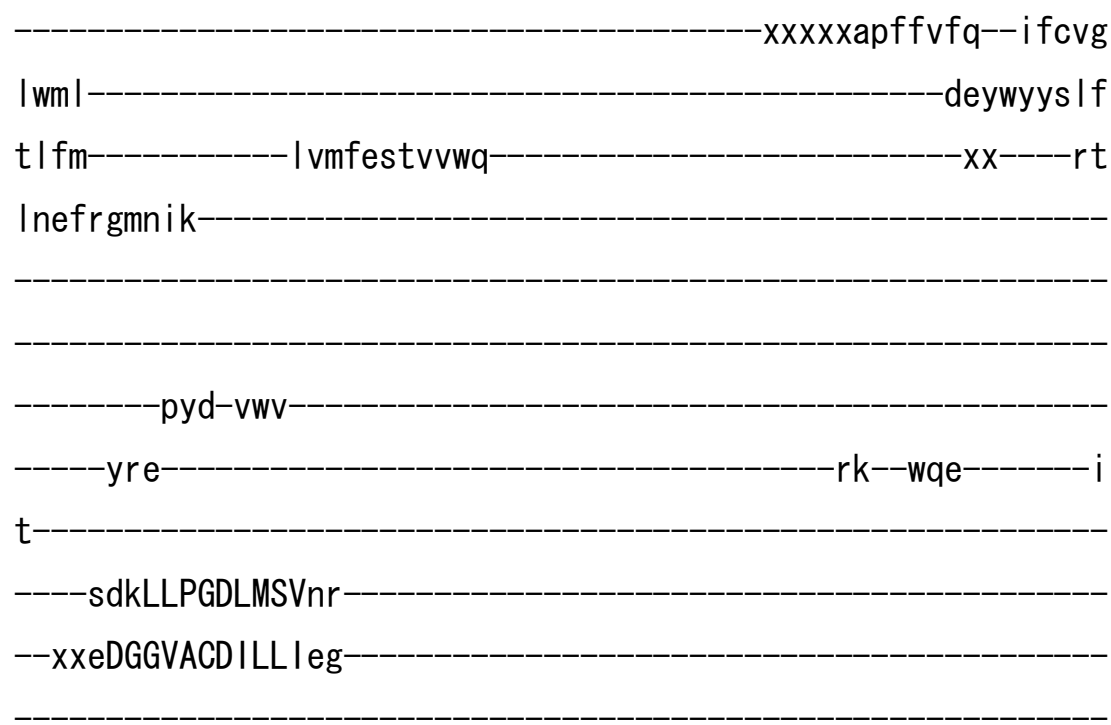

-svi VNEAMLSGESTPLLKes-

$-x X X X X X X X X X X X X X$

Idk

-AFVHG--GTKVI qi $x x x x x x x x x x s q q$

kt-----skvg----------------xxxxxxxxgVVVKTGFETSQGs I vr tmi

xtervsannaea I I f I f I I ifal aaswy

-vwqegvskdr

$-k r s k$ IIIdcVLIITSV

VPPELPME I SLAVNTSLA-ALskfa-

-iFCT-EPFRIPFAGRVDVACFDKTG

-TLTGEDLVVx$x x x x|a| g q$

$\mathrm{XXXXXXXXX}$

ahte Iskxxxxxx $-d t$

t|vlasa-----halv $-k \mid \operatorname{deg}$ 


\section{$-x v v g d p m e--k a t$ l qw-}

|gwtxxk--xxx|ts

$x x x x x x x x x x x x x q v k r r f q f s s a l k-r q s t x x x x v x x x r k$

$-x x x x x x s x x x$

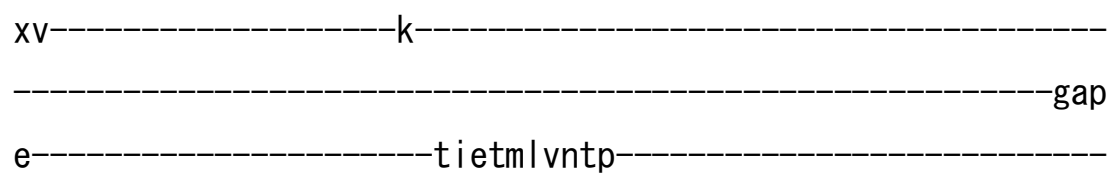

-pny-eetfkyf--trn----garvLALAYKy I

-xxxxe I sqgr innytrexi ese I i fAGFLVLQCPLKDDAIKAVRMLNEss-HRV

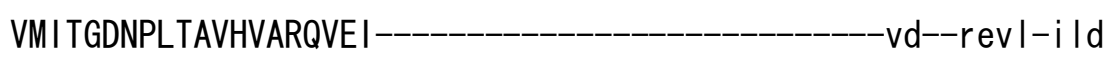

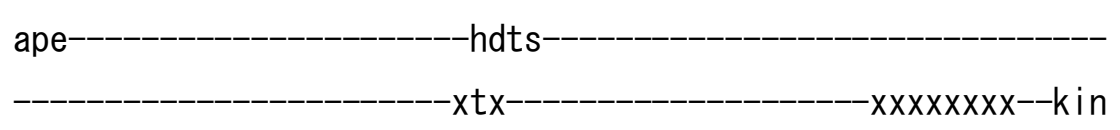
idvdptkp I-

$-d k e$ ixktkd-

icitghala-

$-k f k--d-q k a l p d-$

I rht--wVYA--RVSPKQKED ILLGLk

--- da--gy-

-TTLMCGDGTNDVGALKQAHVGVALXX

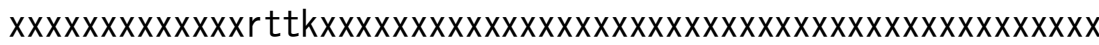

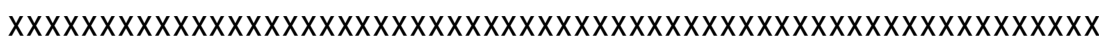

$x x x x x x x x x x x x x x x x x x$ ASVAAPFTSK I-ANV I AIPn i LRQGRCTLVAT I QMY-------K 
ila-Inc-I-isays Isviyldg

ikfgdgqut is

gmImsvcf-Isisraksv-eg

Iskerpq-------xnifnvyi

i-gsvlgqfaixxat l iylsnyvys i eprdxx

vdxxxxfeps I Insaiy I lql iq-qi stfs inyqgrpfre-----si renkamywg I vaa

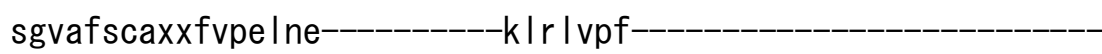
-snefk----vt--| tv Imaldyagc-

-wvienvl

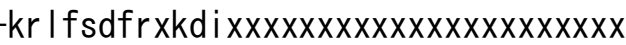

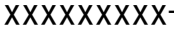

a

$>$ tpaV_V101 XP_763395.1

m-

-xxxxxxxt Iykyrsnqalyir Ispvf।Isvlal If 


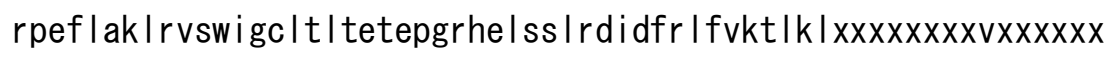

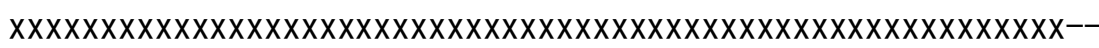
nrtnrv|lplyrtgfi yyihdxxxxxxxxxxxxxepvehfdevqvpkv

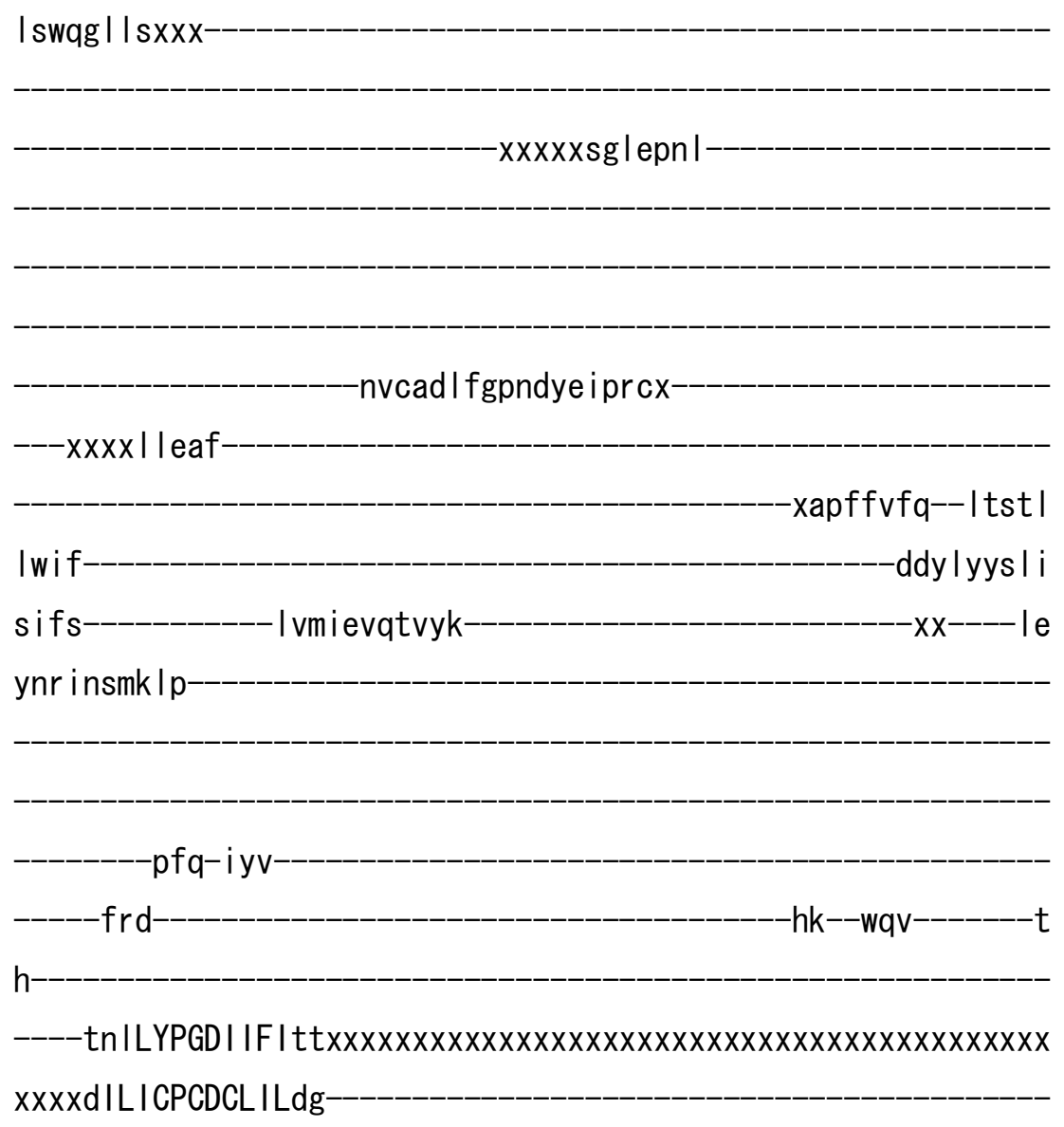

ev iVDESILTGESVPQFKts-

$x X X X X X$

nar

-STIFS--GTSI i vtx----------nva

vt----gtpaxxxxxxxxxxxxxxxxxxxxxxcLVIRTGFESYQGr I vhs i In-

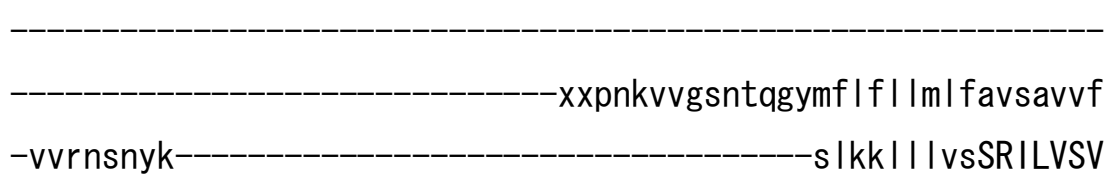

IPPEFPVT i SMAVT I GL I-QLrkkg- 
- YYCT-APNRLPLAANIDVIAFDKTG-

$-T L T Q D Q M Y L x-$

xxxxsdsvn

$x X X X X X X X X X x$

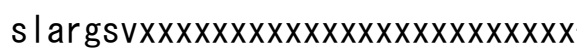

-ys

klviagc-----hslt-

----------------fvnga------1------

$-x x x x x x x x x x x x k i \mid k r w r f t s e l g-r m s t x x x$

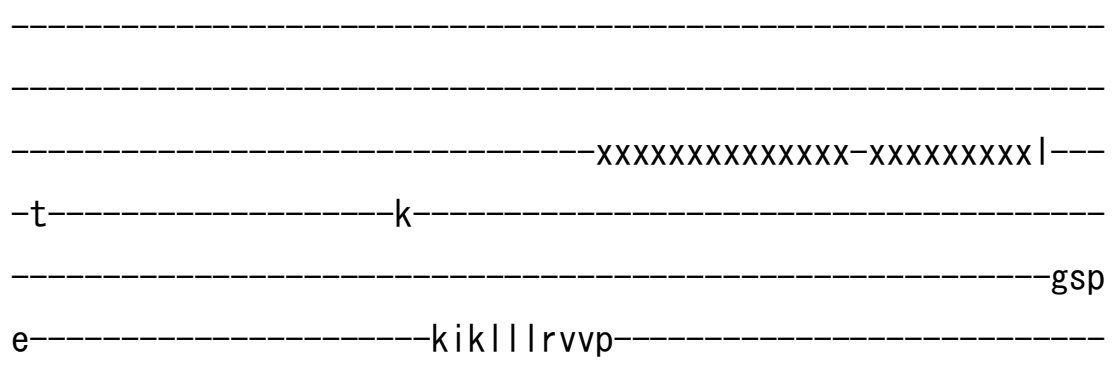

-syf-devchdl--tik----g|rvLCLAYKr I

-xdipvnal i tidrtxvekd l efCGFLALEAPIKKSSKQCMKRLKN---FKK

IMI TGDN I LTACYVTQQ I NM--_-_-_-_-_-_-_-_-_-_-_-_-_-_-vnxxttcpxnfs

qkn-------- xxxxxxxxxxxxxxhs If $x x x x x x x x-x x x x x x x x x x x x x x x x x x x x x x x x$

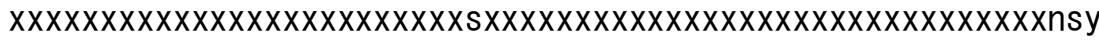

kkf lescpf $x x x x x x x x x x x x x x x x x x x x x x x x x x x x x x x x x x x x x x x x x x x x x x x x x x x x x$

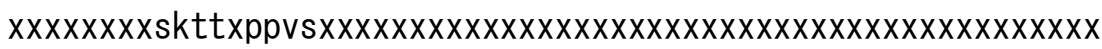

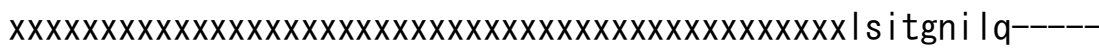

- I Innnxtn--------- xxxxxxxxxxxxxxxxxxxxxxxxxxx 


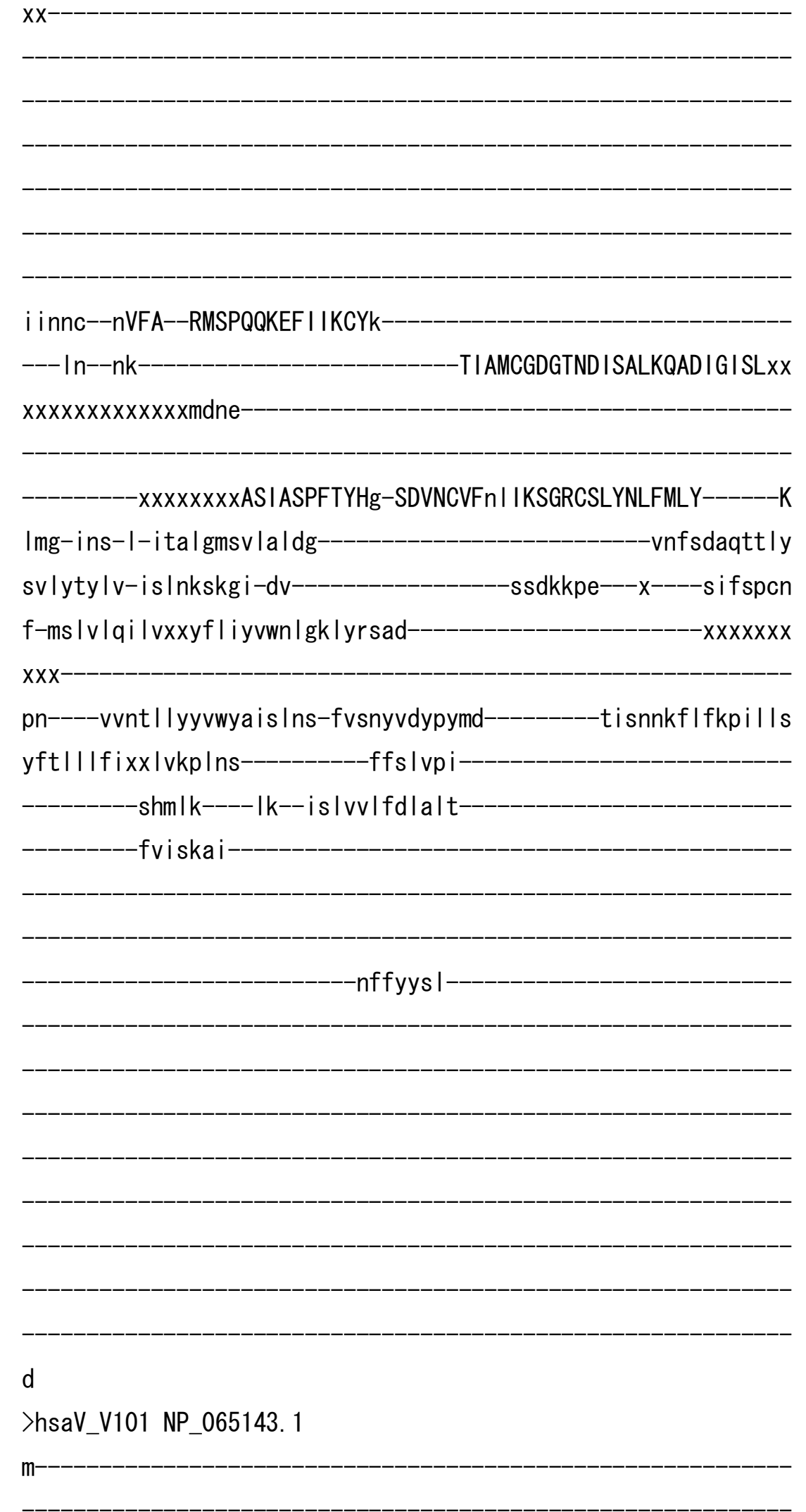


dpskatfvkvvptpnngste Ixxxxxxxxxxxxxv I sfefqkikysyd alekkqfIxxx

\section{xxxxxxxxxxxxxrgfqeds}

-xi raaekkfgsnkaemvvpx

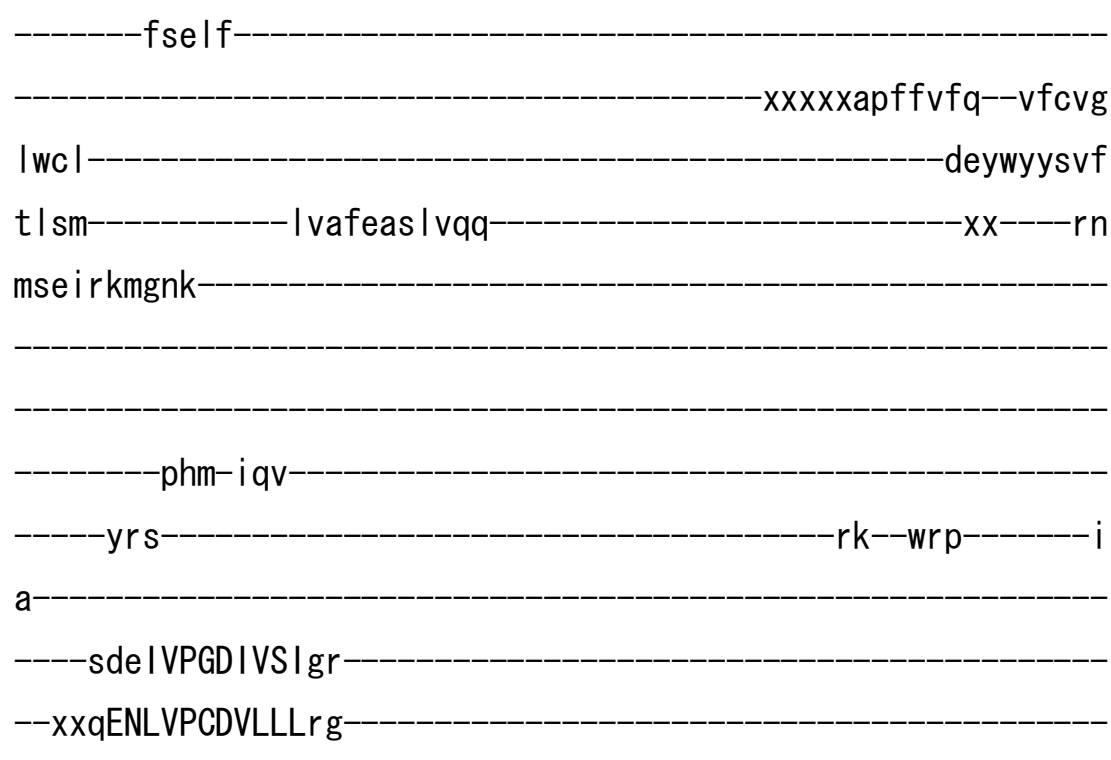


-rc iVDEAMLTGESVPQMKep-

$x x x x x x x x x x x x x x x x x x$

$-H V I F G--G T K V v q h x$

$-p p q$

ka----ttgl--------------xxxxxxxxaYVLRTGFNTSQGk I r r i If

-xvkrvtannletfifilfIIvfaiaaaay

-vwi egtkdpsx

-nryk IflecTLILTSV

VPPELPIE I SLAVNTSL I-ALak I y-

-mYCT-EPFRIPFAGKVEVCCFDKTG

TLTSDSLVVx

xxxx Irdgk

$-X X X$

pvssipv

et

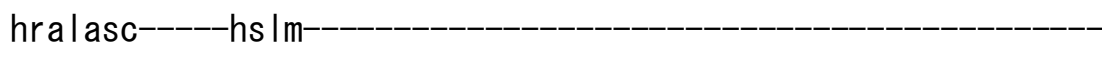

$q l d d g$

$x|\operatorname{vgdp}| e^{--k a m} \mid$ ta

vdwtxxk--xxxvfp-

-xxxxxxxxkihqrfhfasalk-rmsvxxxxexxxst

$-x x x x x=--$

-v--------------------- $\mathrm{k}$

gap

e-

tI hsmfsqcp 
-pdy-hhihte i--sre----garvLALGYKe I

-xhI thqqar evkrex l ecs I kfVGF IVVSCPLKADSKAVIREI QNas-HRV VMITGDNPLTACHVAQELHF i e--kaht-li |

qpp---_--_-_-_

-hlqatd-pqq I r

I iphv--qVFA--RVAPKQKEFVITSLk

$---e$ l--gy-

-VTLMCGDGTNDVGALKHADVGVAL $x x$ xxxxxxxxxxxxxydsp------------------------- xxxxxxxxxxxxxxxxxxxx xxxxxxxxxxxx------------------------------------------- $\mathrm{xxxx}$ $x x x x x x x x x x x x x x x x x$ ASIAAPFTSKI-SSIQCIChv IKQGRCTLVTTLQMF------K

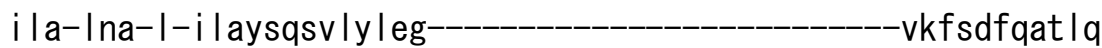

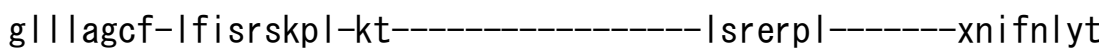
i-|tvm|qffvxx|s|vylyreaqarspekxxxx

vdxxxxfeps I vnstvy i mamam-qmatfa i nykgppfme------s I penkp I vws I avs I la i I I Ixxspdfnsqfglvdi -pvefk----|v--iaqvi||ldfcla- ladrv| 
S

>ddiV_V101 XP_629288. 1

$-x x x x x x x x x x x x x x x x x x x|h| n v i$

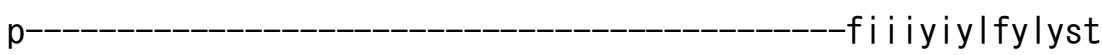

tp i ep i innnnnnngtt I iksndss i ggd i sndt i innnnnstes $x x x x x x x x q x x----$

$-x x x x x x x x x x x x x x x x x x x x x x x$

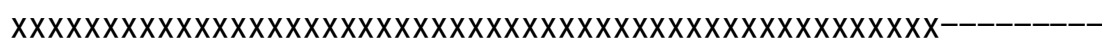

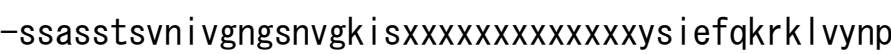
dkkqfekixxx

$-x x x x x x x x-x x x x r$ syetde

-x i e l aamkyg I nrfd i p i px

$-f|a| y$ 

eeyvfyclf

$\mathrm{s} \mid \mathrm{fm}$ Ilvfeatvvks

$-\mathrm{xx}----\mathrm{sn}$

Ins Irnmssk

-gcVVNEAMLTGESTPHHKes-

$n-$

----tte ILPGDIVSIgr

xxxxaTSTLPCDMILLsg

$-x x x x x x$

nek

HILYG--GTTI vqhx-

pse

kl----arvs-

-xxxxxxxxaYAYKTGFNTNQGr Imrtiwf

xservtannkesfIfi IfIItfaiaasay

-I fnkg i rennx-

-skyk I I Inc IMVITSV

VPPELPME I SLAVNNSLI-SLik Ig-

- YYCT-EPFRIPFAGKVDVCCFDKTG

$-T L T T D D L V L x-$ xxxxcpkky

$-x x x x x x x x x x x x x x$

tttdttt $x x x x x x x x x x x x x x x x x x x x x x x x x x x x x x x x x x x x x$

$-v i$

qf i lagc-----hs Iv

l idnk- 


\section{- | vgdpme--mag |ks}

ipftxxa--xxxshq

-xxxxxxeivhryhfsselk-rmttxxxxvxxxn I

$-x x x x x f---$

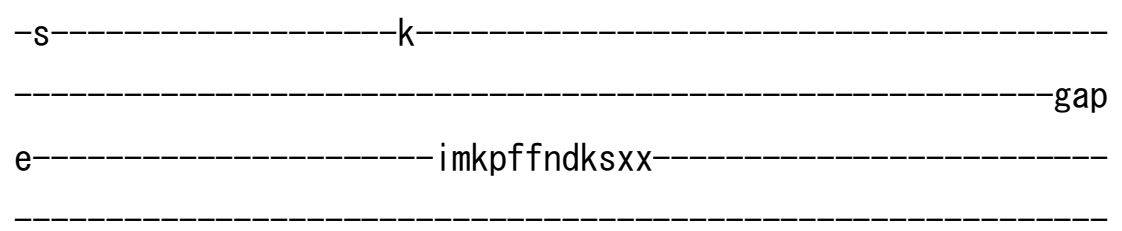

-dny-dqcfksy--srq----gsrvLALGYKrf

-xxx Invsqyksmerds-vesn I efGGF I IFDCPLKPDSKESIEMLMNss-HR I

VMITGDNSLTACHVGKQLGF$-v q x x k q t i-i l q$

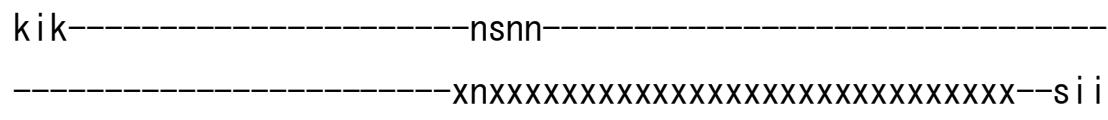

ip lengnenx

Inql xkdyn

|ciggns Id

lvi--g-dknlek

d l y l v--kVFA--RVSPEQKQMILTNFk

$---\mathrm{vn}--\mathrm{gh}-$

-YTLMAGDGTNDVGALKQAHVG IAI Xx

$x x x x x x x x x x x x x$ ifn

$-x x x x x x x x x x x x x x x$

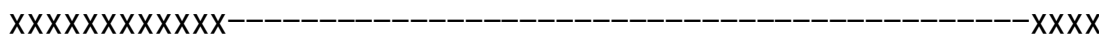

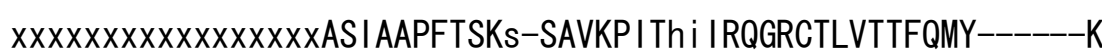




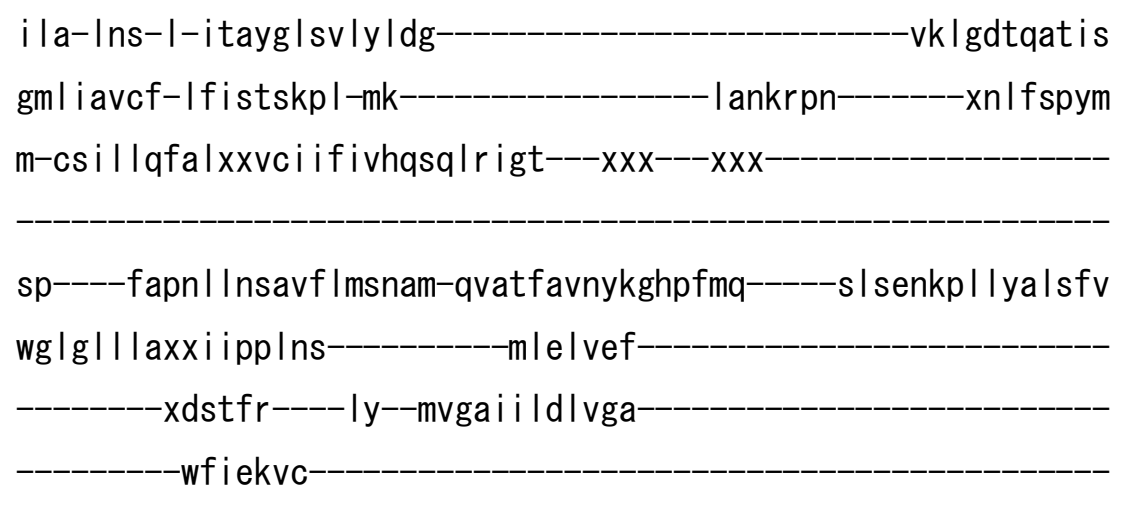

svl Ikn

>cneV_V101 XP_568028. 1

$\mathrm{m}-$ 
pkkgrgkge i vp Idrktapgaxxxxxxxxxxxxxyvynradnvftp ip ypcdssppxxx

$-x x x x$ gi i thp

----xxxxxxxxxxxxxxxlealkatyg Inechipipx

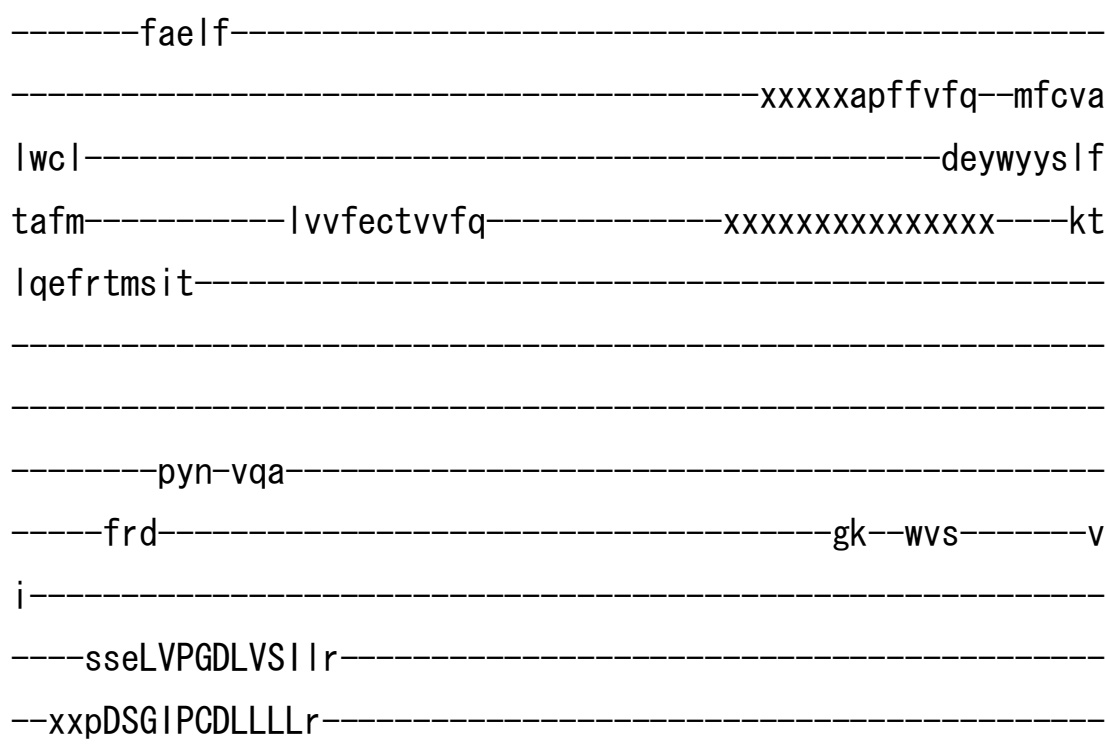

$-x-x---c i$ VNEAMLSGESTPLLKes-

$-X X X X X X X X X$

$x x x x$

$\operatorname{adr}$

$-x$

-NVLFS--GTKAI qvxxxxxxxx---mtt

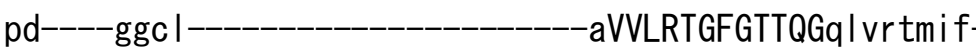

-xtervsantfeaf I figf I I ifaiaasay

-vwikglergm -vkgk I I IdcVLI ITSV

VPPELPME I SLAVNASLV-ALqkya 
iFCT-EPFRIPWAGRVDVCCFDKTG-

-TITGEDLVVx-

xxxxvnsad

$-X X X X X$

pvtesnk-

et

t la laaa-----halv

$-1 \mid d d g$

$-x$ i vgdpme--ktt laa

Idwkxxr--xxxisp

--xxxxxxxxxxxhirrryqfssalk-rmstxxxxsxxxgk

$x \times x \times a---$

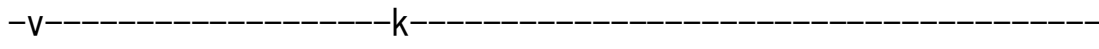

-gap

e----------------------t I ksmyvqvp

-dwy-eetyrwy--trr----gsrvLALGVKym

-dvqadkinqihrdxveck I sfAGFLVFHCPLKPDAVETLKMLNDss-HRC

VMI TGDNPLTAVHVARDVE I---_-_-_-_-_-_-_-_-_-_-----vd--revm-i Id

Ike----------------------gtts-------------------------------

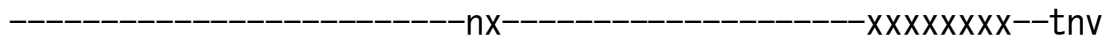

ipvnssepf-

-dqs I xknyd

icitgaalk

-qyd--a- |psvtd- 
I ikht--yVYA--RVSPAQKEF I ITTLr

$---s \mid--g y-$

- ITLMAGDGTNDVGALKAAHIGVAL $x x$

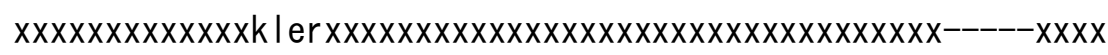
$x x x x x x x x x x x x$ $-x x x x x x x x x x x x x x$ $x x x x x x x x x x x x x x x x x$ ASCAAPFTSK I-SNVSAISn i IRQGRCTLVAT I QMY------K i la-Inc-l-itays I svay ldg--_-_-_--_-_-_-_-_-_-_-_--ikfgdyqut it

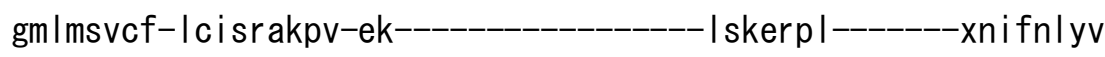
I-Isvl Iqfaixxvalvyitglsksledrgx

idxxxxfept I Intaiy | |g | sq-qvstfv Infqgrpfre-----gi renpp I yyg I I gv savaycgaxxfvpelnr- w q q vem-

-ttsfr----vk--Itismvldf i lc -wai ektc-

-kal fadlexvefxxxxxxxxxxxxxxxxxxxxxx $x x x x x x x x x x x x x$

q >athV_V101 NP_197752. 1

$\mathrm{m}-$ 
$x x x x x x x x x x x x x x x x x x x x x x x w r \mid d v w$

failytvwlttivpsidfsda

cialgg|safhi IvIIfttwsvdfkcfvqfskvnsidqadackvtxxx

-fsgskevvp Ihfrsqmtdsasxxxxxxxxxxxxxqrfi iyske Igafsk Ipyptketxxx

xxxxtghgtea

-xiatatekwgrnvfdypqpx

fqk Im

$-x x x x x e p f f v f q--v f c v g$

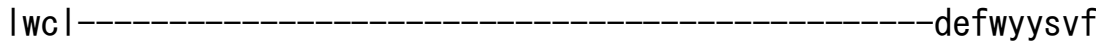

t I fm--_-_-_-_-_-- I fmfestmaks--_-_-_-_-_-_-_-_-_-_-_-_---xx----'kt

Itdlrsvrvd-

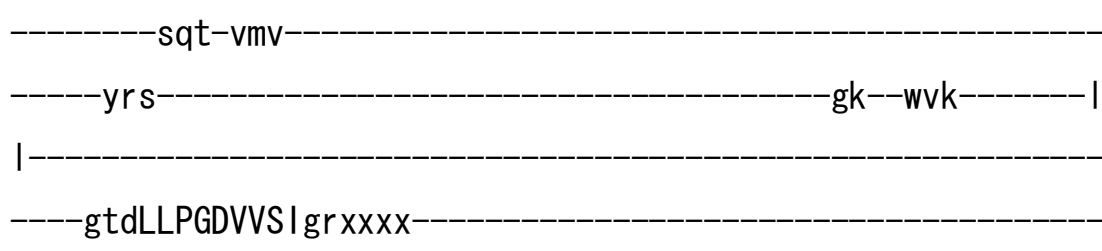

$-x x x e D K T V P A D M L L L v g$ 
-sa iVNEAILTGESTPQWKvp-

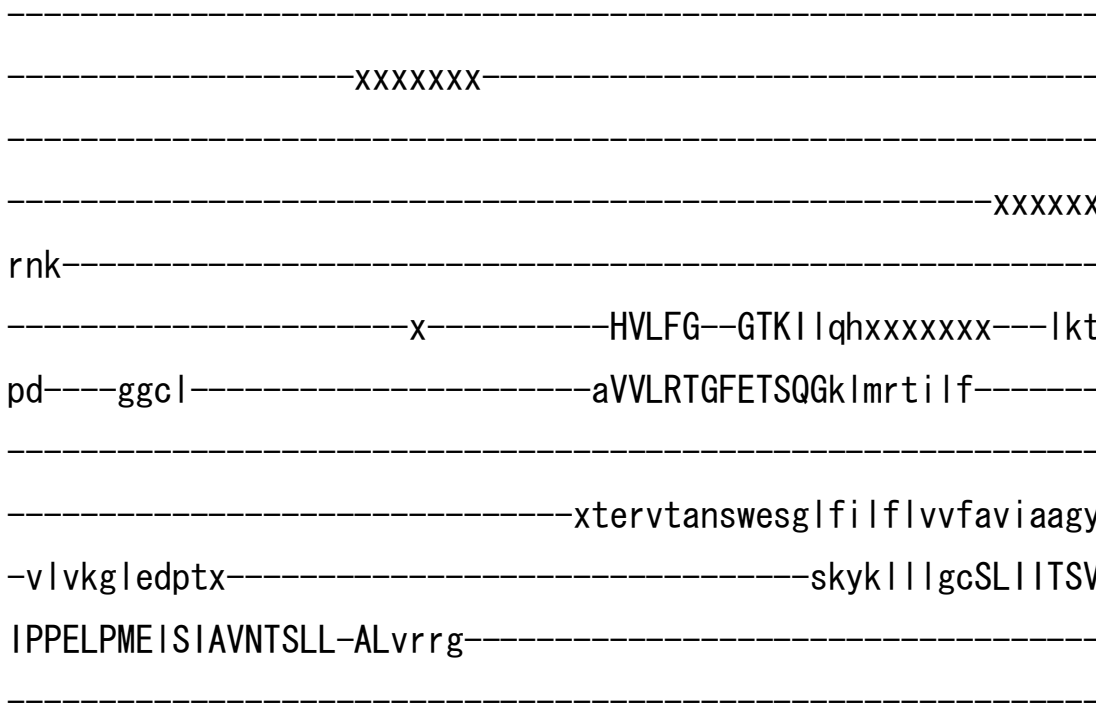

iFCT-EPFRIPFAGKVDLCCFDKTG

TLTSDDMEFx

xxxx I snce

$-X X X X$

dmskvpv

$-r t$

le ilasc-----halv-

fvenk

- Ivgdp le--kaa lkg

idwsxxa--xxxalp

-xxxxxxxxqimqryhfashlk-rmsvxxxx-

$-x x x x x f---$

-v---------------------k

gap

e------_--_-_--_--_-_---ti i ger I vdvp 
-aqy-i etykry--trq----gsrvLALAYKr I

-xdmmvseardmdrdxvesd I tfAGFAVFNCPIRPDSAPVLLELKNss-HDL

VMITGDQALTACHVAGQVHI $-v s--n p v|-i| g$

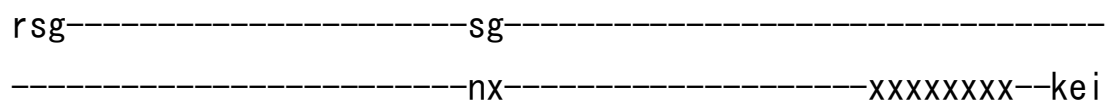

ipyseke-

-i etl xethd

lciggdsie

$-m|q--a-t s a v| r$

vipfv--kVFA--RVAPQQKELILTTFk

$---a v--g r$

-GTLMCGDGTNDVGALKQAHVGVALXX

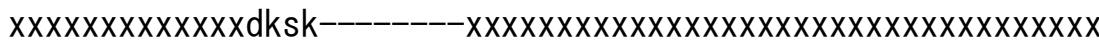

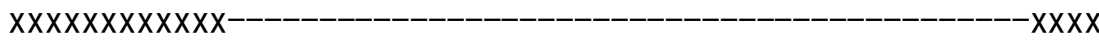

$x x x x x x x x x x x x x x x x x$ ASMASPFTAKh-ASVAPVTd $i$ IRQGRSTLVTTLQMF-------K

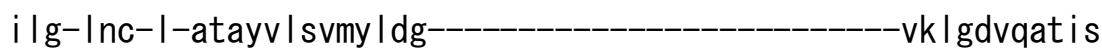

gv| taaff-Ifi sharp |-qt--_-_-_-_-_-_-_-_--| saerph-_-_-_--xsvfsvy |

f-Is I igqfavxxtf I vysvkeaekhmpeexxxxx-_-_-_-_-_-_-_-_-_-_-_-_-_-

as-----fhpn I vntvsymvsmm I-qvatfavnymghpfnq-----si renkpffyal i ag

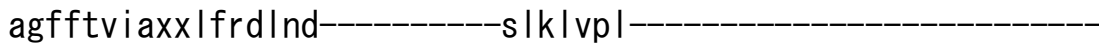

-pqg | r-----dk-- I I iwas Imf i ic

-yswer II 
>mmuV_V101 NP_573487.1

$$
\text { m- }
$$

$-x x x x x x x x x x x x x$

$x X X X X X X X X X X X$ $\mathrm{p}$

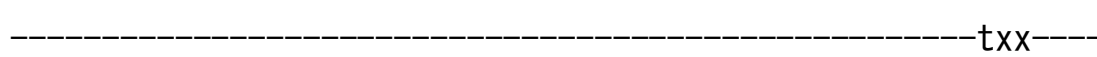

$-x x x x x x x x x x x x x x x x x x x x x x x$

\section{$x X X X X X X X X X X X X X X X$}

dpnkvtfvkvvptpnngste $|x x x x x x x x x x x x x v|$ sfefqkikysyd alekkqf I xxx

$-x x x x x x x x x x x x x r g f q e d s$

-xi raaekkfgsnkaemvvpx

$-f$ self 


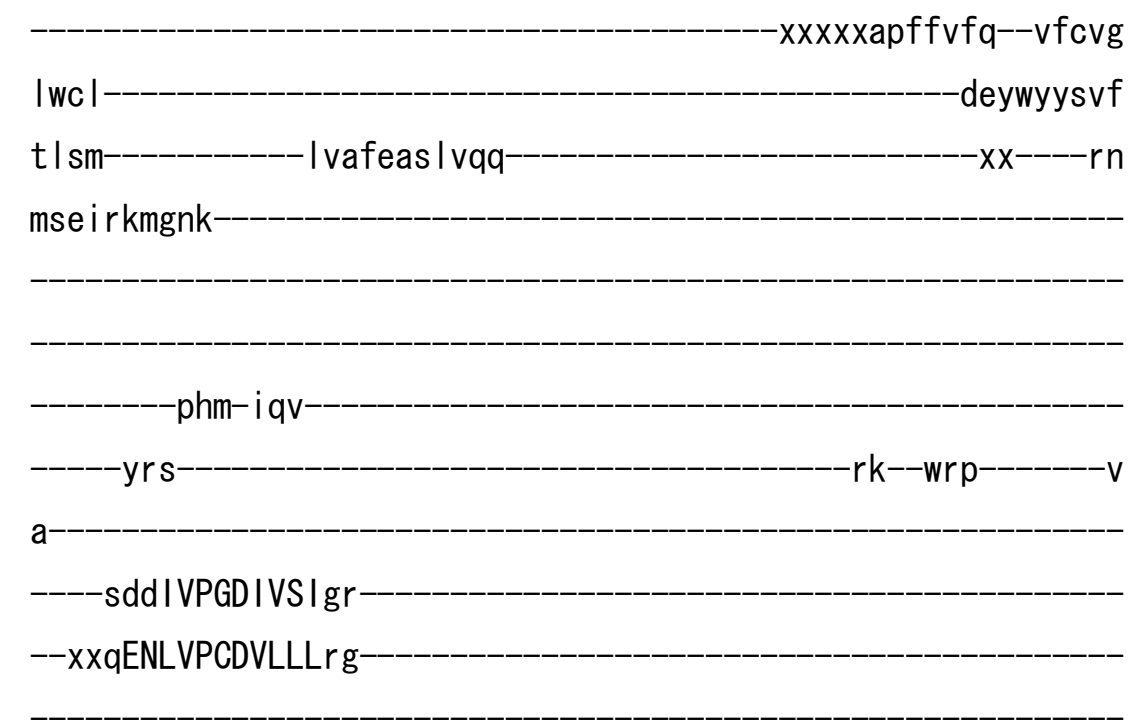

-rciVDEAMLTGESVPQMKep-

$-x \times X X X X X X X X X X X X X X X$

$-H V I F G--G T K V v q h x-$ $-p p q$

ka----tsg|--_-_-_-_-_-_xxxxxxxxaFVLRTGFNTSQGr I Irti i f

-xvkrvtannletfifilfIIvfaiaaaay

-vwvegtkdpsx

nryk IflecTLILTSV

VPPELPIE ISLAVNTSL I-ALak I y

-mYCT-EPFRIPFAGKVEVCCFDKTG

-TLTSDSLVVx---------------xxxx I rdgk-

$-x x x$

pvss i pi--_-_-_-_-_-_-_-_-_-_-_-_-_-_-_

hralasc-----hs $1 \mathrm{~m}-$

$-q \mid d d g$ 
$-v d w t x x k--x x x v f p$

-xxxxxxxxkihqrfhfasalk-rmsvxxxxexxxst

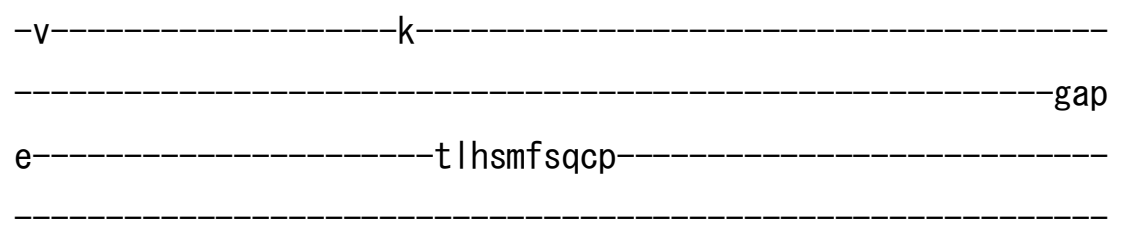

-pdy-hhihte i--sre----garvLALGYKe I

-xhI thqqar e ikrex l ecs I kfVGF IVVSCPLKADSKAVIREI QNas-HRV

VMI TGDNPLTACHVAQELHFid--kaht-li |

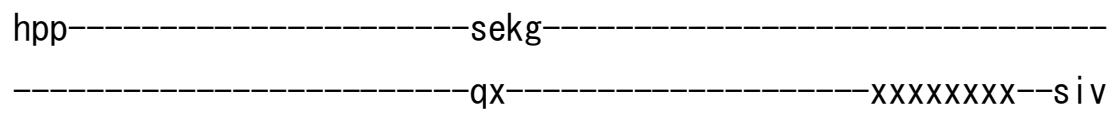

\section{Ip|t|gs}

-pkalx leha

$|c| \operatorname{tgdg} \mid a$

hlqavd-pqq I lc

I i phv--qVFA--RVAPKQKEFVITSLk

---el--gy-

-VTLMCGDGTNDVGALKHADVGVAL $x x$

$x x x x x x x x x x x x x r d s p$

$-x x x x x x x x-x x x x x x x x x x x$

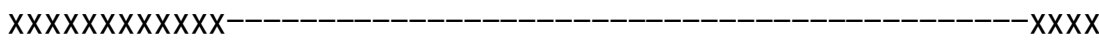

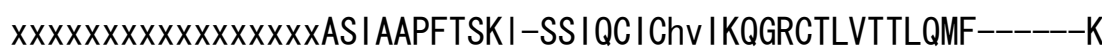


i la-Ina-I-i l aysqsv ly leg--_-_-_-_-_-_-_-_-_-_-_--_vfsdfqat la

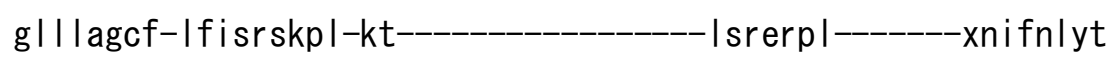

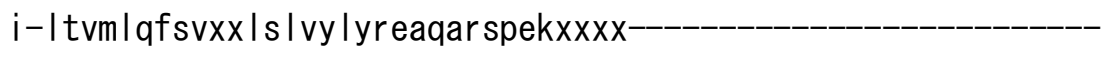

vdxxxxfeps I vnstvy imamam-qmatfa inykgppfme------s I penkp I vws I avs I lai ig | |xxsspdfns---_-_--_---qfg I vdi-_-_-_-_-_-_-_-_-_-_-_-_-_-_ -pvefk----|v--igqvlaldfcla

- ladrvl

qff Igtpkxrvp

S

>rnoV_V101 XP_214310.4

$\mathrm{m}-$

$-\mathrm{XXXXXXXXXXXX}$ 
$x x x x x x x x x x x x x x x x$

dpnkvtfvkvvptpnngste $|x x x x x x x x x x x x x v|$ sfefqkikysyd

al ekkaf I $x x x$

xxxxxxxxxxxxxrgfqeds-

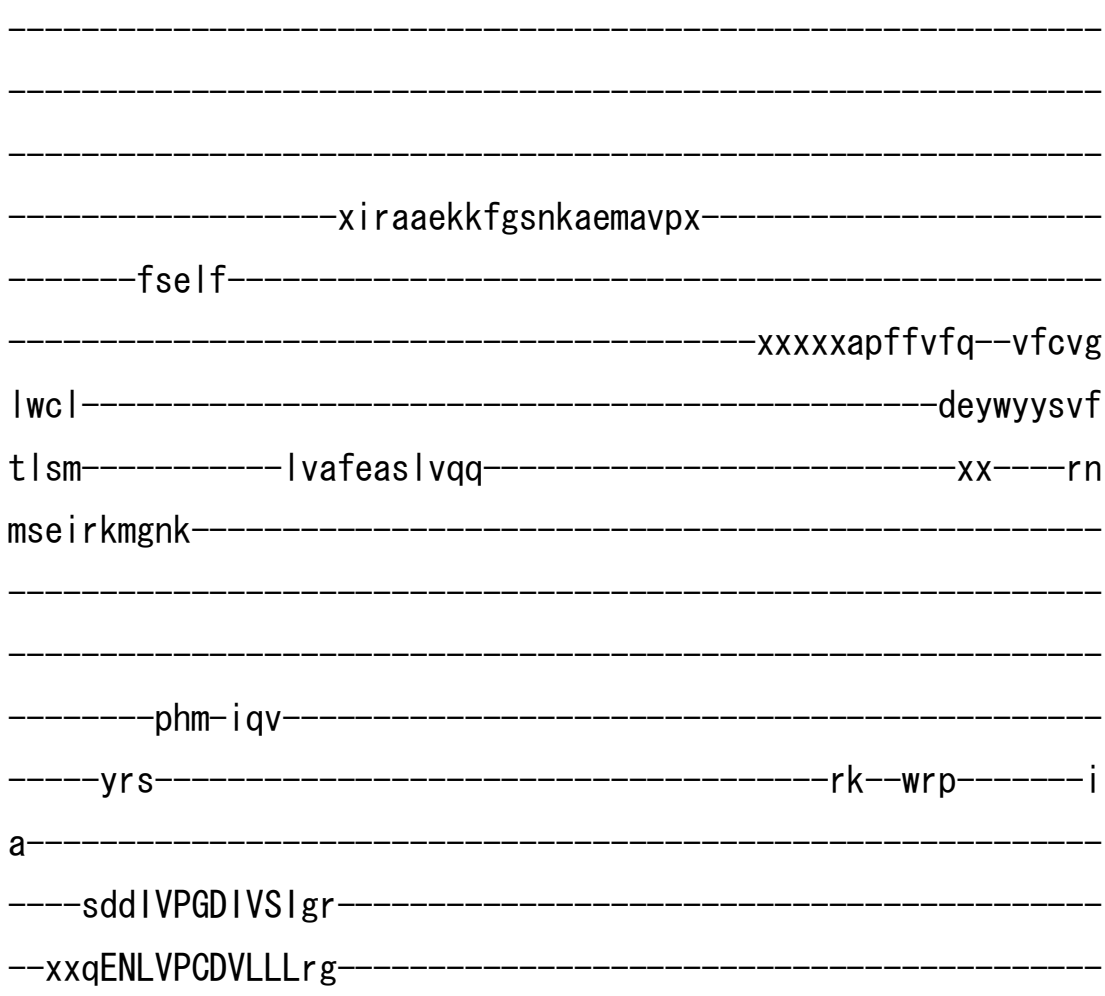

rciVDEAMLTGESVPQMKep-

$x x x x x x x x x x x x x x x x x x$

$-H V I F G--G T K V v q h x$ $-p p q$

ka-----tsg|--_-_-_-_-_---xxxxxxxxaFVLRTGFNTSQGk I rt i If

xvkrvtannletfifilfIIvfaiaaaay

-vwi egtkdpsx -nryk IflecTLILTSV

VPPELPIE ISLAVNTSLI-ALak Iy 
-mYCT-EPFRIPFAGKVEVCCFDKTG-

$-T L T S D S L V V x$ $-x x x$ I rdgk

$-x x x$

pvssipi

$-\mathrm{et}$

hralasc-----hs Im-

qlddg-

$-x|v g d p| e^{--k a m} \mid$ ta

$-v d w t x x k--x x x v f p$

-xxxxxxxxkihqrfhfasalk-rmsvxxxxexxxst

$-x x x x x a---$

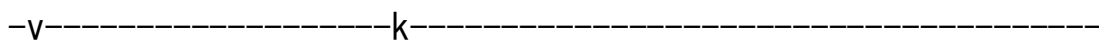

-gap

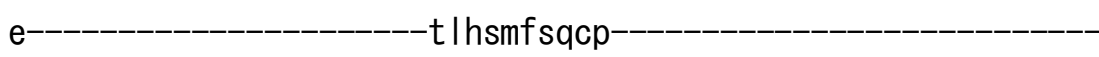

-pdy-hhihtei--sre-----garvLALGYKe I

-xh I thqqar evkrex lecs I kfVGF IVVSCPLKADSKAVIREI QNas-HRV

VMITGDNPLTACHVAQELHF-id--kaht- I i

hpp sekg$q x$ $x x x x x x x x^{--}$s i v

Iplt|gs

$-p k a|x|$ eha

|c | tgdg |a

hlqavd-pqq I h 
I iphv--qVFA--RVAPKQKEFVITSLk

$---\mathrm{el-}-\mathrm{gy}$

VTLMCGDGTNDVGALKHADVGVALXX

$x x x x x x x x x x x x x r d s p$

$-x x x x x x x x-x x x x x x x x x x x$

$x x x x x x x x x x x y$

$x x x x$

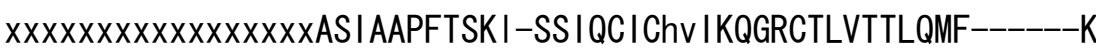

i la-Ina-I-i laysqsv ly leg--_-_-_-_-_-_-_-_-_-_-_---vkfsdfqat la

gl I lagcf-Ifi srskp|-kt--_-_-_-_-_-_-_-_--| srerp|-_-_----xnifnlyt

i-|tvm|qfsvxx|s|vylyreaqarspekxxxx

vdxxxxfeps I vnstvy i mamam-qmatfa i nykgppfme-----s I penkp I vws I avs Imaimg | |xxsspdfnsqfglvdi

-pvefk----|v--igqvlaldfgla-

- | |adrv|

qff Igtpkxrvp

S

>ceIV_V101 NP_502165. 2

m 
$-x x x x x x x x x x x x x x x x x x$ th $\mid y v p$

fti i taiwtyvwlnifgyee

$-x x x x x x x x x x x x x x x x x x x x x x$

\section{$x x x x x x x x x x x x x x x x$}

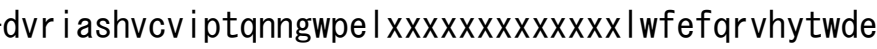
esrefatkxxx

xxxxxxxxxxxhgfevee

-xvkdaky I Igdnktemi ivpx

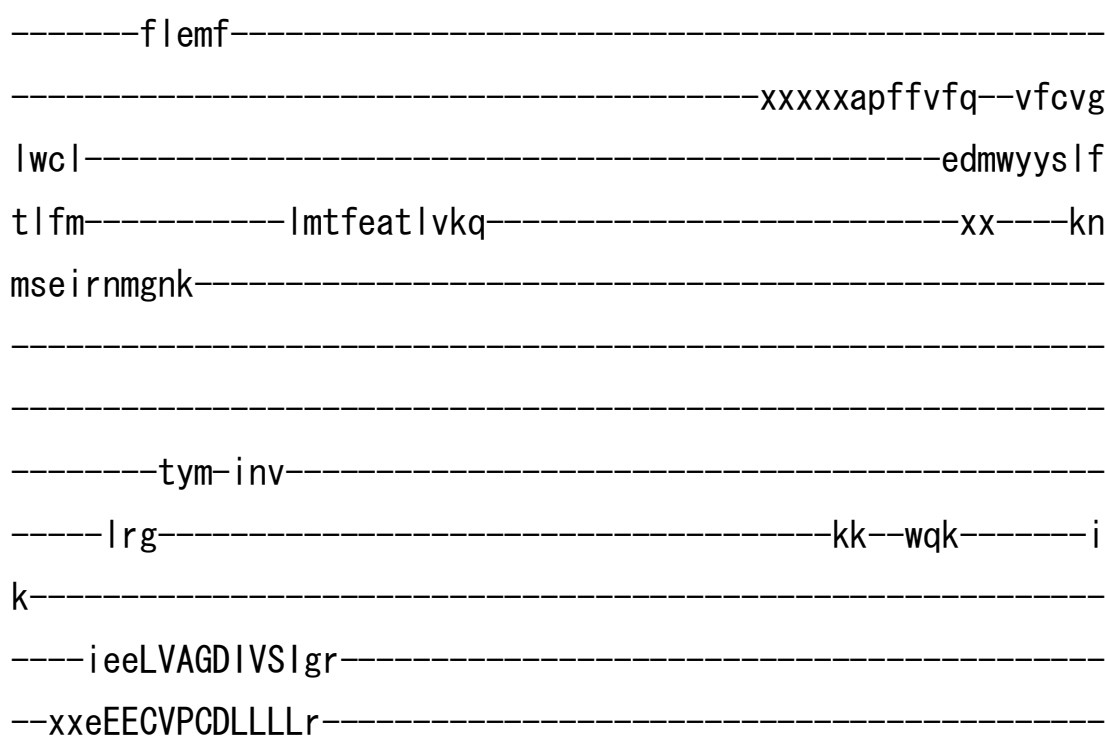


$-x-x---c i V D E S M L T G E S V P Q M K e p-$

$-x x x x x x x x x x x x x x x x x x$

-HVIFG--GTKI vqhxxxxxxxxxxvks

pd----gnci---_---_-_-_-_-_-_-_-_-cYVIRTGFNTSQGk I I rt imf-

-xvkkatannletfcfilfllifaiaaay

-Iwikgsvdetx

skyk IflecTLILTSV

IPPELPIE ISLAVNSSLM-ALqk I g-

-iFCT-EPFRIPFAGKVDICCFDKTG

$-T L T T D N L V V x-$

xxxxnnqke

$-X X X X$

naed I ph

Iqvlasc-----hs Iv-

rfeed-

$|v g d p| e^{--k a c} \mid$ sw-

cgwnxxk--xxxvmp

--xxxxxxxxxxxk i fhryhfssamk-rmtvxxxxqxxxts

$-x x x x x x a---$

-v---------------------- k

e

-v I rnmyad I p 
-sdy-detytr I--trq----gsrvLAMG IRk I

-xetrvge I rdkkrexfend I afAGFVVISCPLKSDTKTMIRE IMDss-HVV

AMI TGDNPLTACHVSKVLKF $-\mathrm{tk}-\mathrm{ks}|\mathrm{p}-\mathrm{t}| \mathrm{v}$

I de--------_--------------padg-

$x x x x x x x x--t i e$

Iplkpetknx

xxrkafxnshe

fcltgsafh

-hI vhne-htfIre-

I i l hv--kVFA--RMAPKQKERI I NELk

$---s \mid--g k$

-VTLMCGDGTNDVGALKHANVGVAL $x x$

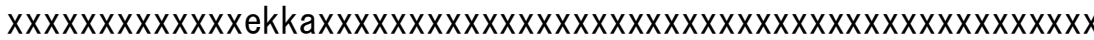

xxxxxxxxxxxx--------------------------------------------- $\mathrm{xxxx}$

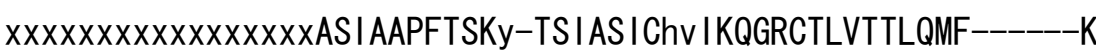

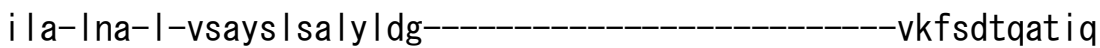

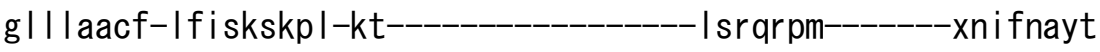

I-Itvt I qfi ivxxsc I | y ivg | aheantekxx-_-_-_-_-_-_-_-_-_-_-_-_-_-_-_

vdxxxxftpni Inttvy i i smal-qvctfavnyrgrpfme-----s I fenkam I ys imfs

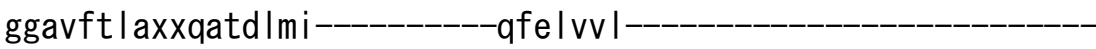

-peal r----na--I Imcvtad I vic-

-yi idrgl

$-n f|| g d m-$ 
$f$

>ggaV_V101 XP_423767. 2

$-x x x x x x x x x x x x x x x x x x x x x x x x x x|h g t v|$

p------_--_-_-_-_-_-_-_-_-_-_-_-_-_fvag I ypaw I w I wgp r vwaawr aaaeee eaqgpgpappeaa | | a | aa igvvh | | ta | sg I wsvhahswt l aaexxxxxxxxpxx----

$-x x x x x x x x x x x x x x x x x x x x x x$ $x x x x x x x x x x x x x x x x-$

-spkkat lakvvptpnngsae I xxxxxxxxxxxxxalsfefqkikysye ingkkqf I xxx

$-x x x x x x x x x x x x x r g y q e d k-$

-xiraaekkygtnkaemvvpx-

$-f l e l f$ 


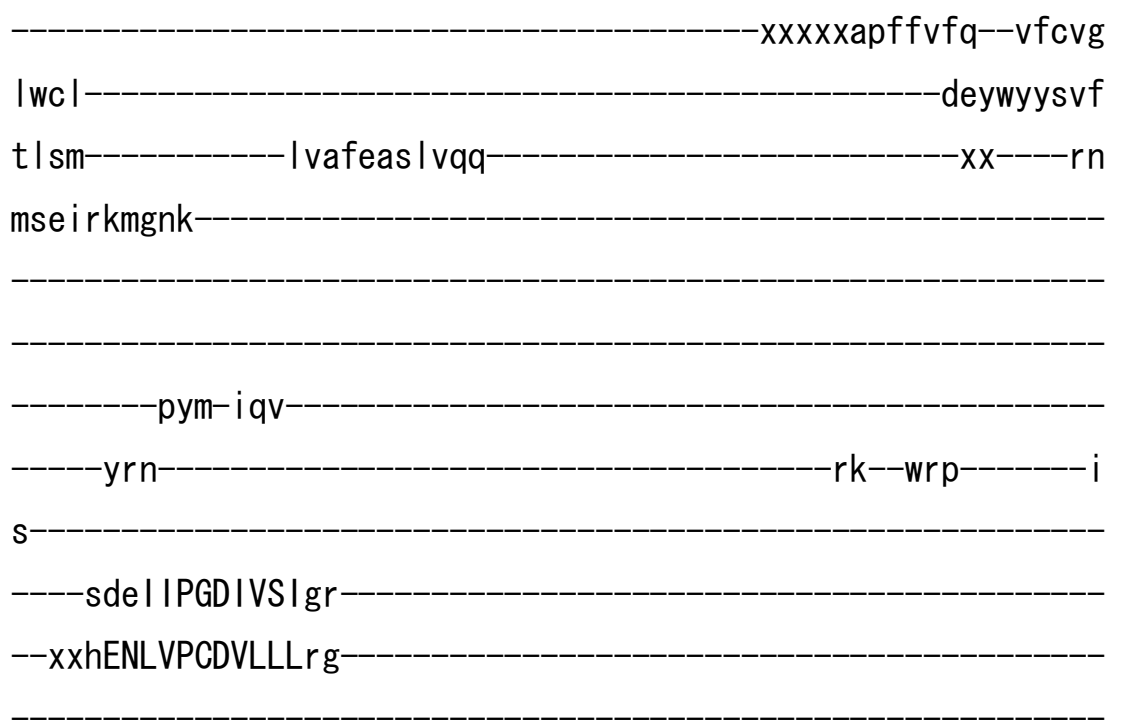

rciVDEAMLTGESVPQMKep-

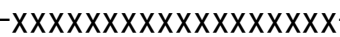

-HI IFG--GTKVvqhx----------ppq

ka----stg|---------_-----xxxxxxxxaYALRTGFNTSQGk I Irti If

-xvkrvtannletfifilfIIvfavaaasy

-vwi egtkdpsx----------------------------------nryk I f I ecTL ILTSV

VPPELPIE I SLAVNTSL I-ALak I y

-vYCT-EPFRIPFAGKVEVCCFDKTG

TLTSDHLVVx-------------xxxx I rdgk

pvsdipi

et

hraiatc-----hs Iv-

qlddg- 
$-v d w t x x k--x x x v f p$

-xxxxxxxxkihqrfhfasalk-rmsvxxxxexxxaa

$-x \times x \times x \times a---$

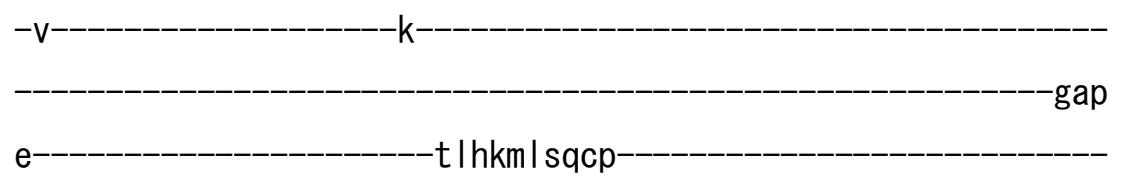

-sny-navhte i--she----garvLALGYKe I

-xh I thqqv remkr ex I ecd I rfAGF IVVSCPLKTDSRSVIREI QNas-HHV

VMI TGDNPLTACHVARELHF-

|q--reht-li |

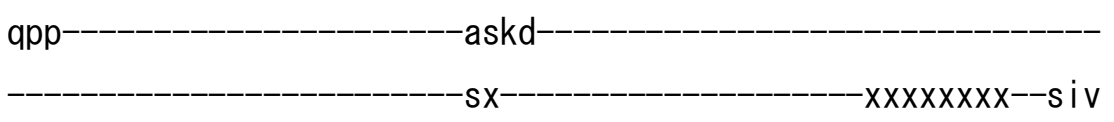

fpilpss

- relxahyd

|cvtgeg|s

hlqaln-rqq| Ir

I i phi--qVFA--RVVPKQKEFVITTLk

---sl--gy-

-VTLMCGDGTNDVGALKHADVGVALXx

$x x x x x x x x x x x x x r d g p$

$-X X X X X X X X X X X X X X X X X X X X X X X X X X X X$

xxxxxxxxxxxx------------------------------------------------- $x x x$

$x x x x x x x x x x x x x x x x x$ ASI AAPFTSKI-SS IQCIChv IKQGRCTLVTTLQMF-------K 


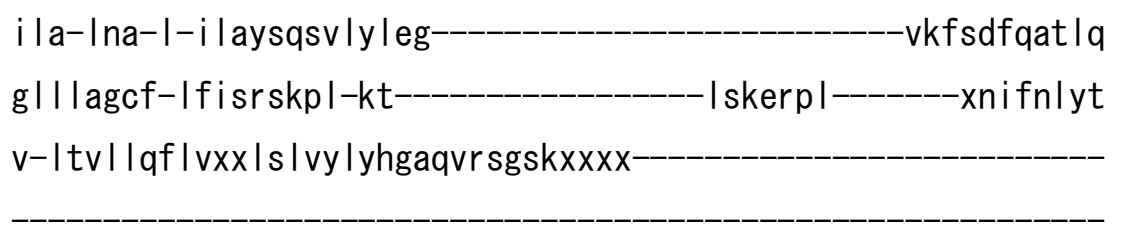

vdxxxxfeps I vnstvy imsmam-qmatfa i nykghpfme-----s I qenkp I I ws i i Is

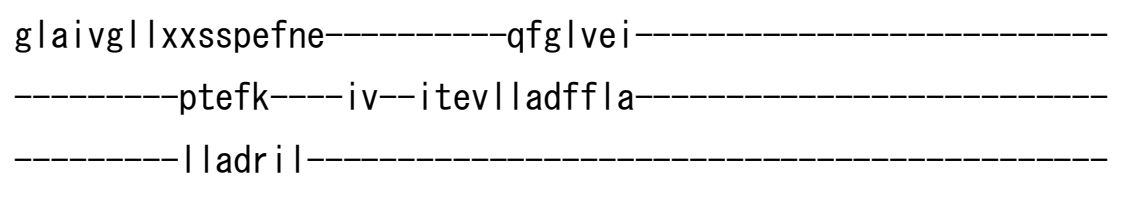

qf I I grakxkvp-

\section{$\mathrm{S}$}

>dmeV_V101 NP_609490.1

m-

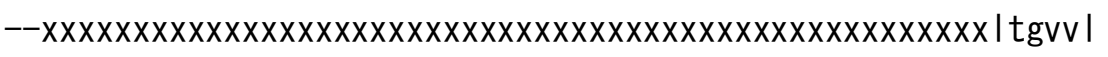
$\mathrm{p}-$ 
-fvp I y l safy I w invtggqendttnndv itadxxxxxxxxtxx----

$-x X X X X X X X X X X X X X X X X X X X X$

$x x x x x x x x x x x x x x x x$

I pganv lakvvptpnngnsk ixxxxxxxxxxxxxqyyfvfqktkyvwn

edrktfraxxx

xxxxxxxxxxxxrgletee

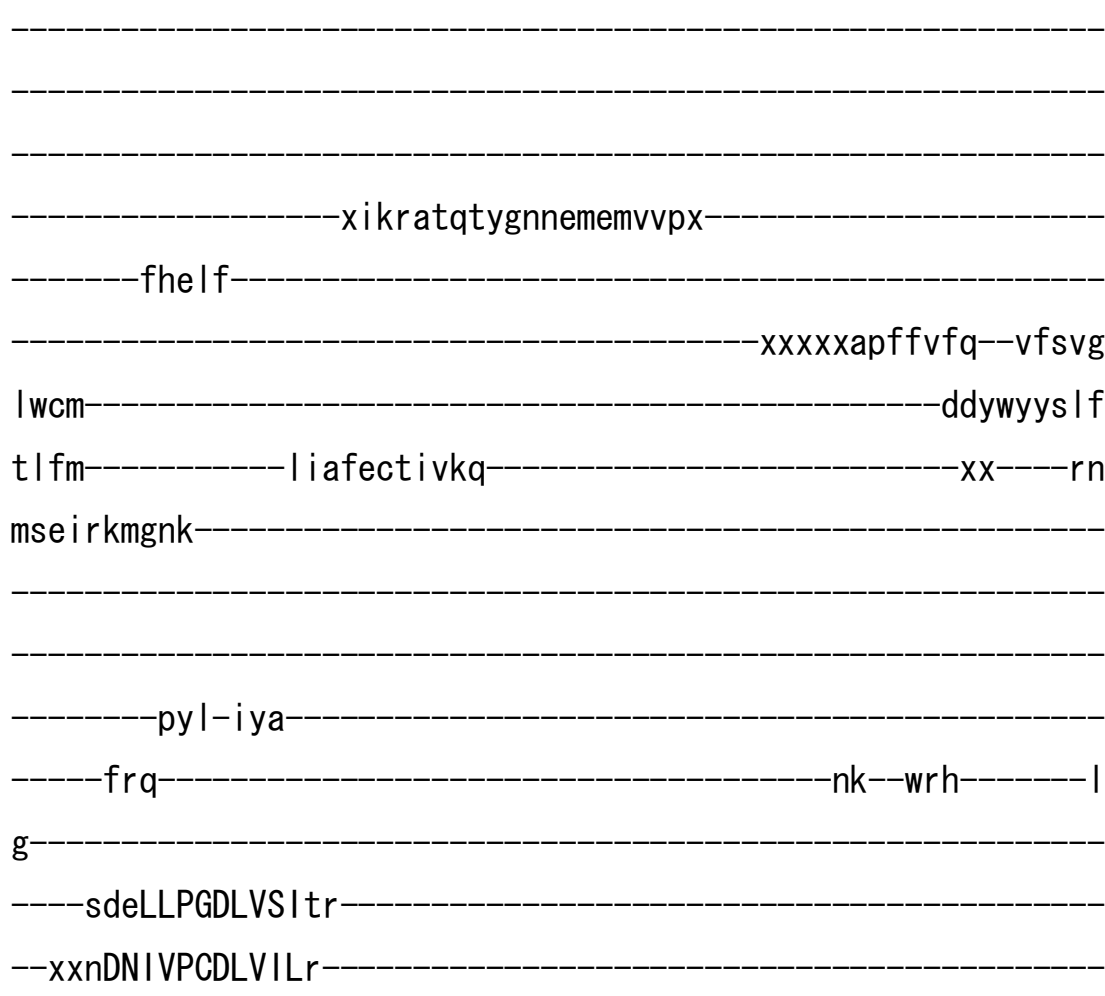

$-x-x---c i$ VDESMLTGESVPLMKes-

$-x x x x x x x x x x x x x x x x x x x$

-FVLFG--GTKVvqh $x x x x x x x---\mid$ ra

pd-----ggci-_--_-_-_-_-_-_-_-_-_-_-_gYVIRTGFNTSQGk I I rt i I f-

xanratennvetfafiaf Imvfavaaasy

-vwvkgsedpex

-nryk|f|ecTLILTSI

IPPDLPIE I TLAVNTSL I-QLtk I f 
-vFCT-EPFRIPFAGKVQI CCFDKTG-

$-T L T T D N L M V x-$

xxxxlapng

$x X X$

piekaeg

$-n t$

vqvlacc-----hsla-

$-1 \mid d d g$

- |vgdple--kat laa

vdwtxxk--xxxvip-

-xxxxxxxxki iqryhfssalk-rmsvxxxx|xxxsn

$-x x x x x=---$

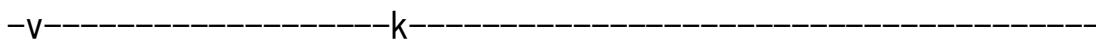

-gap

e

-v i qkm I revp

-ady-ekvy l ey--arr----garvLALGIKdI

-xt I gaqrvremkrexvecd I tfAGFLI ISCPMKPDSKSV IKEL I Qss-HKV

VMI TGDSPLTACHVARELRF$-\mathrm{tr}-\mathrm{kk} \mathrm{i}-\mathrm{ilt}$

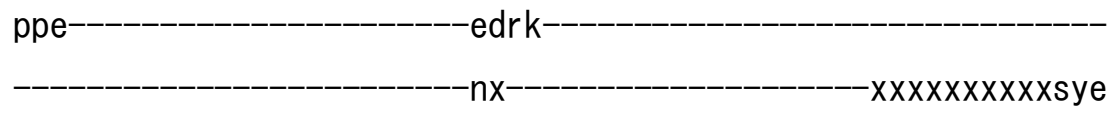

Idtkpgsk

$-x \mid$ shl xathd

Icitgegla 
I lpqi--tVCA--RFAPKQKELVITQLK

$---q \mid--g y-$

CTLMCGDGTNDVGALKHANVGVSLXX

$x x x x x x x x x x x x x q q a a$

$-x x x x x x x x x x x x x x x x x x x x x x x x x x$

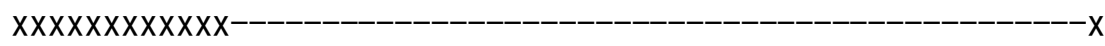

$x x x x x x x x x x x x x x x x x$ ASI AAPFTSKs-SS IMCVNh i IKQGRCTLVTTLQMF-------K

i la-Ina-I-iqaycqsv ly idg--_-_-_-_-_-_-_-_-_-_-_-_-_-_ikfsdtqatmq

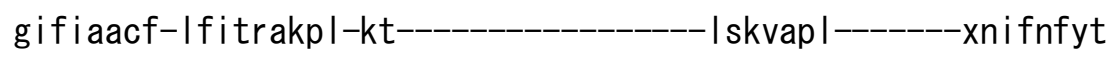

i-st i I sqfavxxgt l yy I tsqan i lapprxxxxxxxxxxx--------------------

aexxxxydpnivsstvy i ic|s|-qvatiavnykgypfme------s|rsnrmlmyaigas aal vi I I sxx lape I te---_--_----_ffe i idf--_-_-_-_-_-_-_-_-_-_-_-_-_ -ptdfr----kt--I Igv|vldivga-

$-f I l d r i c-$

sf Ifgetrxkskxxx

C

$>$ tanV_V101 XP_955061. 1

$\mathrm{m}-$ 
$-x x x x x x x x x x x x x x x x x x x x x x x x x x x x x x x x x x x x x p e s g \mid r$

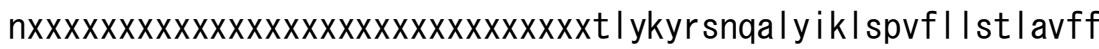

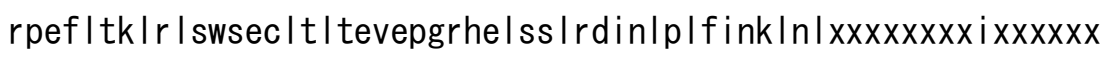
$x x x x x x x x x x x x x x x x x x x x x x x x x x x x x x x x x x x x x x x x x x x x x x x x x x x x x x x x x x x--$ nrtnk| I|plyrtgfi yyihdxxxxxxxxxxxxxepvehfdevqvpqi Inwrg | |sxxx $-x x x x x c g f e p n I$

-xapffIfq--vtst I

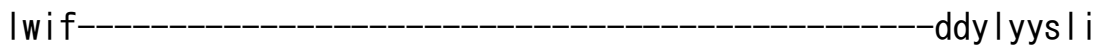

sifs--_-_-_-_---mv l i evqmvyk-_-_-_-_-_-_-_-_-_-_-_-_--xx----ie ynr insmk Ip-

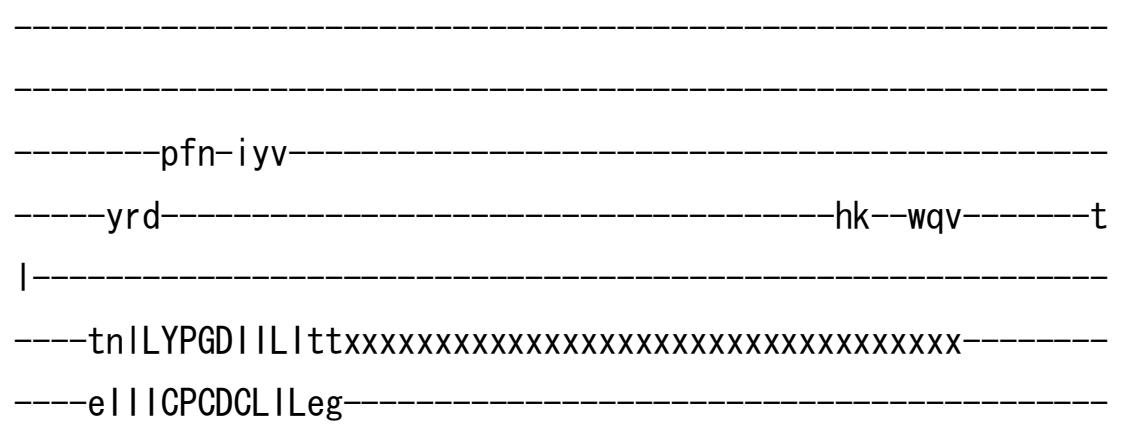


eviVDESILTGESIPQFKts-

$-X X X X X X$

nar

$x-$

-STIFS--GTTI i Itxxxxxxxxxxtss

tsxxxxagas $x x x x x x x x x x x x x x x x x x x x x x x c L$ VIRTGFESYQGr I vhs i In-

-xxpnkvvgsnaqgymf I I I |v Ifavaavvv

-vvrnsnyk -

-svkk I I IvtSRILVSV

IPPEFPVT I SMAVT I GL I-QLrkkg-

iYCT-EPNRLPLAANIDV IAFDKTG

TLTQDQMYLX

xxxxsste

ys

klviggc-----hs It

-nvnga

itgdpme--kisfth

- fnnqxxr--xxxniv

$-x x x x-x x x x x x x k i \mid k r w r f t s e$ lg-rmstxxx-

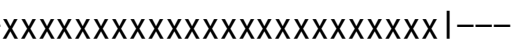

$-t$

$\mathrm{e}$

$-k v k m \mid I r I v p$ 
syf-eevchdl--tik----g|rvLCLAYKr I

-xdip inal i tidrnxvekd l efCGFLALEAPI KNSSKLCMKRLKN---FKK

IMI TGDN ILTACYVTQQ I NM-vnxxsti i ixpin

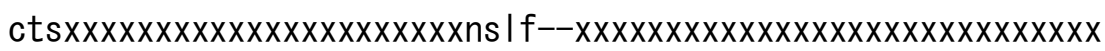
$x x x x x x x x x x x x x x x x x x x x x x x x x x s x x x x x x x x x x x x x x x x x x x x x x x x x x x x---x n s y$ kkfletcpf $x x x x x x x x x x x x x x x x x x x x x x x x x x x x x x x x x x x x x$

-xxsgvsxskvs $x x x x x x x x x x x x x x x x x x x x x x x x x x x x x x x x x x x x x x x x x x x$

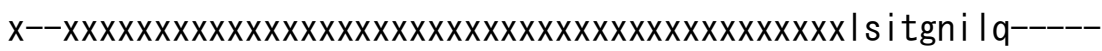
- I Insnxtptnttxxxxxxxxxxxxxxxxxxxxxxxxxxxxxxxx $\mathrm{xx}$

i innc--sVFA--RMSPQQKEF I IKCYk

$---\mid n--n k$ -TI AMCGDGTND ISALKQAD I G ISL XX $x x x x x x x x x x x x x \mid d n e$ $-x x x x x x x x$ ASI ASPFTYHg-SDVNCVFn I IKSGRCSLYNLFMLY $-K$

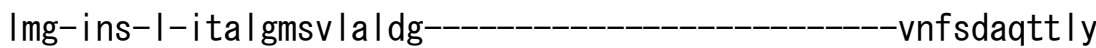

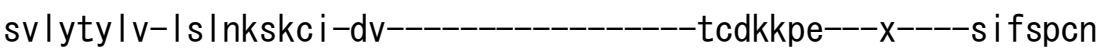

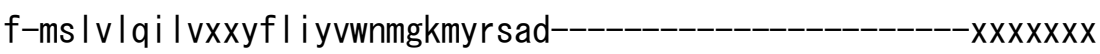
xxx--_-_-_-_-_-_-_-_-_-_-_-_-_-_-_-_-_-_-_-_-_-_-_-_-_-_-_-_-_-_-_-_-_-_ pn----vvnt I lyyvwya in Ins-fvsny i dypymd---_--_---ti vnnkf I fkpv Ifs yftml If ixx Ivkp Ins---_-_-_--_ffs I vpi--_-_-_-_-_-_-_-_-_-_-_-_-_-nht lk----Ik--itavvfldfiltfviskli 
d

>dmeV_V201 NP_726536. 2

$X X X X X X X X X X X X X X X X X X X X X X X m k v g$

-xxxxxxgfcwacif |tgg | |r|v|hwwrh|y|ya

tcsqcs l eeaeqv I v-tedyggkhkmyhvkqi qv Itssn I kt I lexxxxxxxxtxx----

$-x x x x x x x x x x x x x x x x x x$

-qfkkcssirifrckqlvyawn

-nntnrfaringldl

nipcsyyhxxx

glpvhe

qisrrivfgdneitvp|r

$-x x x x x f|e v|$ 
-npfyvfq--Ifsvi

Iwft--_-_-_-_-_-_-_-_-_-_-_-_-_-_-_-_-_-_-_-_-_-_ydyyyyacv

i I Im------------svfgi tvsv lq--------------xx-------------------kn

qdvlqktvyn-

nc iLDESMLTGESVPVTKtp-

$\mathrm{XXXXX}$

$-X X X X X X$

eha

$x-$

-HTLFC--GTKViqt

ryi

gs-----kkv l

aFVINTGNI TAKGel irsi Iy

-x--ppvdykfeqdsykfiqflai iacvgfiy

-tlvtkilrgt

dpvkiavesLDLITIV

VPPALPAAmTVGRFYAQK-RLktse-

iFCI-SPRS INVAGS INCCCFDKTG-

TLTEDGLDM-

-wgvvpx-

$x-x x x$

ip--Iksxxxxx

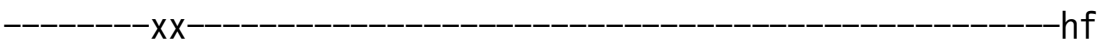

Ifgmvtc------hs it

i Ingr 


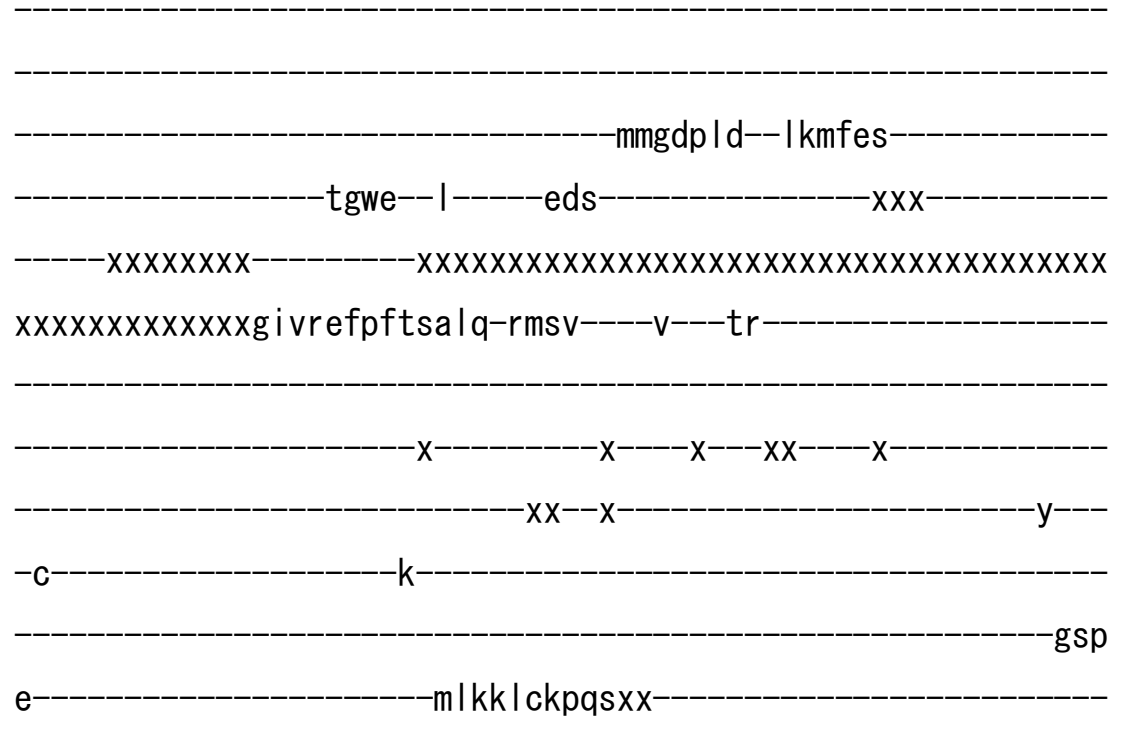

$$
\text { -dny-sqq I sef--akk----gyr i IAI AFKa I }
$$

-xxxmnytkvqr I sree-vennmefLGFVILENRLKPDTTKVINALNAak-IRT IM I TGDN ILTAI SVARDCG I-----_-_-_--_-_-_-_-_-_--_--vsxxqsv i-tvh adp--_-_-_-_-_-_-_-_-_-_igds $x x-x x x x x x x x x x x x x x x x x x x x x x x x x x x x x x$

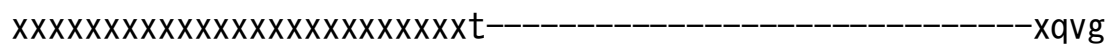
kt ifhmest $x$

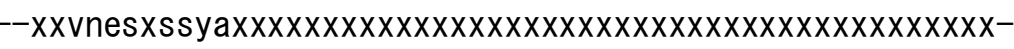
-famdgktwq

ivkdyf-peeme i

I $\operatorname{trg}$--s IYA--RMSPDQKQALVIELq

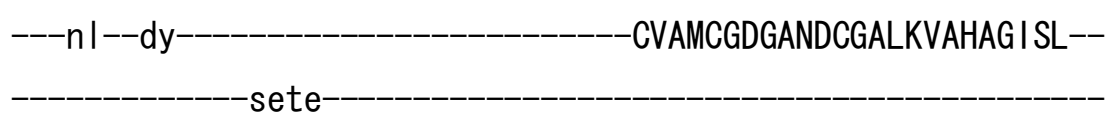
$-K$ 


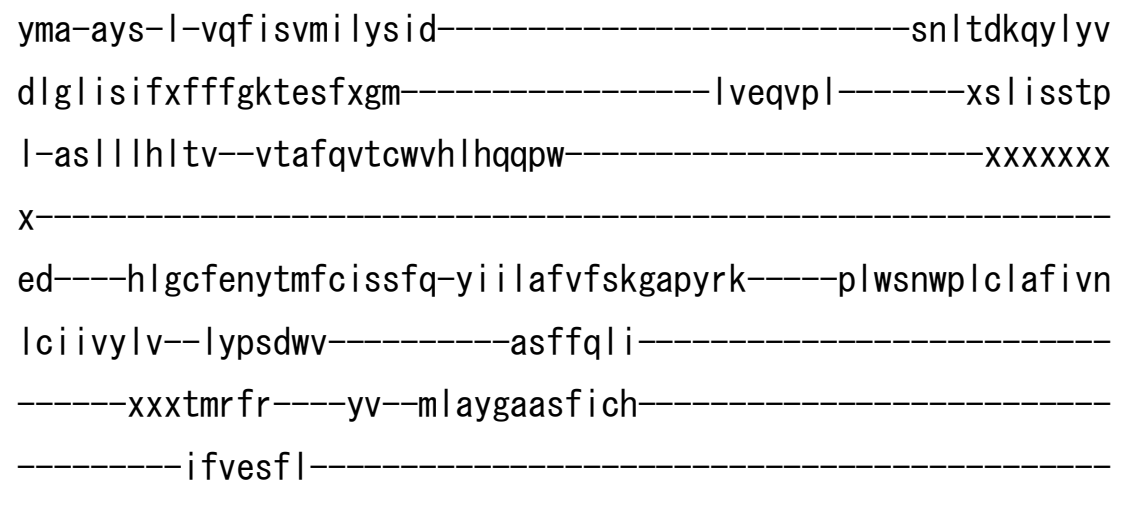

-veylvfkkxqvkxxxxxx-

$-x x x x x x x x x x x x x x x x x x x x x x x x x x x x x x x x x x x x x x x x x x$ $x x x x x x x x x x x x x x x x x x x$

C

$>$ tanV_V201 XP_953853. 1

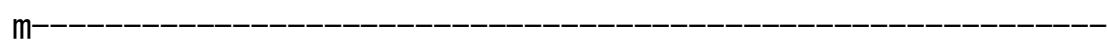

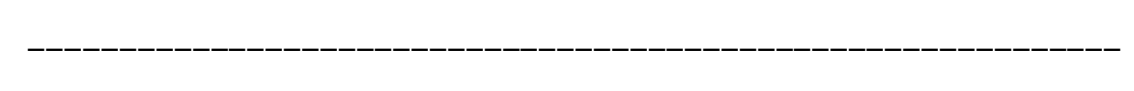

$-x x x x x$

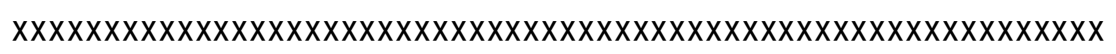

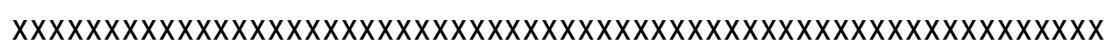
$x x x x x x x x x x x x x x x x x x x x x x x x x x x x x x x x x x x x x x x x x x x x x x x x x x x x x x x x x p$ snt i $x x x x x x x x x x x x x x x x x x x x x x x x x x x x x x x$ | $v$ cne $\mid c$ ipmvssveywdsrakpf $\mid f$ sw 
Ivgfft I I isflvrntrvgkh I fvirvpletct ivkird Inksst $x x x x x x x x \mid x x^{----}$ $-x x x x x x x x x x x x x x x x x x x x--$

pfekykvakncyvpvvtaesxxxxxxxxxxxxxnneescf Idssnr I nsy Imstnxxx

xxxxgg| tese-

sarrsedigpne ip i ek I

$-x x x x x x y r e v s$

dpvffmqxx|ttks

iywr

syitapiw

gtttxx-

-tiykkvki iyd

$x x$

$-n d$

Iynlttssnq

$-x-x---a$ iVDESSI TGESIPLKKrk-

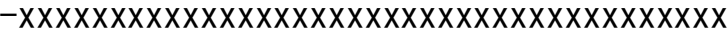

$x x x x x x x x x x$

$-x x x x x x$

$\sin$

$-x$

-NLLKS--GTKV i svxx---------nqe

ts

aVVIATGVYTTKGkqikgv If

-pnqfr lkydtalplvflltfiyalfcs

-yyqirflg -wnm i s i fys I GTLSQV

VPVWTSTI iSISQSRACQ-RLsksex 
iYCI-APSRIAVCGKLRVMCFDKTG-

TLTNNKL IF-

-ngsky

$X X X X X X X X X X X X X X X X X X X$

$-X X X X X X X X X X X X X X X$

$-v i$

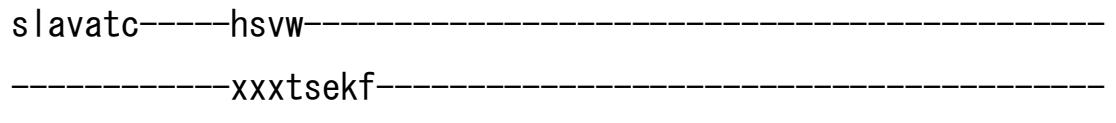

$-x x x v d k s m f e x x g c v v d q x x x x x x-x---$

-nrry--i--xxxqne

$-x x-$

-xxevinsfdfdyqkk-Issvxxxxe---ve

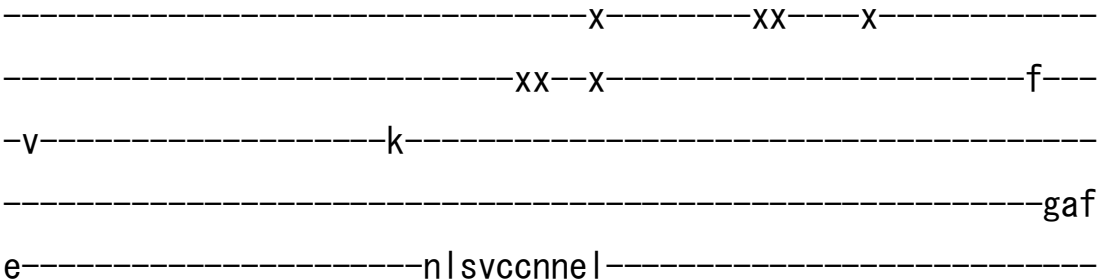

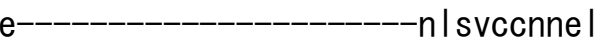

-en I-sv I snne---ssn-----gsyvLGLAYKv I--xxx-

-xxxtdrn I---_-_---mesn I am I SLL IFNNQVRPESKEV I QTLNEam-VRT

VILTGDNIPASKYVARKCNM--_-_-_-_-_-_-_-_-_-_-_-_-_iqxxnst i-_-_

$-x x x x x x q x x x x x x x x x x x x x x x x x x x x x x x x x x x x x x x d e$

$k \mid f f s e e f s x$

i smtgdvfd

-y i ennwxe i lary 
$-x x x x x x x x x x x x x x x x$

f Imk i--r IFA--RLNPNQKVRV INSFk-

$---r \mid--g i-$

- ITGMCGDGTNDCLALQASHAGISL-$-t k g v$

SSMVSPFSSMt-NKLESV I y ILREGRGSLVTCLACF $-K$

fml-Ifgxm-iafvkvt Ifr Icr-_-_-_-_-_-_-_-_-_-_-_-_-_-_gvmpewgy I $\mathrm{fi}$

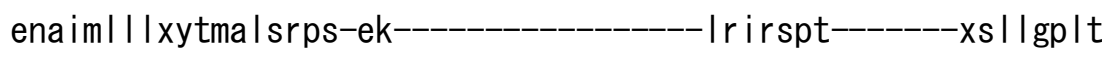
I-Isvgi

-i sdnfeaptvcfwl cyq-vvntalvfsfggvfre-----sihrnigfstvwlfi nsfltylt--|skptk|-----------tclfrin $x x x x x x x x x x x x x x x x x x x x x x x x x x x$

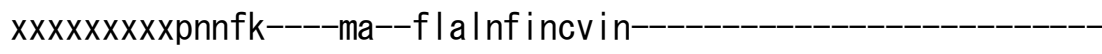
cliaksf

i

>mgrV_V201 XP_370428. 1

$\mathrm{m}-$ 


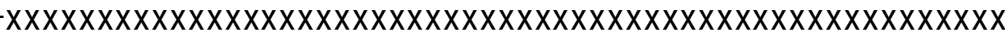

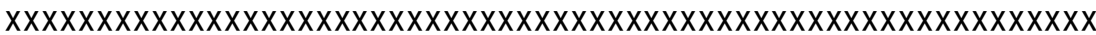

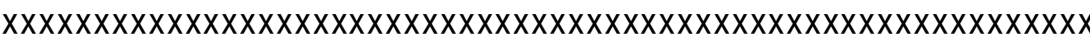

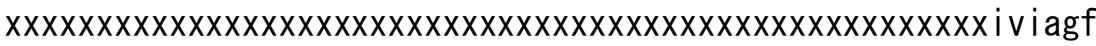

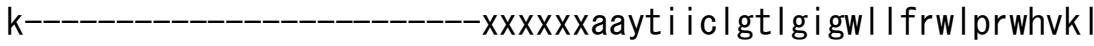
I gqpcp I recewvv i enqwgema i I dvqsrpygrs I stvfgqpgkxxxxxxxxd-

sdp il tel rvvdyryvrfyfa $-p|r d k f m| c y g w k d$ pnwtdvhsxxx

gidsee

-kshrdlvfgtnl idi eqk

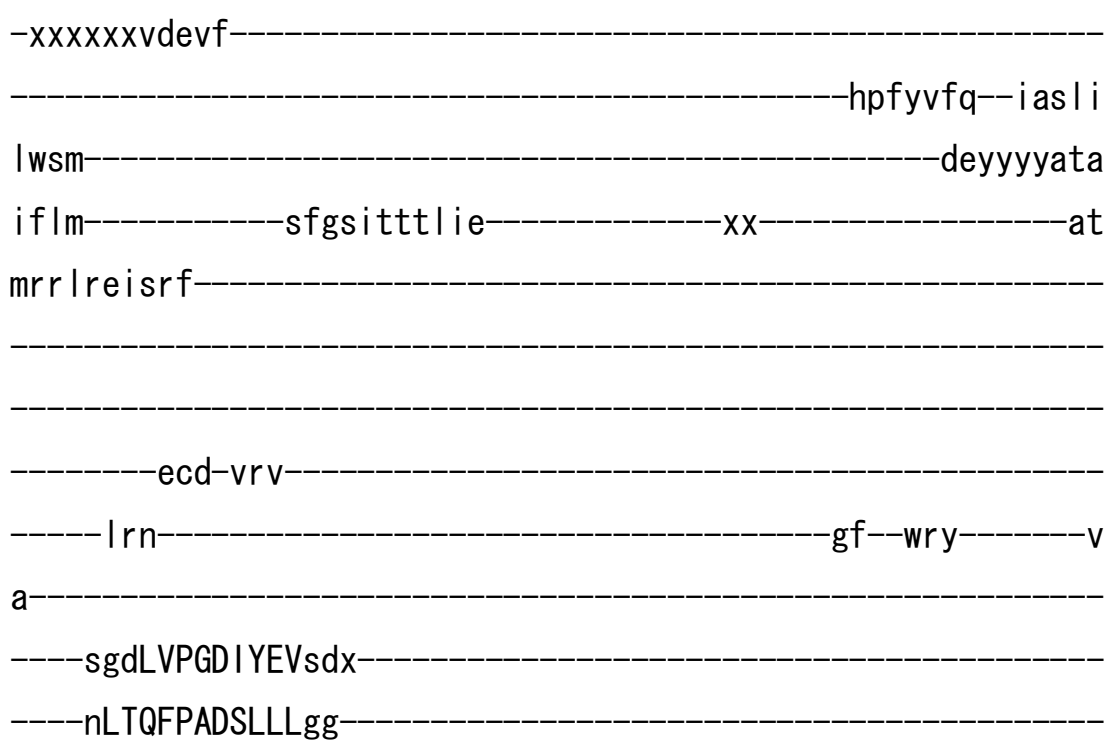


-dc iVNESMLTGESVPVSK ip-

$-x x x x x x x x x x x x x x x x x x x x x x x$

-HFLFC--GTKI i rax-

$-r p v$

eg----gege---------_--------XxxxaMVVRTGFNTTKGs I vrsm I f

-x--kpsgfkfyrdsfryi svmacvamvgfia -sfinfvrlel awh I i i vraLDL ITIV

VPPALPAT IQIGTNFALS-RLkkkq-

iYCI-SPQRVNVGGKIDIMCFDKTG

TLTEEGLDV

I gvrvx

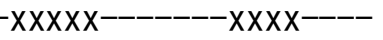

$-x x x x x--x x x x$

dlagdsrxxxxx

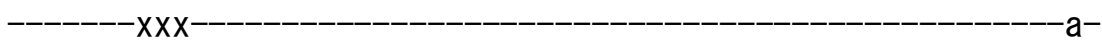

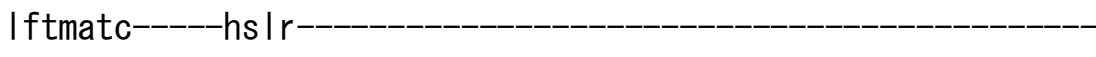

svdge

|vgdp |d--|kmfef

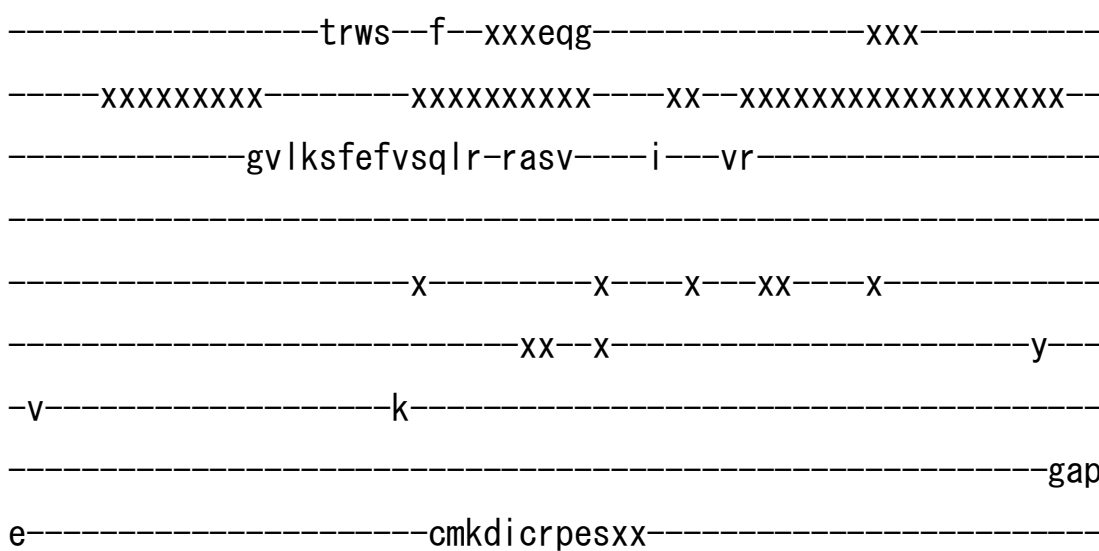




$$
\text { -vdy-de I l syy--thk----gyrv IGCATKh i----x-- }
$$

-x-----xxxvkaqkmkre--- - -aesd I efVGF I IFENKLKPTTAAVLEELVKsn-IGA VMVTGDNILTAISVARESNL idxxahcf-ipr

fingnst xxxxxxxxxxx $-x m p \mid$ ppppqsdas $x$

-xxdisnxrnys

iavsgdifr

-wvvdyaxpev Ihr

mlvcg--kVFA--RMSPDEKHELVEKLq-

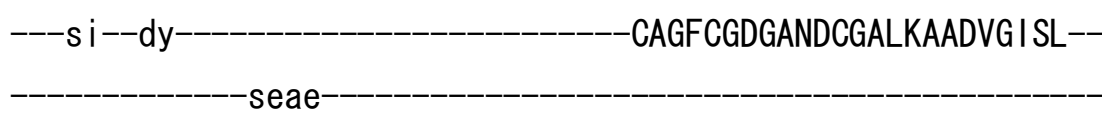

ASVAAPFTSRv-FDIRCVPev IREGRAALVTSFSCF $-K$

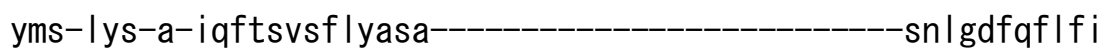

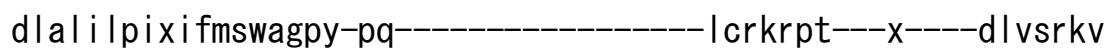
I-tp I I gqmc i--c i a i qavvf i avrkqsw-------------------------xxx---$-x x x x x x$

es----nitnsents Ifl i scfe-y i lagvv inagrpfrq-----gml knwpfvat i sit I lat lymv--|qparpl------------tdImelt -xxaldfk----fy--i i vmgfvyl i ia-ytaehya 
a

$>$ tpaV_V201 XP_766443. 1

$\mathrm{m}$

xxxfvcne I c ipmvssveywdsrakpf Ifsw

Ivgfft I | isf I vrntrvgkh Ifvirvpletctivkvrdfnksstxxxxxxxx | xx

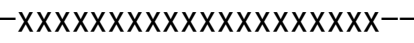

- -

-ipfekykvakycyvpvveadsxxxxxxxxxxxxxxdseescf Idssnr I nsy Imstnxxx

$-x x x x g g \mid$ tese-

sar rsedigpne i p i ek I 
dpvffmqxxIttks

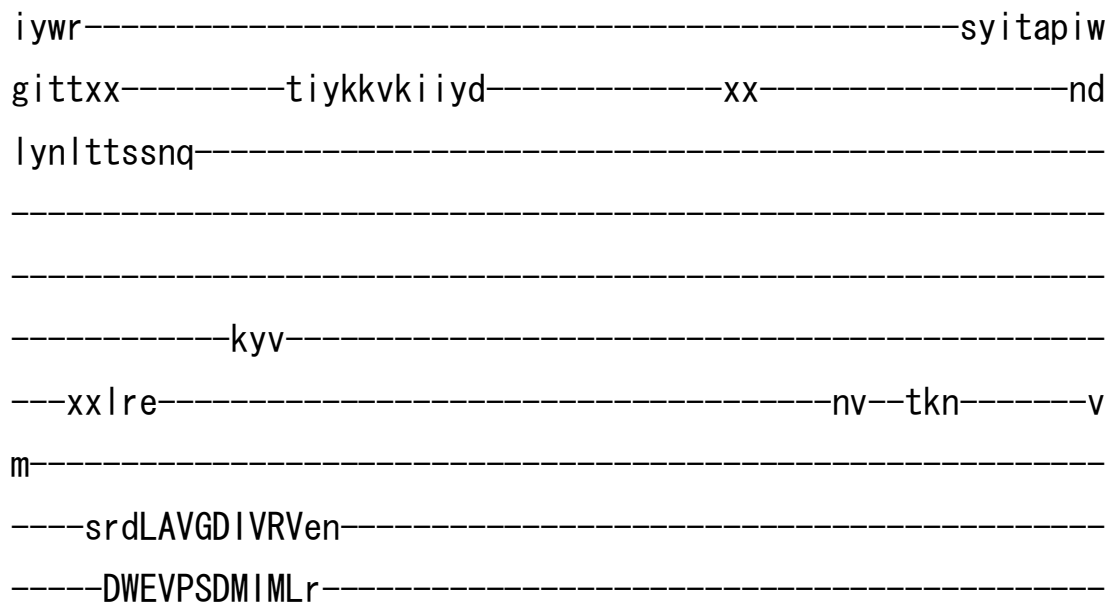

$-x-x---a$ iVDESSI TGESIPLKKrk-

XXXXXXXXXXXXXXXXXXXXXXXXXXXXXXXXXXXXXXX

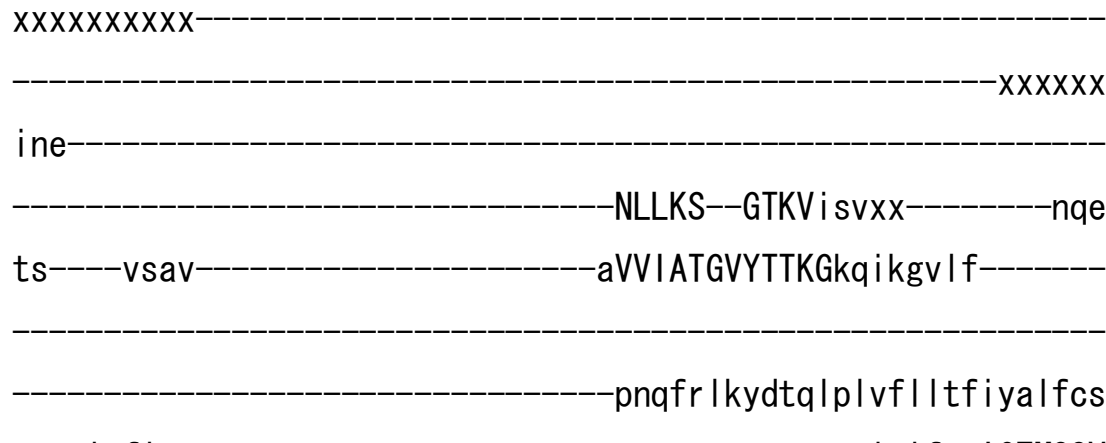

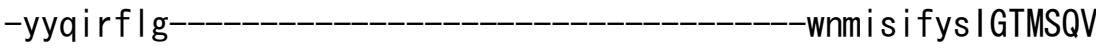

VPVWTST I iSISQSRACQ-RLsksex

iYCI-APSRIAVCGKLRVMCFDKTG

TLTNNKLIF-

ngsky

$-x X X X X X X X X X X X X X X X X X X$

$x x x x x x x x x x x x x x x x$

$-v i$

slavatc-----hsvw-

xxxtseqf 
$-x x x v d k s m f d x x g c v v d q x x x x x x-x$

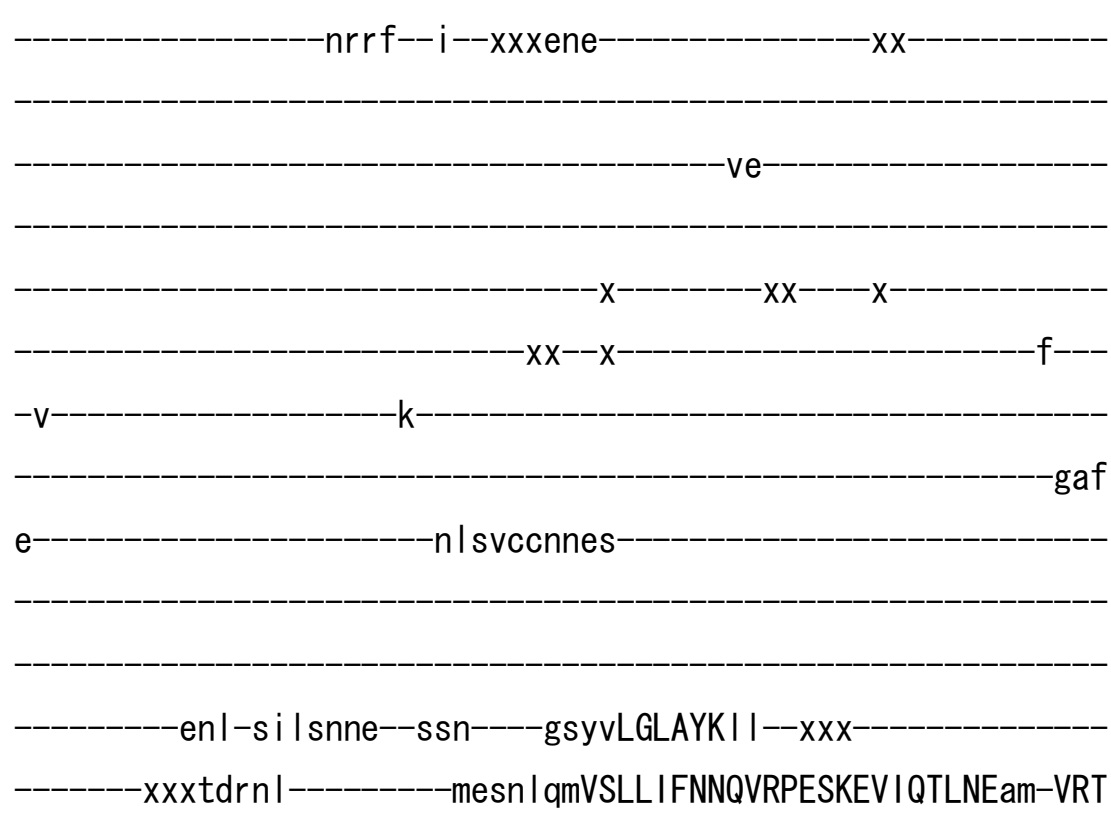

VILTGDNIPASQYVARKCNM--_-_-_-_-_-_-_-_-_-_-_-_-_-_-_-_iqxxqdst---_-

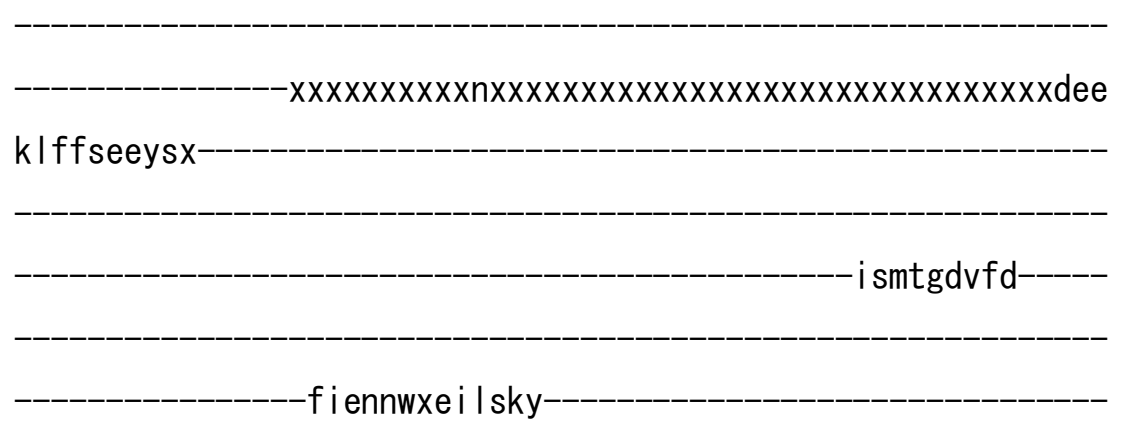

$x x x x x x x x x x x x x x x x$

fImki--r IFA--RLNPNQKVRVINSFk

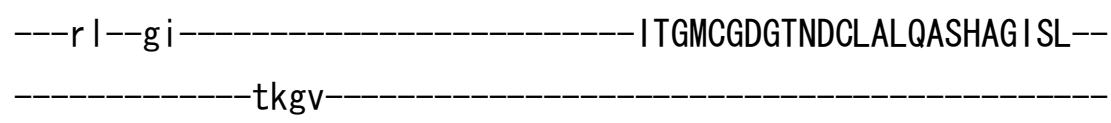

SSMVSPFSSMt-NKLESV I y I LREGRGSLVTCLACF------K 
fml-Ifgxm-iafvkvtlfrlor -gvmpewgylfi

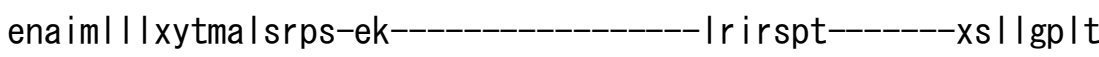
I-Isvgimf i ixxafIIIIfelyhr Igiptxxxxxxxxx-

paxxxx Isdnfeaptvcfwlcyq-vvntalvfsfggvfre-----sihrntgfstvwlfi nsfltylt--|skpnk|--_-_-_-_--tclfrinxxxxxxxxxxxxxxxxxxxxxxxxxxxx xxxxxxxxxpknfk----ia--fIgInfvncvin--_-_-_-_-_-_-_-_-_-_-_-_ cliaksf

i

>hsaV_V201 NP_071372. 1

$\mathrm{m}$

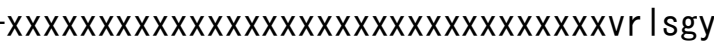
c -xxxxxx igyhvvvwmmag ip | | frwkp | wgvr | 
rIrpcnlahaet I vi e i rdkedsswq I ftvqvqtea i gegs l eps $x x x x x x x x r x x----$ $-x x x x x x x x x x x x x x x x x x x x x x$

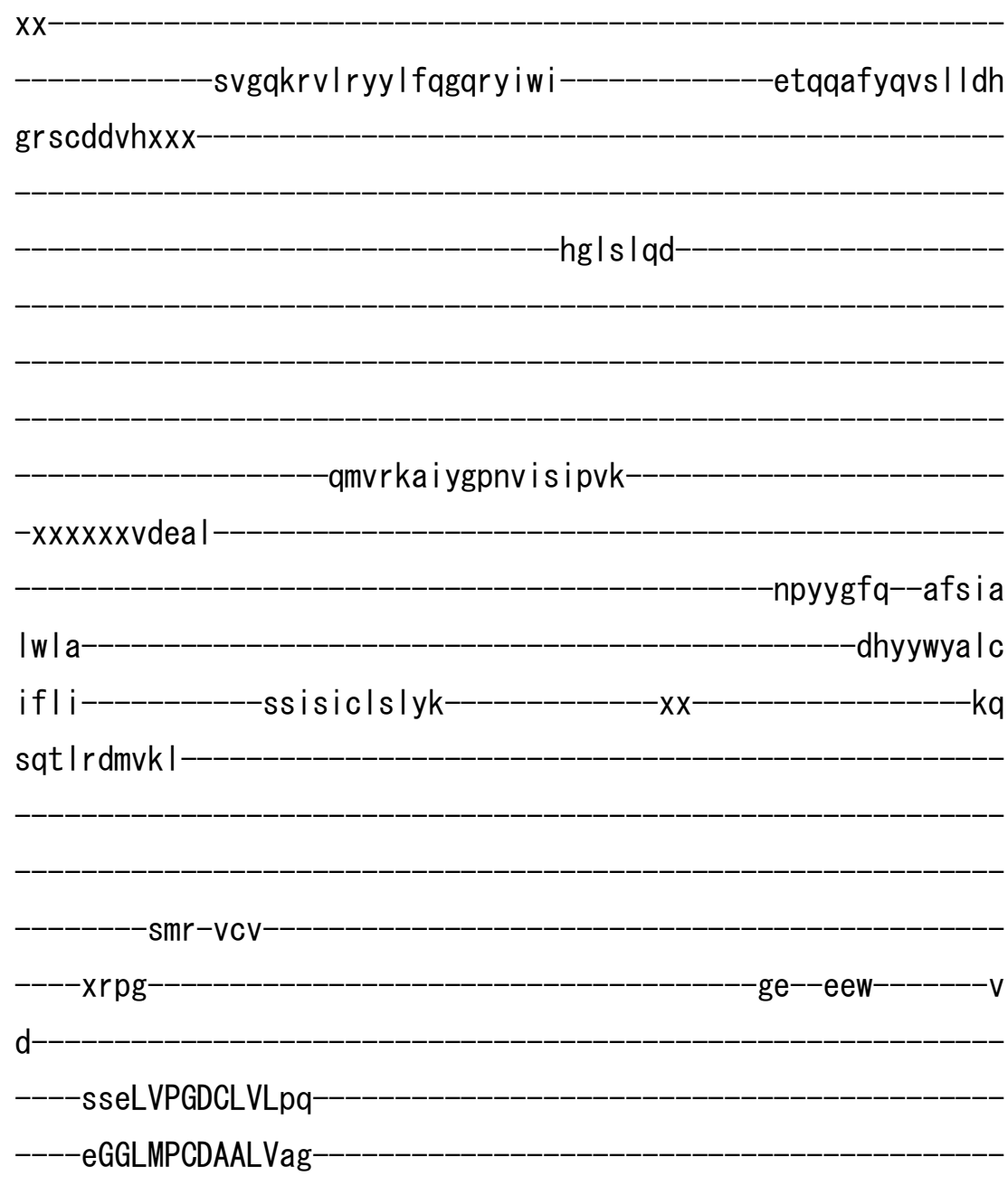

ecmVNESSLTGESIPVLKta-

$x x x x x$

$-x x x x x x$

thr

ray

vg----phv aVVTRTGFCTAKGg I vss i lh

-x--rpinfkfykhsmkfvaalsvlal Igtiy -sifilyrnrv -p Ine iviraLDLVTVV

VPPALPAAmTVCTLYAQS-RLr rqg- 
iFCI-HPLRINLGGKLQLVCFDKTG-

TLTEDGLDV

-mgvvp-

$-x-x x x$

$\mathrm{pl--vpexxxxx}$

$-x x-$

Iralatc-----hals

rlqdt-

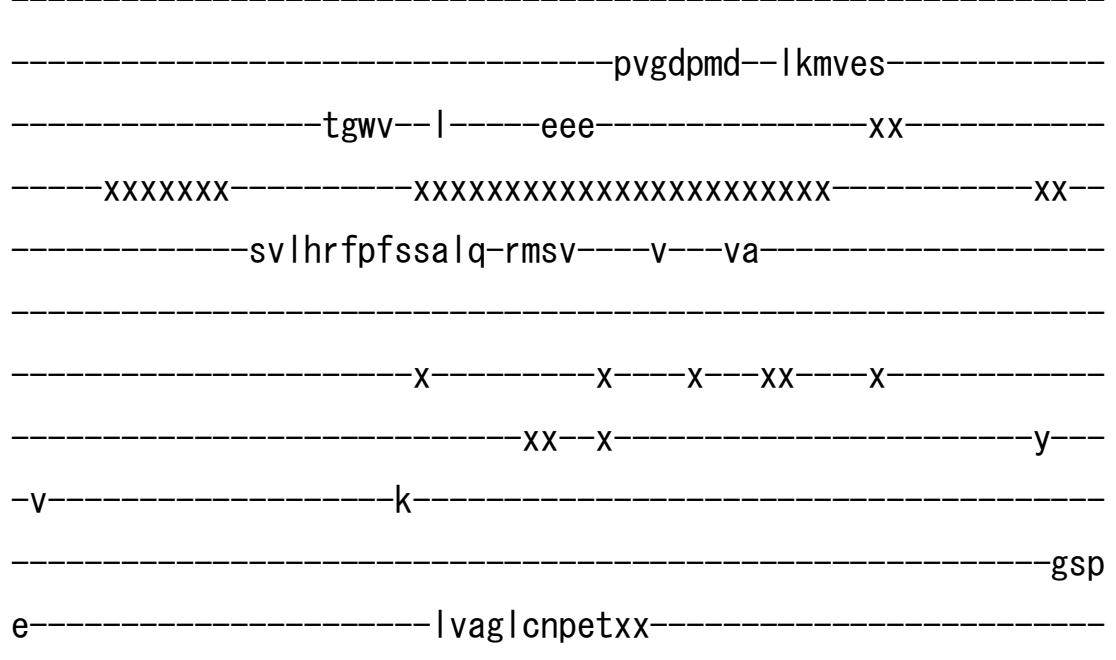

-tdf-aqm I qsy--taa----gyrvVALASKp I

-xxvps I eaaqq I trdxvegd I s I LGLLVMRNLLKPQTTPVIQALRRtr-IRA

VMVTGDNLQTAVTVARGCGM-

vaxxehli-ivh

ath pergxx-xxx-

xgvk

dpdqaasytx-

$-x x d p r s x---h$

lal sgptfg-

- i vkhf-pk I lpk 
vIvqg--tVFA--RMAPEQKTELVCELq-

---k l--qy--_-_-_-_-_-_-_-_-_-_-_-_GMCGDGANDCGALKAADVG I SL--

ASVVSPFTSSm-ASIECVPmV IREGRCSLDTSFSVF------K yma-Iys-I-tqfisvl i lyt in-_-_-_-_-_-_-_-_-_-_-_-_-_-_tn I gdlqfla

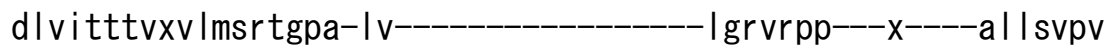
I-ss I | I qmv |--vtgvq I ggyf I t l aqpw------------------------ xxxx---

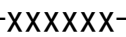

pd----n I pnyentvvfs Issfa-y l i laaavskgapfrr-----p l ytnvpf I vala I I ssv|vg|v--|vpg| |q----------gp la | rn

-------xxdtgfk----|I--|l g Ivt Infvga-

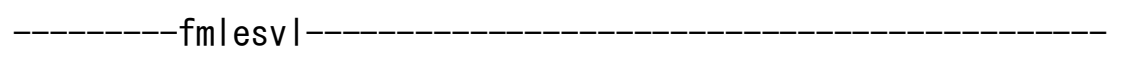

dqc $\mid$ pac $|x r| r x--x x x x$

$-x x x--x x x x x x x x x x x x-x x x x x x x x x x$

>hsaV_V202 XP_937041.2

$\mathrm{m}-$ 
$-x x x x x x x x x x x x x x x m e$ i ygy

n-------------------------Xxxxxxa i vs I gv i csggf I I I I ywmpewrvka

tcvraaikdcevv| Irttdefkmwfcakirvls let-ypvsspksxxxxxxxxhxx----

$x x x x x x x x x x x x x x x x x-$

sqtesqqi ryfthhsvkyfwn

dt ihnfdf IkgIde

gvsctsiy

-xxxxagltkgm-

-hayrk I lygvne iavkvp

$-x x x x x x i k e v \mid$

-npfyifq--Ifsvi

Iwst

deyyyyala

i vvm------------s i vs i vss I ys---------------xx-------------------kq

yvm Ihdmvat-

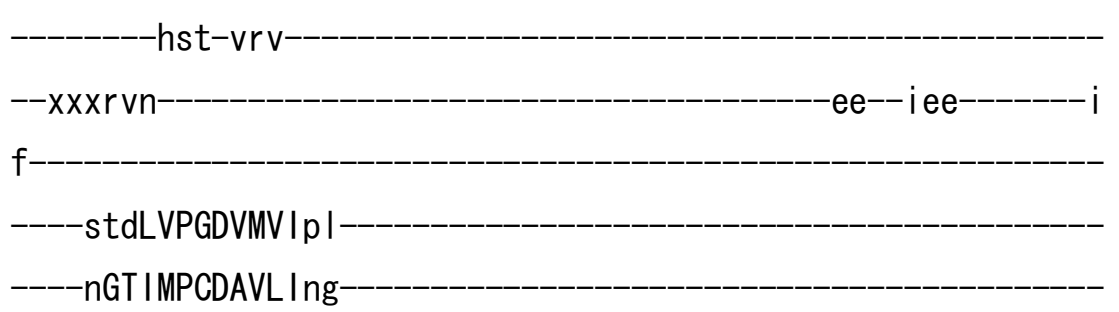


tc iVNESMLTGESVPVTKtn-

$x x x x x x x x x x x x x$

$-X X X X X X$

thk

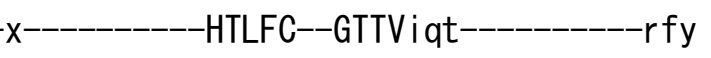

tg----e l vk-_-_-_-_-_-_-_-_-_-_-_-_a IVRTTGFSTSKGq I vrs i l y

-x--kptdfk I yrday If I I / vavag i gf i y

-ti insilnev -qvgvi i i esLDIITIT

VPPALPAAmTAG IVYAQR-RLkkig-

iFCI-SPQR IN I CGQLNLVCFDKTG

TLTEDGLDL

wg i qr

$-x-x x x-$

$x \times x$

speenvcxxxxx

$-x x$

qf

vacmatc-----hs $\mid \mathrm{t}-$

-ki egv-

$\mid$ sgdp |d--|kmfea

i gwi-- I

$-x x$

$x x x x x x x x$

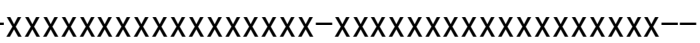
-givrqfpfssalq-rmsv-----v---ar

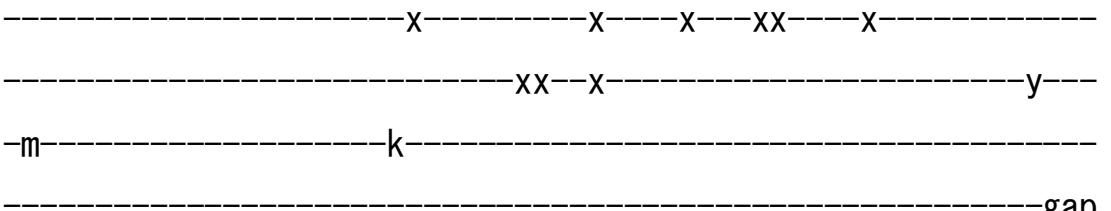

$\mathrm{e}$

-aiag l ckpetxx- 
-vdf-qnv l edf--tkq----gfrv lALAHRk I

-xxx I twhkvqn i srda-i ennmdfMGL I IMQNKLKQETPAVLEDLHKan-IRT

VMVTGDSMLTAVSVARDCGM

ilxxdkvi-iae

alp

$-p k d g x x-x x x x x x$

$x x x x x x x x x x x-a$

-xdpe

a ipvk Ivhdx

$x x d$ l qm-tryh

famngksfs

vi lehf-qd lvpk

Imlhg--tVFA--RMAPDQKTQLIEALq

$---n v--d y$

-FVGMCGDGANDCGALKRAHGG ISL-sele-

ASVASPFTSKt-PSI SCVPn I IREGRAALI TSFCVF

$-K$

fma-lys-i-iqyfsvt I lys i I-_-_-_-_-_-_-_-_-_-_-_-_-_-_n I gdfaf If $\mathrm{i}$

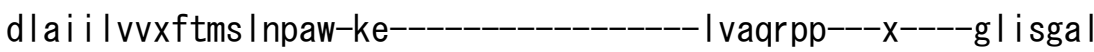

I-fsvlsqi i i--c i gfqs I gffwvkqqpw----------------------- $x x x x x x x$

$x x x x x x x x x x x x x x x x x----------x x x x x x$

eh----niqnyenttvffissfq-yl iva iafskgkpfrq-----pcykn- 


\section{>hsaV_V203 NP_115655. 2}

$$
\mathrm{m}-
$$

$x-x \operatorname{xx} x \operatorname{xx} x \operatorname{xx} x \operatorname{xx} x x x x x$

-me ifgy

r--_-_-_-_-_-_-_-_-_-_-_-_xxxxxs I c lags i fsfg i I p I vfywrpawhvwa hcvpcs I qeadtv I Irttdefqi yswkkviwiy I sa Insafg I tpxxxxxxxxexx----

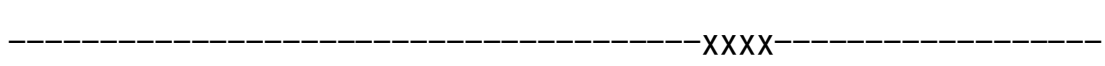

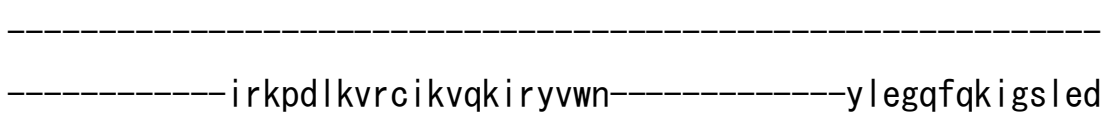
w Issakih$-x x x x s g \mid t r e e-$ -qe irr l icgpntidvevt

$-x x x x x$ ikevl 


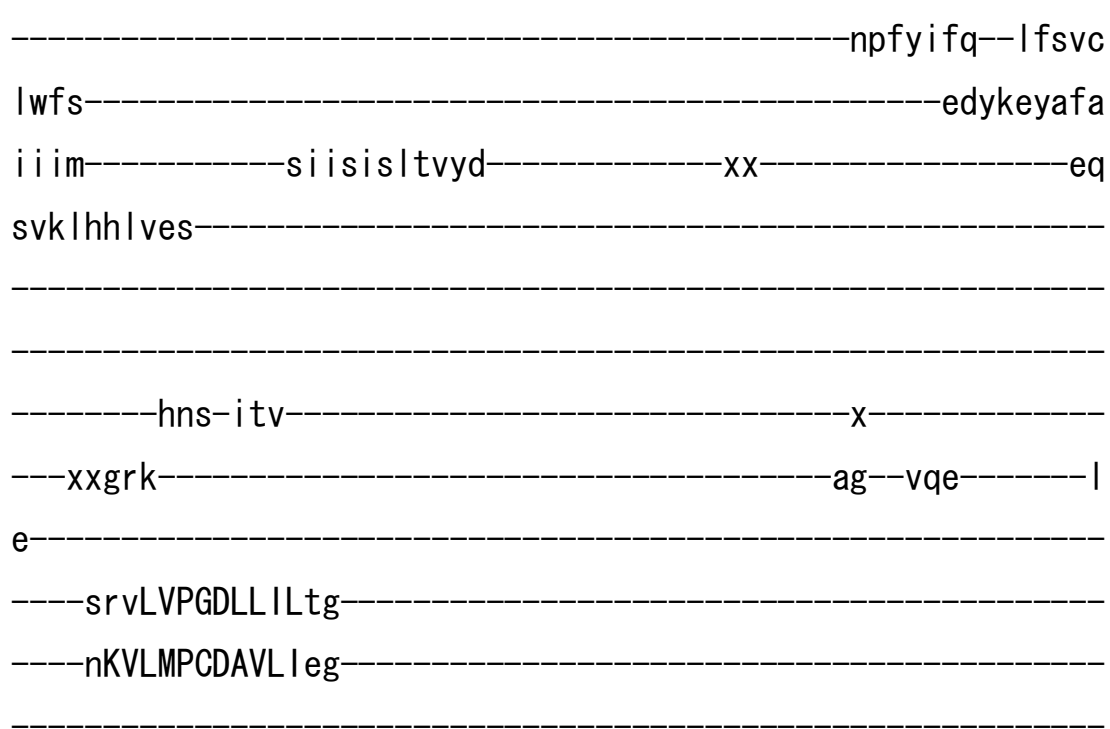

scVVDEGMLTGESIPVTKtp-

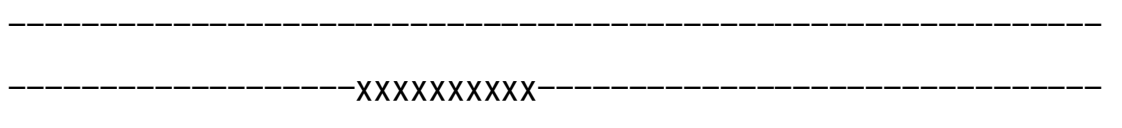

$-x x x x x x$

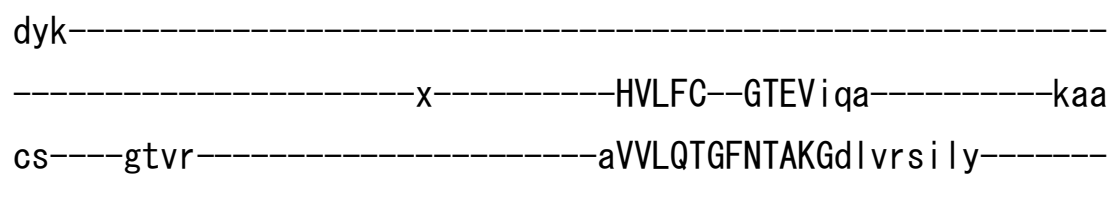

-x--kpvnfqlyrdairfl lc lvgtatigmi y

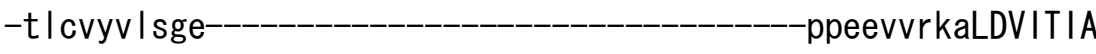

VPPALPAA I TTGI I YAQR-RLkkrg-

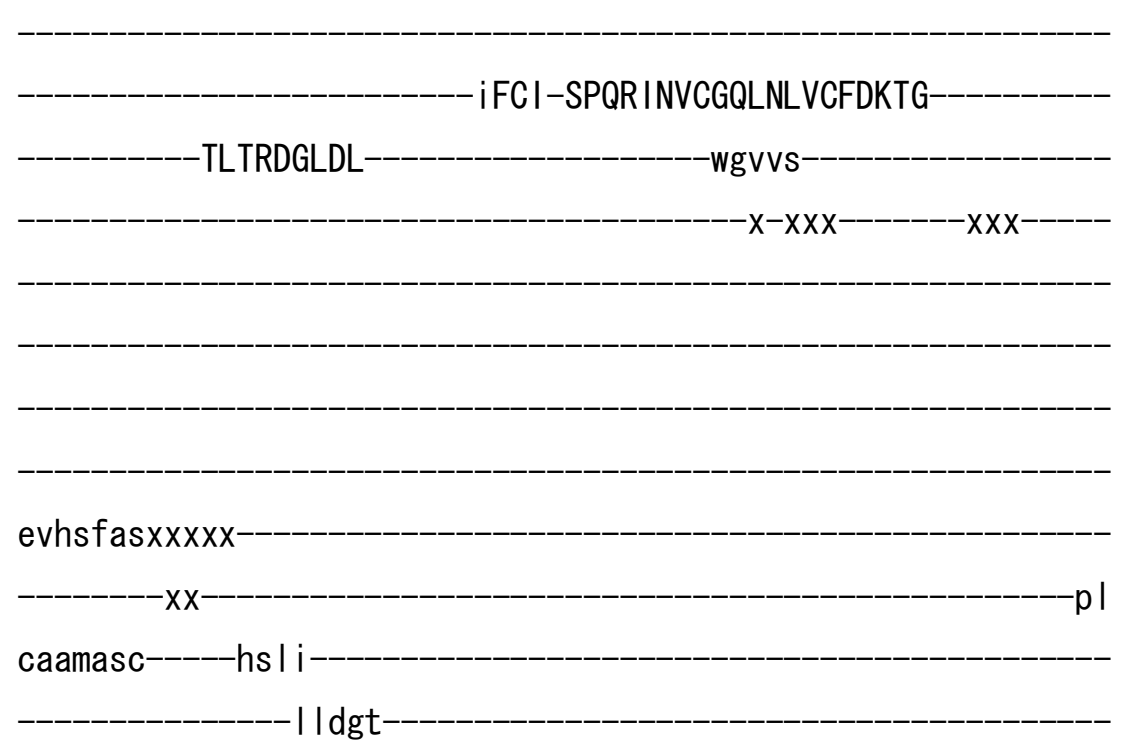




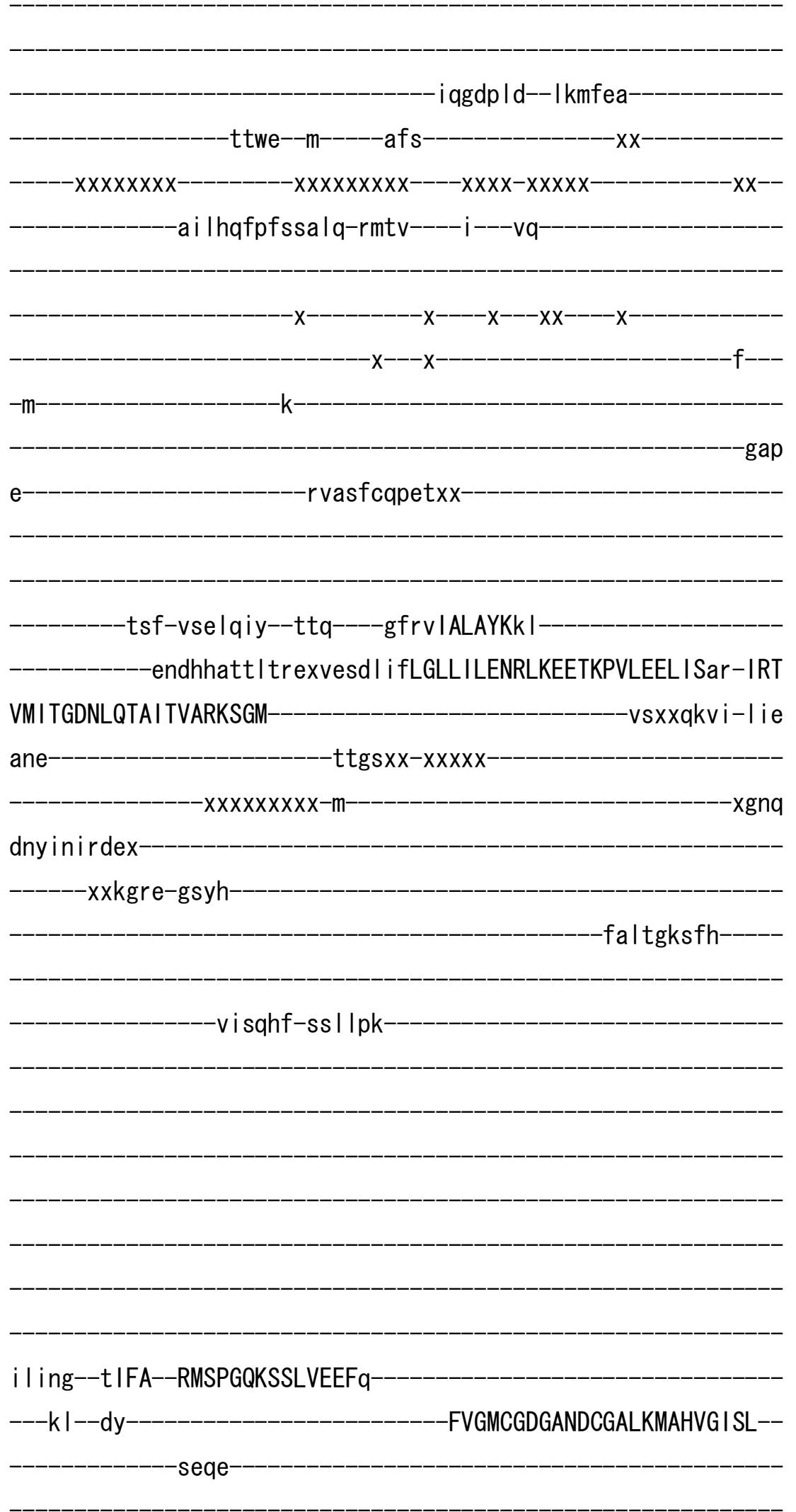

-ASVASPFTSKt-PNIECVPhI I KEGRAALVTSFCMF------K 


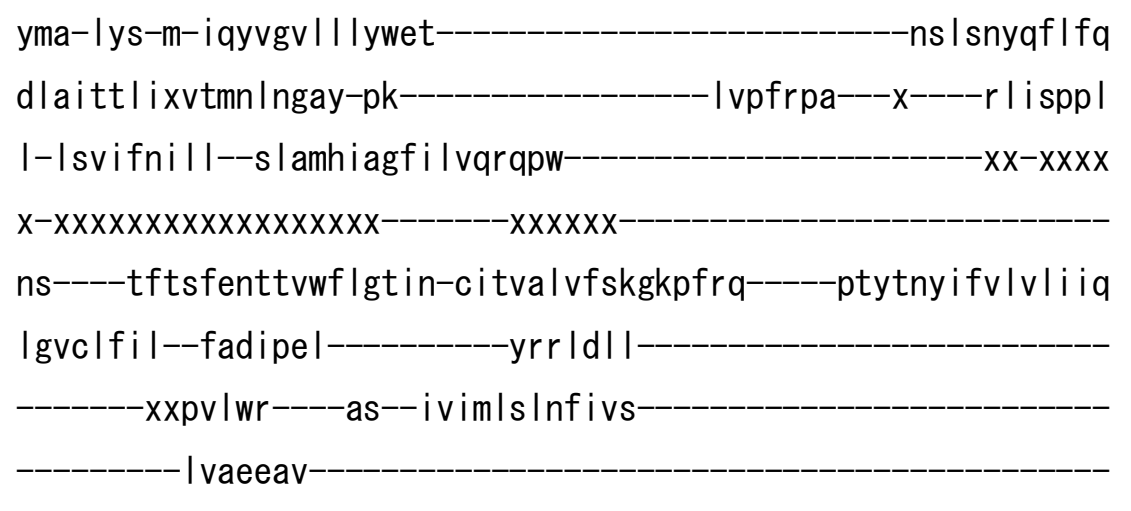

-ienralwm-mikxxxxxxx-

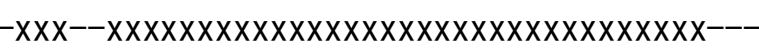
$-x x x x x x x-x x x x x-$

I

>hsaV_V204 NP_940907. 2

m-

$\operatorname{XXXXXXXXXXXXXXXXX-10}$

levfgy

r-_-_-_-_-_-_-_-_-_-_-_-_-_--xxxxxxafc I vasv I tcgg I I I v fywr pqwrvwa 
nc ipcp I qeadtv I |rttdefarymrkkvfcly Ist Ikfpvskkwxxxxxxxxxhxx-----

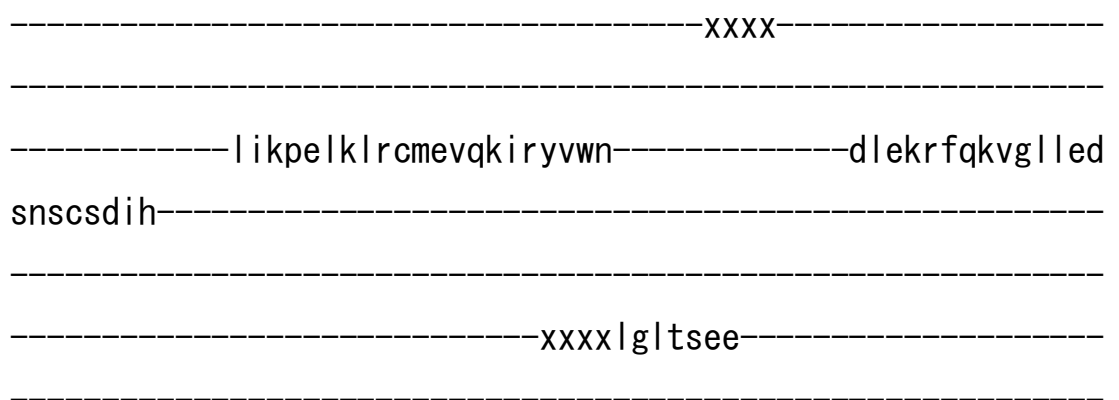

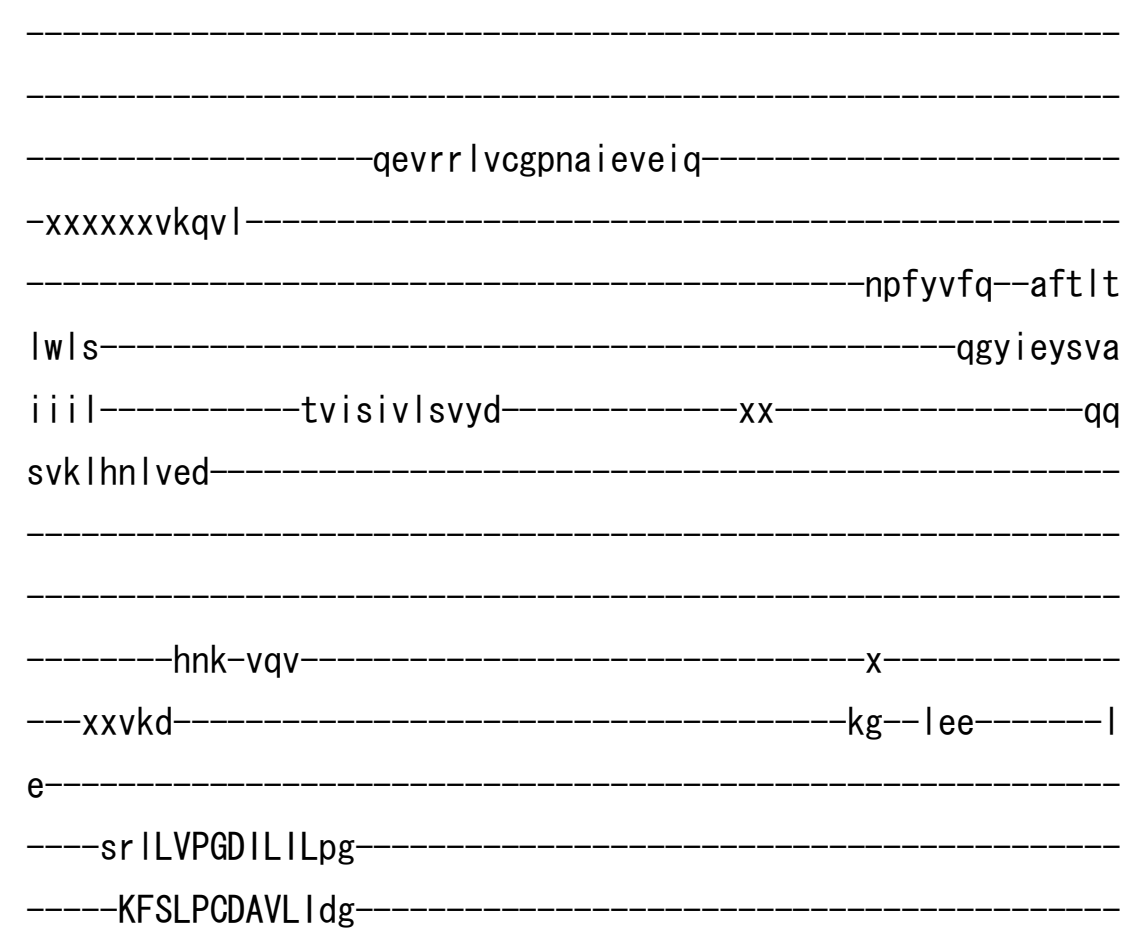

-scVVNEGMLTGESIPVTKtp-

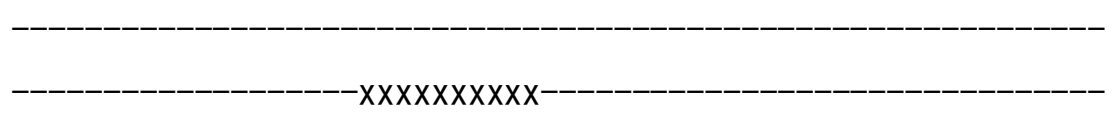

$-x x x x x x$

dy

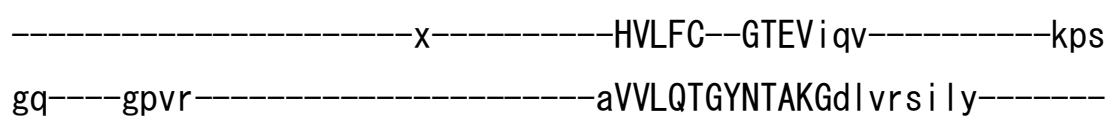

-x--rp Infk Iysdafkfivf Iac I gvmgffy

-al gvymyhgv -ppkdtvtmaL ILLTVT

VPPVLPAAITIGNVYAQK-RLkkkk- 
iFCI-SPQR INMCGQ INLVCFDKTG-

TLTEDGLDL

-wgtvp

$-x-x x x$

eahsfas $x x x x x$

$-x x-$

caamasc-----hs I i

l lngt-

qgdp I d--|kmfeg

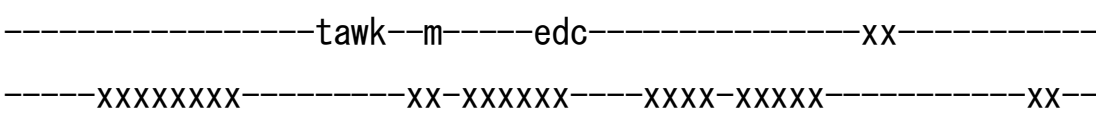

it l cqfpfsss Iq-rmsv----i---aq

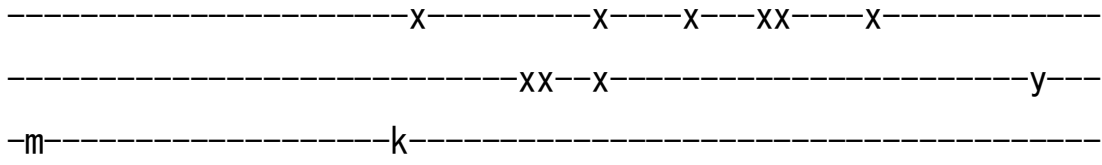

gap

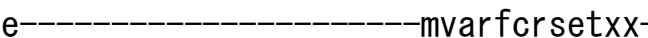

-knf-pqe I rsy--tvq----gfrv IALAHKt I

-xmgn I seveh I ar exvese I tfLGLLI MENRLKKETKLVLKELSEar-IRT

VMI TGDNLQTAITVAKNSEM ipxxsqvi-ive

adepeefxx-xxxxx

gkk

e i ymhtgnsx

$x x x x x x x x x-p$

------xxrgegxscyh

famsgksyq

-vifahf-ns I lpk 
i l vng--tVFA--RMSPGQKSSLIEEFq

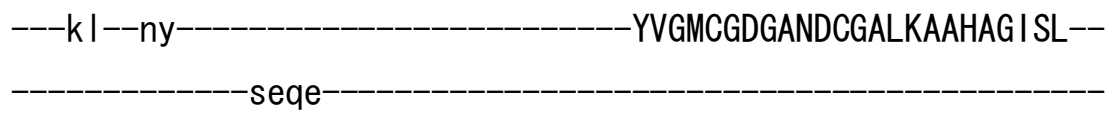

-ASVASPFTSKt-TNI QCVPh I IREGRAALVSSFGVF------K

y lt-myg-i-iqfi sal I lywq I-_-_-_-_-_-_-_-_-_-_-_-_-_---q I fgnyqy Imq

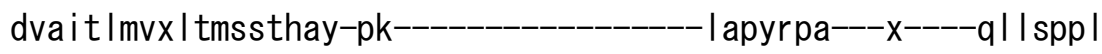

I-I sifInscf--scivqi saf I yvkqqpw---_-_-_-_-_-_-_-_--_xxxxxxx

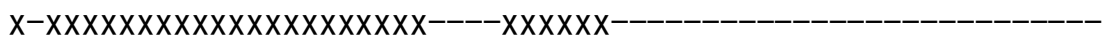

pg----si Isfettt I wp itt in-yitvaf i fskgkpfrk------pi ytny ifsf I I laa

Igltifil---fsdfqvi-_-_-_-_---yrgmeli-_-_-_-_-_-_-_-_-_-_-_-_-_-_

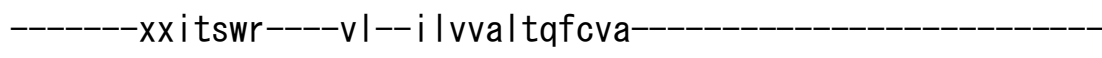

ffreds i

|qnhe $|w|-\mid i k x x x x x x x$

$-x x x--x x x x x x x x x x x x x x x x x x x x x x x x x x x x x x x x x x x x x$

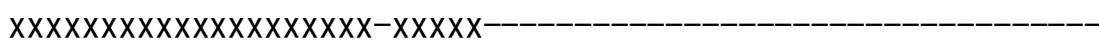

-_-_-_-_-_-_-_-_-_-_-_-_-_-_-_-_-_-_-_-_-_-_-_-_-_-_-_-_-_-_-_-_-_

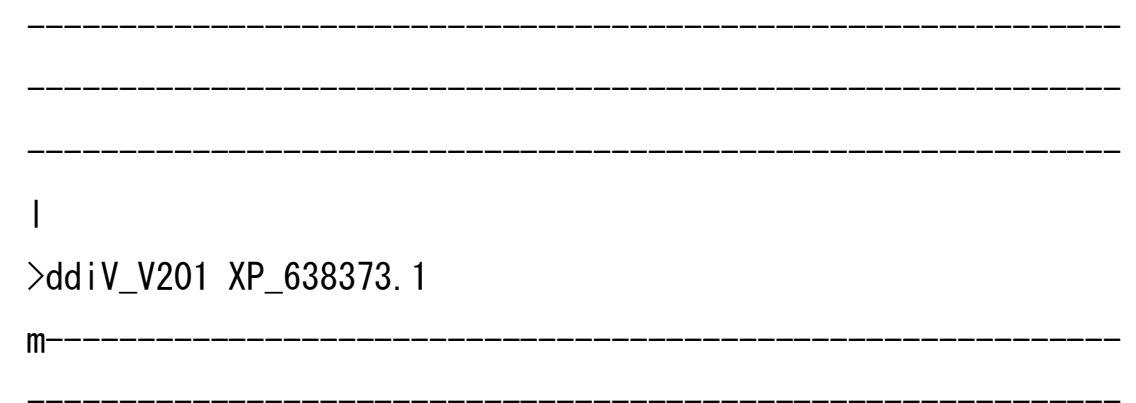


gnee i gene I ep I I vnngsgd I sy I kkfekqen i denggddm I I vxxxxxxxx i xxxxxx $x x x x-$ $x x x x x x x x x x x x x x x x x x x x x x x x x x x-x x x x x x x x x x x x x x x x x x x$ $x x x x x x$

yekidfIrxxx

$-n g \mid$ senn

- armkgrfgsne infpvkx

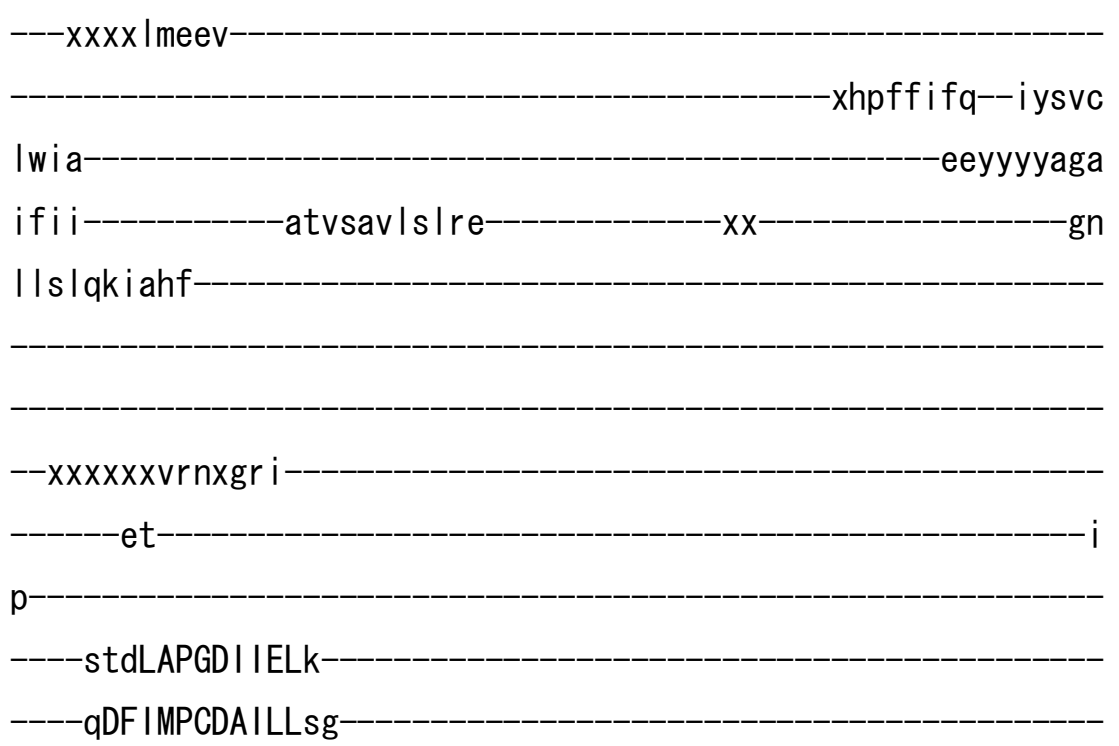


-qa iLNESMLTGESI PVNKys-

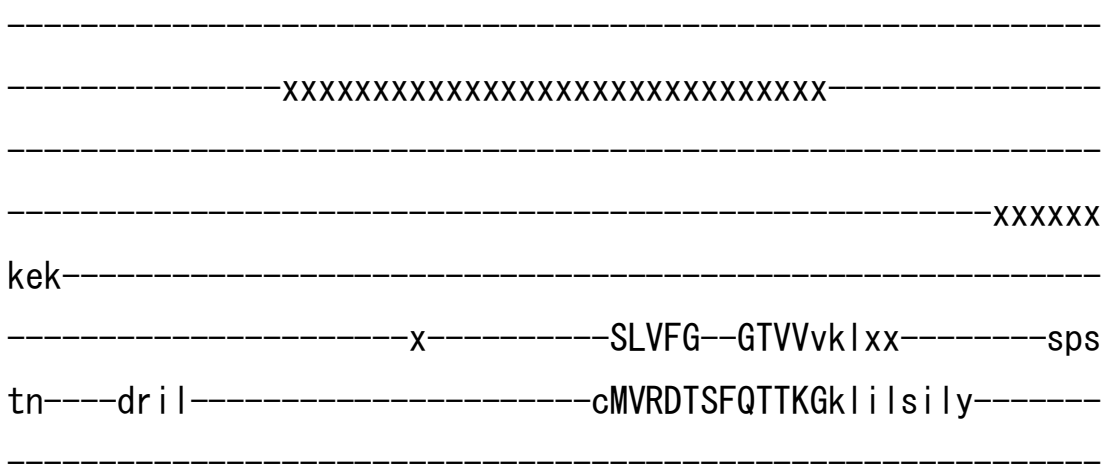

-xkkshfkffmes lkfvgflcflavigfci -svwk Ins Ig i -dfgtiairaLDLITIV

VPPALPMAmTVGTGFGLS-RLrktk-

-vFCI-SPPRLNMAGK I QVFCFDKTG-

-TLTEEGLDL $-\lg$ i i sx-

$-X X X$

tvkhvdnxxxxx

$-x x x x x x x x x x$

$x x x x x x x x x x$

$-m f$

rvImasc-----hs It

-vings

-vsgdple--ikifes

tnst--i--xx

$x x x x x x x x$

$-x x x x x x x x x x x x x x-x x$

-sy lenfdfqsk l q-rmsv----i----vq

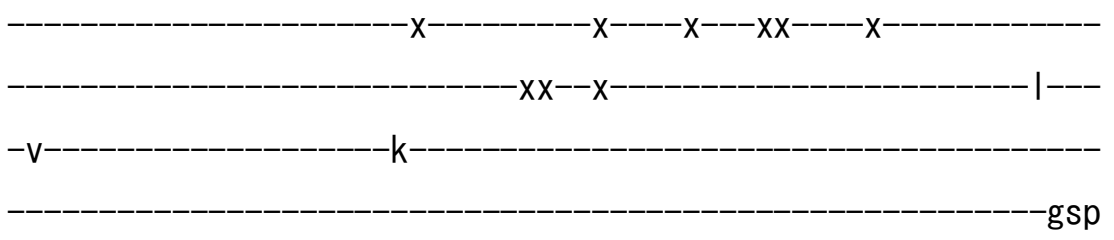

e----------------------mvkg l cf qht xx 
-ndy-dsq l amy--tek----gyrvLACAYRsw

-xptv I fpkre I I rkxsesn I hfLGF I IMENRVKPQSPP I I QRLQSan-IRT

VMVTGDNGLTATSVAKQCGI ikxxtIIf-mg-

I i $-x x x--x x x x x x x x x x x x x g$

xxxxhk |

dpttIIIdex

xrdyn

livsgpvfk

qi ynhyxatgspr

$\mathrm{XXX}$

mIrrg--iVYA--RMTPDEKQSLIEELq-

$---r i--g \mid$

-YVGMCGDGANDCGALKAAHVGISL--

sese

-ASI AAPFTST $i-T D I S C C P n$ I I KEGRASLAVSFKLF------Q

fmg-iysxixftsvifcy

xxxsv I gnway lya

dl ivifp Ixif I gmtepcxk-

Isvkrps

$-x r \mid$ is Inm

i-gs I IshitvxxvfII vvfhIvqskswfdx

plxxxxn ifnyitts Ififgcfq-ysimlfvfsfgkpflk

-aiytnrylflvyivt

lianli ilxxgfeklyn

flqurvi

-pvswr----st--mfgm i i gn I van-

clielsf

-yfyksrskxkkixxxxxxxxxxxxxxxxxxxxxx

$\operatorname{xx} x \operatorname{xx} x \times x x x x x x x x$ 
>ddiV_V202 XP_639952. 1

m

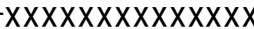

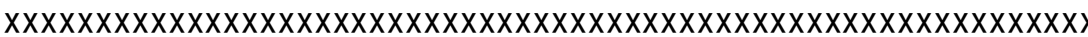
$x x x x x x x x x x x x x x x x x x x x x x x x x x x x x x x x x x x x x x x x x x x x x x x x x x x x x x x a n k k \mid k$

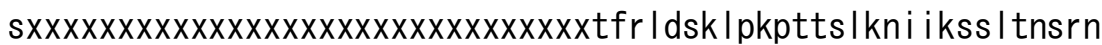

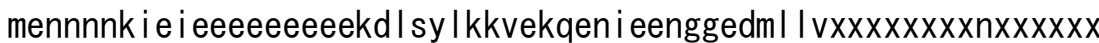

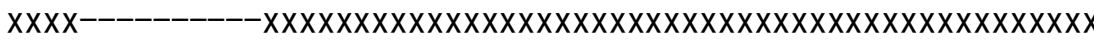
$x x x x x x x x x x x x x x x x x x$

-nqytekyi iyrfxxxxxxekiqeyektkIvnq yekiell rxxx

-ngl skes lkk Ikqrfgene infpvkx

$---x x x x \mid m e e v$ 
-xhpffifq--iysvc

Iwia eeyyyyaaa

ifli atvsa i is I re

$x x$ -gn

IIslqkishf-

$--x x x x x x \mid r n x g e i$

et

s

----steLVPGD I IELr

--- qDF IMPCDVILLsg

-qa iLNESMLTGESIPVNKys-

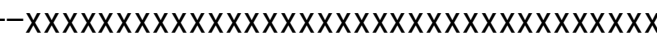

$-x x x x x x$

kek

$x-$

-SL IYG--GTVVvk I xx-sts

tn----dkifgMVRDTSFQTTKGk I i s i ly

-xkkshfkffmes lkfvgvlcvlafagfci -svwr lkt I gv -tnhi ial raLDLITIV

VPPALPMAmTVGTGFGLS-RLrksk-

iFCI-SPPRLNMAGK I QVFCFDKTG

TLTEEGLDL Ig i i sxxxxxxxxxxxxxxxxxx

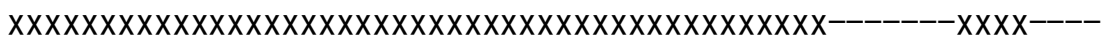

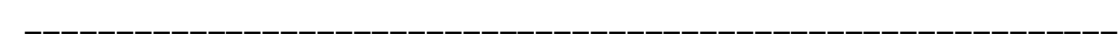

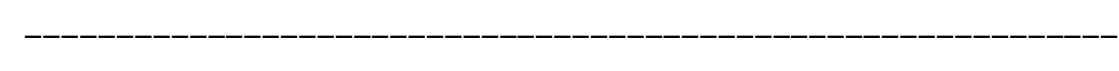

$-x x x x x--x x x x$

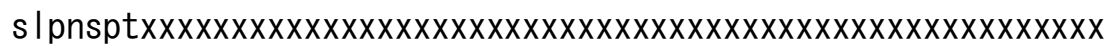

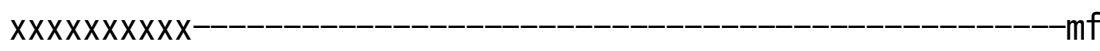
rl|mstc-----hs|t-vings- 
-vsgdple--ikifes

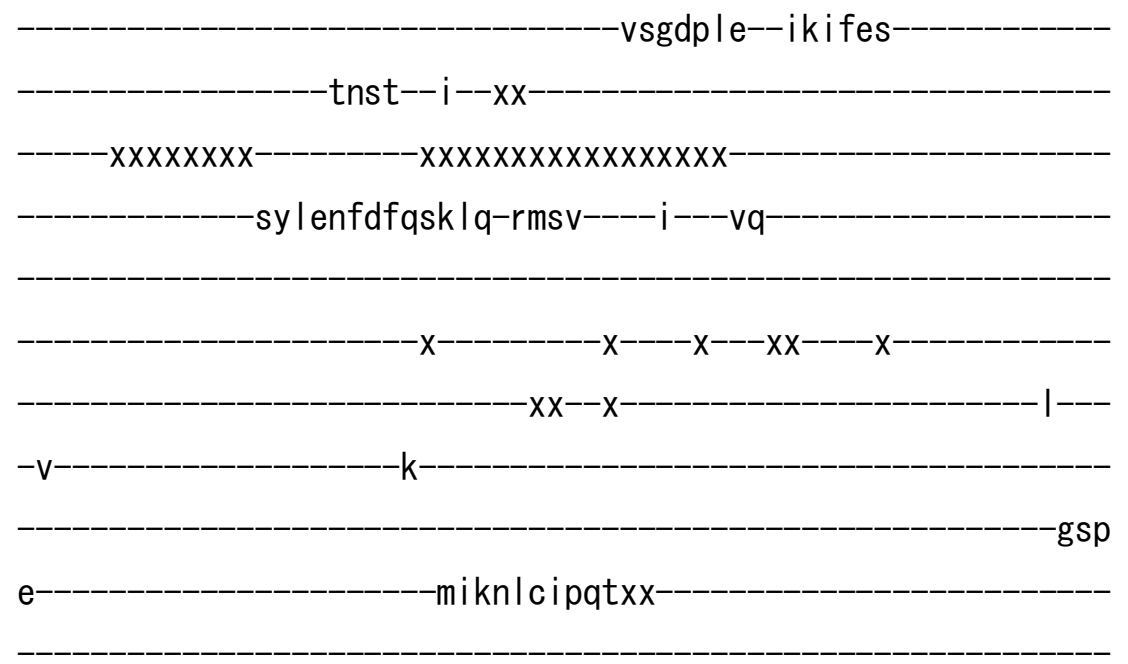

-kdy-dsq l a i y--tek----gyrvLACAYRkw

-xptf I fpkrdi I rkxsesn l efLGF I MENR IKPQSPP I IKKLQKan-IRT

VMVTGDNGLTATSVAKQCGI -

-ikxxsilf-mg-

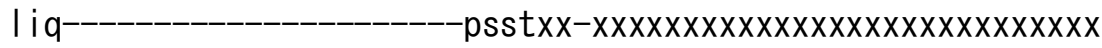

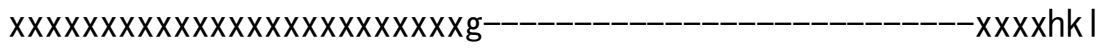

dpnt I I Idd

$-x r d y n$

livtgpvfk-

qi ynhyxatgstr

$-x X X$

m $/$ rrg--vVYA--RMTPDEKQTLIEELe-

$---r i--g \mid$

-YVGMCGDGANDCGALKAAHVGISL--

sese 


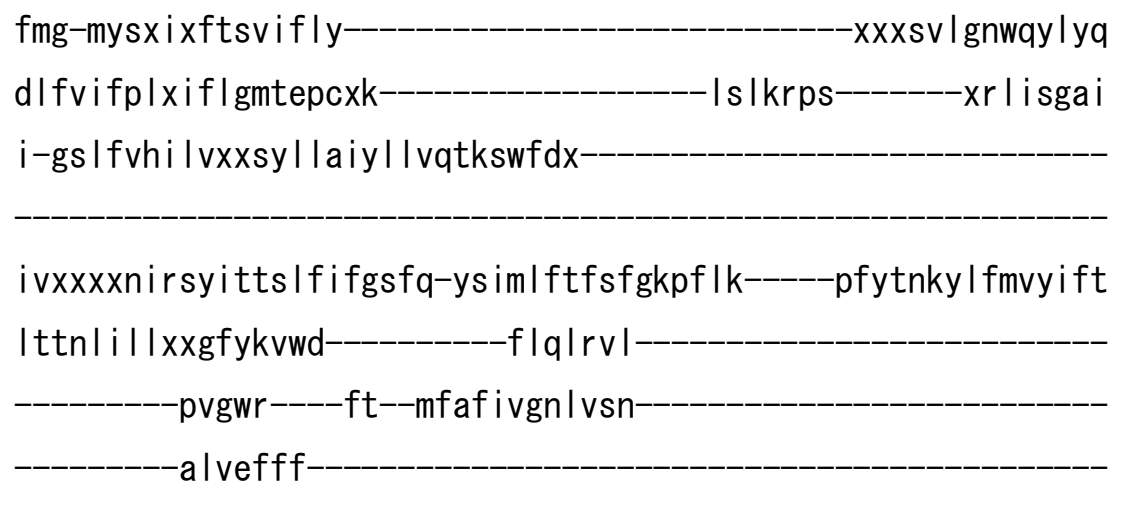

$-y$ lyksrskxkki ixxxxxxxxxxxxxxxxxxxx-x $x X X X X X X X X X X$

>ddiV_V203 XP_641833. 1

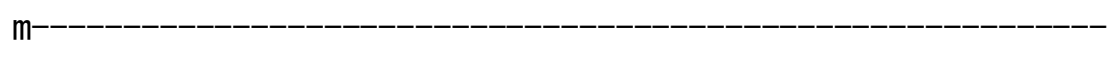

-xxxxxxxxxxxxxxxxxxxxxxxxxxxxdymrgy

$q^{-}$

-xxxxxx i lhyg I i i lsag I I I lvyhwvprwgikm 
rfkeck Indanyvyvkakdkkye I sqvkhv I igkkd le

fsesekkdky i efkftr I fyn

-vekd I firpisntd

yhrskinexxx

$-\operatorname{tg} \mid$ smnk

-yqegrd Ifgpnl isvplk

$-x x x x x \mid$ de $i$

-hpffifq--iysi i

Iwct

edyyvyaga

ilfi-

-alvtaiftlre

$\mathrm{xx}$

$-n n$

IIrIneiaty

-tv iLNESMLTGESIPVTKys-

$X X X X X X X X X X X X X X X$

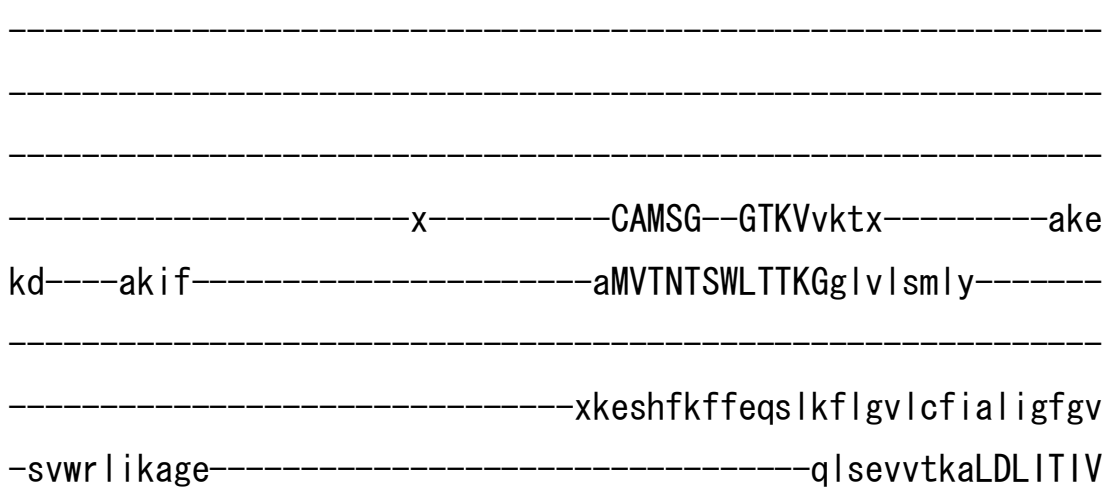

VPPALPMAqSVGTGFSIV-RLkank- 
iFCI-SPPRVNMAGKVEVYCFDKTG-

TLTEDGLDL

$-f g v \mid p x$

$-x x x x x$

$\mathrm{XXX}----$

gfgtvikxxxxx

$x x x-$

$-p \mid$

fl tmatc-----ht la

hideq-

-vsgdp l e--ek i fta-

-tnak--I--xxxgef

$-x x x$

$x x x x x x x$

$-X X X X X X X X X X X X X X X X X X X X X X X$

-al verfefasal q-rmsv-----v---ir

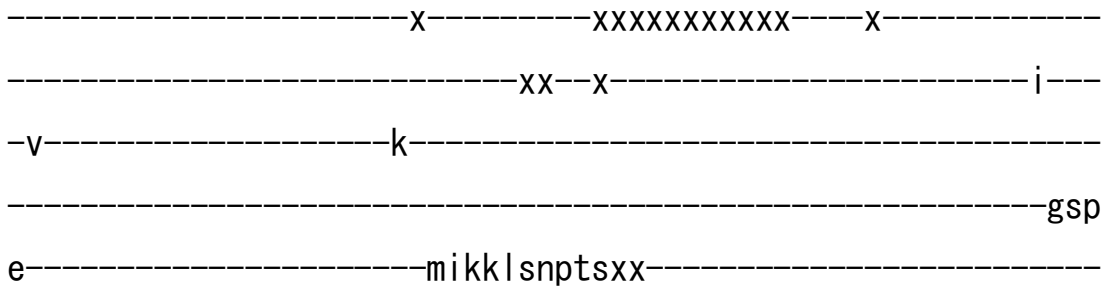

mikkl snptsxx

-sdf-ehv I sty--tkk----gyrvLGCGYKew

-xxsds i gktkde i rsxverd ImfLGF I IMENK IKPETAGALKVLKDan-IR I

IMVTGDNVLTAVSVSRECGL-

vtxxkt if----

$-s s q \mid x x x x x x x x x x x x x x x x x x x x x x x x x x x x x x x$

$x x x x x x x x x x x x x x x x x x$

lavtgdvwk

-yydqfxangqpt 


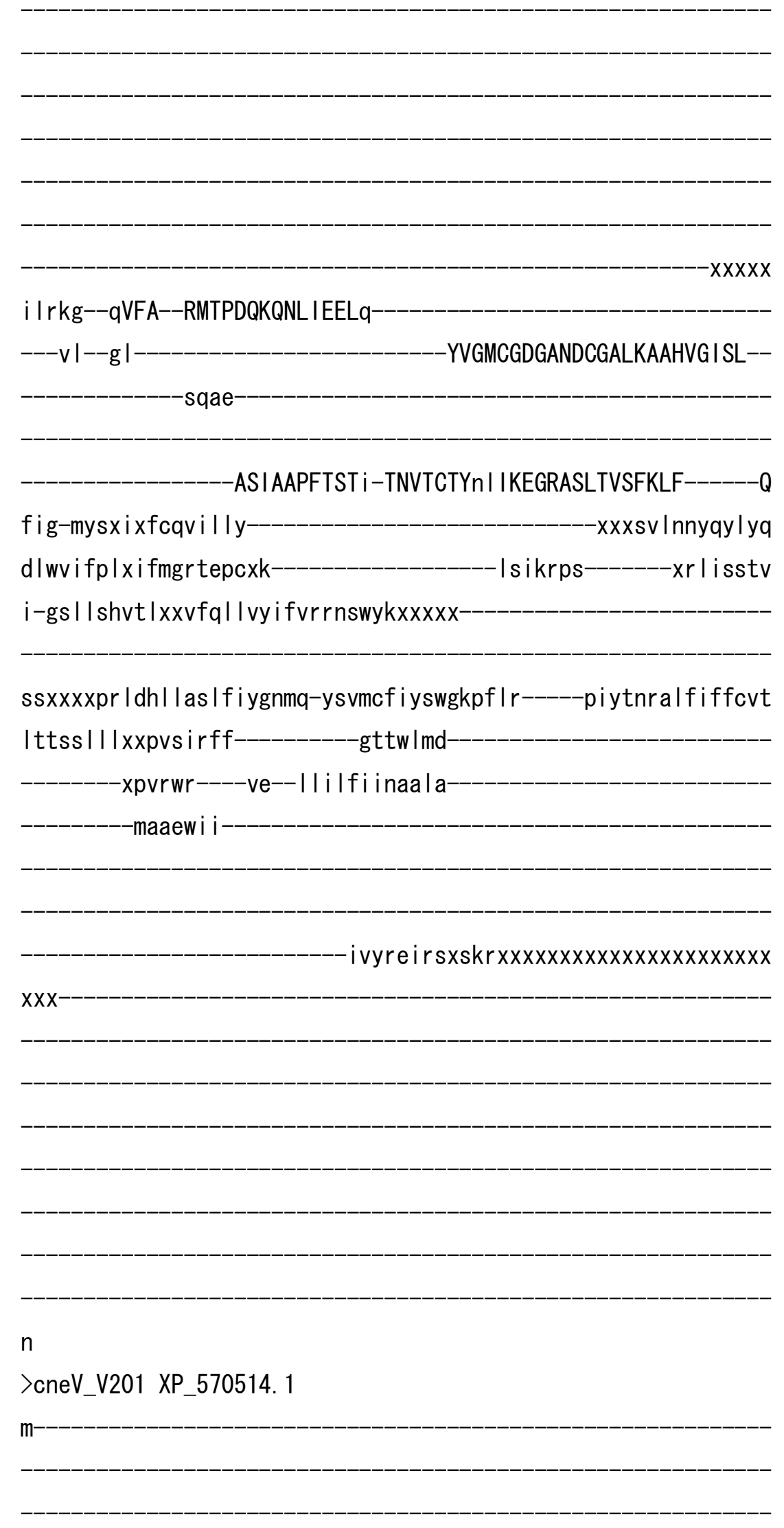




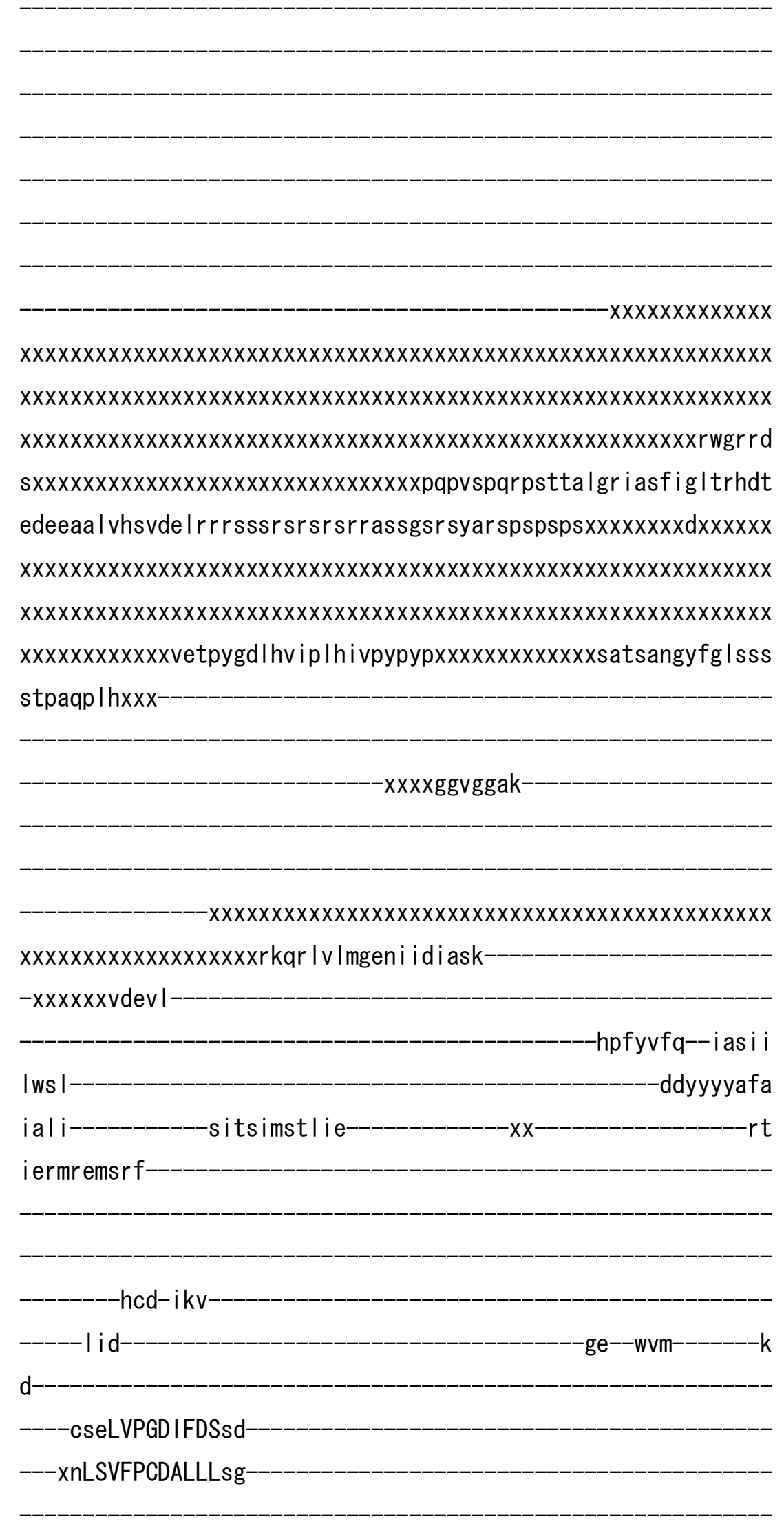


-da iVNESMLTGESVPVSK ip-

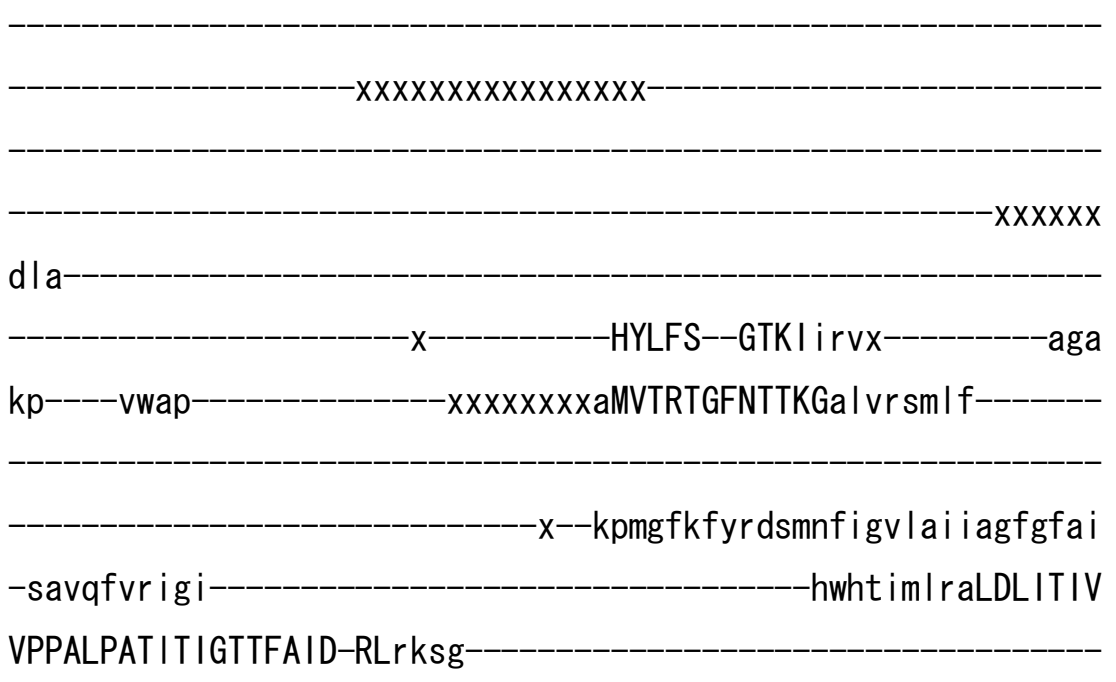

iFCI-SPNRVN I GGK I NVVCFDKTG-

TLTEDGLDV

Igartx-

$x x x x x$

XXX-ーーー

$-\mathrm{XXXX}$

diadvpixxxxx

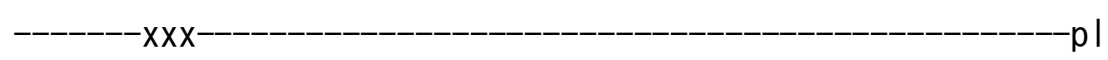

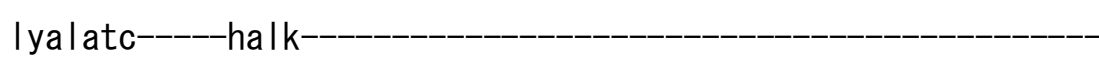

lidge-

i igdp Id--ikmfey-

$-\operatorname{tgwt}--1--x x$

$x-$

XXXXXXXXXXXXXXXXXXXXXXXXXXXXXXXXX--XXXX--XXXXXXXXXXXX--gvirtfdfvsalr-rmsv----i----vk-

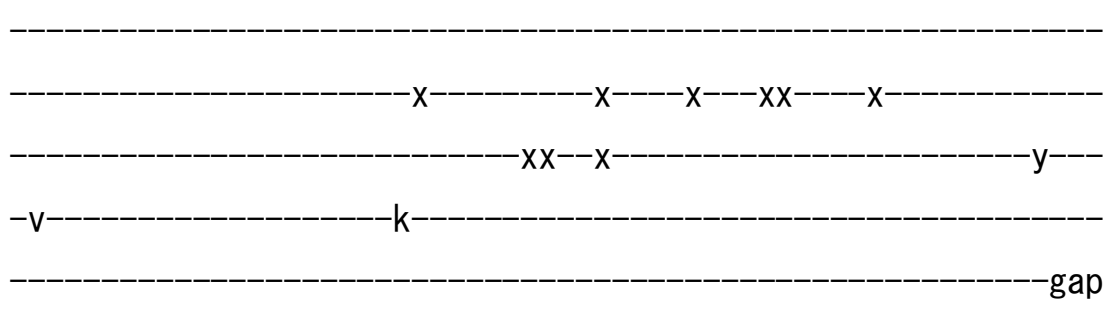

e--------_-_-_-_-_-_----vmpe i cdpssxx- 
-hdy-ddm I syy--trn----gfrvIAIAGKs i

xx-----xxx I raqrmr rdv----aesd I qfLGF IVFENKLKPGTAPN IHTLRAah-LAC

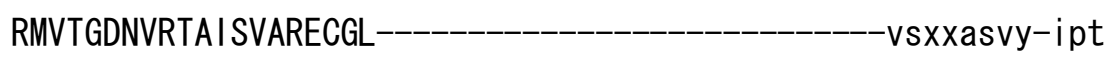

fip--_-_-_-_-_-_-_-_-_---_gtgvxxxxxxxxxxx--_-_-_-_-_-_-_-_-_-_-

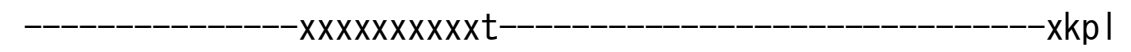

tn--qvgvax-------------------------------------------------------

xxaeaexhdyq-

laltgdvfr

-wmleyaxfetmer

mIvkg--vIFA--RMSPDEKAELVERLq-

---s।--gy--_-_-_-_-_-_-_-_-_-_--_TVAFCGDGANDCGALKAADVGVSL--

-seae-

ASVAAPFTSQ i-PDI SCMV i IKEGRAALVTSFSCF------K

yma-Iys-m-iqfmtvt I lysfa--_-_-_-_-_-_-_-_-_-_-_-_--_ss I gdfaf I y $\mathrm{i}$

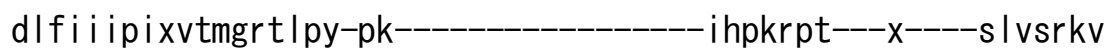

I-is i igqi l i--naa iqvfvfvwvrkqpw-_-_-_-_-_-_-_-_-_-_-_-- $x x x--x x$

xxx--_-_-_-_-_-_-_-_-_-_-_-_-_-_-_-_X-_-_-_-_-_-_-_-_-_-_-_-_-_-_-

kl----etfnfensalf I vscfq-y i Ivagvfsvgppyrk-----p |ytnps Ivic|tv I

tsfsay i I--Ispaas i-_-_-_-_----aai I dit--_-_-_-_-_-_-_-_-_-_-_-_-_-_

--_-----xxkftfr-----lq--| lavaavni las-_-_-_-_-_-_-_-_-_-_-_-_-_-_

faferfa- 


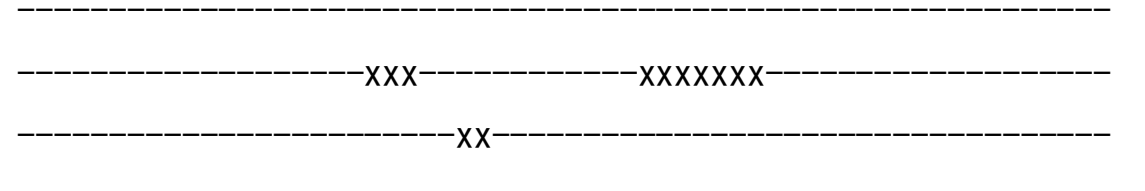

>pfaV_V201 XP_001351598. 1

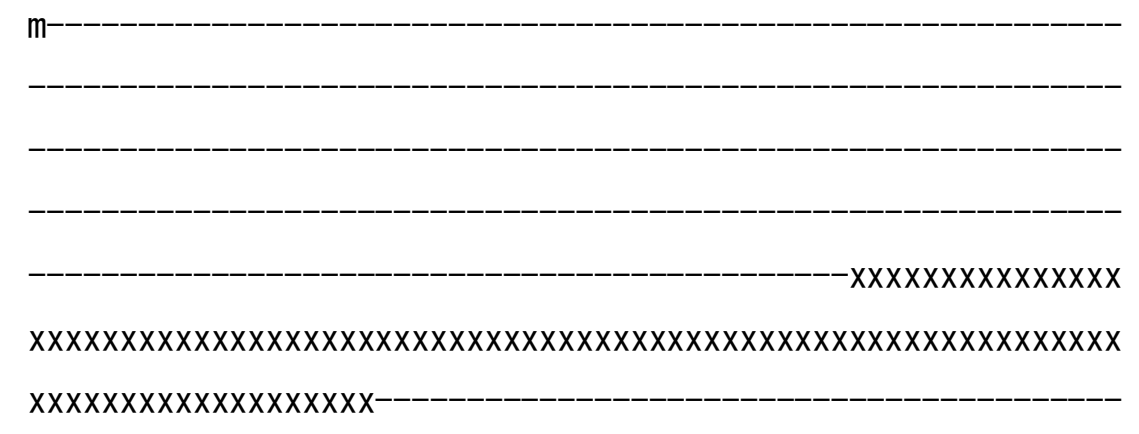

$\operatorname{xxXXXXXXXXX}$

XXXXXXXXXXXXXXXXXXXXXXXXXXXXXXXXXXXXXXXXXXXXXXXXXXXXXXXXXXXXXXXXXXX

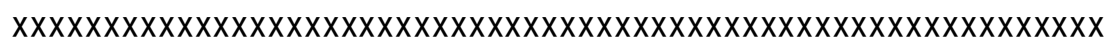

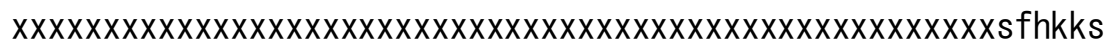

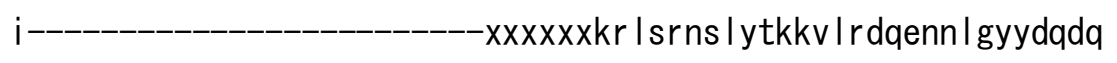
ne i nkmns i dhi yn i ds I nv i dqgndknydneeke i nkkypfnrnxxxxxxxxdxx---$-x x x x x x x x x x x x x x x x x x x x x x$ XXXXXXXXXXXXXXXXXXXXXXXXXXXXXXXXXXXXXXX $x x x x x x x x x x x x$ rey i ennlkvhkvkvr ineknxxxxxxxxxxxxxneekdgfyn $i$ shs $i$ eekvknldxxx $-x x x x g g$ Innnei innineygynnihlefy$-x x x x x x k r e I 1$ 


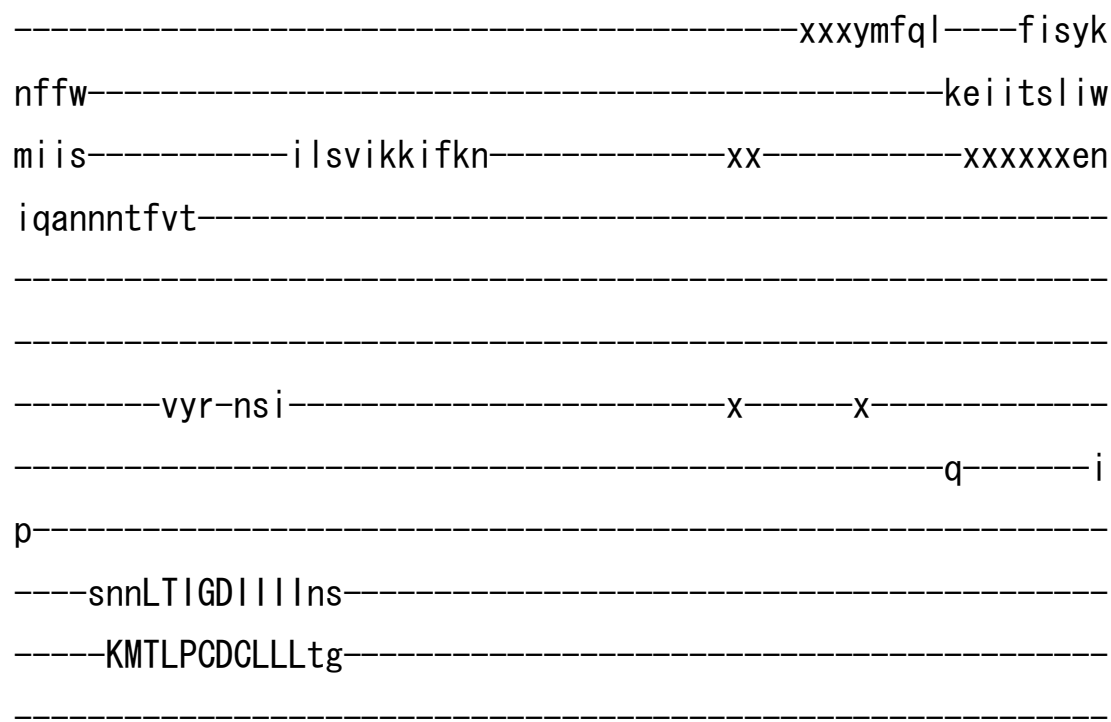

-nv IVDESLLTGESRPMKK i c-

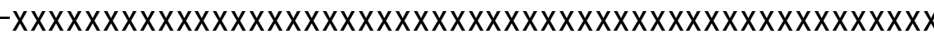

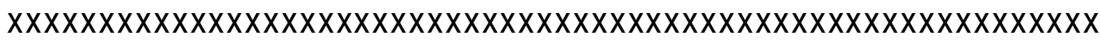

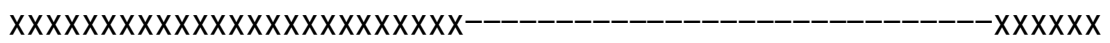

fnt -NILYA--GTDV----x----------st I

nf-----ten -xaIVINVSIYTYKGkymqnv I $f$

-pnp I Ifkydsqlpivfiftimfsfvc I

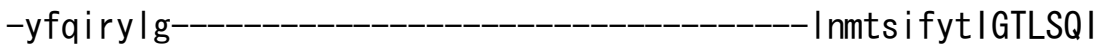

LPVWTPVV INIGLNISTN-RLkkekx

-iCCI-APSRIPICGK IRVFFFDKTG

TLTDHKIEFx xxxxcnni Ixxxxxxxxxxxxxxxxxx

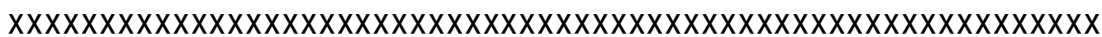

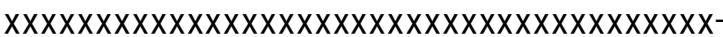
$-x x x x x--x x x x$

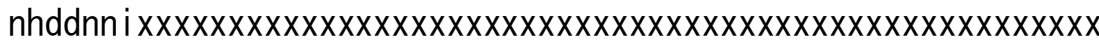

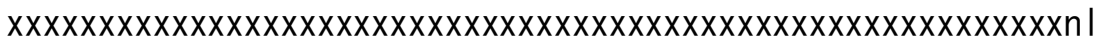
nekeq I $x x x x x n$ e $i k x x x x x x x x x x x x x x x x x x x x x x x x x x x x x x x x x x x x x x x x x x x x x x$

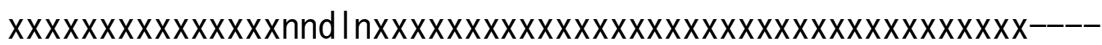




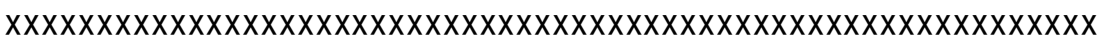

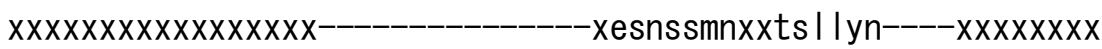 xxxxxxxxxxxxxxxxxagcsxxy--Xxxnni--------------- $x x-----------$}

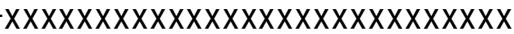

$x x x x x x x x x x x x x d v|k t y e f d y y t k x s t t| x x x x f x x x e k$

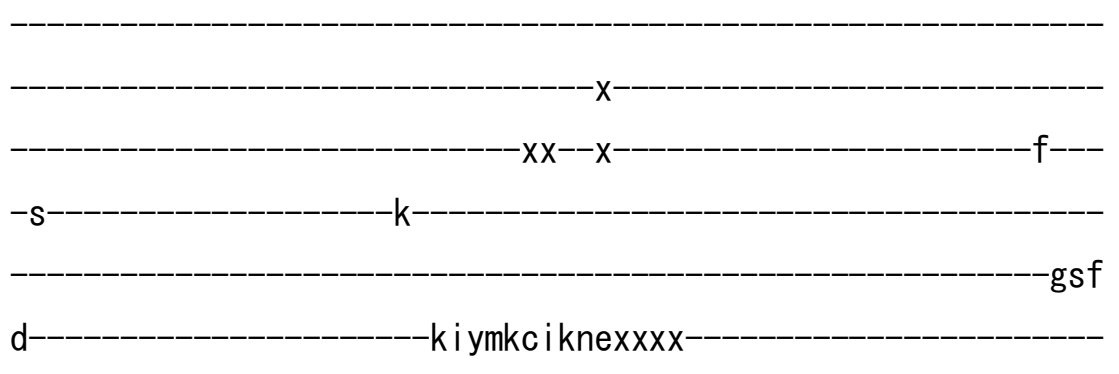

-f-kkkeqey--skn----gfyv IALAFK i i

-xnmkheh i Id Iprqx I ekdmnfLSL IMFNNHIKKDASMVIQTLKGss-IRP

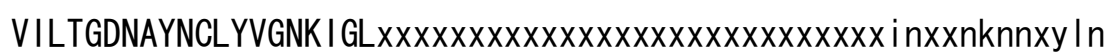

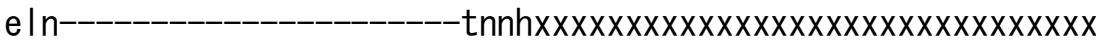

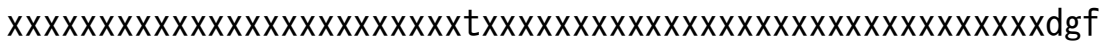

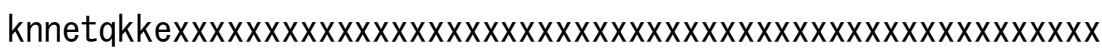
$x x x x x x x x$ h $x x x x x x x x x x x x x x x x x x x x x x x x x x x x x x x x x x x x x x x x x x x x x x i$ i I tgeayt

firyhvxqiktpd-

$-x x x x x x x---x x x x x x$

fIIri--rIFS--RLTPNNKIEVIRDF i

$---k f--d y$ - ISGMCGDGSNDCGALK ISHAGLAL-snld 


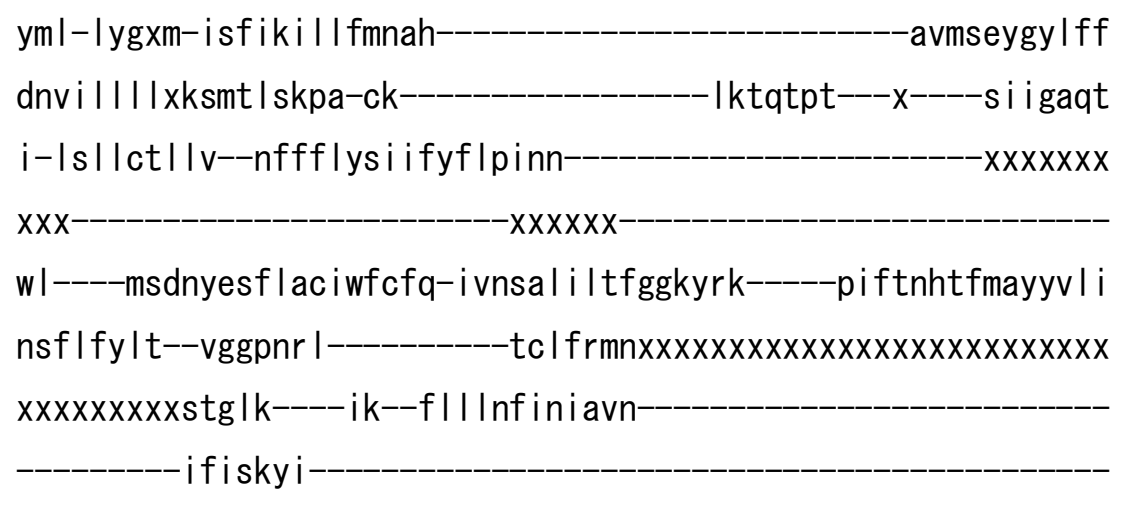

- Ices I yxvvrxxxxxx-

$-x x x x x-$

i

>pfaV_V202 XP_001351718. 1

$\mathrm{m}-$

xxxxxxxxxxikkety

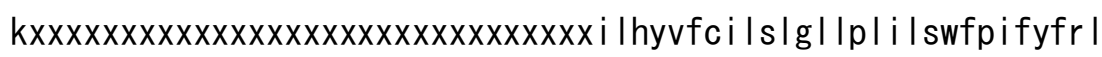


Ihsact Inecekvl i itkdgnky ikkikkikfnhnvcyvmndvffxxxxxxxxxmxx---$x x x x x x x x x x x x x x x x x x x x x x x$

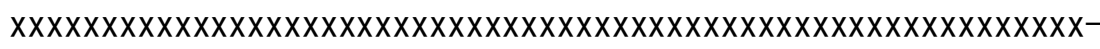
kdn infkds i ypcde i I hvdnxxxxxxxxxxxxxxpfdetteynksr lq dnnrity ixxx

$-x x x x x x x x x x x x x x x x x x x x x x x x x x x x x x x x x$ tg $\mid r n e k-$

-xindrki lygecnlniksd

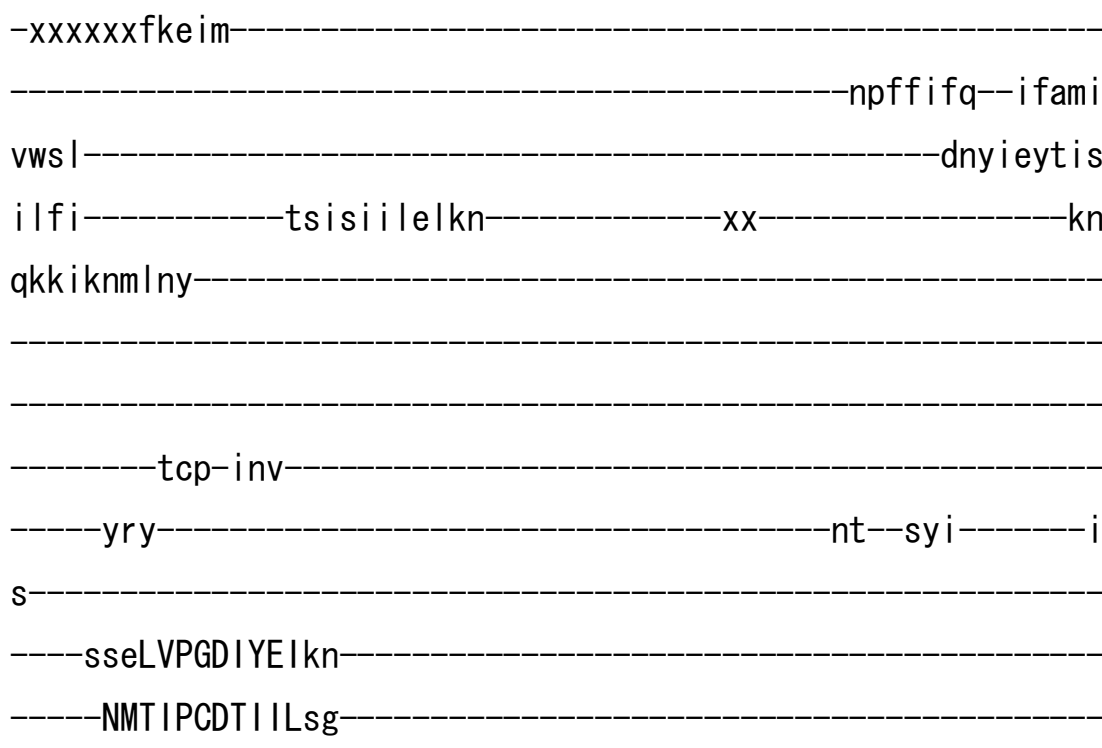

-svtMSEHMLTGESVPIHKeqx

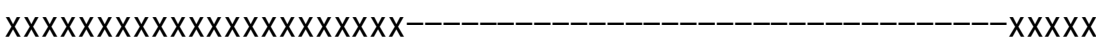

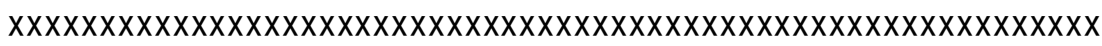

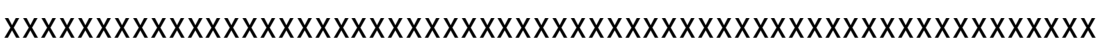

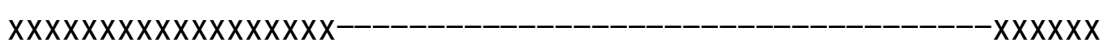

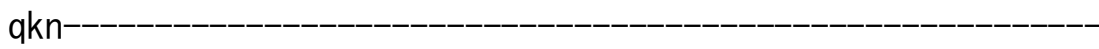
$-x x x x x x x x x x x Y M L Y G--G T Y V|s| x x x x x x x x--n n k$

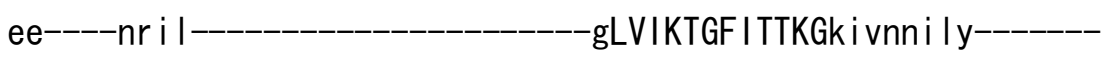
-x--kkkelnl indsykfl i i l i iyaffsvf i - I ly it I snne--_-_-_-_-_-_-_-_-_-_-_-_-_-_-_-_-_tnh i i ikcLD I ITDA IPPALPTTITVGISIAIS-RLkkkfx 
- iSCL-CPHK IN I AGQ INTMVFDKTG-

TLTENNLQFx-

$-x x x x q n k k n$

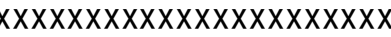

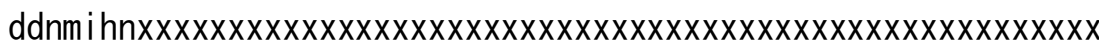
$x x x x x x x x x x-$

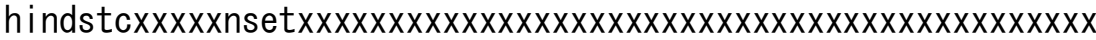

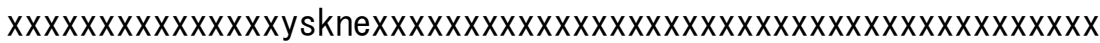

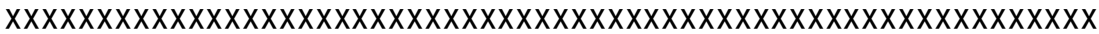

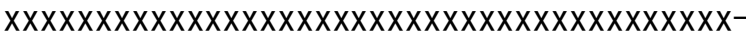

$-x i m g d v l e--i \mid m f n f$

xxxxxxxnnnsxxi--xxxkkn-

$-X X X X X X X X X X X X X X X X$

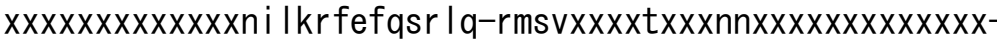

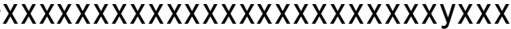

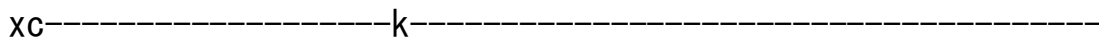

gsp

e---_-_-_-_-_-_-_-_-_-_--kikelclkskxxxxx--_-_-_-_-_-_-_-_-_-_-_

de i Inky--tkq----gmr iLSISYKrv

-ksknin I Invkrsxvesn I hfLGFLIFTNNMEKNAPDI IHNLQTsg-CQC

IMSTGDNVLTSIHVAKKCGI--_-_-_-_-_-_-_-_-_-_-_-_-_-_-_inxxves i-i i g

dv i------------------------pvvgxx---------------- xxxxxxxxxxxxx

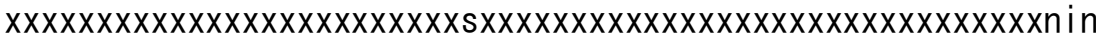
gdningdn i $x x x x x x x x x x x x x x x x x x x x x x x x x x x x x x x x x x x x x x x x x x x x x x x x x x$

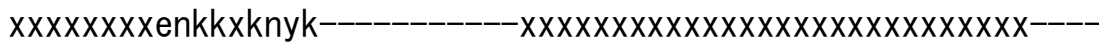
$-x x x i v \mid$ tgkaf $i$

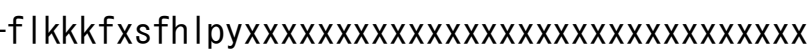




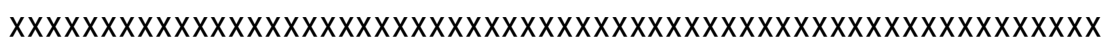

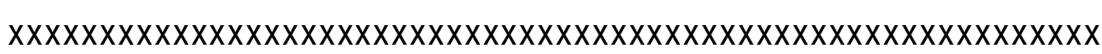

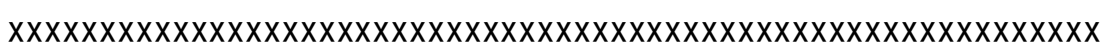

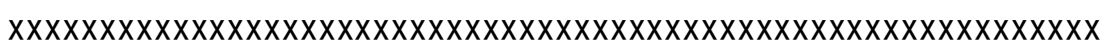

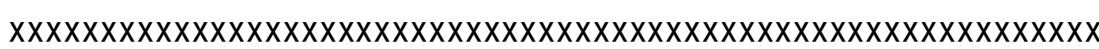

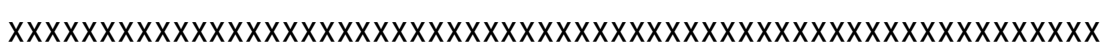

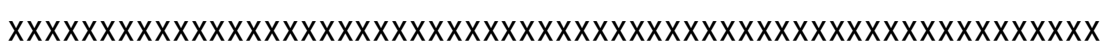

i Irtc--tVYA--RMKPKDKSDLILSLk--_-_-_-_-_-_-_-_-_-_-_-_-_-_-_-

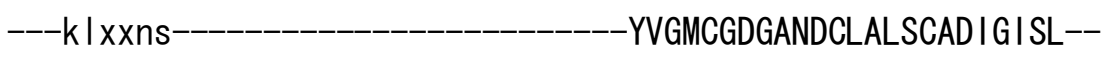

$-x n n n e-$
\end{abstract}

SSICSSFTSNk-LCLHSIVh iLIEGRASLVNSFQLF------K

fis-I ysxmxcsqv l i lys i snkx--_-_-_-_-_-_-_-_-_-_-_-_-_-_tdnqy i $\mathrm{i}$

divt i Ip Ixi fmcwtsasxk--_-_-_-_-_-_-_-_-_ sknipi-_-_-_--xk Ifsfpi

I-is iygqi i ixxffvmi slv| Imn l sfykxxxxxxxx-_-_-_-__-__-_-_-_-_-_

ekxxxxy I ykaqnt I ly i Icsfa-n I fmc i I In iknewrk-----svftn i af i i wmsf I

I Imntcitxxsse if I vxxxx--_-_--dI I kqy I-_-_-_-_-_-_-_-_-_-_-_-_-_-_

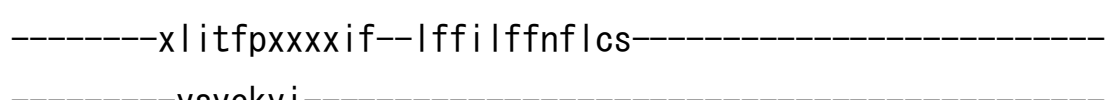

$-y s y e k y \mathrm{i}$

ikyfekrexqkkxxxxxxxxxxxxxxxxxxxxxx

$\mathrm{XXX}$

$\mathrm{n}$

>spoV_V201 NP_594863.1

$\mathrm{m}-$ 


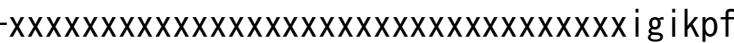

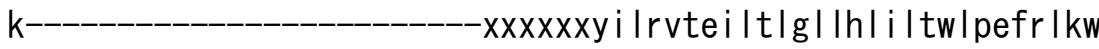
i eapcsnedvefvai sdpsgtss i ekvss i c lkndiqtss

fvlpsgktryfeykk|rfy lep Inlawv Imp le tsays Ivtxxx -xxxngldtft

iak I rqvygsns Ivstkk

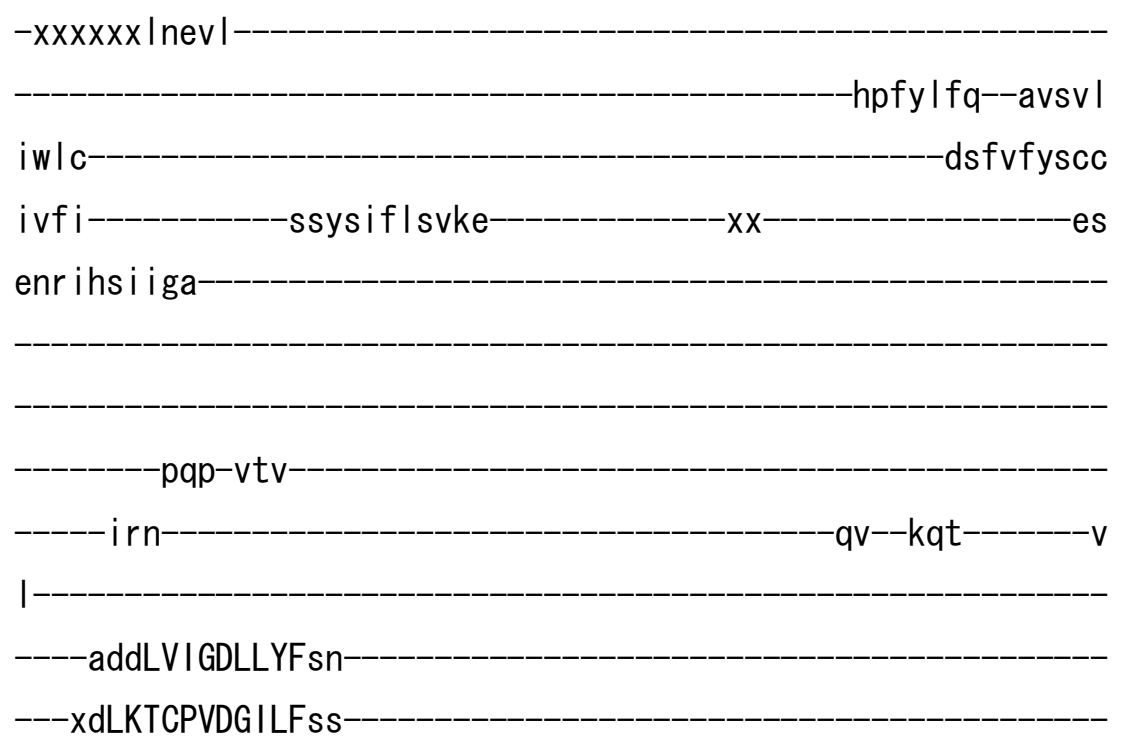


-sc ILDESMVTGESVPARKfp-

$-x x x x x x x x x x$

$-X X X X X X$

$\mathrm{fsp}$

-HL IHA--GTKFI $\mathrm{ki}$

-dst

ps-----tpcl-

iSVVRTGFRSNKGq I irn l y

-x--n|rpsqly|dsmsflktmai Isfvsivf

-iaiylnlyna

-sfghvv|rsLDVLTIL

VPPALPAT ISVG I ANSIA-RLsra I

iYTT-SPESIHNAGCLSTFVFDKTG

TLTENSVQLX

xxxxksgsn

$-x X X X X X X X X X X$

sldstk|x

ah

ayrvatc------sqs I

Xxxxxx | vgdp le--vt I ftq

fngtxxa--xxxasn

$-x x-$

$-x x x x x$

xxxxxxxxxxxxxs i ykalefdpv|r-rmsv----i

$x x x x x x$

$x \times x \times x+---$

$-t$

k

gap

e

s i la i ssqqs xxxx 


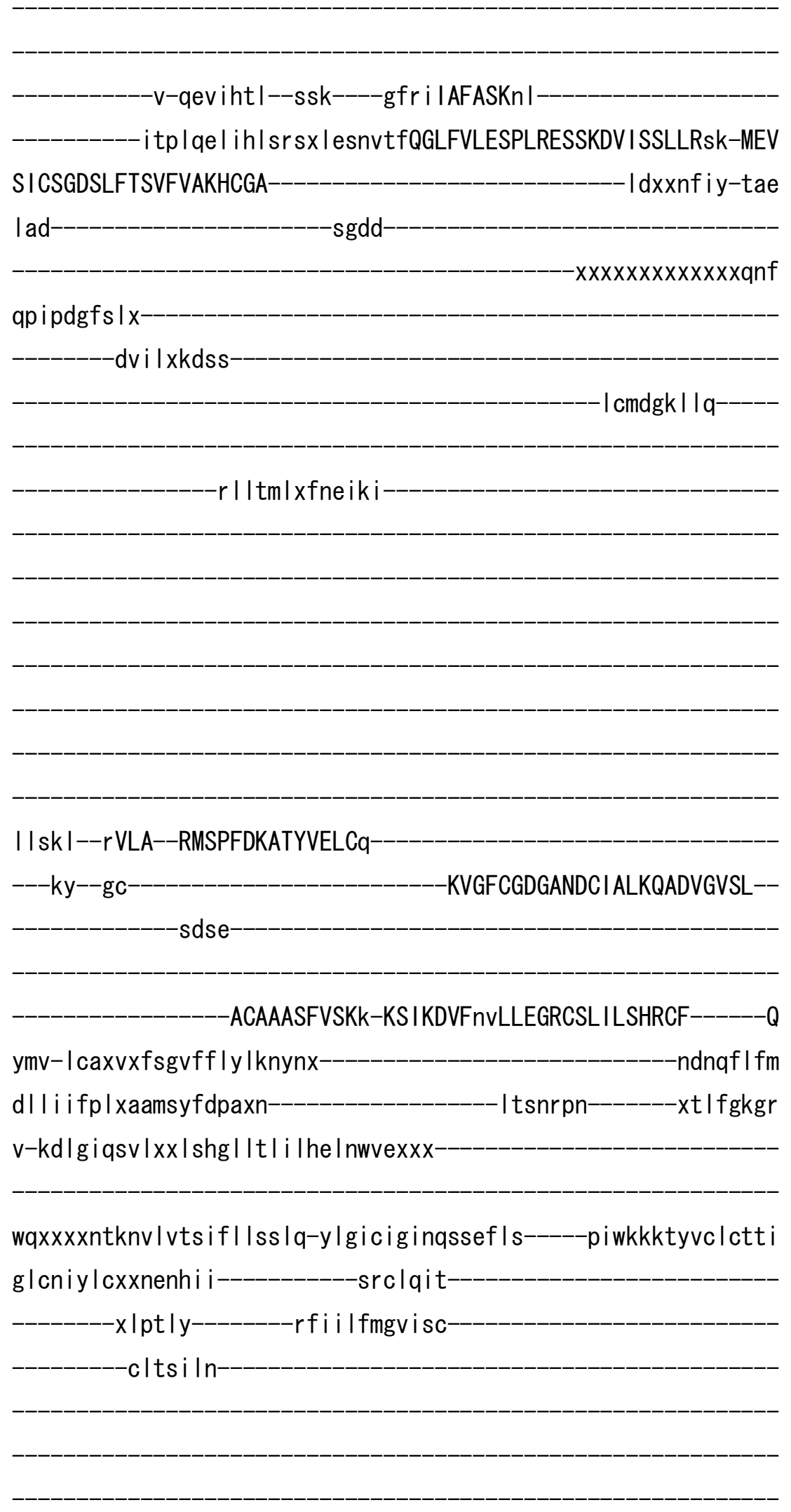


m

>mmuV_V201 NP_083373.1

$$
\mathrm{m}-
$$

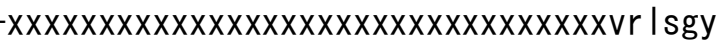
$-x x x x x x$ i gyhaavwm l ag i pw I I frwkp | wgvr |

C-

rlkpcs Iahaet I vie ikdkegssrqlftvqvqteavvqgs le Ipxxxxxxxxrxx----

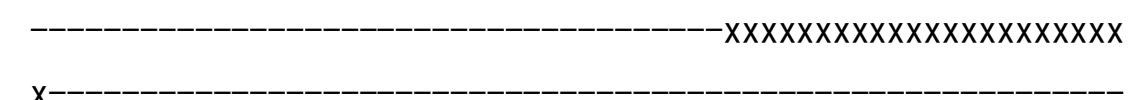

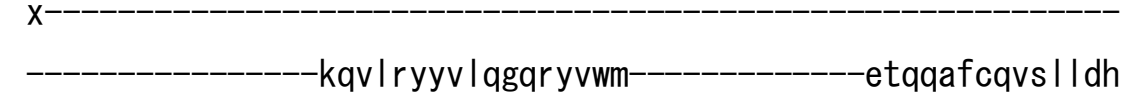
grtcddvhxxx-

$-s g|s| q d-$

-qatrkt i ygpnv i s i pvk

-xxxxxxadeal 
-npyygfq--afsia

$|w| a$ dhyywyalc

ifli

saisiclalyk

$x x$

$-k q$

slt|rdmvk|

ecvVNESSLTGESTPVLKta-

$-x x x x x$

$-X X X X X X$

thr

$x-$

-HTLFC--GTLI I qa

ray

vg----prv|------------------------aVVTRTGFCTAKGg I vss i l h--------

-x--rpi sfkfykhsmkfvaal sv la l I gtvy

-si i i lyrnrv

-pvreiviraLDLVTVV

VPPALPAAmTVCTLYAQS-RLrtgg-

iFCI-HPLRINLGGKLRLVCFDKTG

TLTEDGLDV

-mgvvp

$x-x x x$

$\mathrm{pl--vpexxxxx}$

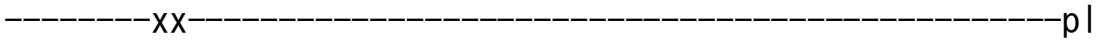

Iralatc-----hals-

qlhdt 


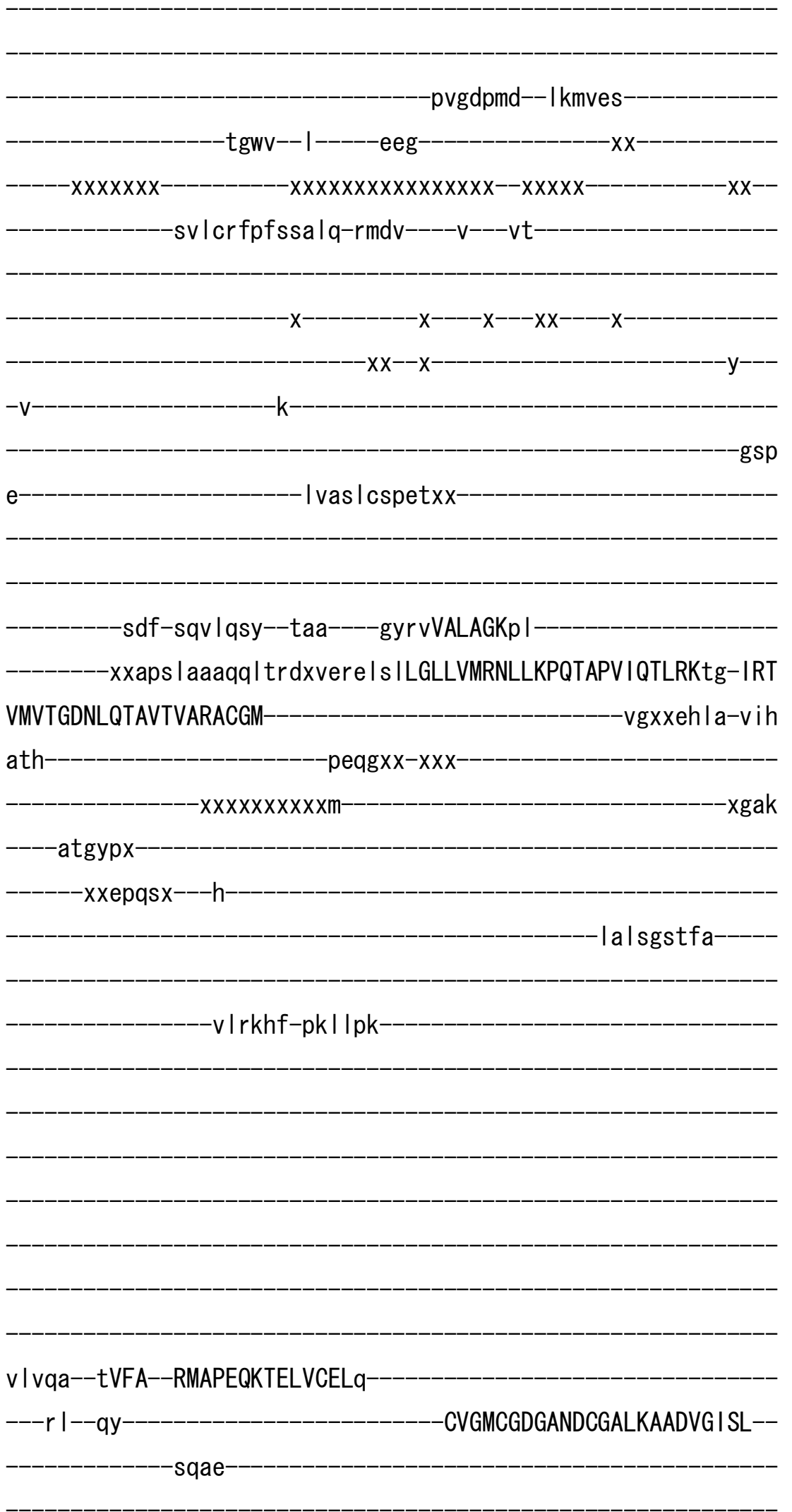

-ASVVSPFTSSm-ASIECVPtv IREGRCSLDTSFSVF---_--K 


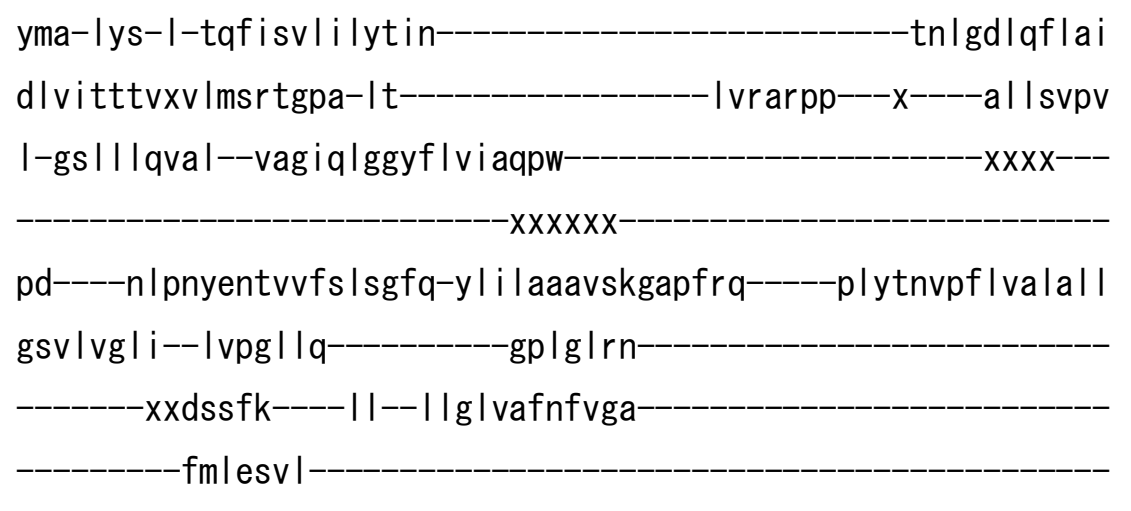

$-d q c|p a c| x w \mid r x--x x x x$

$-x x x--x x x x x x x x x x x x-x x x x x x x x x y$

>mmuV_V202 XP_999996. 1

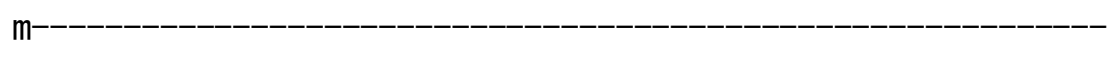

xxxxxxxxxxxxxxxme ihgy

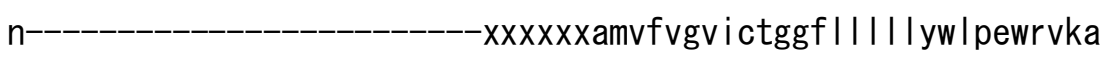


tcvraavkdcevv I Irttdefrvwfcak ihf I pven-qpn I nakcxxxxxxxxxhxx----

$-x x x x x x x x x x x x x-$

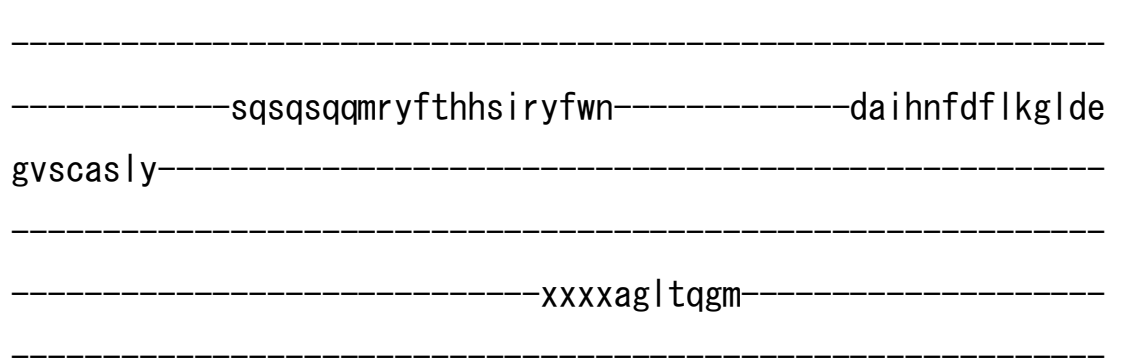

-hayrk I iygvne iavkvp

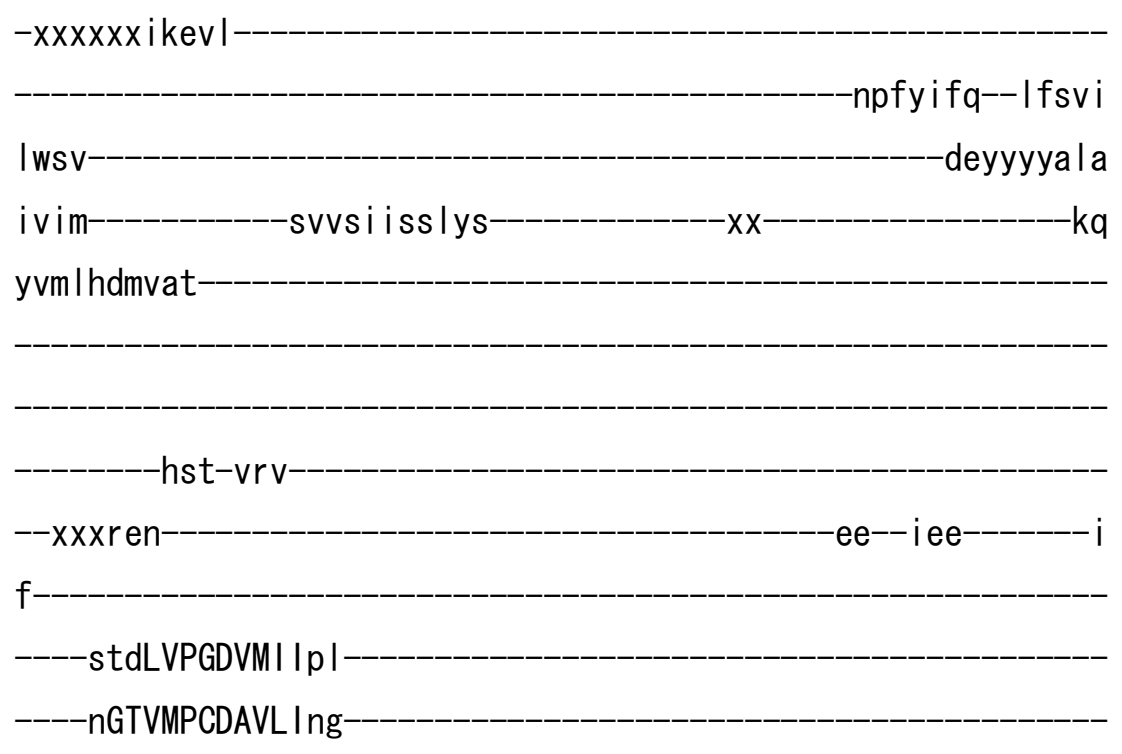

tc iVNESMLTGESVPVTKtn-

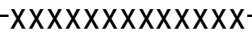

$-x x x x x x$

thk

-----------HTLFC--GTTV iqt-----------rfy

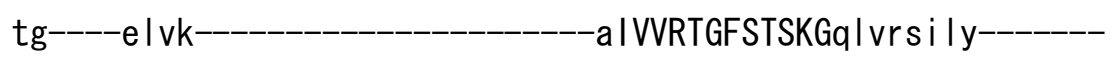

-x--kptdfk I yrday I I I c I vvvag i gf i y

-ti insi Inek -evqe i i iksLDIITIT

VPPALPAAmTAG IVYAQR-RLkkvg 
iFCI-SPQRINICGQLNLVCFDKTG-

TLTEDGLDL

-wg i qr

$-x-x x x$

I pednvcxxxxx

$-x x-$

qf

vacmatc-----hs $\mid \mathrm{t}$

$-k i e g v$

$\mid$ sgdp |d--|kmfea-

$$
\text { i gwi--I-----eea- }
$$

$-x x-$

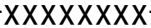

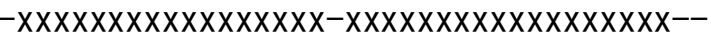

givrqfpfssalq-rmsv

$-\mathrm{V}---\mathrm{ar}$

$-\mathrm{x}----\mathrm{x}---\mathrm{xX}-----\mathrm{X}-$

$-x x--x$

- ---

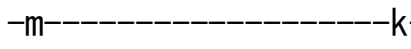

$k-$

gap

e-

-vvas I ckpetxx-

-vdf-ekv ledy--tkq----gfrv IALAHRk I

-xxx I twhkvqh isrda-i ennmdfMGL I IMQNKLKQETPAVLEDLHKan-IRT

VMVTGDNML TAVSVARDCGM-_-_-_-_-_-_-_-_-_-_-_-_-_-_-_ i x x dkv i-i ae

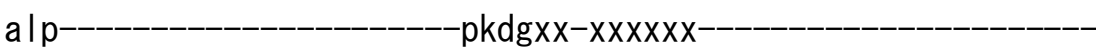

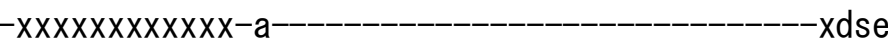

aipiklahdx

------xxdlev-tryh

famngksfs

-vi lehf-qd I vpk- 
Im Ihg--tVFA--RMAPDQKTQLVEALq-

$---n v--d y$

-FVGMCGDGANDCGALKRAHGG ISL-sele-

-ASVASPFTSKt-PSISCVPnI IREGRAALMTSFCVF$-K$

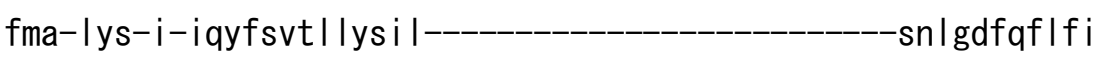

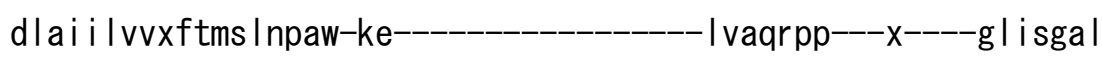

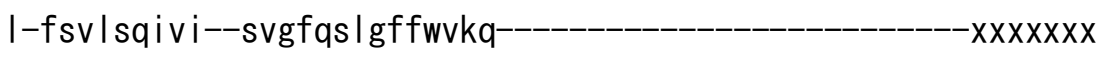

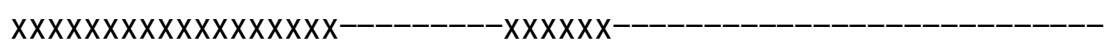
sc----ki iqnyenttvff issfa-y I tvavafskgkpfrq-----pcyknyffvisvi i I yvfilf im--Ihpvasv-_-_-_-_-_-_dqvle im--_-_-_-_-_-_-_-_-_-_-_-_-_-_-------xxpyqwr----iy--ml i i vl inafvs---------------------------

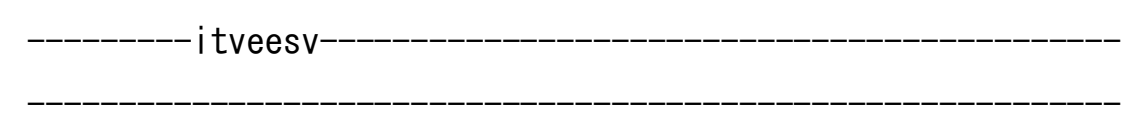
-drwgkcc I xwa I xxxxxxx-

$-x x x--x x x x x x x x x x x x x x x x x x x x x x x x x x x x x x x x---$ $-x-x x x x-$

a >mmuV_V203 NP_766201.2

m- 


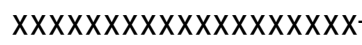

-me ifgy

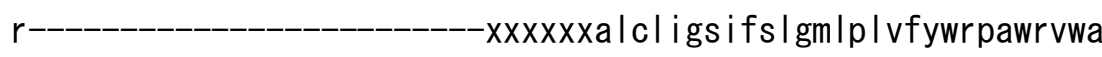
ncvpcs I qeadvv I lkttdefki yswkkviwis I salsstsg| tpxxxxxxxxgxx----_-_-_-_-_-_-_-_-_-_-_-_-_-_-_-_-_-_-_xxx-_-_-_-_-_-_-_-_-_

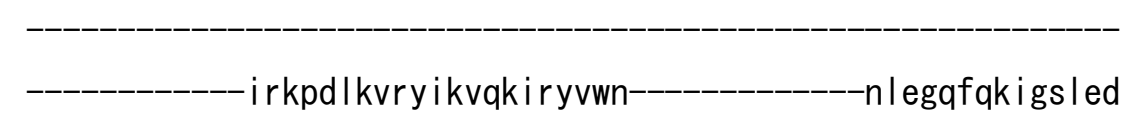
wlssakih-

$$
-x x x|g| \text { tsee- }
$$

\section{-qe i rr l i cgpna idveit}

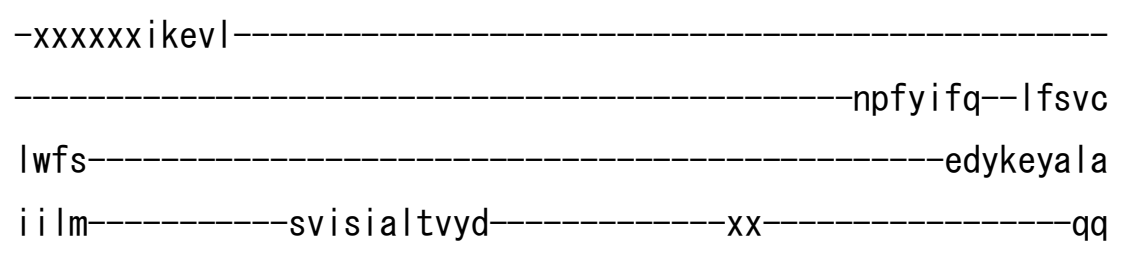

svklhhlves-

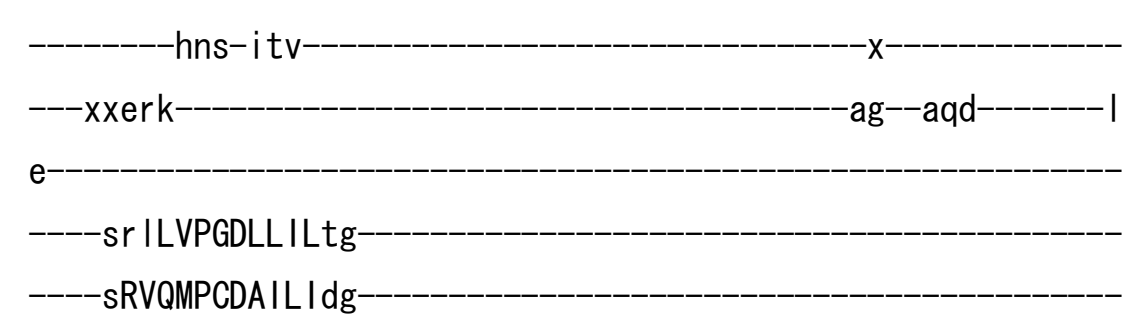




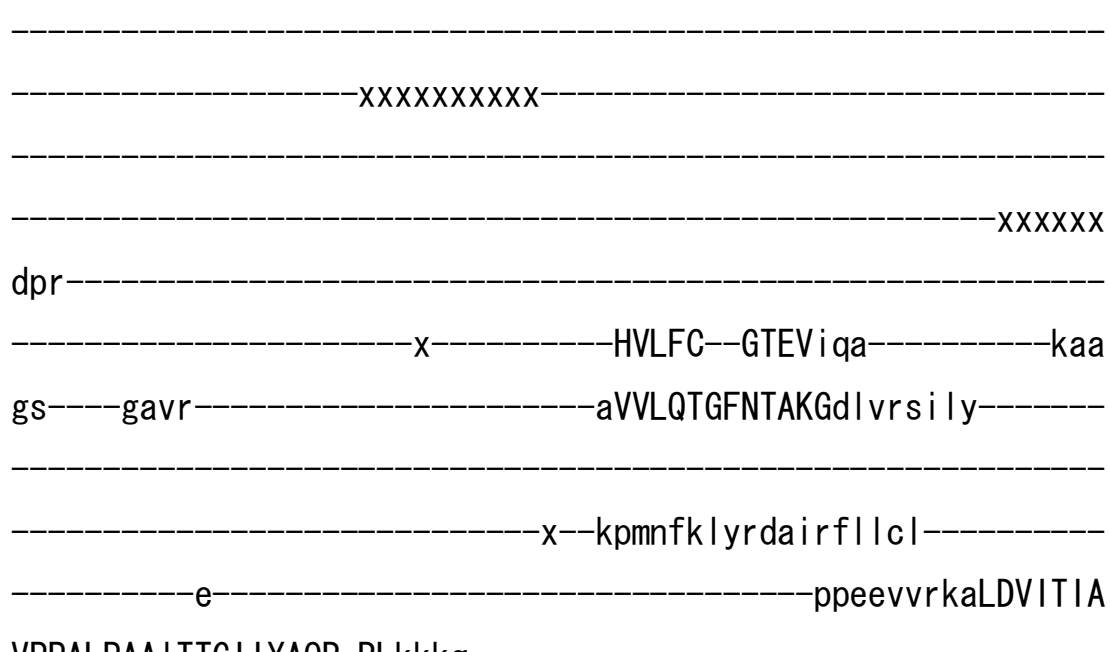

VPPALPAA I TTGI IYAQR-RLkkkg-

iFCI-SPQR INVCGQLNLVCFDKTG-

-TLTRGGLDP

-wgvvp-

$x-x x x$ $-x \times x-----$

avhsfasxxxxx

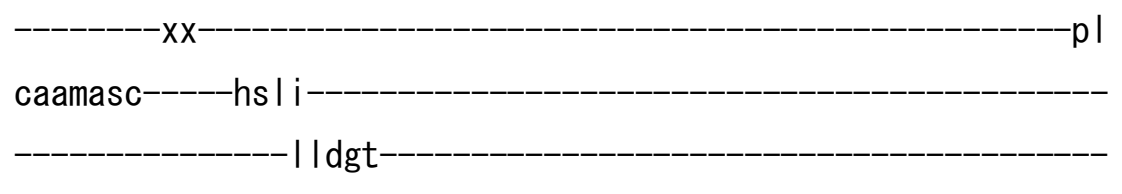
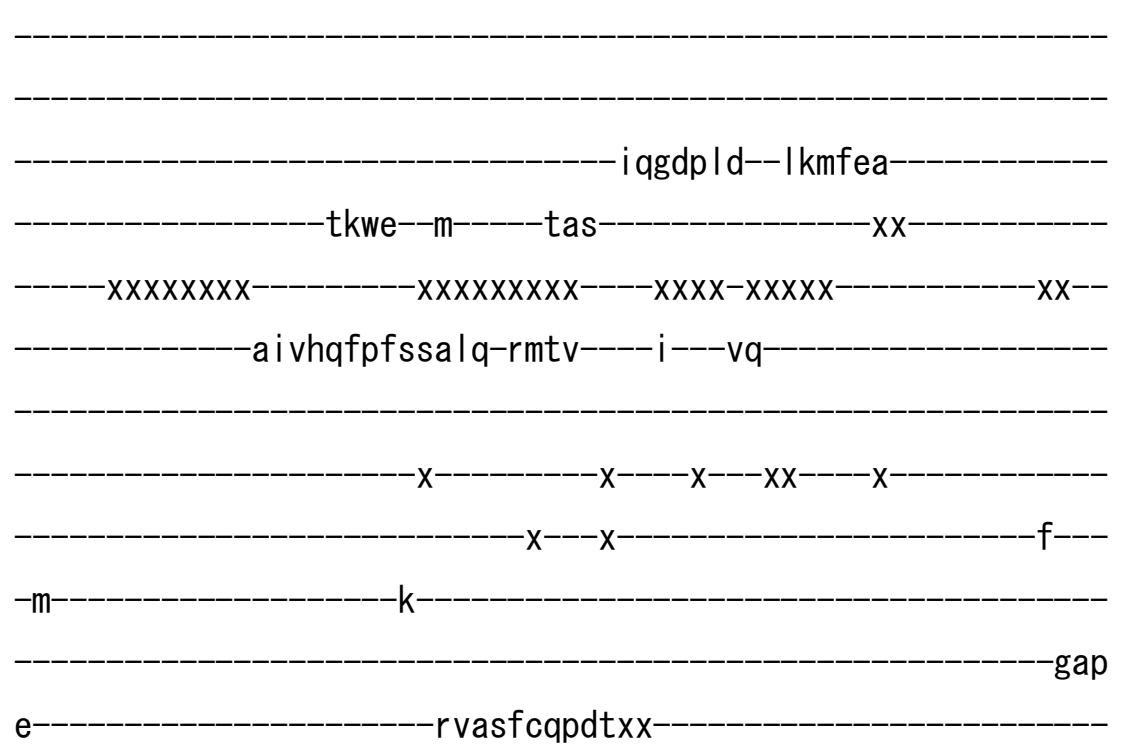
-tsf-i se lqiy--ttq----gfrvIALAYKk I

-emdcptta Imr exvesd I vfLGLLILENRLKEETKPVLEEL I Sar-IRT

VMI TGDNLQTAI TVARKSGM-

-vsxxqkvi-Ive

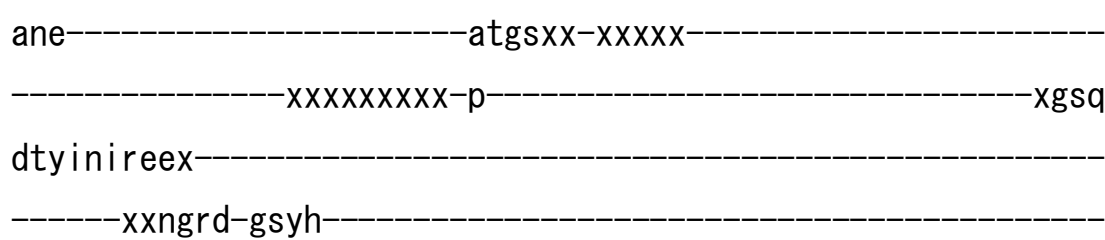

falsgksfh

-visqyf-ss। Ipk-

i I ing--tIFA--RMSPGQKSSLVEEFq

$---\mathrm{k} \mid--\mathrm{dy}$

-FVGMCGDGANDCGALKMAHVGISL--

seqe

ASVASPFTSKt-PNIECVPh I IKEGRAALVTSFCMF

$-K$

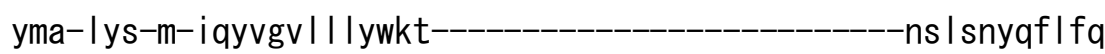

d laitt I i xvtmn Ingan-pk--_-_-_-_-_-_-_-_-- | vpfrpa----x----r l i spp |

I-Isvv In i I I--s I amh i vgf $\mathrm{i}$ I vqkqpw-------------------- $x x-x x x x$

$x-x x x x x x x x x x x x x x x x x x-------x x x x x$

ns----tfasfenttiwf Igtin-cifvalvfskgkpfrq-----ptytny ifv Ivli lq mgvc Ifi I--fadipem-----------hrr IdI I

-------xxpv I wr-----vy--i l imi ssnfvvs--_-_-_-_-_-_-_-_-_-_-_-_-_-_

- lavekai 


$$
\text { I }
$$

>mmuV_V204 NP_783581. 1

$x X X X X X X X X X X X X X X X X X X$

levfgy

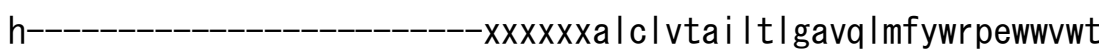
sc ipcp I qeadt i I Irttdefrrymrkkvfc lh Ist Ikfp i sknpxxxxxxxxhxx----

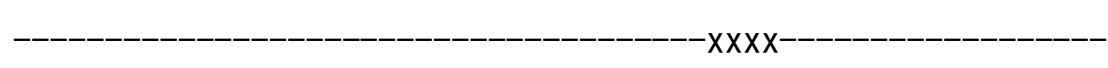
---------------------------------------------------------vmkpelkIrciqvqkiryvwd$-f|k k r f q k v g| l e d$ snscfdih$-x x x x|g|$ tnee-qevrr Ivcgpns i eveiq $-x x x x x x v k q v \mid$ 
-npfyvfq--aftlt

$|w| s$

-ggy i eysva

i i il

tvisivlsvyd

$x x$

qq

svklhklvee-

scvVNEGMLTGESIPVTKtp-

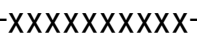

$-x X X X X X$

dyr

-HVLFC--GTEV i qv------------kps

$\mathrm{aq}----\mathrm{g} \mid \mathrm{vr}$

aVVLQTGYNTAKGdI vrsi ly

-x--rp Infk I yndafkfmvf l acvgvvgffy

-al gvymyhev

-ppretatmaLILLSAT

VPPVLPAA I T IGNVYAQK-RLkkek-

iFCI-SPQR INMCGQ INLVCFDKTG

TLTEDGLDL

-wgtvp

$x-x x x$

avhsfas $x x x x x$

$x x-$

caamtsc-----hs l i

I ldgt- 


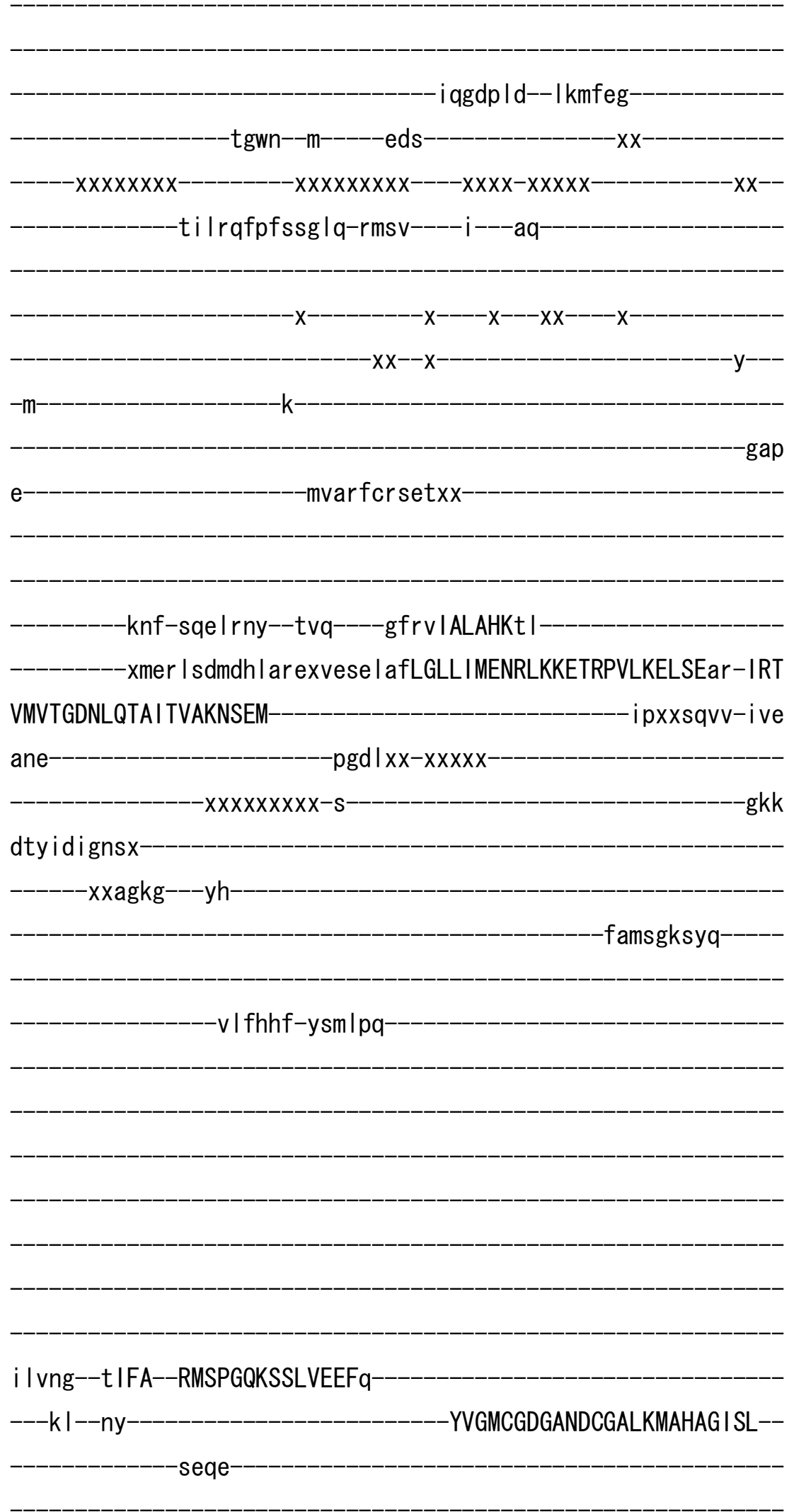

-ASVASPFTSKt-AN IECVPh I IREGRAALVSSFGVF---_--K 


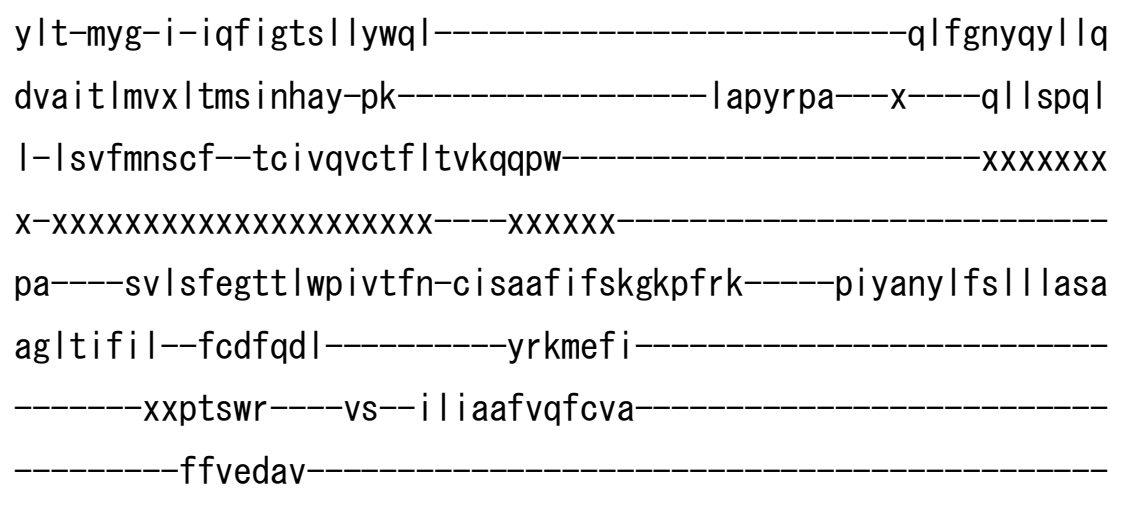

\section{- qnrelw|-fikxxxxxxx-}

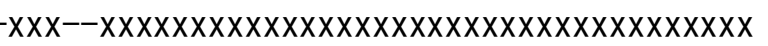

।

>rnoV_V201 XP_342963. 3

m-

$-x x x x x x x x x x x x x x x x x x x x x x x x x x x x x x x x x y r$ Isgy

c-_-_-_-_-_-_-_-_-_-_-_-_-_xxxxxx i gyhaavwm l ag i pw I I frwkp I wgvr I 
r|rpcs laraet I vie irdkegssrq Iftvqvqteavvdgs le I pxxxxxxxxrxx----

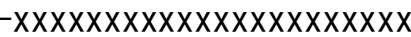

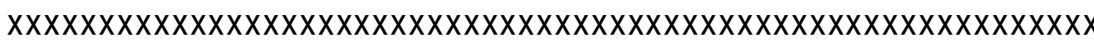
xxxxxxxxxxxxs | tqkqv| ryy i l qgaryvwm--------------etqqafcqvs I I dh gracddihxxx

$-s g|s| q d-$

-qavrkt i ygpnvi i i pvk

$-x x x x x x v d e a l$

-npyggfq--afsia

$|w| a$ dhyywyavc

ifli saisiclslyk $\mathrm{xx}$ $-k q$

smt |rdmvk |

ecVVNESSLTGESIPVLKta-

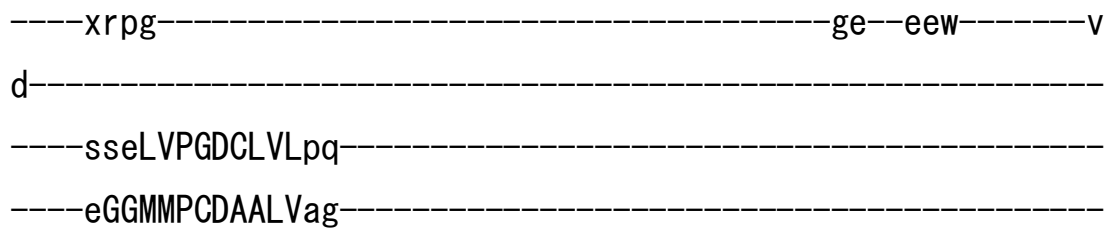

$-x x x x x$

$-x x x x x x$

thr

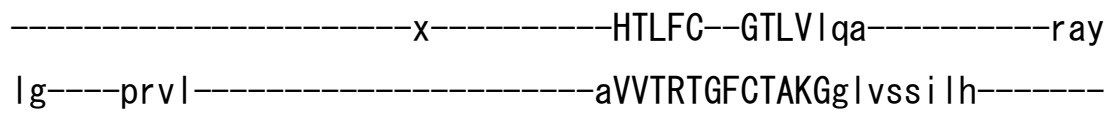

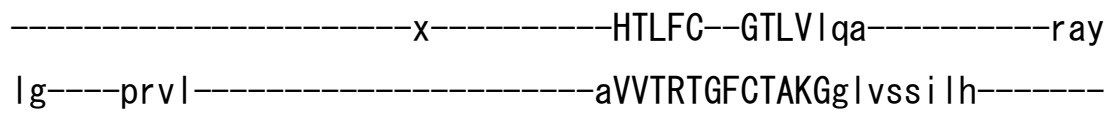

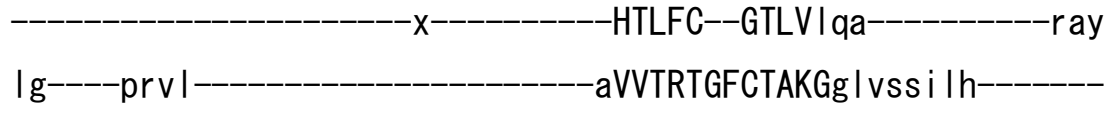
-x--rpisfkfyrhsmkfvaalsvlal Igtiy -si i i lyrnrv -pvke iviraLDLVTVV

VPPALPAAmTVCTLYAQS-RLrtgg- 
iFCI-HPLRINLGGKLRLVCFDKTG

TLTEDGLD

-mgvvp

$-x-x \times x-10+0$

$\mathrm{XXX-----}$

$\mathrm{pl--vpexxxxx}$

$-x x-$

Iralvtc-----hals

qlhdt-

$--\mid$ vgdpmd--I kmves
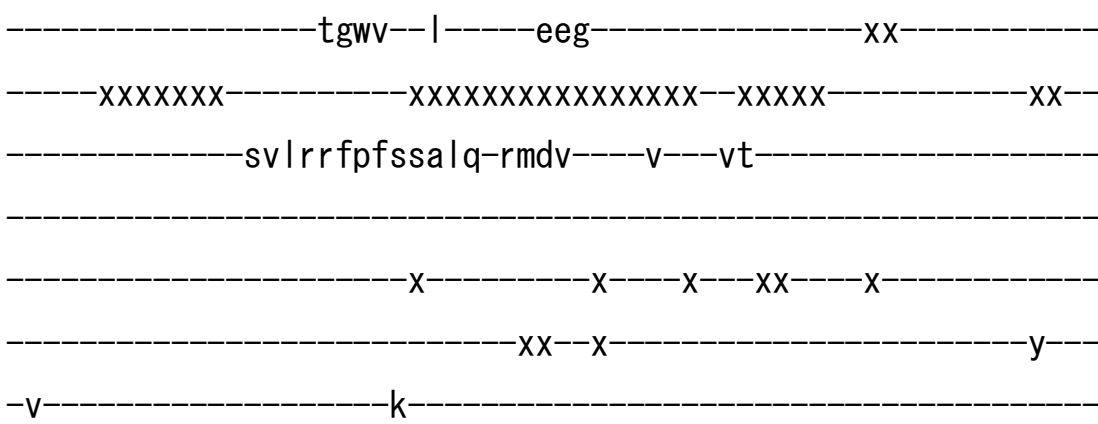

gsp

e-

| vas I cspetxx-

rdf-pqv l qsy--taa----gyrvVALAGKp I

-xxaps I eaaqq I trdxveqe I s I LGLLVMRNLLKPQTTPVIQTLRKtg-IRT

VMVTGDNLQTAVTVAKACGM-

vgxxehla-i ih

ath- peqgxx $-x x x$

-xgak

----atdypx$x x x x x x x x x x m$ - -xxesqsx---h

lalsgstfa

$-v|q k h f-p k| l p k$ 
vIvqa--tVFA--RMAPEQKTQLVCELq-

$---r \mid--q y$

CVGMCGDGANDCGALKAADVGISL--sqae

ASVVSPFTSSm-ASIECVPtvIREGRCSLDTSFSVF$-K$ yma-Iys-I-tafi isvl i lyt in--_-_-_-_-_-_-_-_-_-_-_-_-_n I gdlafla

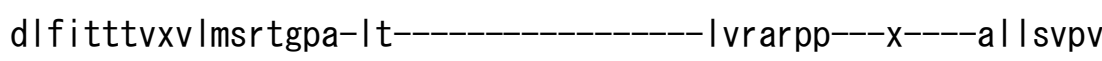
I-gs I I lqval--vagiql ggyf Iviaqpw---_-_-_-_-_-_-_-_-_---Xxxx--$-x x x x x$

pd----n I pnyentvifs Isgfa-y l i laaavskgapfrq-----p lytnvpf I vala I I gsvlvg|i--|vpg||q----------gp|g|rn-

-------xxdssfk----||--|lg I vafnfvga-

$-\mathrm{fm} \mid \mathrm{esv} \mathbf{1}$

dqc $\mid$ pac $|x w| r x--x x x x$

$-x x x--x x x x x x x x x x x x-x x x x x x x x x y$

>rnoV_V202 XP_001053119.1

$\mathrm{m}-$ 
xxxxxxxxxxxxxxxmeihgy n--------------------------Xxxxxxamvcvgv i ctggf | | | | | ywmpewrvka tcvraavkdcevvIIrttdefr iwfcakihf Is len-qpn Inaksxxxxxxxxhxx---$x x x x x x x x x x x x x-$ sqsqsqamryfthhs i ryfwn da ihnfdf I kgI de gvscasmy

\section{xxxxag | tkgm-}

-hayrk I Iygvne i avkvp

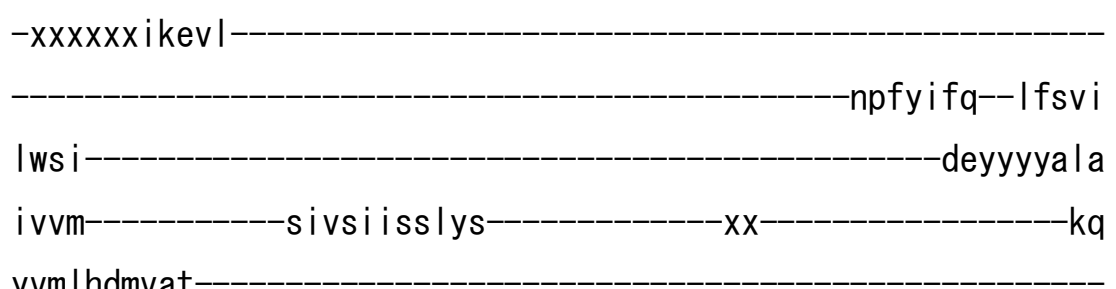

yvmlhdmvat

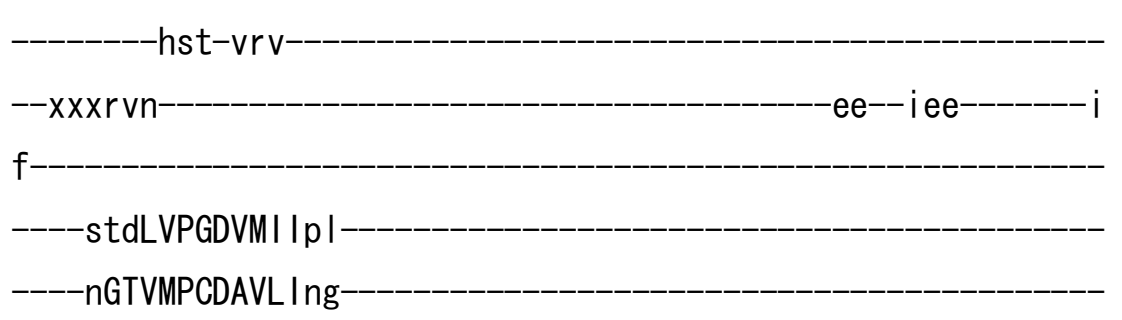


tc iVNESMLTGESVPVTKtn-
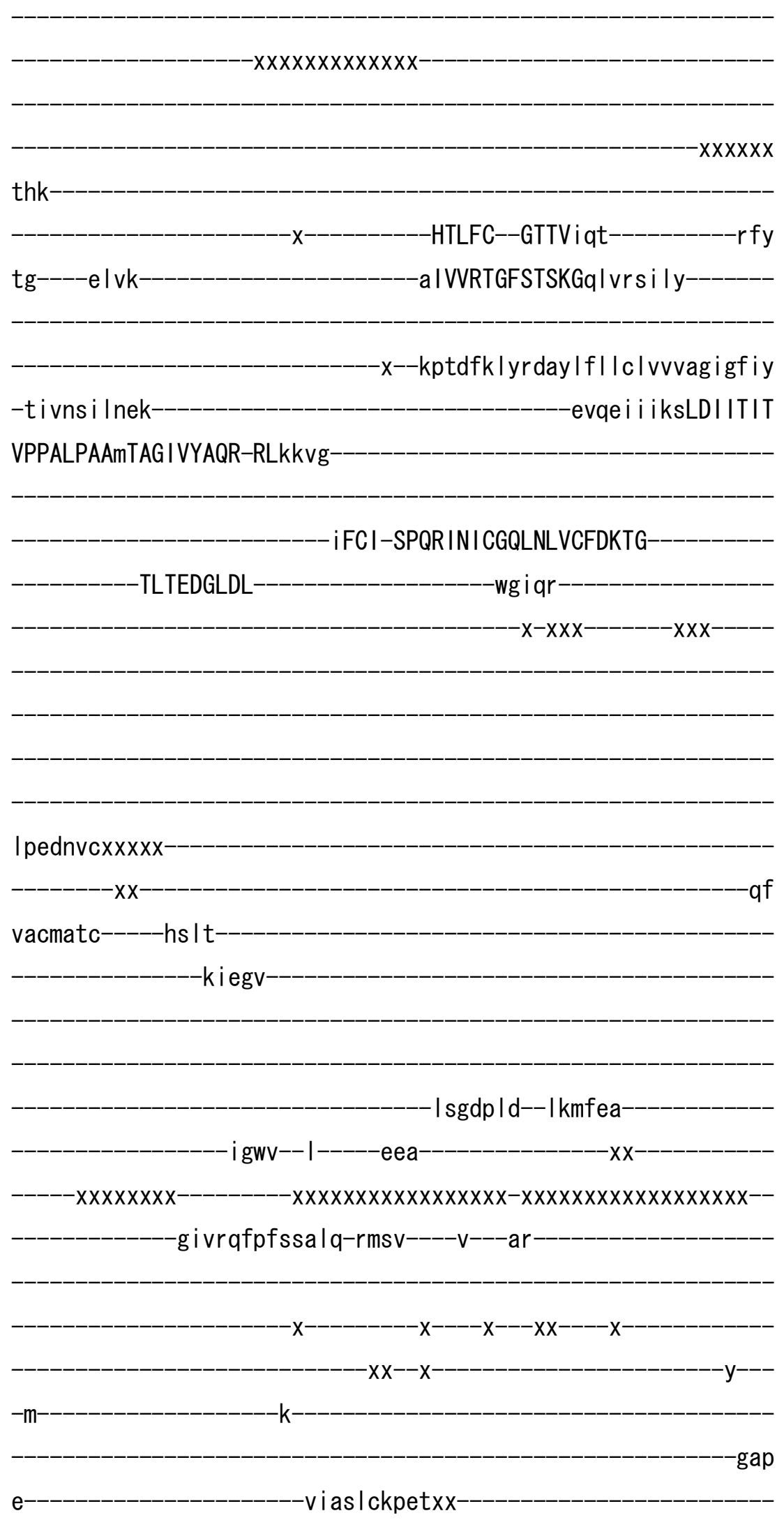
-vdf-ekv ledy--tkq----gfrv lALAHRk I

-xxx I twhkvqhvsrda-i ennmdfMGL I I MQNKLKQETPAVLEDLHKan-IRT

VMVTGDNMLTAVSVARDCGM-

il xxdkvi-iae

alp

$p k d g x x-x x x x x x$

$x x x x x x x x x x x-a$

-xdse

a ipiklahdx

xxdl qv-tryh

famngksfs

vi lehf-qd lvpk

Imlhg--tVFA--RMAPDQKTQLVEALq

$---n v--d y$

-FVGMCGDGANDCGALKRAHGG ISL-sefe-

ASVASPFTSKt-PSISCVPnI IREGRAALMTSFCVF

$-K$

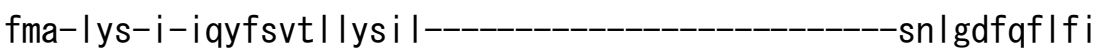

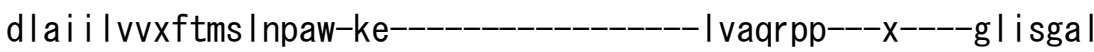

I-fsv| sqi vi--svgfqs I gffwvkq------------------------ $x x x x x x x$

$x x x x x x x x x x x x x x x x x----------x x x x x x$

sc----ki qnyenttvff issfa-y I tvavafskgkpfrq--_---pcyknyffvi svi i I yvfilfim--Ihpvasv-----------dql le im-

-------xxpyqwr----iy--ml i ivltnafvs-

-itveesv 


\section{a}

>rnoV_V203 XP_221355. 4

$\mathrm{m}$

$X X X X X X X X X X X X X X X X X X X$

-me ifgy

$$
r
$$
xxxxxxa|cligsifs Igm|p|vfywrpawrvwa

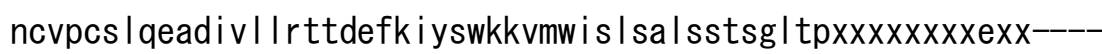

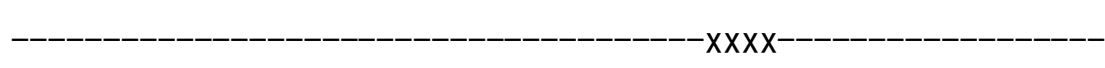

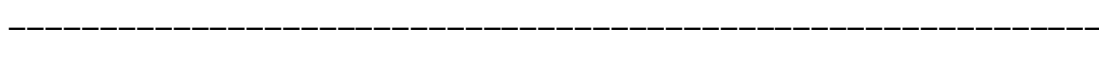
clssakih---------------------------------------------------$-x x x x|g| t s e e$ qe irr l icgpnaidvei i $-x x x x x x v k e v \mid$ 


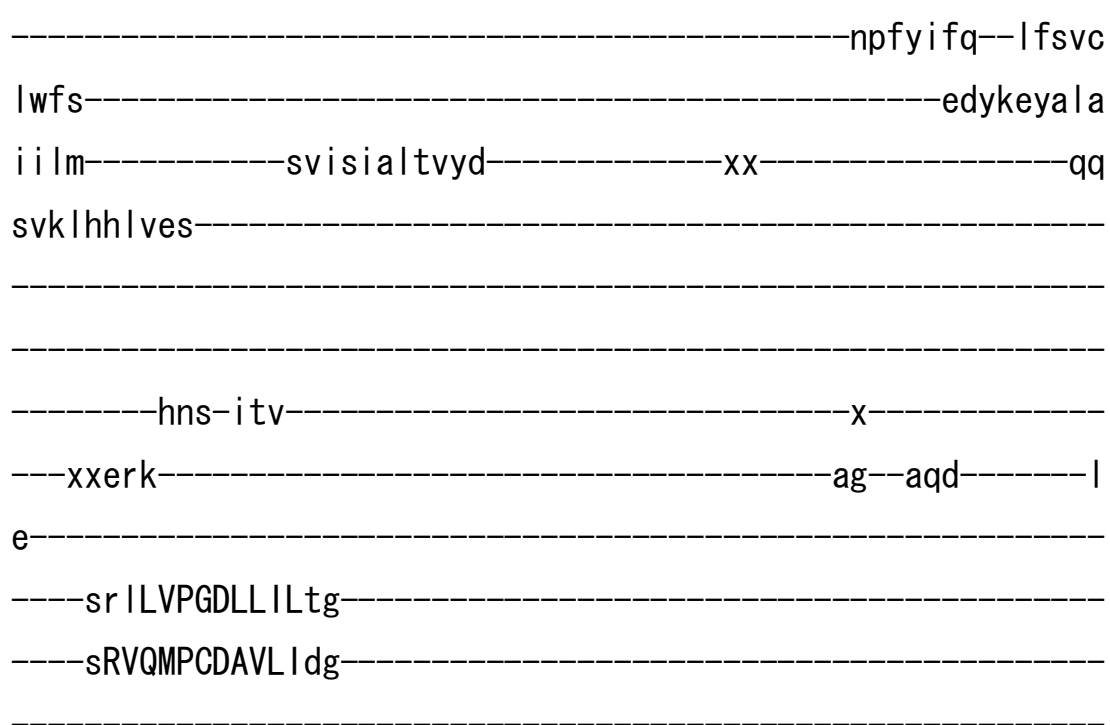

scvVDEGMLTGESIPVTKtp-

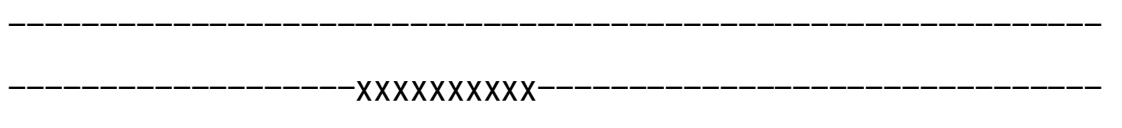

$-x x x x x x$

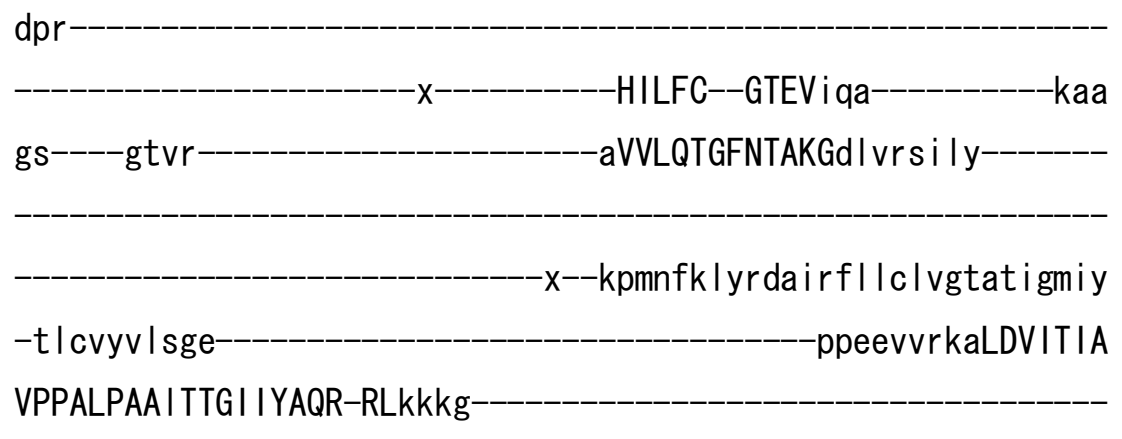

iFCI-SPQR INVCGQLNLVCFDKTG-

TLTRGGLDL-

- wgvvp

$-x-x x x$

$-x x x$

avhsfasxxxxx

$-x x-$

$-p \mid$

caamatc-----hs I i

l ldgt- 
iqgdp Id--Ikmfea-

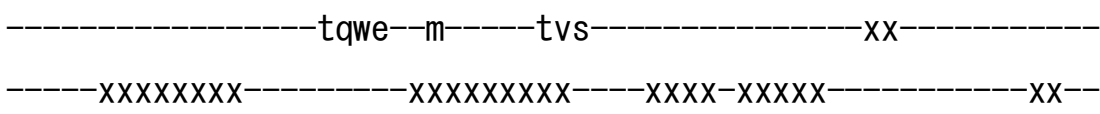

-ai lhqfpfssalq-rmtv-----i---vq

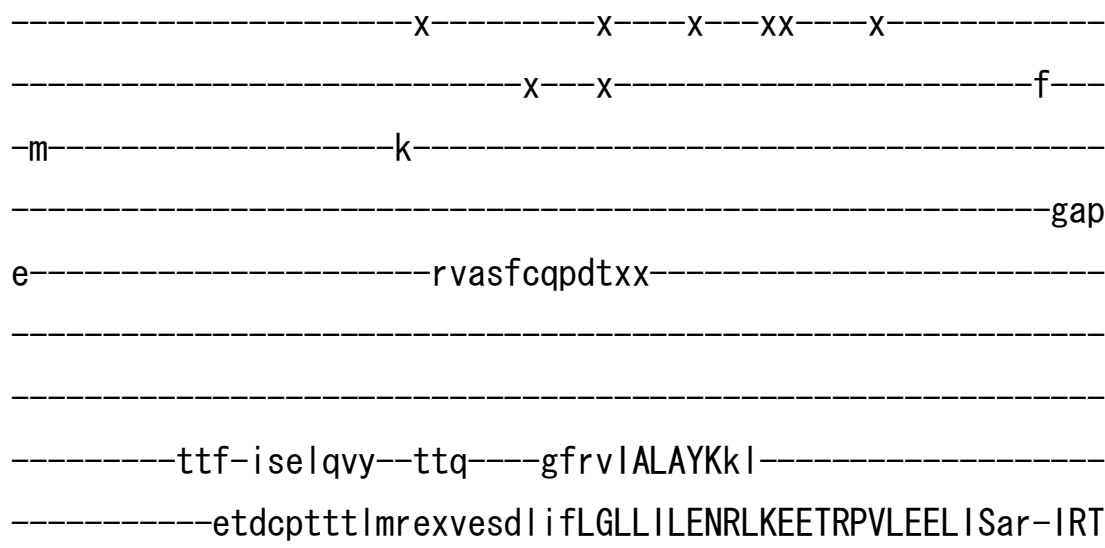 AM I TGDNLQTAI TVARKSGM--_-_-_-_-_-_-_-_-_-_-_-----vsxxqkvi-Ive

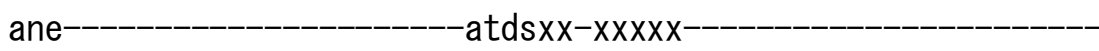

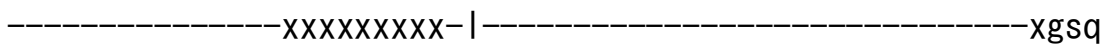
dty in i reex-xxngre-gnyh falsgksfa -visqhf-ss I lpk

il ing--tIFA--RMSPGQKSSLVEEFq

$---\mathrm{k} \mid--\mathrm{dy}$ -FVGMCGDGANDCGALKMAHVGVSL-seqe 


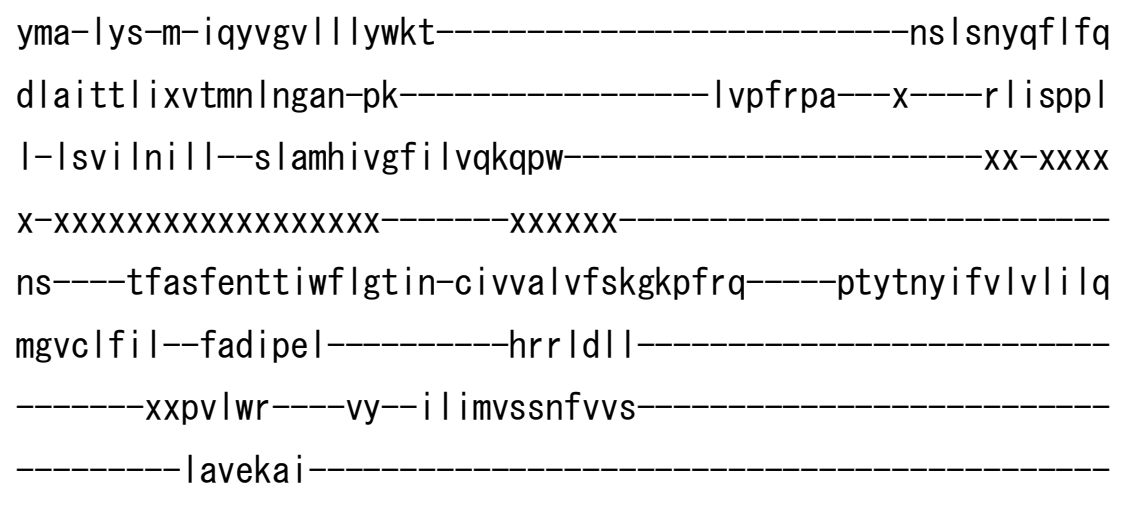

-ienralwt-avkxxxxxxx-

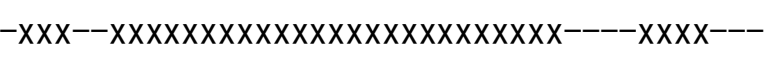
$-x x x x x x x-x x x x x-$

I

>rnoV_V204 XP_221354. 4

$$
\mathrm{m}-
$$

q $-x x x x x x a|c| v t a i \mid$ t l gavq Imfywrpewwvwt 
scipcp Iqeadivl Irttdefrrymrkkvfc lhIst lkip i sknpxxxxxxxxhxx----

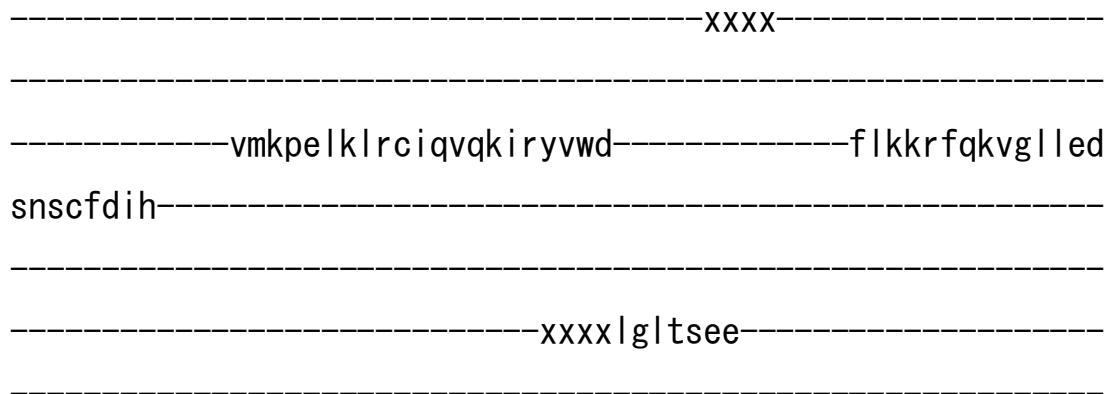

-qevrr Ivcgpnai eveiq

$-x x x x x x v k q v \mid$

-npfyvfq--aftlt

$|w| s$

-qgy i eysva

i i i I

tvisivlsvyd

$x x$

$-q q$

svklhklved-

-scvVNEGMLTGESIPVTKtp-

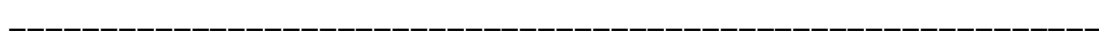

$-x x x x x x x x x-$

$-x x x x x$

dy

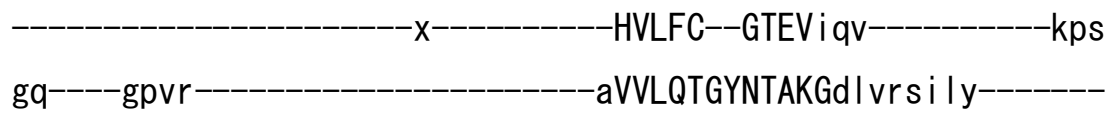

-x--rp Infk I yndafkfmvf lacigvvgffy

-al gvylyhev -ppretvtmaL ILLTAT

VPPVLPAA I I IGNVYAQK-RLkkkk- 
iFCI-SPQR INMCGQ INLVCFDKTG-

TLTEDGLDL

-wgtvp

$-x-x \times x-10+0$

$x X X----$

avhsfas $x x x x x$

$-x x-$

$-p \mid$

caamasc-----hs l i

- l dgt

qgdp I d--| kmfeg

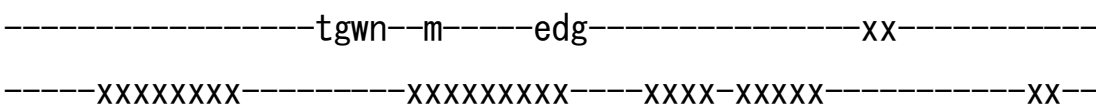

-ti Irqfpfssglq-rmsv----i---aq

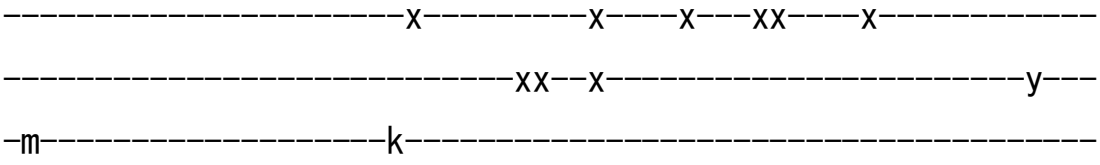

gap

e--_-_-_-_-_-_-_-_-_-_-_----mvarfcssetxx

-knf-pqe I rny--tvq----gfrv IALAHKt I

-xmekvsdver I arexvese I afLGLL IMENRLKNETRPVLQELSEar-IRT

VMVTGDNLQTAI TVAKNSEM-

ipxxsqvi-ive

anepgd I xx-xxxxx

ekk

dty idigns $x$

$x x x x x x x x x-s$

------xxcgkg---yh-

famsgksyq

vlfhhf-ysm I pq 
i l vng--tVFA--RMSPGQKSSLVEEFq

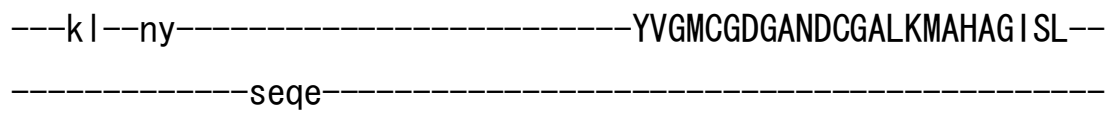

ASVASPFTSKv-ANIECVPh I IREGRAALVSSFGVF------K

y lt-myg-i-iqfigts | lywq I-_-_-_-_-_-_-_-_-_-_-_-_-_-_--q I fgnyqy I lq

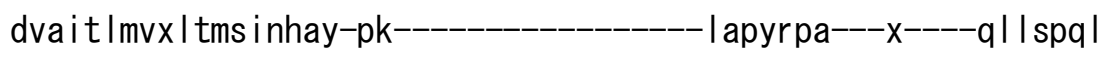

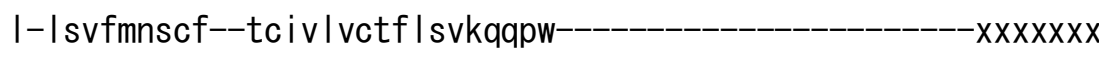

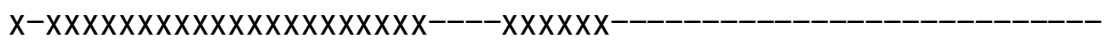

pa----sv Isfegtt I wp ivtfn-c i saaf i fskgkpfrk-----pi ytny Ifs I I Itsa

vgltvfi I--fsdfqdi-_-_-_-_-_--yrrmefi-_-_-_-_-_-_-_-_-_-_-_-_-_-_-

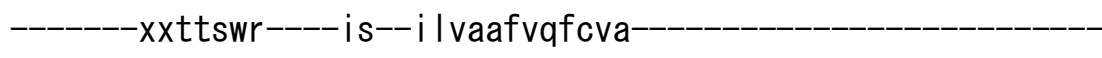

ffvedai

-qunre|w|-fikxxxxxxx-

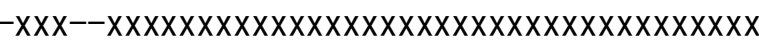

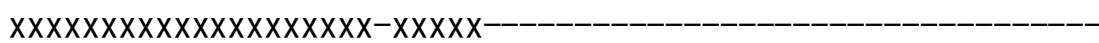

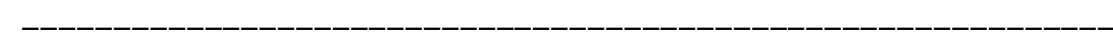

।

>dreV_V201 XP_697103.2

$\mathrm{m}-$ 
$-X X X X X X X X X X X X X X X X X X X X X X X X X X X X X X X X X X X X m d v q g y$

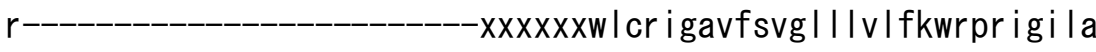

rckscp i smadv I I lkdrygqqfvvdvi teeveegs I dfavgdadxxxxxxxxqx

-hsekkt I ryyvfegiryiwi

skkgafckasvlse

gwtcadl hxxx

qg $\mid$ srad-

-qstrkq i fgani idvpvk-

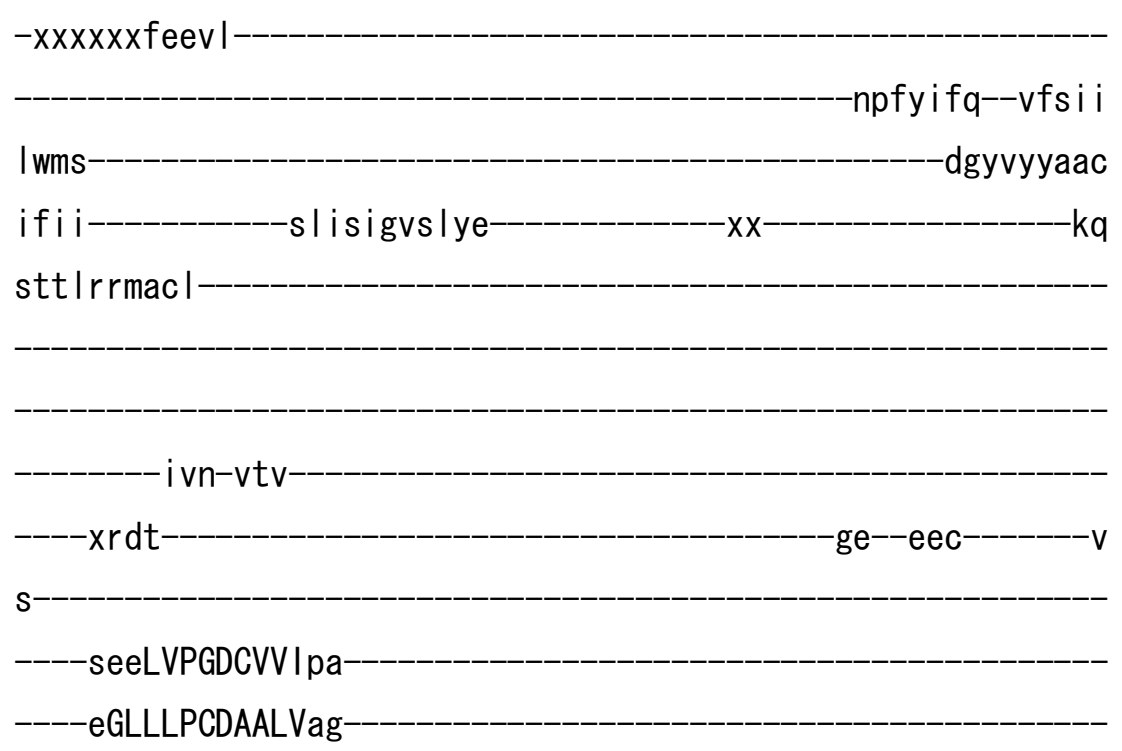



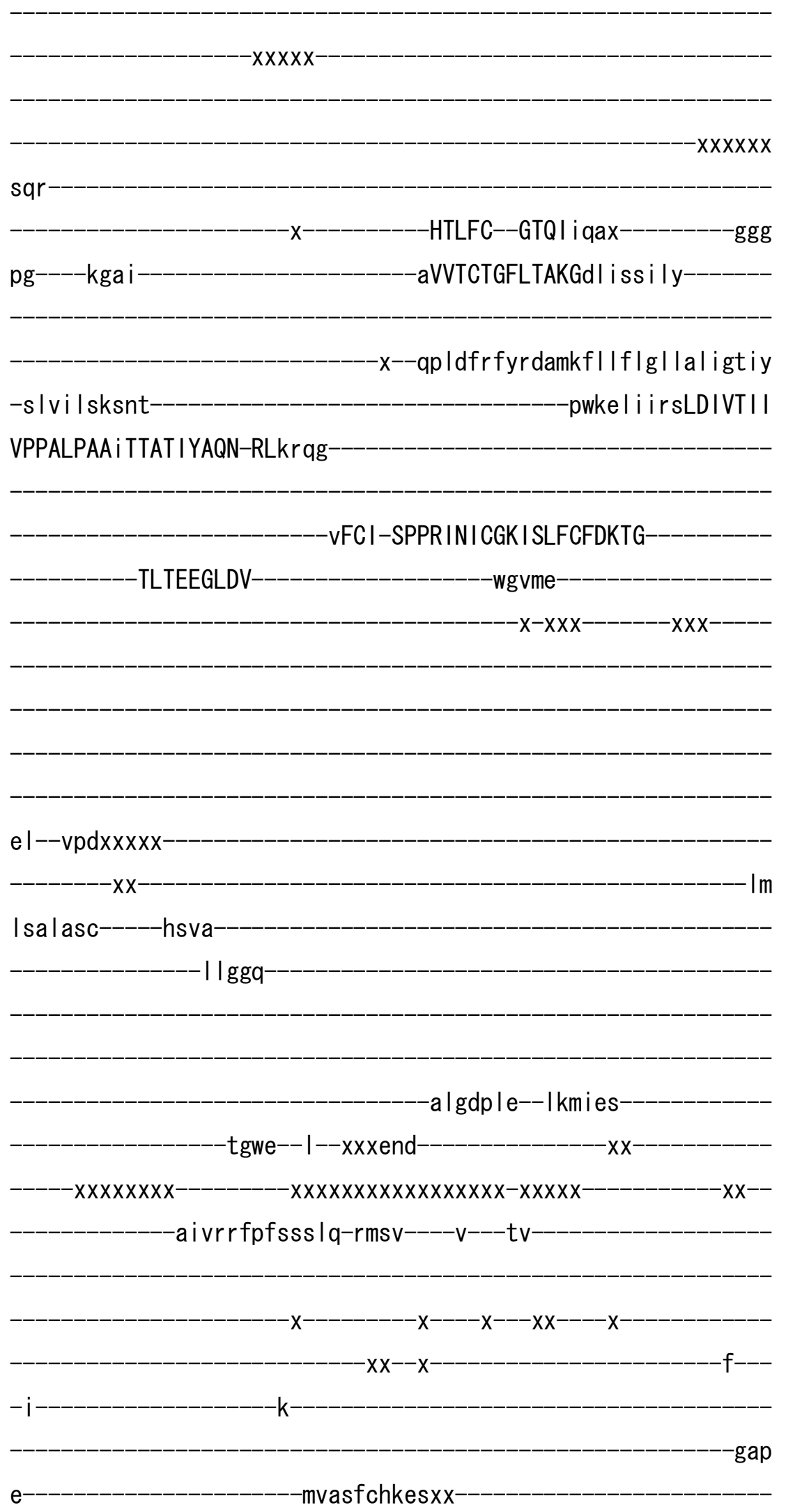
shf-sht I rey--asq----gfrvLGLAYKh I -aketd I stvervxvekgmnfLGLLVMKNQVKPESAEVIQTLTLaq-LRP VMVTGDNILTAVNVARVCGM$-v p x x e k v i-f v h$

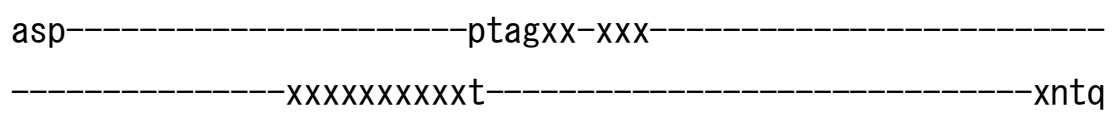

---qtidipxxxqyqsxvgyh-

la ingmsfa

alcdhf-pey lpk-

v Imrg--tIYA--RMTPEQKTQLVKALq-

$---\mathrm{k} \mid--\mathrm{ny}$

-RVGMCGDGANDCGALRAADVGVSL--

seae

-ASVASPFTSKs-DN I SCVP I I IKEGRCSLVTSFSLF $-K$

yma-Iys-I-iqfasvl i lytek--_-_-_-_-_-_-_-_-_-_-_-_-_nlgd laf Iff

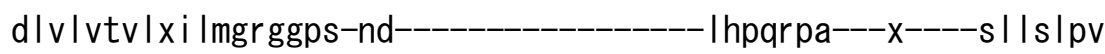
|-as | |mhtv|--| i laqvsg | | i tmsqdw--_-_-_-_-_-_-_-_-_-_-_---xxxx---

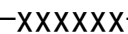

aa----n I pnmedtsvfavsgfq-yi imsvvi tkgfpykk-----p l yhnv I fvcalvf I falmswlv--Ifrhti i-_-_-_-_---hrvls ly-_-_-_-_-_-_-_-_-_-_-_-_-_-_ ------xxxdmsyk----||--|vaiaalnfficflleffi 
S

>celV_V201 NP_501755. 2

m

xxxxxxxx|t|fay

$$
r
$$
xxxxxxi I fyaltvltIgifr I i lhwkqkwdvkm rmvpctfeaaey iy i idnhnvse lapv I rksnat ipteng

-emrkvpe I rwfvyrkleyvwi xxxxxxxxxxxxxdndncwktsfe ian ripcrs IIxxx

$$
\text { xxxfg|t|se- }
$$

$$
\text { -isrrlefygrneivvqlr }
$$

$-x x x x x x v m e v i$ 


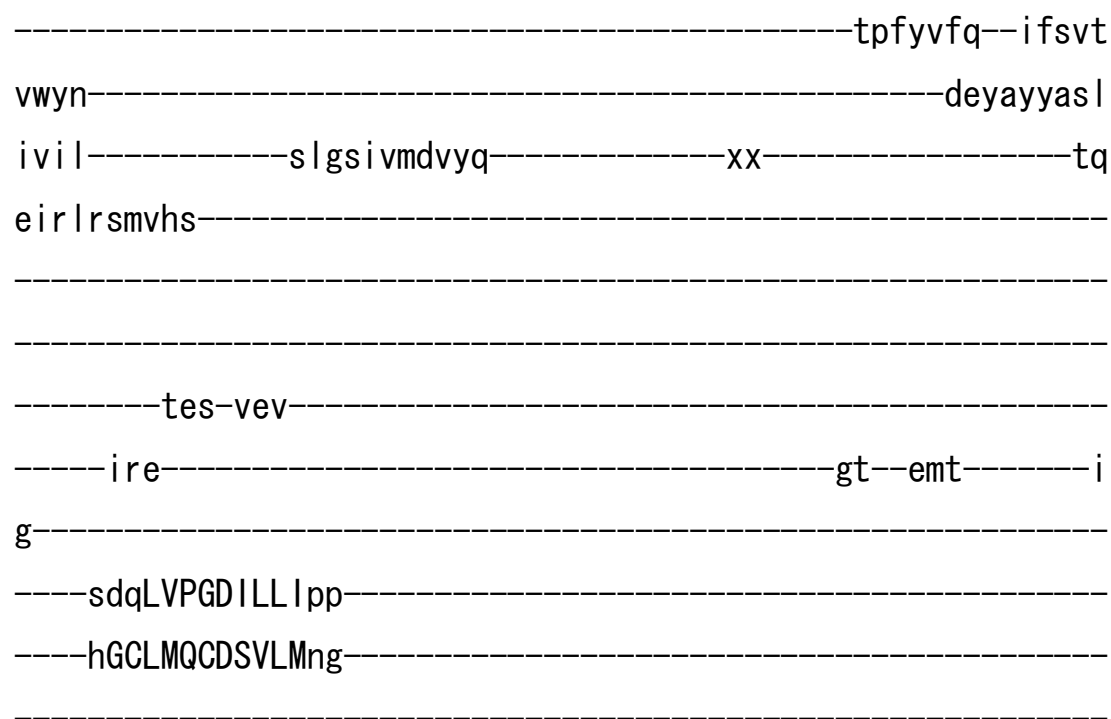

-tviVNESVLTGESVPITKva-

$-\mathrm{XXXXXX}$

XXXXXX

kns

NVLFC--GTQVI qt rfy

rg-----kkvka IVLRTAYSTLKGqI vrs imy

-x--kpvdfrftkdlfkfi Iflac isgcgfi y

-ti i vmimrgn -tIrriivrsLDIITIT

VPPALPAAmSVGI INAQL-RLkkke-

iFCI-SPST INTCGA INVVCFDKTG

TLTEDGLDF hvvrpx

$x-x x-$

eiqkvk Ixxxxx-

$-x x x x x x x x x x$

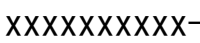

$-d l$

vkaiatc-----hs It

ringv 


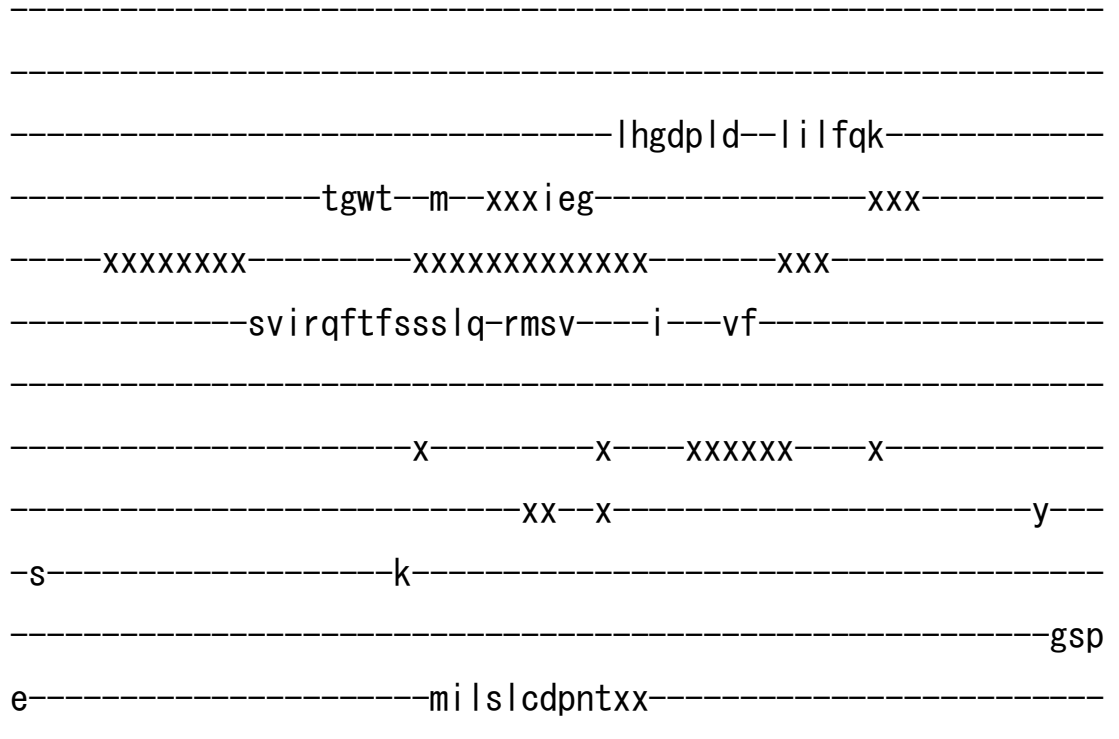

-edy- | I qvnsy--aqh----gfr I I AVARRp I

dInfnkaskvkrdxvecd I emLGL IVMENRVKPVTLGV INQLNRan-IRT VMVTGDNLLTGLSVARECGI---_-_-_-_-_-_-_-_-_-_-_-_--_irxxkraf-Ive

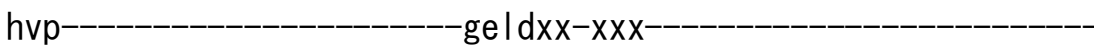

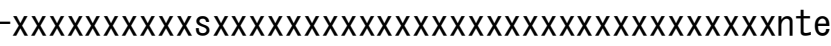
vetpnpvtax-

$-x x g h l i x s s y h$

laisgptfa

vivhey-pelvdq

Icsvc--dVFA--RMAPDQKQSLVEQLq

$---q i--d y-$ -TVAMCGDGANDCAALKAAHAGISL-sdae 


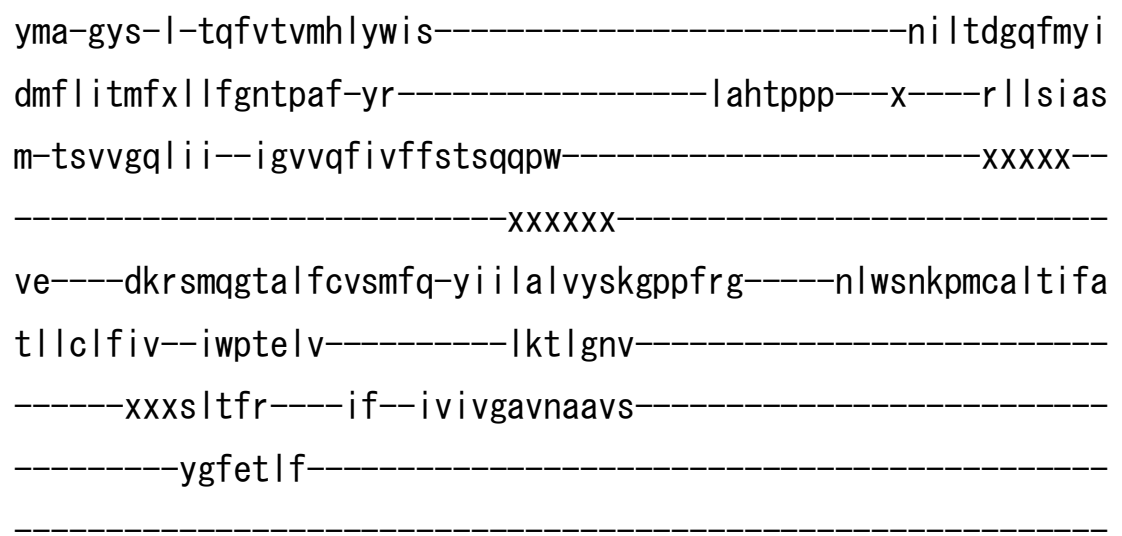

-vdff| Igy--wexxxxxxxxxxxxxxxxxxxxxxx

$x x x x x x x x x x x x x x x x-$

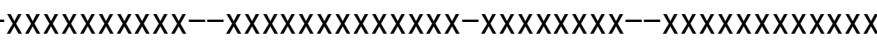
$x x x x x x x x x x x x x x x x x----x x x x x-$

y

>celV_V202 NP_001024768. 1

m

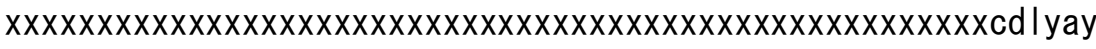
$k$ -xxxxxxi|fw| |tivt Igfyql laywvks | fvkv 
rfaptshdeceyvmvedihgtqt ikevfkaesdvg larptrsg

kqekvkvmrfftyrkiky i wy---------------ekdqew I npadmds

aapfni $y q x x x$

-xxxiglkeqd-

-viasrkiynmnalalalt

$-x x x x x x k e v l$

gpfylfq--cfsva

Iwys

dnyayyasv

ivi i

tvgsaavavyq

$x x$

$-a q$

ekrirnmvgd-

-tv iVNEAMLTGESVPVTKas-

$-\mathrm{XXXXXXX}$

$\mathrm{XXXXXX}$

ehn

$-x-$

-HTLFS--GTTV Iqt rny

kg-----qpvm------_-----------------aRVIRTGFSTLKGq I vr s i my

-x--kpqekealkdvmvfi Iv Igfial igfiy

-tvi emvsrge

slkhi i irsLDIITIV

VPPALPAAmSVGI INANS-RLkkkk- 
iFCT-SPTTVNVCGL INVACFDKTG-

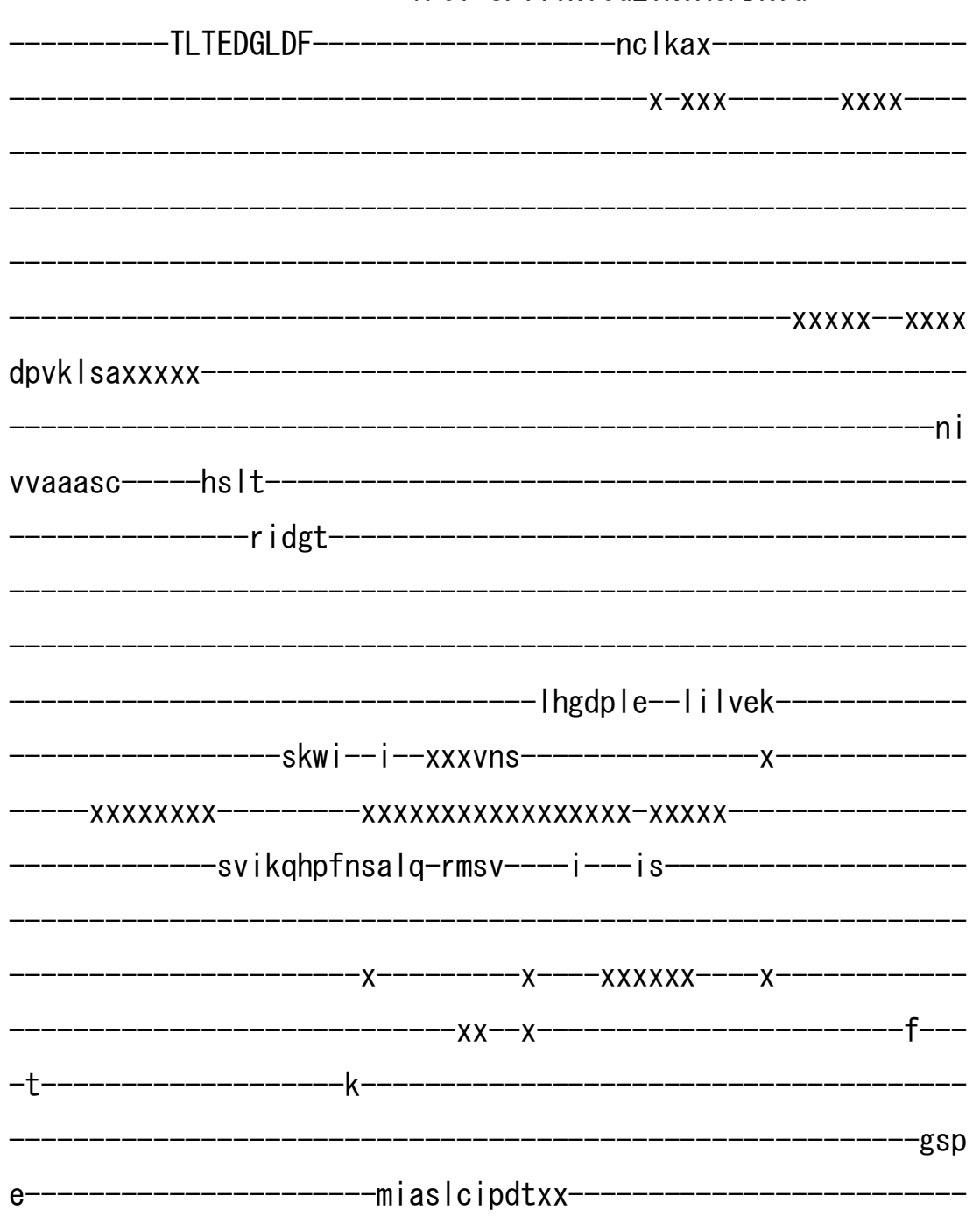

edy-mevvdey--aqr----gfr I I AVASKav

- Infakalktprdi-mese l efLGLIVMENRLKDVTLSVINELSVan-IRC VMVTGDNLLTAMSVARECGI--_-_-_-_-_-_-_-_-_-_-_-_-_-_-_irxxkkaf-l it

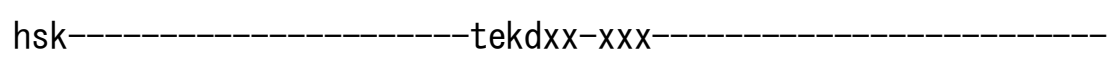

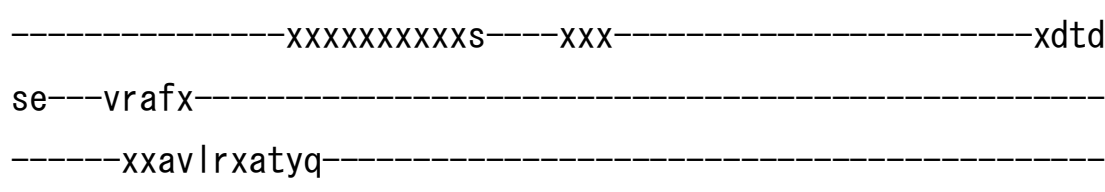
-ma i agptysvithey-pelvdr 


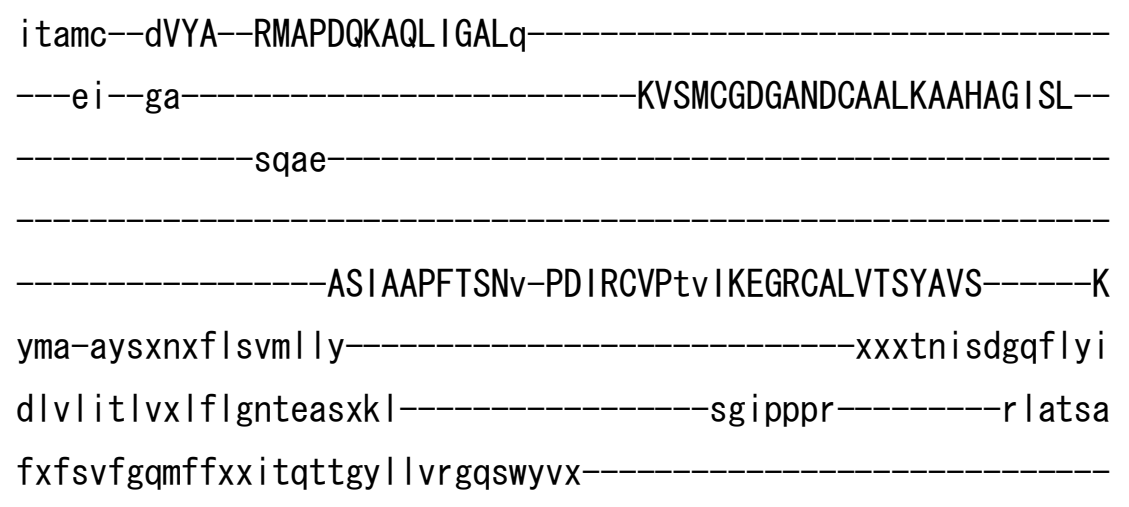




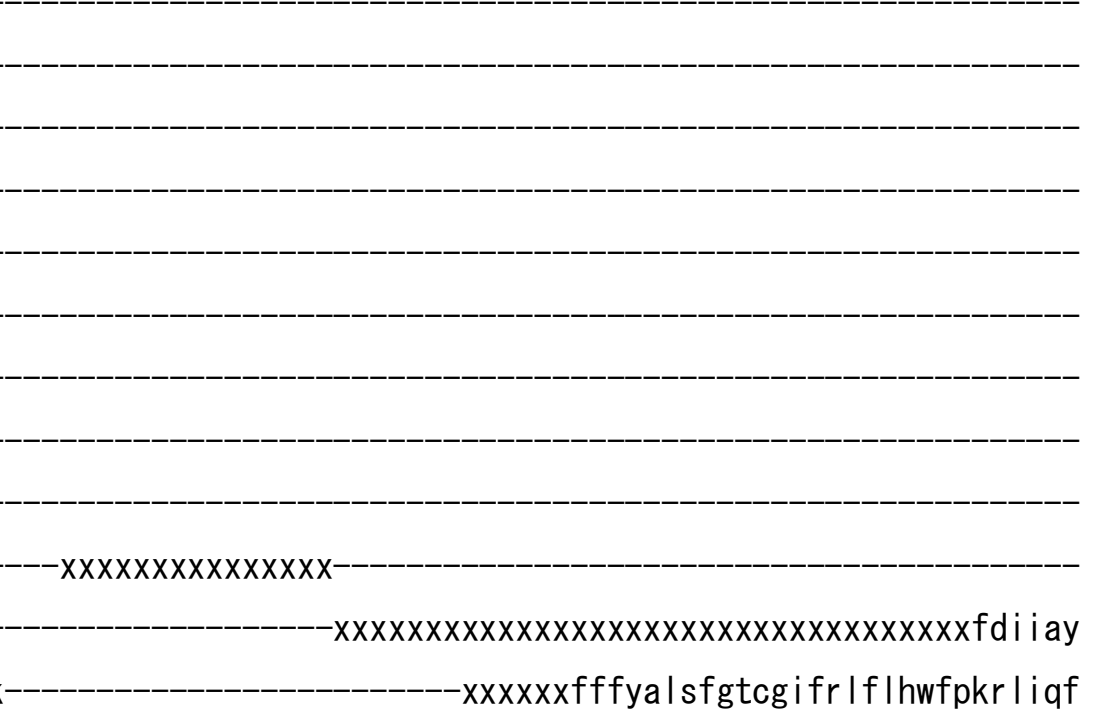

rgkrcsvenad Iv I vvdnhnrydi cnvyyrnksgtdhtvvantdg-

-nlaeldel rwfkyrklaytwi

dgewstpsrays

hvtpenlax

\section{xxxxtg|kadd-}

-val rrtyfgpnvmpvk Is

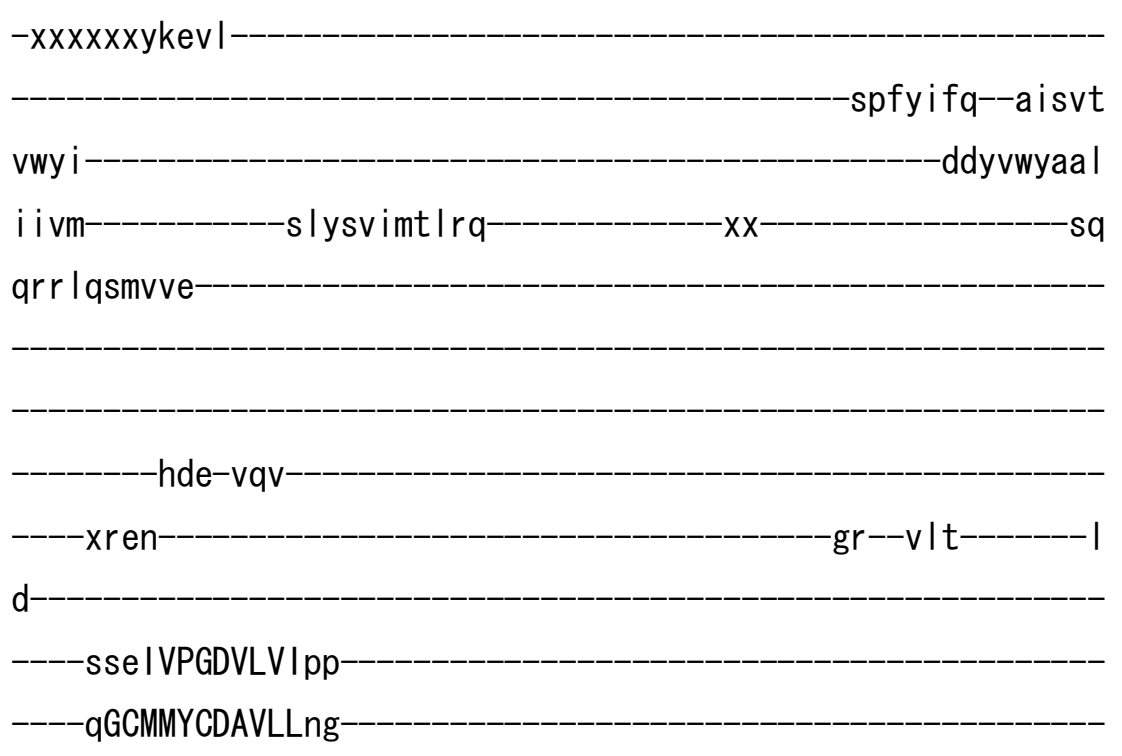


tc iVNESMLTGESIPITKsa-

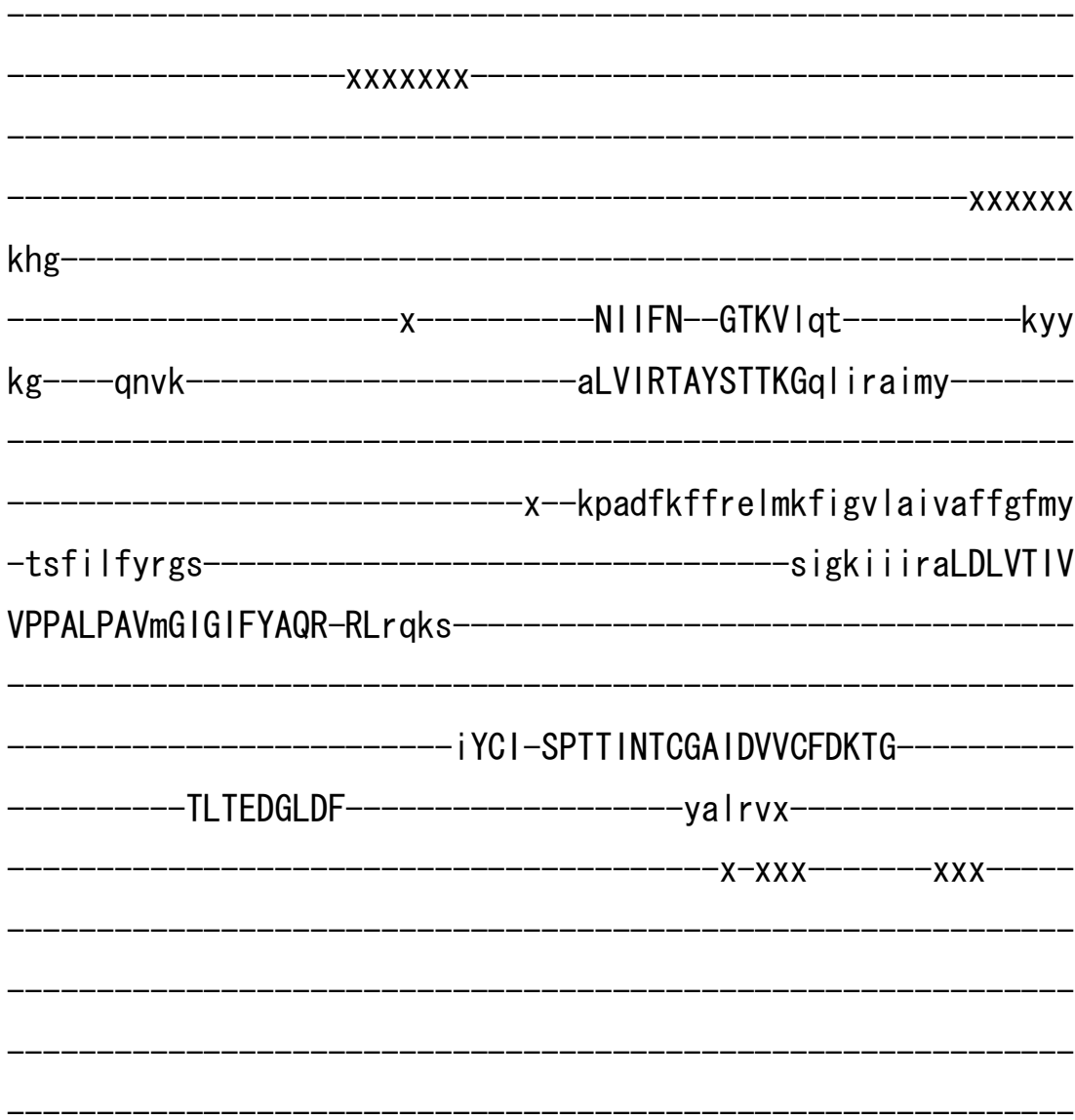

nivqi aaxxxxx

nv

vra iatc--_---ht I s--_-_-_-_-_-_-_-_-_-_-_-_-_-_-_-_-_-_-_-_-_-_-_-_-_-

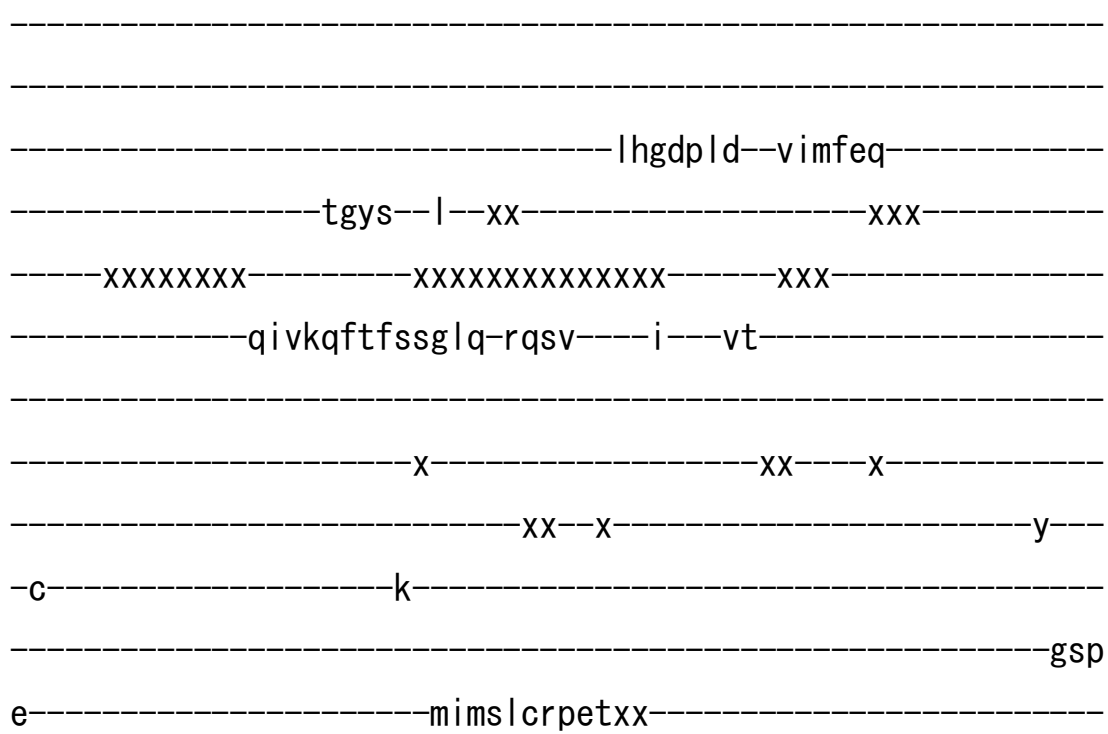


-enf-hd i veey--sqh----gyr I IAVAEKe I

-vvgsevqktprqxi ecd I t I IGLVALENRLKPVTTEVIQKLNEan-IRS

VMVTGDNLLTALSVARECGI

ivxxksay-l ie

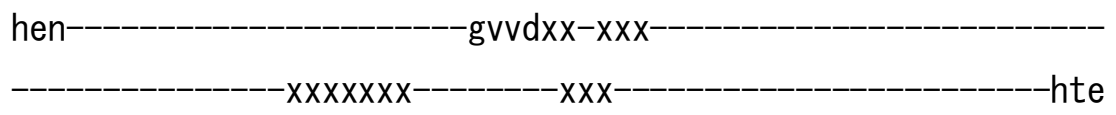

rq---pkivx-

$-x t k m t x k d c q$

faisgstfs

vvthey-pd I Idq

I v lvc--nVFA--RMAPEQKQLLVEHLq

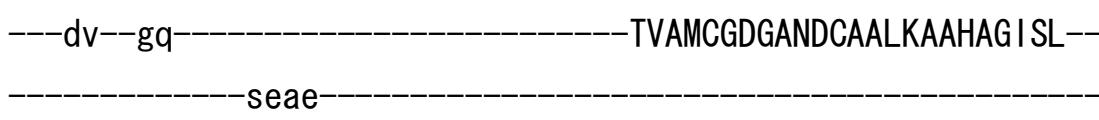

ASI AAPFTSKv-ADIRCVI I I SEGRAALVTSYSAF

\section{$L$}

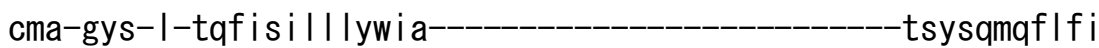

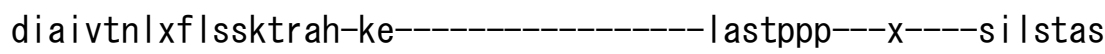

m-vs I fgq la i--ggmaqvavfc l i tmqsw--_-_-_-_-_-_-_-_-_---- xxxxx--

$-x x x x x x-$

de-----drks lqgta i fyvs I fh-y i v l yfvfaagppyra-----s i asnkaf I i smi gv tvtciaiv--vfyvtpi----------qyf Igc I

--------xxpqefr----_i---i lavatvtavi s-_-_-_-_-_-_-_-_-_-_-_-_-_-_-_

$-\mathrm{i}$ iydrcv 
$-\mathrm{XXX}$

$-\mathrm{x}$

a

>ggaV_V201 XP_422709. 2

$\mathrm{m}$

xxxxxxxxxwe iygy

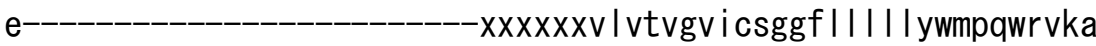

tckktt Ikdcevv I Irttdefk i wfcakvrvmps I gasp I qnpdpxxxxxxxxhxx

$x X X X X X X X X X X X X X X X X-$

-_-_-_-_-_-_-_-_-_-_-_-_-_-_-_-_-_-_-_-_-_-_-_-_-_-_-_-_-_-_-_-_-_-_

- pvsraairyfthhsvkyfwn

dsaqsfdvvrgldd

stfossih-

xxxxrg|tkem-

-hdyrkmffg ine i avkvp

-xxxxxxikevl 


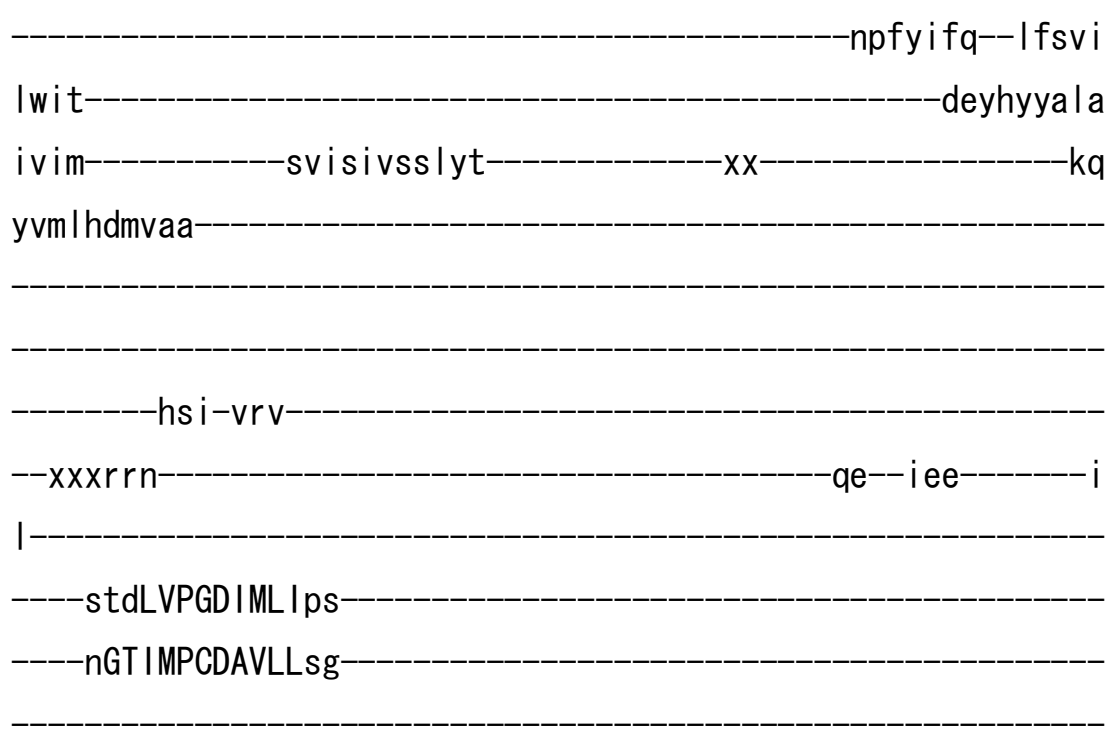

-tc iVNESMLTGESVPVTK in-

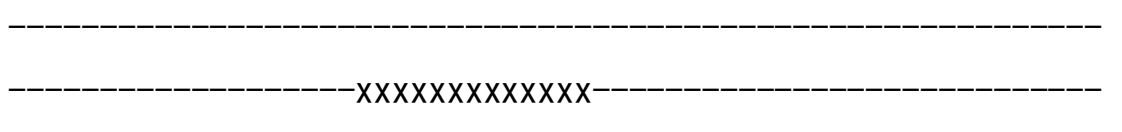

$-x x x x x x$

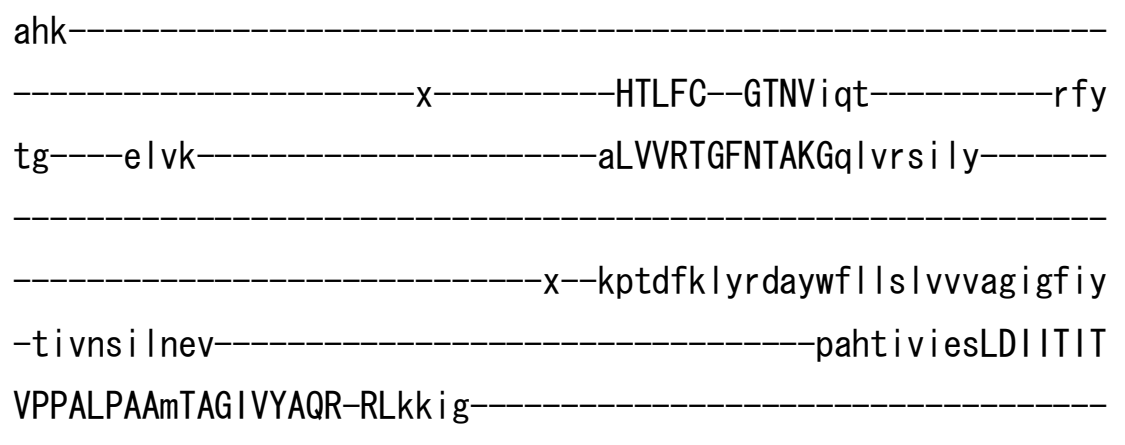

VPPALPAAmTAG IVYAQR-RLkki g--_-_-_-_-_-_-_-_-_-_-_-_-_-_-_-_-_

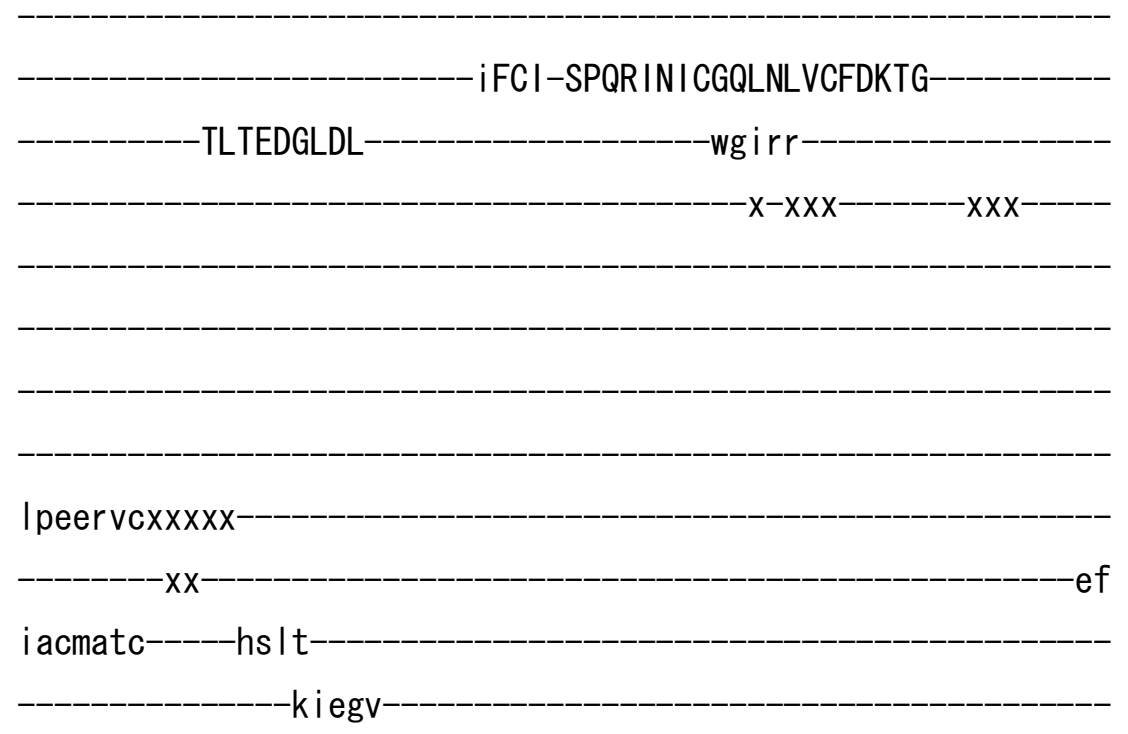




\section{$-\mid$ sgdp |d--|kmfea-}

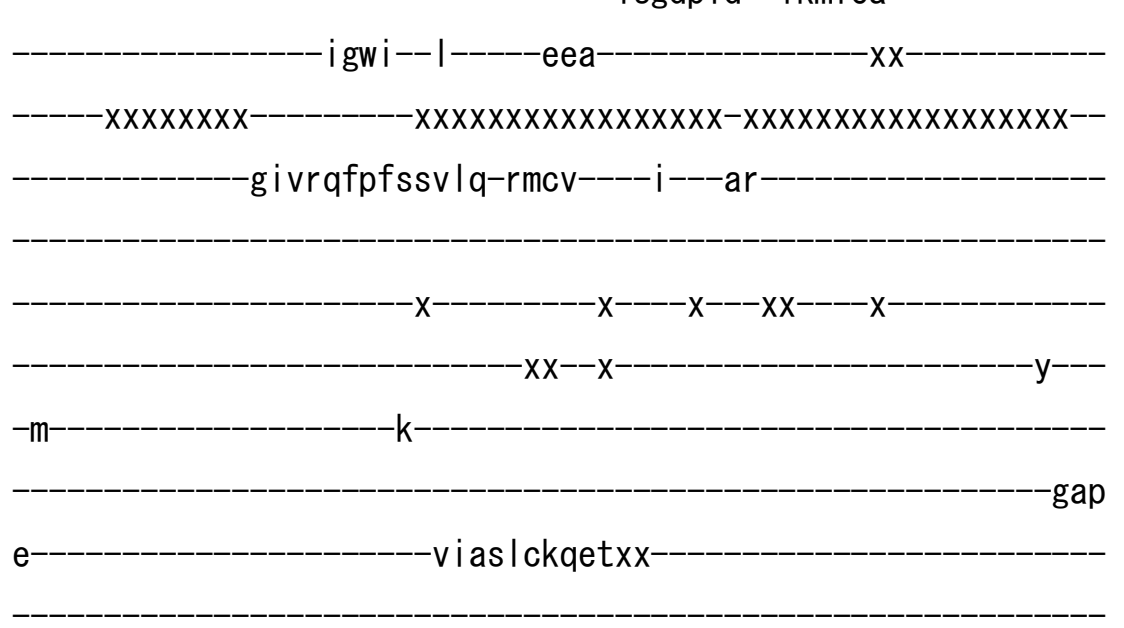

-vdf-esv leey--tkq----gfrv lALAHRk I

-xxx I twhkvqt inrda-i esnmdfMGL I IMQNKLKQETPAVLEDLHKan-IRT

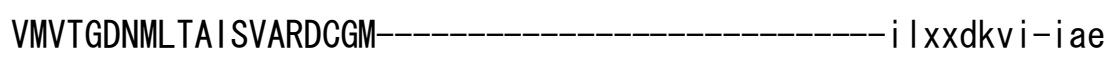

vi lehf-qd lvpk

Iv lhg--tVFA--RMAPDQKTQLVEALq

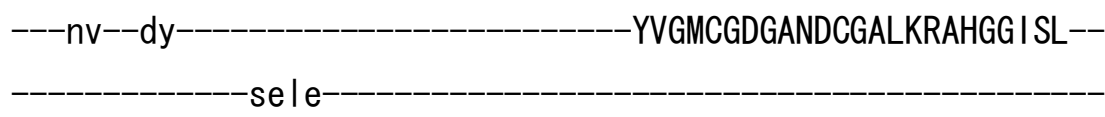




$$
\begin{aligned}
& \text { fma-lys-i-iqyi svt l lysi I--_-_-_-_-_-_-_-_-_-_-_-_-_-_n I gdhqf If } \mathrm{i}
\end{aligned}
$$

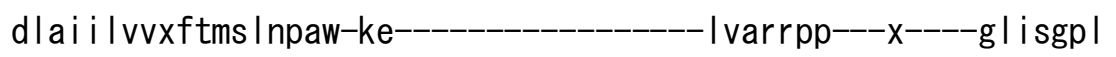

$$
\begin{aligned}
& \text { I-csvlsqi i i--clvfqtfgf I wvkqqpw----------------------- } x x x x x x x
\end{aligned}
$$

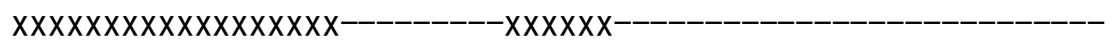

$$
\begin{aligned}
& \text { eh----niknyentt Iffissfa-y l ivai vfskgkpfrq-----pcyknf I fvtsvvv | }
\end{aligned}
$$

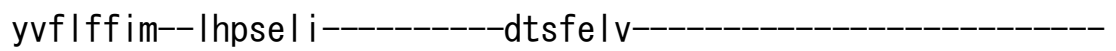

$$
\begin{aligned}
& \text {--------xxpyewr-----Ir--i I i ivivna ivs---_-_-_-_-_-_-_-_-_-_-_--- } \\
& -v \mid m e e t v-
\end{aligned}
$$

drogvcf $|x y| f x x x x x x x$

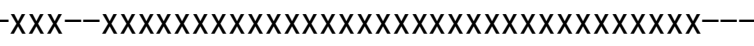
$-x-x x x x x$

$\mathrm{t}$

>ggaV_V202 NP_001026485. 1

m-

$X X X X X X X X X X X X X X X X X X X$

-me ifgy

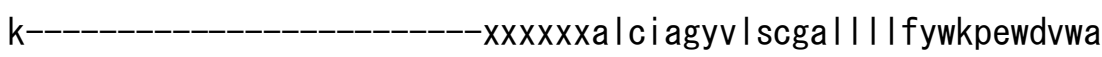




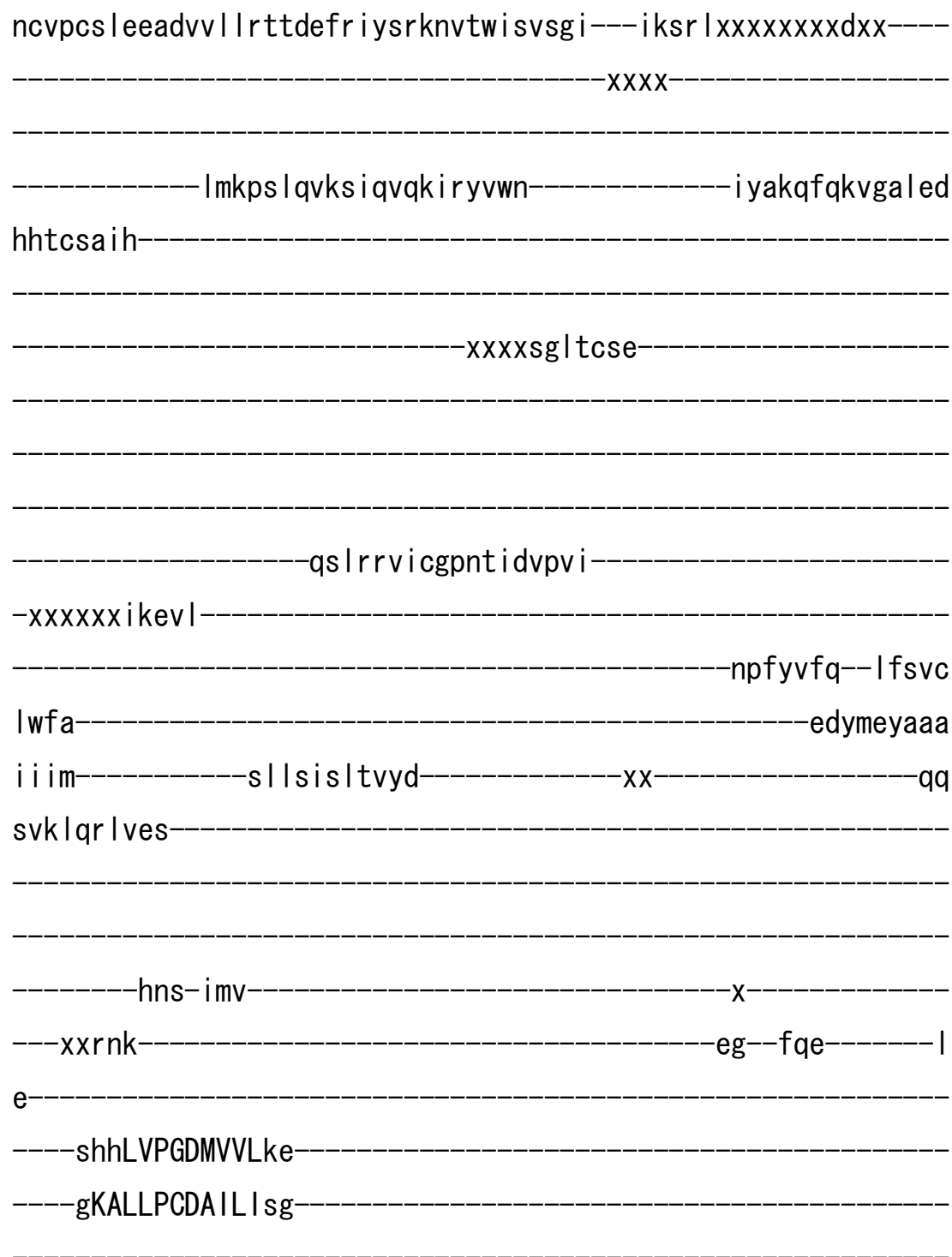

-qc iVNESMLTGESIPVTKtq-

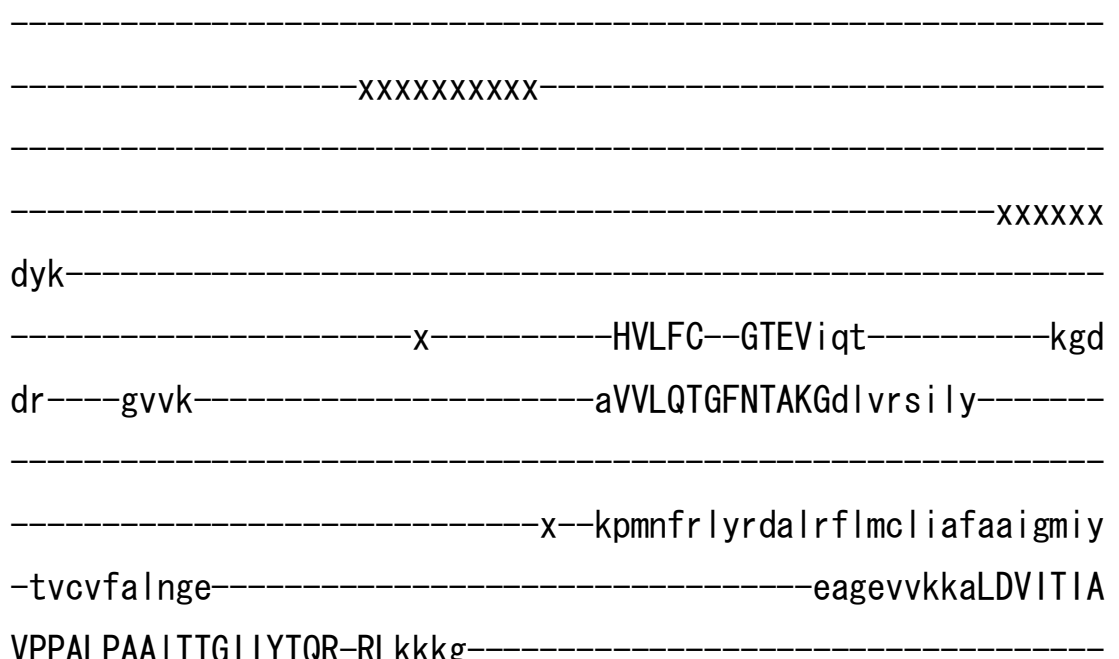

VPPALPAA ITTGI IYTQR-RLkkkg- 
iFCI-SPQR INMCGQLNL I CFDKTG-

TLTEDGLDL

$-w g|| p$

$-x-x x x$

$-X X X----$

dvrrfpaxxxxx

$-x x-$

- pa

framvvc-----hs li

vlegk-

iqgdp I d--vkmfea-

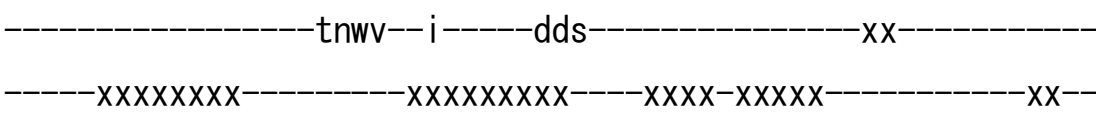

-tilhqfpfssalq-rmsv----i---aq

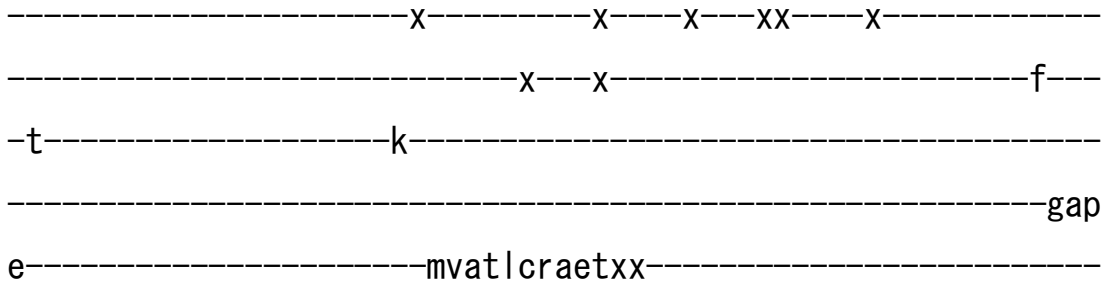

-snf-esk I I fy--taq----gfrv IGLAYKs I

-qsgkqstdI trexvesdItfLGLLIMENRLKRETKPVLEELSAah-IRS

VMVTGDNIQTAVTVAKNAGMisxxnrvi-Ive

ank ipgs $x x-x x x x x$

$-x x x x x x x x x-d$

-xgn I

dsgsqtgrrx

xxaaep-gqfh

famsgksyq

-vvaqyf-shl Ipk 
I I Ina--tVFA--RMSPSQKSSLVEEFq-

$---\mathrm{k} \mid--\mathrm{dy}$

-FVGMCGDGANDCGALKVAHAGISL--seqe

-ASVASPFTSRT-PSI ACVPe I I REGRAALVTSFCMF K yma- Iys-t-iqy | gv | | |ywq |--_-_-_-_-_-_-_-_-_-_-_-_-_--nsfgnyqf I fa

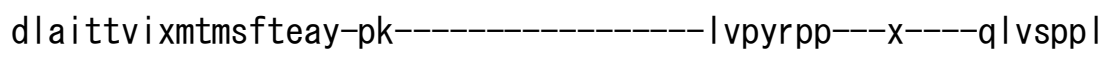
I-Isvi Ini If--s I gmq i gf Imvqkqpw---_-_-_-_-_-_-_-_-_xxxxx

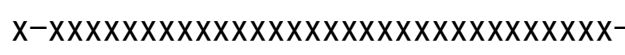

dn----gyksyenttvw I Ist in-cli ial vfskgkpfrq-----pi ytnyvfi Iv Ivgq

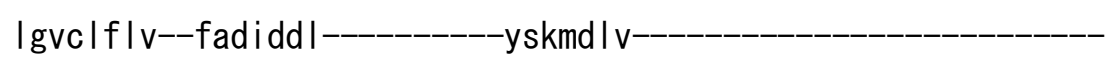

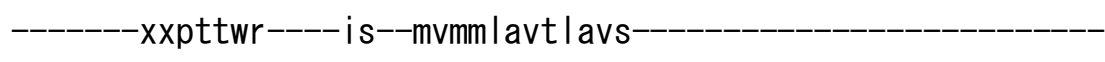
flveeai -ienra|w|-w|kxxxxxxx-

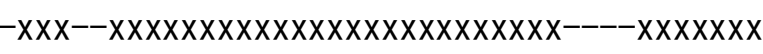

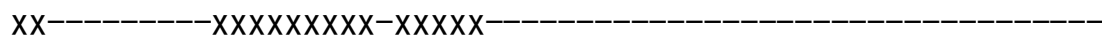
। >ggaV_V203 XP_422713. 2

$\mathrm{m}-$ 


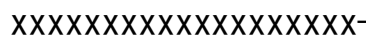

leafgy

k--_-_-_-_-_-_-_-_-_-_xxxxxa I c i agy i I scga I I I I fywkpewdvwa ncvpcs leeadvi IIrttdefakytrkkvtwidlst|t|ptgsnsxxxxxxxxddxx---_

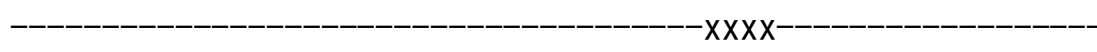
imkpq I kmrciqvqki iry iwd fsvkefrkvgsled

sntchsih

xxxxag|tgee-

-rkisqlvcgpnai eveir

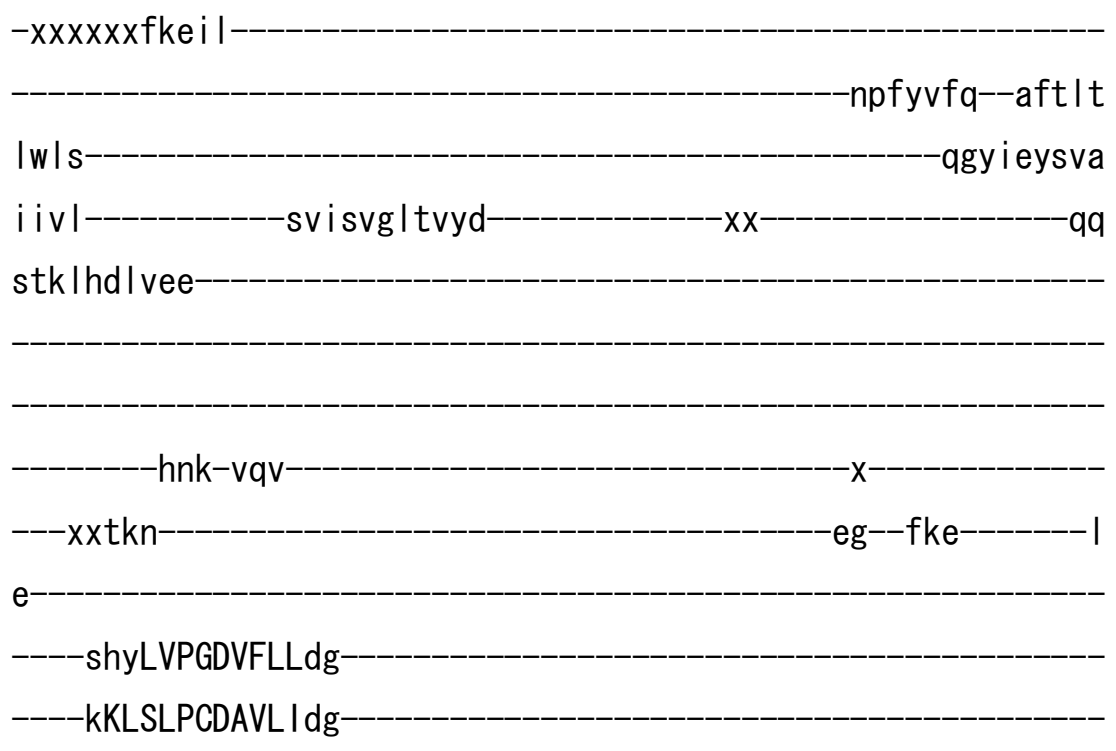


sc i VNEGMLTGESI PVMKt I-
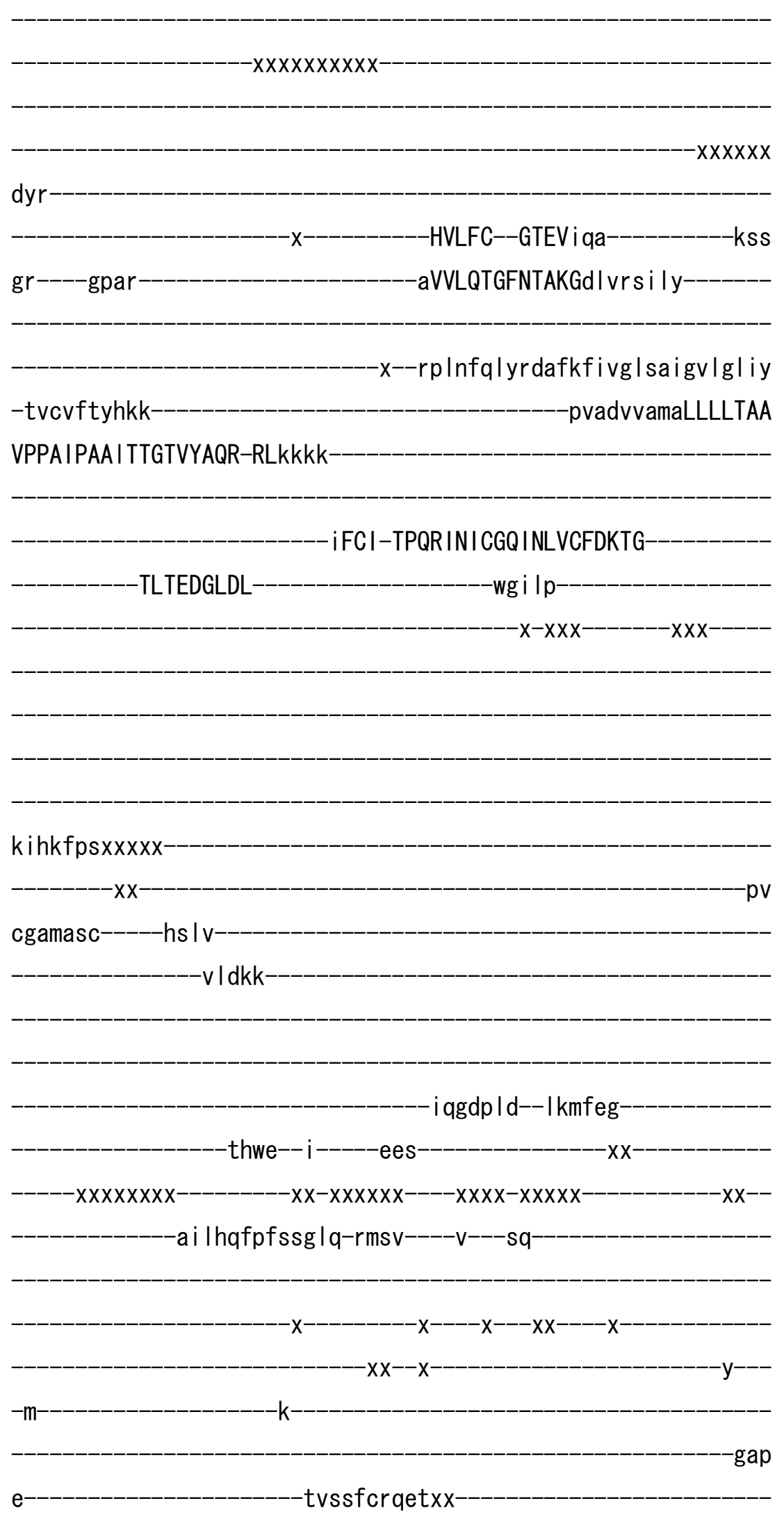
-sdf-leelkty--tsq----gfrvlALAHKv I

- I pedvdvsd l erexaesg I efLGLLVMENRLKPETKPVLRELAAar-IRS

IMVTGDNLQTAVTVARNADM-

ihxxskvi-ive

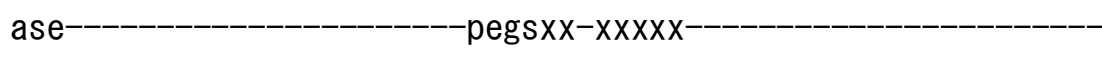

-xxxxxxxxx-a-------------------------------------aаp

tvcantqekx-

xxgges-snyh

famngksyq

$-v|v k h f-y s| l p k$

i I Ina--tVFA--RMSPSQKSSLVEELq

$---\mathrm{k} \mid--\mathrm{dy}$

-YVGMCGDGANDCGALKMAHAGISL--

seqe

ASVASPFTSQi-PNI QCVPe I IREGRAALVASFAVV

$-K$

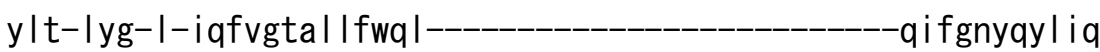

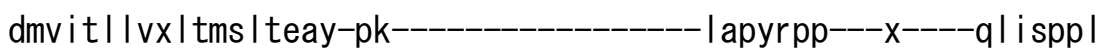

I-I svi Invcf--t i amqtcgf I I vkkqpw--------------------- xxxxxxx

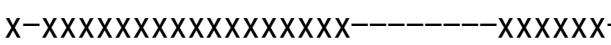

hs----tv I syegtt I wp I ssvn-ci i vafvfskgkpfrk-----pi ytnyvfsal It lq latc If If--ladmdav-----------ysgmqf I-

-------xxpviwr-----vy--v| Iml Ivtfcvs-

fftedvI 
$-x x x--x x x x x x x x x x x x x x x x x x x x x x x x x x x x x x x x x x x x x y$

$\mathrm{xxx}$

d 
strict rule

\#Supplemental Data 1

\#Molecular Biology Reports

\#Evolutionary dynamics of spliceosomal intron revealed by in silico analyses of the P-Type ATPase superfan \#Toshiyuki Oda, Ryosuke L. Ohniwa, Masatsugu Denawa, Hideyuki Okamura and Kunio Takeyasu

\begin{tabular}{|c|c|c|c|c|c|}
\hline 6507 & 6508 drelV_IV03 & drelV_IV09 & rnolV_IV12 & rnolV_IV13 & mmulV_IV12 \\
\hline 6519 & 6520 anillI_IIIA02 & & & & \\
\hline 6525 & 6526 athIV_IV03 & & & & \\
\hline 6676 & 6677 hsall_IIB01 & hsall_IIB02 & hsall_IIB03 & hsall_IIB04 & rnoll IIB01 \\
\hline 6678 & 6679 athl_IBZ202 & & & & \\
\hline 6681 & 6682 athl_IB101 & osal_IB101 & & & \\
\hline 6684 & 6685 osall_llBO2 & osall_IIB05 & athII_IIB05 & athII_IIB06 & athII_IIB07 \\
\hline 6690 & 6691 drelV_IV01 & drelV_IV02 & dreIV_IV04 & drelV_IIV07 & rnolV_IV02 \\
\hline 6694 & 6695 cnelV_IV04 & & & & \\
\hline 6696 & 6697 athl_IB̄204 & osal_IB203 & & & \\
\hline 6701 & 6702 anill_IID01 & anill_IID02 & & & \\
\hline 6702 & 6703 cnellI_IIIA01 & & & & \\
\hline 7152 & 7153 ddilV_IV02 & athIV_IV02 & athIV_IV03 & osalV_IV07 & \\
\hline 7155 & 7156 athIII_IIIA04 & athIII_IIIA10 & athIII_IIIA11 & osallI_IIIA02 & osallI_IIIA03 \\
\hline 7159 & 7160 athIII_IIIA07 & & & & \\
\hline 7168 & 7169 hsaV_V201 & hsaV_V202 & hsaV_V203 & hsaV_V204 & rnoll_IIA101 \\
\hline 7169 & 7170 drelV_IV01 & dreIV_IV02 & dreIV_IV04 & drelV_IV07 & cnelV̄_IV01 \\
\hline 7182 & 7183 osall_IIBO2 & osall_IIB05 & athll_IIB05 & athlI_IIB06 & athlI_IIB07 \\
\hline 7185 & 7186 drelV_IV03 & dreIV_IV09 & rnolV__IV12 & rnolV__IV13 & mmūV_IV12 \\
\hline 8205 & 8206 osal_IB202 & & & & \\
\hline 8221 & 8222 hsall_IIB01 & hsall_IIB02 & hsall_IIB03 & hsall_IIB04 & cellV_IV04 \\
\hline 8382 & 8383 athl_IB101 & osal_IB101 & osall_IIB02 & osall_IIB05 & athlI_IIB05 \\
\hline 8395 & 8396 hsaV̄_V201 & hsaV̄_V202 & hsaV_V203 & hsaV_V204 & $m m \bar{u}$ V_V201 \\
\hline 8409 & 8410 athIV_IV03 & athIV_IV10 & athIV_IV11 & & \\
\hline 8412 & 8413 mmuV̄_V101 & rnoV_V101 & ggaV_V101 & hsaV_V101 & \\
\hline 8980 & 8981 cnell_IIA201 & & & & \\
\hline 8984 & 8985 mmull_IIA201 & mmull_IIA202 & rnoll_IIA201 & rnoll_IIA202 & hsall_IIA201 \\
\hline 8991 & 8992 athl_IBz202 & athI_IB204 & osal_IB203 & & \\
\hline 8994 & 8995 dreIV_IV05 & dreIV_IIV08 & cellV_IV06 & drelV_IV10 & dreIV_IV12 \\
\hline 8997 & 8998 tanV_V201 & tpaV_V201 & & & \\
\hline 9007 & 9008 anill_IIB03 & anill_IIB05 & & & \\
\hline 9010 & 9011 rnoll- IIA101 & hsal|-\|A101 & drell_IIA101 & drell_IIA102 & drell_IIA105 \\
\hline 9011 & 9012 drelV_IIV13 & cnelV̄_IV01 & rnolV̄_IV08 & mmüV_IV07 & mmūV_IV08 \\
\hline 9018 & 9019 athIV_IV02 & osalV_IV07 & & & \\
\hline 9019 & 9020 celV_V̄201 & celV_V̄203 & & & \\
\hline 9020 & 9021 ggalV__IV05 & & & & \\
\hline 9023 & 9024 drelV_IV01 & drelV_IV02 & dreIV_IV04 & dreIV_IV07 & rnolV_IV02 \\
\hline 9030 & 9031 dmelV_IV04 & ggall_IIC01 & ggall_IIC02 & mmull_IIC01 & mmull_IIC02 \\
\hline 9032 & 9033 athlI_IIAA103 & & & & \\
\hline 9035 & 9036 osall_IIB02 & osall_IIB05 & athlI_IIB05 & athII_IIB06 & athII_IIB07 \\
\hline 9042 & 9043 cnelV__IV02 & athl_IB202 & & & \\
\hline 9048 & 9049 cellV_IV04 & & & & \\
\hline 9061 & 9062 athl_IB204 & osal_IB203 & & & \\
\hline 9441 & 9442 osall_IIB02 & osall_IIB05 & athlI_IIB05 & athII_IIB06 & athlI_IIB07 \\
\hline 9446 & 9447 mgrlI_IID01 & & & & \\
\hline 9452 & 9453 athIV_IV02 & osalV_IV07 & & & \\
\hline 9461 & 9462 spoV_V201 & & & & \\
\hline 9475 & 9476 mmull_IIA201 & mmull_IIA202 & rnoll_IIA201 & rnoll_IIA202 & hsall_IIA201 \\
\hline 9480 & 9481 athV_V̄101 & drel_IB̈201 & drel_IB203 & cell_IB201 & drelV_IV03 \\
\hline
\end{tabular}


strict rule

\begin{tabular}{|c|c|c|c|c|c|}
\hline 9489 & 9490 celV_V201 & celV_V202 & celV_V203 & & \\
\hline 9504 & 9505 hsaV_V201 & hsaV_V202 & hsaV_V203 & hsaV_V204 & cnell_IID01 \\
\hline 9508 & 9509 drelV_IV13 & cnelV_IV04 & rnolV_IV08 & cnell_IIA101 & mmulv_IV07 \\
\hline 9578 & 9579 ehilV_IV08 & & & & \\
\hline 9582 & 9583 mgrll_IIC01 & & & & \\
\hline 9586 & 9587 cnelV_IV03 & ddilV_IV07 & anilV_IV03 & & \\
\hline 9588 & 9589 cellV_IV06 & osall_IIB02 & osall_IIB05 & athll_IIB05 & athll_IIB06 \\
\hline 9591 & 9592 athV_V101 & osaV_V101 & & & \\
\hline 13963 & 13964 ggall_IIC01 & ggall_IIC02 & mmull_IIC01 & mmull_IIC02 & mmull_IIC03 \\
\hline 13965 & 13966 hsal_IB203 & & & & \\
\hline 14137 & 14138 dreIV_IV05 & drelV_IV08 & drelV_IV10 & drelV_IV12 & rnolV_IV09 \\
\hline 14142 & 14143 athII_IIA103 & & & & \\
\hline 14145 & 14146 athV_V101 & osaV_V101 & & & \\
\hline 14154 & 14155 osall_IIA101 & osall_IIB02 & osall_IIB05 & athlI_IIA101 & athll_IIB05 \\
\hline 14159 & 14160 drelV_IV03 & drelV_IV09 & rnolV_IV12 & rnolV__IV13 & mmūlV_IV12 \\
\hline 14166 & 14167 athIV_IV02 & osalV_IV07 & & & \\
\hline 14169 & 14170 pfalV_IV03 & cellV_IV04 & & & \\
\hline 14172 & 14173 hsall_IIB01 & hsall_IIB02 & hsall_IIB03 & hsall_IIB04 & cellV_IV02 \\
\hline 14181 & 14182 cnellI_IIIA01 & & & & \\
\hline 14192 & 14193 osal_IB203 & & & & \\
\hline 14201 & 14202 celV_V201 & celV_V203 & & & \\
\hline 14217 & 14218 ggall_IIC01 & ggall_IIC02 & mmuV_V101 & mmull_IIC01 & mmull_IIC02 \\
\hline 14220 & 14221 athV_V101 & osaV_V101 & & & \\
\hline 14233 & 14234 hsaV_V201 & hsaV_V202 & hsaV_V203 & hsaV_V204 & anill_IIB05 \\
\hline 14244 & 14245 athlI_IIA103 & & & & \\
\hline 14245 & 14246 dmeIV_IV04 & drelV_IV01 & drelV_IV02 & drelV_IV04 & drelV_IV07 \\
\hline 14263 & 14264 mmull_IIA201 & mmull_IIA202 & rnoll_IIA201 & drelV_IV06 & rnoll_IIA202 \\
\hline 14264 & 14265 athIV IV04 & athIV IV05 & athIV IV06 & athIV IV07 & athIV IV08 \\
\hline 14269 & 14270 athIV_IV03 & & & & \\
\hline 14271 & 14272 drel_IB201 & drel_IB203 & athl_IB102 & athl_IB103 & rnol_IB202 \\
\hline 16951 & 16952 cneIV_IV01 & & & & \\
\hline 16952 & 16953 anilV_IV02 & & & & \\
\hline 16961 & 16962 drelV_IV06 & athlI_IIA103 & mmulV_IV01 & mmulV_IV02 & ggalV_IV01 \\
\hline 16972 & 16973 athIII_IIIA01 & athIII_IIIA02 & athIII_IIIA03 & athIII_IIIA04 & athIII_IIIA05 \\
\hline 16974 & 16975 tanV_V201 & tpaV_V201 & & & \\
\hline 16980 & 16981 mmū__V101 & rnoV_V101 & cnell_IID01 & ggaV_V101 & hsaV_V101 \\
\hline 16986 & 16987 dreIV_IV03 & drelV_IV09 & rnolV_IV12 & rnolv_IV13 & athIV_IV02 \\
\hline 16995 & 16996 mmuli_IIA201 & mmull_IIA202 & 2 rnoll_IIIA201 & rnoll_IIA202 & hsall_IIA201 \\
\hline 16996 & 16997 hsall_IIB01 & hsall_IIB02 & hsall_IIB03 & hsall_IIB04 & rnoll_llB01 \\
\hline 16998 & 16999 cnelV_IV02 & & & & \\
\hline 17205 & 17206 at & & & & \\
\hline 17214 & 17215 rnoll_llA101 & rnoll_IIA102 & rnoll_IIA103 & hsall_IIA101 & hsall_IIA102 \\
\hline 17218 & 17219 dreIV_IV05 & drelV__IV08 & drelV̄_IV10 & drelV_IV12 & drelV_IV13 \\
\hline 17224 & 17225 athIV_IV01 & osalV_IV01 & & & \\
\hline 17241 & 17242 hsaV_V202 & hsaV_V203 & hsaV_V204 & mmuV_V202 & mmuV_V203 \\
\hline 17250 & 17251 cellV_IV06 & & & & \\
\hline 17251 & 17252 dmelV_IV04 & drelV_IV01 & drelV_IV02 & dreIV_IV04 & drelV_IV07 \\
\hline 17253 & 17254 athV_V101 & osall_IIB02 & osall_IIB05 & osaV_V101 & athll_IIB05 \\
\hline 17263 & 17264 mmuV_V101 & rnoV_V101 & ggaV_V101 & cnelV_IV03 & celV_V201 \\
\hline 17269 & 17270 drelV_IV03 & drelV_IV09 & rnolV_IV12 & rnolV_IV13 & mmūV_IV12 \\
\hline 17697 & 17698 athlI_IIA103 & & & & \\
\hline 17700 & 17701 dmell_IIC01 & & & & \\
\hline 17741 & 17742 cellV_IV04 & & & & \\
\hline 17742 & 17743 athl_IB202 & athl_IB204 & osal_IB203 & & \\
\hline 17743 & 17744 mmūV_V101 & rnoV__V101 & celV_V101 & ggaV_V101 & dmeV_V101 \\
\hline 17747 & 17748 mmull_IIA201 & mmull_IIA202 & rnoll_IIA201 & rnoll_IIA202 & dmell_IIA201 \\
\hline 17759 & 17760 hsaV_V̄201 & hsaV_V̄202 & hsaV_V203 & hsaV_V204 & mmuV_V201 \\
\hline 17764 & 17765 ggall_IIC01 & ggall_IIC02 & mmull_IIC01 & mmull_IIC02 & mmull_IIC03 \\
\hline
\end{tabular}

$$
\text { ページ } 2
$$


strict rule

17784

17793

17785 athl_IB101

17794 anill_IID03

osal_IB101

17796

17797 athIV_IV04 athIV_IV03

athIV_IV05 17820 osall_IIA101 athII_IIA101 athlI_IIA102

17819 
strict rule

ily genes

mmulV_IV13 mmulV_IV14 ggalV_IV10 ggalV_IV12 hsalV_IV12 hsalV_IV13 hsalV_IV14

rnoll_IIB03 rnoll_IIB04 drell_IIB03 drell_IIB04 drell_IIB06 ggall_IIB01 ggall_IIB02

rnolV_IV03 rnolV_IV04 rnolV_IV06 rnolV_IV07 mmulV_IV03 mmulV_IV05 mmulV_IV06

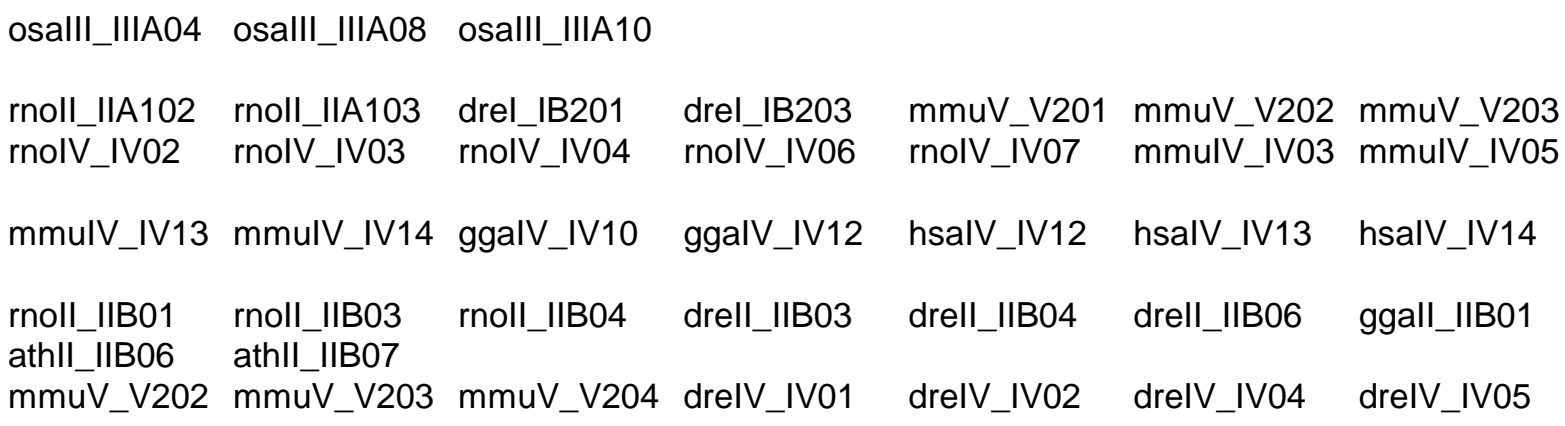

hsall_IIA202 drell_IIA201 ggall_IIA201

rnolV_IV09 rnolV_IV10 rnolV_IV11 mmulV_IV09 mmulV_IV10 ggalV_IV07 ggalV_IV08

mmull_IIA101

ggalV_IV06 hsalV_IV07 hsalV_IV08

rnolV_IV03 rnolV_IV04 rnolV_IV06 rnolV_IV07 mmulV_IV03 mmulV_IV05 mmulV_IV06 mmull_IICO3 mmull_IIC04 cellI_IIC02 hsall_IIC01 hsall_IIC02 hsall_IICO3 hsall_IIC04

hsall_IIA202 drell_IIA201 ggall_IIA201

drelV_IV06 drelV_IV09 rnol_IB202 athI_IB203 osal_IB202 rnolV_IV12 nnolV_IV13 
strict rule

mmuV_V201 mmuV_V202 mmuV_V203 mmuV_V204 rnoV_V201

mmulV_IV08 cnell_IIA201 ggalV_IV06 hsalV_IV07 hsalV_IV08

rnoV_V202

rnoV_V203

cnell_IIB01

athII_IIB07

mmull_IIC04 hsall_IIC01 hsall_IIC02 hsall_IIC03 hsall_IIC04 hsall_IIC05 hsall_IIC06

rnolV_IV10 rnolV_IV11 mmulV_IV09 mmulV_IV10 ggalV_IV07 ggalV_IV08 ggalV_IV09

athlI_IIB06 athlI_IIB07

mmulV_IV13 mmulV_IV14 ggalV_IV10 ggalV_IV12 hsalV_IV12 hsalV_IV13 hsalV_IV14

rnoll_IIB01 rnoll_IIB03 rnoll_IIB04 athIV_IV04 athIV_IV05 athIV_IV06 drell_IIB03

mmull_IIC03 mmull_IIC04 rnoV_V101 ggaV_V101 dreIV_IV13 cneIV_IV01 hsall_IIC01

mmuV_V201 mmuV_V202 mmuV_V203 mmuV_V204 rnoV_V201 rnoV_V202 rnoV_V203

rnolV_IV02 rnolV_IV03 rnolV_IV04 rnolV_IV06 rnolV_IV07 mmulV_IV03 mmulV_IV05

dmell_IIA201 cellIIIA201 hsall_IIA201 hsall_IIA202 mmuIV_IV01 mmulV_IV02 drell_IIĀ201

athIV_IV09 athIV_IV10 athIV_IV11 athIV_IV12

mgrll_IID02 athI_IB201 osal_IB201 osal_IB204 mmul_IB201 mmul_IB202 ggal_IB201

ggalV_IV02 hsalV_IV01

athIII_IIIA06 athIII_IIIA07 athIII_IIIA08 athIII_IIIA09 athIII_IIIA10 athIII_IIIA11 osalII_IIIA02

athIV_IV03 athIV_IV05 athIV_IV06 athIV_IV07 athIV_IV08 athIV_IV09 osalV_IV07

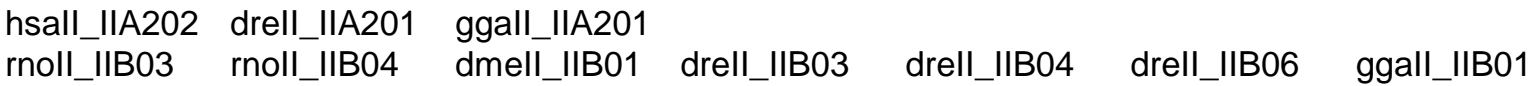

hsall_IIA103 drell_IIA101 drell_IIA102 drell_IIA103 drell_IIA104 drell_IIA105 drell_IIA106

rnolV_IV08 rnolV_IV09 molV_IV10 nolV_IV11 mmulV_IV07 mmulV_IV08 mmulV_IV09

mmuV_V204 rnoV_V202 rnoV_V203 rnoV_V204 ggaV_V201 ggaV_V202 ggaV_V203

rnolV_IV02 rnolV_IV03 rnolV_IV04 rnolV_IV06 rnolV_IV07 mmulV_IV03 mmulV_IV05

athll_IIB06 athll_IIB07

hsaV $\vee 101$

mmulV_IV13 mmulV_IV14 ggalV_IV10 ggalV_IV12 hsalV_IV12 hsalV_IV13 hsalV_IV14

hsaV V101 cneV V101

hsall_IIA201 hsall_IIA202 drell_IIA201 ggall_IIA201

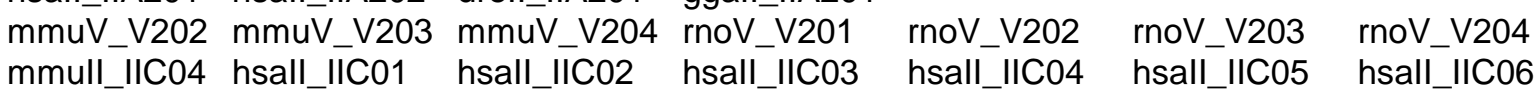


strict rule

athIV_IV09 athIV_IV10 athIV_IV11 athIV_IV12 
strict rule

ggall_IIB03 mmull_IIB01 mmull_IIB02 mmull_IIB03 mmull_IIB04

ggalV_IV04 ggalV_IV05 hsalV_IV03 hsalV_IV04 hsalV_IV05 hsalV_IV06

mmuV_V204 hsall_IIA101 hsall_IIA102 hsall_IIA103 mgrll_IIC01 rnoV_V201 rnoV_V202

mmulV_IV06 ggalV_IV04 ggalV_IV05 hsalV_IV03 hsalV_IV04 hsalV_IIV05 hsalV_IV06

ggall_IIB02 ggall_IIB03 mmull_IIB01 mmull_IIB02 mmull_IIB03 mmull_IIB04

drelV_IV07 dreIV_IV08 dreIV_IV10 drelV_IV12 rnoV_V201 rnoV_V202 rnoV_V203

ggalV_IV09 hsalV_IV09 hsalV_IV10 hsalV_IV11

ggalV_IV04 hsalV_IV03 hsalV_IV04 hsalV_IV05 hsalV_IV06

hsall_IIC05 hsall_IIC06 rnoll_IIC01 rnoll_IIC02 rnoll_IIC03 rnoll_IIC04 rnoll_IIC05

mmul_IB201 mmul_IB202 athIV_IV03 osaV_V101 mmulV_IV01 mmulV_IV02 ggal_IB201 
strict rule

rnoV_V204 dreV_V201 ggaV_V201 ggaV_V202 ggaV_V203 athI_IB204 osal_IB203

rnoll_IIC01 rnoll_IIC02 rnoll_IIC03 rnoll_IIC04 rnoll_IIC05 drell_IIC01 drell_IIC02

hsalV_IV09 hsalV_IV10 hsalV_IV11

athIV_IV07 drell_IIB04 athIV_IV08 athIV_IV09 drell_IIB06 athIV_IV10 athIV_IV11

hsall_IIC02 hsall_IIC03 hsall_IIC04 hsall_IIC05 hsall_IIC06 osall_IIB02 osall_IIB05

rnoV_V204 dreV_V201 ggaV_V201 ggaV_V202 ggaV_V203 athIIIIIIA01 athIII_IIIA02

mmulV_IV06 ggalV_IV04 ggalV_IV05 hsalV_IV03 hsalV_IV04 hsalV_IV05 hsalV_IV06

ggalV_IV01 ggalV_IV02 ggall_IIA201 hsalV_IV01

ggal_IB202 hsal_IB201 hsal_IB202 hsal_IB203 dmel_IB201

osallI_IIIA04 osallIIIIA05 osallIIIIA06 osallI_IIIA08 osallI_IIIA10

athIV_IV10 athIV_IV11 athIV_IV12 mmulV_IV12 mmulV_IV13 mmulV_IV14 ggalV_IV10

ggall_IIB02 ggall_IIB03 mmull_IIB01 mmull_IIB02 mmull_IIB03 mmull_IIB04

athl_IB204 osal_IB203 ggall_IIA101 ggall_IIA102 mmull_IIA101 mmull_IIA102 mmull_IIA103

mmülV_IV10 ggalV_IV06 ggalV_IV07 ggalV_IV08 ggalV_IV09 hsalV_IV07 hsalV_IV08

mmulV_IV06 ggalV_IV04 ggalV_IV05 hsalV_IV03 hsalV_IV04 hsalV_IV05 hsalV_IV06

dreV_V201 ggaV_V201 ggaV_V202 ggaV_V203 tanV_V201 tpaV_V201

rnoll_IIC01 rnoll_IIC02 rnoll_IIC03 rnoll_IIC04 rnoll_IIC05 drell_IIC01 drell_IIC02 
strict rule

ページ 9 
strict rule

rnoV_V203 rnoV_V204 dreV_V201 rnol_IB202 ggaV_V201 athII_IIA103 ggaV_V202

rnoV_V204 dreV_V201 ggaV_V201 ggaV_V202 ggaV_V203 rnolV_IV02 rnolV_IV03

athIV_IV03 drell_IIC01 drell_IIC02 drell_IIC03 drell_IIC04 drell_IIC05 drell_IIC06

ggal_IB202 hsal_IB201 mmulV_IV12 hsal_IB202 mmulV_IV13 hsal_IB203 mmulV_IV14 ページ 10 
strict rule

dmeV_V201

drell_IIC03 drell_IIC04 drell_IIC05 drell_IIC06 drell_IIC07 drell_IIC08

athIV_IV12 ggall_IIB01 ggall_IIB02 ggall_IIB03 mmull_IIB01 mmull_IIB02 mmull_IIB03

rnolV_IV08 rnoll_IIC01 rnoll_IIC02 rnoll_IIC03 rnoll_IIC04 rnoll_IIC05 mmulV_IV07

athIIIIIIA03 athIII_IIIA04 athIIIIIIA05 drell_IIA101 athIIIIIIA07 drell_IIA102 drell_IIA105

ggalV_IV12 hsalV_IV12 hsalV_IV13 hsalV_IV14

hsalV_IV09 hsalV_IV10 hsalV_IV11

drell_IIC03 drell_IIC04 drell_IIC05 drell_IIC06 drell_IIC07 drell_IIC08 
strict rule

ページ 12 
strict rule

ggaV_V203 drell_IIA101 drell_IIA102 drell_IIA103 drell_IA104 drell_IIA106 ggall_IIA101

rnolV_IV04 rnolV_IV06 rnolV_IV07 rnolV_IV09 rnolV_IV10 rnolV_IV11 athIV_IV02

drell_IIC07 drell_IIC08

ggalV_IV01 ggalV_IV02 hsalV_IV01 ggalV_IV10 ggalV_IV12 dmel_IB201 hsalV_IV12 ページ 13 
strict rule

mmull_IIB04

mmulV_IV08 athlIIIBO5 athII_IIB06 athII_IIB07 hsaV_V101 ggalV_IV06 drell_IIC01

athIII_IIIA10 athIII_IIIA11 osallI_IIIA02 osallIIIIA03 osallI_IIIA04 osallIIIIA05 osallI_IIIA08 
strict rule

ページ 15 
strict rule

ggall_IIA102 mmul_IB201 mmul_IB202 ggal_IB201 ggal_IB202 hsal_IB201 hsal_IB202

osalV_IV07 mmulV_IV03 mmulV_IV05 mmulV_IV06 mmulV_IV09 mmulV_IV10 ggalV_IV04

hsalV_IV13 hsalV_IV14 
strict rule

drell_IIC02 drell_IIC03 drell_IIC04 hsalV_IV07 drell_IIC05 hsalV_IV08 drell_IIC06 osallI_IIIA10 
strict rule

ページ 18 
strict rule

hsal_IB203 mmull_IIA101 mmull_IIA102 mmulI_IIA103

ggalV_IV05 ggalV_IV07 ggalV_IV08 ggalV_IV09 hsalV_IV03 hsalV_IV04 hsalV_IV05 
strict rule

cneV_V101 
strict rule

ページ 21 
strict rule

hsalV_IV06 hsalV_IV09 hsalV_IV10 hsalV_IV11 